\title{
Guarantee Testing Results from the Greenidge Multi-Pollutant Control Project
}

Topical Report of Work Performed

March 28, 2007 - May 4, 2007

Daniel P. Connell

James E. Locke

March 2008

U.S. Department of Energy Cooperative Agreement

DE-FC26-06NT41426

Submitted By

CONSOL Energy Inc.

Research \& Development

4000 Brownsville Road

South Park, PA 15129 


\section{DISCLAIMER}

This report was prepared as an account of work sponsored by an agency of the United States Government. Neither the United States Government nor any agency thereof, nor any of their employees, makes any warranty, express or implied, or assumes any legal liability or responsibility for the accuracy, completeness, or usefulness of any information, apparatus, product, or process disclosed, or represents that its use would not infringe privately owned rights. Reference herein to any specific commercial product, process, or service by trade name, trademark, manufacturer, or otherwise does not necessarily constitute or imply its endorsement, recommendation, or favoring by the United States Government or any agency thereof. The views and opinions of authors expressed herein do not necessarily state or reflect those of the United States Government or any agency thereof. 


\section{GUARANTEE TEST REPORT \\ MULTI-POLLUTANT CONTROL PROJECT \\ AES GREENIDGE UNIT 4 \\ DRESDEN, NEW YORK}

\section{Oxides of Nitrogen, Ammonia, Sulfur Dioxide, Sulfur Trioxide, Mercury, Hydrogen Chloride, and Hydrogen Fluoride Measurements}

March 28 - May 4, 2007

\section{LONSOLENERGY}

\begin{tabular}{|c|c|c|}
\hline $\begin{array}{l}\text { Source(s) } \\
\text { Owner } \\
\text { Address }\end{array}$ & & $\begin{array}{l}\text { Unit } 4 \text { (Boiler 6) } \\
\text { AES Greenidge } \\
590 \text { Plant Road } \\
\text { Dresden, NY } 14441\end{array}$ \\
\hline Test Company & & $\begin{array}{l}\text { CONSOL Energy Inc. } \\
\text { Research \& Development } \\
4000 \text { Brownsville Road } \\
\text { South Park, PA 15129-9566 }\end{array}$ \\
\hline Principal Investigator & & $\begin{array}{l}\text { Daniel P. Connell } \\
412-854-6559 \text { (phone) } \\
\text { danielconnell@consolenergy.com (e-mail) }\end{array}$ \\
\hline Sampling Team Leader & & $\begin{array}{l}\text { James E. Locke } \\
412-854-6607 \text { (phone) } \\
\text { imlocke@consolenergy.com }\end{array}$ \\
\hline Contract Coordinator & & $\begin{array}{l}\text { Robert G. Munro } \\
412-854-6620 \text { (phone) } \\
412-854-6613 \text { (fax) } \\
\text { bobmunro@consolenergy.com (e-mail) }\end{array}$ \\
\hline Repo & & March 26, 2008 (Revision 02) \\
\hline
\end{tabular}




\section{TABLE OF CONTENTS}

\section{SECTION}

PAGE

SUMMARY

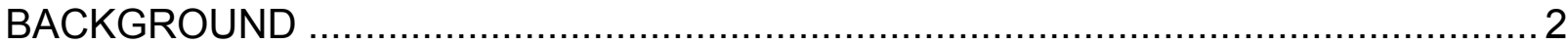

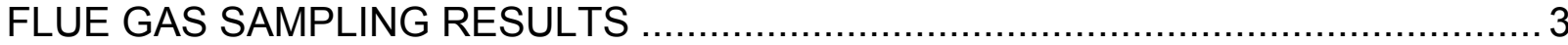

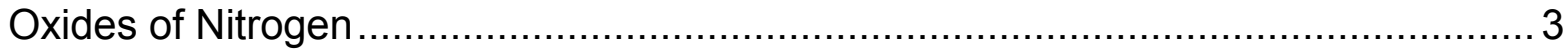

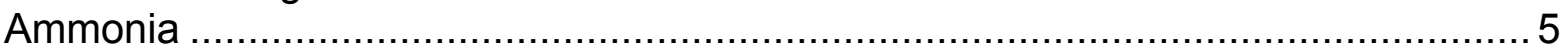

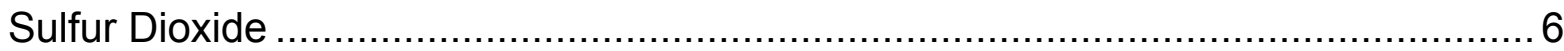

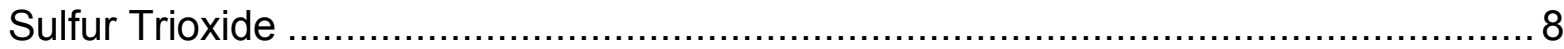

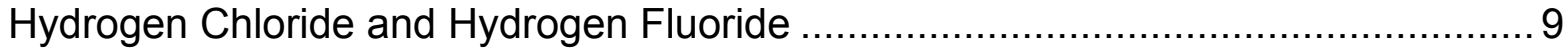

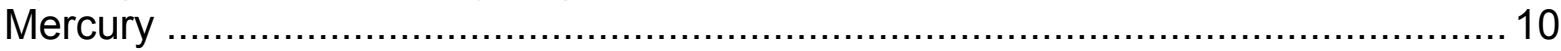

SOLID AND LIQUID PROCESS SAMPLE ANALYSIS RESULTS …..................... 13

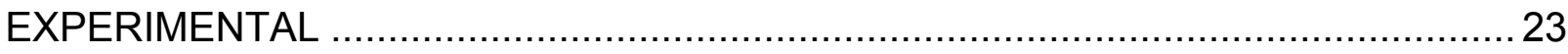

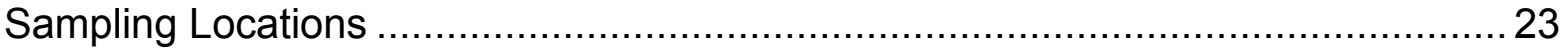

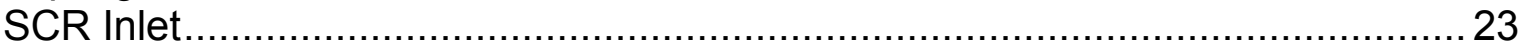

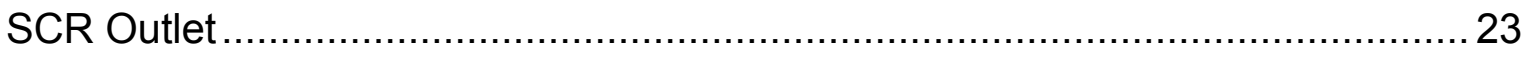

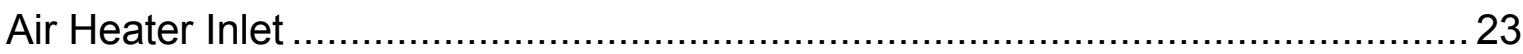

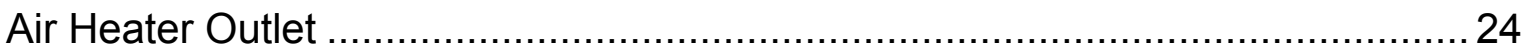

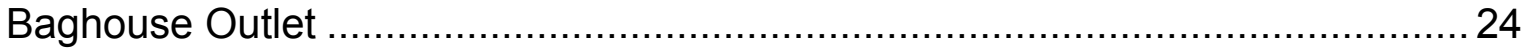

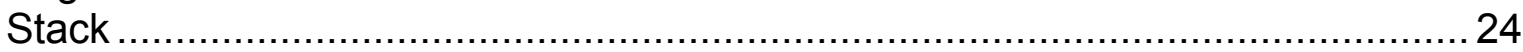

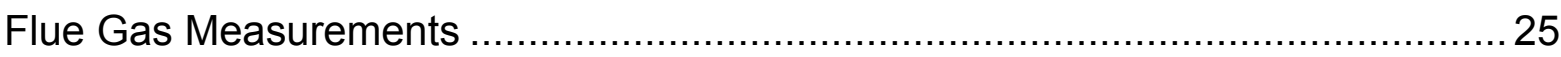

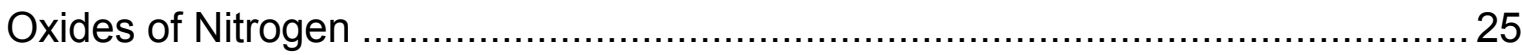

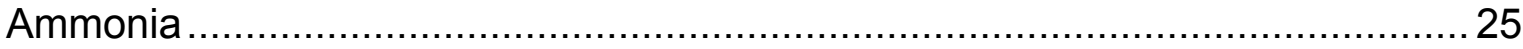

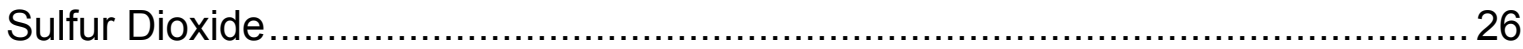

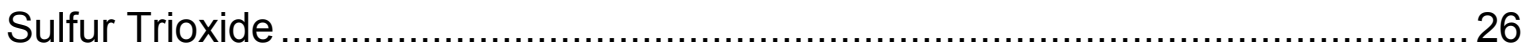

Hydrogen Chloride and Hydrogen Fluoride .............................................. 27

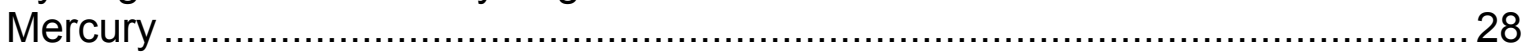

Solid and Liquid Process Sample Analyses .....................................................2 29

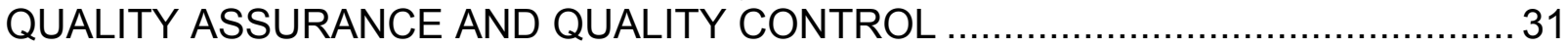

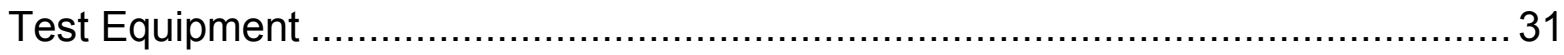

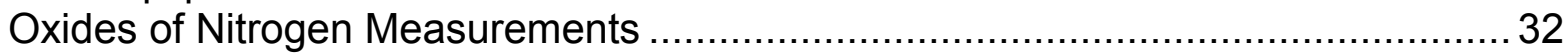

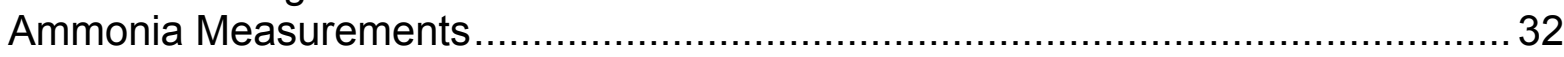

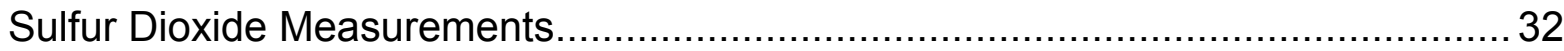

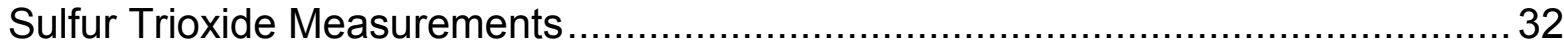

Hydrogen Chloride and Hydrogen Fluoride Measurements.................................. 32

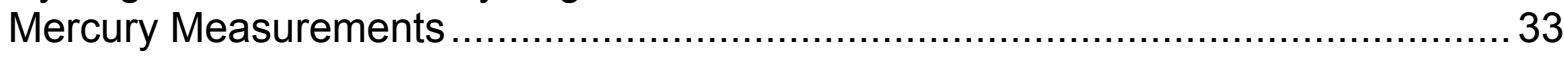

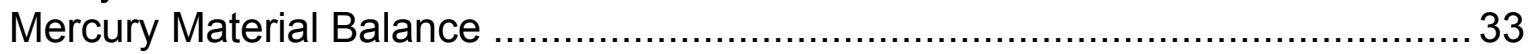

\section{TABLES}

Table 1. Performance Guarantee Results (March Testing) ..................................

Table 2. Performance Guarantee Results (May Testing) ..................................... 2

Table 3. Summary of $\mathrm{NO}_{x}$ Emission Rates Measured by the Unit 4 Stack CEM ..... 
Table 4. Summary of $\mathrm{NO}_{x}$ Measurements at the SCR Inlet and Outlet ...................5

Table 5. $\quad \mathrm{NH}_{3}$ Concentrations Measured at the SCR Outlet / Air Heater Inlet ............6

Table 6. Summary of $\mathrm{SO}_{2}$ Measurements at the Air Heater Outlet and Baghouse Outlet.

Table 7. Summary of $\mathrm{SO}_{2}$ Emission Rates Measured by the Unit 4 Stack CEM and $\mathrm{SO}_{2}$ Removal Efficiencies from the Air Heater Outlet to the Stack

Table 8. Gas Phase $\mathrm{SO}_{3}$ Concentrations at the Air Heater Outlet and Stack............9

Table 9. $\mathrm{HCl}$ and $\mathrm{HF}$ Concentrations at the Air Heater Outlet and Stack 10

Table 10. Summary of Mercury Measurements at the Air Heater Outlet and Stack - Testing Without Activated Carbon Injection

Table 11. Summary of Mercury Measurements at the Air Heater Outlet and Stack - Testing With Activated Carbon Injection ..................................12

Table 12. Summary of Coal-to-Stack Mercury Removal ..........................................13

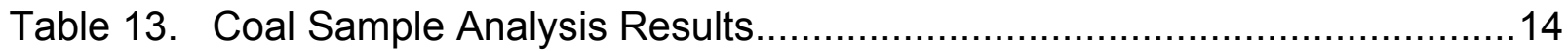

Table 14. Air Heater Outlet Fly Ash Sample Analysis Results .............................16

Table 15. Turbosorp ${ }^{\circledR}$ Hopper Ash Sample Analysis Results ................................17

Table 16. Turbosorp ${ }^{\circledR} /$ Baghouse Product Ash Sample Analysis Results .................18

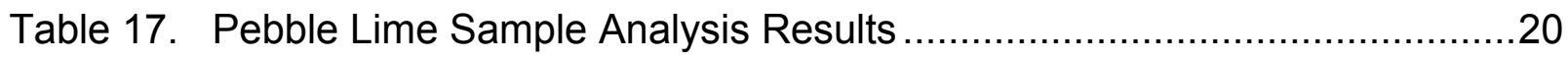

Table 18. Hydrated Lime Sample Analysis Results...........................................21

Table 19. Activated Carbon Sample Analysis Results ........................................22

Table 20. Urea Sample Analysis Results ...................................................22

Table 21. Summary of Material Balance Closure for $\mathrm{Hg}$ Measurements

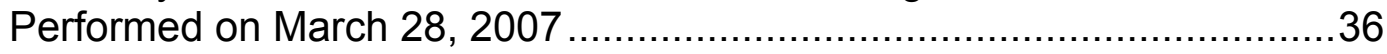

Table 22. Summary of Material Balance Closure for $\mathrm{Hg}$ Measurements Performed on March 30, 2007 36

Table 23. Comparison of $\mathrm{Hg}$ Mass Flow Rates in the Coal and at the Air Heater Outlet for $\mathrm{Hg}$ Measurements Performed on March 28, 2007

Table 24. Comparison of $\mathrm{Hg}$ Mass Flow Rates in the Coal and at the Air Heater Outlet for $\mathrm{Hg}$ Measurements Performed on March 30, 2007

\section{FIGURES}

Figure 1. Sampling locations 
Figure 2. SCR Inlet Sampling Location with Clean Air Engineering's $\mathrm{NO}_{\mathrm{x}}$ Multi-Point Automated Sampling System ..............................................39

Figure 3. SCR Outlet Sampling Location ........................................................... 40

Figure 4. Air Heater Inlet Sampling Location .................................................. 41

Figure 5. Air Heater Outlet Sampling Location ................................................42

Figure 6. Baghouse Outlet Sampling Location .................................................. 43

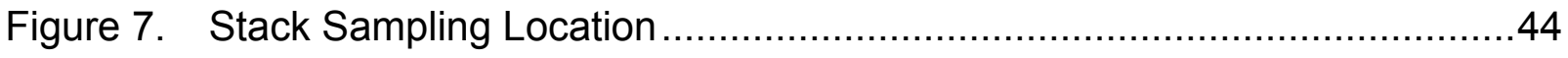

Figure 8. Stack Sampling Dimensions and Traverse Points..................................45

\section{APPENDICES}

APPENDIX A Clean Air Engineering Report $\left(\mathrm{NO}_{x} \& \mathrm{SO}_{2}\right.$ Sampling)

APPENDIX B CTL Group Report (Process Sample Analyses)

APPENDIX C Field Data Sheets

APPENDIX D Laboratory Analyses

APPENDIXE Unit 4 Stack CEM Data 


\section{ACRONYMS AND ABBREVIATIONS}

\begin{tabular}{|c|c|}
\hline $\mathrm{ACl}$ & activated carbon injection \\
\hline ASTM & American Society for Testing and Materials \\
\hline $\mathrm{BaCl}_{2}$ & barium chloride \\
\hline BPEI & Babcock Power Environmental Inc. \\
\hline CAE & Clean Air Engineering \\
\hline CEM & continuous emission monitor \\
\hline $\mathrm{Cl}^{-}$ & chloride \\
\hline $\mathrm{cm}^{3}$ & cubic centimeter \\
\hline $\mathrm{CO}_{2}$ & carbon dioxide \\
\hline CONSOL R\&D & CONSOL Energy Inc., Research \& Development \\
\hline CTM & Conditional Test Method \\
\hline CVAA & cold vapor atomic absorption spectroscopy \\
\hline DOE & U.S. Department of Energy \\
\hline dscf & dry standard cubic feet \\
\hline $\begin{array}{l}\text { dscm } \\
\text { EPA }\end{array}$ & dry standard cubic meter \\
\hline $\begin{array}{l}\text { EPA } \\
\text { EPC }\end{array}$ & U.S. Environmental Protection Agency \\
\hline $\begin{array}{l}\text { EPC } \\
\mathrm{F}^{-}\end{array}$ & $\begin{array}{l}\text { engineering, procurement, and construction } \\
\text { fluoride }\end{array}$ \\
\hline $\mathrm{ft}^{3}$ & cubic feet \\
\hline g & grams \\
\hline gr & grains \\
\hline $\mathrm{h}$ & hour \\
\hline $\mathrm{HCl}$ & hydrogen chloride \\
\hline $\mathrm{HF}$ & hydrogen fluoride \\
\hline $\mathrm{Hg}$ & mercury \\
\hline $\mathrm{Hg}^{\text {part }}$ & mercury in particulate form \\
\hline $\mathrm{Hg}^{\text {total }}$ & total mercury in particulate, oxidized, and elemental forms \\
\hline $\mathrm{Hg}^{++}$ & mercury in oxidized form \\
\hline $\mathrm{Hg}^{\circ}$ & mercury in elemental form \\
\hline HHV & higher heating value \\
\hline $\mathrm{HNO}_{3}$ & nitric acid \\
\hline $\mathrm{H}_{2} \mathrm{O}_{2}$ & hydrogen peroxide \\
\hline $\begin{array}{l}\mathrm{H}_{2} \mathrm{SO}_{4} \\
\mathrm{IC}\end{array}$ & sulfuric acid \\
\hline ICP-AES & $\begin{array}{l}\text { Ion chromatograpny } \\
\text { inductively coupled plasma-atomic emission spectrometry }\end{array}$ \\
\hline ICPMS & inductively coupled plasma mass spectrometry \\
\hline ICV & independent calibration verification \\
\hline IPA & isopropyl alcohol \\
\hline $\mathrm{KCl}$ & potassium chloride \\
\hline $\mathrm{KMnO}_{4}$ & potassium permanganate \\
\hline $\mathrm{L}$ & liter \\
\hline lb & pound \\
\hline $\begin{array}{l}M \\
m^{2}\end{array}$ & molar \\
\hline & square mete \\
\hline
\end{tabular}




\section{ACRONYMS AND ABBREVIATIONS}

$\begin{array}{ll}\text { MASS } & \text { Multipoint Automated Sampling System } \\ \mathrm{mg} & \text { milligram } \\ \mathrm{mL} & \text { milliliter } \\ \mathrm{mmBtu} & \text { million British Thermal Units } \\ \mathrm{MW} & \text { megawatt } \\ \mathrm{N} & \text { normal } \\ \mathrm{NH}_{3} & \text { ammonia } \\ \mathrm{NIST} & \text { National Institute of Standards and Technology } \\ \mathrm{NO}_{x} & \text { oxides of nitrogen } \\ \mathrm{O}_{2} & \text { oxygen } \\ \mathrm{pH} & \text { measurement of acidity or alkalinity of a solution } \\ \mathrm{ppm} & \text { parts per million } \\ \mathrm{ppmvd} & \text { parts per million by volume dry } \\ \mathrm{QA} / \mathrm{QC} & \text { quality assurance/quality control } \\ \mathrm{R}^{2} & \text { coefficient of determination } \\ \mathrm{SCR} & \text { selective catalytic reduction } \\ \mathrm{SM} & \text { Standard Method } \\ \mathrm{SNCR} & \text { selective non-catalytic reduction } \\ \mathrm{SO} \mathrm{F}_{2} & \text { sulfur dioxide } \\ \mathrm{SO} & \text { sulfur trioxide } \\ \mathrm{SRM} & \text { Standard Reference Material } \\ \mathrm{TBtu} & \text { trillion British Thermal Units } \\ \mathrm{TSS} & \text { total suspended solids } \\ \mathrm{U} . \mathrm{S} . & \text { United States } \\ \mu \mathrm{g} & \text { microgram }\end{array}$




\section{SUMMARY}

CONSOL Energy Inc. Research \& Development (CONSOL R\&D) performed flue gas sampling at AES Greenidge to verify the performance of the multi-pollutant control system recently installed by Babcock Power Environmental Inc. (BPEI) on the 107megawatt (MW) Unit 4 (Boiler 6). The multi-pollutant control system includes combustion modifications and a hybrid selective non-catalytic reduction (SNCR) / induct selective catalytic reduction (SCR) system to reduce $\mathrm{NO}_{x}$ emissions, followed by a Turbosorp ${ }^{\circledR}$ circulating fluidized bed dry scrubber system and baghouse to reduce emissions of $\mathrm{SO}_{2}, \mathrm{SO}_{3}, \mathrm{HCl}, \mathrm{HF}$, and particulate matter. Mercury removal is provided via the co-benefits afforded by the in-duct SCR, dry scrubber, and baghouse and by injection of activated carbon upstream of the scrubber, as required. Testing was conducted through ports located at the inlet and outlet of the SCR reactor to evaluate the performance of the hybrid $\mathrm{NO}_{x}$ control system, as well as through ports located at the air heater outlet and baghouse outlet or stack to determine pollutant removal efficiencies across the Turbosorp ${ }^{\circledR}$ scrubber and baghouse. Data from the unit's stack continuous emission monitor (CEM) were also used for determining attainment of the performance targets for $\mathrm{NO}_{\mathrm{x}}$ emissions and $\mathrm{SO}_{2}$ removal efficiency. The testing performed, by location, was:

SCR Inlet / SCR Outlet / Stack CEM:

Oxides of nitrogen

SCR Outlet / Air Heater Inlet:

Ammonia

\section{Air Heater Outlet / Baghouse Outlet / Stack CEM: \\ Sulfur dioxide}

Air Heater Outlet / Stack:

Acid gases $\left(\mathrm{SO}_{3}, \mathrm{HCl}\right.$, and $\left.\mathrm{HF}\right)$

Mercury

Testing was initially conducted on March 28,29 , and 30,2007 . During this period, Clean Air Engineering (CAE) sampled for $\mathrm{NO}_{x}$ and $\mathrm{SO}_{2}$, and CONSOL R\&D sampled for all of the other parameters listed above. Three separate test runs were completed for each parameter while the boiler was operating at or near design load and firing an approximately $2.5 \%$-sulfur eastern U.S. bituminous coal. Mercury removal was measured both with and without activated carbon injection (ACl). Results of these tests, which were performed in accordance with the methods specified in BPEl's Acceptance Test Procedure (Document 100276-100103100-01, dated March 22, 2007), are summarized in Table 1 below; the reported results represent the average of the three test runs (unless otherwise noted). 
The results for ammonia slip, $\mathrm{SO}_{3}$ removal, and $\mathrm{HF}$ removal obtained during the March tests were inconsistent with the performance targets and/or inconclusive. Hence, CONSOL R\&D repeated the tests for these parameters (as well as for $\mathrm{HCl}$, for which only two valid test runs were completed in March) on May 1, 2, and 4, 2007. The sampling and/or analytical methods for determining $\mathrm{NH}_{3}$ and $\mathrm{SO}_{3}$ were modified to improve the accuracy and representativeness of the measurements. Results of the May tests are summarized in Table 2. During these tests, the boiler fired an approximately 3.0\%-sulfur eastern U.S. bituminous coal, and it was again operated at or near design load.

Table 1. Performance Guarantee Results (March Testing)

\begin{tabular}{|c|c|c|}
\hline Performance Category & Performance Target & Measured Result \\
\hline SCR Outlet NOx & $\leq 0.10 \mathrm{lb} / \mathrm{mmBtu}$ & $0.095 \mathrm{lb} / \mathrm{mmBtu}^{\mathrm{a}}$ \\
\hline Ammonia Slip @ SCR Outlet & $\leq 2 \mathrm{ppmvd} @ 3 \% \mathrm{O}_{2}$ & 9.9 ppmvd @ 3\% O \\
\hline $\mathrm{SO}_{2}$ Removal Efficiency & $\geq 95 \%$ removal & $96.0 \%$ removal $^{b}$ \\
\hline Acid Gas Removal & $\geq 95 \%$ removal & $\begin{array}{c}\text { Invalid }\left(64.4 \% \text { removal }^{\mathrm{c}}\right. \\
94.8 \% \text { removal }^{\mathrm{d}} \\
\text { Indeterminate }^{\mathrm{e}}\end{array}$ \\
\hline $\begin{array}{r}\text { Mercury Reduction (coal-to-stack) } \\
\text { Without } \mathrm{ACl} \\
\text { With } \mathrm{ACl}\end{array}$ & $\geq 90 \%$ removal & $\begin{array}{l}>95.3 \% \text { removal } \\
>93.8 \% \text { removal }\end{array}$ \\
\hline
\end{tabular}

${ }^{a}$ Measured by the unit's stack CEM. ${ }^{b}$ Based on inlet $\mathrm{SO}_{2}$ measured at the air heater outlet by CAE and outlet $\mathrm{SO}_{2}$ measured by the unit's stack CEM. 'Measurement considered to be invalid because of possible ammonia interference. ${ }^{\mathrm{d}}$ Average of two test runs. ${ }^{\mathrm{e}} \mathrm{HF}$ concentrations were below the limit of detection at both the air heater outlet and stack, making it impossible to calculate a removal percentage.

Table 2. Performance Guarantee Results (May Testing)

\begin{tabular}{|c|c|c|}
\hline Performance Category & Performance Target & Measured Result \\
\hline Ammonia Slip @ Air Heater Inlet & $\leq 2 p p m v d @ 3 \% \mathrm{O}_{2}$ & 4.2 ppmvd @ 3\% $\mathrm{O}_{2}{ }^{\mathrm{a}}$ \\
\hline Acid Gas Removal & $\geq 95 \%$ removal & $\begin{array}{l}97.1 \% \text { removal }^{b} \\
97.2 \% \text { removal }^{\mathrm{b}} \\
\text { Indeterminate }^{\mathrm{c}, \mathrm{d}}\end{array}$ \\
\hline
\end{tabular}

${ }^{a}$ Average of four measurements at the air heater inlet. The average concentration measured simultaneously at the eastern side of the SCR outlet was 11.3 ppmvd @ $3 \% \mathrm{O}_{2}$. ${ }^{\mathrm{b}}$ Average of three measurements. ${ }^{\mathrm{C}}$ Average of two measurements. ${ }^{\mathrm{d}} \mathrm{HF}$ concentrations were below the limit of detection at both the air heater outlet and stack, making it impossible to calculate a removal percentage.

\section{BACKGROUND}

CONSOL Energy Inc. Research \& Development, AES Greenidge LLC, and Babcock Power Environmental Inc. (BPEI) were awarded a cooperative agreement from the U.S. Department of Energy (DOE) to install and test an integrated multi-pollutant control system on the 107-MW AES Greenidge Unit 4. The project seeks to be the first to demonstrate: 
- Full-load $\mathrm{NO}_{x}$ emissions of $\leq 0.10 \mathrm{lb} / \mathrm{mmBtu}$ using a hybrid selective non-catalytic reduction / selective catalytic reduction (hybrid SNCR/SCR) system, in combination with low- $\mathrm{NO}_{x}$ combustion technology, on a unit firing coal and biomass

- $\mathrm{SO}_{2}$ removal of $\geq 95 \%$ using a Turbosorp ${ }^{\circledR}$ circulating fluidized bed dry scrubber (including a new baghouse) on a unit firing greater than $2 \%$-sulfur bituminous coal

- Mercury reduction of $\geq 90 \%$ via the co-benefits afforded by the in-duct SCR, Turbosorp ${ }^{\circledR}$ scrubber, and baghouse, and by the addition of activated carbon into the Turbosorp ${ }^{\circledR}$ system, as required

- Acid gas $\left(\mathrm{SO}_{3}, \mathrm{HCl}, \mathrm{HF}\right)$ removal of $\geq 95 \%$ in the Turbosorp ${ }^{\circledR}$ system

The goal of the project is to demonstrate substantial improvements in mercury, $\mathrm{SO}_{3}$, and particulate matter control, and substantial reductions in the cost for $\mathrm{NO}_{\mathrm{x}}$ and $\mathrm{SO}_{2}$ control, compared to conventional technologies when applied to the large number of smaller coal-fired generating units in the United States.

CONSOL R\&D performed guarantee testing to verify the ability of the multi-pollutant control system to meet the performance targets set forth in the Engineering, Procurement, and Construction (EPC) agreement between AES Greenidge and Babcock Power Environmental Inc. Described herein are the results of the guarantee measurements and the methods used.

\section{FLUE GAS SAMPLING RESULTS}

Six locations, the SCR inlet, SCR outlet, air heater inlet, air heater outlet, baghouse outlet, and stack, were sampled while AES Greenidge Unit 4 was operating at or near design load ( 105 $\mathrm{MW}_{\text {gross }}$ or $\left.97 \mathrm{MW}_{\text {net }}\right)$ and firing an approximately 2.5-3.0\%-sulfur eastern U.S. bituminous coal. To determine the performance of the SNCR/SCR system, $\mathrm{NO}_{x}$ sampling was performed at the SCR inlet and SCR outlet locations, and ammonia sampling was performed at the SCR outlet and air heater inlet locations. The performance of the Turbosorp ${ }^{\circledR}$ system (including the baghouse) was determined by sampling for $\mathrm{SO}_{2}, \mathrm{SO}_{3}, \mathrm{HCl}, \mathrm{HF}$, and mercury at the air heater outlet and stack or baghouse outlet locations. Data from the unit's stack CEM were also used for determining attainment of the performance targets for $\mathrm{NO}_{x}$ emissions and $\mathrm{SO}_{2}$ removal efficiency. The sampling for ammonia at the SCR outlet and for mercury, $\mathrm{HCl}$, and $\mathrm{HF}$ was performed at an isokinetic sampling rate, and the sampling for ammonia at the air heater inlet and for $\mathrm{NO}_{\mathrm{x}}, \mathrm{SO}_{2}$, and $\mathrm{SO}_{3}$ was performed at a constant sampling rate. The measured results at each location are described below. The sampling and analytical methods used to perform the measurements are described in the Experimental section later in this report. All times reported herein are local (i.e., Eastern Daylight) times.

\section{Oxides of Nitrogen}

Attainment of the performance target for $\mathrm{NO}_{\mathrm{x}}$ was demonstrated by monitoring the $\mathrm{NO}_{\mathrm{x}}$ emission rates measured by the plant's stack continuous emission monitor during three 72-minute periods on March 28. These $\mathrm{NO}_{x}$ emission rates are summarized in Table 3. The one-minute CEM data from the test period, which were provided by AES 
and averaged by CONSOL R\&D to derive the values presented in Table 3 , are included in Appendix $E$ to this report.

Table 3. Summary of $\mathrm{NO}_{x}$ Emission Rates Measured by the Unit 4 Stack CEM

\begin{tabular}{||r|r|r||}
\hline Test No. & $\begin{array}{c}\text { Date \& Time } \\
\text { (MM/DD/YY: hhmm-hhmm) }\end{array}$ & $\begin{array}{c}\text { NO }_{\mathbf{x}} \text { Emissions @ Stack, } \\
\text { Ib/mmBtu }^{\mathbf{a}}\end{array}$ \\
\hline \hline Test \#1 & 03/28/07: 0912-1023 & 0.096 \\
\hline Test \#2 & 03/28/07: 1112-1223 & 0.095 \\
\hline Test \#3 & 03/28/07: 1248-1359 & 0.095 \\
\hline \multicolumn{2}{|r|}{ Average } & 0.095 \\
\hline
\end{tabular}

${ }^{a}$ Calculated by CONSOL R\&D as the arithmetic mean of 1-minute CEM data provided by AES.

To supplement the $\mathrm{NO}_{x}$ measurements made by the unit's CEM, Clean Air Engineering (CAE) simultaneously performed $\mathrm{NO}_{x}$ testing using their Multipoint Automated Sampling System (MASS) at the SCR inlet and outlet sampling grids. Each 72-minute test included three complete traverses of each grid; both grids were sampled at the same time. Table 4 summarizes the average $\mathrm{NO}_{x}$ concentrations measured at the SCR inlet and outlet, the average $\mathrm{NO}_{x}$ removal efficiency measured across the SCR catalyst, and the $\mathrm{NO}_{x}$ emission rate measured at the SCR outlet during each of the three $\mathrm{NO}_{x}$ testing periods on March 28. ( $\mathrm{NO}_{x}$ concentrations were converted to a standard oxygen basis of $3 \%$ using $\mathrm{O}_{2}$ concentrations measured by CAE at the SCR inlet and outlet grids using EPA Method 3A. NO $\mathrm{NO}_{x}$ emission rates at the SCR outlet were computed by CONSOL R\&D using $\mathrm{NO}_{x}$ and $\mathrm{CO}_{2}$ concentrations measured by CAE at the SCR outlet grid and assuming a $\mathrm{CO}_{2}$-based fuel factor of $\mathrm{F}_{\mathrm{c}}=$ $1800 \mathrm{scf} / \mathrm{mmBtu}$, per EPA Method 19 guidelines for bituminous coal). A more detailed presentation of the results of $\mathrm{NO}_{x}$ sampling at the SCR inlet and outlet is provided in the CAE report that is included as Appendix $A$ to this report.

The average $\mathrm{NO}_{x}$ concentration measured by CAE at the SCR outlet was about $25 \%$ less than the average $\mathrm{NO}_{x}$ concentration measured by the plant's stack CEM during the three $\mathrm{NO}_{x}$ measurement periods. The cause of this disparity is unknown. Possible causes include measurement error (although both the stack CEM and CAE $\mathrm{NO}_{x}$ analyzer were calibrated prior to the tests) or flow stratification at the SCR outlet. (The $\mathrm{NO}_{x}$ emission rate at the SCR outlet is calculated from the unweighted average of the $\mathrm{NO}_{x}$ concentrations measured at the $24 \mathrm{SCR}$ outlet grid points. This method assumes that the volumetric flow rates at all of these points are equal. If this assumption is violated, then the $\mathrm{NO}_{\mathrm{x}}$ emission rate measured at the SCR outlet could differ from that at the stack. Flow rate measurements were not available at the SCR outlet to confirm or refute this assumption). Because the unit's permit limit for $\mathrm{NO}_{x}$ and the controls for the hybrid SNCR/SCR system are both based on the $\mathrm{NO}_{x}$ emission rate measured by the stack CEM, this emission rate was used to determine attainment of the performance target for $\mathrm{NO}_{x}$ emissions. 
Table 4. Summary of $\mathrm{NO}_{x}$ Measurements at the SCR Inlet and Outlet

\begin{tabular}{|c|c|c|c|c|c|}
\hline \multirow[b]{2}{*}{ Test No. } & \multirow{2}{*}{$\begin{array}{c}\text { Date \& Time } \\
\text { (MM/DD/YY: hhmm-hhmm) }\end{array}$} & \multicolumn{3}{|c|}{ SCR NO ${ }_{x}$ Data @ 3\% $\mathrm{O}_{2}$} & \multirow{2}{*}{$\begin{array}{l}\text { SCR Outlet } \\
\text { NO }_{x} \\
\text { Emissions, }^{\text {bmission }}\end{array}$} \\
\hline & & $\begin{array}{l}\text { SCR Inlet, } \\
\text { ppmvd }\end{array}$ & $\begin{array}{l}\text { SCR Outlet, } \\
\text { ppmvd }\end{array}$ & $\underset{\%^{\mathrm{a}}}{\text { Removal, }}$ & \\
\hline Test \#1 & 03/28/07: 0912-1023 & 92.6 & 52.7 & 43.1 & 0.073 \\
\hline Test \#2 & 03/28/07: 1112-1223 & 86.3 & 52.7 & 38.9 & 0.072 \\
\hline Test \#3 & 03/28/07: 1248-1359 & 84.6 & 50.1 & 40.8 & 0.069 \\
\hline & Average & 87.8 & 51.8 & 41.0 & 0.071 \\
\hline
\end{tabular}

${ }^{a}$ Percent removal calculated by CONSOL R\&D based on overall average inlet and outlet concentrations reported by CAE for each test. ${ }^{b}$ Emission rate calculated by CONSOL R\&D based on the average $\mathrm{NO}_{\mathrm{x}}$ and $\mathrm{CO}_{2}$ concentrations at the SCR outlet reported by $\mathrm{CAE}$ for each test, and assuming a $\mathrm{CO}_{2}$-based fuel factor of $F_{c}=1800 \mathrm{scf} / \mathrm{mmBtu}$.

\section{Ammonia}

Ammonia testing was conducted on March 28 and May 1, 2007. On March 28, ammonia sampling was performed at the SCR outlet through the northernmost of the two ports located on the eastern wall of the SCR outlet duct at the 498' level. On May 1 , ammonia sampling was performed at the SCR outlet through both ports on the eastern wall of the SCR outlet duct (reported results are for composite samples drawn from both ports), and additional ammonia sampling was performed at the air heater inlet through a single port located on the south side of each of the eastern and western air heater inlet ducts (reported results are for composite samples drawn from both ports). Three approximately one-hour-long test runs were performed at the SCR outlet on March 28, and four approximately one-hour-long test runs were performed at the SCR outlet and air heater inlet (both locations sampled simultaneously) on May 1. The ammonia concentrations measured during these tests are reported in Table 5. $\left(\mathrm{NH}_{3}\right.$ concentrations were converted to a standard oxygen basis of $3 \%$ using $\mathrm{O}_{2}$ concentrations measured by CONSOL R\&D at the sampling train exhaust using a Teledyne Max $\mathrm{V}$ portable electrochemical $\mathrm{O}_{2}$ analyzer). 
Table 5. $\quad \mathrm{NH}_{3}$ Concentrations Measured at the SCR Outlet / Air Heater Inlet

\begin{tabular}{|c|c|c|c|}
\hline \multirow{2}{*}{ Test No. } & \multirow{2}{*}{$\begin{array}{c}\text { Date \& Time } \\
\text { (Mm/DD/YY: hhmm-hhmm) }\end{array}$} & \multicolumn{2}{|c|}{$\begin{array}{l}\mathrm{NH}_{3} \text { Concentration, } \\
\text { ppmvd @ } 3 \% \mathrm{O}_{2}\end{array}$} \\
\hline & & $\begin{array}{l}\text { SCR } \\
\text { Outlet }\end{array}$ & $\begin{array}{c}\text { Air Heater } \\
\text { Inlet }\end{array}$ \\
\hline Test \#1 & 03/28/07: 0914-1014 & 4.6 & \\
\hline Test \#2 & 03/28/07: 1115-1215 & 11.4 & \\
\hline Test \#3 & 03/28/07: $1300-1400$ & 13.5 & \\
\hline \multicolumn{2}{|r|}{ Average } & 9.9 & \\
\hline Test \#1 & 05/01/07: 0903-1006 & 11.6 & 3.6 \\
\hline Test \#2 & 05/01/07: 1121-1235 & 9.7 & 5.1 \\
\hline Test \#3 & 05/01/07: 1335-1438 & 14.3 & 4.0 \\
\hline Test \#4 & 05/01/07: 1530-1635 & 9.8 & 4.2 \\
\hline \multicolumn{2}{|r|}{ Average } & 11.3 & 4.2 \\
\hline
\end{tabular}

\section{Sulfur Dioxide}

Sulfur dioxide removal efficiency testing was conducted on March 29. Three approximately 60-minute-long tests were performed. During each test, $\mathrm{SO}_{2}$ concentrations at the air heater outlet and baghouse outlet were measured continuously by $\mathrm{CAE}$, and $\mathrm{SO}_{2}$ concentrations at the stack were measured continuously by the plant's stack CEM.

Table 6 summarizes the average $\mathrm{SO}_{2}$ concentrations measured during these tests by $\mathrm{CAE}$ at the air heater outlet and baghouse outlet. $\left(\mathrm{SO}_{2}\right.$ concentrations were converted to a standard oxygen basis of $3 \%$ using $\mathrm{O}_{2}$ concentrations measured by CAE at the air heater outlet and baghouse outlet using EPA Method 3A). During the first two tests, at each location, flue gas was sampled from a single point located approximately in the center of the duct. During the third test, sampling was conducted using a three-point traverse through a single port at each location. A more detailed presentation of the $\mathrm{SO}_{2}$ sampling results from the air heater outlet and baghouse outlet is provided in the CAE report that is included as Appendix A to this report.

Table 7 summarizes the average $\mathrm{SO}_{2}$ emission rates measured by the unit's stack CEM during each test, as well as the average $\mathrm{SO}_{2}$ removal efficiency across the Turbosorp ${ }^{\circledR}$ system (including the baghouse) calculated using the $\mathrm{SO}_{2}$ measured at the air heater outlet by CAE and the $\mathrm{SO}_{2}$ measured at the stack by the unit's CEM. (For purposes of this calculation, the $\mathrm{SO}_{2}$ concentrations measured by CAE at the air heater outlet were converted to emission rates in units of $\mathrm{lb} / \mathrm{mmBtu}$ based on the $\mathrm{CO}_{2}$ 
concentrations measured by CAE at the air heater outlet using EPA Method $3 \mathrm{~A}$ and assuming a $\mathrm{CO}_{2}$-based fuel factor of $\mathrm{F}_{\mathrm{c}}=1800 \mathrm{scf} / \mathrm{mmBtu}$, per EPA Method 19 guidelines for bituminous coal). The one-minute stack CEM data from the test period, which were provided by AES and averaged by CONSOL to derive the values presented in Table 7, are included in Appendix E to this report.

Table 6. Summary of $\mathrm{SO}_{2}$ Measurements at the Air Heater Outlet and Baghouse Outlet

\begin{tabular}{|c|c|c|c|c|}
\hline \multirow{2}{*}{ Test No. } & \multirow{2}{*}{$\begin{array}{c}\text { Date \& Time } \\
\text { (MM/DD/YY: hhmm-hhmm) }\end{array}$} & \multicolumn{2}{|c|}{$\begin{array}{l}\mathrm{SO}_{2} \text { Concentration, } \\
\text { ppmvd @ } 3 \% \mathrm{O}_{2}\end{array}$} & \multirow{2}{*}{$\begin{array}{c}\text { Removal, } \\
\%\end{array}$} \\
\hline & & $\begin{array}{l}\text { Air Heater } \\
\text { Outlet }\end{array}$ & $\begin{array}{c}\text { Baghouse } \\
\text { Outlet }\end{array}$ & \\
\hline Test \#1 & 03/29/07: 0959-1100 & 1854.76 & 103.89 & 94.40 \\
\hline Test \#2 & 03/29/07: 1216-1317 & 1829.81 & 90.83 & 95.04 \\
\hline Test \#3 & 03/29/07: 1513-1613 & 1837.92 & 132.12 & 92.81 \\
\hline & Average & 1840.83 & 108.95 & 94.08 \\
\hline
\end{tabular}

Table 7. Summary of $\mathrm{SO}_{2}$ Emission Rates Measured by the Unit 4 Stack CEM and $\mathrm{SO}_{2}$ Removal Efficiencies from the Air Heater Outlet to the Stack

\begin{tabular}{||c|c|c|c|c||}
\hline \multirow{2}{*}{ Test No. } & $\begin{array}{c}\text { Date \& Time } \\
\text { (MMIDD/YY: hhmm-hhmm) }\end{array}$ & \multicolumn{2}{|c||}{$\begin{array}{c}\mathrm{SO}_{2} \text { Emission Rate, } \\
\text { Ib/mmBtu }\end{array}$} & \multirow{2}{*}{$\begin{array}{c}\text { Removal, } \\
\%\end{array}$} \\
\cline { 3 - 4 } & $\begin{array}{c}\text { Air Heater } \\
\text { Outlet }^{\mathrm{a}}\end{array}$ & $\begin{array}{c}\text { Stack } \\
\text { CEM }^{\mathrm{b}}\end{array}$ & \\
\hline \hline Test \#1 & 03/29/07: 0959-1100 & 3.650 & 0.137 & 96.2 \\
\hline Test \#2 & 03/29/07: 1216-1317 & 3.598 & 0.128 & 96.4 \\
\hline Test \#3 & 03/29/07: 1513-1613 & 3.583 & 0.173 & 95.2 \\
\hline & Average & 3.610 & 0.146 & 96.0 \\
\hline
\end{tabular}

${ }^{a}$ Calculated by CONSOL R\&D based on the average $\mathrm{SO}_{2}$ and $\mathrm{CO}_{2}$ concentrations at the air heater outlet reported by CAE for each test, and assuming a $\mathrm{CO}_{2}$-based fuel factor of $\mathrm{F}_{\mathrm{c}}=1800 \mathrm{scf} / \mathrm{mmBtu}$. ${ }^{\mathrm{b}}$ Calculated by CONSOL R\&D as the arithmetic mean of 1-minute CEM data provided by AES.

The three-test average $\mathrm{SO}_{2}$ removal efficiency of $96.0 \%$ calculated using the unit's stack CEM as the measure of $\mathrm{SO}_{2}$ emissions downstream of the baghouse is slightly greater than the three-test average removal efficiency of $94.1 \%$ calculated using the $\mathrm{CAE}$ measurements at the baghouse outlet as the measure of $\mathrm{SO}_{2}$ emissions. The cause of this discrepancy is unknown. However, because the unit's permit limit for 
$\mathrm{SO}_{2}$ and the controls for the Turbosorp ${ }^{\circledR}$ system are both based on the $\mathrm{SO}_{2}$ emission rate measured by the stack CEM, this emission rate was used to determine attainment of the performance target for $\mathrm{SO}_{2}$ emissions.

\section{Sulfur Trioxide}

Sulfur trioxide was sampled at the air heater outlet and stack to determine the removal efficiency across the Turbosorp ${ }^{\circledR}$ system (including the baghouse). Three test runs were performed on March 29 at the air heater outlet (40-minute tests) and stack (60minute tests). However, for all of these tests, in which $\mathrm{SO}_{3}$ concentrations were determined by controlled condensation with $\mathrm{BaCl}_{2}$ titration, results were likely biased by $\mathrm{NH}_{3}$ interfering with the titration endpoint, as discussed in EPA Method 8. The data presented in Table 5 show that ammonia was present downstream of the SCR reactor during the March testing period. Hence, the results from the March $29 \mathrm{SO}_{3}$ tests, which are shown in Table 8 below, are considered to be invalid. $\left(\mathrm{SO}_{3}\right.$ concentrations at the air heater outlet were converted to a standard oxygen basis of $3 \%$ using $\mathrm{O}_{2}$ concentrations measured by CONSOL R\&D at the sampling train exhaust using a Teledyne Max $\mathrm{V}$ portable electrochemical $\mathrm{O}_{2}$ analyzer. $\mathrm{SO}_{3}$ concentrations at the stack were converted to a standard oxygen basis of $3 \%$ using $\mathrm{O}_{2}$ concentrations calculated from the average $\mathrm{CO}_{2}$ concentration measured by the plant's stack CEM during each test run and the ultimate analysis of coal samples collected during the tests).

To obtain valid results for $\mathrm{SO}_{3}$ removal across the Turbosorp ${ }^{\circledR}$ system, CONSOL R\&D repeated the tests for $\mathrm{SO}_{3}$ at the air heater outlet and stack on May 2 using a modified procedure. To avoid possible $\mathrm{NH}_{3}$ interference, the controlled condensation samples were analyzed by ion chromatography (IC) rather than by $\mathrm{BaCl}_{2}$ titration. The use of IC was also expected to improve the sensitivity of the method for determining the lowlevel $\mathrm{SO}_{3}$ concentrations encountered at the stack. To further improve the sensitivity and representativeness of the measurements, the sampling durations were increased to 60 minutes at the air heater outlet and to 90 minutes at the stack, and traverses were performed at both locations (sampling in March was conducted at a single point at each location). The results of the three test runs completed on May 2 are summarized in Table $8 . \quad\left(\mathrm{SO}_{3}\right.$ concentrations at the stack were converted to a standard oxygen basis of $3 \%$ using $\mathrm{O}_{2}$ concentrations calculated from the average $\mathrm{CO}_{2}$ concentration measured by the plant's stack CEM during each test run and the ultimate analysis of coal samples collected during the tests. $\mathrm{O}_{2}$ concentrations measured by CONSOL at the air heater outlet were anomalously greater than $\mathrm{O}_{2}$ concentrations at the stack. This likely resulted from air in-leakage at the meterbox exhaust rather than from a leak in the sampling train, as pre- and post-test leak checks indicated no sampling train leaks. Hence, for the oxygen basis conversions, the $\mathrm{O}_{2}$ concentration at the air heater outlet was assumed to equal that at the stack). 
Table 8. Gas Phase $\mathrm{SO}_{3}$ Concentrations at the Air Heater Outlet and Stack

\begin{tabular}{|c|c|c|c|}
\hline \multirow[t]{2}{*}{ Test No. } & \multirow{2}{*}{$\begin{array}{c}\text { Date \& Time } \\
\text { (MM/DD/YY: hhmm-hhmm) }\end{array}$} & \multicolumn{2}{|c|}{$\begin{array}{l}\text { Gas Phase } \mathrm{SO}_{3} \\
\text { Concentration, } \\
\text { ppmvd @ } 3 \% \mathrm{O}_{2}\end{array}$} \\
\hline & & $\begin{array}{l}\text { Air Heater } \\
\text { Outlet }\end{array}$ & Stack \\
\hline Test \#1 & 03/29/07: 1000-1103 & $6.1^{a}$ & $2.4^{\mathrm{a}}$ \\
\hline Test \#2 & 03/29/07: 1220-1330 & $7.4^{\mathrm{a}}$ & $2.3^{\mathrm{a}}$ \\
\hline Test \#3 & 03/29/07: 1515-1617 & $6.2^{\mathrm{a}}$ & $2.3^{\mathrm{a}}$ \\
\hline & Average Concentration & Invalid & Invalid \\
\hline & Average Removal & \multicolumn{2}{|c|}{ Invalid } \\
\hline Test \#1 & 05/02/07: 1044-1215 & 25.5 & 0.8 \\
\hline Test \#2 & 05/02/07: 1312-1443 & 28.7 & 0.8 \\
\hline Test \#3 & 05/02/07: 1517-1648 & 25.4 & 0.7 \\
\hline & Average Concentration & 26.5 & 0.8 \\
\hline & Average Removal & \multicolumn{2}{|c|}{$97.1 \%$} \\
\hline
\end{tabular}

${ }^{a}$ Result considered to be invalid because of possible $\mathrm{NH}_{3}$ interference.

In addition to the $\mathrm{SO}_{3}$ measurements performed at the air heater outlet and stack, three $\mathrm{SO}_{3}$ tests including simultaneous sampling at the SCR inlet and SCR outlet were conducted on March 30 in an attempt to evaluate $\mathrm{SO}_{2}$-to- $\mathrm{SO}_{3}$ conversion across the SCR catalyst. These results are invalid because of certain ammonia interference, however, and therefore they are not included in this report.

\section{Hydrogen Chloride and Hydrogen Fluoride}

Hydrogen chloride and hydrogen fluoride were sampled at the air heater outlet and stack to determine the removal efficiency for these components across the Turbosorp ${ }^{\circledR}$ system (including the baghouse). Three approximately 60-minute-long tests including simultaneous sampling at these locations were completed on March 29. The samples from the first test, however, were contaminated by residual hydrochloric acid that had been used to rinse the probes during previous mercury testing. Hence, the $\mathrm{HCl}$ concentrations determined during the first test are invalid. Moreover, HF concentrations determined at both locations during each of the three tests on March 29 were less than the method detection limit, making it impossible to calculate a removal efficiency.

To confirm the results of the March 29 testing, additional $\mathrm{HCl}$ and $\mathrm{HF}$ measurements were performed on May 4. Two approximately 60-minute-long test runs including 
simultaneous sampling at the air heater outlet and stack were performed. (A third test was planned, but it could not be completed because of combustion problems that forced a drop in unit load). Again, HF concentrations were less than the method detection limit at both the air heater outlet and the stack during each test run, preventing the determination of a removal efficiency.

Table 9 summarizes the results of the $\mathrm{HCl}$ and $\mathrm{HF}$ tests performed on March 29 and May 4. $(\mathrm{HCl}$ and $\mathrm{HF}$ concentrations at the air heater outlet were converted to a standard oxygen basis of $3 \%$ using $\mathrm{O}_{2}$ concentrations measured by CONSOL at the sampling train exhaust using a Teledyne Max $\mathrm{V}$ portable electrochemical $\mathrm{O}_{2}$ analyzer. $\mathrm{HCl}$ and $\mathrm{HF}$ concentrations at the stack were converted to a standard oxygen basis of $3 \%$ using $\mathrm{O}_{2}$ concentrations calculated from the average $\mathrm{CO}_{2}$ concentration measured by the plant's stack CEM during each test run and the ultimate analysis of coal samples collected during the tests).

Table 9. $\mathrm{HCl}$ and $\mathrm{HF}$ Concentrations at the Air Heater Outlet and Stack

\begin{tabular}{|c|c|c|c|c|c|}
\hline \multirow{2}{*}{ Test No. } & \multirow{2}{*}{$\begin{array}{c}\text { Date \& Time } \\
\text { (MM/DD/YY: hhmm-hhmm) }\end{array}$} & \multicolumn{2}{|c|}{$\begin{array}{l}\mathrm{HCl} \text { Concentration, } \\
\text { ppmvd @ 3\% } \mathrm{O}_{2}\end{array}$} & \multicolumn{2}{|c|}{$\begin{array}{l}\text { HF Concentration, } \\
\text { ppmvd @ } 3 \% \mathrm{O}_{2}\end{array}$} \\
\hline & & $\begin{array}{l}\text { Air Heater } \\
\text { Outlet }\end{array}$ & Stack & $\begin{array}{c}\text { Air Heater } \\
\text { Outlet }\end{array}$ & Stack \\
\hline Test \#1 & 03/29/07: 1000-1125 & $216.1^{a}$ & $73.8^{\mathrm{a}}$ & $<0.34$ & $<0.20$ \\
\hline Test \#2 & 03/29/07: 1220-1344 & 36.0 & 2.8 & $<0.34$ & $<0.23$ \\
\hline Test \#3 & 03/29/07: 1515-1637 & 38.6 & 1.1 & $<0.35$ & $<0.21$ \\
\hline \multicolumn{2}{|r|}{ Average Concentration } & $37.3^{\mathrm{b}}$ & $1.9^{b}$ & $<0.34$ & $<0.21$ \\
\hline \multicolumn{2}{|r|}{ Average Removal } & \multicolumn{2}{|c|}{$94.8 \%^{\mathrm{b}}$} & \multicolumn{2}{|c|}{ Indeterminate } \\
\hline Test \#1 & 05/04/07: 0831-0940 & 40.4 & 1.3 & $<0.25$ & $<0.16$ \\
\hline Test \#2 & 05/04/07: 1015-1130 & 39.9 & 1.0 & $<0.21$ & $<0.16$ \\
\hline \multicolumn{2}{|r|}{ Average Concentration } & 40.1 & 1.1 & $<0.23$ & $<0.16$ \\
\hline & Average Removal & \multicolumn{2}{|c|}{$97.2 \%$} & \multicolumn{2}{|c|}{ Indeterminate } \\
\hline
\end{tabular}

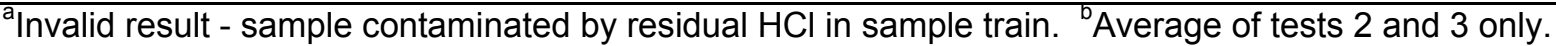

\section{Mercury}

Mercury measurements were performed at the air heater outlet and stack locations. Three approximately two-hour-long test runs including simultaneous sampling at these locations were performed on March 28, when the activated carbon injection system was not in service. This testing protocol was repeated on March 30, except that activated carbon was injected downstream of the air heater outlet sampling location 
(upstream of the Turbosorp ${ }^{\circledR}$ scrubber inlet) on that day. The mercury concentrations measured at each location on March 28 and March 30 are summarized in Tables 10 and 11, respectively. ( $\mathrm{Hg}$ concentrations at the air heater outlet were converted to a standard oxygen basis of $3 \%$ using $\mathrm{O}_{2}$ concentrations measured by CONSOL at the sampling train exhaust using a Teledyne Max $\mathrm{V}$ portable electrochemical $\mathrm{O}_{2}$ analyzer. $\mathrm{Hg}$ concentrations at the stack were converted to a standard oxygen basis of $3 \%$ using $\mathrm{O}_{2}$ concentrations calculated from the average $\mathrm{CO}_{2}$ concentration measured by the plant's stack CEM during each test run and the ultimate analysis of coal samples collected during the tests).

As shown in Tables 10 and 11, the average total $\mathrm{Hg}$ concentrations measured at the air heater outlet were $10.06 \mu \mathrm{g} / \mathrm{dscf}$ on March 28 and $9.38 \mu \mathrm{g} / \mathrm{dscf}$ on March 30 (both concentrations corrected to $3 \% \mathrm{O}_{2}$ ). On both days, $\mathrm{Hg}$ concentrations at the stack were less than the analytical limit of detection. Thus, $\mathrm{Hg}$ removal efficiencies across the Turbosorp ${ }^{\circledR}$ system (including the baghouse) were $>95.1 \%$ on March 28 , when no activated carbon was being injected into the system, and $>93.7 \%$ on March 30 , when activated carbon was being injected.

Table 10. Summary of Mercury Measurements at the Air Heater Outlet and Stack - Testing Without Activated Carbon Injection

\begin{tabular}{|c|c|c|c|c|}
\hline \multicolumn{5}{|c|}{ Air Heater Outlet } \\
\hline Test No. & 1 & 2 & 3 & \multirow{2}{*}{ Average } \\
\hline Date/Time (MM/DD/YY: hhmm-hhmm) & $\begin{array}{c}\text { 03/28/07: } \\
0905-1132\end{array}$ & $\begin{array}{c}03 / 28 / 07: \\
1245-1502\end{array}$ & $\begin{array}{c}03 / 28 / 07: \\
1558-1817\end{array}$ & \\
\hline Particulate $\mathrm{Hg}\left[\mu \mathrm{g} / \mathrm{dscm} @ 3 \% \mathrm{O}_{2}\right]$ & 5.32 & 3.72 & 4.89 & 4.64 \\
\hline Oxidized $\mathrm{Hg}\left[\mu \mathrm{g} / \mathrm{dscm} @ 3 \% \mathrm{O}_{2}\right]$ & 3.72 & 6.12 & 4.79 & 4.88 \\
\hline Elemental $\mathrm{Hg}\left[\mu \mathrm{g} / \mathrm{dscm} @ 3 \% \mathrm{O}_{2}\right]$ & 0.63 & 0.40 & 0.60 & 0.54 \\
\hline Total Hg [ $\left.\mu \mathrm{g} / \mathrm{dscm} @ 3 \% \mathrm{O}_{2}\right]$ & 9.67 & 10.23 & 10.28 & 10.06 \\
\hline \multicolumn{5}{|c|}{ Stack } \\
\hline Test No. & 1 & 2 & 3 & \multirow{2}{*}{ Average } \\
\hline Date/Time (MM/DD/YY: hhmm-hhmm) & $\begin{array}{c}03 / 28 / 07: \\
0910-1133\end{array}$ & $\begin{array}{c}03 / 28 / 07: \\
1243-1507\end{array}$ & $\begin{array}{c}\text { 03/28/07: } \\
\text { 1600-1814 }\end{array}$ & \\
\hline Particulate $\mathrm{Hg}\left[\mu \mathrm{g} / \mathrm{dscm} @ 3 \% \mathrm{O}_{2}\right]$ & 0.00 & 0.00 & 0.00 & 0.00 \\
\hline Oxidized $\mathrm{Hg}\left[\mu \mathrm{g} / \mathrm{dscm} @ 3 \% \mathrm{O}_{2}\right]$ & $<0.21$ & $<0.23$ & $<0.27$ & $<0.24$ \\
\hline Elemental $\mathrm{Hg}\left[\mu \mathrm{g} / \mathrm{dscm} @ 3 \% \mathrm{O}_{2}\right]$ & $<0.25$ & $<0.25$ & $<0.26$ & $<0.25$ \\
\hline Total Hg [ $\left.\mu \mathrm{g} / \mathrm{dscm} @ 3 \% \mathrm{O}_{2}\right]$ & $<0.46$ & $<0.49$ & $<0.54$ & $<0.50$ \\
\hline
\end{tabular}


Table 11. Summary of Mercury Measurements at the Air Heater Outlet and Stack - Testing With Activated Carbon Injection

\begin{tabular}{|c|c|c|c|c|}
\hline \multicolumn{5}{|c|}{ Air Heater Outlet } \\
\hline Test No. & 4 & 5 & 6 & \multirow[b]{2}{*}{ Average } \\
\hline Date/Time (MM/DD/YY: hhmm-hhmm) & $\begin{array}{c}\text { 03/30/07: } \\
0855-1110\end{array}$ & $\begin{array}{c}\text { 03/30/07: } \\
1243-1502\end{array}$ & $\begin{array}{c}\text { 03/30/07: } \\
1552-1805\end{array}$ & \\
\hline Particulate Hg [ $\left.\mu \mathrm{g} / \mathrm{dscm} @ 3 \% \mathrm{O}_{2}\right]$ & 4.41 & 4.83 & 4.13 & 4.46 \\
\hline Oxidized Hg [ $\left.\mu \mathrm{g} / \mathrm{dscm} @ 3 \% \mathrm{O}_{2}\right]$ & 4.77 & 4.36 & 4.11 & 4.41 \\
\hline Elemental $\mathrm{Hg}\left[\mu \mathrm{g} / \mathrm{dscm} @ 3 \% \mathrm{O}_{2}\right]$ & $<0.41$ & $<0.43$ & 0.70 & 0.52 \\
\hline |Total Hg [ $\left.\mu \mathrm{g} / \mathrm{dscm} @ 3 \% \mathrm{O}_{2}\right]$ & 9.59 & 9.63 & 8.93 & 9.38 \\
\hline \multicolumn{5}{|c|}{ Stack } \\
\hline Test No. & 4 & 5 & 6 & \multirow{2}{*}{ Average } \\
\hline Date/Time (MM/DD/YY: hhmm-hhmm) & $\begin{array}{c}\text { 03/30/07: } \\
0855-1110\end{array}$ & $\begin{array}{c}\text { 03/30/07: } \\
1243-1454\end{array}$ & $\begin{array}{c}\text { 03/30/07: } \\
1552-1803\end{array}$ & \\
\hline Particulate $\mathrm{Hg}\left[\mu \mathrm{g} / \mathrm{dscm} @ 3 \% \mathrm{O}_{2}\right]$ & 0.00 & 0.00 & 0.00 & 0.00 \\
\hline Oxidized $\mathrm{Hg}\left[\mu \mathrm{g} / \mathrm{dscm} @ 3 \% \mathrm{O}_{2}\right]$ & $<0.30$ & $<0.30$ & $<0.32$ & $<0.30$ \\
\hline Elemental $\mathrm{Hg}\left[\mu \mathrm{g} / \mathrm{dscm} @ 3 \% \mathrm{O}_{2}\right]$ & $<0.29$ & $<0.29$ & $<0.28$ & $<0.28$ \\
\hline |Total Hg [ $\left.\mu \mathrm{g} / \mathrm{dscm} @ 3 \% \mathrm{O}_{2}\right]$ & $<0.59$ & $<0.59$ & $<0.60$ & $<0.59$ \\
\hline
\end{tabular}

The results presented in Tables 10 and 11 include a breakdown of $\mathrm{Hg}$ concentrations by $\mathrm{Hg}$ species (i.e., particulate, oxidized, and elemental), as determined according to the Ontario Hydro method. However, these speciation results should be interpreted cautiously. Whereas total $\mathrm{Hg}$ concentrations determined using the Ontario Hydro method are expected to be reliable, even in high-dust locations, $\mathrm{Hg}$ speciation results determined using that method can be biased in high-dust locations by adsorption of $\mathrm{Hg}$ onto the fly ash collected on the sample filter or by reaction of $\mathrm{Hg}$ with the fly ash. This can lead to artificially high particulate and/or oxidized mercury concentrations, and artificially low elemental and/or oxidized mercury concentrations. (The direction of the bias in the oxidized mercury results depends upon the extent to which mercury is adsorbed as opposed to oxidized by the fly ash). Hence, it is likely that the mercury speciation observed at the air heater outlet, which included $\sim 47 \%$ particulate $\mathrm{Hg}$ on average, was biased high relative to actual flue gas composition as a result of this artifact. The air heater outlet is a high-dust location, containing 2.2-2.5 gr/dscf of particulate matter, and the high unburned carbon content of the fly ash sampled there (15-21\%) would tend to promote adsorption of gas-phase $\mathrm{Hg}$ onto the ash.

Table 12 summarizes the mercury removal efficiency of the multi-pollutant control system based on the total amount of $\mathrm{Hg}$ fed to the system in the coal and the total amount measured at the stack using the Ontario Hydro method. The amount of $\mathrm{Hg}$ in 
the coal was determined by laboratory analysis of coal samples collected during each test. Mercury mass flow rates determined at the stack by the Ontario Hydro method were converted to a heat input basis using average stack $\mathrm{O}_{2}$ concentrations and $\mathrm{O}_{2}-$ based fuel factors $\left(F_{d}\right)$ derived from the analysis of coal samples collected during each test.

Table 12. Summary of Coal-to-Stack Mercury Removal

\begin{tabular}{||l|c|c|c|c||}
\hline \multicolumn{1}{|c|}{ No Activated Carbon Injection } & Test \#1 & Test \#2 & Test \#3 & Average \\
\hline Hg in coal [lb/TBtu] & 7.34 & 8.19 & 6.86 & 7.46 \\
\hline Hg in flue gas leaving stack [lb/TBtu] & $<0.32$ & $<0.34$ & $<0.38$ & $<0.35$ \\
\hline Hg removal, fuel-to-stack [\%] & $>95.6$ & $>95.9$ & $>94.5$ & $>95.3$ \\
\hline \hline \multicolumn{1}{|c|}{ With Activated Carbon Injection } & Test \#4 & Test \#5 & Test \#6 & Average \\
\hline Hg in coal [lb/TBtu] & 6.92 & 6.45 & 6.38 & 6.58 \\
\hline Hg in flue gas leaving stack [lb/TBtu] & $<0.40$ & $<0.41$ & $<0.42$ & $<0.41$ \\
\hline Hg removal, fuel-to-stack [\%] & $>94.2$ & $>93.7$ & $>93.5$ & $>93.8$ \\
\hline
\end{tabular}

\section{SOLID AND LIQUID PROCESS SAMPLE ANALYSIS RESULTS}

During the flue gas sampling on March 28-30 and May 1-4, various solid and liquid process samples were collected by AES Greenidge personnel for analysis. For the March 28-30 testing, these samples included coal, Turbosorp ${ }^{\circledR}$ hopper ash (i.e., fly ash collected in the hopper at the inlet to the Turbosorp ${ }^{\circledR}$ absorber vessel), Turbosorp ${ }^{\circledR} /$ baghouse product ash (i.e., the mixture of fly ash and solid scrubber products leaving the baghouse), pebble lime, hydrated lime, urea (sampled prior to dilution), and activated carbon. For the May 1-4 testing, these samples included coal and fly ash collected at the air heater outlet. The samples were analyzed by CONSOL R\&D (or by CTL Group under subcontract to CONSOL) to determine various chemical and physical parameters specified in BPEl's Acceptance Test Procedure (Document 100276-100103100-01, dated March 22, 2007). Results are summarized in Tables 13-20. (Particle size distribution results for hydrated lime and Turbosorp ${ }^{\circledR} /$ baghouse product ash samples are presented in the CTL Group report that is included as Appendix $B$ to this report, and trace element results for coal samples collected during testing on March 28-30 are presented in the laboratory analysis sheets that are included in Appendix $D$ to this report). The methods used to analyze the process samples are described in the Experimental section of this report. 
Table 13. Coal Sample Analysis Results

\begin{tabular}{|c|c|c|c|c|c|c|c|c|c|c|}
\hline Analytical Number & 20071795 & 20071796 & 20071797 & 20071798 & 20071814 & 20071815 & 20071816 & 20071829 & 20071830 & 20071831 \\
\hline Test Identification & TEST 1 & TEST 2 & TEST 3 & TEST 3 & TEST 1 & TEST 2 & TEST 3 & TEST $1^{\mathrm{b}}$ & TEST $2^{c}$ & TEST $3^{\mathrm{d}}$ \\
\hline Date \& Time ${ }^{a}$ & $\begin{array}{c}3 / 28 / 2007 \\
\text { 09:00-10:00 }\end{array}$ & $\begin{array}{c}3 / 28 / 2007 \\
13: 00-14: 00\end{array}$ & $\begin{array}{c}3 / 28 / 2007 \\
16: 15\end{array}$ & $\begin{array}{c}3 / 28 / 2007 \\
18: 25\end{array}$ & $\begin{array}{c}3 / 29 / 2007 \\
9: 30\end{array}$ & $\begin{array}{c}3 / 29 / 2007 \\
13: 30\end{array}$ & $\begin{array}{c}3 / 29 / 2007 \\
16: 30\end{array}$ & $\begin{array}{l}3 / 30 / 2007 \\
9: 05-10: 35\end{array}$ & $\begin{array}{c}3 / 30 / 2007 \\
12: 45-14: 00\end{array}$ & $\begin{array}{c}3 / 28 / 2007 \\
15: 45-16: 45\end{array}$ \\
\hline Total Moisture, $\%$ & 6.62 & 6.28 & 6.54 & 6.71 & 4.20 & 4.60 & 4.61 & 6.66 & 4.97 & 4.87 \\
\hline As Determined Moisture, \% & 1.31 & 1.29 & 1.43 & 1.41 & 1.75 & 1.61 & 1.70 & 1.30 & 1.69 & 1.50 \\
\hline Volatile Matter, \% dry & 39.95 & 39.95 & 39.92 & 40.20 & 39.78 & 40.36 & 39.66 & 40.30 & 40.32 & 40.45 \\
\hline Carbon, \% dry & 76.14 & 75.66 & 75.86 & 76.58 & 76.59 & 76.62 & 76.23 & 76.02 & 76.41 & 76.35 \\
\hline Hydrogen, \% dry & 5.12 & 5.04 & 5.01 & 5.01 & 4.86 & 4.93 & 4.97 & 4.98 & 4.83 & 4.91 \\
\hline Nitrogen, \% dry & 1.41 & 1.45 & 1.44 & 1.46 & 1.41 & 1.50 & 1.46 & 1.48 & 1.40 & 1.42 \\
\hline Sulfur, \% dry & 2.68 & 2.69 & 2.57 & 2.68 & 2.79 & 2.50 & 2.66 & 2.61 & 2.65 & 2.65 \\
\hline Chlorine, \% dry & 0.069 & 0.075 & 0.074 & 0.071 & 0.072 & 0.073 & 0.076 & 0.075 & 0.075 & 0.070 \\
\hline Mercury, ppm dry & 0.101 & 0.113 & 0.097 & 0.094 & 0.116 & 0.097 & 0.108 & 0.097 & 0.090 & 0.089 \\
\hline Selenium, ppm dry & 1.26 & 1.15 & 1.25 & 1.12 & 1.10 & 1.04 & 1.12 & 1.01 & 1.02 & 0.99 \\
\hline HHV, Btu/lb dry & 13768 & 13799 & 13916 & 13939 & 13893 & 13838 & 13982 & 14015 & 13956 & 13948 \\
\hline \multicolumn{11}{|l|}{ Major Ash Elements, \% of ash } \\
\hline $\mathrm{SiO}_{2}$ & 44.98 & 42.69 & 44.62 & 42.22 & 42.34 & 41.41 & 43.52 & 41.81 & 42.67 & 42.38 \\
\hline $\mathrm{Al}_{2} \mathrm{O}_{3}$ & 20.75 & 20.16 & 22.46 & 21.80 & 20.94 & 20.78 & 21.48 & 20.75 & 20.97 & 20.88 \\
\hline $\mathrm{TiO}_{2}$ & 0.92 & 0.88 & 0.99 & 0.92 & 0.90 & 0.86 & 0.91 & 0.87 & 0.90 & 0.89 \\
\hline $\mathrm{Fe}_{2} \mathrm{O}_{3}$ & 20.51 & 22.46 & 20.52 & 20.84 & 23.67 & 18.98 & 23.20 & 21.95 & 21.39 & 21.46 \\
\hline $\mathrm{CaO}$ & 4.98 & 5.29 & 4.59 & 5.71 & 4.59 & 8.19 & 4.28 & 6.42 & 5.59 & 4.99 \\
\hline $\mathrm{MgO}$ & 0.85 & 0.85 & 0.92 & 1.36 & 1.12 & 1.06 & 0.86 & 0.95 & 0.98 & 0.94 \\
\hline $\mathrm{Na}_{2} \mathrm{O}$ & 0.80 & 0.76 & 0.81 & 0.86 & 0.81 & 0.82 & 0.83 & 0.87 & 0.84 & 0.86 \\
\hline $\mathrm{K}_{2} \mathrm{O}$ & 1.46 & 1.39 & 1.57 & 1.57 & 1.58 & 1.51 & 1.61 & 1.53 & 1.46 & 1.71 \\
\hline $\mathrm{P}_{2} \mathrm{O}_{5}$ & 0.36 & 0.38 & 0.48 & 0.37 & 0.34 & 0.37 & 0.40 & 0.37 & 0.38 & 0.37 \\
\hline $\mathrm{SO}_{3}$ & 4.81 & 4.97 & 3.78 & 5.31 & 4.29 & 5.83 & 3.83 & 5.14 & 4.92 & 4.82 \\
\hline
\end{tabular}

${ }^{\mathrm{a}}$ If a range of times is listed, result is for a composite of samples collected at the beginning and end of the range. ${ }^{\mathrm{b}}$ Mercury Test \#4. ${ }^{\mathrm{c}} \mathrm{Mercury}$ Test \#5. ${ }^{\mathrm{d}}$ Mercury Test \#6. 
Table 13. Coal Sample Analysis Results (continued)

\begin{tabular}{|c|c|c|c|c|c|c|c|c|c|}
\hline Analytical Number & 20072513 & 20072514 & 20072515 & 20072516 & 20072517 & 20072518 & 20072519 & 20072520 & 20072521 \\
\hline Test Identification & TEST 1 & TEST 2 & TEST 3 & TEST 4 & TEST 1 & TEST 2 & TEST 3 & TEST 1 & TEST 2 \\
\hline Date \& Time & $5 / 1 / 2007$ & $5 / 1 / 2007$ & $5 / 1 / 2007$ & $5 / 1 / 2007$ & $5 / 2 / 2007$ & $5 / 2 / 2007$ & $5 / 2 / 2007$ & $5 / 4 / 2007$ & $5 / 4 / 2007$ \\
\hline Total Moisture, \% & 6.04 & 6.14 & 5.87 & 5.85 & 5.51 & 5.66 & 5.58 & 5.94 & 5.97 \\
\hline As Determined Moisture, $\%$ & 1.26 & 1.32 & 1.26 & 1.20 & 1.19 & 1.16 & 1.30 & 1.30 & 1.32 \\
\hline Volatile Matter, \% dry & 40.81 & 41.02 & 41.11 & 41.07 & 41.30 & 41.37 & 41.27 & 41.39 & 41.31 \\
\hline Ash, $\%$ dry & 8.64 & 8.95 & 8.53 & 8.71 & 8.53 & 8.75 & 9.04 & 8.60 & 8.85 \\
\hline Carbon, \% dry & 75.37 & 75.59 & 75.69 & 75.61 & 75.19 & 75.14 & 75.94 & 74.24 & 75.13 \\
\hline Hydrogen, $\%$ dry & 5.26 & 5.01 & 5.00 & 5.26 & 5.12 & 4.84 & 4.94 & 4.83 & 5.01 \\
\hline Nitrogen, $\%$ dry & 1.38 & 1.38 & 1.38 & 1.37 & 1.39 & 1.35 & 1.37 & 1.35 & 1.39 \\
\hline Sulfur, \% dry & 3.05 & 3.14 & 3.11 & 3.08 & 3.20 & 3.38 & 3.26 & 3.32 & 3.25 \\
\hline Chlorine, \% dry & 0.080 & 0.068 & 0.076 & 0.088 & 0.064 & 0.088 & 0.094 & 0.066 & 0.096 \\
\hline \multicolumn{10}{|l|}{ Mercury, ppm dry } \\
\hline \multicolumn{10}{|l|}{ Fluorine, ppm dry } \\
\hline \multicolumn{10}{|l|}{ Selenium, ppm dry } \\
\hline HHV, Btu/lb dry & 13796 & 13765 & 13818 & 13784 & 13819 & 13741 & 13685 & 13732 & 13762 \\
\hline \multicolumn{10}{|l|}{ Major Ash Elements, \% of ash } \\
\hline $\mathrm{SiO}_{2}$ & 42.89 & 43.12 & 42.72 & 43.03 & 43.40 & 42.03 & 43.00 & 43.14 & 43.06 \\
\hline $\mathrm{Al}_{2} \mathrm{O}_{3}$ & 21.86 & 21.81 & 21.69 & 21.76 & 22.08 & 21.33 & 21.67 & 22.03 & 21.81 \\
\hline $\mathrm{TiO}_{2}$ & 0.90 & 0.90 & 0.89 & 0.90 & 0.91 & 0.86 & 0.87 & 0.90 & 0.87 \\
\hline $\mathrm{Fe}_{2} \mathrm{O}_{3}$ & 21.64 & 21.37 & 20.91 & 19.69 & 20.48 & 22.42 & 19.99 & 21.13 & 20.24 \\
\hline $\mathrm{CaO}$ & 4.98 & 4.87 & 4.58 & 4.45 & 4.36 & 4.42 & 5.17 & 4.77 & 4.99 \\
\hline $\mathrm{MgO}$ & 0.81 & 0.79 & 0.79 & 0.81 & 0.78 & 0.76 & 0.87 & 0.83 & 0.81 \\
\hline $\mathrm{Na}_{2} \mathrm{O}$ & 0.79 & 0.80 & 0.79 & 0.99 & 0.76 & 0.88 & 1.20 & 0.75 & 0.99 \\
\hline $\mathrm{K}_{2} \mathrm{O}$ & 1.46 & 1.41 & 1.38 & 1.42 & 1.40 & 1.38 & 1.46 & 1.40 & 1.43 \\
\hline $\mathrm{P}_{2} \mathrm{O}_{5}$ & 0.26 & 0.27 & 0.24 & 0.24 & 0.25 & 0.23 & 0.25 & 0.27 & 0.24 \\
\hline $\mathrm{SO}_{3}$ & 4.88 & 4.93 & 3.74 & 4.43 & 4.16 & 4.56 & 4.66 & 3.88 & 4.66 \\
\hline
\end{tabular}


Table 14. Air Heater Outlet Fly Ash Sample Analysis Results

\begin{tabular}{|c|c|c|c|c|c|c|c|c|c|}
\hline Analytical Number & 20072522 & 20072523 & 20072524 & 20072525 & 20072526 & 20072527 & 20072528 & 20072529 & 20072530 \\
\hline Test Identification & TEST 1 & TEST 2 & TEST 3 & TEST 4 & TEST 1 & TEST 2 & TEST 3 & TEST 1 & TEST 2 \\
\hline Date \& Time & $5 / 1 / 2007$ & $5 / 1 / 2007$ & $5 / 1 / 2007$ & $5 / 1 / 2007$ & $5 / 2 / 2007$ & $5 / 2 / 2007$ & $5 / 2 / 2007$ & $5 / 4 / 2007$ & $5 / 4 / 2007$ \\
\hline As Determined Moisture, \% & 0.20 & 1.07 & 1.08 & 0.38 & 0.17 & 0.11 & 0.79 & 0.19 & 0.91 \\
\hline Ash, \% dry & 77.06 & 83.09 & 84.20 & 80.32 & 75.73 & 74.67 & 79.08 & 78.76 & 80.01 \\
\hline Carbon, \% dry & 23.79 & 16.26 & 15.09 & 20.83 & 24.71 & 26.20 & 21.10 & 19.63 & 17.71 \\
\hline Sulfur, \% dry & 0.44 & 0.54 & 0.53 & 0.52 & 0.51 & 0.50 & 0.52 & 0.50 & 0.65 \\
\hline Chlorine, \% dry & $<0.0005$ & 0.0138 & 0.0065 & 0.0041 & 0.0025 & 0.0018 & 0.0137 & 0.0023 & 0.0060 \\
\hline \multicolumn{10}{|l|}{ Major Ash Elements, \% dry } \\
\hline $\mathrm{SiO}_{2}$ & 34.53 & 39.20 & 39.63 & 38.90 & 35.99 & 34.71 & 37.21 & 37.65 & 38.72 \\
\hline $\mathrm{Al}_{2} \mathrm{O}_{3}$ & 16.86 & 19.74 & 20.07 & 19.38 & 18.33 & 17.06 & 17.91 & 17.52 & 18.73 \\
\hline $\mathrm{TiO}_{2}$ & 0.72 & 0.89 & 0.90 & 0.87 & 0.82 & 0.75 & 0.80 & 0.81 & 0.89 \\
\hline $\mathrm{Fe}_{2} \mathrm{O}_{3}$ & 14.85 & 14.74 & 14.87 & 15.29 & 16.16 & 16.49 & 14.82 & 14.86 & 12.99 \\
\hline $\mathrm{CaO}$ & 3.72 & 3.90 & 4.07 & 4.01 & 3.85 & 3.75 & 4.12 & 4.17 & 4.23 \\
\hline $\mathrm{MgO}$ & 0.61 & 0.73 & 0.75 & 0.69 & 0.65 & 0.60 & 0.71 & 0.70 & 0.76 \\
\hline $\mathrm{Na}_{2} \mathrm{O}$ & 0.62 & 0.78 & 0.79 & 0.71 & 0.67 & 0.64 & 0.72 & 0.68 & 0.75 \\
\hline $\mathrm{K}_{2} \mathrm{O}$ & 1.09 & 1.35 & 1.38 & 1.26 & 1.20 & 1.08 & 1.31 & 1.21 & 1.32 \\
\hline $\mathrm{P}_{2} \mathrm{O}_{5}$ & 0.15 & 0.22 & 0.21 & 0.18 & 0.18 & 0.14 & 0.19 & 0.16 & 0.18 \\
\hline $\mathrm{SO}_{3}$ & 1.11 & 1.34 & 1.30 & 1.29 & 1.27 & 1.24 & 1.30 & 1.24 & 1.60 \\
\hline
\end{tabular}


Table 15. Turbosorp ${ }^{\circledR}$ Hopper Ash Sample Analysis Results

\begin{tabular}{|c|c|c|c|c|c|c|}
\hline Analytical Number & 20071805 & 20071806 & 20071807 & 20071835 & 20071836 & 20071837 \\
\hline Test Identification & TEST 1 & TEST 2 & TEST 3 & TEST $1^{\mathrm{a}}$ & TEST $2^{\mathrm{b}}$ & TEST $3^{c}$ \\
\hline Date \& Time & $\begin{array}{c}\text { MARCH } 28 \\
11: 30 \\
\end{array}$ & $\begin{array}{c}\text { MARCH } 28 \\
16: 00 \\
\end{array}$ & $\begin{array}{c}\text { MARCH } 28 \\
20: 00 \\
\end{array}$ & $\begin{array}{c}\text { MARCH } 30 \\
10: 00 \\
\end{array}$ & $\begin{array}{c}\text { MARCH } 30 \\
13: 45 \\
\end{array}$ & $\begin{array}{c}\text { MARCH } 30 \\
16: 45 \\
\end{array}$ \\
\hline As Determined Moisture, \% & 0.10 & 0.09 & 0.12 & 0.06 & 0.08 & 0.35 \\
\hline Ash, \% dry & 81.48 & 82.45 & 79.20 & 74.83 & 82.78 & 81.88 \\
\hline Carbon, \% dry & 19.10 & 17.99 & 21.20 & 26.38 & 16.36 & 17.48 \\
\hline Chlorine, \% dry & 0.027 & 0.018 & 0.016 & 0.023 & 0.016 & 0.018 \\
\hline Mercury, ppm dry & 0.134 & 0.123 & 0.141 & 0.139 & 0.105 & 0.107 \\
\hline Fluorine, ppm dry & 58.16 & 59.55 & 57.27 & 53.13 & 53.34 & 53.39 \\
\hline \multicolumn{7}{|l|}{ Major Ash Elements, \% dry } \\
\hline $\mathrm{SiO}_{2}$ & 25.86 & 25.48 & 26.31 & 26.34 & 26.73 & 26.05 \\
\hline $\mathrm{Al}_{2} \mathrm{O}_{3}$ & 11.66 & 10.36 & 11.96 & 12.01 & 12.18 & 11.73 \\
\hline $\mathrm{TiO}_{2}$ & 0.46 & 0.46 & 0.47 & 0.45 & 0.48 & 0.46 \\
\hline $\mathrm{Fe}_{2} \mathrm{O}_{3}$ & 37.54 & 40.28 & 32.91 & 29.96 & 34.00 & 34.54 \\
\hline $\mathrm{CaO}$ & 4.91 & 7.06 & 5.10 & 5.46 & 8.09 & 7.04 \\
\hline $\mathrm{MgO}$ & 0.46 & 0.54 & 0.49 & 0.48 & 0.54 & 0.52 \\
\hline $\mathrm{Na}_{2} \mathrm{O}$ & 0.38 & 0.31 & 0.38 & 0.42 & 0.42 & 0.40 \\
\hline $\mathrm{K}_{2} \mathrm{O}$ & 0.69 & 0.54 & 0.70 & 0.71 & 0.71 & 0.68 \\
\hline $\mathrm{P}_{2} \mathrm{O}_{5}$ & 0.28 & 0.35 & 0.32 & 0.28 & 0.26 & 0.25 \\
\hline $\mathrm{SO}_{3}$ & 1.50 & 2.10 & 1.52 & 1.47 & 2.38 & 2.46 \\
\hline
\end{tabular}

${ }^{\mathrm{a}}$ Mercury test \#4. ${ }^{\mathrm{b}}$ Mercury test \#5. ${ }^{\mathrm{c}}$ Mercury test \#6. 
Table 16. Turbosorp ${ }^{\circledR} /$ Baghouse Product Ash Sample Analysis Results

\begin{tabular}{|c|c|c|c|c|c|c|}
\hline Analytical Number & 20071799 & 20071800 & 20071801 & 20071802 & 20071803 & 20071804 \\
\hline Test Identification & TEST 1 & TEST 1 & TEST 2 & TEST 2 & TEST 3 & TEST 3 \\
\hline Date \& Time & $\begin{array}{c}\text { MARCH } 28 \\
10: 00\end{array}$ & $\begin{array}{c}\text { MARCH } 28 \\
11: 00\end{array}$ & $\begin{array}{c}\text { MARCH } 28 \\
14: 00\end{array}$ & $\begin{array}{c}\text { MARCH } 28 \\
15: 50\end{array}$ & $\begin{array}{c}\text { MARCH } 28 \\
17: 15\end{array}$ & $\begin{array}{c}\text { MARCH } 28 \\
19: 20\end{array}$ \\
\hline $\begin{array}{l}\text { As Determined } \\
\text { Moisture, } \%\end{array}$ & 0.79 & 0.91 & 0.65 & 0.80 & 0.75 & 0.84 \\
\hline Ash, \% dry & 84.57 & 84.58 & 84.37 & 84.77 & 84.78 & 84.75 \\
\hline Carbon, \% dry & 8.05 & 8.18 & 8.13 & 7.67 & 7.61 & 7.47 \\
\hline Chlorine, \% dry & 0.276 & 0.296 & 0.291 & 0.268 & 0.278 & 0.296 \\
\hline Mercury, ppm dry & 0.346 & 0.347 & 0.360 & 0.367 & 0.363 & 0.372 \\
\hline Fluorine, ppm dry & 80.74 & 92.54 & 83.95 & 83.06 & 87.36 & 91.17 \\
\hline \multicolumn{7}{|l|}{$\begin{array}{l}\text { Major Ash Elements, } \\
\% \text { dry }\end{array}$} \\
\hline $\mathrm{SiO}_{2}$ & 12.28 & 12.18 & 11.85 & 11.65 & 11.82 & 11.37 \\
\hline $\mathrm{Al}_{2} \mathrm{O}_{3}$ & 6.08 & 6.06 & 5.93 & 5.85 & 5.94 & 5.71 \\
\hline $\mathrm{TiO}_{2}$ & 0.26 & 0.26 & 0.25 & 0.25 & 0.26 & 0.25 \\
\hline $\mathrm{Fe}_{2} \mathrm{O}_{3}$ & 4.55 & 4.55 & 4.40 & 4.29 & 4.35 & 4.23 \\
\hline $\mathrm{CaO}$ & 37.53 & 37.78 & 38.14 & 38.06 & 37.77 & 37.47 \\
\hline $\mathrm{MgO}$ & 0.64 & 0.65 & 0.64 & 0.64 & 0.63 & 0.63 \\
\hline $\mathrm{Na}_{2} \mathrm{O}$ & 0.32 & 0.32 & 0.30 & 0.31 & 0.30 & 0.30 \\
\hline $\mathrm{K}_{2} \mathrm{O}$ & 0.45 & 0.45 & 0.43 & 0.43 & 0.43 & 0.44 \\
\hline $\mathrm{P}_{2} \mathrm{O}_{5}$ & 0.14 & 0.12 & 0.12 & 0.13 & 0.11 & 0.11 \\
\hline $\mathrm{SO}_{3}$ & 22.79 & 22.79 & 23.11 & 23.16 & 23.16 & 22.62 \\
\hline
\end{tabular}


Table 16. Turbosorp ${ }^{\circledR} /$ Baghouse Product Ash Sample Analysis Results (continued)

\begin{tabular}{|c|c|c|c|c|c|c|}
\hline Analytical Number & 20071817 & 20071818 & 20071819 & 20071832 & 20071833 & 20071834 \\
\hline Test Identification & TEST 1 & TEST 2 & TEST 3 & TEST $1^{\mathrm{a}}$ & TEST $2^{b}$ & TEST $3^{\mathrm{c}}$ \\
\hline Date \& Time & $\begin{array}{l}\text { MARCH } 29 \\
9: 30\end{array}$ & $\begin{array}{c}\text { MARCH } 29 \\
13: 30\end{array}$ & $\begin{array}{c}\text { MARCH } 29 \\
16: 30\end{array}$ & $\begin{array}{c}\text { MARCH } 30 \\
11: 15\end{array}$ & $\begin{array}{c}\text { MARCH } 30 \\
13: 45\end{array}$ & $\begin{array}{c}\text { MARCH } 30 \\
16: 45\end{array}$ \\
\hline $\begin{array}{l}\text { As Determined } \\
\text { Moisture, } \%\end{array}$ & 0.98 & 0.98 & 0.66 & 0.56 & 0.63 & 0.51 \\
\hline Ash, \% dry & 83.72 & 84.23 & 83.30 & 83.24 & 83.64 & 83.69 \\
\hline Carbon, \% dry & 8.23 & 8.07 & 7.96 & 8.34 & 8.16 & 8.12 \\
\hline Chlorine, \% dry & 0.270 & 0.249 & 0.261 & 0.213 & 0.270 & 0.280 \\
\hline Mercury, ppm dry & 0.369 & 0.377 & 0.395 & 0.379 & 0.349 & 0.352 \\
\hline Fluorine, ppm dry & 79.58 & 76.45 & 72.58 & 91.81 & 82.12 & 80.31 \\
\hline \multicolumn{7}{|l|}{$\begin{array}{c}\text { Major Ash Elements, } \\
\% \text { dry }\end{array}$} \\
\hline $\mathrm{SiO}_{2}$ & 12.41 & 12.06 & 12.03 & 12.93 & 12.59 & 12.12 \\
\hline $\mathrm{Al}_{2} \mathrm{O}_{3}$ & 6.17 & 6.05 & 6.06 & 6.61 & 6.28 & 6.06 \\
\hline $\mathrm{TiO}_{2}$ & 0.26 & 0.26 & 0.26 & 0.29 & 0.27 & 0.26 \\
\hline $\mathrm{Fe}_{2} \mathrm{O}_{3}$ & 4.62 & 4.49 & 4.33 & 4.52 & 4.73 & 4.57 \\
\hline $\mathrm{CaO}$ & 38.84 & 38.45 & 38.18 & 37.10 & 38.21 & 38.77 \\
\hline $\mathrm{MgO}$ & 0.66 & 0.65 & 0.63 & 0.65 & 0.66 & 0.66 \\
\hline $\mathrm{Na}_{2} \mathrm{O}$ & 0.32 & 0.31 & 0.31 & 0.33 & 0.33 & 0.33 \\
\hline $\mathrm{K}_{2} \mathrm{O}$ & 0.46 & 0.45 & 0.44 & 0.48 & 0.48 & 0.47 \\
\hline $\mathrm{P}_{2} \mathrm{O}_{5}$ & 0.11 & 0.13 & 0.14 & 0.13 & 0.13 & 0.12 \\
\hline $\mathrm{SO}_{3}$ & 21.14 & 21.90 & 21.91 & 20.92 & 21.57 & 21.84 \\
\hline
\end{tabular}

${ }^{\mathrm{a}}$ Mercury test \#4. ${ }^{\mathrm{b}}$ Mercury test \#5. ${ }^{\mathrm{c}}$ Mercury test \#6. 
Table 17. Pebble Lime Sample Analysis Results

\begin{tabular}{|c|c|c|c|c|c|c|c|c|c|}
\hline Analytical Number & 20071811 & 20071812 & 20071813 & 20071826 & 20071827 & 20071828 & 20071841 & 20071842 & 20071843 \\
\hline Test Identification & TEST 1 & TEST 2 & TEST 3 & TEST 1 & TEST 2 & TEST 3 & TEST $1^{a}$ & TEST $2^{\mathrm{b}}$ & TEST $3^{\mathrm{C}}$ \\
\hline Date \& Time & $\begin{array}{c}3 / 28 / 2007 \\
10: 00 \\
\end{array}$ & $\begin{array}{c}3 / 28 / 2007 \\
14: 30 \\
\end{array}$ & $\begin{array}{c}3 / 28 / 2007 \\
17: 15 \\
\end{array}$ & $\begin{array}{c}3 / 29 / 2007 \\
9: 30 \\
\end{array}$ & $\begin{array}{c}3 / 29 / 2007 \\
13: 30 \\
\end{array}$ & $\begin{array}{c}/ 29 / 2007 \\
16: 30 \\
\end{array}$ & $\begin{array}{c}\text { 3/30/2007 } \\
10: 00 \\
\end{array}$ & $\begin{array}{c}3 / 30 / 2007 \\
13: 45 \\
\end{array}$ & $\begin{array}{c}3 / 30 / 2007 \\
16: 45 \\
\end{array}$ \\
\hline As Determined Moisture, $\%$ & $<0.01$ & $<0.01$ & $<0.01$ & $<0.01$ & $<0.01$ & $<0.01$ & $<0.01$ & $<0.01$ & $<0.01$ \\
\hline Ash, $\%$ dry & 97.74 & 95.88 & 98.69 & 97.87 & 97.51 & 96.01 & 97.95 & 98.11 & 99.08 \\
\hline Carbon, \% dry & 0.37 & 1.36 & 0.14 & 0.31 & 0.30 & 0.38 & 0.62 & 0.48 & 0.31 \\
\hline Available Lime, \% as $\mathrm{CaO}$ & \multicolumn{3}{|c|}{$89.38^{\mathrm{d}, \mathrm{g}}$} & \multicolumn{3}{|c|}{$88.07^{\mathrm{e}, \mathrm{g}}$} & \multicolumn{3}{|c|}{$92.57^{f, g}$} \\
\hline $\begin{array}{c}\text { Slaking Rate, temperature rise } \\
\text { in } 30 \mathrm{sec} .,{ }^{\circ} \mathrm{C}\end{array}$ & \multicolumn{3}{|c|}{$32.0^{\mathrm{d}, \mathrm{g}}$} & \multicolumn{3}{|c|}{$30.0^{\mathrm{e}, \mathrm{g}}$} & \multicolumn{3}{|c|}{$33.0^{\mathrm{f}, \mathrm{g}}$} \\
\hline $\begin{array}{l}\text { Slaking Rate, temperature rise } \\
\text { in } 3 \mathrm{~min} .,{ }^{\circ} \mathrm{C}\end{array}$ & \multicolumn{3}{|c|}{$52.0^{\mathrm{d}, \mathrm{g}}$} & \multicolumn{3}{|c|}{$50.5^{\mathrm{e}, \mathrm{g}}$} & \multicolumn{3}{|c|}{$52.0^{\mathrm{f}, \mathrm{g}}$} \\
\hline Slaking Residue, \% & \multicolumn{3}{|c|}{$0.97^{\mathrm{d}, \mathrm{g}}$} & \multicolumn{3}{|c|}{$3.79^{e, g}$} & \multicolumn{3}{|c|}{$2.06^{f, g}$} \\
\hline \multicolumn{10}{|l|}{ Major Ash Elements, \% dry } \\
\hline $\mathrm{SiO}_{2}$ & 1.06 & 1.07 & 0.98 & 1.03 & 1.61 & 1.33 & 0.65 & 1.81 & 2.34 \\
\hline $\mathrm{Al}_{2} \mathrm{O}_{3}$ & 0.48 & 0.49 & 0.48 & 0.54 & 0.49 & 0.43 & 0.44 & 0.46 & 0.46 \\
\hline $\mathrm{TiO}_{2}$ & 0.02 & 0.02 & 0.02 & $<0.03$ & $<0.03$ & $<0.03$ & $<0.03$ & $<0.03$ & $<0.03$ \\
\hline $\mathrm{Fe}_{2} \mathrm{O}_{3}$ & 0.20 & 0.25 & 0.21 & 0.23 & 0.21 & 0.19 & 0.18 & 0.18 & 0.18 \\
\hline $\mathrm{CaO}$ & 95.22 & 91.37 & 96.28 & 95.21 & 96.64 & 95.51 & 96.09 & 95.10 & 95.17 \\
\hline $\mathrm{MgO}$ & 1.11 & 2.88 & 1.04 & 1.04 & 1.05 & 0.10 & 0.96 & 0.95 & 0.99 \\
\hline $\mathrm{Na}_{2} \mathrm{O}$ & 0.08 & 0.06 & 0.08 & 0.05 & 0.09 & 0.08 & 0.06 & 0.05 & 0.06 \\
\hline $\mathrm{K}_{2} \mathrm{O}$ & 0.07 & 0.06 & 0.06 & $<0.03$ & 0.07 & 0.06 & 0.04 & 0.03 & $<0.03$ \\
\hline $\mathrm{P}_{2} \mathrm{O}_{5}$ & 0.01 & 0.01 & 0.01 & $<0.03$ & $<0.03$ & $<0.03$ & $<0.03$ & $<0.03$ & $<0.03$ \\
\hline $\mathrm{SO}_{3}$ & 0.16 & 0.18 & 0.17 & 0.14 & 0.15 & 0.17 & 0.09 & 0.10 & 0.12 \\
\hline
\end{tabular}

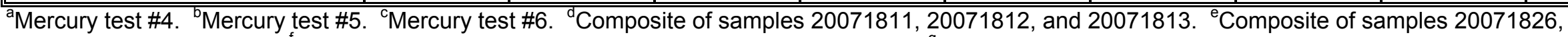
20071827, and 20071828. ' Composite of samples 20071841, 20071842, and 20071843. 'Analysis performed by CTL Group. 
Table 18. Hydrated Lime Sample Analysis Results

\begin{tabular}{|c|c|c|c|c|c|c|c|c|c|}
\hline Analytical Number & 20071808 & 20071809 & 20071810 & 20071823 & 20071824 & 20071825 & 20071838 & 20071839 & 20071840 \\
\hline Test Identification & TEST 1 & TEST 2 & TEST 3 & TEST 1 & TEST 2 & TEST 3 & TEST $1^{\mathrm{a}}$ & TEST $2^{\mathrm{b}}$ & TEST $3^{\mathrm{C}}$ \\
\hline Date \& Time & $\begin{array}{c}3 / 28 / 2007 \\
11: 00 \\
\end{array}$ & $\begin{array}{c}3 / 28 / 2007 \\
14: 30 \\
\end{array}$ & $\begin{array}{c}3 / 28 / 2007 \\
17: 00 \\
\end{array}$ & $\begin{array}{c}3 / 29 / 2007 \\
9: 30\end{array}$ & $\begin{array}{c}3 / 29 / 2007 \\
13: 30 \\
\end{array}$ & $\begin{array}{c}3 / 29 / 2007 \\
16: 30 \\
\end{array}$ & $\begin{array}{c}3 / 30 / 2007 \\
10: 00 \\
\end{array}$ & $\begin{array}{c}3 / 30 / 2007 \\
13: 45\end{array}$ & $\begin{array}{c}3 / 30 / 2007 \\
16: 45 \\
\end{array}$ \\
\hline As Determined Moisture, $\%$ & 0.27 & 0.29 & 0.35 & 0.09 & $<0.01$ & $<0.01$ & $<0.01$ & $<0.01$ & 0.06 \\
\hline Ash, \% dry & 76.43 & 76.39 & 76.39 & 75.73 & 75.52 & 75.92 & 75.74 & 76.00 & 76.32 \\
\hline Carbon, \% dry & 0.32 & 0.30 & 0.32 & 0.44 & 0.58 & 0.42 & 0.39 & 0.45 & 0.39 \\
\hline Mercury, ppm dry & 0.005 & $<0.005$ & $<0.005$ & & & & 0.006 & 0.005 & 0.004 \\
\hline Apparent Density - Loose, lb/ft ${ }^{3}$ & \multicolumn{3}{|c|}{$22.32^{\mathrm{d}, \mathrm{g}}$} & \multicolumn{3}{|c|}{$22.83^{\mathrm{e}, \mathrm{g}}$} & \multicolumn{3}{|c|}{$22.14^{\mathrm{f}, \mathrm{g}}$} \\
\hline Apparent Density - Packed, lb/ft ${ }^{3}$ & \multicolumn{3}{|c|}{$39.58^{\mathrm{d}, \mathrm{g}}$} & \multicolumn{3}{|c|}{$37.17^{\mathrm{e}, \mathrm{g}}$} & \multicolumn{3}{|c|}{$36.78^{\mathrm{f}, \mathrm{g}}$} \\
\hline BET Surface Area, $\mathrm{m}^{2} / \mathrm{g}$ & \multicolumn{3}{|c|}{$15.73^{\mathrm{d}, \mathrm{g}}$} & \multicolumn{3}{|c|}{$17.29^{\mathrm{e}, \mathrm{g}}$} & \multicolumn{3}{|c|}{$17.62^{\mathrm{f}, \mathrm{g}}$} \\
\hline \multicolumn{10}{|l|}{ Major Ash Elements, \% dry } \\
\hline $\mathrm{SiO}_{2}$ & 0.83 & 0.73 & 0.71 & 1.31 & 1.31 & 1.31 & 1.31 & 1.34 & 1.25 \\
\hline $\mathrm{Al}_{2} \mathrm{O}_{3}$ & 0.40 & 0.40 & 0.41 & 0.38 & 0.38 & 0.37 & 0.36 & 0.36 & 0.35 \\
\hline $\mathrm{TiO}_{2}$ & 0.02 & 0.02 & 0.02 & 0.02 & 0.02 & 0.02 & 0.01 & 0.01 & 0.01 \\
\hline $\mathrm{Fe}_{2} \mathrm{O}_{3}$ & 0.16 & 0.16 & 0.16 & 0.14 & 0.18 & 0.14 & 0.16 & 0.14 & 0.14 \\
\hline $\mathrm{CaO}$ & 75.45 & 74.30 & 74.19 & 70.01 & 69.05 & 70.83 & 70.94 & 71.24 & 71.27 \\
\hline $\mathrm{MgO}$ & 0.82 & 0.82 & 0.81 & 0.80 & 0.80 & 0.81 & 0.80 & 0.80 & 0.80 \\
\hline $\mathrm{Na}_{2} \mathrm{O}$ & 0.08 & 0.07 & 0.07 & 0.01 & 0.02 & 0.02 & 0.03 & 0.02 & 0.02 \\
\hline $\mathrm{K}_{2} \mathrm{O}$ & 0.05 & 0.05 & 0.05 & 0.04 & 0.04 & 0.04 & 0.06 & 0.05 & 0.04 \\
\hline $\mathrm{P}_{2} \mathrm{O}_{5}$ & 0.01 & 0.01 & 0.01 & 0.01 & 0.02 & 0.01 & 0.01 & $<0.01$ & $<0.03$ \\
\hline $\mathrm{SO}_{3}$ & 0.13 & 0.12 & 0.17 & 0.20 & 0.21 & 0.18 & 0.20 & 0.22 & 0.17 \\
\hline
\end{tabular}

${ }^{a}$ Mercury test \#4. ${ }^{b}$ Mercury test \#5. ${ }^{c}$ Mercury test \#6. ${ }^{d}$ Composite of samples 20071808, 20071809, and 20071810. ${ }^{e}$ Composite of samples 20071823 , 20071824, and 20071825. 'Composite of samples 20071838, 20071839, and 20071840. ${ }^{9}$ Analysis performed by CTL Group. 
Table 19. Activated Carbon Sample Analysis Results

\begin{tabular}{||c|c|c||}
\hline Analytical Number & 20071845 & 20071844 \\
\hline Test Identification & NA & NA \\
\hline Date \& Time & $3 / 29 / 0715: 30$ & $3 / 30 / 0712: 00$ \\
\hline As Determined Moisture \% & 1.21 & 1.17 \\
\hline Volatile Matter, \% dry & 4.63 & 4.50 \\
\hline Ash, \% dry & 8.24 & 8.33 \\
\hline Carbon, \% dry & 90.95 & 90.66 \\
\hline Hydrogen, \% dry & $<0.01$ & $<0.01$ \\
\hline Nitrogen, \% dry & 0.37 & 0.40 \\
\hline Sulfur, \% dry & 0.37 & 0.39 \\
\hline Mercury, ppm dry & 0.005 & 0.004 \\
\hline Major Ash Elements, \% dry & & 1.02 \\
\hline $\mathrm{SiO}_{2}$ & 2.11 & 3.53 \\
\hline $\mathrm{Al}_{2} \mathrm{O}_{3}$ & 4.10 & 0.33 \\
\hline $\mathrm{TiO}_{2}$ & 0.35 & 13.27 \\
\hline $\mathrm{Fe}_{2} \mathrm{O}_{3}$ & 13.52 & 39.96 \\
\hline $\mathrm{CaO}^{\mathrm{MgO}}$ & 40.93 & 20.14 \\
\hline $\mathrm{Na}_{2} \mathrm{O}$ & 19.20 & 5.80 \\
\hline $\mathrm{K}_{2} \mathrm{O}$ & 4.04 & 0.50 \\
\hline $\mathrm{P}_{2} \mathrm{O}_{5}$ & 0.31 & $<0.01$ \\
\hline $\mathrm{SO}_{3}$ & 0.03 & 14.61 \\
\hline
\end{tabular}

Table 20. Urea Sample Analysis Results

\begin{tabular}{||c|c|c|c|c|c|c|c||}
\hline \hline $\begin{array}{c}\text { Analytical } \\
\text { Number }\end{array}$ & $\begin{array}{c}\text { Sample } \\
\text { Identification }\end{array}$ & $\mathbf{p H}$ & $\begin{array}{c}\mathbf{H g}, \\
\boldsymbol{\mu g} / \mathbf{L}\end{array}$ & $\begin{array}{c}\text { TSS, } \\
\mathbf{m g} / \mathbf{L}\end{array}$ & $\begin{array}{c}\text { Ammonia, } \\
\mathbf{m g} / \mathbf{L} \text { as N }\end{array}$ & $\begin{array}{c}\text { Phosphate, } \\
\mathbf{m g} / \mathbf{L}\end{array}$ & $\begin{array}{c}\text { Density, } \\
\mathbf{g}^{\mathbf{3}} \mathbf{c m}^{\mathbf{3}}\end{array}$ \\
\hline 20071971 & $\begin{array}{c}\text { AES Greenidge Urea } \\
\text { Composite 3/28/07 }\end{array}$ & 9.56 & $<0.35$ & $<6$ & 595 & 45.20 & 1.14 \\
\hline 20071972 & $\begin{array}{c}\text { AES Greenidge Urea } \\
\text { Composite 3/30/07 }\end{array}$ & 9.40 & $<0.35$ & $<6$ & 635 & 79.20 & 1.13 \\
\hline
\end{tabular}




\section{EXPERIMENTAL}

\section{Sampling Locations}

Figure 1 presents a schematic showing the locations where flue gas sampling was conducted at AES Greenidge. These sampling locations are described below.

\section{SCR Inlet}

$\mathrm{NO}_{x}$ tests were conducted at the SCR inlet. The eastern wall of the SCR inlet duct is outfitted with four, four-inch flange ports, each of which contains six permanent sampling tubes. The six tubes installed through each of the four ports terminate at different depths in the duct, creating a 24-point grid of sample points covering the cross section of the duct. Clean Air Engineering performed $\mathrm{NO}_{\mathrm{x}}$ testing using an automated sampling system that sequentially extracted samples from the grid points. Each of the 24 grid points was sampled for three, one-minute intervals during each test. Figure 2 presents a photograph of the SCR inlet $\mathrm{NO}_{x}$ sampling location and the CAE sampling setup. A diagram showing the locations of the SCR inlet grid sampling points is provided in the CAE report that is included as Appendix $A$ to this report.

\section{SCR Outlet}

$\mathrm{NO}_{x}$ and $\mathrm{NH}_{3}$ tests were conducted at the SCR outlet. The $\mathrm{NH}_{3}$ samples were extracted from ports located on the eastern wall of the SCR outlet duct at the 498' level. The March 28 ammonia samples were drawn through the northernmost of the two four-inch ports at this location; the port was traversed to a depth of approximately 10 feet using three sample points. Figure 3 presents a photograph showing ammonia sampling at the SCR outlet. Each ammonia test was 60 minutes in duration. The ammonia samples collected on May 1 were drawn through both the northern and southern ports on the eastern wall of the SCR outlet duct; each port was sampled for 30 minutes per test using a three-point traverse to a depth of approximately 10 feet. All ammonia samples at the SCR outlet were drawn at an isokinetic sampling rate.

The eastern wall of the SCR outlet duct is also outfitted with four, four-inch flange ports, each of which contains six permanent sampling tubes. The six tubes installed through each of the four ports terminate at different depths in the duct, creating a 24point grid of sample points covering the cross section of the duct. Clean Air Engineering performed $\mathrm{NO}_{x}$ testing using an automated sampling system that sequentially extracted samples from the grid points. Each of the 24 grid points was sampled for three, one-minute intervals during each test. A diagram showing the locations of the SCR outlet grid sampling points is provided in the CAE report that is included as Appendix A to this report.

\section{Air Heater Inlet}

Ammonia sampling at the air heater inlet was conducted through two ports located immediately upstream of the unit's air heaters. One port was located at approximately the center (horizontal dimension) of the south wall of the eastern air heater inlet duct, 
and the other port was located at approximately the center of the south wall of the western air heater inlet duct. Samples were drawn at a constant rate from a single point in each duct. Each 60-minute test consisted of 30 minutes of sampling in the eastern duct and 30 minutes of sampling in the western duct. Figure 4 presents a photograph of the air heater inlet sampling location.

\section{Air Heater Outlet}

$\mathrm{SO}_{2}, \mathrm{SO}_{3}, \mathrm{HCl}, \mathrm{HF}$, and $\mathrm{Hg}$ tests were conducted at the air heater outlet. Figure 5 presents a photograph of this location. For $\mathrm{HCl}, \mathrm{HF}$, and $\mathrm{Hg}$ sampling, traverses were performed through three ports located in the flue gas ductwork between the air heater outlet and the Turbosorp ${ }^{\circledR}$ inlet, just upstream of the activated carbon injection point. Each port was sampled at three points during the testing in late March and at four points during the testing in early May (if applicable), resulting in nine- and twelve-point traverses, respectively. Test durations were 55-63 minutes for $\mathrm{HCl}$ and $\mathrm{HF}$, and 126 minutes for mercury. All $\mathrm{HCl}, \mathrm{HF}$, and mercury sampling was performed isokinetically.

For the March 29 testing, $\mathrm{SO}_{3}$ samples were drawn at a constant rate from a single point in one port located in the air heater outlet duct, just upstream of the activated carbon injection point. Each $\mathrm{SO}_{3}$ test at the air heater outlet on March 29 was 40 minutes in duration. For the May 2 testing, $\mathrm{SO}_{3}$ samples were drawn via a twelvepoint traverse, as described above for $\mathrm{HCl}$ and $\mathrm{HF}$. The $\mathrm{SO}_{3}$ sampling was performed at a constant rate, rather than isokinetically, however. Each $\mathrm{SO}_{3}$ test at the air heater outlet on May 2 was 60 minutes in duration.

Sulfur dioxide samples were drawn from a single port located immediately downstream of the three ports described above. For the first two tests on March 29, the sample was drawn from a single point in this port. For the third test on that day, a three-point traverse was performed through the same port. Each $\mathrm{SO}_{2}$ test was 60 minutes in duration.

\section{Baghouse Outlet}

Sulfur dioxide samples were also drawn from a single port located in the verticallyoriented ductwork between the baghouse outlet and the booster fan inlet. This sampling location is shown in Figure 6. For the first two tests on March 29, the sample was drawn from a single point in this port. For the third test on that day, a three-point traverse was performed through the same port. Each $\mathrm{SO}_{2}$ test was 60 minutes in duration.

\section{Stack}

$\mathrm{SO}_{3}, \mathrm{HCl}, \mathrm{HF}$, and $\mathrm{Hg}$ tests were conducted at the stack, which is shown in Figure 7. (As discussed above, measurements made by the unit's stack CEM were also used in determining attainment of the performance targets for $\mathrm{NO}_{x}$ and $\mathrm{SO}_{2}$ ). For $\mathrm{HCl}, \mathrm{HF}$, and $\mathrm{Hg}$ sampling, traverses were performed through four ports located at the 83-foot level platform. Each port was sampled at four points during each test, resulting in a sixteenpoint traverse, as shown in Figure 8. Test durations were 64 minutes for $\mathrm{HCl}$ and $\mathrm{HF}$, 
and 120 minutes for mercury. All $\mathrm{HCl}, \mathrm{HF}$, and mercury sampling was performed isokinetically.

For the March 29 testing, $\mathrm{SO}_{3}$ samples were drawn at a constant rate from a single point in one of the four ports at the 83-foot level. Each $\mathrm{SO}_{3}$ test at the stack on March 29 was 60 minutes in duration. For the May 2 testing, $\mathrm{SO}_{3}$ samples were drawn via a sixteen-point traverse, as described above for the other parameters. The $\mathrm{SO}_{3}$ sampling was performed at a constant rate, rather than isokinetically, however. Each $\mathrm{SO}_{3}$ test at the stack on May 2 was 90 minutes in duration.

\section{Flue Gas Measurements}

\section{Oxides of Nitrogen}

$\mathrm{NO}_{x}$ measurements at the SCR inlet and SCR outlet were performed by Clean Air Engineering using U.S. EPA Method 7E, modified to incorporate the use of CAE's Multipoint Automated Sampling System (MASS). A complete description of the methodology is provided in the CAE report that is included as Appendix $A$ to this report. $\mathrm{NO}_{\mathrm{x}}$ measurements at the stack were made using the unit's stack CEM.

\section{Ammonia}

Flue gas ammonia concentrations were measured in accordance with the procedures specified in U.S. EPA Conditional Test Method (CTM) 027 . Because of space constraints at the sampling locations, however, complete isokinetic traverses could not be completed (as discussed above, sampling was conducted using an incomplete traverse at an isokinetic sampling rate at the SCR outlet and at a constant sampling rate at the air heater inlet), and a heated Teflon sample line had to be used to connect the sampling probe to the impingers. Given the presence of $\mathrm{SO}_{2}$ and $\mathrm{SO}_{3}$ in the flue gas and the temperature of the heated line (ca. $250{ }^{\circ} \mathrm{F}$ ), some of the collected ammonia was expected to condense out of the sample stream as ammonium bisulfate in the Teflon line, before reaching the impingers. Hence, the contents of line were collected by rinsing with deionized water, and this line rinse was analyzed to account for the ammonia collected there. The sampling procedure is described in more detail below.

Samples were collected by pulling flue gas through a temperature-controlled quartzlined probe and an in-stack filter assembly. The probe temperature was maintained at ca. $250{ }^{\circ} \mathrm{F}$. Upon exiting the probe, the flue gas passed through a heated Teflon sample line (maintained at ca. $250{ }^{\circ} \mathrm{F}$ ) to the impinger train, where it flowed through a series of chilled impingers. The first two impingers were Greenburg-Smith design, each containing $100 \mathrm{~mL}$ of a 0.1 normal $(\mathrm{N})$ sulfuric acid $\left(\mathrm{H}_{2} \mathrm{SO}_{4}\right)$ solution. The third impinger, also containing $0.1 \mathrm{~N} \mathrm{H}_{2} \mathrm{SO}_{4}$, was a Greensburg-Smith with the tip removed and was used to evaluate $\mathrm{NH}_{3}$ breakthrough. The final impinger, a Greensburg-Smith without the tip, was filled with approximately $200 \mathrm{~g}$ of silica desiccant for moisture removal. After exiting the impingers, the gas sample passed through a dry test meter where its volume was measured. The $\mathrm{O}_{2}$ concentration in the gas exiting the dry test meter was monitored using a Teledyne Max $\mathrm{V}$ portable electrochemical $\mathrm{O}_{2}$ analyzer. 
Prior to sampling, the system was leak checked under a vacuum of approximately 10" of $\mathrm{Hg}$. The sample probe was placed at the proper location in the sample port; the sample port opening was sealed, and gas was sampled for 40-60 minutes. The following data were recorded: (1) starting gas volume, (2) interval gas volume, (3) final gas volume, (4) probe temperature, (5) heated line temperature, (6) meter differential pressure setting, (7) dry test meter temperature, (8) system vacuum, (9) exit gas $\mathrm{O}_{2}$ concentration, (10) barometric pressure, and (11) sampling time. Copies of the field data sheets are provided in Appendix C. After sampling, the probe was removed from the duct, leak checked under a vacuum equal to or greater than the highest vacuum observed during testing, and the train components were disassembled for sample recovery. The sample train components were recovered in the following manner:

Sample Probe and In-Stack Filter Assembly - The quartz probe liner was rinsed with deionized water, and the rinse was collected in an approved sample container. The filter was collected, but not analyzed, and the filter assembly was cleaned with deionized water. (The filter assembly rinse was not recovered). The probe rinse was diluted to a known volume and kept refrigerated prior to analysis.

Heated line - The heated Teflon line was rinsed with deionized water, and the rinse was collected in an approved sample container. The rinse was then diluted to a known volume and kept refrigerated prior to analysis.

Impingers - The contents of the first three impingers and connecting glassware (including rinses of these sample train components with deionized water) were collected in approved sample containers. These samples were then diluted to known volumes and kept refrigerated prior to analysis.

Samples were analyzed for ammonium ion by ion chromatography per CTM 027 . The concentration of ammonia in the flue gas was calculated based on the mass of ammonium ion collected (sum of masses determined in the probe, heated line, and impingers) and the volume of flue gas sampled during the test.

\section{Sulfur Dioxide}

$\mathrm{SO}_{2}$ measurements at the air heater outlet and baghouse outlet were performed by Clean Air Engineering using U.S. EPA Method 6C. A complete description of the methodology is provided in the CAE report that is included as Appendix A to this report. $\mathrm{SO}_{2}$ measurements at the stack were made using the unit's stack CEM.

\section{Sulfur Trioxide}

Flue gas $\mathrm{SO}_{3}$ concentrations were determined using a controlled condensation method originally developed by the U.S. EPA and modified by CONSOL R\&D. In this method, flue gas is pulled through a temperature-controlled quartz-lined probe that is fitted with a quartz wool plug to remove particulate matter. The probe temperature is maintained at ca. $550{ }^{\circ} \mathrm{F}$ to minimize $\mathrm{SO}_{3}$ condensation and $\mathrm{SO}_{2}$ oxidation. After the filter, the gas sample passes through a $140{ }^{\circ} \mathrm{F}$, temperature-controlled condenser that is loosely packed with glass wool. Essentially all of the $\mathrm{SO}_{3}$ is collected in the condenser. The sample gas exits the condenser and enters a series of miniature impingers. The first 
two impingers contain a $3 \% \mathrm{H}_{2} \mathrm{O}_{2}$ solution, which captures the $\mathrm{SO}_{2}$. The gas next passes through an empty impinger, and finally through a silica gel-filled impinger for moisture removal. The gas is then conveyed through a rotameter, a vacuum pump, and a dry test meter. The $\mathrm{O}_{2}$ concentration in the gas exiting the dry test meter is monitored using a Teledyne Max $\mathrm{V}$ portable electrochemical $\mathrm{O}_{2}$ analyzer.

Prior to sampling, the system is leak checked under a vacuum of 10 " of $\mathrm{Hg}$. The sample probe is then positioned at the proper location and gas is sampled for at least 30 minutes. The following data are recorded: (1) starting gas volume, (2) interval gas volume, (3) final gas volume, (4) probe temperature, (5) condenser temperature, (6) water bath temperature, (7) flue gas duct temperature, (8) dry test meter temperature, (9) flow meter setting, (10) system vacuum, (11) exit gas $\mathrm{O}_{2}$ concentration, (12) barometric pressure, and (13) sampling time. Copies of the field data sheets are provided in Appendix C. After sampling, the probe is removed from the duct, leak checked under a vacuum of 10 " of $\mathrm{Hg}$, purged with ambient air for 10 minutes, and the train components are disassembled for sample recovery.

During the $\mathrm{SO}_{3}$ tests on March 29, the sample train components were recovered using isopropyl alcohol (IPA) rinses and analyzed by $\mathrm{BaCl}_{2}$ titration in the conventional way. The quartz plug was removed from the probe tip, placed in a glass bottle, and extracted with $20 \mathrm{~mL}$ of isopropyl alcohol . The solids were filtered and the filtrate was diluted to a known volume prior to analysis. The quartz probe liner was rinsed with IPA into a glass bottle and diluted to a known volume. The condenser interior was also rinsed (via three complete rinses) with IPA into a glass bottle and the rinses were diluted to a known volume prior to analysis. Each of these samples was analyzed by a $\mathrm{BaCl}_{2}$ titration to a thorin endpoint as described in EPA Method 6. A blank IPA sample was titrated with the same $\mathrm{BaCl}_{2}$ titrant for comparison. The quartz plug contains $\mathrm{SO}_{3}$ that was absorbed onto the solid particles prior to collection in the sampling train. The gas phase $\mathrm{SO}_{3}$ value is the sum of the probe and condenser rinses. In most cases, the majority of the gas phase $\mathrm{SO}_{3}$ is found in the condenser rinse. The $\mathrm{SO}_{3}$ values are reported in ppmvd at duct conditions and at 3\% oxygen. For the testing at AES Greenidge, the contents of the miniature impingers were not analyzed, because $\mathrm{SO}_{2}$ concentrations were measured using continuous emission monitors.

As discussed earlier in this report, it is possible that ammonia in the sampled flue gas interfered with the $\mathrm{BaCl}_{2}$ titrations for some or all of the $\mathrm{SO}_{3}$ samples collected on March 29, potentially biasing the results. Therefore, to avoid possible $\mathrm{NH}_{3}$ interference, the $\mathrm{SO}_{3}$ samples collected on May 2 were analyzed by ion chromatography rather than by $\mathrm{BaCl}_{2}$ titration. IPA poses a problem for the IC analysis; hence, samples were recovered using deionized water instead of IPA.

\section{Hydrogen Chloride and Hydrogen Fluoride}

Flue gas acid gas measurements were obtained using a combined U.S. EPA Method $17 / 26$ A sampling train. In this method, gas is extracted isokinetically from the flue gas stream through an in-stack filter assembly, heated glass-lined probe, and heated sample line. Probe and line temperatures are maintained at $\sim 250^{\circ} \mathrm{F}$. 
The flue gas exits the sample line and passes through a series of chilled impingers. The first two impingers are Greenburg-Smith design, each containing $100 \mathrm{~mL}$ of a 0.1 normal sulfuric acid $\left(\mathrm{H}_{2} \mathrm{SO}_{4}\right)$ solution that collects the hydrogen halide portion of the sample, which is solubilized in the acidic solution and forms chloride $\left(\mathrm{Cl}^{-}\right)$and fluoride $\left(\mathrm{F}^{-}\right)$ions. Because reductions in emissions of $\mathrm{Cl}_{2}$ and $\mathrm{F}_{2}$ were not targeted in the program at AES Greenidge, the Method 26A impinger train was altered to exclude the impingers specified for capturing those compounds. The next impinger is initially empty to catch any excess moisture. The gas exits the impinger train through a silica gel-filled impinger that removes uncondensed moisture from the sample gas.

The sampling train design results in the following collection sequence:

\begin{tabular}{lc} 
Sampling Train Component & Species Measured \\
\hline Probe and Sample Line Rinse & $\mathrm{HCl} \& \mathrm{HF}$ \\
Impingers & $\mathrm{HCl} \& \mathrm{HF}$
\end{tabular}

Sampling was performed isokinetically. Oxygen readings were monitored at the outlet of the sampling train using a Teledyne Model Max $\mathrm{V}$ portable electrochemical $\mathrm{O}_{2}$ analyzer. Copies of the field data sheets are provided in Appendix $\mathrm{C}$. The $\mathrm{Cl}^{-}$and $\mathrm{F}^{-}$ concentrations of the impinger solutions were determined by ion chromatography as specified in EPA Method 26A. Copies of the laboratory analyses are provided in Appendix D.

\section{Mercury}

Flue gas mercury measurements were performed using the Ontario-Hydro $\mathrm{Hg}$ speciation method (ASTM D 6784-02). Gas was extracted isokinetically from the flue gas stream through an in-stack filter assembly, heated glass-lined probe, and heated sample line. Mercury collected in the filter is assumed to be particle-bound $\mathrm{Hg}\left(\mathrm{Hg}^{\text {part }}\right)$. The flue gas exits the sample line and passes through a series of chilled impingers. The first three impingers are filled with $100 \mathrm{~mL}$ of a $1 \mathrm{M}$ potassium chloride $(\mathrm{KCl})$ solution. Mercury captured in these impingers and in the probe and sample line, which are rinsed with $\mathrm{HNO}_{3}$ and $\mathrm{HCl}$, is reported as oxidized $\mathrm{Hg}\left(\mathrm{Hg}^{++}\right)$. The next impinger is filled with $100 \mathrm{~mL}$ of $5 \%$ nitric acid and $10 \% \mathrm{H}_{2} \mathrm{O}_{2}$ solution to remove $\mathrm{SO}_{2}$ from the flue gas and preserve the oxidizing strength of the subsequent permanganate impingers. The next two impingers are filled with $100 \mathrm{~mL}$ of an acidic potassium permanganate $\left(\mathrm{KMnO}_{4}\right)$ solution. Mercury captured in the nitric acid impinger and the potassium permanganate impingers is reported as elemental mercury $\left(\mathrm{Hg}^{0}\right)$. The next impinger is blank to catch any excess moisture. The gas exits the impinger train through a silica gel-filled impinger that removes uncondensed moisture from the sample gas.

Oxygen readings were monitored at the outlet of the sampling train using a Teledyne Max $\mathrm{V}$ portable electrochemical $\mathrm{O}_{2}$ analyzer. Copies of the field data sheets are provided in Appendix C.

The $\mathrm{Hg}$ concentration of the individual impinger solutions and sampling component rinses was determined by cold vapor atomic absorption spectroscopy (CVAA) as specified in the Ontario-Hydro Method. Analyses were conducted using a Thermo 
Unicam 969 CVAA. The concentration of $\mathrm{Hg}$ on the filter solids was determined according to the procedures described in ASTM D 6414 or ASTM D 6722.

\section{Solid and Liquid Process Sample Analyses}

Solid and liquid process samples were analyzed using the following methods:

ASTM D 2013 Preparing Coal Samples for Analysis. This standard practice covers the reduction and division of gross or divided samples up to and including the individual portions used for laboratory analysis.

ASTM D 5142 Proximate Analysis of the Analysis Sample of Coal and Coke by Instrumental Procedures. Moisture, volatile matter and ash are determined sequentially in a single instrumental procedure by establishing the loss in mass of the analysis specimen when heated under rigidly controlled conditions of temperature, time, atmosphere, and specimen mass.

ASTM D 5373 Instrumental Determination of Carbon, Hydrogen, and Nitrogen in Laboratory Samples of Coal and Coke. Carbon, Hydrogen, and Nitrogen are determined concurrently in a single instrumental procedure. The conversion of the subject materials in each sample occurs during combustion of the sample at an elevated temperature in an atmosphere of purified oxygen.

ASTM D 4239 Sulfur in the Analysis Sample of Coal and Coke Using HighTemperature Combustion and Infrared Absorption. The test specimen is heated in a tube furnace in a stream of oxygen to oxidize the sulfur to sulfur dioxide. The gas stream containing the sulfur dioxide is passed through a cell where it is measured at a precise wavelength by an infrared absorption detector.

ASTM D 5865 Gross Calorific Value of Coal and Coke. A weighed sample of coal is burned under controlled conditions in an oxygen bomb calorimeter. The higher heating value is calculated from the temperature rise of the water in the calorimeter vessel and the effective heat capacity of the system. Corrections are made for the heat released by the ignition of the fuse and the thermochemical reactions forming nitric and sulfuric acids.

ASTM D 6721 Determination of Chlorine by Oxidative Hydrolysis Microcoulometry. A weighed sample is combusted with tungsten accelerator in a humidified oxygen gas flow, at $900{ }^{\circ} \mathrm{C}$. Halogens are oxidized and converted to hydrogenated halides, which are flushed into a titration cell where they accumulate. Chlorine is converted to hydrochloric acid. Once the chloride is captured in the electrolyte of the titration cell, it can be quantitatively determined by microcoulometry.

ASTM D 6349 Determination of Major and Minor Elements in Coal, Coke, and Solid Residues from Combustion of Coal and Coke by Inductively Coupled Plasma-Atomic Emission Spectrometry. The sample to be analyzed is ashed under standard 
conditions and ignited to a constant weight. The ash is digested in a mixture of hydrofluoric, nitric, and hydrochloric acids. The solution is then analyzed by ICP-AES, in which characteristic line emission spectra are produced by a radio-frequency inductively coupled plasma. The intensity of these emissions is proportional to the concentration of analyte in the sample.

ASTM D 3302 Standard Test Method for Total Moisture in Coal. All of the moisture in and on a sample of coal is determined based on the loss of weight in a coal sample in an air atmosphere under rigidly controlled conditions of temperature, time, and air flow.

ASTM D 5987 Total Fluorine in Coal and Coke by Pyrohydrolytic Extraction and Ion Chromatography. A weighed sample is subjected to pyrohydrolytic combustion conditions. Fluorine is quantitatively released from the sample matrix and retained in the pyrohydrolysate that is gravimetrically processed. The total concentration in the sample is then determined by ion chromatography.

ASTM D 6722 Total Mercury in Coal and Coal Combustion Residues by Direct Combustion Analysis. The analysis sample is heated under oxidative conditions and chemically decomposed. Flowing oxygen carries the decomposition products to a gold amalgamator that selectively traps mercury. The amalgamator is rapidly heated, releasing mercury vapor that is carried through an atomic absorption sprectrophotometer. Mercury concentration is measured as a function of absorbance peak area.

ASTM D 5967 Digestion with ASTM D 6357 Analysis for the Determination of Se. A weighed sample is subjected to pyrohydrolytic combustion conditions. Selenium is quantitatively released from the sample matrix and subsequently analyzed by inductively coupled plasma mass spectrometry (ICPMS).

$\mathrm{SM} 4500-\mathrm{H}$ B for the Determination of $\mathrm{pH}$. The activity of hydrogen ions is determined by potentiometric measurement using a standard hydrogen electrode and a reference electrode.

SM 2540D for the Determination of Total Suspended Solids. A well mixed sample is filtered through a weighed standard glass-fiber filter and the residue retained on the filter is dried to a constant weight. The increase in the weight of the filter represents the total suspended solids.

SM 4500-NH3 E for the Determination of Ammonia. An ammonia selective electrode equipped with a hydrophobic gas permeable membrane separates the sample solution from an electrode internal solution of ammonium chloride. Ammonia diffuses through the membrane and potentiometric measurements are subsequently made by an ion selective electrode. 
SM 3112B for the Determination of Mercury. The mercury in an aqueous sample is reduced to vaporous elemental mercury and is determined by cold vapor atomic absorption or by cold vapor atomic fluorescence.

SM 4110B for the Determination of Anions by lon Chromatography. Phosphate in a portion of test solution is determined by ion chromatography with chemical suppression of eluent conductivity.

Density using the Paar Digital Density Meter DMA35. The sample is passed through a vibrating tube. The sample density is proportional to the period of vibration in the tube.

In addition to the methods listed above, which were used by CONSOL R\&D to analyze solid and liquid process samples, CTL Group analyzed pebble lime, hydrated lime, and Turbosorp ${ }^{\circledR} /$ baghouse product ash samples by the following methods (see Appendix B for additional details):

Pebble Lime

Available $\mathrm{CaO}$

ASTM C 25, Section 28

Slaking Rate / Residue

ASTM C 110-05, Section 11

Hydrated Lime

Density

Particle Size Distribution

Surface Area

ASTM C 110, Sections 19 and 20

Laser Diffraction (wet dispersion in isopropanol)

$\mathrm{BET}\left(\mathrm{N}_{2}\right.$, single point)

Product Ash

Particle Size Distribution Laser Diffraction (wet dispersion in isopropanol)

\section{QUALITY ASSURANCE AND QUALITY CONTROL}

\section{Test Equipment}

The CONSOL field sampling team uses standard EPA-type sampling equipment. The majority of the equipment used in the manual sampling methods was obtained from Thermo-Andersen (formerly known as Andersen Instruments, Graseby-Nutech, and Nutech) and conforms to all applicable test codes. CONSOL used a Mettler electronic balance for pre- and post-test dust weights. This balance is checked daily with class "S" calibration weights.

All sampling trains were leak checked prior to each test and again at the end of each test. All sample trains, with the exception of CTM 027 sample trains, were purged with air for ten minutes and then disassembled and transported to the lab trailer for recovery.

A Teledyne Max $\mathrm{V}$ portable gas analyzer was used for determining flue gas composition at the sample train discharge. The meter operator uses the readings to 
assess the operation of the sampling train and the unit operation. Past experience has indicated the accuracy of the oxygen determination by this analyzer is equal to or better than that of a manual Orsat analyzer.

\section{Oxides of Nitrogen Measurements}

Quality assurance / quality control information for the $\mathrm{NO}_{x}$ measurements at the SCR inlet and outlet is provided in the Clean Air Engineering report that is included as Appendix A to this report.

\section{Ammonia Measurements}

Although not required by method, samples were run in duplicate by ion chromatography. The average result for each sample was reported. In all cases, the duplicate results had a relative percent difference of $\leq 10 \%$. An independent quality control standard with a concentration of approximately the midpoint of the calibration curve range was run immediately after calibration and after every 10 samples. In all cases, recovery of the quality control standard was between $90 \%$ and $110 \%$. One sample per batch was spiked at $2 \mathrm{mg} / \mathrm{L} \mathrm{NH}_{3}$ as $\mathrm{N}$ to assess any possible matrix issues; none were discovered. Blank samples of the deionized water and $0.1 \mathrm{~N} \mathrm{H}_{2} \mathrm{SO}_{4}$ reagents used for sample recovery were also run to confirm that their ammonia content was less than the analytical limit of detection $\left(0.1 \mathrm{mg} / \mathrm{L} \mathrm{NH}_{3}\right.$ as $\left.\mathrm{N}\right)$.

\section{Sulfur Dioxide Measurements}

Quality assurance / quality control information for the $\mathrm{SO}_{2}$ measurements at the air heater outlet and baghouse outlet is provided in the Clean Air Engineering Report that is included as Appendix $A$ to this report.

\section{Sulfur Trioxide Measurements}

For the IC analyses, immediately after calibration of the ion chromatograph, an independent calibration verification (ICV) sample was analyzed in duplicate. Recovery of the ICV sample met the $100 \pm 10 \%$ criterion, and the duplicate results had a relative percent difference of $\leq 5 \%$. An independent, secondary QC standard was analyzed in duplicate after every $10^{\text {th }}$ sample. In all cases, recovery of the secondary QC standard was $100 \pm 10 \%$, and replicate results agreed within $5 \%$. All samples were run in duplicate. If duplicate results were within $5 \%$ of each other, then the average of the duplicates was reported. If results did not agree within $5 \%$, the samples were run again in duplicate and the average of the four replicates was reported. A blank sample of the deionized water used for sample recovery was also included in the run to verify that background $\mathrm{SO}_{4}{ }^{2-}$ concentrations were low.

\section{Hydrogen Chloride and Hydrogen Fluoride Measurements}

$\mathrm{HCl}$ and $\mathrm{HF}$ samples were analyzed per EPA Method 26A. Immediately after calibration of the ion chromatograph, an ICV sample was analyzed in duplicate. Recovery was $100 \pm 10 \%$ for both $\mathrm{Cl}^{-}$and $\mathrm{F}^{-}$, and the duplicate results had relative percent differences of $\leq 5 \%$ for both species. An independent, secondary QC sample 
was analyzed in duplicate after every 10 samples. In all cases, recovery of $\mathrm{Cl}^{-}$and $\mathrm{F}^{-}$ in the secondary QC standard was $100 \pm 10 \%$, and replicate results agreed within $5 \%$. All samples were run in duplicate. If duplicate results agreed within $5 \%$, then the average of the duplicates was reported. If results were not within $5 \%$ of one another, the samples were run again in duplicate and the average of the 4 replicates was reported, per Method 26A. Although not required by method, one sample per batch was spiked at $2 \mathrm{mg} / \mathrm{L} \mathrm{Cl}^{-}$and $2 \mathrm{mg} / \mathrm{L} \mathrm{F}^{-}$to assess any possible matrix issues; none were discovered. A blank sample of the $0.1 \mathrm{~N} \mathrm{H}_{2} \mathrm{SO}_{4}$ impinger solution was also analyzed by ion chromatography. Any blank value in excess of the detection limit (0.2 $\mathrm{mg} / \mathrm{L}$ for both $\mathrm{Cl}^{-}$and $\mathrm{F}^{-}$) was subtracted from the $\mathrm{HCl}$ or $\mathrm{HF}$ analysis results, as permitted by EPA Method 26A.

\section{Mercury Measurements}

Samples were prepared and analyzed as outlined in ASTM D 6784-02 (Ontario Hydro Method). Analysis was completed on a Thermo Unicam 969 CVAA, which has a detection limit of $0.14 \mu \mathrm{g} / \mathrm{L}$. $R^{2}$ values for all instrument calibration curves were $>0.999$. An independent calibration verification sample, NIST SRM 1641D, prepared to an $\mathrm{Hg}$ concentration of $4.0 \mu \mathrm{g} / \mathrm{L}$, was analyzed immediately after calibration. If recovery of $\mathrm{Hg}$ in the ICV was not within $100 \pm 10 \%$, then the analyzer was re-calibrated before proceeding. The ICV was reanalyzed after every $10^{\text {th }}$ sample; if it did not satisfy the $100 \pm 10 \%$ criterion, then the run was stopped, the analyzer was recalibrated, and the affected samples were reanalyzed. All samples were run in duplicate with an acceptance criterion of $10 \%$ relative percent difference. (Four samples were slightly above 10\% RPD). One in 10 samples was analyzed in triplicate with an acceptance criterion of $10 \%$ relative standard deviation. Matrix spikes were included at a 1-in-10 sample frequency with an acceptance criterion of $100 \pm 10 \%$ spike recovery. Although not required by ASTM D 6784-02, (3) digestion duplicates and (3) digestion spikes were included to assess the efficiency of the digestions.

Particulate-impregnated filters and blank filter thimbles were digested according to ASTM D 6414 and then analyzed in duplicate with an acceptance criterion of 10\% relative percent difference. Matrix spikes were included at a 1-in-10 sample frequency, with an acceptance criterion of $100 \pm 10 \%$ spike recovery. NIST SRM 1633B was digested and analyzed with the batch of filters; recovery of $\mathrm{Hg}$ in the SRM was required to be $100 \pm 10 \%$. Loose particulate from filter thimbles was analyzed according to ASTM D 6722. One out of every ten samples was analyzed in duplicate with an acceptance criterion of $10 \%$ relative percent difference. Again, a sample of NIST SRM 1633B was analyzed with the batch of samples; $100 \pm 10 \%$ recovery of $\mathrm{Hg}$ in the SRM was required.

\section{Mercury Material Balance}

Mercury material balances were performed to confirm the quality of the mercury measurements made on March 28 and 30. These material balances, which were performed independently for each test run, compare the total amount of mercury leaving the process during the test with the total amount of mercury entering the 
process during the test. The mercury material balance closure is the total mercury output from the process divided by the total mercury input, expressed as a percentage.

For AES Greenidge Unit 4, potential mercury input streams include the coal, urea, activated carbon (on March 30 ), process water, and hydrated lime. Potential mercury output streams include the bottom ash, Turbosorp ${ }^{\circledR}$ hopper ash, Turbosorp ${ }^{\circledR} /$ baghouse product ash, and stack flue gas.

For purposes of the $\mathrm{Hg}$ material balances, the amount of mercury entering or exiting the process via the urea, activated carbon, process water, bottom ash, and Turbosorp ${ }^{\circledR}$ hopper ash streams was assumed to be negligible. Based on mercury concentrations determined in the urea samples that were collected during each of the mercury tests performed on March 28 and 30 (all less than the analytical detection limit of $0.35 \mu \mathrm{g} / \mathrm{L}$ ) and the average urea feed rate recorded by the plant during these test periods $(47 \mathrm{gal} / \mathrm{h}$, or $178 \mathrm{~L} / \mathrm{h}$ ), the urea contributed less than $0.01 \%$ of the total $\mathrm{Hg}$ input to the process. The activated carbon also contributed less than $0.01 \%$ of the total $\mathrm{Hg}$ input, based on the $\mathrm{Hg}$ concentration of $0.005 \mathrm{ppm}$ determined in activated carbon samples collected during testing and on the design carbon injection rate of 89 $\mathrm{lb} / \mathrm{h}$. Process water samples were not collected during the tests on March 28 and 30. However, even if the $\mathrm{Hg}$ concentration in the process water equaled the EPA drinking water standard of $2 \mu \mathrm{g} / \mathrm{L}$ (likely a high estimate), the process water would have contributed less than $2 \%$ of the total $\mathrm{Hg}$ input to the process, assuming the design process water flow rate of $121 \mathrm{gal} / \mathrm{h}$ (combination of urea dilution water and water injected into the Turbosorp ${ }^{\circledR}$ system). Bottom ash samples also were not collected during the testing on March 28 and 30; hence, the exact $\mathrm{Hg}$ content of the bottom ash is not known. However, during baseline testing at AES Greenidge in November 2004, the bottom ash contained less than $0.1 \%$ of the total $\mathrm{Hg}$ output from the unit. Finally, the flow rate of ash leaving the Turbosorp ${ }^{\circledR}$ hopper is not known, but it is insignificant relative to the flow rate of Turbosorp ${ }^{\circledR} /$ baghouse product ash leaving the process. The concentration of $\mathrm{Hg}$ in the Turbosorp ${ }^{\circledR}$ hopper ash was also only about $35 \%$ as great as that in the Turbosorp ${ }^{\circledR} /$ baghouse product ash. Hence, it is reasonable to assume that the urea, activated carbon, process water, bottom ash, and Turbosorp ${ }^{\circledR}$ hopper ash were not significant sources or sinks of mercury.

Methods used to measure or estimate the amount of mercury fed to or removed from the system via the remaining process streams are summarized below.

\section{Mercury Input from Coal}

Coal feed rates during the tests were recorded and provided by the plant. Coal samples were collected at approximately the beginning and middle of each test; the samples from each test were composited and analyzed for mercury. The mercury input from coal was computed as the product of the coal feed rate and the mercury concentration in the coal.

Mercury Input from Hydrated Lime

The mass flow rate of hydrated lime being fed to the Turbosorp ${ }^{\circledR}$ absorber vessel was estimated for each test by performing a calcium balance around the Turbosorp ${ }^{\circledR}$ system. The mass flow rate of $\mathrm{Ca}$ entering the Turbosorp ${ }^{\circledR}$ system via the fly ash was 
estimated from the coal feed rate, coal ash content, and coal ash composition; per the assumptions made during baseline testing, which were based on historic plant data, we assumed that $90.5 \%$ of the coal ash is converted to fly ash. The mass flow rate of Ca exiting the Turbosorp ${ }^{\circledR}$ system via the product ash was estimated from the product ash mass flow rate (estimated as described below) and the Ca content of the product ash sample(s) collected during the test. The required mass flow rate of $\mathrm{Ca}$ in the hydrated lime stream was then calculated by subtracting the flow rate of $\mathrm{Ca}$ in the fly ash stream from the flow rate of $\mathrm{Ca}$ in the product ash stream, and the total mass flow rate of hydrated lime was computed by dividing this result by the weight percentage of $\mathrm{Ca}$ determined in the hydrated lime sample collected during the test. To compute the mercury input from hydrated lime, the mercury concentration determined in the hydrated lime sample was multiplied by the estimated hydrated lime mass flow rate.

\section{Mercury Output via Product Ash}

The mass flow rate of Turbosorp ${ }^{\circledR} /$ baghouse product ash being discharged from the process was estimated for each test by performing a sulfur balance around the Turbosorp ${ }^{\circledR}$ system. The mass flow rate of sulfur entering the Turbosorp ${ }^{\circledR}$ system was estimated from the coal feed rate and coal sulfur content measured during the test, and the mass flow rate of sulfur exiting the system via the stack flue gas was calculated using the average stack flue gas flow rate and $\mathrm{SO}_{2}$ concentration measured during the test. $\left(\mathrm{SO}_{2}\right.$ concentrations were obtained from the plant's stack $\mathrm{CEM}$, and flue gas flow rates were computed as the average of values measured by the stack CEM and by CONSOL as part of the Ontario Hydro method. Plant flow rate measurements were converted to a dry basis using flue gas moisture concentrations determined as part of the Ontario Hydro method). The required mass flow rate of sulfur in the product ash stream was then calculated by subtracting the flow rate of sulfur in the stack flue gas stream from the flow rate of sulfur entering the Turbosorp ${ }^{\circledR}$ system, and the total mass flow rate of product ash was computed by dividing this result by the weight percentage of sulfur determined in the product ash sample(s) collected during the test. To compute the mercury output via product ash, the mercury concentration determined in the product ash sample(s) was multiplied by the estimated product ash mass flow rate.

\section{Mercury Output via Stack Flue Gas}

The mercury output via the stack flue gas was measured using the Ontario Hydro method. For all tests, the concentration of mercury in the flue gas was less than the detection limit of the method; for purposes of the $\mathrm{Hg}$ material balances, the $\mathrm{Hg}$ concentration was assumed to be equal to the detection limit value.

Mercury material balance results for the tests conducted on March 28 and March 30 are summarized in Tables 21 and 22, respectively. For the tests performed on March 28 , during which no activated carbon was being injected into the system, the material balance closures ranged from $93-109 \%$ for the individual tests, with a three-test average of $101 \%$. For the tests performed on March 30, during which activated carbon was being injected into the system, the material balance closures ranged from $119-121 \%$ for the individual tests, with a three-test average of $120 \%$. CONSOL's QA/QC criterion for material balance closure for a single test is $100 \pm 30 \%$. The 
criterion for a three-test average is $100 \pm 20 \%$. Hence, all of the tests on both days satisfied these criteria.

Table 21. Summary of Material Balance Closure for $\mathrm{Hg}$ Measurements Performed on March 28, 2007

\begin{tabular}{|c|c|c|c|c|}
\hline CONSOL Test No. & 1 & 2 & 3 & Average \\
\hline $\mathrm{Hg}$ Input from Coal (lb/h) & 7.45E-03 & 8.30E-03 & 7.01E-03 & 7.59E-03 \\
\hline $\mathrm{Hg}$ Input from Hydrated Lime (lb/h) & 4.98E-05 & $5.03 \mathrm{E}-05$ & 4.85E-05 & 4.95E-05 \\
\hline Total Hg Input (lb/h) & $7.50 \mathrm{E}-03$ & 8.35E-03 & 7.05E-03 & 7.63E-03 \\
\hline Hg Output in Product Ash (lb/h) & $7.18 \mathrm{E}-03$ & $7.42 \mathrm{E}-03$ & 7.29E-03 & 7.30E-03 \\
\hline Hg Output in Stack Gas (Ib/h) & $3.41 \mathrm{E}-04^{\mathrm{a}}$ & $3.59 \mathrm{E}-04^{\mathrm{a}}$ & $3.89 \mathrm{E}-04^{\mathrm{a}}$ & $3.63 E-04^{a}$ \\
\hline Total Hg Output (lb/h) & $7.52 \mathrm{E}-03$ & 7.77E-03 & 7.68E-03 & 7.66E-03 \\
\hline $\begin{array}{l}\text { Hg Material Balance Closure } \\
\text { (output / input) = }\end{array}$ & $100 \%$ & $93 \%$ & $109 \%$ & $101 \%$ \\
\hline
\end{tabular}

${ }^{a}$ Measured to be less than detection limit; for this calculation, assumed to equal detection limit.

Table 22. Summary of Material Balance Closure for $\mathrm{Hg}$ Measurements Performed on March 30, 2007

\begin{tabular}{||c|c|c|c|c||}
\hline \multicolumn{1}{|c|}{ CONSOL Test No. } & 4 & 5 & 6 & Average \\
\hline \hline Hg Input from Coal (lb/h) & $7.23 \mathrm{E}-03$ & $6.79 \mathrm{E}-03$ & $6.64 \mathrm{E}-03$ & $6.88 \mathrm{E}-03$ \\
\hline Hg Input from Hydrated Lime (Ib/h) & $6.54 \mathrm{E}-05$ & $5.73 \mathrm{E}-05$ & $4.63 \mathrm{E}-05$ & $5.61 \mathrm{E}-05$ \\
\hline Total Hg Input (Ib/h) & $7.29 \mathrm{E}-03$ & $6.84 \mathrm{E}-03$ & $6.68 \mathrm{E}-03$ & $6.94 \mathrm{E}-03$ \\
\hline Hg Output in Product Ash (Ib/h) & $8.25 \mathrm{E}-03$ & $7.74 \mathrm{E}-03$ & $7.63 \mathrm{E}-03$ & $7.88 \mathrm{E}-03$ \\
\hline Hg Output in Stack Gas (Ib/h) & $4.08 \mathrm{E}-04^{\mathrm{a}}$ & $4.19 \mathrm{E}-04^{\mathrm{a}}$ & $4.31 \mathrm{E}-04^{\mathrm{a}}$ & $4.19 \mathrm{E}-04^{\mathrm{a}}$ \\
\hline \multicolumn{1}{|c|}{ Total Hg Output $(\mathrm{lb} / \mathrm{h})$} & $8.66 \mathrm{E}-03$ & $8.16 \mathrm{E}-03$ & $8.06 \mathrm{E}-03$ & $8.29 \mathrm{E}-03$ \\
\hline $\begin{array}{l}\text { Hg Material Balance Closure } \\
\text { (output / input) }=\end{array}$ & $119 \%$ & $119 \%$ & $121 \%$ & $120 \%$ \\
\hline
\end{tabular}

${ }^{a}$ Measured to be less than detection limit; for this calculation, assumed to equal detection limit.

The March 30 tests, however, exhibited a consistent positive deviation from 100\% closure and narrowly satisfied the criterion for a three-test average. For each of the tests conducted that day, the calculated amount of $\mathrm{Hg}$ exiting the system was about $20 \%$ greater than the calculated amount of $\mathrm{Hg}$ entering the system. This deviation from $100 \%$ closure could have arisen partially from the assumption that the concentration of $\mathrm{Hg}$ in the stack flue gas equaled the detection limit of the Ontario 
Hydro method during the tests. If it is assumed that the $\mathrm{Hg}$ concentration in the stack gas was zero (rather than the detection limit value of $\sim 0.6 \mu \mathrm{g} / \mathrm{m}^{3}$ ), then the three-test average closure decreases to $114 \%$. This is still insufficient to fully account for the positive deviation from $100 \%$ closure, however. Hence, it is likely that this deviation is attributable to an overestimation of the product ash mass flow rate, which could have resulted from errors in one or more of the measurements (i.e., coal sulfur content, coal feed rate, stack $\mathrm{SO}_{2}$ concentration, stack flow rate, stack moisture, product ash sulfur content) used to estimate this flow rate. Material balances were performed for $\mathrm{SiO}_{2}$ and $\mathrm{Al}_{2} \mathrm{O}_{3}$ using the same assumptions that were used for the $\mathrm{Hg}$ material balances. The three-test average closures on March 30 were $111 \%$ for $\mathrm{SiO}_{2}$ and $117 \%$ for $\mathrm{Al}_{2} \mathrm{O}_{3}$, consistent with the results for $\mathrm{Hg}$. (For comparison, the three-test average closures for the March 28 tests were $97 \%$ for $\mathrm{SiO}_{2}$ and $100 \%$ for $\mathrm{Al}_{2} \mathrm{O}_{3}$ ). This supports the notion that the deviation from $100 \%$ closure resulted from a misestimation of the flow rate of one or more process streams, rather than from an error in the mercury concentration measurements.

The $\mathrm{Hg}$ material balance closure results presented in Tables 21 and 22 provide no information about the quality of the $\mathrm{Hg}$ concentrations measured at the air heater outlet using the Ontario Hydro method. Under the assumption that the amount of $\mathrm{Hg}$ exiting the system via the bottom ash is negligible, the mass flow rate of $\mathrm{Hg}$ at the air heater outlet should approximately equal the mass flow rate of $\mathrm{Hg}$ in the coal. Tables 23 and 24 compare these flow rates for the tests that were conducted on March 28 and March 30, respectively. For all of the tests conducted on both days, the mass flow rate of $\mathrm{Hg}$ measured at the air heater outlet agreed with the mass flow rate of $\mathrm{Hg}$ in the coal to within $\pm 8 \%$, lending support to the quality of the measurements.

Table 23. Comparison of $\mathrm{Hg}$ Mass Flow Rates in the Coal and at the Air Heater Outlet for Hg Measurements Performed on March 28, 2007

\begin{tabular}{||c|c|c|c||}
\hline \multicolumn{1}{|c|}{ CONSOL Test No. } & 1 & 2 & 3 \\
\hline \hline Hg Input from Coal $(\mathrm{Ib} / \mathrm{h})$ & $7.45 \mathrm{E}-03$ & $8.30 \mathrm{E}-03$ & $7.01 \mathrm{E}-03$ \\
\hline Hg at Air Heater Outlet $(\mathrm{Ib} / \mathrm{h})$ & $7.07 \mathrm{E}-03$ & $7.76 \mathrm{E}-03$ & $7.54 \mathrm{E}-03$ \\
\hline Percent Difference from Coal $\mathrm{Hg}$ & $-5.1 \%$ & $-6.5 \%$ & $7.6 \%$ \\
\hline
\end{tabular}

Table 24. Comparison of $\mathrm{Hg}$ Mass Flow Rates in the Coal and at the Air Heater Outlet for Hg Measurements Performed on March 30, 2007

\begin{tabular}{||c|c|c|c||}
\hline CONSOL Test No. & 4 & 5 & 6 \\
\hline \hline $\mathrm{Hg}$ Input from Coal $(\mathrm{lb} / \mathrm{h})$ & $7.23 \mathrm{E}-03$ & $6.79 \mathrm{E}-03$ & $6.64 \mathrm{E}-03$ \\
\hline Hg at Air Heater Outlet $(\mathrm{b} / \mathrm{h})$ & $6.92 \mathrm{E}-03$ & $7.01 \mathrm{E}-03$ & $6.48 \mathrm{E}-03$ \\
\hline Percent Difference from Coal Hg & $-4.3 \%$ & $3.3 \%$ & $-2.4 \%$ \\
\hline
\end{tabular}




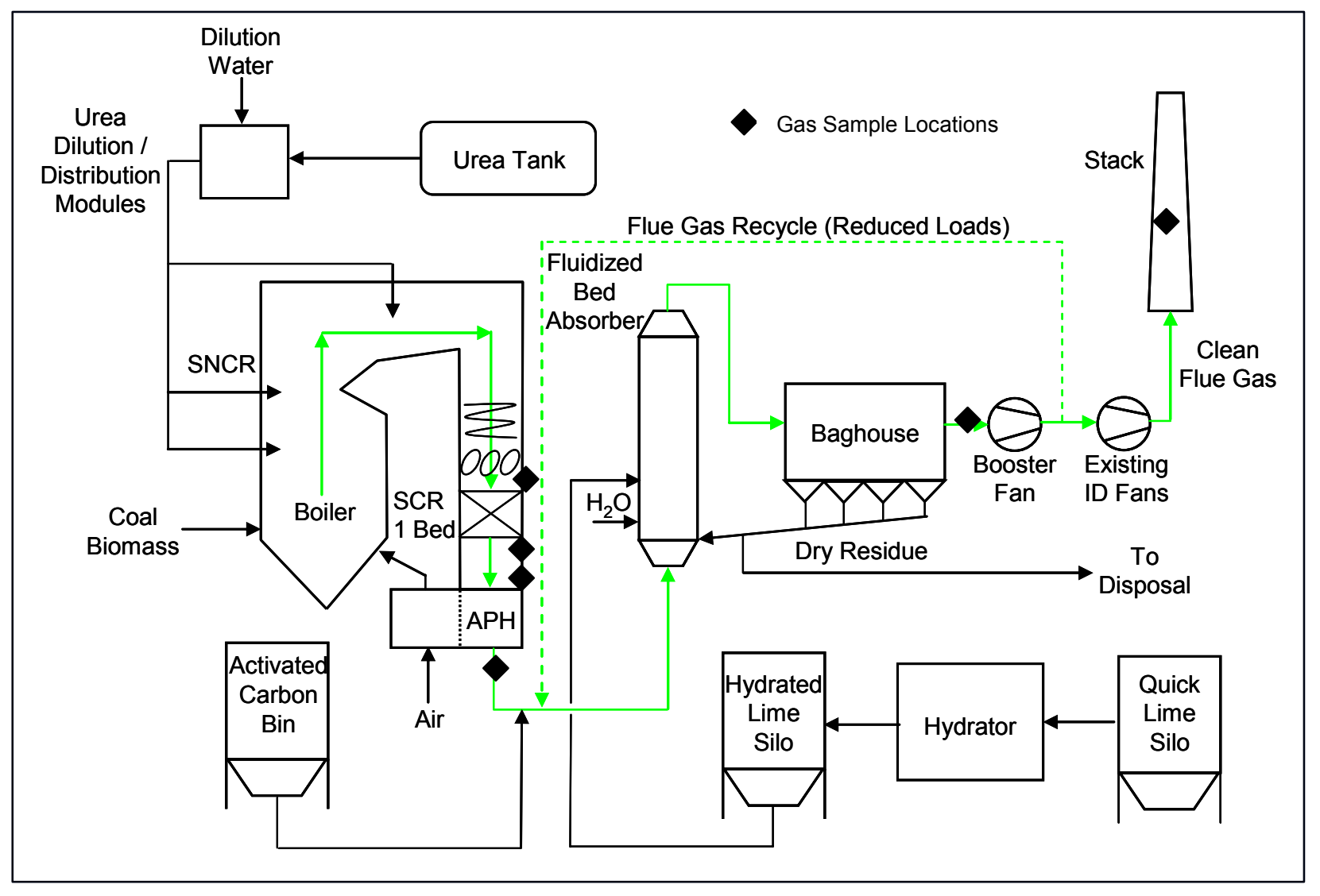

Figure 1. Sampling locations 


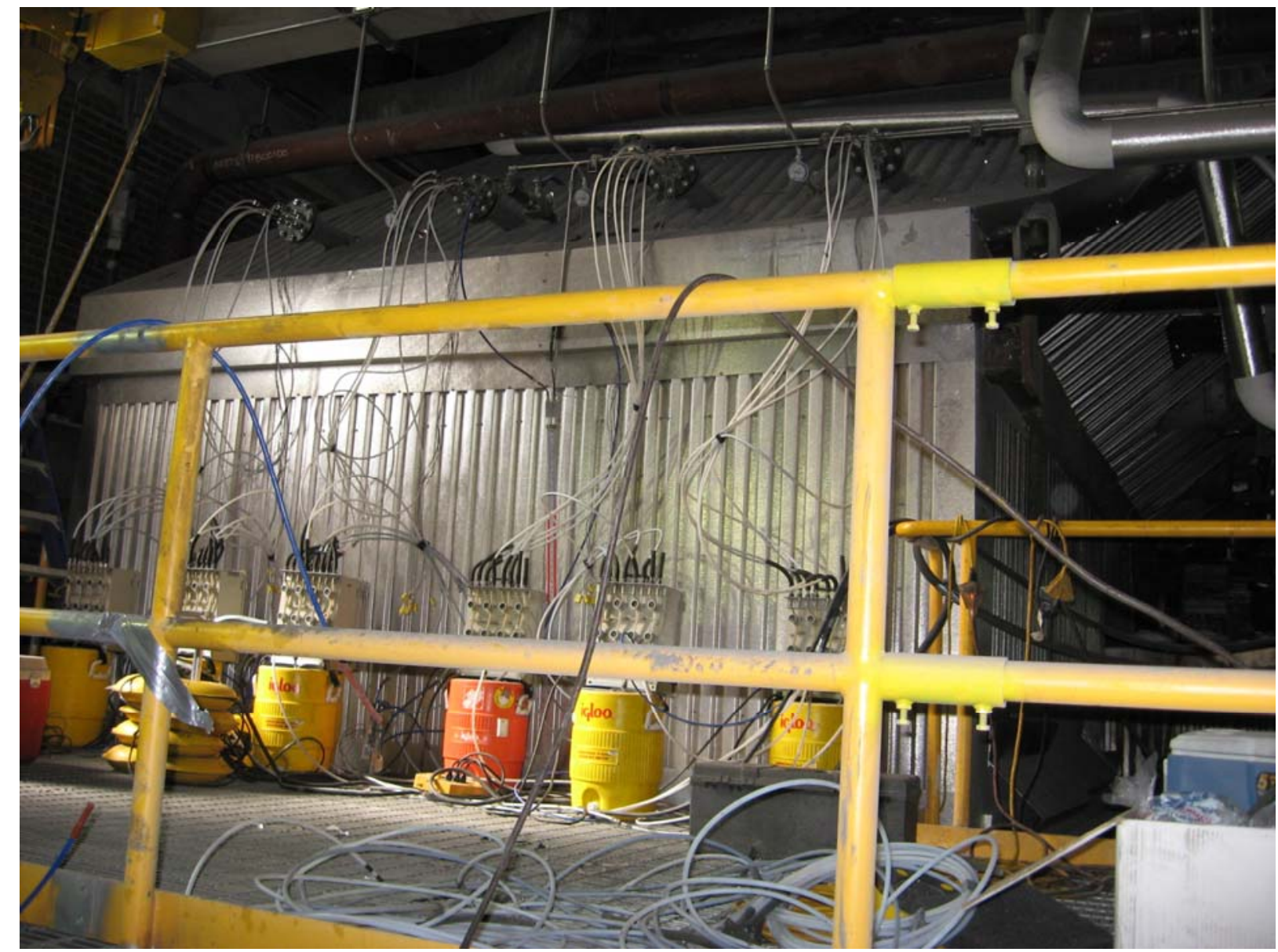

Figure 2. $\quad$ SCR Inlet Sampling Location with Clean Air Engineering's $\mathrm{NO}_{x}$ MultiPoint Automated Sampling System 


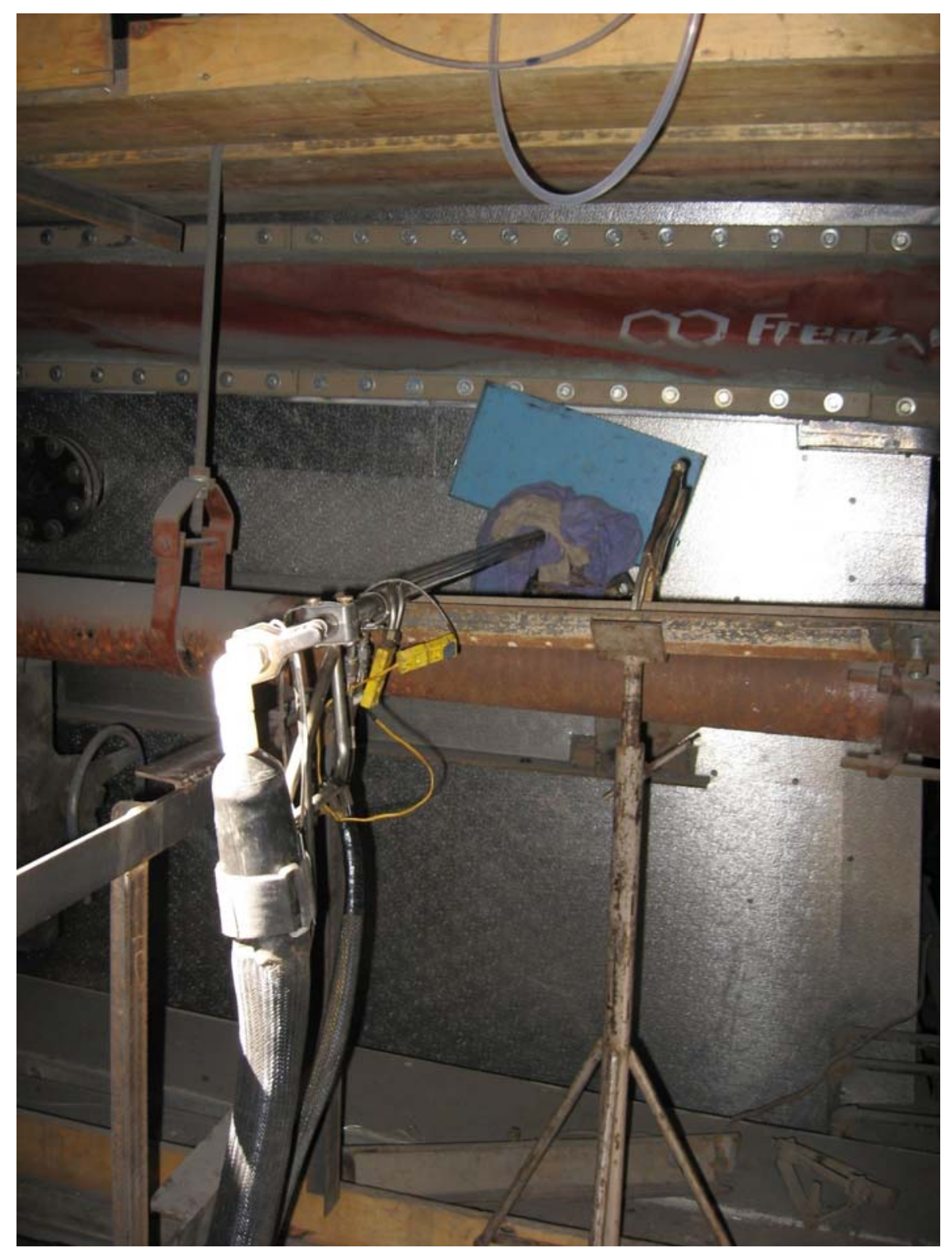

Figure 3. SCR Outlet Sampling Location 


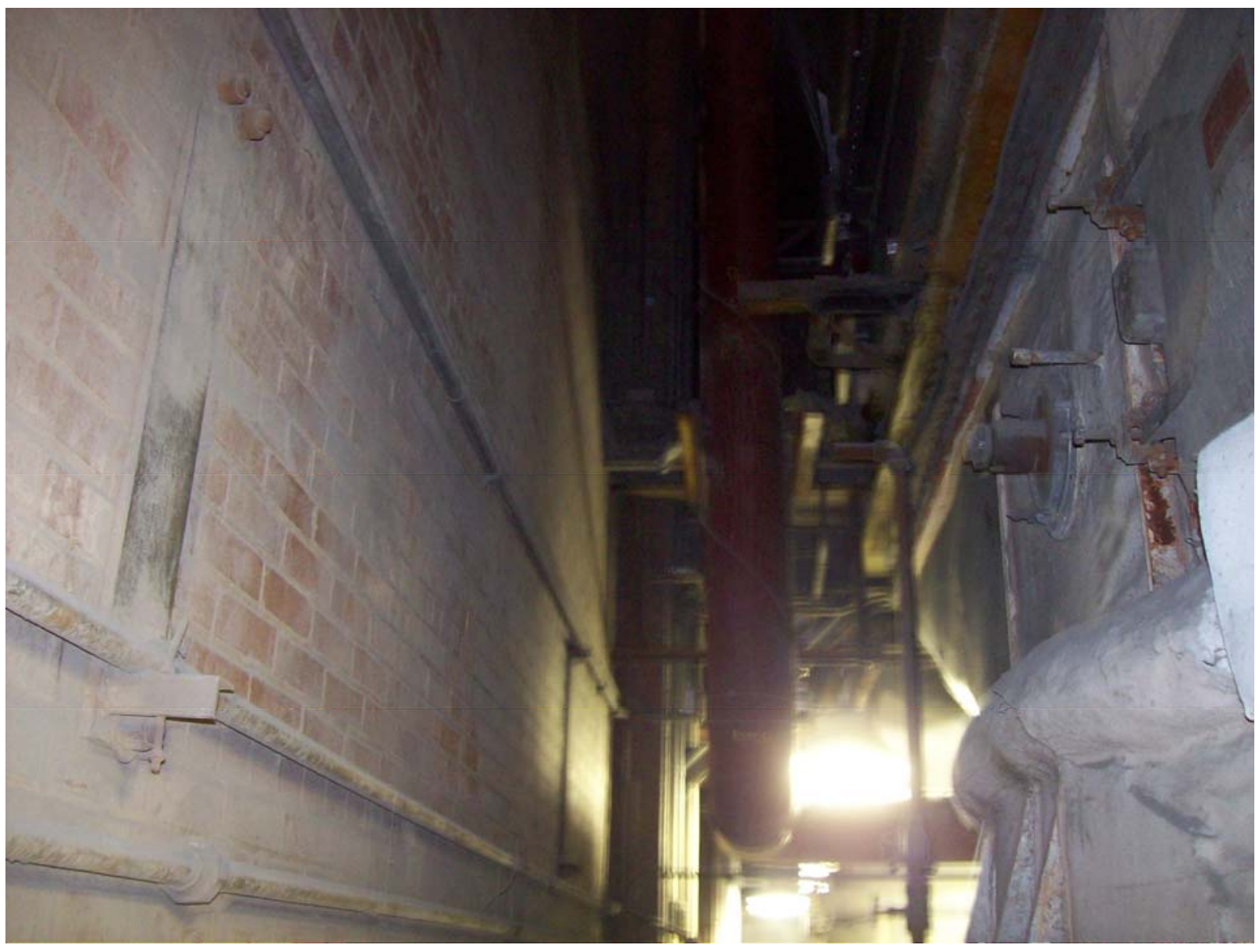

Figure 4. Air Heater Inlet Sampling Location 


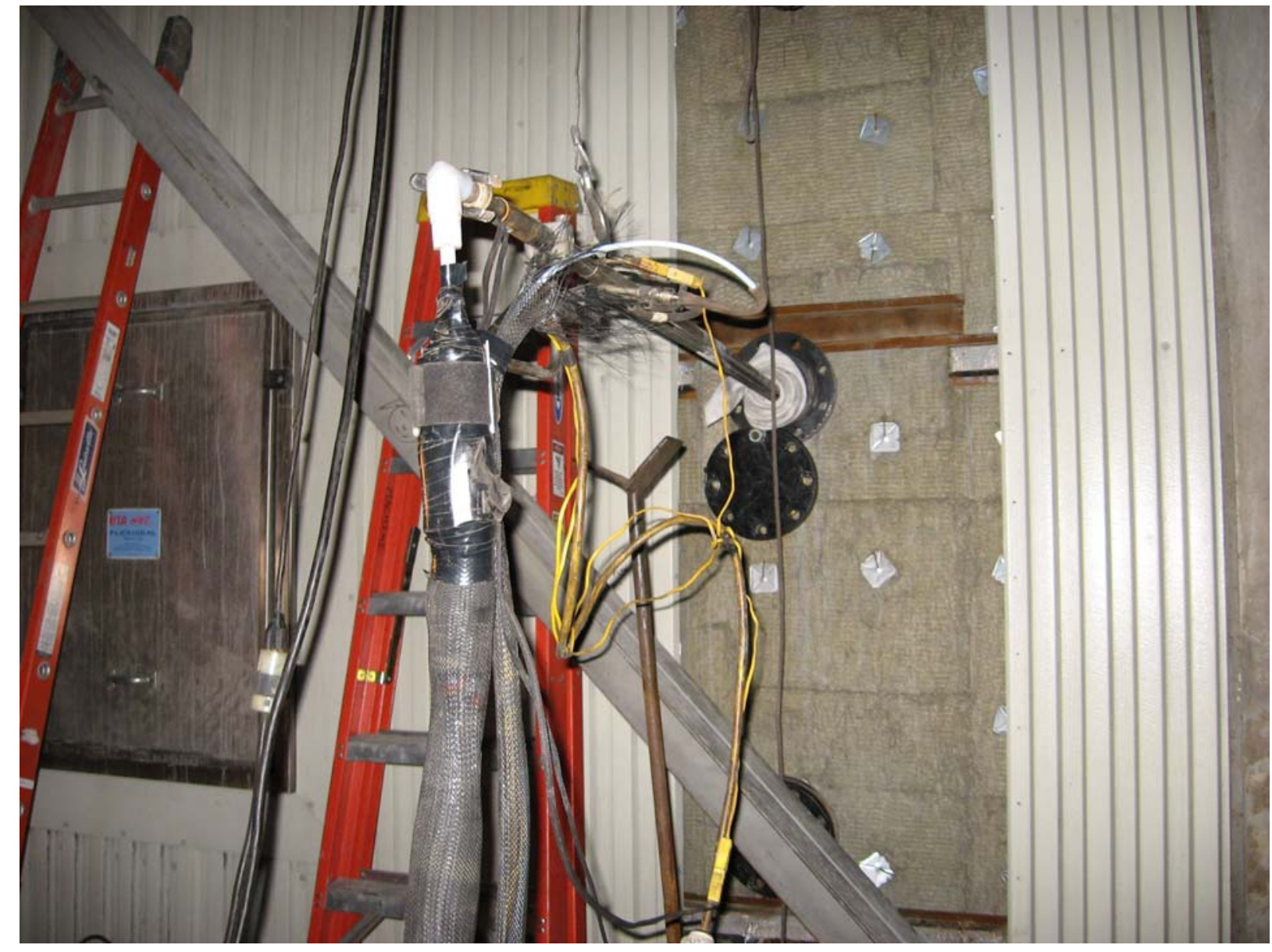

Figure 5. Air Heater Outlet Sampling Location 


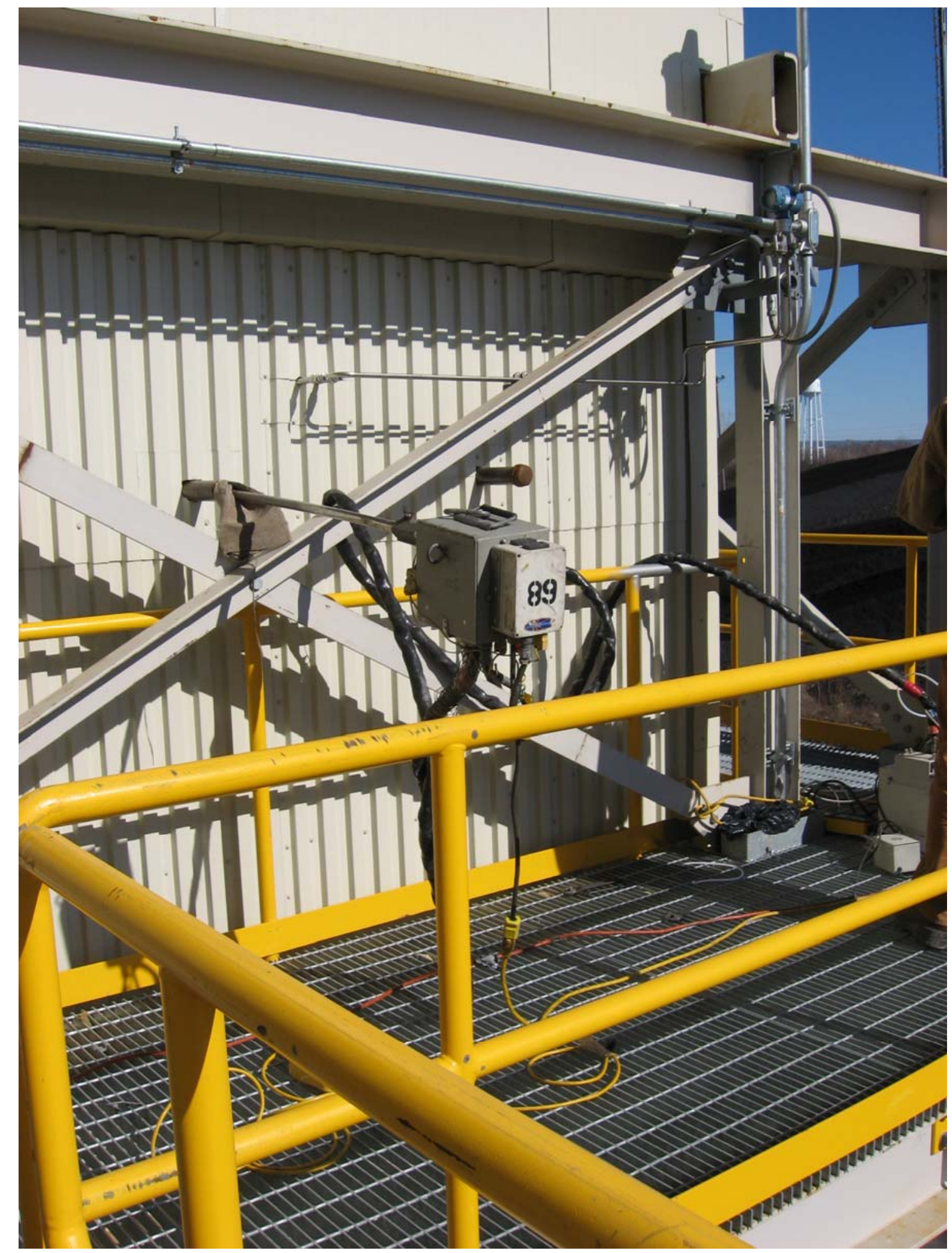

Figure 6. Baghouse Outlet Sampling Location 


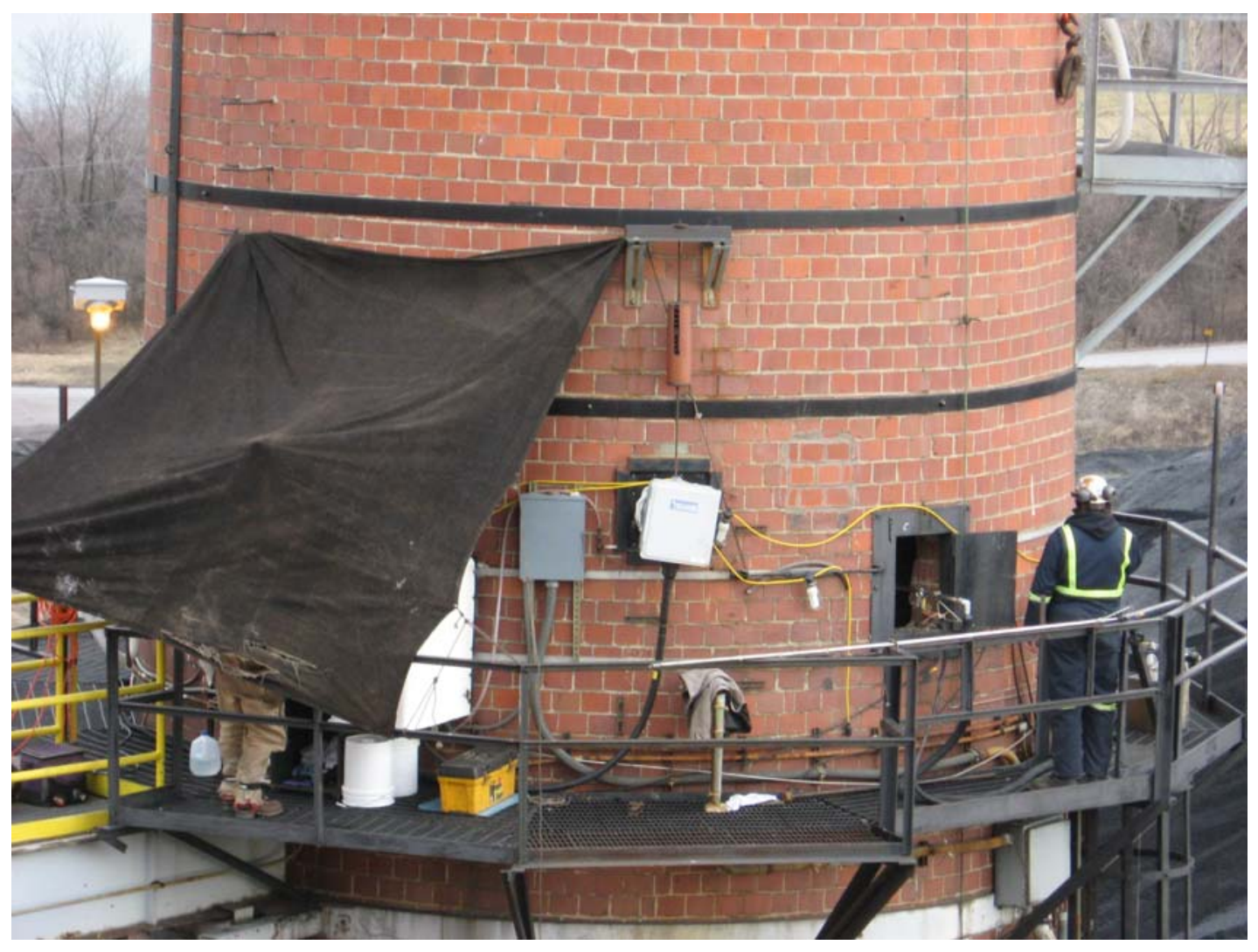

Figure 7. Stack Sampling Location 

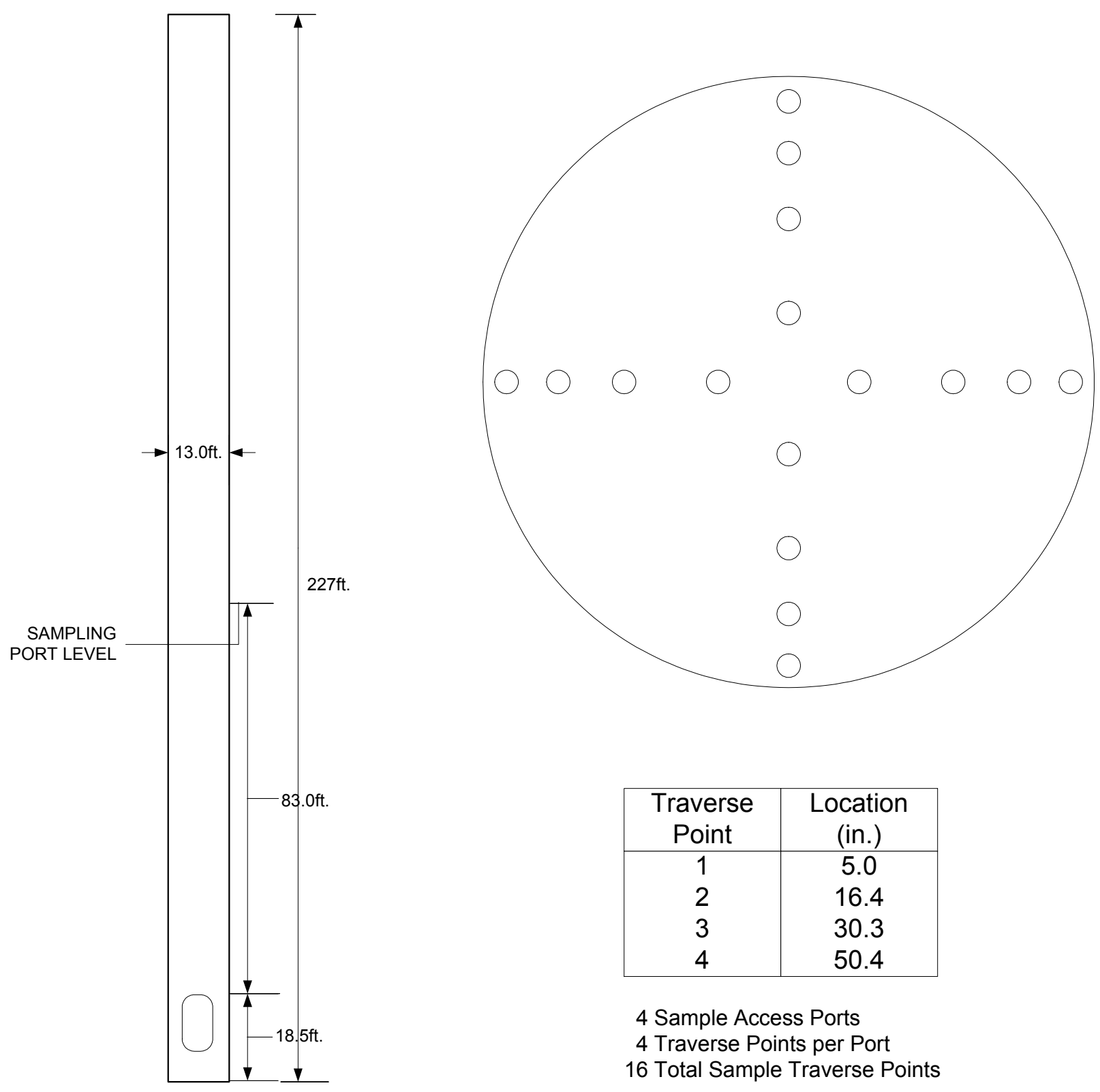

\begin{tabular}{|c|c|}
\hline $\begin{array}{c}\text { Traverse } \\
\text { Point }\end{array}$ & $\begin{array}{c}\text { Location } \\
\text { (in.) }\end{array}$ \\
\hline 1 & 5.0 \\
2 & 16.4 \\
3 & 30.3 \\
4 & 50.4 \\
\hline
\end{tabular}

4 Sample Access Ports 4 Traverse Points per Port 16 Total Sample Traverse Points

Figure 8. Stack Sampling Dimensions and Traverse Points 


\section{APPENDIX A}

Clean Air Engineering Report ( $\mathrm{NO}_{x} \& \mathrm{SO}_{2}$ Sampling) 
Consol Energy Inc. 1800 Washington Road Pittsburgh, PA 15241

\section{REPORT ON PERFORMANCE TESTING}

\section{Performed for: \\ CONSOL ENERGY INC. \\ UNIT 4 SCR OUTLET, SCR INLET, AIR HEATER OUTLET, AND \\ BAGHOUSE OUTLET AT THE \\ AES GREENIDGE STATION}

Client Reference No: 4700140111

CleanAir Project No: 10192

Revision 0: May 18, 2007

Revision 1: July 9, 2007

To the best of our knowledge, the data presented in this report are accurate, complete, error free, legible and representative of the actual emissions during the test program.

Submitted by,

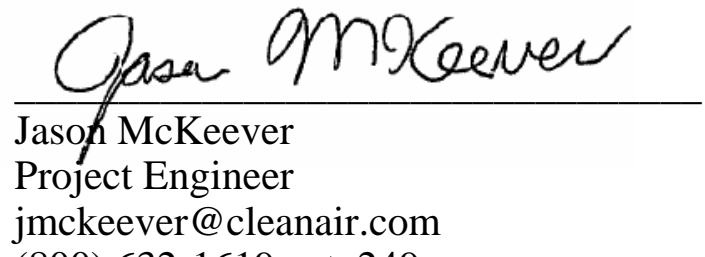

(800) 632-1619 ext. 249
Reviewed by,

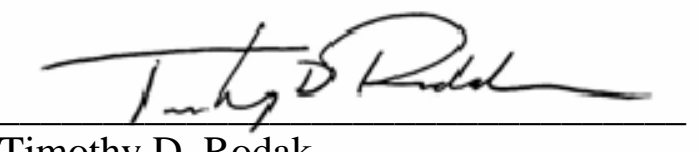

Timothy D. Rodak

Leader, Eastern Engineering Group

trodak@cleanair.com

(800) 632-1619 ext. 225 


\section{CleanAir.}

CONSOL ENERGY INC.

Client Reference No: 4700140111

AES GREENIDGE STATION

CleanAir Project No: 10192

\section{REVISION HISTORY}

ii

\section{REPORT ON PERFORMANCE TESTING}

Revision History

\begin{tabular}{|c|c|c|l|}
\hline Revision No: & Date & Pages & Comments \\
\hline 0 & $05 / 18 / 07$ & All & Original version of document. \\
\hline 1 & $7 / 9 / 07$ & All & Comments revision \\
\hline
\end{tabular}




\section{CleanAir.}

\section{CONTENTS}

Table 1-1: Unit 4 Schedule of Activities.............................................................. 1-2

Table 1-2: Summary of Nitrogen Oxides Test Results ........................................... 1-3

Table 1-3: Summary of Nitrogen Oxides Test Result in (Ib/MMBtu) .......................... 1-3

Table 1-4: Summary of Sulfur Dioxide Test Results .............................................. 1-4

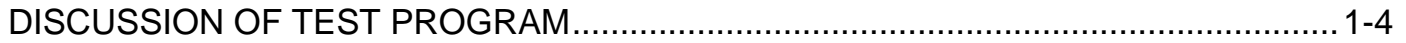

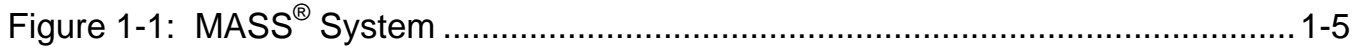

2 RESULTS

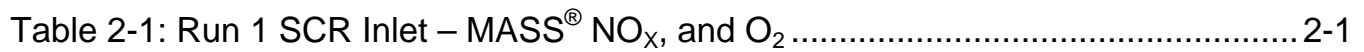

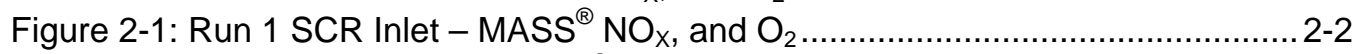

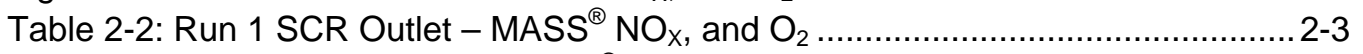

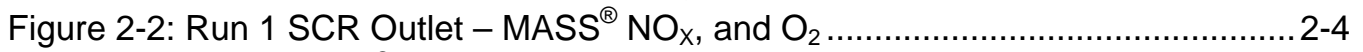

Table 2-3: Run 1 MASS $^{\circledR}-\mathrm{NO}_{x}$ Removal Efficiency .............................................. 2-5

Figure 2-3: Run 1 MASS $^{\circledR}-\mathrm{NO}_{x}$ Removal Efficiency.............................................. 2-5

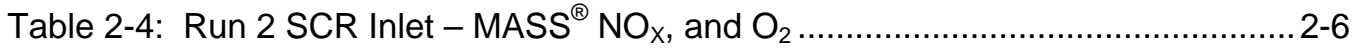

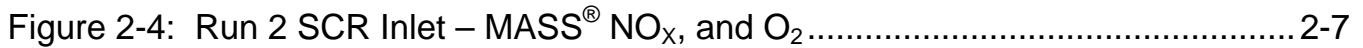

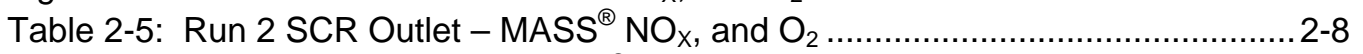

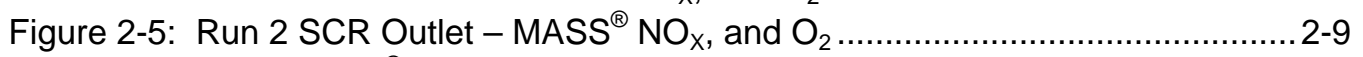

Table 2-6: Run 2 MASS $^{\circledR}$ - NOx Removal Efficiency .............................................. 2-10

Figure 2-6: Run 2: $\mathrm{MASS}^{\circledR}-\mathrm{NO}_{\mathrm{X}}$ Removal Efficiency............................................ 2-10

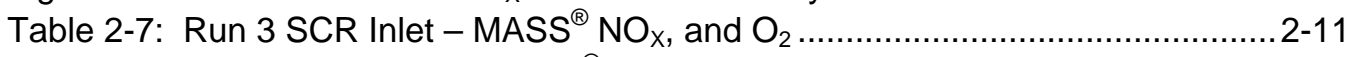

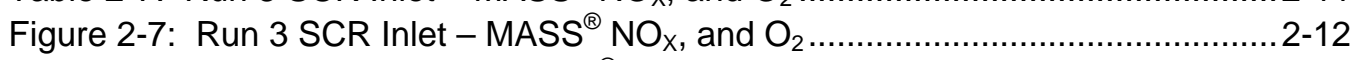

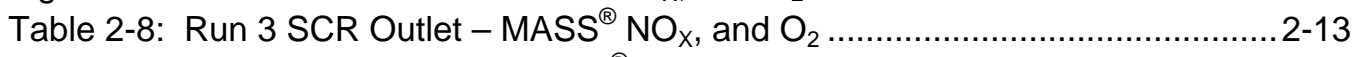

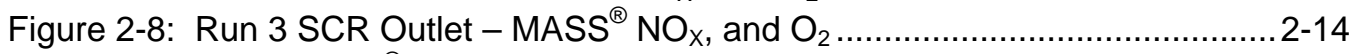

Table 2-9: Run 3 MASS $^{\circledR}-\mathrm{NO}_{x}$ Removal Efficiency ............................................. 2-15

Figure 2-9: Run 3 MASS $^{\circledR}$ - Removal Effiency .................................................. 2-15

Table 2-10: Sulfur Dioxide Reduction Efficiency Results ...........................................2-16

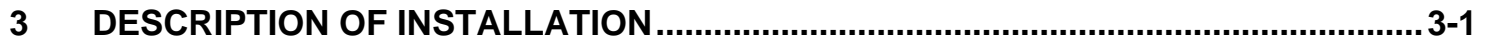

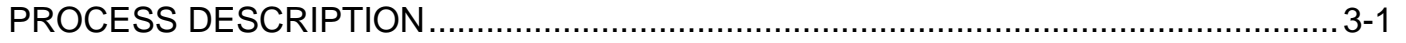

DESCRIPTION OF SAMPLING LOCATION(S) ….............................................

Table 3-1: Sampling Points ................................................................................. 3-1

Figure 3-1: SCR Inlet - Sampling Point Determination (EPA Method 1) ................... 3-2

Figure 3-2: SCR Outlet - Sampling Point Determination (EPA Method 1) .................. 3-3

4 METHODOLOGY

Table 4-1: Summary of Sampling Procedures .................................................... 4-1

5 APPENDIX

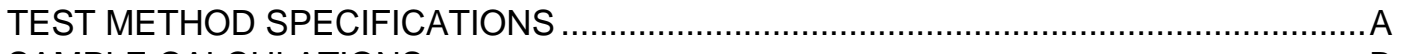

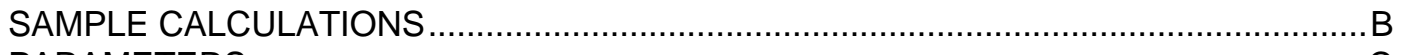

PARAMETERS

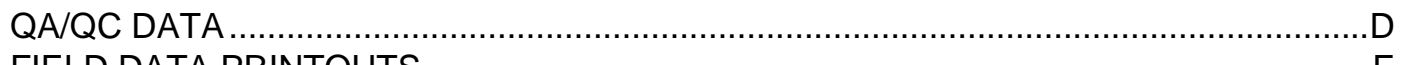

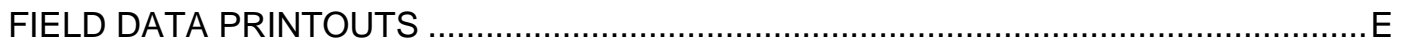




\section{CleanAir.}

CONSOL ENERGY INC.

\section{PROJECT OVERVIEW}

1-1

Consol Energy Inc. (Consol) contracted Clean Air Engineering (CleanAir) to perform guarantee testing at the AES Greenidge Station in Dresden, NY. The testing was conducted in order to demonstrate a cost effective emission control technology.

All testing was performed in accordance with regulations set forth by the United States Environmental Protection Agency (USEPA).

The test included the following constituents:

$\underline{\text { SCR Inlet and Outlet }}$

- $\quad$ nitrogen oxides $\left(\mathrm{NO}_{\mathrm{x}}\right)$

- flue gas composition $\left(\mathrm{O}_{2}, \mathrm{CO}_{2}\right)$

$\underline{\text { Air Heater Outlet and Baghouse Outlet }}$

- $\quad$ sulfur dioxide $\left(\mathrm{SO}_{2}\right)$

- flue gas composition $\left(\mathrm{O}_{2}, \mathrm{CO}_{2}\right)$

The testing took place on Greenidge Unit 4 on March 28 and 29, 2007. Coordinating the field testing were:
J. Locke - Consol Energy Inc.
D. Connell - Consol Energy Inc.
S. Lehmann - Clean Air Engineering
J. McKeever - Clean Air Engineering

Table 1-1 outlines the schedule adhered to during the test program. Tables 1-2 and 1-3 summarize the results of the test program. A more detailed presentation of the test conditions and results of analysis are shown on tables 2-1 through 2-10 and Figures 2-1 through 2-9. 


\section{CleanAir.}

CONSOL ENERGY INC.

Client Reference No: 4700140111

AES GREENIDGE STATION

CleanAir Project No: 10192

\section{PROJECT OVERVIEW}

\section{1-2}

Table 1-1:

Unit 4 Schedule of Activities

\begin{tabular}{|c|c|c|c|c|c|c|}
\hline $\begin{array}{c}\text { Run } \\
\text { Number }\end{array}$ & Location & Method & Analyte & Date & $\begin{array}{l}\text { Start } \\
\text { Time } \\
\end{array}$ & $\begin{array}{l}\text { End } \\
\text { Time } \\
\end{array}$ \\
\hline 1 & Unit 4 SCR Inlet/Outlet & USEPA Method 3A, 7E & $\mathrm{O}_{2}, \mathrm{CO}_{2}, \mathrm{NO}_{\mathrm{X}}$ & 03/28/07 & 09:12 & $10: 23$ \\
\hline 2 & Unit 4 SCR Inlet/Outlet & USEPA Method 3A, 7E & $\mathrm{O}_{2}, \mathrm{CO}_{2}, \mathrm{NO}_{\mathrm{X}}$ & $03 / 28 / 07$ & $11: 12$ & $12: 23$ \\
\hline 3 & Unit 4 SCR Inlet/Outlet & USEPA Method 3A, 7E & $\mathrm{O}_{2}, \mathrm{CO}_{2}, \mathrm{NO}_{\mathrm{x}}$ & $03 / 28 / 07$ & $12: 48$ & $13: 59$ \\
\hline 1 & Unit 4 AH Outlet/ BH Outlet & USEPA Method 3A, 6C & $\mathrm{O}_{2}, \mathrm{CO}_{2}, \mathrm{SO}_{2}$ & 03/29/07 & 09:59 & $11: 00$ \\
\hline 2 & Unit $4 \mathrm{AH}$ Outlet/ $\mathrm{BH}$ Outlet & USEPA Method 3A, 6C & $\mathrm{O}_{2}, \mathrm{CO}_{2}, \mathrm{SO}_{2}$ & $03 / 29 / 07$ & $12: 16$ & 13:17 \\
\hline 3 & Unit $4 \mathrm{AH}$ Outlet/ $\mathrm{BH}$ Outlet & USEPA Method 3A, 6C & $\mathrm{O}_{2}, \mathrm{CO}_{2}, \mathrm{SO}_{2}$ & $03 / 29 / 07$ & $15: 13$ & $16: 13$ \\
\hline
\end{tabular}




\section{CleanAir}

CONSOL ENERGY INC.

\section{PROJECT OVERVIEW}

Table 1-2:

Summary of Nitrogen Oxides Test Results

Source

Constituent

Unit 4 SCR Inlet

$\mathrm{NO}_{\mathrm{X}}(\mathrm{ppmdv} @$ 3\% O

$\mathrm{O}_{2}$ (\%dv)

$\mathrm{CO}_{2}(\% \mathrm{dv})$

Unit 4 SCR Outlet

$\mathrm{NO}_{\mathrm{X}}\left(\mathrm{ppmdv} @ 3 \% \mathrm{O}_{2}\right.$ )

$\mathrm{O}_{2}(\% \mathrm{dv})$

$\mathrm{CO}_{2}(\% \mathrm{dv})$

Unit $4 \mathrm{NO}_{x}$ Reduction

$\mathrm{NO}_{\mathrm{x}}$ Reduction (\% Efficiency)

\section{Sampling \\ Method}

EPA M7E

EPA M3A

EPA M3A

EPA M7E

EPA M3A

EPA M3A
Average

Emission

87.8

4.7

14

51.8

4.9

13.9

41.0

Table 1-3:

Summary of Nitrogen Oxides Test Result in (Ib/MMBtu)

Source

Constituent

Unit 4 SCR Outlet

$\mathrm{NO}_{\mathrm{X}}\left(\mathrm{ppmdv} @ 3 \% \mathrm{O}_{2}\right.$ )

Fd Factor (dscf/MMBtu)

$\mathrm{NO}_{\mathrm{x}}\left(\mathrm{lb} / \mathrm{MMBtu} @ 3 \% \mathrm{O}_{2}\right.$ )
Run 1

52.7

9737

0.0711
Run 2

52.7

9618

0.0709
50.1

9535

0.0674
Average

Run 3

Emission

51.8

0.0698

Note: Fd factor was determined by short prox/ultimate analysis (provided by Consol Energy Inc.) 


\section{PROJECT OVERVIEW}

Summary of Sulfur Dioxide Test Results

Source

Constituent

Unit 4 Air Heater Outlet

$\mathrm{SO}_{2}$ (ppmdv @ 3\% $\mathrm{O}_{2}$ )

$\mathrm{O}_{2}$ (\%dv)

$\mathrm{CO}_{2}(\% \mathrm{dv})$

Unit 4 Baghouse Outlet

$\mathrm{SO}_{2}$ (ppmdv @ 3\% $\mathrm{O}_{2}$ )

$\mathrm{O}_{2}(\% \mathrm{dv})$

$\mathrm{CO}_{2}(\% \mathrm{dv})$
Sampling

Method

EPA M6C

EPA M3A

EPA M3A

EPA M6C

EPA M3A

EPA M3A
Average

Emission

1840.8

7.32

11.56

108.9

7.30

11.57

Unit $4 \mathrm{SO}_{2}$ Reduction

$\mathrm{SO}_{2}$ Reduction (\% Efficiency)

94.1

\section{DISCUSSION OF TEST PROGRAM}

Testing on AES Greenidge Unit 4 consisted of several USEPA methods and conformed to Part 60 of the Code of Federal Regulations. The testing at all four locations was conducted using extractive methods. These tests were performed with the units running at full load.

The $\mathrm{SO}_{2}$ testing was based on a modified Method 6C. USEPA Code of Federal Regulations part 60 was followed with the following exceptions. During the sampling a single test point was used during the first two runs, this point was chosen by selecting a point in the center of the duct. For the third $\mathrm{SO}_{2}$ run a single port was traversed using 3 points across the duct.

To collect $\mathrm{NO}_{\mathrm{x}}, \mathrm{CO}_{2}$, and $\mathrm{O}_{2}$ emissions data, Clean Air used its Multipoint Automated Sampling Systems (MASS). Using the MASS, Clean Air was able to establish a sampling grid and develop a detailed $\mathrm{NO}_{\mathrm{x}}$, and $\mathrm{O}_{2}$ profile for both the inlet and outlet ducts. This proprietary system allows a large number of sample points to be individually analyzed in a short amount of time giving a more complete view of the gas profile within the duct. Not only are overall concentrations in the duct discovered, but also spatial stratification or temporal variation.

This tool takes much of the wait out of boiler or SCR tuning and optimization by creating an efficient method of data collection. Decision making time is reduced and 


\section{CleanAir.}

\section{PROJECT OVERVIEW}

enhanced with expedient, accurate, and reliable data. The expanded data capacity allows for increased analytical ability and a better "big picture” of unit operation.

\section{Description of the MASS ${ }^{\circledR}$}

The Clean Air Engineering's MASS ${ }^{\circledR}$ (Multi-Point Automated Sampling System) uses a series of automated 8-point sample modules with integrated programmable logic controllers (PLC's) to sequentially and rapidly cycle through a multi-point test grid. For this program, a 24-point permanent outlet grid was tested using an 8 module system. Using a bank of two (2) $\mathrm{O}_{2} / \mathrm{CO}_{2}$ monitors and (2) $\mathrm{NO}_{\mathrm{X}}$ monitors, the 24-point traverse was completed in 24 minutes. This is a considerable time savings over the typical 2 hour manual traverse.

The MASS ${ }^{\circledR}$ has been programmed to automatically cycle through and analyze each point on the grid. The key is the dynamic switching which allows for a vented "prepurge" prior the actual analysis allowing for zero wasted analyzer time. This modular and expandable system is capable of analyzing 128 individual sample points in 32 minutes or less. A diagram of the system can be seen in Figure 1-1 below. This shows a general arrangement process flow diagram for a “typical” 64-point MASS ${ }^{\circledR}$ system arrangement.

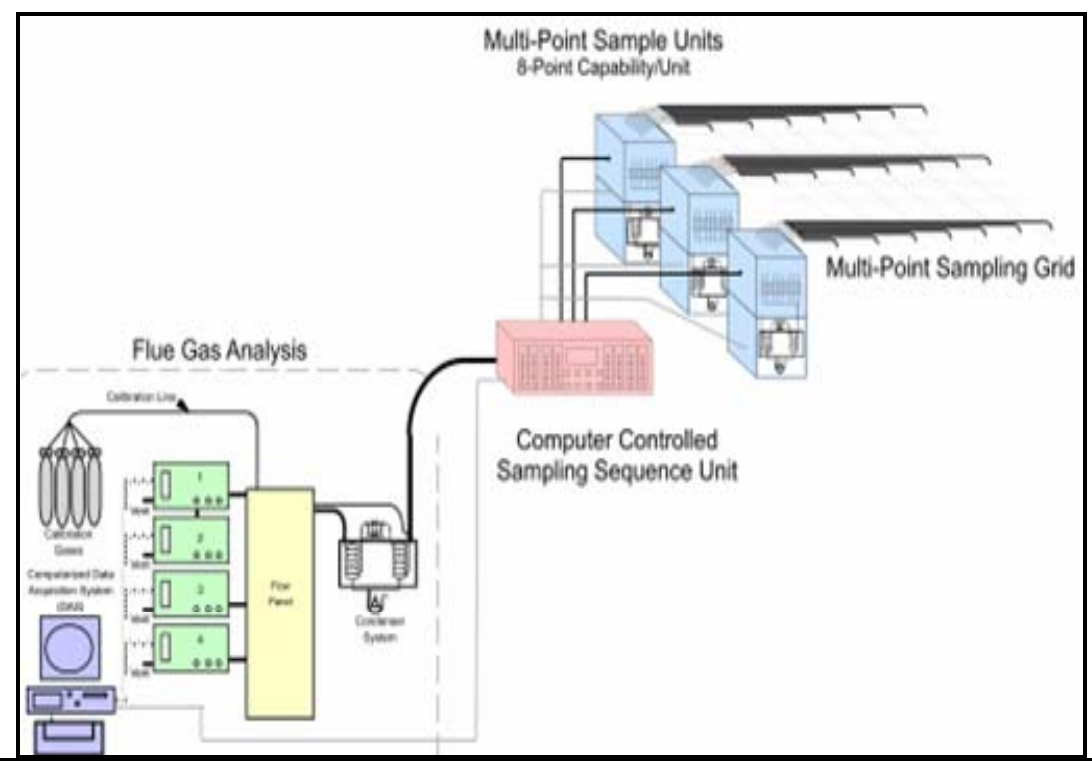

Figure 1-1: MASS $^{\circledR}$ System 


\section{CleanAir.}

CONSOL ENERGY INC.

AES GREENIDGE STATION
Client Reference No: 4700140111

CleanAir Project No: 10192

\section{RESULTS}

2-1

Table 2-1:

Run 1 SCR Inlet - MASS ${ }^{\circledR} \mathrm{NO}_{\mathrm{X}}$, and $\mathrm{O}_{2}$

Date: $3 / 28 / 2007$

Start Time: $\quad 9: 12$

End Time: $\quad$ 10:23

Inlet - Nitrogen Oxides (ppmdv @ 3\% $\mathrm{O}_{2}$ )

\begin{tabular}{|c|c|c|c|c|c|}
\hline AVG & 98.0 & 91.4 & 93.7 & 87.1 & \\
\hline 6 & 108.9 & 107.0 & 107.7 & 87.7 & 102.8 \\
\hline 5 & 80.4 & 72.8 & 77.5 & 69.5 & 75.1 \\
\hline 4 & 89.0 & 82.4 & 84.7 & 82.2 & 84.6 \\
\hline 3 & 100.5 & 100.5 & 104.2 & 97.8 & 100.8 \\
\hline 2 & 105.5 & 85.9 & 86.9 & 87.4 & 91.5 \\
\hline 1 & 103.8 & 99.7 & 101.4 & 98.2 & 100.8 \\
\hline & 1 & 2 & 3 & 4 & 92.6 \\
\hline
\end{tabular}

Inlet - Oxygen (\%dv)

\begin{tabular}{|c|c|c|c|c|}
\multicolumn{1}{c|}{ AVG } & 5.5 & 4.9 & 4.1 & 4.4 \\
\hline 6 & 8.6 & 6.8 & 6.3 & 6.6 \\
\hline 5 & 4.8 & 4.5 & 4.5 & 4.5 \\
\hline 4 & 4.4 & 3.9 & 3.7 & 3.7 \\
\hline 3 & 5.0 & 5.3 & 3.3 & 3.2 \\
\hline 2 & 2.9 & 3.0 & 2.9 & 2.5 \\
\hline 1 & 7.5 & 6.0 & 4.0 & 5.7 \\
\hline & 1 & 2 & 3 & 4 \\
\hline
\end{tabular}




\section{CleanAir.}

CONSOL ENERGY INC.

Client Reference No: 4700140111

AES GREENIDGE STATION

CleanAir Project No: 10192

\section{RESULTS}

2-2

Figure 2-1:

Run 1 SCR Inlet - MASS ${ }^{\circledR} \mathrm{NO}_{\mathrm{X}}$, and $\mathrm{O}_{2}$

Run 1: Inlet - Nitrogen Oxides (ppm dv (1) 3\% 02)

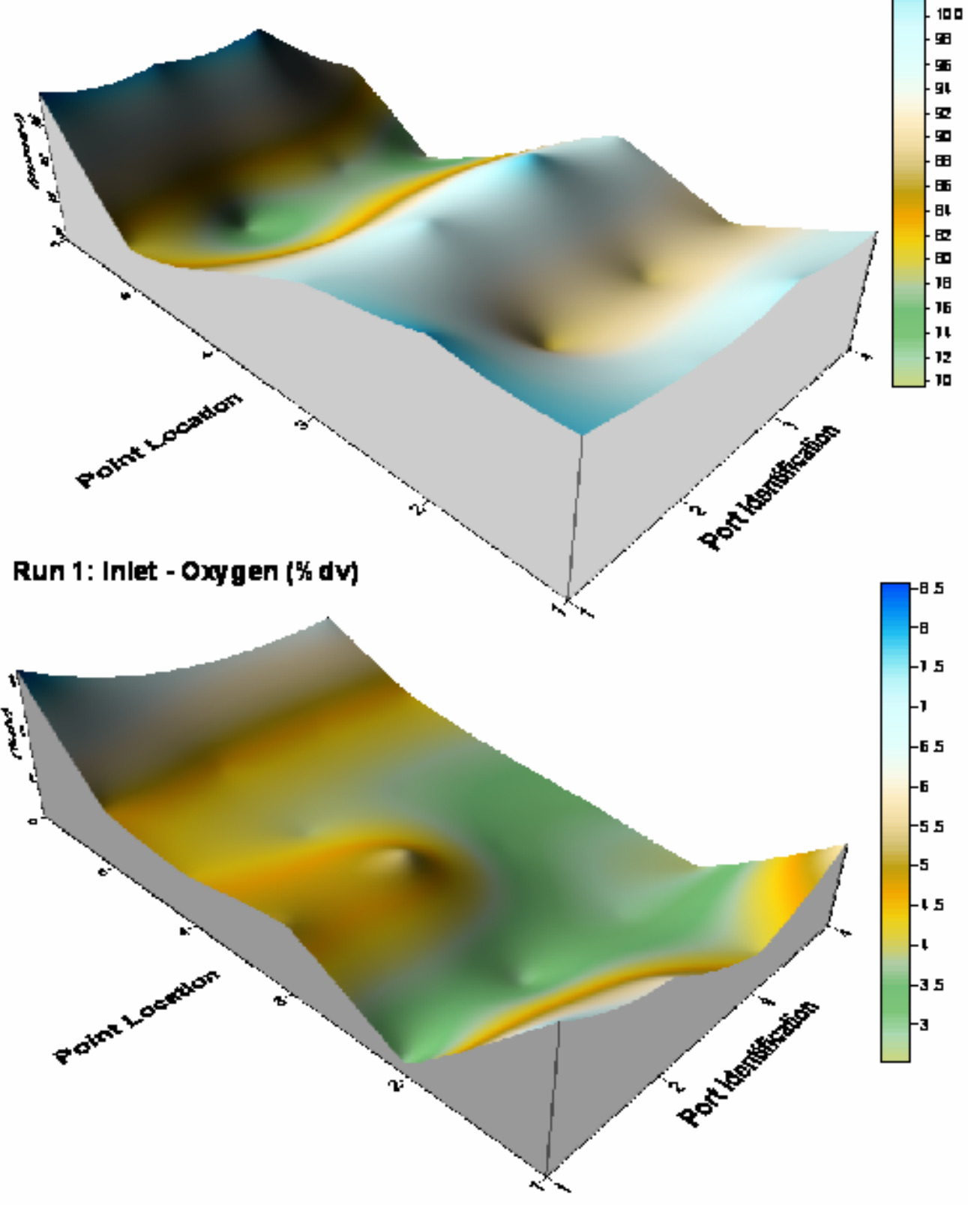




\section{CleanAir.}

CONSOL ENERGY INC.

AES GREENIDGE STATION
Client Reference No: 4700140111

CleanAir Project No: 10192

\section{RESULTS}

2-3

Table 2-2:

Run 1 SCR Outlet - MASS ${ }^{\circledR} \mathrm{NO}_{\mathrm{X}}$, and $\mathrm{O}_{2}$

Date: $3 / 28 / 2007$

Start Time: $\quad 9: 12$

End Time: $\quad$ 10:23

Outlet - Nitrogen Oxides (ppmdv @ 3\% $\mathrm{O}_{2}$ )

\begin{tabular}{|c|c|c|c|c|}
\multicolumn{1}{c}{ AVG } & 69.9 & 58.2 & 52.0 & 30.9 \\
\hline $\mathbf{6}$ & 55.9 & 59.0 & 28.1 & 6.2 \\
\hline $\mathbf{5}$ & 61.7 & 27.3 & 32.8 & 4.8 \\
\hline $\mathbf{4}$ & 68.8 & 85.8 & 67.2 & 39.4 \\
\hline $\mathbf{3}$ & 65.2 & 77.2 & 85.0 & 75.3 \\
\hline $\mathbf{2}$ & 91.8 & 62.3 & 73.4 & 51.8 \\
\hline $\mathbf{1}$ & 75.9 & 37.4 & 25.4 & 7.8 \\
\hline & $\mathbf{1}$ & $\mathbf{2}$ & $\mathbf{3}$ & $\mathbf{4}$ \\
\hline
\end{tabular}

37.3

31.7

65.3

75.7

69.8

36.6

52.7

Outlet - Oxygen (\%dv)

\begin{tabular}{|c|c|c|c|c|}
\multicolumn{1}{|c}{ AVG } & 5.7 & 5.3 & 4.5 & 4.0 \\
\hline $\mathbf{6}$ & 7.7 & 6.7 & 5.5 & 5.6 \\
\hline $\mathbf{5}$ & 6.3 & 4.9 & 4.3 & 4.0 \\
\hline $\mathbf{4}$ & 4.7 & 5.9 & 4.9 & 4.5 \\
\hline $\mathbf{3}$ & 5.3 & 4.7 & 4.4 & 3.5 \\
\hline $\mathbf{2}$ & 5.6 & 4.6 & 4.3 & 2.7 \\
\hline $\mathbf{1}$ & 4.9 & 5.0 & 3.7 & 3.5 \\
\hline & $\mathbf{1}$ & $\mathbf{2}$ & $\mathbf{3}$ & $\mathbf{4}$ \\
\hline
\end{tabular}




\section{CleanAir.}

CONSOL ENERGY INC.

Client Reference No: 4700140111

AES GREENIDGE STATION

CleanAir Project No: 10192

\section{RESULTS}

2-4

Figure 2-2:

Run 1 SCR Outlet - MASS ${ }^{\circledR} \mathrm{NO}_{\mathrm{X}}$, and $\mathrm{O}_{2}$

Run 1: Outlet - Nitrogen Oxides (ppm dv@ $3 \%$ 02)
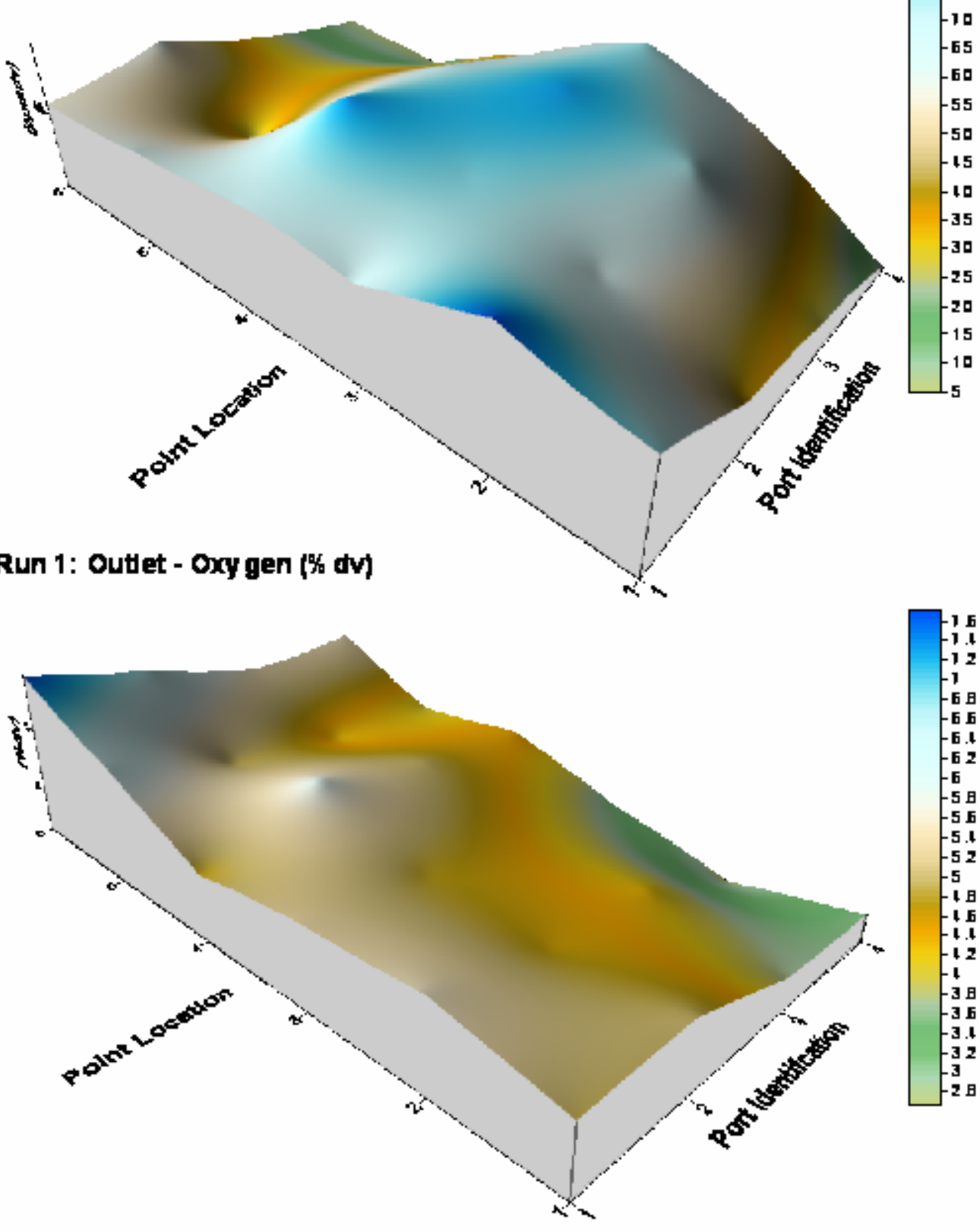


\section{CleanAir.}

\section{RESULTS}

Table 2-3:

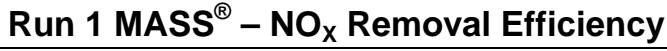

Date: $3 / 28 / 2007$

Start Time: $\quad$ 9:12

End Time: $\quad$ 10:23

\begin{tabular}{|c|c|c|c|c|c|}
\hline \multicolumn{5}{|c|}{ Removal Efficiency (@ 3\% O } & \multirow[b]{3}{*}{$65.1 \%$} \\
\hline AVG & $28.3 \%$ & $36.1 \%$ & $43.6 \%$ & $65.6 \%$ & \\
\hline 6 & $48.7 \%$ & $44.8 \%$ & $73.9 \%$ & $92.9 \%$ & \\
\hline 5 & $23.3 \%$ & $62.5 \%$ & $57.7 \%$ & $93.1 \%$ & $59.1 \%$ \\
\hline 4 & $22.8 \%$ & $-4.1 \%$ & $20.7 \%$ & $52.0 \%$ & $22.8 \%$ \\
\hline 3 & $35.1 \%$ & $23.2 \%$ & $18.4 \%$ & $23.0 \%$ & $24.9 \%$ \\
\hline 2 & $13.0 \%$ & $27.5 \%$ & $15.6 \%$ & $40.8 \%$ & $24.2 \%$ \\
\hline 1 & $26.8 \%$ & $62.5 \%$ & $75.0 \%$ & $92.1 \%$ & $64.1 \%$ \\
\hline & 1 & 2 & 3 & 4 & $43.4 \%$ \\
\hline
\end{tabular}

Run 1: NOx Removal Efficiency

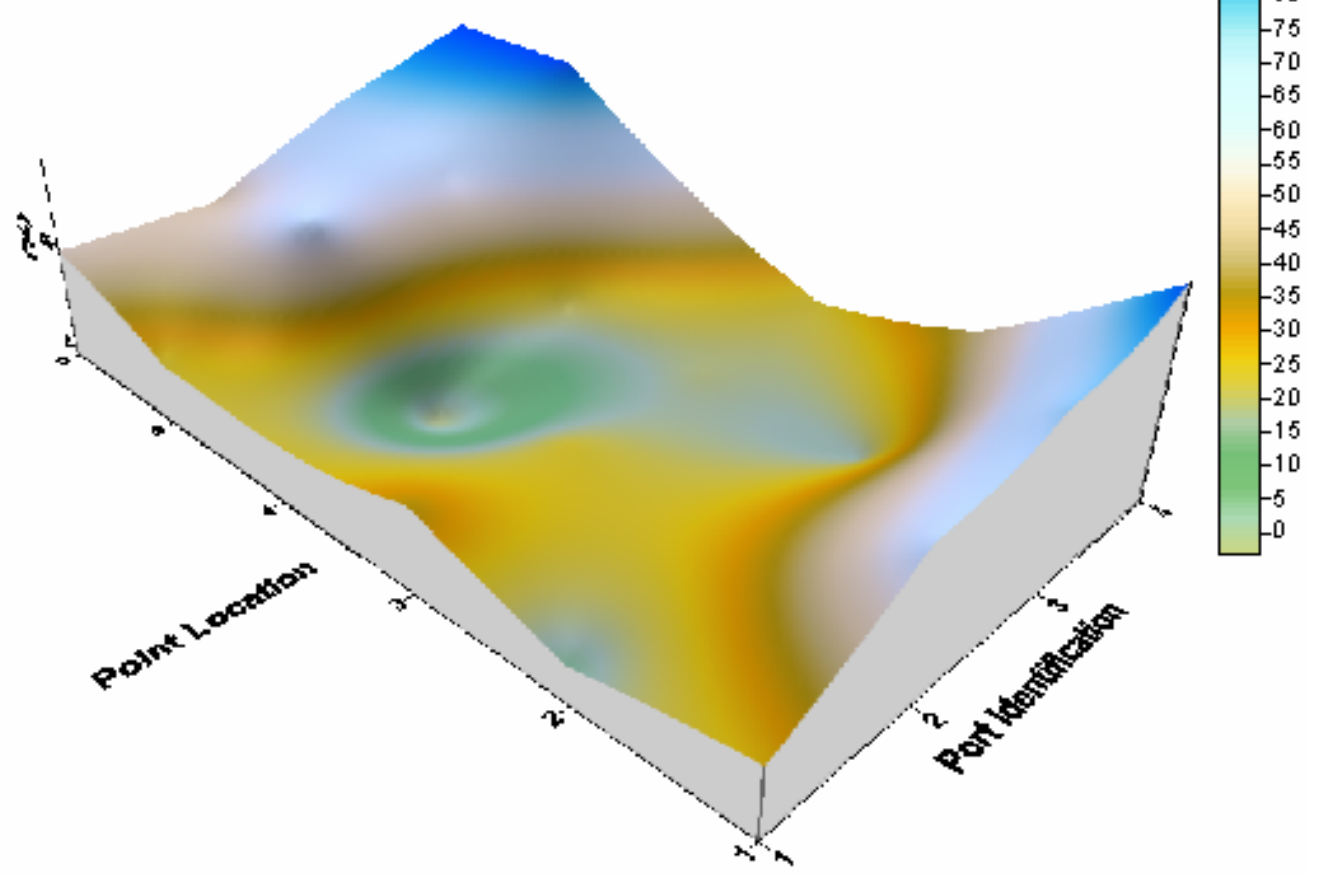

Figure 2-3:

Run 1 MASS $^{\circledR}-$ NO $_{x}$ Removal Efficiency 


\section{CleanAir.}

CONSOL ENERGY INC.

AES GREENIDGE STATION
Client Reference No: 4700140111

CleanAir Project No: 10192

\section{RESULTS}

2-6

Table 2-4:

Run 2 SCR Inlet - MASS ${ }^{\circledR} \mathrm{NO}_{\mathrm{X}}$, and $\mathrm{O}_{2}$

Date: $3 / 28 / 2007$

Start Time: $\quad 11: 12$

End Time: $12: 23$

Inlet - Nitrogen Oxides (ppmdv @ 3\% $\mathrm{O}_{2}$ )

\begin{tabular}{|c|c|c|c|c|c|}
\hline AVG & 93.4 & 84.7 & 86.7 & 80.3 & \\
\hline 6 & 102.6 & 96.6 & 98.2 & 77.2 & 93.6 \\
\hline 5 & 73.5 & 63.0 & 69.9 & 61.7 & 67.0 \\
\hline 4 & 86.3 & 77.6 & 80.4 & 77.5 & 80.4 \\
\hline 3 & 96.1 & 95.6 & 99.5 & 94.5 & 96.4 \\
\hline 2 & 99.6 & 80.1 & 78.6 & 78.3 & 84.1 \\
\hline \multirow[t]{2}{*}{1} & 102.3 & 95.4 & 93.6 & 92.8 & 96.0 \\
\hline & 1 & 2 & 3 & 4 & 86.3 \\
\hline
\end{tabular}

Inlet - Oxygen (\%dv)

\begin{tabular}{|c|c|c|c|c|}
\multicolumn{1}{c|}{ AVG } & 5.5 & 4.9 & 4.1 & 4.4 \\
\hline 6 & 8.6 & 6.8 & 6.3 & 6.4 \\
\hline 5 & 4.6 & 4.3 & 4.4 & 4.3 \\
\hline 4 & 4.3 & 3.8 & 3.6 & 3.5 \\
\hline 3 & 5.0 & 5.4 & 3.3 & 3.1 \\
\hline 2 & 3.1 & 3.2 & 3.0 & 2.7 \\
\hline 1 & 7.6 & 6.1 & 4.2 & 6.3 \\
\hline & 1 & 2 & 3 & 4 \\
\hline
\end{tabular}




\section{CleanAir.}

CONSOL ENERGY INC.

Client Reference No: 4700140111

AES GREENIDGE STATION

CleanAir Project No: 10192

\section{RESULTS}

2-7

Figure 2-4:

Run 2 SCR Inlet - MASS ${ }^{\circledR} \mathrm{NO}_{\mathrm{X}}$, and $\mathrm{O}_{2}$

Run 2: Inlet - Nitrogen Oxides (ppm dv @ 3\% 02)
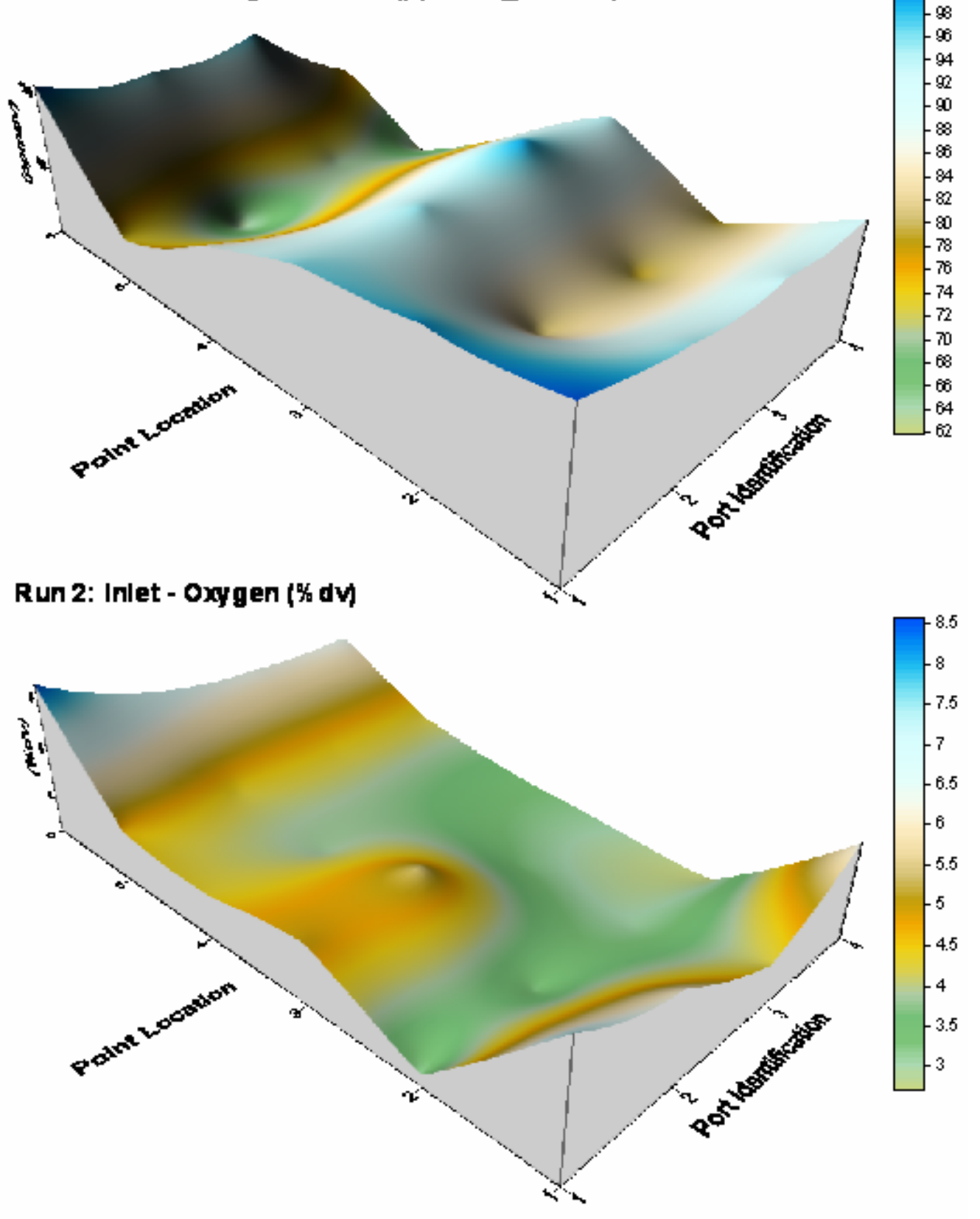


\section{CleanAir.}

CONSOL ENERGY INC.

AES GREENIDGE STATION
Client Reference No: 4700140111

CleanAir Project No: 10192

\section{RESULTS}

2-8

Table 2-5:

Run 2 SCR Outlet - MASS ${ }^{\circledR} \mathrm{NO}_{\mathrm{X}}$, and $\mathrm{O}_{2}$

Date: $3 / 28 / 2007$

Start Time: $\quad 11: 12$

End Time: $\quad 12: 23$

Outlet - Nitrogen Oxides (ppmdv @ 3\% $\mathrm{O}_{2}$ )

\begin{tabular}{|c|c|c|c|c|}
\multicolumn{1}{c}{ AVG } & 73.0 & 56.2 & 51.4 & 30.0 \\
\hline $\mathbf{6}$ & 67.3 & 52.8 & 24.9 & 5.1 \\
\hline $\mathbf{5}$ & 60.4 & 24.7 & 31.7 & 4.2 \\
\hline $\mathbf{4}$ & 74.0 & 87.3 & 69.9 & 42.5 \\
\hline $\mathbf{3}$ & 66.5 & 78.9 & 86.2 & 76.5 \\
\hline $\mathbf{2}$ & 92.1 & 58.5 & 72.6 & 45.1 \\
\hline $\mathbf{1}$ & 78.0 & 35.3 & 23.2 & 6.8 \\
\hline & $\mathbf{1}$ & $\mathbf{2}$ & $\mathbf{3}$ & $\mathbf{4}$ \\
\hline
\end{tabular}

37.5

30.3

68.4

77.0

67.1

35.8

52.7

Outlet - Oxygen (\%dv)

\begin{tabular}{|c|c|c|c|c|}
\multicolumn{1}{c}{ AVG } & 5.7 & 5.3 & 4.5 & 3.9 \\
\hline $\mathbf{6}$ & 8.0 & 6.7 & 5.7 & 5.4 \\
\hline $\mathbf{5}$ & 6.1 & 4.8 & 4.2 & 3.9 \\
\hline $\mathbf{4}$ & 4.4 & 5.8 & 4.8 & 4.4 \\
\hline $\mathbf{3}$ & 5.2 & 4.8 & 4.3 & 3.3 \\
\hline $\mathbf{2}$ & 5.7 & 4.6 & 4.3 & 2.7 \\
\hline $\mathbf{1}$ & 4.8 & 5.0 & 3.8 & 3.8 \\
\hline & $\mathbf{1}$ & $\mathbf{2}$ & $\mathbf{3}$ & $\mathbf{4}$ \\
\hline
\end{tabular}

6.5

4.7

4.8

4.4

4.3

4.3

4.9 


\section{CleanAir.}

CONSOL ENERGY INC.

Client Reference No: 4700140111

AES GREENIDGE STATION

CleanAir Project No: 10192

\section{RESULTS}

Figure 2-5:

Run 2 SCR Outlet - MASS ${ }^{\circledR} \mathrm{NO}_{\mathrm{X}}$, and $\mathrm{O}_{2}$

Run 2: Outlet - Nitrogen Oxides (ppm dv @3\% 02)

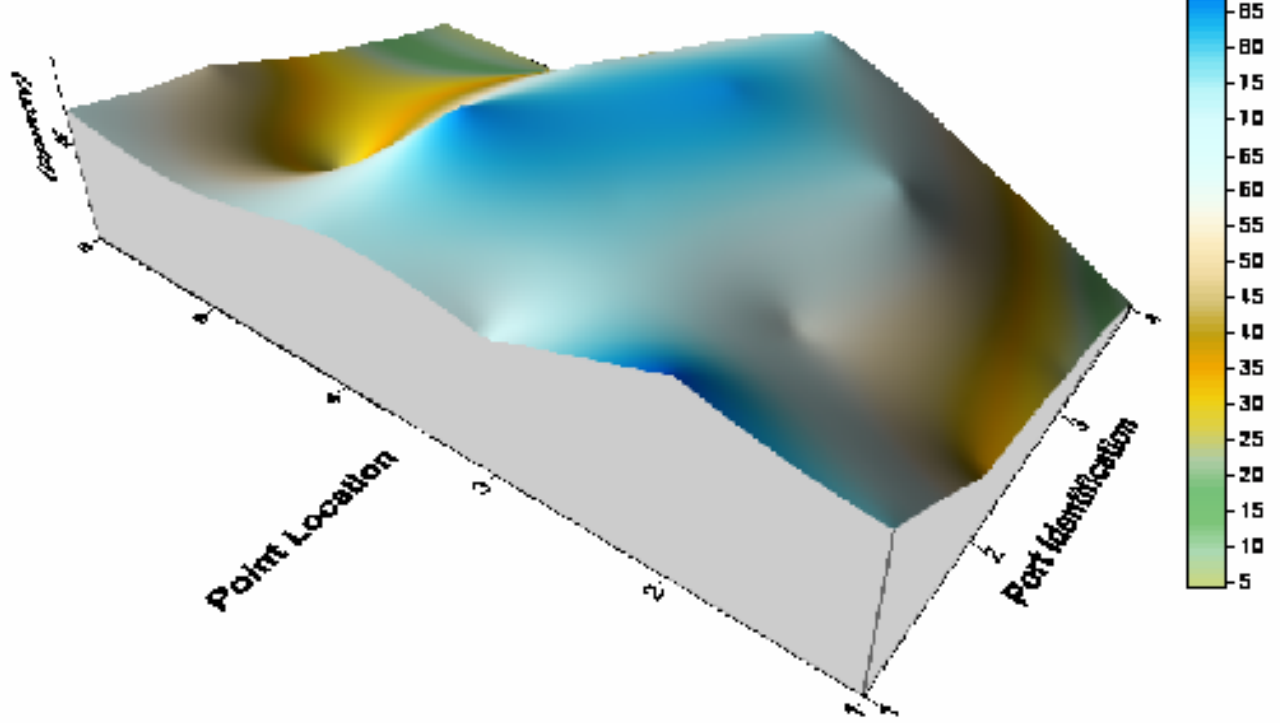

Run 2: Outtet - Oxygen (\% dV)

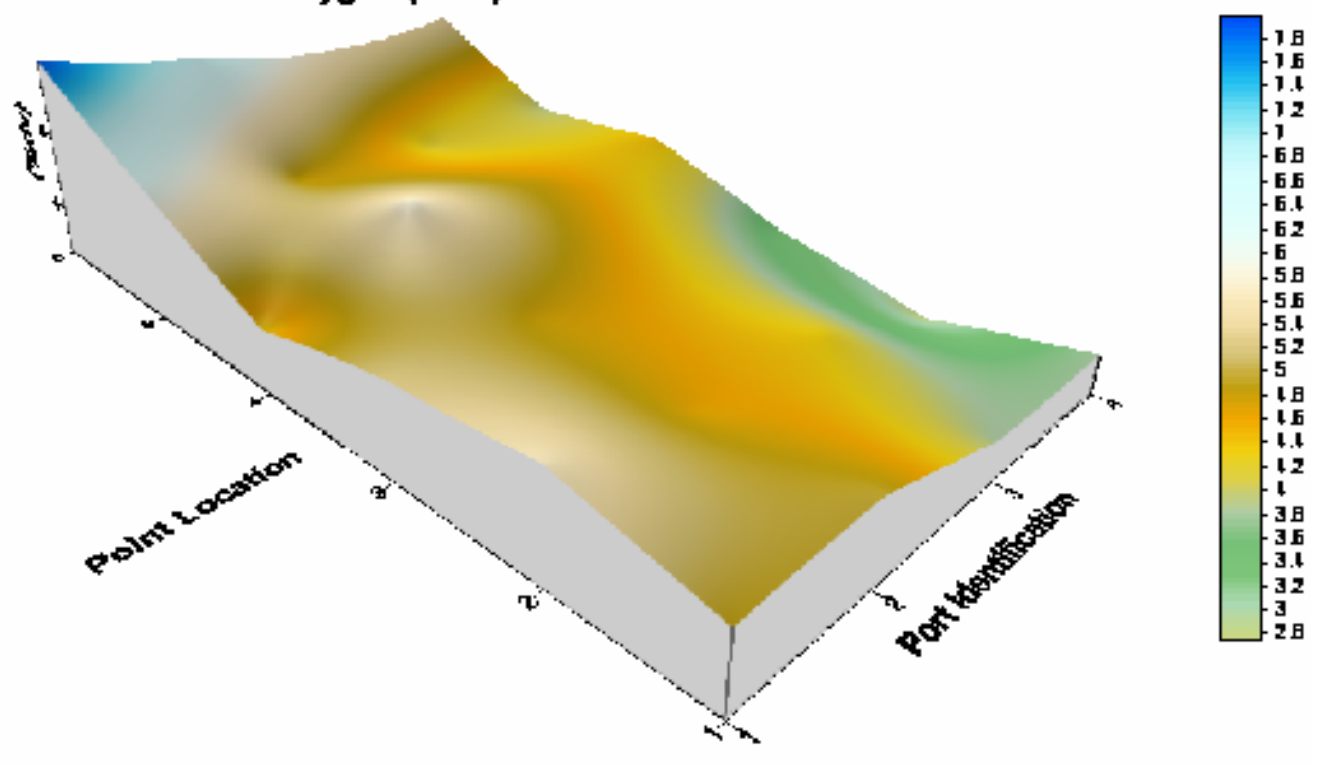




\section{CleanAir.}

\section{RESULTS}

Date: $3 / 28 / 2007$

Start Time: $\quad 11: 12$

End Time: $\quad$ 12:23

Removal Efficiency (ppmdv @ 3\% $\mathrm{O}_{2}$ )

\begin{tabular}{|c|c|c|c|c|}
\multicolumn{1}{c|}{ AVG } & $21.4 \%$ & $33.5 \%$ & $39.8 \%$ & $64.3 \%$ \\
\hline $\mathbf{6}$ & $34.5 \%$ & $45.3 \%$ & $74.6 \%$ & $93.4 \%$ \\
\hline $\mathbf{5}$ & $17.8 \%$ & $60.9 \%$ & $54.7 \%$ & $93.1 \%$ \\
\hline $\mathbf{4}$ & $14.2 \%$ & $-12.5 \%$ & $13.1 \%$ & $45.1 \%$ \\
\hline $\mathbf{3}$ & $30.8 \%$ & $17.5 \%$ & $13.4 \%$ & $19.0 \%$ \\
\hline $\mathbf{2}$ & $7.5 \%$ & $27.0 \%$ & $7.6 \%$ & $42.4 \%$ \\
\hline $\mathbf{1}$ & $23.8 \%$ & $63.0 \%$ & $75.3 \%$ & $92.7 \%$ \\
\hline & $\mathbf{1}$ & $\mathbf{2}$ & $\mathbf{3}$ & $\mathbf{4}$ \\
\cline { 2 - 5 }
\end{tabular}

Run 2: NOx Removal Efficiency (@ 3\% 02)

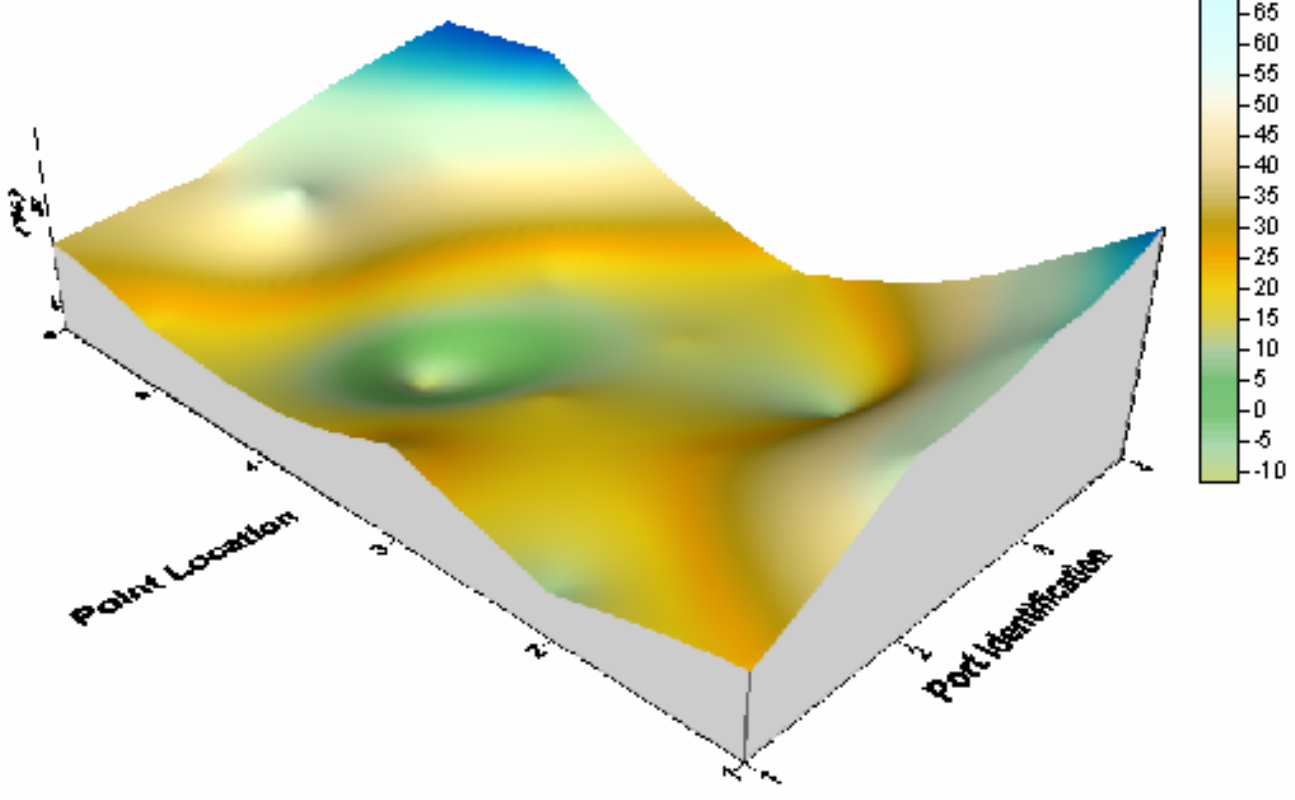

Run 2: MASS ${ }^{\circledR}$ - NOx Removal Efficiency 


\section{CleanAir.}

CONSOL ENERGY INC.

AES GREENIDGE STATION
Client Reference No: 4700140111

CleanAir Project No: 10192

\section{RESULTS}

2-11

Table 2-7:

Run 3 SCR Inlet - MASS ${ }^{\circledR} \mathrm{NO}_{\mathrm{X}}$, and $\mathrm{O}_{2}$

Date: $3 / 28 / 2007$

Start Time: $\quad 12: 48$

End Time: $\quad$ 13:59

Inlet - Nitrogen Oxides (ppmdv @ 3\% O

\begin{tabular}{|c|c|c|c|c|}
\multicolumn{1}{c|}{ AVG } & 91.1 & 83.7 & 85.0 & 78.5 \\
\hline $\mathbf{6}$ & 100.5 & 97.8 & 96.0 & 74.8 \\
\hline $\mathbf{5}$ & 68.8 & 59.5 & 66.6 & 58.8 \\
\hline $\mathbf{4}$ & 81.5 & 74.1 & 78.7 & 75.4 \\
\hline $\mathbf{3}$ & 94.1 & 96.1 & 98.3 & 94.4 \\
\hline $\mathbf{2}$ & 101.6 & 77.9 & 76.4 & 76.5 \\
\hline $\mathbf{1}$ & 100.1 & 96.5 & 93.8 & 91.1 \\
\hline & $\mathbf{1}$ & $\mathbf{2}$ & $\mathbf{3}$ & $\mathbf{4}$ \\
\hline
\end{tabular}

92.3

63.4

77.4

95.7

83.1

95.4

84.6

\begin{tabular}{|c|c|c|c|c|}
\hline \multicolumn{5}{|c|}{ Inlet - Oxygen (\%dv) } \\
\hline AVG & 5.6 & 4.9 & 4.1 & 4.3 \\
\hline 6 & 8.6 & 6.9 & 6.3 & 6.3 \\
\hline 5 & 4.5 & 4.2 & 4.3 & 4.1 \\
\hline$\overline{4}$ & 4.3 & 3.6 & 3.5 & 3.4 \\
\hline 3 & 5.0 & 5.2 & 3.3 & 3.1 \\
\hline 2 & 3.2 & 3.3 & 3.2 & 2.8 \\
\hline 1 & 7.8 & 6.2 & 4.3 & 6.1 \\
\hline & 1 & 2 & 3 & 4 \\
\hline
\end{tabular}

7.0

4.2

3.7

4.2

3.1

6.1

4.7 


\section{CleanAir.}

CONSOL ENERGY INC.

Client Reference No: 4700140111

AES GREENIDGE STATION

CleanAir Project No: 10192

\section{RESULTS}

2-12

Figure 2-7:

Run 3 SCR Inlet - MASS ${ }^{\circledR} \mathrm{NO}_{\mathrm{x}}$, and $\mathrm{O}_{2}$

Run 3: Inlet - Nitrogen Oxides (ppm dv @3\% 02)

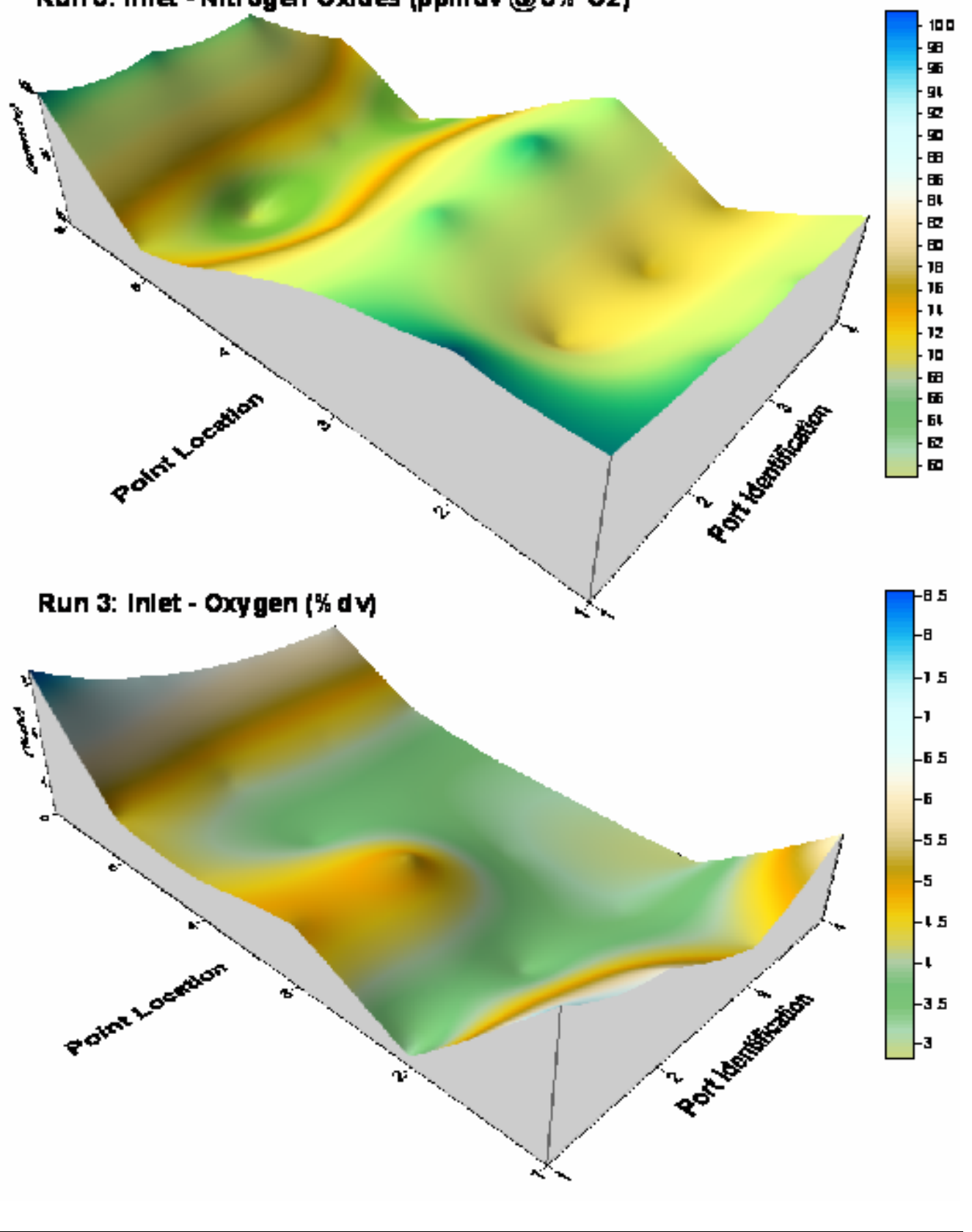




\section{CleanAir.}

CONSOL ENERGY INC.

AES GREENIDGE STATION
Client Reference No: 4700140111

CleanAir Project No: 10192

\section{RESULTS}

2-13

Table 2-8:

Run 3 SCR Outlet - MASS ${ }^{\circledR} \mathrm{NO}_{\mathrm{X}}$, and $\mathrm{O}_{2}$

Date: $3 / 28 / 2007$

Start Time: $\quad 12: 48$

End Time: $\quad 13: 59$

Outlet - Nitrogen Oxides (ppmdv @ 3\% $\mathrm{O}_{2}$ )

\begin{tabular}{|c|c|c|c|c|}
\multicolumn{1}{c}{ AVG } & 68.3 & 53.1 & 48.6 & 30.2 \\
\hline $\mathbf{6}$ & 68.5 & 48.2 & 24.6 & 4.8 \\
\hline $\mathbf{5}$ & 60.3 & 34.8 & 42.3 & 3.7 \\
\hline $\mathbf{4}$ & 70.3 & 80.4 & 69.1 & 42.0 \\
\hline $\mathbf{3}$ & 60.8 & 71.3 & 79.6 & 78.5 \\
\hline $\mathbf{2}$ & 78.7 & 51.5 & 57.1 & 45.7 \\
\hline $\mathbf{1}$ & 71.3 & 32.5 & 18.9 & 6.4 \\
\hline & $\mathbf{1}$ & $\mathbf{2}$ & $\mathbf{3}$ & $\mathbf{4}$ \\
\hline
\end{tabular}

36.5

35.3

65.5

72.6

58.3

32.3

50.1

Outlet - Oxygen (\%dv)

\begin{tabular}{|c|c|c|c|c|}
\multicolumn{1}{c}{ AVG } & 5.9 & 5.3 & 4.5 & 3.9 \\
\hline $\mathbf{6}$ & 7.9 & 6.6 & 5.4 & 5.3 \\
\hline $\mathbf{5}$ & 5.8 & 5.0 & 4.4 & 3.7 \\
\hline $\mathbf{4}$ & 4.9 & 5.7 & 4.7 & 4.3 \\
\hline $\mathbf{3}$ & 5.4 & 5.0 & 4.6 & 3.4 \\
\hline $\mathbf{2}$ & 5.4 & 4.3 & 3.9 & 2.8 \\
\hline $\mathbf{1}$ & 5.6 & 5.3 & 4.2 & 3.9 \\
\hline & $\mathbf{1}$ & $\mathbf{2}$ & $\mathbf{3}$ & $\mathbf{4}$ \\
\hline
\end{tabular}

6.3

4.7

4.9

4.6

4.1

4.8

4.9 


\section{CleanAir.}

CONSOL ENERGY INC.

Client Reference No: 4700140111

AES GREENIDGE STATION

CleanAir Project No: 10192

\section{RESULTS}

2-14

Figure 2-8:

Run 3 SCR Outlet - MASS ${ }^{\circledR} \mathrm{NO}_{\mathrm{X}}$, and $\mathrm{O}_{2}$

Run 3: Outlet - Nitrogen Oxides (ppm dv@3\% 02)

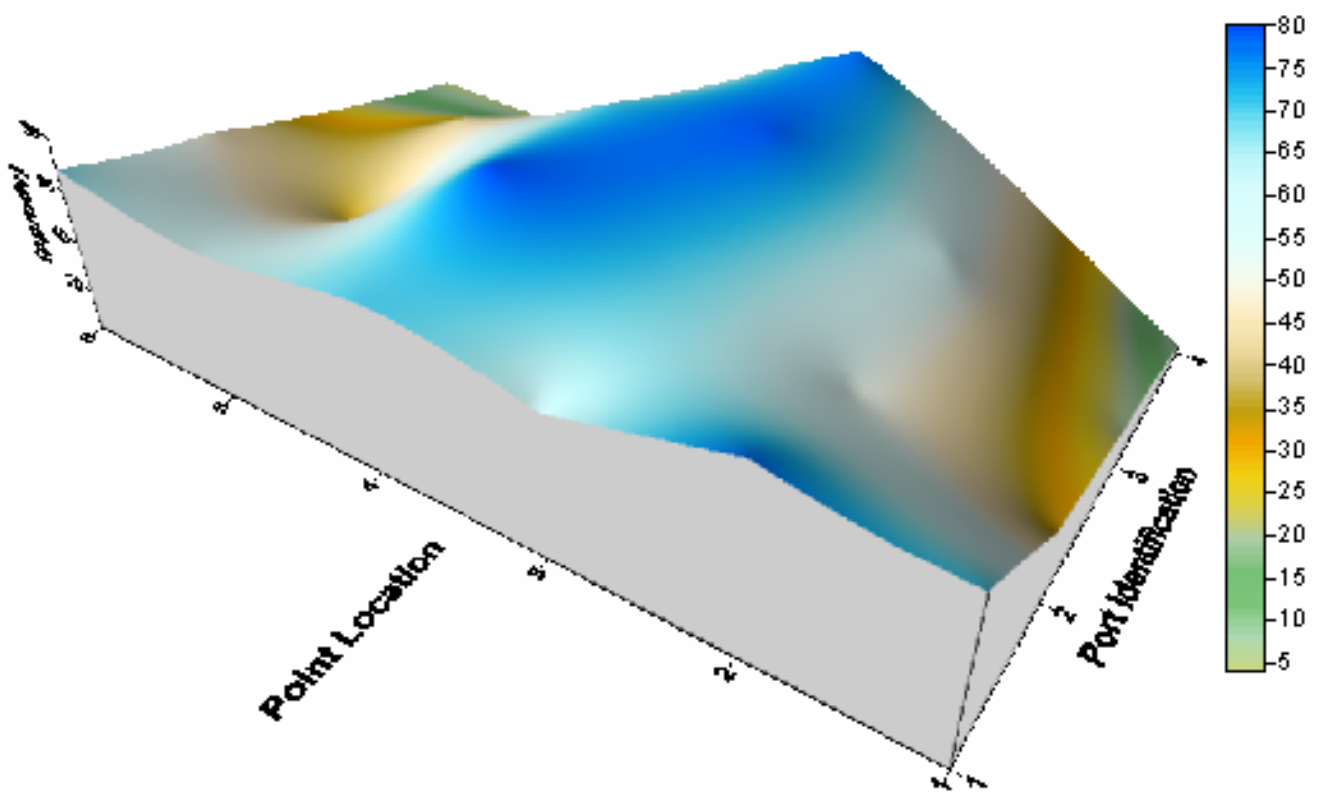

Run 3: Outlet - Oxygen ( $\%$ dv)

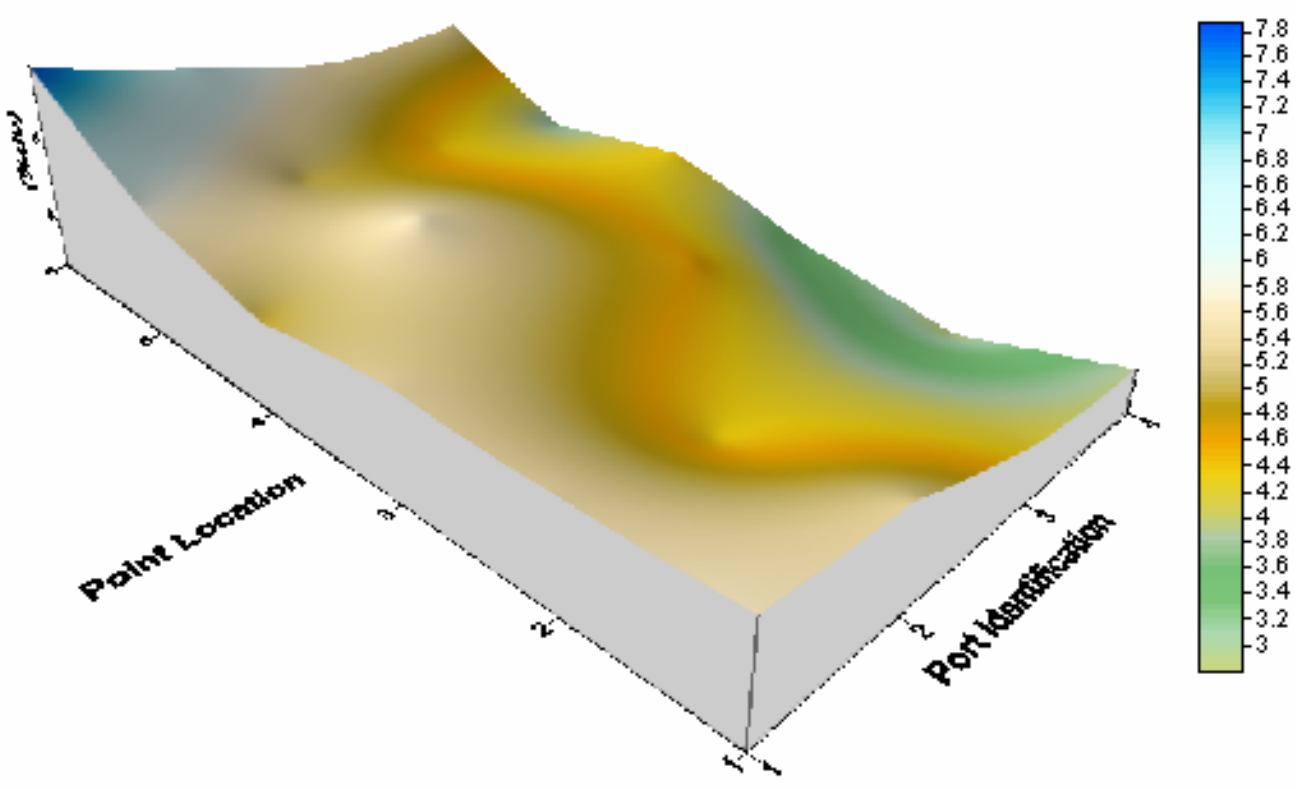




\section{CleanAir.}

\section{RESULTS}

Table 2-9:

Run 3 MASS $^{\circledR}$ - NOx Removal Efficiency

Date: $3 / 28 / 2007$

Start Time: $\quad$ 12:48

End Time: $\quad 13: 59$

Removal Efficiency (ppmdv @ 3\% $\mathrm{O}_{2}$ )

\begin{tabular}{|c|c|c|c|c|}
\multicolumn{1}{c|}{ AVG } & $\mathbf{2 4 . 1 \%}$ & $35.0 \%$ & $\mathbf{4 1 . 2} \%$ & $\mathbf{6 3 . 6 \%}$ \\
\hline $\mathbf{6}$ & $31.9 \%$ & $50.8 \%$ & $74.4 \%$ & $93.6 \%$ \\
\hline $\mathbf{5}$ & $12.4 \%$ & $41.6 \%$ & $36.4 \%$ & $93.6 \%$ \\
\hline $\mathbf{4}$ & $13.7 \%$ & $-8.5 \%$ & $12.2 \%$ & $44.3 \%$ \\
\hline $\mathbf{3}$ & $35.3 \%$ & $25.8 \%$ & $19.0 \%$ & $16.8 \%$ \\
\hline $\mathbf{2}$ & $22.5 \%$ & $34.0 \%$ & $25.2 \%$ & $40.2 \%$ \\
\hline $\mathbf{1}$ & $28.8 \%$ & $66.3 \%$ & $79.9 \%$ & $92.9 \%$ \\
\hline & $\mathbf{1}$ & $\mathbf{2}$ & $\mathbf{3}$ & $\mathbf{4}$ \\
\cline { 2 - 5 }
\end{tabular}

Run 3: NOx Rem oval Effieieney 3\% 02)

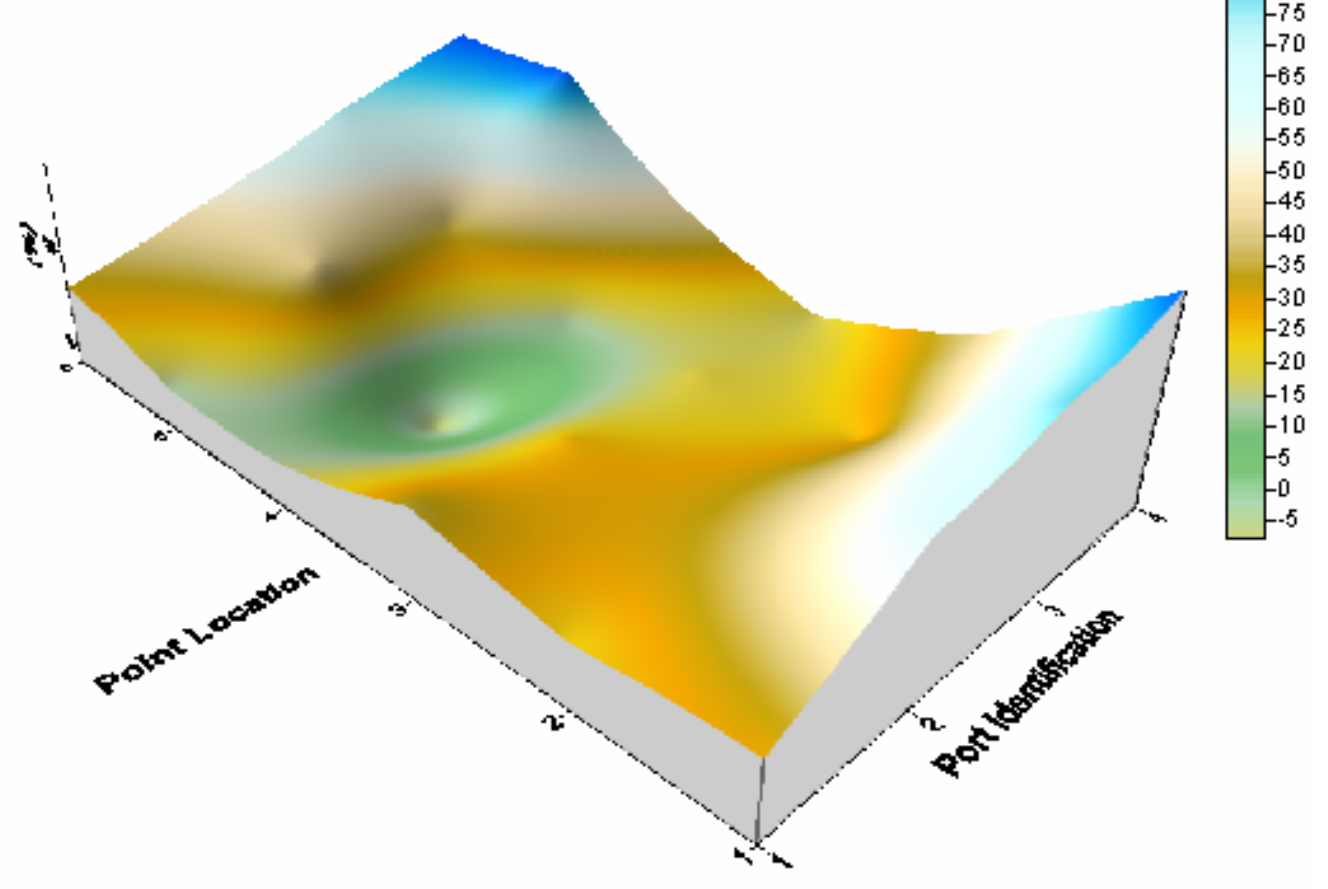

Figure 2-9:

Run 3 MASS $^{\circledR}$ - Removal Effiency 


\section{CleanAir.}

CONSOL ENERGY INC.

Client Reference No: 4700140111

AES GREENIDGE STATION

CleanAir Project No: 10192

\section{RESULTS}

2-16

Table 2-10:

Sulfur Dioxide Reduction Efficiency Results

$\begin{array}{lccc}\text { Run } & \mathbf{1} & \mathbf{2} & \mathbf{3} \\ \text { Date (2007) } & & & \\ \text { Start Time } & 29-M a r & 29-M a r & 29-M a r \\ \text { Stop Time } & 9: 59 & 12: 16 & 15: 13 \\ & 11: 00 & 13: 17 & 16: 13\end{array}$

Air Heater Outlet

$\begin{array}{lcccr}\mathrm{SO}_{2}\left(\mathrm{ppmdv} @ 3 \% \mathrm{O}_{2}\right) & 1854.76 & 1829.81 & 1837.92 & 1840.83 \\ \mathrm{O}_{2}(\% \mathrm{dv}) & 7.30 & 7.22 & 7.45 & 7.32 \\ \mathrm{CO}_{2}(\% \mathrm{dv}) & 11.54 & 11.61 & 11.52 & 11.56\end{array}$

\section{Baghouse Outlet}

$\begin{array}{lcccr}\mathrm{SO}_{2}\left(\mathrm{ppmdv} @ 3 \% \mathrm{O}_{2}\right) & 103.89 & 90.83 & 132.12 & 108.95 \\ \mathrm{O}_{2}(\% \mathrm{dv}) & 7.21 & 7.16 & 7.51 & 7.30 \\ \mathrm{CO}_{2}(\% \mathrm{dv}) & 11.49 & 11.71 & 11.51 & 11.57\end{array}$

Reduction Efficiency

$\begin{array}{lllll}\mathrm{SO}_{2}(\%) & 94.40 & 95.04 & 92.81 & 94.08\end{array}$




\section{CleanAir.}

\section{DESCRIPTION OF INSTALLATION}

AES Greenidge Unit 4 is representative of 492 coal-fired electricity generating units in the United States with capacities of 50-300 MWe. AES Greenidge Unit 4 is a 104-MW coal-fired unit. The unit had an integrated multipollutant control system installed on it, which is going to reduce emissions in an economically viable way. This new technology utilizes a hybrid selective non-catalytic reduction / selective catalytic reduction system for $\mathrm{NO}_{\mathrm{X}}$ reduction and a circulating fluidized-bed dry scrubber for $\mathrm{SO}_{2}$ and Mercury Reduction.

The testing was performed at the SCR inlet and outlet for $\mathrm{NO}_{\mathrm{x}}$ and $\mathrm{O}_{2} / \mathrm{CO}_{2}$. Testing was also conducted at the Air Heater Outlet and Baghouse Outlet for $\mathrm{SO}_{2}$ and $\mathrm{O}_{2} / \mathrm{CO}_{2}$.

\section{DESCRIPTION OF SAMPLING LOCATION(S)}

Sampling points for the MASS system were located on a grid at both the SCR Inlet and SCR Outlet location. The grid consisted of four ports with six points per port from which $\mathrm{NO}_{\mathrm{x}}, \mathrm{O}_{2}$, and $\mathrm{CO}_{2}$ were measured.

Sampling points for the $\mathrm{SO}_{2}$ testing were selected at a central location in the duct of the Air Heater Outlet and Baghouse Outlet for the first two runs. The third $\mathrm{SO}_{2}$ run consisted of a three point traverse of that same port.

Table 3-1 outlines the sampling point configurations. Figure 3-1 through 3-2 illustrates the sampling points and orientation of sampling ports for each of the sources tested in the program.

Table 3-1:

Sampling Points

\begin{tabular}{|c|c|c|c|c|c|c|c|c|}
\hline Location & Constituent & Method & $\begin{array}{l}\text { Run } \\
\text { No. }\end{array}$ & Ports & $\begin{array}{c}\text { Points } \\
\text { per Port }\end{array}$ & $\begin{array}{l}\text { Minutes } \\
\text { per Point }\end{array}$ & $\begin{array}{c}\text { Total } \\
\text { Minutes }\end{array}$ & Figure \\
\hline SCR Inlet & $\mathrm{NO}_{\mathrm{X}}, \mathrm{O}_{2}, \mathrm{CO}_{2}$ & $3 \mathrm{~A}, 7 \mathrm{E}$ & $1-3$ & 4 & 6 & 1 & $72^{1}$ & 3-1 \\
\hline SCR Outlet & $\mathrm{NO}_{\mathrm{X}}, \mathrm{O}_{2}, \mathrm{CO}_{2}$ & $3 \mathrm{~A}, 7 \mathrm{E}$ & $1-3$ & 4 & 6 & 1 & $72^{1}$ & $3-2$ \\
\hline Air Heater Outlet & $\mathrm{SO}_{2}, \mathrm{O}_{2}, \mathrm{CO}_{2}$ & $3 \mathrm{~A}, 6 \mathrm{C}$ & $1-2$ & 1 & 1 & 60 & 60 & N/A \\
\hline Air Heater Outlet & $\mathrm{SO}_{2}, \mathrm{O}_{2}, \mathrm{CO}_{2}$ & $3 \mathrm{~A}, 6 \mathrm{C}$ & 3 & 1 & 3 & 20 & 60 & $\mathrm{~N} / \mathrm{A}$ \\
\hline Baghouse Outlet & $\mathrm{SO}_{2}, \mathrm{O}_{2}, \mathrm{CO}_{2}$ & $3 \mathrm{~A}, 6 \mathrm{C}$ & $1-2$ & 1 & 1 & 60 & 60 & N/A \\
\hline Baghouse Outlet & $\mathrm{SO}_{2}, \mathrm{O}_{2}, \mathrm{CO}_{2}$ & $3 \mathrm{~A}, 6 \mathrm{C}$ & 3 & 1 & 3 & 20 & 60 & $\mathrm{~N} / \mathrm{A}$ \\
\hline
\end{tabular}

\footnotetext{
${ }^{1}$ Each cycle of the MASS took 24 minutes, 3 cycles were combined for each run.
} 


\section{CleanAir.}

CONSOL ENERGY INC.

Client Reference No: 4700140111

AES GREENIDGE STATION

CleanAir Project No: 10192

\section{DESCRIPTION OF INSTALLATION}

3-2

DESCRIPTION OF SAMPLING LOCATION (CONTINUED)

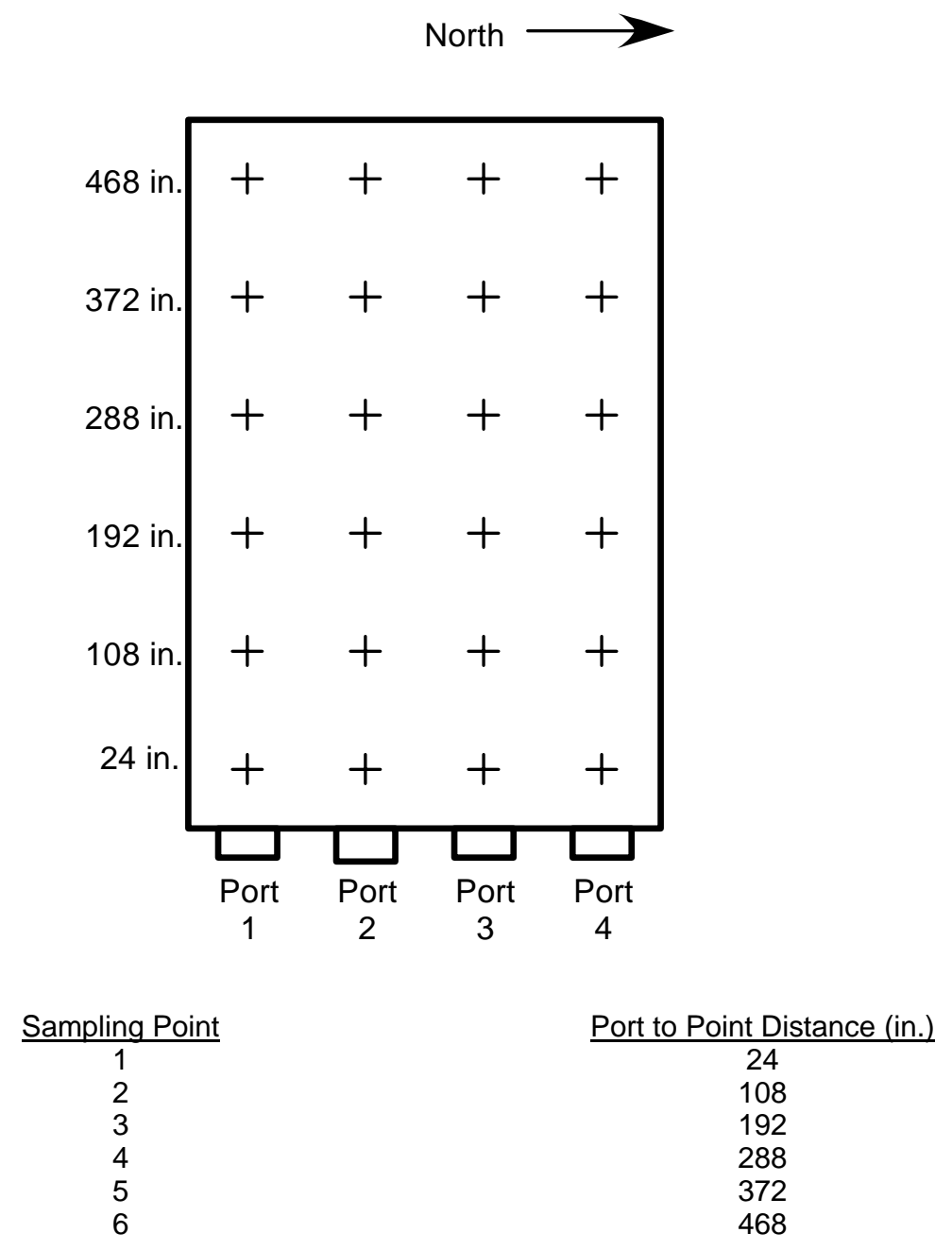

Figure 3-1: SCR Inlet - Sampling Point Determination (EPA Method 1) 


\section{CleanAir.}

CONSOL ENERGY INC.

Client Reference No: 4700140111

AES GREENIDGE STATION

CleanAir Project No: 10192

\section{DESCRIPTION OF INSTALLATION}

3-3

DESCRIPTION OF SAMPLING LOCATION (CONTINUED)

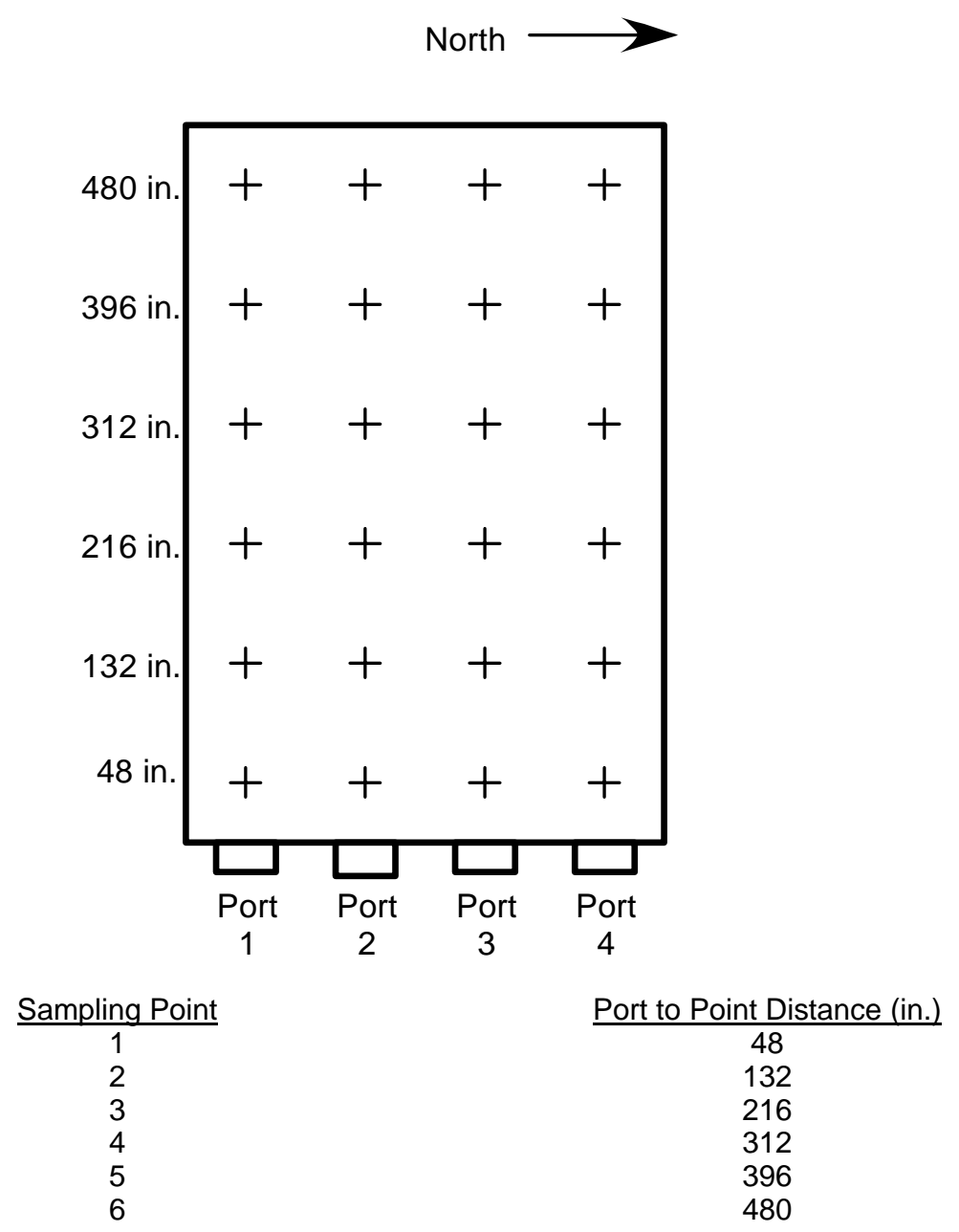

Figure 3-2: SCR Outlet - Sampling Point Determination (EPA Method 1) 


\section{METHODOLOGY}

Clean Air Engineering followed procedures as detailed in U.S. Environmental Protection Agency (EPA) Methods 3A, 6C, 7E, as well as Clean A Proprietary Method MASS $^{\circledR}$. The following table summarizes the methods and their respective sources.

Table 4-1:

\section{Summary of Sampling Procedures}

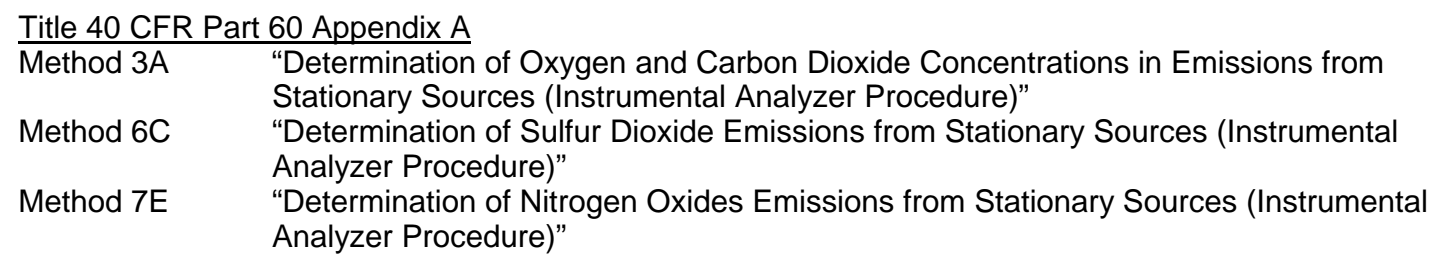

These methods appear in detail in Title 40 of the Code of Federal Regulations (CFR) and on the World Wide Web at http://www.cleanair.com.

Diagrams of the sampling apparatus and major specifications of the sampling, recovery and analytical procedures are summarized for each method in Appendix A.

Clean Air Engineering followed specific quality assurance and quality control (QA/QC) procedures as outlined in the individual methods and in USEPA "Quality Assurance Handbook for Air Pollution Measurement Systems: Volume III Stationary Source-Specific Methods”, EPA/600/R-94/038C. Additional QA/QC methods as prescribed in Clean Air's internal Quality Manual were also followed. Results of all QA/QC activities performed by Clean Air Engineering are summarized in Appendix D. 


\section{CleanAir.}

CONSOL ENERGY INC.

AES GREENIDGE STATION
Client Reference No: 4700140111

CleanAir Project No: 10192

\section{APPENDIX}

TEST METHOD SPECIFICATIONS

5-1

SAMPLE CALCULATIONS.

PARAMETERS

QA/QC DATA

FIELD DATA PRINTOUTS 
CONSOL ENERGY INC.

AES GREENIDGE STATION
Client Reference No: 4700140111

CleanAir Project No: 10192

\section{TEST METHOD SPECIFICATIONS}

A 
This Page Intentionally Left Blank

A -2 


\section{Specification Sheet for}

Source Location Name(s)

Pollutant(s) to be Determined

Other Parameters to be Determined from Train

\section{Pollutant Sampling Information}

Duration of Run

No. of Sample Traverse Points

Sample Time per Point

Sampling Rate

\section{Sampling Probe}

Nozzle Material

Nozzle Design

Probe Liner Material

Effective Probe Length

Probe Temperature Set-Point

\section{Particulate Filter}

In-Stack Filter

In-Stack Filter Material

External Filter

External Filter Material

External Filter Set-Point

\section{Sample Delivery System}

Heated Sample Line Material

Heated Sample Line Set-Point

Heated Sample Line Connections

Moisture Removal System

Sample Pump Type

Sample Pump Material

Sample Flow Control

Non-Heated Sample Line Material

Non-Heated Sample Line Connections

Additional Filters

Additional Filter Type

Additional Filter Location

Filter Material

\section{Analyzer Description}

Oxygen $\left(\mathrm{O}_{2}\right)$

Carbon Dioxide $\left(\mathrm{CO}_{2}\right)$

Sulfur Dioxide $\left(\mathrm{SO}_{2}\right)$

Nitrogen Oxides $\left(\mathrm{NO}_{\mathrm{x}}\right)$

Carbon Monoxide (CO)

Total Hydrocarbon (THC)

Hydrogen Chloride $(\mathrm{HCl})$

Ammonia $\left(\mathrm{NH}_{3}\right)$

\title{
EPA Method 6C
}

\author{
Air Heater Outlet
}

Sulfur Dioxide $\left(\mathrm{SO}_{2}\right)$

$\mathrm{O} 2$ and $\mathrm{CO} 2$ (EPA Method $3 \mathrm{~A}$ )

\section{Standard Method Specification}

N/A
N/A
N/A
Constant Rate

N/A

N/A

Stainless Steel or Pyrex Glass

Sufficient to Traverse Points

Prevent Condensation

Yes

Non-reactive to gas

Yes

Borosilicate, Quartz Glass Wool or Fiber Mat

Prevent Condensation

Stainless Steel or Teflon

Prevent Condensation

Probe Exit to Moisture Removal System

Refrigerator-type condenser or similar

Leak-Free, minimal response time

Non-reactive to sample gases

Constant Rate

Stainless Steel or Teflon

Moisture Removal to Sample Gas Manifold

Optional

N/A

Optional

Non-reactive to sample gases

EPA Method 3A (Paramagnetic)

EPA Method 3A (NDIR)

EPA Method 6C (UV, NDIR or Fluorescence)

N/A

N/A

N/A

N/A

N/A
Actual Specification Used

60 minutes

1

60 minutes

Constant Rate

None

N/A

Stainless Steel

8 feet

$248^{\circ} \mathrm{F} \pm 25^{\circ} \mathrm{F}$

Yes

Fritted Stainless Steel

Yes

Borosilicate Glass Fiber Mat

$248^{\circ} \mathrm{F} \pm 25^{\circ} \mathrm{F}$

Tefion

$248^{\circ} \mathrm{F} \pm 25^{\circ} \mathrm{F}$

Probe to Moisture Removal System

Refrigerator-type condenser

Diaphragm

Teflon

Constant Rate $( \pm 10 \%)$

Teflon

Moisture Removal to Sample Gas Manifold

Yes

Particulate Removal

Entrance to Sample Manifold

Glass Fiber

EPA Method 3A (Paramagnetic)

EPA Method 3A (NDIR)

EPA Method 6C (UV Absorption) 


\section{Specification Sheet for}

\author{
Instrument Span Range \\ Oxygen $\left(\mathrm{O}_{2}\right)$ \\ Carbon Dioxide $\left(\mathrm{CO}_{2}\right)$ \\ Sulfur Dioxide $\left(\mathrm{SO}_{2}\right)$ \\ Nitrogen Oxides $\left(\mathrm{NO}_{\mathrm{x}}\right)$ \\ Carbon Monoxide (CO) \\ Total Hydrocarbon (THC) \\ Hydrogen Chloride $(\mathrm{HCl})$ \\ Ammonia $\left(\mathrm{NH}_{3}\right)$
}

\section{Data Acquisition}

Data Recorder

Recorder Resolution

Data Storage

Measurement Freq. $<60 \mathrm{~min}$. Sample Time Recording Freq. $<60 \mathrm{~min}$. Sample Time Measurement Freq. $>60 \mathrm{~min}$. Sample Time Recording Freq. $>60 \mathrm{~min}$. Sample Time

\section{Calibration Gas Specifications}

\section{Oxygen $\left(\mathrm{O}_{2}\right)$}

Carbon Dioxide $\left(\mathrm{CO}_{2}\right)$

Sulfur Dioxide $\left(\mathrm{SO}_{2}\right)$

Nitrogen Oxides $\left(\mathrm{NO}_{\mathrm{x}}\right)$

Carbon Monoxide (CO)

Total Hydrocarbon (THC)

Hydrogen Chloride $(\mathrm{HCl})$

Ammonia $\left(\mathrm{NH}_{3}\right)$

\section{EPA Method 6C}

Standard Method Specification

Actual Specification Used

$\begin{array}{ll}\leq 1.33 \times \text { Expected Maximum } & 0-15 \% \\ \leq 1.33 \times \text { Expected Maximum } & 0-15 \% \\ \leq 1.33 \times \text { Expected Maximum } & 0-5,000 \mathrm{ppm} \\ \text { N/A } & \text { N/A } \\ \text { N/A } & \text { N/A } \\ \text { N/A } & \text { N/A } \\ \text { N/A } & \text { N/A } \\ \text { N/A } & \text { N/A }\end{array}$

Strip chart, Analog Computer or Digital Recorder

Analog Computer

0.5 Percent of Span

0.1 Percent of Span

Manually or Automatic

Manually

1 -min. intervals or 30 measurements (less restrictive) One reading per second

1 -min. intervals or 30 measurements (less restrictive) One Minute Average $(60,1$ second readings)

2-min. intervals or 96 measurements (less restrictive) N/A

2-min. intervals or 96 measurements (less restrictive) N/A

EPA Protocol 1

EPA Protocol 1

EPA Protocol 1

EPA Protocol 1

EPA Protocol 1

EPA Protocol 1

N/A

N/A

N/A

N/A

N/A

A - 4 


\section{Specification Sheet for}

Source Location Name(s)

Pollutant(s) to be Determined

Other Parameters to be Determined from Train $\mathrm{O} 2$ and CO2 (EPA Method 3A)

\section{Pollutant Sampling Information}

Duration of Run

No. of Sample Traverse Points

Sample Time per Point

Sampling Rate

\section{Sampling Probe}

Nozzle Material

Nozzle Design

Probe Liner Material

Effective Probe Length

Probe Temperature Set-Point

\section{Particulate Filter}

In-Stack Filter

In-Stack Filter Material

External Filter

External Filter Material

External Filter Set-Point

\section{Sample Delivery System}

Heated Sample Line Material

Heated Sample Line Set-Point

Heated Sample Line Connections

Moisture Removal System

Sample Pump Type

Sample Pump Material

Sample Flow Control

Non-Heated Sample Line Material

Non-Heated Sample Line Connections

Additional Filters

Additional Filter Type

Additional Filter Location

Filter Material

\section{Analyzer Description}

Oxygen $\left(\mathrm{O}_{2}\right)$

Carbon Dioxide $\left(\mathrm{CO}_{2}\right)$

Sulfur Dioxide $\left(\mathrm{SO}_{2}\right)$

Nitrogen Oxides $\left(\mathrm{NO}_{\mathrm{x}}\right)$

Carbon Monoxide (CO)

Total Hydrocarbon (THC)

Hydrogen Chloride $(\mathrm{HCl})$

Ammonia $\left(\mathrm{NH}_{3}\right)$

\section{EPA Method 6C}

\author{
Baghouse Outlet
}

Sulfur Dioxide $\left(\mathrm{SO}_{2}\right)$

N/A

N/A

Stainless Steel or Pyrex Glass

Sufficient to Traverse Points

Prevent Condensation

Yes

Non-reactive to gas

Yes

Prevent Condensation

Stainless Steel or Teflon

Prevent Condensation

Non-reactive to sample gases

EPA Method 3A (NDIR)

$N / A$

N/A

$N / A$

N/A

N/A
Standard Method Specification

Actual Specification Used

$\begin{array}{ll}\text { N/A } & 60 \text { minutes } \\ \text { N/A } & 1 \\ \text { N/A } & 60 \text { minutes } \\ \text { Constant Rate } & \text { Constant Rate }\end{array}$

None

N/A

Stainless Steel

8 feet

$248^{\circ} \mathrm{F} \pm 25^{\circ} \mathrm{F}$

Yes

Fritted Stainless Steel

Yes

Borosilicate, Quartz Glass Wool or Fiber Mat

Borosilicate Glass Fiber Mat

$248^{\circ} \mathrm{F} \pm 25^{\circ} \mathrm{F}$

Teflon

Probe Exit to Moisture Removal System

$248^{\circ} \mathrm{F} \pm 25^{\circ} \mathrm{F}$

Probe to Moisture Removal System

Refrigerator-type condenser

Diaphragm

Teflon

Constant Rate $( \pm 10 \%)$

Teflon

Moisture Removal to Sample Gas Manifold

Yes

Particulate Removal

Entrance to Sample Manifold

Glass Fiber

EPA Method 3A (Paramagnetic)

EPA Method 6C (UV, NDIR or Fluorescence)
EPA Method $3 A$ (Paramagnetic)

EPA Method 3A (NDIR)

EPA Method 6C (UV Absorption) 
Specification Sheet for

\section{Instrument Span Range}

Oxygen $\left(\mathrm{O}_{2}\right)$

Carbon Dioxide $\left(\mathrm{CO}_{2}\right)$

Sulfur Dioxide $\left(\mathrm{SO}_{2}\right)$

Nitrogen Oxides $\left(\mathrm{NO}_{\mathrm{x}}\right)$

Carbon Monoxide (CO)

Total Hydrocarbon (THC)

Hydrogen Chloride ( $\mathrm{HCl})$

Ammonia $\left(\mathrm{NH}_{3}\right)$

\section{Calibration Gas Specifications}

Oxygen $\left(\mathrm{O}_{2}\right)$

Carbon Dioxide $\left(\mathrm{CO}_{2}\right)$

Sulfur Dioxide $\left(\mathrm{SO}_{2}\right)$

\section{EPA Method 6C}

Standard Method Specification

Actual Specification Used

$\leq 1.33 \times$ Expected Maximum
$\leq 1.33 \times$ Expected Maximum
$\leq 1.33 \times$ Expected Maximum
N/A
N/A
N/A
N/A
N/A

$0-15 \%$

$0-15 \%$

0-150 ppm

$\mathrm{N} / \mathrm{A}$

N/A

$\mathrm{N} / \mathrm{A}$

N/A

N/A

EPA Protocol 1

EPA Protocol 1

EPA Protocol 1

EPA Protocol 1
EPA Protocol 1 


\section{EPA Methods 3A, 6C Sampling Train Configuration Air Heater Outlet \& Baghouse Outlet}

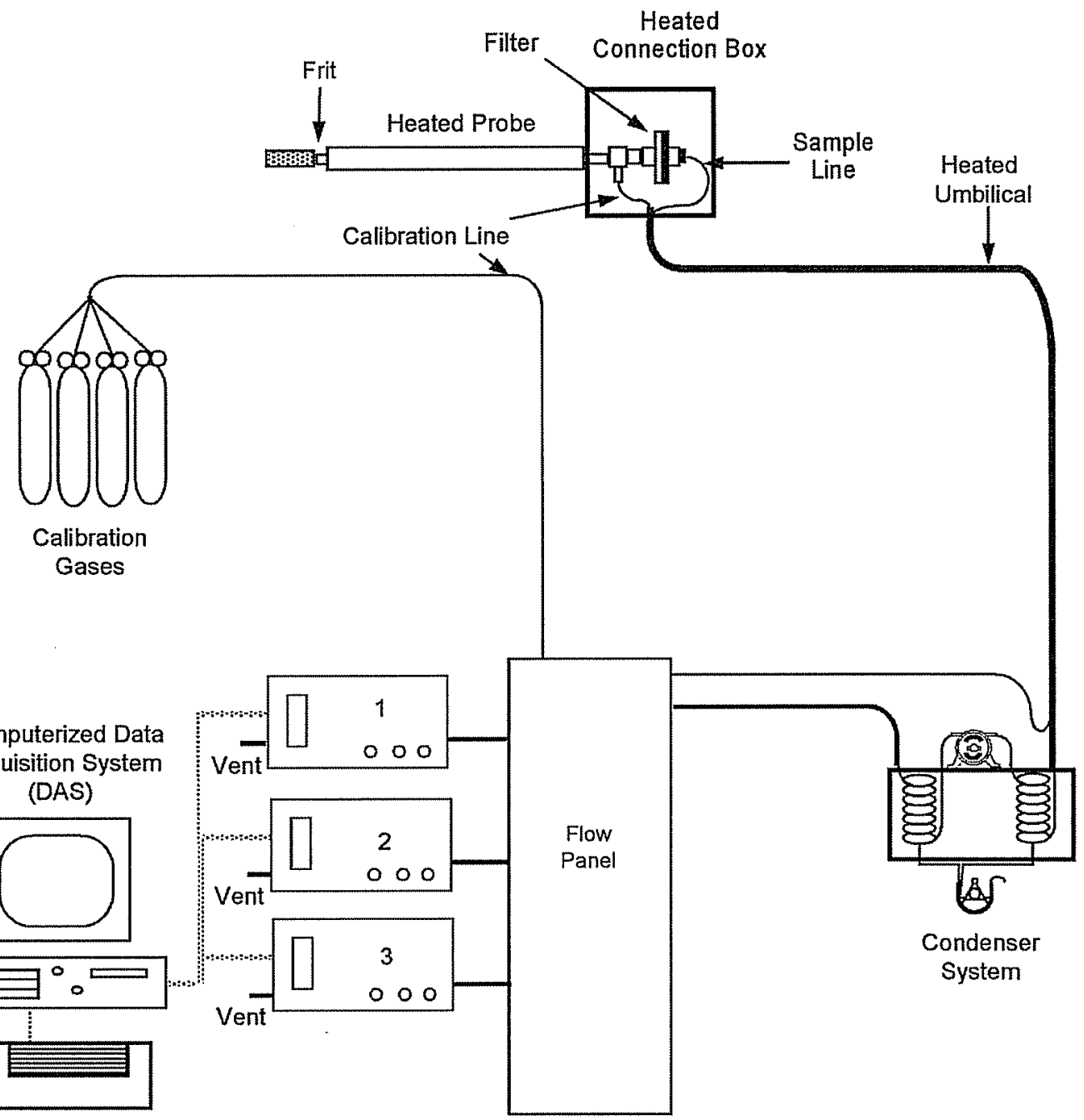

Printer

\begin{tabular}{|c|c|c|c|c|}
\hline Number & Gas & Monitor & Range Used & $\begin{array}{c}\text { Calibration Gas } \\
\text { Concentrations }\end{array}$ \\
\hline 1 Air Heater Outlet & $\mathrm{O}_{2}$ & Servomex 1420B Paramanetic & $0-15 \%$ & $6.04 \%, 14.1 \%$ \\
\hline 2 Air Heater Outlet & $\mathrm{CO}_{2}$ & Servomex 1415 NDIR & $0-15 \%$ & $6.02,13.98 \%$ \\
\hline 3 Air Heater Outlet & $\mathrm{SO}_{2}$ & Western Research 921 UV & $0-5000 \mathrm{ppm}$ & $121.6 \mathrm{ppm}, 2479 \mathrm{ppm}$ \\
\hline 1 Baghouse Outlet & $\mathrm{O}_{2}$ & Servomex 1420B Paramanetic & $0-15 \%$ & $6.04 \%, 14.1 \%$ \\
\hline 2 Baghouse Outlet & $\mathrm{CO}_{2}$ & Servomex 1415 NDIR & $0-15 \%$ & $6.02,13.98 \%$ \\
\hline 3 Baghouse Outlet & $\mathrm{SO}_{2}$ & Western Research 921 UV & $0-150 \mathrm{ppm}$ & $49.35 \mathrm{ppm}, 121.6 \mathrm{ppm}$ \\
\hline
\end{tabular}




\section{Specification Sheet for}

Source Location Name(s)

Pollutant(s) to be Determined

Other Parameters to be Determined from Train

\section{Pollutant Sampling Information}

Duration of Run

No. of Sample Traverse Points

Sample Time per Point

Sampling Rate

\section{Sampling Probe}

Nozzle Material

Nozzle Design

Probe Liner Material

Effective Probe Length

Probe Temperature Set-Point

\section{Particulate Filter}

In-Stack Filter

In-Stack Filter Material

External Filter

External Filter Material

External Filter Set-Point

\section{Sample Delivery System}

Heated Sample Line Material

Heated Sample Line Set-Point

Heated Sample Line Connections

Moisture Removal System

Sample Pump Type

Sample Pump Material

Sample Flow Control

Non-Heated Sample Line Material

Non-Heated Sample Line Connections

Additional Filters

Additional Filler Type

Additional Filter Location

Filter Material

\section{Analyzer Description}

Oxygen $\left(\mathrm{O}_{2}\right)$

Carbon Dioxide $\left(\mathrm{CO}_{2}\right)$

Sulfur Dioxide $\left(\mathrm{SO}_{2}\right)$

Nitrogen Oxides $\left(\mathrm{NO}_{x}\right)$

Carbon Monoxide (CO)

Total Hydrocarbon (THC)

Hydrogen Chloride $(\mathrm{HCl})$

Ammonia $\left(\mathrm{NH}_{3}\right)$

\section{EPA Method 3A and 7E with MASS}

SCR Inlet/Outlet Test Grid

Determination of Nitrogen Oxides $\left(\mathrm{NO}_{x}\right)$ Emissions

O2 (EPA Method 3A)
Actual Specification Used

N/A

N/A

N/A

Constant Rate

N/A

N/A

Stainless Steel or Pyrex Glass

Sufficient to Traverse Points

Prevent Condensation

Yes

Non-reactive to gas

Yes

Borosilicate, Quartz Glass Wool or Fiber Mat

Prevent Condensation

Stainless Steel or Teflon

Prevent Condensation

Probe Exit to Moisture Removal System

Refrigerator-type condenser or similar

Leak-Free, minimal response time

Non-reactive to sample gases

Constant Rate

Stainless Steel or Teflon

Moisture Removal to Sample Gas Manifold

Optional

N/A

Optional

Non-reactive to sample gases

N/A

N/A

N/A

EPA Method 7E (Chemiluminescent)

N/A

N/A

N/A

N/A
72 minutes

24 ( 3 cycles per run)

1 minute

Constant Rate

None

N/A

Test Grid

See Report Section 3 for point locations Stack Temp

No

N/A

Yes

Borosilicate Glass Fiber Mat Ambient

Teflon

Ambient

N/A

Coil - Condenser Type

Piston

Teflon

Constant Rate $( \pm 10 \%)$

Teflon

Probe to Sample Gas Manifold

Yes

Particulate Removal

Entrance to Sample Manifold

Glass Fiber

EPA Method 3A (Paramagnetic)

N/A

N/A

EPA Method 7E (Chemiluminescent)

N/A

N/A

N/A

N/A 
Specification Sheet for

\author{
Instrument Span Range \\ Oxygen $\left(\mathrm{O}_{2}\right)$ \\ Carbon Dioxide $\left(\mathrm{CO}_{2}\right)$ \\ Sulfur Dioxide $\left(\mathrm{SO}_{2}\right)$ \\ Nitrogen Oxides $\left(\mathrm{NO}_{\mathrm{x}}\right)$ \\ Carbon Monoxide (CO) \\ Total Hydrocarbon (THC) \\ Hydrogen Chloride $(\mathrm{HCl})$ \\ Ammonia $\left(\mathrm{NH}_{3}\right)$
}

\section{Data Acquisition}

Data Recorder

Recorder Resolution

Data Storage

Measurement Freq. $<60 \mathrm{~min}$. Sample Time Recording Freq. $<60 \mathrm{~min}$. Sample Time Measurement Freq. $>60 \mathrm{~min}$. Sample Time Recording Freq. $>60 \mathrm{~min}$. Sample Time

\section{Calibration Gas Specifications}

Oxygen $\left(\mathrm{O}_{2}\right)$

Carbon Dioxide $\left(\mathrm{CO}_{2}\right)$

Sulfur Dioxide $\left(\mathrm{SO}_{2}\right)$

Nitrogen Oxides $\left(\mathrm{NO}_{x}\right)$

Carbon Monoxide (CO)

Total Hydrocarbon (THC)

Hydrogen Chloride $(\mathrm{HCl})$

Ammonia $\left(\mathrm{NH}_{3}\right)$

\section{EPA Method 3A and 7E with MASS}

Standard Method Specification

Actual Specification Used

$\begin{array}{ll}\leq 1.33 \times \text { Expected Maximum } & 0-15 \% \\ \leq 1.33 \times \text { Expected Maximum } & 0-15 \% \\ & \text { N/A } \\ \leq 1.33 \times \text { Expected Maximum } & 0-250 \mathrm{ppm} \\ \leq 1.33 \times \text { Expected Maximum } & \text { N/A } \\ \text { N/A } & \text { N/A } \\ \text { N/A } & \text { N/A } \\ \text { N/A } & \text { N/A }\end{array}$

Strip chart, Analog Computer or Digital Recorder

Analog Computer

0.5 Percent of Span

0.1 Percent of Span

Manually or Automatic

Automatic

1 -min. intervals or 30 measurements (less restrictive) One reading per second

1 -min. intervals or 30 measurements (less restrictive) One Minute Average $(60,1$ second readings)

2-min. intervals or 96 measurements (less restrictive) N/A

2-min. intervals or 96 measurements (less restrictive) N/A

EPA Protocol 1
N/A
N/A
EPA Protocol 1
N/A
N/A
N/A
N/A

EPA Protocol 1

EPA Protocol 1

EPA Protocol 1 
CONSOL ENERGY INC. AES GREENIDGE STATION
Client Reference Number: $\mathbf{4 7 0 0 1 4 0 1 1 1}$

Clean Air Project Number: 10192

\section{Multi-Point Automated Sampling System}

The NOx and $\mathrm{O}_{2}$ distributions at the SCR Inlet and Outlet were measured simultaneously using an extractive continuous emission monitoring (CEM) package contained in a mobile emission laboratory. The system is comprised of three basic subsystems, including; 1) a flue gas sample acquisition and conditioning system, 2) a calibration gas system, and 3) the electronic gas analyzers.

In order to assess local NOx reductions, CleanAir has developed a proprietary flue gas profiling system called the Multi-Point Automated Sampling System (MASS). The MASS system allows duct emission profiles to be characterized in a matter of minutes, as opposed much longer sampling times for traditional duct emission traverses using "manual" traversing techniques.

Clean Air Engineering's MASS system uses a series of automated 8-point sample modules with integrated programmable logic controllers (PLC's) to sequentially and rapidly cycle through a multi-point sample grid. A diagram of the sampling system is provided in Figure 1.

Figure 1:

CleanAir's Multi-Point Automated Sampling System

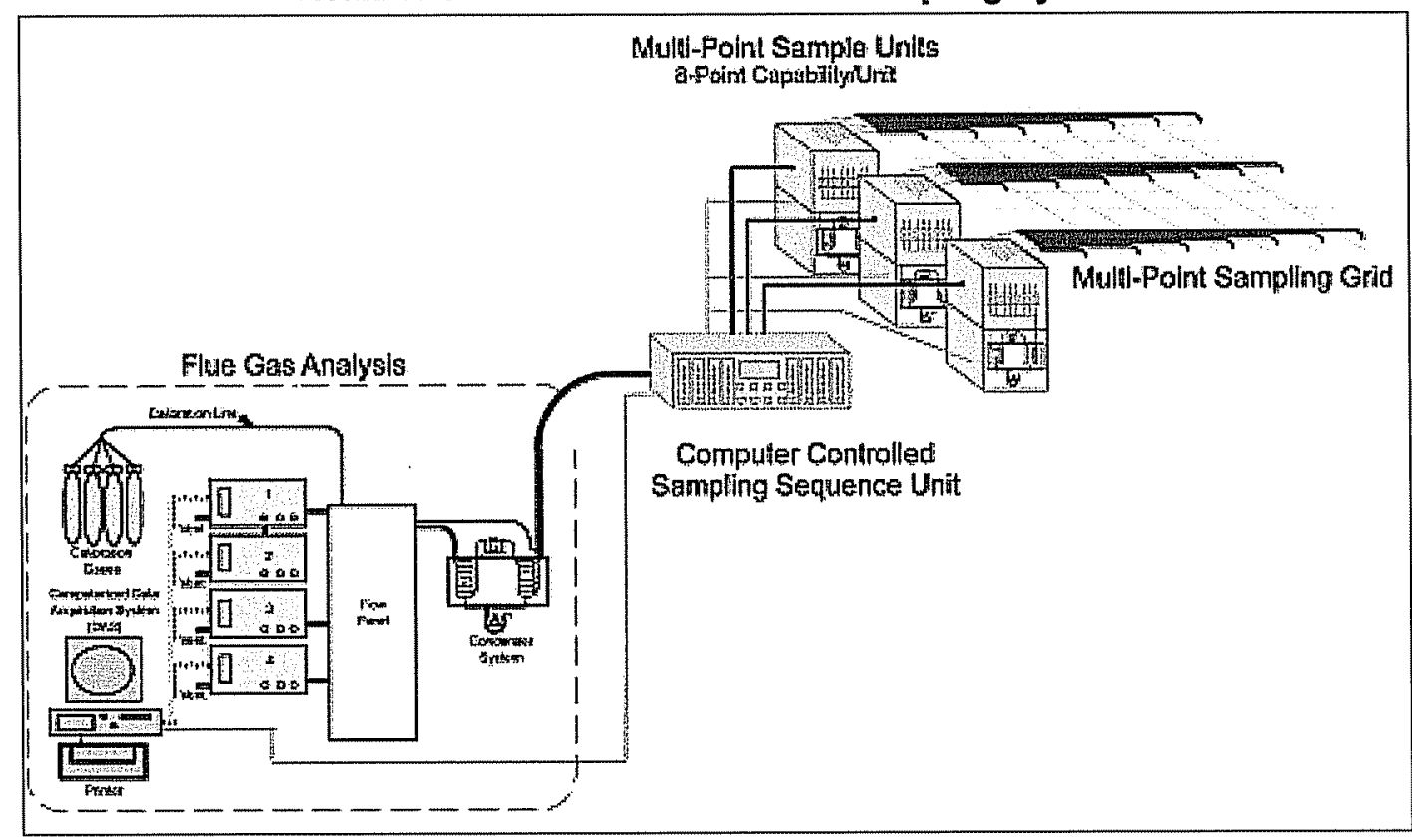

Based on a 24 point grid system (Inlet and Outlet) for the reactor module, three (3) cycles were performed to obtain a test duration of 72 minutes. A total of three test runs were performed concurrently at both the SCR Inlet and Outlet test grid locations. 
CONSOL ENERGY INC.

AES GREENIDGE STATION
Client Reference No: 4700140111

CleanAir Project No: 10192

SAMPLE CALCULATIONS

B 
This Page Intentionally Left Blank

B -2 


\section{CEM Field Sample Calculations for SO2 AH Outlet}

Sample data taken from Run 1].WW

and Channel 2

Note: The tables presenting the results are generated electronically from raw data. It may not be possible to exactly duplicate these results using a calculator. The reference method data, results and all calculations are carried to sixteen decimal places throughout. The final table is formatted to an appropriate number of significant figures.

051507140613

1. Average of a calibration series

$$
C_{\text {mce }}=\frac{\left(C_{1}+C_{2}+C_{3}\right)}{3}
$$

Where:

$$
\begin{aligned}
\mathrm{C}_{1}, \mathrm{C}_{2}, \mathrm{C}_{3}= & \text { concentrations of } 3 \text { consecutive gas samples that are } \\
& \text { representative of the calibration gas } \\
\mathrm{C}_{\mathrm{mce}} & \text { average concentration of a calibration series } \\
& \text { In this case the low cal series for channel } 2
\end{aligned}
$$

ppmdv

2a. Calibration Error Check for Hydrocarbons ( $5 \%$ of actual calibration gas value error allowed by Method 25A)

$$
\begin{aligned}
& \begin{array}{c}
E_{H C} \\
\text { Where: }
\end{array}=a b s\left|\frac{C_{m c e}-C_{m a}}{C_{m a}}\right| \leq l_{c a l} \\
& \mathrm{C}_{\mathrm{mce}} \quad=\text { average concentration of a calibration series } \\
& \text { In this case the low cal series for channel } 2 \\
& \mathrm{C}_{\mathrm{ma}} \quad=\text { concentration of actual calibration gas value } \\
& \mathrm{I}_{\mathrm{cal}} \quad=\text { limit for calibration error for hydrocarbons } \\
& E_{H C} \quad=\text { calibration error check value }
\end{aligned}
$$

$\begin{array}{lcc}= & 133.235 & \text { ppmdv } \\ = & 121.600 & \text { ppmdv } \\ = & 5.0 \% & \\ & & \end{array}$

2b. Calibration Error Check for non-Hydrocarbons (2\% of Instrument Span)

$$
\begin{aligned}
& E=a b s\left|\frac{C_{m c e}-C_{m a}}{\operatorname{Span}}\right| \leq l_{c a l} \\
& \mathrm{C}_{\text {mce }} \quad=\text { average concentration of a calibration series } \\
& \text { In this case the low cal series for channel } 2 \\
& \mathrm{C}_{\mathrm{ma}} \quad=\text { concentration of actual calibration gas value } \\
& \text { Span } \quad=\text { instrument span value } \\
& \text { I } \quad=\text { limit for calibration error for non-hydrocarbons } \\
& \mathrm{E} \quad=\text { calibration error check value }
\end{aligned}
$$

$\begin{array}{lcc}= & 133.235 & \text { ppmdv } \\ = & 121.600 & \text { ppmdv } \\ = & 2479.000 & \\ = & 2.0 \% & \\ & & \text { Pass }\end{array}$

3. System Bias as Percent of Span Value ( $5 \%$ is allowed)

$$
E_{\text {Bias }}=a b s\left|\frac{C_{m f}-C_{m c e}}{\operatorname{Span}}\right| \leq l_{\text {bias }}
$$

Where:

$$
\begin{aligned}
& \mathrm{C}_{\text {mce }}=\text { average concentration of a calibration series } \\
& \text { in this case the High cal series for channel } 2 \\
& \mathrm{C}_{\mathrm{mt}} \quad=\text { calibration error response concentration for Cal01 } \\
& \text { Span } \quad=\text { instrument span value } \\
& \text { lbias } \quad=\text { limit for system bias error } \\
& \text { E } \quad=\text { calibration bias error check value }
\end{aligned}
$$

$\begin{array}{lcl}= & 2482.035 & \text { ppmdv } \\ = & 2411.901 & \text { ppmdv } \\ = & 2479.000 & \text { ppmdv } \\ = & 5.0 \% & \\ =\quad 2.83 \% & \text { Pass }\end{array}$


4. System Drift as Percent of Span Value (3\%)

$$
E_{D r i f t}=a b s\left|\frac{C_{m f}-C_{m i}}{S p a n}\right| \leq l_{d r i f t}
$$

\begin{tabular}{|c|c|c|c|c|}
\hline \multicolumn{5}{|l|}{ Where: } \\
\hline $\mathrm{C}_{\mathrm{mt}}$ & $=$ calibration error response concentration for Cal01 (final) & $=$ & 2411.901 & ppmdv \\
\hline $\mathrm{C}_{\mathrm{mi}}$ & $=$ calibration error response concentration for Cal00 (initial) & $=$ & 2432.641 & ppmdv \\
\hline Span & $=$ instrument span value & $=$ & 2479.000 & ppmdv \\
\hline$I_{\text {drift }}$ & $=$ limit for system drift error & $=$ & $3.0 \%$ & \\
\hline$E_{\text {drift }}$ & $=$ calibration drift error check value & $=$ & $0.84 \%$ & Pass \\
\hline
\end{tabular}

5. Average Concentration for an entire Run

$$
\begin{array}{cl}
C & = \\
\text { Where: } & =\text { All concentration readings for the entirety of Run } 1 \\
\mathrm{C}_{\mathrm{i}} & \text { for the monitor looking for SO2 on channel } 2 \\
\mathrm{~N} & =\text { total number of readings in Run } 1
\end{array}
$$

6. Drift-Corrected Average Concentration for an entire Run

$\begin{array}{lcl} & \mathrm{i}=1 & \\ = & 1391.429 & \text { ppmdv } \\ = & 61 & \\ = & 1384.546 & \text { ppmdv }\end{array}$

$\mathrm{C}_{\mathrm{ma}} \quad=$ concentration of actual calibration gas value

$\begin{array}{ll}\mathrm{C} & =\text { average SO2 concentration for Run } 1 \\ \mathrm{C}_{\mathrm{mf}} & =\text { calibration error response concentration for Cal01 (final) }\end{array}$

$\mathrm{C}_{\mathrm{m} l} \quad=$ calibration error response concentration for Cal00 (initial)

$\mathrm{C}_{\mathrm{of}} \quad=$ calibration error response concentration for Cal01 (final) for zero gas

$\mathrm{C}_{\mathrm{ol}} \quad=$ calibration error response concentration for Cal00 (initial) for zero gas

$\mathrm{C}_{\mathrm{DC}} \quad=$ drift corrected average concentration for Run 1
$=2479.000 \quad$ ppmdv

$=1384.546 \quad$ ppmdv

$=2411.901 \quad \mathrm{ppmdv}$

$=2432.641 \quad$ ppmdv

$=13.789 \quad$ ppmdv

$=19.829 \quad$ ppmdv

$=1409.550 \quad$ ppmdv 


\section{CEM Emissions Sample Calculations for $\mathrm{SO} 2 \mathrm{AH}$ Outlet}

\section{Sample data taken from Run 1}

and Channel 2

Note: The tables presenting the results are generated electronically from raw data. It may not be possible to exactly duplicate these results using a calculator. The reference method data, results and all calculations are carried to sixteen decimal places throughout. The final table is formatted to an appropriate number of significant figures.

051507143419

1. SO2 concentration (ppmdv)

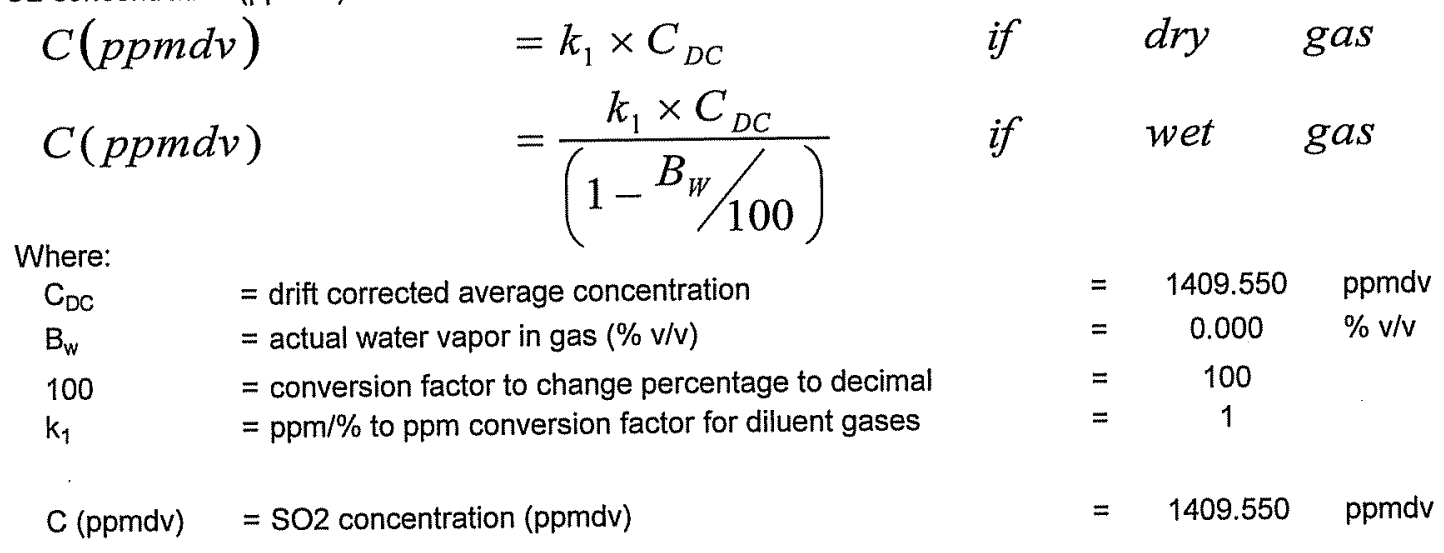

2. SO2 concentration (ppmwv)

\begin{tabular}{|c|c|c|c|}
\hline$C(p p m w r)$ & $=k_{1} \times C_{D C}$ & if & wet \\
\hline$(p p m w r)$ & $=k_{1} \times C_{D C} \times$ & if & $d r y$ \\
\hline
\end{tabular}

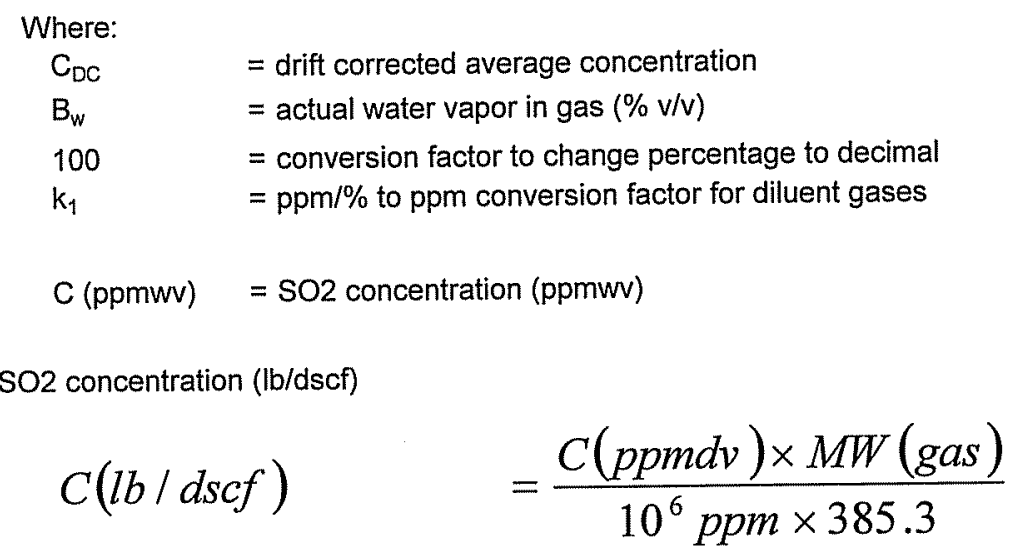

Where:

$\begin{array}{ll}\mathrm{C}(\mathrm{ppmdv}) & =\mathrm{SO} 2 \text { concentration (ppmdv) } \\ \mathrm{MW} & =\text { Molecular Weight of } \mathrm{SO} 2 \text { gas } \\ 10^{6} & =\text { conversion factor from decimal to } \mathrm{ppm} \\ 385.3 & =\text { molar volume } \\ \mathrm{C}(\mathrm{lb} / \mathrm{dscf}) & =\mathrm{SO} 2 \text { concentration }(\mathrm{lb} / \mathrm{dscf})\end{array}$

$=1409.550 \quad$ ppmdv

$=64.0628 \quad \mathrm{lb} / \mathrm{lb}-$ mole

$=1.00 \mathrm{E}+06$

$=\quad 385.3 \mathrm{dscf} / \mathrm{b}-\mathrm{b}$-mole

$=2.344 \mathrm{E}-04 \mathrm{lb} / \mathrm{dscf}$ 
CleanAir Project No. 10192

\section{Dresden, New York}

Stack, AH Outlet

4. $\mathrm{SO} 2$ concentration ( $\mathrm{lb} / \mathrm{scf})$

$$
C(l b / s c f)=C(l b / d s c f) \times \frac{Q_{s t d}}{Q_{s}}
$$

Where:

$\begin{array}{lllcl}\mathrm{C}(\mathrm{lb} / \mathrm{dscf}) & =\mathrm{SO} 2 \text { concentration }(\mathrm{lb} / \mathrm{dscf}) & 2.344 \mathrm{E}-04 & \mathrm{lb} / \mathrm{dscf} \\ \mathrm{Q}_{\mathrm{std}} & =\text { volumetric flow rate at standard conditions, dry basis (dscfm) } & = & 0 & \mathrm{dscf} / \mathrm{min} \\ \mathrm{Q}_{\mathrm{s}} & =\text { volumetric flow rate (standard cubic feet/min) } & = & 0 & \mathrm{scf} / \mathrm{min} \\ \mathrm{C}(\mathrm{lb} / \mathrm{scf}) & =\mathrm{SO} 2 \text { concentration }(\mathrm{lb} / \mathrm{scf}) & = & \mathrm{N} / \mathrm{A} & \mathrm{lb} / \mathrm{scf}\end{array}$

5. $\mathrm{SO} 2$ concentration (Ib/acf)

$$
C(l b / a c f)=C(l b / d s c f) \times \frac{Q_{s i d}}{Q_{a}}
$$

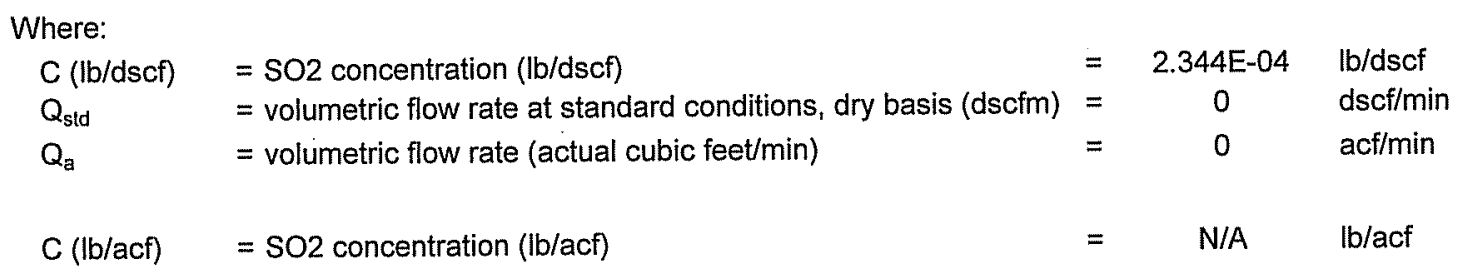

6. $\mathrm{SO} 2$ concentration ( $\% \mathrm{dv})$

$$
C(\% d v) \quad=C(p p m d v) \times \frac{100}{10^{6}}
$$

Where:

$\begin{array}{ll}C(\mathrm{ppmdv}) & =\mathrm{SO} 2 \text { concentration }(\mathrm{ppmdv}) \\ 100 & =\text { conversion factor from decimal to percentage } \\ 10^{6} & =\text { conversion factor from decimal to } \mathrm{ppm} \\ \mathrm{C}(\% \mathrm{dv}) & =\mathrm{SO} 2 \text { concentration }(\% \mathrm{dv})\end{array}$

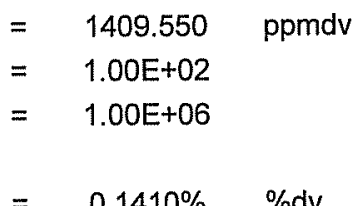

7. $\mathrm{SO} 2$ concentration (mg/dscm)

$$
C(\mathrm{mg} / \mathrm{dscm}) \quad=C(\mathrm{lb} / \mathrm{dscf}) \times k_{2} \times 35.31
$$

\footnotetext{
Where:
}

$\mathrm{C}(\mathrm{lb} / \mathrm{dscf})=\mathrm{SO} 2$ concentration $(\mathrm{lb} / \mathrm{dscf})$

$\mathrm{k}_{2} \quad=$ conversion factor from $\mathrm{lb}$ to $\mathrm{mg}$

$35.31=$ conversion factor from $\mathrm{dscf}$ to $\mathrm{dscm}$

$=2.344 \mathrm{E}-04 \mathrm{lb} / \mathrm{dscf}$

$=453515 \mathrm{mg} / \mathrm{lb}$

$=\quad 35.31 \quad \mathrm{ft}^{3} / \mathrm{m}^{3}$

$\mathrm{C}(\mathrm{mg} / \mathrm{dscm})=\mathrm{SO} 2$ concentration $(\mathrm{mg} / \mathrm{dscm})$

$=3752.982 \mathrm{mg} / \mathrm{dscm}$ 
CleanAir Project No. 10192

Dresden, New York

Stack, AH Outlet

8. $\mathrm{SO} 2$ concentration ( $\mathrm{mg} / \mathrm{Nm} 3$ dry)

$$
C\left(m g / N m^{3} d r y\right) \quad=C(l b / d s c f) \times k_{2} \times 35.31 \times\left(\frac{68+460}{32+460}\right)
$$

Where:

$\begin{array}{ll}\mathrm{C}(\mathrm{lb} / \mathrm{dscf}) & =\mathrm{SO} 2 \text { concentration }(\mathrm{lb} / \mathrm{dscf}) \\ \mathrm{k}_{2} & =\text { conversion factor from } \mathrm{lb} \text { to } \mathrm{mg} \\ 35.31 & =\text { conversion factor from dscf to } \mathrm{dscm} \\ 68 & =\text { standard temperature }\left({ }^{\circ} \mathrm{F}\right) \\ 32 & =\text { normal temperature }\left({ }^{\circ} \mathrm{F}\right) \\ 460 & ={ }^{\circ} \mathrm{F} \text { to }{ }^{\circ} \mathrm{R} \text { conversion constant }\end{array}$

$\mathrm{C}(\mathrm{mg} / \mathrm{Nm} 3 \mathrm{dr}=\mathrm{SO} 2$ concentration $(\mathrm{mg} / \mathrm{Nm} 3 \mathrm{dry})$

$\begin{array}{lcl}= & 2.344 \mathrm{E}-04 & \mathrm{lb} / \mathrm{dscf} \\ = & 453515 & \mathrm{mg} / \mathrm{lb} \\ = & 35.31 & \mathrm{ft}^{3} / \mathrm{m}^{3} \\ = & 68 & { }^{\circ} \mathrm{F} \\ = & 32 & { }^{\circ} \mathrm{F} \\ = & 460 & \\ =4027.591 & \mathrm{mg} / \mathrm{Nm}^{3} \mathrm{dry}\end{array}$

9. $\mathrm{SO} 2$ concentration corrected to $3 \% \mathrm{O} 2$ (ppmdv example)

$$
\begin{aligned}
& C(p p m d v @ x \% 02)=C(p p m d v) \times\left(\frac{20.9-x}{20.9-O_{2}}\right) \\
& \text { Where: } \\
& \mathrm{C}(\mathrm{ppmdv}) \quad=\mathrm{SO} 2 \text { concentration (ppmdv) } \\
& \mathrm{x} \quad \text { oxygen content of corrected gas (\%) } \\
& \mathrm{O}_{2} \quad \text { = proportion of oxygen in the gas stream by volume }(\%) \\
& 20.9=\text { oxygen content of ambient air (\%) } \\
& \mathrm{C}(\mathrm{ppmdv}-\mathrm{O} z=\mathrm{SO} 2 \text { concentration corrected to } 3 \% \mathrm{O} 2 \text { (ppmdv example) }=1841.848 \quad \mathrm{ppmdv} @ 3 \% \mathrm{O} 2
\end{aligned}
$$

$\begin{array}{lcl}= & 1409.550 & \text { ppmdv } \\ = & 3.00 & \% \\ = & 7.201 & \% \\ = & 20.9 & \% \\ = & 1841.848 & \text { ppmdv @ 3\%O2 }\end{array}$

10. SO2 concentration corrected to $12 \% \mathrm{CO} 2$ (ppmdv example)

$$
C\left(p p m d v @ y \% C 0_{2}\right)=C(p p m d v) \times\left(\frac{y}{C O_{2}}\right)
$$

Where:

$$
\begin{array}{lllcl}
\mathrm{C}(\mathrm{ppmdv}) & =\mathrm{SO} 2 \text { concentration (ppmdv) } & 1409.550 & \mathrm{ppmdv} & \\
\mathrm{y} & =\text { carbon dioxide content of corrected gas }(\%) & 12.00 & \% & \% \\
\mathrm{CO}_{2} & =\text { proportion of carbon dioxide in the gas stream by volume }(\%)= & 11.491 & \% \\
\mathrm{C}(\mathrm{ppmdv}-\mathrm{CO}=\mathrm{SO} 2 \text { concentration corrected to } 12 \% \mathrm{CO} 2 \text { (ppmdv example) } & = & 1472.001 & \text { ppmdv @ 12\%CO2 }
\end{array}
$$

11. SO2 Removal Efficiency\% (no data on this channel for example)

$$
R E \quad=\frac{\left(C_{i}-C_{o}\right)}{C_{i}}
$$

$\begin{array}{llll}\text { Where: } & =\text { Removal Channel Number } & \text { N/A } \\ \text { RC } & \text { Unit Index Number } & = & \\ \text { UI } & =\text { Initial concentration } & = & 1.842 E+03 \\ C_{i} & =\text { Final concentration } & = & 1.046 E+02 \\ C_{0} & =\text { Recovery Efficiency }(\%) & = & 94.32 \% \\ \text { RE } & & \end{array}$




\section{CEM Emissions Sample Calculations for NOX 0}

\section{Sample data taken from Run 1 and Channel 2}

Note: The tables presenting the results are generated electronically from raw data. It may not be possible to exactly duplicate these results using a calculator. The reference method data, results and all calculations are carried to sixteen decimal places throughout. The final table is formatted to an appropriate number of significant figures.

070607080136

1. NOX concentration corrected to $3 \% \mathrm{O} 2$ (ppmdv example)

$$
C(p p m d v @ x \% 02)=C(p p m d v) \times\left(\frac{20.9-x}{20.9-O_{2}}\right)
$$

Where:

$\begin{array}{lllll}\mathrm{C}(\mathrm{ppmdv}) & =\text { NOX concentration (ppmdv) } & =46.900 & \mathrm{ppmdv} \\ \mathrm{x} & =\text { oxygen content of corrected gas }(\%) & = & 3.00 & \% \\ \mathrm{O}_{2} & =\text { proportion of oxygen in the gas stream by volume }(\%) & = & 4.900 & \% \\ 20.9 & = & 20.9 & \% \\ & = & & \end{array}$

2. NOX concentration corrected to $12 \% \mathrm{CO} 2$ (ppmdv example)

$$
C\left(p p m d v @ y \% C 0_{2}\right)=C(p p m d v) \times\left(\frac{y}{\mathrm{CO}_{2}}\right)
$$

Where:

$\begin{array}{lllcl}\mathrm{C}(\mathrm{ppmdv}) & =\text { NOX concentration (ppmdv) } & =46.900 & \mathrm{ppmdv} \\ \mathrm{y} & =\text { carbon dioxide content of corrected gas }(\%) & = & 12.00 & \% \\ \mathrm{CO}_{2} & =\text { proportion of carbon dioxide in the gas stream by volume }(\%) & = & 13.900 & \%\end{array}$

$\mathrm{C}(\mathrm{ppmdv}-\mathrm{CO}=\mathrm{NOX}$ concentration corrected to $12 \% \mathrm{CO} 2$ (ppmdv example) $=40.500 \quad$ ppmdv @ $12 \% \mathrm{CO} 2$

\begin{tabular}{|c|c|c|c|c|c|}
\hline$E_{F d}$ & $=C(l b / d s c f) \times F_{d} \times$ & $\left(\frac{20.9}{20.9-O_{2}}\right)$ & & & \\
\hline \multicolumn{6}{|l|}{ Where: } \\
\hline & = ratio of gas volume to heat col & ntent of fuel (dscf/MMBtu) & $=$ & 9737 & dscf/MMBtu \\
\hline $\mathrm{O}_{2}$ & $=$ proportion of oxygen in the ga & s stream by volume (\%) & $=$ & 4.900 & $\%$ \\
\hline 20.9 & $=$ oxygen content of ambient air & $(\%)$ & $=$ & 20.9 & $\%$ \\
\hline$E_{F d}$ & $=$ NOX Fd-based emission rate & (lb/MMBtu) & $=$ & 0.0711 & Ib/MMBtu \\
\hline
\end{tabular}

3. NOX Fd-based emission rate (Ib/MMBtu) 


\title{
CEM Field Sample Calculations for NOX 0
}

\author{
Sample data taken from Run 1 W \\ and Channel 2
}

Note: The tables presenting the results are generated electronically from raw data. It may not be possible to exactly duplicate these results using a calculator. The reference method data, results and all calculations are carried to sixteen decimal places throughout. The final table is formatted to an appropriate number of significant figures.

070607073506

1. Average Concentration for an entire Run

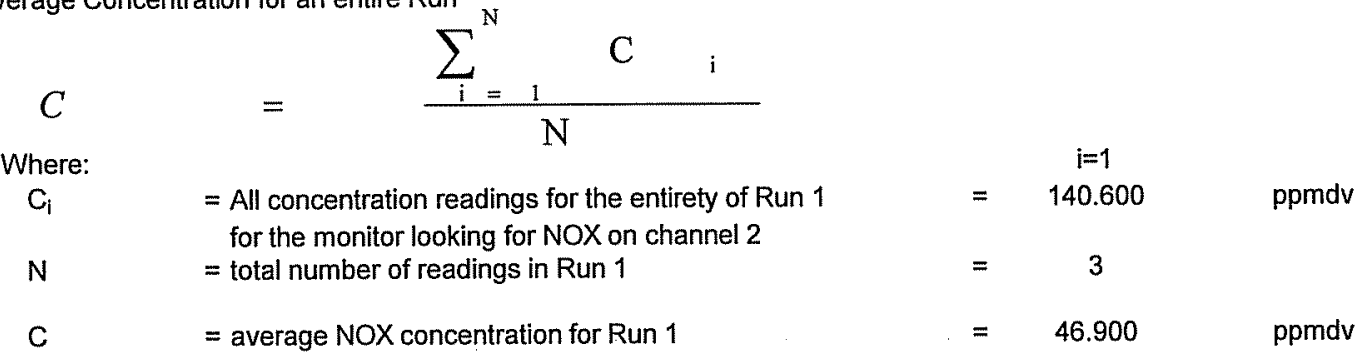


This Page Intentionally Left Blank 
CONSOL ENERGY INC.

AES GREENIDGE STATION
Client Reference No: 4700140111

CleanAir Project No: 10192

\section{PARAMETERS}

C 
This Page Intentionally Left Blank

C - 2 


\section{TEST LOG}

Client: Consol Energy Dresden, New York

CleanAir Project No. 10192

\begin{tabular}{|c|c|c|c|c|c|c|c|}
\hline $\begin{array}{c}\text { Run } \\
\text { Number }\end{array}$ & Location & Method & Analyte & Date & $\begin{array}{l}\text { Start } \\
\text { Time }\end{array}$ & End Time & Notes \\
\hline 1 & SCR Inlet & MASS: 3A, 7E & $\mathrm{O}_{2} / \mathrm{CO}_{2}, \mathrm{NO}_{\mathrm{x}}$ & $3 / 28 / 07$ & $09: 12$ & $10: 23$ & \\
\hline 2 & SCR Inlet & MASS: 3A, 7E & $\mathrm{O}_{2} / \mathrm{CO}_{2}, \mathrm{NO}_{\mathrm{x}}$ & $3 / 28 / 07$ & $11: 12$ & $12: 23$ & \\
\hline 3 & SCR Inlet & MASS: 3A, 7E & $\mathrm{O}_{2} / \mathrm{CO}_{2}, \mathrm{NO}_{\mathrm{x}}$ & $3 / 28 / 07$ & $12: 48$ & $13: 59$ & \\
\hline 1 & SCR Outlet & MASS: 3A, 7E & $\mathrm{O}_{2} / \mathrm{CO}_{2}, \mathrm{NO}_{\mathrm{x}}$ & $3 / 28 / 07$ & $09: 12$ & $10: 23$ & \\
\hline 2 & SCR Outlet & MASS: $3 A, 7 E$ & $\mathrm{O}_{2} / \mathrm{CO}_{2}, \mathrm{NO}_{\mathrm{x}}$ & $3 / 28 / 07$ & $11: 12$ & $12: 23$ & \\
\hline 3 & SCR Outlet & MASS: $3 A, 7 E$ & $\mathrm{O}_{2} / \mathrm{CO}_{2}, \mathrm{NO}_{\mathrm{x}}$ & $3 / 28 / 07$ & $12: 48$ & $13: 59$ & \\
\hline
\end{tabular}


Clean Air Engineering Project \#10192

Consol Energy

AES Dresden

Date:

$3 / 28 / 2007$

$9: 12$

Run \# (Cycle \#) 1 (Avg)

End Time: $\quad 10: 23$

\begin{tabular}{|c|c|c|c|c|c|c|c|}
\hline $\begin{array}{l}\text { Inlet } \\
\text { Point }\end{array}$ & Time Sampled & $\begin{array}{c}\text { NOx } \\
\text { ppmdv }\end{array}$ & $\begin{array}{l}\text { PPM Dev } \\
\text { from Avg. }\end{array}$ & $\begin{array}{c}\mathrm{O2} \\
\% \mathrm{dv}\end{array}$ & $\begin{array}{l}\text { CO2 } \\
\% d v\end{array}$ & $\begin{array}{c}\text { NOx } \\
@ 3 \% \text { O2 }\end{array}$ & $\begin{array}{l}\text { PPM Dev } \\
\text { from Avg. }\end{array}$ \\
\hline $1-1$ & & 77.6 & -5.6 & 7.5 & 11.6 & 103.8 & 11.2 \\
\hline $1-2$ & & 105.9 & 22.7 & 2.9 & 15.4 & 105.5 & 12.9 \\
\hline $1-3$ & & 89.1 & 5.9 & 5.0 & 13.7 & 100.5 & 8.0 \\
\hline $1-4$ & & 82.2 & -1.0 & 4.4 & 14.3 & 89.0 & -3.5 \\
\hline $1-5$ & & 72.5 & -10.7 & 4.8 & 13.9 & 80.4 & -12.1 \\
\hline 1-6 & & 75.0 & -8.2 & 8.6 & 10.7 & 108.9 & 16.3 \\
\hline $2-1$ & & 83.3 & 0.1 & 6.0 & 12.9 & 99.7 & 7.1 \\
\hline $2-2$ & & 86.2 & 3.0 & 3.0 & 15.5 & 85.9 & -6.6 \\
\hline $2-3$ & & 87.4 & 4.2 & 5.3 & 13.5 & 100.5 & 8.0 \\
\hline $2-4$ & & 78.4 & -4.8 & 3.9 & 14.7 & 82.4 & -10.1 \\
\hline $2-5$ & & 66.5 & -16.7 & 4.5 & 14.1 & 72.8 & -19.8 \\
\hline $2-6$ & & 84.1 & 0.9 & 6.8 & 12.2 & 107.0 & 14.4 \\
\hline $3-1$ & & 95.7 & 12.5 & 4.0 & 14.5 & 101.4 & 8.8 \\
\hline $3-2$ & & 87.4 & 4.2 & 2.9 & 15.5 & 86.9 & -5.6 \\
\hline $3-3$ & & 102.4 & 19.2 & 3.3 & 15.1 & 104.2 & 11.7 \\
\hline $3-4$ & & 81.5 & -1.7 & 3.7 & 14.9 & 84.7 & -7.9 \\
\hline $3-5$ & & 70.9 & -12.3 & 4.5 & 14.1 & 77.5 & -15.1 \\
\hline $3-6$ & & 87.7 & 4.5 & 6.3 & 12.6 & 107.7 & 15.1 \\
\hline $4-1$ & & 83.4 & 0.2 & 5.7 & 13.1 & 98.2 & 5.6 \\
\hline $4-2$ & & 89.7 & 6.5 & 2.5 & 15.8 & 87.4 & -5.1 \\
\hline $4-3$ & & 96.9 & 13.7 & 3.2 & 15.3 & 97.8 & 5.2 \\
\hline 4-4 & & 79.0 & -4.2 & 3.7 & 14.8 & 82.2 & -10.4 \\
\hline $4-5$ & & 63.6 & -19.6 & 4.5 & 14.2 & 69.5 & -23.1 \\
\hline $4-6$ & & 70.2 & -13.0 & 6.6 & 12.4 & 87.7 & -4.9 \\
\hline
\end{tabular}

Inlet Averages

83.2

$\begin{array}{lll}4.7 & 13.9 & 92.6\end{array}$

\begin{tabular}{|c|c|c|c|c|c|c|c|c|c|}
\hline $\begin{array}{l}\text { Outlet } \\
\text { Point }\end{array}$ & Time Sampled & $\begin{array}{c}\text { NOx } \\
\text { ppmdv }\end{array}$ & $\begin{array}{l}\text { PPM Dev } \\
\text { from Avg. }\end{array}$ & $\begin{array}{c}\mathrm{O} 2 \\
\% \mathrm{dv}\end{array}$ & $\begin{array}{l}\mathrm{CO} 2 \\
\% d v\end{array}$ & $\begin{array}{c}\text { NOx } \\
\text { @ } 3 \% 02\end{array}$ & $\begin{array}{l}\text { PPM Dev } \\
\text { from Avg. }\end{array}$ & $\begin{array}{l}\text { Removal } \\
\text { Efficiency }\end{array}$ & $\begin{array}{c}\text { Removal Eff } \\
\text { W/ O2 correction }\end{array}$ \\
\hline $1-1$ & & 68.0 & 21.1 & 4.9 & 13.9 & 75.9 & 23.2 & 9.7 & 24.6 \\
\hline $1-2$ & & 78.6 & 31.8 & 5.6 & 13.3 & 91.8 & 39.1 & 25.4 & 12.5 \\
\hline $1-3$ & & 56.9 & 10.1 & 5.3 & 13.6 & 65.2 & 12.5 & 33.6 & 33.1 \\
\hline $1-4$ & & 62.2 & 15.4 & 4.7 & 14.0 & 68.8 & 16.0 & 31.1 & 29.2 \\
\hline 1.5 & & 50.4 & 3.5 & 6.3 & 12.7 & 61.7 & 9.0 & 29.8 & 23.4 \\
\hline $1-6$ & & 40.7 & -6.2 & 7.7 & 10.3 & 55.9 & 3.1 & 66.8 & 70.0 \\
\hline $2-1$ & & 33.3 & -13.5 & 5.0 & 13.8 & 37.4 & -15.3 & 60.0 & 62.4 \\
\hline $2-2$ & & 56.7 & 9.8 & 4.6 & 14.1 & 62.3 & 9.6 & 31.9 & 25.1 \\
\hline $2-3$ & & 69.7 & 22.8 & 4.7 & 14.0 & 77.2 & 24.5 & 18.2 & 21.3 \\
\hline $2-4$ & & 71.9 & 25.0 & 5.9 & 13.0 & 85.8 & 33.1 & 5.6 & -7.1 \\
\hline $2-5$ & & 24.4 & -22.4 & 4.9 & 13.9 & 27.3 & -25.4 & 63.5 & 62.9 \\
\hline $2-6$ & & 46.9 & 0.0 & 6.7 & 12.3 & 59.0 & 6.3 & 36.4 & 37.2 \\
\hline $3-1$ & & 24.4 & -22.5 & 3.7 & 14.9 & 25.4 & -27.4 & 75.2 & 75.6 \\
\hline $3-2$ & & 68.1 & 21.3 & 4.3 & 14.4 & 73.4 & 20.7 & 18.8 & 12.8 \\
\hline $3-3$ & & 78.6 & 31.7 & 4.4 & 14.3 & 85.0 & 32.3 & 20.5 & 15.6 \\
\hline $3-4$ & & 59.9 & 13.0 & 4.9 & 13.9 & 67.2 & 14.4 & 26.0 & 20.2 \\
\hline $3-5$ & & 30.5 & -16.4 & 4.3 & 14.4 & 32.8 & -19.9 & 56.1 & 57.0 \\
\hline 3-6 & & 24.1 & -22.7 & 5.5 & 13.4 & 28.1 & -24.7 & 70.3 & 71.9 \\
\hline $4-1$ & & 7.6 & -39.3 & 3.5 & 15.1 & 7.8 & -44.9 & 91.0 & 92.2 \\
\hline $4-2$ & & 52.8 & 5.9 & 2.7 & 15.9 & 51.8 & -0.9 & 38.5 & 38.1 \\
\hline $4-3$ & & 73.3 & 26.5 & 3.5 & 15.2 & 75.3 & 22.6 & 24.0 & 22.6 \\
\hline 4-4 & & 36.1 & -10.8 & 4.5 & 14.2 & 39.4 & -13.3 & 53.6 & 50.9 \\
\hline $4-5$ & & 4.5 & -42.3 & 4.0 & 14.6 & 4.8 & -47.9 & 92.7 & 92.9 \\
\hline 4-6 & & 5.3 & -41.5 & 5.6 & 13.3 & 6.2 & -46.5 & 91.7 & 92.2 \\
\hline \multirow{2}{*}{\multicolumn{2}{|c|}{ Outlet Averages }} & 46.9 & & 4.9 & 13.9 & 52.7 & & 44.9 & 43.4 \\
\hline & & 9737.0 & Fd & & & & & & \\
\hline
\end{tabular}


Clean Air Engineering Project \#10192

Consol Energy

AES Dresden

Date:

Start Time:

$3 / 28 / 2007$

Run \# (Cycle \#)

2 (Avg)

End Time:

$11: 12$
$12: 23$

\begin{tabular}{|c|c|c|c|c|c|c|c|}
\hline $\begin{array}{l}\text { Inlet } \\
\text { Point }\end{array}$ & Time Sampled & $\begin{array}{c}\text { NOx } \\
\text { ppmdiv }\end{array}$ & $\begin{array}{l}\text { PPM Dev } \\
\text { from Avg. }\end{array}$ & $\begin{array}{c}\mathrm{O2} \\
\% \mathrm{dv}\end{array}$ & $\begin{array}{l}\mathrm{CO2} \\
\% \mathrm{dv}\end{array}$ & $\begin{array}{c}\text { NOx } \\
@ 3 \% 02\end{array}$ & $\begin{array}{l}\text { PPM Dev } \\
\text { from Avg. }\end{array}$ \\
\hline $1-1$ & & 76.3 & -1.1 & 7.6 & 11.6 & 102.3 & 16.0 \\
\hline $1-2$ & & 99.0 & 21.5 & 3.1 & 15.3 & 99.6 & 13.3 \\
\hline $1-3$ & & 85.4 & 8.0 & 5.0 & 13.8 & 96.1 & 9.8 \\
\hline $1-4$ & & 79.9 & 2.4 & 4.3 & 14.3 & 86.3 & 0.0 \\
\hline $1-5$ & & 66.9 & -10.5 & 4.6 & 14.1 & 73.5 & -12.8 \\
\hline $1-6$ & & 70.6 & -6.8 & 8.6 & 10.7 & 102.6 & 16.4 \\
\hline $2-1$ & & 79.1 & 1.7 & 6.1 & 12.8 & 95.4 & 9.1 \\
\hline $2-2$ & & 79.2 & 1.8 & 3.2 & 15.3 & 80.1 & -6.2 \\
\hline $2-3$ & & 82.8 & 5.4 & 5.4 & 13.4 & 95.6 & 9.3 \\
\hline $2-4$ & & 74.1 & -3.3 & 3.8 & 14.7 & 77.6 & -8.7 \\
\hline $2-5$ & & 58.4 & -19.1 & 4.3 & 14.3 & 63.0 & -23.2 \\
\hline 2-6 & & 75.9 & -1.5 & 6.8 & 12.2 & 96.6 & 10.3 \\
\hline$\overline{3-1}$ & & 87.5 & 10.0 & 4.2 & 14.4 & 93.6 & 7.3 \\
\hline $3-2$ & & 78.4 & 1.0 & 3.0 & 15.4 & 78.6 & -7.7 \\
\hline $3-3$ & & 98.0 & 20.6 & 3.3 & 15.2 & 99.5 & 13.2 \\
\hline $3-4$ & & 77.7 & 0.2 & 3.6 & 14.9 & 80.4 & -5.8 \\
\hline $3-5$ & & 64.4 & -13.0 & 4.4 & 14.3 & 69.9 & -16.4 \\
\hline $3-6$ & & 79.8 & 2.4 & 6.3 & 12.6 & 98.2 & 11.9 \\
\hline $4-1$ & & 75.7 & -1.7 & 6.3 & 12.6 & 92.8 & 6.5 \\
\hline $4-2$ & & 79.7 & 2.3 & 2.7 & 15.7 & 78.3 & -8.0 \\
\hline $4-3$ & & 94.2 & 16.7 & 3.1 & 15.4 & 94.5 & 8.2 \\
\hline 4-4 & & 75.5 & -2.0 & 3.5 & 15.0 & 77.5 & -8.8 \\
\hline 4-5 & & 57.3 & -20.1 & 4.3 & 14.4 & 61.7 & -24.5 \\
\hline 4-6 & & 62.5 & -15.0 & 6.4 & 12.5 & 77.2 & -9.0 \\
\hline
\end{tabular}

Inlet Averages

77.4

$\begin{array}{lll}4.7 & 14.0 & 86.3\end{array}$

\begin{tabular}{|c|c|c|c|c|c|c|c|c|c|}
\hline $\begin{array}{l}\text { Outlet } \\
\text { Point }\end{array}$ & Time Sampled & $\begin{array}{c}\text { NOx } \\
\text { ppmdv }\end{array}$ & $\begin{array}{l}\text { PPM Dev } \\
\text { from Avg. }\end{array}$ & $\begin{array}{c}\mathrm{O2} \\
\% \mathrm{dv}\end{array}$ & $\begin{array}{l}\mathrm{CO} 2 \\
\% d v\end{array}$ & $\begin{array}{c}\text { NOx } \\
@ 3 \% \text { O2 }\end{array}$ & $\begin{array}{l}\text { PPM Dev } \\
\text { from Avg. }\end{array}$ & $\begin{array}{l}\text { Removal } \\
\text { Efficiency }\end{array}$ & $\begin{array}{c}\text { Removal Eff } \\
\text { W/O2 correction }\end{array}$ \\
\hline $1-1$ & & 69.9 & 23.1 & 4.8 & 13.9 & 78.0 & 25.3 & 9.7 & 24.6 \\
\hline $1-2$ & & 78.4 & 31.5 & 5.7 & 13.3 & 92.1 & 39.4 & 25.4 & 12.5 \\
\hline $1-3$ & & 58.2 & 11.3 & 5.2 & 13.6 & 66.5 & 13.8 & 33.6 & 33.1 \\
\hline $1-4$ & & 68.4 & 21.6 & 4.4 & 14.4 & 74.0 & 21.4 & 31.1 & 29.2 \\
\hline $1-5$ & & 49.8 & 3.0 & 6.1 & 12.8 & 60.4 & 7.7 & 29.8 & 23.4 \\
\hline $1-6$ & & 48.5 & 1.7 & 8.0 & 11.1 & 67.3 & 14.6 & 66.8 & 70.0 \\
\hline $2-1$ & & 31.4 & -15.4 & 5.0 & 13.8 & 35.3 & -17.4 & 60.0 & 62.4 \\
\hline $2-2$ & & 53.1 & 6.3 & 4.6 & 14.1 & 58.5 & 5.8 & 31.9 & 25.1 \\
\hline $2-3$ & & 71.1 & 24.3 & 4.8 & 14.0 & 78.9 & 26.2 & 18.2 & 21.3 \\
\hline $2-4$ & & 73.4 & 26.6 & 5.8 & 13.1 & 87.3 & 34.6 & 5.6 & -7.1 \\
\hline $2-5$ & & 22.2 & -24.6 & 4.8 & 14.0 & 24.7 & -28.0 & 63.5 & 62.9 \\
\hline 2-6 & & 41.7 & -5.1 & 6.7 & 12.2 & 52.8 & 0.1 & 36.4 & 37.2 \\
\hline$\overline{3-1}$ & & 22.1 & -24.7 & 3.8 & 14.9 & 23.2 & -29.5 & 75.2 & 75.6 \\
\hline $3-2$ & & 67.2 & 20.4 & 4.3 & 14.4 & 72.6 & 19.9 & 18.8 & 12.8 \\
\hline $3-3$ & & 79.7 & 32.9 & 4.3 & 14.4 & 86.2 & 33.5 & 20.5 & 15.6 \\
\hline $3-4$ & & 63.1 & 16.3 & 4.8 & 14.0 & 69.9 & 17.2 & 26.0 & 20.2 \\
\hline $3-5$ & & 29.6 & -17.2 & 4.2 & 14.5 & 31.7 & -21.0 & 56.1 & 57.0 \\
\hline 3-6 & & 21.2 & -25.6 & 5.7 & 13.2 & 24.9 & -27.7 & 70.3 & 71.9 \\
\hline 4-1 & & 6.5 & -40.3 & 3.8 & 14.9 & 6.8 & -45.9 & 91.0 & 92.2 \\
\hline 4-2 & & 45.7 & -1.1 & 2.7 & 15.8 & 45.1 & -7.6 & 38.5 & 38.1 \\
\hline $4-3$ & & 75.0 & 28.2 & 3.3 & 15.3 & 76.5 & 23.8 & 24.0 & 22.6 \\
\hline $4-4$ & & 39.1 & -7.7 & 4.4 & 14.3 & 42.5 & -10.2 & 53.6 & 50.9 \\
\hline $4-5$ & & 4.0 & -42.8 & 3.9 & 14.8 & 4.2 & -48.4 & 92.7 & 92.9 \\
\hline 4-6 & & 4.4 & -42.4 & 5.4 & 13.4 & 5.1 & -47.6 & 91.7 & 92.2 \\
\hline \multirow{3}{*}{\multicolumn{2}{|c|}{ Outlet Averages }} & 46.8 & & 4.9 & 13.9 & 52.7 & & 41.2 & 39.7 \\
\hline & & 9737.0 & $\mathrm{Fd}$ & & & & & & \\
\hline & & 0.0709 & lb/Mbtu & & & & & & \\
\hline
\end{tabular}

C - 5 
Clean Air Engineering Project \#10192

Consol Energy

AES Dresden

Date:

Start Time:

End Time:
$3 / 28 / 2007$

$12: 48$

13:59
Run \# (Cycle \#) 3 (Avg)

\begin{tabular}{|c|c|c|c|c|c|c|c|}
\hline $\begin{array}{l}\text { Inlet } \\
\text { Point }\end{array}$ & Time Sampled & $\begin{array}{c}\text { NOx } \\
\text { ppmdv }\end{array}$ & $\begin{array}{l}\text { PPM Dev } \\
\text { from Avg. }\end{array}$ & $\begin{array}{c}02 \\
\% d v\end{array}$ & $\begin{array}{l}\mathrm{CO2} \\
\% \mathrm{dv}\end{array}$ & $\begin{array}{c}\text { NOx } \\
@ 3 \% \text { O2 }\end{array}$ & $\begin{array}{l}\text { PPM Dev } \\
\text { from Avg. }\end{array}$ \\
\hline $1-1$ & & 73.3 & -2.6 & 7.8 & 11.4 & 100.1 & 15.5 \\
\hline $1-2$ & & 100.6 & 24.7 & 3.2 & 15.2 & 101.6 & 17.0 \\
\hline $1-3$ & & 83.5 & 7.6 & 5.0 & 13.7 & 94.1 & 9.5 \\
\hline $1-4$ & & 75.6 & -0.3 & 4.3 & 14.3 & 81.5 & -3.0 \\
\hline $1-5$ & & 63.1 & -12.8 & 4.5 & 14.2 & 68.8 & -15.7 \\
\hline $1-6$ & & 69.3 & -6.6 & 8.6 & 10.7 & 100.5 & 16.0 \\
\hline $2-1$ & & 79.3 & 3.4 & 6.2 & 12.7 & 96.5 & 11.9 \\
\hline $2-2$ & & 76.5 & 0.6 & 3.3 & 15.2 & 77.9 & -6.6 \\
\hline $2-3$ & & 84.1 & 8.2 & 5.2 & 13.6 & 96.1 & 11.6 \\
\hline $2-4$ & & 71.5 & -4.4 & 3.6 & 14.9 & 74.1 & -10.5 \\
\hline $2-5$ & & 55.6 & -20.3 & 4.2 & 14.5 & 59.5 & -25.1 \\
\hline $2-6$ & & 76.6 & 0.7 & 6.9 & 12.1 & 97.8 & 13.3 \\
\hline $3-1$ & & 87.3 & 11.4 & 4.3 & 14.4 & 93.8 & 9.3 \\
\hline $3-2$ & & 75.5 & -0.4 & 3.2 & 15.3 & 76.4 & -8.2 \\
\hline $3-3$ & & 96.7 & 20.8 & 3.3 & 15.2 & 98.3 & 13.7 \\
\hline $3-4$ & & 76.4 & 0.5 & 3.5 & 15.0 & 78.7 & -5.9 \\
\hline $3-5$ & & 61.9 & -14.0 & 4.3 & 14.4 & 66.6 & -18.0 \\
\hline 3-6 & & 78.6 & 2.7 & 6.3 & 12.7 & 96.0 & 11.5 \\
\hline $4-1$ & & 75.5 & -0.4 & 6.1 & 12.8 & 91.1 & 6.6 \\
\hline 4-2 & & 77.2 & 1.3 & 2.8 & 15.6 & 76.5 & -8.1 \\
\hline $4-3$ & & 94.1 & 18.2 & 3.1 & 15.4 & 94.4 & 9.9 \\
\hline $4-4$ & & 73.8 & -2.1 & 3.4 & 15.1 & 75.4 & -9.2 \\
\hline 4.5 & & 55.3 & -20.6 & 4.1 & 14.6 & 58.8 & -25.7 \\
\hline 4-6 & & 60.9 & -15.0 & 6.3 & 12.6 & 74.8 & -9.8 \\
\hline
\end{tabular}

Intet Averages

75.9

$\begin{array}{lll}4.7 & 14.0 & 84.6\end{array}$

\begin{tabular}{|c|c|c|c|c|c|c|c|c|c|}
\hline $\begin{array}{l}\text { Outlet } \\
\text { Point }\end{array}$ & Time Sampled & $\begin{array}{c}\text { NOx } \\
\text { ppmdv }\end{array}$ & $\begin{array}{l}\text { PPM Dev } \\
\text { from Avg. }\end{array}$ & $\begin{array}{c}\mathrm{O2} \\
\% \mathrm{dv}\end{array}$ & $\begin{array}{l}\mathrm{CO} 2 \\
\% \mathrm{dv}\end{array}$ & $\begin{array}{c}\text { NOx } \\
@ 3 \% \text { O2 }\end{array}$ & $\begin{array}{l}\text { PPM Dev } \\
\text { from Avg. }\end{array}$ & $\begin{array}{l}\text { Removal } \\
\text { Efficiency }\end{array}$ & $\begin{array}{c}\text { Removal Eff } \\
\text { w/ } 02 \text { correction }\end{array}$ \\
\hline $1-1$ & & 61.3 & 17.0 & 5.6 & 13.2 & 71.3 & 21.2 & 9.7 & 24.6 \\
\hline $1-2$ & & 67.6 & 23.2 & 5.4 & 13.5 & 78.7 & 28.7 & 25.4 & 12.5 \\
\hline $1-3$ & & 52.3 & 8.0 & 5.4 & 13.5 & 60.8 & 10.8 & 33.6 & 33.1 \\
\hline $1-4$ & & 62.9 & 18.6 & 4.9 & 13.9 & 70.3 & 20.3 & 31.1 & 29.2 \\
\hline $1-5$ & & 50.8 & 6.4 & 5.8 & 13.1 & 60.3 & 10.3 & 29.8 & 23.4 \\
\hline $1-6$ & & 49.8 & 5.5 & 7.9 & 11.2 & 68.5 & 18.4 & 66.8 & 70.0 \\
\hline $2-1$ & & 28.2 & -16.1 & 5.3 & 13.5 & 32.5 & -17.6 & 60.0 & 62.4 \\
\hline $2-2$ & & 47.4 & 3.0 & 4.3 & 14.4 & 51.5 & 1.4 & 31.9 & 25.1 \\
\hline $2-3$ & & 63.5 & 19.1 & 5.0 & 13.8 & 71.3 & 21.2 & 18.2 & 21.3 \\
\hline $2-4$ & & 68.4 & 24.1 & 5.7 & 13.2 & 80.4 & 30.3 & 5.6 & -7.1 \\
\hline $2-5$ & & 30.8 & -13.6 & 5.0 & 13.8 & 34.8 & -15.3 & 63.5 & 62.9 \\
\hline $2-6$ & & 38.5 & -5.8 & 6.6 & 12.4 & 48.2 & -1.9 & 36.4 & 37.2 \\
\hline $3-1$ & & 17.6 & -26.7 & 4.2 & 14.5 & 18.9 & -31.2 & 75.2 & 75.6 \\
\hline $3-2$ & & 54.2 & 9.8 & 3.9 & 14.8 & 57.1 & 7.1 & 18.8 & 12.8 \\
\hline $3-3$ & & 72.4 & 28.0 & 4.6 & 14.1 & 79.6 & 29.5 & 20.5 & 15.6 \\
\hline $3-4$ & & 62.7 & 18.3 & 4.7 & 14.1 & 69.1 & 19.1 & 26.0 & 20.2 \\
\hline $3-5$ & & 38.7 & -5.6 & 4.4 & 14.3 & 42.3 & -7.7 & 56.1 & 57.0 \\
\hline $3-6$ & & 21.2 & -23.2 & 5.4 & 13.4 & 24.6 & -25.5 & 70.3 & 71.9 \\
\hline 4-1 & & 6.1 & -38.2 & 3.9 & 14.8 & 6.4 & -43.6 & 91.0 & 92.2 \\
\hline 4-2 & & 46.2 & 1.9 & 2.8 & 15.8 & 45.7 & -4.3 & 38.5 & 38.1 \\
\hline 4-3 & & 77.0 & 32.6 & 3.4 & 15.3 & 78.5 & 28.5 & 24.0 & 22.6 \\
\hline $4-4$ & & 38.9 & -5.4 & 4.3 & 14.4 & 42.0 & -8.0 & 53.6 & 50.9 \\
\hline $4-5$ & & 3.6 & -40.8 & 3.7 & 15.0 & 3.7 & -46.3 & 92.7 & 92.9 \\
\hline $4-6$ & & 4.1 & -40.2 & 5.3 & 13.6 & 4.8 & -45.3 & 91.7 & 92.2 \\
\hline \multirow{3}{*}{\multicolumn{2}{|c|}{ Outlet Averages }} & 44.4 & & 4.9 & 13.9 & 50.1 & & 42.6 & 41.0 \\
\hline & & 9737.0 & Fd & & & & & & \\
\hline & & 0.0674 & Ib/Mbtu & & & & & & \\
\hline
\end{tabular}




\section{TEST LOG}

Client: Consol Energy Dresden, New York

CleanAir Project No. 10192

\begin{tabular}{|c|c|c|c|c|c|c|c|}
\hline $\begin{array}{c}\text { Run } \\
\text { Number }\end{array}$ & Location & Method & Analyte & Date & $\begin{array}{l}\text { Start } \\
\text { Time }\end{array}$ & End Time & Notes \\
\hline 1 & AH Outlet & $3 \mathrm{~A}, 6 \mathrm{C}$ & $\mathrm{O} 2 / \mathrm{CO} 2, \mathrm{SO} 2$ & $3 / 29 / 07$ & $09: 59$ & $11: 00$ & \\
\hline 2 & AH Outlet & $3 A, 6 C$ & $\mathrm{O} 2 / \mathrm{CO} 2, \mathrm{SO} 2$ & $3 / 29 / 07$ & $12: 16$ & $13: 17$ & \\
\hline 3 & $\mathrm{AH}$ Outlet & $3 \mathrm{~A}, 6 \mathrm{C}$ & $\mathrm{O} 2 / \mathrm{CO} 2, \mathrm{SO} 2$ & $3 / 29 / 07$ & $15: 13$ & $16: 13$ & \\
\hline 1 & BH Outlet & $3 A, 6 C$ & $\mathrm{O} 2 / \mathrm{CO} 2, \mathrm{SO} 2$ & $3 / 29 / 07$ & $09: 59$ & $11: 00$ & \\
\hline 2 & BH Outlet & $3 \mathrm{~A}, 6 \mathrm{C}$ & $\mathrm{O} 2 / \mathrm{CO} 2, \mathrm{SO} 2$ & $3 / 29 / 07$ & $12: 16$ & $13: 17$ & \\
\hline 3 & BH Outlet & $3 A, 6 C$ & $\mathrm{O} 2 / \mathrm{CO} 2, \mathrm{SO} 2$ & $3 / 29 / 07$ & $15: 13$ & $16: 13$ & \\
\hline
\end{tabular}

Notes:

None

061407143432 
Consol Energy

CleanAir Project No. 10192

Dresden, New York

BH Outlet, AH Outlet

Run Number

Date (2007)

Start Time

End Time

Elapsed Time (hh:mm)

Channel

Parameter

Location

Measurement Units

Measured Average (drift-corrected)

Concentration (ppmdv)

Concentration (\%dv)

Concentration @3\%O2 (ppm)

Concentration @12\%CO2 (ppm)

Concentration @3\%O2 (lb/scf)

Concentration @12\%CO2 (b/scf)

Concentration @3\%O2 (\%v)

Concentration @12\%CO2 (\%v)

Concentration @3\%O2 ( $\mathrm{mg} / \mathrm{scm})$

Concentration @12\%CO2 (mg/scm)

Concentration @3\%O2 (mg/ $\mathrm{Nm} 3)$

Concentration @12\%CO2 (mg/Nm3)

Removal Efficiency (\%)

Consol Energy

Clean Air Project No. 10192

Dresden, New York

BH Outlet, AH Outlet

\section{Run Number}

Date (2007)

Start Time

End Time

Elapsed Time (hh:mm)

\section{Channel}

Parameter

Location

Measurement Units

Measured Average (drift-corrected)

Concentration (ppmdv)

Concentration (\%dv)

Concentration @3\%O2 (ppm)

Concentration @12\%CO2 (ppm)

Concentration @3\%O2 (lb/scf)

Concentration @12\%CO2 (lb/scf)

Concentration @3\%O2 (\%v)

Concentration @12\%CO2 (\%v)

Concentration @3\%O2 (mg/scm)

Concentration @12\% CO2 (mg/scm)

Concentration @3\%O2 (mg/Nm3)

Concentration @12\%CO2 (mg/Nm3)

Removal Efficiency (\%)

\section{Continuous Emissions Monitoring Parameters}

1
Mar 29
$9: 59$
$11: 00$
$01: 01$

\begin{tabular}{|c|c|c|c|c|c|}
\hline $\begin{array}{c}2 \\
\mathrm{SO}_{2} \\
\text { AH Outlet } \\
\text { ppmdv }\end{array}$ & $\begin{array}{c}4 \\
\text { O2 } \\
\text { AH Outlet } \\
\% d v\end{array}$ & $\begin{array}{c}5 \\
\mathrm{CO} 2 \\
\text { AH Outlet } \\
\% \text { dv }\end{array}$ & $\begin{array}{c}7 \\
\text { SO2 } \\
\text { BH Outlet } \\
\text { ppmdv }\end{array}$ & $\begin{array}{c}8 \\
\text { O2 } \\
\text { BH Outlet } \\
\% d v\end{array}$ & $\begin{array}{c}9 \\
\text { CO2 } \\
\text { BH Outlet } \\
\% d v\end{array}$ \\
\hline 1409.55 & 7.30 & 11.54 & 79.50 & 7.20 & 11.49 \\
\hline $\begin{array}{c}1409.55 \\
0.141\end{array}$ & 7.297 & 11538 & $\begin{array}{l}79.50 \\
0.008\end{array}$ & 7.201 & 11.491 \\
\hline 1854.76 & & & 103.89 & & \\
\hline $\begin{array}{c}1466.04 \\
3.084 E-04\end{array}$ & & & 83.03 & & \\
\hline $\begin{array}{l}3.084 E-04 \\
2.438 E-04\end{array}$ & & & 1.727E-05 & & \\
\hline $2.438 \mathrm{E}-04$ & & & $1.380 \mathrm{E}-05$ & & \\
\hline 0.185 & & & 0.010 & & \\
\hline 0.147 & & & 0.008 & & \\
\hline 4938.36 & & & 276.60 & & \\
\hline 3903.40 & & & 221.06 & & \\
\hline 5299.71 & & & 296.84 & & \\
\hline 4189.01 & & & 237.24 & & \\
\hline & & & $94.40 \%$ & & \\
\hline
\end{tabular}

\section{Continuous Emissions Monitoring Parameters}

2

Mar 29

$12: 16$

13:17

01:01

\begin{tabular}{|c|c|c|c|c|c|}
\hline $\begin{array}{c}2 \\
\mathrm{SO}_{2} \\
\text { AH Outlet } \\
\text { ppmdv }\end{array}$ & $\begin{array}{c}4 \\
\text { O2 } \\
\text { AH Outlet } \\
\% d v\end{array}$ & $\begin{array}{c}5 \\
\text { CO2 } \\
\text { AH Outlet } \\
\% d v\end{array}$ & $\begin{array}{c}7 \\
\text { SO2 } \\
\text { BH Outlet } \\
\text { ppmdv }\end{array}$ & $\begin{array}{c}8 \\
\text { O2 } \\
\text { BH Outlet } \\
\% d v\end{array}$ & $\begin{array}{c}9 \\
\text { CO2 } \\
\text { BH Outlet } \\
\% d v\end{array}$ \\
\hline 1398.11 & 7.22 & 11.61 & 69.70 & 7.16 & 11.71 \\
\hline 1398.11 & & & 69.70 & & \\
\hline 0.140 & 7.223 & 11.610 & 0.007 & 7.164 & 11.711 \\
\hline $\begin{array}{l}1829.81 \\
1445.01\end{array}$ & & & $\begin{array}{l}90.83 \\
71.42\end{array}$ & & \\
\hline 3.042E-04 & & & $1.510 \mathrm{E}-05$ & & \\
\hline 2.403E-04 & & & $1.187 E-05$ & & \\
\hline 0.183 & & & 0.009 & & \\
\hline 0.145 & & & 0.007 & & \\
\hline 4871.95 & & & 241.83 & & \\
\hline 3847.40 & & & 190.16 & & \\
\hline 5228.43 & & & 259.52 & & \\
\hline 4128.92 & & & 204.07 & & \\
\hline & & & $95.04 \%$ & & \\
\hline
\end{tabular}


Consol Energy

Clean Air Project No. 10192

Dresden, New York

BH Outlet, AH Outlet

Run Number

Date (2007)

Start Time

End Time

Elapsed Time (hh:mm)

Channel

Parameter

Location

Measurement Units

Measured Average (drift-corrected)

Concentration (ppmdv)

Concentration (\%dv)

Concentration @3\%O2 (ppm)

Concentration @12\%CO2 (ppm)

Concentration @3\%O2 (b/scf)

Concentration @12\%CO2 (lb/scf)

Concentration @3\%O2 (\%v)

Concentration @12\%CO2 (\%v)

Concentration @3\%O2 (mg/scm)

Concentration @12\%CO2 (mg/scm)

Concentration @3\%O2 (mg/Nm3)

Concentration@12\%CO2 (mg/Nm3)

Removal Efficiency (\%)

\section{Continuous Emissions Monitoring Parameters}

$$
3
$$

Mar 29

$15: 13$

$16: 13$

01:00

$\begin{array}{cccccc}2 & 4 & 5 & 7 & 8 & 9 \\ \begin{array}{c}\text { SO2 } \\ \text { AH Outlet } \\ \text { ppmdv }\end{array} & \begin{array}{c}\text { O2 } \\ \text { AH Outlet } \\ \% d v\end{array} & \begin{array}{c}\text { CO2 } \\ \text { AH Outlet } \\ \% d v\end{array} & \begin{array}{c}\text { SO2 } \\ \text { BH Outlet } \\ \text { ppmdv }\end{array} & \begin{array}{c}\text { O2 } \\ \text { BH Outlet } \\ \% d v\end{array} & \begin{array}{c}\text { CO2 } \\ \text { BH Outlet } \\ \% d v\end{array} \\ 1381.35 & 7.45 & 11.52 & 98.81 & 7.51 & 11.51 \\ & & & & & \\ 1381.35 & & & 98.81 & & \\ 0.138 & 7.447 & 11.523 & 0.010 & 7.513 & 11.507 \\ 1837.92 & & & 132.12 & & \\ 1438.51 & & & 103.05 & & \\ 3.056 \mathrm{E}-04 & & & 2.197 \mathrm{E}-05 & & \\ 2.392 \mathrm{E}-04 & & & 1.713 \mathrm{E}-05 & & \\ 0.184 & & & 0.013 & & \\ 0.144 & & & 0.010 & & \\ 4893.53 & & & 351.78 & & \\ 3830.09 & & & 274.37 & & \\ 5251.59 & & & 377.52 & & \\ 4110.34 & & & 294.44 & & \\ & & & 92.81 \% & & \end{array}$


This Page Intentionally Left Blank 
CONSOL ENERGY INC.

AES GREENIDGE STATION
Client Reference No: 4700140111

CleanAir Project No: 10192

QAVC DATA

D 
This Page Intentionally Left Blank

D - 2 


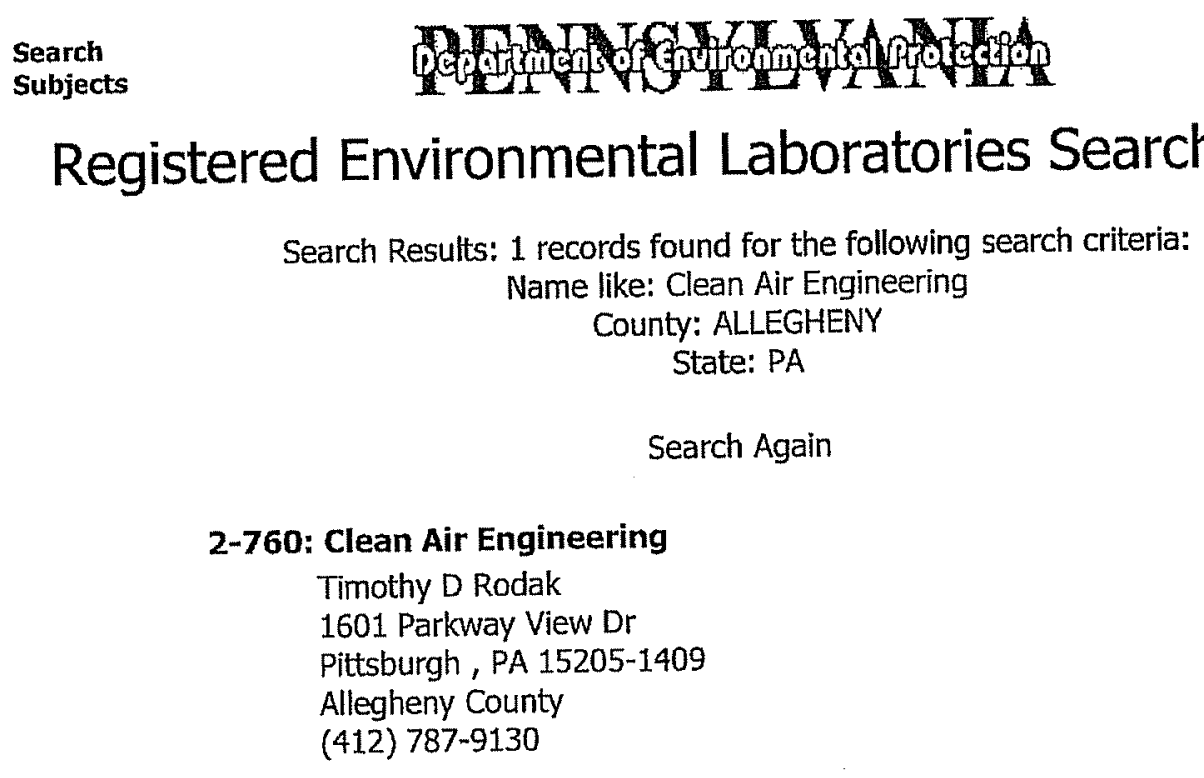

Search Again

PA Home Site | Ask DEP | Plug-Ins | Home Page 


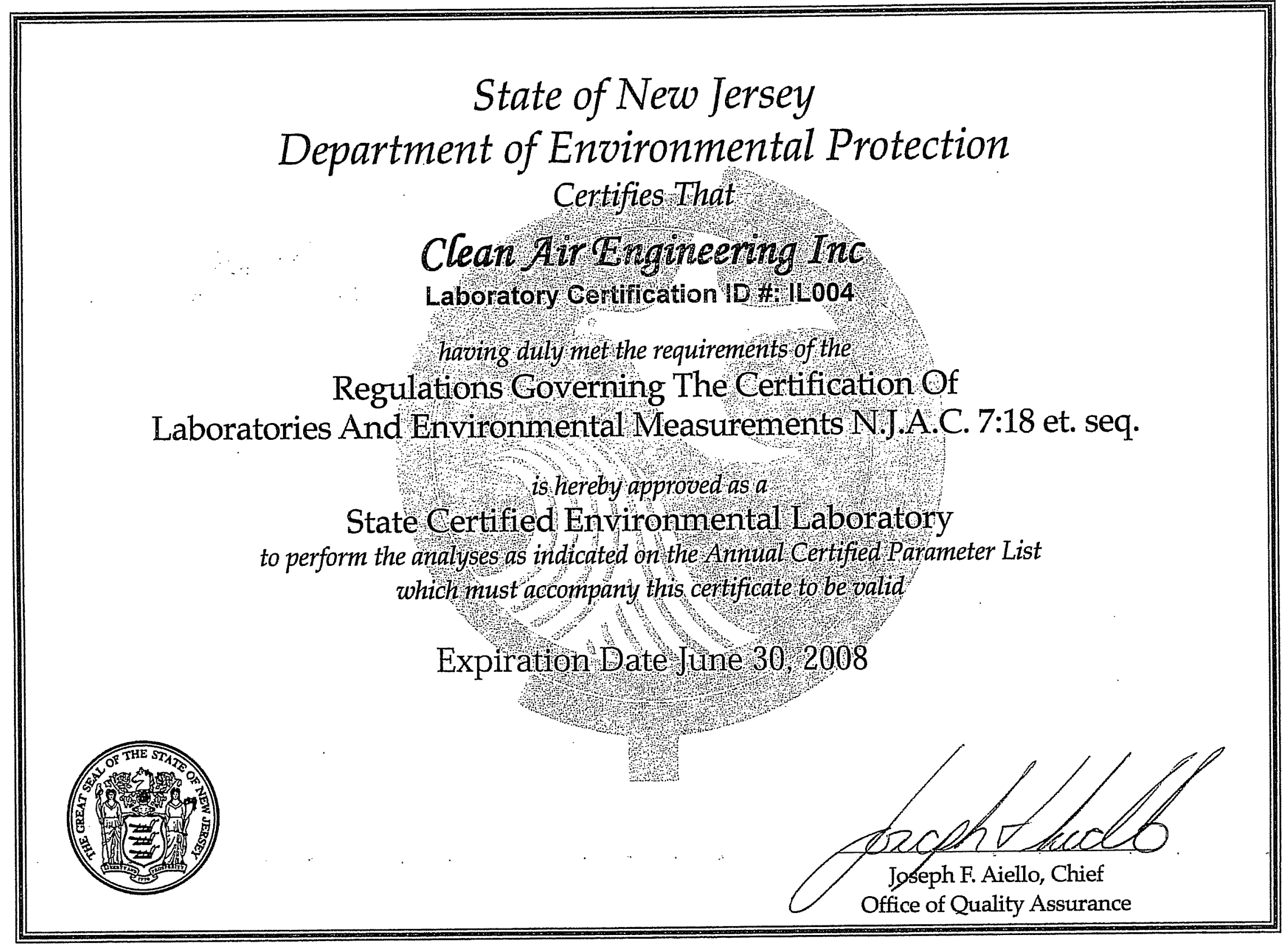

THIS CERTIFICATE IS TO BE CONSPICUOUSLY DISPLAYED AT THE LABORATORY WITH THE ANNUAL CERTIFIED PARAMETER LIST IN A LOCATION ON THE PREMISES VISIBLE TO THE PUBLIC 
New Jersey Department of Environmental Protection

Environmental Laboratory Certification Program

\section{ANNUAL CERTIFIED PARAMETER LIST AND CURRENT STATUS}

Effective as of $07 / 01 / 2007$ until $06 / 30 / 2008$

Laboratory Name: CLEAN AIR ENGINEERING INC Laboratory Number: IL004 Activity ID: SLC070001

500 WEST WOOD ST

PALATINE, IL 60067

\begin{tabular}{|c|c|c|c|c|c|}
\hline $\begin{array}{l}\text { Category } \\
\text { Status }\end{array}$ & $\begin{array}{l}\text { CAP01 - Atmos. } \\
\text { Code }\end{array}$ & $\begin{array}{l}\text { Inorg. Par } \\
\text { Matrix }\end{array}$ & $\begin{array}{l}\text { on-Metals } \\
\text { Technique Description }\end{array}$ & Approved Method & Parameter Description \\
\hline Dropped & CAP01.00005 & $A E$ & Thermal Conductivity & [EPA 3C] & Carbon Dioxide \\
\hline Dropped & CAP01.00015 & $\mathrm{AE}$ & Spectrophotometric & [EPA 10A] & Carbon monoxide \\
\hline Dropped & CAP01.00020 & $\mathrm{AE}$ & Gas Chromatography / FPD & [EPA 15] & Cárbon disulfide \\
\hline Dropped & CAP01.00025 & $\mathrm{AE}$ & Gas Chromatography / FPD & [EPA 15] & Carbon oxysulfide (Carbonyl sulfide) \\
\hline Dropped & CAP01.00035 & $\mathrm{AE}$ & Specific Ion Electrode & [EPA 13B] & Fluoride \\
\hline Dropped & CAP01.00040 & $\mathrm{AE}$ & Primary Aluminum Plants & [EPA 14A] & Fluoride \\
\hline Certified & CAP01.00045 & $A E$ & Ion Chromatography & [EPA 26] [EPA 26A] & Hydrogen chloride, Halides and Halogens \\
\hline Dropped & CAP01.00050 & $\mathrm{AE}$ & Gas Chromatography / FPD & [EPA 15] & Hydrogen sulfide \\
\hline Applied & CAP01.00055 & $\mathrm{AE}$ & Emission Sampling Train & [EPA 0051, Rev 0, 12/96] & Impinger $\mathrm{HCl} / \mathrm{Cl} 2$ \\
\hline Dropped & CAP01.00060 & $\overline{\mathrm{AE}}$ & Gravimetric & [EPA 24A] & Inks and coatings \\
\hline Certified & CAP01.00065 & $\mathrm{AE}$ & Emission Sampling Train & [EPA 0050, Rev 0, 12/96] & Isokinetic $\mathrm{HCl} / \mathrm{Cl} 2$ \\
\hline Applied & CAP01.00068 & $\mathrm{AE}$ & Thermal Conductivity & {$[\mathrm{EPA} 3 \mathrm{C}]$} & Methane \\
\hline Applied & CAP01.00070 & $\mathrm{AE}$ & Thermal Conductivity & [EPA 3C] & Nitrogen \\
\hline Applied & CAP01.00100 & $\mathrm{AE}$ & Thermal Conductivity & [EPA 3C] & Oxygen \\
\hline Certified & CAP01.00105 & $\mathrm{AE}$ & Gravimetric & [EPA 5] [EPA 17] & Particulate Matter \\
\hline Certified & .CAP01.00110 & $\mathrm{AE}$ & Gravimetric & [EPA 5A] & Particulate Matter \\
\hline Certified & CAP01.00115 & $\mathrm{AE}$ & Gravimetric & [EPA.5B] & Particulate Matter \\
\hline Certified & CAP01.00120 & $\mathrm{AE}$ & Gravimetric & [EPA 5E] & Particulate Matter \\
\hline Certified & CAP01.00125 & $\mathrm{AE}$ & Gravimetric & [EPA 5F] & Particulate Matter \\
\hline Certified & CAP01.00145 & $\mathrm{AE}$ & Gravimetric & [EPA 201A] & Particulate Matter \\
\hline Certified & CAP01.00150 & $\overline{A E}$ & Gravimetric & [EPA 202] & Particulate Malter \\
\hline Dropped & CAP01.00180 & $\mathrm{AE}$ & Standard Methods & [EPA 24] & Surface coatings \\
\hline Dropped & CAP01.00185 & $\mathrm{AE}$ & Barium-Thorin Titration & [EPA 15A] & Sulfur, Total Reduced \\
\hline Dropped & CAP01.00190 & $\mathrm{AE}$ & Barium-Thorin Titration & [EPA 16A] & Sulfur, Total Reduced \\
\hline Dropped & CAP01.00195 & $\mathrm{AE}$ & Gas Chromatography / FPD & [EPA 16B] & Sulfur, Total Reduced \\
\hline Applied & CAP01.00200 & $\mathrm{AE}$ & Barium-Thorin Titration & [EPA 6] & Sulfur Dioxide \\
\hline Applied & CAP01.00210 & $\mathrm{AE}$ & Barium-Thorin Titration & [EPA 8] & Sulfuric acid \\
\hline Category & CAP03 - Atmos & heric Orga & eters & & \\
\hline Status & Code & Matrix & Technique Description & Approved Method & Parameter Description \\
\hline Dropped & CAP03.00025 & $\overline{A E}$ & GC/FID & [EPA 308] & Methyl alcohol (Methanol) \\
\hline
\end{tabular}

KEY: $\mathrm{AE}=$ Air and Emissions, $\mathrm{BT}=$ Biological Tissues, $\mathrm{DW}=$ Drinking Water, NPW = Non-Potable Water, $\mathrm{SCM}=$ Solid and Chemical Materials 
New Jersey Department of Environmental Protection

Environmental Laboratory Certification Program

\section{ANNUAL CERTIFIED PARAMETER LIST AND CURRENT STATUS}

Effective as of $07 / 01 / 2007$ until $06 / 30 / 2008$

Laboratory Name: CLEAN AIR ENGINEERING INC Laboratory Number: IL004 Activity ID: SLC070001

500 WEST WOOD ST

PALATINE, IL 60067

Category: CAP03 - Atmospheric Organic Parameters

$\begin{array}{llll}\text { Categorion } & \text { Tode }\end{array}$

Approved Method

Parameter Description

Certified CAP03.00040 AE

GC

[EPA 18]

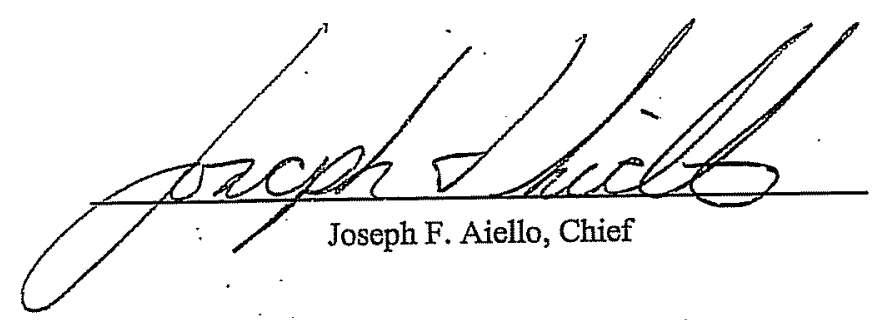

KEY: $\mathrm{AE}=$ Air and Emissions, $\mathrm{BT}=$ Biological Tissues, $\mathrm{DW}=$ Drinking Water, NPW $=$ Non-Potable Water, $\mathrm{SCM}=$ Solid and Chemical Materials

- Annual Certified Parameters List - Effective as of 07/01/2007 until 06/30/2008 
New Jersey Department of Environmental Protection

Environmental Laboratory Certification Program

LABORATORY PERSONNEL LIST

Effective as of: 07/01/2007

Laboratory Name: CLEAN AIR ENGINEERING INC Laboratory Number: IL004 Activity ID: SLC070001 500 WEST WOOD ST

PALATINE, IL 60067

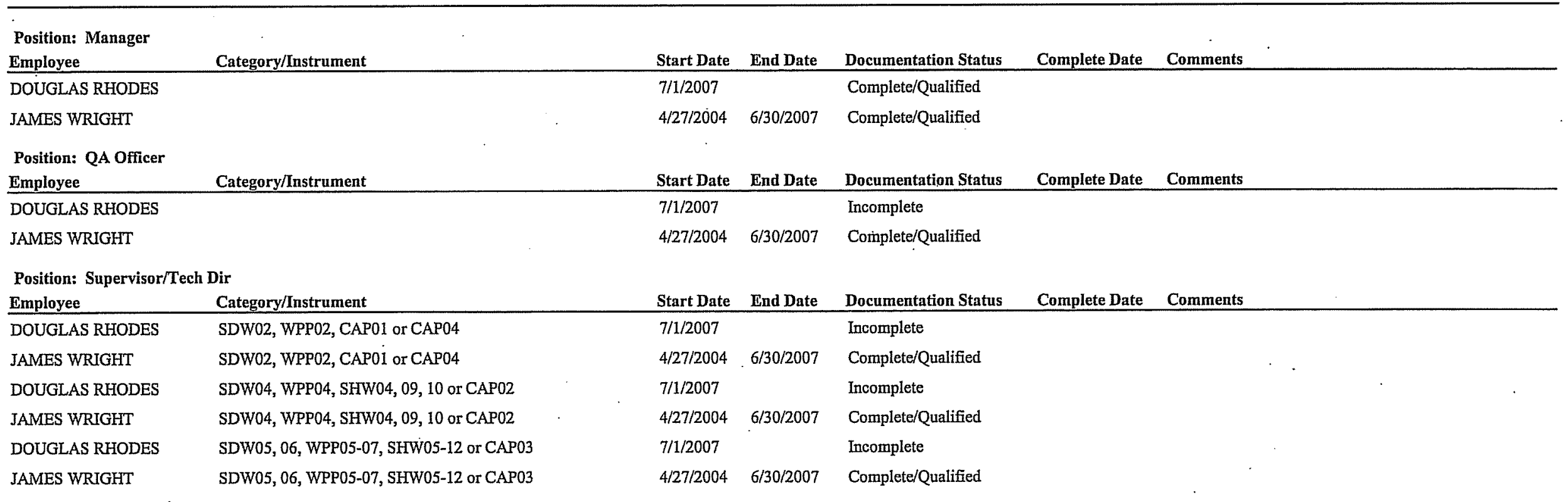




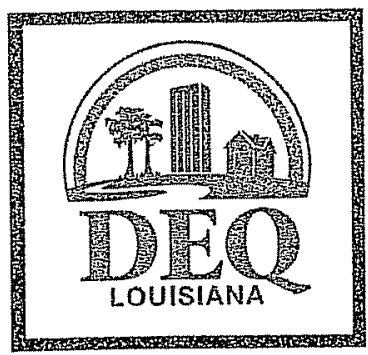

\section{DEPARTMENT OF ENMRONMENTAL QUALTY}

KATHLEEN BABINEAUX BLANCO

GOVERNOR

MIKE D. MCDANIEL, Ph.D.

SECRETARY

CERTIFIED MAIL \#7007 0710000561081458

Return Receipt Requested

June 30, 2007

AI \#85668

LELAP Certificate \#03099

Mr. Robert Doran

Clean Air Engineering

321 Century Plaza \#130

Houston, TX 77073

\section{RE: Accreditation Certificate}

Dear Mr. Doran:

In accordance with Louisiana Administrative Code, Title 33, Part I, Subpart 3, Laboratory Accreditation, the State of Louisiana formally recognizes that this laboratory has successfully completed the accreditation process and is technically competent to perform the environmental analyses listed on the scope of accreditation detailed in the attachment. Accreditation does not constitute an endorsement of the suitability of the listed methods for any specific purpose. Parameters or analytes that the laboratory has applied for accreditation not included in the scope of accreditation attachment are not accredited.

NELAP accreditation is granted only for those methods/analytes for which "NELAP" is indicated as the type of accreditation. "STATE" is indicated as the type of accreditation for those methods/analytes for which NELAP accreditation is not available. Accreditation is dependent on the laboratory's successful ongoing compliance with regulations as outlined in the Louisiana Administrative Code, Title 33, Part I, Subpart 3, Laboratory Accreditation.

The enclosed accreditation certificate is property of the State of Louisiana. Should a change in accreditation status occur, the Department may recall the original accreditation certificate and attachments. The recalled certificate and attachments should be returned 
Re: Accreditation Certificate

June 30, 2007

Page 2 of 2

to: Office of Environmental Assessment, Louisiana Environmental Laboratory Accreditation Program, P.O. Box 4314, Baton Rouge, LA 70821-4314, Attention: Dr. David L. Boucher.

LAC 33:I.5313.A requires that the laboratory report must include all relevant information. Therefore, the certificate number shall be placed in the upper right corner of all laboratory reports. If the test report includes results of any test for which the laboratory is not accredited, the unaccredited results must be clearly identified as such.

Please be advised that it is your responsibility to examine the scope of accreditation attachment for accuracy and completeness. If you find that an analyte for which you expected to be accredited is not listed, please examine your records to ensure that:

1. You have met the requirements for successful participation in proficiency test studies as outlined in LAC 33:I.4711 and in the NELAC Standard 2.7.2.

2. In the case of accreditation by recognition, the requested analyte must be listed for the requested method and matrix on both the certificate issued by the Primary AA and on the Louisiana application form.

If you have any questions, please contact the Louisiana Environmental Laboratory Accreditation Program at (225) 219-9800.

Sincerely,

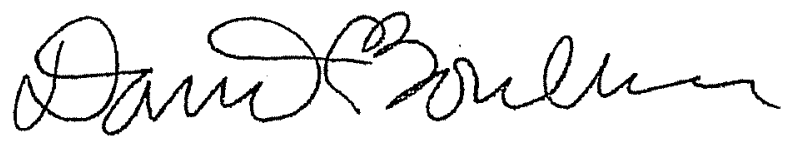

Dr. David L. Boucher, Acting Supervisor

Louisiana Environmental Laboratory Accreditation Program

$\mathrm{db}$

Enclosure 


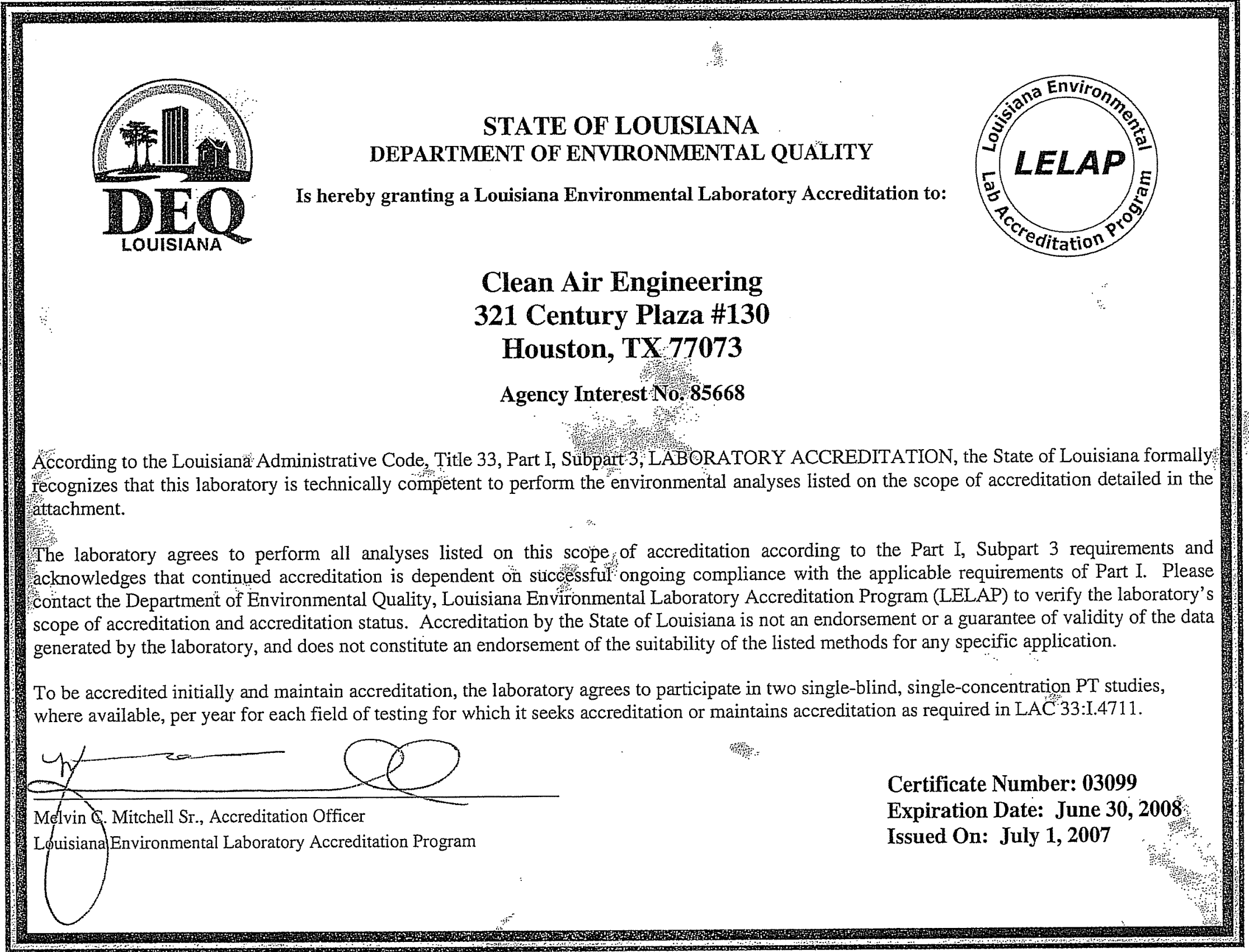

According to the Louisiana Administrative Code, Title 33, Part I, Sübpart 3, LABORATORY ACCREDITATION, the State of Louisiana formally: cognizes that this laboratory is technically competent to perform the environmental analyses listed on the scope of accreditation detailed in the attachment.

The laboratory agrees to perform all analyses listed on this scope of accreditation according to the Part I, Subpart 3 requirements and

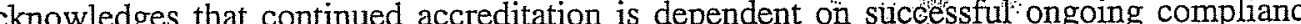
act the Department of Environmental Quality, Lowisiana Environmental Laboratory Accreditation Program (LELAI scope of accreditation and accreditation status. Accreditation by the State of Louisiana is not an endorsement or a guarantee of validity of the data generated by the laboratory, and does not constitute an endorsement of the suitability of the listed methods for any specific application.

To be accredited initially and maintain accreditation, the laboratory agrees to participate in two single-blind, single-concentration PT studies,

Melvin \&. Mitchell Sr., Accreditation Office

Louisianal Environmental Laboratory Accreditation Program

Expiration Date: June 30, 2008

Issued On: July 1, 2007 


\section{Organization}

\section{9}

Clean Air Engineering

321 Century Plaza \#130

Houston, TX 77073

\begin{tabular}{|c|c|c|c|c|c|c|}
\hline $\begin{array}{l}\text { Method } \\
\text { Code }\end{array}$ & Method Ref & Analyte & Status & Date Effective & Type & AA \\
\hline 895 & NCASI DI/MEOH 94.03 & Methanol & Accredited & $7 / 1 / 2003$ & STATE & LA \\
\hline 1217 & Method 140 CFR 60 App. A & Traverse Points & Accredited & $7 / 1 / 2003$ & STATE & LA \\
\hline 1218 & Method 1040 CFR $60 \mathrm{App}$. A & Carbon monoxide $(\mathrm{CO})$ & Accredited & $7 / 1 / 2003$ & STATE & $L A$ \\
\hline 1232 & Method 10A 40 CFR 60 App. A & Carbon monoxide (CO) & Accredited & $7 / 1 / 2003$ & STATE & LA \\
\hline 1233 & Method 10B 40 CFR 60 App. A & Carbon monoxide (CO) & Accredited & $7 / 1 / 2003$ & STATE & LA \\
\hline 1248 & Method 1A 40 CFR 60 App. A & Traverse Points & Accredited & $7 / 1 / 2003$ & STATE & LA \\
\hline 1249 & Method 240 CFR 60 App. A & Stack gas velocity volume flow rate & Accredited & $7 / 1 / 2003$ & STATE & LA \\
\hline 1250 & Method 2040 CFR 60 App. A & Nitrogen Oxides (NOX) & Accredited & $7 / 1 / 2003$ & STATE & LA \\
\hline 1250 & Method 2040 CFR 60 App. A & Oxygen & Accredited & $7 / 1 / 2003$ & STATE & LA \\
\hline 1250 & Method 2040 CFR 60 App. A & Sulfur dioxide & Accredited & $7 / 1 / 2003$ & STATE & LA \\
\hline 1251 & Method 201 A 40 CFR 51 App. M & Particulates $<10 \mathrm{um}$ & Accredited & $7 / 1 / 2003$ & STATE & LA \\
\hline 1252 & Method 20240 CFR 51 App. M & Particulate Matter <2.5 um & Accredited & $7 / 1 / 2003$ & STATE & LA \\
\hline 1262 & Method 2240 CFR 60 App. A & Visible emissions from coke oven batteries & Accredited & $7 / 1 / 2003$ & STATE & LA \\
\hline 1271 & Method 2A 40 CFR 60 App. A & Stack gas velocity volume flow rate in small stacks/ducts & Accredited & $7 / 1 / 2003$ & STATE & LA \\
\hline 1272 & Method 2B 40 CFR 60 App. A & Stack gas velocity volume flow rate & Accredited & $7 / 1 / 2003$ & STATE & LA \\
\hline 1273 & Method 2C 40 CFR 60 App. A & Stack gas velocity volume flow rate in small stacks/ducts & Accredited & $7 / 1 / 2003$ & STATE & LA \\
\hline 1274 & Method 2D 40 CFR 60 App. A & Stack gas velocity volume flow rate in small stacks/ducts & Accredited & $7 / 1 / 2003$ & STATE & LA \\
\hline 1275 & Method 2E 40 CFR 60 App. A & Stack gas velocity volume flow rate & Accredited & $7 / 1 / 2003$ & STATE & LA \\
\hline 1276 & Method 2 F 40 CFR 60 App. A & Stack gas velocity volume flow rate & Accredited & $7 / 1 / 2003$ & STATE & LA \\
\hline 1277 & Method 2G 40 CFR 60 App. A & Stack gas velocity volume flow rate & Accredited & $7 / 1 / 2003$ & STATE & LA \\
\hline 1278 & Method $2 \mathrm{H} 40$ CFR $60 \mathrm{App}$. A & Stack gas velocity volume flow rate & Accredited & $7 / 1 / 2003$ & STATE & LA \\
\hline 1279 & Method 340 CFR 60 App. A & Carbon dioxide oxygen dry molecular weight & Accredited & $7 / 1 / 2003$ & STATE & LA \\
\hline 1296 & Method 3A 40 CFR 60 App. A & Carbon dioxide & Accredited & $7 / 1 / 2003$ & STATE & LA \\
\hline 1296 & Method 3A 40 CFR 60 App. A & Oxygen & Accredited & $7 / 1 / 2003$ & STATE & LA \\
\hline 1297 & Method 3B 40 CFR 60 App. A & Emission Rate Correction Factors & Accredited & $7 / 1 / 2003$ & STATE & LA \\
\hline 1298 & Method 3C 40 CFR 60 App. A & Carbon dioxide & Accredited & $7 / 1 / 2003$ & STATE & LA \\
\hline 1302 & Method 440 CFR 60 App. A & Moisture content & Accredited & $7 / 1 / 2003$ & STATE & LA \\
\hline
\end{tabular} \\ (281) $443-6400$}

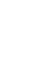




\section{Organization}

03099

(281) 443-6400

Clean Air Engineering

321 Century Plaza \#130

Houston, TX 77073

\begin{tabular}{|c|c|c|c|c|c|c|}
\hline $\begin{array}{l}\text { Method } \\
\text { Code }\end{array}$ & Method Ref & nalyte & Status & Date Effective & Type & AA \\
\hline$\frac{\text { Code }}{1303}$ & Method 540 CFR 60 App. A & Particulates & Accredited & $7 / 1 / 2003$ & STATE & LA \\
\hline 1304 & Method 5A 40 CFR 60 App. A & Particulates from asphalt processing & Accredited & $7 / 1 / 2003$ & STATE & LA \\
\hline 1305 & Method 5B 40 CFR 60 App. A & Particulates & Accredited & $7 / 1 / 2003$ & STATE & LA \\
\hline 1306 & Method 5D 40 CFR 60 App. A & Particulates from fabric filters & Accredited & $7 / 1 / 2003$ & STATE & LA \\
\hline 1307 & Method 5E 40 CFR 60 App. A & Particulates from wool fiberglass insulation & Accredited & $7 / 1 / 2003$ & STATE & LA \\
\hline 1308 & Method 5F 40 CFR 60 App. A & Particulates & Accredited & $7 / 1 / 2003$ & STATE & LA \\
\hline 1315 & Method 6C 40 CFR 60 App. A & Sulfur dioxide & Accredited & $7 / 1 / 2003$ & STATE & LA \\
\hline 1321 & Method 7E 40 CFR 60 App. A & Nitrogen Oxides (NOX) & Accredited & $7 / 1 / 2003$ & STATE & LA \\
\hline 1761 & Method 101A 40 CFR 61 App. B (Sample Only) & Mercury & Accredited & $7 / 1 / 2003$ & STATE & LA \\
\hline 1789 & Method 1140 CFR 60 App. A (Sample Only) & Hydrogen sulfide & Accredited & $7 / 1 / 2003$ & STATE & LA \\
\hline 1793 & Method 1240 CFR 60 App. A (Sample Only) & Lead & Accredited & $7 / 1 / 2003$ & STATE & LA \\
\hline 1797 & Method 13B 40 CFR 60 App. A (Sample Only) & Fluoride & Accredited & $7 / 1 / 2003$ & STATE & $L A$ \\
\hline 1799 & Method 1440 CFR 60 App. A (Sample Only) & Fluoride & Accredited & $7 / 1 / 2003$ & STATE & LA \\
\hline 1801 & Method 1540 CFR 60 App. A (Sample Only) & Carbon disulfide & Accredited & $7 / 1 / 2003$ & STATE & LA \\
\hline 1801 & Method 1540 CFR 60 App. A (Sample Only) & Carbonyl sulfide & Accredited & $7 / 1 / 2003$ & STATE & LA \\
\hline 1801 & Method 1540 CFR 60 App. A (Sample Only) & Hydrogen sulfide & Accredited & $7 / 1 / 2003$ & STATE & LA \\
\hline 1803 & Method 15A 40 CFR 60 App. A (Sample Only) & Total reduced sulfur & Accredited & $7 / 1 / 2003$ & STATE & LA \\
\hline 1805 & Method 1640 CFR 60 App. A (Sample Only) & Dimethyl Disulfide & Accredited & $7 / 1 / 2003$ & STATE & LA \\
\hline 1805 & Method 1640 CFR 60 App. A (Sample Only) & Dimethyl Sulfide & Accredited & $7 / 1 / 2003$ & STATE & LA \\
\hline 1805 & Method 1640 CFR 60 App. A (Sample Only) & Hydrogen sulfide & Accredited & $7 / 1 / 2003$ & STATE & LA \\
\hline 1805 & Method 1640 CFR 60 App. A (Sample Only) & Methyl Mercaptan & Accredited & $7 / 1 / 2003$ & STATE & LA \\
\hline 1807 & Method $16 A 40$ CFR 60 App. A (Sample Only) & Total reduced sulfur & Accredited & $7 / 1 / 2003$ & STATE & LA \\
\hline 1809 & Method $16 \mathrm{~B} 40$ CFR 60 App. A (Sample Only) & Total reduced sulfur & Accredited & $7 / 1 / 2003$ & STATE & LA \\
\hline 1811 & Method 1740 CFR 60 App. A (Sample Only) & Particulates & Accredited & $7 / 1 / 2003$ & STATE & LA \\
\hline 1813 & Method 1840 CFR 60 App. A (Sample Only) & Gaseous Organic Compound Emissions & Accredited & $7 / 1 / 2003$ & STATE & LA \\
\hline 1815 & Method 1940 CFR 60 App. A (Sample Only) & Nitrogen Oxides (NOX) & Accredited & $7 / 1 / 2003$ & STATE & LA \\
\hline 1815 & Method 1940 CFR 60 App. A (Sample Only) & Particulates SO2 NOx sulfur removal efficiency & Accredited & $7 / 1 / 2003$ & STATE & LA \\
\hline
\end{tabular}

Issue Date: July 1, 200?

Expiration Date: Jure 30, 2008 


\section{Organization}

03099

(281) $443-6400$

Clean Air Engineering

321 Century Plaza \#130

Houston, TX 77073

\begin{tabular}{|c|c|c|c|c|c|c|}
\hline $\begin{array}{l}\text { Method } \\
\text { Code }\end{array}$ & Method Ref & Analyte & Status & Date Effective & Type & \\
\hline 1815 & Method 1940 CFR 60 App. A (Sample Only) & Sulfur dioxide & Accredited & $7 / 1 / 2003$ & STATE & LA \\
\hline 1847 & Method 2340 CFR 60 App. A (Sample Only) & Dioxins/Furans & Accredited & $7 / 1 / 2003$ & STATE & LA \\
\hline 1849 & Method 2540 CFR 60 App. A (Sample Only) & Gaseous Nonmethane Organic Emissions & Accredited & $7 / 1 / 2003$ & STATE & LA \\
\hline 1851 & Method 25A 40 CFR 60 App. A (Sample Only) & Gaseous Organic Emissions & Accredited & $7 / 1 / 2003$ & STATE & LA \\
\hline 1857 & Method 2640 CFR 60 App. A (Sample Only) & Bromine $(\mathrm{B} r 2)$ & Accredited & $7 / 1 / 2003$ & STATE & LA \\
\hline 1857 & Method 2640 CFR 60 App. A (Sample Only) & Chlorine & Accredited & $7 / 1 / 2003$ & STATE & LA \\
\hline 1857 & Method 2640 CFR 60 App. A (Sample Only) & Hydrachloric acid (Hydrogen chloride (gas only)) & Accredited & $7 / 1 / 2003$ & STATE & $L A$ \\
\hline 1857 & Method 2640 CFR 60 App. A (Sample Only) & Hydrogen Bromide $(\mathrm{HBr})$ & Accredited & $7 / 1 / 2003$ & STATE & LA \\
\hline 1857 & Method 2640 CFR 60 App. A (Sample Only) & Hydrogen fluoride (Hydrofluoric acid) & Accredited & $7 / 1 / 2003$ & STATE & LA \\
\hline 1859 & Method 26A 40 CFR 60 App. A (Sample Only) & Bromine $(\mathrm{Br} 2)$ & Accredited & $7 / 1 / 2003$ & STATE & LA \\
\hline 1859 & Method $26 \mathrm{~A} 40$ CFR 60 App. A (Sample Only) & Chlorine & Accredited & $7 / 1 / 2003$ & STATE & LA \\
\hline 1859 & Method 26A 40 CFR 60 App. A (Sample Only) & Hydrochloric acid (Hydrogen chloride (gas only)) & Accredited & $7 / 1 / 2003$ & STATE & LA \\
\hline 1859 & Method 26A 40 CFR 60 App. A (Sample Only) & Hydrogen Bromide ( $\mathrm{HBr})$ & Accredited & $7 / 1 / 2003$ & STATE & LA \\
\hline 1859 & Method 26A 40 CFR 60 App. A (Sample Only) & Hydrogen fluoride (Hydrofluoric acid) & Accredited & $7 / 1 / 2003$ & STATE & LA \\
\hline 1861 & Method 2940 CFR 60 App. A (Sample Only) & Antimany & Accredited & $7 / 1 / 2003$ & STATE & LA \\
\hline 1861 & Method 2940 CFR 60 App. A (Sample Only) & Arsenic & Accredited & $7 / 1 / 2003$ & STATE & LA \\
\hline 1861 & Method 2940 CFR 60 App. A (Sample Only) & Barium & Accredited & $7 / 1 / 2003$ & STATE & LA \\
\hline 1861 & Method 2940 CFR 60 App. A (Sample Only) & Beryllium & Accredited & $7 / 1 / 2003$ & STATE & LA \\
\hline 1861 & Method 2940 CFR 60 App. A (Sample Only) & Cadmium & Accredited & $7 / 1 / 2003$ & STATE & LA \\
\hline 1861 & Method 2940 CFR 60 App. A (Sample Only) & Chromium & Accredited & $7 / 1 / 2003$ & STATE & LA \\
\hline 1861 & Method 2940 CFR 60 App. A (Sample Only) & Cobalt & Accredited & $7 / 1 / 2003$ & STATE & LA \\
\hline 1861 & Method 2940 CFR 60 App. A (Sample Only) & Copper & Accredited & $7 / 1 / 2003$ & STATE & LA \\
\hline 1861 & Method 2940 CFR 60 App. A (Sample Only) & Lead & Accredited & $7 / 1 / 2003$ & STATE & $L A$ \\
\hline 1861 & Method 2940 CFR 60 App. A (Sample Only) & Manganese & Accredited & $7 / 1 / 2003$ & STATE & LA \\
\hline 1861 & Method 2940 CFR 60 App. A (Sample Only) & Mercury & Accredited & $7 / 1 / 2003$ & STATE & LA \\
\hline 1861 & Method 2940 CFR 60 App. A (Sample Only) & Nickel & Accredited & $7 / 1 / 2003$ & STATE & LA \\
\hline 1861 & Method 2940 CFR 60 App. A (Sample Only) & Phosphorus total & Accredited & $7 / 1 / 2003$ & STATE & LA \\
\hline
\end{tabular}

Issue Date: July 1,2007

Expiration Date: Jure 30, 2008 


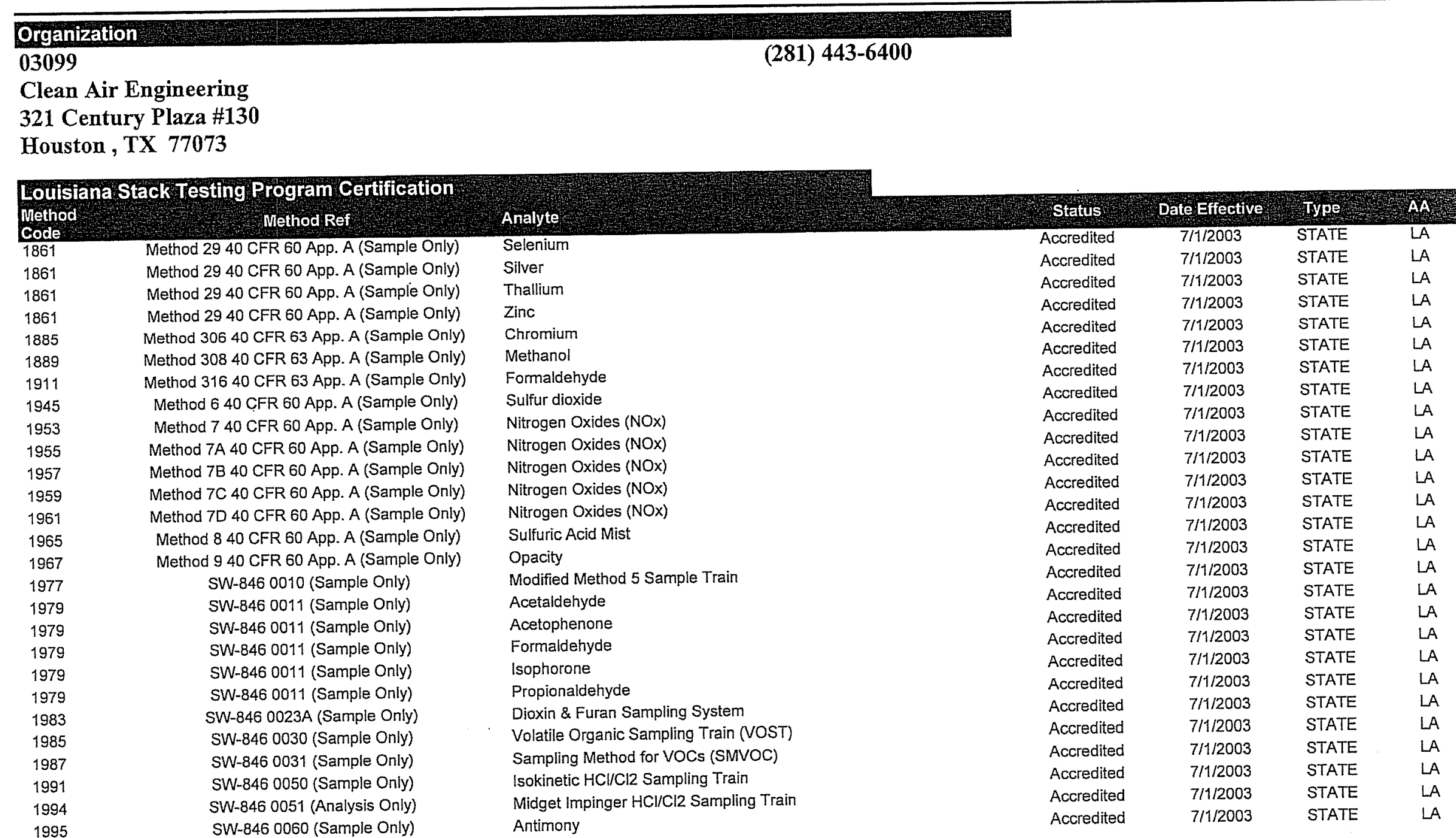




\begin{tabular}{|c|c|c|c|c|c|c|}
\hline \multicolumn{7}{|c|}{ Organization } \\
\hline \multirow{4}{*}{\multicolumn{7}{|c|}{$\begin{array}{l}03099 \\
\text { Clean Air Engineering } \\
321 \text { Century Plaza \#130 } \\
\text { Houston, TX } 77073\end{array}$}} \\
\hline & & & & & & \\
\hline & & & & & & \\
\hline & & & & & & \\
\hline \multicolumn{7}{|c|}{ Louisiana Stack Testing Program Certification } \\
\hline $\begin{array}{l}\text { Method } \\
\text { Code }\end{array}$ & Method Ref & Analyte & Status & Date Effective & Type & AA \\
\hline $1995:-1$ & SW-846 0060 (Sample Only) & Airsenic & Accredited & $7 / 1 / 2003$ & STATE & LA \\
\hline 1995 & SW-846 0060 (Sample Only) & Barium & Accredited & $7 / 1 / 2003$ & STATE & LA \\
\hline 1995 & SW-846 0060 (Sample Only) & Beryllium & Accredited & $7 / 1 / 2003$ & STATE & LA \\
\hline 1995 & SW-846 0060 (Sample Only) & Cadmium & Accredited & $7 / 1 / 2003$ & STATE & LA \\
\hline 1995 & SW-846 0060 (Sample Only) & Cobalt & Accredited & $7 / 1 / 2003$ & STATE & LA \\
\hline 1995 & SW-846 0060 (Sample Only) & Copper & Accredited & $7 / 1 / 2003$ & STATE & LA \\
\hline 1995 & SW-846 0060 (Sample Only) & Lead & Accredited & $7 / 1 / 2003$ & STATE & LA \\
\hline 1995 & SW-846 0060 (Sample Only) & Manganese & Accredited & $7 / 1 / 2003$ & STATE & LA \\
\hline 1995 & SW-846 0060 (Sample Only) & Mercury & Accredited & $7 / 1 / 2003$ & STATE & LA \\
\hline 1995 & SW-846 0060 (Sample Only) & Nickel & Accredited & $7 / 1 / 2003$ & STATE & LA \\
\hline 1995 & SW-846 0060 (Sample Only) & Particulates & Accredited & $7 / 1 / 2003$ & STATE & $\angle A$ \\
\hline 1995 & SW-846 0060 (Sample Only) & Phosphorus total & Accredited & $7 / 1 / 2003$ & STATE & $L A$ \\
\hline 1995 & SW-846 0060 (Sample Only) & Selenium & Accredited & $7 / 1 / 2003$ & STATE & LA \\
\hline 1995 & SW-846 0060 (Sample Only) & Silver & Accredited & $7 / 1 / 2003$ & STATE & LA \\
\hline 1995 & SW-846 0060 (Sample Only) & Thallium & Accredited & $7 / 1 / 2003$ & STATE & LA \\
\hline 1995 & SW-846 0060 (Sample Only) & Total chromium & Accredited & $7 / 1 / 2003$ & STATE & LA \\
\hline 1995 & SW-846 0060 (Sample Only) & Zinc & Accredited & $7 / 1 / 2003$ & STATE & LA \\
\hline 1997 & SW-846 0061 (Sample Only) & Chromium VI & Accredited & $7 / 1 / 2003$ & STATE & LA \\
\hline \multicolumn{7}{|c|}{ Non Potable Water Gertification } \\
\hline $\begin{array}{l}\text { Method } \\
\text { Cade }\end{array}$ & WhodRef & & & Date Effective & Type & AA \\
\hline 10053006 & EPA 300.0 & Chloride & Accredited & $1 / 25 / 2006$ & STATE & LA \\
\hline 10053006 & EPA 300.0 & Fluoride & Accredited & $1 / 25 / 2006$ & STATE & LA \\
\hline 10053006 & EPA 300.0 & Nitrite & Accredited & $1 / 25 / 2006$ & STATE & LA \\
\hline 10053006 & EPA 300.0 & Sulfate & Accredited & $1 / 25 / 2006$ & STATE & LA \\
\hline
\end{tabular}

Issue Date: July 1, 2007 
Clean Air Engineering Project \#10192

Consol Energy

AES Dresden

\begin{tabular}{|c|c|c|c|c|c|}
\hline Cal Range & 1 & 2 & & & \\
\hline High NOx & 233.8 & 91.4 & $\mathrm{ppm}$ & & \\
\hline Low NOx & 91.4 & 47.4 & $\mathrm{ppm}$ & & \\
\hline High $\mathrm{O} 2$ & 14.1 & 14.1 & $\%$ & & \\
\hline Low O2 & 6.04 & 6.04 & $\%$ & & \\
\hline High $\mathrm{CO} 2$ & 13.98 & 13.98 & & & \\
\hline Low CO2 & 6.02 & 6.02 & & & \\
\hline Date: & $3 / 28 / 2007$ & & Date: & $3 / 28 / 2007$ & \\
\hline Start Time: & $7: 50$ & & Start Time: & $7: 50$ & \\
\hline End Time: & $8: 15$ & & End Time: & $8: 15$ & \\
\hline $\begin{array}{l}\text { Cal Range } \\
\text { HIGH }\end{array}$ & 1 & & $\begin{array}{l}\text { Cal Range } \\
\text { HIGH }\end{array}$ & 2 & \\
\hline Instrument & $\begin{array}{c}\text { Response } \\
\text { (1 min avg.) }\end{array}$ & $\begin{array}{l}\text { Cal. Error } \\
(\%)\end{array}$ & Instrument & $\begin{array}{l}\text { Response } \\
\text { (1 min avg.) }\end{array}$ & $\begin{array}{c}\text { Cal. Error } \\
(\%)\end{array}$ \\
\hline Nox In & 233.6 & 0.09 & Nox Out & 91.92 & 0.57 \\
\hline $\mathrm{O} 2 \mathrm{In}$ & 14.1 & 0.00 & O2 Out & 14.1 & 0.00 \\
\hline $\mathrm{CO} 2 \ln$ & 14 & 0.14 & $\mathrm{CO} 2$ Out & 14 & 0.14 \\
\hline $\begin{array}{l}\text { LOW } \\
\text { Instrument }\end{array}$ & $\begin{array}{l}\text { Response } \\
\text { (1 min avg.) }\end{array}$ & $\begin{array}{l}\text { Cal. Error } \\
(\%)\end{array}$ & $\begin{array}{l}\text { LOW } \\
\text { Instrument }\end{array}$ & $\begin{array}{l}\text { Response } \\
\text { (1 min avg.) }\end{array}$ & $\begin{array}{l}\text { Cal. Error } \\
(\%)\end{array}$ \\
\hline Nox In & 92.3 & 0.38 & Nox Out & 47.56 & 0.18 \\
\hline $\mathrm{O} 2 \ln$ & 5.9 & 0.99 & O2 Out & 6 & 0.28 \\
\hline $\mathrm{CO} 2 \mathrm{ln}$ & 6.1 & 0.57 & $\mathrm{CO} 2$ Out & 6.2 & 1.29 \\
\hline $\begin{array}{l}\text { ZERO } \\
\text { Instrument }\end{array}$ & $\begin{array}{l}\text { Response } \\
\text { (1 min avg.) }\end{array}$ & $\begin{array}{l}\text { Cal. Error } \\
(\%)\end{array}$ & $\begin{array}{l}\text { ZERO } \\
\text { Instrument }\end{array}$ & $\begin{array}{l}\text { Response } \\
\text { (1 min avg.) }\end{array}$ & $\begin{array}{c}\text { Cal. Error } \\
(\%)\end{array}$ \\
\hline Nox in & 2 & 0.86 & Nox Out & 0.9 & 0.98 \\
\hline $\mathrm{O} 2 \mathrm{In}$ & -0.1 & 0.71 & O2 Out & 0 & 0.00 \\
\hline $\mathrm{CO} 2 \mathrm{In}$ & 0 & 0.00 & $\mathrm{CO} 2$ Out & 0 & 0.00 \\
\hline BIAS & (uses low gas) & & BIAS & (uses low gas) & \\
\hline Instrument & $\begin{array}{l}\text { Response } \\
\text { (1 min avg.) }\end{array}$ & $\begin{array}{l}\text { Cal. Error } \\
(\%)\end{array}$ & Instrument & $\begin{array}{l}\text { Response } \\
\text { (1 min avg.) }\end{array}$ & $\begin{array}{c}\text { Cal. Error } \\
(\%)\end{array}$ \\
\hline Nox In & 90.5 & 1.80 & Nox Out & 46.9 & 0.66 \\
\hline $\mathrm{O} 2 \mathrm{In}$ & 5.8 & 1.00 & O2 Out & 5.9 & 1.00 \\
\hline $\mathrm{O} 2 \_2$ & 14 & 0.00 & $\mathrm{O} 22$ & 14.1 & 1.00 \\
\hline DRIFT & (end of day) & & DRIFT & (end of day) & \\
\hline Instrument & $\begin{array}{l}\text { Response } \\
\text { (1 min avg.) }\end{array}$ & $\begin{array}{l}\text { Cal. Error } \\
(\%)\end{array}$ & Instrument & $\begin{array}{l}\text { Response } \\
(1 \mathrm{~min} \text { avg })\end{array}$ & $\begin{array}{c}\text { Cal. Error } \\
(\%)\end{array}$ \\
\hline NOx 1 & 85.6 & 4.90 & NOX 1 & 45.1 & 1.80 \\
\hline NOX 2 & & 0.00 & NO 2 & & 0.00 \\
\hline $\mathrm{O} 2 \_1$ & 5.8 & 0.00 & $\mathrm{O} 2 \_1$ & 6 & 1.00 \\
\hline $\mathrm{O} 2{ }_{2} 2$ & 14 & 0.00 & $\mathrm{O} 2 \_2$ & 14.1 & 0.00 \\
\hline
\end{tabular}


CALIBRATION ERROR

$$
\text { Date: }
$$

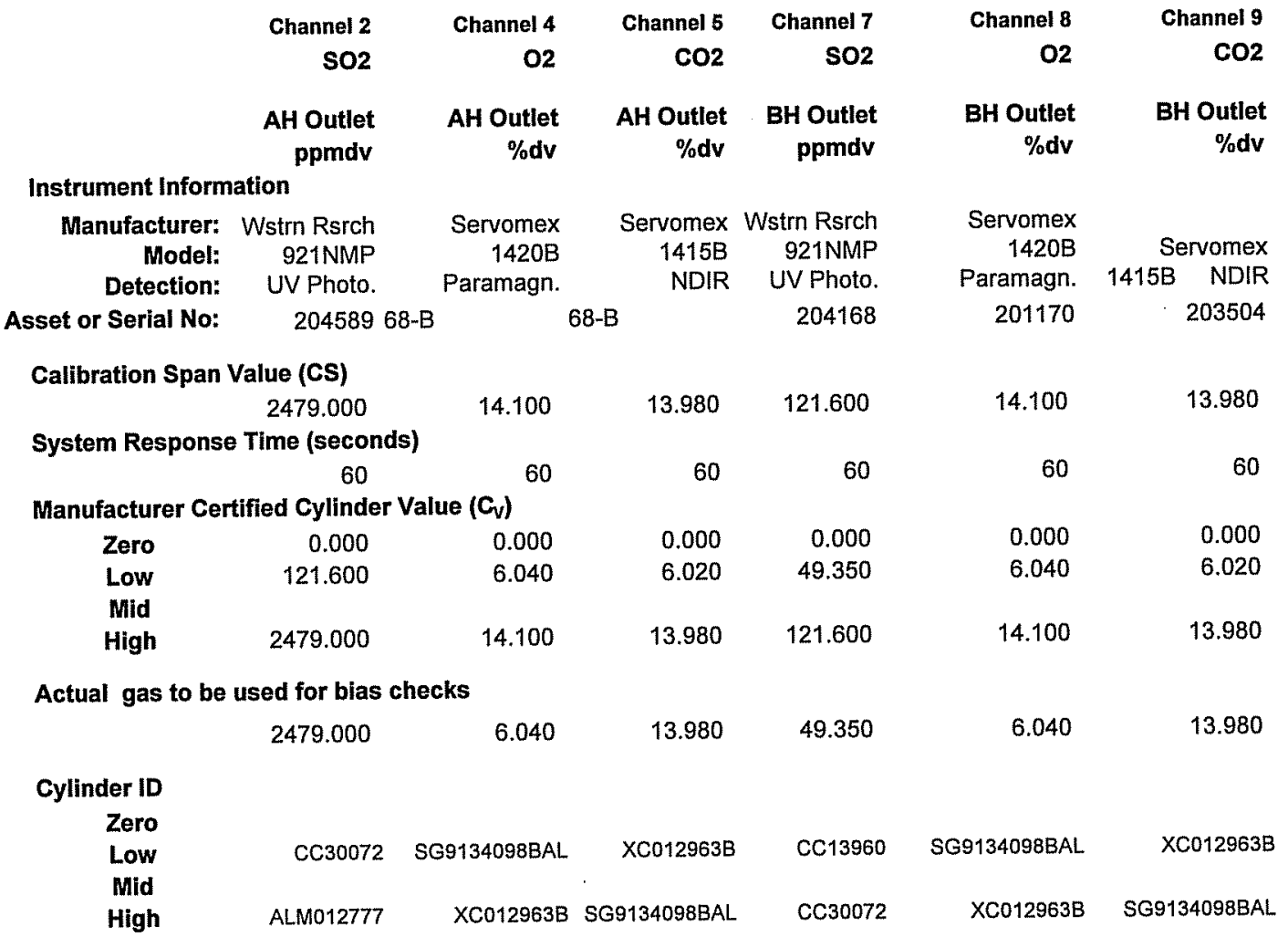

Analyzer Calibration Response ( $\mathrm{C}_{\text {Dir }}$ )

\begin{tabular}{|c|c|c|c|c|c|c|}
\hline Zero & 3.452 & -0.010 & 0.148 & -0.056 & 0.002 & 0.003 \\
\hline Low & 133.235 & 6.061 & 6.041 & 48.630 & 6.050 & 5.940 \\
\hline Mid & & & 0.209 & & & \\
\hline High & 2482.035 & 14.189 & 14.059 & 121.848 & 14.140 & 13.966 \\
\hline
\end{tabular}

Analyzer Calibration Error (ACE) (Limit $=\mathbf{2} \%$, Method $25 \mathrm{~A}$ limit $=\mathbf{5} \%$ of gas value)

$\begin{array}{lcccccc}\text { Zero } & 0.1 \% & -0.1 \% & 1.1 \% & 0.0 \% & 0.0 \% & 0.0 \% \\ \text { Low } & 0.5 \% & 0.2 \% & 0.1 \% & -0.6 \% & 0.1 \% & -0.6 \% \\ \text { Mid } & \text { N/A } & \text { N/A } & \text { N/A } & \text { N/A } & \text { N/A } & \text { N/A } \\ \text { High } & 0.1 \% & 0.6 \% & 0.6 \% & 0.2 \% & 0.3 \% & -0.1 \%\end{array}$

Calibration Error Status

$\begin{array}{lrrrrrr}\text { Zero } & \text { OK } & \text { OK } & \text { OK } & \text { OK } & \text { OK } & \text { OK } \\ \text { Low } & \text { OK } & \text { OK } & \text { OK } & \text { OK } & \text { OK } & \text { OK } \\ \text { Mid } & \text { N/A } & \text { N/A } & \text { N/A } & \text { N/A } & \text { N/A } & \text { N/A } \\ \text { High } & \text { OK } & \text { OK } & \text { OK } & \text { OK } & \text { OK } & \text { OK }\end{array}$

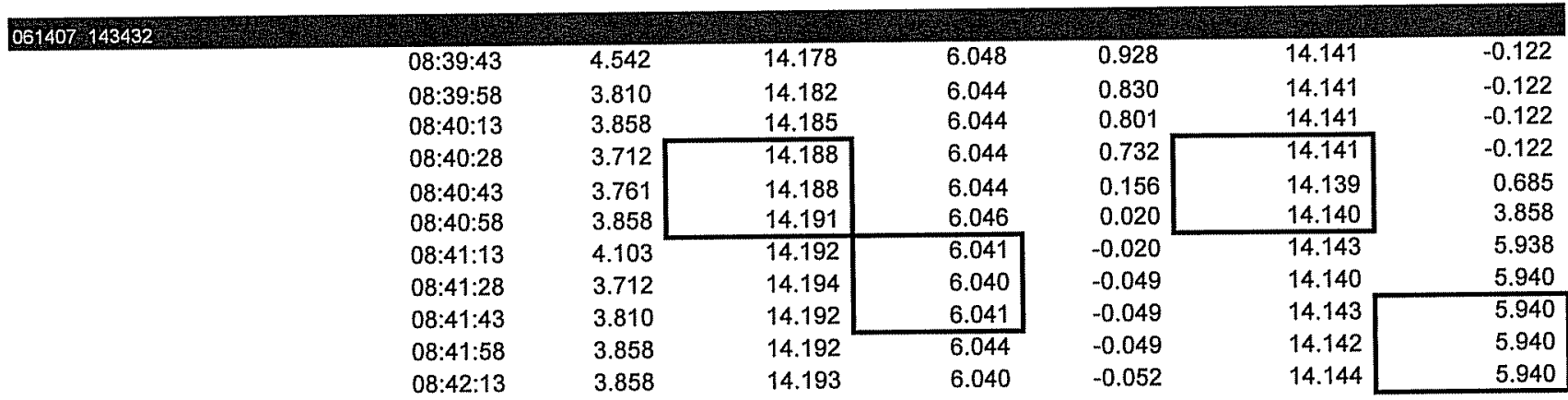




\section{Date: \\ Start Time
Stop Time
CALIBRATION ERROR \\ Start Time
Stop Time
CALIBRATION ERROR \\ Start Tim
Stop Tim
CALIBRATION ERROR \\ 8:39 \\ 9:01 \\ March 29, 2007 \\ (1)}

\begin{tabular}{|c|c|c|c|c|c|c|}
\hline & $\begin{array}{r}\text { Channel } 2 \\
\text { SO2 }\end{array}$ & $\begin{array}{r}\text { Channel } 4 \\
\mathrm{O} 2\end{array}$ & $\begin{array}{r}\text { Channel } 5 \\
\mathrm{CO} 2\end{array}$ & $\begin{array}{r}\text { Channel } 7 \\
\mathrm{SO}_{2}\end{array}$ & $\begin{array}{r}\text { Channel } 8 \\
\mathrm{O} 2\end{array}$ & $\begin{array}{r}\text { Channel } 9 \\
\mathrm{CO} 2\end{array}$ \\
\hline & $\begin{array}{l}\text { AH Outlet } \\
\text { ppmdv }\end{array}$ & $\begin{array}{r}\text { AH Outlet } \\
\% d v\end{array}$ & $\begin{array}{r}\text { AH Outlet } \\
\% \mathrm{dv}\end{array}$ & $\begin{array}{l}\text { BH Outlet } \\
\text { ppmdv }\end{array}$ & $\begin{array}{r}\text { BH Outlet } \\
\% \text { dv }\end{array}$ & $\begin{array}{r}\text { BH Outlet } \\
\% d v\end{array}$ \\
\hline $08: 42: 28$ & 3.858 & 14.191 & 6.043 & -0.078 & 14.145 & 5.940 \\
\hline $\begin{array}{l}08: 42: 43 \\
08: 42: 58\end{array}$ & $\begin{array}{l}3.321 \\
3.175\end{array}$ & $\begin{array}{l}13.657 \\
13.620\end{array}$ & $\begin{array}{l}5.869 \\
5.764\end{array}$ & $\begin{array}{l}-0.235 \\
-0.156\end{array}$ & $\begin{array}{l}13.872 \\
13.912\end{array}$ & $\begin{array}{l}5.864 \\
5.862\end{array}$ \\
\hline $\begin{array}{l}08: 43: 13 \\
08: 43: 28\end{array}$ & $\begin{array}{r}11.673 \\
7.570\end{array}$ & $\begin{array}{l}9.785 \\
6.261\end{array}$ & $\begin{array}{r}9.526 \\
13.762 \\
\end{array}$ & $\begin{array}{l}0.088 \\
0.059\end{array}$ & $\begin{array}{l}8.958 \\
6.163\end{array}$ & $\begin{array}{l}10.066 \\
13.902\end{array}$ \\
\hline $08: 43: 43$ & 4.396 & 6.071 & 14.059 & -0.078 & 6.069 & 14.064 \\
\hline $\begin{array}{l}08: 43: 58 \\
08: 44: 13\end{array}$ & $\begin{array}{l}3.175 \\
1.807\end{array}$ & $\begin{array}{l}5.890 \\
5.874\end{array}$ & $\begin{array}{l}14.069 \\
14.047 \\
\end{array}$ & $\begin{array}{l}-0.219 \\
-0.482\end{array}$ & $\begin{array}{l}5.930 \\
5.737\end{array}$ & $\begin{array}{l}14.033 \\
14.003\end{array}$ \\
\hline $08: 44: 28$ & 4.298 & 6.063 & 14.084 & -0.153 & 6.064 & 14.077 \\
\hline $08: 44: 43$ & 4.249 & 6.061 & 14.086 & -0.179 & 6.062 & 14.078 \\
\hline $\begin{array}{l}08: 44: 58 \\
08: 45: 13\end{array}$ & $\begin{array}{l}4.298 \\
4.396\end{array}$ & $\begin{array}{l}6.060 \\
6.062\end{array}$ & $\begin{array}{l}14.087 \\
14.088\end{array}$ & $\begin{array}{l}-0.225 \\
-0.215\end{array}$ & $\begin{array}{l}6.062 \\
6.062\end{array}$ & $\begin{array}{l}14.080 \\
14.082\end{array}$ \\
\hline $08: 45: 28$ & 4.396 & 6.062 & 14.087 & -0.212 & 6.061 & 14.083 \\
\hline $\begin{array}{l}08: 45: 43 \\
08: 45: 58\end{array}$ & $\begin{array}{r}81.954 \\
1661.587\end{array}$ & $\begin{array}{l}5.765 \\
0.866\end{array}$ & $\begin{array}{r}13.924 \\
2.886\end{array}$ & $\begin{array}{l}-0.212 \\
-0.169\end{array}$ & $\begin{array}{l}6.053 \\
6.050 \\
\end{array}$ & $\begin{array}{l}14.023 \\
13.949\end{array}$ \\
\hline $08: 46: 13$ & 2388.718 & 0.017 & 0.307 & -0.160 & 6.050 & 13.924 \\
\hline $08: 46: 28$ & 2469.011 & -0.016 & 0.167 & -0.098 & 6.050 & 13.904 \\
\hline $08: 46: 43$ & 2471.306 & -0.019 & 0.152 & -0.078 & 6.050 & 13.892 \\
\hline $08: 46: 58$ & 2473.407 & -0.018 & 0.148 & -0.049 & 6.050 & 13.887 \\
\hline $08: 47: 13$ & 2487.863 & -0.021 & 0.143 & -0.013 & 6.051 & 13.881 \\
\hline $08: 47: 28$ & 2492.161 & -0.129 & -3.269 & 0.124 & 6.008 & 13.703 \\
\hline $08: 47: 43$ & 2491.575 & -2.002 & -8.243 & -0.238 & 5.808 & 13.832 \\
\hline $\begin{array}{l}08: 47: 58 \\
08: 48: 13\end{array}$ & $\begin{array}{l}2491.526 \\
2495.385\end{array}$ & $\begin{array}{l}-0.099 \\
-0.040\end{array}$ & $\begin{array}{l}-3.216 \\
-1.541\end{array}$ & $\begin{array}{r}-0.006 \\
0.075 \\
\end{array}$ & $\begin{array}{l}5.975 \\
6.025\end{array}$ & $\begin{array}{l}13.804 \\
13.826\end{array}$ \\
\hline $08: 48: 28$ & 2494.066 & -0.019 & 0.135 & 0.147 & 6.059 & 13.850 \\
\hline $08: 48: 43$ & 2487.277 & -0.020 & 0.134 & 0.212 & 6.060 & 13.846 \\
\hline $08: 48: 58$ & 2483.956 & -0.022 & 0.132 & 0.257 & 6.062 & 13.839 \\
\hline $08: 49: 13$ & 2482.637 & -0.020 & 0.131 & 0.293 & 6.062 & 13.835 \\
\hline $08: 49: 28$ & 2484.688 & -0.020 & 0.130 & 0.335 & 6.064 & 13.832 \\
\hline $08: 49: 43$ & 2484.542 & -0.020 & 0.131 & 0.420 & 6.066 & 13.826 \\
\hline $08: 49: 58$ & 2485.079 & -0.022 & 0.130 & 0.453 & 6.067 & 13.821 \\
\hline $08: 50: 13$ & 2485.568 & -0.019 & 0.128 & 0.495 & 6.070 & 13.818 \\
\hline $\begin{array}{l}08: 50: 28 \\
08: 50: 43\end{array}$ & $\begin{array}{l}2479.512 \\
2481.026\end{array}$ & $\begin{array}{l}-0.014 \\
-0.011\end{array}$ & $\begin{array}{l}0.132 \\
0.139\end{array}$ & $\begin{array}{l}0.537 \\
0.544\end{array}$ & $\begin{array}{l}6.074 \\
6.085\end{array}$ & $\begin{array}{l}13.813 \\
13.810\end{array}$ \\
\hline $08: 50: 58$ & 2481.661 & -0.011 & 0.147 & 0.596 & 6.204 & 13.806 \\
\hline $08: 51: 13$ & 2480.977 & -0.012 & 0.156 & 0.677 & 6.244 & 13.803 \\
\hline $08: 51: 28$ & 2481.026 & -0.009 & 0.166 & 0.733 & 6.172 & 13.799 \\
\hline $08: 51: 43$ & 2478.730 & -0.009 & 0.175 & 0.775 & 6.145 & 13.797 \\
\hline $\begin{array}{l}08: 51: 58 \\
08: 52: 13\end{array}$ & $\begin{array}{l}2478.388 \\
2478.535\end{array}$ & $\begin{array}{l}-0.008 \\
-0.013 \\
\end{array}$ & $\begin{array}{r}0.185 \\
-1.486\end{array}$ & $\begin{array}{l}0.781 \\
0.853\end{array}$ & $\begin{array}{l}6.150 \\
6.167\end{array}$ & $\begin{array}{l}13.795 \\
13.736\end{array}$ \\
\hline $08: 52: 28$ & 2477.070 & -0.003 & 0.206 & 0.872 & 6.662 & 13.790 \\
\hline $08: 52: 43$ & 2478.486 & -0.006 & 0.216 & 0.964 & 6.598 & 13.785 \\
\hline $08: 52: 58$ & 2476.532 & -0.003 & 0.227 & 0.990 & 6.572 & 13.782 \\
\hline $08: 53: 13$ & 2475.897 & 0.022 & 0.229 & 3.754 & 6.309 & 13.318 \\
\hline $08: 53: 28$ & 1136.264 & 0.106 & 0.208 & 94.382 & 0.766 & 1.783 \\
\hline $08: 53: 43$ & 181.294 & -0.017 & 0.133 & 120.651 & 0.004 & -0.098 \\
\hline 08:53:58 & 134.554 & -0.025 & 0.129 & 121.218 & -0.006 & -0.114 \\
\hline $\begin{array}{l}08: 54: 13 \\
08: 54: 28\end{array}$ & $\begin{array}{l}133.333 \\
133.089\end{array}$ & $\begin{array}{l}-0.034 \\
-0.036\end{array}$ & $\begin{array}{l}0.128 \\
0.126\end{array}$ & $\begin{array}{l}121.696 \\
122.631 \\
\end{array}$ & $\begin{array}{l}-0.038 \\
-0.054\end{array}$ & $\begin{array}{l}-0.121 \\
-0.122\end{array}$ \\
\hline $08: 54: 43$ & 133.284 & -0.024 & 0.130 & 122.348 & -0.023 & -0.121 \\
\hline $08: 54: 58$ & 133.138 & -0.014 & 0.130 & 121.371 & -0.001 & -0.090 \\
\hline $08: 55: 13$ & 133.333 & -0.015 & 0.134 & 121.100 & 0.002 & -0.033 \\
\hline $08: 55: 28$ & 133.382 & -0.002 & 0.138 & 121.035 & 0.005 & 0.007 \\
\hline $08: 55: 43$ & 200.977 & 1.428 & 1.554 & 120.984 & 0.013 & 0.035 \\
\hline
\end{tabular}

QA/QC JM

Date $6 \longdiv { 1 4 1 0 7 }$
Consol Energy

CleanAir Project No. 10192

Dresden, New York

BH Outlet, AH Outlet

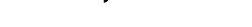


Consol Energy

CleanAir Project No. 10192

Dresden, New York

BH Outlet, AH Outlet

\begin{tabular}{|c|c|c|c|c|}
\hline \multirow{4}{*}{\multicolumn{2}{|c|}{$\begin{array}{r}\text { Date: } \\
\text { Start Time } \\
\text { Stop Time } \\
\text { CALIBRATION ERROR }\end{array}$}} & \multicolumn{3}{|c|}{ March 29, 2007} \\
\hline & & $8: 39$ & & \\
\hline & & $9: 01$ & & \\
\hline & & & & \\
\hline Channel 4 & Channel 5 & Channel 7 & Channel 8 & Channel 9 \\
\hline 02 & $\mathrm{CO} 2$ & so2 & 02 & $\mathrm{CO} 2$ \\
\hline $\begin{array}{c}\text { AH Outlet } \\
\% d v\end{array}$ & $\begin{array}{r}\text { AH Outlet } \\
\% d v\end{array}$ & $\begin{array}{c}\text { BH Outlet } \\
\text { ppmdv }\end{array}$ & $\begin{array}{r}\text { BH Outlet } \\
\% d v\end{array}$ & $\begin{array}{r}\text { BH Outlet } \\
\% d v\end{array}$ \\
\hline 7.373 & 11.410 & 115.034 & 0.105 & 0.247 \\
\hline 7.376 & 11.389 & 61.164 & 0.017 & -0.097 \\
\hline 7.379 & 11.363 & 49.091 & -0.002 & -0.130 \\
\hline 7.371 & 11.338 & 48.420 & -0.006 & -0.129 \\
\hline 7.376 & 11.313 & 48.378 & -0.004 & -0.130 \\
\hline 7.379 & 11.286 & 48.449 & -0.001 & -0.131 \\
\hline 7.381 & 11.264 & 48.449 & 0.003 & -0.128 \\
\hline
\end{tabular}

$\begin{array}{ll}08: 59: 51 & 1353.553 \\ 09: 00: 06 & 1353.553 \\ 09: 00: 21 & 1353.651 \\ 09: 00: 36 & 1353.651 \\ 09: 00: 51 & 1353.553 \\ 09: 01: 06 & 1353.553 \\ 09: 01: 21 & 1353.602\end{array}$

$\begin{array}{r}\text { Channel } 2 \\ \text { SO2 }\end{array}$
AH Outlet
ppmdv

1353.553
1353.553
1353.651
1353.651
1353.553
1353.553
1353.602

7.381

$-0.128$ 


\section{Certificate of Analysis: EPA Protocol Gas Mixture}

Cylinder Number: SG9134098BAL

Cylinder Pressure: 2000.6 PSIG

Certification Date: $9 / 19 / 2006$

\section{Certified Concentrations}

\section{Component}

OXYGEN

CARBON DIOXIDE

NITROGEN
Reference Number: $32-112503706-4$

Expiration Date: $\quad$ 9/19/2009

Laboratory:
MIC - Royal Oak - MI

\section{Concentration}

$6.040 \%$

$13.98 \%$

Balance

\section{Accuracy}

$+1-1 \%$

$+1 / .1 \%$

Airgas Great Lakes, Inc. 2009 Bellaire Ave. Royal Oak, Ml 48067 Ph: (248) 399-9150 Fax: (248) $584-2540$ http://www.airgas.com

Certification performed in accordance with "EPA Traceability Protocol (Sept. 1997)" using the assay procedures listed. Analytical Methodology does not require correction for analytical interferences.

Notes: CREIGHTON

Do not use cylinder below 150 psig.

Analytical Principle

Paramagnetic (Para)

Nondispersive Infrared (NDIR)
Procedure

G1

G1

\section{Reference Standard Information}

Type

NTRiv 82745

NTRM 82658x

Analytical Results

\section{Ist Component}

1st Analysis Date:

R $3.51 \quad S 3.02$

S 3.02

$\mathrm{Z} 0.00$

$\mathrm{Z} 0.00$

$\begin{array}{ll}\text { Balance Gas } & \text { Component } \\ \text { NITROGEN } & \text { CARBON DIOXIDE } \\ \text { NITROGEN } & \text { OXYGEN }\end{array}$

NITROGEN

\section{OXYGEN}

$09 / 19 / 2006$

Z 0.00

R 3.51

S 3.02

Conc $6.040 \%$
Conc $6.040 \%$
Approval Signature

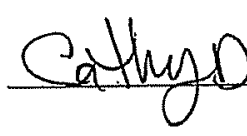

$\begin{array}{ll}\text { Cyl.Number } & \text { Concentration } \\ \text { SG9183197BAL } & 15.862 \% \\ \text { SG9160230BAL } & 7.015 \%\end{array}$

2nd Component

CARBON DIOXIDE

1st Analysis Date:

R $7.93 \quad S 6.99$

$S 6.99 \quad Z 0.00$

$\mathrm{Z} 0.00$

R 7.93
09/19/2006

Z $0.00 \quad$ Conc $13.98 \%$

R 7.93 Conc $13.98 \%$

S 6.99 Conc $13.98 \%$

AVG: $13.98 \%$ 


\section{Certificate of Analysis: EPA Protocol Gas Mixture}

Cylinder Number: XC012963B Reference Number: 32-112503706-3

Cylinder Pressure: 2000.6 PSIG Expiration Date: 9/19/2009

Certification Date: 9/19/2006 Laboratory: MIC-Royal Oak - MI
Airgas Great Lakes, Inc. 2009 Bellaire Ave. Royal Oak, MI 48067 Ph: (248) 399-9150 Fax: (248) 584-2540 http://www.airgas.com

Certified Concentrations

\begin{tabular}{lllll} 
Component & Concentration & Accuracy & Analytical Principle & Procedure \\
CARBON DIOXIDE & $6.020 \%$ & $+1-1 \%$ & Nondispersive Infrared (NDIR) & G1 \\
OXYGEN & $14.10 \%$ & $+1.1 \%$ & Paramagnetic (Para) & G1 \\
NITROGEN & Balance & & & \\
\hline
\end{tabular}

Certification performed in accordance with "EPA Traceability Protocol (Sept. 1997)" using the assay procedures listed. Analytical Methodology does not require correction for analytical interferences.

Notes: CREIGHTON

Do not use cylinder below 150 psig.

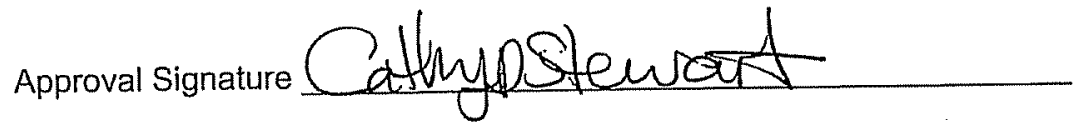

\section{Reference Standard Information}

$\begin{array}{lllll}\text { Type } & \text { Balance Gas } & \text { Component } & \text { Cyl.Number } & \text { Concentration } \\ \text { NTRM 81674 } & \text { NITROGEN } & \text { CARBON DIOXIDE } & \text { XC018562B } & 6.89 \% \\ \text { NTRM 82659X } & \text { NITROGEN } & \text { OXYGEN } & \text { XC024399B } & 22.60 \%\end{array}$

Analytical Results 1st Component

1st Analysis Date:

$\begin{array}{ll}R 6.89 & \text { S } 6.02 \\ \text { S } 6.02 & \text { Z } 0.00 \\ \text { Z } 0.00 & \text { R } 6.89\end{array}$

\section{CARBON DIOXIDE}

09/19/2006

Z $0.00 \quad$ Conc $6.020 \%$

R 6.89 Conc $6.020 \%$

S6.02. Conc $6.020 \%$

\section{2nd Component}

1st Analysis Date:

R 4.52

S 2.82

$\mathrm{Z} 0.00$
$S 2.82$

$Z 0.00$

R 4.52
OXYGEN

09/19/2006

Z $0.00 \quad$ Conc $14.10 \%$

R 4.52 Conc $14.10 \%$

S 2.82 Conc $14.10 \%$

AVG: $14.10 \%$ 


\section{Certificate of Analysis: EPA Protocol Gas Mixture}

Airgas Great Lakes, Inc. 2009 Bellaire Ave.

Royal Oak, Ml 48067

Ph: (248) 399-9150

Cylinder Number: CC13960 Reference Number: 32-112503706-6

Fax: (248) 584-2540

Cylinder Pressure: 2000.6 PSIG Expiration Date: 9/25/2008

Certification Date: 9/25/2006 Laboratory: MIC - Royal Oak - MI

http://www.airgas.com

\section{Certified Concentrations}

\begin{tabular}{|lllll|}
\hline Component & Concentration & Accuracy & Analytical Principle & Procedure \\
SULFUR DIOXIDE & $49.35 \mathrm{PPM}$ & $+1-1 \%$ & Nondispersive Ultraviolet (NDUV) & G1 \\
CARBON MONOXIDE & $123.83 \mathrm{PPM}$ & $+1-1 \%$ & Nondispersive Infrared (NDIR) & G1 \\
NITRIC OXIDE & $126.49 \mathrm{PPM}$ & $+1-1 \%$ & Chemiluminescence (Chemi) & G1 \\
NITROGEN & Balance & & & \\
\hline
\end{tabular}

Total oxides of nitrogen

126.9 PPM

Certification performed in accordance with "EPA Traceability Protocol (Sept. 1997)" using the assay procedures listed. Analytical Methodology does not require correction for analytical interferences.

\section{Notes:CREIGHTON}

Do not use cylinder below 150 psig.

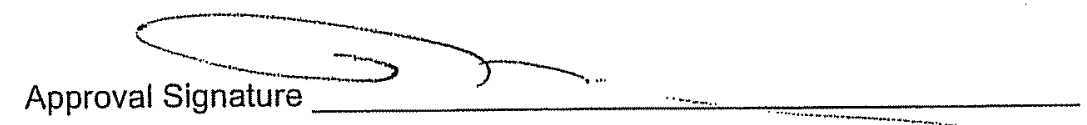

\section{Reference Standard Information}

Type
NTRM 82636
NTRM $81661 X$
NTRM 81694
NTRM 81685
ytical Results
omponent

\section{Analytical Results}

1st Analysis Date:

R $98.5 \quad S 19.7$

$S 19.7 \quad Z 0.0$

$Z 0.0 \quad R 98.5$

2nd Analysis Date:

R 97.541 S 49.227

$S 49.267 \quad Z-0.123$

Z-0.119 R $\$ 7.669$

Balance Gas

Component

CARBON MONOXIDE

SULFUR DIOXIDE

NITROGEN

NITROGEN

SULFUR DIOXIDE

NITRIC OXIDE

\section{SULFUR DIOXIDE}

$09 / 73 / 2006$

Z 0.0 Conc 49.2 PPM

R 98.5 Conc 49.2 PPM

S 19.7 Conc 49.2 PPM

AVG: 49.2 PPM

09/25/2006

Z-0.123 Conc 49.57 PPM

R 97.757 Conc 49.45 PPM

S 49.273 Conc $49.50 \mathrm{PPM}$

,AVG: 49.50 PPM

3rd Component

1st Analysis Date:

$\begin{array}{ll}\text { R } 4.89 & S 2.52 \\ S 2.52 & \text { Z } 0.0 \\ \text { Z } 0.0 & \text { R } 4.89\end{array}$

2nd Analysis Date:

R 249.12

$S 129.58$

S 129.62

$\mathrm{Z} 0.381$

Z 0.468

R 249.28

\section{NITPIC OXIOE}

09/18/2006

$\begin{array}{ll}\text { Z0.0 } & \text { Conc 126.1 PPM } \\ \text { R } 4.89 & \text { Conc 126.1 PPM } \\ \text { S } 2.52 & \text { Conc 126.1 PPM } \\ & \text { AVG: 126.1 PPM }\end{array}$

$09 / 25 / 2006$

$\mathrm{Z} 0.443$

R 249.89

S 129.58

\section{Cyl. Number}

XC014180B

$\mathrm{XC006260B}$

$X C 0192828$

XC024156B

2nd Component

1st Aralysis Date:

$R 4.93 \quad S 2.48$

$S 2.48 \quad Z 0.0$

$\mathrm{Z} 0.0 \quad \mathrm{R} 4.93$

2nd Analysis Date:

R 248.08 S 124.52

$S 124.76$

Z -0.124

$\mathrm{Z}-0.204$

R 247.63
Concentration
246.2 PPM
246.1 PPM
98.0 PPM
244.6 PPM

CARBON MONOXIDE

$09 / 18 / 2006$

Z $0.0 \quad$ Conc 123.8 PPM

R4.93 Conc 123.8 PPM

S 2.48 Conc 123.8 PPM

AVG: 123.8 PPM

09/25/2006

$Z-0.032$ Conc 123.59 PPM

R 247.98 Conc 123.92 PPM

S 124.71 Conc 124.09 PPM

AVG: 123.86 PPM
Conc 127.02 PPM

Conc 125.70 PPM

Conc 126.93 PPM

AVG: 126.88 PPM 


\section{CERTIFICATE OF ANALYSIS Grade of Product: EPA Protocol}

Airgas Great Lakes, Inc. 2009 Bellaire Ave. Royal Dak, MI 48067 Ph: (248) 399-9150 Fax: (248) $584-2540$. http://www.airgas.com

Customer: CREIGHTON

Part Number:

E02NI99E15A0914

Reference Number: $32-112534012-2$

Cylinder Number: CC30072

Laboratory: $\quad$ MIC - Royal Oak - MI

Cylinder Volume: 144 Cu.Ft.

Analysis Date: Feb 19, 2007

Cylinder Pressure: 2015 PSIG

Valve Outlet: . 660

Expiration Date: Feb 19, 2009

\begin{tabular}{|c|c|c|c|c|}
\hline \multicolumn{5}{|c|}{ ANALYTICAL RESULTS } \\
\hline Component & $\begin{array}{l}\text { Requested } \\
\text { Concentration }\end{array}$ & $\begin{array}{l}\text { Actual } \\
\text { Concentration }\end{array}$ & $\begin{array}{l}\text { Protocol } \\
\text { Method }\end{array}$ & $\begin{array}{l}\text { Total Relative } \\
\text { Uncertainity }\end{array}$ \\
\hline
\end{tabular}

\begin{tabular}{|c|c|c|c|c|}
\hline \multicolumn{5}{|c|}{ CALIBRATION STANDARDS } \\
\hline NTRM & 98061120 & $\mathrm{XC006250B}$ & $\begin{array}{l}\text { 246.1PPM SULFUR DIOXIDE/NITROGEN } \\
\text { NALYTICAL. EQUIPMIENT }\end{array}$ & Jun 01, 2007. \\
\hline Instru & t/Make/Mc & & Analytical Principle & Last Multipoint Calibration \\
\hline $\mathrm{E} / \mathrm{N} 50$ & ppmFS SC & $721-\mathrm{M}$ & Nondispersive Ultraviolet (NDUV) & $\operatorname{Jan} 29,2007$ \\
\hline
\end{tabular}

Triad Data Available Upon Request

Notes:

AFM

QA Approval 


\section{Scott Specialty Gases}

RATA CLASS

1290 COMBERMERE STREET,TROY,MI 48083

Dual-Analyzed Calibration Standard

Phone: 248-589-2950

Fax: $248-589-213$

\section{CERTIFICATE OF ACCURACY: EPA Protocol Gas}

\section{Assay Laboratory}

SCOTT SPECIALTY GASES

1290 COMBERMERE STREET

TROY,MI 48083
P.O. No.: 54830-71-65000

Project No.: 05-41817-014
Customer

CLEAN AIR INSTRUMENT RENTAL

GARY ZAPEL

500 WEST WOOD STREET

PALATINE IL 60067

\section{ANALYTICAL INFORMATION}

This certification was performed according to EPA Traceability Protocol For Assay \& Certification of Gaseous Calibration Standards; Procedure G-1; September, 1997.
Cylinder Number:
ALM012777
Certification Date:
04Apr 2006
Exp. Date: 03Apr2009

Cylinder Pressure ${ }^{* * *}$ : 1884 PSIG

\section{COMPONENT}

SULFUR DIOXIDE *

NITROGEN

\begin{tabular}{l} 
CERTIFIED CONCENTRATION (Moles) \\
\hline $2,479 \quad$ PPM \\
BALANCE
\end{tabular}

\section{ANALYTICAL}

\section{ACCURACY* *} $+1-1 \%$
TRACEABILITY

Direct NIST and NMi

** Do not use when cylinder pressure is below 150 psig.

* Analytical accuracy is based on the requirements of EPA Protocol Procedure G1, September 1997.

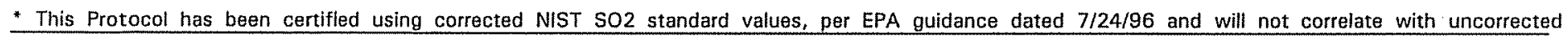
REFERENCE STANDARD

$\frac{\text { TYPE/SRM NO. }}{\text { NTRM } 1664} \quad \frac{\text { EXPIRATION DATE }}{19 \text { Apr2007 }} \frac{\text { CYLINDER NUMBER }}{\text { ALMO43727 }} \quad \frac{\text { CONCENTRATION }}{2402 . \text { PPM }} \quad \frac{\text { COMPONENT }}{\text { SULFUR DIOXIDE }}$

\section{INSTRUMENTATION}

INSTRUMENT/MODEL/SERIAL\#

FTIR//0928621
DATE LAST CALIBRATED

$16 \mathrm{Mar} 2006$
ANALYTICAL PRINCIPLE FTIR

\section{ANALYZER READINGS}

$$
\text { ( } Z=\text { Zero Gas } R=\text { Reference Gas } \quad T=\text { Test Gas } r=\text { Correlation Coefficient) }
$$

First Triad Analysis

Second Triad Analysis

Calibration Curve

\section{SULFUR DIOXIDE *}

\begin{tabular}{lcc|}
\hline Date: 28Mar2006 & Response Unit:PPM \\
$\mathrm{Z} 1=0.47230$ & $\mathrm{R} 1=2400.719$ & $\mathrm{~T} 1=2477.829$ \\
$\mathrm{R} 2=2401.982$ & $\mathrm{Z} 2=1.43331$ & $\mathrm{~T} 2=2477.837$ \\
$\mathrm{Z}=1.48831$ & $\mathrm{~T} 3=2481.002$ & $\mathrm{R} 3=2402.695$ \\
Avg. Concentration: & 2479. & $\mathrm{PPM}$ \\
\hline
\end{tabular}

\begin{tabular}{|lcc|}
\hline Date: 04Apr2006 & \multicolumn{2}{c|}{ Response Unit: PPM } \\
$Z 1=0.25354$ & $\mathrm{R} 1=2400.160$ & $\mathrm{~T} 1=2476.662$ \\
$\mathrm{R} 2=2401.880$ & $\mathrm{Z} 2=1.29722$ & $\mathrm{~T} 2=2477.193$ \\
$Z 3=1.74568$ & $\mathrm{~T} 3=2480.275$ & $\mathrm{R} 3=2402.152$ \\
Avg. Concentration: & 2479. & PPM \\
\hline
\end{tabular}

Concentration $=\mathrm{A}+\mathrm{BX}+\mathrm{C} \times 2+\mathrm{D} \times 3+\mathrm{E} \times 4$ $r=9.99984 \mathrm{E}-1$

Constants:

$B=1.03923 E+0$

$A=0.00000 E+0$

$\mathrm{C}=4.00000 \mathrm{E}-6$

$D=0.00000 E+0 \quad E=0.00000 E+0$ 


\section{CERTIFICATE OF ANALYSIS Grade of Product: EPA Protocol}

Airgas Great Lakes, inc. 2009 Bellairs Ave. Royal Dak, Ml 48067 Ph: (248) 399-9150 Fax: (248) 584-2540 http://www.airgas.com

Customer:

Part Number:

Cylinder Number:

Laboratory:

Analysis Date:

\section{AIRGAS CREIGHTION}

E04NI99E15A70B4

CC96201

MIC - Royal Oak - MI

Feb 19, 2007

$\begin{array}{ll}\text { Reference Number: } & 32-112534012-5 \\ \text { Cylinder Volume: } & 144 \text { Cu.Ft. } \\ \text { Cylinder Pressure: } & 2015 \text { PSIG } \\ \text { Valve Outlet: } & 660\end{array}$

Expiration Date: Feb 19, 2009

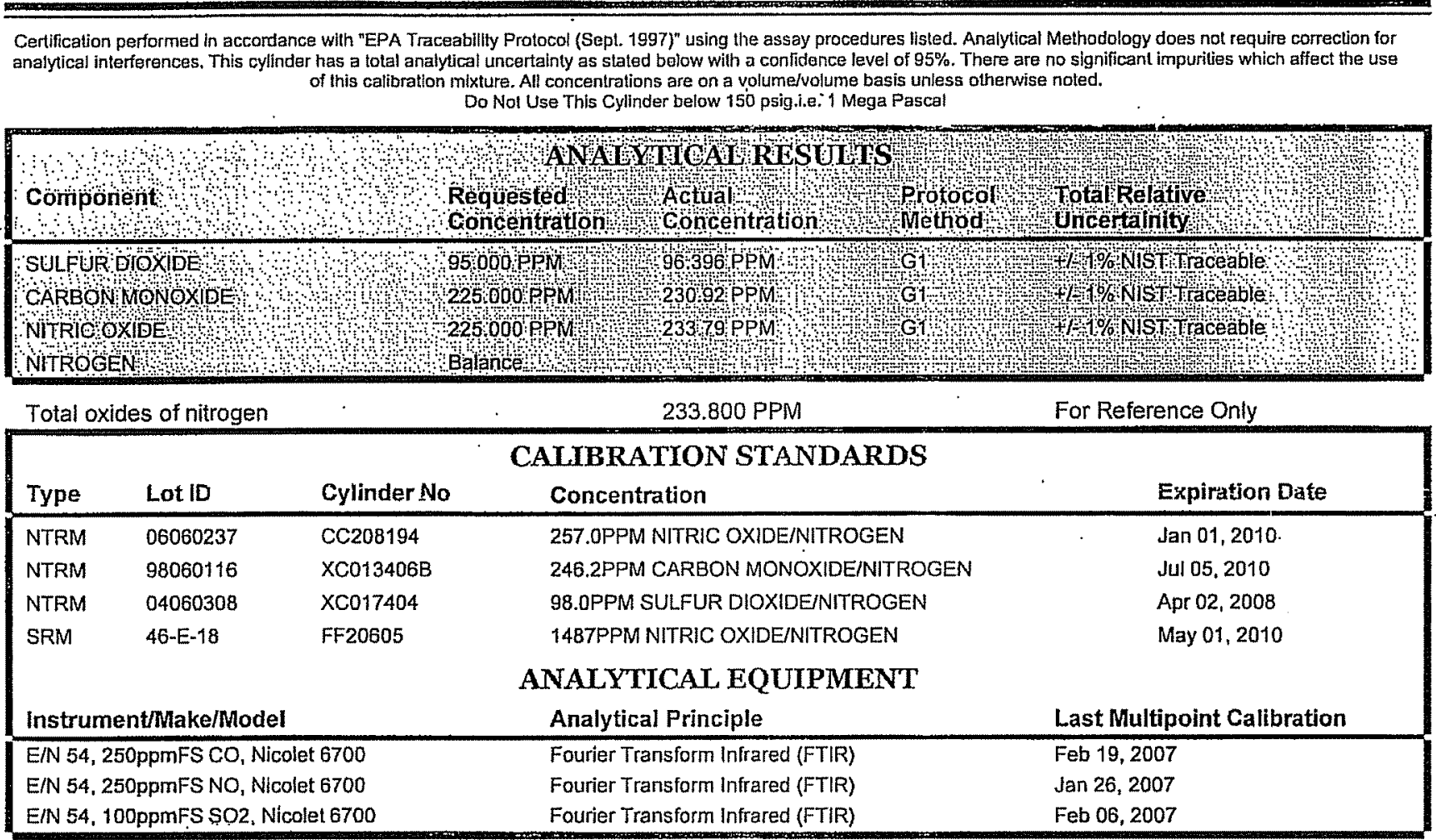

Triad Data Available Upon Request

Notes:NOX: 233.8

ORDER 108610

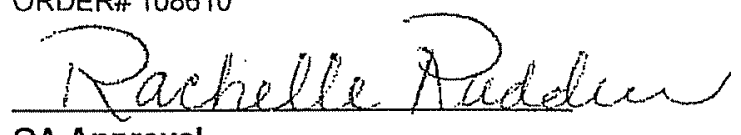

QA Approval 


\section{CERTIFICATE OF ANALYSIS Grade of Product: EPA Protocol}

Airgas Great Lakes, Inc. 2009 Bellaire Ave. Royal Oak, Ml 48067 Ph: (248) $399-9150$ Fax: (248) $584-2540$

Customer: CREIGHTON

Part Number: $\quad$ E02NI99E15A03D9

Cylinder Number: XC023749B

Laboratory: $\quad$ MIC - Royal Oak - MI

Analysis Date: Feb 05, 2007

http://www.airgas.com

\section{Expiration Date: Feb 05, 2009}
Reference Number: $32-112531795-4$
Cylinder Volume:
144 Cu.Ft.
Cylinder Pressure:
Valve Outlet:
2015 PSIG
660

\begin{tabular}{|c|c|c|c|c|c|c|}
\hline \multicolumn{7}{|c|}{$\begin{array}{l}\text { Certification performed in accordance with "EPA Traceability Protocol (Sept. 1997)" using the assay procedures listed. Analytical Methodology does not require correction fo } \\
\text { analytical interferences. This cylinider has a lotal analytical uncertainty as stated below with a confidence level of 95\%. There are no significant impurities which affect the us } \\
\text { of this calibralion mixture. All concentrations are on a volume/volume basis unless otherwise noted. } \\
\text { Do Not Use This.Cylinder below } 150 \text { psig. i.e. } 1 \text { Mega Pascal }\end{array}$} \\
\hline \multirow{2}{*}{\multicolumn{2}{|c|}{ Component }} & \multicolumn{4}{|c|}{ ANALYTTCAL RESULTS } & . \\
\hline & & & $\begin{array}{l}\text { Requested } \\
\text { Concentration }\end{array}$ & $\begin{array}{l}\text { Actual } \\
\text { Concentration }\end{array}$ & $\begin{array}{l}\text { Protocol } \\
\text { Method }\end{array}$ & $\begin{array}{l}\text { Total Relative } \\
\text { Uncerfainity }\end{array}$ \\
\hline \multicolumn{2}{|c|}{$\begin{array}{l}\text { NITRIC OXXIDE } \\
\text { NITROGEN }\end{array}$} & $\cdot$ & $\begin{array}{l}90.000 \text { PPM } \\
\text { Balance }\end{array}$ & $91.0 \mathrm{PPM}$ & G1 & $+/-1 \%$ NIST Traceable \\
\hline \multicolumn{3}{|c|}{ Total oxides of nitrogen } & \multicolumn{2}{|r|}{91.400 PPM } & . & For Reference Only \\
\hline \multicolumn{7}{|c|}{ CALIBRATION STANDARDS } \\
\hline \multicolumn{2}{|c|}{ NTRM 06060407} & CC208042 & \multicolumn{3}{|c|}{$\begin{array}{l}\text { 93.2PPM NITRIC OXIDEINITROGEN } \\
\text { ANALYTICAL EQUUTPMENT }\end{array}$} & $\begin{array}{l}\text { Jan } 01,2010 \\
\text { Last Multipoint Calibration }\end{array}$ \\
\hline \multicolumn{3}{|c|}{ EN 160, 100ppmFS NO, Beckman 951A } & \multicolumn{2}{|c|}{ Chemiluminescence (Chemi) } & & $\operatorname{Jan} 19,2007$ \\
\hline
\end{tabular}

Triad Data Available Upon Request Notes:

$$
\frac{A F P}{\text { QA Approval }}
$$




\section{Certificate of Analysis: EPA Protocol Gas Mixture}

Cylinder Number: SG9165466BAL Reference Number: 32-112441658-2

Cylinder Pressure: 2000.6 PSIG

Expiration Date: $\quad 12 / 5 / 2007$

Certification Date: 12/5/2005

Laboratory: $\quad$ MIC - Royal Oak - MI

Airgas Great Lakes, Inc. 2009 Bellaire Ave.

Royal Oak, Ml 48067

Ph: (248) 399-9150

Fax: (248) 584-2540

Certified Concentrations

\begin{tabular}{|llllll}
\hline Component & Concentration & Accuracy & Analytical Principle & Procedure \\
NITRIC OXIDE & 47.4 PPM & $+1-1 \%$ & Chemiluminescence (Chemi) & G1 \\
NITROGEN & Balance & & & \\
\hline
\end{tabular}

Total oxides of nitrogen

47.8 PPM

Certification performed in accordance with "EPA Traceability Protocol (Sept. 1997)" using the assay procedures listed. Analytical Methodology does not require correction for analytical interferences.

Notes: CLEVELAND

Do not use cylinder below 150 psig.

Approval Signature A.F. Wheneremel

\section{Reference Standard Information}

$\begin{array}{lllll}\text { Type } & \text { Balance Gas } & \text { Component } & \text { Cyl.Number } & \text { Concentration } \\ \text { GMIS 1684B } & \text { NITROGEN } & \text { NITRIC OXIDE } & \text { XN000401B } & 99.4 \text { PPM }\end{array}$

Analytical Results

1st Component NITRIC OXIDE

1st Analysis Date: $11 / 28 / 2005$

\begin{tabular}{|c|c|c|c|}
\hline R 4.97 & S 2.37 & $z 0.00$ & Conc 47.4 PPM \\
\hline S 2.37 & $z 0.00$ & R 4.97 & Conc 47.4 PPM \\
\hline$Z 0.00$ & R 4.97 & S 2.37 & $\begin{array}{l}\text { Conc } 47.4 \text { PPM } \\
\text { AVG: } 47.4 \text { PPM }\end{array}$ \\
\hline
\end{tabular}

2nd Analysis Date: $\quad 12 / 05 / 2005$

$\begin{array}{llll}R 4.97 & \mathrm{~S} 2.37 & \mathrm{Z} 0.0 & \text { Conc 47.4 PPM } \\ \mathrm{S} 2.37 & \mathrm{Z} 0.0 & \mathrm{R} 4.97 & \text { Conc 47.4 PPM } \\ \mathrm{Z} 0.0 & \mathrm{R} 4.97 & \mathrm{~S} 2.37 & \text { Conc 47.4 PPM }\end{array}$

AVG: 47.4 PPM 
This Page Intentionally Left Blank 
CONSOL ENERGY INC.

AES GREENIDGE STATION
Client Reference No: 4700140111

CleanAir Project No: 10192

FIELD DATA PRINTOUTS

E 
This Page Intentionally Left Blank

E - 2 


\section{CALIBRATION BIAS 00}

Start Time $\quad 9: 06$

Stop Time $\quad 9: 43$

March 29, 2007

Channel 2 Channel 4

Channel 5

Channel 7

Channel 8

Channel 9

$\mathrm{SO} 2$

02

$\mathrm{CO} 2$

$\mathrm{SO} 2$

$\mathrm{O} 2$

$\mathrm{CO} 2$

\section{AH Outlet}

ppmdv

AH Outlet

$\% d v$

AH Outlet

$\% d v$

\section{BH Outlet}

ppmdv

\section{BH Outlet}

$\% d v$
BH Outlet

\%dv

System Response to Calibration Gasses $\left(C_{S}\right)$

$\mathrm{C}_{\text {of }}$ Zero gas

$\mathrm{C}_{\text {uf }}$ Upscale gas

\begin{tabular}{|r|r|}
\hline 19.829 & -0.021 \\
\hline
\end{tabular}

Analyzer Calibration Error Reponses ( $C_{\text {Dir }}$ )

$\mathrm{C}_{\text {oce }}$ Zero gas
$\mathrm{C}_{\text {mce }}$ Upscale gas

3.452

6.006

|

2482.035

$-0.010$

6.061

0.178

13.997

1.423

45.648

0.013

6.049

0.280

Actual Upscale Gas Value $\left(\mathrm{C}_{\mathrm{MA}}\right)$

$C_{\text {ma Upscale gas }}$ Ualue (CS)
Calibration Span Valu

2479.000

6.040

\subsection{8}

$-0.056$

0.002

13.991

$\begin{array}{ccc}2479.000 & 14.100 & 13.980 \\ \text { of Calibration Span Value (SB) } & (\mathbf{5} \%)\end{array}$

System Bias as Percent of Calibration Span Value (SB) (5\%)

$\begin{array}{lllllll}\text { Zero gas } & 0.7 \% & -0.1 \% & 0.2 \% & 1.2 \% & 0.1 \% & 2.0 \% \\ \text { Upscale gas } & -2.0 \% & -0.4 \% & -0.4 \% & -2.5 \% & 0.0 \% & 0.2 \%\end{array}$

System Bias Status

\begin{tabular}{|c|c|c|c|c|c|c|}
\hline Zero gas & OK & OK & OK & OK & OK & OK \\
\hline Upscale gas & OK & OK & OK & OK & OK & OK \\
\hline \multicolumn{7}{|c|}{ vious System Response to Calibration Gases $\left(C_{\mathrm{s}}\right)$} \\
\hline Zero gas & N/A & $\mathrm{N} / \mathrm{A}$ & N/A & N/A & N/A & $N / A$ \\
\hline Upscale gas & N/A & $N / A$ & N/A & N/A & N/A & $N / A$ \\
\hline
\end{tabular}

Drift Assessment as Percent of Calibration Span Value (D) (3\%)

$\begin{array}{cllllll}\begin{array}{l}\text { Zero gas } \\ \text { Upscale gas }\end{array} & \text { N/A } & \text { N/A } & \text { N/A } & \text { N/A } & \text { N/A } & \text { N/A } \\ \text { Nrift Assessment Status } & \text { N/A } & \text { N/A } & \text { N/A } & \text { N/A } & \text { N/A } & \text { N/A } \\ \text { Zero gas } & & & & & & \text { N/A } \\ \text { Upscale gas } & \text { N/A } & \text { N/A } & \text { N/A } & \text { N/A } & \text { N/A } & \text { N/A }\end{array}$

\begin{tabular}{|c|c|c|c|}
\hline $09: 06: 57$ & 1330.012 & 8.243 & 11.180 \\
\hline $09: 07: 12$ & 725.568 & 10.421 & 8.557 \\
\hline $09: 07: 27$ & 224.664 & 6.309 & 13.388 \\
\hline $09: 07: 42$ & 102.662 & 6.020 & 13.939 \\
\hline $09: 07: 57$ & 64.176 & 6.004 & 13.982 \\
\hline 09:08:12 & 46.154 & 6.008 & 13.999 \\
\hline 09:08:27 & 35.751 & 6.006 & 14.010 \\
\hline 09:08:42 & 29.206 & 6.004 & 13.998 \\
\hline 09:08:57 & 24.762 & 6.007 & 14.012 \\
\hline $09: 09: 12$ & 21.929 & 6.010 & 14.019 \\
\hline $09: 09: 27$ & 19.927 & 5.999 & 14.023 \\
\hline $09: 09: 42$ & 17.631 & 6.001 & 14.029 \\
\hline $09: 09: 57$ & 96.899 & 5.129 & 12.948 \\
\hline $09: 10: 12$ & 1457.436 & 0.515 & 2.165 \\
\hline $09: 10: 27$ & 2197.069 & -0.008 & 0.374 \\
\hline $09: 10: 42$ & 2336.752 & -0.026 & 0.267 \\
\hline $09: 10: 57$ & 2381.538 & -0.030 & 0.235 \\
\hline 09:11:12 & 2401.612 & -0.035 & 0.217 \\
\hline $09: 11: 27$ & 2412.210 & -0.035 & 0.204 \\
\hline
\end{tabular}

$\begin{array}{lll}48.840 & 0.013 & 0.234 \\ 48.840 & 0.017 & 0.260 \\ 48.840 & 0.015 & 0.281 \\ 48.840 & 0.016 & 0.299 \\ 48.840 & 0.018 & 0.311 \\ 48.840 & 0.023 & 0.325 \\ 48.840 & 0.022 & 0.336 \\ 48.850 & 0.019 & 0.344 \\ 48.879 & 0.026 & 0.351 \\ 48.889 & 0.029 & 0.359 \\ 48.856 & 0.031 & 0.366 \\ 48.889 & 0.032 & 0.369 \\ 48.886 & 0.032 & 0.369 \\ 48.886 & 0.036 & 0.370 \\ 48.889 & 0.041 & 0.372 \\ 48.889 & 0.037 & 0.373 \\ 48.889 & 0.041 & 0.373 \\ 48.889 & 0.042 & 0.370 \\ 48.889 & 0.047 & 0.369\end{array}$


Consol Energy

CleanAir Project No. 10192

Dresden, New York

BH Outlet, AH Outlet
March 29, 2007

Start Time $\quad 9: 06$

Stop Time $\quad 9: 43$

CALIBRATION BIAS 00

\begin{tabular}{|c|c|c|c|c|c|c|}
\hline & Channel 2 & Channel 4 & $\begin{array}{r}\text { Channel } 5 \\
\end{array}$ & Channel 7 & $\begin{array}{r}\text { Channel } 8 \\
02\end{array}$ & $\begin{array}{r}\text { Channel } 9 \\
\mathrm{CO} 2\end{array}$ \\
\hline & $\begin{array}{c}\text { AH Outlet } \\
\text { ppmdv }\end{array}$ & $\begin{array}{r}\text { AH Outlet } \\
\% d v\end{array}$ & $\begin{array}{r}\text { AH Outlet } \\
\% d v\end{array}$ & $\begin{array}{c}\text { BH Outlet } \\
\text { ppmdv }\end{array}$ & $\begin{array}{r}\text { BH Outlet } \\
\% \text { dv }\end{array}$ & $\begin{array}{r}\text { BH Outlet } \\
\% d v\end{array}$ \\
\hline $\begin{array}{l}09: 11: 42 \\
09 \cdot 11: 57\end{array}$ & $\begin{array}{l}2422.418 \\
2425.299\end{array}$ & $\begin{array}{l}-0.037 \\
-0.038\end{array}$ & $\begin{array}{l}0.194 \\
0.188\end{array}$ & $\begin{array}{l}48.892 \\
48.909\end{array}$ & $\begin{array}{l}0.052 \\
0.053\end{array}$ & $\begin{array}{l}0.369 \\
0.368\end{array}$ \\
\hline $\begin{array}{l}09: 11: 57 \\
09: 12: 12\end{array}$ & $\frac{2425.299}{2426.667}$ & $\begin{array}{l}-0.038 \\
-0.039\end{array}$ & $\frac{0.188}{0.182}$ & $\begin{array}{l}48.909 \\
48.931\end{array}$ & 0.055 & $\begin{array}{l}0.368 \\
0.369\end{array}$ \\
\hline $09: 12: 27$ & 2430.183 & -0.039 & 0.178 & 48.964 & 0.057 & 0.368 \\
\hline $09: 12: 42$ & 2441.074 & -0.039 & 0.174 & 48.967 & 0.057 & 0.364 \\
\hline $09: 35: 03$ & 1403.468 & 7.234 & 11.643 & 93.428 & 7.268 & 11.705 \\
\hline $09: 35: 18$ & 1404.884 & 7.253 & 11.639 & 80.248 & 12.165 & 7.800 \\
\hline $09: 35: 33$ & 1402.637 & 7.192 & 11.683 & 48.104 & 6.954 & 12.344 \\
\hline $09: 35: 48$ & 1409.035 & 7.177 & 11.707 & 24.840 & 6.100 & 13.877 \\
\hline $09: 36: 03$ & 1402.637 & 7.308 & 11.600 & 14.291 & 6.062 & 13.973 \\
\hline $09: 36: 18$ & 1393.016 & 7.357 & 11.558 & 9.296 & 6.057 & 13.996 \\
\hline $09: 36: 33$ & 1394.432 & 7.270 & 11.607 & 6.535 & 6.055 & 14.004 \\
\hline $09: 36: 48$ & 1403.468 & 7.245 & 11.674 & 4.884 & 6.053 & 14.007 \\
\hline $09: 37: 03$ & 1393.065 & 7.264 & 11.617 & 3.897 & 6.053 & 14.014 \\
\hline $09: 37: 18$ & 1400.147 & 7.206 & 11.684 & 3.184 & 6.054 & 14.017 \\
\hline $09: 37: 33$ & 1406.300 & 7.145 & 11.718 & 2.716 & 6.053 & 14.020 \\
\hline $09: 37: 48$ & 1413.236 & 7.182 & 11.722 & 2.354 & 6.053 & 14.024 \\
\hline $09: 38: 03$ & 1403.175 & 7.332 & 11.573 & 2.067 & 6.050 & 14.027 \\
\hline $09: 38: 18$ & 1404.054 & 7.241 & 11.646 & 1.872 & 6.049 & 14.028 \\
\hline $09: 38: 33$ & 1405.910 & 7.264 & 11.634 & 1.693 & 6.048 & 14.029 \\
\hline $09: 38: 48$ & 1407.863 & 7.243 & 11.650 & 1.563 & 6.051 & 14.031 \\
\hline 09:39:03 & 1402.979 & 7.396 & 11.516 & 1.455 & 6.049 & 14.038 \\
\hline 09:39:18 & 1397.265 & 7.317 & 11.571 & 1.250 & 4.972 & 12.747 \\
\hline $09: 39: 33$ & 1395.458 & 7.282 & 11.589 & 3.487 & 0.539 & 2.009 \\
\hline $09: 39: 48$ & 1402.393 & 7.183 & 11.677 & 19.878 & 0.052 & 0.193 \\
\hline $09: 40: 03$ & 1411.478 & 7.133 & 11.717 & 30.974 & 0.028 & 0.043 \\
\hline 09:40:18 & 1414.799 & 7.076 & 11.768 & 36.317 & 0.026 & 0.004 \\
\hline $09: 40: 33$ & 1408.547 & 7.246 & 11.646 & 39.108 & 0.022 & -0.018 \\
\hline $09: 40: 48$ & 1401.319 & 7.211 & 11.664 & 40.759 & 0.019 & -0.036 \\
\hline $09: 41: 03$ & 1396.532 & 7.323 & 11.579 & 41.833 & 0.019 & -0.047 \\
\hline $09: 41: 18$ & 1389.109 & 7.330 & 11.570 & 42.335 & 0.018 & -0.057 \\
\hline $09: 41: 33$ & 1388.913 & 7.277 & 11.612 & 43.054 & 0.015 & -0.063 \\
\hline $09: 41: 48$ & 1396.777 & 7.208 & 11.647 & 43.858 & 0.016 & -0.070 \\
\hline $09: 42: 03$ & 1403.272 & 7.314 & 11.606 & 44.444 & 0.014 & -0.076 \\
\hline $09: 42: 18$ & 1396.581 & 7.299 & 11.593 & 44.848 & 0.014 & -0.081 \\
\hline $09: 42: 33$ & 1404.347 & 7.309 & 11.590 & 45.180 & 0.014 & -0.085 \\
\hline $09: 42: 48$ & 1401.612 & 7.227 & 11.654 & 45.372 & 0.013 & -0.087 \\
\hline 09:43:03 & 1402.247 & 7.272 & 11.615 & 45.509 & 0.014 & -0.089 \\
\hline $09: 43: 18$ & 1408.645 & 7.170 & 11.695 & 45.623 & 0.014 & -0.094 \\
\hline $09: 43: 33$ & 1404.738 & 7.297 & 11.615 & 45.812 & 0.013 & -0.094 \\
\hline $09: 43: 48$ & 1397.021 & 7.294 & 11.615 & 54.268 & 2.266 & 2.149 \\
\hline
\end{tabular}

Prepared by Clean Air Engineering Proprietary Software SS CEM Version 06-2004a

Copyright $\odot 2004$ Clean Air Engineering Inc.
QA/QC JM

Date $\longdiv { 6 / 1 4 / 0 7 }$ 
Consol Energy

CleanAir Project No. 10192

Dresden, New York

BH Outlet, AH Outlet

March 29, 2007

Start Time $\quad 9: 59$

Stop time $\quad 11: 00$

REFERENCE METHOD RUN 1

Channel 2

$\mathrm{SO} 2$

AH Outlet

ppmdv

Calibration Checks

$\mathrm{C}_{\text {oi }}$ Initial zero

$\mathrm{C}_{\mathrm{ui}}$ Initial upscale

$\mathrm{C}_{\text {of }}$ Final zero

19.829
2432.641

13.789

$\mathrm{C}_{\text {uf }}$ Final upscale

2411.901

$\mathrm{C}_{\text {ma }}$ Actual gas value

2479.000

Analyzer Averages (concentrations)

$\mathrm{C}_{\text {Avg }}$ Average conc. $\quad 1384.546$

$\mathrm{C}_{\mathrm{Gas}}$ Bias adjusted

1409.550
Channel 4
02

AH Outlet

$\% d v$

$-0.021$

6.006

$-0.017$

6.124

6.040

7.331

7.297
Channel 5

$\mathrm{CO} 2$

AH Outlet

$\% d v$

0.178

13.997

0.154

14.012

13.980
Channel 7

$\mathrm{SO} 2$

BH Outlet

ppmdv

1.423

45.648

2.554

47.500

49.350

11.587

11.538
73.817

79.504
Channel 8

02

BH Outlet

$\% d v$

BH Outlet $\% d v$

Clock Time (at end of sample period)

\section{2}

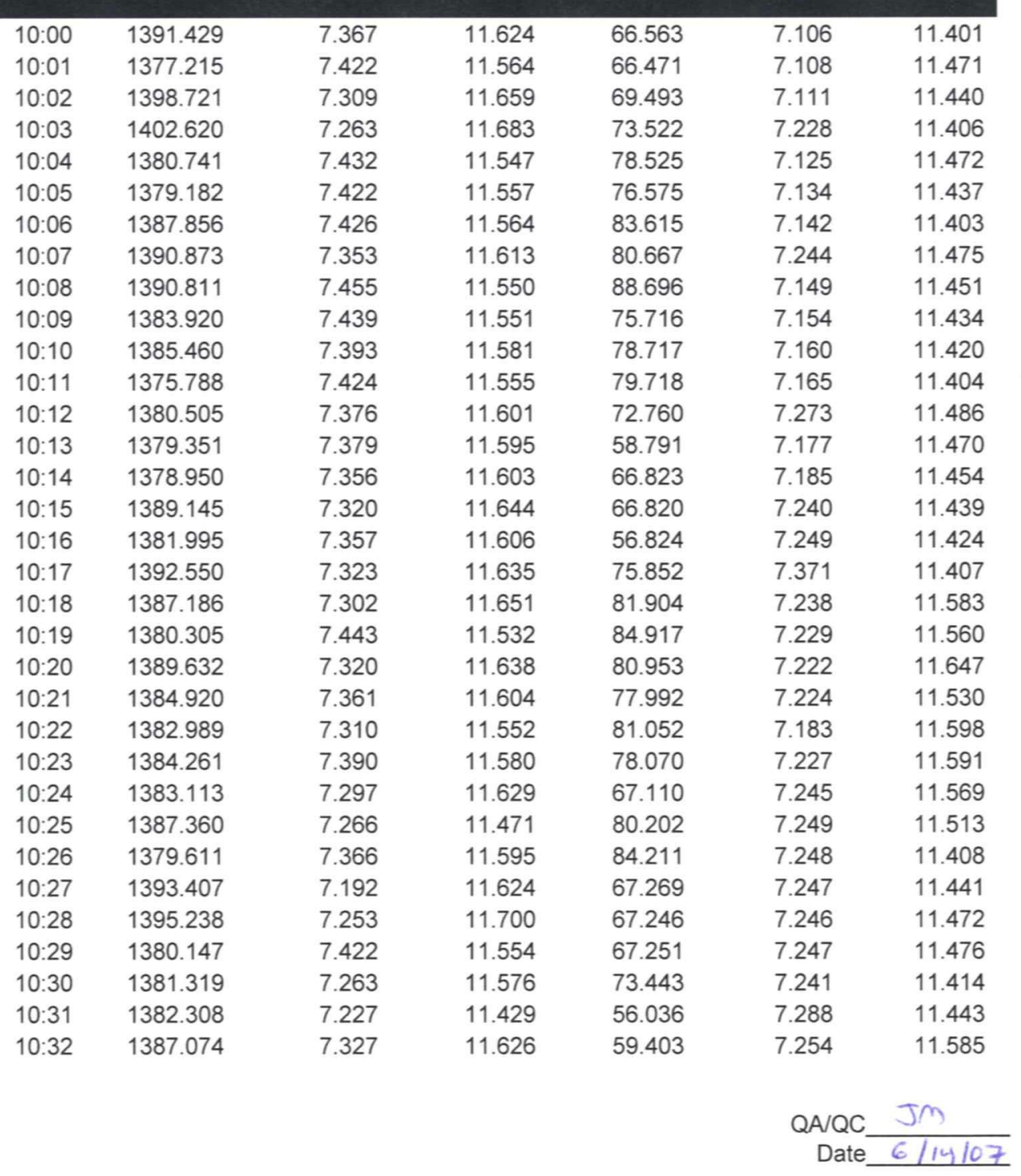


Consol Energy

CleanAir Project No. 10192

Dresden, New York

BH Outlet, AH Outlet

March 29, 2007

Start Time

Stop time

9:59

$11: 00$

REFERENCE METHOD RUN 1

$$
\text { Channel } 2
$$

$\mathrm{SO} 2$

AH Outlet

ppmdv

$10: 33$

$10: 34$

10:35

$10: 36$

$10: 37$

$10: 38$

$10: 39$

$10: 40$

$10: 41$

$10: 42$

$10: 43$

$10: 44$

$10: 45$

$10: 46$

$10: 47$

$10: 48$

$10: 49$

$10: 50$

$10: 51$

$10: 52$

$10: 53$

$10: 54$

$10: 55$

$10: 56$

10:57

10:58

$10: 59$

11:00

\subsection{7}

1390.598

1387.900

1381.966

1377.717

1380.195

1388.010

1392.002

1381.795

1380.147

1390.794

1384.392

1380.916

1389.930

1371.441

1389.292

1390.708

1384.032

1375.141

1384.777

1372.308

1378.928

1386.602

1375.299

1387.881

1387.509

1381.758

1370.473

$$
\text { Channel } 4
$$

$\mathrm{O} 2$

\section{AH Outlet}

$$
\% d v
$$

7.305

7.336

7.212

7.351

7.428

7.406

7.269

7.141

7.407

7.348

7.259

7.349

7.346

7.056

7.327

7.320

7.302

7.340

7.384

7.361

7.459

7.303

7.297

7.409

7.303

7.208

6.949

7.432
Channel 5

\section{AH Outlet}

$\% d v$

11.643

11.630

11.503

11.576

11.547

11.562

11.667

11.622

11.567

11.611

11.692

11.614

11.613

11.592

11.462

11.650

11.653

11.616

11.571

11.610

11.530

11.644

11.639

11.555

11.644

11.560

11.179

11.553

\section{Channel 7 $\mathrm{SO} 2$ \\ BH Outlet ppmdv}

58.366

62.291

68.523

73.514

73.552

69.655

73.757

75.923

77.497

82.058

82.354

78.069

34.178

81.915

86.468

89.452

74.138

96.678

92.073

84.916

76.514

64.884

91.870

77.200

83.634

58.589

53.180
Channel 8

\section{BH Outlet}

$\% d v$

7.246

7.128

7.241

7.149

7.294

7.224

7.201

7.234

7.273

7.091

7.180

7.208

7.215

7.281

7.252

7.266

7.284

7.321

7.267

7.295

7.273

7.284

7.256

7.285

7.269

7.277

7.186

7.237
Channel 9 $\mathrm{CO} 2$

BH Outlet $\% d v$

11.465

11.441

11.430

11.495

11.540

11.536

11.571

11.592

11.630

11.683

11.644

11.563

11.671

11.769

11.555

11.661

11.655

11.625

11.593

11.660

11.590

11.564

11.673

11.600

11.620

11.671

11.550

11.568 
Consol Energy

CleanAir Project No. 10192

Dresden, New York

BH Outlet, AH Outlet

March 29, 2007

Start Time $\quad 11: 10$

Stop Time $\quad 11: 22$

CALIBRATION BIAS 01

\begin{tabular}{|c|c|c|c|c|c|}
\hline Channel 2 & Channel 4 & Channel 5 & Channel 7 & Channel 8 & Channel 9 \\
\hline $\mathrm{SO} 2$ & $\mathrm{O} 2$ & $\mathrm{CO} 2$ & SO2 & $\mathrm{O} 2$ & $\mathrm{CO} 2$ \\
\hline $\begin{array}{l}\text { Outlet } \\
\text { ppmdv }\end{array}$ & $\begin{array}{r}\text { AH Outlet } \\
\% d v\end{array}$ & $\begin{array}{r}\text { AH Outlet } \\
\% d v\end{array}$ & $\begin{array}{l}\text { BH Outlet } \\
\text { ppmdv }\end{array}$ & $\begin{array}{r}\text { BH Outlet } \\
\% d v\end{array}$ & $\begin{array}{r}\text { BH Outlet } \\
\% d v\end{array}$ \\
\hline
\end{tabular}

System Response to Calibration Gasses $\left(\mathbf{C}_{\mathrm{S}}\right)$

$\mathrm{C}_{\text {of }}$ Zero gas

$\mathrm{C}_{\text {uf }}$ Upscale gas

\begin{tabular}{|r|r|}
\hline 13.789 & -0.01 \\
\hline
\end{tabular}

Analyzer Calibration Error Reponses $\left(\mathrm{C}_{\text {Dir }}\right)$

$\begin{array}{lrrrrrr}C_{\text {oce }} \text { Zero gas } & 3.452 & -0.010 & 0.148 & -0.056 & 0.002 & 0.003 \\ C_{\text {mce }} \text { Upscale gas } & 2482.035 & 6.061 & 14.059 & 48.630 & 6.050 & 13.966\end{array}$

Actual Upscale Gas Value ( $\left.C_{\text {MA }}\right)$

$\begin{array}{lllllll}\mathrm{C}_{\text {ma }} \text { Upscale gas } & 2479.000 & 6.040 & 13.980 & 49.350 & 6.040 & 13.980\end{array}$

Calibration Span Value (CS)

$\begin{array}{llllll}2479.000 & 14.100 & 13.980 & 121.600 & 14.100 & 13.980\end{array}$

System Bias as Percent of Calibration Span Value (SB) (5\%)

\begin{tabular}{|c|c|c|c|c|c|}
\hline Zero gas & $0.4 \%$ & $-0.1 \%$ & $0.0 \%$ & $2.1 \%$ & $0.0 \%$ \\
\hline Upscale gas & $-2.8 \%$ & $0.4 \%$ & $-0.3 \%$ & $-0.9 \%$ & $0.2 \%$ \\
\hline
\end{tabular}

System Bias Status

$\begin{array}{lllllll}\text { Zerogas } & \text { OK } & \text { OK } & \text { OK } & \text { OK } & \text { OK } & \text { OK } \\ \text { Upscale gas } & \text { OK } & \text { OK } & \text { OK } & \text { OK } & \text { OK } & \text { OK }\end{array}$

Previous System Response to Calibration Gases $\left(\mathbf{C}_{\mathbf{s}}\right)$

$\begin{array}{lllllll}\mathrm{C}_{\text {oi }} \quad \text { Zero gas } & 19.829 & -0.021 & 0.178 & 1.423 & 0.013 & 0.280\end{array}$

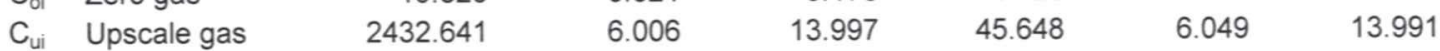

Drift Assessment as Percent of Calibration Span Value (D) (3\%)

$\begin{array}{lllllll}\text { Zero gas } & -0.2 \% & 0.0 \% & -0.2 \% & 0.9 \% & 0.0 \% & -2.0 \% \\ \text { Upscale gas } & -0.8 \% & 0.8 \% & 0.1 \% & 1.5 \% & 0.2 \% & 0.1 \%\end{array}$

Drift Assessment Status

$\begin{array}{lllllll}\text { Zero gas } & \text { OK } & \text { OK } & \text { OK } & \text { OK } & \text { OK } & \text { OK } \\ \text { Upscale gas } & \text { OK } & \text { OK } & \text { OK } & \text { OK } & \text { OK } & \text { OK }\end{array}$

061407143432

\begin{tabular}{|c|c|c|c|c|c|c|}
\hline $11: 10: 53$ & 1316.923 & 9.283 & 10.790 & 42.188 & 8.295 & 11.460 \\
\hline 11:11:08 & 630.965 & 12.004 & 7.344 & 38.235 & 11.212 & 8.182 \\
\hline $11: 11: 23$ & 195.897 & 6.328 & 13.493 & 23.043 & 6.359 & 13.514 \\
\hline 11:11:38 & 90.501 & 6.031 & 13.983 & 14.323 & 6.101 & 13.974 \\
\hline 11:11:53 & 57.240 & 6.012 & 14.021 & 10.123 & 6.087 & 14.013 \\
\hline $11: 12: 08$ & 42.002 & 6.007 & 14.032 & 7.645 & 6.079 & 14.026 \\
\hline $11: 12: 23$ & 33.602 & 6.005 & 14.040 & 6.128 & 6.078 & 14.034 \\
\hline $11: 12: 38$ & 28.425 & 6.000 & 14.044 & 5.105 & 6.076 & 14.042 \\
\hline $11: 12: 53$ & 25.055 & 5.994 & 14.043 & 4.389 & 6.072 & 14.047 \\
\hline $11: 13: 08$ & 21.831 & 5.993 & 14.045 & 3.881 & 6.072 & 14.050 \\
\hline $11: 13: 23$ & 20.318 & 5.986 & 14.045 & 3.533 & 6.071 & 14.054 \\
\hline $11: 13: 38$ & 19.585 & 5.979 & 14.037 & 3.259 & 6.071 & 14.054 \\
\hline $11: 13: 53$ & 18.462 & 5.977 & 14.031 & 3.064 & 6.073 & 14.054 \\
\hline $11: 14: 08$ & 16.606 & 5.978 & 14.039 & 2.933 & 6.070 & 14.054 \\
\hline $11: 14: 23$ & 15.873 & 5.981 & 14.042 & 2.787 & 6.074 & 14.056 \\
\hline $11: 14: 38$ & 14.896 & 5.982 & 14.051 & 2.706 & 6.078 & 14.056 \\
\hline $11: 14: 53$ & 13.919 & 5.987 & 14.052 & 2.631 & 6.080 & 14.057 \\
\hline $11: 15: 08$ & 13.577 & 5.945 & 13.770 & 2.354 & 6.004 & 13.996 \\
\hline $11: 15: 23$ & 13.870 & 5.783 & 13.797 & 2.677 & 6.058 & 14.066 \\
\hline
\end{tabular}


Consol Energy

CleanAir Project No. 10192

Dresden, New York

BH Outlet, AH Outlet
March 29, 2007

11:10

$11: 22$

CALIBRATION BIAS 01

\begin{tabular}{|c|c|c|c|c|c|c|}
\hline & $\begin{array}{r}\text { Channel } 2 \\
\text { SO2 }\end{array}$ & $\begin{array}{r}\text { Channel } 4 \\
02\end{array}$ & $\begin{array}{r}\text { Channel } 5 \\
\mathrm{CO} 2\end{array}$ & $\begin{array}{r}\text { Channel } 7 \\
\text { SO2 }\end{array}$ & $\begin{array}{r}\text { Channel } 8 \\
02\end{array}$ & $\begin{array}{r}\text { Channel } 9 \\
\mathrm{CO} 2\end{array}$ \\
\hline & $\begin{array}{l}\text { AH Outlet } \\
\text { ppmdv }\end{array}$ & $\begin{array}{r}\text { AH Outlet } \\
\% d v\end{array}$ & $\begin{array}{r}\text { AH Outlet } \\
\% d v\end{array}$ & $\begin{array}{r}\text { BH Outlet } \\
\text { ppmdv }\end{array}$ & $\begin{array}{r}\text { BH Outlet } \\
\% d v\end{array}$ & $\begin{array}{r}\text { BH Outlet } \\
\% d v\end{array}$ \\
\hline $\begin{array}{l}11: 15: 38 \\
11 \cdot 15 \cdot 53\end{array}$ & $\begin{array}{r}75.995 \\
1329280\end{array}$ & $\begin{array}{l}5.453 \\
0.689\end{array}$ & $\begin{array}{r}13.180 \\
2596\end{array}$ & $\begin{array}{r}2.497 \\
13.183\end{array}$ & $\begin{array}{l}6.698 \\
4.069\end{array}$ & $\begin{array}{r}13.258 \\
9.421\end{array}$ \\
\hline $11: 16: 08$ & 2145.446 & 0.001 & 0.409 & 23.010 & 0.232 & 0.854 \\
\hline $11: 16: 23$ & 2299.292 & -0.024 & 0.282 & 33.472 & 0.030 & 0.107 \\
\hline $11: 16: 38$ & 2347.008 & -0.029 & 0.247 & 38.128 & 0.016 & 0.024 \\
\hline $11: 16: 53$ & 2363.907 & -0.031 & 0.227 & 40.573 & 0.015 & -0.005 \\
\hline $11: 17: 08$ & 2375.385 & -0.032 & 0.214 & 42.028 & 0.011 & -0.023 \\
\hline $11: 17: 23$ & 2381.099 & -0.036 & 0.202 & 43.116 & 0.017 & -0.039 \\
\hline $11: 17: 38$ & 2384.664 & -0.037 & 0.195 & 43.910 & 0.025 & -0.051 \\
\hline $11: 17: 53$ & 2391.600 & -0.037 & 0.191 & 44.523 & 0.030 & -0.059 \\
\hline $11: 18: 08$ & 2395.653 & -0.037 & 0.187 & 44.975 & 0.032 & -0.065 \\
\hline $11: 18: 23$ & 2397.411 & -0.037 & 0.181 & 45.395 & 0.028 & -0.070 \\
\hline $11: 18: 38$ & 2403.419 & -0.038 & 0.177 & 45.740 & 0.025 & -0.075 \\
\hline $11: 18: 53$ & 2404.493 & -0.039 & 0.174 & 46.027 & 0.019 & -0.082 \\
\hline $11: 19: 08$ & 2403.419 & -0.041 & 0.171 & 46.252 & 0.015 & -0.083 \\
\hline $11: 19: 23$ & 2403.370 & -0.037 & 0.168 & 46.411 & 0.017 & -0.086 \\
\hline $11: 19: 38$ & 2402.930 & -0.040 & 0.165 & 46.567 & 0.012 & -0.091 \\
\hline $11: 19: 53$ & 2405.372 & -0.041 & 0.165 & 46.694 & 0.010 & -0.093 \\
\hline $11: 20: 08$ & 2404.542 & -0.042 & 0.163 & 46.831 & 0.010 & -0.095 \\
\hline $11: 20: 23$ & 2406.349 & -0.041 & 0.160 & 46.912 & 0.010 & -0.096 \\
\hline $11: 20: 38$ & 2406.984 & -0.043 & 0.159 & 47.010 & 0.010 & -0.098 \\
\hline $11: 20: 53$ & 2407.619 & -0.041 & 0.159 & 47.134 & 0.022 & -0.099 \\
\hline $11: 21: 08$ & 2410.598 & -0.041 & 0.158 & 47.231 & 0.019 & -0.104 \\
\hline $11: 21: 23$ & 2410.012 & -0.042 & 0.156 & 47.297 & 0.015 & -0.107 \\
\hline $11: 21: 38$ & 2408.449 & -0.040 & 0.153 & 47.375 & 0.012 & -0.106 \\
\hline $11: 21: 53$ & 2406.789 & -0.038 & 0.154 & 47.375 & 0.012 & -0.107 \\
\hline $11: 22: 08$ & 2401.612 & -0.041 & 0.153 & 47.424 & 0.006 & -0.108 \\
\hline $11: 22: 23$ & 2405.323 & -0.042 & 0.154 & 47.476 & 0.008 & -0.111 \\
\hline $11: 22: 38$ & 2428.767 & -0.040 & 0.155 & 47.599 & 0.012 & -0.112 \\
\hline $11: 22: 53$ & 2319.023 & 3.659 & 4.580 & 75.477 & 5.393 & 5.709 \\
\hline
\end{tabular}


Consol Energy

CleanAir Project No. 10192

Dresden, New York

BH Outlet, AH Outlet
March 29, 2007

Start Time

Stop time

REFERENCE METHOD RUN 2

Channel 2

$\mathrm{SO} 2$

Channel 4

$\mathrm{O} 2$

Channel 5

$\mathrm{CO} 2$

AH Outlet

ppmdv

AH Outlet

AH Outlet

$\% d v$

Calibration Checks

$\mathrm{C}_{\mathrm{oi}}$ Initial zero

$\mathrm{C}_{\mathrm{ui}}$ Initial upscale

$\mathrm{C}_{\text {of }}$ Final zero

$\mathrm{C}_{\text {uf }}$ Final upscale

$\mathrm{C}_{\mathrm{ma}}$ Actual gas value

$\begin{array}{rr}13.789 & -0.017 \\ 2411.901 & 6.124 \\ 11.005 & 0.028 \\ 2389.955 & 5.990 \\ 2479.000 & 6.040\end{array}$

0.154

14.012

0.146

13.997

13.980

7.242

11.656

11.610
$\begin{array}{ll}\mathrm{C}_{\text {Avg }} \text { Average conc. } & 1359.480 \\ \mathbf{C}_{\text {Gas }} \text { Bias adjusted } & \mathbf{1 3 9 8 . 1 0 6}\end{array}$
7.223
66.122

69.699

2.554

47.500

2.847

47.711

49.350
Channel 8

$\mathrm{O} 2$

Channel 9

$\mathrm{SO} 2$

BH Outlet

ppmdv

BH Outlet

$\% d v$

BH Outlet $\% d v$

$\begin{array}{ll}0.009 & -0.001 \\ 6.071 & 14.004 \\ 0.000 & -0.002 \\ 6.036 & 13.987 \\ 6.040 & 13.980\end{array}$

7.180

11.724

7.164

11.711

Clock Time (at end of sample period)

\section{2}

\begin{tabular}{|c|c|c|c|c|c|c|}
\hline $12: 17$ & 1364.664 & 7.254 & 11.675 & 55.117 & 7.121 & 11.779 \\
\hline $12: 18$ & 1361.966 & 7.292 & 11.648 & 72.509 & 7.142 & 11.757 \\
\hline $12: 19$ & 1373.822 & 7.220 & 11.697 & 64.044 & 7.084 & 11.808 \\
\hline $12: 20$ & 1364.005 & 7.268 & 11.651 & 61.744 & 7.091 & 11.811 \\
\hline $12: 21$ & 1353.663 & 7.337 & 11.591 & 62.497 & 7.201 & 11.710 \\
\hline $12: 22$ & 1367.155 & 7.219 & 11.692 & 59.941 & 7.138 & 11.755 \\
\hline $12: 23$ & 1369.695 & 7.204 & 11.705 & 53.649 & 7.088 & 11.803 \\
\hline $12: 24$ & 1357.607 & 7.319 & 11.606 & 53.478 & 7.182 & 11.733 \\
\hline $12: 25$ & 1358.327 & 7.297 & 11.625 & 49.334 & 7.193 & 11.702 \\
\hline $12: 26$ & 1364.188 & 7.269 & 11.646 & 45.573 & 7.173 & 11.724 \\
\hline $12: 27$ & 1370.610 & 7.188 & 11.707 & 57.184 & 7.093 & 11.797 \\
\hline $12: 28$ & 1364.237 & 7.244 & 11.662 & 59.284 & 7.139 & 11.760 \\
\hline $12: 29$ & 1361.685 & 7.256 & 11.642 & 56.007 & 7.167 & 11.730 \\
\hline $12: 30$ & 1360.965 & 7.224 & 11.668 & 56.487 & 7.186 & 11.710 \\
\hline $12: 31$ & 1397.778 & 7.096 & 11.795 & 45.052 & 7.103 & 11.796 \\
\hline $12: 32$ & 1374.896 & 7.207 & 11.698 & 57.928 & 7.226 & 11.673 \\
\hline $12: 33$ & 1376.606 & 7.176 & 11.717 & 66.098 & 7.229 & 11.666 \\
\hline $12: 34$ & 1381.062 & 7.148 & 11.735 & 64.527 & 7.208 & 11.686 \\
\hline $12: 35$ & 1367.644 & 7.266 & 11.639 & 51.045 & 7.184 & 11.715 \\
\hline $12: 36$ & 1355.568 & 7.310 & 11.600 & 56.625 & 7.207 & 11.700 \\
\hline $12: 37$ & 1340.012 & 7.397 & 11.519 & 71.093 & 7.270 & 11.644 \\
\hline $12: 38$ & 1352.808 & 7.296 & 11.601 & 58.999 & 7.354 & 11.658 \\
\hline $12: 39$ & 1363.761 & 7.184 & 11.693 & 67.587 & 7.210 & 11.692 \\
\hline $12: 40$ & 1357.631 & 7.249 & 11.648 & 55.478 & 7.153 & 11.767 \\
\hline $12: 41$ & 1354.908 & 7.256 & 11.641 & 70.853 & 7.201 & 11.719 \\
\hline $12: 42$ & 1357.412 & 7.216 & 11.676 & 73.363 & 7.202 & 11.705 \\
\hline $12: 43$ & 1358.095 & 7.262 & 11.645 & 70.869 & 7.173 & 11.745 \\
\hline $12: 44$ & 1353.041 & 7.289 & 11.621 & 70.698 & 7.233 & 11.684 \\
\hline $12: 45$ & 1363.199 & 7.218 & 11.669 & 75.281 & 7.205 & 11.704 \\
\hline $12: 46$ & 1368.315 & 7.210 & 11.685 & 61.355 & 7.141 & 11.768 \\
\hline $12: 47$ & 1364.860 & 7.212 & 11.682 & 67.165 & 7.166 & 11.750 \\
\hline $12: 48$ & 1360.513 & 7.257 & 11.644 & 50.511 & 7.147 & 11.768 \\
\hline $12: 49$ & 1361.539 & 7.206 & 11.682 & 63.435 & 7.189 & 11.729 \\
\hline $12: 50$ & 1355.226 & 7.267 & 11.634 & 64.125 & 7.185 & 11.726 \\
\hline & & & & & $\begin{array}{r}\text { QAVQ } \\
\text { Da }\end{array}$ & $\frac{\operatorname{tm}}{6 / 14 / 07}$ \\
\hline
\end{tabular}


Consol Energy CleanAir Project No. 10192 Dresden, New York BH Outlet, AH Outlet
March 29, 2007

12:16

13:17

REFERENCE METHOD RUN 2

\begin{tabular}{|c|c|c|c|c|c|c|}
\hline & $\begin{array}{r}\text { Channel } 2 \\
\text { SO2 }\end{array}$ & $\begin{array}{r}\text { Channel } 4 \\
02\end{array}$ & $\begin{array}{r}\text { Channel } 5 \\
\text { CO22 }\end{array}$ & $\begin{array}{r}\text { Channel } 7 \\
\text { SO2 }\end{array}$ & $\begin{array}{r}\text { Channel } 8 \\
02\end{array}$ & $\begin{array}{r}\text { Channel } 9 \\
\mathrm{CO} 2\end{array}$ \\
\hline & $\begin{array}{l}\text { AH Outlet } \\
\text { ppmdv }\end{array}$ & $\begin{array}{r}\text { AH Outlet } \\
\% \text { dv }\end{array}$ & $\begin{array}{r}\text { AH Outlet } \\
\% \text { dv }\end{array}$ & $\begin{array}{l}\text { BH Outlet } \\
\text { ppmdv }\end{array}$ & $\begin{array}{r}\text { BH Outlet } \\
\% \text { dv }\end{array}$ & $\begin{array}{r}\text { BH Outlet } \\
\% d v\end{array}$ \\
\hline $12: 51$ & 1355.739 & 7.297 & 11.613 & 67.337 & 7.188 & 11.725 \\
\hline $12: 52$ & 1360.379 & 7.224 & 11.676 & 67.175 & 7.173 & 11.725 \\
\hline $12: 53$ & 1361.673 & 7.194 & 11.694 & 62.870 & 7.131 & 11.778 \\
\hline $12: 54$ & 1351.514 & 7.299 & 11.595 & 63.604 & 7.182 & 11.737 \\
\hline $12: 55$ & 1351.966 & 7.235 & 11.645 & 79.328 & 7.168 & 11.751 \\
\hline $12: 56$ & 1351.612 & 7.235 & 11.653 & 83.057 & 7.167 & 11.742 \\
\hline $12: 57$ & 1357.436 & 7.180 & 11.704 & 68.204 & 7.150 & 11.750 \\
\hline $12: 58$ & 1357.070 & 7.262 & 11.645 & 47.138 & 7.148 & 11.765 \\
\hline $12: 59$ & 1348.010 & 7.309 & 11.592 & 69.689 & 7.242 & 11.674 \\
\hline $13: 00$ & 1353.712 & 7.253 & 11.646 & 71.374 & 7.173 & 11.736 \\
\hline $13: 01$ & 1357.741 & 7.215 & 11.680 & 64.848 & 7.150 & 11.760 \\
\hline $13: 02$ & 1355.385 & 7.225 & 11.666 & 60.493 & 7.190 & 11.718 \\
\hline $13: 03$ & 1350.550 & 7.295 & 11.620 & 67.607 & 7.178 & 11.737 \\
\hline $13: 04$ & 1341.478 & 7.356 & 11.561 & 85.670 & 7.260 & 11.655 \\
\hline $13: 05$ & 1351.148 & 7.243 & 11.654 & 79.551 & 7.204 & 11.696 \\
\hline $13: 06$ & 1355.556 & 7.221 & 11.670 & 72.598 & 7.156 & 11.755 \\
\hline 13:07 & 1353.443 & 7.210 & 11.667 & 71.383 & 7.190 & 11.721 \\
\hline $13: 08$ & 1344.957 & 7.276 & 11.602 & 71.493 & 7.243 & 11.659 \\
\hline $13: 09$ & 1342.003 & 7.331 & 11.565 & 72.107 & 7.229 & 11.683 \\
\hline $13: 10$ & 1349.255 & 7.221 & 11.655 & 89.273 & 7.222 & 11.675 \\
\hline $13: 11$ & 1350.391 & 7.253 & 11.639 & 73.191 & 7.170 & 11.708 \\
\hline $13: 12$ & 1354.591 & 7.233 & 11.652 & 61.389 & 7.141 & 11.736 \\
\hline $13: 13$ & 1338.999 & 7.318 & 11.583 & 84.348 & 7.266 & 11.611 \\
\hline $13: 14$ & 1341.258 & 7.295 & 11.603 & 77.016 & 7.237 & 11.626 \\
\hline $13: 15$ & 1351.990 & 7.225 & 11.652 & 85.978 & 7.198 & 11.657 \\
\hline $13: 16$ & 1390.806 & 6.985 & 11.863 & 82.170 & 7.111 & 11.737 \\
\hline $13: 17$ & 1392.161 & 7.067 & 11.806 & 85.589 & 7.104 & 11.764 \\
\hline
\end{tabular}


Consol Energy

CleanAir Project No. 10192

Dresden, New York

BH Outlet, AH Outlet

March 29, 2007

Start Time $\quad 13: 18$

Stop Time 13:34

\title{
CALIBRATION BIAS 02
}

$\begin{array}{rrrrrr}\begin{array}{r}\text { Channel 2 } \\ \text { SO2 }\end{array} & \begin{array}{r}\text { Channel 4 } \\ \text { O2 }\end{array} & \begin{array}{r}\text { Channel 5 } \\ \text { CO2 }\end{array} & \begin{array}{r}\text { Channel 7 } \\ \text { SO2 }\end{array} & \begin{array}{r}\text { Channel 8 } \\ \text { O2 }\end{array} & \begin{array}{r}\text { Channel 9 } \\ \text { CO2 }\end{array} \\ \begin{array}{r}\text { AH Outlet } \\ \text { ppmdv }\end{array} & \begin{array}{r}\text { AH Outlet } \\ \% d v\end{array} & \begin{array}{r}\text { AH Outlet } \\ \% d v\end{array} & \begin{array}{r}\text { BH Outlet } \\ \text { ppmdv }\end{array} & \begin{array}{r}\text { BH Outlet } \\ \% d v\end{array} & \begin{array}{r}\text { BH Outlet } \\ \% d v\end{array}\end{array}$

System Response to Calibration Gasses $\left(\mathrm{C}_{\mathrm{S}}\right)$

$\mathrm{C}_{\text {of }}$ Zero gas

$\mathrm{C}_{\text {uf }}$ Upscale gas

\begin{tabular}{|r|r}
\hline 11.005 \\
\hline 2389.955 \\
\hline
\end{tabular}

0.028

5.990

0.146

13.997

2.847

47.711

\begin{tabular}{l|l}
0.000 & -0.002 \\
\hline 6.036 & 13.987 \\
\hline
\end{tabular}

Analyzer Calibration Error Reponses $\left(\mathbf{C}_{\text {Dir }}\right)$

$\mathrm{C}_{\text {oce }}$ Zero gas

3.452

$-0.010$

0.148

14.059

$-0.056$

0.002

13.987

$\mathrm{C}_{\text {mce }}$ Upscale gas

2482.035

6.061

48.630

6.050

0.003

Actual Upscale Gas Value ( $\left.\mathrm{C}_{\mathrm{MA}}\right)$

$\mathrm{C}_{\text {ma }}$ Upscale gas
Calibration Span Value (CS)

2479.000

6.040

13.980

49.350

6.040

13.980

2479.000

14.100

13.980

121.600

14.100

13.980

System Bias as Percent of Calibration Span Value (SB) (5\%) Zero gas
Upscale gas

System Bias Status

$$
\text { Zero gas }
$$

$0.3 \%$

$0.3 \%$

$0.0 \%$

$-0.5 \%$

$-0.4 \%$

$2.4 \%$
$-0.8 \%$

$0.0 \%$

$-0.1 \%$

$0.0 \%$

OK

OK

OK

$\mathrm{OK}$

OK

$0.2 \%$

Upscale gas

OK OK

$\left(C_{s}\right)$

$\begin{array}{lll}\mathrm{C}_{\mathrm{oi}} \quad \text { Zero gas } & 13.789 & -0.017\end{array}$

$\mathrm{C}_{\mathrm{ui}} \quad$ Upscale gas

2411.901

6.124

0.154

14.012

OK

Drift Assessment as Percent of Calibration Span Value (D) (3\%)

Zero gas

Upscale gas

$-0.1 \%$

$-0.9 \%$

$0.3 \%$

$-1.0 \%$

$-0.1 \%$

$-0.1 \%$

Drift Assessment Status

Upscale gas

OK OK

OK

OK
OK

\begin{abstract}
2.554
47.500
\end{abstract}

0.009

6.071

$-0.001$

14.004

$\begin{array}{llr}0.2 \% & -0.1 \% & 0.0 \% \\ 0.2 \% & -0.2 \% & -0.1 \%\end{array}$

\section{2}

$\begin{array}{lr}13: 18: 30 & 1257.192 \\ 13: 18: 45 & 572.454 \\ 13: 19: 00 & 195.409 \\ 13: 19: 15 & 97.729 \\ 13: 19: 30 & 62.173 \\ 13: 19: 45 & 45.617 \\ 13: 20: 00 & 35.018 \\ 13: 20: 15 & 28.913 \\ 13: 20: 30 & 24.420 \\ 13: 20: 45 & 22.173 \\ 13: 21: 00 & 19.927 \\ 13: 21: 15 & 18.608 \\ 13: 21: 30 & 16.948 \\ 13: 21: 45 & 16.459 \\ 13: 22: 00 & 15.336 \\ 13: 22: 15 & 14.652 \\ 13: 22: 30 & 14.163 \\ 13: 22: 45 & 13.724 \\ 13: 23: 00 & 12.503\end{array}$

OK

$\begin{array}{lll}\text { OK } & \text { OK } & \text { OK } \\ \text { OK } & \text { OK } & \text { OK }\end{array}$

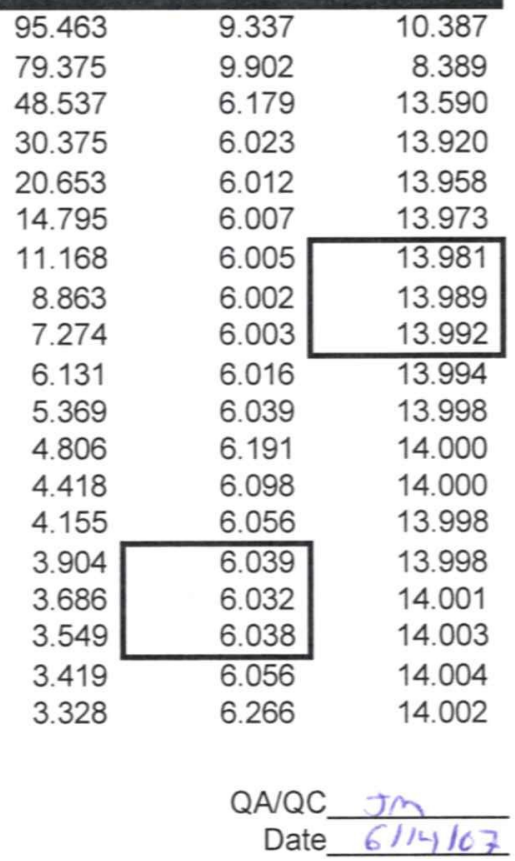


Consol Energy

CleanAir Project No. 10192

Dresden, New York

BH Outlet, AH Outlet
March 29, 2007

CALIBRATION BIAS 02

$\begin{array}{ll}\text { Start Time } & 13: 18 \\ \text { Stop Time } & 13: 34\end{array}$

\begin{tabular}{|c|c|c|c|c|c|c|}
\hline & $\begin{array}{r}\text { Channel } 2 \\
\text { SO2 }\end{array}$ & $\begin{array}{r}\text { Channel } 4 \\
\text { O2 }\end{array}$ & $\begin{array}{r}\text { Channel } 5 \\
\mathrm{CO} 2\end{array}$ & $\begin{array}{r}\text { Channel } 7 \\
\text { SO2 }\end{array}$ & $\begin{array}{r}\text { Channel } 8 \\
\text { O2 }\end{array}$ & $\begin{array}{r}\text { Channel } 9 \\
\mathrm{CO} 2\end{array}$ \\
\hline & $\begin{array}{c}\text { AH Outlet } \\
\text { ppmdv }\end{array}$ & $\begin{array}{r}\text { AH Outlet } \\
\% \text { dv }\end{array}$ & $\begin{array}{r}\text { AH Outlet } \\
\% d v\end{array}$ & $\begin{array}{r}\text { BH Outlet } \\
\text { ppmdv }\end{array}$ & $\begin{array}{r}\text { BH Outlet } \\
\% d v\end{array}$ & $\begin{array}{r}\text { BH Outlet } \\
\% d v\end{array}$ \\
\hline $13: 23: 15$ & 12.308 & 5.976 & 14.030 & 3.223 & 6.416 & 14.003 \\
\hline $13: 23: 30$ & 11.917 & 5.976 & 14.035 & 3.159 & 6.020 & 14.005 \\
\hline $13: 23: 45$ & 11.966 & 5.974 & 14.034 & 3.110 & 6.009 & 14.005 \\
\hline $13: 24: 00[$ & 11.184 & 5.975 & 14.033 & 3.051 & 6.006 & 14.007 \\
\hline $13: 24: 15$ & 10.842 & 5.976 & 14.039 & 2.986 & 6.005 & 14.008 \\
\hline $13: 24: 30$ & 10.989 & 5.979 & 14.041 & 2.930 & 6.003 & 14.009 \\
\hline $13: 24: 45$ & 271.941 & 3.319 & 9.172 & 2.624 & 2.437 & 7.480 \\
\hline $13: 25: 00$ & 1552.088 & 0.133 & 0.883 & 9.846 & 0.102 & 0.520 \\
\hline $13: 25: 15$ & 2093.578 & -0.019 & 0.332 & 24.404 & 0.012 & 0.091 \\
\hline $13: 25: 30$ & 2228.767 & -0.030 & 0.269 & 32.869 & 0.004 & 0.023 \\
\hline $13: 25: 45$ & 2300.269 & -0.034 & 0.240 & 37.682 & 0.002 & -0.006 \\
\hline $13: 26: 00$ & 2326.496 & -0.037 & 0.224 & 40.668 & 0.000 & -0.024 \\
\hline $13: 26: 15$ & 2342.076 & -0.037 & 0.212 & 42.471 & 0.000 & -0.04 \\
\hline $13: 26: 30$ & 2351.062 & -0.036 & 0.201 & 43.604 & 0.000 & -0.050 \\
\hline $13: 26: 45$ & 2354.969 & -0.037 & 0.195 & 44.405 & 0.000 & -0.058 \\
\hline $13: 27: 00$ & 2358.730 & -0.041 & 0.190 & 45.017 & -0.001 & -0.064 \\
\hline $13: 27: 15$ & 2364.005 & -0.039 & 0.185 & 45.438 & 0.000 & -0.071 \\
\hline $13: 27: 30$ & 2370.891 & -0.042 & 0.182 & 45.766 & -0.002 & -0.077 \\
\hline $13: 27: 45$ & 2366.593 & -0.040 & 0.179 & 46.001 & 0.000 & -0.081 \\
\hline $13: 28: 00$ & 2367.619 & -0.041 & 0.176 & 46.304 & 0.000 & -0.086 \\
\hline $13: 28: 15$ & 2370.208 & -0.040 & 0.171 & 46.480 & 0.000 & -0.089 \\
\hline $13: 28: 30$ & 2375.922 & -0.039 & 0.169 & 46.606 & 0.000 & -0.091 \\
\hline $13: 28: 45$ & 2371.477 & -0.040 & 0.167 & 46.707 & -0.001 & -0.09 \\
\hline $13: 29: 00$ & 2371.477 & -0.040 & 0.165 & 46.786 & 0.000 & -0.098 \\
\hline $13: 29: 15$ & 2371.624 & -0.042 & 0.164 & 46.902 & 0.000 & -0.101 \\
\hline $13: 29: 30$ & 2377.729 & -0.041 & 0.161 & 46.997 & -0.001 & -0.101 \\
\hline $13: 29: 45$ & 2377.143 & -0.045 & 0.160 & 47.105 & -0.001 & -0.104 \\
\hline $13: 30: 00$ & 2373.968 & -0.043 & 0.159 & 47.153 & 0.000 & -0.106 \\
\hline $13: 30: 15$ & 2377.778 & -0.043 & 0.158 & 47.179 & -0.001 & -0.107 \\
\hline $13: 30: 30$ & 2374.017 & -0.044 & 0.156 & 47.179 & -0.002 & -0.107 \\
\hline $13: 30: 45$ & 2371.917 & -0.047 & 0.154 & 47.225 & -0.005 & -0.107 \\
\hline $13: 31: 00$ & 2371.282 & -0.043 & 0.153 & 47.310 & -0.002 & -0.111 \\
\hline $13: 31: 15$ & 2375.385 & -0.045 & 0.153 & 47.401 & -0.002 & -0.112 \\
\hline $13: 31: 30$ & 2376.215 & -0.043 & 0.151 & 47.424 & 0.000 & -0.113 \\
\hline $13: 31: 45$ & 2374.896 & -0.041 & 0.151 & 47.424 & -0.001 & -0.113 \\
\hline $13: 32: 00$ & 2374.750 & -0.043 & 0.148 & 47.434 & -0.003 & -0.113 \\
\hline $13: 32: 15$ & 2375.824 & -0.043 & 0.149 & 47.483 & -0.004 & -0.117 \\
\hline $13: 32: 30$ & 2374.554 & -0.042 & 0.149 & 47.521 & -0.004 & -0.118 \\
\hline $13: 32: 45$ & 2376.215 & -0.041 & 0.147 & 47.473 & -0.005 & -0.119 \\
\hline $13: 33: 00$ & 2379.390 & -0.042 & 0.147 & 47.473 & -0.008 & -0.119 \\
\hline $13: 33: 15$ & 2382.076 & -0.043 & 0.146 & 47.528 & -0.005 & -0.118 \\
\hline $13: 33: 30$ & 2377.778 & -0.046 & 0.146 & 47.570 & -0.008 & -0.12 \\
\hline $13: 33: 45[$ & 2376.215 & -0.040 & 0.147 & 47.612 & -0.004 & -0.120 \\
\hline $13: 34: 00$ & 2379.438 & -0.043 & 0.146 & 47.730 & -0.008 & -0.11 \\
\hline $13: 34: 15$ & 2414.212 & -0.006 & 0.151 & 47.792 & 0.142 & -0.106 \\
\hline $13: 34: 30$ & 2165.812 & 4.877 & 5.991 & 70.838 & 5.452 & 7.428 \\
\hline
\end{tabular}


Consol Energy

CleanAir Project No. 10192

Dresden, New York

BH Outlet, AH Outlet
March 29, 2007

$15: 13$

16:13

\section{REFERENCE METHOD RUN 3}

$\begin{array}{rrrrrr}\begin{array}{r}\text { Channel 2 } \\ \text { SO2 }\end{array} & \begin{array}{r}\text { Channel 4 } \\ \text { O2 }\end{array} & \begin{array}{r}\text { Channel 5 } \\ \text { CO2 }\end{array} & \begin{array}{r}\text { Channel 7 } \\ \text { SO2 }\end{array} & \begin{array}{r}\text { Channel 8 } \\ \text { O2 }\end{array} & \begin{array}{r}\text { Channel 9 } \\ \text { CO2 }\end{array} \\ \begin{array}{r}\text { AH Outlet } \\ \text { ppmdv }\end{array} & \begin{array}{r}\text { AH Outlet } \\ \% d v\end{array} & \begin{array}{r}\text { AH Outlet } \\ \% d v\end{array} & \begin{array}{r}\text { BH Outlet } \\ \text { ppmdv }\end{array} & \begin{array}{r}\text { BH Outlet } \\ \% d v\end{array} & \begin{array}{r}\text { BH Outlet } \\ \% \text { dv }\end{array}\end{array}$

\section{Calibration Checks}

$\mathrm{C}_{\text {oi }}$ Initial zero

11.005

0.028

0.146

13.997

2.847

0.000

$-0.002$

$\mathrm{C}_{\text {ui }}$ Initial upscale

2389.955

12.177

$\mathrm{C}_{\text {of }}$ Final zero

$-0.019$

0.150

47.711

3.055

6.036

13.987

6.032

14.042

47.506

$-0.006$

$-0.012$

6.040

13.980

49.350

6.041

13.981

2479.000

Analyzer Averages (concentrations)

$\mathrm{C}_{\text {Avg }}$ Average conc. $\quad 1328.389$

$\mathrm{C}_{\mathrm{Gas}}$ Bias adjusted

1381.352
11.582

7.447

11.523
92.367

7.512

11.509

98.811

7.513

11.507

Clock Time (at end of sample period)

\section{2}

\begin{tabular}{|c|c|c|c|c|c|c|}
\hline $15: 14$ & 1341.746 & 7.394 & 11.607 & 85.943 & 7.699 & 11.548 \\
\hline $15: 15$ & 1329.561 & 7.383 & 11.544 & 101.783 & 7.758 & 11.488 \\
\hline $15: 16$ & 1335.543 & 7.301 & 11.619 & 99.989 & 7.729 & 11.492 \\
\hline $15: 17$ & 1338.706 & 7.300 & 11.633 & 95.828 & 7.517 & 11.577 \\
\hline $15: 18$ & 1336.288 & 7.289 & 11.643 & 86.194 & 7.215 & 11.569 \\
\hline $15: 19$ & 1334.982 & 7.311 & 11.625 & 72.071 & 7.205 & 11.589 \\
\hline $15: 20$ & 1325.031 & 7.409 & 11.543 & 71.788 & 7.280 & 11.523 \\
\hline $15: 21$ & 1325.324 & 7.384 & 11.565 & 71.992 & 7.422 & 11.454 \\
\hline $15: 22$ & 1344.359 & 7.265 & 11.681 & 70.107 & 7.435 & 11.555 \\
\hline $15: 23$ & 1338.706 & 7.340 & 11.674 & 96.813 & 7.329 & 11.480 \\
\hline $15: 24$ & 1352.051 & 7.387 & 12.114 & 92.204 & 7.230 & 11.513 \\
\hline $15: 25$ & 1349.109 & 7.337 & 11.762 & 90.066 & 7.275 & 11.534 \\
\hline $15: 26$ & 1347.265 & 7.324 & 11.888 & 98.562 & 7.323 & 11.490 \\
\hline $15: 27$ & 1342.943 & 7.276 & 12.118 & 103.508 & 7.294 & 11.523 \\
\hline $15: 28$ & 1336.142 & 7.344 & 11.747 & 107.680 & 7.346 & 11.471 \\
\hline $15: 29$ & 1334.042 & 7.368 & 11.705 & 91.079 & 7.331 & 11.455 \\
\hline $15: 30$ & 1338.376 & 7.598 & 11.671 & 100.676 & 7.881 & 11.553 \\
\hline $15: 31$ & 1333.968 & 7.392 & 11.764 & 86.242 & 7.790 & 11.505 \\
\hline $15: 32$ & 1322.283 & 7.379 & 11.629 & 84.263 & 7.785 & 11.494 \\
\hline $15: 33$ & 1316.178 & 7.454 & 11.534 & 113.180 & 7.871 & 11.420 \\
\hline $15: 34$ & 1325.702 & 7.401 & 11.566 & 105.572 & 7.756 & 11.513 \\
\hline $15: 35$ & 1327.558 & 7.393 & 11.574 & 89.464 & 7.757 & 11.523 \\
\hline $15: 36$ & 1325.714 & 7.418 & 11.555 & 83.420 & 7.763 & 11.517 \\
\hline $15: 37$ & 1323.346 & 7.463 & 11.510 & 96.301 & 7.759 & 11.492 \\
\hline $15: 38$ & 1327.631 & 7.390 & 11.556 & 97.468 & 7.750 & 11.502 \\
\hline $15: 39$ & 1340.452 & 7.351 & 11.606 & 78.756 & 7.494 & 11.540 \\
\hline $15: 40$ & 1340.086 & 7.386 & 11.575 & 78.554 & 7.480 & 11.490 \\
\hline $15: 41$ & 1326.642 & 7.449 & 11.527 & 106.330 & 7.405 & 11.425 \\
\hline $15: 42$ & 1339.084 & 7.341 & 11.612 & 103.406 & 7.336 & 11.447 \\
\hline $15: 43$ & 1332.992 & 7.448 & 11.532 & 85.951 & 7.244 & 11.514 \\
\hline $15: 44$ & 1328.230 & 7.441 & 11.529 & 93.355 & 7.194 & 11.584 \\
\hline
\end{tabular}


Consol Energy

CleanAir Project No. 10192

Dresden, New York

BH Outlet, AH Outlet
March 29, 2007

15:13

$16: 13$

REFERENCE METHOD RUN 3

$\begin{array}{rrrrrrr} & \begin{array}{r}\text { Channel 2 } \\ \text { SO2 }\end{array} & \begin{array}{rrrrr}\text { Channel 4 } \\ \text { O2 }\end{array} & \begin{array}{r}\text { Channel 5 } \\ \text { CO2 }\end{array} & \begin{array}{r}\text { Channel 7 } \\ \text { SO2 }\end{array} & \begin{array}{r}\text { Channel 8 } \\ \text { O2 }\end{array} & \begin{array}{r}\text { Channel 9 } \\ \text { CO2 }\end{array} \\ & & & & & & \\ & \text { AH Outlet } & \text { AH Outlet } & \text { AH Outlet } & \text { BH Outlet } & \text { BH Outlet } & \text { BH Outlet } \\ \text { ppmdv } & \text { \%dv } & \text { \%dv } & \text { ppdv } & \text { \%dv } \\ & & & & & & \\ 15: 45 & 1319.475 & 7.460 & 11.510 & 106.243 & 7.320 & 11.449 \\ 15: 46 & 1325.837 & 7.424 & 11.534 & 84.945 & 7.268 & 11.501 \\ 15: 47 & 1342.332 & 7.250 & 11.670 & 96.186 & 7.202 & 11.549 \\ 15: 48 & 1344.286 & 7.280 & 11.654 & 79.721 & 7.203 & 11.561 \\ 15: 49 & 1345.458 & 7.296 & 11.646 & 72.390 & 7.187 & 11.581 \\ 15: 50 & 1341.062 & 7.362 & 11.586 & 83.234 & 7.634 & 11.515 \\ 15: 51 & 1327.094 & 7.505 & 11.459 & 75.178 & 7.523 & 11.407 \\ 15: 52 & 1333.040 & 7.424 & 11.527 & 95.417 & 7.672 & 11.421 \\ 15: 53 & 1328.926 & 7.337 & 11.288 & 100.889 & 7.554 & 11.466 \\ 15: 54 & 1326.178 & 7.466 & 11.496 & 106.206 & 7.422 & 11.501 \\ 15: 55 & 1324.811 & 7.423 & 11.542 & 91.772 & 7.375 & 11.544 \\ 15: 56 & 1326.655 & 7.435 & 11.535 & 89.937 & 7.388 & 11.572 \\ 15: 57 & 1315.727 & 7.536 & 11.448 & 83.850 & 7.504 & 11.514 \\ 15: 58 & 1322.247 & 7.448 & 11.514 & 99.317 & 7.650 & 11.514 \\ 15: 59 & 1315.836 & 7.449 & 11.509 & 91.258 & 7.737 & 11.520 \\ 16: 00 & 1318.742 & 7.474 & 11.501 & 94.484 & 7.752 & 11.540 \\ 16: 01 & 1316.471 & 7.460 & 11.508 & 101.659 & 7.497 & 11.510 \\ 16: 02 & 1328.266 & 7.392 & 11.570 & 89.159 & 7.546 & 11.572 \\ 16: 03 & 1317.460 & 7.581 & 11.420 & 111.626 & 7.637 & 11.522 \\ 16: 04 & 1296.349 & 7.670 & 11.318 & 87.416 & 7.526 & 11.484 \\ 16: 05 & 1311.074 & 7.487 & 11.472 & 99.113 & 7.566 & 11.458 \\ 16: 06 & 1314.701 & 7.467 & 11.491 & 85.098 & 7.762 & 11.477 \\ 16: 07 & 1312.466 & 7.498 & 11.471 & 90.090 & 7.864 & 11.469 \\ 16: 08 & 1318.352 & 7.431 & 11.469 & 97.488 & 7.524 & 11.479 \\ 16: 09 & 1313.126 & 7.517 & 11.459 & 105.937 & 7.609 & 11.456 \\ 16: 10 & 1312.527 & 7.439 & 11.524 & 105.612 & 7.600 & 11.513 \\ 16: 11 & 1311.514 & 7.449 & 11.522 & 94.792 & 7.782 & 11.545 \\ 16: 12 & 1315.482 & 7.434 & 11.537 & 96.286 & 7.418 & 11.537 \\ 16: 13 & 1317.802 & 7.461 & 11.517 & 88.195 & 7.352 & 11.570\end{array}$

\begin{tabular}{|c|c|c|c|c|c|c|}
\hline & Channel 2 & Channel 4 & Channel 5 & Channel 7 & Channel 8 & Channel 9 \\
\hline & $\mathrm{SO} 2$ & $\mathrm{O} 2$ & $\mathrm{CO} 2$ & $\mathrm{SO} 2$ & $\mathrm{O} 2$ & $\mathrm{CO} 2$ \\
\hline & $\begin{array}{c}\text { AH Outlet } \\
\text { ppmdv }\end{array}$ & $\begin{array}{c}\text { AH Outlet } \\
\% d v\end{array}$ & $\begin{array}{r}\text { AH Outlet } \\
\% d v\end{array}$ & $\begin{array}{r}\text { BH Outlet } \\
\text { ppmdv }\end{array}$ & $\begin{array}{r}\text { BH Outlet } \\
\% d v\end{array}$ & $\begin{array}{r}\text { BH Outlet } \\
\% d v\end{array}$ \\
\hline $15: 45$ & 1319.475 & 7.460 & 11.510 & 106.243 & 7.320 & 11.449 \\
\hline $15: 46$ & 1325.837 & 7.424 & 11.534 & 84.945 & 7.268 & 11.501 \\
\hline $15: 47$ & 1342.332 & 7.250 & 11.670 & 96.186 & 7.202 & 11.549 \\
\hline $15: 48$ & 1344.286 & 7.280 & 11.654 & 79.721 & 7.203 & 11.561 \\
\hline $15: 49$ & 1345.458 & 7.296 & 11.646 & 72.390 & 7.187 & 11.581 \\
\hline $15: 50$ & 1341.062 & 7.362 & 11.586 & 83.234 & 7.634 & 11.515 \\
\hline $15: 51$ & 1327.094 & 7.505 & 11.459 & 75.178 & 7.523 & 11.407 \\
\hline $15: 52$ & 1333.040 & 7.424 & 11.527 & 95.417 & 7.672 & 11.421 \\
\hline $15: 53$ & 1328.926 & 7.337 & 11.288 & 100.889 & 7.554 & 11.466 \\
\hline $15: 54$ & 1326.178 & 7.466 & 11.496 & 106.206 & 7.422 & 11.501 \\
\hline $15: 55$ & 1324.811 & 7.423 & 11.542 & 91.772 & 7.375 & 11.544 \\
\hline $15: 56$ & 1326.655 & 7.435 & 11.535 & 89.937 & 7.388 & 11.572 \\
\hline $15: 57$ & 1315.727 & 7.536 & 11.448 & 83.850 & 7.504 & 11.514 \\
\hline $15: 58$ & 1322.247 & 7.448 & 11.514 & 99.317 & 7.650 & 11.514 \\
\hline $15: 59$ & 1315.836 & 7.449 & 11.509 & 91.258 & 7.737 & 11.520 \\
\hline $16: 00$ & 1318.742 & 7.474 & 11.501 & 94.484 & 7.752 & 11.540 \\
\hline $16: 01$ & 1316.471 & 7.460 & 11.508 & 101.659 & 7.497 & 11.510 \\
\hline $16: 02$ & 1328.266 & 7.392 & 11.570 & 89.159 & 7.546 & 11.572 \\
\hline $16: 03$ & 1317.460 & 7.581 & 11.420 & 111.626 & 7.637 & 11.522 \\
\hline $16: 04$ & 1296.349 & 7.670 & 11.318 & 87.416 & 7.526 & 11.484 \\
\hline $16: 05$ & 1311.074 & 7.487 & 11.472 & 99.113 & 7.566 & 11.458 \\
\hline $16: 06$ & 1314.701 & 7.467 & 11.491 & 85.098 & 7.762 & 11.477 \\
\hline $16: 07$ & 1312.466 & 7.498 & 11.471 & 90.090 & 7.864 & 11.469 \\
\hline $16: 08$ & 1318.352 & 7.431 & 11.469 & 97.488 & 7.524 & 11.479 \\
\hline $16: 09$ & 1313.126 & 7.517 & 11.459 & 105.937 & 7.609 & 11.456 \\
\hline $16: 10$ & 1312.527 & 7.439 & 11.524 & 105.612 & 7.600 & 11.513 \\
\hline $16: 11$ & 1311.514 & 7.449 & 11.522 & 94.792 & 7.782 & 11.545 \\
\hline $16: 12$ & 1315.482 & 7.434 & 11.537 & 96.286 & 7.418 & 11.537 \\
\hline $16: 13$ & 1317.802 & 7.461 & 11.517 & 88.195 & 7.352 & 11.570 \\
\hline
\end{tabular}




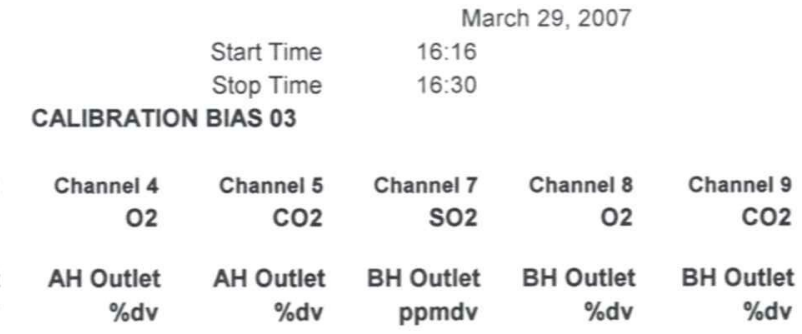

System Response to Calibration Gasses ( $C_{\mathrm{s}}$ )

\begin{tabular}{|c|c|c|c|c|c|c|c|}
\hline & & & & & & & \\
\hline & Zero gas & 12.177 & -0.019 & 0.150 & 3.055 & -0.006 & -0.012 \\
\hline $\mathrm{C}_{\mathrm{uf}}$ & Upscale gas & 2359.526 & 6.032 & 14.042 & 47.506 & 6.041 & 13.981 \\
\hline \multicolumn{8}{|c|}{ Analyzer Calibration Error Reponses $\left(\mathrm{C}_{\text {Dir }}\right)$} \\
\hline $\mathrm{C}_{\text {oce }}$ & Zero gas & 3.452 & -0.010 & 0.148 & -0.056 & 0.002 & 0.003 \\
\hline $\mathrm{C}_{\text {mce }}$ & Upscale gas & 2482.035 & 6.061 & 14.059 & 48.630 & 6.050 & 13.966 \\
\hline \multicolumn{8}{|c|}{ Actual Upscale Gas Value $\left(\mathrm{C}_{\mathrm{MA}}\right)$} \\
\hline $\mathrm{C}_{\mathrm{ma}}$ & Upscale gas & 2479.000 & 6.040 & 13.980 & 49.350 & 6.040 & 13.980 \\
\hline \multicolumn{8}{|c|}{ Calibration Span Value (CS) } \\
\hline & & 2479.000 & 14.100 & 13.980 & 121.600 & 14.100 & 13.980 \\
\hline
\end{tabular}

System Bias as Percent of Calibration Span Value (SB) (5\%)

\begin{tabular}{lllllll} 
Zero gas & $0.4 \%$ & $-0.1 \%$ & $0.0 \%$ & $2.6 \%$ & $-0.1 \%$ & $-0.1 \%$ \\
Upscale gas & $-4.9 \%$ & $-0.2 \%$ & $-0.1 \%$ & $-0.9 \%$ & $-0.1 \%$ & $0.1 \%$ \\
\hline stem Bias Status & & & & & &
\end{tabular}

System Bias Status

\begin{tabular}{|c|c|c|c|c|c|c|}
\hline Zero gas & OK & OK & OK & OK & OK & OK \\
\hline Upscale gas & OK & OK & OK & OK & OK & OK \\
\hline \multicolumn{7}{|c|}{ vious System Response to Calibration Gases $\left(C_{s}\right)$} \\
\hline Zero gas & 11.005 & 0.028 & 0.146 & 2.847 & 0.000 & -0.002 \\
\hline Upscale gas & 2389.955 & 5.990 & 13.997 & 47.711 & 6.036 & 13.987 \\
\hline \multicolumn{7}{|c|}{ ft Assessment as Percent of Calibration Span Value (D) $(3 \%)$} \\
\hline Zero gas & $0.0 \%$ & $-0.3 \%$ & $0.0 \%$ & $0.2 \%$ & $0.0 \%$ & $-0.1 \%$ \\
\hline Upscale gas & $-1.2 \%$ & $0.3 \%$ & $0.3 \%$ & $-0.2 \%$ & $0.0 \%$ & $0.0 \%$ \\
\hline
\end{tabular}

Drift Assessment Status

$\begin{array}{lllllll}\text { Zerogas } & \text { OK } & \text { OK } & \text { OK } & \text { OK } & \text { OK } & \text { OK }\end{array}$

Upscale gas

$\begin{array}{lllll}O K & O K & O K & O K & O K\end{array}$

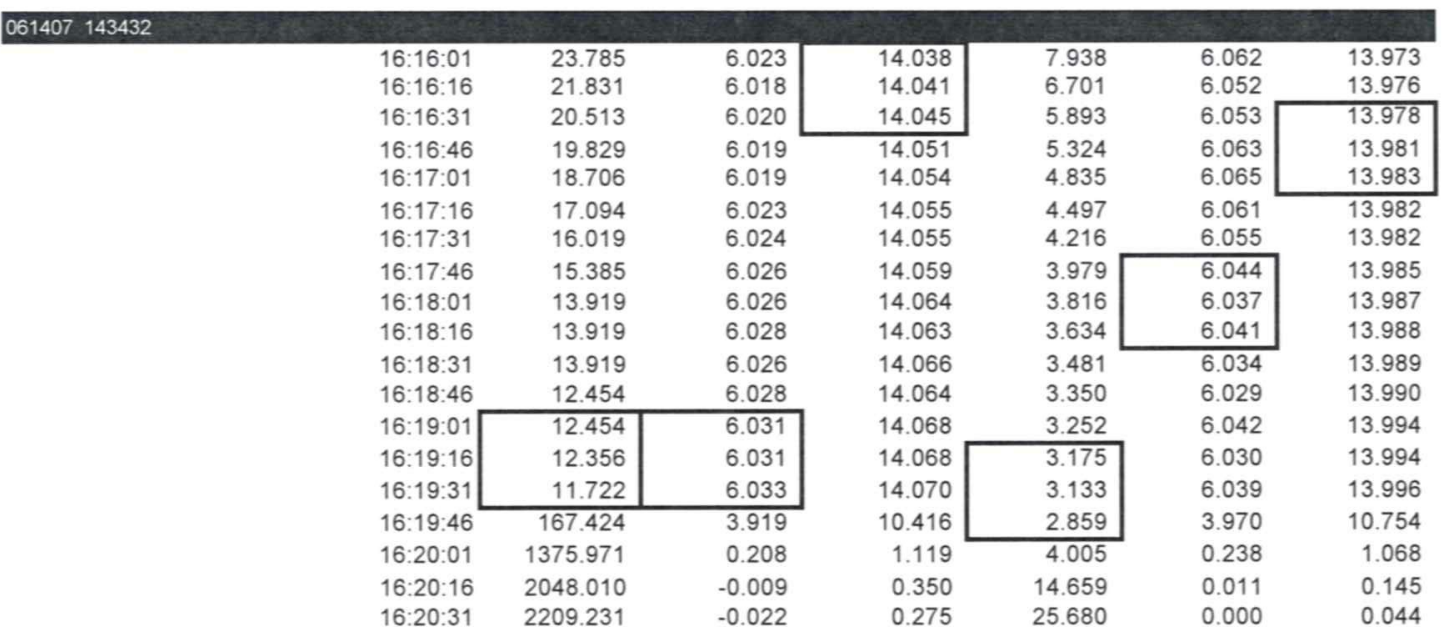


Consol Energy

CleanAir Project No. 10192

Dresden, New York

BH Outlet, AH Outlet

\section{March 29, 2007 \\ Start Time $\quad 16: 16$ \\ Stop Time $\quad 16: 30$ \\ CALIBRATION BIAS 03}

\begin{tabular}{|c|c|c|c|c|c|c|}
\hline & $\begin{array}{r}\text { Channel } 2 \\
\text { SO2 }\end{array}$ & $\begin{array}{r}\text { Channel } 4 \\
02\end{array}$ & $\begin{array}{r}\text { Channel } 5 \\
\mathrm{CO} 2\end{array}$ & $\begin{array}{r}\text { Channel } 7 \\
\text { SO2 }\end{array}$ & $\begin{array}{r}\text { Channel } 8 \\
\mathrm{O} 2\end{array}$ & $\begin{array}{r}\text { Channel } 9 \\
\mathrm{CO} 2\end{array}$ \\
\hline & $\begin{array}{l}\text { AH Outlet } \\
\text { ppmdv }\end{array}$ & $\begin{array}{r}\text { AH Outlet } \\
\% \text { dv }\end{array}$ & $\begin{array}{r}\text { AH Outlet } \\
\% \text { dv }\end{array}$ & $\begin{array}{r}\text { BH Outlet } \\
\text { ppmdv }\end{array}$ & $\begin{array}{r}\text { BH Outlet } \\
\% d v\end{array}$ & $\begin{array}{r}\text { BH Outlet } \\
\% \text { dv }\end{array}$ \\
\hline $16: 20: 46$ & 2265.641 & -0.025 & 0.247 & 32.847 & -0.003 & 0.007 \\
\hline $\begin{array}{l}16: 21: 01 \\
16: 21: 16\end{array}$ & $\begin{array}{l}2288.596 \\
2304.762\end{array}$ & $\begin{array}{l}-0.029 \\
-0.028\end{array}$ & $\begin{array}{l}0.229 \\
0.215\end{array}$ & $\begin{array}{l}37.327 \\
40.111\end{array}$ & $\begin{array}{l}-0.003 \\
-0.005\end{array}$ & $\begin{array}{l}-0.013 \\
-0.030\end{array}$ \\
\hline $16: 21: 31$ & 2310.965 & -0.030 & 0.205 & 41.885 & -0.011 & -0.041 \\
\hline $16: 21: 46$ & 2321.123 & -0.027 & 0.197 & 43.048 & -0.010 & -0.049 \\
\hline 16:22:01 & 2323.370 & -0.027 & 0.193 & 43.842 & -0.011 & -0.058 \\
\hline $16: 22: 16$ & 2326.838 & -0.027 & 0.189 & 44.438 & -0.010 & -0.064 \\
\hline $16: 22: 31$ & 2330.842 & -0.025 & 0.183 & 44.894 & -0.011 & -0.070 \\
\hline $16: 22: 46$ & 2334.603 & -0.031 & 0.181 & 45.242 & -0.014 & -0.073 \\
\hline $16: 23: 01$ & 2335.141 & -0.029 & 0.177 & 45.522 & -0.013 & -0.076 \\
\hline $16: 23: 16$ & 2336.166 & -0.034 & 0.174 & 45.743 & -0.014 & -0.081 \\
\hline $16: 23: 31$ & 2332.943 & -0.034 & 0.170 & 45.900 & -0.016 & -0.086 \\
\hline $16: 23: 46$ & 2334.017 & -0.034 & 0.167 & 46.046 & -0.015 & -0.088 \\
\hline $16: 24: 01$ & 2340.513 & -0.034 & 0.166 & 46.138 & -0.013 & -0.091 \\
\hline $16: 24: 16$ & 2340.317 & -0.031 & 0.164 & 46.242 & -0.013 & -0.092 \\
\hline $16: 24: 31$ & 2355.507 & -0.026 & 0.165 & 46.329 & -0.012 & -0.095 \\
\hline $16: 24: 46$ & 2349.109 & -0.031 & 0.165 & 46.378 & -0.014 & -0.097 \\
\hline $16: 25: 01$ & 2343.394 & -0.031 & 0.162 & 46.401 & -0.014 & -0.097 \\
\hline $16: 25: 16$ & 2346.862 & -0.039 & 0.161 & 46.496 & -0.014 & -0.100 \\
\hline $16: 25: 31$ & 2342.125 & -0.037 & 0.160 & 46.606 & -0.013 & -0.101 \\
\hline $16: 25: 46$ & 2340.610 & -0.040 & 0.157 & 46.733 & -0.015 & -0.101 \\
\hline $16: 26: 01$ & 2342.320 & -0.039 & 0.156 & 46.825 & -0.016 & -0.104 \\
\hline $16: 26: 16$ & 2341.783 & -0.039 & 0.156 & 46.893 & -0.017 & -0.104 \\
\hline $16: 26: 31$ & 2343.590 & -0.037 & 0.156 & 46.932 & -0.015 & -0.106 \\
\hline $16: 26: 46$ & 2343.785 & -0.037 & 0.154 & 46.984 & -0.016 & -0.107 \\
\hline $16: 27: 01$ & 2344.078 & -0.037 & 0.153 & 46.984 & -0.014 & -0.108 \\
\hline $16: 27: 16$ & 2343.834 & -0.037 & 0.153 & 47.004 & -0.016 & -0.111 \\
\hline $16: 27: 31$ & 2344.957 & -0.039 & 0.153 & 47.033 & -0.015 & -0.110 \\
\hline $16: 27: 46$ & 2347.644 & -0.028 & 0.152 & 47.066 & -0.012 & -0.113 \\
\hline 16:28:01 & 2346.667 & -0.041 & 0.151 & 47.092 & -0.014 & -0.113 \\
\hline $16: 28: 16$ & 2335.824 & -0.037 & 0.150 & 47.176 & -0.013 & -0.114 \\
\hline $16: 28: 31$ & 2339.048 & -0.037 & 0.149 & 47.355 & -0.012 & -0.116 \\
\hline $16: 28: 46$ & 2347.399 & -0.037 & 0.151 & 47.987 & 1.545 & 1.116 \\
\hline $16: 29: 01$ & 2354.041 & -0.036 & 0.151 & 68.142 & 6.632 & 9.983 \\
\hline $16: 29: 16$ & 2358.974 & -0.036 & 0.149 & 83.386 & 7.038 & 11.492 \\
\hline $16: 29: 31$ & 2358.265 & -0.035 & 0.151 & 79.873 & 7.151 & 11.537 \\
\hline $16: 29: 46$ & 2357.093 & -0.036 & 0.150 & 85.333 & 7.147 & 11.561 \\
\hline $16: 30: 01$ & 2357.018 & -0.035 & 0.150 & 99.087 & 7.178 & 11.529 \\
\hline $16: 30: 16$ & 2347.839 & -0.044 & 0.144 & 102.004 & 7.204 & 11.522 \\
\hline $16: 30: 31$ & 2339.292 & -0.112 & -0.110 & 90.273 & 7.072 & 11.514 \\
\hline $16: 30: 46$ & 2359.563 & -6.703 & -6.561 & 83.930 & 7.118 & 11.483 \\
\hline $16: 29: 01$ & 2360.041 & -0.036 & 0.151 & 68.142 & 6.632 & 9.983 \\
\hline $16: 29: 16$ & 2358.974 & -0.036 & 0.149 & 83.386 & 7.038 & 11.492 \\
\hline $16: 29: 31$ & 2357.265 & -0.035 & 0.151 & 79.873 & 7.151 & 11.537 \\
\hline $16: 29: 46$ & 2356.093 & -0.036 & 0.150 & 85.333 & 7.147 & 11.561 \\
\hline $16: 30: 01$ & 2355.018 & -0.035 & 0.150 & 99.087 & 7.178 & 11.529 \\
\hline $16: 30: 16$ & 2347.839 & -0.044 & 0.144 & 102.004 & 7.204 & 11.522 \\
\hline 16:30:31 & 2339.292 & -0.112 & -0.110 & 90.273 & 7.072 & 11.514 \\
\hline $16: 30: 46$ & 2361.563 & -6.703 & -6.561 & 83.930 & 7.118 & 11.48 \\
\hline
\end{tabular}


Clean Air Engineering Project \#10192

Consol Energy

AES Dresden

$\begin{array}{lrr}\text { Date: } & 3 / 28 / 2007 & \text { Run \#(Cycle \#) } \\ \text { Start Time: } & 9: 12 & 1(1) \\ \text { End Time: } & 9: 35 & \end{array}$

\begin{tabular}{|c|c|c|c|c|c|c|c|c|c|c|}
\hline $\begin{array}{l}\text { Inlet } \\
\text { Point }\end{array}$ & Time Sampled & \multicolumn{2}{|c|}{$\begin{array}{cc}\text { NOx } & \text { NOx } \\
\text { ppmdv } & \text { Port Avg }\end{array}$} & $\begin{array}{l}\text { PPM Dev } \\
\text { from Avg. }\end{array}$ & $\begin{array}{c}\mathrm{O} 2 \\
\% d v\end{array}$ & $\begin{array}{c}\text { O2 } \\
\text { Port Avg }\end{array}$ & $\begin{array}{l}\mathrm{CO2} \\
\% \mathrm{dv}\end{array}$ & $\begin{array}{c}\mathrm{CO2} \\
\text { Port Avg }\end{array}$ & $\begin{array}{c}\text { NOx } \\
\text { @ } 3 \% 02 \\
\end{array}$ & $\begin{array}{l}\text { PPM Dev } \\
\text { from Avg. }\end{array}$ \\
\hline $1-1$ & $9: 12$ & 79.9 & & -5.6 & 7.5 & & 11.6 & & 106.3 & 11.2 \\
\hline $1-2$ & $9: 16$ & 108.9 & & 23.4 & 3.0 & & 15.4 & & 108.7 & 13.6 \\
\hline $1-3$ & $9: 20$ & 91.3 & 86.0 & 5.8 & 5.0 & 5.5 & 13.7 & 13.3 & 102.7 & 7.6 \\
\hline $1-4$ & $9: 24$ & 83.2 & & -2.3 & 4.4 & & 14.2 & & 90.3 & -4.8 \\
\hline $1-5$ & $9: 28$ & 75.1 & & -10.4 & 4.8 & & 13.9 & & 83.7 & -11.5 \\
\hline $1-6$ & $9: 32$ & 77.8 & & -7.7 & 8.6 & & 10.7 & & 112.9 & 17.7 \\
\hline $2-1$ & $9: 13$ & 86.9 & & 1.4 & 5.9 & & 12.9 & & 103.7 & 8.5 \\
\hline $2-2$ & $9: 17$ & 90.6 & & 5.1 & 3.0 & & 15.4 & & 90.8 & -4.4 \\
\hline $2-3$ & $9: 21$ & 90.3 & 83.8 & 4.8 & 5.3 & 4.9 & 13.5 & 13.8 & 103.7 & 8.6 \\
\hline $2-4$ & $9: 25$ & 80.7 & & -4.8 & 3.9 & & 14.7 & & 84.9 & -10.3 \\
\hline $2-5$ & $9: 29$ & 69.6 & & -15.9 & 4.5 & & 14.1 & & 76.1 & -19.1 \\
\hline $2-6$ & 9:33 & 84.6 & & -0.9 & 6.7 & & 12.2 & & 106.9 & 11.8 \\
\hline $3-1$ & $9: 14$ & 98.5 & & 13.0 & 4.0 & & 14.5 & & 104.5 & 9.3 \\
\hline $3-2$ & $9: 18$ & 90.3 & & 4.8 & 2.9 & & 15.5 & & 89.7 & -5.4 \\
\hline $3-3$ & $9: 22$ & 104.9 & 89.9 & 19.4 & 3.3 & 4.1 & 15.2 & 14.4 & 106.4 & 11.3 \\
\hline $3-4$ & 9:26 & 83.3 & & -2.2 & 3.7 & & 14.8 & & 86.8 & -8.3 \\
\hline $3-5$ & $9: 30$ & 73.3 & & -12.2 & 4.6 & & 14.1 & & 80.4 & -14.7 \\
\hline $3-6$ & $9: 34$ & 88.9 & & 3.4 & 6.4 & & 12.5 & & 109.4 & 14.2 \\
\hline $4-1$ & $9: 15$ & 85.8 & & 0.3 & 5.7 & & 13.1 & & 100.8 & 5.7 \\
\hline $4-2$ & $9: 19$ & 92.1 & & 6.6 & 2.5 & & 15.8 & & 89.8 & -5.4 \\
\hline 4-3 & $9: 23$ & 98.0 & 82.3 & 12.5 & 3.3 & 4.4 & 15.2 & 14.2 & 99.4 & 4.3 \\
\hline $4-4$ & $9: 27$ & 79.5 & & -6.0 & 3.8 & & 14.7 & & 83.4 & -11.8 \\
\hline $4-5$ & $9: 31$ & 66.3 & & -19.2 & 4.6 & & 14.1 & & 72.6 & -22.6 \\
\hline 4-6 & 9:35 & 72.3 & & -13.2 & 6.5 & & 12.4 & & 89.8 & -5.4 \\
\hline
\end{tabular}

\begin{tabular}{|c|c|c|c|c|c|c|c|c|c|c|c|c|}
\hline \multirow[b]{2}{*}{$\begin{array}{l}\text { Outlet } \\
\text { Point }\end{array}$} & \multirow[b]{2}{*}{ Time Sampled } & \multirow{2}{*}{\multicolumn{2}{|c|}{$\begin{array}{cc}\text { NOx } & \text { NOx } \\
\text { ppmdv } & \text { Port Avg }\end{array}$}} & \multirow[b]{2}{*}{$\begin{array}{l}\text { PPM Dev } \\
\text { from Avg. }\end{array}$} & & \multirow[b]{2}{*}{$\begin{array}{l}\text { PPM Dev } \\
\text { from Avg. }\end{array}$} & \\
\hline & & & & & $\begin{array}{c}\text { O2 } \\
\% d v\end{array}$ & $\begin{array}{c}\mathrm{O} 2 \\
\text { Port Avg } \\
\end{array}$ & $\begin{array}{l}\mathrm{CO2} \\
\% \mathrm{dv} \\
\end{array}$ & $\begin{array}{c}\mathrm{CO} 2 \\
\text { Port Avg } \\
\end{array}$ & $\begin{array}{c}\mathrm{NOx} \\
\text { (Q) } 3 \% 02 \\
\end{array}$ & & \begin{tabular}{c|} 
Removal \\
Efficiency \\
\end{tabular} & $\begin{array}{c}\text { Removal Eff } \\
\text { W/ O2 correction } \\
\end{array}$ \\
\hline $1-1$ & $9: 12$ & 68.8 & & 21.5 & 4.8 & & 14.0 & & 76.3 & 22.9 & 13.9 & 28.3 \\
\hline $1-2$ & $9: 16$ & 78.7 & & 31.4 & 5.6 & & 13.3 & & 92.3 & 38.9 & 27.7 & 15.1 \\
\hline $1-3$ & $9: 20$ & 56.5 & 61.5 & 9.2 & 5.3 & 5.8 & 13.5 & 13.1 & 64.9 & 11.5 & 38.1 & 36.8 \\
\hline $1-4$ & $9: 24$ & 64.7 & & 17.4 & 4.7 & & 14.0 & & 71.5 & 18.2 & 22.2 & 20.8 \\
\hline $1-5$ & $9: 28$ & 50.8 & & 3.5 & 6.4 & & 12.6 & & 62.8 & 9.4 & 32.4 & 25.0 \\
\hline $1-6$ & 9:32 & 49.4 & & 2.1 & 8.0 & & 11.1 & & 68.8 & 15.4 & 36.5 & 39.1 \\
\hline $2-1$ & $9: 13$ & 33.3 & & -14.0 & 4.9 & & 13.9 & & 37.2 & -16.2 & 61.7 & 64.1 \\
\hline $2-2$ & $9: 17$ & 56.8 & & 9.5 & 4.8 & & 14.0 & & 63.0 & 9.6 & 37.3 & 30.6 \\
\hline $2-3$ & $9: 21$ & 70.8 & 50.5 & 23.5 & 4.7 & 5.3 & 14.1 & 13.5 & 78.3 & 24.9 & 21.6 & 24.5 \\
\hline $2-4$ & $9: 25$ & 72.9 & & 25.6 & 5.9 & & 13.0 & & 86.9 & 33.6 & 9.7 & -2.4 \\
\hline 2.5 & $9: 29$ & 25.8 & & -21.5 & 4.9 & & 13.9 & & 28.8 & -24.6 & 62.9 & 62.1 \\
\hline $2-6$ & $9: 33$ & 43.4 & & -3.9 & 6.6 & & 12.4 & & 54.4 & 1.1 & 48.7 & 49.1 \\
\hline $3-1$ & $9: 14$ & 25.1 & & -22.2 & 3.6 & & 15.0 & & 26.0 & -27.4 & 74.5 & 75.2 \\
\hline $3-2$ & $9: 18$ & 66.1 & & 18.8 & 4.4 & & 14.3 & & 71.7 & 18.3 & 26.8 & 20.1 \\
\hline $3-3$ & $9: 22$ & 76.8 & 47.3 & 29.5 & 4.4 & 4.5 & 14.3 & 14.2 & 83.1 & 29.7 & 26.8 & 22.0 \\
\hline $3-4$ & $9: 26$ & 61.1 & & 13.8 & 4.9 & & 13.9 & & 68.5 & 15.1 & 26.7 & 21.1 \\
\hline $3-5$ & 9:30 & 31.3 & & -16.0 & 4.3 & & 14.4 & & 33.8 & -19.6 & 57.3 & 58.0 \\
\hline $3-6$ & $9: 34$ & 23.4 & & -23.9 & 5.4 & & 13.5 & & 27.0 & -26.4 & 73.7 & 75.3 \\
\hline $4-1$ & $9: 15$ & 7.7 & & -39.6 & 3.4 & & 15.2 & & 7.9 & -45.5 & 91.0 & 92.2 \\
\hline 4-2 & $9: 19$ & 53.4 & & 6.1 & 2.7 & & 15.9 & & 52.4 & -0.9 & 42.0 & 41.6 \\
\hline $4-3$ & $9: 23$ & 73.7 & 30.0 & 26.4 & 3.5 & 4.0 & 15.1 & 14.7 & 75.9 & 22.6 & 24.8 & 23.6 \\
\hline $4-4$ & $9: 27$ & 35.2 & & -12.1 & 4.6 & & 14.2 & & 38.7 & -14.7 & 55.7 & 53.6 \\
\hline 4-5 & $9: 31$ & 4.8 & & -42.5 & 4.1 & & 14.5 & & 5.1 & -48.3 & 92.8 & 92.9 \\
\hline 4-6 & $9: 35$ & 5.3 & & -42.0 & 5.5 & & 13.4 & & 6.1 & -47.2 & 92.7 & 93.2 \\
\hline
\end{tabular}


CONSOL ENERGY INC.

AES GREENIDGE STATION
Client Reference No: 4700140111

CleanAir Project No:10192

Run 1: Inlet - Set 1

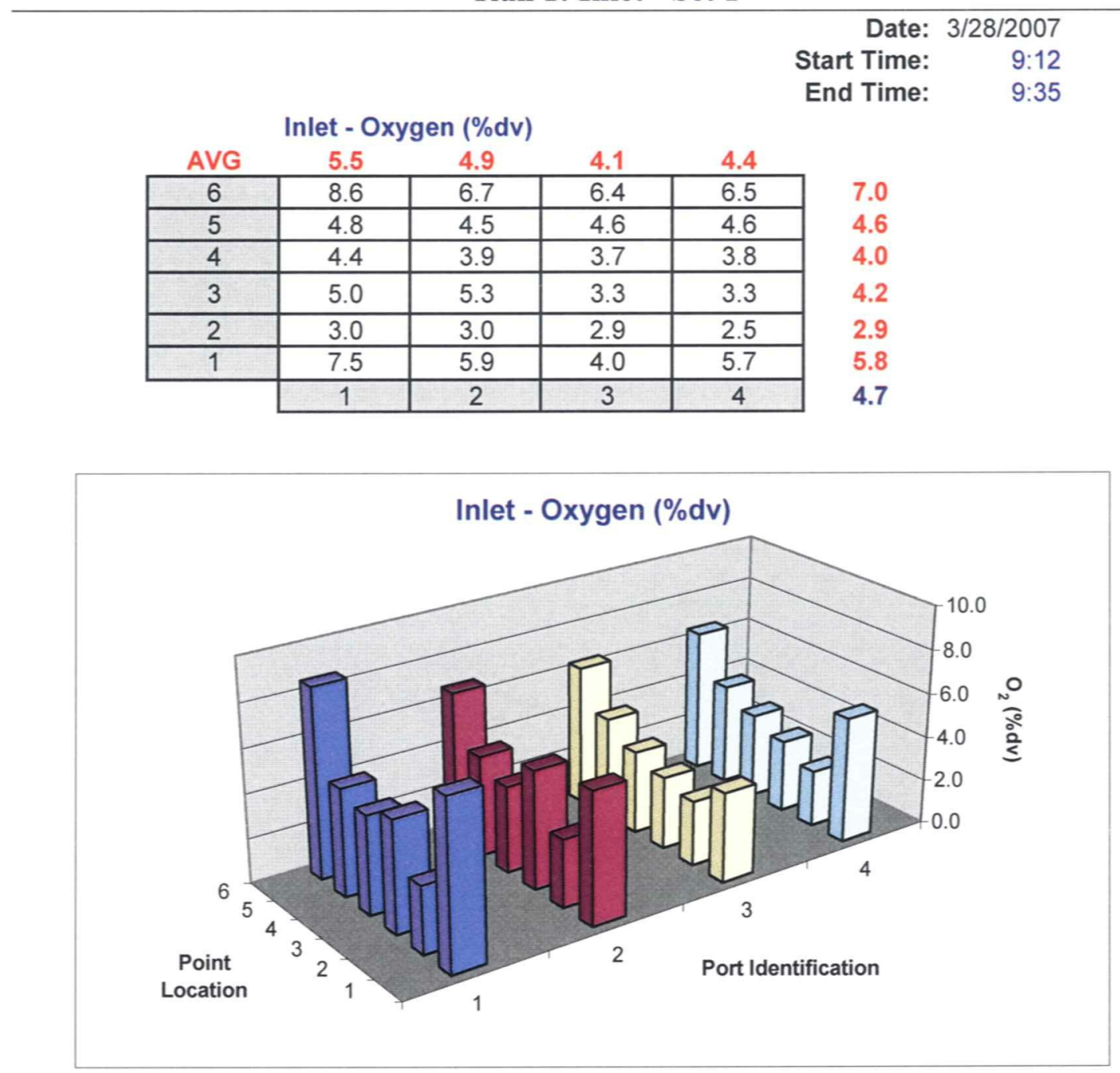


Run 1: Inlet - Set 1

Date: $3 / 28 / 2007$

Start Time: $\quad 9: 12$

End Time: $\quad 9: 35$

\begin{tabular}{|c|c|c|c|c|c|}
\hline \multicolumn{5}{|c|}{ Inlet - Carbon Dioxide (\%dv) } & \multirow[b]{3}{*}{12.0} \\
\hline AVG & 13.3 & 13.8 & 14.4 & 14.2 & \\
\hline 6 & 10.7 & 12.2 & 12.5 & 12.4 & \\
\hline 5 & 13.9 & 14.1 & 14.1 & 14.1 & 14.0 \\
\hline 4 & 14.2 & 14.7 & 14.8 & 14.7 & 14.6 \\
\hline 3 & 13.7 & 13.5 & 15.2 & 15.2 & 14.4 \\
\hline 2 & 15.4 & 15.4 & 15.5 & 15.8 & 15.5 \\
\hline 1 & 11.6 & 12.9 & 14.5 & 13.1 & 13.0 \\
\hline & 1 & 2 & 3 & 4 & 13.9 \\
\hline
\end{tabular}

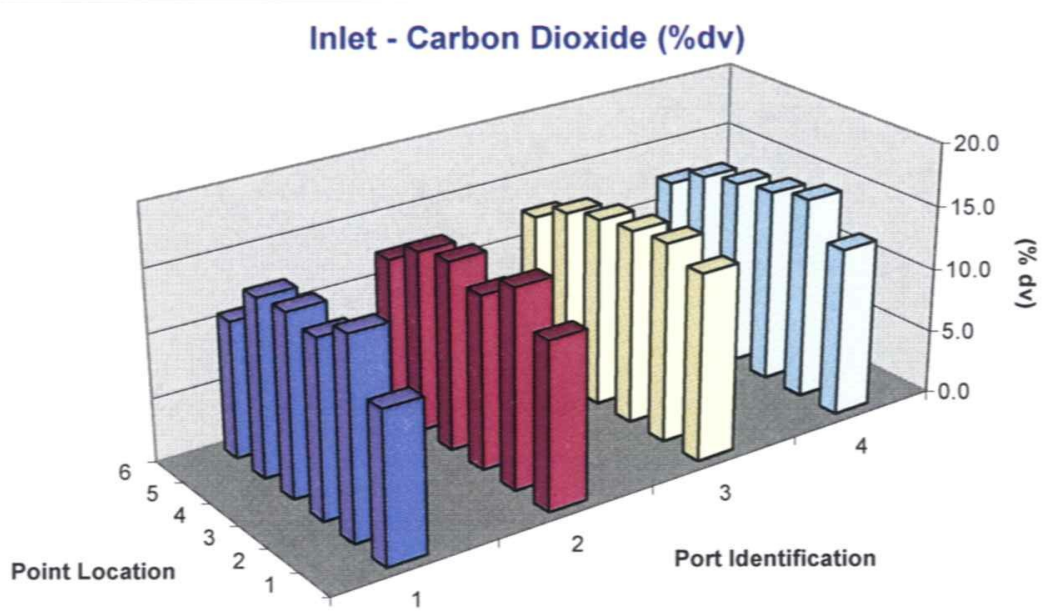


Run 1: Inlet - Set 1

Date: $3 / 28 / 2007$

Start Time: $\quad 9: 12$

End Time: $\quad 9: 35$

Inlet - Nitrogen Oxides (ppmdv), uncorrected

\begin{tabular}{|c|c|c|c|c|}
\multicolumn{1}{c}{ AVG } & 86.0 & 83.8 & 89.9 & 82.3 \\
\hline $\mathbf{6}$ & 77.8 & 84.6 & 88.9 & 72.3 \\
\hline $\mathbf{5}$ & 75.1 & 69.6 & 73.3 & 66.3 \\
\hline $\mathbf{4}$ & 83.2 & 80.7 & 83.3 & 79.5 \\
\hline $\mathbf{3}$ & 91.3 & 90.3 & 104.9 & 98.0 \\
\hline $\mathbf{2}$ & 108.9 & 90.6 & 90.3 & 92.1 \\
\hline $\mathbf{1}$ & 79.9 & 86.9 & 98.5 & 85.8 \\
\hline
\end{tabular}

80.9

71.1

81.7

96.1

95.5

87.8

85.5

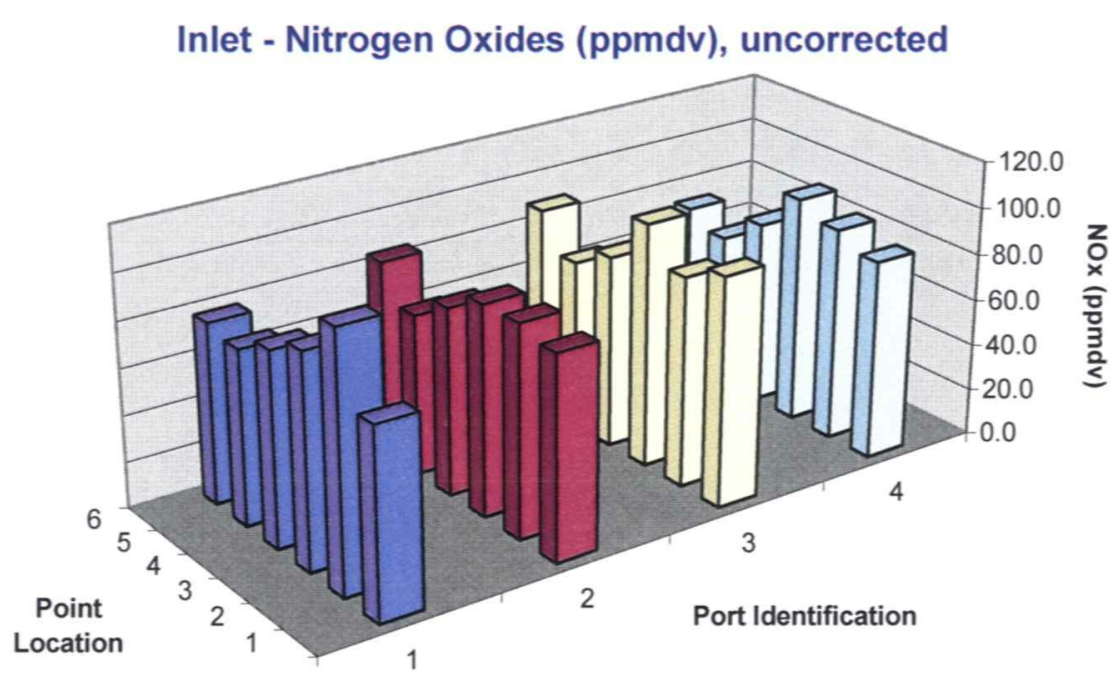


Run 1: Inlet - Set 1

Date: $3 / 28 / 2007$

Start Time: $\quad$ 9:12

End Time: $\quad 9: 35$

Inlet - Nitrogen Oxides (ppmdv @ 3\% $\mathrm{O}_{2}$ )

\begin{tabular}{|c|c|c|c|c|c|}
\hline AVG & 100.8 & 94.4 & 96.2 & 89.3 & \\
\hline 6 & 112.9 & 106.9 & 109.4 & 89.8 & 104.7 \\
\hline 5 & 83.7 & 76.1 & 80.4 & 72.6 & 78.2 \\
\hline 4 & 90.3 & 84.9 & 86.8 & 83.4 & 86.3 \\
\hline 3 & 102.7 & 103.7 & 106.4 & 99.4 & 103.1 \\
\hline 2 & 108.7 & 90.8 & 89.7 & 89.8 & 94.8 \\
\hline \multirow[t]{2}{*}{1} & 106.3 & 103.7 & 104.5 & 100.8 & 103.8 \\
\hline & 1 & 2 & 3 & 4 & 95.2 \\
\hline
\end{tabular}

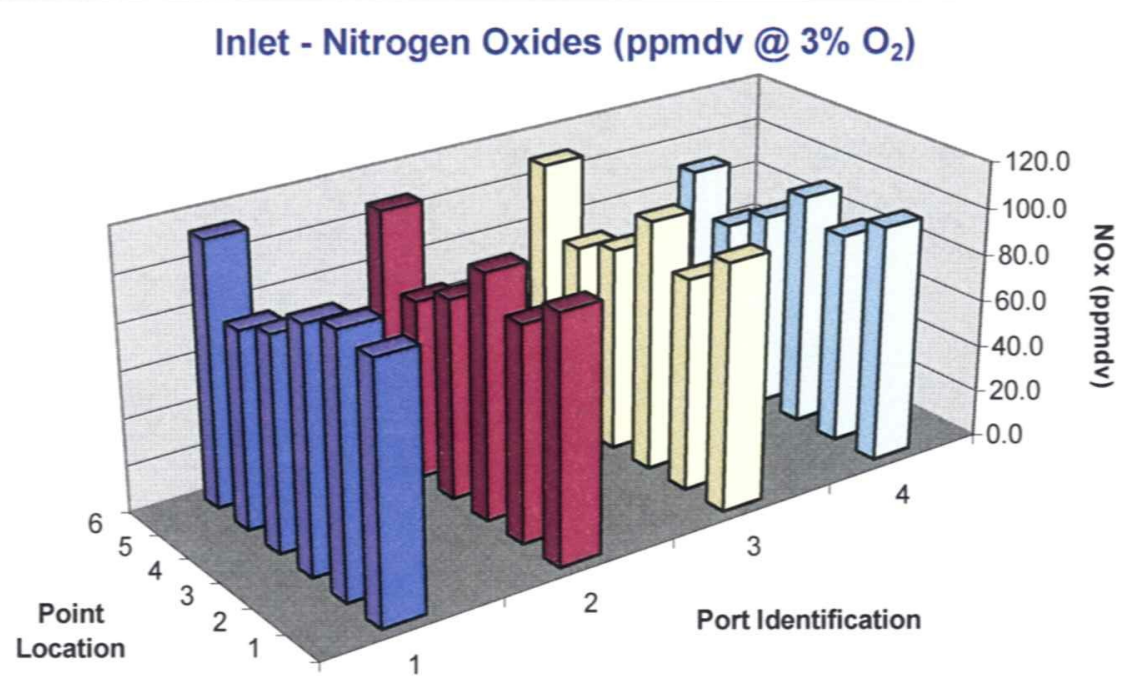


Run 1: Outlet - Set 1

Date: $3 / 28 / 2007$

$\begin{array}{cc}\text { Start Time: } & 9: 12 \\ \text { End Time: } & 9: 35\end{array}$

\begin{tabular}{|c|c|c|c|c|c|}
\hline \multicolumn{5}{|c|}{ Outlet - Oxygen (\%dv) } & \multirow[b]{3}{*}{6.4} \\
\hline AVG & 5.8 & 5.3 & 4.5 & 4.0 & \\
\hline 6 & 8.0 & 6.6 & 5.4 & 5.5 & \\
\hline 5 & 6.4 & 4.9 & 4.3 & 4.1 & 4.9 \\
\hline 4 & 4.7 & 5.9 & 4.9 & 4.6 & 5.0 \\
\hline 3 & 5.3 & 4.7 & 4.4 & 3.5 & 4.5 \\
\hline 2 & 5.6 & 4.8 & 4.4 & 2.7 & 4.4 \\
\hline 1 & 4.8 & 4.9 & 3.6 & 3.4 & 4.2 \\
\hline & 1 & 2 & 3 & 4 & 4.9 \\
\hline
\end{tabular}

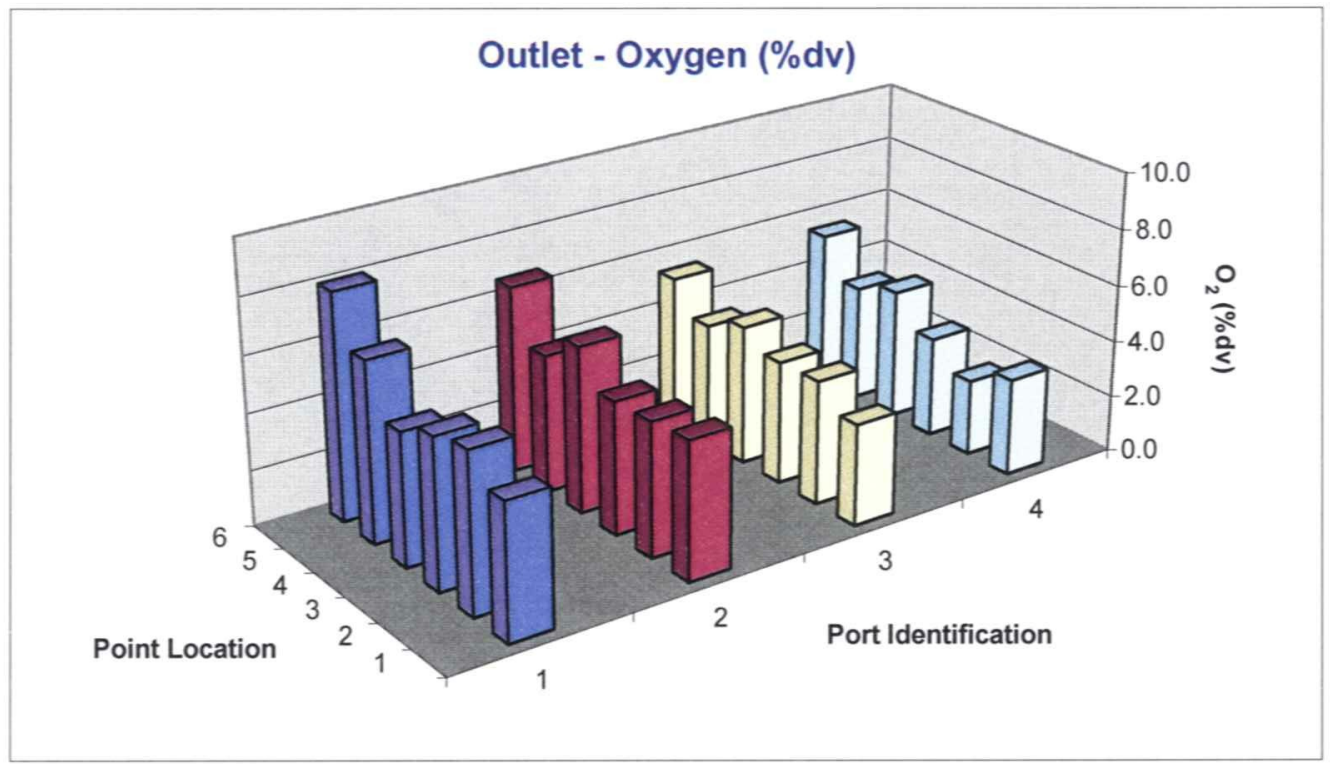


Run 1: Outlet - Set 1

Date: $3 / 28 / 2007$

Start Time: $\quad 9: 12$

End Time: $\quad 9: 35$

Outlet - Carbon Dioxide (\%dv)

\begin{tabular}{|c|c|c|c|c|}
\hline AVG & 13.1 & 13.5 & 14.2 & 14.7 \\
\hline $\mathbf{6}$ & 11.1 & 12.4 & 13.5 & 13.4 \\
\hline $\mathbf{5}$ & 12.6 & 13.9 & 14.4 & 14.5 \\
\hline $\mathbf{4}$ & 14.0 & 13.0 & 13.9 & 14.2 \\
\hline $\mathbf{3}$ & 13.5 & 14.1 & 14.3 & 15.1 \\
\hline $\mathbf{2}$ & 13.3 & 14.0 & 14.3 & 15.9 \\
\hline $\mathbf{1}$ & 14.0 & 13.9 & 15.0 & 15.2 \\
\hline & $\mathbf{1}$ & $\mathbf{2}$ & $\mathbf{3}$ & $\mathbf{4}$ \\
\hline
\end{tabular}

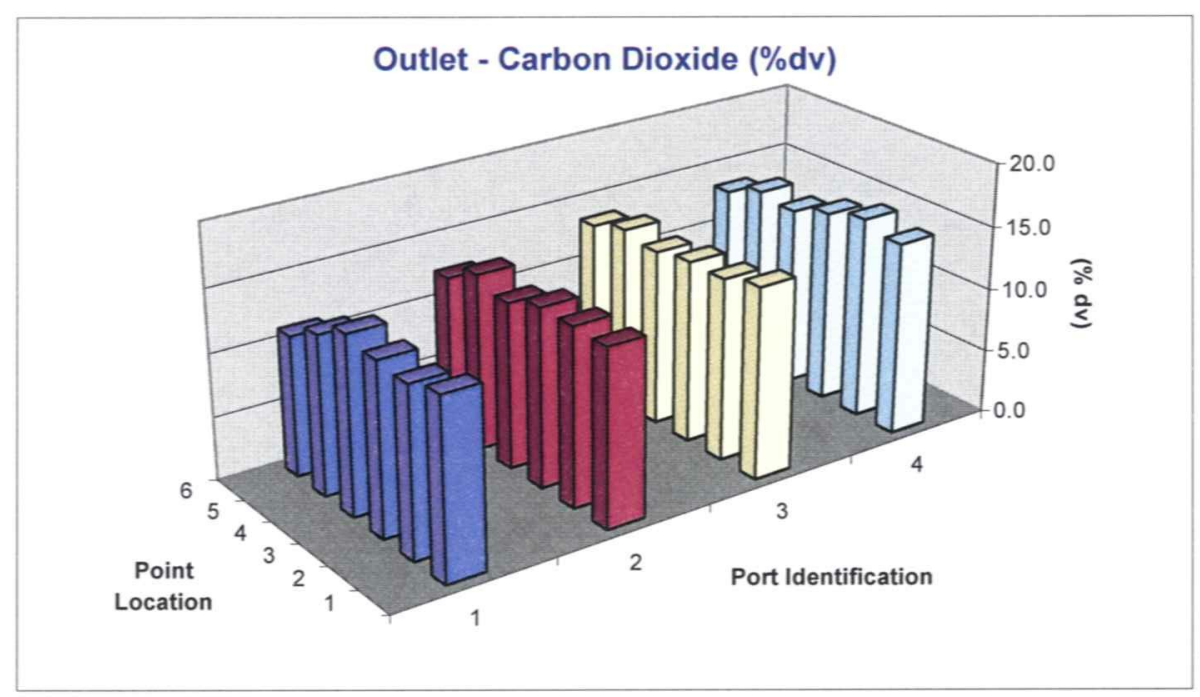


Date: $3 / 28 / 2007$

Start Time: $\quad 9: 12$

End Time: $\quad 9: 35$

Outlet - Nitrogen Oxides (ppmdv), uncorrected

\begin{tabular}{|c|c|c|c|c|}
\multicolumn{1}{c}{ AVG } & 61.5 & 50.5 & 47.3 & 30.0 \\
\hline $\mathbf{6}$ & 49.4 & 43.4 & 23.4 & 5.3 \\
\hline $\mathbf{5}$ & 50.8 & 25.8 & 31.3 & 4.8 \\
\hline $\mathbf{4}$ & 64.7 & 72.9 & 61.1 & 35.2 \\
\hline $\mathbf{3}$ & 56.5 & 70.8 & 76.8 & 73.7 \\
\hline $\mathbf{2}$ & 78.7 & 56.8 & 66.1 & 53.4 \\
\hline $\mathbf{1}$ & 68.8 & 33.3 & 25.1 & 7.7 \\
\hline & $\mathbf{1}$ & $\mathbf{2}$ & $\mathbf{3}$ & $\mathbf{4}$ \\
\hline
\end{tabular}

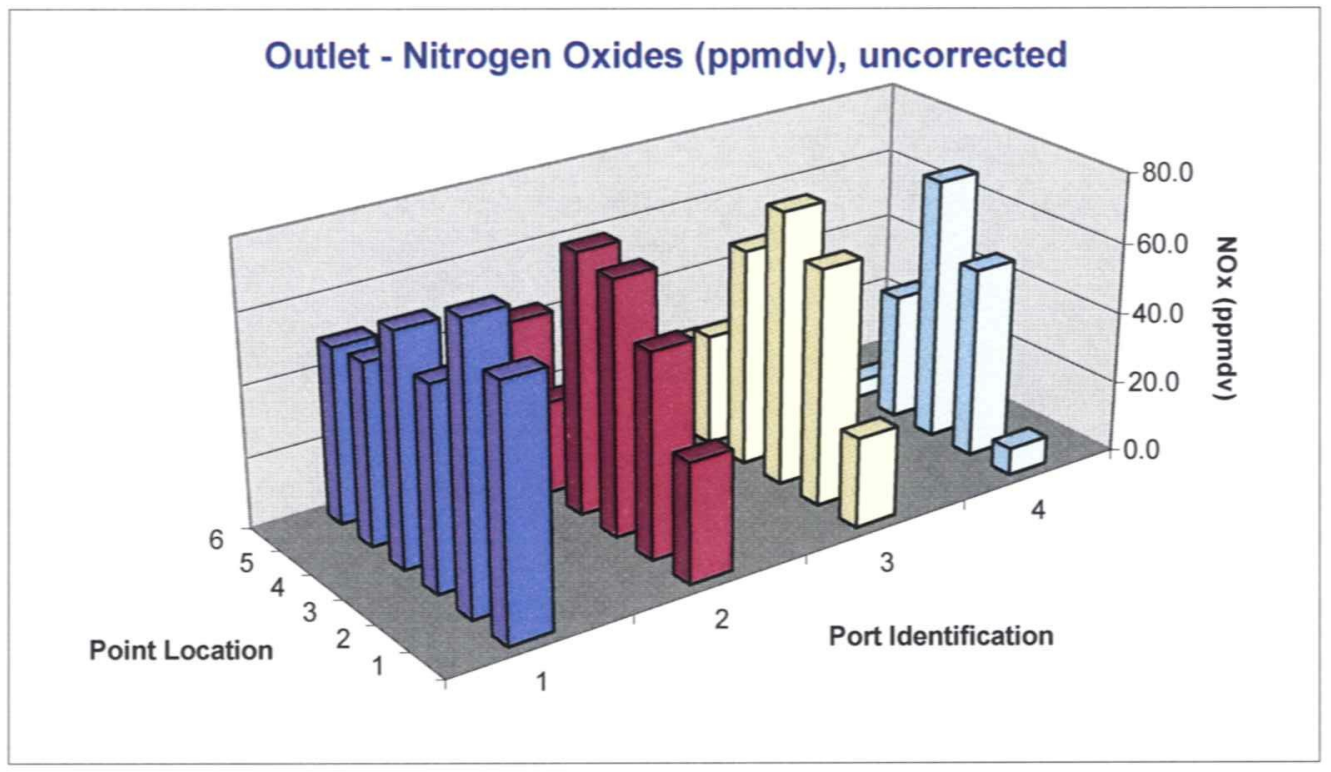


Date: $3 / 28 / 2007$

Start Time: $\quad 9: 12$

End Time: $\quad 9: 35$

Outlet - Nitrogen Oxides (ppmdv @ 3\% O

\begin{tabular}{|c|c|c|c|c|}
\multicolumn{1}{c}{ AVG } & 72.7 & 58.1 & 51.7 & 31.0 \\
\hline $\mathbf{6}$ & 68.8 & 54.4 & 27.0 & 6.1 \\
\hline $\mathbf{5}$ & 62.8 & 28.8 & 33.8 & 5.1 \\
\hline $\mathbf{4}$ & 71.5 & 86.9 & 68.5 & 38.7 \\
\hline $\mathbf{3}$ & 64.9 & 78.3 & 83.1 & 75.9 \\
\hline $\mathbf{2}$ & 92.3 & 63.0 & 71.7 & 52.4 \\
\hline $\mathbf{1}$ & 76.3 & 37.2 & 26.0 & 7.9 \\
\hline & $\mathbf{1}$ & $\mathbf{2}$ & $\mathbf{3}$ & $\mathbf{4}$ \\
\hline
\end{tabular}

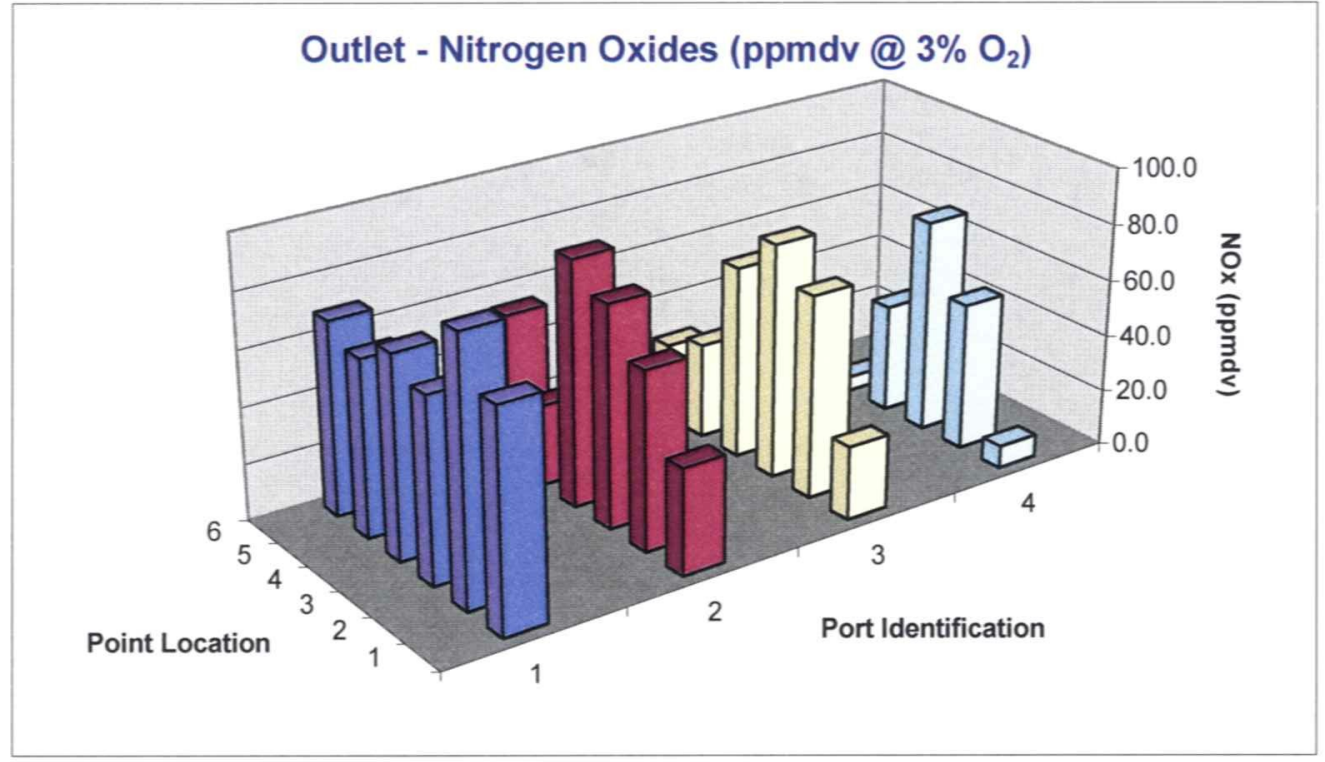


Client Reference No: 4700140111

CleanAir Project No:10192

Run 1: Outlet - Set 1

Date: $3 / 28 / 2007$

Start Time: $\quad 9: 12$

End Time: $\quad 9: 35$

Removal Efficiency (ppmdv @ 3\% $\mathrm{O}_{2}$ )

\begin{tabular}{|c|c|c|c|c|}
\multicolumn{1}{c}{ AVG } & $27.5 \%$ & $38.0 \%$ & $45.3 \%$ & $66.2 \%$ \\
\hline $\mathbf{6}$ & $39.1 \%$ & $49.1 \%$ & $75.3 \%$ & $93.2 \%$ \\
\hline $\mathbf{5}$ & $25.0 \%$ & $62.1 \%$ & $58.0 \%$ & $92.9 \%$ \\
\hline $\mathbf{4}$ & $20.8 \%$ & $-2.4 \%$ & $21.1 \%$ & $53.6 \%$ \\
\hline $\mathbf{3}$ & $36.8 \%$ & $24.5 \%$ & $22.0 \%$ & $23.6 \%$ \\
\hline $\mathbf{2}$ & $15.1 \%$ & $30.6 \%$ & $20.1 \%$ & $41.6 \%$ \\
\hline $\mathbf{1}$ & $28.3 \%$ & $64.1 \%$ & $75.2 \%$ & $92.2 \%$ \\
\hline & $\mathbf{1}$ & $\mathbf{2}$ & $\mathbf{3}$ & $\mathbf{4}$ \\
\hline
\end{tabular}

$64.2 \%$

$59.5 \%$

$23.3 \%$

$26.7 \%$

$26.9 \%$

$64.9 \%$

$44.3 \%$

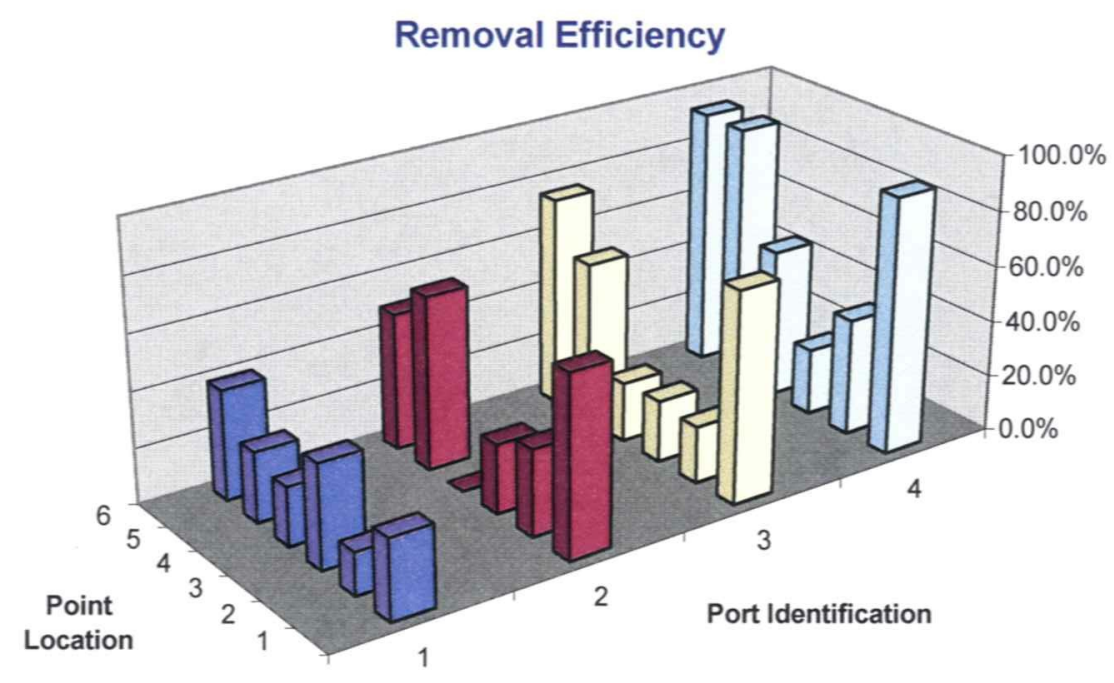


Clean Air Engineering Project \#10192

Consol Energy

AES Dresden

$\begin{array}{lrr}\text { Date: } & 3 / 28 / 2007 & \text { Run \# (Cycle \#) } 1(2) \\ \text { Start Time: } & 9: 36 & \\ \text { End Time: } & 9: 59 & \end{array}$

\begin{tabular}{|c|c|c|c|c|c|c|c|c|c|c|}
\hline $\begin{array}{l}\text { Inlet } \\
\text { Point }\end{array}$ & Time Sampled & $\begin{array}{c}\text { NOx } \\
\text { ppmdv }\end{array}$ & $\begin{array}{c}\text { NOx } \\
\text { Port Avg }\end{array}$ & $\begin{array}{l}\text { PPM Dev } \\
\text { from Avg. }\end{array}$ & $\begin{array}{c}\text { O2 } \\
\% d v\end{array}$ & $\begin{array}{c}\mathrm{O} 2 \\
\text { Port Avg }\end{array}$ & $\begin{array}{l}\text { CO2 } \\
\% d v\end{array}$ & $\begin{array}{c}\mathrm{CO} 2 \\
\text { Port Avg }\end{array}$ & $\begin{array}{c}\mathrm{NOx} \\
\text { (1) 3\% O2 }\end{array}$ & $\begin{array}{l}\text { PPM Dev } \\
\text { from Avg. }\end{array}$ \\
\hline $1-1$ & $9: 36$ & 77.9 & & -5.5 & 7.6 & & 11.5 & & 104.5 & 11.6 \\
\hline $1-2$ & $9: 40$ & 105.1 & & 21.7 & 2.9 & & 15.5 & & 104.5 & 11.7 \\
\hline $1-3$ & $9: 44$ & 89.7 & 84.3 & 6.3 & 5.1 & 5.5 & 13.7 & 13.3 & 101.4 & 8.5 \\
\hline $1-4$ & $9: 48$ & 83.2 & & -0.2 & 4.4 & & 14.3 & & 90.0 & -2.8 \\
\hline $1-5$ & 9:52 & 73.9 & & -9.5 & 4.8 & & 14.0 & & 81.9 & -10.9 \\
\hline $1-6$ & $9: 56$ & 76.0 & & -7.4 & 8.7 & & 10.6 & & 111.1 & 18.3 \\
\hline $2-1$ & $9: 37$ & 83.1 & & -0.3 & 6.0 & & 12.8 & & 99.8 & 6.9 \\
\hline $2-2$ & $9: 41$ & 85.4 & & 2.0 & 2.9 & & 15.5 & & 85.1 & -7.8 \\
\hline $2-3$ & $9: 45$ & 88.0 & 80.8 & 4.6 & 5.3 & 4.9 & 13.5 & 13.8 & 100.8 & 8.0 \\
\hline $2-4$ & $9: 49$ & 79.0 & & -4.4 & 3.9 & & 14.7 & & 83.1 & -9.8 \\
\hline $2-5$ & $9: 53$ & 66.7 & & -16.7 & 4.5 & & 14.1 & & 72.9 & -19.9 \\
\hline $2-6$ & $9: 57$ & 82.3 & & -1.1 & 6.9 & & 12.1 & & 104.9 & 12.0 \\
\hline $3-1$ & $9: 38$ & 96.2 & & 12.8 & 4.0 & & 14.5 & & 101.9 & 9.0 \\
\hline 3-2 & $9: 42$ & 87.4 & & 4.0 & 2.9 & & 15.5 & & 87.0 & -5.8 \\
\hline $3-3$ & $9: 46$ & 102.8 & 87.5 & 19.4 & 3.3 & 4.1 & 15.1 & 14.5 & 104.6 & 11.8 \\
\hline 3-4 & $9: 50$ & 81.3 & & -2.1 & 3.6 & & 14.9 & & 84.1 & -8.7 \\
\hline $3-5$ & $9: 54$ & 70.9 & & -12.5 & 4.5 & & 14.2 & & 77.1 & -15.7 \\
\hline $3-6$ & $9: 58$ & 86.4 & & 3.0 & 6.3 & & 12.6 & & 105.6 & 12.7 \\
\hline $4-1$ & $9: 39$ & 83.1 & & -0.3 & 5.7 & & 13.1 & & 97.7 & 4.9 \\
\hline $4-2$ & $9: 43$ & 90.6 & & 7.2 & 2.6 & & 15.8 & & 88.6 & -4.2 \\
\hline $4-3$ & $9: 47$ & 97.4 & 81.2 & 14.0 & 3.2 & 4.4 & 15.2 & 14.2 & 98.4 & 5.6 \\
\hline 4-4 & $9: 51$ & 79.3 & & -4.1 & 3.7 & & 14.8 & & 82.6 & -10.3 \\
\hline $4-5$ & $9: 55$ & 65.5 & & -17.9 & 4.6 & & 14.1 & & 71.7 & -21.1 \\
\hline $4-6$ & $9: 59$ & 71.2 & & -12.2 & 6.6 & & 12.4 & & 89.1 & -3.7 \\
\hline
\end{tabular}

\begin{tabular}{|c|c|c|c|c|c|c|c|c|c|c|c|c|c|}
\hline \multirow[b]{2}{*}{$\begin{array}{l}\text { Outlet } \\
\text { Point }\end{array}$} & \multirow[b]{2}{*}{ Nidtllept } & \multirow[b]{2}{*}{ Time Sampled } & \multirow{2}{*}{\multicolumn{2}{|c|}{$\begin{array}{cc}\text { NOx } & \text { NOx } \\
\text { ppmdv } & \text { Port Avg } \\
\end{array}$}} & \multirow[b]{2}{*}{$\begin{array}{l}\text { PPM Dev } \\
\text { from Avg. }\end{array}$} & & \multirow[b]{2}{*}{$\begin{array}{l}\text { PPM Dev } \\
\text { from Avg. }\end{array}$} & \\
\hline & & & & & & $\begin{array}{c}\text { O2 } \\
\text { \%dv }\end{array}$ & $\begin{array}{c}\mathrm{O} 2 \\
\text { Port Avg }\end{array}$ & $\begin{array}{l}\mathrm{CO2} \\
\% \mathrm{dv} \\
\end{array}$ & $\begin{array}{c}\mathrm{CO} 2 \\
\text { Port Avg }\end{array}$ & $\begin{array}{c}\text { NOx } \\
\text { (Q) 3\% O2 } \\
\end{array}$ & & $\begin{array}{l}\text { Removal } \\
\text { Efficiency }\end{array}$ & $\begin{array}{c}\text { Removal Eff } \\
\text { w/ } \mathrm{O} 2 \text { correction }\end{array}$ \\
\hline $1-1$ & & $9: 36$ & 67.6 & & 20.1 & 4.9 & & 13.8 & & 75.8 & 22.3 & 13.2 & 27.4 \\
\hline $1-2$ & & $9: 40$ & 79.9 & & 32.4 & 5.5 & & 13.4 & & 92.9 & 39.3 & 24.0 & 11.1 \\
\hline $1-3$ & & 9:44 & 57.0 & 62.1 & 9.5 & 5.4 & 5.8 & 13.5 & 13.1 & 65.6 & 12.1 & 36.5 & 35.3 \\
\hline $1-4$ & & $9: 48$ & 66.8 & & 19.3 & 4.6 & & 14.2 & & 73.4 & 19.8 & 19.7 & 18.5 \\
\hline $1-5$ & & $9: 52$ & 52.2 & & 4.7 & 6.4 & & 12.6 & & 64.3 & 10.8 & 29.4 & 21.5 \\
\hline $1-6$ & & 9:56 & 49.0 & & 1.5 & 8.0 & & 11.1 & & 68.2 & 14.6 & 35.5 & 38.7 \\
\hline $2-1$ & & 9:37 & 34.8 & & -12.7 & 5.0 & & 13.8 & & 39.1 & -14.4 & 58.1 & 60.8 \\
\hline $2-2$ & & $9: 41$ & 57.0 & & 9.5 & 4.6 & & 14.2 & & 62.6 & 9.0 & 33.3 & 26.4 \\
\hline $2-3$ & & $9: 45$ & 69.6 & 50.0 & 22.1 & 4.7 & 5.3 & 14.1 & 13.5 & 76.9 & 23.4 & 20.9 & 23.7 \\
\hline $2-4$ & & $9: 49$ & 71.5 & & 24.0 & 6.0 & & 13.0 & & 85.6 & 32.1 & 9.5 & -3.0 \\
\hline $2-5$ & & $9: 53$ & 24.4 & & -23.1 & 4.9 & & 13.9 & & 27.3 & -26.2 & 63.4 & 62.5 \\
\hline $2-6$ & & 9:57 & 42.9 & & -4.6 & 6.7 & & 12.3 & & 54.0 & 0.5 & 47.9 & 48.5 \\
\hline $3-1$ & & $9: 38$ & 25.1 & & -22.4 & 3.7 & & 14.8 & & 26.2 & -27.4 & 73.9 & 74.3 \\
\hline $3-2$ & & $9: 42$ & 69.8 & & 22.3 & 4.3 & & 14.4 & & 75.2 & 21.7 & 20.1 & 13.6 \\
\hline 3-3 & & $9: 46$ & 79.9 & 47.9 & 32.4 & 4.3 & 4.5 & 14.4 & 14.2 & 86.2 & 32.6 & 22.3 & 17.6 \\
\hline $3-4$ & & $9: 50$ & 59.4 & & 11.9 & 5.0 & & 13.8 & & 66.7 & 13.2 & 26.9 & 20.7 \\
\hline 3-5 & & $9: 54$ & 30.1 & & -17.4 & 4.3 & & 14.4 & & 32.4 & -21.2 & 57.5 & 58.0 \\
\hline $3-6$ & & $9: 58$ & 22.9 & & -24.6 & 5.6 & & 13.3 & & 26.8 & -26.7 & 73.5 & 74.6 \\
\hline $4-1$ & & $9: 39$ & 7.7 & & -39.8 & 3.6 & & 15.0 & & 8.0 & -45.6 & 90.7 & 91.8 \\
\hline $4-2$ & & $9: 43$ & 51.8 & & 4.3 & 2.7 & & 15.8 & & 51.1 & -2.5 & 42.8 & 42.4 \\
\hline $4-3$ & & $9: 47$ & 73.8 & 29.9 & 26.3 & 3.6 & 4.0 & 15.1 & 14.7 & 76.1 & 22.6 & 24.2 & 22.7 \\
\hline 4-4 & & $9: 51$ & 36.7 & & -10.8 & 4.5 & & 14.2 & & 40.1 & -13.4 & 53.7 & 51.4 \\
\hline $4-5$ & & $9: 55$ & 4.5 & & -43.0 & 4.1 & & 14.6 & & 4.8 & -48.8 & 93.1 & 93.3 \\
\hline $4-6$ & & $9: 59$ & 5.1 & & -42.4 & 5.6 & & 13.3 & & 6.0 & -47.6 & 92.8 & 93.3 \\
\hline
\end{tabular}


Run 1: Inlet - Set 2

Date: $3 / 28 / 2007$

Start Time: $\quad 9: 36$

End Time: $\quad 9: 59$

Inlet - Oxygen (\%dv)

\begin{tabular}{|c|c|c|c|c|}
\hline AVG & 5.5 & 4.9 & 4.1 & 4.4 \\
\hline 6 & 8.7 & 6.9 & 6.3 & 6.6 \\
\hline 5 & 4.8 & 4.5 & 4.5 & 4.6 \\
\hline 4 & 4.4 & 3.9 & 3.6 & 3.7 \\
\hline 3 & 5.1 & 5.3 & 3.3 & 3.2 \\
\hline 2 & 2.9 & 2.9 & 2.9 & 2.6 \\
\hline 1 & 7.6 & 6.0 & 4.0 & 5.7 \\
\hline & 1 & 2 & 3 & 4 \\
\hline
\end{tabular}

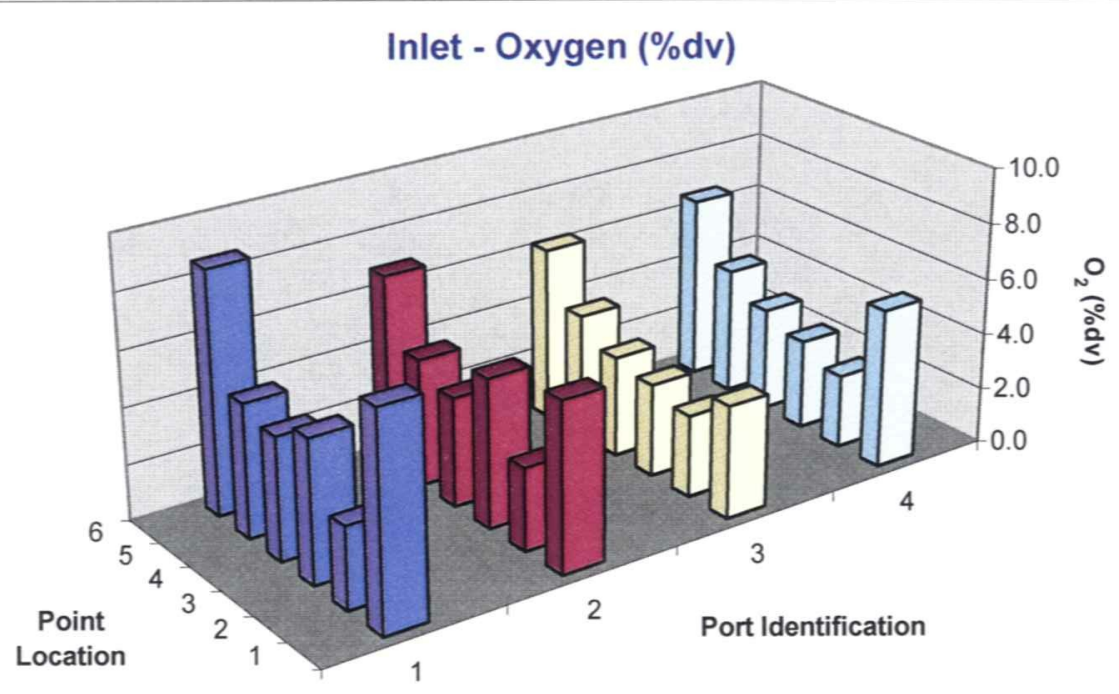


Run 1: Inlet - Set 2

Date: $3 / 28 / 2007$

$\begin{array}{rr}\text { Start Time: } & 9: 36 \\ \text { End Time: } & 9: 59\end{array}$

Inlet - Carbon Dioxide (\%dv)

\begin{tabular}{|c|c|c|c|c|}
\hline AVG & 13.3 & 13.8 & 14.5 & 14.2 \\
\hline 6 & 10.6 & 12.1 & 12.6 & 12.4 \\
\hline 5 & 14.0 & 14.1 & 14.2 & 14.1 \\
\hline 4 & 14.3 & 14.7 & 14.9 & 14.8 \\
\hline 3 & 13.7 & 13.5 & 15.1 & 15.2 \\
\hline 2 & 15.5 & 15.5 & 15.5 & 15.8 \\
\hline \multirow[t]{2}{*}{1} & 11.5 & 12.8 & 14.5 & 13.1 \\
\hline & 1 & 2 & 3 & 4 \\
\hline
\end{tabular}

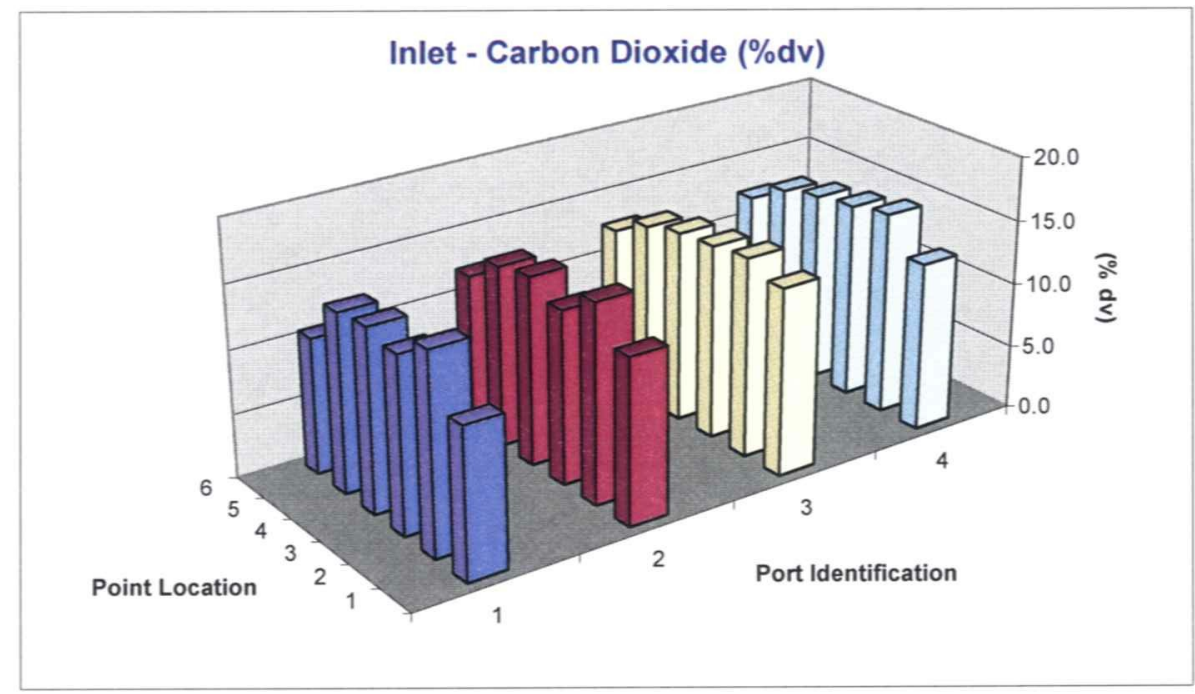


Run 1: Inlet - Set 2

Date: $3 / 28 / 2007$

Start Time: $\quad 9: 36$

End Time: $\quad 9: 59$

Inlet - Nitrogen Oxides (ppmdv), uncorrected

\begin{tabular}{|c|c|c|c|c|}
\multicolumn{1}{|c|}{ AVG } & 84.3 & 80.8 & 87.5 & 81.2 \\
\hline $\mathbf{6}$ & 76.0 & 82.3 & 86.4 & 71.2 \\
\hline $\mathbf{5}$ & 73.9 & 66.7 & 70.9 & 65.5 \\
\hline $\mathbf{4}$ & 83.2 & 79.0 & 81.3 & 79.3 \\
\hline $\mathbf{3}$ & 89.7 & 88.0 & 102.8 & 97.4 \\
\hline $\mathbf{2}$ & 105.1 & 85.4 & 87.4 & 90.6 \\
\hline $\mathbf{1}$ & 77.9 & 83.1 & 96.2 & 83.1 \\
\hline & $\mathbf{1}$ & $\mathbf{2}$ & $\mathbf{3}$ & $\mathbf{4}$ \\
\hline
\end{tabular}

79.0

69.3

80.7

94.5

92.1

85.1

83.4

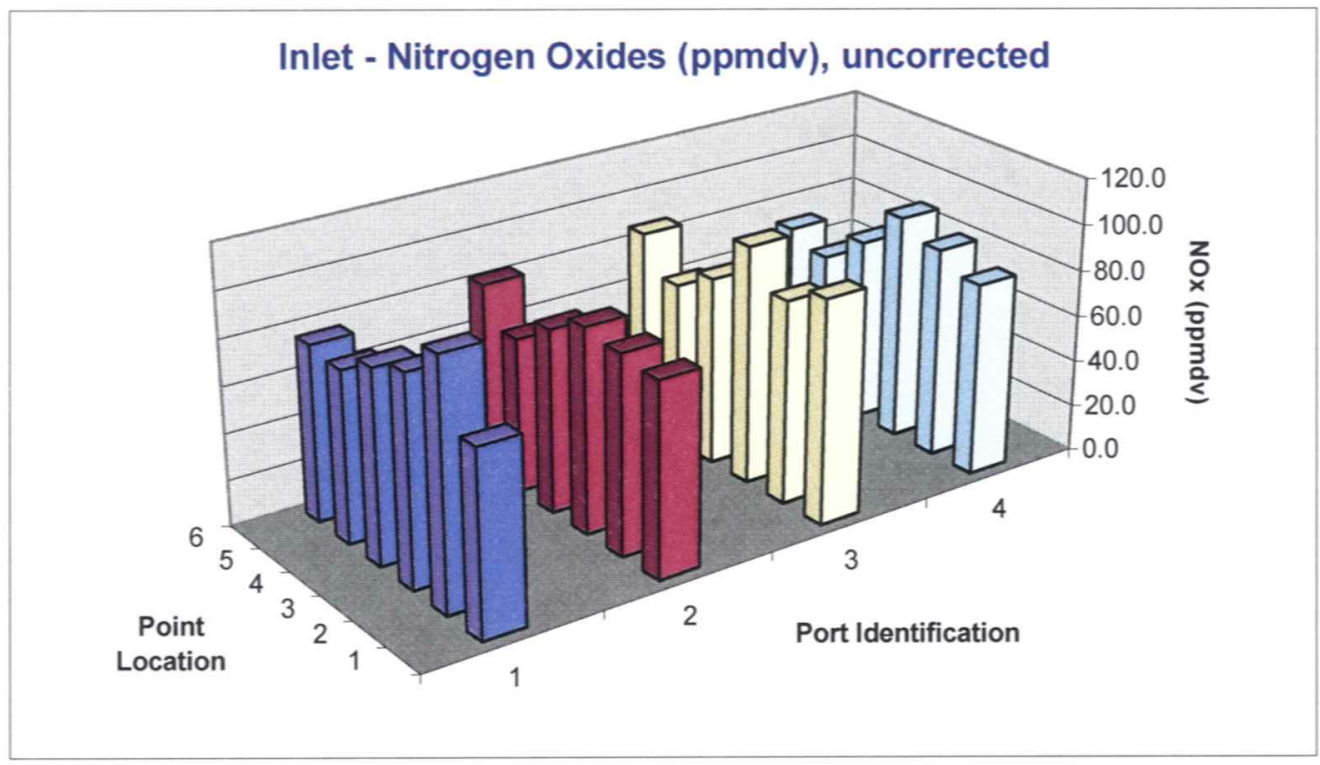


Run 1: Inlet - Set 2

Date: $3 / 28 / 2007$

Start Time: $\quad$ 9:36

End Time: $\quad 9: 59$

Inlet - Nitrogen Oxides (ppmdv @ $3 \% \mathrm{O}_{2}$ )

\begin{tabular}{|c|c|c|c|c|c|}
\hline AVG & 98.9 & 91.1 & 93.4 & 88.0 & \\
\hline 6 & 111.1 & 104.9 & 105.6 & 89.1 & 102.7 \\
\hline 5 & 81.9 & 72.9 & 77.1 & 71.7 & 75.9 \\
\hline 4 & 90.0 & 83.1 & 84.1 & 82.6 & 85.0 \\
\hline 3 & 101.4 & 100.8 & 104.6 & 98.4 & 101.3 \\
\hline 2 & 104.5 & 85.1 & 87.0 & 88.6 & 91.3 \\
\hline 1 & 104.5 & 99.8 & 101.9 & 97.7 & 101.0 \\
\hline & 1 & 2 & 3 & 4 & 92.9 \\
\hline
\end{tabular}

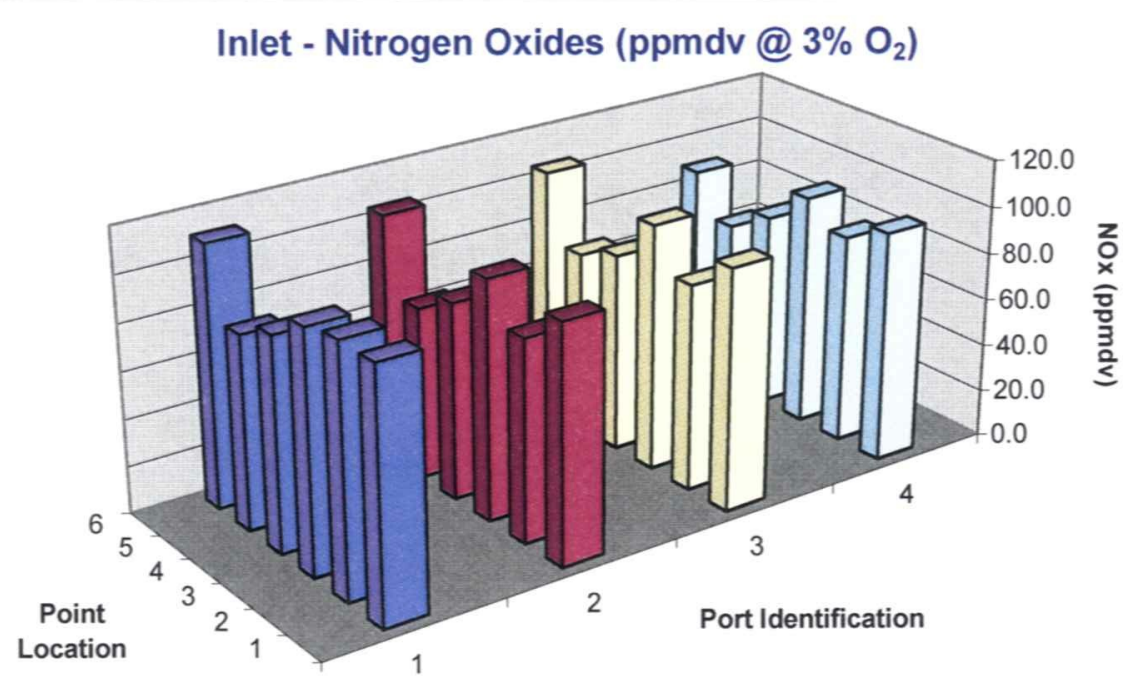


Client Reference No: 4700140111

CleanAir Project No:10192

Run 1: Outlet - Set 2

Date: $3 / 28 / 2007$

Start Time: $\quad 9: 36$

End Time: $\quad 9: 59$

\begin{tabular}{|c|c|c|c|c|c|}
\hline \multicolumn{5}{|c|}{ Outlet - Oxygen (\%dv) } & \\
\hline AVG & 5.8 & 5.3 & 4.5 & 4.0 & \\
\hline 6 & 8.0 & 6.7 & 5.6 & 5.6 & 6.5 \\
\hline 5 & 6.4 & 4.9 & 4.3 & 4.1 & 4.9 \\
\hline 4 & 4.6 & 6.0 & 5.0 & 4.5 & 5.0 \\
\hline 3 & 5.4 & 4.7 & 4.3 & 3.6 & 4.5 \\
\hline 2 & 5.5 & 4.6 & 4.3 & 2.7 & 4.3 \\
\hline 1 & 4.9 & 5.0 & 3.7 & 3.6 & 4.3 \\
\hline & 1 & 2 & 3 & $\overline{4}$ & 4.9 \\
\hline
\end{tabular}

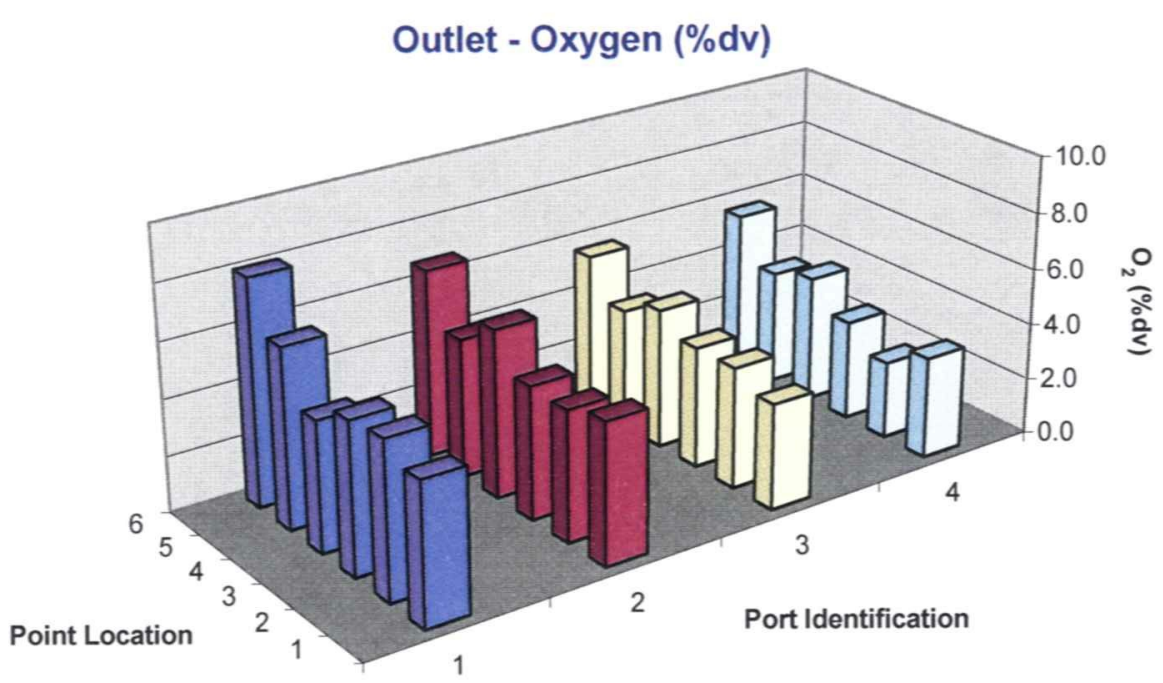


Run 1: Outlet - Set 2

Date: $3 / 28 / 2007$

Start Time: $\quad 9: 36$

End Time: $\quad 9: 59$

Outlet - Carbon Dioxide (\%dv)

\begin{tabular}{|c|c|c|c|c|}
\multicolumn{1}{|c|}{ AVG } & 13.1 & 13.5 & 14.2 & 14.7 \\
\hline $\mathbf{6}$ & 11.1 & 12.3 & 13.3 & 13.3 \\
\hline $\mathbf{5}$ & 12.6 & 13.9 & 14.4 & 14.6 \\
\hline $\mathbf{4}$ & 14.2 & 13.0 & 13.8 & 14.2 \\
\hline $\mathbf{3}$ & 13.5 & 14.1 & 14.4 & 15.1 \\
\hline $\mathbf{2}$ & 13.4 & 14.2 & 14.4 & 15.8 \\
\hline $\mathbf{1}$ & 13.8 & 13.8 & 14.8 & 15.0 \\
\hline & $\mathbf{1}$ & $\mathbf{2}$ & $\mathbf{3}$ & $\mathbf{4}$ \\
\hline
\end{tabular}

12.5

13.9

13.8

14.3

14.4

14.4

13.9

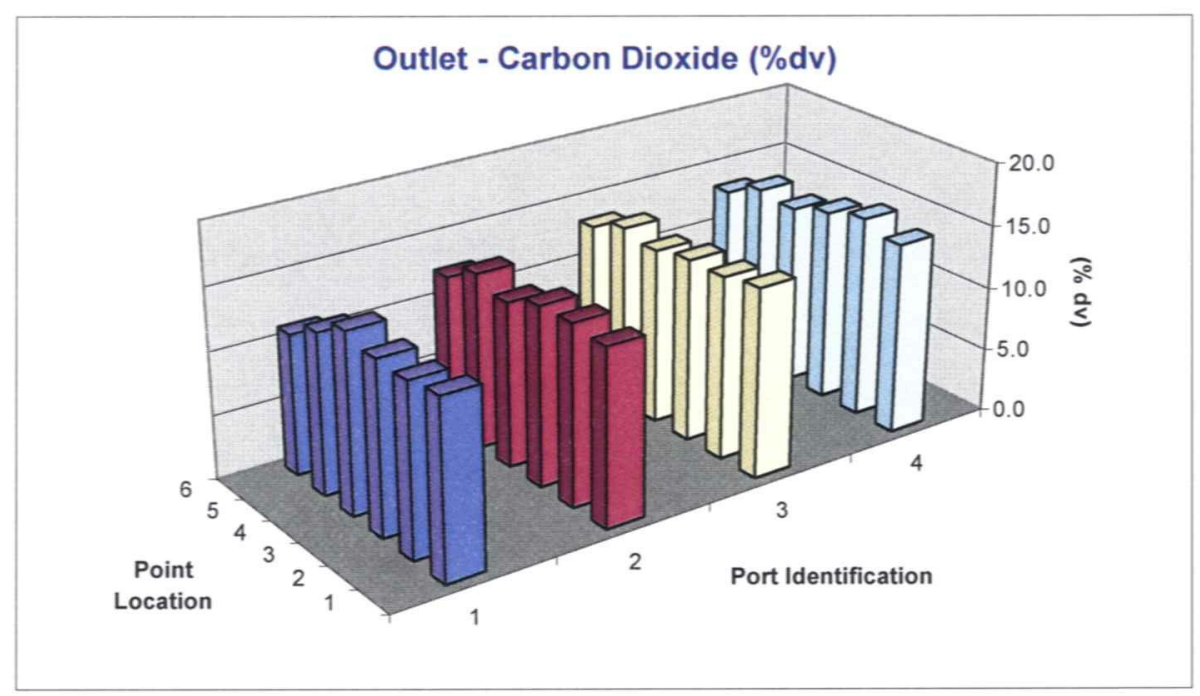


Date: $3 / 28 / 2007$

Start Time: $\quad 9: 36$

End Time: $\quad 9: 59$

Outlet - Nitrogen Oxides (ppmdv), uncorrected

\begin{tabular}{|c|c|c|c|c|}
\hline AVG & 62.1 & 50.0 & 47.9 & 29.9 \\
\hline 6 & 49.0 & 42.9 & 22.9 & 5.1 \\
\hline 5 & 52.2 & 24.4 & 30.1 & 4.5 \\
\hline 4 & 66.8 & 71.5 & 59.4 & 36.7 \\
\hline 3 & 57.0 & 69.6 & 79.9 & 73.8 \\
\hline 2 & 79.9 & 57.0 & 69.8 & 51.8 \\
\hline 1 & 67.6 & 34.8 & 25.1 & 7.7 \\
\hline & & 2 & 3 & 4 \\
\hline
\end{tabular}

30.0

27.8

58.6

70.1

64.6

33.8

47.5

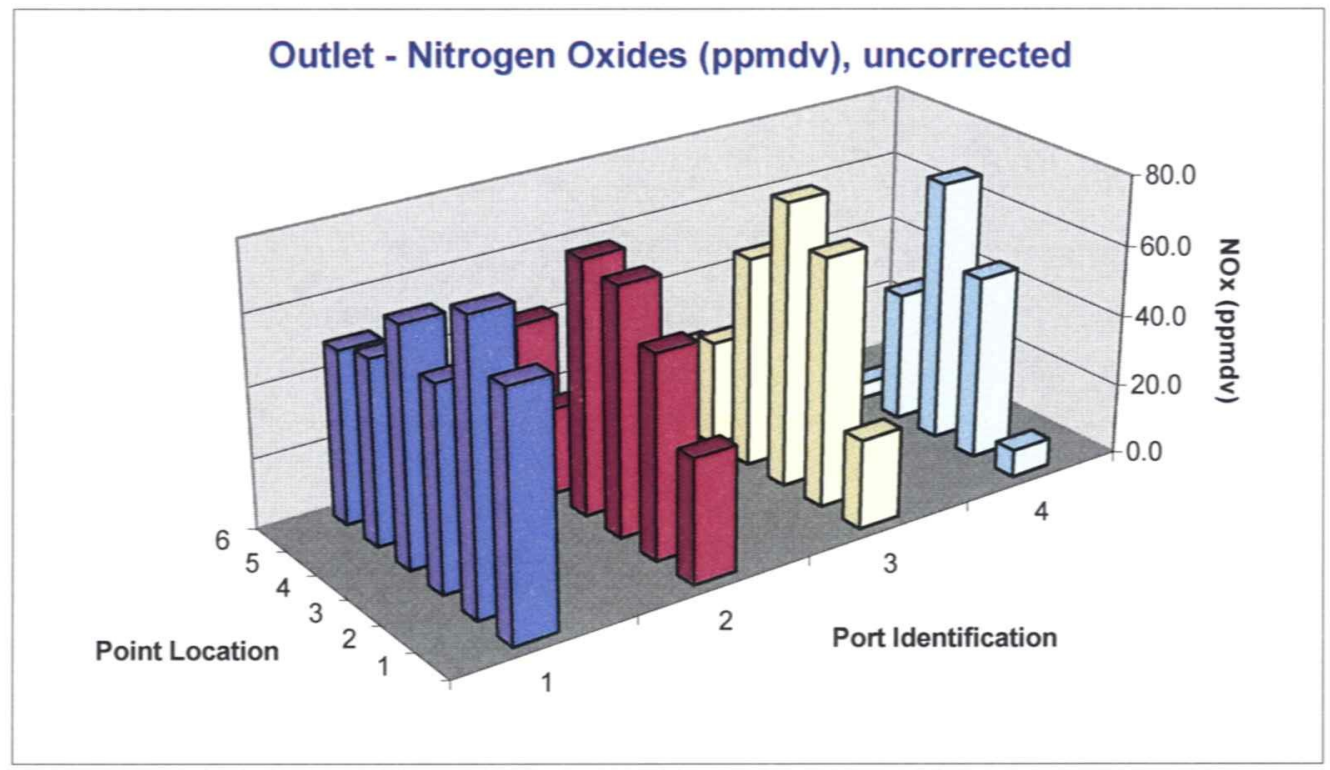


Run 1: Outlet - Set 2

Date: $3 / 28 / 2007$

Start Time: $\quad 9: 36$

End Time: $\quad 9: 59$

Outlet - Nitrogen Oxides (ppmdv @ 3\% $\mathrm{O}_{2}$ )

\begin{tabular}{|c|c|c|c|c|}
\multicolumn{1}{c}{ AVG } & 73.4 & 57.6 & 52.2 & 31.0 \\
\hline $\mathbf{6}$ & 68.2 & 54.0 & 26.8 & 6.0 \\
\hline $\mathbf{5}$ & 64.3 & 27.3 & 32.4 & 4.8 \\
\hline $\mathbf{4}$ & 73.4 & 85.6 & 66.7 & 40.1 \\
\hline $\mathbf{3}$ & 65.6 & 76.9 & 86.2 & 76.1 \\
\hline $\mathbf{2}$ & 92.9 & 62.6 & 75.2 & 51.1 \\
\hline $\mathbf{1}$ & 75.8 & 39.1 & 26.2 & 8.0 \\
\hline & $\mathbf{1}$ & $\mathbf{2}$ & $\mathbf{3}$ & $\mathbf{4}$ \\
\hline
\end{tabular}

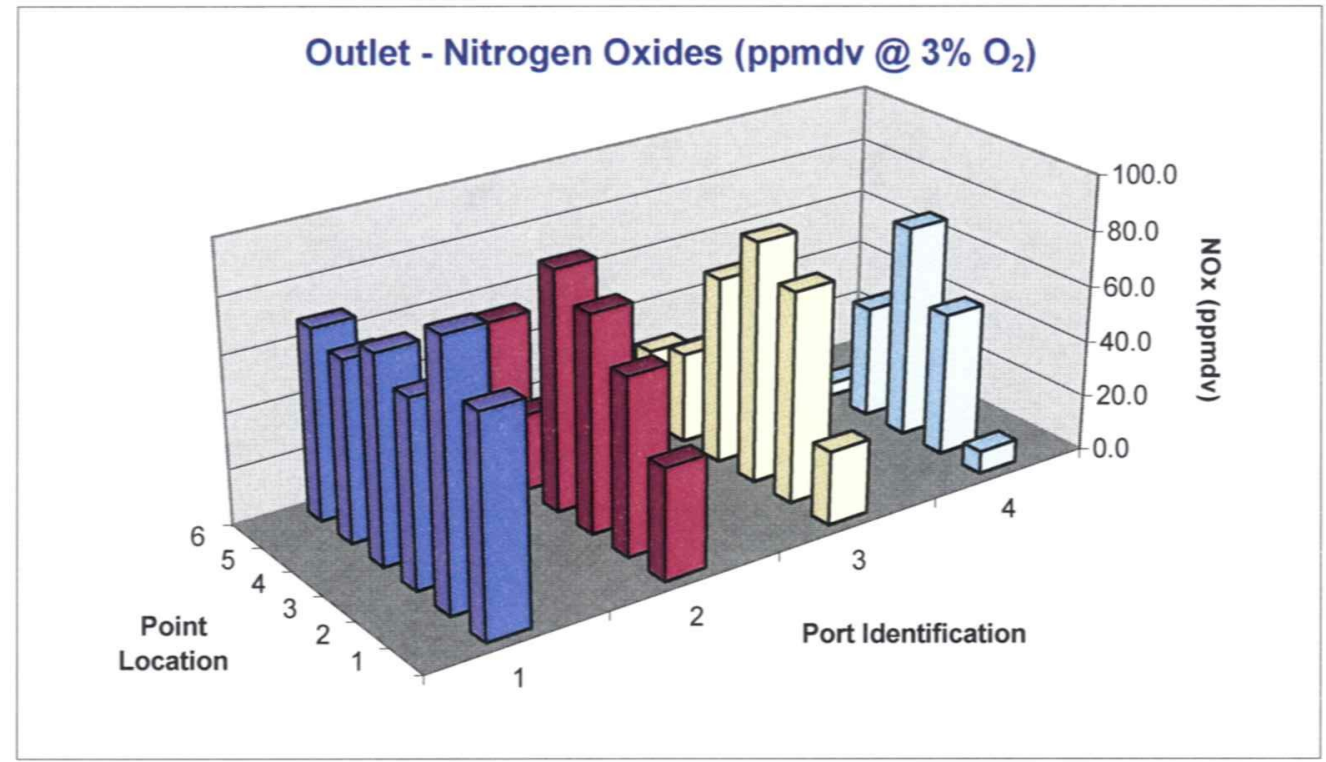


Run 1: Outlet - Set 2

Date: $3 / 28 / 2007$

Start Time: $\quad 9: 36$

End Time: $\quad 9: 59$

Removal Efficiency (ppmdv @ 3\% $\mathrm{O}_{2}$ )

\begin{tabular}{|c|c|c|c|c|}
\multicolumn{1}{c}{ AVG } & $25.4 \%$ & $36.5 \%$ & $43.1 \%$ & $65.8 \%$ \\
\hline $\mathbf{6}$ & $38.7 \%$ & $48.5 \%$ & $74.6 \%$ & $93.3 \%$ \\
\hline $\mathbf{5}$ & $21.5 \%$ & $62.5 \%$ & $58.0 \%$ & $93.3 \%$ \\
\hline $\mathbf{4}$ & $18.5 \%$ & $-3.0 \%$ & $20.7 \%$ & $51.4 \%$ \\
\hline $\mathbf{3}$ & $35.3 \%$ & $23.7 \%$ & $17.6 \%$ & $22.7 \%$ \\
\hline $\mathbf{2}$ & $11.1 \%$ & $26.4 \%$ & $13.6 \%$ & $42.4 \%$ \\
\hline $\mathbf{1}$ & $27.4 \%$ & $60.8 \%$ & $74.3 \%$ & $91.8 \%$ \\
\hline & $\mathbf{1}$ & $\mathbf{2}$ & $\mathbf{3}$ & $\mathbf{4}$ \\
\hline
\end{tabular}

$63.8 \%$

$58.8 \%$

$21.9 \%$

$24.8 \%$

$23.4 \%$

$63.6 \%$

$42.7 \%$

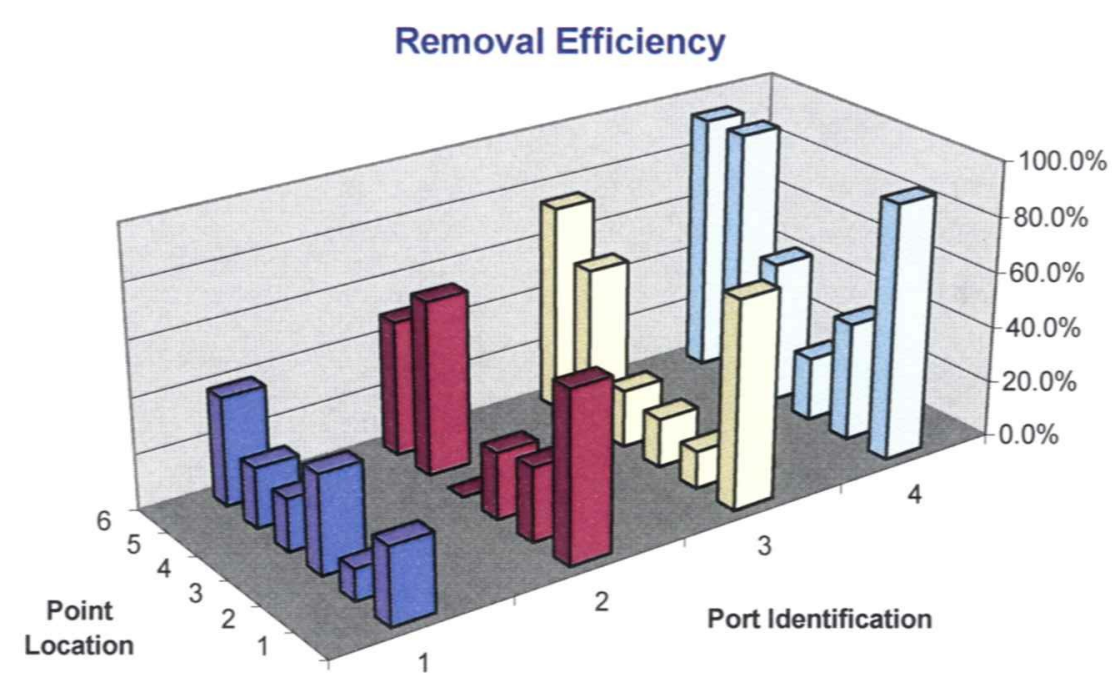


Clean Air Engineering Project \#10192

Consol Energy

AES Dresden

Date:

Start Time:

$3 / 28 / 2007$

$10: 00$

Run \# (Cycle \#)

$1(3)$

End Time:

\begin{tabular}{|c|c|c|c|c|c|c|c|c|c|c|}
\hline $\begin{array}{l}\text { Inlet } \\
\text { Point }\end{array}$ & Time Sampled & $\begin{array}{c}\text { NOx } \\
\text { ppmdv }\end{array}$ & $\begin{array}{c}\text { NOx } \\
\text { Port Avg } \\
\end{array}$ & $\begin{array}{l}\text { PPM Dev } \\
\text { from Avg. }\end{array}$ & $\begin{array}{c}\text { O2 } \\
\% d v\end{array}$ & $\begin{array}{c}\mathrm{O} 2 \\
\text { Port Avg }\end{array}$ & $\begin{array}{l}\mathrm{CO2} \\
\% d v\end{array}$ & $\begin{array}{c}\mathrm{CO} 2 \\
\text { Port Avg }\end{array}$ & $\begin{array}{c}\mathrm{NOx} \\
\text { (9) } 3 \% 02 \\
\end{array}$ & $\begin{array}{l}\text { PPM Dev } \\
\text { from Avg. }\end{array}$ \\
\hline $1-1$ & $10: 00$ & 74.9 & & -5.7 & 7.6 & & 11.5 & & 100.5 & 10.8 \\
\hline $1-2$ & $10: 04$ & 103.6 & & 23.0 & 2.9 & & 15.5 & & 103.3 & 13.6 \\
\hline $1-3$ & 10:08 & 86.3 & 80.8 & 5.7 & 5.1 & 5.5 & 13.7 & 13.3 & 97.5 & 7.8 \\
\hline $1-4$ & 10:12 & 80.1 & & -0.5 & 4.4 & & 14.3 & & 86.7 & -3.0 \\
\hline $1-5$ & $10: 16$ & 68.5 & & -12.1 & 4.7 & & 14.0 & & 75.7 & -14.0 \\
\hline $1-6$ & $10: 20$ & 71.3 & & -9.3 & 8.5 & & 10.8 & & 102.6 & 12.9 \\
\hline $2-1$ & $10: 01$ & 79.8 & & -0.8 & 6.0 & & 12.9 & & 95.6 & 5.9 \\
\hline $2-2$ & $10: 05$ & 82.5 & & 1.9 & 2.9 & & 15.5 & & 82.0 & -7.7 \\
\hline $2-3$ & 10:09 & 84.0 & 78.4 & 3.4 & 5.4 & 4.9 & 13.4 & 13.8 & 97.0 & 7.3 \\
\hline $2-4$ & $10: 13$ & 75.5 & & -5.1 & 3.9 & & 14.7 & & 79.3 & -10.4 \\
\hline $2-5$ & $10: 17$ & 63.3 & & -17.3 & 4.6 & & 14.1 & & 69.4 & -20.3 \\
\hline $2-6$ & $10: 21$ & 85.4 & & 4.8 & 6.9 & & 12.1 & & 109.3 & 19.6 \\
\hline $3-1$ & $10: 02$ & 92.3 & & 11.7 & 4.0 & & 14.5 & & 97.9 & 8.2 \\
\hline $3-2$ & 10:06 & 84.4 & & 3.8 & 2.9 & & 15.5 & & 84.1 & -5.6 \\
\hline $3-3$ & $10: 10$ & 99.4 & 85.4 & 18.8 & 3.4 & 4.1 & 15.1 & 14.4 & 101.6 & 11.9 \\
\hline $3-4$ & 10:14 & 80.0 & & -0.6 & 3.7 & & 14.9 & & 83.1 & -6.6 \\
\hline $3-5$ & $10: 18$ & 68.4 & & -12.2 & 4.6 & & 14.1 & & 74.9 & -14.8 \\
\hline 3-6 & $10: 22$ & 87.9 & & 7.3 & 6.3 & & 12.6 & & 108.1 & 18.4 \\
\hline $4-1$ & $10: 03$ & 81.3 & & 0.7 & 5.7 & & 13.1 & & 96.0 & 6.3 \\
\hline $4-2$ & $10: 07$ & 86.5 & & 5.9 & 2.4 & & 15.9 & & 83.9 & -5.8 \\
\hline $4-3$ & $10: 11$ & 95.4 & 77.9 & 14.8 & 3.0 & 4.3 & 15.4 & 14.3 & 95.6 & 5.9 \\
\hline $4-4$ & $10: 15$ & 78.2 & & -2.4 & 3.5 & & 15.0 & & 80.6 & -9.1 \\
\hline 4-5 & $10: 19$ & 59.0 & & -21.6 & 4.5 & & 14.2 & & 64.2 & -25.5 \\
\hline 4-6 & $10: 23$ & 67.2 & & -13.4 & 6.6 & & 12.4 & & 84.1 & -5.6 \\
\hline
\end{tabular}

Inlet Averages

80.6

4.7

14.0

89.7

\begin{tabular}{|c|c|c|c|c|c|c|c|c|c|c|c|c|}
\hline $\begin{array}{l}\text { Outlet } \\
\text { Point }\end{array}$ & Time Sampled & $\begin{array}{c}\text { NOx } \\
\text { ppmdv }\end{array}$ & $\begin{array}{c}\text { NOx } \\
\text { Port Avg } \\
\end{array}$ & $\begin{array}{l}\text { PPM Dev } \\
\text { from Avg. }\end{array}$ & $\begin{array}{c}\mathrm{O} 2 \\
\% d v\end{array}$ & $\begin{array}{c}\mathrm{O} 2 \\
\text { Port Avg }\end{array}$ & $\begin{array}{l}\mathrm{CO2} \\
\% d v\end{array}$ & $\begin{array}{c}\mathrm{CO} 2 \\
\text { Port Avg }\end{array}$ & $\begin{array}{c}\mathrm{NOx} \\
\text { @ } 3 \% \mathrm{O} 2 \\
\end{array}$ & $\begin{array}{l}\text { PPM Dev } \\
\text { from Avg. }\end{array}$ & \begin{tabular}{c|} 
Removal \\
Efficiency
\end{tabular} & $\begin{array}{c}\text { Removal Eff } \\
\text { W/ O2 correction }\end{array}$ \\
\hline $1-1$ & $10: 00$ & 67.6 & & 21.8 & 4.9 & & \begin{tabular}{|l|l|}
13.8 & \\
\end{tabular} & & 75.8 & 24.5 & 9.7 & 24.6 \\
\hline $1-2$ & $10: 04$ & 77.3 & & 31.5 & 5.6 & & 13.3 & & 90.4 & 39.1 & 25.4 & 12.5 \\
\hline $1-3$ & $10: 08$ & 57.3 & 54.9 & 11.5 & 5.2 & 5.6 & 13.6 & 12.7 & 65.2 & 13.9 & 33.6 & 33.1 \\
\hline $1-4$ & $10: 12$ & 55.2 & & 9.4 & 4.8 & & 13.9 & & 61.4 & 10.1 & 31.1 & 29.2 \\
\hline $1-5$ & $10: 16$ & 48.1 & & 2.3 & 6.1 & & 12.9 & & 58.0 & 6.7 & 29.8 & 23.4 \\
\hline $1-6$ & $10: 20$ & 23.7 & & -22.1 & 7.1 & & 8.8 & & 30.7 & -20.5 & 66.8 & 70.0 \\
\hline $2-1$ & $10: 01$ & 31.9 & & -13.9 & 5.0 & & 13.8 & & 35.9 & -15.3 & 60.0 & 62.4 \\
\hline $2-2$ & 10:05 & 56.2 & & 10.4 & 4.5 & & 14.3 & & 61.4 & 10.1 & 31.9 & 25.1 \\
\hline $2-3$ & $10: 09$ & 68.7 & 50.9 & 22.9 & 4.8 & 5.3 & 14.0 & 13.6 & 76.4 & 25.1 & 18.2 & 21.3 \\
\hline $2-4$ & $10: 13$ & 71.3 & & 25.5 & 5.9 & & 13.1 & & 85.0 & 33.7 & 5.6 & -7.1 \\
\hline $2-5$ & $10: 17$ & 23.1 & & -22.7 & 4.9 & & 14.0 & & 25.8 & -25.5 & 63.5 & 62.9 \\
\hline $2-6$ & $10: 21$ & 54.3 & & 8.5 & 6.7 & & 12.3 & & 68.6 & 17.4 & 36.4 & 37.2 \\
\hline $3-1$ & $10: 02$ & 22.9 & & -22.9 & 3.8 & & 14.9 & & 23.9 & -27.3 & 75.2 & 75.6 \\
\hline $3-2$ & $10: 06$ & 68.5 & & 22.7 & 4.2 & & 14.5 & & 73.3 & 22.1 & 18.8 & 12.8 \\
\hline 3-3 & $10: 10$ & 79.0 & 47.6 & 33.2 & 4.4 & 4.5 & 14.3 & 14.2 & 85.8 & 34.5 & 20.5 & 15.6 \\
\hline $3-4$ & $10: 14$ & 59.2 & & 13.4 & 4.9 & & 13.9 & & 66.3 & 15.0 & 26.0 & 20.2 \\
\hline $3-5$ & $10: 18$ & 30.0 & & -15.8 & 4.2 & & 14.4 & & 32.2 & -19.1 & 56.1 & 57.0 \\
\hline $3-6$ & $10: 22$ & 26.1 & & -19.7 & 5.5 & & 13.4 & & 30.3 & -20.9 & 70.3 & 71.9 \\
\hline $4-1$ & $10: 03$ & 7.3 & & -38.5 & 3.5 & & 15.1 & & 7.5 & -43.7 & 91.0 & 92.2 \\
\hline $4-2$ & $10: 07$ & 53.2 & & 7.4 & 2.6 & & 16.0 & & 51.9 & 0.6 & 38.5 & 38.1 \\
\hline $4-3$ & $10: 11$ & 72.5 & 29.9 & 26.7 & 3.4 & 3.9 & 15.3 & 14.8 & 73.9 & 22.7 & 24.0 & 22.6 \\
\hline $4-4$ & $10: 15$ & 36.3 & & -9.5 & 4.5 & & 14.3 & & 39.6 & -11.7 & 53.6 & 50.9 \\
\hline $4-5$ & $10: 19$ & 4.3 & & -41.5 & 3.9 & & 14.8 & & 4.5 & -46.7 & 92.7 & 92.9 \\
\hline 4-6 & $10: 23$ & 5.6 & & -40.2 & 5.6 & & 13.3 & & 6.6 & -44.7 & 91.7 & 92.2 \\
\hline
\end{tabular}

Outlet Averages

45.8

4.8

13.8

51.3

44.6

43.2 
Client Reference No: 4700140111

CleanAir Project No:10192

Run 1: Inlet - Set 3

Date: $3 / 28 / 2007$

Start Time: $\quad 10: 00$

End Time: $\quad 10: 23$

Inlet - Oxygen (\%dv)

\begin{tabular}{|c|c|c|c|c|}
\hline AVG & 5.5 & 4.9 & 4.1 & 4.3 \\
\hline 6 & 8.5 & 6.9 & 6.3 & 6.6 \\
\hline 5 & 4.7 & 4.6 & 4.6 & 4.5 \\
\hline 4 & 4.4 & 3.9 & 3.7 & 3.5 \\
\hline 3 & 5.1 & 5.4 & 3.4 & 3.0 \\
\hline 2 & 2.9 & 2.9 & 2.9 & 2.4 \\
\hline \multirow[t]{2}{*}{1} & 7.6 & 6.0 & 4.0 & 5.7 \\
\hline & 1 & 2 & 3 & 4 \\
\hline
\end{tabular}

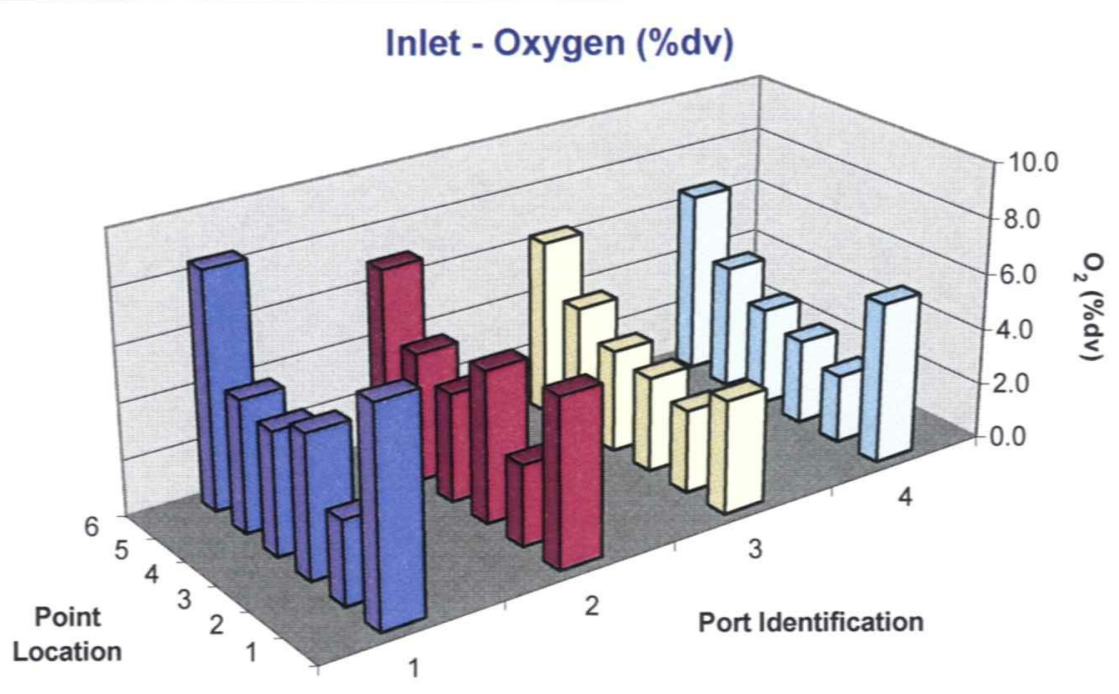


Run 1: Inlet - Set 3

Date: $3 / 28 / 2007$

Start Time: $\quad 10: 00$

End Time: $\quad 10: 23$

Inlet - Carbon Dioxide (\%dv)

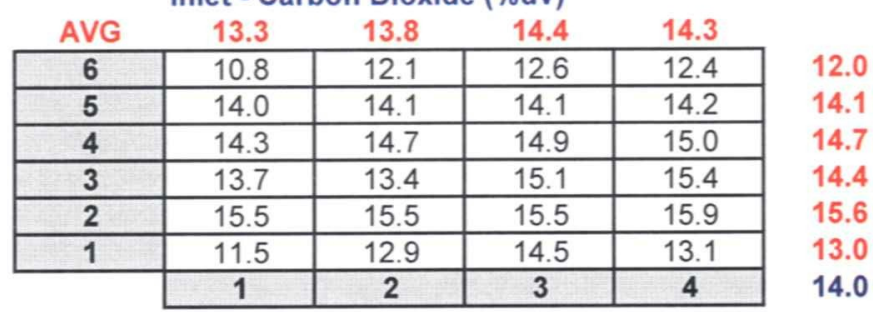

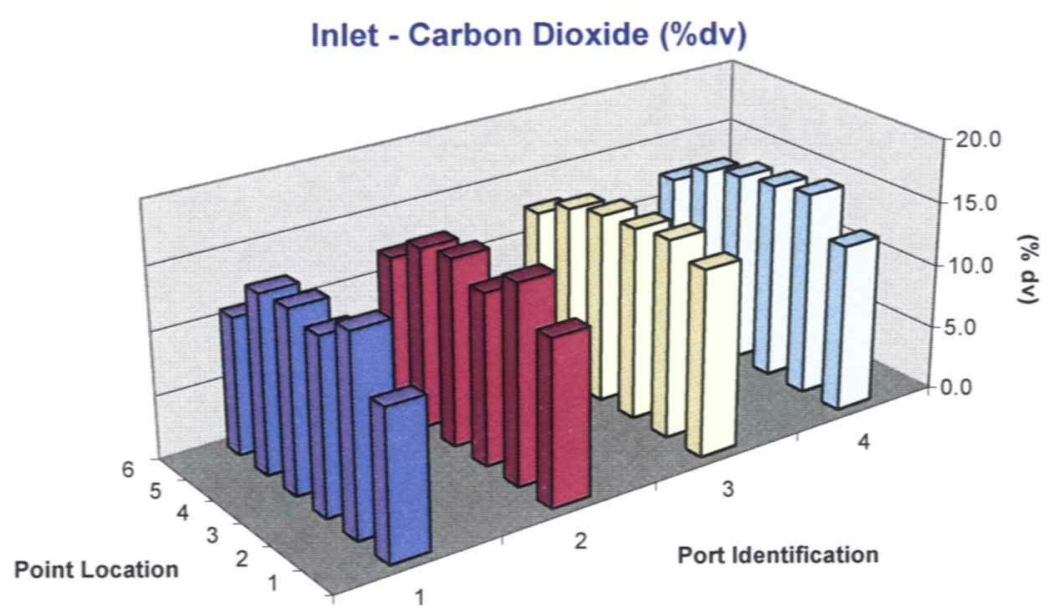


Run 1: Inlet - Set 3

Date: $3 / 28 / 2007$

Start Time: $\quad 10: 00$

End Time: $\quad 10: 23$

Inlet - Nitrogen Oxides (ppmdv), uncorrected

\begin{tabular}{|c|c|c|c|c|}
\multicolumn{1}{c|}{ AVG } & 80.8 & 78.4 & 85.4 & 77.9 \\
\hline $\mathbf{6}$ & 71.3 & 85.4 & 87.9 & 67.2 \\
\hline $\mathbf{5}$ & 68.5 & 63.3 & 68.4 & 59.0 \\
\hline $\mathbf{4}$ & 80.1 & 75.5 & 80.0 & 78.2 \\
\hline $\mathbf{3}$ & 86.3 & 84.0 & 99.4 & 95.4 \\
\hline $\mathbf{2}$ & 103.6 & 82.5 & 84.4 & 86.5 \\
\hline $\mathbf{1}$ & 74.9 & 79.8 & 92.3 & 81.3 \\
\hline & $\mathbf{1}$ & $\mathbf{2}$ & $\mathbf{3}$ & $\mathbf{4}$ \\
\hline
\end{tabular}

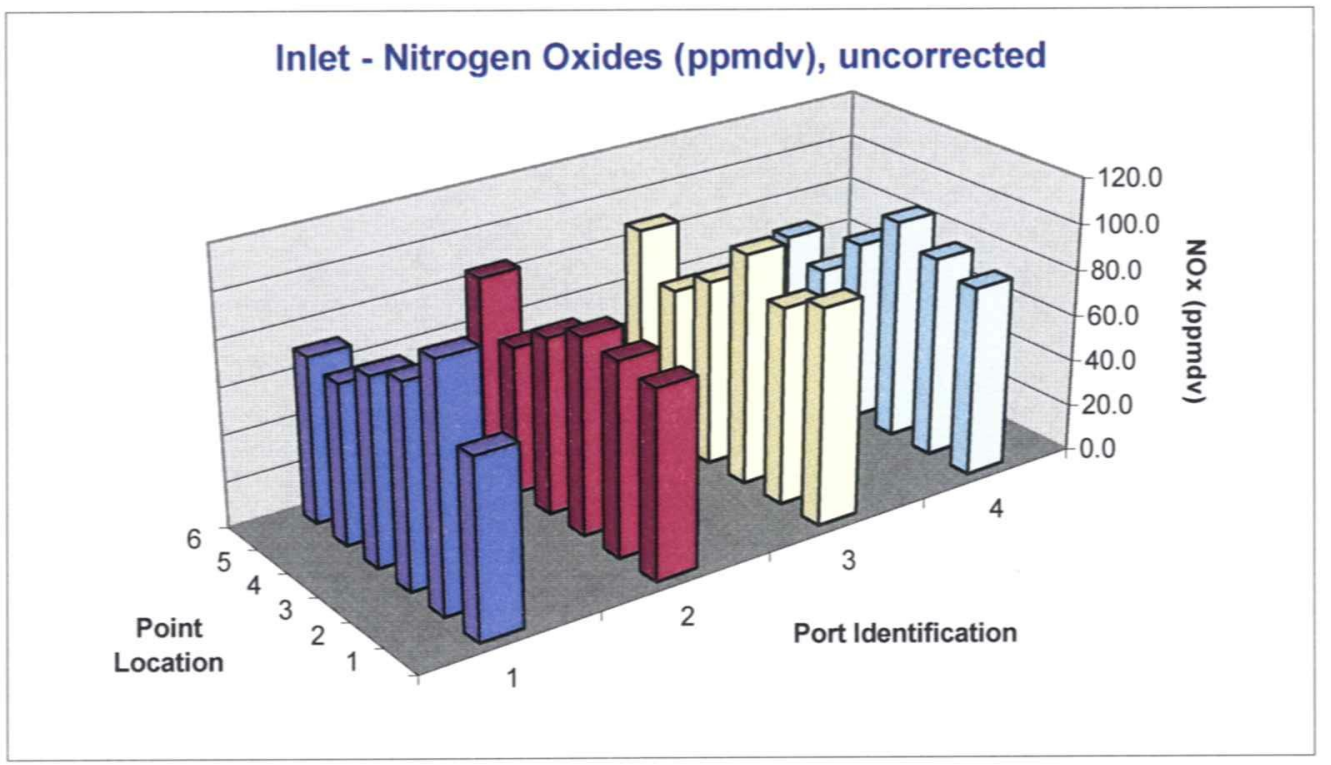


Run 1: Inlet - Set 3

Date: $3 / 28 / 2007$

Start Time: $\quad 10: 00$

End Time: $\quad 10: 23$

Inlet - Nitrogen Oxides (ppmdv @ 3\% $\mathrm{O}_{2}$ )

\begin{tabular}{|c|c|c|c|c|c|}
\multicolumn{1}{c|}{ AVG } & 94.4 & 88.8 & 91.6 & 84.1 & \\
\hline $\mathbf{6}$ & 102.6 & 109.3 & 108.1 & 84.1 & 101.0 \\
\hline $\mathbf{5}$ & 75.7 & 69.4 & 74.9 & 64.2 & 71.1 \\
\hline $\mathbf{4}$ & 86.7 & 79.3 & 83.1 & 80.6 & 82.4 \\
\hline $\mathbf{3}$ & 97.5 & 97.0 & 101.6 & 95.6 & 97.9 \\
\hline $\mathbf{2}$ & 103.3 & 82.0 & 84.1 & 83.9 & 88.3 \\
\hline $\mathbf{1}$ & 100.5 & 95.6 & 97.9 & 96.0 & 97.5 \\
\cline { 2 - 5 } & $\mathbf{1}$ & $\mathbf{2}$ & $\mathbf{3}$ & $\mathbf{4}$ & $\mathbf{8 9 . 7}$ \\
\cline { 2 - 5 } & & & & &
\end{tabular}

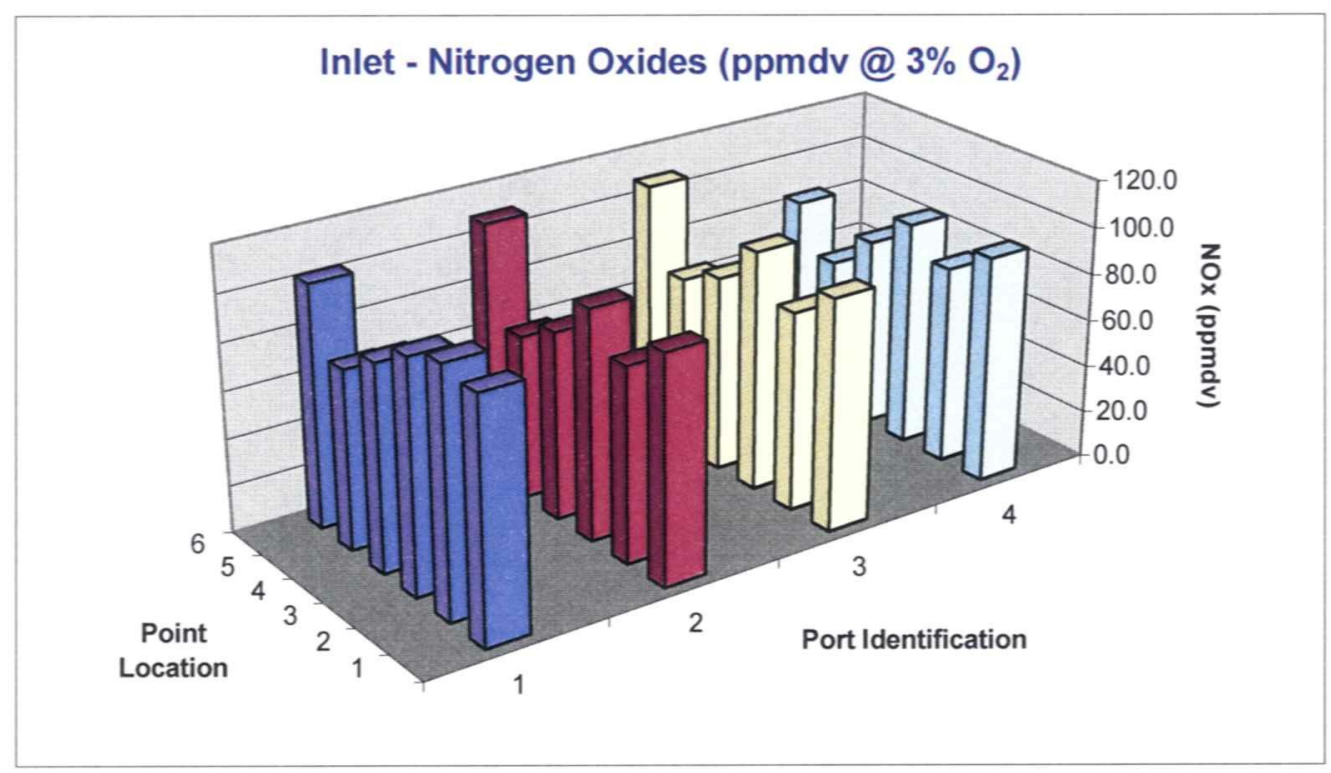


Run 1: Outlet - Set 3

Date: $3 / 28 / 2007$

Start Time: $\quad 10: 00$

End Time: $\quad 10: 23$

\begin{tabular}{|c|c|c|c|c|c|}
\hline \multicolumn{5}{|c|}{ Outlet - Oxygen (\%dv) } & \multirow[b]{3}{*}{6.2} \\
\hline AVG & 5.6 & 5.3 & 4.5 & 3.9 & \\
\hline 6 & 7.1 & 6.7 & 5.5 & 5.6 & \\
\hline 5 & 6.1 & 4.9 & 4.2 & 3.9 & 4.8 \\
\hline 4 & 4.8 & 5.9 & 4.9 & 4.5 & 5.0 \\
\hline 3 & 5.2 & 4.8 & 4.4 & 3.4 & 4.4 \\
\hline 2 & 5.6 & 4.5 & 4.2 & 2.6 & 4.2 \\
\hline 1 & 4.9 & 5.0 & 3.8 & 3.5 & 4.3 \\
\hline & 1 & 2 & 3 & 4 & 4.8 \\
\hline
\end{tabular}

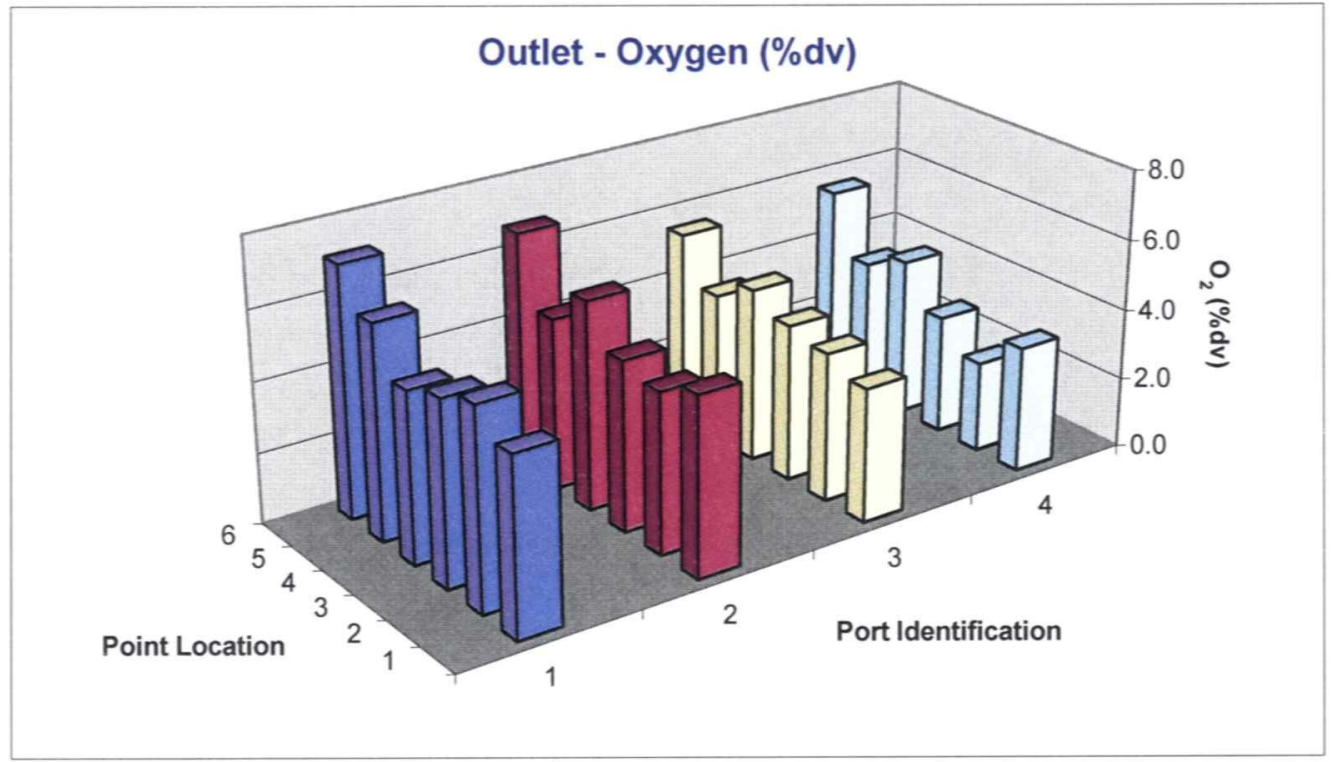


Run 1: Outlet - Set 3

Date: $3 / 28 / 2007$

Start Time: $\quad 10: 00$

End Time: $\quad 10: 23$

Outlet - Carbon Dioxide (\%dv)

\begin{tabular}{|c|c|c|c|c|}
\multicolumn{1}{|c|}{ AVG } & 12.7 & 13.6 & 14.2 & 14.8 \\
\hline $\mathbf{6}$ & 8.8 & 12.3 & 13.4 & 13.3 \\
\hline $\mathbf{5}$ & 12.9 & 14.0 & 14.4 & 14.8 \\
\hline $\mathbf{4}$ & 13.9 & 13.1 & 13.9 & 14.3 \\
\hline $\mathbf{3}$ & 13.6 & 14.0 & 14.3 & 15.3 \\
\hline $\mathbf{2}$ & 13.3 & 14.3 & 14.5 & 16.0 \\
\hline $\mathbf{1}$ & 13.8 & 13.8 & 14.9 & 15.1 \\
\hline & $\mathbf{1}$ & $\mathbf{2}$ & $\mathbf{3}$ & $\mathbf{4}$ \\
\hline
\end{tabular}

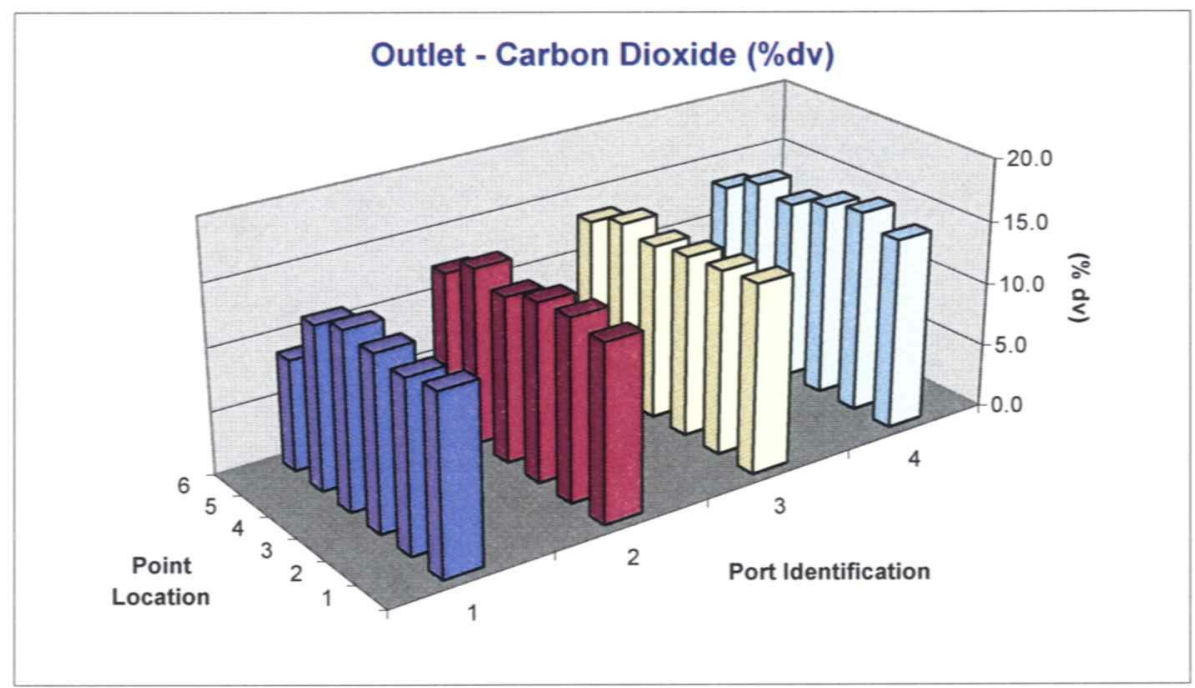


Date: $3 / 28 / 2007$

Start Time: $\quad$ 10:00

End Time: $\quad 10: 23$

Outlet - Nitrogen Oxides (ppmdv), uncorrected

\begin{tabular}{|c|c|c|c|c|}
\multicolumn{1}{c}{ AVG } & 54.9 & 50.9 & 47.6 & 29.9 \\
\hline $\mathbf{6}$ & 23.7 & 54.3 & 26.1 & 5.6 \\
\hline $\mathbf{5}$ & 48.1 & 23.1 & 30.0 & 4.3 \\
\hline $\mathbf{4}$ & 55.2 & 71.3 & 59.2 & 36.3 \\
\hline $\mathbf{3}$ & 57.3 & 68.7 & 79.0 & 72.5 \\
\hline $\mathbf{2}$ & 77.3 & 56.2 & 68.5 & 53.2 \\
\hline $\mathbf{1}$ & 67.6 & 31.9 & 22.9 & 7.3 \\
\hline & $\mathbf{1}$ & $\mathbf{2}$ & $\mathbf{3}$ & $\mathbf{4}$ \\
\hline
\end{tabular}

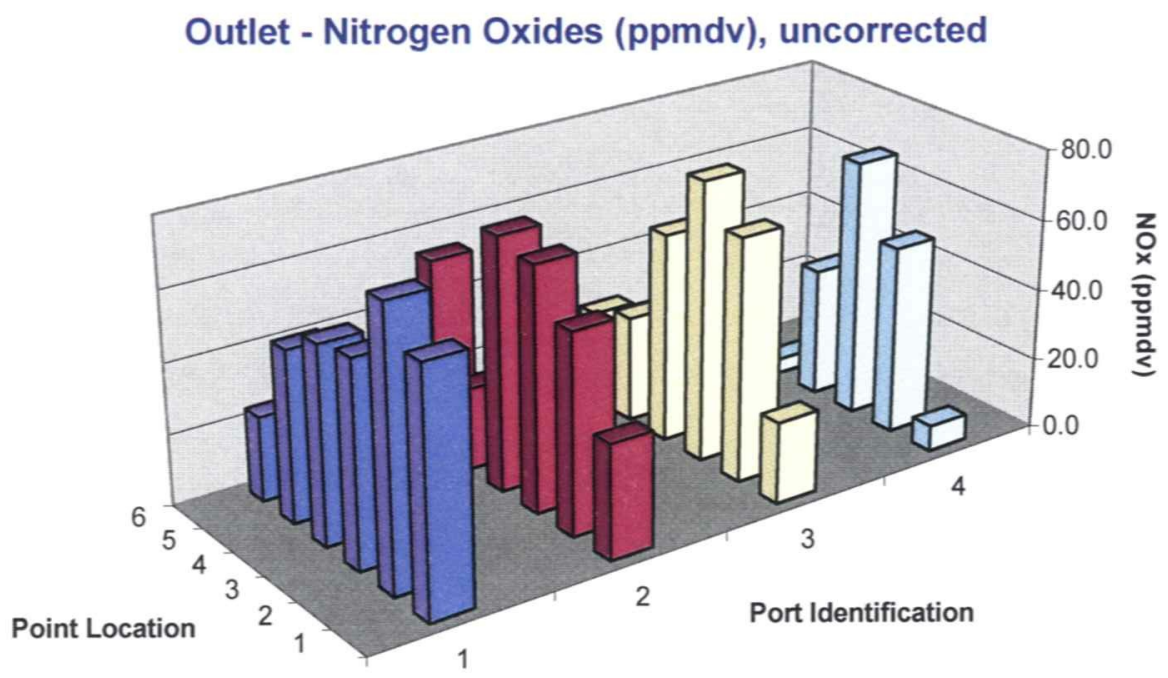


Date: $3 / 28 / 2007$

Start Time: $\quad 10: 00$

End Time: $\quad 10: 23$

Outlet - Nitrogen Oxides (ppmdv @ 3\% $\mathrm{O}_{2}$ )

\begin{tabular}{|c|c|c|c|c|}
\multicolumn{1}{c|}{ AVG } & 63.6 & 58.8 & 52.0 & 30.7 \\
\hline $\mathbf{6}$ & 30.7 & 68.6 & 30.3 & 6.6 \\
\hline $\mathbf{5}$ & 58.0 & 25.8 & 32.2 & 4.5 \\
\hline $\mathbf{4}$ & 61.4 & 85.0 & 66.3 & 39.6 \\
\hline $\mathbf{3}$ & 65.2 & 76.4 & 85.8 & 73.9 \\
\hline $\mathbf{2}$ & 90.4 & 61.4 & 73.3 & 51.9 \\
\hline $\mathbf{1}$ & 75.8 & 35.9 & 23.9 & 7.5 \\
\hline & $\mathbf{1}$ & $\mathbf{2}$ & $\mathbf{3}$ & $\mathbf{4}$ \\
\hline
\end{tabular}

34.1

30.1

63.0

75.3

69.2

35.8

51.3

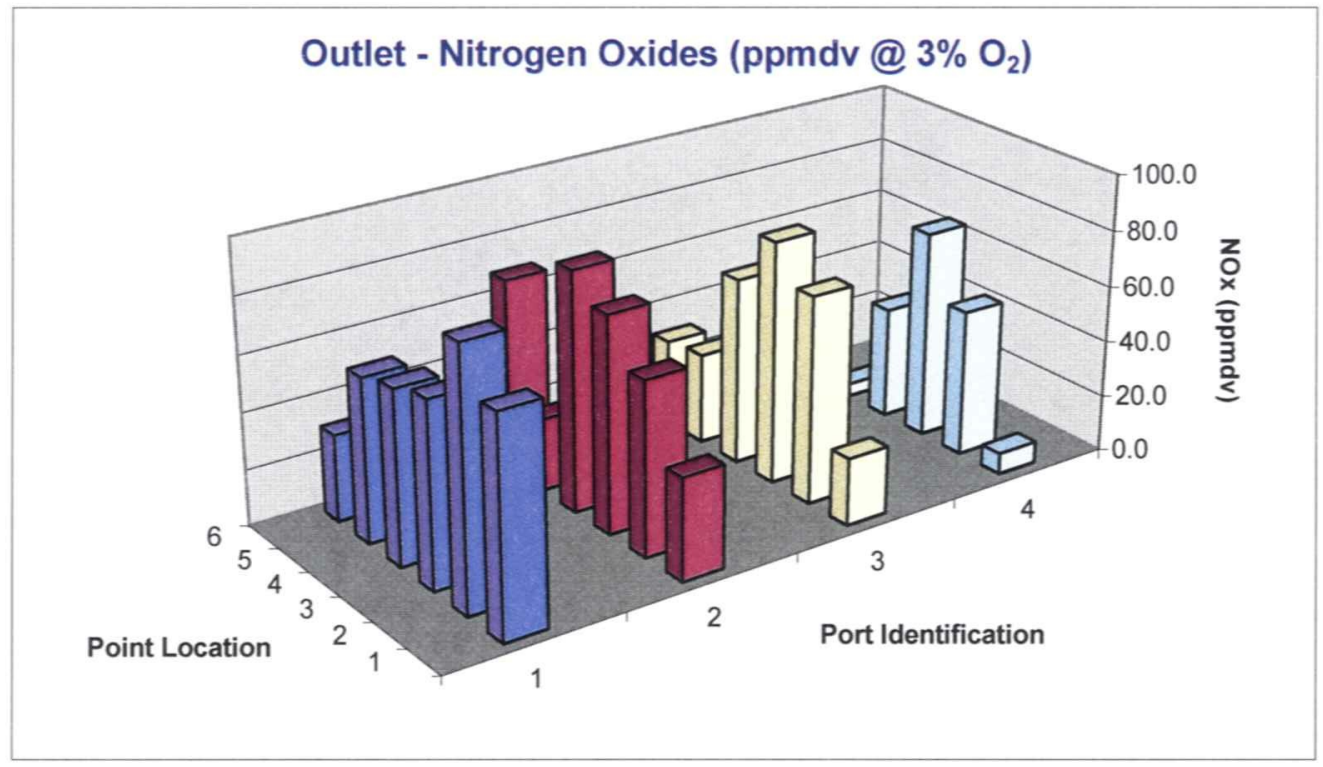


CONSOL ENERGY INC.
Client Reference No: 4700140111

CleanAir Project No:10192

Run 1: Outlet - Set 3

Date: $3 / 28 / 2007$

Start Time: $\quad 10: 00$

End Time: $\quad 10: 23$

Removal Efficiency (ppmdv @ 3\% $\mathrm{O}_{2}$ )

\begin{tabular}{|c|c|c|c|c|}
\multicolumn{1}{c}{ AVG } & $32.1 \%$ & $33.6 \%$ & $42.2 \%$ & $64.8 \%$ \\
\hline $\mathbf{6}$ & $70.0 \%$ & $37.2 \%$ & $71.9 \%$ & $92.2 \%$ \\
\hline $\mathbf{5}$ & $23.4 \%$ & $62.9 \%$ & $57.0 \%$ & $92.9 \%$ \\
\hline $\mathbf{4}$ & $29.2 \%$ & $-7.1 \%$ & $20.2 \%$ & $50.9 \%$ \\
\hline $\mathbf{3}$ & $33.1 \%$ & $21.3 \%$ & $15.6 \%$ & $22.6 \%$ \\
\hline $\mathbf{2}$ & $12.5 \%$ & $25.1 \%$ & $12.8 \%$ & $38.1 \%$ \\
\hline $\mathbf{1}$ & $24.6 \%$ & $62.4 \%$ & $75.6 \%$ & $92.2 \%$ \\
\hline & $\mathbf{1}$ & $\mathbf{2}$ & $\mathbf{3}$ & $\mathbf{4}$ \\
\hline
\end{tabular}

$67.8 \%$

$59.0 \%$

$23.3 \%$

$23.1 \%$

$22.1 \%$

$63.7 \%$

$43.2 \%$

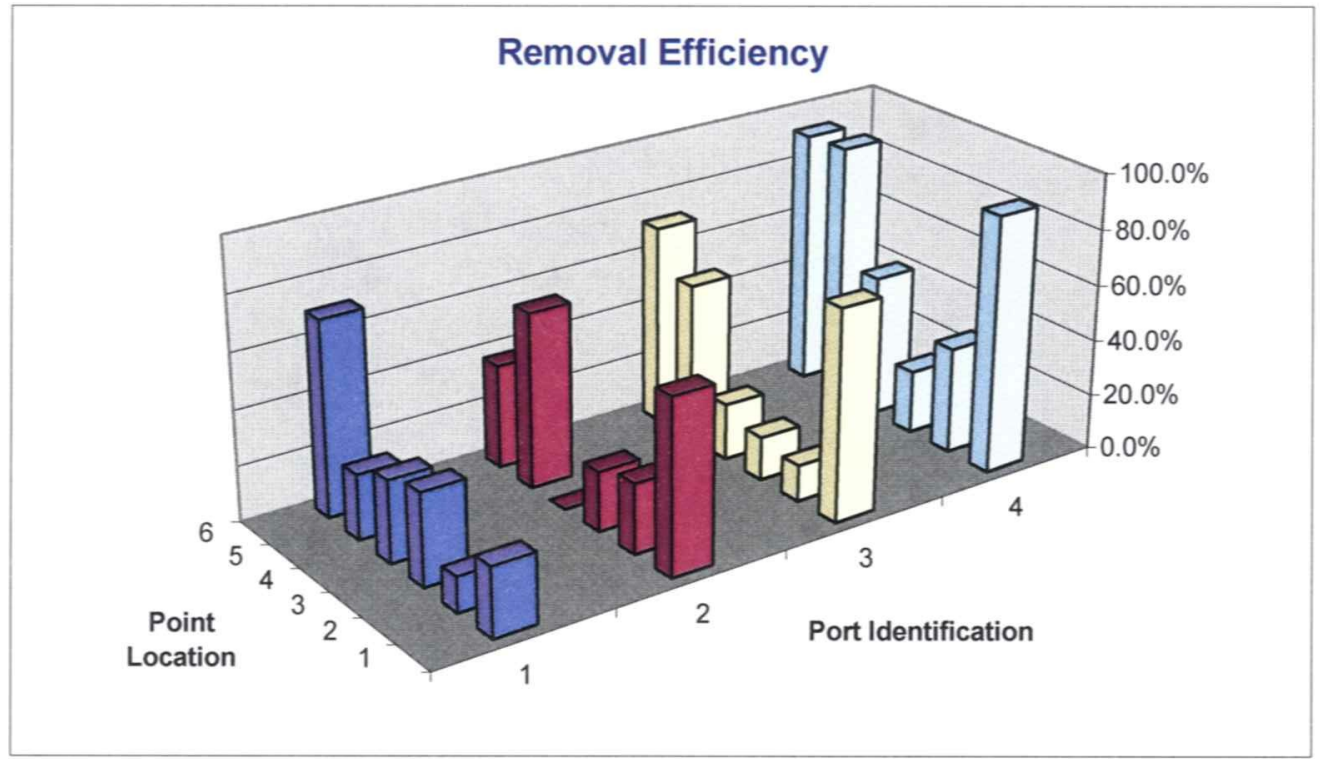


Clean Air Engineering Project \#10192

Consol Energy

AES Dresden

$\begin{array}{lrr}\text { Date: } & 3 / 28 / 2007 & \text { Run \# (Cycle \#) } 2(1) \\ \text { Start Time: } & 11: 12 & \\ \text { End Time: } & 11: 35 & \end{array}$

\begin{tabular}{|c|c|c|c|c|c|c|c|c|c|c|}
\hline $\begin{array}{l}\text { Inlet } \\
\text { Point }\end{array}$ & Time Sampled & $\begin{array}{c}\text { NOx } \\
\text { ppmdv }\end{array}$ & $\begin{array}{c}\text { NOx } \\
\text { Port Avg }\end{array}$ & $\begin{array}{l}\text { PPM Dev } \\
\text { from Avg. }\end{array}$ & $\begin{array}{c}\mathrm{O} 2 \\
\% d v\end{array}$ & $\begin{array}{c}\mathrm{O} 2 \\
\text { Port Avg }\end{array}$ & $\begin{array}{l}\mathrm{CO2} \\
\% d v\end{array}$ & $\begin{array}{c}\mathrm{CO} 2 \\
\text { Port Avg }\end{array}$ & $\begin{array}{c}\mathrm{NOx} \\
\text { (1) } 3 \% 02\end{array}$ & $\begin{array}{l}\text { PPM Dev } \\
\text { from Avg. }\end{array}$ \\
\hline $1-1$ & $11: 12$ & 78.6 & & -0.3 & 7.3 & & 11.8 & & 103.1 & 15.5 \\
\hline $1-2$ & 11:16 & 102.0 & & 23.1 & 2.9 & & 15.5 & & 101.2 & 13.5 \\
\hline $1-3$ & $11: 20$ & 84.5 & 81.4 & 5.6 & 5.0 & 5.4 & 13.8 & 13.4 & 95.0 & 7.3 \\
\hline $1-4$ & $11: 24$ & 80.9 & & 2.0 & 4.4 & & 14.3 & & 87.7 & 0.0 \\
\hline $1-5$ & $11: 28$ & 69.6 & & -9.3 & 4.6 & & 14.1 & & 76.4 & -11.3 \\
\hline $1-6$ & $11: 32$ & 72.9 & & -6.0 & 8.6 & & 10.7 & & 106.3 & 18.6 \\
\hline $2-1$ & $11: 13$ & 82.3 & & 3.4 & 5.9 & & 12.9 & & 98.4 & 10.7 \\
\hline $2-2$ & 11:17 & 79.9 & & 1.0 & 3.1 & & 15.3 & & 80.3 & -7.4 \\
\hline $2-3$ & $11: 21$ & 84.1 & 76.5 & 5.2 & 5.2 & 4.9 & 13.6 & 13.8 & 96.0 & 8.3 \\
\hline $2-4$ & $11: 25$ & 75.4 & & -3.5 & 3.9 & & 14.7 & & 79.3 & -8.4 \\
\hline $2-5$ & $11: 29$ & 59.4 & & -19.5 & 4.3 & & 14.4 & & 64.0 & -23.7 \\
\hline $2-6$ & $11: 33$ & 77.9 & & -1.0 & 6.8 & & 12.2 & & 99.0 & 11.4 \\
\hline $3-1$ & $11: 14$ & 87.4 & & 8.5 & 4.0 & & 14.5 & & 92.7 & 5.0 \\
\hline $3-2$ & 11:18 & 79.0 & & 0.1 & 2.8 & & 15.6 & & 78.3 & -9.4 \\
\hline $3-3$ & $11: 22$ & 98.7 & 82.0 & 19.8 & 3.2 & 4.1 & 15.3 & 14.5 & 99.6 & 11.9 \\
\hline $3-4$ & $11: 26$ & 79.4 & & 0.5 & 3.7 & & 14.9 & & 82.5 & -5.1 \\
\hline $3-5$ & $11: 30$ & 66.3 & & -12.6 & 4.5 & & 14.2 & & 72.4 & -15.3 \\
\hline $3-6$ & $11: 34$ & 81.0 & & 2.1 & 6.3 & & 12.6 & & 99.4 & 11.8 \\
\hline $4-1$ & 11:15 & 77.8 & & -1.1 & 5.9 & & 13.0 & & 93.0 & 5.3 \\
\hline $4-2$ & 11:19 & 81.6 & & 2.7 & 2.6 & & 15.8 & & 79.6 & -8.1 \\
\hline $4-3$ & $11: 23$ & 95.0 & 75.9 & 16.1 & 3.1 & 4.4 & 15.4 & 14.3 & 95.4 & 7.7 \\
\hline $4-4$ & 11:27 & 76.9 & & -2.0 & 3.5 & & 15.0 & & 79.3 & -8.4 \\
\hline $4-5$ & 11:31 & 60.1 & & -18.8 & 4.6 & & 14.1 & & 65.9 & -21.8 \\
\hline $4-6$ & $11: 35$ & 63.7 & & -15.2 & 6.6 & & 12.4 & & 79.5 & -8.2 \\
\hline
\end{tabular}

\begin{tabular}{|c|c|c|c|c|c|c|c|c|c|c|c|c|}
\hline $\begin{array}{l}\text { Outlet } \\
\text { Point }\end{array}$ & Time Sampled & $\begin{array}{l}\text { NOx } \\
\text { ppmdv }\end{array}$ & $\begin{array}{c}\text { NOx } \\
\text { Port Avg }\end{array}$ & $\begin{array}{l}\text { PPM Dev } \\
\text { from Avg. }\end{array}$ & $\begin{array}{c}\mathrm{O} 2 \\
\% d v\end{array}$ & $\begin{array}{c}\text { O2 } \\
\text { Port Avg }\end{array}$ & $\begin{array}{l}\mathrm{CO} 2 \\
\% \text { dv }\end{array}$ & $\begin{array}{c}\mathrm{CO} 2 \\
\text { Port Avg }\end{array}$ & $\begin{array}{c}\mathrm{NOx} \\
\text { (Q) } 3 \% \mathrm{O} 2 \\
\end{array}$ & $\begin{array}{l}\text { PPM Dev } \\
\text { from Avg. }\end{array}$ & $\begin{array}{l}\text { Removal } \\
\text { Efficiency }\end{array}$ & $\begin{array}{c}\text { Removal Eff } \\
\text { W/ O2 correction }\end{array}$ \\
\hline $1-1$ & $11: 12$ & 71.2 & & 25.2 & 4.6 & & 14.2 & & 78.1 & 26.7 & 9.4 & 24.3 \\
\hline $1-2$ & $11: 16$ & 76.7 & & 30.7 & 5.4 & & 13.5 & & 88.7 & 37.3 & 24.8 & 12.3 \\
\hline $1-3$ & $11: 20$ & 58.1 & 61.4 & 12.1 & 5.3 & 5.6 & 13.6 & 13.3 & 66.6 & 15.2 & 31.2 & 29.9 \\
\hline $1-4$ & $11: 24$ & 65.9 & & 19.9 & 4.5 & & 14.2 & & 72.1 & 20.6 & 18.5 & 17.8 \\
\hline $1-5$ & $11: 28$ & 47.1 & & 1.1 & 5.8 & & 13.2 & & 55.6 & 4.2 & 32.3 & 27.1 \\
\hline $1-6$ & $11: 32$ & 49.1 & & 3.1 & 7.9 & & 11.2 & & 67.5 & 16.0 & 32.6 & 36.5 \\
\hline $2-1$ & $11: 13$ & 33.3 & & -12.7 & 4.8 & & 14.0 & & 36.9 & -14.5 & 59.5 & 62.5 \\
\hline $2-2$ & $11: 17$ & 51.4 & & 5.4 & 4.5 & & 14.3 & & 56.0 & 4.6 & 35.7 & 30.2 \\
\hline $2-3$ & $11: 21$ & 69.4 & 48.3 & 23.4 & 4.7 & 5.2 & 14.1 & 13.6 & 76.5 & 25.1 & 17.5 & 20.3 \\
\hline $2-4$ & $11: 25$ & 72.6 & & 26.6 & 5.9 & & 13.1 & & 86.5 & 35.0 & 3.7 & -9.1 \\
\hline $2-5$ & $11: 29$ & 22.7 & & -23.3 & 4.7 & & 14.1 & & 25.1 & -26.4 & 61.8 & 60.8 \\
\hline $2-6$ & $11: 33$ & 40.6 & & -5.4 & 6.7 & & 12.3 & & 51.1 & -0.3 & 47.9 & 48.4 \\
\hline $3-1$ & $11: 14$ & 24.2 & & -21.8 & 3.6 & & 15.0 & & 25.0 & -26.4 & 72.3 & 73.0 \\
\hline $3-2$ & $11: 18$ & 58.3 & & 12.3 & 4.0 & & 14.6 & & 61.8 & 10.4 & 26.2 & 21.0 \\
\hline $3-3$ & $11: 22$ & 73.2 & 45.7 & 27.2 & 4.3 & 4.4 & 14.4 & 14.3 & 78.7 & 27.3 & 25.8 & 21.0 \\
\hline $3-4$ & $11: 26$ & 67.4 & & 21.4 & 4.6 & & 14.1 & & 74.2 & 22.8 & 15.1 & 10.1 \\
\hline $3-5$ & $11: 30$ & 30.5 & & -15.5 & 4.1 & & 14.5 & & 32.5 & -18.9 & 54.0 & 55.1 \\
\hline $3-6$ & $11: 34$ & 20.7 & & -25.3 & 5.6 & & 13.3 & & 24.2 & -27.3 & 74.4 & 75.7 \\
\hline $4-1$ & $11: 15$ & 6.8 & & -39.2 & 3.6 & & 15.0 & & 7.0 & -44.4 & 91.3 & 92.4 \\
\hline $4-2$ & $11: 19$ & 44.8 & & -1.2 & 2.5 & & 16.0 & & 43.7 & -7.7 & 45.1 & 45.1 \\
\hline 4-3 & $11: 23$ & 73.1 & 28.7 & 27.1 & 3.3 & 3.9 & 15.3 & 14.8 & 74.3 & 22.9 & 23.1 & 22.1 \\
\hline $4-4$ & $11: 27$ & 39.1 & & -6.9 & 4.5 & & 14.3 & & 42.6 & -8.8 & 49.2 & 46.3 \\
\hline 4-5 & $11: 31$ & 4.1 & & -41.9 & 4.1 & & 14.6 & & 4.4 & -47.1 & 93.2 & 93.4 \\
\hline 4-6 & $11: 35$ & 4.4 & & -41.6 & 5.6 & & 13.3 & & 5.1 & -46.3 & 93.1 & 93.5 \\
\hline
\end{tabular}

Outlet Averages

46.0

4.8

14.0

51.4

43.2

42.1 
Run 2: Inlet - Set 1

Date: $3 / 28 / 2007$

Start Time: $\quad 11: 12$

End Time: 11:35

Inlet - Oxygen (\%dv)

\begin{tabular}{|c|c|c|c|c|}
\multicolumn{1}{|c|}{ AVG } & 5.4 & 4.9 & 4.1 & 4.4 \\
\hline 6 & 8.6 & 6.8 & 6.3 & 6.6 \\
\hline 5 & 4.6 & 4.3 & 4.5 & 4.6 \\
\hline 4 & 4.4 & 3.9 & 3.7 & 3.5 \\
\hline 3 & 5.0 & 5.2 & 3.2 & 3.1 \\
\hline 2 & 2.9 & 3.1 & 2.8 & 2.6 \\
\hline 1 & 7.3 & 5.9 & 4.0 & 5.9 \\
\hline
\end{tabular}

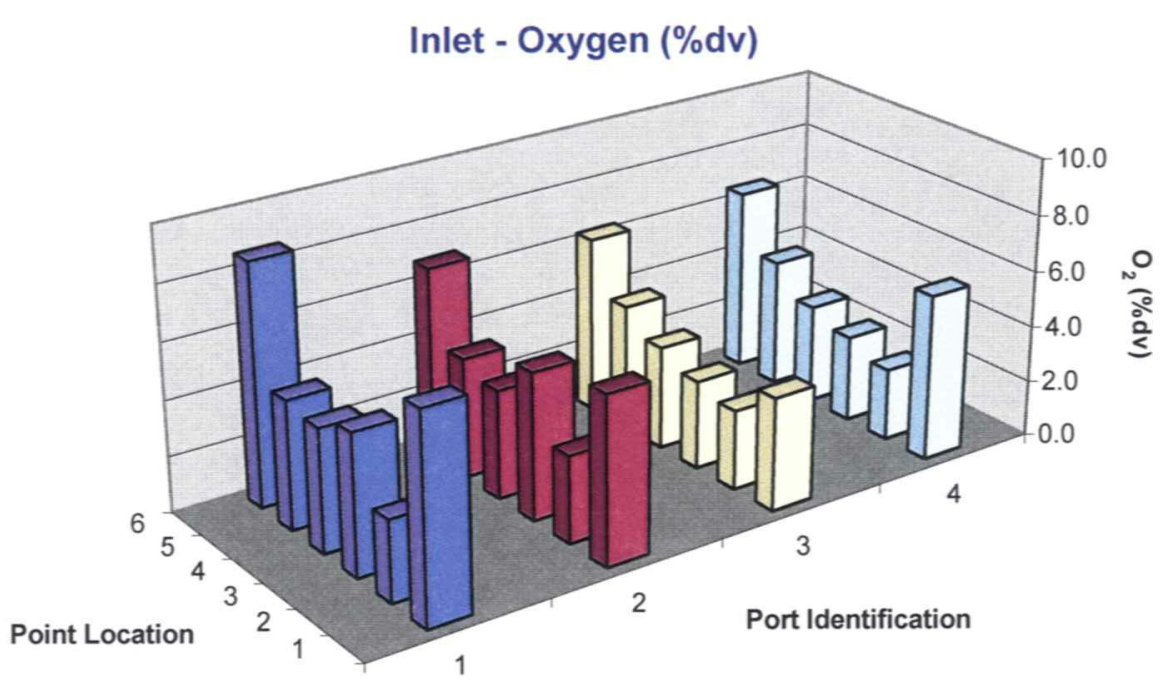


Run 2: Inlet - Set 1

Date: $3 / 28 / 2007$

Start Time: $\quad 11: 12$

End Time: $\quad 11: 35$

\begin{tabular}{|c|c|c|c|c|c|}
\hline \multicolumn{5}{|c|}{ Inlet - Carbon Dioxide (\%dv) } & \\
\hline AVG & 13.4 & 13.8 & 14.5 & 14.3 & \\
\hline 6 & 10.7 & 12.2 & 12.6 & 12.4 & 12.0 \\
\hline 5 & 14.1 & 14.4 & 14.2 & 14.1 & 14.2 \\
\hline 4 & 14.3 & 14.7 & 14.9 & 15.0 & 14.7 \\
\hline 3 & 13.8 & 13.6 & 15.3 & 15.4 & 14.5 \\
\hline 2 & 15.5 & 15.3 & 15.6 & 15.8 & 15.6 \\
\hline 1 & 11.8 & 12.9 & 14.5 & 13.0 & 13.0 \\
\hline & 1 & 2 & 3 & 4 & 14.0 \\
\hline
\end{tabular}

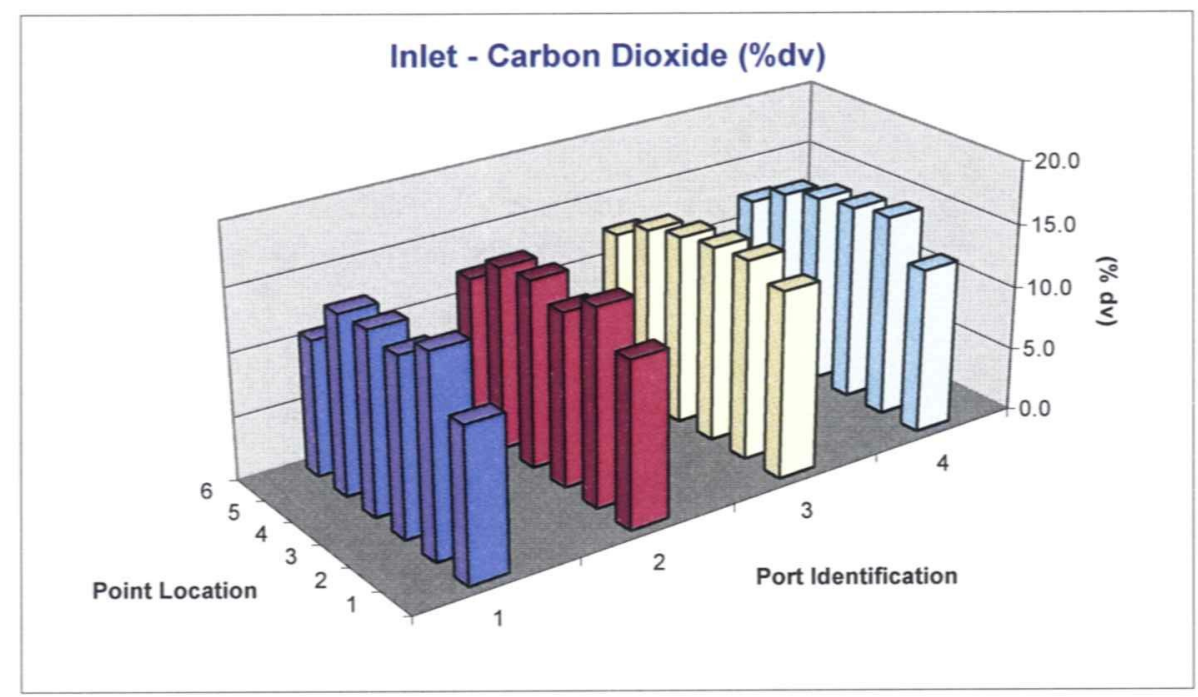


Run 2: Inlet - Set 1

Date: $3 / 28 / 2007$

Start Time: $\quad 11: 12$

End Time: $\quad 11: 35$

Inlet - Nitrogen Oxides (ppmdv), uncorrected

\begin{tabular}{|c|c|c|c|c|}
\multicolumn{1}{|c}{ AVG } & 81.4 & 76.5 & 82.0 & 75.9 \\
\hline $\mathbf{6}$ & 72.9 & 77.9 & 81.0 & 63.7 \\
\hline $\mathbf{5}$ & 69.6 & 59.4 & 66.3 & 60.1 \\
\hline $\mathbf{4}$ & 80.9 & 75.4 & 79.4 & 76.9 \\
\hline $\mathbf{3}$ & 84.5 & 84.1 & 98.7 & 95.0 \\
\hline $\mathbf{2}$ & 102.0 & 79.9 & 79.0 & 81.6 \\
\hline $\mathbf{1}$ & 78.6 & 82.3 & 87.4 & 77.8 \\
\hline
\end{tabular}

73.9

63.9

78.2

90.6

85.6

81.5

78.9

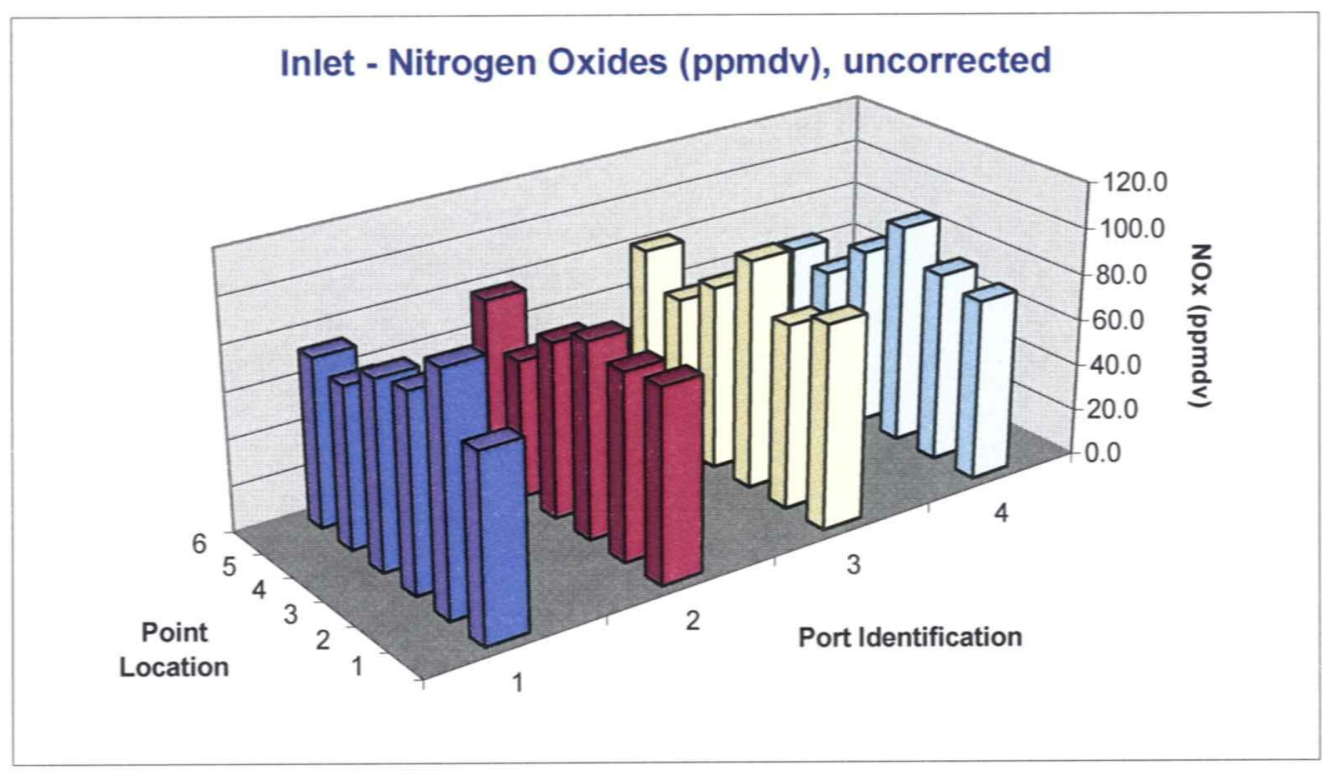


Run 2: Inlet - Set 1

Date: $3 / 28 / 2007$

Start Time: $\quad 11: 12$

End Time: $\quad 11: 35$

Inlet - Nitrogen Oxides (ppmdv @ 3\% $\mathrm{O}_{2}$ )

\begin{tabular}{|c|c|c|c|c|}
\multicolumn{1}{|c}{ AVG } & 94.9 & 86.2 & 87.5 & 82.1 \\
\hline $\mathbf{6}$ & 106.3 & 99.0 & 99.4 & 79.5 \\
\hline $\mathbf{5}$ & 76.4 & 64.0 & 72.4 & 65.9 \\
\hline $\mathbf{4}$ & 87.7 & 79.3 & 82.5 & 79.3 \\
\hline $\mathbf{3}$ & 95.0 & 96.0 & 99.6 & 95.4 \\
\hline $\mathbf{2}$ & 101.2 & 80.3 & 78.3 & 79.6 \\
\hline $\mathbf{1}$ & 103.1 & 98.4 & 92.7 & 93.0 \\
\hline & $\mathbf{1}$ & $\mathbf{2}$ & $\mathbf{3}$ & $\mathbf{4}$ \\
\hline
\end{tabular}

96.1

69.7

82.2

96.5

84.8

96.8

87.7

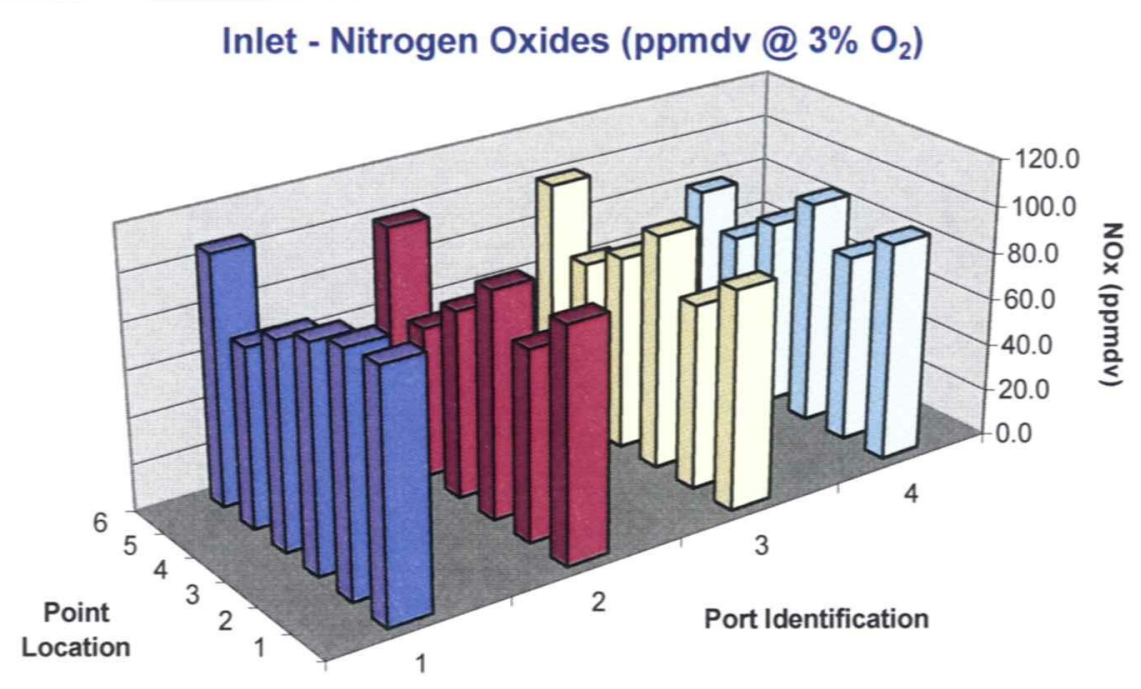


CONSOL ENERGY INC.
Client Reference No: 4700140111

CleanAir Project No:10192

Run 2: Outlet - Set 1

Date: $3 / 28 / 2007$

Start Time: $\quad 11: 12$

End Time: $\quad 11: 35$

Outlet - Oxygen (\%dv)

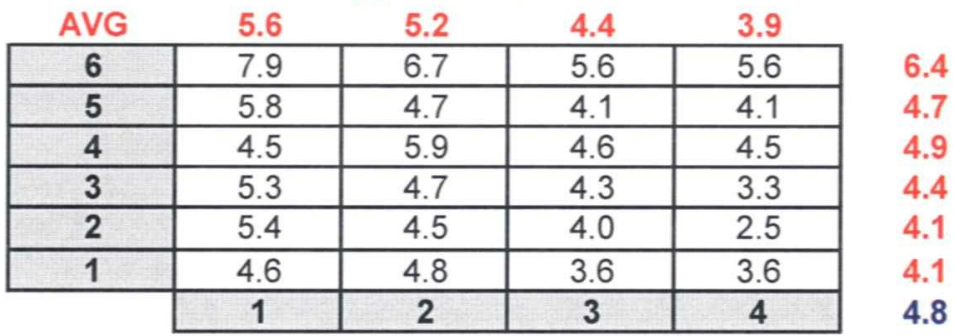

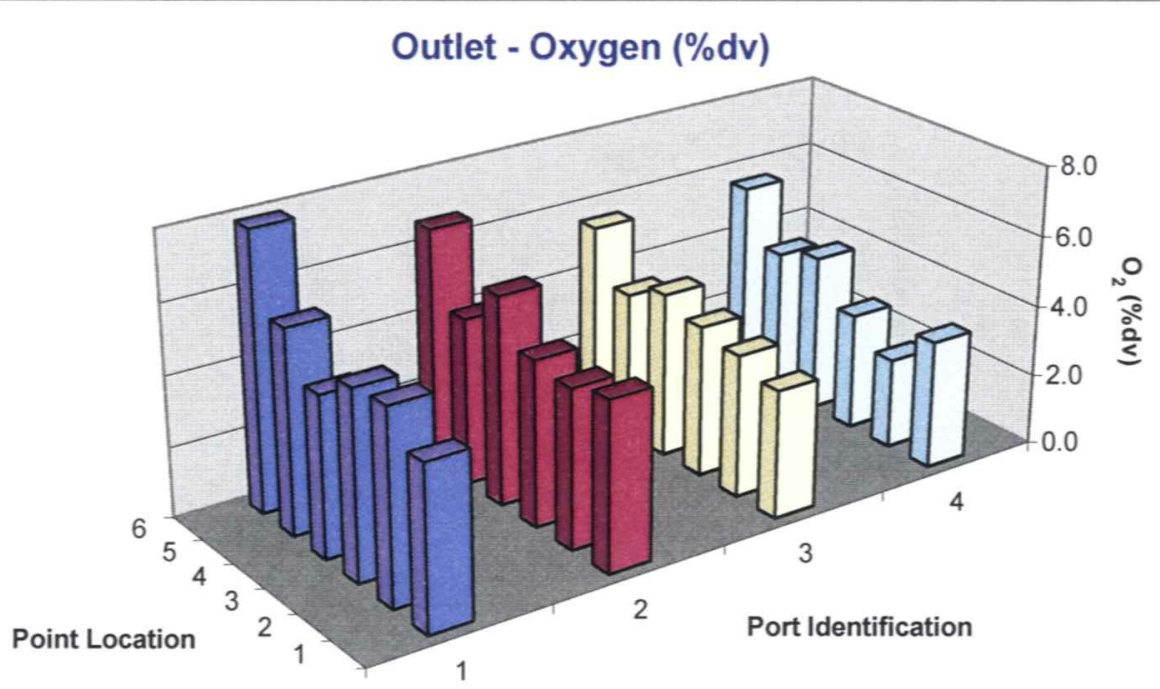


Run 2: Outlet - Set 1

Date: $3 / 28 / 2007$

Start Time: $\quad 11: 12$

End Time: $\quad 11: 35$

Outlet - Carbon Dioxide (\%dv)

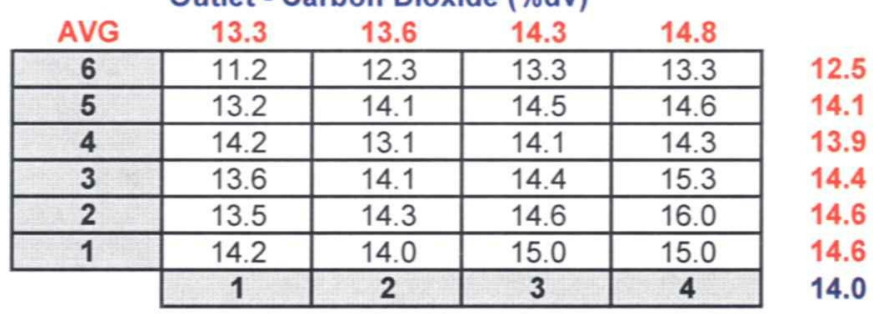

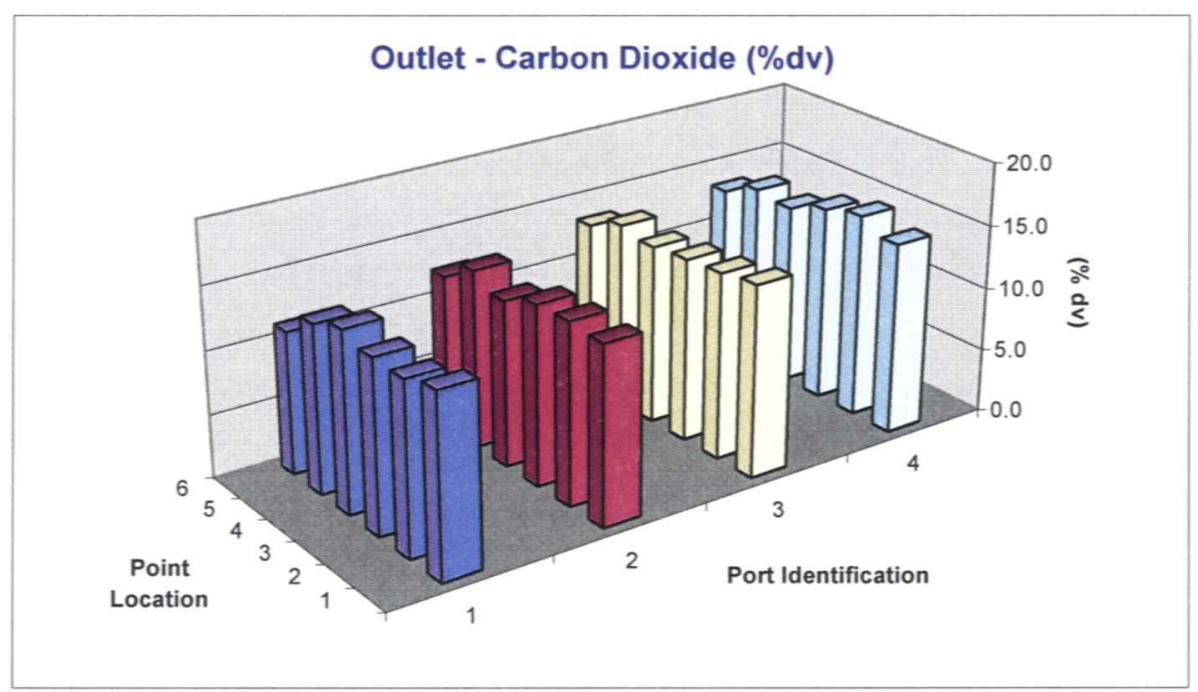


Date: $3 / 28 / 2007$

Start Time: $\quad 11: 12$

End Time: $\quad 11: 35$

Outlet - Nitrogen Oxides (ppmdv), uncorrected

\begin{tabular}{|c|c|c|c|c|}
\multicolumn{1}{c}{ AVG } & 61.4 & 48.3 & 45.7 & 28.7 \\
\hline $\mathbf{6}$ & 49.1 & 40.6 & 20.7 & 4.4 \\
\hline $\mathbf{5}$ & 47.1 & 22.7 & 30.5 & 4.1 \\
\hline $\mathbf{4}$ & 65.9 & 72.6 & 67.4 & 39.1 \\
\hline $\mathbf{3}$ & 58.1 & 69.4 & 73.2 & 73.1 \\
\hline $\mathbf{2}$ & 76.7 & 51.4 & 58.3 & 44.8 \\
\hline $\mathbf{1}$ & 71.2 & 33.3 & 24.2 & 6.8 \\
\hline & $\mathbf{1}$ & $\mathbf{2}$ & $\mathbf{3}$ & $\mathbf{4}$ \\
\hline
\end{tabular}

28.7

26.1

61.3

68.5

57.8

33.9

46.0

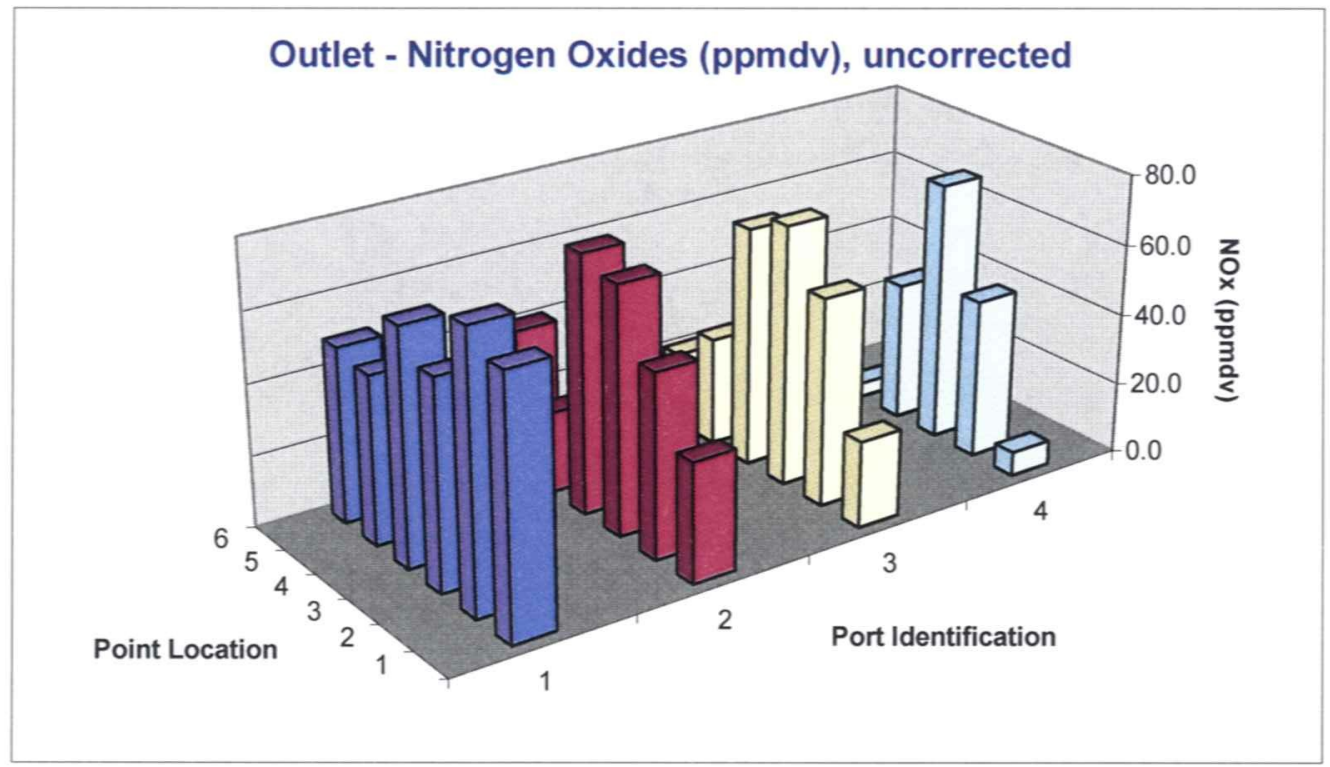


Date: $3 / 28 / 2007$

Start Time: $\quad 11: 12$

End Time: $\quad 11: 35$

Outlet - Nitrogen Oxides (ppmdv @ 3\% $\mathrm{O}_{2}$ )

\begin{tabular}{|c|c|c|c|c|}
\multicolumn{1}{|c}{ AVG } & 71.4 & 55.4 & 49.4 & 29.5 \\
\hline $\mathbf{6}$ & 67.5 & 51.1 & 24.2 & 5.1 \\
\hline $\mathbf{5}$ & 55.6 & 25.1 & 32.5 & 4.4 \\
\hline $\mathbf{4}$ & 72.1 & 86.5 & 74.2 & 42.6 \\
\hline $\mathbf{3}$ & 66.6 & 76.5 & 78.7 & 74.3 \\
\hline $\mathbf{2}$ & 88.7 & 56.0 & 61.8 & 43.7 \\
\hline $\mathbf{1}$ & 78.1 & 36.9 & 25.0 & 7.0 \\
\hline & $\mathbf{1}$ & $\mathbf{2}$ & $\mathbf{3}$ & $\mathbf{4}$ \\
\hline
\end{tabular}

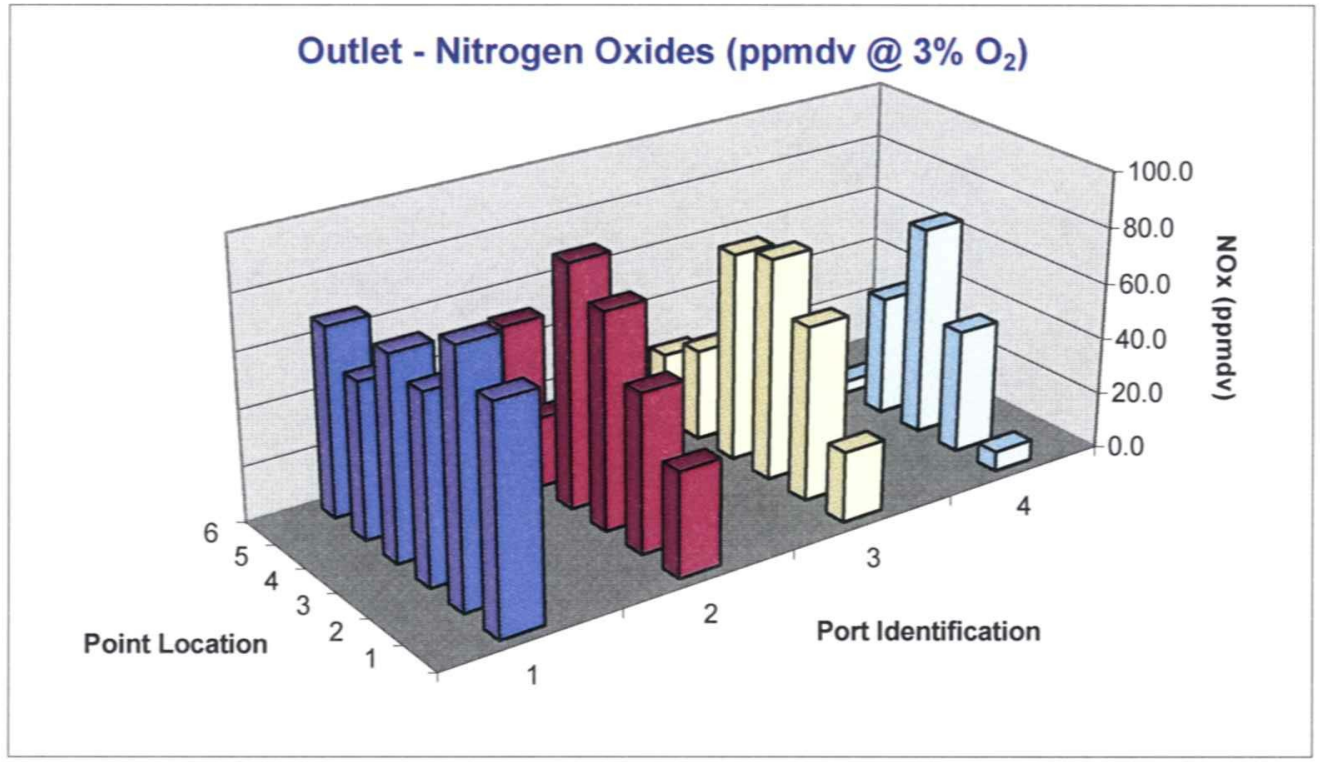


CONSOL ENERGY INC.
Client Reference No: 4700140111

CleanAir Project No:10192

Run 2: Outlet - Set 1

Date: $3 / 28 / 2007$

Start Time: $\quad 11: 12$

End Time: $\quad 11: 35$

Removal Efficiency (ppmdv @ 3\% O

\begin{tabular}{|c|c|c|c|c|}
\multicolumn{1}{c}{ AVG } & $24.7 \%$ & $35.5 \%$ & $42.7 \%$ & $65.5 \%$ \\
\hline $\mathbf{6}$ & $36.5 \%$ & $48.4 \%$ & $75.7 \%$ & $93.5 \%$ \\
\hline $\mathbf{5}$ & $27.1 \%$ & $60.8 \%$ & $55.1 \%$ & $93.4 \%$ \\
\hline $\mathbf{4}$ & $17.8 \%$ & $-9.1 \%$ & $10.1 \%$ & $46.3 \%$ \\
\hline $\mathbf{3}$ & $29.9 \%$ & $20.3 \%$ & $21.0 \%$ & $22.1 \%$ \\
\hline $\mathbf{2}$ & $12.3 \%$ & $30.2 \%$ & $21.0 \%$ & $45.1 \%$ \\
\hline $\mathbf{1}$ & $24.3 \%$ & $62.5 \%$ & $73.0 \%$ & $92.4 \%$ \\
\hline & $\mathbf{1}$ & $\mathbf{2}$ & $\mathbf{3}$ & $\mathbf{4}$ \\
\hline
\end{tabular}

$63.5 \%$

$59.1 \%$

$16.3 \%$

$23.3 \%$

$27.2 \%$

$63.0 \%$

$42.1 \%$

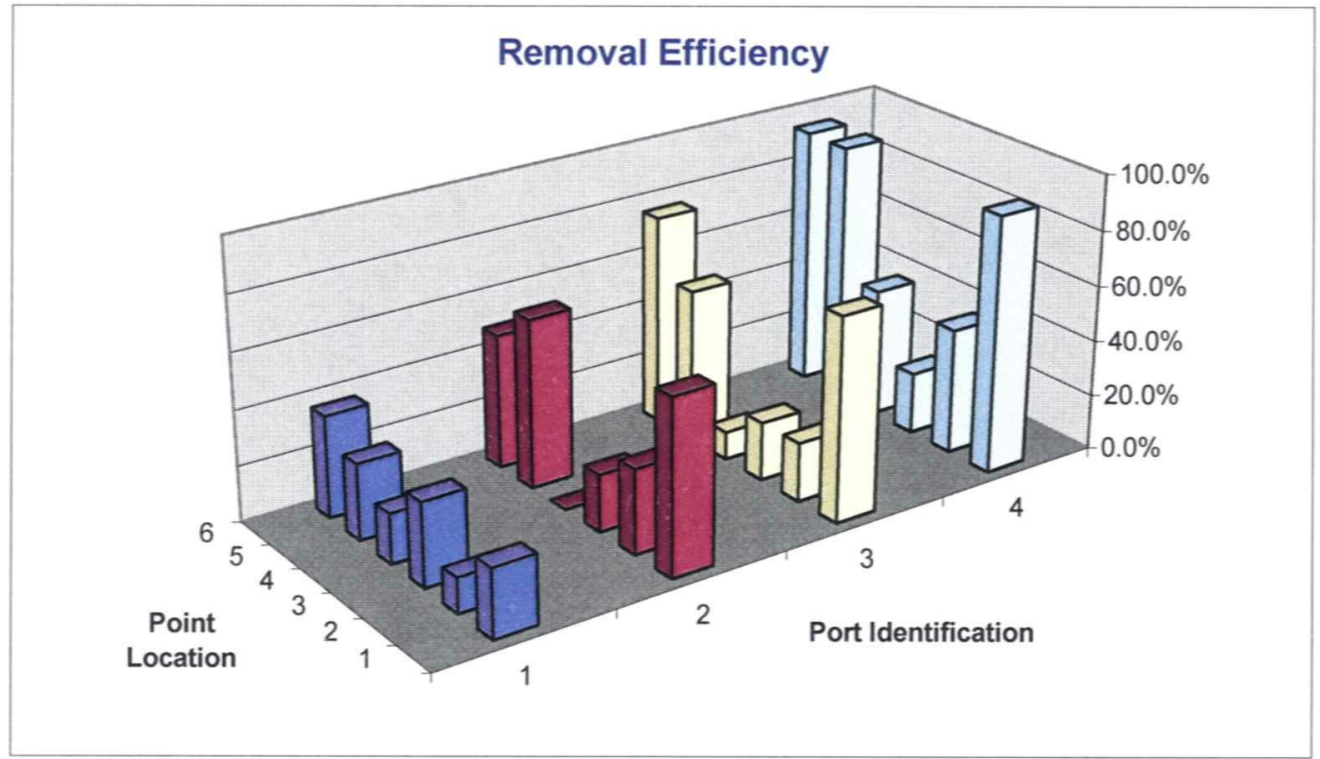


Clean Air Engineering Project \#10192 Consol Energy

AES Dresden

$\begin{array}{lrr}\text { Date: } & 3 / 28 / 2007 & \text { Run \# (Cycle \#) } 2(\mathbf{2}) \\ \text { Start Time: } & 11: 36 & \\ \text { End Time: } & 11: 59 & \end{array}$

\begin{tabular}{|c|c|c|c|c|c|c|c|c|c|c|}
\hline $\begin{array}{l}\text { Inlet } \\
\text { Point }\end{array}$ & Time Sampled & $\begin{array}{l}\text { NOx } \\
\text { ppmdv }\end{array}$ & $\begin{array}{c}\text { NOx } \\
\text { Port Avg }\end{array}$ & $\begin{array}{l}\text { PPM Dev } \\
\text { from Avg. }\end{array}$ & $\begin{array}{c}\text { O2 } \\
\% d v\end{array}$ & $\begin{array}{c}02 \\
\text { Port Avg }\end{array}$ & $\begin{array}{l}\mathrm{CO2} \\
\% d v\end{array}$ & $\begin{array}{c}\mathrm{CO} 2 \\
\text { Port Avg }\end{array}$ & $\begin{array}{c}\text { NOx } \\
\text { @ } 3 \% \text { O2 }\end{array}$ & $\begin{array}{l}\text { PPM Dev } \\
\text { from Avg. }\end{array}$ \\
\hline $1-1$ & $11: 36$ & 75.8 & & -1.0 & 7.7 & & 11.5 & & 102.4 & 16.8 \\
\hline $1-2$ & $11: 40$ & 96.4 & & 19.6 & 3.1 & & 15.3 & & 97.2 & 11.6 \\
\hline $1-3$ & $11: 44$ & 86.3 & 78.9 & 9.5 & 5.0 & 5.6 & 13.8 & 13.3 & 97.0 & 11.4 \\
\hline $1-4$ & $11: 48$ & 79.0 & & 2.2 & 4.3 & & 14.3 & & 85.1 & -0.5 \\
\hline $1-5$ & $11: 52$ & 65.8 & & -11.0 & 4.7 & & 14.1 & & 72.5 & -13.1 \\
\hline $1-6$ & $11: 56$ & 70.1 & & -6.7 & 8.6 & & 10.6 & & 102.3 & 16.8 \\
\hline $2-1$ & $11: 37$ & 77.8 & & 1.0 & 6.1 & & 12.8 & & 94.2 & 8.6 \\
\hline $2-2$ & $11: 41$ & 79.9 & & 3.1 & 3.2 & & 15.3 & & 80.8 & -4.8 \\
\hline $2-3$ & $11: 45$ & 82.4 & 73.8 & 5.6 & 5.5 & 5.0 & 13.3 & 13.8 & 96.0 & 10.4 \\
\hline $2-4$ & $11: 49$ & 73.2 & & -3.6 & 3.8 & & 14.8 & & 76.4 & -9.1 \\
\hline $2-5$ & $11: 53$ & 56.5 & & -20.3 & 4.4 & & 14.3 & & 61.2 & -24.4 \\
\hline $2-6$ & $11: 57$ & 72.8 & & -4.0 & 6.8 & & 12.2 & & 92.4 & 6.8 \\
\hline $3-1$ & $11: 38$ & 88.1 & & 11.3 & 4.2 & & 14.4 & & 94.5 & 8.9 \\
\hline $3-2$ & $11: 42$ & 78.8 & & 2.0 & 3.1 & & 15.4 & & 79.2 & -6.4 \\
\hline $3-3$ & $11: 46$ & 99.0 & 80.9 & 22.2 & 3.3 & 4.2 & 15.1 & 14.4 & 100.9 & 15.3 \\
\hline $3-4$ & $11: 50$ & 76.8 & & 0.0 & 3.6 & & 14.9 & & 79.4 & -6.2 \\
\hline $3-5$ & $11: 54$ & 63.3 & & -13.5 & 4.4 & & 14.3 & & 68.6 & -17.0 \\
\hline $3-6$ & $11: 58$ & 79.4 & & 2.6 & 6.4 & & 12.5 & & 98.2 & 12.6 \\
\hline $4-1$ & $11: 39$ & 75.0 & & -1.8 & 6.1 & & 12.8 & & 90.9 & 5.3 \\
\hline 4-2 & $11: 43$ & 79.3 & & 2.5 & 2.7 & & 15.7 & & 78.0 & -7.6 \\
\hline 4-3 & $11: 47$ & 93.5 & 73.5 & 16.7 & 3.0 & 4.3 & 15.5 & 14.3 & 93.4 & 7.9 \\
\hline 4-4 & $11: 51$ & 74.8 & & -2.0 & 3.4 & & 15.0 & & 76.7 & -8.9 \\
\hline 4-5 & $11: 55$ & 56.2 & & -20.6 & 4.2 & & 14.4 & & 60.2 & -25.4 \\
\hline 4-6 & $11: 59$ & 62.3 & & -14.5 & 6.4 & & 12.6 & & 76.7 & -8.9 \\
\hline
\end{tabular}

\begin{tabular}{|c|c|c|c|c|c|c|c|c|c|c|c|c|}
\hline $\begin{array}{l}\text { Outlet } \\
\text { Point }\end{array}$ & Time Sampled & $\begin{array}{c}\text { NOx } \\
\text { ppmdv }\end{array}$ & $\begin{array}{c}\text { NOx } \\
\text { Port Avg }\end{array}$ & $\begin{array}{l}\text { PPM Dev } \\
\text { from Avg. }\end{array}$ & $\begin{array}{c}\text { O2 } \\
\% d v\end{array}$ & $\begin{array}{c}\mathrm{O} 2 \\
\text { Port Avg }\end{array}$ & $\begin{array}{l}\mathrm{CO2} \\
\% d v\end{array}$ & $\begin{array}{c}\mathrm{CO} 2 \\
\text { Port Avg }\end{array}$ & $\begin{array}{c}\text { NOx } \\
\text { @ 3\% 02 }\end{array}$ & $\begin{array}{l}\text { PPM Dev } \\
\text { from Avg. }\end{array}$ & $\begin{array}{l}\text { Removal } \\
\text { Efficiency }\end{array}$ & $\begin{array}{c}\text { Removal Eff } \\
\text { w/ O2 correction }\end{array}$ \\
\hline $1-1$ & $11: 36$ & 69.9 & & 22.6 & 4.9 & & 13.9 & & 78.2 & 24.8 & 7.8 & 23.7 \\
\hline $1-2$ & $11: 40$ & 78.3 & & 31.0 & 5.7 & & 13.2 & & 92.0 & 38.7 & 18.8 & 5.3 \\
\hline $1-3$ & $11: 44$ & 58.5 & 62.8 & 11.2 & 5.1 & 5.7 & 13.7 & 13.2 & 66.4 & 13.1 & 32.2 & 31.5 \\
\hline $1-4$ & $11: 48$ & 71.3 & & 24.0 & 4.0 & & 14.7 & & 75.4 & 22.1 & 9.7 & 11.4 \\
\hline 1.5 & $11: 52$ & 51.0 & & 3.7 & 6.4 & & 12.6 & & 63.0 & 9.7 & 22.5 & 13.1 \\
\hline $1-6$ & $11: 56$ & 47.5 & & 0.2 & 8.2 & & 10.9 & & 66.9 & 13.6 & 32.2 & 34.6 \\
\hline $2-1$ & $11: 37$ & 30.5 & & -16.8 & 5.0 & & 13.8 & & 34.3 & -19.0 & 60.8 & 63.5 \\
\hline $2-2$ & $11: 41$ & 54.7 & & 7.4 & 4.7 & & 14.1 & & 60.5 & 7.2 & 31.5 & 25.1 \\
\hline $2-3$ & $11: 45$ & 71.9 & 48.9 & 24.6 & 4.9 & 5.3 & 14.0 & 13.5 & 80.2 & 26.9 & 12.7 & 16.4 \\
\hline $2-4$ & $11: 49$ & 73.7 & & 26.4 & 5.8 & & 13.1 & & 87.5 & 34.2 & -0.7 & -14.4 \\
\hline $2-5$ & $11: 53$ & 21.7 & & -25.6 & 4.8 & & 14.0 & & 24.2 & -29.1 & 61.6 & 60.5 \\
\hline $2-6$ & $11: 57$ & 41.1 & & -6.2 & 6.8 & & 12.2 & & 52.2 & -1.1 & 43.5 & 43.5 \\
\hline $3-1$ & $11: 38$ & 21.8 & & -25.5 & 4.0 & & 14.7 & & 23.0 & -30.3 & 75.3 & 75.6 \\
\hline $3-2$ & $11: 42$ & 70.8 & & 23.5 & 4.4 & & 14.3 & & 76.9 & 23.6 & 10.2 & 2.8 \\
\hline $3-3$ & $11: 46$ & 82.3 & 47.9 & 35.0 & 4.5 & 4.6 & 14.3 & 14.2 & 89.6 & 36.3 & 16.9 & 11.3 \\
\hline $3-4$ & $11: 50$ & 63.3 & & 16.0 & 4.7 & & 14.0 & & 70.0 & 16.7 & 17.6 & 11.9 \\
\hline $3-5$ & $11: 54$ & 28.9 & & -18.4 & 4.2 & & 14.5 & & 31.0 & -22.3 & 54.3 & 54.8 \\
\hline $3-6$ & $11: 58$ & 20.1 & & -27.2 & 5.8 & & 13.2 & & 23.8 & -29.5 & 74.7 & 75.8 \\
\hline $4-1$ & $11: 39$ & 6.5 & & -40.8 & 3.7 & & 14.9 & & 6.8 & -46.5 & 91.3 & 92.5 \\
\hline $4-2$ & $11: 43$ & 48.0 & & 0.7 & 2.8 & & 15.9 & & 47.4 & -5.9 & 39.5 & 39.3 \\
\hline $4-3$ & $11: 47$ & 77.4 & 29.8 & 30.1 & 3.3 & 3.9 & 15.3 & 14.8 & 78.9 & 25.6 & 17.2 & 15.6 \\
\hline $4-4$ & 11:51 & 38.3 & & -9.0 & 4.4 & & 14.3 & & 41.6 & -11.7 & 48.8 & 45.7 \\
\hline 4-5 & 11:55 & 4.2 & & -43.1 & 3.9 & & 14.8 & & 4.4 & -48.9 & 92.5 & 92.7 \\
\hline $4-6$ & $11: 59$ & 4.4 & & -42.9 & 5.4 & & 13.4 & & 5.1 & -48.2 & 92.9 & 93.4 \\
\hline
\end{tabular}


Run 2: Inlet - Set 2

Inlet - Oxygen (\%dv)

\begin{tabular}{|c|c|c|c|c|}
\multicolumn{1}{c|}{ AVG } & 5.6 & 5.0 & 4.2 & 4.3 \\
\hline 6 & 8.6 & 6.8 & 6.4 & 6.4 \\
\hline 5 & 4.7 & 4.4 & 4.4 & 4.2 \\
\hline 4 & 4.3 & 3.8 & 3.6 & 3.4 \\
\hline 3 & 5.0 & 5.5 & 3.3 & 3.0 \\
\hline 2 & 3.1 & 3.2 & 3.1 & 2.7 \\
\hline 1 & 7.7 & 6.1 & 4.2 & 6.1 \\
\hline & 1 & 2 & 3 & 4 \\
\hline
\end{tabular}

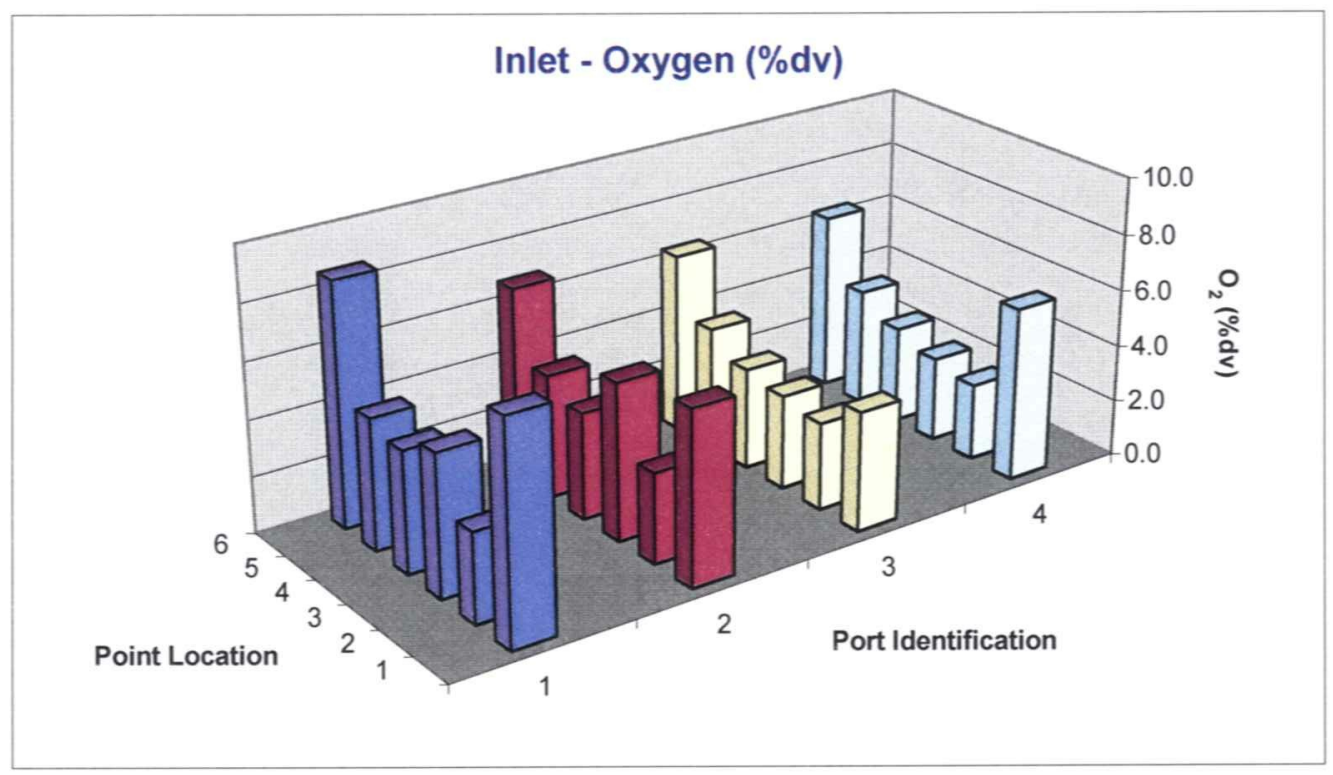

Date: $3 / 28 / 2007$

Start Time: $11: 36$

End Time: $\quad 11: 59$

1
4
.8
2
.0
0
.7


Run 2: Inlet - Set 2

Date: $3 / 28 / 2007$

Start Time: $\quad 11: 36$

End Time: $\quad 11: 59$

\begin{tabular}{|c|c|c|c|c|c|}
\hline \multicolumn{5}{|c|}{ Inlet - Carbon Dioxide (\%dv) } & \\
\hline AVG & 13.3 & 13.8 & 14.4 & 14.3 & \\
\hline 6 & 10.6 & 12.2 & 12.5 & 12.6 & 12.0 \\
\hline 5 & 14.1 & 14.3 & 14.3 & 14.4 & 14.3 \\
\hline 4 & 14.3 & 14.8 & 14.9 & 15.0 & 14.8 \\
\hline 3 & 13.8 & 13.3 & 15.1 & 15.5 & 14.4 \\
\hline 2 & 15.3 & 15.3 & 15.4 & 15.7 & 15.4 \\
\hline 1 & 11.5 & 12.8 & 14.4 & 12.8 & 12.9 \\
\hline & 1 & 2 & 3 & 4 & 13.9 \\
\hline
\end{tabular}

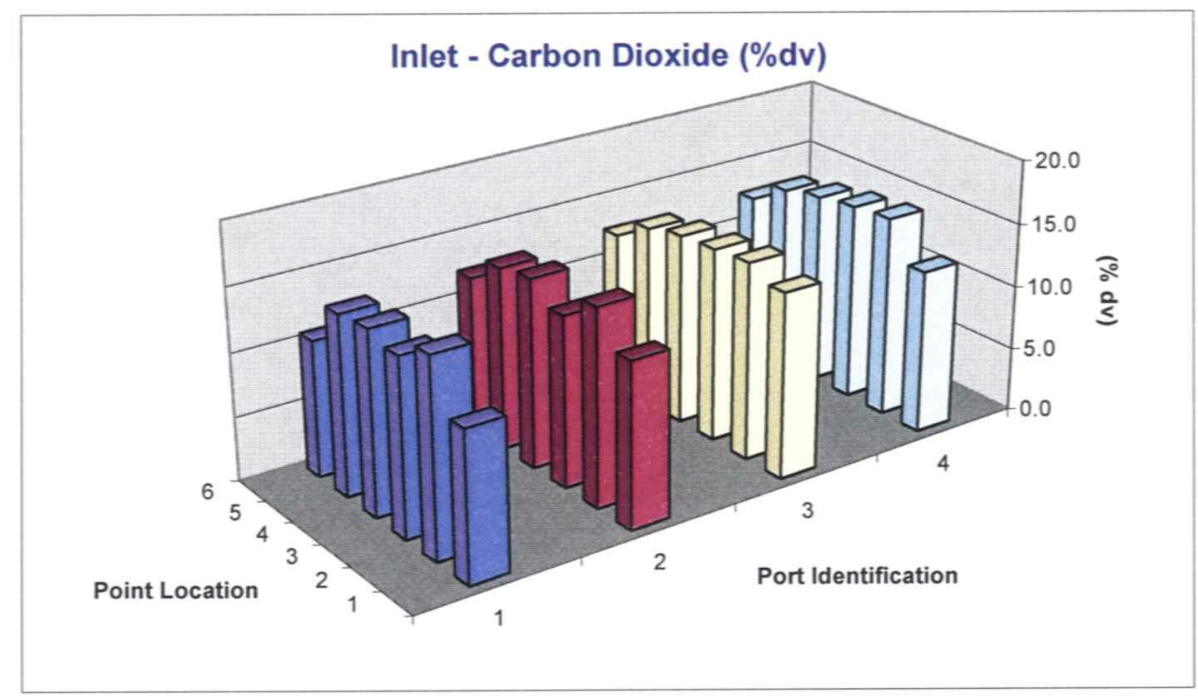


CONSOL ENERGY INC.

AES GREENIDGE STATION
Client Reference No: 4700140111

CleanAir Project No:10192

Run 2: Inlet - Set 2

Date: $3 / 28 / 2007$

Start Time: $\quad 11: 36$

End Time: $\quad 11: 59$

Inlet - Nitrogen Oxides (ppmdv), uncorrected

\begin{tabular}{|c|c|c|c|c|}
\multicolumn{1}{c|}{ AVG } & 78.9 & 73.8 & 80.9 & 73.5 \\
\hline $\mathbf{6}$ & 70.1 & 72.8 & 79.4 & 62.3 \\
\hline $\mathbf{5}$ & 65.8 & 56.5 & 63.3 & 56.2 \\
\hline $\mathbf{4}$ & 79.0 & 73.2 & 76.8 & 74.8 \\
\hline $\mathbf{3}$ & 86.3 & 82.4 & 99.0 & 93.5 \\
\hline $\mathbf{2}$ & 96.4 & 79.9 & 78.8 & 79.3 \\
\hline $\mathbf{1}$ & 75.8 & 77.8 & 88.1 & 75.0 \\
\hline & $\mathbf{1}$ & $\mathbf{2}$ & $\mathbf{3}$ & $\mathbf{4}$ \\
\hline
\end{tabular}

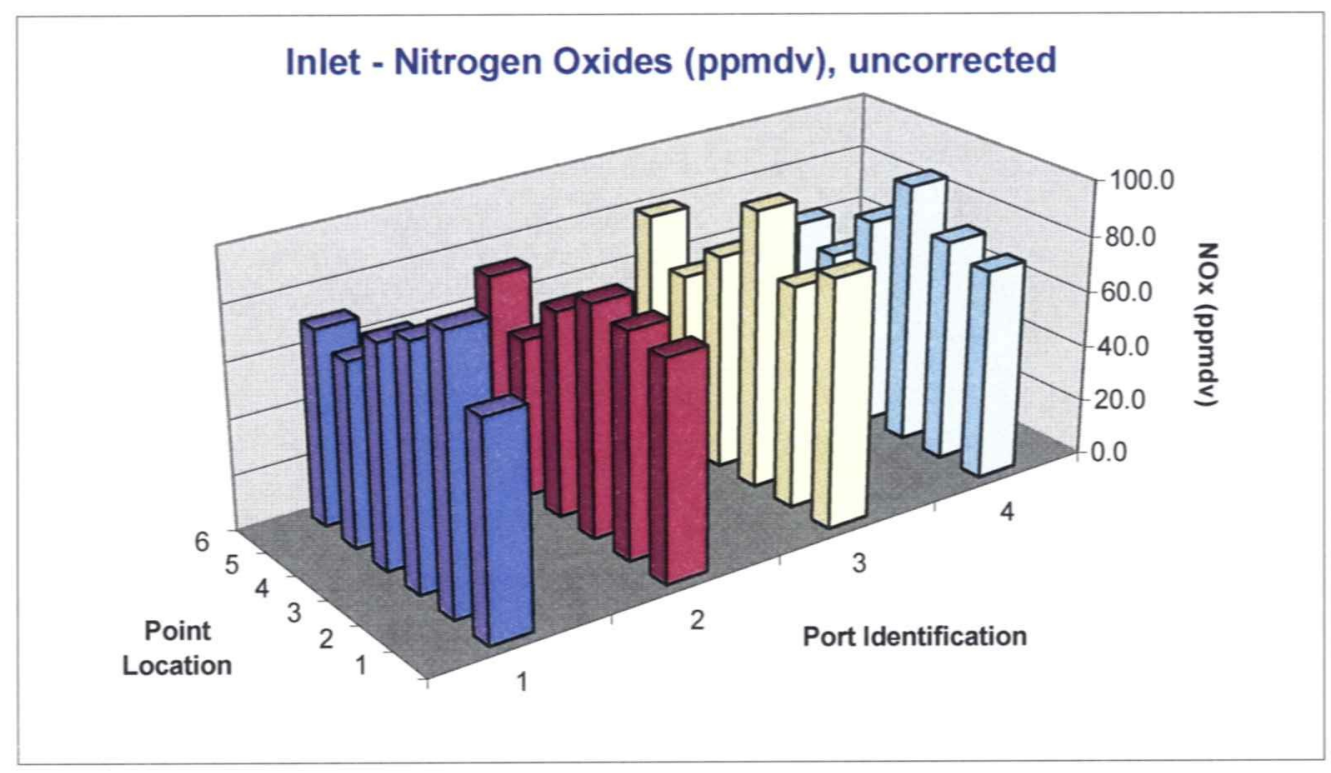


Run 2: Inlet - Set 2

Date: $3 / 28 / 2007$

Start Time: $\quad 11: 36$

End Time: $\quad 11: 59$

Inlet - Nitrogen Oxides (ppmdv @ 3\% $\mathrm{O}_{2}$ )

\begin{tabular}{|c|c|c|c|c|}
\multicolumn{1}{c|}{ AVG } & 92.7 & 83.5 & 86.8 & 79.3 \\
\hline $\mathbf{6}$ & 102.3 & 92.4 & 98.2 & 76.7 \\
\hline $\mathbf{5}$ & 72.5 & 61.2 & 68.6 & 60.2 \\
\hline $\mathbf{4}$ & 85.1 & 76.4 & 79.4 & 76.7 \\
\hline $\mathbf{3}$ & 97.0 & 96.0 & 100.9 & 93.4 \\
\hline $\mathbf{2}$ & 97.2 & 80.8 & 79.2 & 78.0 \\
\hline $\mathbf{1}$ & 102.4 & 94.2 & 94.5 & 90.9 \\
\hline & $\mathbf{1}$ & $\mathbf{2}$ & $\mathbf{3}$ & $\mathbf{4}$ \\
\hline
\end{tabular}

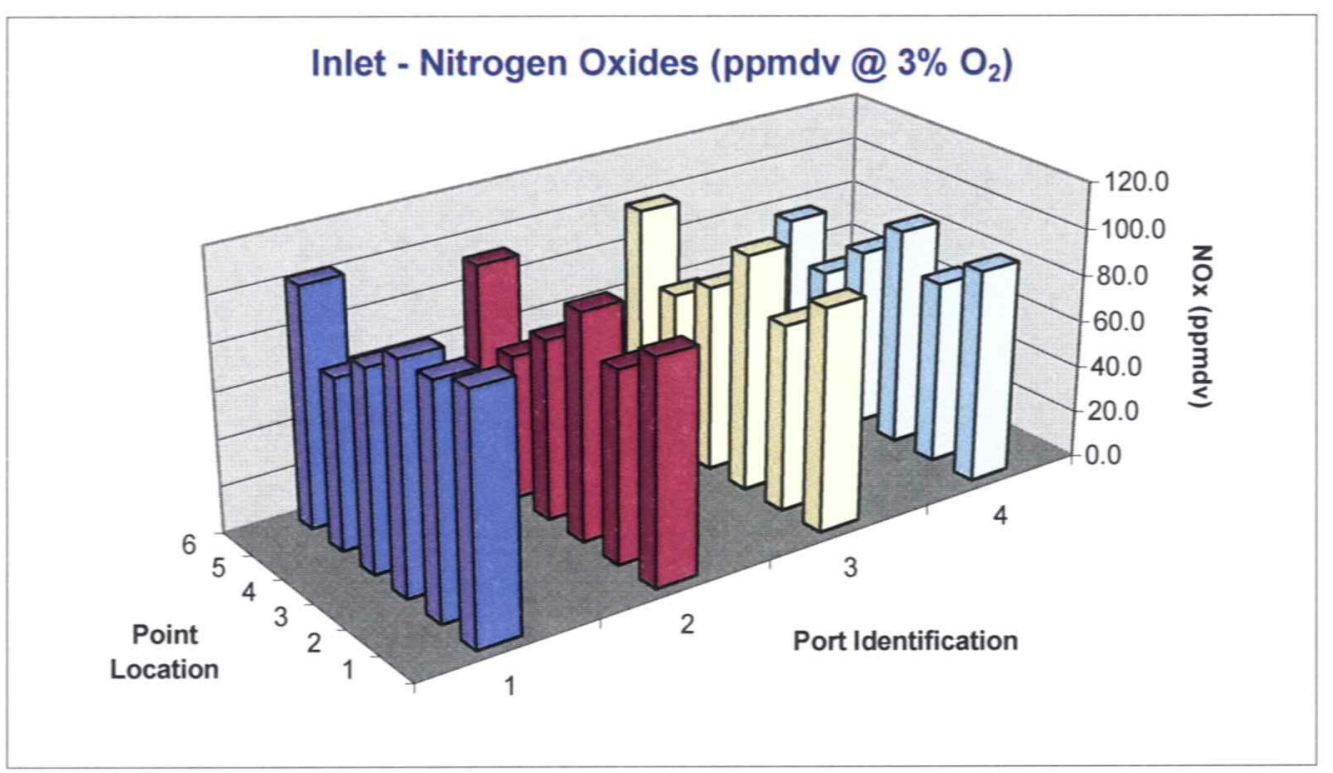


Run 2: Outlet - Set 2

Date: $3 / 28 / 2007$

Start Time: $\quad 11: 36$

End Time: $\quad 11: 59$

\begin{tabular}{|c|c|c|c|c|c|}
\hline \multicolumn{5}{|c|}{ Outlet - Oxygen (\%dv) } & \\
\hline AVG & 5.7 & 5.3 & 4.6 & 3.9 & \\
\hline 6 & 8.2 & 6.8 & 5.8 & 5.4 & 6.5 \\
\hline 5 & 6.4 & 4.8 & 4.2 & 3.9 & 4.8 \\
\hline 4 & 4.0 & 5.8 & 4.7 & 4.4 & 4.7 \\
\hline 3 & 5.1 & 4.9 & 4.5 & 3.3 & 4.4 \\
\hline 2 & 5.7 & 4.7 & 4.4 & 2.8 & 4.4 \\
\hline 1 & 4.9 & 5.0 & 4.0 & 3.7 & 4.4 \\
\hline & 1 & 2 & 3 & 4 & 4.9 \\
\hline
\end{tabular}

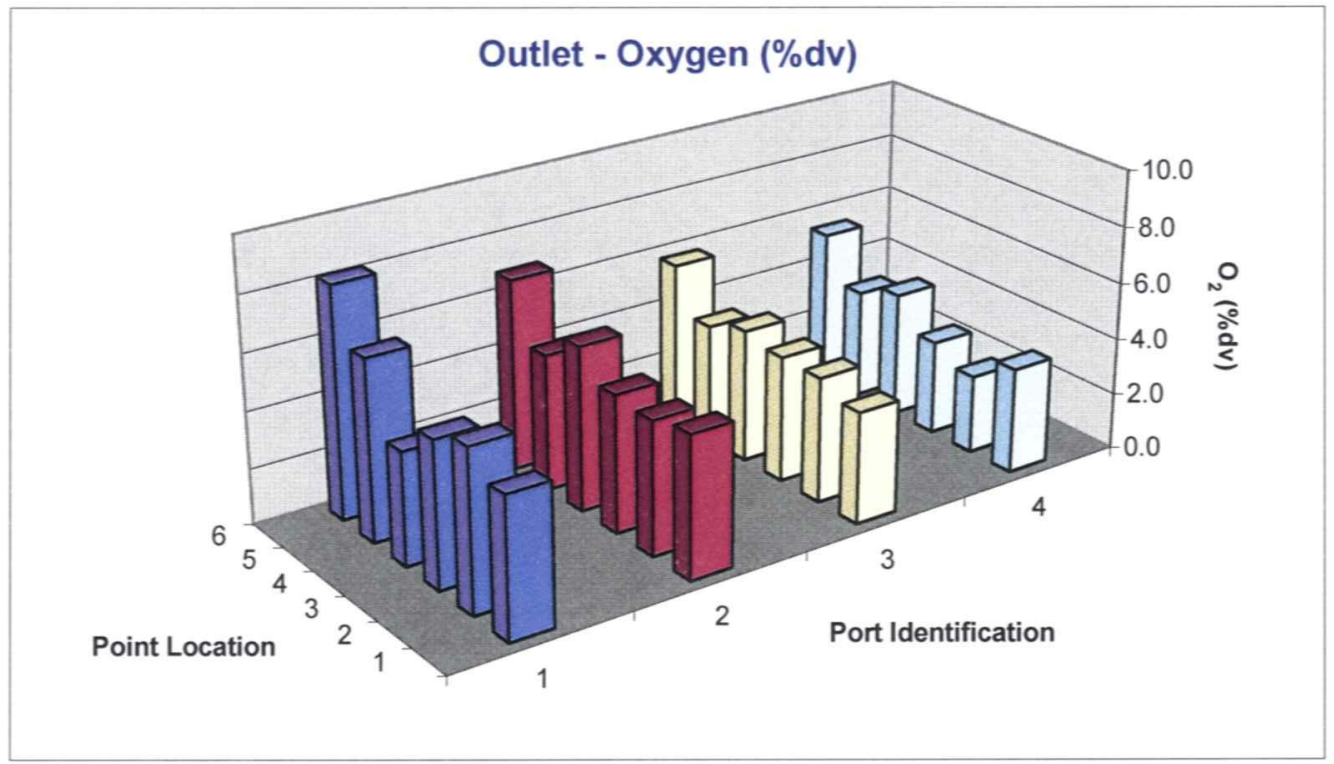


Run 2: Outlet - Set 2

Date: $3 / 28 / 2007$

Start Time: $\quad 11: 36$

End Time: 11:59

Outlet - Carbon Dioxide (\%dv)

\begin{tabular}{|c|c|c|c|c|}
\hline \multicolumn{1}{|c|}{ AVG } & 13.2 & 13.5 & 14.2 & 14.8 \\
\hline $\mathbf{6}$ & 10.9 & 12.2 & 13.2 & 13.4 \\
\hline $\mathbf{5}$ & 12.6 & 14.0 & 14.5 & 14.8 \\
\hline $\mathbf{4}$ & 14.7 & 13.1 & 14.0 & 14.3 \\
\hline $\mathbf{3}$ & 13.7 & 14.0 & 14.3 & 15.3 \\
\hline $\mathbf{2}$ & 13.2 & 14.1 & 14.3 & 15.9 \\
\hline $\mathbf{1}$ & 13.9 & 13.8 & 14.7 & 14.9 \\
\hline & $\mathbf{1}$ & $\mathbf{2}$ & $\mathbf{3}$ & $\mathbf{4}$ \\
\hline
\end{tabular}

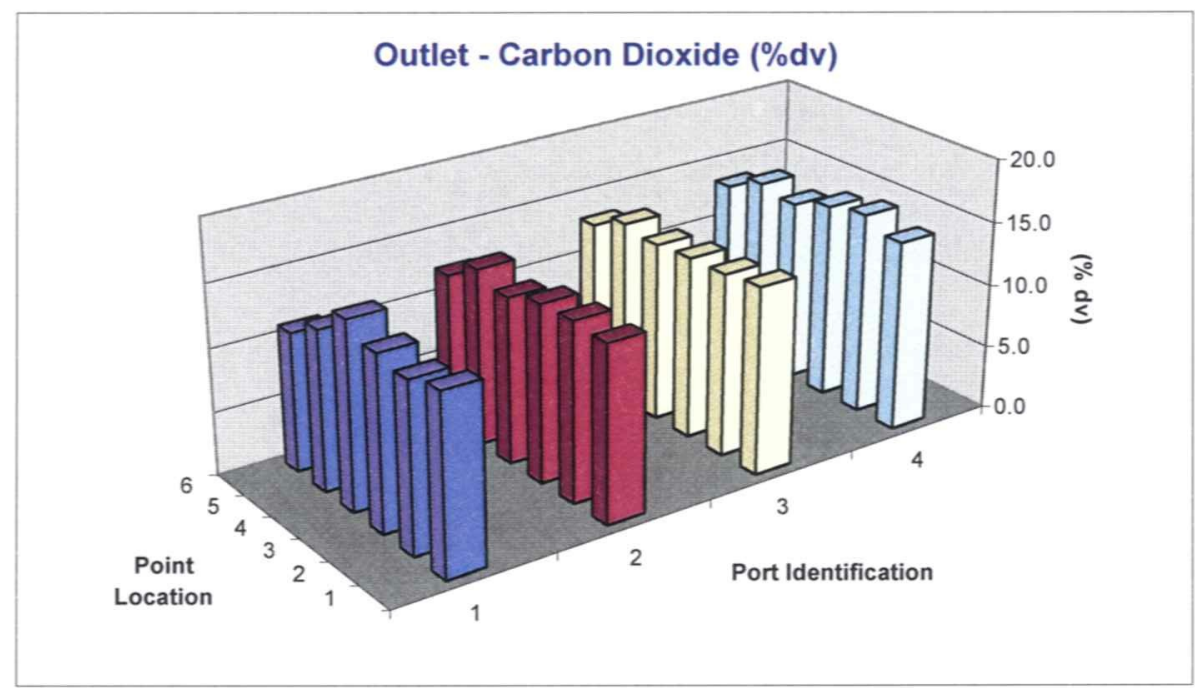


Run 2: Outlet - Set 2

Date: $3 / 28 / 2007$

Start Time: $\quad 11: 36$

End Time: $\quad 11: 59$

Outlet - Nitrogen Oxides (ppmdv), uncorrected

\begin{tabular}{|c|c|c|c|c|}
\multicolumn{1}{c}{ AVG } & 62.8 & 48.9 & 47.9 & 29.8 \\
\hline $\mathbf{6}$ & 47.5 & 41.1 & 20.1 & 4.4 \\
\hline $\mathbf{5}$ & 51.0 & 21.7 & 28.9 & 4.2 \\
\hline $\mathbf{4}$ & 71.3 & 73.7 & 63.3 & 38.3 \\
\hline $\mathbf{3}$ & 58.5 & 71.9 & 82.3 & 77.4 \\
\hline $\mathbf{2}$ & 78.3 & 54.7 & 70.8 & 48.0 \\
\hline $\mathbf{1}$ & 69.9 & 30.5 & 21.8 & 6.5 \\
\hline & $\mathbf{1}$ & $\mathbf{2}$ & $\mathbf{3}$ & $\mathbf{4}$ \\
\hline
\end{tabular}

28.3

26.5

61.7

72.5

63.0

32.2

47.3

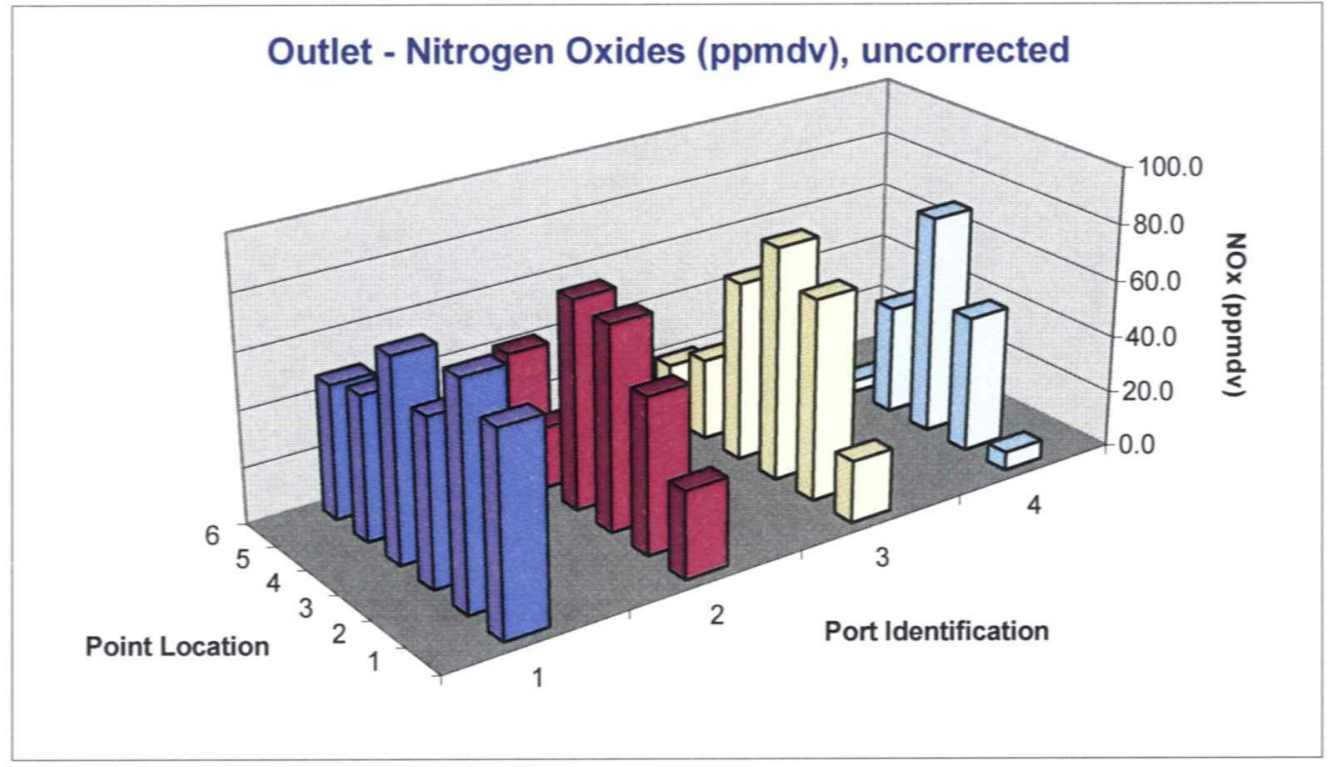


Date: $3 / 28 / 2007$

Start Time: $\quad 11: 36$

End Time: $\quad 11: 59$

Outlet - Nitrogen Oxides (ppmdv @ 3\% $\mathrm{O}_{2}$ )

\begin{tabular}{|c|c|c|c|c|}
\multicolumn{1}{c}{ AVG } & 73.7 & 56.5 & 52.4 & 30.7 \\
\hline $\mathbf{6}$ & 66.9 & 52.2 & 23.8 & 5.1 \\
\hline $\mathbf{5}$ & 63.0 & 24.2 & 31.0 & 4.4 \\
\hline $\mathbf{4}$ & 75.4 & 87.5 & 70.0 & 41.6 \\
\hline $\mathbf{3}$ & 66.4 & 80.2 & 89.6 & 78.9 \\
\hline $\mathbf{2}$ & 92.0 & 60.5 & 76.9 & 47.4 \\
\hline $\mathbf{1}$ & 78.2 & 34.3 & 23.0 & 6.8 \\
\hline & $\mathbf{1}$ & $\mathbf{2}$ & $\mathbf{3}$ & $\mathbf{4}$ \\
\hline
\end{tabular}

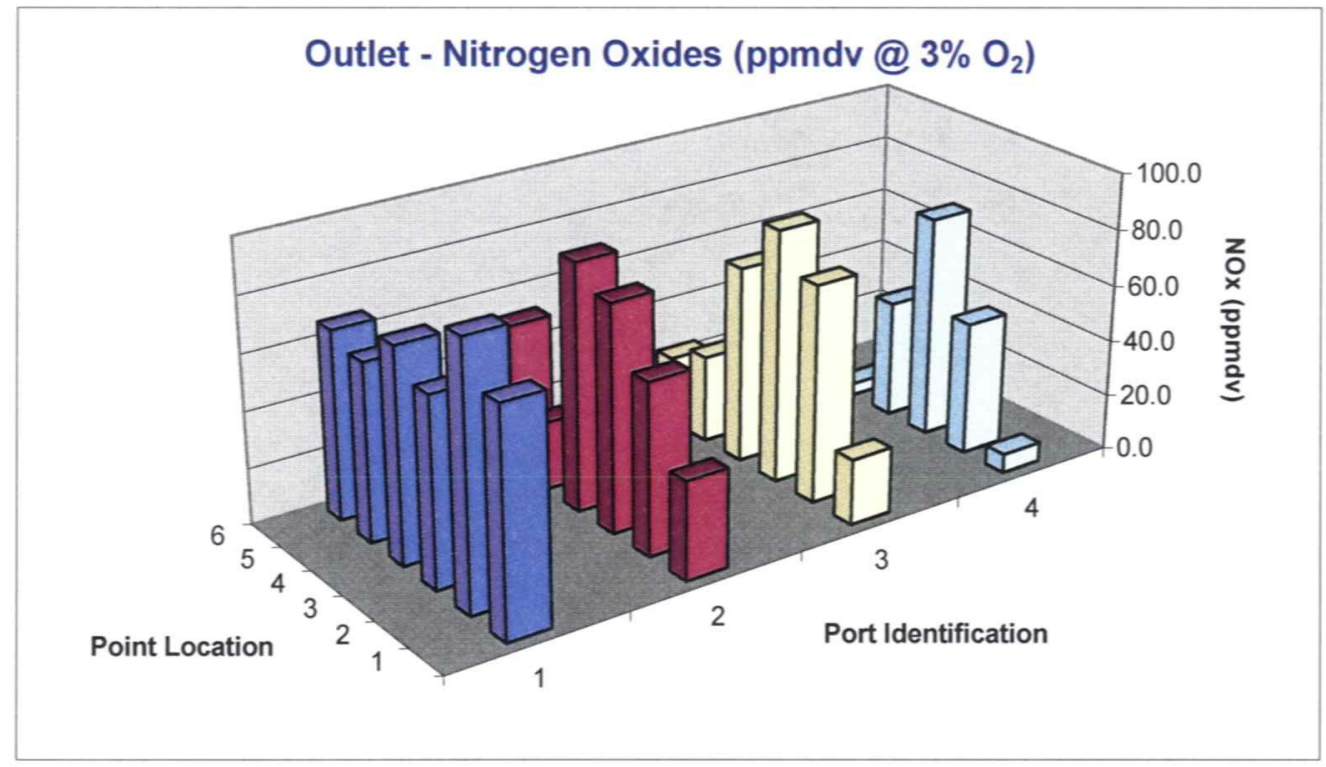


CONSOL ENERGY INC.
Client Reference No: 4700140111

CleanAir Project No:10192

Run 2: Outlet - Set 2

Date: $3 / 28 / 2007$

Start Time: $\quad 11: 36$

End Time: $\quad 11: 59$

Removal Efficiency (ppmdv @ 3\% O $\mathrm{O}_{2}$ )

\begin{tabular}{|c|c|c|c|c|}
\multicolumn{1}{c}{ AVG } & $19.9 \%$ & $32.4 \%$ & $38.7 \%$ & $63.2 \%$ \\
\hline $\mathbf{6}$ & $34.6 \%$ & $43.5 \%$ & $75.8 \%$ & $93.4 \%$ \\
\hline $\mathbf{5}$ & $13.1 \%$ & $60.5 \%$ & $54.8 \%$ & $92.7 \%$ \\
\hline $\mathbf{4}$ & $11.4 \%$ & $-14.4 \%$ & $11.9 \%$ & $45.7 \%$ \\
\hline $\mathbf{3}$ & $31.5 \%$ & $16.4 \%$ & $11.3 \%$ & $15.6 \%$ \\
\hline $\mathbf{2}$ & $5.3 \%$ & $25.1 \%$ & $2.8 \%$ & $39.3 \%$ \\
\hline $\mathbf{1}$ & $23.7 \%$ & $63.5 \%$ & $75.6 \%$ & $92.5 \%$ \\
\hline & $\mathbf{1}$ & $\mathbf{2}$ & $\mathbf{3}$ & $\mathbf{4}$ \\
\hline
\end{tabular}

$61.8 \%$

$55.3 \%$

$13.6 \%$

$18.7 \%$

$18.1 \%$

$63.8 \%$

$38.6 \%$

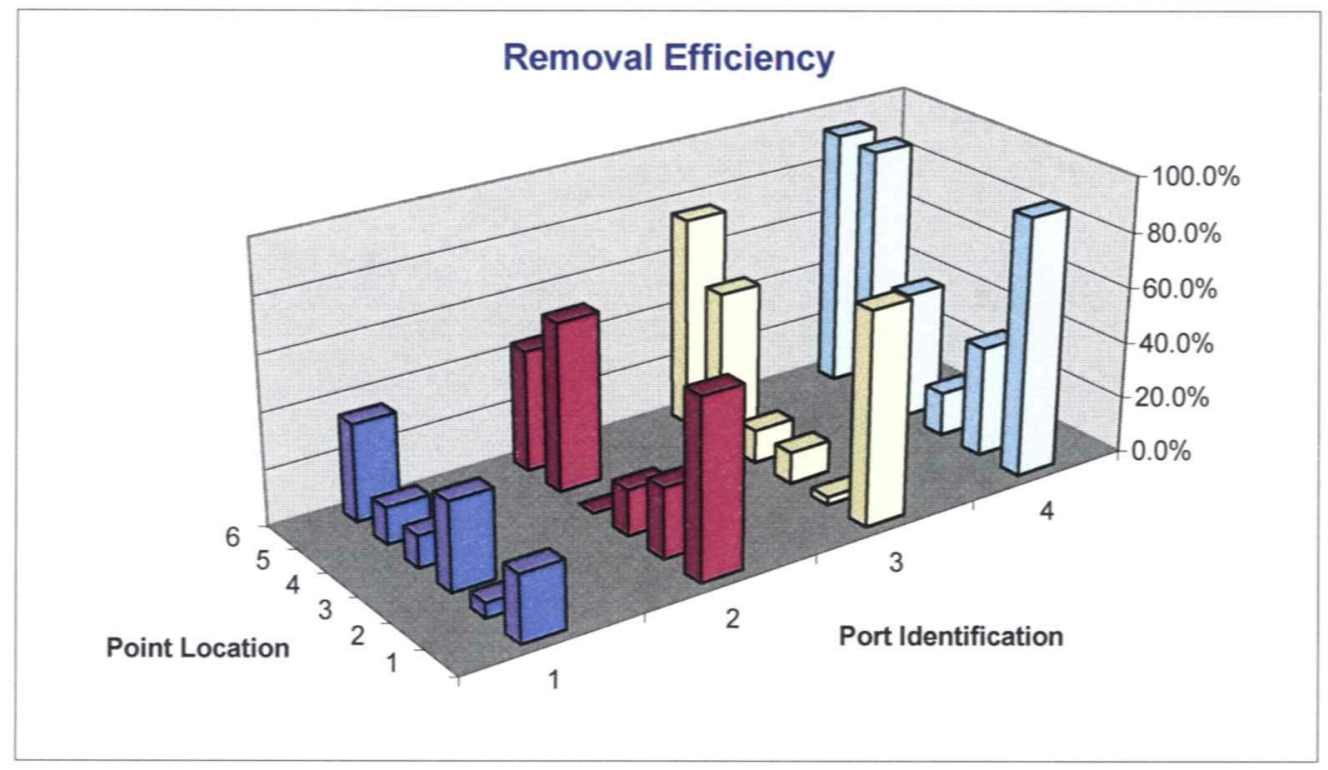


Clean Air Engineering Project \#10192

Consol Energy

AES Dresden

Date:

Start Time:

$3 / 28 / 2007$

$12: 00$
$12: 23$

Run \# (Cycle \#) 2 (3)

\begin{tabular}{|c|c|c|c|c|c|c|c|c|c|c|}
\hline \multirow[b]{2}{*}{$\begin{array}{l}\text { Inlet } \\
\text { Point }\end{array}$} & \multirow[b]{2}{*}{ Time Sampled } & & \multirow[b]{2}{*}{$\begin{array}{l}\text { PPM Dev } \\
\text { from Avg. }\end{array}$} & & & & & & \multirow[b]{2}{*}{$\begin{array}{l}\text { PPM Dev } \\
\text { from Avg. }\end{array}$} \\
\hline & & $\begin{array}{c}\text { NOx } \\
\text { ppmdv }\end{array}$ & $\begin{array}{c}\text { NOx } \\
\text { Port Avg }\end{array}$ & & $\begin{array}{c}\text { O2 } \\
\% d v\end{array}$ & $\begin{array}{c}02 \\
\text { Port Avg }\end{array}$ & $\begin{array}{l}\mathrm{CO} 2 \\
\% d v\end{array}$ & $\begin{array}{c}\mathrm{CO} 2 \\
\text { Port Avg }\end{array}$ & $\begin{array}{c}\mathrm{NOx} \\
\text { (4) 3\% } 02\end{array}$ & \\
\hline $1-1$ & $12: 00$ & 74.5 & & -2.1 & 7.7 & & 11.4 & & 101.3 & 15.7 \\
\hline $1-2$ & $12: 04$ & 98.5 & & 21.9 & 3.3 & & 15.1 & & 100.4 & 14.8 \\
\hline $1-3$ & $12: 08$ & 85.5 & 78.8 & 8.9 & 5.0 & 5.6 & 13.8 & 13.3 & 96.4 & 10.8 \\
\hline $1-4$ & $12: 12$ & 79.7 & & 3.1 & 4.3 & & 14.3 & & 86.1 & 0.5 \\
\hline $1-5$ & $12: 16$ & 65.4 & & -11.2 & 4.5 & & 14.2 & & 71.5 & -14.1 \\
\hline $1-6$ & $12: 20$ & 68.9 & & -7.7 & 8.5 & & 10.8 & & 99.3 & 13.7 \\
\hline $2-1$ & $12: 01$ & 77.3 & & 0.7 & 6.1 & & 12.7 & & 93.7 & 8.1 \\
\hline $2-2$ & $12: 05$ & 77.8 & & 1.2 & 3.3 & & 15.2 & & 79.2 & -6.4 \\
\hline $2-3$ & 12:09 & 81.9 & 74.5 & 5.3 & 5.4 & 5.0 & 13.4 & 13.8 & 94.7 & 9.1 \\
\hline $2-4$ & $12: 13$ & 73.8 & & -2.8 & 3.8 & & 14.8 & & 77.1 & -8.5 \\
\hline $2-5$ & 12:17 & 59.2 & & -17.4 & 4.3 & & 14.4 & & 63.9 & -21.7 \\
\hline $2-6$ & $12: 21$ & 77.1 & & 0.5 & 6.9 & & 12.2 & & 98.3 & 12.7 \\
\hline $3-1$ & $12: 02$ & 86.9 & & 10.3 & 4.3 & & 14.4 & & 93.6 & 8.0 \\
\hline $3-2$ & $12: 06$ & 77.4 & & 0.8 & 3.2 & & 15.2 & & 78.4 & -7.2 \\
\hline 3-3 & $12: 10$ & 96.4 & 80.0 & 19.8 & 3.3 & 4.2 & 15.2 & 14.4 & 98.0 & 12.4 \\
\hline $3-4$ & 12:14 & 76.8 & & 0.2 & 3.6 & & 14.9 & & 79.4 & -6.2 \\
\hline 3-5 & $12: 18$ & 63.6 & & -13.0 & 4.4 & & 14.3 & & 68.8 & -16.8 \\
\hline $3-6$ & $12: 22$ & 79.0 & & 2.4 & 6.3 & & 12.6 & & 96.9 & 11.3 \\
\hline $4-1$ & $12: 03$ & 74.4 & & -2.2 & 6.8 & & 12.2 & & 94.6 & 9.0 \\
\hline $4-2$ & $12: 07$ & 78.2 & & 1.6 & 2.8 & & 15.6 & & 77.3 & -8.3 \\
\hline $4-3$ & $12: 11$ & 94.0 & 73.1 & 17.4 & 3.1 & 4.4 & 15.4 & 14.2 & 94.6 & 9.0 \\
\hline 4-4 & 12:15 & 74.7 & & -1.9 & 3.4 & & 15.1 & & 76.4 & -9.2 \\
\hline $4-5$ & $12: 19$ & 55.6 & & -21.0 & 4.1 & & 14.5 & & 59.2 & -26.4 \\
\hline 4-6 & $12: 23$ & 61.4 & & -15.2 & 6.4 & & 12.6 & & 75.6 & -10.0 \\
\hline
\end{tabular}

\begin{tabular}{|c|c|c|c|c|c|c|c|c|c|c|c|c|}
\hline $\begin{array}{l}\text { Outlet } \\
\text { Point }\end{array}$ & Time Sampled & $\begin{array}{c}\text { NOx } \\
\text { ppmdv }\end{array}$ & $\begin{array}{c}\text { NOx } \\
\text { Port Avg }\end{array}$ & $\begin{array}{l}\text { PPM Dev } \\
\text { from Avg. }\end{array}$ & $\begin{array}{c}\mathrm{O} 2 \\
\% d v\end{array}$ & $\begin{array}{c}\text { O2 } \\
\text { Port Avg }\end{array}$ & $\begin{array}{l}\mathrm{CO2} \\
\% d v\end{array}$ & $\begin{array}{c}\mathrm{CO} 2 \\
\text { Port Avg }\end{array}$ & $\begin{array}{c}\text { NOx } \\
\text { (1) 3\% 02 }\end{array}$ & $\begin{array}{l}\text { PPM Dev } \\
\text { from Avg. }\end{array}$ & $\begin{array}{l}\text { Removal } \\
\text { Efficiency }\end{array}$ & $\begin{array}{c}\text { Removal Eff } \\
\text { w/ O2 correction }\end{array}$ \\
\hline $1-1$ & $12: 00$ & 68.7 & & 21.5 & 5.1 & & 13.8 & & 77.6 & 24.3 & 7.8 & 23.4 \\
\hline $1-2$ & $12: 04$ & 80.1 & & 32.9 & 5.9 & & 13.1 & & 95.5 & 42.2 & 18.7 & 4.9 \\
\hline $1-3$ & $12: 08$ & 57.9 & 62.5 & 10.7 & 5.3 & 5.8 & 13.6 & 13.1 & 66.4 & 13.1 & 32.3 & 31.1 \\
\hline $1-4$ & $12: 12$ & 68.1 & & 20.9 & 4.6 & & 14.2 & & 74.6 & 21.3 & 14.6 & 13.4 \\
\hline 1.5 & $12: 16$ & 51.3 & & 4.1 & 6.2 & & 12.7 & & 62.6 & 9.3 & 21.6 & 12.4 \\
\hline $1-6$ & $12: 20$ & 49.0 & & 1.8 & 7.9 & & 11.2 & & 67.4 & 14.1 & 28.9 & 32.1 \\
\hline $2-1$ & $12: 01$ & 30.5 & & -16.7 & 5.1 & & 13.7 & & 34.6 & -18.7 & 60.5 & 63.1 \\
\hline $2-2$ & $12: 05$ & 53.3 & & 6.1 & 4.7 & & 14.1 & & 58.9 & 5.6 & 31.5 & 25.6 \\
\hline $2-3$ & $12: 09$ & 72.1 & 49.3 & 24.9 & 4.7 & 5.3 & 14.0 & 13.5 & 79.8 & 26.5 & 12.0 & 15.7 \\
\hline $2-4$ & $12: 13$ & 74.0 & & 26.8 & 5.8 & & 13.1 & & 87.9 & 34.6 & -0.3 & -14.0 \\
\hline $2-5$ & $12: 17$ & 22.3 & & -24.9 & 4.8 & & 14.0 & & 24.8 & -28.5 & 62.3 & 61.2 \\
\hline $2-6$ & $12: 21$ & 43.5 & & -3.7 & 6.7 & & 12.2 & & 55.0 & 1.7 & 43.6 & 44.1 \\
\hline $3-1$ & $12: 02$ & 20.4 & & -26.8 & 3.8 & & 14.8 & & 21.4 & -31.9 & 76.5 & 77.1 \\
\hline $3-2$ & $12: 06$ & 72.6 & & 25.4 & 4.5 & & 14.3 & & 79.0 & 25.7 & 6.2 & -0.9 \\
\hline $3-3$ & $12: 10$ & 83.6 & 47.9 & 36.4 & 4.3 & 4.6 & 14.4 & 14.2 & 90.2 & 36.9 & 13.3 & 7.9 \\
\hline $3-4$ & $12: 14$ & 58.6 & & 11.4 & 4.9 & & 13.9 & & 65.6 & 12.3 & 23.7 & 17.4 \\
\hline $3-5$ & $12: 18$ & 29.5 & & -17.7 & 4.2 & & 14.5 & & 31.6 & -21.7 & 53.6 & 54.1 \\
\hline $3-6$ & $12: 22$ & 22.9 & & -24.3 & 5.6 & & 13.2 & & 26.8 & -26.5 & 71.0 & 72.3 \\
\hline $4-1$ & $12: 03$ & 6.2 & & -41.0 & 4.0 & & 14.7 & & 6.6 & -46.7 & 91.7 & 93.1 \\
\hline $4-2$ & $12: 07$ & 44.4 & & -2.8 & 2.9 & & 15.7 & & 44.2 & -9.1 & 43.2 & 42.8 \\
\hline $4-3$ & $12: 11$ & 74.6 & 28.9 & 27.4 & 3.4 & 4.0 & 15.3 & 14.7 & 76.3 & 23.0 & 20.6 & 19.4 \\
\hline $4-4$ & $12: 15$ & 39.9 & & -7.3 & 4.4 & & 14.4 & & 43.3 & -10.0 & 46.6 & 43.4 \\
\hline 4.5 & $12: 19$ & 3.8 & & -43.4 & 3.7 & & 15.0 & & 4.0 & -49.3 & 93.2 & 93.3 \\
\hline $4-6$ & $12: 23$ & 4.4 & & -42.8 & 5.3 & & 13.6 & & 5.1 & -48.2 & 92.8 & 93.3 \\
\hline
\end{tabular}


Run 2: Inlet - Set 3

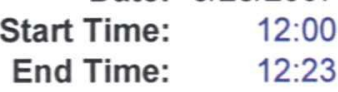

Inlet - Oxygen (\%dv)

\begin{tabular}{|c|c|c|c|c|c|}
\multicolumn{1}{c|}{ AVG } & 5.6 & 5.0 & 4.2 & 4.4 & \\
\hline 6 & 8.5 & 6.9 & 6.3 & 6.4 \\
\hline 5 & 4.5 & 4.3 & 4.4 & 4.1 & 4.3 \\
\hline 4 & 4.3 & 3.8 & 3.6 & 3.4 & 3.8 \\
\hline 3 & 5.0 & 5.4 & 3.3 & 3.1 \\
\hline 2 & 3.3 & 3.3 & 3.2 & 2.8 & 4.2 \\
\hline 1 & 7.7 & 6.1 & 4.3 & 6.8 & 6.2 \\
\hline & 1 & 2 & 3 & 4 & 4.8 \\
\cline { 2 - 5 } & & & & & \\
\end{tabular}

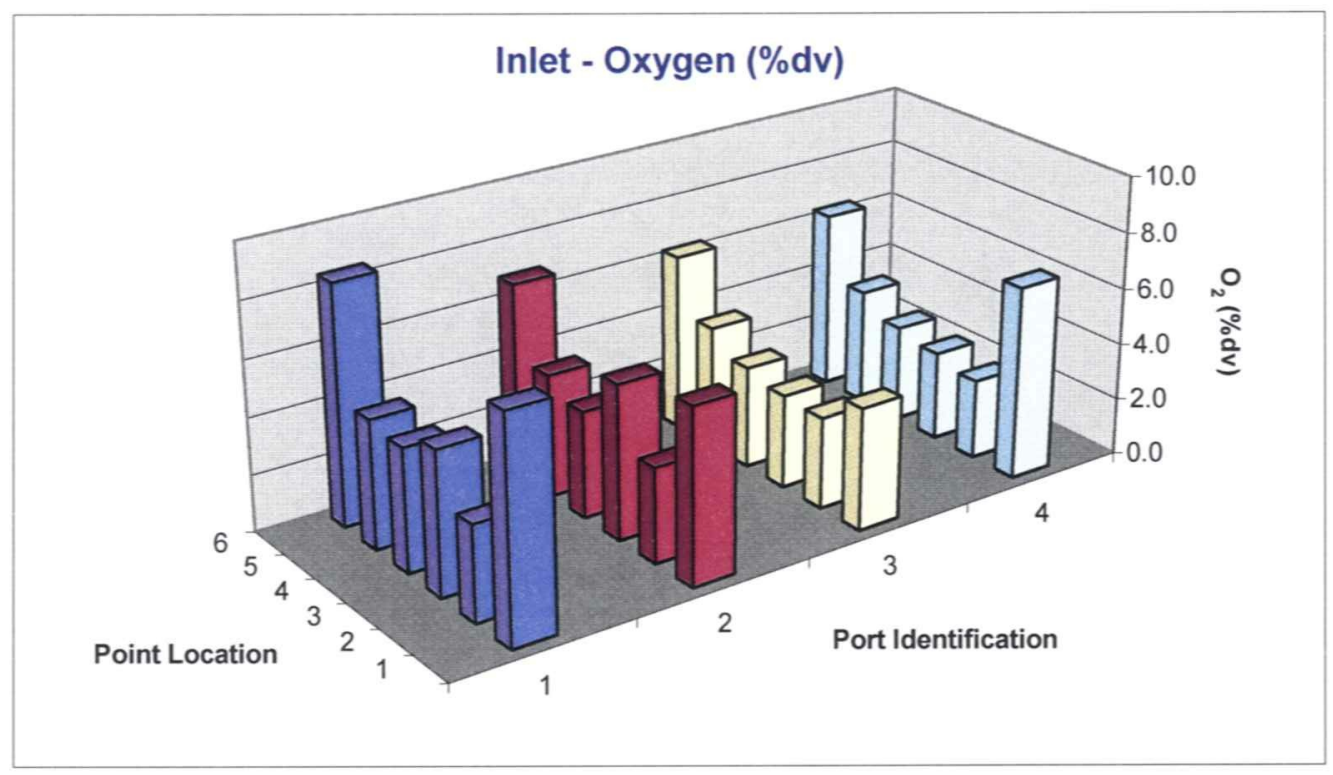


Run 2: Inlet - Set 3

Date: $3 / 28 / 2007$

Start Time: $\quad 12: 00$

End Time: $\quad 12: 23$

Inlet - Carbon Dioxide (\%dv)

\begin{tabular}{|c|c|c|c|c|r|}
\hline AVG & 13.3 & 13.8 & 14.4 & 14.2 & \\
\hline $\mathbf{6}$ & 10.8 & 12.2 & 12.6 & 12.6 & 12.0 \\
\hline $\mathbf{5}$ & 14.2 & 14.4 & 14.3 & 14.5 & 14.3 \\
\hline $\mathbf{4}$ & 14.3 & 14.8 & 14.9 & 15.1 & 14.8 \\
\hline $\mathbf{3}$ & 13.8 & 13.4 & 15.2 & 15.4 & 14.4 \\
\hline $\mathbf{2}$ & 15.1 & 15.2 & 15.2 & 15.6 & 15.3 \\
\hline $\mathbf{1}$ & 11.4 & 12.7 & 14.4 & 12.2 & 12.7 \\
\cline { 2 - 5 } & $\mathbf{1}$ & $\mathbf{2}$ & $\mathbf{3}$ & $\mathbf{4}$ & $\mathbf{1 3}$ \\
\cline { 2 - 5 } & & & &
\end{tabular}

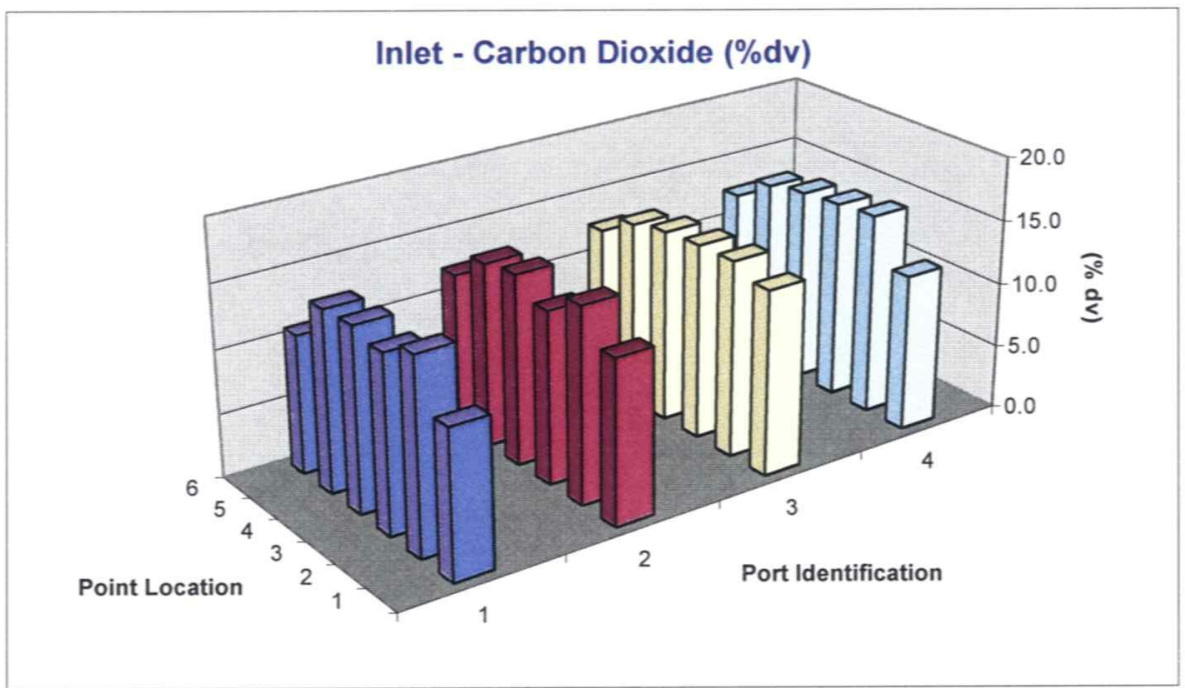


Run 2: Inlet - Set 3

ate: $3 / 28 / 2007$

Start Time: $\quad 12: 00$

End Time: $\quad 12: 23$

Inlet - Nitrogen Oxides (ppmdv), uncorrected

\begin{tabular}{|c|c|c|c|c|}
\multicolumn{1}{|c}{ AVG } & 78.8 & 74.5 & 80.0 & 73.1 \\
\hline $\mathbf{6}$ & 68.9 & 77.1 & 79.0 & 61.4 \\
\hline $\mathbf{5}$ & 65.4 & 59.2 & 63.6 & 55.6 \\
\hline $\mathbf{4}$ & 79.7 & 73.8 & 76.8 & 74.7 \\
\hline $\mathbf{3}$ & 85.5 & 81.9 & 96.4 & 94.0 \\
\hline $\mathbf{2}$ & 98.5 & 77.8 & 77.4 & 78.2 \\
\hline $\mathbf{1}$ & 74.5 & 77.3 & 86.9 & 74.4 \\
\hline
\end{tabular}

71.6

61.0

76.3

89.5

83.0

78.3

76.6

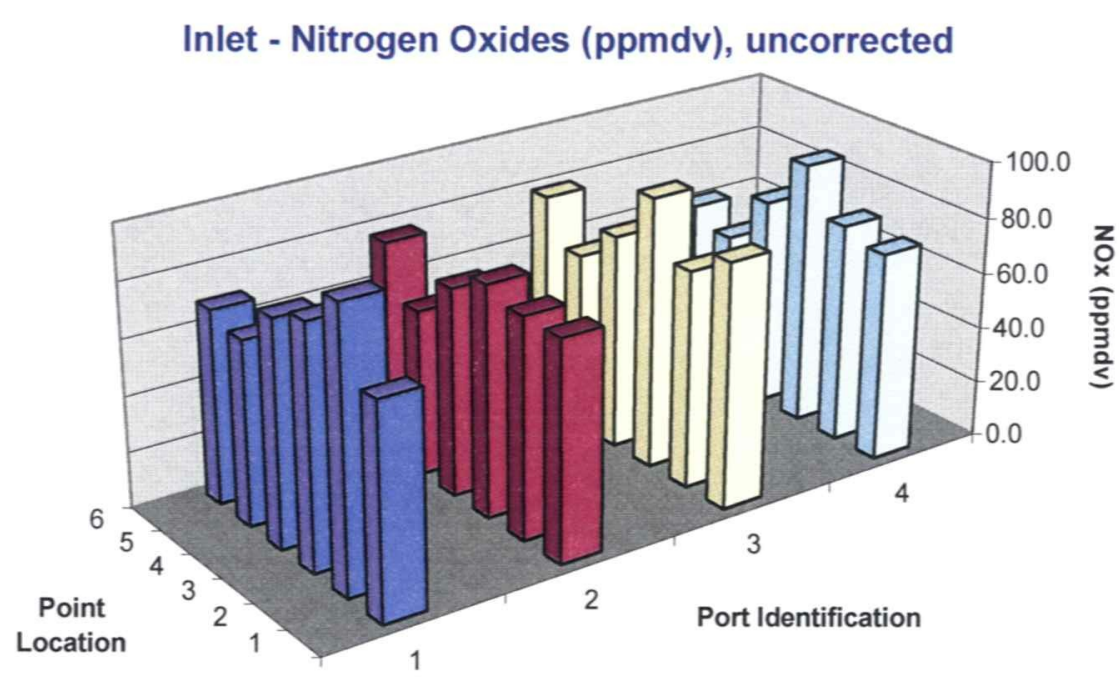


Run 2: Inlet - Set 3

Date: $3 / 28 / 2007$

Start Time: $\quad 12: 00$

End Time: $\quad 12: 23$

Inlet - Nitrogen Oxides (ppmdv @ 3\% $\mathrm{O}_{2}$ )

\begin{tabular}{|c|c|c|c|c|}
\multicolumn{1}{|c}{ AVG } & 92.5 & 84.5 & 85.8 & 79.6 \\
\hline $\mathbf{6}$ & 99.3 & 98.3 & 96.9 & 75.6 \\
\hline $\mathbf{5}$ & 71.5 & 63.9 & 68.8 & 59.2 \\
\hline $\mathbf{4}$ & 86.1 & 77.1 & 79.4 & 76.4 \\
\hline $\mathbf{3}$ & 96.4 & 94.7 & 98.0 & 94.6 \\
\hline $\mathbf{2}$ & 100.4 & 79.2 & 78.4 & 77.3 \\
\hline $\mathbf{1}$ & 101.3 & 93.7 & 93.6 & 94.6 \\
\hline
\end{tabular}

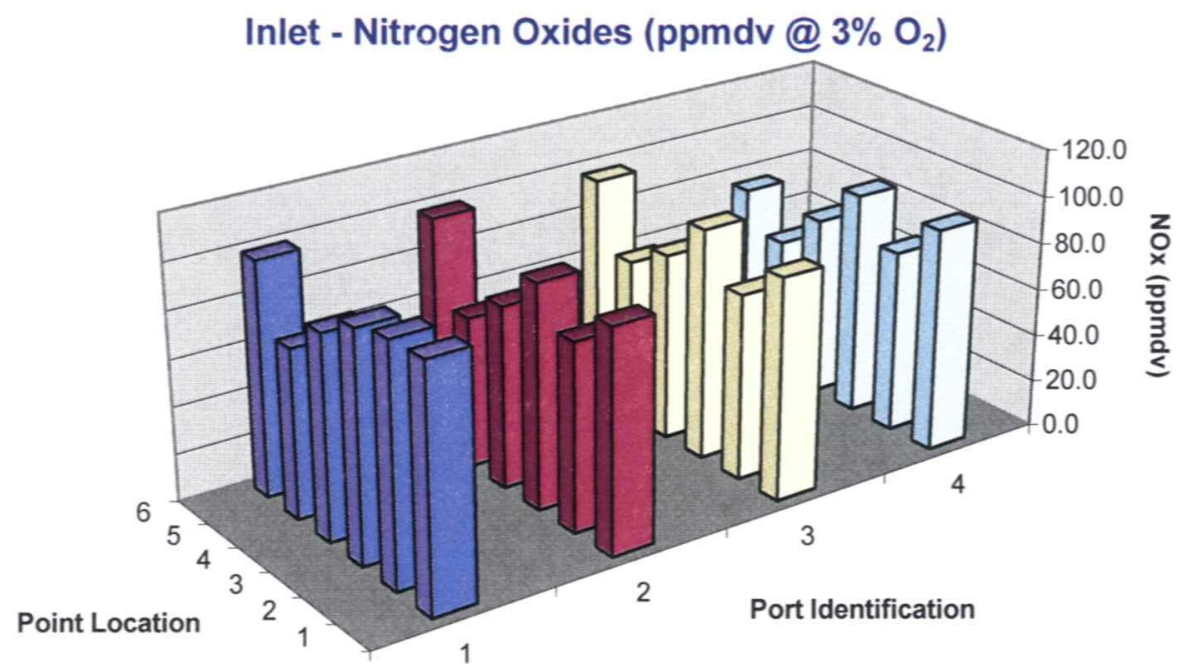


Run 2: Outlet - Set 3

Date: $3 / 28 / 2007$

Start Time: $\quad 12: 00$

End Time: $12: 23$

\begin{tabular}{|c|c|c|c|c|c|}
\hline \multicolumn{5}{|c|}{ Outlet - Oxygen (\%dv) } & \\
\hline AVG & 5.8 & 5.3 & 4.6 & 4.0 & \\
\hline 6 & 7.9 & 6.7 & 5.6 & 5.3 & 6.4 \\
\hline 5 & 6.2 & 4.8 & 4.2 & 3.7 & 4.7 \\
\hline 4 & 4.6 & 5.8 & 4.9 & 4.4 & 4.9 \\
\hline 3 & 5.3 & 4.7 & 4.3 & 3.4 & 4.4 \\
\hline 2 & 5.9 & 4.7 & 4.5 & 2.9 & 4.5 \\
\hline 1 & 5.1 & 5.1 & 3.8 & 4.0 & 4.5 \\
\hline & 1 & 2 & 3 & 4 & 4.9 \\
\hline
\end{tabular}

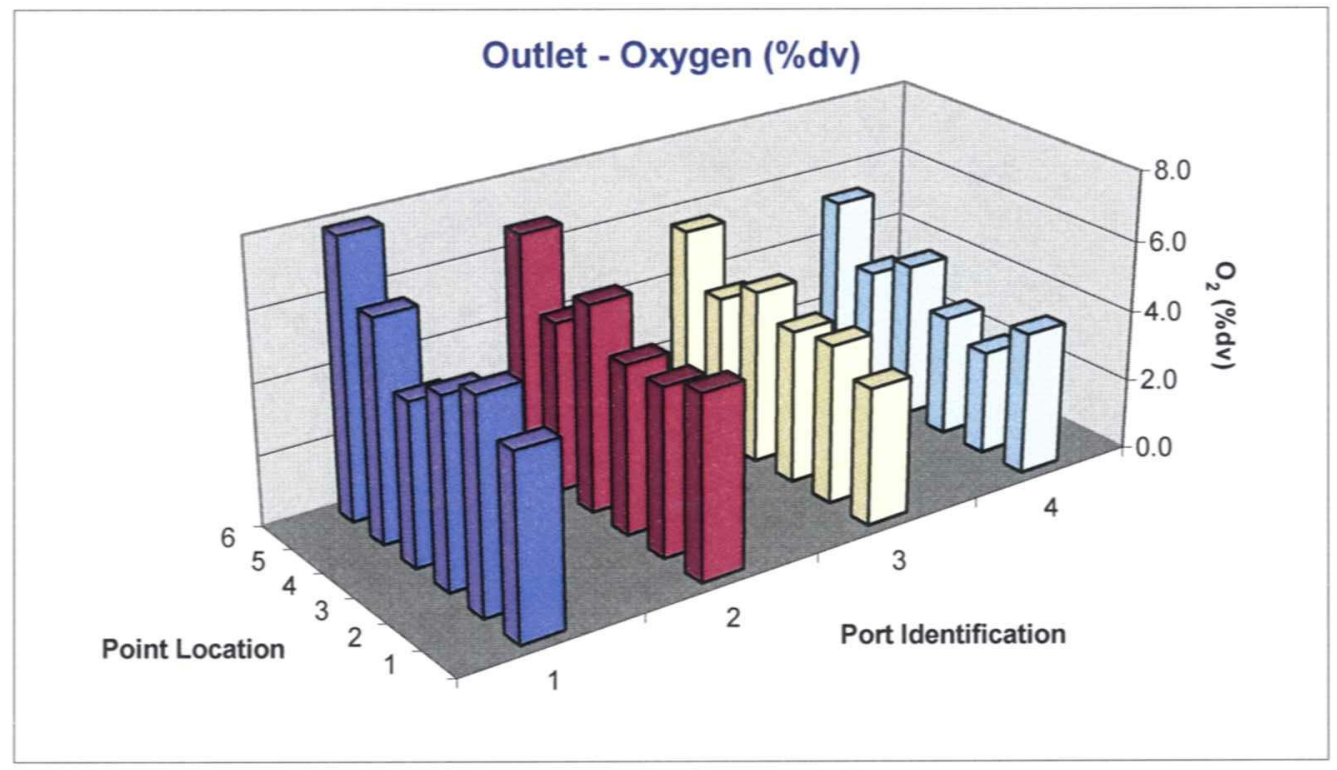


Run 2: Outlet - Set 3

Date: $3 / 28 / 2007$

Start Time: $\quad 12: 00$

End Time: $12: 23$

Outlet - Carbon Dioxide (\%dv)

\begin{tabular}{|c|c|c|c|c|c|}
\hline AVG & 13.1 & 13.5 & 14.2 & 14.7 & \\
\hline $\mathbf{6}$ & 11.2 & 12.2 & 13.2 & 13.6 & 12.6 \\
\hline $\mathbf{5}$ & 12.7 & 14.0 & 14.5 & 15.0 & 14.0 \\
\hline $\mathbf{4}$ & 14.2 & 13.1 & 13.9 & 14.4 & 13.9 \\
\hline $\mathbf{3}$ & 13.6 & 14.0 & 14.4 & 15.3 & 14.3 \\
\hline $\mathbf{2}$ & 13.1 & 14.1 & 14.3 & 15.7 & 14.3 \\
\hline $\mathbf{1}$ & 13.8 & 13.7 & 14.8 & 14.7 & 14.3 \\
\cline { 2 - 5 } & $\mathbf{1}$ & $\mathbf{2}$ & $\mathbf{3}$ & $\mathbf{4}$ & $\mathbf{1 3 . 9}$ \\
\cline { 2 - 5 } & & & & &
\end{tabular}

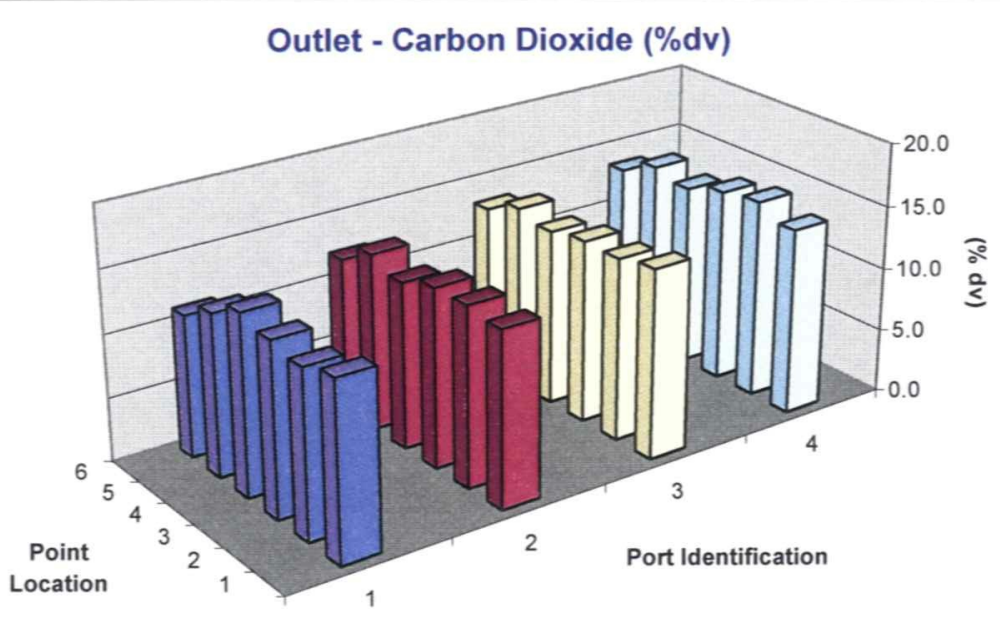


Run 2: Outlet - Set 3

Date: $3 / 28 / 2007$

Start Time: $\quad 12: 00$

End Time: $\quad 12: 23$

Outlet - Nitrogen Oxides (ppmdv), uncorrected

\begin{tabular}{|c|c|c|c|c|}
\hline AVG & 62.5 & 49.3 & 47.9 & 28.9 \\
\hline 6 & 49.0 & 43.5 & 22.9 & 4.4 \\
\hline 5 & 51.3 & 22.3 & 29.5 & 3.8 \\
\hline 4 & 68.1 & 74.0 & 58.6 & 39.9 \\
\hline 3 & 57.9 & 72.1 & 83.6 & 74.6 \\
\hline 2 & 80.1 & 53.3 & 72.6 & 44.4 \\
\hline \multirow[t]{2}{*}{1} & 68.7 & 30.5 & 20.4 & 6.2 \\
\hline & 1 & 2 & 3 & 4 \\
\hline
\end{tabular}

30.0

26.7

60.2

72.1

62.6

31.5

47.2

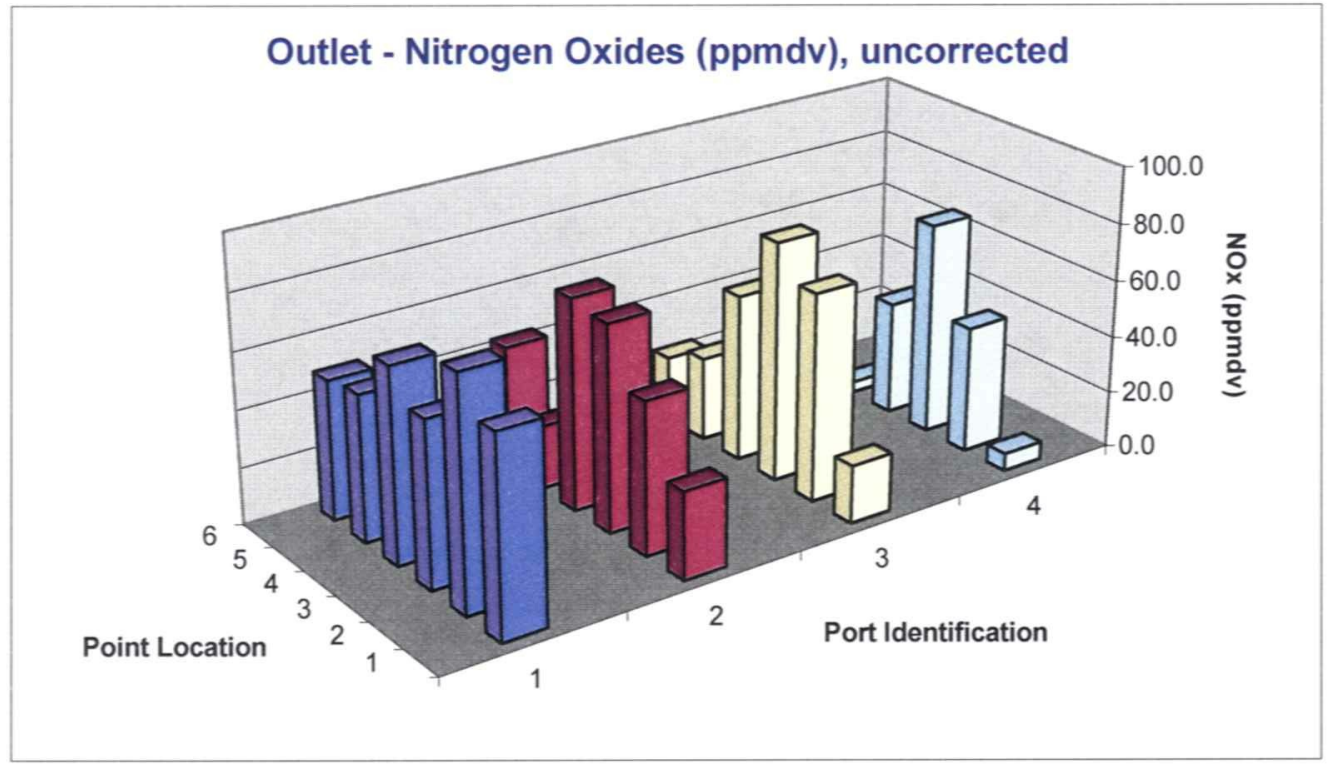


CONSOL ENERGY INC.
Client Reference No: 4700140111

CleanAir Project No:10192

Run 2: Outlet - Set 3

Date: $3 / 28 / 2007$

Start Time: $\quad 12: 00$

End Time: $\quad 12: 23$

Outlet - Nitrogen Oxides (ppmdv @ 3\% O

\begin{tabular}{|c|c|c|c|c|}
\multicolumn{1}{|c}{ AVG } & 74.0 & 56.8 & 52.4 & 29.9 \\
\hline $\mathbf{6}$ & 67.4 & 55.0 & 26.8 & 5.1 \\
\hline $\mathbf{5}$ & 62.6 & 24.8 & 31.6 & 4.0 \\
\hline $\mathbf{4}$ & 74.6 & 87.9 & 65.6 & 43.3 \\
\hline $\mathbf{3}$ & 66.4 & 79.8 & 90.2 & 76.3 \\
\hline $\mathbf{2}$ & 95.5 & 58.9 & 79.0 & 44.2 \\
\hline $\mathbf{1}$ & 77.6 & 34.6 & 21.4 & 6.6 \\
\hline & $\mathbf{1}$ & $\mathbf{2}$ & $\mathbf{3}$ & $\mathbf{4}$ \\
\hline
\end{tabular}

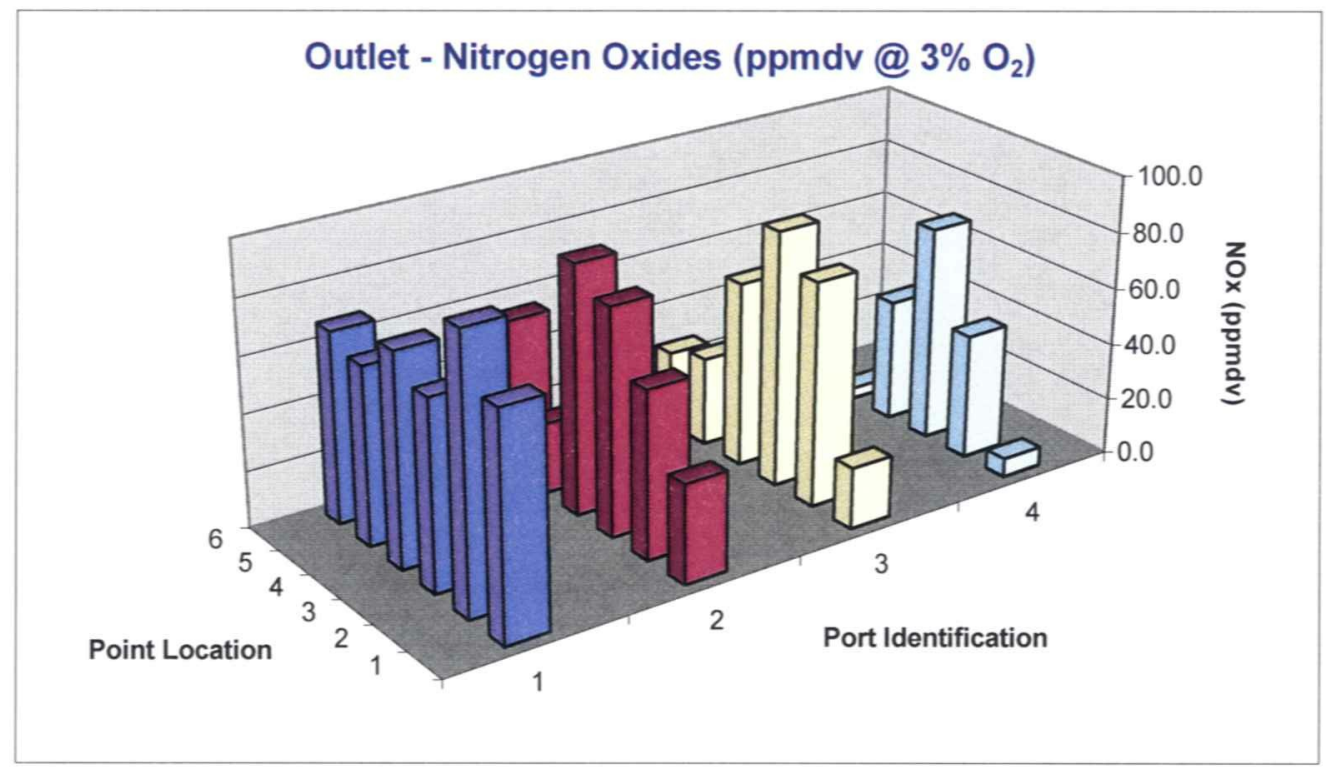


CONSOL ENERGY INC.
Client Reference No: 4700140111

CleanAir Project No:10192

Run 2: Outlet - Set 3

Date: $3 / 28 / 2007$

Start Time: $\quad 12: 00$

End Time: $\quad 12: 23$

Removal Efficiency (ppmdv @ 3\% $\mathrm{O}_{2}$ )

\begin{tabular}{|c|c|c|c|c|}
\multicolumn{1}{c}{ AVG } & $19.5 \%$ & $32.6 \%$ & $38.0 \%$ & $64.2 \%$ \\
\hline $\mathbf{6}$ & $32.1 \%$ & $44.1 \%$ & $72.3 \%$ & $93.3 \%$ \\
\hline $\mathbf{5}$ & $12.4 \%$ & $61.2 \%$ & $54.1 \%$ & $93.3 \%$ \\
\hline $\mathbf{4}$ & $13.4 \%$ & $-14.0 \%$ & $17.4 \%$ & $43.4 \%$ \\
\hline $\mathbf{3}$ & $31.1 \%$ & $15.7 \%$ & $7.9 \%$ & $19.4 \%$ \\
\hline $\mathbf{2}$ & $4.9 \%$ & $25.6 \%$ & $-0.9 \%$ & $42.8 \%$ \\
\hline $\mathbf{1}$ & $23.4 \%$ & $63.1 \%$ & $77.1 \%$ & $93.1 \%$ \\
\hline & $\mathbf{1}$ & $\mathbf{2}$ & $\mathbf{3}$ & $\mathbf{4}$ \\
\hline
\end{tabular}

$60.4 \%$

$55.3 \%$

$15.0 \%$

$18.5 \%$

$18.1 \%$

$64.2 \%$

$38.6 \%$

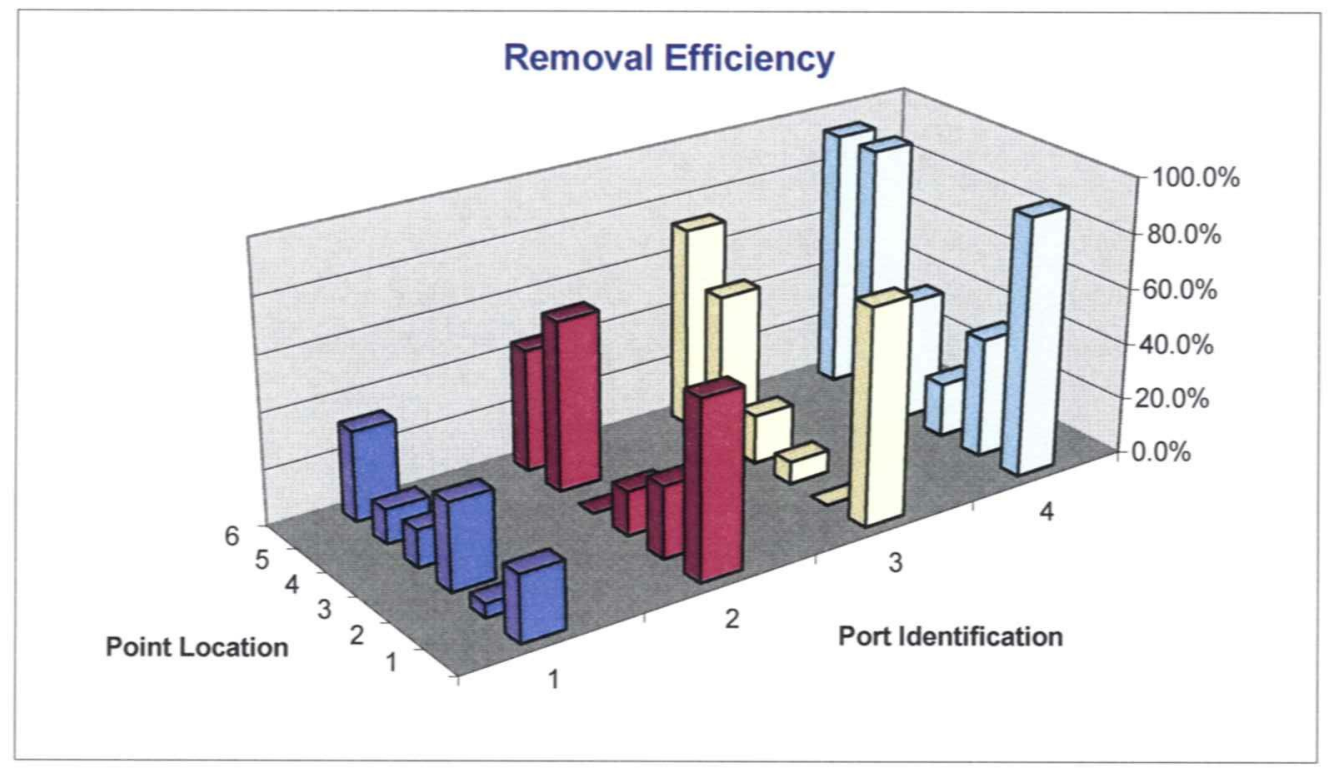


Clean Air Engineering Project \#10192

Consol Energy

AES Dresden

$\begin{array}{lrr}\text { Date: } & 3 / 28 / 2007 & \text { Run \# (Cycle \#) } \\ \text { Start Time: } & 12: 48 & (1) \\ \text { End Time: } & 13: 11 & \end{array}$

\begin{tabular}{|c|c|c|c|c|c|c|c|c|c|c|}
\hline $\begin{array}{l}\text { Inlet } \\
\text { Point }\end{array}$ & Time Sampled & $\begin{array}{c}\text { NOx } \\
\text { ppmdv }\end{array}$ & $\begin{array}{c}\text { NOx } \\
\text { Port Avg }\end{array}$ & $\begin{array}{l}\text { PPM Dev } \\
\text { from Avg. }\end{array}$ & $\begin{array}{c}\mathrm{O} 2 \\
\% d v\end{array}$ & $\begin{array}{c}\mathrm{O} 2 \\
\text { Port Avg }\end{array}$ & $\begin{array}{l}\mathrm{CO2} \\
\% d v\end{array}$ & $\begin{array}{c}\mathrm{CO} 2 \\
\text { Port Avg }\end{array}$ & $\begin{array}{c}\text { NOx } \\
\text { @ } 3 \% \text { O2 }\end{array}$ & $\begin{array}{l}\text { PPM Dev } \\
\text { from Avg. }\end{array}$ \\
\hline $1-1$ & $12: 48$ & 69.2 & & -5.6 & 7.9 & & 11.3 & & 95.2 & 11.9 \\
\hline $1-2$ & $12: 52$ & 101.3 & & 26.5 & 3.0 & & 15.3 & & 101.3 & 18.0 \\
\hline $1-3$ & $12: 56$ & 75.7 & 74.6 & 0.9 & 5.1 & 5.6 & 13.6 & 13.2 & 85.8 & 2.5 \\
\hline 1.4 & 13:00 & 70.5 & & -4.3 & 4.4 & & 14.2 & & 76.4 & -6.9 \\
\hline 1.5 & 13:04 & 61.6 & & -13.2 & 4.5 & & 14.2 & & 67.2 & -16.1 \\
\hline $1-6$ & 13:08 & 69.0 & & -5.8 & 8.5 & & 10.8 & & 99.9 & 16.6 \\
\hline $2-1$ & $12: 49$ & 78.1 & & 3.3 & 6.2 & & 12.7 & & 95.4 & 12.1 \\
\hline $2-2$ & $12: 53$ & 75.4 & & 0.6 & 3.3 & & 15.2 & & 76.6 & -6.7 \\
\hline $2-3$ & $12: 57$ & 81.5 & 73.7 & 6.7 & 5.2 & 4.9 & 13.6 & 13.8 & 93.0 & 9.7 \\
\hline $2-4$ & $13: 01$ & 72.5 & & -2.3 & 3.6 & & 14.9 & & 75.0 & -8.3 \\
\hline $2-5$ & $13: 05$ & 56.0 & & -18.8 & 4.2 & & 14.4 & & 60.1 & -23.2 \\
\hline $2-6$ & $13: 09$ & 78.7 & & 3.9 & 6.8 & & 12.1 & & 100.2 & 16.9 \\
\hline $3-1$ & $12: 50$ & 87.6 & & 12.8 & 4.4 & & 14.3 & & 94.9 & 11.6 \\
\hline $3-2$ & $12: 54$ & 75.2 & & 0.4 & 3.1 & & 15.3 & & 75.7 & -7.6 \\
\hline $3-3$ & $12: 58$ & 94.6 & 78.9 & 19.8 & 3.2 & 4.1 & 15.2 & 14.5 & 95.8 & 12.5 \\
\hline $3-4$ & 13:02 & 75.5 & & 0.7 & 3.5 & & 15.0 & & 77.7 & -5.6 \\
\hline $3-5$ & 13:06 & 61.7 & & -13.1 & 4.2 & & 14.4 & & 66.3 & -17.0 \\
\hline $3-6$ & $13: 10$ & 78.5 & & 3.7 & 6.2 & & 12.7 & & 95.8 & 12.5 \\
\hline $4-1$ & $12: 51$ & 74.3 & & -0.5 & 6.1 & & 12.8 & & 89.9 & 6.6 \\
\hline $4-2$ & $12: 55$ & 75.9 & & 1.1 & 2.8 & & 15.6 & & 75.1 & -8.2 \\
\hline $4-3$ & $12: 59$ & 93.4 & 72.2 & 18.6 & 3.0 & 4.3 & 15.5 & 14.4 & 93.3 & 10.0 \\
\hline $4-4$ & $13: 03$ & 73.0 & & -1.8 & 3.4 & & 15.1 & & 74.5 & -8.8 \\
\hline $4-5$ & $13: 07$ & 54.7 & & -20.1 & 3.9 & & 14.7 & & 57.7 & -25.6 \\
\hline $4-6$ & $13: 11$ & 61.6 & & -13.2 & 6.5 & & 12.5 & & 76.4 & -6.9 \\
\hline
\end{tabular}

\begin{tabular}{|c|c|c|c|c|c|c|c|c|c|c|c|c|}
\hline \multirow[b]{2}{*}{$\begin{array}{l}\text { Outlet } \\
\text { Point }\end{array}$} & \multirow[b]{2}{*}{ Time Sampled } & \multirow{2}{*}{\multicolumn{2}{|c|}{$\begin{array}{cc}\text { NOx } & \text { NOx } \\
\text { ppmdv } & \text { Port Avg }\end{array}$}} & \multirow[b]{2}{*}{$\begin{array}{l}\text { PPM Dev } \\
\text { from Avg. }\end{array}$} & & & & & & \multirow[b]{2}{*}{$\begin{array}{l}\text { PPM Dev } \\
\text { from Avg. }\end{array}$} & \\
\hline & & & & & $\begin{array}{c}\text { O2 } \\
\% d v\end{array}$ & $\begin{array}{c}\text { O2 } \\
\text { Port Avg }\end{array}$ & $\begin{array}{l}\mathrm{CO} 2 \\
\% \mathrm{dv}\end{array}$ & $\begin{array}{c}\text { CO2 } \\
\text { Port Avg }\end{array}$ & $\begin{array}{c}\mathrm{NOx} \\
\text { (Q) 3\% O2 }\end{array}$ & & $\begin{array}{l}\text { Removal } \\
\text { Efficiency }\end{array}$ & $\begin{array}{c}\text { Removal Eff } \\
\text { W/ O2 correction }\end{array}$ \\
\hline $1-1$ & $12: 48$ & 43.7 & & -0.6 & 6.9 & & 12.0 & & 55.9 & 5.1 & 36.8 & 41.3 \\
\hline $1-2$ & $12: 52$ & 78.6 & & 34.3 & 5.6 & & 13.3 & & 92.0 & 41.2 & 22.4 & 9.2 \\
\hline $1-3$ & $12: 56$ & 48.1 & 54.5 & 3.8 & 7.3 & 6.5 & 12.0 & 12.5 & 63.2 & 12.4 & 36.5 & 26.3 \\
\hline $1-4$ & 13:00 & 54.2 & & 9.9 & 5.7 & & 13.2 & & 63.8 & 13.0 & 23.1 & 16.5 \\
\hline $1-5$ & 13:04 & 54.3 & & 10.0 & 5.1 & & 13.8 & & 61.6 & 10.8 & 11.9 & 8.4 \\
\hline $1-6$ & 13:08 & 47.9 & & 3.6 & 8.1 & & 11.0 & & 67.1 & 16.3 & 30.6 & 32.8 \\
\hline $2-1$ & $12: 49$ & 28.3 & & -16.0 & 5.3 & & 13.6 & & 32.4 & -18.4 & 63.8 & 66.1 \\
\hline $2-2$ & $12: 53$ & 54.7 & & 10.4 & 4.6 & & 14.2 & & 60.0 & 9.2 & 27.5 & 21.6 \\
\hline $2-3$ & $12: 57$ & 56.5 & 47.5 & 12.2 & 5.5 & 5.4 & 13.3 & 13.4 & 65.5 & 14.7 & 30.7 & 29.6 \\
\hline $2-4$ & $13: 01$ & 71.8 & & 27.5 & 5.7 & & 13.2 & & 84.6 & 33.8 & 1.0 & -12.7 \\
\hline 2.5 & $13: 05$ & 33.1 & & -11.2 & 5.0 & & 13.7 & & 37.3 & -13.5 & 40.9 & 37.9 \\
\hline $2-6$ & 13:09 & 40.4 & & -3.9 & 6.7 & & 12.4 & & 50.8 & 0.0 & 48.7 & 49.3 \\
\hline $3-1$ & $12: 50$ & 18.6 & & -25.7 & 4.1 & & 14.6 & & 19.8 & -31.0 & 78.8 & 79.2 \\
\hline $3-2$ & $12: 54$ & 67.6 & & 23.3 & 4.1 & & 14.5 & & 72.2 & 21.4 & 10.1 & 4.6 \\
\hline $3-3$ & $12: 58$ & 72.3 & 45.3 & 28.0 & 5.2 & 4.7 & 13.6 & 14.1 & 82.5 & 31.7 & 23.6 & 13.9 \\
\hline $3-4$ & 13:02 & 60.3 & & 16.0 & 4.8 & & 14.0 & & 67.0 & 16.2 & 20.1 & 13.7 \\
\hline $3-5$ & $13: 06$ & 30.7 & & -13.6 & 4.2 & & 14.5 & & 32.9 & -17.9 & 50.2 & 50.3 \\
\hline $3-6$ & $13: 10$ & 22.1 & & -22.2 & 5.9 & & 13.1 & & 26.3 & -24.5 & 71.8 & 72.6 \\
\hline $4-1$ & $12: 51$ & 6.3 & & -38.0 & 3.9 & & 14.8 & & 6.6 & -44.2 & 91.5 & 92.6 \\
\hline $4-2$ & $12: 55$ & 47.6 & & 3.3 & 2.8 & & 15.8 & & 47.2 & -3.6 & 37.3 & 37.2 \\
\hline $4-3$ & $12: 59$ & 78.3 & 29.9 & 34.0 & 3.3 & 3.9 & 15.4 & 14.8 & 79.5 & 28.7 & 16.2 & 14.8 \\
\hline $4-4$ & $13: 03$ & 39.1 & & -5.2 & 4.3 & & 14.4 & & 42.2 & -8.6 & 46.4 & 43.4 \\
\hline $4-5$ & $13: 07$ & 3.8 & & -40.5 & 3.5 & & 15.2 & & 3.9 & -46.9 & 93.1 & 93.2 \\
\hline 4-6 & $13: 11$ & 4.3 & & -40.0 & 5.4 & & 13.5 & & 5.0 & -45.8 & 93.0 & 93.5 \\
\hline
\end{tabular}


Run 3: Inlet - Set 1

\begin{tabular}{|c|c|c|c|c|c|}
\hline \multicolumn{5}{|c|}{ Inlet - Oxygen (\%dv) } & \multirow[b]{3}{*}{7.0} \\
\hline AVG & 5.6 & 4.9 & 4.1 & 4.3 & \\
\hline 6 & 8.5 & 6.8 & 6.2 & 6.5 & \\
\hline 5 & 4.5 & 4.2 & 4.2 & 3.9 & 4.2 \\
\hline 4 & 4.4 & 3.6 & 3.5 & 3.4 & 3.7 \\
\hline 3 & 5.1 & 5.2 & 3.2 & 3.0 & 4.1 \\
\hline 2 & 3.0 & 3.3 & 3.1 & 2.8 & 3.1 \\
\hline \multirow[t]{2}{*}{1} & 7.9 & 6.2 & 4.4 & 6.1 & 6.2 \\
\hline & 1 & 2 & 3 & 4 & 4.7 \\
\hline
\end{tabular}

Date: $3 / 28 / 2007$

Start Time: $\quad 12: 48$

End Time: $\quad 13: 16$

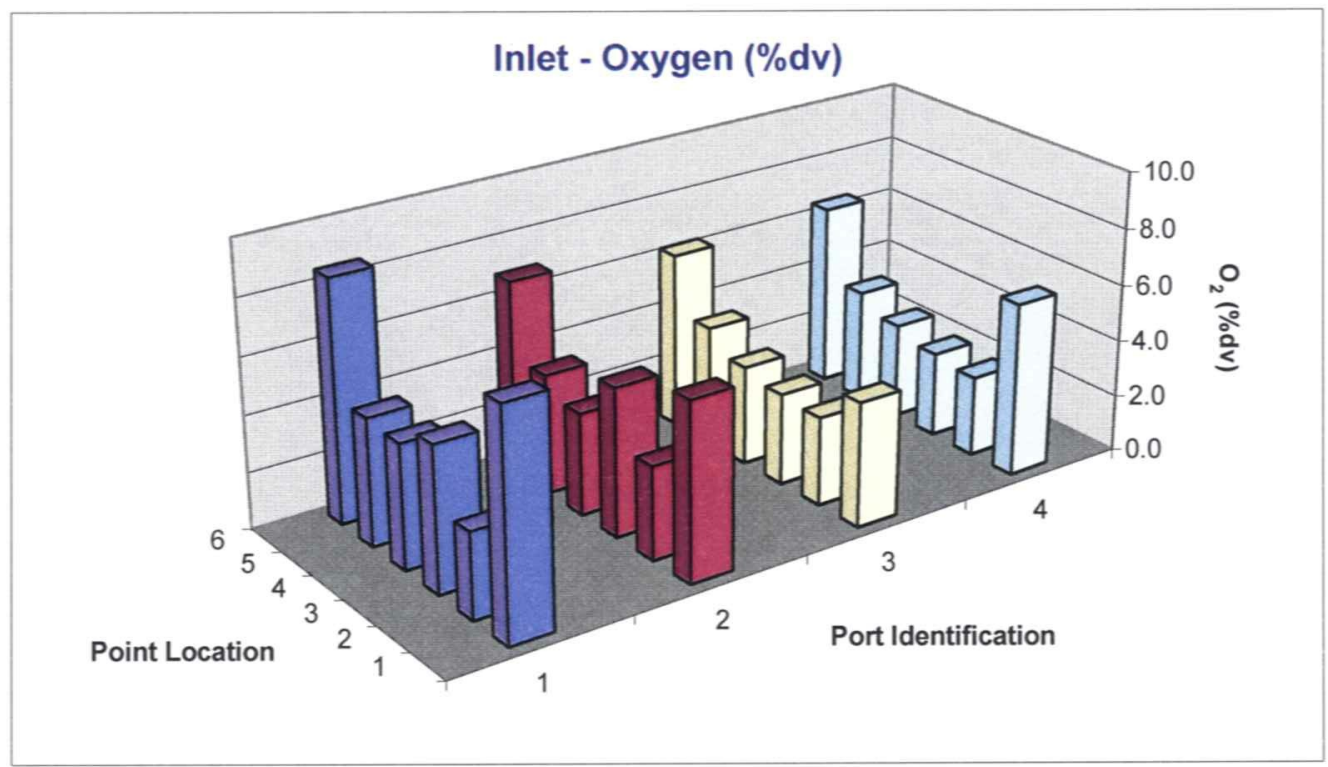


Date: $3 / 28 / 2007$

Start Time: $\quad 12: 48$

End Time: $\quad 13: 16$

\begin{tabular}{|c|c|c|c|c|c|}
\hline \multicolumn{5}{|c|}{ Inlet - Carbon Dioxide (\%dv) } & \multirow[b]{3}{*}{12.0} \\
\hline AVG & 13.2 & 13.8 & 14.5 & 14.4 & \\
\hline 6 & 10.8 & 12.1 & 12.7 & 12.5 & \\
\hline 5 & 14.2 & 14.4 & 14.4 & 14.7 & 14.4 \\
\hline 4 & 14.2 & 14.9 & 15.0 & 15.1 & 14.8 \\
\hline 3 & 13.6 & 13.6 & 15.2 & 15.5 & 14.5 \\
\hline 2 & 15.3 & 15.2 & 15.3 & 15.6 & 15.4 \\
\hline 1 & 11.3 & 12.7 & 14.3 & 12.8 & 12.8 \\
\hline & 1 & 2 & 3 & 4 & 14.0 \\
\hline
\end{tabular}

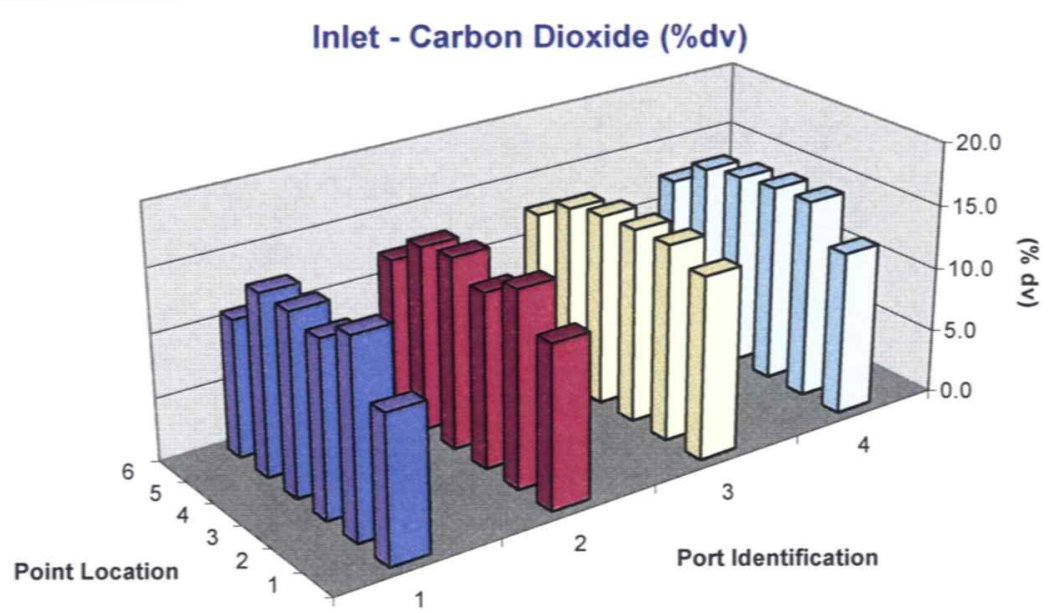


Run 3: Inlet - Set 1

Date: $3 / 28 / 2007$

Start Time: $\quad 12: 48$

End Time: $\quad 13: 16$

Inlet - Nitrogen Oxides (ppmdv), uncorrected

\begin{tabular}{|c|c|c|c|c|c|}
\hline AVG & 74.6 & 73.7 & 78.9 & 72.2 & \\
\hline 6 & 69.0 & 78.7 & 78.5 & 61.6 & 72.0 \\
\hline 5 & 61.6 & 56.0 & 61.7 & 54.7 & 58.5 \\
\hline 4 & 70.5 & 72.5 & 75.5 & 73.0 & 72.9 \\
\hline 3 & 75.7 & 81.5 & 94.6 & 93.4 & 86.3 \\
\hline 2 & 101.3 & 75.4 & 75.2 & 75.9 & 82.0 \\
\hline \multirow[t]{2}{*}{1} & 69.2 & 78.1 & 87.6 & 74.3 & 77.3 \\
\hline & 1 & 2 & 3 & 4 & 74.8 \\
\hline
\end{tabular}

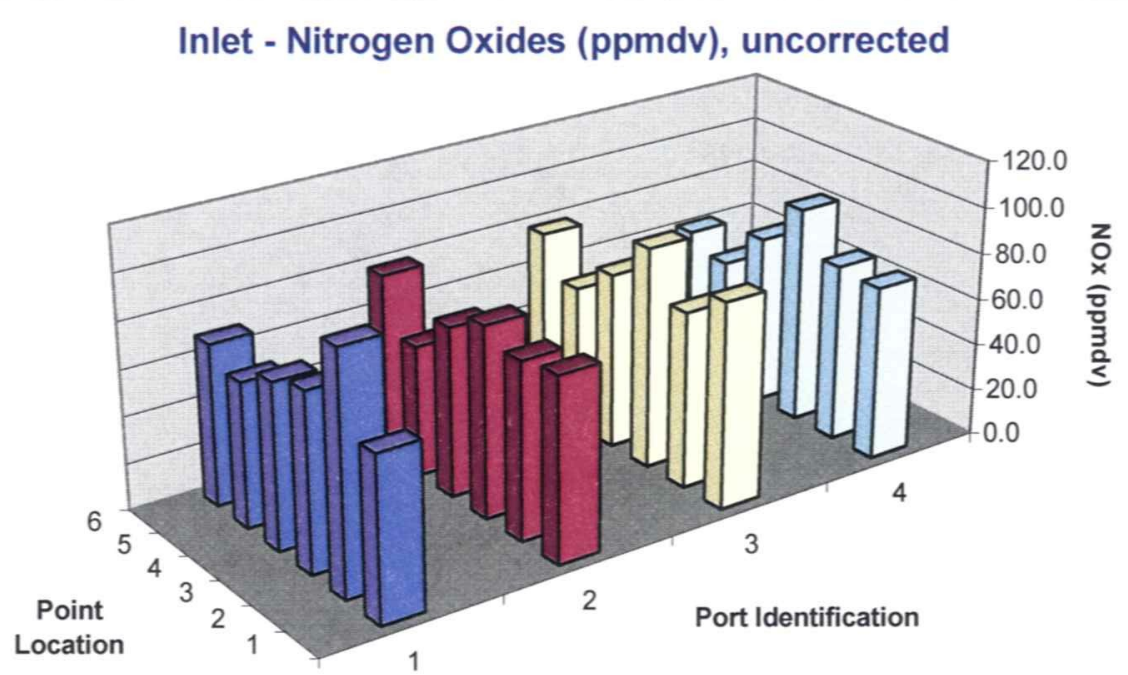


CONSOL ENERGY INC.
Client Reference No: 4700140111

CleanAir Project No:10192

Run 3: Inlet - Set 1

ate: $3 / 28 / 2007$

Start Time: $\quad 12: 48$

End Time: $\quad 13: 16$

Inlet - Nitrogen Oxides (ppmdv @ 3\% $\mathrm{O}_{2}$ )

\begin{tabular}{|c|c|c|c|c|}
\multicolumn{1}{c|}{ AVG } & 87.6 & 83.4 & 84.4 & 77.8 \\
\hline $\mathbf{6}$ & 99.9 & 100.2 & 95.8 & 76.4 \\
\hline $\mathbf{5}$ & 67.2 & 60.1 & 66.3 & 57.7 \\
\hline $\mathbf{4}$ & 76.4 & 75.0 & 77.7 & 74.5 \\
\hline $\mathbf{3}$ & 85.8 & 93.0 & 95.8 & 93.3 \\
\hline $\mathbf{2}$ & 101.3 & 76.6 & 75.7 & 75.1 \\
\hline $\mathbf{1}$ & 95.2 & 95.4 & 94.9 & 89.9 \\
\hline & $\mathbf{1}$ & $\mathbf{2}$ & $\mathbf{3}$ & $\mathbf{4}$ \\
\cline { 2 - 5 }
\end{tabular}

93.1

62.8

75.9

92.0

82.2

93.9

83.3

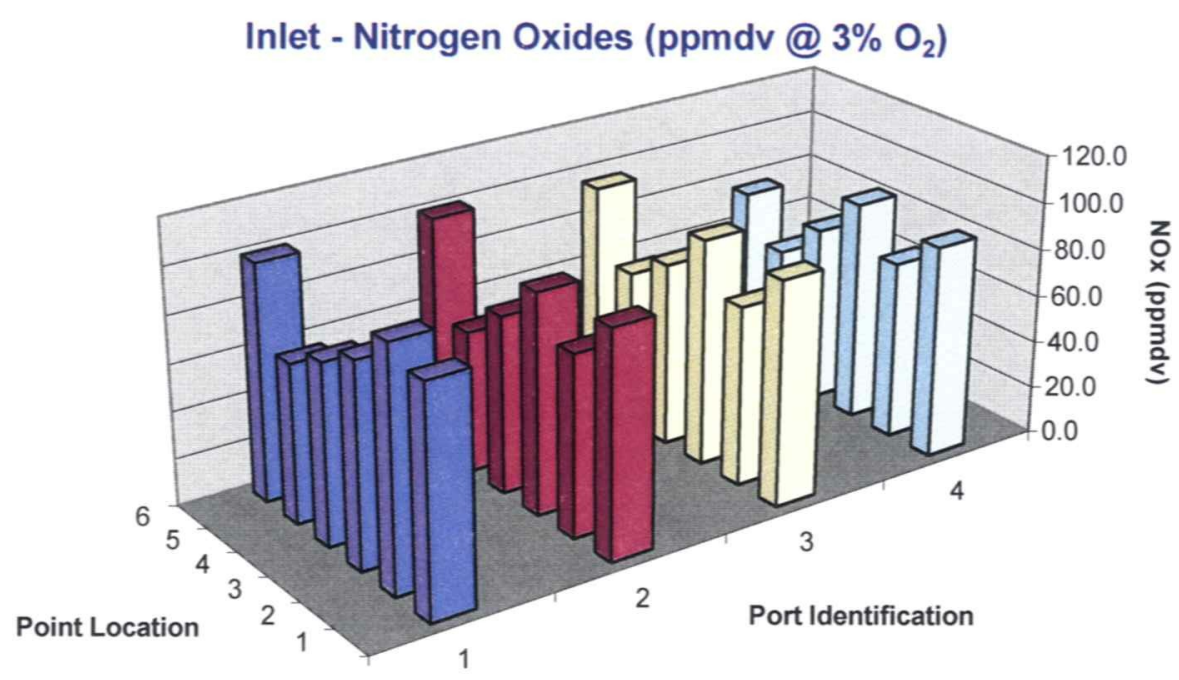


Date: $3 / 28 / 2007$

Start Time: $12: 48$

End Time: $13: 16$

\begin{tabular}{|c|c|c|c|c|c|}
\hline \multicolumn{5}{|c|}{ Outlet - Oxygen (\%dv) } & \multirow[b]{3}{*}{6.5} \\
\hline AVG & 6.5 & 5.4 & 4.7 & 3.9 & \\
\hline 6 & 8.1 & 6.7 & 5.9 & 5.4 & \\
\hline 5 & 5.1 & 5.0 & 4.2 & 3.5 & 4.5 \\
\hline 4 & 5.7 & 5.7 & 4.8 & 4.3 & 5.1 \\
\hline 3 & 7.3 & 5.5 & 5.2 & 3.3 & 5.3 \\
\hline 2 & 5.6 & 4.6 & 4.1 & 2.8 & 4.3 \\
\hline 1 & 6.9 & 5.3 & 4.1 & 3.9 & 5.0 \\
\hline & 1 & 2 & 3 & 4 & 5.1 \\
\hline
\end{tabular}

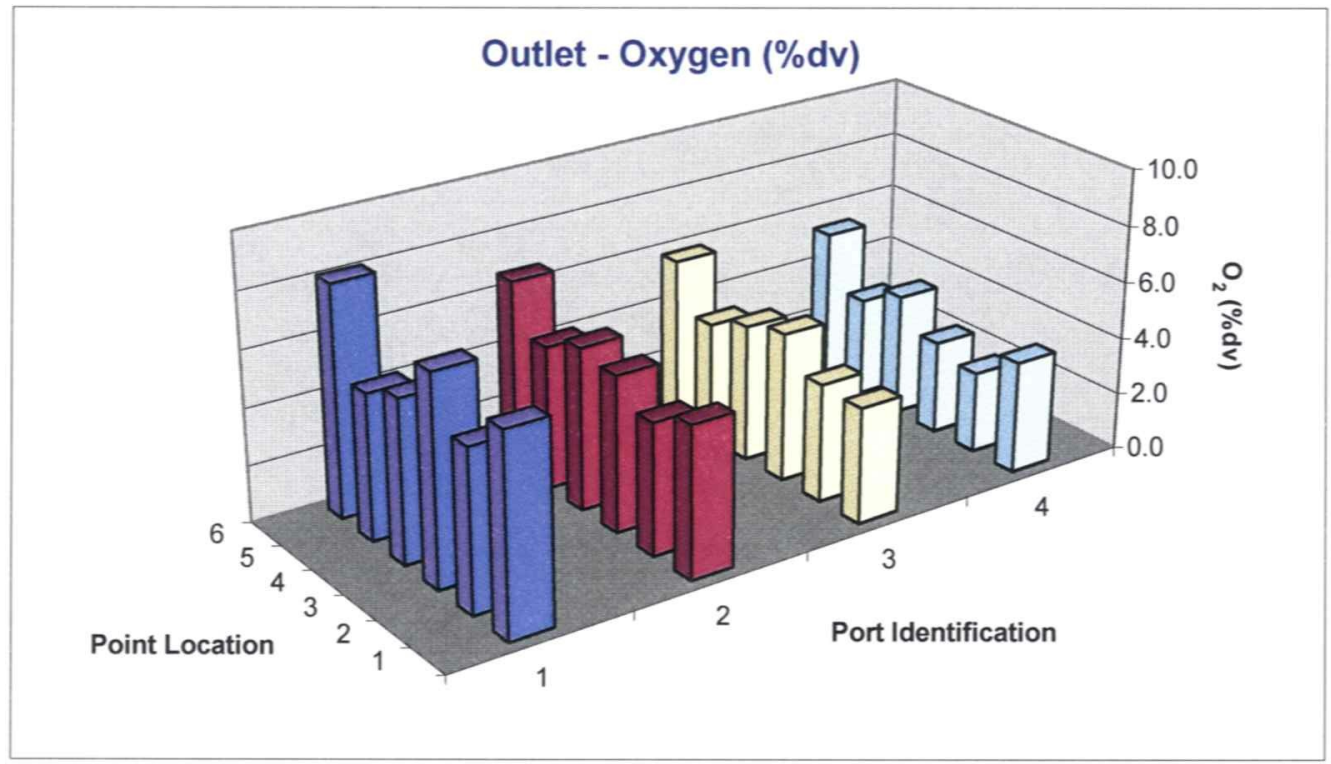




\section{Run 3: Outlet - Set 1}

Date: $3 / 28 / 2007$

Start Time: $\quad 12: 48$

End Time: $\quad 13: 16$

Outlet - Carbon Dioxide (\%dv)

\begin{tabular}{|c|c|c|c|c|}
\hline AVG & 12.5 & 13.4 & 14.1 & 14.8 \\
\hline $\mathbf{6}$ & 11.0 & 12.4 & 13.1 & 13.5 \\
\hline $\mathbf{5}$ & 13.8 & 13.7 & 14.5 & 15.2 \\
\hline $\mathbf{4}$ & 13.2 & 13.2 & 14.0 & 14.4 \\
\hline $\mathbf{3}$ & 12.0 & 13.3 & 13.6 & 15.4 \\
\hline $\mathbf{2}$ & 13.3 & 14.2 & 14.5 & 15.8 \\
\hline $\mathbf{1}$ & 12.0 & 13.6 & 14.6 & 14.8 \\
\hline & $\mathbf{1}$ & $\mathbf{2}$ & $\mathbf{3}$ & $\mathbf{4}$ \\
\hline
\end{tabular}

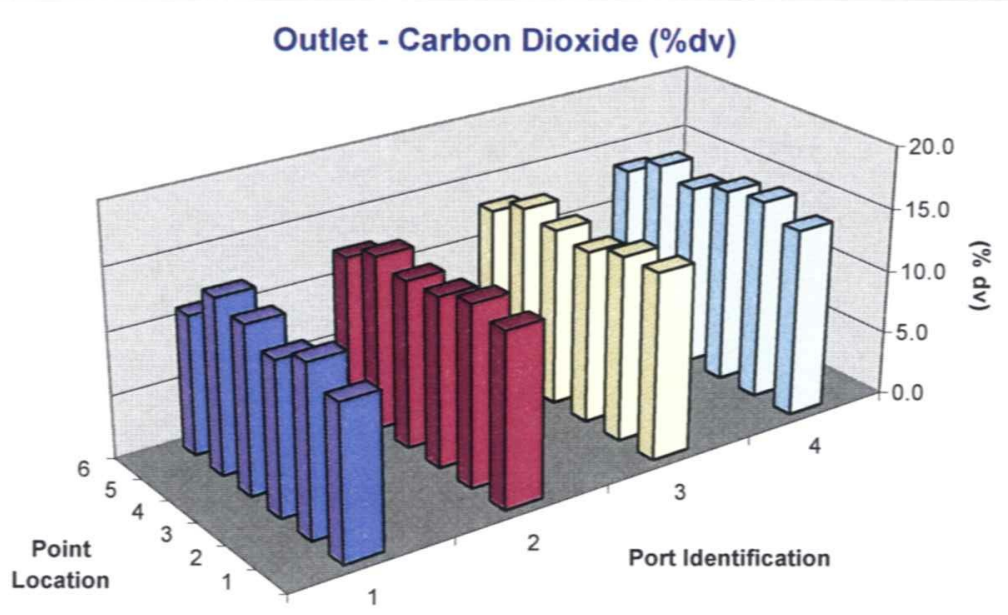


Date: $3 / 28 / 2007$

Start Time: $\quad 12: 48$

End Time: $\quad 13: 16$

Outlet - Nitrogen Oxides (ppmdv), uncorrected

\begin{tabular}{|c|c|c|c|c|}
\multicolumn{1}{c}{ AVG } & 54.5 & 47.5 & 45.3 & 29.9 \\
\hline $\mathbf{6}$ & 47.9 & 40.4 & 22.1 & 4.3 \\
\hline $\mathbf{5}$ & 54.3 & 33.1 & 30.7 & 3.8 \\
\hline $\mathbf{4}$ & 54.2 & 71.8 & 60.3 & 39.1 \\
\hline $\mathbf{3}$ & 48.1 & 56.5 & 72.3 & 78.3 \\
\hline $\mathbf{2}$ & 78.6 & 54.7 & 67.6 & 47.6 \\
\hline $\mathbf{1}$ & 43.7 & 28.3 & 18.6 & 6.3 \\
\hline & $\mathbf{1}$ & $\mathbf{2}$ & $\mathbf{3}$ & $\mathbf{4}$ \\
\hline
\end{tabular}

28.7

30.5

56.4

63.8

62.1

24.2

44.3

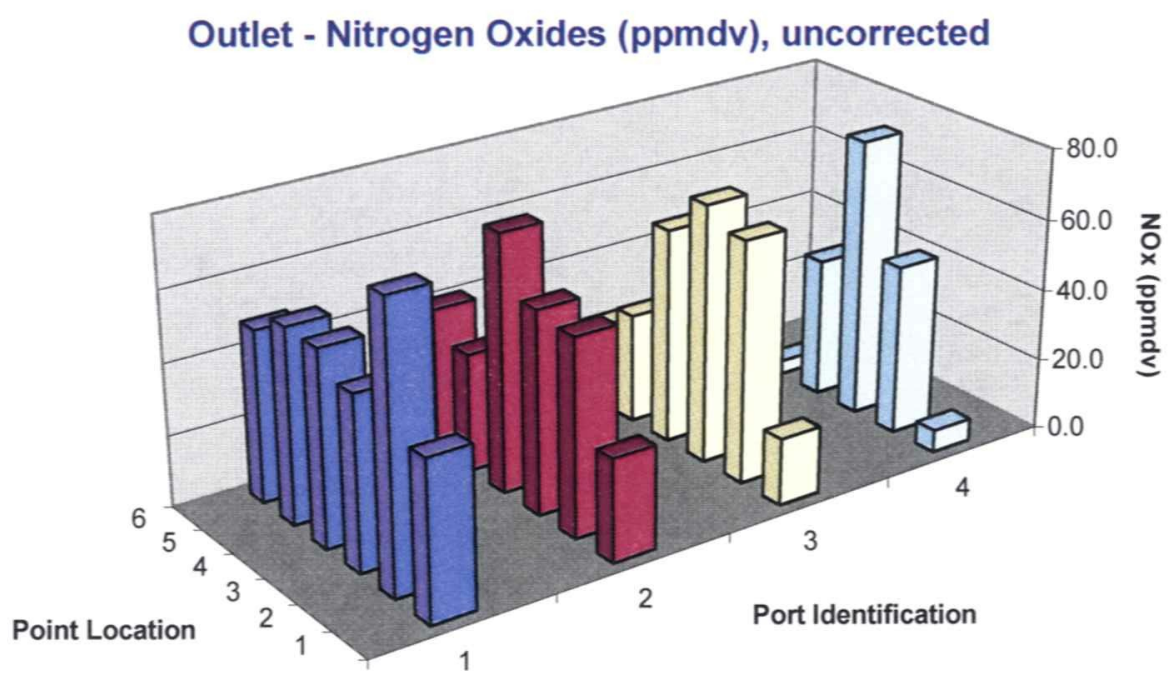


CONSOL ENERGY INC.
Client Reference No: 4700140111

CleanAir Project No:10192

Run 3: Outlet - Set 1

Date: $3 / 28 / 2007$

Start Time: $\quad 12: 48$

End Time: $\quad 13: 16$

Outlet - Nitrogen Oxides (ppmdv @ 3\% $\mathrm{O}_{2}$ )

\begin{tabular}{|c|c|c|c|c|}
\multicolumn{1}{|c}{ AVG } & 67.3 & 55.1 & 50.1 & 30.7 \\
\hline $\mathbf{6}$ & 67.1 & 50.8 & 26.3 & 5.0 \\
\hline $\mathbf{5}$ & 61.6 & 37.3 & 32.9 & 3.9 \\
\hline $\mathbf{4}$ & 63.8 & 84.6 & 67.0 & 42.2 \\
\hline $\mathbf{3}$ & 63.2 & 65.5 & 82.5 & 79.5 \\
\hline $\mathbf{2}$ & 92.0 & 60.0 & 72.2 & 47.2 \\
\hline $\mathbf{1}$ & 55.9 & 32.4 & 19.8 & 6.6 \\
\hline & $\mathbf{1}$ & $\mathbf{2}$ & $\mathbf{3}$ & $\mathbf{4}$ \\
\hline
\end{tabular}

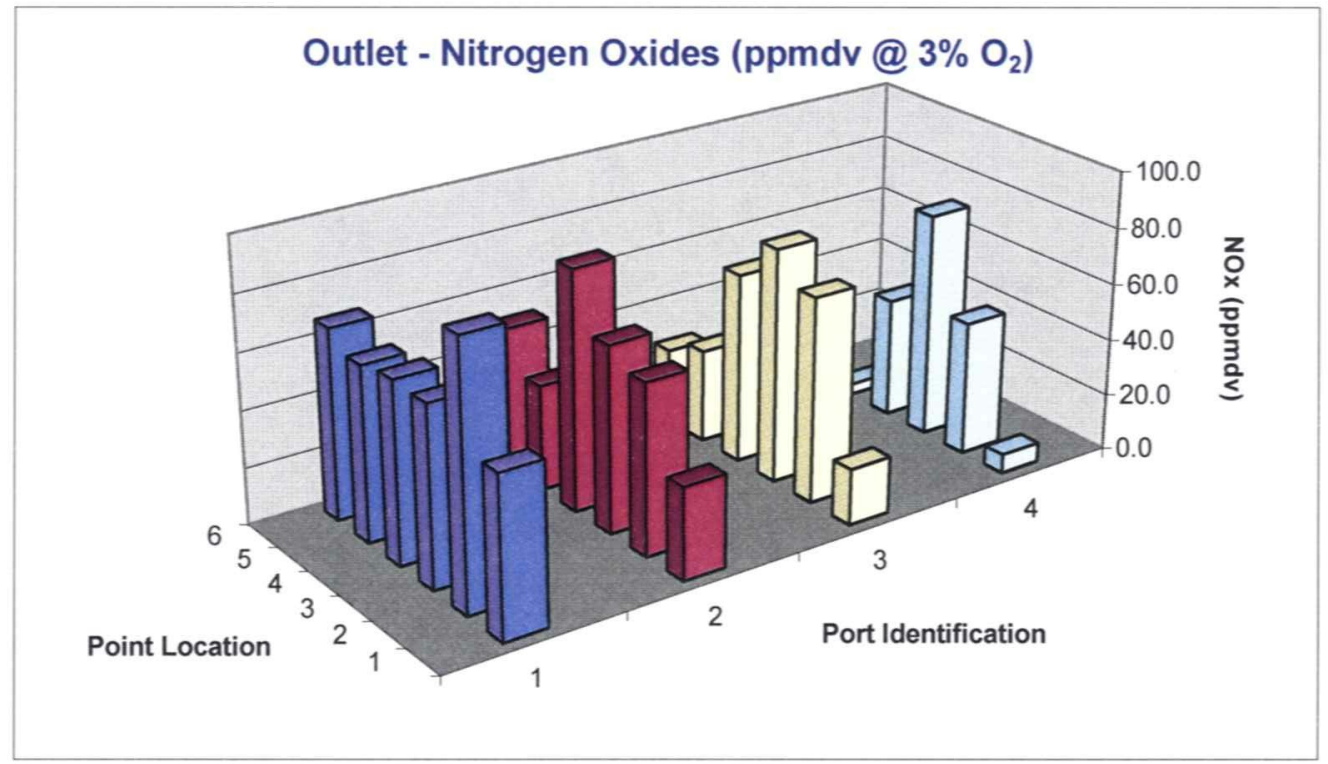


CONSOL ENERGY INC.
Client Reference No: 4700140111

CleanAir Project No:10192

Run 3: Outlet - Set 1

Date: $3 / 28 / 2007$

Start Time: $\quad 12: 48$

End Time: $\quad 13: 16$

Removal Efficiency (ppmdv @ 3\% $\mathrm{O}_{2}$ )

\begin{tabular}{|c|c|c|c|c|}
\multicolumn{1}{c}{ AVG } & $22.4 \%$ & $32.0 \%$ & $39.0 \%$ & $62.5 \%$ \\
\hline $\mathbf{6}$ & $32.8 \%$ & $49.3 \%$ & $72.6 \%$ & $93.5 \%$ \\
\hline $\mathbf{5}$ & $8.4 \%$ & $37.9 \%$ & $50.3 \%$ & $93.2 \%$ \\
\hline $\mathbf{4}$ & $16.5 \%$ & $-12.7 \%$ & $13.7 \%$ & $43.4 \%$ \\
\hline $\mathbf{3}$ & $26.3 \%$ & $29.6 \%$ & $13.9 \%$ & $14.8 \%$ \\
\hline $\mathbf{2}$ & $9.2 \%$ & $21.6 \%$ & $4.6 \%$ & $37.2 \%$ \\
\hline $\mathbf{1}$ & $41.3 \%$ & $66.1 \%$ & $79.2 \%$ & $92.6 \%$ \\
\hline & $\mathbf{1}$ & $\mathbf{2}$ & $\mathbf{3}$ & $\mathbf{4}$ \\
\hline
\end{tabular}

$62.1 \%$

$47.5 \%$

$15.2 \%$

$21.1 \%$

$18.2 \%$

$69.8 \%$

$39.0 \%$

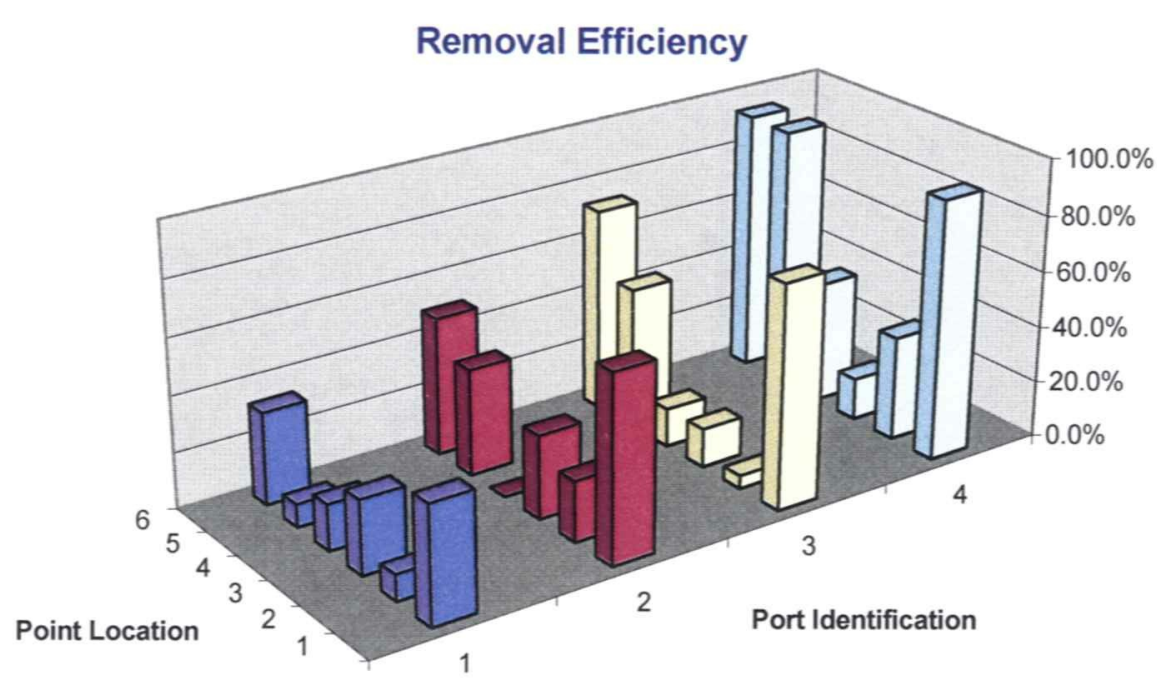


Clean Air Engineering Project \#10192

Consol Energy

AES Dresden

Date:

Start Time:

$3 / 28 / 2007$

End Time:

Run \# (Cycle \#) 3 (2)

\begin{tabular}{|c|c|c|c|c|c|c|c|c|c|c|}
\hline \multirow[b]{2}{*}{$\begin{array}{l}\text { Inlet } \\
\text { Point }\end{array}$} & \multirow[b]{2}{*}{ Time Sampled } & & \multirow[b]{2}{*}{$\begin{array}{l}\text { PPM Dev } \\
\text { from Avg. }\end{array}$} & & \multirow[b]{2}{*}{$\begin{array}{l}\text { PPM Dev } \\
\text { from Avg. }\end{array}$} \\
\hline & & $\begin{array}{c}\text { NOx } \\
\text { ppmdv }\end{array}$ & $\begin{array}{c}\text { NOx } \\
\text { Port Avg }\end{array}$ & & $\begin{array}{l}\mathrm{O} 2 \\
\% d v\end{array}$ & $\begin{array}{c}\mathrm{O} 2 \\
\text { Port Avg }\end{array}$ & $\begin{array}{l}\mathrm{CO2} \\
\% d v\end{array}$ & $\begin{array}{c}\mathrm{CO} 2 \\
\text { Port Avg }\end{array}$ & $\begin{array}{c}\text { NOx } \\
\text { @ } 3 \% \text { O2 }\end{array}$ & \\
\hline $1-1$ & $13: 12$ & 75.3 & & -1.3 & 7.8 & & 11.4 & & 102.7 & 17.3 \\
\hline $1-2$ & $13: 16$ & 99.3 & & 22.7 & 3.3 & & 15.2 & & 100.8 & 15.3 \\
\hline $1-3$ & $13: 20$ & 88.2 & 79.4 & 11.6 & 5.0 & 5.5 & 13.8 & 13.3 & 99.0 & 13.6 \\
\hline $1-4$ & $13: 24$ & 79.9 & & 3.3 & 4.2 & & 14.4 & & 85.7 & 0.3 \\
\hline $1-5$ & $13: 28$ & 62.9 & & -13.7 & 4.5 & & 14.2 & & 68.5 & -16.9 \\
\hline $1-6$ & $13: 32$ & 70.6 & & -6.0 & 8.5 & & 10.8 & & 101.7 & 16.2 \\
\hline $2-1$ & $13: 13$ & 79.2 & & 2.6 & 6.2 & & 12.7 & & 96.6 & 11.2 \\
\hline $2-2$ & $13: 17$ & 77.4 & & 0.8 & 3.5 & & 15.1 & & 79.4 & -6.0 \\
\hline $2-3$ & 13:21 & 86.4 & 74.3 & 9.8 & 5.2 & 4.9 & 13.6 & 13.8 & 98.6 & 13.2 \\
\hline $2-4$ & $13: 25$ & 70.1 & & -6.5 & 3.7 & & 14.8 & & 72.8 & -12.6 \\
\hline $2-5$ & $13: 29$ & 54.0 & & -22.6 & 4.2 & & 14.5 & & 57.7 & -27.7 \\
\hline $2-6$ & $13: 33$ & 78.7 & & 2.1 & 6.9 & & 12.1 & & 100.8 & 15.3 \\
\hline $3-1$ & $13: 14$ & 86.5 & & 9.9 & 4.2 & & 14.5 & & 92.4 & 7.0 \\
\hline $3-2$ & $13: 18$ & 76.3 & & -0.3 & 3.3 & & 15.1 & & 77.8 & -7.7 \\
\hline $3-3$ & $13: 22$ & 98.5 & 79.7 & 21.9 & 3.3 & 4.2 & 15.2 & 14.4 & 100.0 & 14.6 \\
\hline $3-4$ & $13: 26$ & 76.7 & & 0.1 & 3.5 & & 15.0 & & 79.0 & -6.4 \\
\hline $3-5$ & $13: 30$ & 62.9 & & -13.7 & 4.3 & & 14.3 & & 68.0 & -17.4 \\
\hline $3-6$ & $13: 34$ & 77.3 & & 0.7 & 6.3 & & 12.6 & & 94.6 & 9.2 \\
\hline $4-1$ & $13: 15$ & 75.4 & & -1.2 & 6.1 & & 12.8 & & 91.4 & 5.9 \\
\hline $4-2$ & $13: 19$ & 77.1 & & 0.5 & 2.9 & & 15.6 & & 76.7 & -8.8 \\
\hline $4-3$ & $13: 23$ & 93.9 & 73.1 & 17.3 & 3.1 & 4.3 & 15.4 & 14.3 & 94.2 & 8.7 \\
\hline $4-4$ & $13: 27$ & 74.3 & & -2.3 & 3.4 & & 15.1 & & 76.1 & -9.3 \\
\hline $4-5$ & 13:31 & 57.0 & & -19.6 & 4.2 & & 14.5 & & 60.9 & -24.5 \\
\hline $4-6$ & $13: 35$ & 61.1 & & -15.5 & 6.3 & & 12.7 & & 74.7 & -10.7 \\
\hline
\end{tabular}

\begin{tabular}{|c|c|c|c|c|c|c|c|c|c|c|c|c|}
\hline $\begin{array}{l}\text { Outlet } \\
\text { Point }\end{array}$ & Time Sampled & $\begin{array}{c}\text { NOx } \\
\text { ppmdv }\end{array}$ & $\begin{array}{c}\text { NOx } \\
\text { Port Avg }\end{array}$ & $\begin{array}{l}\text { PPM Dev } \\
\text { from Avg. }\end{array}$ & $\begin{array}{c}\text { O2 } \\
\% d v\end{array}$ & $\begin{array}{c}\text { O2 } \\
\text { Port Avg }\end{array}$ & $\begin{array}{l}\mathrm{CO2} \\
\% \mathrm{dv}\end{array}$ & $\begin{array}{c}\mathrm{CO2} \\
\text { Port Avg }\end{array}$ & $\begin{array}{c}\text { NOx } \\
\text { @ } 3 \% 02\end{array}$ & $\begin{array}{l}\text { PPM Dev } \\
\text { from Avg. }\end{array}$ & $\begin{array}{l}\text { Removal } \\
\text { Efficiency }\end{array}$ & $\begin{array}{c}\text { Removal Eff } \\
\text { w/ O2 correction }\end{array}$ \\
\hline $1-1$ & $13: 12$ & 70.9 & & 24.7 & 5.0 & & 13.8 & & 80.0 & 27.8 & 5.8 & 22.2 \\
\hline $1-2$ & 13:16 & 82.0 & & 35.8 & 5.8 & & 13.2 & & 97.2 & 45.0 & 17.4 & 3.5 \\
\hline $1-3$ & $13: 20$ & 56.4 & 63.0 & 10.2 & 5.2 & 5.8 & 13.6 & 13.1 & 64.5 & 12.3 & 36.1 & 34.9 \\
\hline $1-4$ & $13: 24$ & 68.3 & & 22.1 & 4.5 & & 14.3 & & 74.5 & 22.3 & 14.5 & 13.2 \\
\hline $1-5$ & $13: 28$ & 48.8 & & 2.6 & 6.3 & & 12.7 & & 59.7 & 7.5 & 22.4 & 12.9 \\
\hline $1-6$ & $13: 32$ & 51.7 & & 5.5 & 7.8 & & 11.3 & & 70.9 & 18.7 & 26.8 & 30.3 \\
\hline $2-1$ & $13: 13$ & 30.1 & & -16.1 & 5.5 & & 13.3 & & 35.0 & -17.2 & 62.0 & 63.8 \\
\hline $2-2$ & $13: 17$ & 51.2 & & 5.0 & 4.9 & & 13.9 & & 57.1 & 4.9 & 33.9 & 28.1 \\
\hline $2-3$ & $13: 21$ & 64.7 & 48.1 & 18.5 & 4.8 & 5.4 & 14.0 & 13.4 & 71.7 & 19.5 & 25.1 & 27.3 \\
\hline $2-4$ & $13: 25$ & 63.8 & & 17.6 & 5.5 & & 13.4 & & 74.2 & 22.0 & 9.0 & -1.9 \\
\hline $2-5$ & $13: 29$ & 37.8 & & -8.4 & 5.2 & & 13.6 & & 43.1 & -9.1 & 30.0 & 25.3 \\
\hline $2-6$ & $13: 33$ & 40.7 & & -5.5 & 6.7 & & 12.3 & & 51.4 & -0.8 & 48.3 & 49.0 \\
\hline $3-1$ & $13: 14$ & 17.9 & & -28.3 & 4.5 & & 14.2 & & 19.5 & -32.7 & 79.3 & 78.9 \\
\hline $3-2$ & $13: 18$ & 41.8 & & -4.4 & 4.1 & & 14.5 & & 44.6 & -7.6 & 45.2 & 42.7 \\
\hline $3-3$ & $13: 22$ & 65.1 & 45.1 & 18.9 & 4.4 & 4.6 & 14.4 & 14.1 & 70.4 & 18.2 & 33.9 & 29.6 \\
\hline $3-4$ & $13: 26$ & 69.5 & & 23.3 & 4.4 & & 14.2 & & 75.4 & 23.2 & 9.4 & 4.6 \\
\hline $3-5$ & $13: 30$ & 55.4 & & 9.2 & 4.9 & & 13.9 & & 61.9 & 9.7 & 11.9 & 9.0 \\
\hline $3-6$ & $13: 34$ & 20.8 & & -25.4 & 5.3 & & 13.5 & & 23.9 & -28.3 & 73.1 & 74.7 \\
\hline $4-1$ & $13: 15$ & 6.1 & & -40.1 & 3.9 & & 14.8 & & 6.4 & -45.8 & 91.9 & 93.0 \\
\hline $4-2$ & $13: 19$ & 45.5 & & -0.7 & 2.8 & & 15.9 & & 44.9 & -7.3 & 41.0 & 41.4 \\
\hline $4-3$ & $13: 23$ & 75.7 & 28.7 & 29.5 & 3.3 & 3.9 & 15.4 & 14.8 & 77.1 & 24.9 & 19.4 & 18.1 \\
\hline $4-4$ & $13: 27$ & 37.3 & & -8.9 & 4.4 & & 14.4 & & 40.4 & -11.8 & 49.8 & 46.9 \\
\hline $4-5$ & $13: 31$ & 3.6 & & -42.6 & 3.8 & & 14.9 & & 3.8 & -48.4 & 93.7 & 93.8 \\
\hline 4-6 & $13: 35$ & 4.2 & & -42.0 & 5.3 & & 13.6 & & 4.8 & -47.4 & 93.1 & 93.6 \\
\hline
\end{tabular}

46.2

13.9

52.2

40.5

39.0 
Run 3: Inlet - Set 2

Date: $3 / 28 / 2007$

Start Time: $\quad 13: 12$

End Time: $13: 35$

Inlet - Oxygen (\%dv)

\begin{tabular}{|c|c|c|c|c|}
\multicolumn{1}{|c}{ AVG } & 5.5 & 4.9 & 4.2 & 4.3 \\
\hline 6 & 8.5 & 6.9 & 6.3 & 6.3 \\
\hline 5 & 4.5 & 4.2 & 4.3 & 4.2 \\
\hline 4 & 4.2 & 3.7 & 3.5 & 3.4 \\
\hline 3 & 5.0 & 5.2 & 3.3 & 3.1 \\
\hline 2 & 3.3 & 3.5 & 3.3 & 2.9 \\
\hline 1 & 7.8 & 6.2 & 4.2 & 6.1 \\
\hline & 1 & 2 & 3 & 4 \\
\hline
\end{tabular}

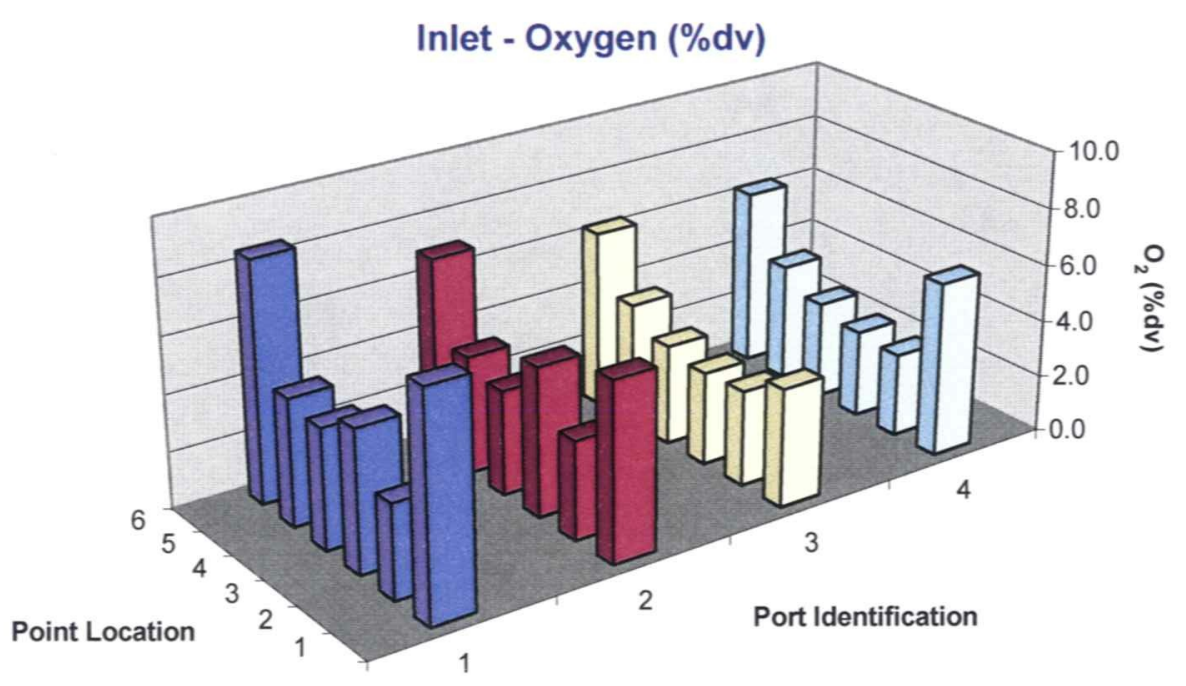


Run 3: Inlet - Set 2

Date: $3 / 28 / 2007$

Start Time: $\quad 13: 12$

End Time: $\quad 13: 35$

Inlet - Carbon Dioxide (\%dv)

\begin{tabular}{|c|c|c|c|c|}
\multicolumn{1}{c|}{ AVG } & 13.3 & 13.8 & 14.4 & 14.3 \\
\hline $\mathbf{6}$ & 10.8 & 12.1 & 12.6 & 12.7 \\
\hline $\mathbf{5}$ & 14.2 & 14.5 & 14.3 & 14.5 \\
\hline $\mathbf{4}$ & 14.4 & 14.8 & 15.0 & 15.1 \\
\hline $\mathbf{3}$ & 13.8 & 13.6 & 15.2 & 15.4 \\
\hline $\mathbf{2}$ & 15.2 & 15.1 & 15.1 & 15.6 \\
\hline $\mathbf{1}$ & 11.4 & 12.7 & 14.5 & 12.8 \\
\hline & $\mathbf{1}$ & $\mathbf{2}$ & $\mathbf{3}$ & $\mathbf{4}$ \\
\hline
\end{tabular}

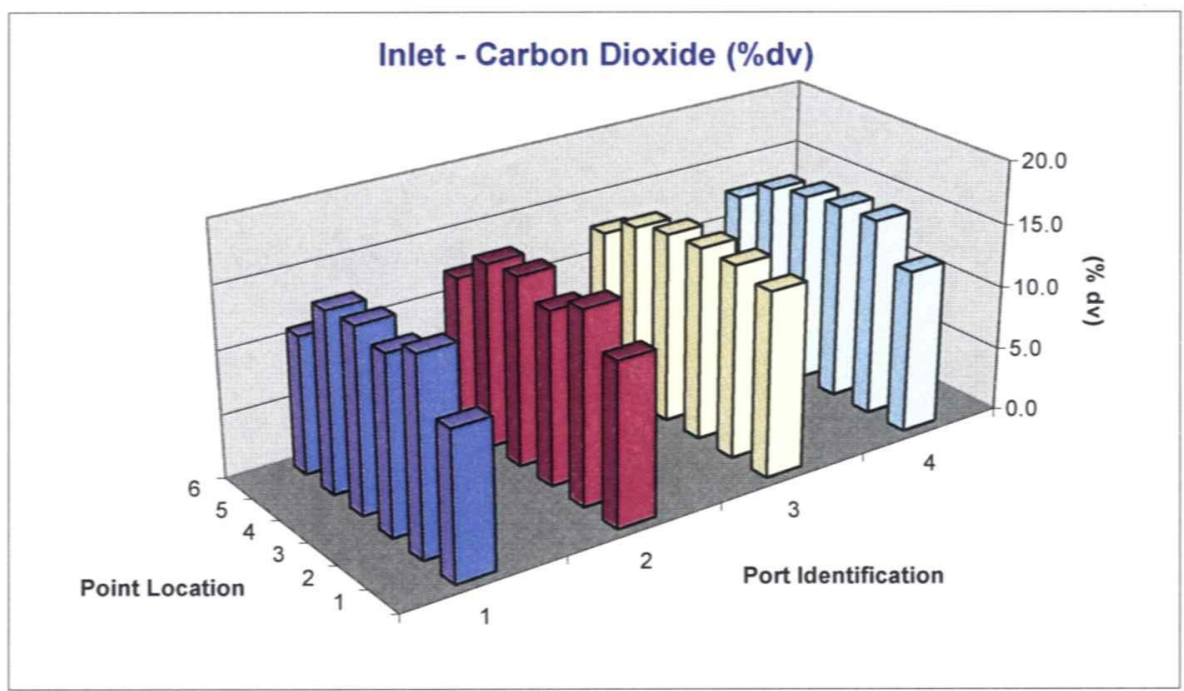


Run 3: Inlet - Set 2

Date: $3 / 28 / 2007$

Start Time: $\quad 13: 12$

End Time: $\quad 13: 35$

Inlet - Nitrogen Oxides (ppmdv), uncorrected

\begin{tabular}{|c|c|c|c|c|}
\multicolumn{1}{c|}{ AVG } & 79.4 & 74.3 & 79.7 & 73.1 \\
\hline $\mathbf{6}$ & 70.6 & 78.7 & 77.3 & 61.1 \\
\hline $\mathbf{5}$ & 62.9 & 54.0 & 62.9 & 57.0 \\
\hline $\mathbf{4}$ & 79.9 & 70.1 & 76.7 & 74.3 \\
\hline $\mathbf{3}$ & 88.2 & 86.4 & 98.5 & 93.9 \\
\hline $\mathbf{2}$ & 99.3 & 77.4 & 76.3 & 77.1 \\
\hline $\mathbf{1}$ & 75.3 & 79.2 & 86.5 & 75.4 \\
\hline & $\mathbf{1}$ & $\mathbf{2}$ & $\mathbf{3}$ & $\mathbf{4}$ \\
\hline
\end{tabular}

71.9

59.2

75.3

91.8

82.5

79.1

76.6

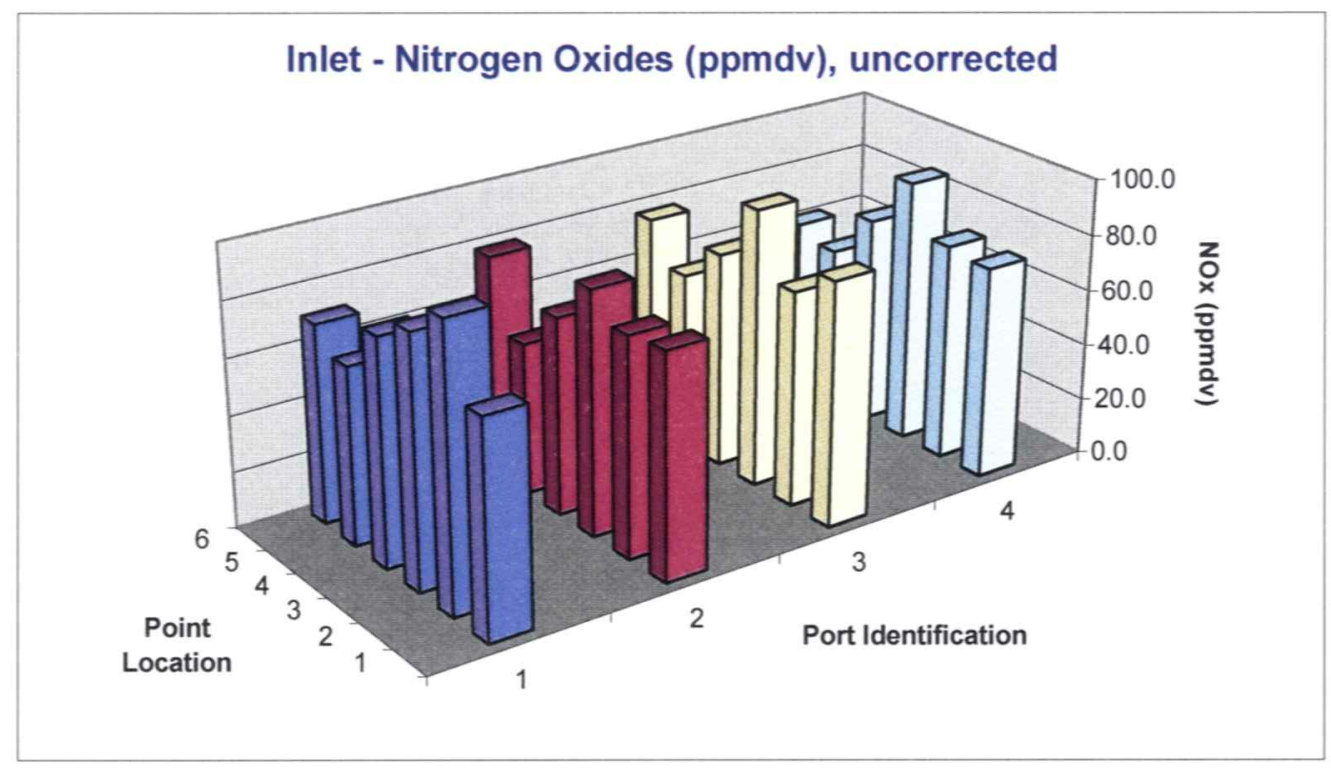


Run 3: Inlet - Set 2

Date: $3 / 28 / 2007$

Start Time: $\quad 13: 12$

End Time: $\quad 13: 35$

Inlet - Nitrogen Oxides (ppmdv @ 3\% $\mathrm{O}_{2}$ )

\begin{tabular}{|c|c|c|c|c|}
\multicolumn{1}{c|}{ AVG } & 93.1 & 84.3 & 85.3 & 79.0 \\
\hline $\mathbf{6}$ & 101.7 & 100.8 & 94.6 & 74.7 \\
\hline $\mathbf{5}$ & 68.5 & 57.7 & 68.0 & 60.9 \\
\hline $\mathbf{4}$ & 85.7 & 72.8 & 79.0 & 76.1 \\
\hline $\mathbf{3}$ & 99.0 & 98.6 & 100.0 & 94.2 \\
\hline $\mathbf{2}$ & 100.8 & 79.4 & 77.8 & 76.7 \\
\hline $\mathbf{1}$ & 102.7 & 96.6 & 92.4 & 91.4 \\
\hline & $\mathbf{1}$ & $\mathbf{2}$ & $\mathbf{3}$ & $\mathbf{4}$ \\
\hline
\end{tabular}

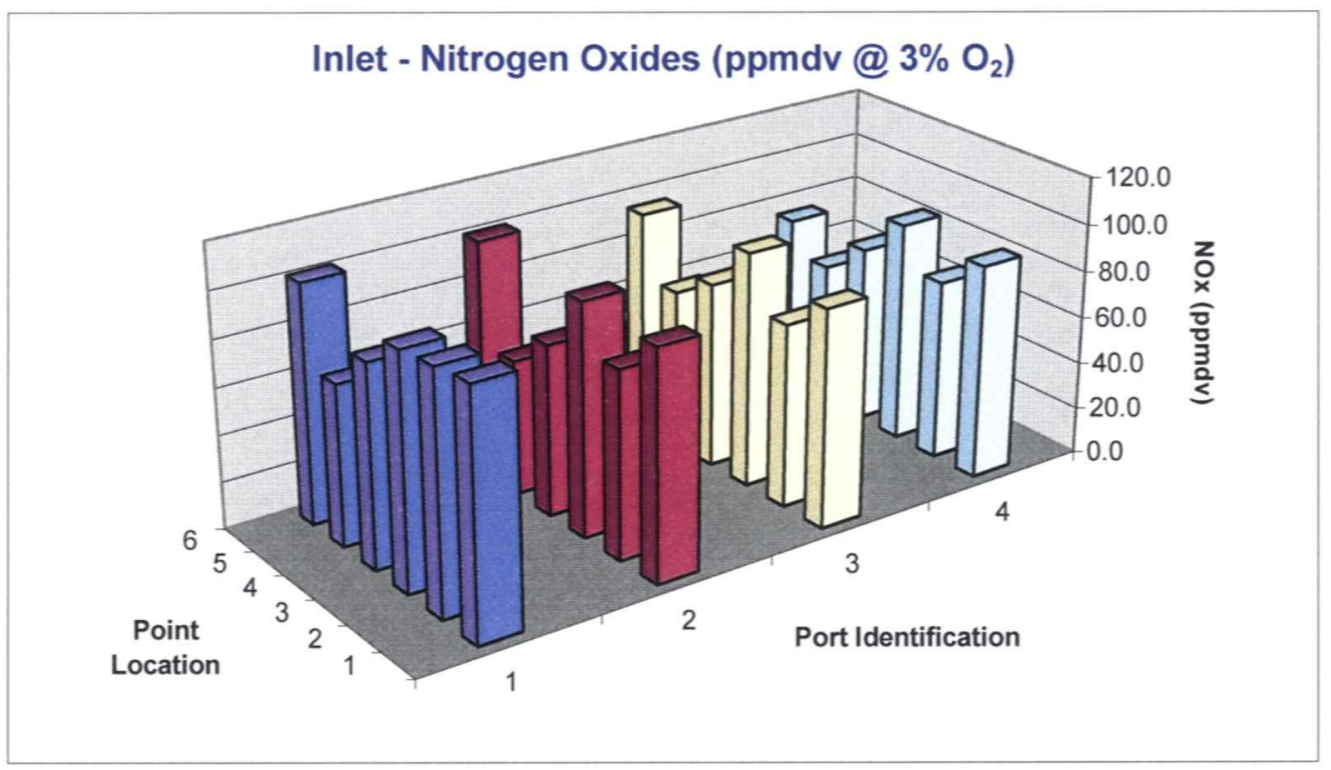


Run 3: Outlet -Set 2

Date: $3 / 28 / 2007$

Start Time: $\quad 13: 12$

End Time: $\quad 13: 35$

\begin{tabular}{|c|c|c|c|c|c|}
\hline \multicolumn{5}{|c|}{ Outlet - Oxygen (\%dv) } & \multirow[b]{3}{*}{6.3} \\
\hline AVG & 5.8 & 5.4 & 4.6 & 3.9 & \\
\hline 6 & 7.8 & 6.7 & 5.3 & 5.3 & \\
\hline 5 & 6.3 & 5.2 & 4.9 & 3.8 & 5.0 \\
\hline 4 & 4.5 & 5.5 & 4.4 & 4.4 & 4.7 \\
\hline 3 & 5.2 & 4.8 & 4.4 & 3.3 & 4.4 \\
\hline 2 & 5.8 & 4.9 & 4.1 & 2.8 & 4.4 \\
\hline 1 & 5.0 & 5.5 & 4.5 & 3.9 & 4.7 \\
\hline & 1 & 2 & 3 & 4 & 4.9 \\
\hline
\end{tabular}

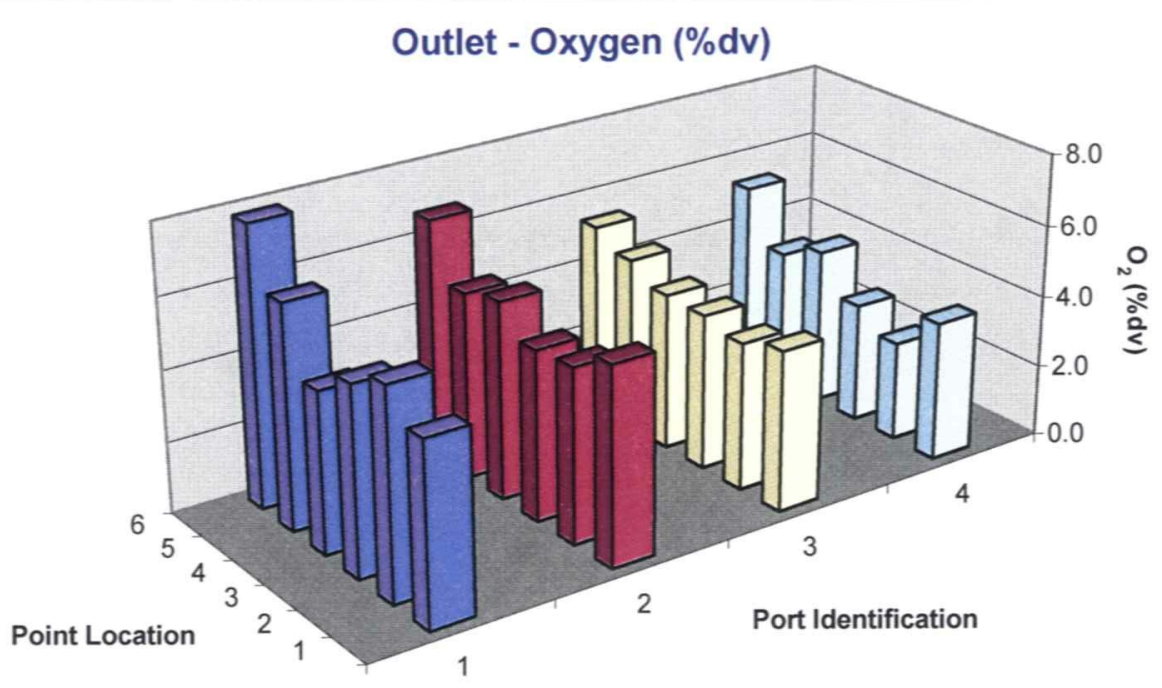


Run 3: Outlet - Set 2

Date: $3 / 28 / 2007$

Start Time: $\quad 13: 12$

End Time: $\quad 13: 35$

Outlet - Carbon Dioxide (\%dv)

\begin{tabular}{|c|c|c|c|c|}
\hline AVG & 13.1 & 13.4 & 14.1 & 14.8 \\
\hline 6 & 11.3 & 12.3 & 13.5 & 13.6 \\
\hline 5 & 12.7 & 13.6 & 13.9 & 14.9 \\
\hline 4 & 14.3 & 13.4 & 14.2 & 14.4 \\
\hline 3 & 13.6 & 14.0 & 14.4 & 15.4 \\
\hline 2 & 13.2 & 13.9 & 14.5 & 15.9 \\
\hline \multirow[t]{2}{*}{1} & 13.8 & 13.3 & 14.2 & 14.8 \\
\hline & 1 & 2 & 3 & 4 \\
\hline
\end{tabular}

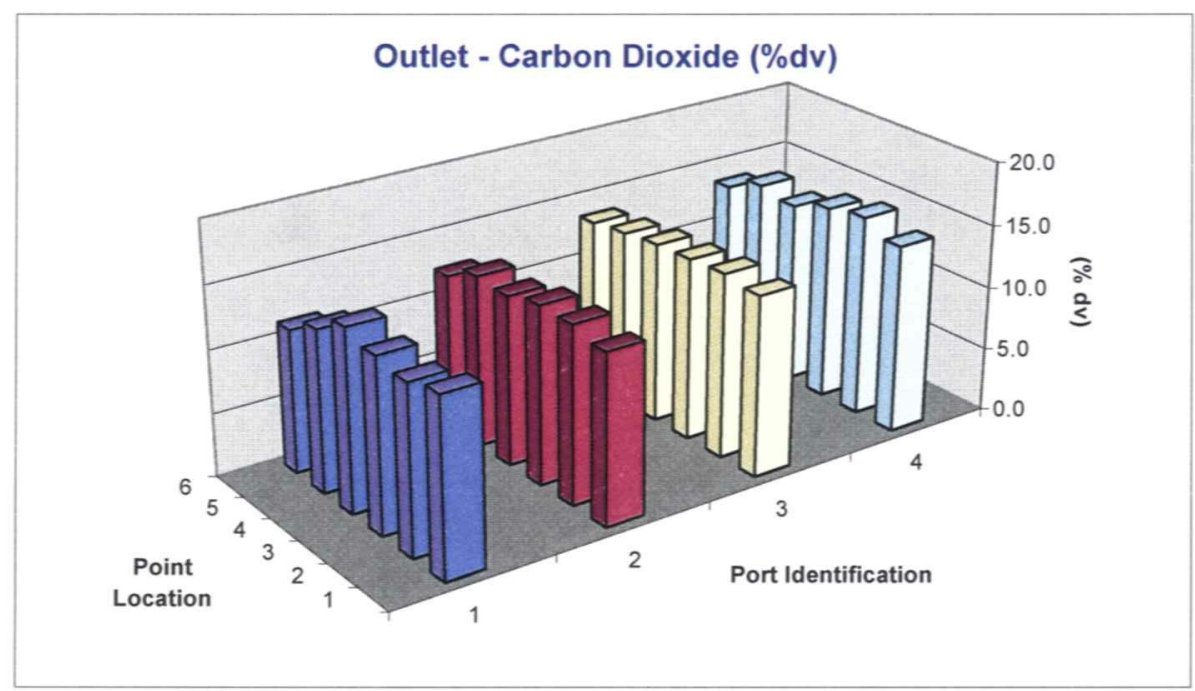


CONSOL ENERGY INC.
Client Reference No: 4700140111

CleanAir Project No:10192

Run 3: Outlet - Set 2

Date: $3 / 28 / 2007$

Start Time: $\quad 13: 12$

End Time: $\quad 13: 35$

Outlet - Nitrogen Oxides (ppmdv), uncorrected

\begin{tabular}{|c|c|c|c|c|}
\multicolumn{1}{|c}{ AVG } & 63.0 & 48.1 & 45.1 & 28.7 \\
\hline $\mathbf{6}$ & 51.7 & 40.7 & 20.8 & 4.2 \\
\hline $\mathbf{5}$ & 48.8 & 37.8 & 55.4 & 3.6 \\
\hline $\mathbf{4}$ & 68.3 & 63.8 & 69.5 & 37.3 \\
\hline $\mathbf{3}$ & 56.4 & 64.7 & 65.1 & 75.7 \\
\hline $\mathbf{2}$ & 82.0 & 51.2 & 41.8 & 45.5 \\
\hline $\mathbf{1}$ & 70.9 & 30.1 & 17.9 & 6.1 \\
\hline & $\mathbf{1}$ & $\mathbf{2}$ & $\mathbf{3}$ & $\mathbf{4}$ \\
\hline
\end{tabular}

29.4

36.4

59.7

65.5

55.1

31.3

46.2

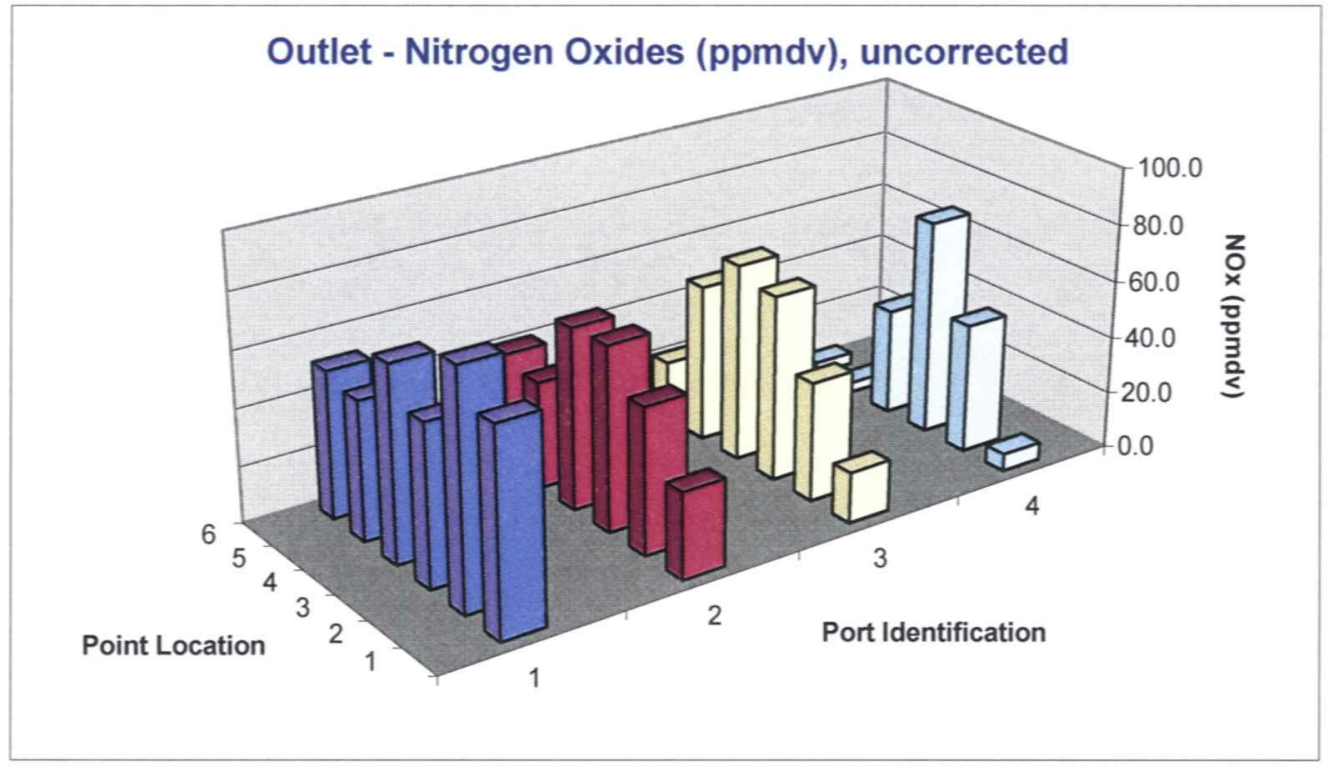


CONSOL ENERGY INC.
Client Reference No: 4700140111

CleanAir Project No:10192

Run 3: Outlet - Set 2

Date: $3 / 28 / 2007$

Start Time: $\quad 13: 12$

End Time: $13: 35$

Outlet - Nitrogen Oxides (ppmdv @ 3\% $\mathrm{O}_{2}$ )

\begin{tabular}{|c|c|c|c|c|}
\hline AVG & 74.4 & 55.4 & 49.3 & 29.6 \\
\hline 6 & 70.9 & 51.4 & 23.9 & 4.8 \\
\hline 5 & 59.7 & 43.1 & 61.9 & 3.8 \\
\hline 4 & 74.5 & 74.2 & 75.4 & 40.4 \\
\hline 3 & 64.5 & 71.7 & 70.4 & 77.1 \\
\hline 2 & 97.2 & 57.1 & 44.6 & 44.9 \\
\hline 1 & 80.0 & 35.0 & 19.5 & 6.4 \\
\hline & 1 & 2 & 3 & 4 \\
\hline
\end{tabular}

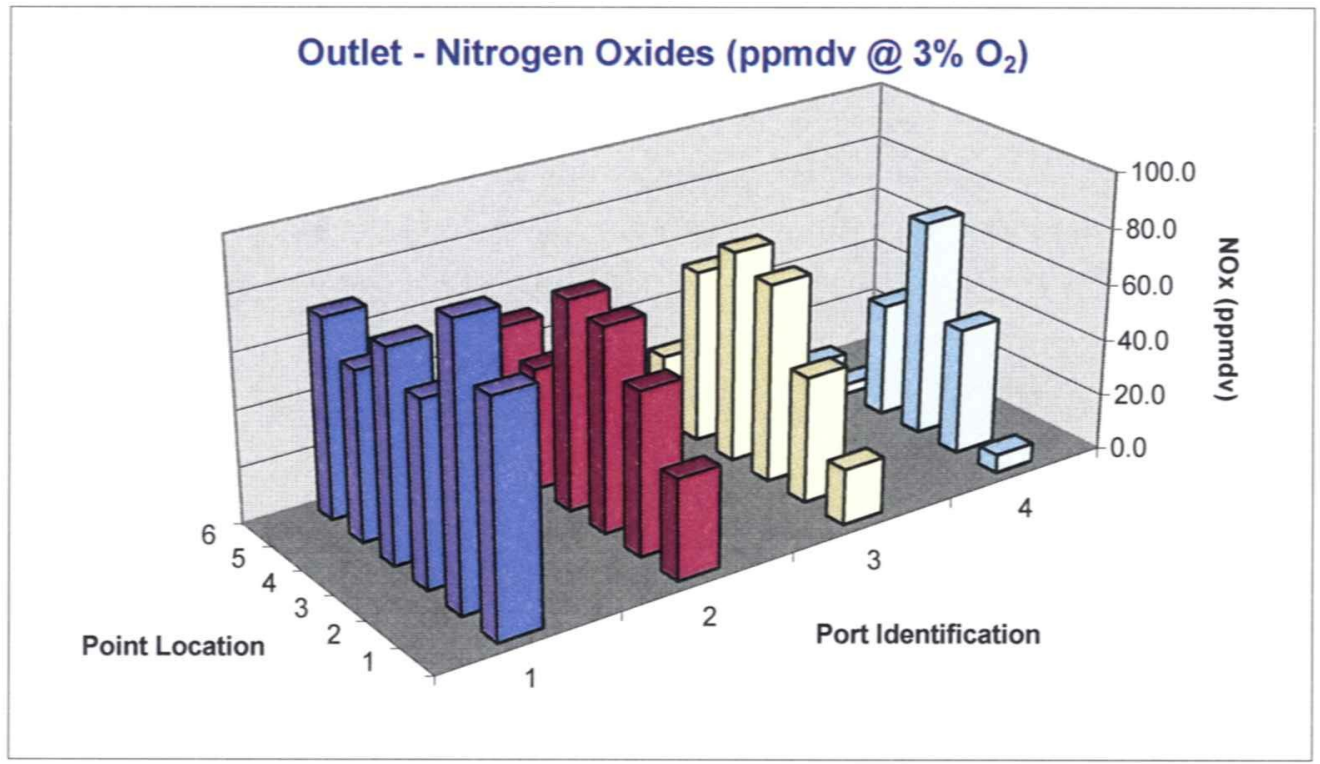


CONSOL ENERGY INC.

AES GREENIDGE STATION
Client Reference No: 4700140111

CleanAir Project No:10192

Run 3: Outlet - Set 2

Date: $3 / 28 / 2007$

Start Time: $\quad 13: 12$

End Time: $\quad 13: 35$

Removal Efficiency (ppmdv @ 3\% $\mathrm{O}_{2}$ )

\begin{tabular}{|c|c|c|c|c|}
\hline AVG & $19.5 \%$ & $31.9 \%$ & $39.9 \%$ & $64.5 \%$ \\
\hline 6 & $30.3 \%$ & $49.0 \%$ & $74.7 \%$ & $93.6 \%$ \\
\hline 5 & $12.9 \%$ & $25.3 \%$ & $9.0 \%$ & $93.8 \%$ \\
\hline 4 & $13.2 \%$ & $-1.9 \%$ & $4.6 \%$ & $46.9 \%$ \\
\hline 3 & $34.9 \%$ & $27.3 \%$ & $29.6 \%$ & $18.1 \%$ \\
\hline 2 & $3.5 \%$ & $28.1 \%$ & $42.7 \%$ & $41.4 \%$ \\
\hline \multirow[t]{2}{*}{1} & $22.2 \%$ & $63.8 \%$ & $78.9 \%$ & $93.0 \%$ \\
\hline & 1 & 2 & 3 & 4 \\
\hline
\end{tabular}

$61.9 \%$

$35.2 \%$

$15.7 \%$

$27.5 \%$

$28.9 \%$

$64.5 \%$

$39.0 \%$

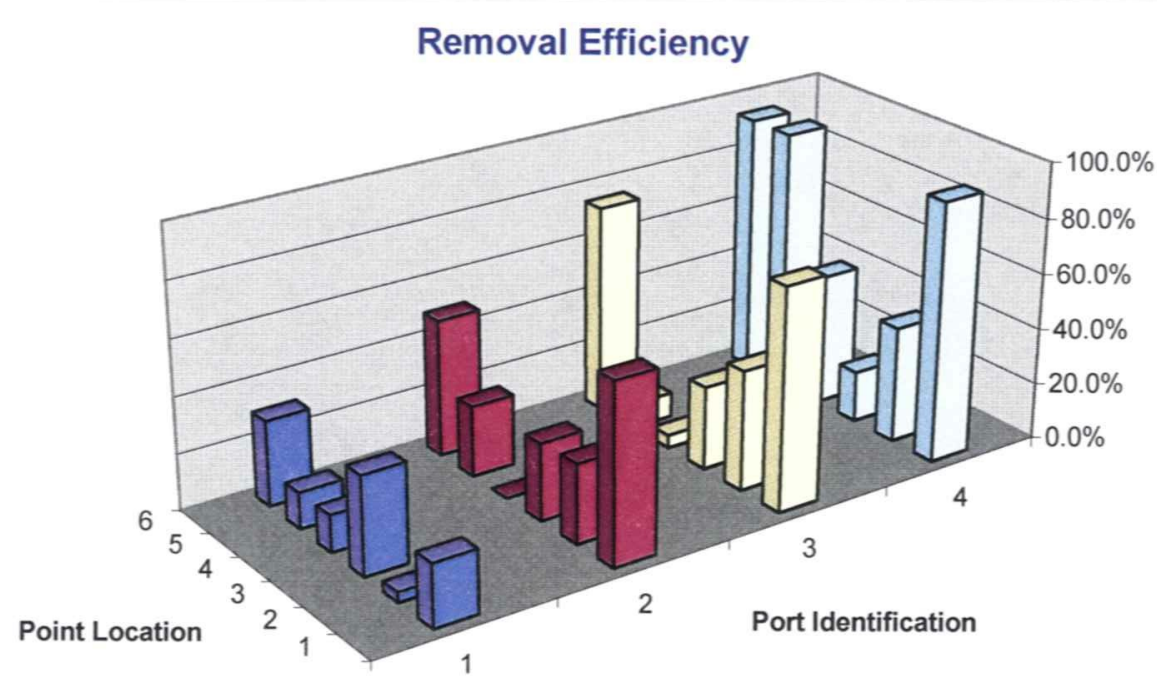


Clean Air Engineering Project \#10192

Consol Energy

AES Dresden

Date:

Start Time:

End Time:
$3 / 28 / 2007$

$13: 36$
$13: 59$
Run \# (Cycle \#) 3 (3)

\begin{tabular}{|c|c|c|c|c|c|c|c|c|c|c|}
\hline $\begin{array}{l}\text { Inlet } \\
\text { Point }\end{array}$ & Time Sampled & $\begin{array}{c}\text { NOx } \\
\text { ppmdv }\end{array}$ & $\begin{array}{c}\text { NOx } \\
\text { Port Avg }\end{array}$ & $\begin{array}{l}\text { PPM Dev } \\
\text { from Avg. }\end{array}$ & $\begin{array}{c}\text { O2 } \\
\% d v\end{array}$ & $\begin{array}{c}\mathrm{O} 2 \\
\text { Port Avg }\end{array}$ & $\begin{array}{l}\mathrm{CO2} \\
\% d v\end{array}$ & $\begin{array}{c}\mathrm{CO} 2 \\
\text { Port Avg }\end{array}$ & $\begin{array}{c}\text { NOx } \\
\text { @ } 3 \% \text { O2 }\end{array}$ & $\begin{array}{l}\text { PPM Dev } \\
\text { from Avg. }\end{array}$ \\
\hline $1-1$ & $13: 36$ & 75.5 & & -0.8 & 7.7 & & 11.5 & & 102.3 & 17.4 \\
\hline $1-2$ & $13: 40$ & 101.1 & & 24.8 & 3.3 & & 15.2 & & 102.6 & 17.7 \\
\hline $1-3$ & $13: 44$ & 86.5 & 78.8 & 10.2 & 5.0 & 5.6 & 13.8 & 13.3 & 97.4 & 12.4 \\
\hline $1-4$ & $13: 48$ & 76.3 & & 0.0 & 4.3 & & 14.3 & & 82.5 & -2.5 \\
\hline 1.5 & $13: 52$ & 64.9 & & -11.4 & 4.5 & & 14.2 & & 70.7 & -14.2 \\
\hline $1-6$ & $13: 56$ & 68.2 & & -8.1 & 8.7 & & 10.6 & & 100.0 & 15.0 \\
\hline $2-1$ & $13: 37$ & 80.5 & & 4.2 & 6.1 & & 12.8 & & 97.4 & 12.4 \\
\hline $2-2$ & $13: 41$ & 76.7 & & 0.4 & 3.3 & & 15.2 & & 77.9 & -7.1 \\
\hline $2-3$ & $13: 45$ & 84.4 & 73.8 & 8.1 & 5.3 & 4.9 & 13.5 & 13.8 & 96.8 & 11.8 \\
\hline $2-4$ & $13: 49$ & 71.9 & & -4.4 & 3.6 & & 14.9 & & 74.4 & -10.6 \\
\hline $2-5$ & $13: 53$ & 56.8 & & -19.5 & 4.2 & & 14.5 & & 60.7 & -24.2 \\
\hline $2-6$ & $13: 57$ & 72.3 & & -4.0 & 6.9 & & 12.1 & & 92.5 & 7.6 \\
\hline $3-1$ & $13: 38$ & 87.7 & & 11.4 & 4.2 & & 14.4 & & 94.1 & 9.2 \\
\hline $3-2$ & $13: 42$ & 74.9 & & -1.4 & 3.2 & & 15.3 & & 75.7 & -9.3 \\
\hline $3-3$ & $13: 46$ & 97.1 & 79.6 & 20.8 & 3.3 & 4.1 & 15.1 & 14.5 & 98.9 & 14.0 \\
\hline $3-4$ & $13: 50$ & 76.9 & & 0.6 & 3.6 & & 15.0 & & 79.3 & -5.6 \\
\hline $3-5$ & $13: 54$ & 61.1 & & -15.2 & 4.2 & & 14.4 & & 65.5 & -19.4 \\
\hline $3-6$ & $13: 58$ & 79.9 & & 3.6 & 6.2 & & 12.7 & & 97.6 & 12.6 \\
\hline $4-1$ & $13: 39$ & 76.8 & & 0.5 & 6.0 & & 12.9 & & 92.0 & 7.1 \\
\hline $4-2$ & $13: 43$ & 78.5 & & 2.2 & 2.8 & & 15.6 & & 77.7 & -7.2 \\
\hline $4-3$ & $13: 47$ & 94.9 & 73.1 & 18.6 & 3.2 & 4.3 & 15.3 & 14.4 & 95.8 & 10.9 \\
\hline $4-4$ & $13: 51$ & 74.2 & & -2.1 & 3.3 & & 15.2 & & 75.6 & -9.4 \\
\hline $4-5$ & $13: 55$ & 54.3 & & -22.0 & 4.1 & & 14.5 & & 57.8 & -27.1 \\
\hline 4-6 & $13: 59$ & 59.9 & & -16.4 & 6.3 & & 12.7 & & 73.3 & -11.6 \\
\hline
\end{tabular}

\begin{tabular}{|c|c|c|c|c|c|c|c|c|c|c|c|c|}
\hline $\begin{array}{l}\text { Outlet } \\
\text { Point }\end{array}$ & Time Sampled & $\begin{array}{c}\text { NOx } \\
\text { ppmdv }\end{array}$ & $\begin{array}{c}\text { NOx } \\
\text { Port Avg }\end{array}$ & $\begin{array}{l}\text { PPM Dev } \\
\text { from Avg. }\end{array}$ & $\begin{array}{l}\text { O2 } \\
\% d v\end{array}$ & $\begin{array}{c}\mathrm{O} 2 \\
\text { Port Avg }\end{array}$ & $\begin{array}{l}\mathrm{CO2} \\
\% \text { dv }\end{array}$ & $\begin{array}{c}\mathrm{CO} 2 \\
\text { Port Avg }\end{array}$ & $\begin{array}{c}\mathrm{NOx} \\
\text { @ } 3 \% \mathrm{O} \text {. }\end{array}$ & $\begin{array}{l}\text { PPM Dev } \\
\text { from Avg. }\end{array}$ & $\begin{array}{c}\text { Removal } \\
\text { Efficiency }\end{array}$ & $\begin{array}{c}\text { Removal Eff } \\
\text { w/ O2 correction }\end{array}$ \\
\hline $1-1$ & $13: 36$ & 69.4 & \multirow{6}{*}{54.9} & 26.8 & 5.0 & \multirow{6}{*}{5.3} & 13.9 & \multirow{6}{*}{13.6} & 78.0 & 30.8 & 8.1 & 23.8 \\
\hline $1-2$ & $13: 40$ & 42.1 & & -0.5 & 4.9 & & 14.0 & & 47.1 & -0.1 & 58.4 & 54.1 \\
\hline $1-3$ & $13: 44$ & 52.5 & & 9.9 & 3.7 & & 15.0 & & 54.8 & 7.6 & 39.3 & 43.8 \\
\hline $1-4$ & $13: 48$ & 66.3 & & 23.7 & 4.6 & & 14.2 & & 72.8 & 25.6 & 13.1 & 11.8 \\
\hline $1-5$ & $13: 52$ & 49.3 & & 6.7 & 6.1 & & 12.9 & & 59.7 & 12.5 & 24.0 & 15.6 \\
\hline $1-6$ & $13: 56$ & 49.9 & & 7.3 & 7.6 & & 11.4 & & 67.4 & 20.2 & 26.8 & 32.6 \\
\hline $2-1$ & $13: 37$ & 26.3 & \multirow{6}{*}{42.9} & -16.3 & 5.2 & \multirow{6}{*}{5.1} & 13.6 & \multirow{6}{*}{13.8} & 30.0 & -17.1 & 67.3 & 69.1 \\
\hline $2-2$ & $13: 41$ & 36.2 & & -6.4 & 3.5 & & 15.1 & & 37.3 & -9.9 & 52.8 & 52.1 \\
\hline $2-3$ & $13: 45$ & 69.3 & & 26.7 & 4.7 & & 14.1 & & 76.7 & 29.5 & 17.9 & 20.7 \\
\hline $2-4$ & $13: 49$ & 69.7 & & 27.1 & 5.8 & & 13.2 & & 82.4 & 35.2 & 3.1 & -10.8 \\
\hline $2-5$ & $13: 53$ & 21.5 & & -21.1 & 4.8 & & 14.0 & & 23.8 & -23.3 & 62.1 & 60.7 \\
\hline $2-6$ & $13: 57$ & 34.5 & & -8.1 & 6.3 & & 12.7 & & 42.3 & -4.9 & 52.3 & 54.3 \\
\hline $3-1$ & $13: 38$ & 16.4 & \multirow{6}{*}{43.1} & -26.2 & 4.1 & & 14.6 & \multirow{6}{*}{14.4} & 17.4 & -29.8 & 81.3 & 81.5 \\
\hline 3.2 & $13: 42$ & 53.2 & & 10.6 & 3.5 & & 15.3 & & 54.6 & 7.4 & 29.0 & 27.9 \\
\hline $3-3$ & $13: 46$ & 79.8 & & 37.2 & 4.3 & 4.3 & 14.5 & & 85.8 & 38.7 & 17.8 & 13.2 \\
\hline $3-4$ & $13: 50$ & 58.3 & & 15.7 & 4.8 & & 14.0 & & 64.9 & 17.7 & 24.2 & 18.2 \\
\hline $3-5$ & $13: 54$ & 30.1 & & -12.5 & 4.2 & & 14.5 & & 32.2 & -15.0 & 50.7 & 50.9 \\
\hline $3-6$ & $13: 58$ & 20.7 & & -21.9 & 5.2 & & 13.7 & & 23.5 & -23.7 & 74.1 & 75.9 \\
\hline $4-1$ & $13: 39$ & 6.0 & \multirow{6}{*}{29.4} & -36.6 & 3.8 & & 14.9 & \multirow{6}{*}{14.8} & 6.3 & -40.9 & 92.2 & 93.2 \\
\hline $4-2$ & $13: 43$ & 45.6 & & 3.0 & 2.8 & & 15.9 & & 45.0 & -2.1 & 41.9 & 42.0 \\
\hline $4-3$ & $13: 47$ & 77.0 & & 34.4 & 3.5 & 3.9 & 15.2 & & 79.0 & 31.8 & 18.9 & 17.5 \\
\hline $4-4$ & $13: 51$ & 40.4 & & -2.2 & 4.3 & & 14.5 & & 43.5 & -3.7 & 45.6 & 42.5 \\
\hline $4-5$ & $13: 55$ & 3.4 & & -39.2 & 3.7 & & 15.0 & & 3.5 & -43.7 & 93.7 & 93.9 \\
\hline $4-6$ & $13: 59$ & 3.9 & & -38.7 & 5.3 & & 13.6 & & 4.5 & -42.7 & 93.5 & 93.9 \\
\hline
\end{tabular}


Run 3: Inlet - Set 3

Date: $3 / 28 / 2007$

Start Time: $\quad 13: 36$

End Time: $\quad 13: 59$

Inlet - Oxygen (\%dv)

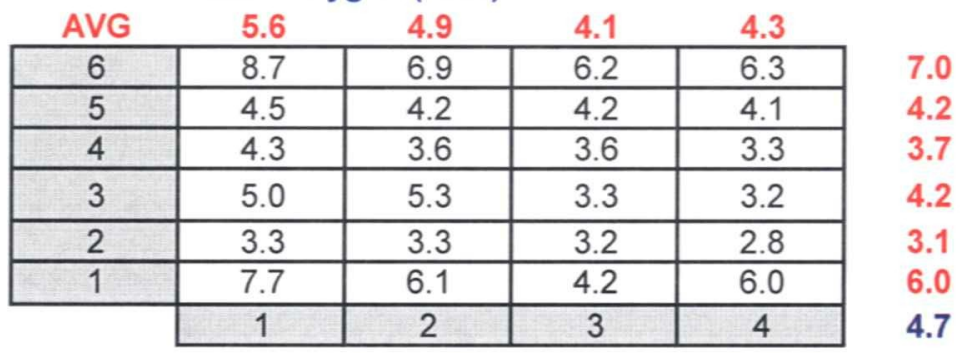

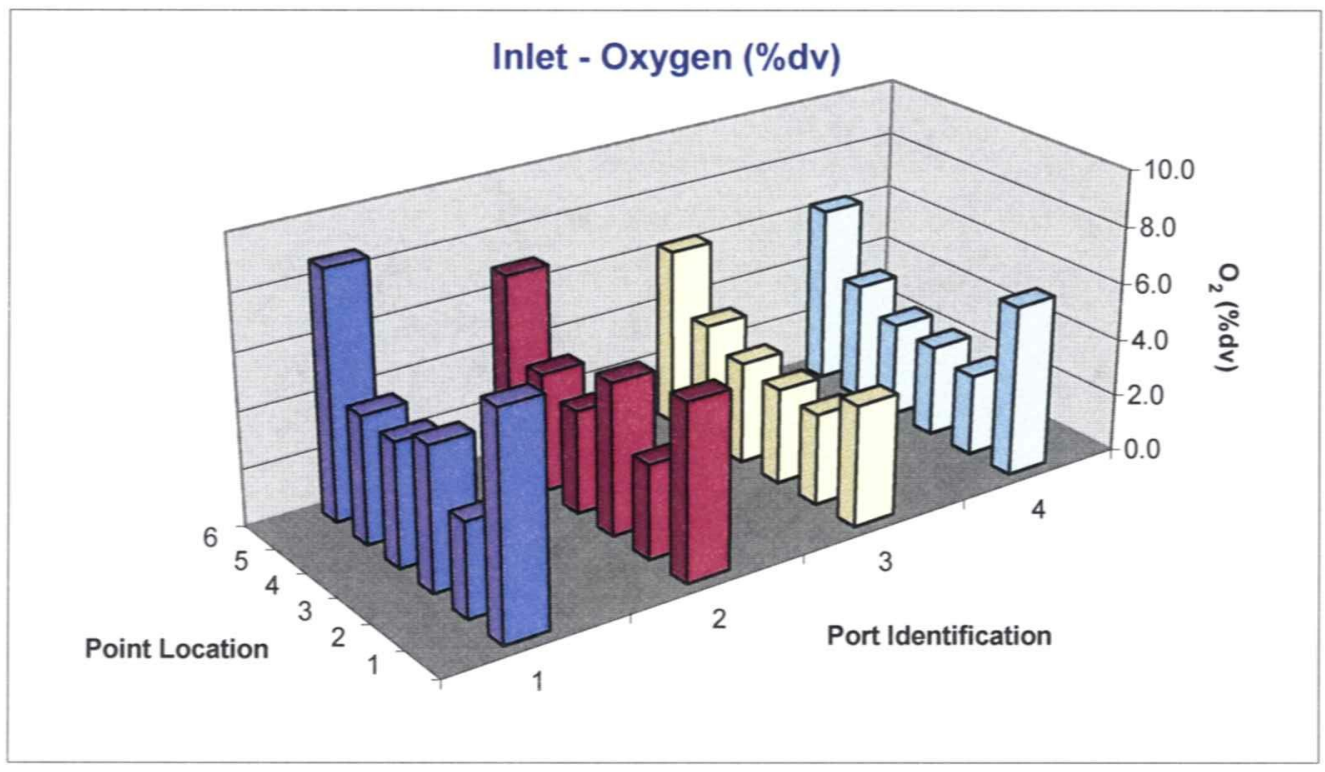


Run 3: Inlet - Set 3

Date: $3 / 28 / 2007$

Start Time: $\quad 13: 36$

End Time: $\quad 13: 59$

Inlet - Carbon Dioxide (\%dv)

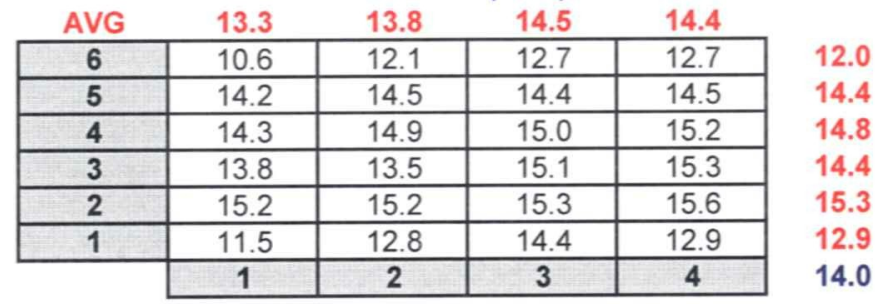

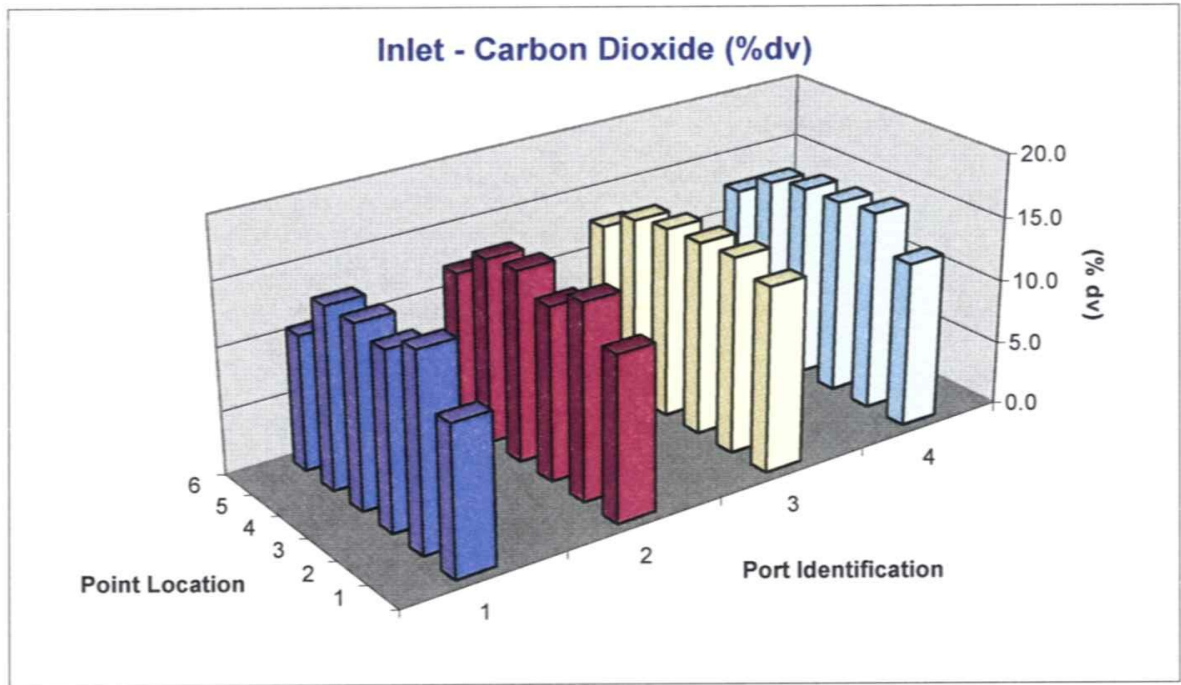


Run 3: Inlet - Set 3

Date: $3 / 28 / 2007$

Start Time: $\quad 13: 36$

End Time: $\quad 13: 59$

Inlet - Nitrogen Oxides (ppmdv), uncorrected

\begin{tabular}{|c|c|c|c|c|}
\multicolumn{1}{|c|}{ AVG } & 78.8 & 73.8 & 79.6 & 73.1 \\
\hline $\mathbf{6}$ & 68.2 & 72.3 & 79.9 & 59.9 \\
\hline $\mathbf{5}$ & 64.9 & 56.8 & 61.1 & 54.3 \\
\hline $\mathbf{4}$ & 76.3 & 71.9 & 76.9 & 74.2 \\
\hline $\mathbf{3}$ & 86.5 & 84.4 & 97.1 & 94.9 \\
\hline $\mathbf{2}$ & 101.1 & 76.7 & 74.9 & 78.5 \\
\hline $\mathbf{1}$ & 75.5 & 80.5 & 87.7 & 76.8 \\
\hline & $\mathbf{1}$ & $\mathbf{2}$ & $\mathbf{3}$ & $\mathbf{4}$ \\
\hline
\end{tabular}

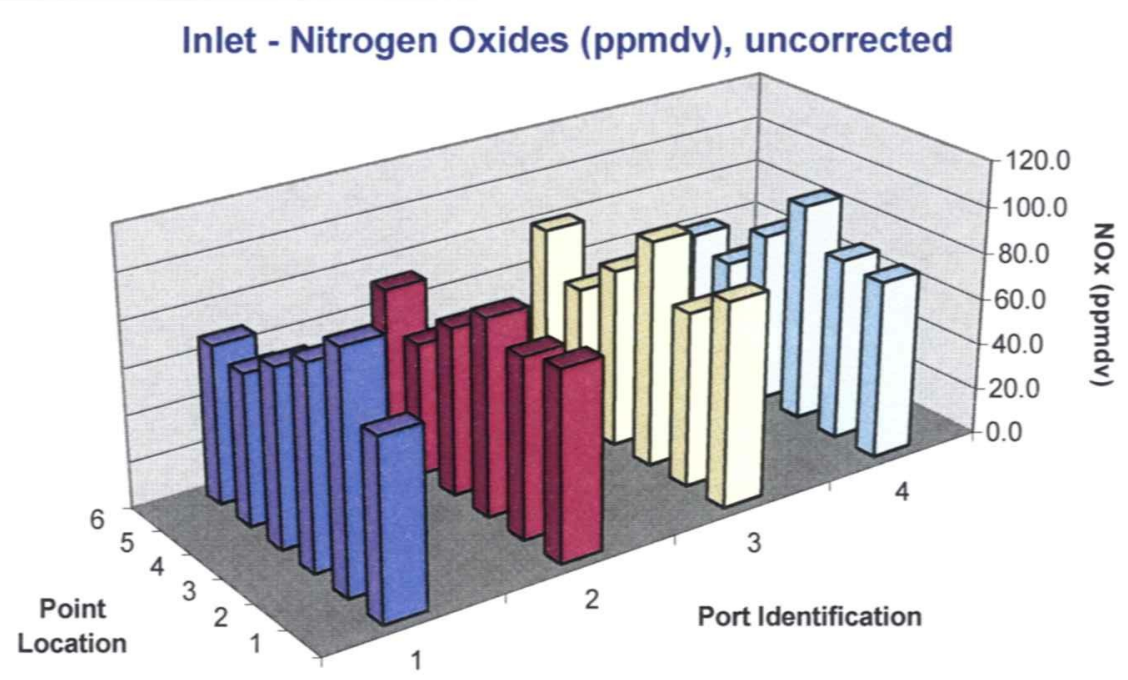


Run 3: Inlet - Set 3

Date: $3 / 28 / 2007$

Start Time: $\quad 13: 36$

End Time: $\quad 13: 59$

Inlet - Nitrogen Oxides (ppmdv @ 3\% $\mathrm{O}_{2}$ )

\begin{tabular}{|c|c|c|c|c|}
\multicolumn{1}{c}{ AVG } & 92.6 & 83.3 & 85.2 & 78.7 \\
\hline $\mathbf{6}$ & 100.0 & 92.5 & 97.6 & 73.3 \\
\hline $\mathbf{5}$ & 70.7 & 60.7 & 65.5 & 57.8 \\
\hline $\mathbf{4}$ & 82.5 & 74.4 & 79.3 & 75.6 \\
\hline $\mathbf{3}$ & 97.4 & 96.8 & 98.9 & 95.8 \\
\hline $\mathbf{2}$ & 102.6 & 77.9 & 75.7 & 77.7 \\
\hline $\mathbf{1}$ & 102.3 & 97.4 & 94.1 & 92.0 \\
\hline & $\mathbf{1}$ & $\mathbf{2}$ & $\mathbf{3}$ & $\mathbf{4}$ \\
\cline { 2 - 5 }
\end{tabular}

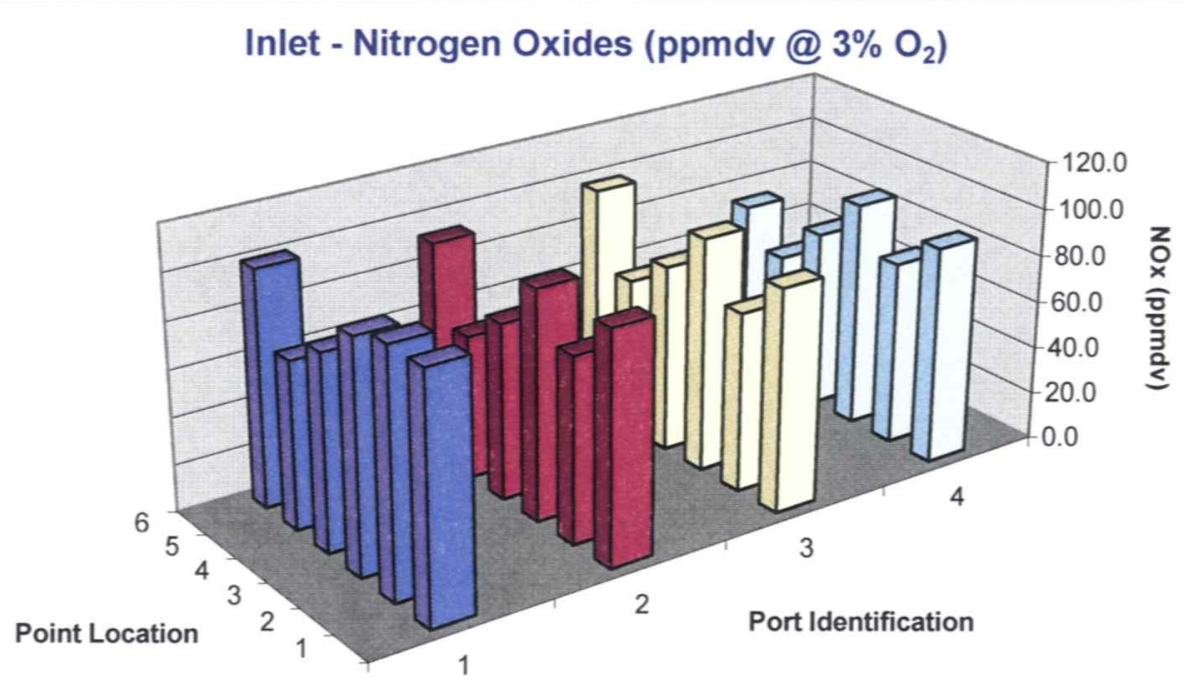


Date: $3 / 28 / 2007$

Start Time: $\quad 13: 36$

End Time: $\quad 13: 59$

\begin{tabular}{|c|c|c|c|c|c|}
\hline \multicolumn{5}{|c|}{ Outlet - Oxygen (\%dv) } & \multirow[b]{3}{*}{6.1} \\
\hline AVG & 5.3 & 5.1 & 4.3 & 3.9 & \\
\hline 6 & 7.6 & 6.3 & 5.2 & 5.3 & \\
\hline 5 & 6.1 & 4.8 & 4.2 & 3.7 & 4.7 \\
\hline 4 & 4.6 & 5.8 & 4.8 & 4.3 & 4.9 \\
\hline 3 & 3.7 & 4.7 & 4.3 & 3.5 & 4.0 \\
\hline 2 & 4.9 & 3.5 & 3.5 & 2.8 & 3.7 \\
\hline 1 & 5.0 & 5.2 & 4.1 & 3.8 & 4.5 \\
\hline & 1 & 2 & 3 & 4 & 4.6 \\
\hline
\end{tabular}

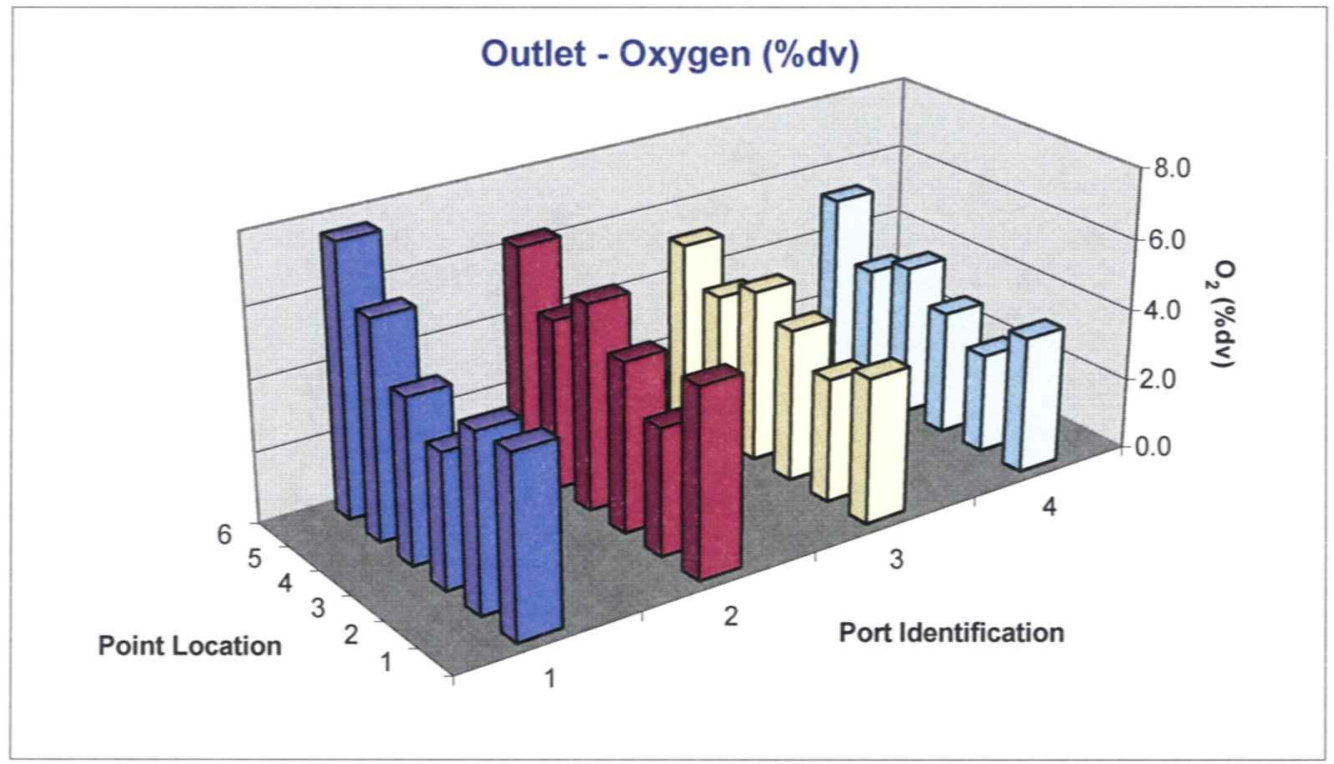


Run 3: Outlet - Set 3

Date: $3 / 28 / 2007$

Start Time: $\quad 13: 36$

End Time: $\quad 13: 59$

Outlet - Carbon Dioxide (\%dv)

\begin{tabular}{|c|c|c|c|c|}
\hline AVG & 13.6 & 13.8 & 14.4 & 14.8 \\
\hline 6 & 11.4 & 12.7 & 13.7 & 13.6 \\
\hline 5 & 12.9 & 14.0 & 14.5 & 15.0 \\
\hline 4 & 14.2 & 13.2 & 14.0 & 14.5 \\
\hline 3 & 15.0 & 14.1 & 14.5 & 15.2 \\
\hline 2 & 14.0 & 15.1 & 15.3 & 15.9 \\
\hline \multirow[t]{2}{*}{1} & 13.9 & 13.6 & 14.6 & 14.9 \\
\hline & 1 & 2 & 3 & 4 \\
\hline
\end{tabular}

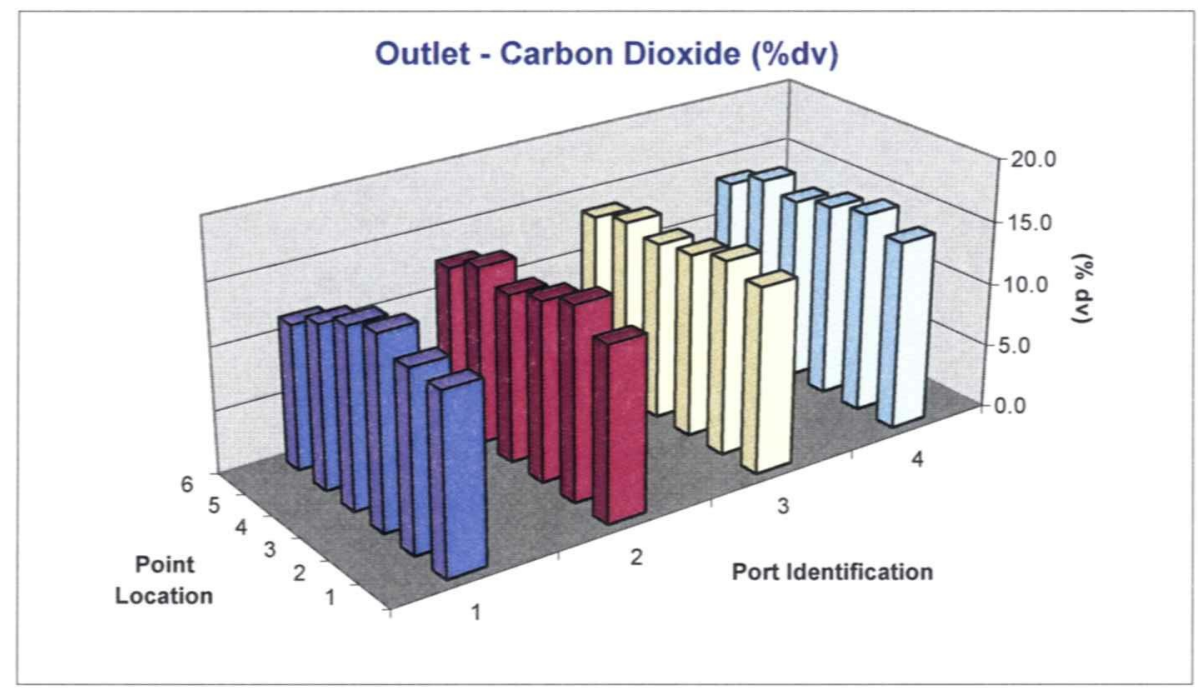


Date: $3 / 28 / 2007$

Start Time: $\quad 13: 36$

End Time: $\quad 13: 59$

Outlet - Nitrogen Oxides (ppmdv), uncorrected

\begin{tabular}{|c|c|c|c|c|}
\multicolumn{1}{c}{ AVG } & 54.9 & 42.9 & 43.1 & 29.4 \\
\hline $\mathbf{6}$ & 49.9 & 34.5 & 20.7 & 3.9 \\
\hline $\mathbf{5}$ & 49.3 & 21.5 & 30.1 & 3.4 \\
\hline $\mathbf{4}$ & 66.3 & 69.7 & 58.3 & 40.4 \\
\hline $\mathbf{3}$ & 52.5 & 69.3 & 79.8 & 77.0 \\
\hline $\mathbf{2}$ & 42.1 & 36.2 & 53.2 & 45.6 \\
\hline $\mathbf{1}$ & 69.4 & 26.3 & 16.4 & 6.0 \\
\hline & $\mathbf{1}$ & $\mathbf{2}$ & $\mathbf{3}$ & $\mathbf{4}$ \\
\hline
\end{tabular}

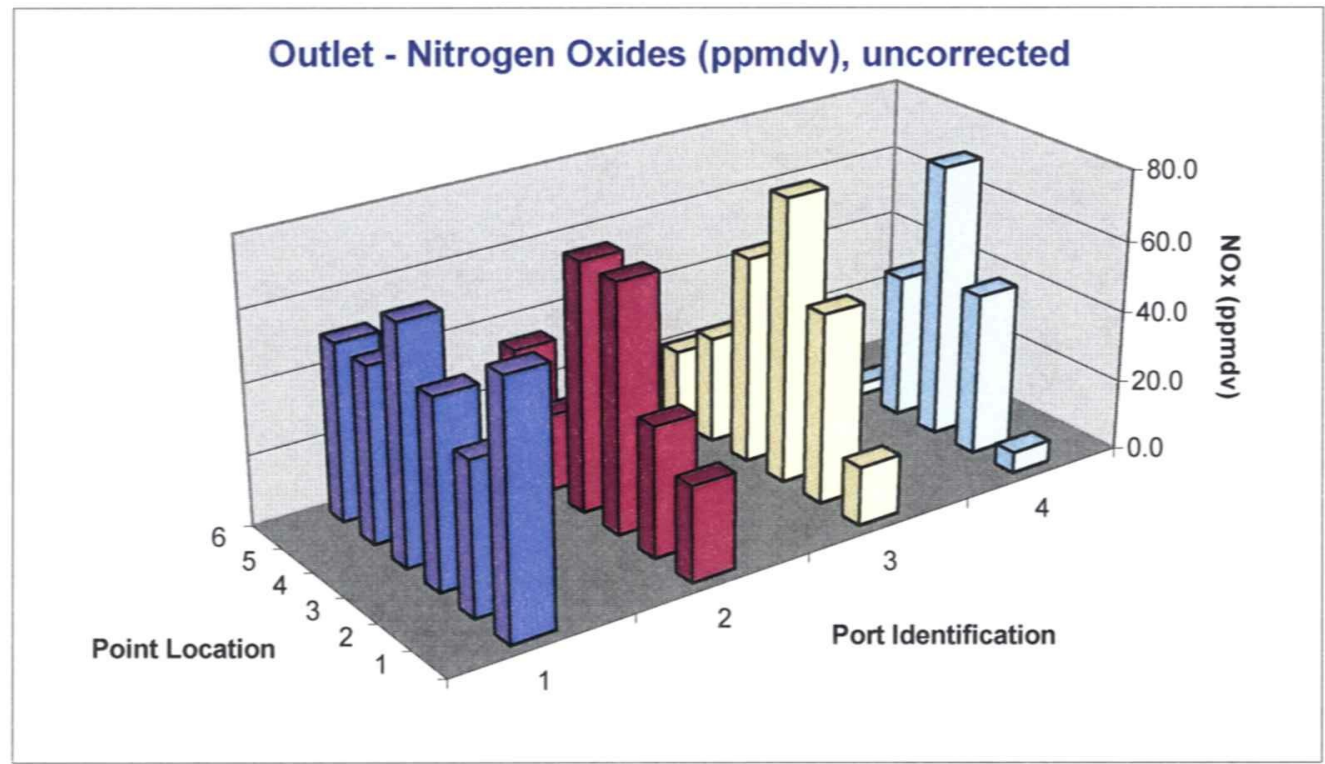


Client Reference No: 4700140111

CleanAir Project No:10192

Run 3: Outlet - Set 3

Date: $3 / 28 / 2007$

Start Time: $\quad 13: 36$

End Time: $\quad 13: 59$

Outlet - Nitrogen Oxides (ppmdv @ 3\% $\mathrm{O}_{2}$ )

\begin{tabular}{|c|c|c|c|c|}
\multicolumn{1}{c|}{ AVG } & 63.3 & 48.8 & 46.4 & 30.3 \\
\hline $\mathbf{6}$ & 67.4 & 42.3 & 23.5 & 4.5 \\
\hline $\mathbf{5}$ & 59.7 & 23.8 & 32.2 & 3.5 \\
\hline $\mathbf{4}$ & 72.8 & 82.4 & 64.9 & 43.5 \\
\hline $\mathbf{3}$ & 54.8 & 76.7 & 85.8 & 79.0 \\
\hline $\mathbf{2}$ & 47.1 & 37.3 & 54.6 & 45.0 \\
\hline $\mathbf{1}$ & 78.0 & 30.0 & 17.4 & 6.3 \\
\hline & $\mathbf{1}$ & $\mathbf{2}$ & $\mathbf{3}$ & $\mathbf{4}$ \\
\hline
\end{tabular}

34.4

29.8

65.9

74.1

46.0

32.9

47.2

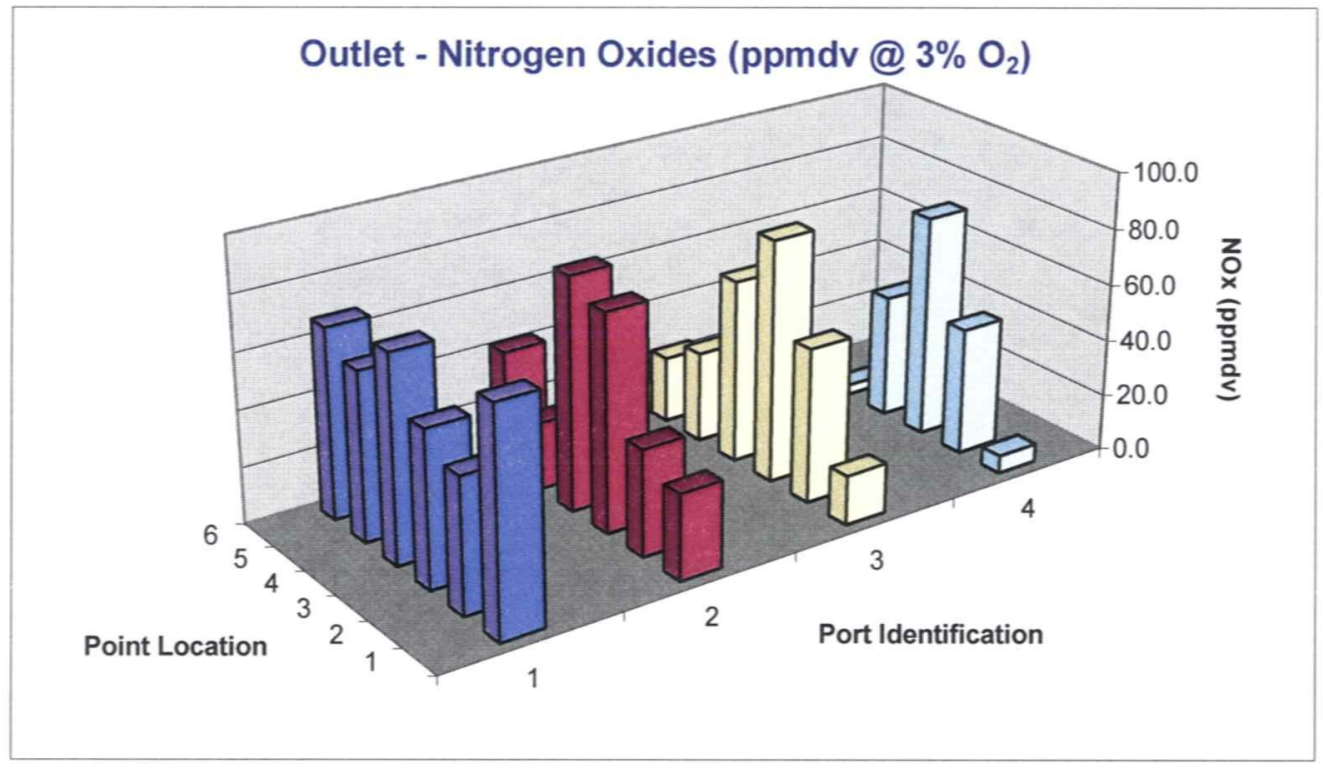


Date: $3 / 28 / 2007$

Start Time: $\quad 13: 36$

End Time: $\quad 13: 59$

Removal Efficiency (ppmdv @ 3\% $\mathrm{O}_{2}$ )

\begin{tabular}{|c|c|c|c|c|}
\hline AVG & $30.3 \%$ & $41.0 \%$ & $44.6 \%$ & $63.8 \%$ \\
\hline 6 & $32.6 \%$ & $54.3 \%$ & $75.9 \%$ & $93.9 \%$ \\
\hline 5 & $15.6 \%$ & $60.7 \%$ & $50.9 \%$ & $93.9 \%$ \\
\hline 4 & $11.8 \%$ & $-10.8 \%$ & $18.2 \%$ & $42.5 \%$ \\
\hline 3 & $43.8 \%$ & $20.7 \%$ & $13.2 \%$ & $17.5 \%$ \\
\hline 2 & $54.1 \%$ & $52.1 \%$ & $27.9 \%$ & $42.0 \%$ \\
\hline 1 & $23.8 \%$ & $69.1 \%$ & $81.5 \%$ & $93.2 \%$ \\
\hline & 1 & 2 & 3 & 4 \\
\hline
\end{tabular}

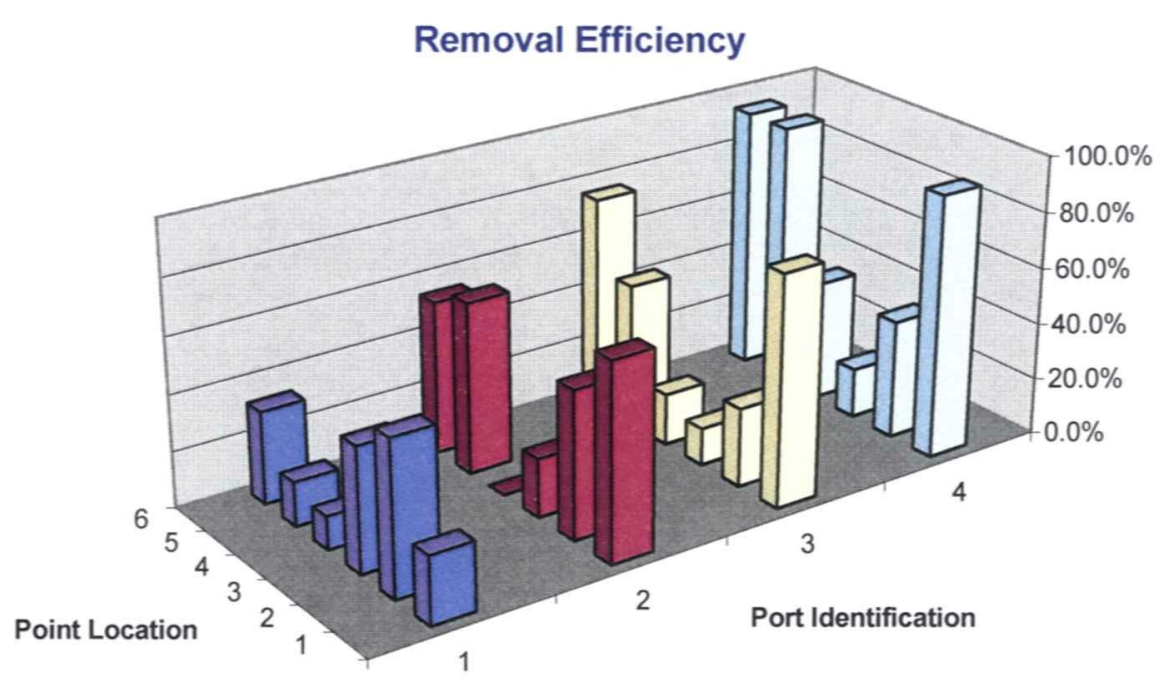


APPENDIX B

CTL Group Report (Process Sample Analyses) 


\section{CONSOL Energy Inc.}

Project No.: 403423

Performance Testing of Air Pollution Control System of Coal-Fired Power Plant

Date:

May 15,2007

Submitted by:

Ella Shkolnik

CTLGroup

5400 Old Orchard Road

Skokie, Illinois 60077-1030

(847) 965-7500

9030 Red Branch Road, Suite 110

Columbia, Maryland 21045

1 Washington Street

Dover New Hampshire 03820

www.CTLGroup.com

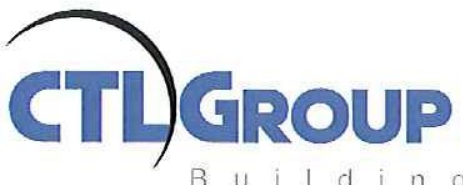


CTL) GROUP

Client: $\quad$ CONSOL Energy, Inc.

Project: Chemical Analysis

Contact: Daniel Connell

Submitter: Daniel Connell

Date Received: April 23, 2007

CONSTRUCTION

TECHNOLOGY LABORATORIES

ENGINEERS \& CONSTRUCTION TECHNOLOGY CONSUITANTS

www.CTLGroup.com

CTL Project No:

CTL Project Mgr.:

Analyst:

Approved:

Date Analyzed:

Date Reported:
403423

Ella Shkolnik

Cecylia Wedzicha

Rifi

May 1, 2007

May 1, 2007

\section{REPORT of AVAILABLE LIME ANALYSIS}

$\begin{array}{ll}\begin{array}{c}\text { Sample Identification } \\ \text { CTL ID }\end{array} & \text { Client ID } \\ 1839004 & \text { PEB032807 } \\ 1839005 & \text { PEB032907 } \\ 1839006 & \text { PEB033007 }\end{array}$

Description

Pebble lime

Pebble lime

Pebble lime
Determined Results (wt. \%) as $\mathrm{CaO}$

$\underline{\text { as } \mathrm{Ca}(\mathrm{OH})_{2}}$

89.38

88.07

92.57
118.07

116.34

122.29

Notes:

1. This analysis represents specifically the samples submitted as received.

2. The results were determined in accordance with ASTM C 25, Section 28.

3. This report may not be reproduced except in its entirety. 
Client: $\quad$ CONSOL Energy, Inc.

Project: Chemical Analysis

Contact: Daniel Connell

Submitter: Daniel Connell

Date Received: April 23, 2007

www.CTLGroup.com

CTL Project No:

CTL Project Mgr.:

Analyst:

Approved:

Date Analyzed:

Date Reported:
403423

Ella Shkolnik

Cecylia Wedzicha

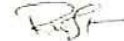

May 1, 2007

May 2, 2007

REPORT of SLAKING RATE ANALYSIS

(ASTM C 110)

Sample Identification

CTL ID: $\quad 1839004$

Client ID: $\quad$ PEB032807

Description: Pebble lime

\begin{tabular}{|c|c|c|}
\hline \multicolumn{2}{|c|}{ Time } & Temperature \\
\hline (minute) & (second) & $\left({ }^{\circ} \mathrm{C}\right)$ \\
\hline 0.0 & 0 & 25.0 \\
\hline 0.5 & 30 & 57.0 \\
\hline 1.0 & 60 & 67.0 \\
\hline 1.5 & 90 & 72.0 \\
\hline 2.0 & 120 & 74.5 \\
\hline 2.5 & 150 & 75.5 \\
\hline 3.0 & 180 & 77.0 \\
\hline 3.5 & 210 & 77.5 \\
\hline 4.0 & 240 & 78.0 \\
\hline 4.5 & 270 & 78.5 \\
\hline 5.0 & 300 & 78.5 \\
\hline 5.5 & 330 & 78.5 \\
\hline 6.0 & 360 & 78.5 \\
\hline 6.5 & 390 & 78.5 \\
\hline 7.0 & 420 & 78.5 \\
\hline 7.5 & 450 & 78.5 \\
\hline 8.0 & 480 & 78.5 \\
\hline 8.5 & 510 & 78.5 \\
\hline 9.0 & 540 & 78.5 \\
\hline 9.5 & 570 & \\
\hline 10.0 & 600 & \\
\hline
\end{tabular}

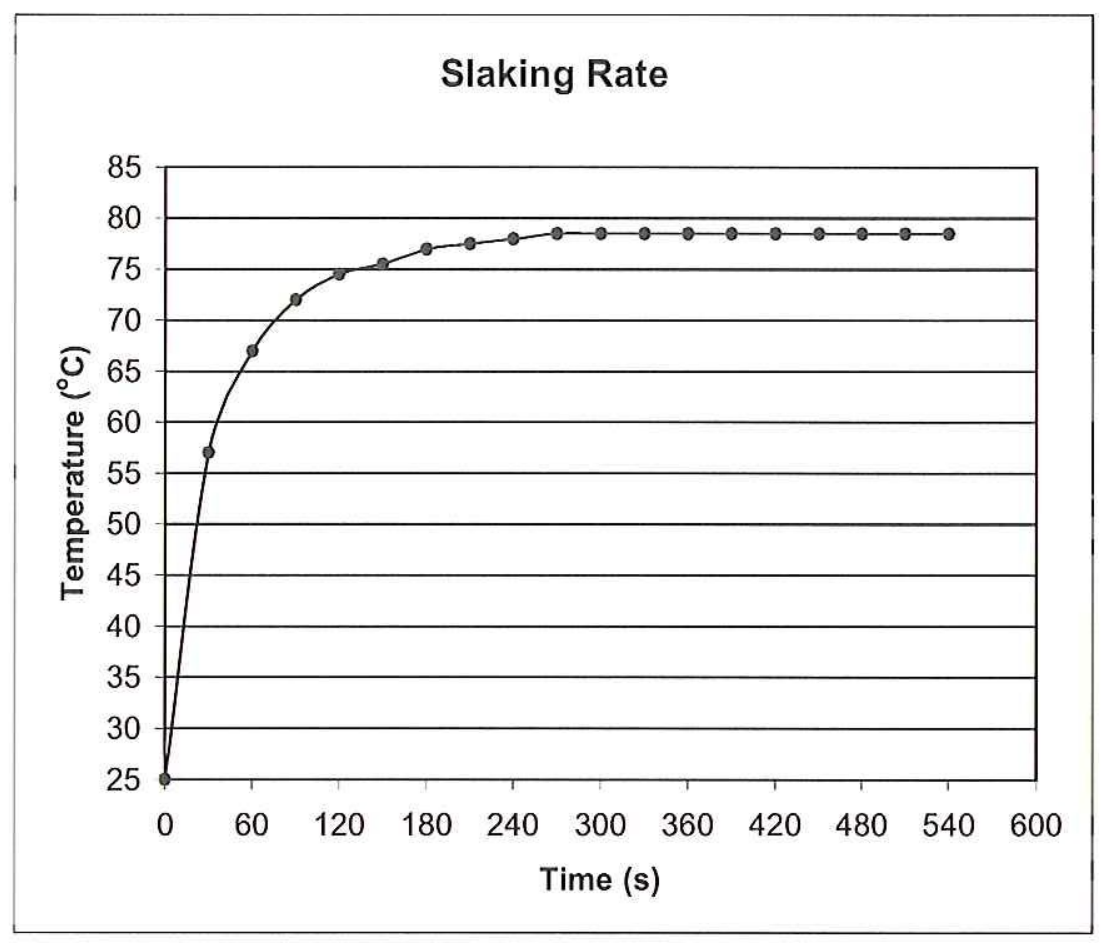

Determined Results:

Total active slaking time (minute)

Final reaction temperature $\left({ }^{\circ} \mathrm{C}\right)$

78.5

Temperature rise in 30 seconds $\left({ }^{\circ} \mathrm{C}\right)$

32.0

Temperature rise in 3 minutes $\left({ }^{\circ} \mathrm{C}\right.$ )

52.0

Total temperature rise $\left({ }^{\circ} \mathrm{C}\right)$

53.5

Residue (wt. \%)

0.97

Notes:

1. This analysis represents specifically the sample(s) submitted as received.

2. The results were determined in accordance with ASTM C 110-05, Sec.11.

3. This report may not be reproduced except in its entirety. 


\begin{tabular}{|c|c|}
\hline $\begin{array}{l}\text { Client: } \\
\text { Project: }\end{array}$ & $\begin{array}{l}\text { CONSOL Energy, Inc. } \\
\text { Chemical Analysis }\end{array}$ \\
\hline Contact: & Daniel Connell \\
\hline Submitter: & Daniel Connell \\
\hline Date Received: & April 23, 2007 \\
\hline
\end{tabular}

CTL Project No:

CTL Project Mgr.:

Analyst:

Approved:

Date Analyzed:

Date Reported:

www.CTLGroup.com

\section{REPORT of SLAKING RATE ANALYSIS (ASTM C 110)}

Sample Identification

$\begin{array}{ll}\text { CTL ID: } & 1839005 \\ \text { Client ID: } & \text { PEB032907 } \\ \text { Description: } & \text { Pebble lime }\end{array}$

\begin{tabular}{|c|c|c|}
\hline \multicolumn{2}{|c|}{ Time } & Temperature \\
\hline (minute) & (second) & $\left({ }^{\circ} \mathrm{C}\right)$ \\
\hline 0.0 & 0 & 25.0 \\
\hline 0.5 & 30 & 55.0 \\
\hline 1.0 & 60 & 65.0 \\
\hline 1.5 & 90 & 71.0 \\
\hline 2.0 & 120 & 73.5 \\
\hline 2.5 & 150 & 74.5 \\
\hline 3.0 & 180 & 75.5 \\
\hline 3.5 & 210 & 76.0 \\
\hline 4.0 & 240 & 76.5 \\
\hline 4.5 & 270 & 77.0 \\
\hline 5.0 & 300 & 77.0 \\
\hline 5.5 & 330 & 77.0 \\
\hline 6.0 & 360 & 77.0 \\
\hline 6.5 & 390 & 77.0 \\
\hline 7.0 & 420 & 77.0 \\
\hline 7.5 & 450 & 77.0 \\
\hline 8.0 & 480 & 77.0 \\
\hline 8.5 & 510 & 77.0 \\
\hline 9.0 & 540 & 77.0 \\
\hline 9.5 & 570 & \\
\hline 10.0 & 600 & \\
\hline
\end{tabular}

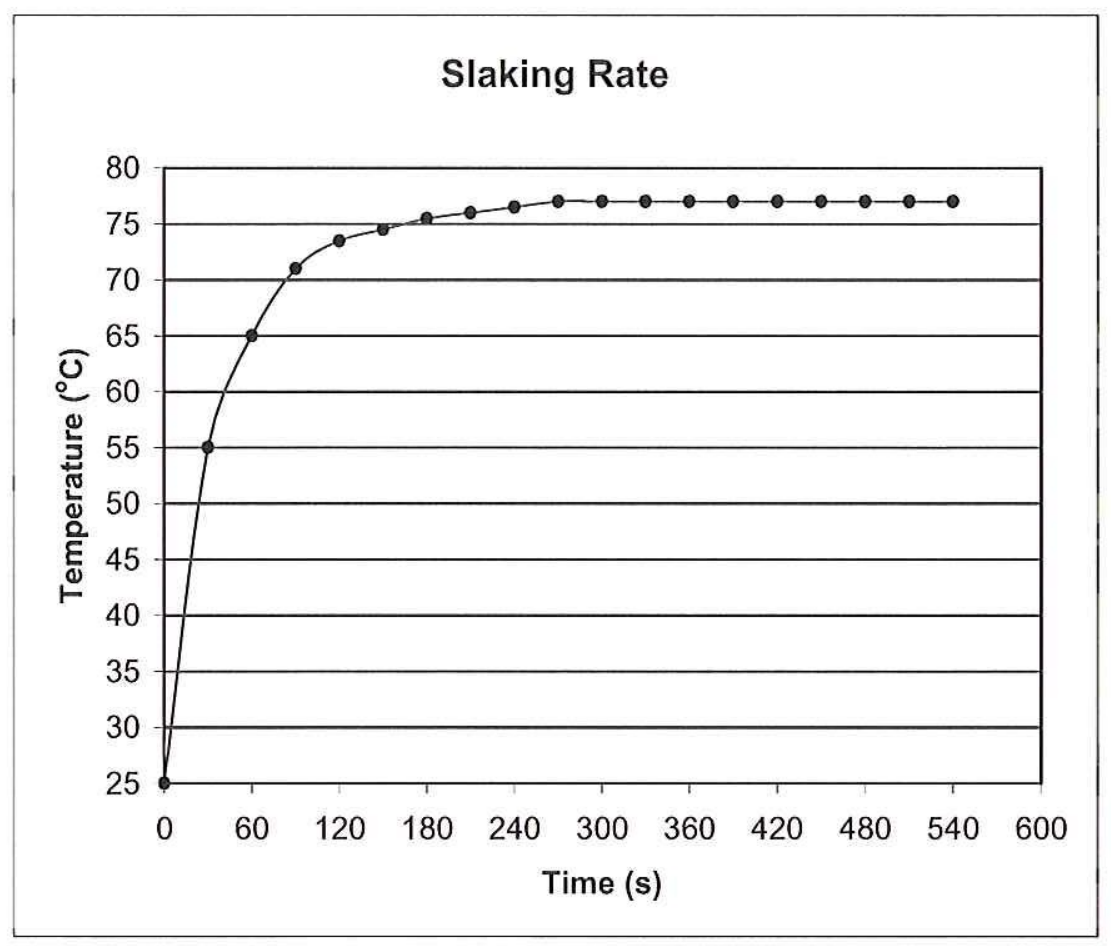

Determined Results

Total active slaking time (minute)

Final reaction temperature $\left({ }^{\circ} \mathrm{C}\right)$

77.0

Temperature rise in 30 seconds $\left({ }^{\circ} \mathrm{C}\right)$

30.0

Temperature rise in 3 minutes $\left({ }^{\circ} \mathrm{C}\right)$

50.5

Total temperature rise $\left({ }^{\circ} \mathrm{C}\right)$

52.0

Residue (wt. \%)

3.79

Notes:

1. This analysis represents specifically the sample(s) submitted as received.

2. The results were determined in accordance with ASTM C 110-05, Sec.11.

3. This report may not be reproduced except in its entirety. 


\begin{tabular}{|c|c|}
\hline $\begin{array}{l}\text { Client: } \\
\text { Project: }\end{array}$ & $\begin{array}{l}\text { CONSOL Energy, Inc. } \\
\text { Chemical Analysis }\end{array}$ \\
\hline Contact: & Daniel Connell \\
\hline Submitter: & Daniel Connell \\
\hline Date Received: & April 23, 2007 \\
\hline
\end{tabular}

CTL Project No:

CTL Project Mgr.:

Analyst:

Approved:

Date Analyzed:

Date Reported:
403423

Ella Shkolnik

Cecylia Wedzicha

May 1, 2007

May 2, 2007

\section{REPORT of SLAKING RATE ANALYSIS}

\section{(ASTM C 110)}

Sample Identification

\begin{tabular}{ll}
\hline CTL ID: & 1839006 \\
Client ID: & PEB033007 \\
Description: & Pebble lime
\end{tabular}

\begin{tabular}{|c|c|c|}
\hline \multicolumn{2}{|c|}{ Time } & Temperature \\
\hline (minute) & (second) & \\
\hline 0.0 & 0 & 25.0 \\
\hline 0.5 & 30 & 58.0 \\
\hline 1.0 & 60 & 68.0 \\
\hline 1.5 & 90 & 73.0 \\
\hline 2.0 & 120 & 74.5 \\
\hline 2.5 & 150 & 76.0 \\
\hline 3.0 & 180 & 77.0 \\
\hline 3.5 & 210 & 77.5 \\
\hline 4.0 & 240 & 78.0 \\
\hline 4.5 & 270 & 79.0 \\
\hline 5.0 & 300 & 79.0 \\
\hline 5.5 & 330 & 79.0 \\
\hline 6.0 & 360 & 79.0 \\
\hline 6.5 & 390 & 79.0 \\
\hline 7.0 & 420 & 79.0 \\
\hline 7.5 & 450 & 79.0 \\
\hline 8.0 & 480 & 79.0 \\
\hline 8.5 & 510 & 79.0 \\
\hline 9.0 & 540 & 79.0 \\
\hline 9.5 & 570 & \\
\hline 10.0 & 600 & \\
\hline
\end{tabular}

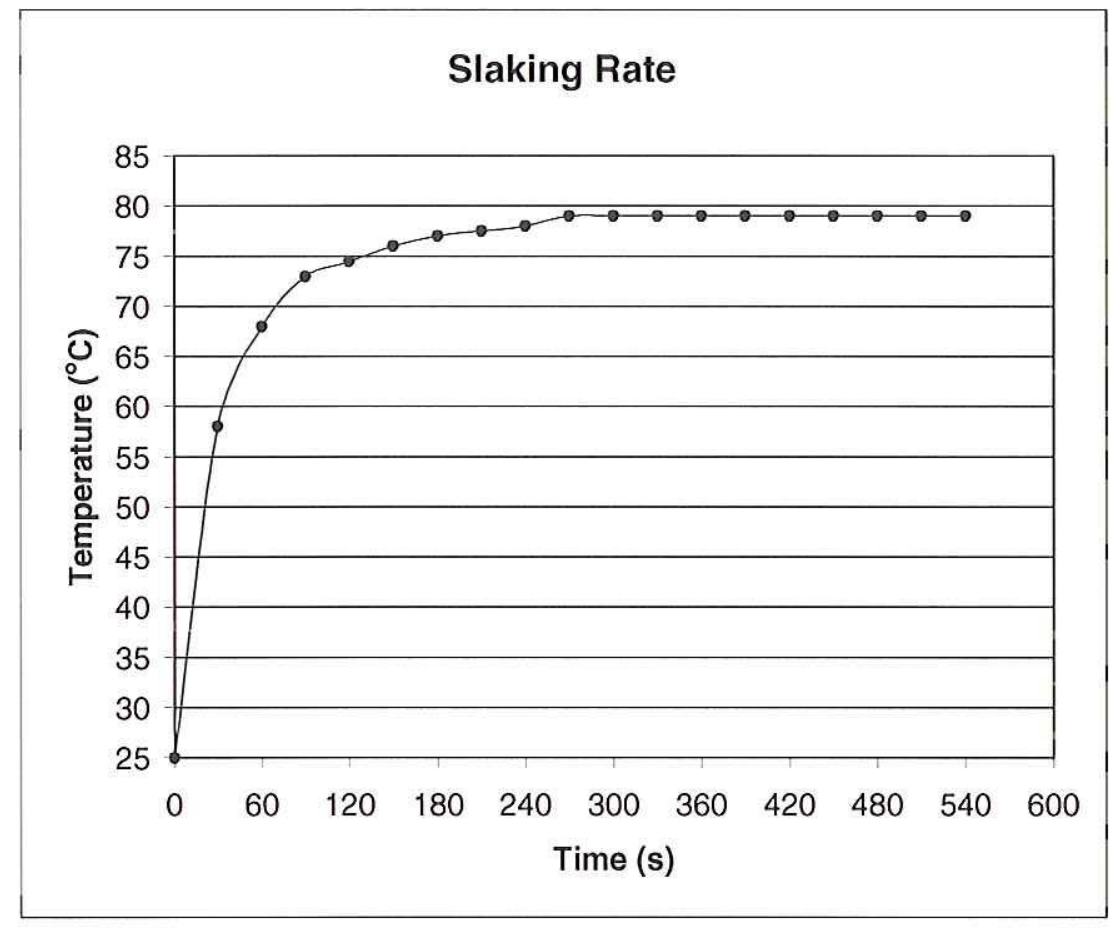

Determined Results

Total active slaking time (minute)

Final reaction temperature $\left({ }^{\circ} \mathrm{C}\right)$

Temperature rise in 30 seconds $\left({ }^{\circ} \mathrm{C}\right)$

33.0

Temperature rise in 3 minutes $\left({ }^{\circ} \mathrm{C}\right)$

52.0

Total temperature rise $\left({ }^{\circ} \mathrm{C}\right)$

54.0

Residue (wt. \%)

2.06

Notes:

1. This analysis represents specifically the samples submitted as received.

2. The results were determined in accordance with ASTM C110-05, Sec.11.

3. This report may not be reproduced except in its entirety. 
Client: $\quad$ CONSOL Energy, Inc.

Project:

ASTM C 110 Density Analysis

Contact:

Submitter:

Date Received:
Daniel Connell

Daniel Connell

April 23, 2007
CTL Project No.:

CTL Proj. Mgr.:

Analyst:

Approved:

Date Analyzed:

Date Reported:
403423

Ella Shkolnik

Charlotte Hernandez

2. Shanceria

April 30, 2007

May 2, 2007

\section{REPORT of DENSITY ANALYSIS}

Client's Sample ID:

CTL Sample ID:

Material:

\section{Apparent Density $^{\text {note } 2}$}

Loose $\left(\mathrm{g} / \mathrm{cm}^{3}\right)$

Loose $\left(\mathrm{lb} / \mathrm{ft}^{3}\right)$

Packed $\left(\mathrm{g} / \mathrm{cm}^{3}\right)$

Packed $\left(\mathrm{lb} / \mathrm{ft}^{3}\right)$

\author{
HYD032807 \\ 1839001 \\ Hydrated lime
}

\author{
HYD032907 \\ 1839002 \\ Hydrated lime
}

HYD033007

1839003

Hydrated lime
0.36

22.32

0.63

39.58
0.37

22.83

0.60

37.17
0.35

22.14

0.59

36.78

Notes:

1. This analysis represents specifically the samples submitted.

2. Loose and packed apparent densities were determined following ASTM C 110, Sections 19 and 20.

3. This report may not be reproduced except in its entirety. 


\begin{tabular}{lll}
$\begin{array}{ll}\text { Client: } \\
\text { Project: }\end{array}$ & $\begin{array}{l}\text { CONSOL Energy, Inc. } \\
\text { Fineness Analysis }\end{array}$ & CTL Project No.: \\
& & CTL Proj. Mgr.: \\
Contact: & Daniel Connell & Approved: \\
Submitter: & Daniel Connell & Date Analyzed: \\
Date Received: & April 23, 2007 & Date Reported: \\
\hline & & \\
& REPORT of PARTICLE SIZE DISTRIBUTION ANALYSIS \\
& & by LASER DIFFRACTION
\end{tabular}

Client's Sample ID:

Material Type:

CTL Sample ID:

Size at $50 \%(\mu \mathrm{m})$
HYD032807

Hydrated lime

1839001

5.54
HYD032907

Hydrated lime

1839002

5.96
403423

Ella Shkolnik Ella Shkolnik

RefH-

May 3, 2007

May 4, 2007

$\underline{\text { Cumulative Volume under Stated Size }}^{\text {note } 2}$

$<45 \mu \mathrm{m}$

88.70

$<30 \mu \mathrm{m}$

83.67

$<10 \mu \mathrm{m}$

67.02

$<7 \mu \mathrm{m}$

$<3 \mu \mathrm{m}$

$<1 \mu \mathrm{m}$
57.60

28.29

6.14
86.91

86.17

81.53

80.78

63.99

63.71

54.84

54.74

27.16

27.36

5.94
HYD033007

Hydrated lime

1839003

5.97 


\section{PARTICLE SIZE DISTRIIBUTIION (PSD) ANALYSIS REPORT}

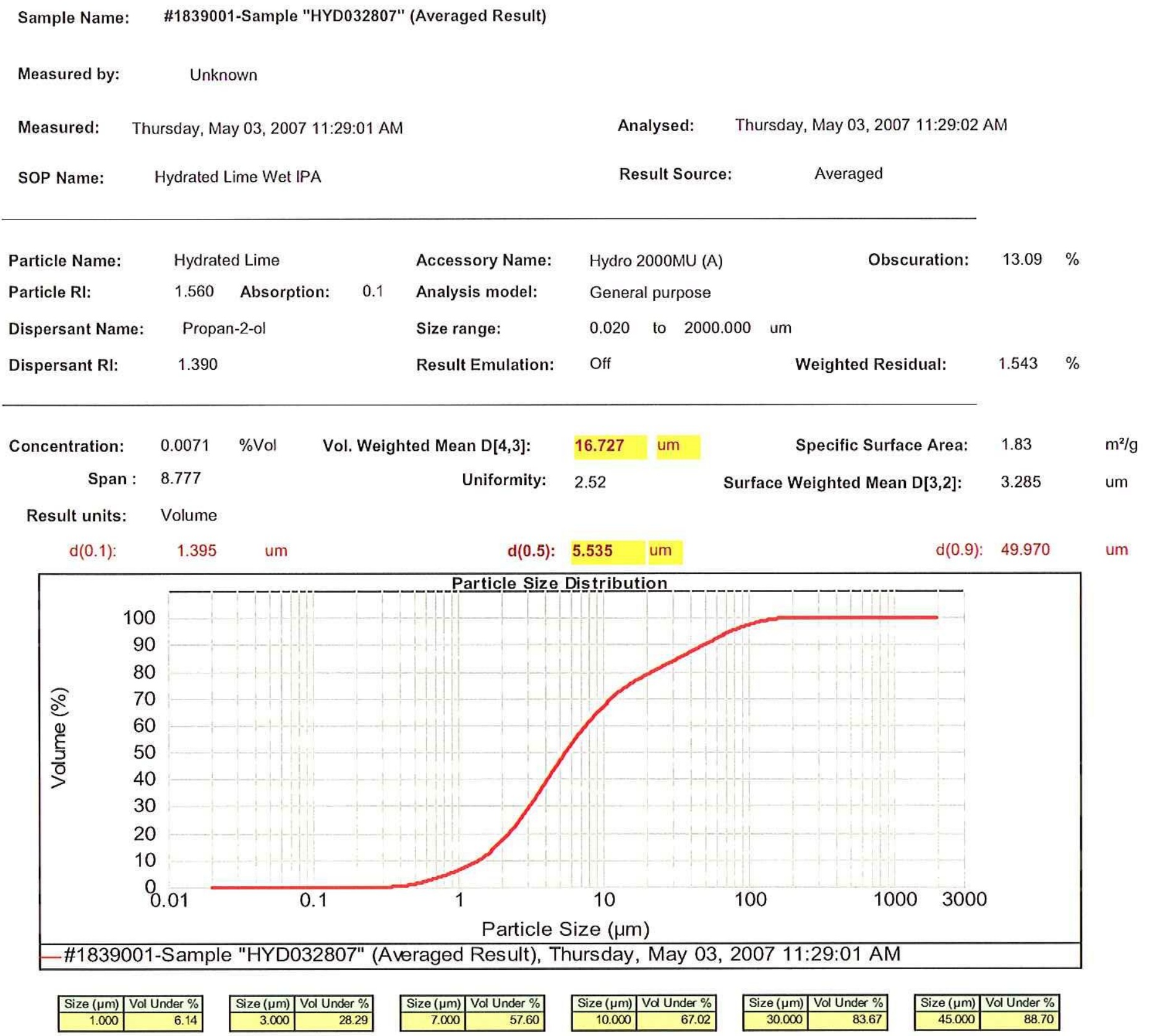

Operator notes: Average of four measurements

File name: Samples 1839001-00 


\section{PARTICLE SIZE DISTRIBUTION (PSD) ANALYSIS REPORT}

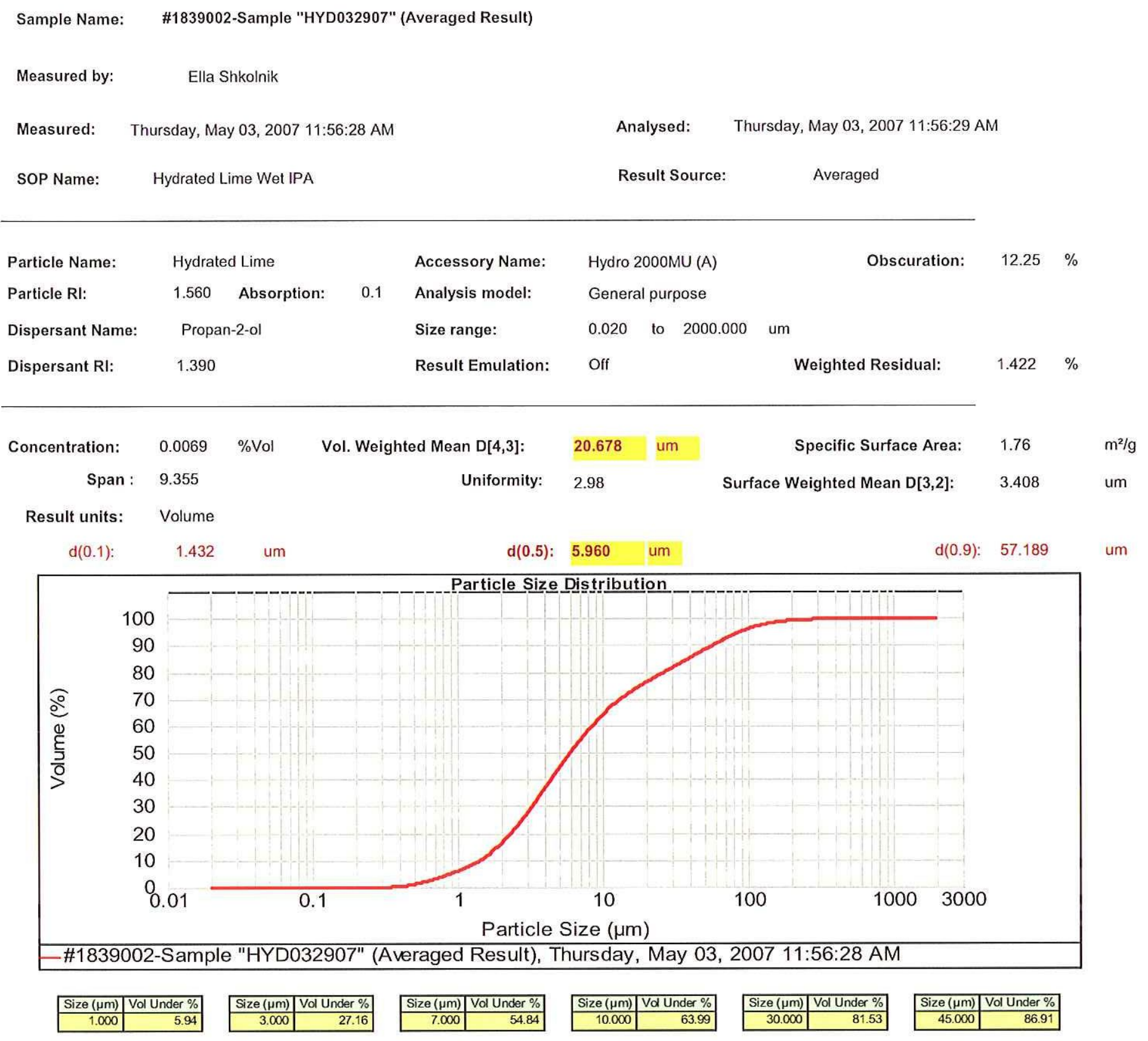

Operator notes: $\quad$ Average of four measurements 


\section{PARTICLE SIZE DISTRIIBUTION (PSD) ANALYSIS REPORT}

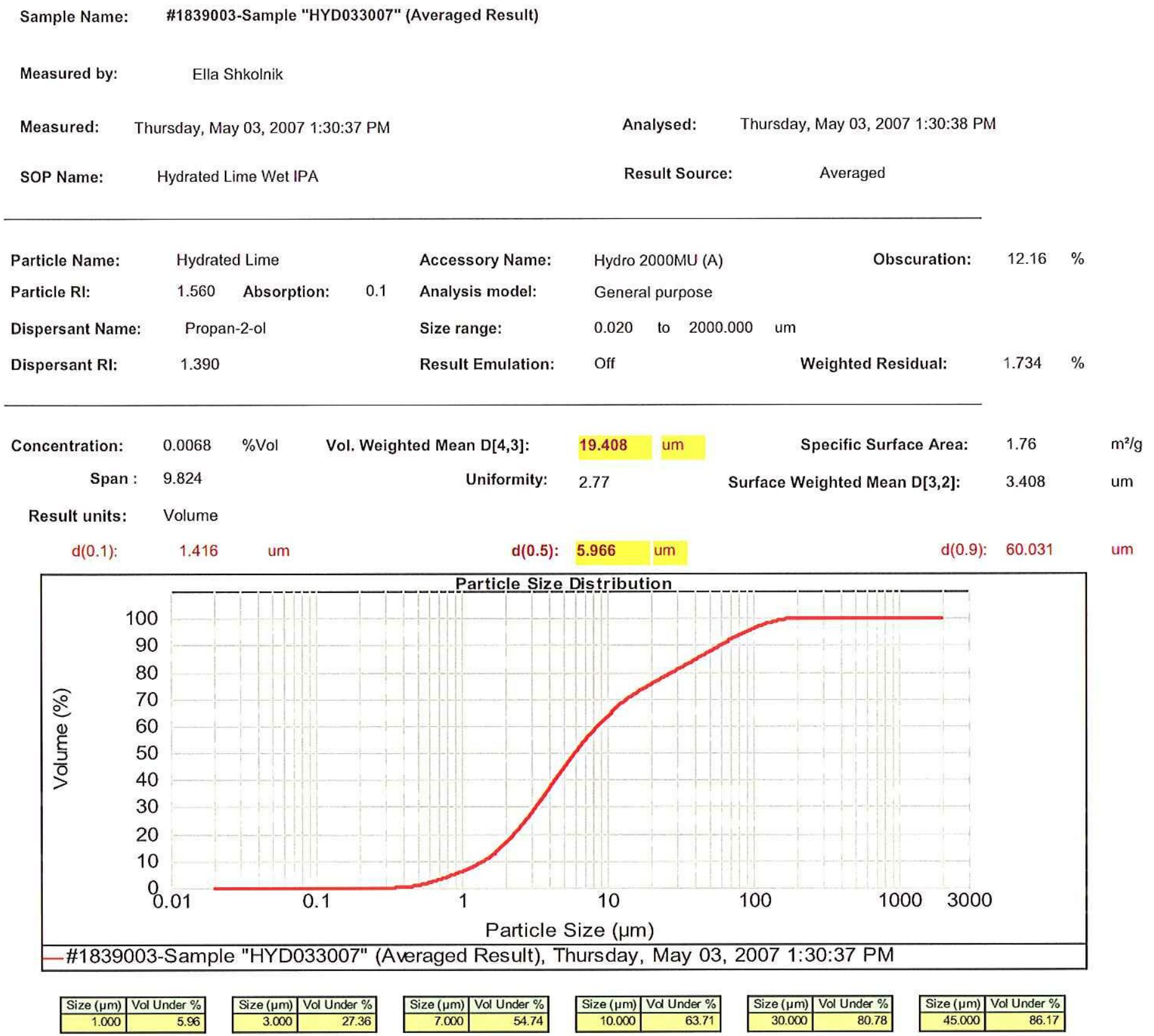




\title{
PARTICLE SIZE DISTRIIBUTIION (PSD) ANALYSIS REPORT
}

\author{
Sample Name: \#1839001-Sample "HYD032807" (Averaged Result) \\ Measured by: Unknown \\ Measured: Thursday, May 03, 2007 11:29:01 AM Analysed: Thursday, May 03, 2007 11:29:02 AM
}

$\begin{array}{lll}\text { SOP Name: } & \text { Hydrated Lime Wet IPA } & \text { Result Source: Averaged }\end{array}$

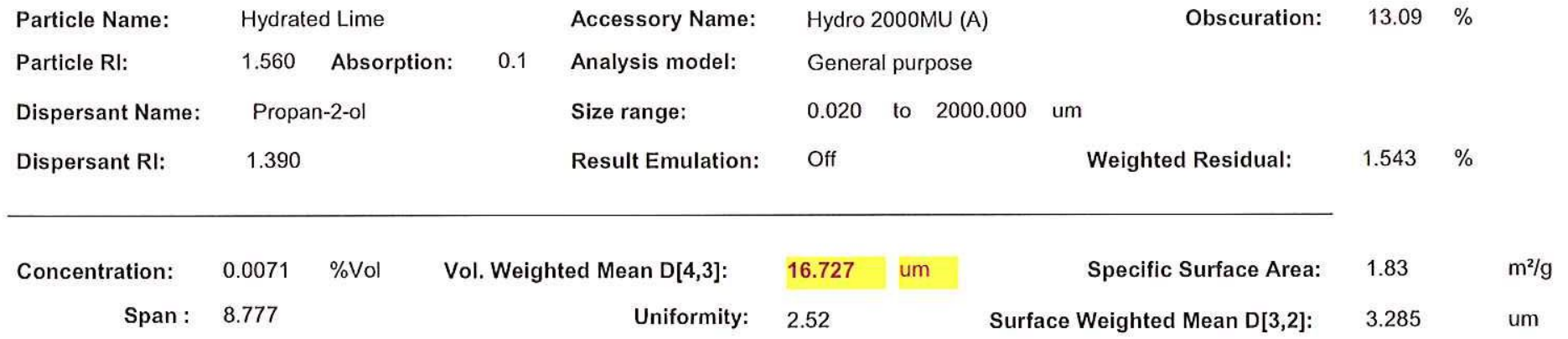

Result units: Volume

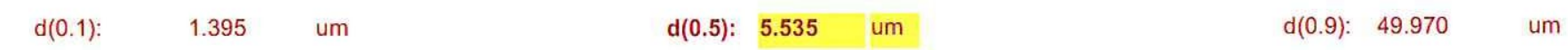

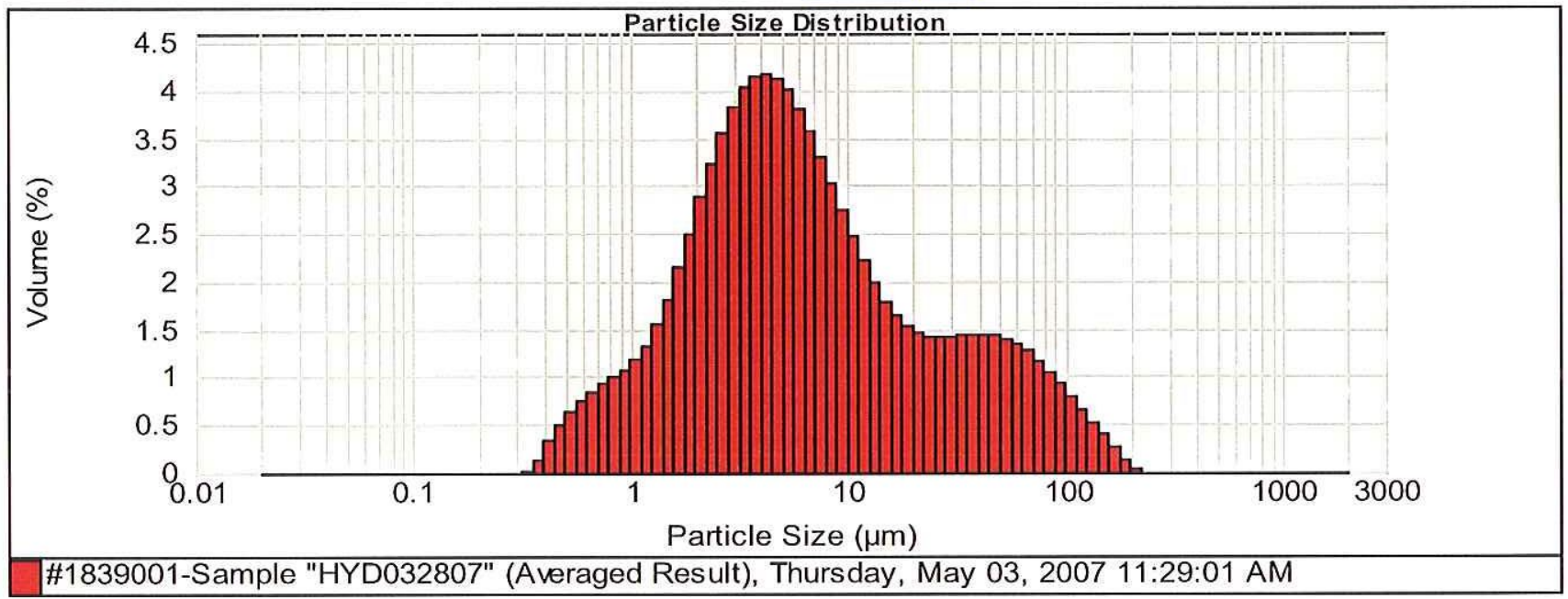

\begin{tabular}{|r|r|}
\hline Size $(\mu \mathrm{m})$ & Vol Under $\%$ \\
\hline 0.020 & 0.00 \\
0.022 & 0.00 \\
0.025 & 0.00 \\
0.028 & 0.00 \\
0.032 & 0.00 \\
0.036 & 0.00 \\
0.040 & 0.00 \\
0.045 & 0.00 \\
0.050 & 0.00 \\
0.056 & 0.00 \\
0.063 & 0.00 \\
0.071 & 0.00 \\
0.080 & 0.00 \\
0.089 & 0.00 \\
0.100 & 0.00 \\
0.112 & 0.00 \\
0.126 & 0.00 \\
\hline
\end{tabular}

\begin{tabular}{|r|r|}
\hline Size $(\mu \mathrm{m})$ & Vol Under \% \\
\hline 0.142 & 0.00 \\
0.159 & 0.00 \\
0.178 & 0.00 \\
0.200 & 0.00 \\
0.224 & 0.00 \\
0.252 & 0.00 \\
0.283 & 0.00 \\
0.317 & 0.00 \\
0.356 & 0.00 \\
0.399 & 0.13 \\
0.448 & 0.46 \\
0.502 & 0.94 \\
0.564 & 1.58 \\
0.632 & 2.33 \\
0.710 & 3.17 \\
0.796 & 4.10 \\
0.893 & 5.09 \\
\hline
\end{tabular}

\begin{tabular}{|r|r|}
\hline Size $(\mu \mathrm{m})$ & Vol Under $\%$ \\
\hline 1.002 & 6.16 \\
1.125 & 7.34 \\
1.262 & 8.68 \\
1.416 & 10.22 \\
1.589 & 12.03 \\
1.783 & 14.17 \\
2.000 & 16.66 \\
2.244 & 19.54 \\
2.518 & 22.77 \\
2.825 & 26.33 \\
3.170 & 30.15 \\
3.557 & 34.18 \\
3.991 & 38.32 \\
4.477 & 42.50 \\
5.024 & 46.62 \\
5.637 & 50.62 \\
6.325 & 54.44 \\
\hline
\end{tabular}

\begin{tabular}{|r|r|}
\hline Size $(\mu \mathrm{m})$ & Vd Under \% \\
\hline 7.096 & 58.01 \\
7.962 & 61.31 \\
8.934 & 64.33 \\
10.024 & 67.07 \\
11.247 & 69.53 \\
12.619 & 71.74 \\
14.159 & 73.72 \\
15.887 & 75.50 \\
17.825 & 77.14 \\
20.000 & 78.66 \\
22.440 & 80.11 \\
25.179 & 81.53 \\
28.251 & 82.93 \\
31.698 & 84.34 \\
35.566 & 85.77 \\
39.905 & 87.20 \\
44.774 & 88.64 \\
\hline
\end{tabular}

\begin{tabular}{|r|r|}
\hline Size $(\mu \mathrm{m})$ & Vol Under \% \\
\hline 50.238 & 90.07 \\
56.368 & 91.46 \\
63.246 & 92.80 \\
70.963 & 94.07 \\
79.621 & 95.24 \\
89.337 & 96.29 \\
100.237 & 97.21 \\
112.468 & 98.00 \\
126.191 & 98.65 \\
141.589 & 99.17 \\
158.866 & 99.57 \\
178.250 & 99.82 \\
200.000 & 99.96 \\
224.404 & 100.00 \\
251.785 & 100.00 \\
282.508 & 100.00 \\
316.979 & 100.00 \\
\hline
\end{tabular}

\begin{tabular}{|r|r|}
\hline Size $(\mu \mathrm{m})$ & Vol Under \% \\
\hline 355.656 & 100.00 \\
399.052 & 100.00 \\
447.744 & 100.00 \\
502.377 & 100.00 \\
563.677 & 100.00 \\
632.456 & 100.00 \\
709.627 & 100.00 \\
796.214 & 100.00 \\
893.367 & 100.00 \\
1002.374 & 100.00 \\
1124.683 & 100.00 \\
1261.915 & 100.00 \\
1415.892 & 100.00 \\
1588.656 & 100.00 \\
1782.502 & 100.00 \\
2000.000 & 100.00 \\
& \\
\hline
\end{tabular}

Operator notes:

Average of four measurements

Malvern Instruments Ltd
File name: Samples 1839001-00 Record Number: 13 03 May 2007 1:43:53 PM 


\title{
PARTICLE SIZE DISTIRIBUTIION (PSD) ANALYSIS REPORT
}

\author{
Sample Name: \#1839002-Sample "HYD032907" (Averaged Result) \\ Measured by: $\quad$ Ella Shkolnik
}

Measured: $\quad$ Thursday, May 03, 2007 11:56:28 AM

Analysed: $\quad$ Thursday, May 03, 2007 11:56:29 AM

Result Source: Averaged

$\begin{array}{llllll}\text { Particle Name: } & \text { Hydrated Lime } & & \text { Accessory Name: } & \text { Hydro 2000MU (A) } \\ \text { Particle RI: } & 1.560 \quad \text { Absorption: } & 0.1 & \text { Analysis model: } & \text { General purpose } & \text { Obscuration: } \\ \text { Dispersant Name: } & \text { Propan-2-ol } & \text { Size range: } & 0.020 & \text { to } & 2000.000 \quad \text { um } \\ \text { Dispersant Rl: } & 1.390 & \text { Result Emulation: } & \text { Off } & \text { Weighted Residual: }\end{array}$

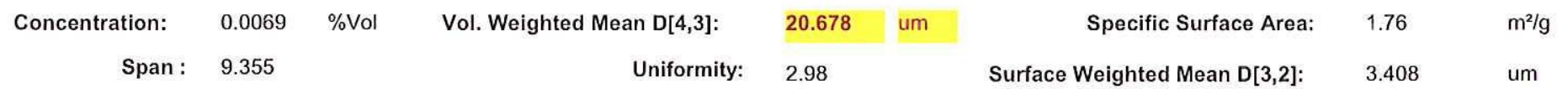

Result units: Volume
$d(0.1)$ :
1.432 um
$d(0.5): \quad 5.960 \quad$ um
$\mathrm{d}(0.9): \quad 57.189$

um

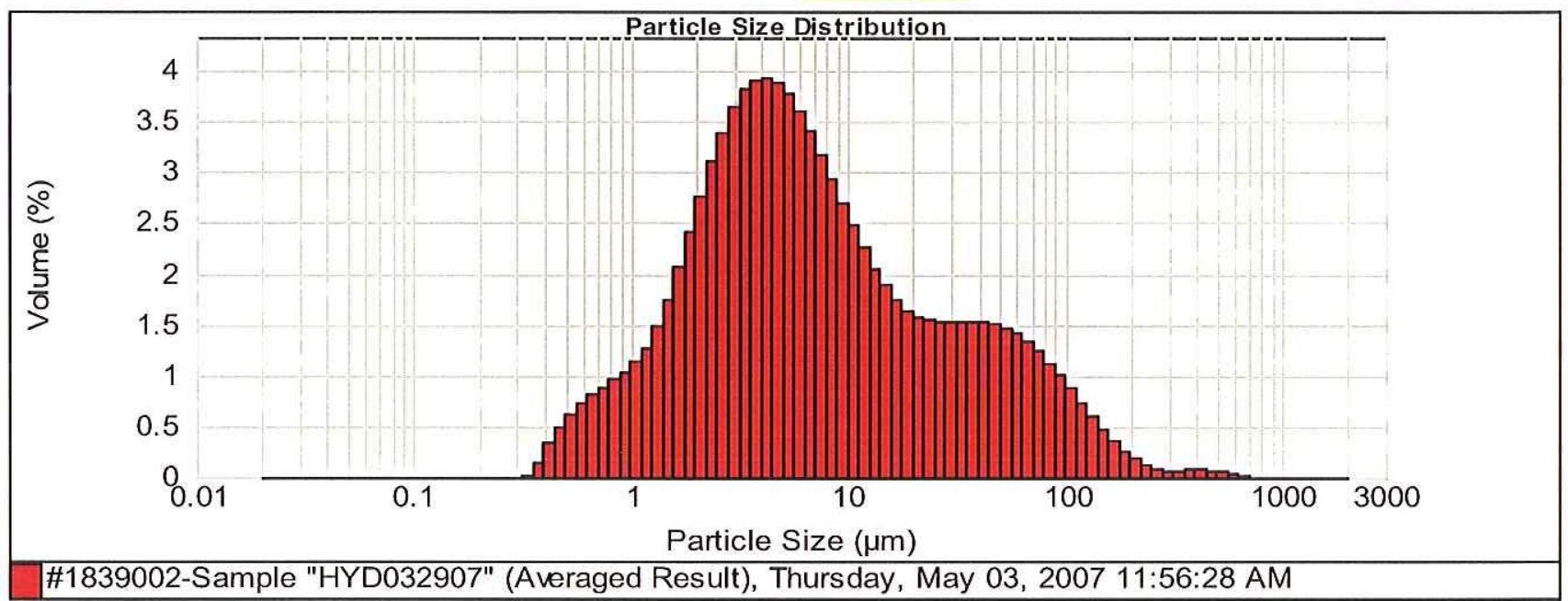

\#1839002-Sample "HYD032907" (Averaged Result), Thurs day, May 03, 2007 11:56:28 AM

\begin{tabular}{|r|r|}
\hline Size $(\mu \mathrm{m})$ & Vol Under $\%$ \\
\hline 0.020 & 0.00 \\
0.022 & 0.00 \\
0.025 & 0.00 \\
0.028 & 0.00 \\
0.032 & 0.00 \\
0.036 & 0.00 \\
0.040 & 0.00 \\
0.045 & 0.00 \\
0.050 & 0.00 \\
0.056 & 0.00 \\
0.063 & 0.00 \\
0.071 & 0.00 \\
0.080 & 0.00 \\
0.089 & 0.00 \\
0.100 & 0.00 \\
0.112 & 0.00 \\
0.126 & 0.00 \\
\hline
\end{tabular}

\begin{tabular}{|r|r|}
\hline Size $(\mu \mathrm{m})$ & Vol Under \% \\
\hline 0.142 & 0.00 \\
0.159 & 0.00 \\
0.178 & 0.00 \\
0.200 & 0.00 \\
0.224 & 0.00 \\
0.252 & 0.00 \\
0.283 & 0.00 \\
0.317 & 0.00 \\
0.356 & 0.00 \\
0.399 & 0.13 \\
0.448 & 0.46 \\
0.502 & 0.93 \\
0.564 & 1.55 \\
0.632 & 2.28 \\
0.710 & 3.09 \\
0.796 & 3.98 \\
0.893 & 4.93 \\
\hline
\end{tabular}

\begin{tabular}{|r|r|}
\hline Size $(\mu \mathrm{m})$ & Vol Under \% \\
\hline 1.002 & 5.96 \\
1.125 & 7.09 \\
1.262 & 8.37 \\
1.416 & 9.85 \\
1.589 & 11.59 \\
1.783 & 13.64 \\
2.000 & 16.04 \\
2.244 & 18.80 \\
2.518 & 21.90 \\
2.825 & 25.29 \\
3.170 & 28.93 \\
3.557 & 32.74 \\
3.991 & 36.65 \\
4.477 & 40.58 \\
5.024 & 44.46 \\
5.637 & 48.23 \\
6.325 & 51.84 \\
\hline
\end{tabular}

\begin{tabular}{|r|r|}
\hline Size $(\mu \mathrm{m})$ & Vo Under $\%$ \\
\hline 7.096 & 55.24 \\
7.962 & 58.41 \\
8.934 & 61.35 \\
10.024 & 64.05 \\
11.247 & 66.51 \\
12.619 & 68.76 \\
14.159 & 70.82 \\
15.887 & 72.70 \\
17.825 & 74.45 \\
20.000 & 76.09 \\
22.440 & 77.67 \\
25.179 & 79.21 \\
28.251 & 80.73 \\
31.698 & 82.26 \\
35.566 & 83.78 \\
39.905 & 85.32 \\
44.774 & 86.84 \\
\hline
\end{tabular}

\begin{tabular}{|r|r|}
\hline Size $(\mu \mathrm{m})$ & Vol Under $\%$ \\
\hline 50.238 & 88.35 \\
56.368 & 89.82 \\
63.246 & 91.23 \\
70.963 & 92.56 \\
79.621 & 93.80 \\
89.337 & 94.93 \\
100.237 & 95.92 \\
112.468 & 96.79 \\
126.191 & 97.53 \\
141.589 & 98.12 \\
158.866 & 98.59 \\
178.250 & 98.95 \\
200.000 & 99.20 \\
224.404 & 99.37 \\
251.785 & 99.49 \\
282.508 & 99.57 \\
316.979 & 99.63 \\
\hline
\end{tabular}

\begin{tabular}{|r|r|}
\hline Size $(\mu \mathrm{m})$ & Vol Under \% \\
\hline 355.656 & 99.69 \\
399.052 & 99.76 \\
447.744 & 99.83 \\
502.377 & 99.89 \\
563.677 & 99.95 \\
632.456 & 99.98 \\
709.627 & 100.00 \\
796.214 & 100.00 \\
893.367 & 100.00 \\
1002.374 & 100.00 \\
1124.683 & 100.00 \\
1261.915 & 100.00 \\
1415.892 & 100.00 \\
1588.656 & 100.00 \\
1782.502 & 100.00 \\
2000.000 & 100.00 \\
& \\
\hline
\end{tabular}

Operator notes:

Average of four measurements

Malvern Instruments Ltd
File name: Samples 1839001-00 Record Number: 14 03 May 2007 1:43:53 PM 


\title{
PARTICLE SIIZE DISTRIIBUTIION (PSD) ANALYSIS REPORT
}

\author{
Sample Name: \#1839003-Sample "HYD033007" (Averaged Result) \\ Measured by: $\quad$ Ella Shkolnik \\ Measured: $\quad$ Thursday, May 03, 2007 1:30:37 PM Analysed: $\quad$ Thursday, May 03, 2007 1:30:38 PM
}

SOP Name: $\quad$ Hydrated Lime Wet IPA Result Source: Averaged

$\begin{array}{llllll}\text { Particle Name: } & \text { Hydrated Lime } & & \text { Accessory Name: } & \text { Hydro 2000MU (A) } \\ \text { Particle RI: } & 1.560 \quad \text { Absorption: } & 0.1 & \text { Analysis model: } & \text { General purpose } \\ \text { Dispersant Name: } & \text { Propan-2-ol } & \text { Size range: } & 0.020 \quad \text { to } & 2000.000 \quad \text { um } \\ \text { Dispersant RI: } & 1.390 & \text { Result Emulation: } & \text { Off } & \text { Weighted Residual: }\end{array}$

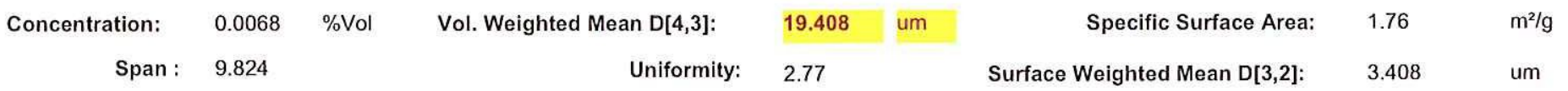

Result units: Volume

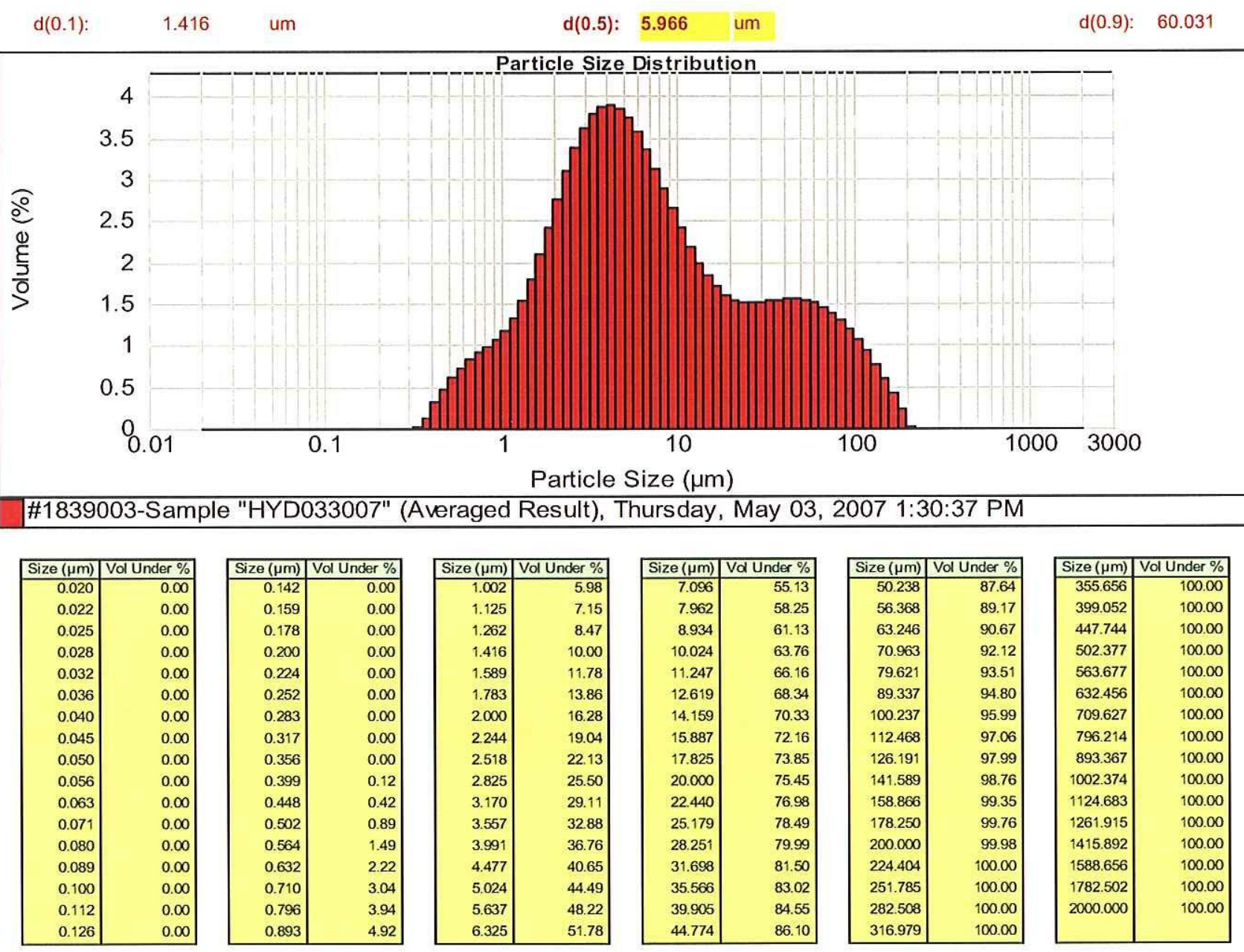

Operator notes: $\quad$ Average of four measurements

File name: Samples 1839001-00 Record Number: 15 03 May 2007 1:43:53 PM 
Client:

Project:

Contact:

Submitter:

Date Received:
CONSOL Energy, Inc.

Fineness Analysis

Daniel Connell

Daniel Connell

April 23, 2007
CTL Project No.:

CTL Proj. Mgr.:

Analyst:

Approved:

Date Analyzed:

Date Reported:
403423

Ella Shkolnik

Ella Shkolnik

Reft-

May 3, 2007

May 4, 2007

\section{REPORT of PARTICLE SIZE DISTRIBUTION ANALYSIS by LASER DIFFRACTION}

Client's Sample ID:

Material Type:

CTL Sample ID:
ASH032807

Fly ash

1839007

6.47
ASH032907

Fly ash

1839008

6.74
ASH033007

Fly ash

1839009

6.49

Cumulative Volume under Stated Size ${ }^{\text {note } 2}$

$<45 \mu \mathrm{m}$

82.59

$<30 \mu \mathrm{m}$

$<10 \mu \mathrm{m}$

$<7 \mu \mathrm{m}$

$<3 \mu \mathrm{m}$

$<1 \mu \mathrm{m}$
76.79

61.96

52.59

21.25

3.97
81.76

83.02

75.84

77.02

60.64

61.70

51.21

52.44

20.77

21.59

3.96

Notes:

1. This analysis represents specifically the samples submitted.

2. The provided results are volume based and expressed in terms of equivalent spheres.

3. This report may not be reproduced except in its entirety. 


\title{
PARTICLE SIZE DISTRIIBUTIION (PSD) ANALYSIS REPORT
}

\author{
Sample Name: \#1839007-Sample "ASH032807" (Averaged Result) \\ Measured by: $\quad$ Ella Shkolnik
}

Measured: Thursday, May 03, 2007 2:04:17 PM

Analysed: $\quad$ Thursday, May 03, 2007 2:04:18 PM

SOP Name:

Fly Ash Wet IPA

Result Source: $\quad$ Averaged

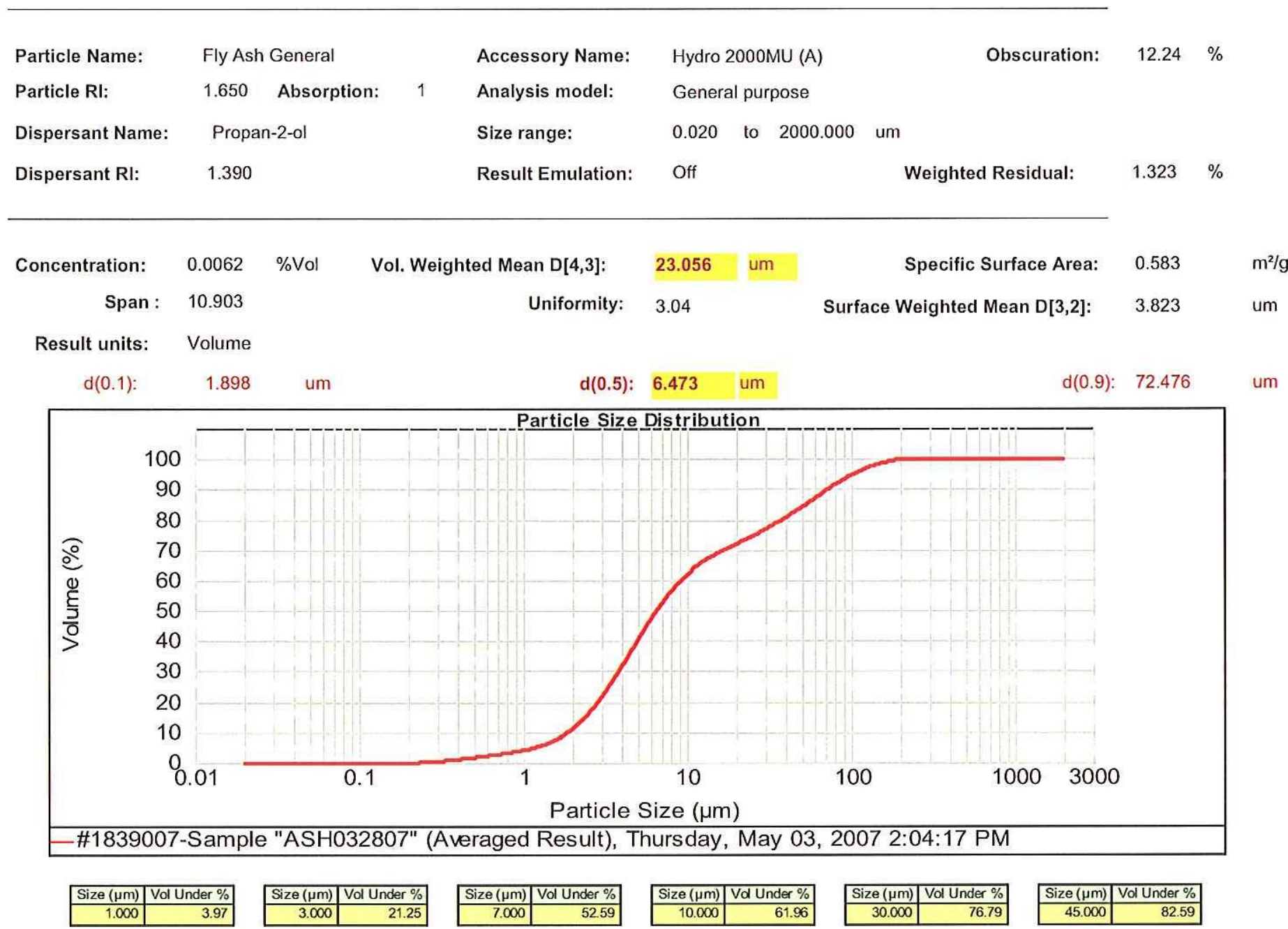

Operator notes: Average of four measurements

File name: Samples $1839007-00$ Record Number: 13 03 May 2007 3:10:21 PM 


\section{PARTICLE SIZE DISTRIBUTION (PSD) ANALYSIS REPORT}

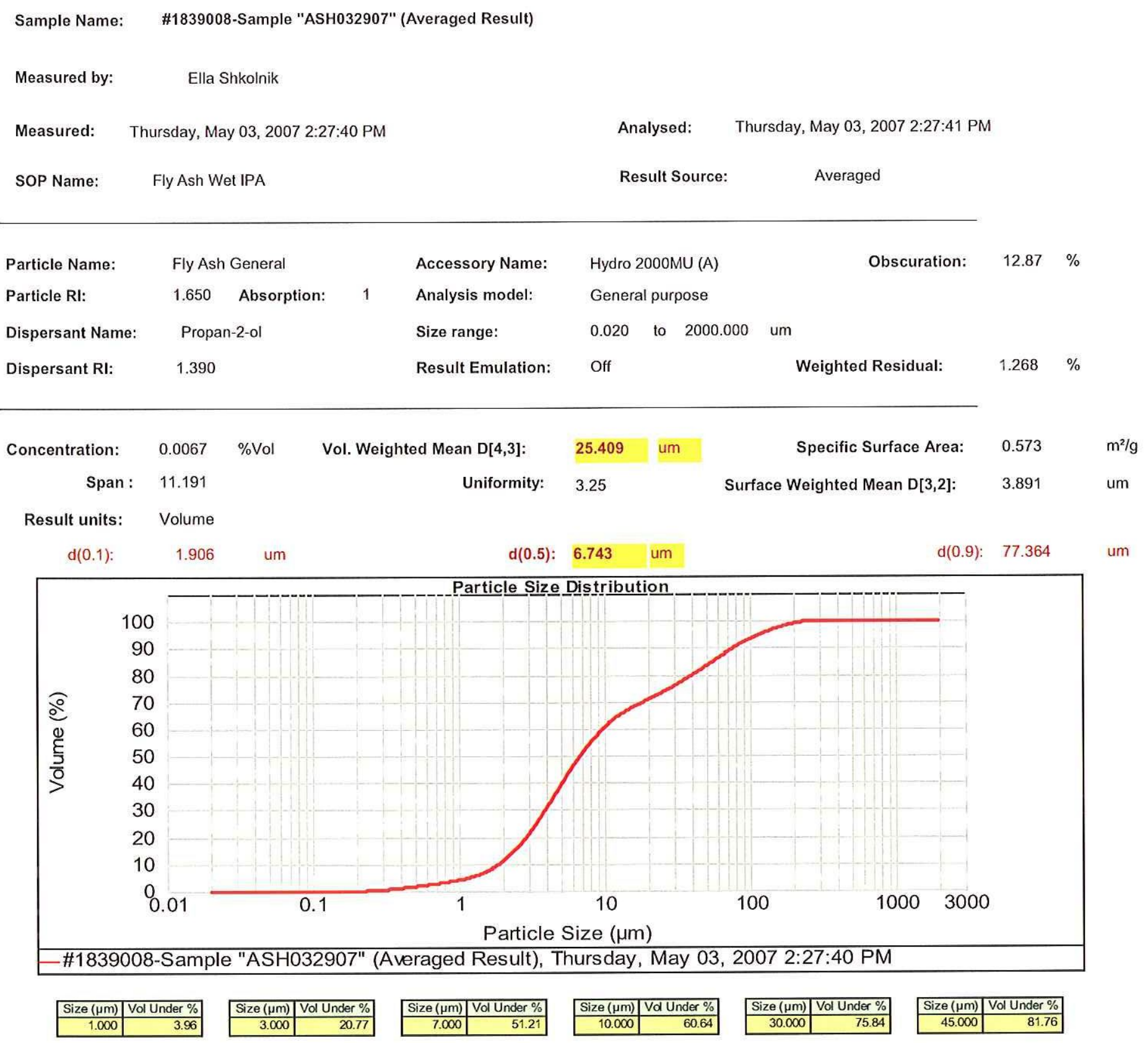

Operator notes: $\quad$ Average of four measurements 


\section{PARTICLE SIZZE DISTRIIBUTION (PSD) ANAL YSIS REPORT}

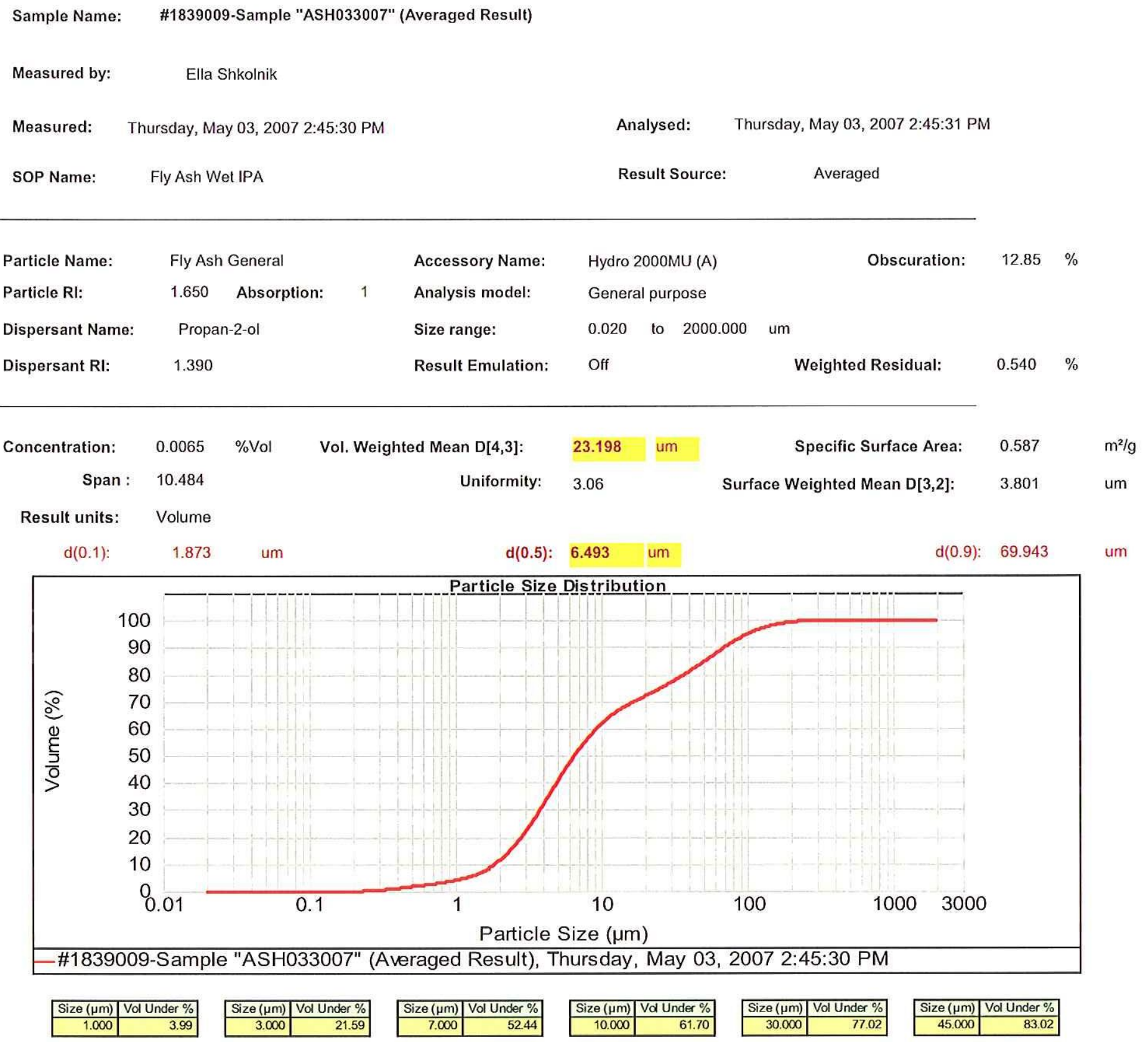

Operator notes: Average of four measurements 


\title{
PARTICLE SIZE DISTRIIBUTIION (PSD) ANALYYSIS REPORT
}

\author{
Sample Name: \#1839007-Sample "ASH032807" (Averaged Result) \\ Measured by: $\quad$ Ella Shkolnik \\ Measured: $\quad$ Thursday, May 03, 2007 2:04:17 PM \\ Analysed: $\quad$ Thursday, May 03, 2007 2:04:18 PM
}

Result Source: Averaged

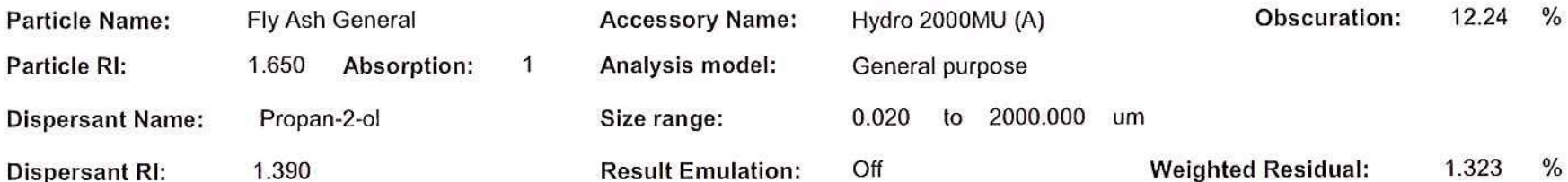

\begin{tabular}{|c|c|c|c|c|c|c|c|c|}
\hline Concentration: & 0.0062 & $\%$ Vol & Vol. Weighted Mean $D[4,3]$ : & 23.056 & um & Specific Surface Area: & 0.583 & $\mathrm{~m}^{2} / \mathrm{g}$ \\
\hline Span : & 10.903 & & Uniformity: & 3.04 & & Surface Weighted Mean $D[3,2]$ : & 3.823 & um \\
\hline
\end{tabular}

Result units: Volume
$d(0.1)$ :
1.898 um
$d(0.5): \quad 6.473 \quad$ um
$d(0.9): \quad 72.476$

um

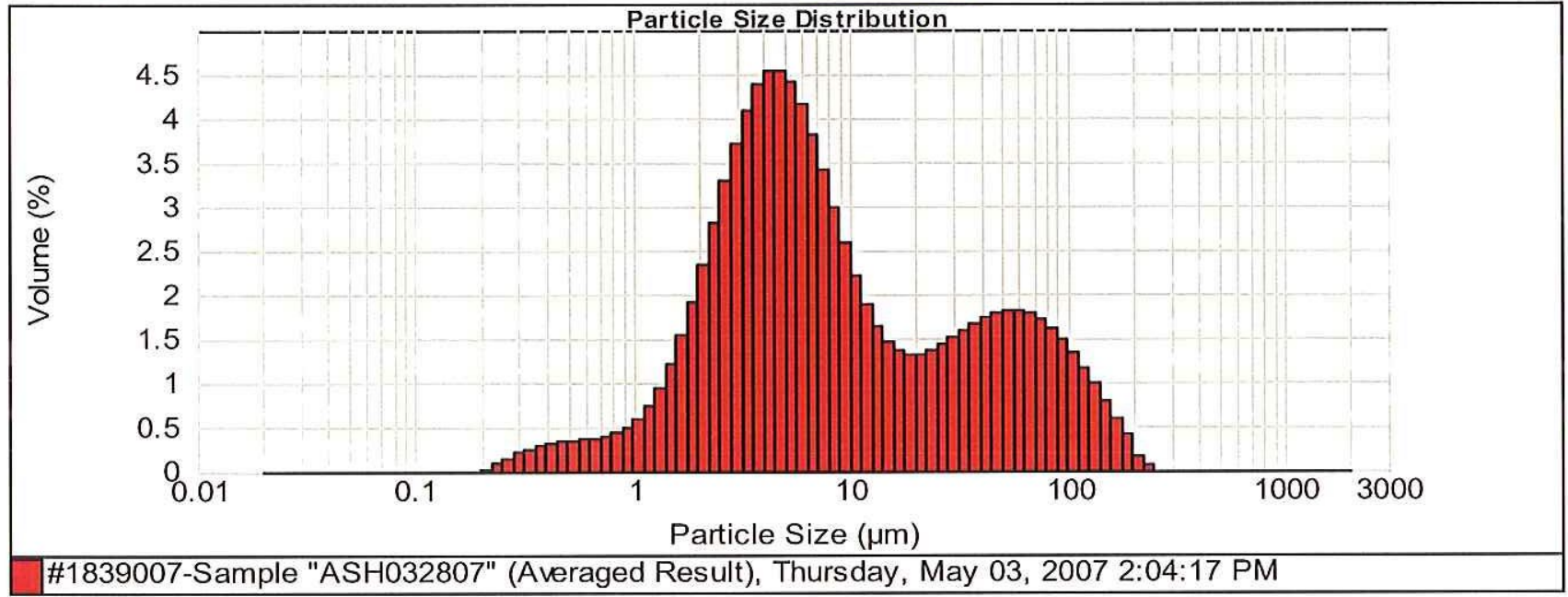

\begin{tabular}{|r|r|}
\hline Size $(\mu \mathrm{m})$ & Vol Under \% \\
\hline 0.020 & 0.00 \\
0.022 & 0.00 \\
0.025 & 0.00 \\
0.028 & 0.00 \\
0.032 & 0.00 \\
0.036 & 0.00 \\
0.040 & 0.00 \\
0.045 & 0.00 \\
0.050 & 0.00 \\
0.056 & 0.00 \\
0.063 & 0.00 \\
0.071 & 0.00 \\
0.080 & 0.00 \\
0.089 & 0.00 \\
0.100 & 0.00 \\
0.112 & 0.00 \\
0.126 & 0.00 \\
\hline
\end{tabular}

\begin{tabular}{|r|r|}
\hline Size $(\mu \mathrm{m})$ & Vol Under \% \\
\hline 0.142 & 0.00 \\
0.159 & 0.00 \\
0.178 & 0.00 \\
0.200 & 0.00 \\
0.224 & 0.00 \\
0.252 & 0.09 \\
0.283 & 0.23 \\
0.317 & 0.44 \\
0.356 & 0.69 \\
0.399 & 0.97 \\
0.448 & 1.28 \\
0.502 & 1.61 \\
0.564 & 1.95 \\
0.632 & 2.30 \\
0.710 & 2.67 \\
0.796 & 3.06 \\
0.893 & 3.49 \\
\hline
\end{tabular}

\begin{tabular}{|r|r|}
\hline Size $(\mu \mathrm{m})$ & Vol Under \% \\
\hline 1.002 & 3.98 \\
1.125 & 4.58 \\
1.262 & 5.33 \\
1.416 & 6.27 \\
1.589 & 7.48 \\
1.783 & 9.01 \\
2.000 & 10.92 \\
2.244 & 13.27 \\
2.518 & 16.08 \\
2.825 & 19.36 \\
3.170 & 23.08 \\
3.557 & 27.17 \\
3.991 & 31.55 \\
4.477 & 36.08 \\
5.024 & 40.62 \\
5.637 & 45.03 \\
6.325 & 49.20 \\
\hline \multicolumn{2}{|c}{}
\end{tabular}

\begin{tabular}{|r|r|}
\hline Size $(\mu \mathrm{m})$ & Vd Under $\%$ \\
\hline 7.096 & 53.02 \\
7.962 & 56.44 \\
8.934 & 59.43 \\
10.024 & 62.01 \\
11.247 & 64.21 \\
12.619 & 66.10 \\
14.159 & 67.75 \\
15.887 & 69.22 \\
17.825 & 70.58 \\
20.000 & 71.89 \\
22.440 & 73.21 \\
25.179 & 74.58 \\
28.251 & 76.01 \\
31.698 & 77.52 \\
35.566 & 79.11 \\
39.905 & 80.78 \\
44.774 & 82.51 \\
\hline
\end{tabular}

\begin{tabular}{|r|r|}
\hline Size $(\mu \mathrm{m})$ & Vol Under $\%$ \\
\hline 50.238 & 84.29 \\
56.368 & 86.10 \\
63.246 & 87.91 \\
70.963 & 89.68 \\
79.621 & 91.39 \\
89.337 & 93.00 \\
100.237 & 94.49 \\
112.468 & 95.83 \\
126.191 & 97.00 \\
141.589 & 97.99 \\
158.866 & 98.78 \\
178.250 & 99.38 \\
200.000 & 99.79 \\
224.404 & 99.95 \\
251.785 & 100.00 \\
282.508 & 100.00 \\
316.979 & 100.00 \\
\hline \multicolumn{2}{|c}{}
\end{tabular}

\begin{tabular}{|r|r|}
\hline Size $(\mu \mathrm{m})$ & Vol Under \% \\
\hline 355.656 & 100.00 \\
399.052 & 100.00 \\
447.744 & 100.00 \\
502.377 & 100.00 \\
563.677 & 100.00 \\
632.456 & 100.00 \\
709.627 & 100.00 \\
796.214 & 100.00 \\
893.367 & 100.00 \\
1002.374 & 100.00 \\
1124.683 & 100.00 \\
1261.915 & 100.00 \\
1415.892 & 100.00 \\
1588.656 & 100.00 \\
1782.502 & 100.00 \\
2000.000 & 100.00 \\
\hline & \\
\hline
\end{tabular}

Operator notes: Average of four measurements

File name: Samples 1839007-00 Record Number: 13 03 May 2007 3:09:43 PM 


\title{
PARTICLE SIZE DISTRIIBUTION (PSD) ANALYSIS REPORT
}

\author{
Sample Name: \#1839008-Sample "ASH032907" (Averaged Result) \\ Measured by: $\quad$ Ella Shkolnik \\ Measured: $\quad$ Thursday, May 03, 2007 2:27:40 PM Analysed: Thursday, May 03, 2007 2:27:41 PM
}

SOP Name: $\quad$ Fly Ash Wet IPA Averaged

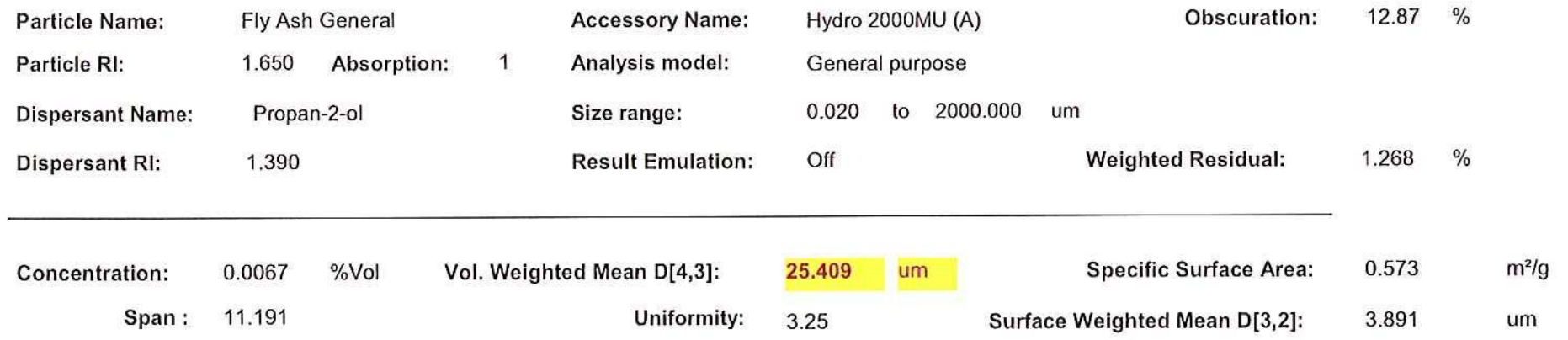

Result units: Volume

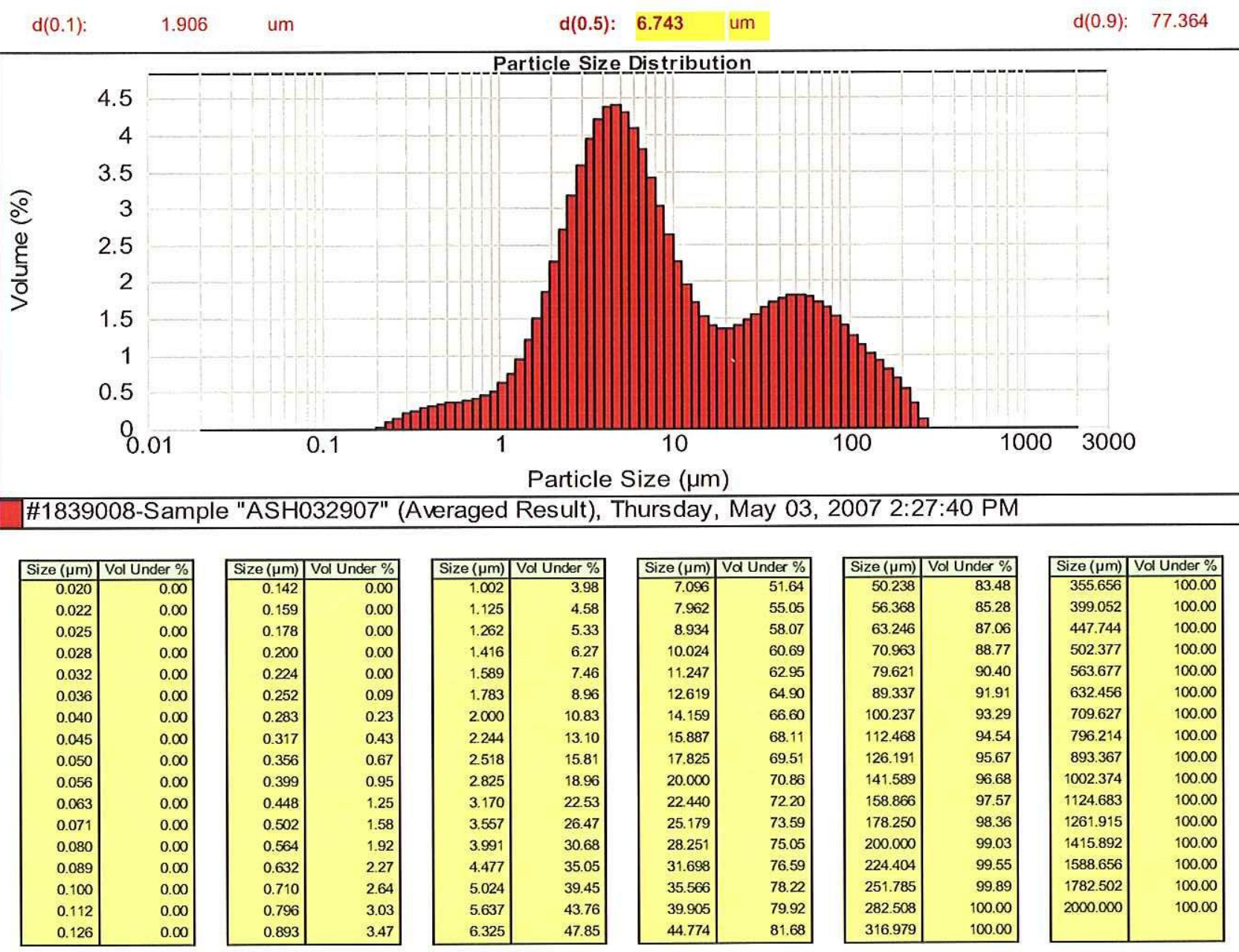

Operator notes: Average of four measurements

File name: Samples $1839007-00$ Record Number: 14 


\title{
PARTICLE SIZE DISTRIIBUTIION (PSD) ANALYSIS REPORT
}

\author{
Sample Name: \#1839009-Sample "ASH033007" (Averaged Result) \\ Measured by: $\quad$ Ella Shkolnik \\ Measured: $\quad$ Thursday, May 03, 2007 2:45:30 PM \\ Analysed: $\quad$ Thursday, May 03, 2007 2:45:31 PM
}

SOP Name:

Fly Ash Wet IPA

Result Source: Averaged

\begin{tabular}{|c|c|c|c|c|c|c|c|c|c|}
\hline Particle Name: & Fly As! & General & & Accessory Name: & Hydro 2000I & $\mathrm{U}(\mathrm{A})$ & & Obscuration: & 12.85 \\
\hline Particle RI: & 1.650 & Absorption: & 1 & Analysis model: & General pur & ose & & & \\
\hline Dispersant Name: & Propa & $-2-\mathrm{ol}$ & & Size range: & 0.020 to & 2000.000 & um & & \\
\hline Dispersant RI: & 1.390 & & & Result Emulation: & Off & & & d Residual: & 0.540 \\
\hline
\end{tabular}

\begin{tabular}{|c|c|c|c|c|c|c|c|c|}
\hline Concentration: & 0.0065 & $\%$ Vol & Vol. Weighted Mean $D[4,3]$ : & 23.198 & um & Specific Surface Area: & 0.587 & $\mathrm{~m}^{2} / \mathrm{g}$ \\
\hline Span : & 10.484 & & Uniformity: & 3.06 & & Surface Weighted Mean D[3,2]: & 3.801 & um \\
\hline
\end{tabular}

Result units: Volume

$d(0.1)$

1.873 um

$d(0.5): \quad 6.493 \quad$ um

$d(0.9): \quad 69.943$

um

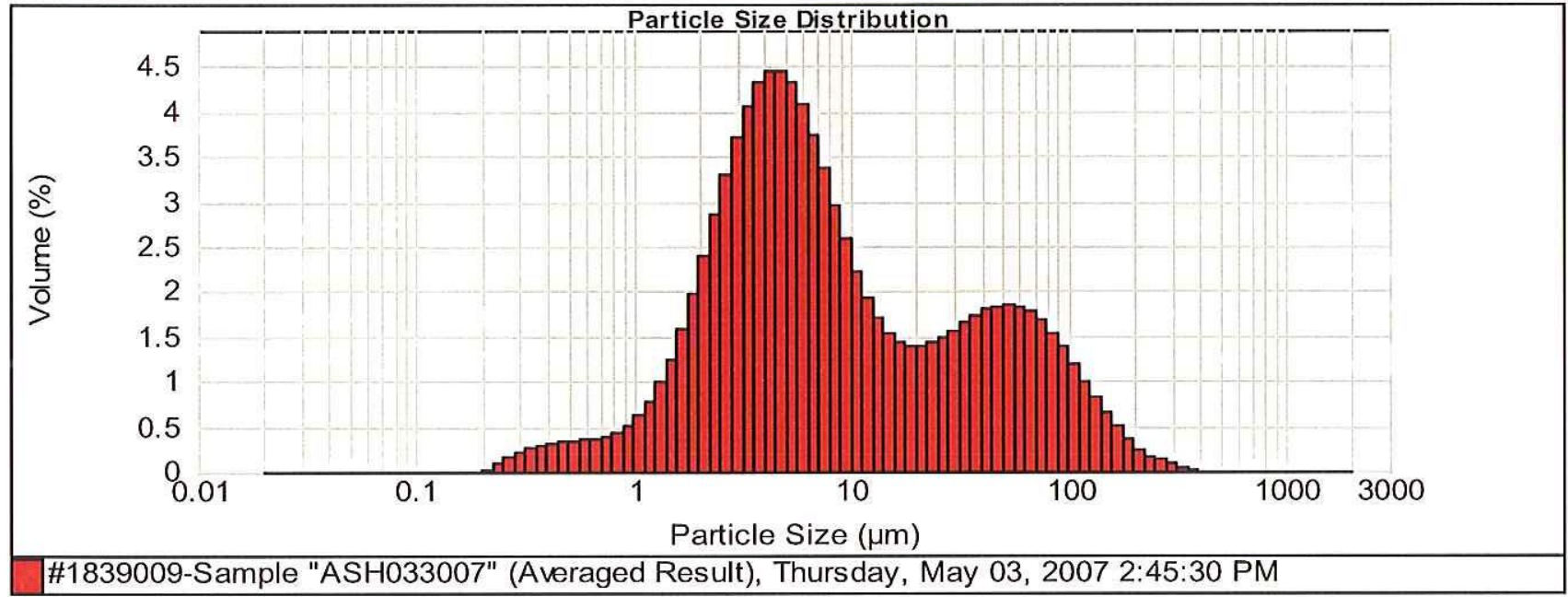

\begin{tabular}{|r|r|}
\hline Size $(\mu \mathrm{m})$ & Vol Under \% \\
\hline 0.020 & 0.00 \\
0.022 & 0.00 \\
0.025 & 0.00 \\
0.028 & 0.00 \\
0.032 & 0.00 \\
0.036 & 0.00 \\
0.040 & 0.00 \\
0.045 & 0.00 \\
0.050 & 0.00 \\
0.056 & 0.00 \\
0.063 & 0.00 \\
0.071 & 0.00 \\
0.080 & 0.00 \\
0.089 & 0.00 \\
0.100 & 0.00 \\
0.112 & 0.00 \\
0.126 & 0.00 \\
\hline
\end{tabular}

\begin{tabular}{|r|r|}
\hline Size $(\mu \mathrm{m})$ & Vol Under $\%$ \\
\hline 0.142 & 0.00 \\
0.159 & 0.00 \\
0.178 & 0.00 \\
0.200 & 0.00 \\
0.224 & 0.00 \\
0.252 & 0.09 \\
0.283 & 0.24 \\
0.317 & 0.45 \\
0.356 & 0.70 \\
0.399 & 0.98 \\
0.448 & 1.29 \\
0.502 & 1.62 \\
0.564 & 1.96 \\
0.632 & 2.31 \\
0.710 & 2.67 \\
0.796 & 3.06 \\
0.893 & 3.50 \\
\hline
\end{tabular}

\begin{tabular}{|r|r|}
\hline Size $(\mu \mathrm{m})$ & Vol Under \% \\
\hline 1.002 & 4.00 \\
1.125 & 4.62 \\
1.262 & 5.39 \\
1.416 & 6.38 \\
1.589 & 7.63 \\
1.783 & 9.21 \\
2.000 & 11.17 \\
2.244 & 13.56 \\
2.518 & 16.40 \\
2.825 & 19.70 \\
3.170 & 23.42 \\
3.557 & 27.48 \\
3.991 & 31.81 \\
4.477 & 36.26 \\
5.024 & 40.71 \\
5.637 & 45.04 \\
6.325 & 49.12 \\
\hline
\end{tabular}

\begin{tabular}{|r|r|}
\hline Size $(\mu \mathrm{m})$ & Vd Under $\%$ \\
\hline 7.096 & 52.86 \\
7.962 & 56.22 \\
8.934 & 59.18 \\
10.024 & 61.75 \\
11.247 & 63.97 \\
12.619 & 65.90 \\
14.159 & 67.59 \\
15.887 & 69.12 \\
17.825 & 70.54 \\
20.000 & 71.92 \\
22.440 & 73.30 \\
25.179 & 74.72 \\
28.251 & 76.21 \\
31.698 & 77.77 \\
35.566 & 79.42 \\
39.905 & 81.15 \\
44.774 & 82.94 \\
\hline
\end{tabular}

\begin{tabular}{|r|r|}
\hline Size $(\mu \mathrm{m})$ & Vol Under $\%$ \\
\hline 50.238 & 84.77 \\
56.368 & 86.62 \\
63.246 & 88.45 \\
70.963 & 90.22 \\
79.621 & 91.89 \\
89.337 & 93.43 \\
100.237 & 94.80 \\
112.468 & 96.00 \\
126.191 & 97.01 \\
141.589 & 97.82 \\
158.866 & 98.47 \\
178.250 & 98.97 \\
200.000 & 99.34 \\
224.404 & 99.58 \\
251.785 & 99.75 \\
282.508 & 99.88 \\
316.979 & 99.96 \\
\hline
\end{tabular}

\begin{tabular}{|r|r|}
\hline Size $(\mu \mathrm{m})$ & Vol Under $\%$ \\
\hline 355.656 & 100.00 \\
399.052 & 100.00 \\
447.744 & 100.00 \\
502.377 & 100.00 \\
563.677 & 100.00 \\
632.456 & 100.00 \\
709.627 & 100.00 \\
796.214 & 100.00 \\
893.367 & 100.00 \\
1002.374 & 100.00 \\
1124.683 & 100.00 \\
1261.915 & 100.00 \\
1415.892 & 100.00 \\
1588.656 & 100.00 \\
1782.502 & 100.00 \\
2000.000 & 100.00 \\
& \\
\hline
\end{tabular}

Operator notes: Average of four measurements

File name: Samples $1839007-00$ Record Number: 15 03 May 2007 3:09:43 PM 


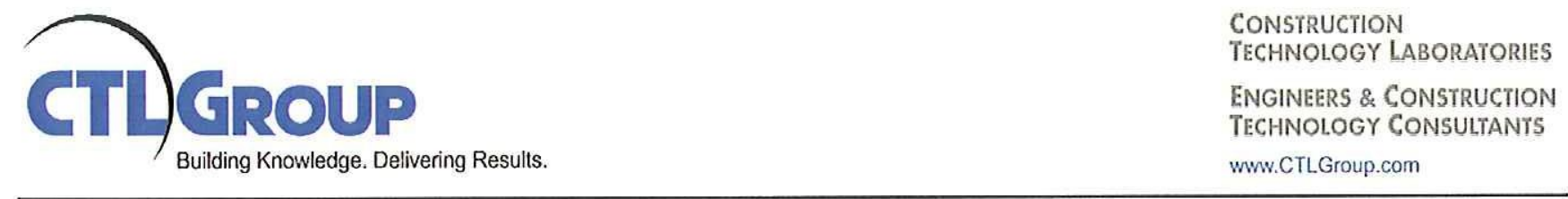

\begin{tabular}{lllr} 
Client: & CONSOL Energy, Inc. & CTL Project No.: & 403423 \\
Project: & BET Analysis & CTL Proj. Mgr.: & Ella Shkolnik \\
& PO \#4700142976 & Analyst: & note 2 \\
Contact: & Daniel Connell & Approved: & May 9, 2007 \\
Submitter: & Daniel Connell & Date Analyzed: & May 10, 2007 \\
Date Received: & April 23, 2007 & Date Reported: & \\
\hline
\end{tabular}

REPORT of BET SURFACE AREA

$\begin{array}{ll}\begin{array}{c}\text { Sample Identification } \\ \text { CTL ID }\end{array} & \underline{\text { Client ID }} \\ 1839001 & \text { HYD032807 } \\ 1839002 & \text { HYD032907 } \\ 1839003 & \text { HYD033007 }\end{array}$

Material
Hydrated Lime
Hydrated Lime
Hydrated Lime

BET Surface Area $\left(\mathrm{m}^{2} / \mathrm{kg}\right)^{\text {note } 2}$

15.73

17.29

17.62

Notes:

1. This analysis represents specifically the samples submitted.

2. Surface area analysis was performed by Particle Technology Laboratories (PTL); the original PTL report is enclosed.

3. This report may not be reproduced except in its entirety. 
May 18, 2007

Ms. Ella Shkolnik

CTLGROUP

5400 Old Orchard Road

Skokie, IL 60077-1030

\section{Subject: B.E.T. Surface Area Analysis of Three Hydrated Lime Samples}

\section{P.O. \#: 39804B}

\section{PTL Project: 14234}

Dear Ms. Shkolnik:

Enclosed are the results from the 1-point B.E.T. surface area analysis conducted on your three Hydrated Lime samples. The sample information is detailed in Table 1 below.

TABLE 1

SAMPLE DETAILS

\begin{tabular}{|c|c|c|}
\hline SAMPLE TYPE & SAMPLE ID & DATE RECEIVED \\
\hline \multirow{3}{*}{ Hydrated Lime } & 1839001 & \multirow{2}{*}{$05 / 02 / 07$} \\
\cline { 2 - 2 } & 1839002 & \\
\cline { 2 - 2 } & 1839003 & \\
\hline
\end{tabular}

The surface area was determined on our Micromeritics ${ }^{\circledR}$ TriStar 3000 static pressure surface area analyzer according to PTL Test Method TS1234.01. Per this method, an aliquot portion of each sample was heat conditioned for 3 hours at $105^{\circ} \mathrm{C}$, under vacuum, and then analyzed according to standard operating procedures for this instrumentation type.

The data has been summarized in Table 2. In addition, the original data pages have been included for your review.

We trust this information will be beneficial for your future use. If there are any questions concerning this data or the methods used to acquire the data, please do not hesitate to contact us here at Particle Technology Labs.

Submitted by,

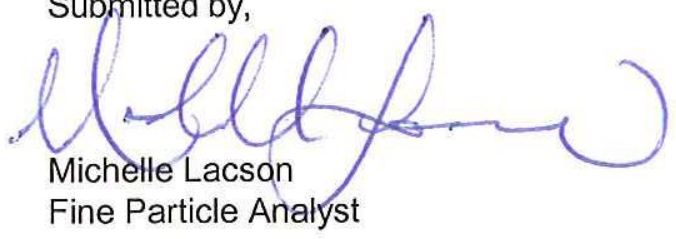

Reviewed by,

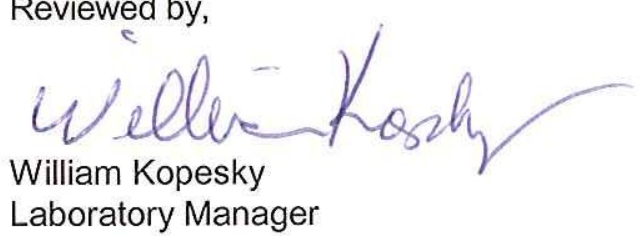

DR:114234.doc:Reports 
TABLE 2

B.E.T. SURFACE AREA DATA SUMMARY

\begin{tabular}{|c|c|}
\hline SAMPLE ID & SPECIFIC SURFACE AREA $\left(\mathbf{m}^{2} / \mathbf{g}\right)$ \\
\hline 1839001 & 15.73 \\
\hline 1839002 & 17.29 \\
\hline 1839003 & 17.62 \\
\hline
\end{tabular}




\section{Particle Technology Labs}

\section{CTL Group}

TriStar Confirm V7.02

Unit 1 Port 1

Serial \#: 119

Page 2

Sample: $14234 \mathrm{~A}$

Operator: ML

Submitter: UNIT \# 231

File Location: Category: Mic. Samples Subcategory: Example

Started: 5/9/2007 1:17:01PM

Completed: 5/9/2007 2:36:08PM

Report Time: 5/9/2007 3:44:13PM

Warm Free Space: $8.0416 \mathrm{~cm}^{3}$ Measured

Equilibration Interval: $20 \mathrm{~s}$

Sample Density: $1.000 \mathrm{~g} / \mathrm{cm}^{3}$

File Created By: Michelle Lacson

Analysis By: Michelle Lacson

Report By: Michelle Lacson
Analysis Adsorptive: N2

Analysis Bath Temp.: $77.300 \mathrm{~K}$

Sample Mass: $0.7716 \mathrm{~g}$

Cold Free Space: $24.2140 \mathrm{~cm}^{3}$ Measured

Low Pressure Dose: None

Automatic Degas: No

File Creation Time: 5/9/2007 11:44:15AM

Coll. Software: TriStar Confirm V7.02

Sample File Version: 6

Comments: CTL GROUP - Hydrated Lime - S55967 - CTL ID:1839001 - TS1234.01 - 58042

\section{Summary Report}

Surface Area

Single point surface area at $\mathrm{P} / \mathrm{Po}=0.306803201: 15.7282 \mathrm{~m}^{2} / \mathrm{g}$ 


\section{Particle Technology Labs \\ CTL Group}

TriStar Confirm V7.02

Unit 1 Port 2

Serial \#: 119

Page 2

Sample: $14234 \mathrm{~B}$

Operator: ML

Submitter: UNIT \# 231

File Location: Category: Mic. Samples Subcategory: Example

Started: 5/9/2007 1:17:01PM

Completed: 5/9/2007 2:36:08PM

Report Time: 5/9/2007 3:32:51PM

Warm Free Space: $8.4716 \mathrm{~cm}^{3}$ Measured

Equilibration Interval: $20 \mathrm{~s}$

Sample Density: $1.000 \mathrm{~g} / \mathrm{cm}^{3}$

File Created By: Michelle Lacson

Analysis By: Michelle Lacson

Report By: Michelle Lacson
Analysis Adsorptive: N2 Analysis Bath Temp.: $77.300 \mathrm{~K}$

Sample Mass: $0.7034 \mathrm{~g}$

Cold Free Space: $25.2163 \mathrm{~cm}^{3}$ Measured Low Pressure Dose: None

Automatic Degas: No

File Creation Time: 5/9/2007 11:49:55AM

Coll. Software: TriStar Confirm V7.02

Sample File Version: 6

Comments: CTL GROUP - Hydrated Lime - TS1234.01 - S55968 - CTL ID: 1839002 - 58043

\section{Summary Report}

\section{Surface Area}

Single point surface area at $\mathrm{P} / \mathrm{Po}=0.306303993: 17.2884 \mathrm{~m}^{2} / \mathrm{g}$ 


\section{Particle Technology Labs}

\section{CTL Group}

TriStar Confirm V7.02

Unit 1 Port 3

Serial \#: 119

Page 2

Sample: $14234 \mathrm{C}$

Operator: ML

Submitter: UNIT \# 231

File Location: Category: Mic. Samples Subcategory: Example

Started: 5/9/2007 1:17:02PM

Completed: 5/9/2007 2:36:09PM

Report Time: 5/9/2007 3:33:59PM

Warm Free Space: $7.8878 \mathrm{~cm}^{3}$ Measured

Equilibration Interval: $20 \mathrm{~s}$

Sample Density: $1.000 \mathrm{~g} / \mathrm{cm}^{3}$

File Created By: Michelle Lacson

Analysis By: Michelle Lacson

Report By: Michelle Lacson
Analysis Adsorptive: N2

Analysis Bath Temp.: $77.300 \mathrm{~K}$ Sample Mass: $0.5703 \mathrm{~g}$

Cold Free Space: $23.6188 \mathrm{~cm}^{3}$ Measured Low Pressure Dose: None

Automatic Degas: No

File Creation Time: 5/9/2007 11:51:37AM

Coll. Software: TriStar Confirm V7.02

Sample File Version: 6

Comments: CTL GROUP - Hydrated Lime - TS1234.01 - S55969 - CTL ID: 1839003 - 58044

\section{Summary Report}

\section{Surface Area}

Single point surface area at $\mathrm{P} / \mathrm{Po}=0.308913595: 17.6179 \mathrm{~m}^{2} / \mathrm{g}$ 
APPENDIX C

Field Data Sheets 
Axial Flow Check

\begin{tabular}{|c|c|c|c|c|}
\hline Location $\frac{\text { SCR OurLe }}{\text { Date }}$ & $\begin{array}{l}\text { - Duct Ht, " } \\
\text { Duct ID," }\end{array}$ & & $\begin{array}{l}\text { Barometric } \\
\text { Static }\end{array}$ & $\begin{array}{r}29.50 \\
-7.9 \\
\end{array}$ \\
\hline Time & Duct Area & & Dry Bulb & \\
\hline Tube I.D. 5.53 & $\% \mathrm{O}_{2}$ & $6.0 \%$ & Wet Bulb & \\
\hline $\begin{array}{l}\text { C-Factor } \frac{807}{\text { Operator(s) } 3 G \& R D}\end{array}$ & $\begin{array}{l}\% \mathrm{CO}_{2} \\
\% \mathrm{~N}_{2}\end{array}$ & $14.0 \%$ & $\begin{array}{l}\% \mathrm{H}_{2} \mathrm{O} \\
\text { W.M.Wt }\end{array}$ & $10 \%$ \\
\hline
\end{tabular}

\begin{tabular}{|c|c|c|c|c|c|c|}
\hline $\begin{array}{l}\text { PORTI } \\
\text { POINT }\end{array}$ & $\begin{array}{l}\text { DISTANCE } \\
\text { [" From Wall] }\end{array}$ & $\begin{array}{l}\text { TEMP } \\
{\left[{ }^{\circ} \mathrm{F}\right]} \\
\end{array}$ & $\begin{array}{c}\text { DELTAP } \\
{\left[" \mathrm{H}_{2} \mathrm{O}\right]}\end{array}$ & $\begin{array}{r}\text { VELOCITY } \\
{[\mathrm{Ft} / \mathrm{Sec}]}\end{array}$ & $\begin{array}{l}\text { Null } \\
\text { Angle }\end{array}$ & \multirow{9}{*}{$\begin{array}{l}-7.8 \\
-8.1 \\
-8.1 \\
-7.6\end{array}$} \\
\hline 11 & & 6.36 & 0.271 & $Q$ & $\approx 0$ & \\
\hline $2^{\prime}$ & & 651 & 0.265 & & & \\
\hline $3^{\prime}$ & & 6.58 & 0.308 & & \pm 0 & \\
\hline $4^{\circ}$ & 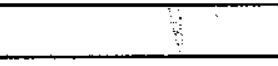 & 636 & 0.224 & & & \\
\hline 51 & & 626 & 0.192 & & $\approx 0$ & \\
\hline $6^{\prime}$ & & 638 & 0.218 & & & \\
\hline $7^{\prime}$ & & 638 & 0.225 & & $\approx 0$ & \\
\hline $8^{\prime}$ & & 625 & 0.229 & & & \\
\hline
\end{tabular}

M4@inlet

$y: 0.983$

$\triangle H Q: 1.790$

\begin{tabular}{||l|l|}
\hline \multicolumn{2}{|c|}{ DATA SUMMARY } \\
\hline Velocity, [fps] & \\
\hline acfm & \\
\hline scfm & \\
\hline dscfm & \\
\hline Ex Air Free cfm & \\
\hline Est. MAM Btu/hr Heat Input & \\
\hline Est. Firing Rate, Ib/hr & \\
\hline
\end{tabular}

M5: r:0.9.9 AH:1.883 EN

Ell-A: 0.833

$D_{N}: 0.313$

KFACT: $4.38,4.70$ 
Axial Flow Check

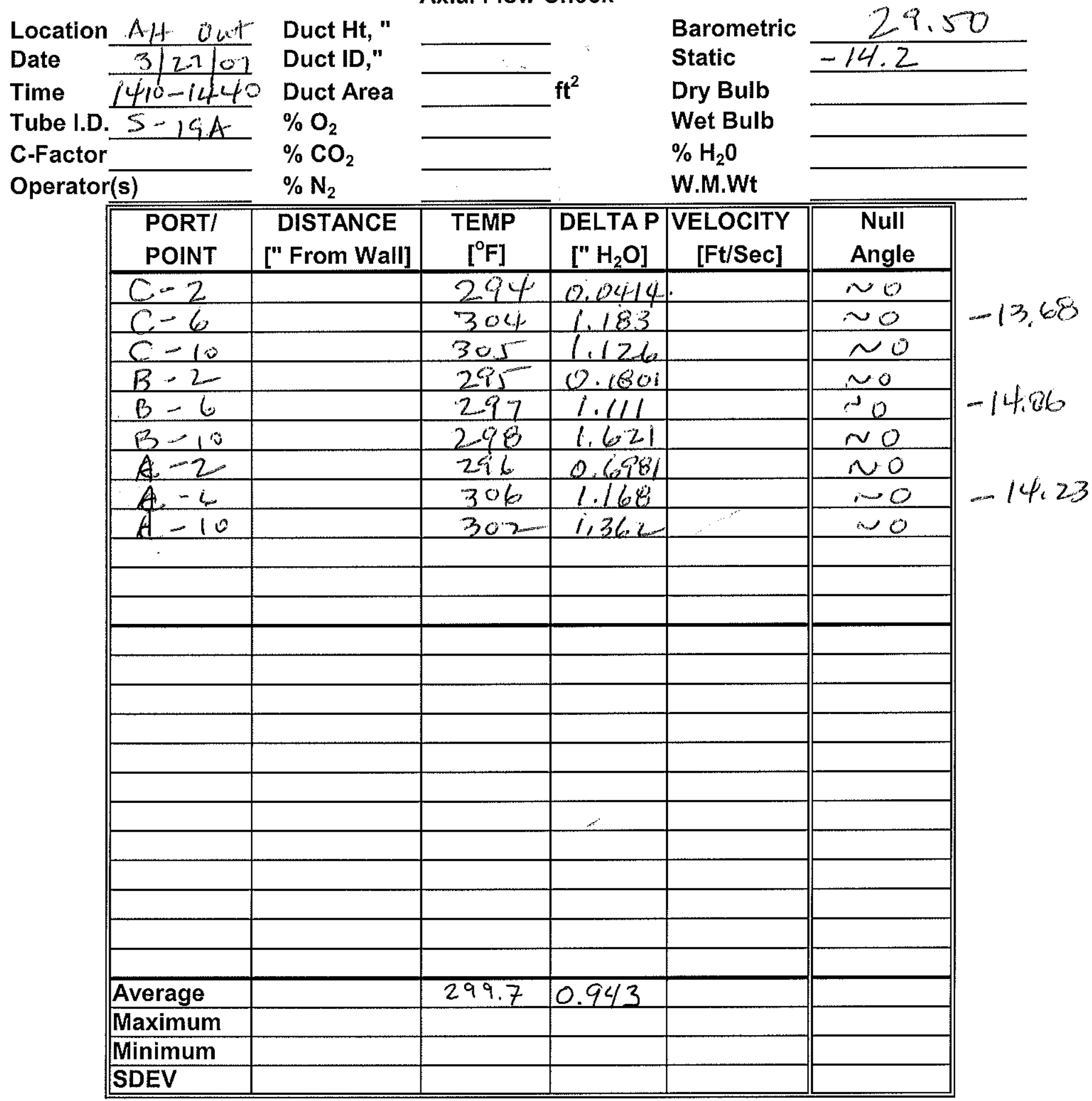

Meter: N1:

$Y: 0.970$

$\triangle H O: 1.894$

E3: 0.840

$D_{N}: 0.184$

KFACT 0.744

\begin{tabular}{|c|c|}
\hline \multicolumn{2}{|c|}{ DATA SUMMARY } \\
\hline Velocity, [fps] & \\
\hline $\mathrm{acfm}$ & \\
\hline scfm & \\
\hline dscfm & \\
\hline Ex Air Free cfm & \\
\hline Est. MM Btu/hr Heat Input & \\
\hline Est. Firing Rate, lb/hr & \\
\hline
\end{tabular}

$N-1$

(CD) A

(d) $B$

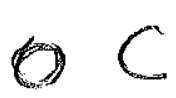




\section{Axial Flow Check}

Location ST $<$. Duct Ht, "

Date $\frac{5 T .37 .07}{3.3 u c t ~ I D, "}$

Time 1520.1535 Duct Area

Tube I.D. $\mathrm{S}-54 \quad \% \mathrm{O}_{2}$

C.Factor

Operator(s) KC, BS $\% \mathrm{~N}_{2}$

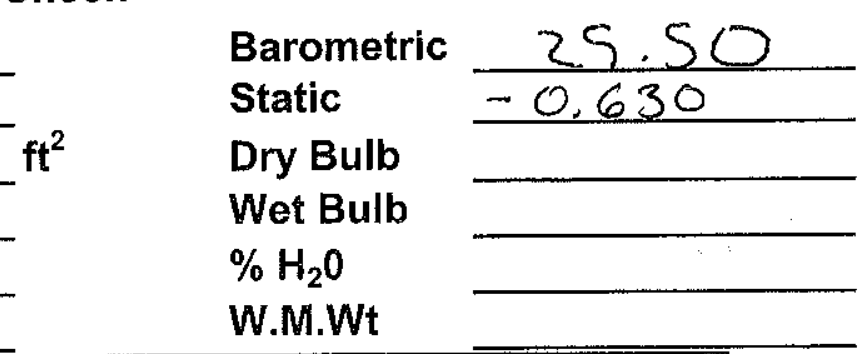

Barometric 25.50

Dry Bulb

Wet Bulb

W.M.Wt

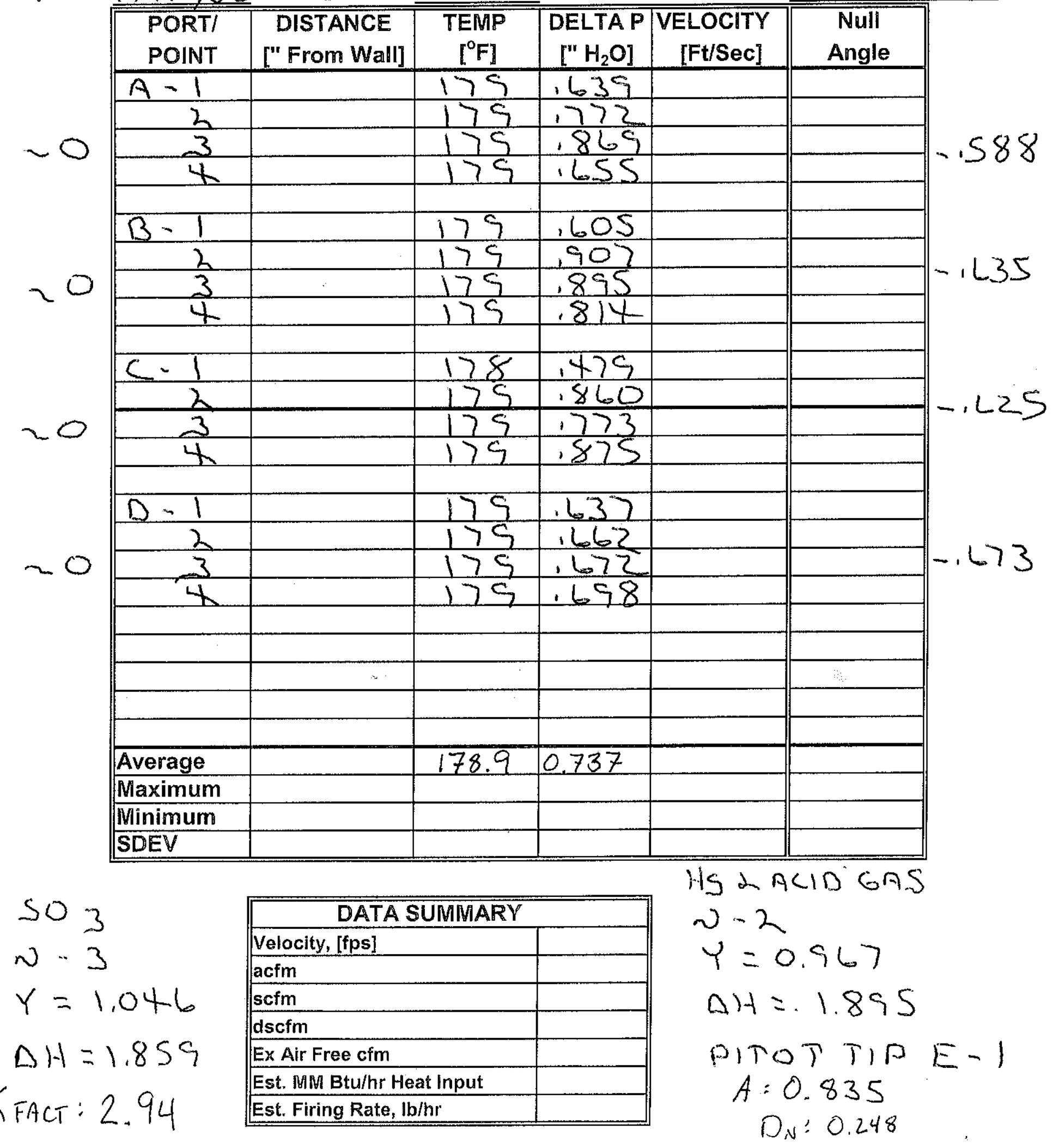




\section{AES GREENIDGE UNIT 4 GUARANTEE TESTING \\ NH3 Summary \\ March 28, 2007}

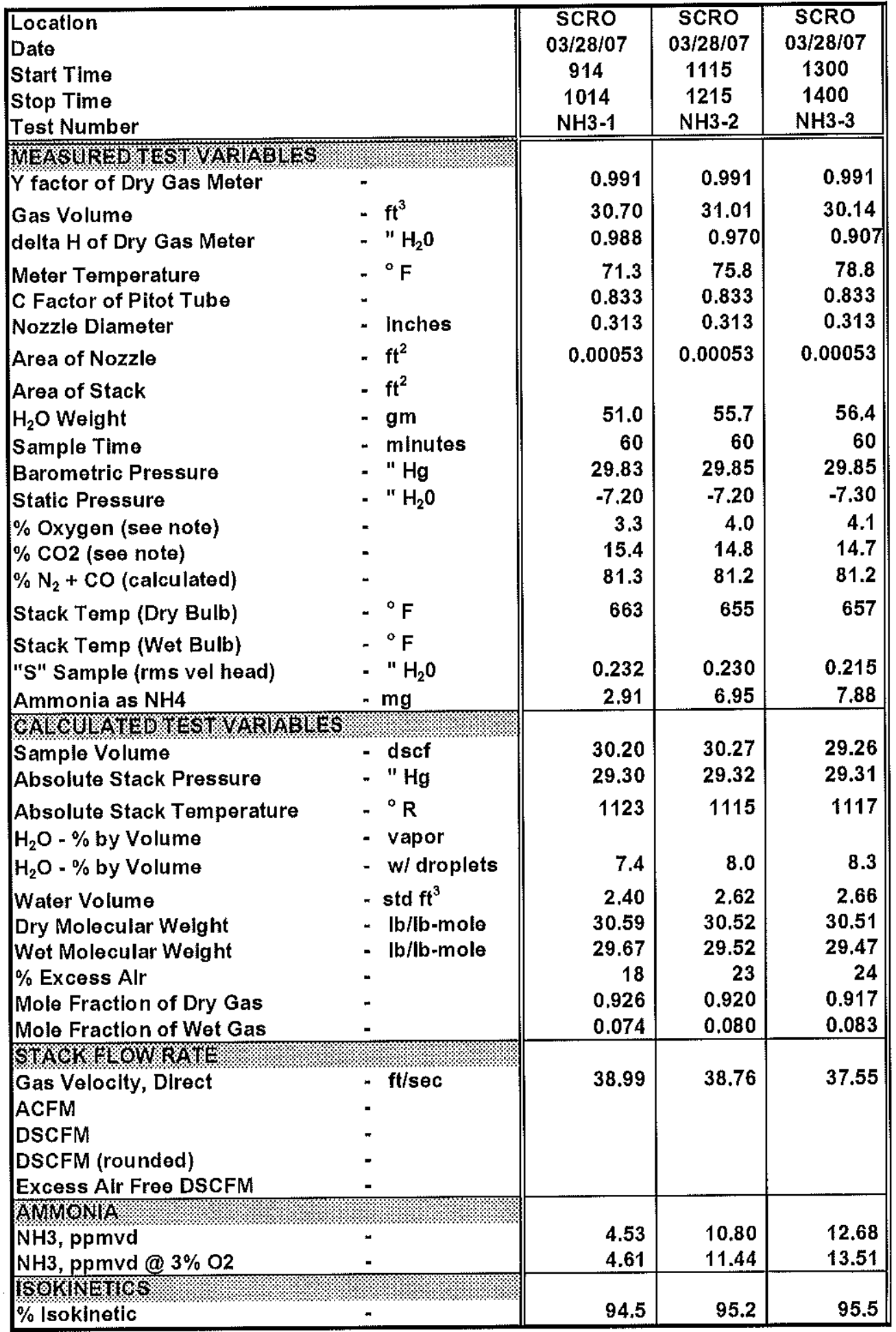

NOTE: The $\% \mathrm{O}_{2}$ was measured by CONSOL using a Teledyne Max 5 portable electrochemical $\mathrm{O}_{2}$ analyzer, and the $\%$ $\mathrm{CO}_{2}$ was calculated from the measured $\mathrm{O}_{2}$ and coal composition. 


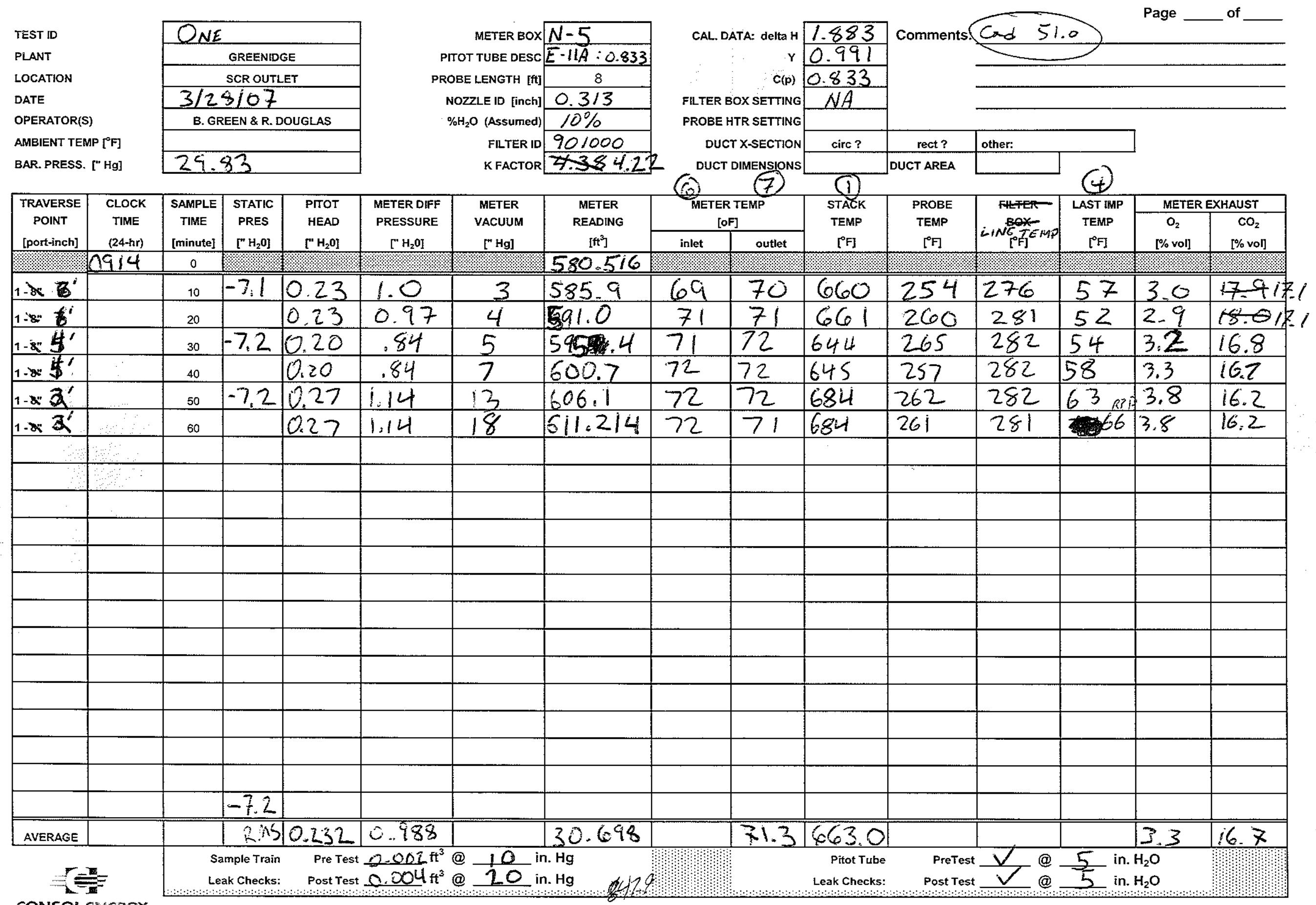




\section{AMMONIA SAMPLING FIELD DATA SHEET}

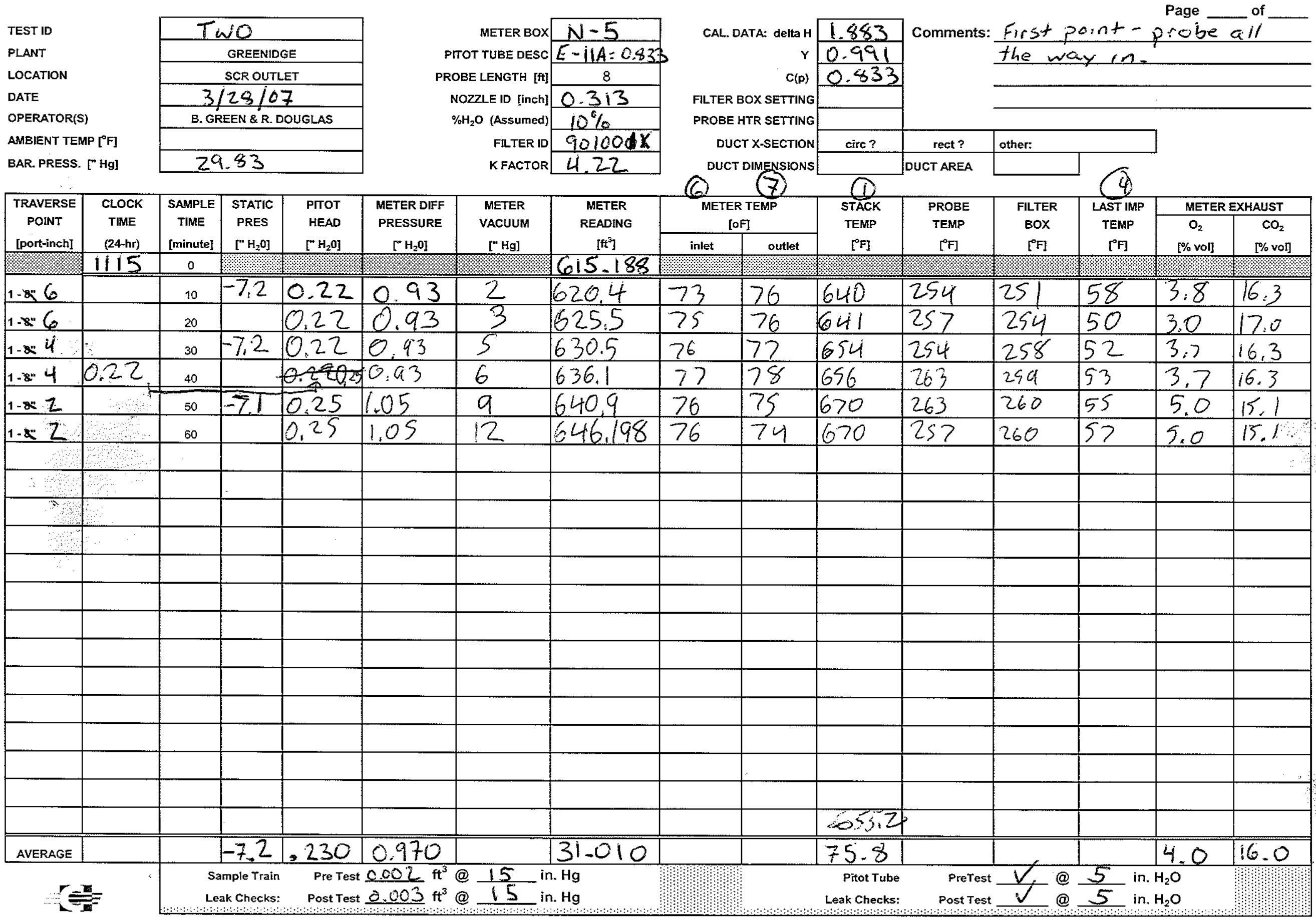




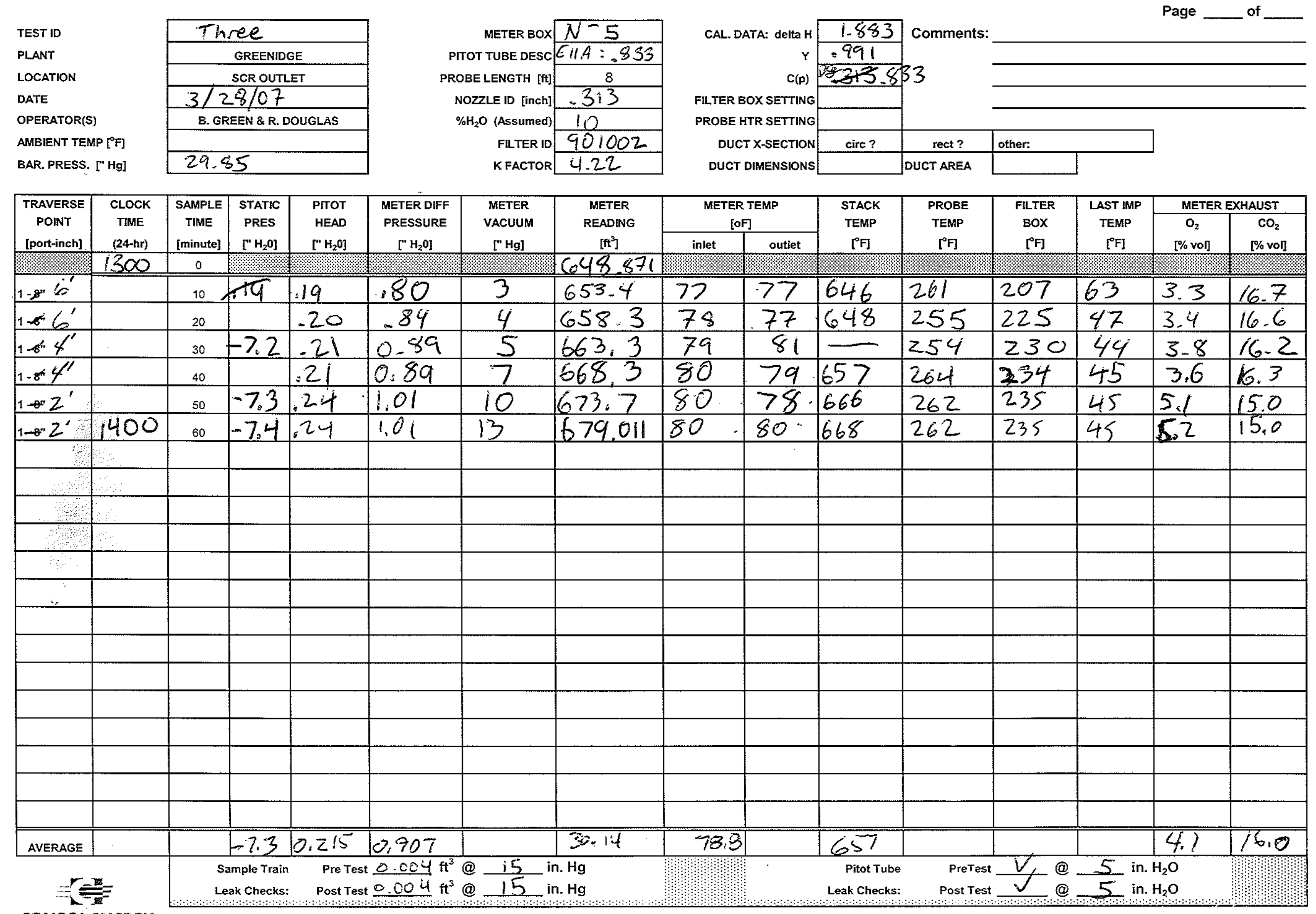
CONSOLENERGY. 


\section{AES GREENIDGE UNIT 4 GUARANTEE TESTING}

NH3 Summary

May 1, 2007

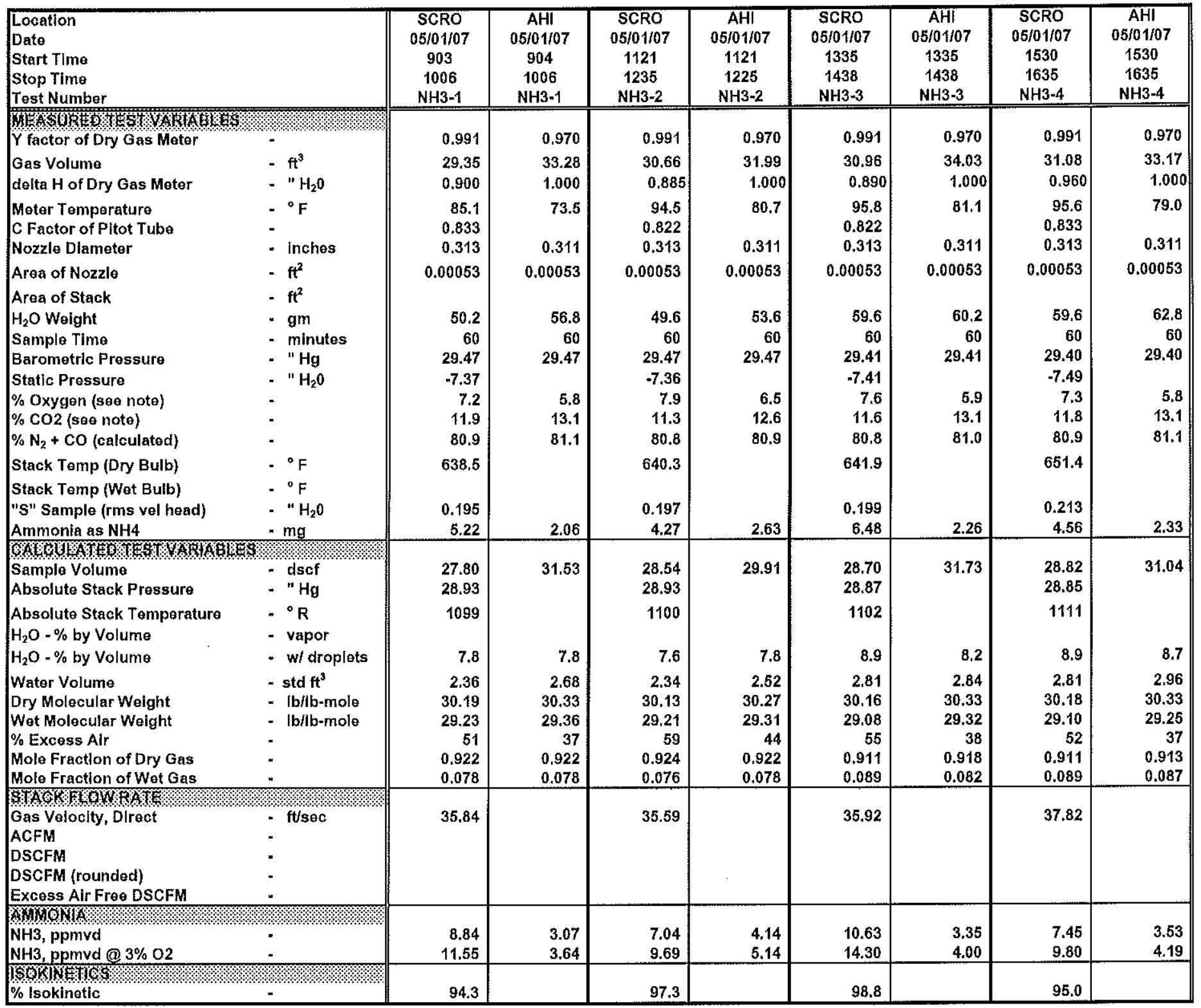

NOTE: The $\% \mathrm{O}_{2}$ was measured by $\mathrm{CONSOL}$ using a Teledyne Max 5 porlable electrochemical $\mathrm{O}_{2}$ analyzer, and the $\% \mathrm{CO}_{2}$ was calculated from the measured $\mathrm{O}_{2}$ and coal compasition. 


\section{AMMONIA SAMPLING FIELD DATA SHEET}

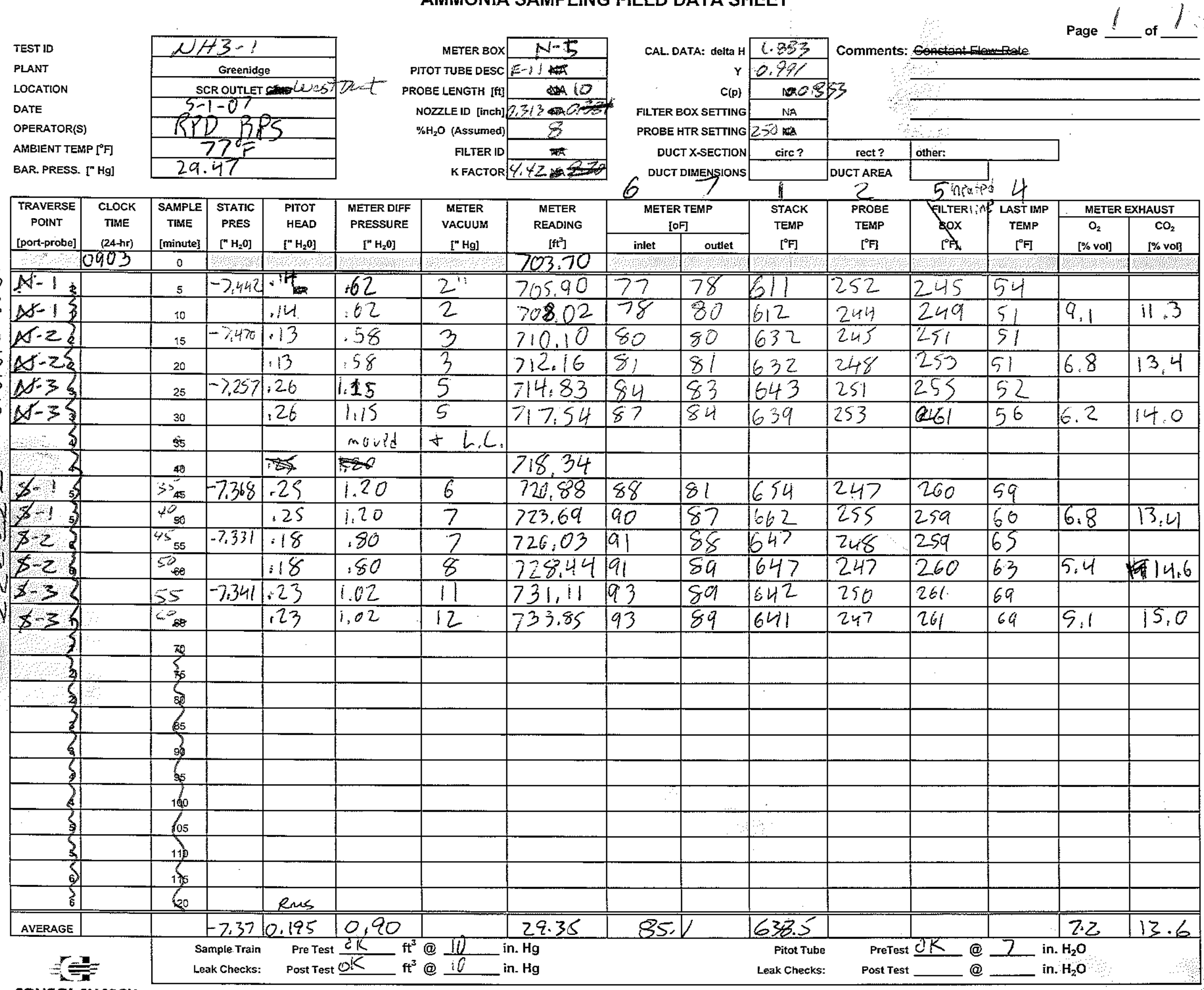

CONSOL ENERGY 


\section{AMMONIA SAMPLING FIELD DATA SHEET}

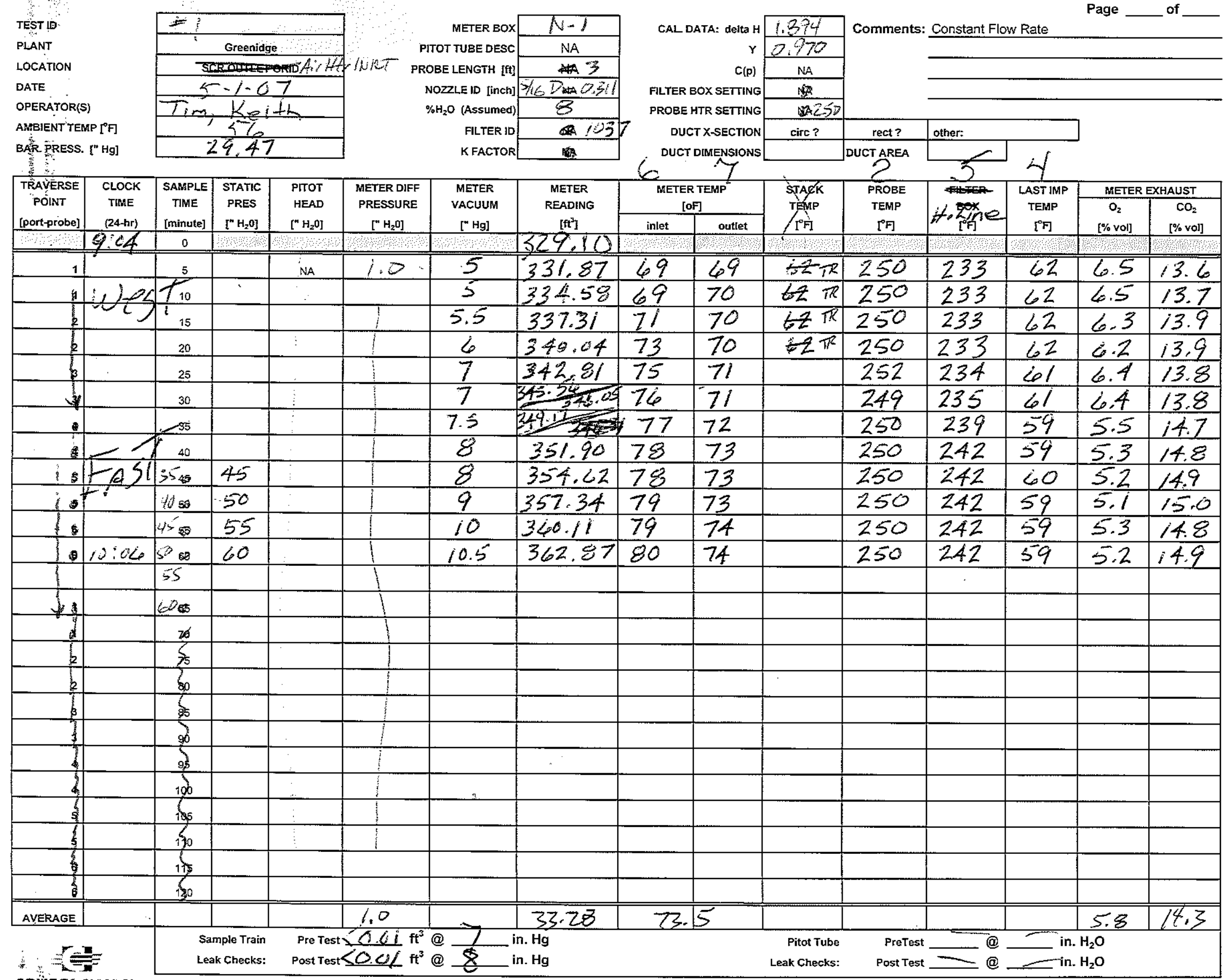




\section{AMMONIA SAMPLING FIELD DATA SHEET}

\begin{tabular}{|c|c|c|c|c|c|c|c|c|c|c|c|c|c|c|c|}
\hline \multirow{7}{*}{\multicolumn{2}{|c|}{$\begin{array}{l}\text { TEST ID } \\
\text { PLANT } \\
\text { LOCATION } \\
\text { DATE } \\
\text { OPERATOR(S) } \\
\text { AMBIENT TEMP [ } \mathrm{F} \text { ] } \\
\text { BAR. PRESS. [" Hg] }\end{array}$}} & \multicolumn{3}{|c|}{ NH3-R } & \multirow{2}{*}{\multicolumn{3}{|c|}{ 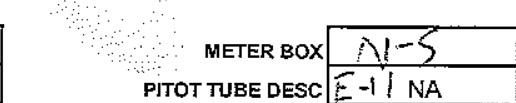 }} & \multirow{2}{*}{\multicolumn{2}{|c|}{$\begin{array}{r}\text { CAL. DATA: deta H } \\
\mathrm{Y}\end{array}$}} & \multirow{2}{*}{\begin{tabular}{|l}
1.883 \\
0.991 \\
\end{tabular}} & \multirow[t]{2}{*}{ Comments } & \multicolumn{2}{|c|}{ Conont Flow Rate } & \multicolumn{2}{|c|}{ Page $I$ of } \\
\hline & & & Greenids & & & & & & & & & & & & \\
\hline & & & ER OUTLET & 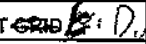 & et & E LENGTH [f & $10 \mathrm{NA}$ & & & $\ldots+0.8$ & 33 & & & & \\
\hline & & $5-$ & $1-07$ & & & ZZLE ID [inch & $313 \mathrm{NA}$ & FILTER & BOX SETTING & $\mathrm{NA}$ & & & & & \\
\hline & & $k 8$ & 13 & 3 & & ${ }_{2}^{O}$ (Assumed & 8 & PROBE & HTR SETTING & $\mathrm{NA} 250$ & & & & & \\
\hline & & 20 & $\frac{20}{147}$ & & & FLTER II & $\frac{N A}{442 N A}$ & & T X-SECTION & circ? & \begin{tabular}{|l|} 
rect? \\
DUCT AREA
\end{tabular} & Oother. & & & \\
\hline & & & & & & & $1.7<\mathrm{NA}$ & 6 & DIMENSIONS & I & $\begin{array}{c}\text { DUCT AREA } \\
?\end{array}$ & 5 & 4 & & \\
\hline \begin{tabular}{|c|} 
TRAVERSE \\
POINT
\end{tabular} & CLOCK & SAMPLE & StATIC & PITOT & METER DIFF & METER & METER & METE & TTEMP & STACK & PROBE & FILTER & LAST IMP & METER E & XHALST \\
\hline POINT & & & PRES & HEAD & PRESSURE & VACUUM & READING & & & TEMP & TEMP & Box & TEMP & $\mathrm{O}_{2}$ & $\mathrm{CO}_{2}$ \\
\hline \begin{tabular}{|l|l|} 
[port-probe] \\
\end{tabular} & $(24-\mathrm{hr})$ & [minute] & {$\left[{ }^{n} \mathrm{H}_{2} \mathrm{O}\right]$} & {$\left[" \mathrm{H}_{2} \mathrm{O}\right]$} & {$\left[" \mathrm{H}_{2} \mathrm{O}\right]$} & {$\left[{ }^{[} \mathrm{Hg}\right]$} & {$\left[\mathrm{ft}^{3}\right]$} & inlet & outlet & {$\left[{ }^{\circ} \mathrm{F}\right]$} & {$[\mathrm{F}]$} & {$[\circ]$} & {$[\mathrm{F}]$} & {$[\%$ voll } & {$[\%$ von } \\
\hline & & 0 & & & & & 747.814 & & & & & & & & \\
\hline$\sqrt{1}$ & 1121 & 5 & $-7,384$ & 1.23 & 1.02 & 2 & 750.95 & 90 & $a d$ & 605 & 242 & 227 & 54 & & \\
\hline$|v| ?$ & & 10 & & .23 & 1,02 & 3 & $753.5 \mathrm{~V}$ & 96 & a1 & 668 & 255 & 226 & 48 & 7,2 & 13.0 \\
\hline$N 2$ & & 15 & $-7,318$ & .17 & .75 & 3 & 755.95 & 96 & 92 & 649 & 250 & 232 & 48 & & 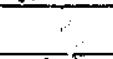 \\
\hline N2? & & 20 & & .17 & .75 & 3 & 758.33 & 97 & 92 & 649 & 249 & 235 & 49 & 6.1 & $\operatorname{lo}_{\mathrm{j}}$ \\
\hline N3 & & 25 & -7.35 & .24 & 1.07 & 4 & 761.07 & 98 & 93 & 663 & 249 & 231 & 50. & & \\
\hline N33 & & 30 & & .24 & 1.07 & 4 & 763.83 & 99 & 93 & 661 & 246 & 231 & 50 & 6.6 & 13.6 \\
\hline 4 & & 35 & & & & & & & & & & & & & \\
\hline$A$ & & 35. & & +5 & +6 & & 16775 & & & & & & & & \\
\hline 5115 & & $40_{45}$ & $-3,32$ & 415 & .66 & 3 & 170,95 & 95 & 92 & 606 & 246 & 233 & 52 & - & \\
\hline 51, & & 45.50 & & $\because 15$ & .66 & 3 & 172.18 & 96 & 93 & 615 & 245 & 235 & 50 & & \\
\hline $52 \%$ & & 505 & -7.311 & .15 & .66 & 4 & 174.45 & 27 & 93 & 632 & 251 & 237 & 219 & 9.8 & 10.2 \\
\hline $52 \%$ & & 60 & & .15 & .66 & 4 & 776,70 & 98 & 93 & $6 n^{6}$ & 255 & 235 & 49 & & \\
\hline 531 & & 55 & -7.45 & $+1,5,26$ & 1.15 & 5 & 779,42 & 98 & 93 & 645 & 256 & 233 & 49 & 9.7 & 10.6 \\
\hline 531 & & $1 \infty 65$ & & $: 26$ & 1.15 & & 182,39 & 99 & 93 & 645 & 248 & 233 & 49 & & \\
\hline t & & 70 & & & & & & & & & & & & & \\
\hline$\%$ & & 75 & & & & & & & & & & & & & \\
\hline$S_{2}$ & & 80 & & & & & & & & & & & & & \\
\hline 3 & & 85 & & & & & & & & & & & & & \\
\hline (3) & & 90 & & & & & & & & & & & & & \\
\hline 4) & & 95 & & & & & & & & & & & & & \\
\hline 4) & & 100 & & & & & & & & & & & & & \\
\hline 5 & & 105 & & & & & & & & & & & & & \\
\hline 5 & & 110 & & & & & & & & & & & & & \\
\hline 6 & & 115 & & & & & & & & & & & & & \\
\hline 6 & & 120 & & Res & & & & & & & & &. & & \\
\hline \begin{tabular}{|l|} 
AVERAGE \\
\end{tabular} & & & -7.36 & 0.197 & 0.885 & & 30.656 & $9 \%$ & & 640.3 & & & & 7.9 & 12.3 \\
\hline $1=$ & & & $\begin{array}{l}\text { mple Train } \\
\text { ak Checks: }\end{array}$ & $\begin{array}{r}\text { Pre Test } \\
\text { Post Test }\end{array}$ & $\mathrm{ft}^{\mathrm{t}} \mathrm{ft}$ & $\bar{\square}$ & $\begin{array}{l}\text { in. } \mathrm{Hg} \\
\text { in. } \mathrm{Hg}\end{array}$ & & & $\begin{array}{r}\text { Pitot Tube } \\
\text { Leak Checks: }\end{array}$ & Pre & 工 & $\bar{Z}$ & $\begin{array}{l}\mathrm{H}_{2} \mathrm{O} \\
\mathrm{H}_{2} \mathrm{O}\end{array}$ & \\
\hline
\end{tabular}




\section{AMMONIA SAMPLING FIELD DATA SHEET}

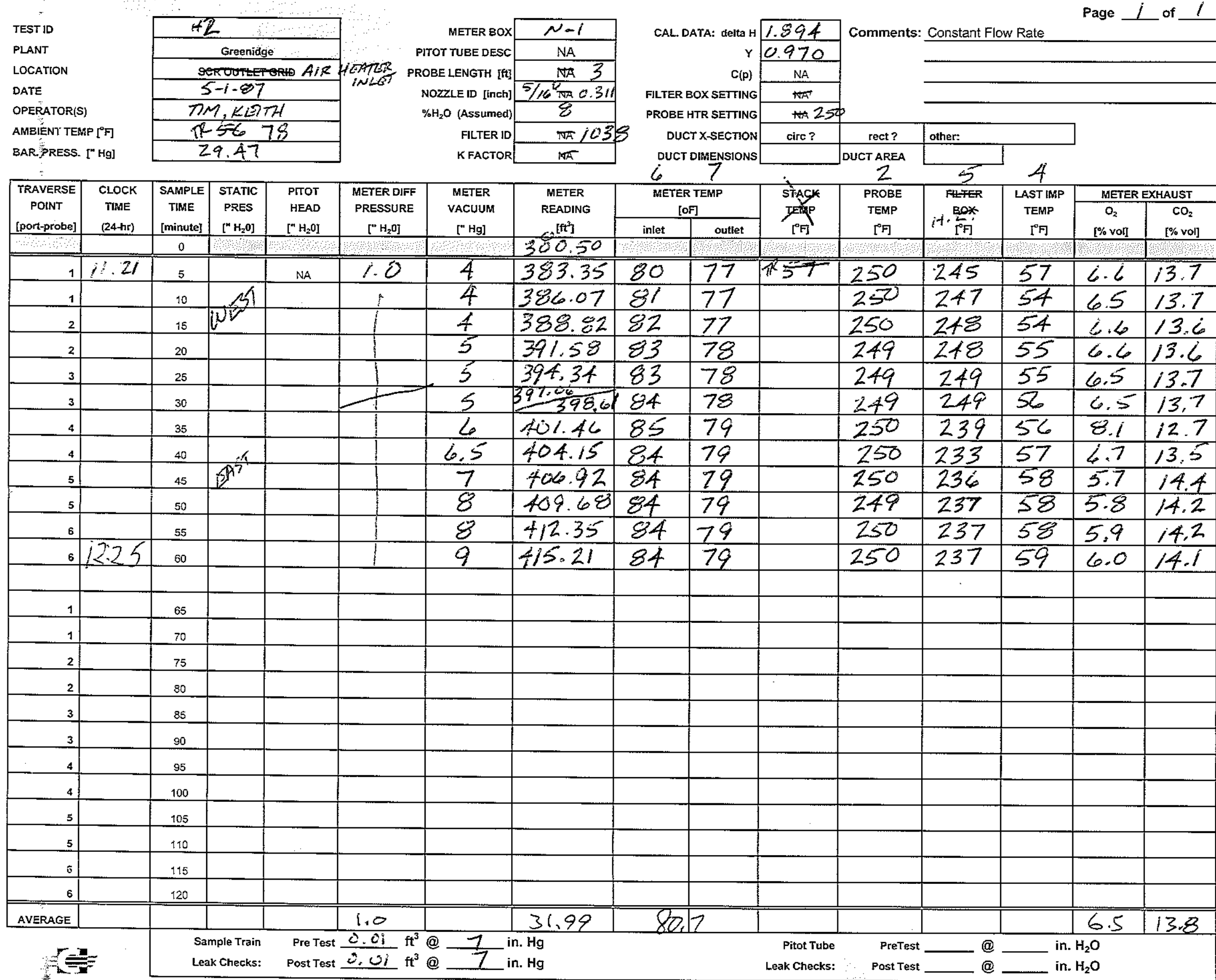




\begin{tabular}{|c|c|c|c|c|c|c|c|c|c|c|c|c|c|c|c|}
\hline & & & & & & & & & & & & & & Page & of \\
\hline TEST ID & & ! & $+3-3$ & & & METER BOX & $N-S$ & & DATA: delta H & 1.883 & Comments & 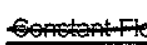 & wrate. & & \\
\hline PLANT & & & Greenids & & & OT TUBE DESC & $E-11 \mathrm{NA}$ & & $\mathbf{Y}$ & 0.091 & & & & & \\
\hline LOCATION & & & CR OUTLET & W.P. & it & BE LENGTH [ft] & $10 \mathrm{NA}$ & & $C(p)$ & A. 83 & 3 & & & & \\
\hline DATE & & & $1-07$ & & & OZZLE ID [inch] & $.31^{3} \mathrm{NA}$ & & BOX SETTING & NA & & & & & \\
\hline OPERATOR(S & & $R P D$ & BPS & & & $\mathrm{H}_{2} \mathrm{O}$ (Assumed) & 8 & PRO & HTR SEITING & NA $2 S \phi$ & & & & & \\
\hline AMBIENT TEN & {$[\circ \mathrm{F}$} & & $80^{\circ}$ & & & FLTER ID & NA & & CT X-SECTION & circ? & rect? & other: & & & \\
\hline BAR. PRESS. & $" \mathrm{Hg}]$ & & $0,4,40$ & & & KFACTOR & 4,42 NA & & DIMENSIONS & & DUCT AREA & & & & \\
\hline TRAVERSE & $\mathrm{CLOCK}$ & SAMPLE & STATIC & PITOT & METER DIFF & METER & METER & & $\frac{1}{R T E M P}$ & $\frac{1}{\text { STACK }}$ & PROBE & FILTER & $\frac{1}{\text { LAST MPP }}$ & METER & XYAUST \\
\hline POINT & TIME & TIME & PRES & HEAD & PRESSURE & VACUUM & READING & & of & TEMP & TEMP & BOX & TEMP & $\mathrm{O}_{2}$ & $\mathrm{CO}_{2}$ \\
\hline [port-probe] & (24-hr\}) & [minute] & {$\left[" \mathrm{H}_{2} \mathrm{O}\right]$} & {$\left[{ }^{[} \mathrm{H}_{2} \mathrm{O}\right]$} & {$\left[{ }^{n} \mathrm{H}_{2} \mathrm{O}\right]$} & {$[" \mathrm{Hg}]$} & {$\left[\mathrm{ft}^{3}\right]$} & inlet & outlet & {$[\mathrm{F}]$} & {$[\circ]$} & {$[\mathrm{F}]$} & {$[\mathrm{F}]$} & {$[\%$ vol $]$} & {$[\%$ vol $]$} \\
\hline 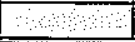 & & 0 & & & & & 780,41 & & & 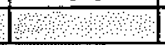 & & आ & & & \\
\hline NI i & & 5 & & 122 & .98 & 2 & 792.23 & 92 & 91 & 1668 & 243 & 263 & 54 & & \\
\hline NI? & & 10 & $-7,39$ & $: 22$ & .98 & 3 & 794.90 & 96 & 92 & 671 & 251 & 265 & 44 & 7,7 & 125 \\
\hline $\mathrm{N}^{2}$ & & 15 & & .18 & .50 & 3 & 797.38 & 98 & 92 & 1652 & 251 & 265 & 44 & & \\
\hline$N_{2}$ & & 20 & -7.379 & .15 & .80 & 3 & 799.81 & 99 & 93 & 651 & 249 & 266 & 44 & 6,3 & 13.8 \\
\hline$N^{3}: 3$ & & 25 & & .25 & 1.10 & 5 & 802.65 & 99 & 93 & 663 & 255 & 264 & 44 & & \\
\hline $\mathrm{N3}_{3}$ & & 30 & $-7,244$ & .25 & 1.10 & 6 & 805.50 & 100 & 94 & 662 & 254 & 265 & 45 & 5.9 & $M, 3$ \\
\hline$\therefore \quad$ & & 35 & & & & & & & & & & & & & \\
\hline 4 & & 40 & & P & 56 & & 506.85 & & & & & & & & \\
\hline 515 & & 45 & & 115 & .66 & 5 & 609.15 & 99 & $\sqrt{15}$ & 614 & 255 & 267 & 46 & & \\
\hline $4 / 5$ & & 50 & $-7,358$ & 15 & .66 & 5 & 810.39 & 99 & 94 & 618 & 246 & 267 & 45 & $9: 9$ & 10.4 \\
\hline 2216 & & 55 & & .26 & 1.15 & 8 & 814.23 & 99 & 94 & 630 & 255 & 266 & 45 & 2.6 & 12.6 \\
\hline $52 / 6$ & & 60 & -1.628 & .26 & 1.15 & 10 & 817,12 & 100 & 94 & 630 & 255 & 266 & 46 & 7.6 & 12.6 \\
\hline 53 & & & & 113 & .58 & 8 & 819,35 & 100 & 94 & 652 & 257 & 266 & 47 & 7.7 & 12,5 \\
\hline 5311 & & 65 & -7.463 & $: 17$ & .75 & 10 & 821.72 & 90 & 94 & 652 & 255 & 266 & 47 & 7.8 & 12.5 \\
\hline 1 & & 70 & & & & & & & & & & & & & \\
\hline 2 & & 75 & & & & & & & & & & & & & \\
\hline 2 & & 80 & & & & & & & & & & & & & \\
\hline 3 & & 85 & & & & & & & & & & & & & \\
\hline 3 & & 90 & & & & & & & & & & & & & \\
\hline 4 & & 95 & & & & & & & & & & & & & \\
\hline 14 & & 100 & & & & & & & & & & & & & \\
\hline 5 & & 105 & & & & & & & & & & & & & \\
\hline 5 & & 110 & & & & & & & & & & & & & \\
\hline 6 & & 115 & & 0.1899 & & & & & & & & & & & \\
\hline 6 & & 120 & & Recs & & & & & & & & & & & \\
\hline AVERAGE & & & -7.4 & artet & 0.89 & & 30.96 & 55 & & 641.8 & & & & 7.6 & 12.7 \\
\hline 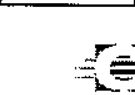 & & & $\begin{array}{l}\text { ample Train } \\
\text { eak Checks: }\end{array}$ & $\begin{array}{l}\text { Pre Test } \\
\text { Post Test }\end{array}$ & $\frac{O K}{O K} \mathrm{ft}^{3}$ & ${ }^{2}{ }^{i}$ & $\begin{array}{l}\text { in. } \mathrm{Hg} \\
\text { in. } \mathrm{Hg}\end{array}$ & & & $\begin{array}{r}\text { Pitot Tube } \\
\text { Leak Checks: }\end{array}$ & $\begin{array}{r}\text { Pret } \\
\text { Post } 7\end{array}$ & $\frac{O K}{O K} @$ & & $\begin{array}{l}\mathrm{H}_{2} \mathrm{O} \\
\mathrm{H}_{2} \mathrm{O}\end{array}$ & \\
\hline
\end{tabular}




\section{AMMONIA SAMPLING FIELD DATA SHEET}

\begin{tabular}{|c|c|c|c|c|c|c|c|c|c|c|c|c|c|c|c|}
\hline & & & & & & & & & & & & & & & \\
\hline TEST ID & & $\mathrm{NH}$ & $3-4$ & & & METER BOX & $N^{-3}$ & CAL. DA & ATA: delta $\mathrm{H}$ & 1.883 & Comments: & & & & \\
\hline PLANT & & & GREENIDC & & & TOT TUBE DESC & $|-1|$ & & & 0.991 & & & & & \\
\hline LOCATION & & & SCR OUTL & ET $h, 1)$ ud & & DBE LENGTH [fft] & 10 & & & .833 & & & & & \\
\hline DATE & & & $-1-07$ & & & NOZZLE ID [inch] & 313 & FILTER B & OOX SETTING & & & & & & \\
\hline OPERATOR(S) & & Pon & $=0$ & Emas UPS & $\% R P D$ & $\mathrm{KH}_{2} \mathrm{O}$ (Assumed) & 8 & PROBE H & ITR SETTING & 250 & & & & & \\
\hline AMBIENT TEM & & & 812 & & & FILTER ID & & Duc & TX-SECTION & circ? & rect? & other: & & & \\
\hline BAR. PRESS. & & & 0.40 & & & KFACTOR & $\dot{H}_{4} 42$ & DUCT & DIMENSIONS & & DUCT AREA & & & & \\
\hline & & & & & & & & 6 & & 1 & 2 & 5 & 4 & & \\
\hline \begin{tabular}{|l|} 
TRAVERSE \\
\end{tabular} & CLOCK & SAMPLE & \begin{tabular}{|l|} 
STATIC \\
\end{tabular} & PाTOT & METER DIFF & METER & METER & METER & TEMP & STACK & PROBE & FILTER & LAST IMP & METER & XHAUST \\
\hline POINT & TIME & TIME & PRES & HEAD & PRESSURE & VACUUM & READING & [of & & TEMP & TEMP & Box & TEMP & $\mathrm{O}_{2}$ & $\mathrm{CO}_{2}$ \\
\hline [port-inch] & (24-hr) & [minute] & {$\left[" \mathrm{H}_{2} \mathrm{O}\right]$} & {$\left[\mathrm{H}_{2} \mathrm{O}\right]$} & {$\left[" \mathrm{H}_{2} \mathrm{O}\right]$} & [" $\mathrm{Hg}]$ & & inlet & outlet & {$[\mathrm{F}]$} & {$[\mathrm{F}]$} & {$[\mathrm{F}]$} & {$[\mathrm{F}]$} & {$[\%$ vol] } & {$[\%$ vol $]$} \\
\hline & & 0 & 17. & , & & ר. & 828.00 & $x_{0}$ & 4.9 & & & & & & \\
\hline $1-N 1$ & & 50 & -7596 & .23 & 1.02 & 2 & 830.88 & 93 & 91 & 672 & 234 & 237 & 5.5 & & \\
\hline $1-8 \mathrm{~N}$ & & 1000 & & .23 & 1.02 & 2 & 83353 & 95 & 92 & 677 & 253 & 240 & 49 & 7.4 & 12.5 \\
\hline$\Leftrightarrow N 2$ & & $15=30$ & $-7,488$ & .19 & .85 & 2 & 836.18 & 98 & 92 & 658 & 251 & 248 & 46 & & \\
\hline 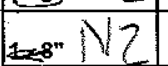 & & 200 & & .19 & .85 & 2 & 838.61 & 98 & 93 & 655 & 254 & 249 & 46 & 6.4 & 13,7 \\
\hline $1 N^{3}$ & & $25 \%$ & $-7,614$ & .25 & 1.10 & 5 & 841.40 & 99 & 93 & 668 & 249 & 243 & 46 & & \\
\hline$\Rightarrow N^{3}$ & & 3000 & & .25 & 1.10 & 5 & 844.16 & 99 & 94 & 672 & 257 & 244 & 46 & 5.4 & 14.7 \\
\hline & & & & & & & & & & & & & & & \\
\hline & & & & & & & 845.65 & 98 & & & & & & & \\
\hline 51 & & 35 & -7.474 & 115 & 17,68 & 5 & 840.05 & 98 & 94 & 618 & 253 & 243 & 48 & & \\
\hline 51 & & 40 & & .15 & .68 & 5 & 850.31 & 98 & 94 & 621 & 250 & 244 & 46 & 9.6 & 10.6 \\
\hline 52 & & 45 & -7.53 & .28 & 1.25 & 9 & 753,23 & 99 & 94 & 635 & 257 & 245 & 45 & & \\
\hline 52 & & 50 & & .28 & 1.25 & 10 & 856.21 & 100 & 94 & 635 & 257 & 245 & 45 & $7.4 x$ & 12.8 \\
\hline 53 & & 55 & $-7,260$ & 19 & .86 & 10 & 858.75 & 100 & 94 & 653 & 248 & 245 & 46 & & \\
\hline $5-3$ & & 60 & & .19 & .86 & 12 & 861.29 & 99 & 94 & 653 & 255 & 245 & 46 & 7.4 & 12.8 \\
\hline & & & & & & & & & & & & $3:$ & & & \\
\hline & & & & & & & & & & & & & & & \\
\hline & & & & & & & & & & & & & & & \\
\hline & & & & & & & & & & & & & & & \\
\hline & & & & & & & & & & & & & & & \\
\hline & & & & & & & & & & & & & & & \\
\hline AVERAGE & & & -7.49 & 0.213 & 0.96 & & 31.80 & 85.6 & & 651.4 & & & & $2 \cdot 3$ & 12.9 \\
\hline z & & & ample Train & $\begin{array}{l}\text { Pre Tes } \\
\text { Post Tes }\end{array}$ & $\mathrm{ft}$ & $@$ & $\begin{array}{l}\text { in. } \mathrm{Hg} \\
\text { in. } \mathrm{Hg}\end{array}$ & & & $\begin{array}{l}\text { Pitot Tube } \\
\text { Leak Checks: }\end{array}$ & $\begin{array}{l}\text { PreTest } \\
\text { Post Test }\end{array}$ & $+{ }^{\infty}$ & in. & $\begin{array}{l}\mathrm{H}_{2} \mathrm{O} \\
\mathrm{H}_{2} \mathrm{O}\end{array}$ & \\
\hline
\end{tabular}




\section{AMMONIA SAMPLING FIELD DATA SHEET}

i.

TESTID

PLANT

LOCATION

DATE

OPERATOR(S)

AMBIENT TEMP $\left[{ }^{\circ} \mathrm{F}\right.$

BAR." PRESS. [" $\mathrm{Hg}]$

B.

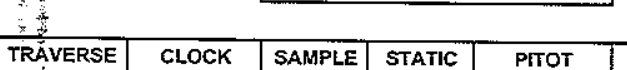

\begin{tabular}{c|c|c|c|c|c|c|c}
\hline TRAVERSE & CLOCK & SAMPLE & STATIC & PITOT & METER DIFF & METER & METER \\
POINT & TIME & TMME & PRES & HEAD & PRESSURE & VACUUM & READING \\
\hline
\end{tabular}

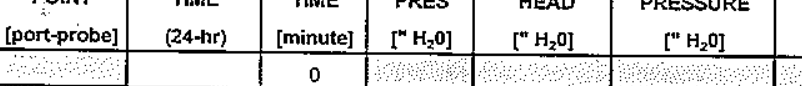

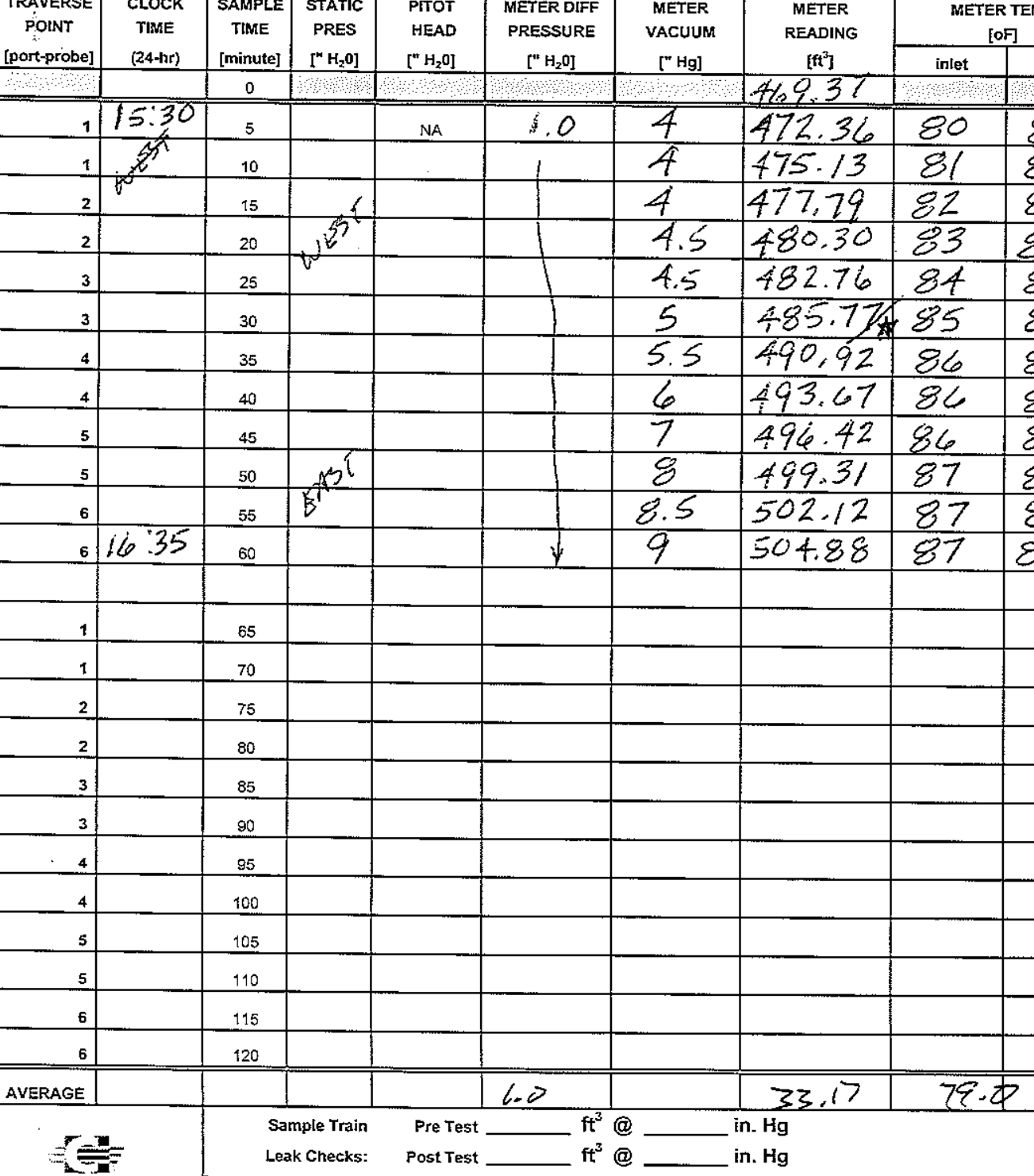

CONSOLENERGY

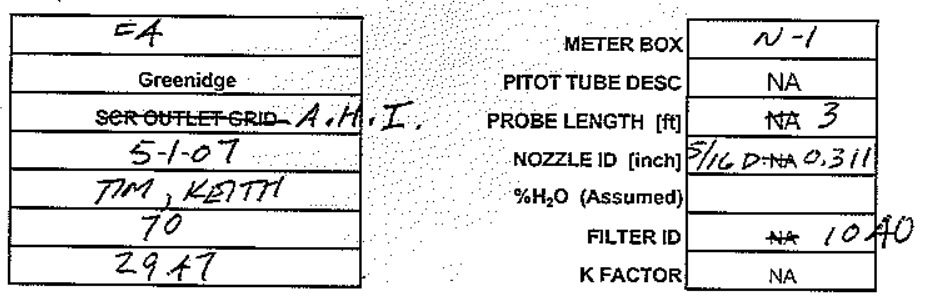

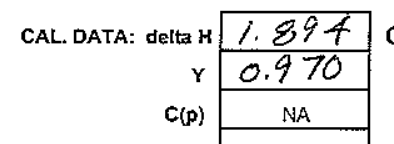

\begin{tabular}{ll|} 
FILTER BOX SETTING & NA \\
\cline { 2 - 3 } PROBE HTR SETTING & AA 270
\end{tabular}

\begin{tabular}{rl|l} 
DUCT X-SECTION & circ? & r \\
\cline { 2 - 3 } DUCT DIMENSIONS & & DUCT
\end{tabular} DUCT AREA
$Z$

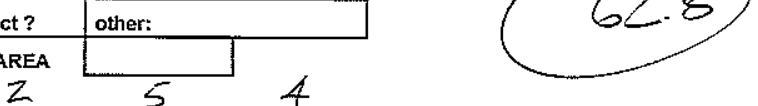

4

Page 1 of 1

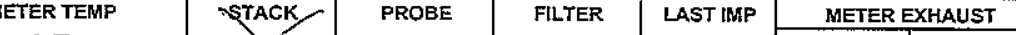

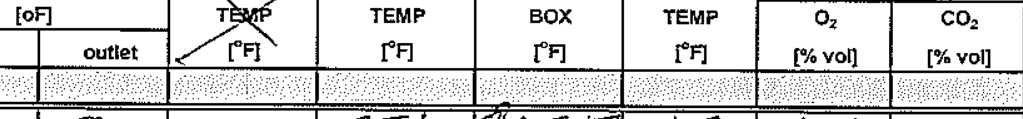

\begin{tabular}{|l|l|l|l|l|}
\hline 251 & 627 & 62 & 6.8 & 13.4 \\
\hline 249 & 249 & 57 & 6.5 & 13.7 \\
\hline 249 & 251 & 58 & 6.7 & 13.5 \\
\hline 250 & 251 & 60 & 6.5 & 13.8 \\
\hline 250 & 252 & 61 & 6.6 & 13.6 \\
\hline 250 & 252 & 61 & 6.3 & 13.9 \\
\hline 249 & 251 & 61 & 5.4 & 14.9 \\
\hline
\end{tabular} TIME OF Z INDORT




\begin{tabular}{|c|c|c|c|c|c|c|}
\hline $\begin{array}{l}\text { DATE } \\
\text { START TIME } \\
\text { END TIME } \\
\text { RUN } \\
\end{array}$ & $\begin{array}{c}03 / 29 / 07 \\
1000 \\
1040 \\
\text { AHO-1 } \\
\end{array}$ & $\begin{array}{c}03 / 29 / 07 \\
1003 \\
1103 \\
\text { STK-1 } \\
\end{array}$ & $\begin{array}{c}03 / 29 / 07 \\
1220 \\
1300 \\
\text { AHO-2 } \\
\end{array}$ & $\begin{array}{c}03 / 29 / 07 \\
1240 \\
1330 \\
\text { STK-2 } \\
\end{array}$ & $\begin{array}{c}03 / 29 / 07 \\
1515 \\
1555 \\
\text { AHO-3 } \\
\end{array}$ & $\begin{array}{c}03 / 29 / 07 \\
1517 \\
1617 \\
\text { STK-3 } \\
\end{array}$ \\
\hline $\begin{array}{l}\text { Titration Inform } \\
\text { FILTER PLUG: } \\
\text { NORMALITY } \\
\text { VOLUME } \\
\text { ALIQUOT } \\
\text { TITRANT } \\
\end{array}$ & $\begin{array}{r}0.0095 \\
100 \\
10.0 \\
0.20 \\
\end{array}$ & $\begin{array}{r}0.0095 \\
100 \\
10.0 \\
0.10 \\
\end{array}$ & $\begin{array}{r}0.0095 \\
100 \\
10.0 \\
0.20 \\
\end{array}$ & $\begin{array}{r}0.0095 \\
100 \\
10.0 \\
0.10 \\
\end{array}$ & $\begin{array}{r}0.0095 \\
100 \\
10.0 \\
0.15 \\
\end{array}$ & $\begin{array}{r}0.0095 \\
100 \\
10.0 \\
0.10 \\
\end{array}$ \\
\hline $\begin{array}{l}\text { PROBE: } \\
\text { NORMALITY } \\
\text { VOLUME } \\
\text { ALLQUOT } \\
\text { ALITRANT } \\
\end{array}$ & $\begin{array}{r}0.0095 \\
100 \\
10.0 \\
0.15 \\
\end{array}$ & $\begin{array}{r}0.0095 \\
100 \\
10.0 \\
0.10 \\
\end{array}$ & $\begin{array}{r}0.0095 \\
100 \\
10.0 \\
0.20 \\
\end{array}$ & $\begin{array}{r}0.0095 \\
100 \\
10.0 \\
0.10 \\
\end{array}$ & $\begin{array}{r}0.0095 \\
100 \\
10.0 \\
0.20 \\
\end{array}$ & $\begin{array}{r}0.0095 \\
100 \\
10.0 \\
0.10 \\
\end{array}$ \\
\hline $\begin{array}{l}\text { CONDENSER: } \\
\text { VOLUME } \\
\text { ALIQUOT } \\
\text { TITRANT }\end{array}$ & $\begin{array}{r}100 \\
10.0 \\
0.30\end{array}$ & $\begin{array}{r}100 \\
10.0 \\
0.15\end{array}$ & $\begin{array}{r}100 \\
10.0 \\
0.35\end{array}$ & $\begin{array}{r}100 \\
10.0 \\
0.10\end{array}$ & $\begin{array}{r}100 \\
10.0 \\
0.28\end{array}$ & $\begin{array}{r}100 \\
10.0 \\
0.13\end{array}$ \\
\hline
\end{tabular}

AES Greenidge Turbosorp $\mathrm{SO}_{3}$ Sampling Results Guarantee Testing - March 29, 2007

\begin{tabular}{|c|c|c|c|c|c|c|}
\hline $\begin{array}{l}\text { DATE } \\
\text { START TIME } \\
\text { END TIME } \\
\text { RUN }\end{array}$ & $\begin{array}{c}3 / 29 / 2007 \\
1000 \\
1040 \\
\mathrm{AHO}-1 \\
\end{array}$ & $\begin{array}{c}3 / 29 / 2007 \\
1003 \\
1103 \\
\text { STK-1 } \\
\end{array}$ & $\begin{array}{c}3 / 29 / 2007 \\
1220 \\
1300 \\
\text { AHO-2 } \\
\end{array}$ & $\begin{array}{c}3 / 29 / 2007 \\
1240 \\
1330 \\
\text { STK-2 } \\
\end{array}$ & $\begin{array}{c}3 / 29 / 2007 \\
1515 \\
1555 \\
\mathrm{AHO}-3 \\
\end{array}$ & $\begin{array}{c}3 / 29 / 2007 \\
1517 \\
1617 \\
\text { STK-3 } \\
\end{array}$ \\
\hline \multicolumn{7}{|l|}{ 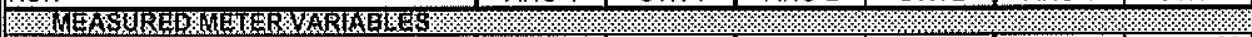 } \\
\hline $\begin{array}{l}\text { SAMPLE TIME [Minules] } \\
\text { BAROMETRIC PRESSURE [" Hg] }\end{array}$ & $\begin{array}{r}40 \\
30.06\end{array}$ & $\begin{array}{r}60 \\
30.06\end{array}$ & $\begin{array}{r}40 \\
30.03\end{array}$ & $\begin{array}{r}60 \\
30.03\end{array}$ & $\begin{array}{r}40 \\
29.97\end{array}$ & $\begin{array}{r}60 \\
29.97\end{array}$ \\
\hline SAMPLE VOLUME $\left(\mathrm{ft}^{3}\right)$ & 3.77 & 5.94 & 3.82 & 4.81 & 4.02 & 5.82 \\
\hline METER TEMPERATURE $\left[{ }^{\circ} \mathrm{F}\right]$ & 41.1 & 56.6 & 45.8 & 59.0 & 50.0 & 74.4 \\
\hline ORIFICE PRESSURE [" $\mathrm{H}_{2} \mathrm{O}$ ] (assumed) & 0.20 & 0.20 & 0.20 & 0.20 & 0.20 & 0.20 \\
\hline $\begin{array}{l}\text { Y FACTOR } \\
\text { DSCF SAMPLED }\end{array}$ & $\begin{array}{l}0.983 \\
3.922\end{array}$ & $\begin{array}{l}1.046 \\
6.385\end{array}$ & $\begin{array}{l}0.983 \\
3.929\end{array}$ & $\begin{array}{l}1.046 \\
5.132\end{array}$ & $\begin{array}{l}0.983 \\
4.097\end{array}$ & $\begin{array}{l}1.046 \\
6.021\end{array}$ \\
\hline APPROX CONDENSER TEMP $\left.{ }^{\circ} \mathrm{F}\right]$ & 142 & 151 & 141 & 149 & 141 & 155 \\
\hline DUCT STATIC PRESSURE, in $\mathrm{H}_{2} \mathrm{O}$ & -13.60 & -0.55 & -13.50 & -0.56 & -13.10 & -0.57 \\
\hline DUCT PR & 29.06 & 30.02 & 29.04 & 29.99 & 29.01 & 29.93 \\
\hline DUCT MC & 7.20 & 10.60 & $\begin{array}{l}7.70 \\
7.10\end{array}$ & $\begin{array}{r}11.00 \\
8.50\end{array}$ & $\begin{array}{r}7.80 \\
7.25\end{array}$ & $\begin{array}{l}10.50 \\
8.94\end{array}$ \\
\hline DUCT OXYGEN [\%] & 7.20 & 9.16 & 7.10 & 8.50 & 7.25 & 8.94 \\
\hline DUCT TEMP DURING TEST $\left[{ }^{\circ} \mathrm{F}\right]$ & 304.0 & 186.3 & 307.3 & 185.6 & 306.3 & 187.5 \\
\hline \multicolumn{7}{|l|}{ W $\%$ wo } \\
\hline \multicolumn{7}{|l|}{$\mathrm{SO}_{3} \ln$ FILTER PLUG } \\
\hline $\begin{array}{l}\mathrm{Ib} / \mathrm{DSCF} \\
\text { PPMYD }\end{array}$ & 4.27E-07 & $\begin{array}{r}1.31 E-07 \\
0.6\end{array}$ & $\begin{array}{r}4.26 \mathrm{E}-07 \\
21\end{array}$ & $\begin{array}{r}1.63 E-07 \\
0.8\end{array}$ & $\begin{array}{r}3.07 E-07 \\
1.5\end{array}$ & $\begin{array}{r}1.39 E-07 \\
0.7\end{array}$ \\
\hline PPMVD, @ 3\% Oxygen & 2.7 & $\begin{array}{l}0.0 \\
1.0\end{array}$ & 2.7 & 1.1 & 1.9 & 1.0 \\
\hline $\mathrm{SO}_{3}$ in PROBE & & & & & & \\
\hline $\mathrm{Ib} / \mathrm{OSCF}$ & $3.20 \mathrm{E}-07$ & 1.31E-07 & 4.26E-07 & $1.63 E-07$ & $4.09 \mathrm{E}-07$ & $1.39 \mathrm{E}-07$ \\
\hline PPMVD, As Sampled & & & 2.1 & 0.8 & 2.0 & 0.7 \\
\hline PPMVD, @3\% Oxygen & 2.0 & 1.0 & 2.7 & 1.1 & 2.6 & 1.0 \\
\hline \multicolumn{7}{|l|}{$\mathrm{SO}_{3}$ in CONDENSER } \\
\hline $\mathrm{Ib} / \mathrm{DSCF}$ & $6.41 \mathrm{E}-07$ & $1.97 \mathrm{E}-07$ & 7.46E-07 & 1.63E.07 & $5.62 \mathrm{E}-07$ & $1.74 \mathrm{E}-07$ \\
\hline $\begin{array}{l}\text { PPMVD, As Sampled } \\
\text { PPMVD, @ 3\% Oxygen }\end{array}$ & $\begin{array}{l}3.1 \\
4.0\end{array}$ & $\begin{array}{l}1.0 \\
1.5\end{array}$ & $\begin{array}{l}3.6 \\
4.7\end{array}$ & & $\begin{array}{l}2.7 \\
3.6\end{array}$ & $\begin{array}{l}0.8 \\
1.3\end{array}$ \\
\hline GAS PHASE $\mathrm{SO}_{3}$ [[b/DSCF] & $9.61 E-07$ & $3.28 \mathrm{E}-07$ & 1.17E-06 & 3.27E-07 & $9.71 \mathrm{E}-07$ & 3.13E-07 \\
\hline GAS PHASE $\mathrm{SO}_{3}$ [As Sampled PPMVD] & 4.6 & 1.6 & 5.7 & 1,6 & 4.7 & 1.5 \\
\hline GAS PHASE $\mathrm{SO}_{3}$, PPMVD @ $3 \% \mathrm{O}_{2}$ & 6.1 & 2.4 & 7.4 & 2,3 & 6.2 & 2.3 \\
\hline TOTAL PHASE SO ${ }_{3}$ [Ib/DSCF] & 1.39E-06 & $4.59 \mathrm{E}-07$ & $1.60 \mathrm{E}-06$ & $4.90 \mathrm{E}-07$ & $1.28 \mathrm{E}-06$ & 4.52E-07 \\
\hline TOTAL PHASE $\mathrm{SO}_{3}$ (AS Sampled PPMVD & 6.7 & 2.2 & 7.7 & 2.4 & 6.2 & 2.2 \\
\hline \multirow{2}{*}{$\begin{array}{l}\text { TOTAL } \mathrm{SO}_{3}, \text { PPMVD @ } 3 \% \mathrm{O}_{2} \\
\% \mathrm{SO}_{3} \text { in SOLIDS [filter plug/total] }\end{array}$} & 8.8 & 3.4 & 10.0 & 3.4 & 8.1 & 3.3 \\
\hline & 30.8 & 28.6 & 26.7 & 33.3 & 24.0 & 30.8 \\
\hline \multicolumn{7}{|l|}{ DEW POINT DETERMINATION } \\
\hline Partial Pressure $\mathrm{H}_{2} \mathrm{O}, \mathrm{mm}$ & 53.14 & 80.83 & 56.79 & 83.79 & 57.47 & 79.82 \\
\hline Partial Pressure $\mathrm{SO}_{3}, \mathrm{mmHg}$ & 0.0050 & 0.0017 & 0.0057 & 0.0018 & 0.0046 & 0.0017 \\
\hline Calculated $\mathrm{SO}_{3}$ Dew Point, ${ }^{\circ} \mathrm{F}$ & 265 & 255 & 269 & 256 & 265 & 254 \\
\hline
\end{tabular}

NOTE: The $\% \mathrm{O}_{2}$ at the air heater outlet was measured by CONSOL using a Teledyne Max 5 porlable electrochemical $\mathrm{O}_{2}$ analyzer, and the $\% \mathrm{O}_{2}$ at the stack was calculated from the $\% \mathrm{CO}_{2}$ measured by the plant's stack CEM and from the coal compostion. 
$\mathrm{SO}_{3} \mathrm{~F} \| \mathrm{ELD} S \mathrm{SM}$ LING DATA SHEET

PLANT

LOCATION

DUCT DIMENSIONS

DUCT AREA

DATE $Q$

SAMPLE BOX

METER BOX

PITOT TUBE DESC

OPERATOR(S)

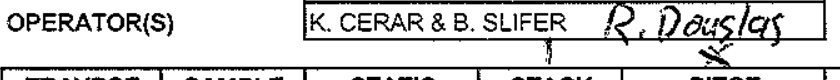

\begin{tabular}{|c|c|c|c|c|c|c|c|c|c|c|c|c|c|c|}
\hline \multicolumn{7}{|c|}{ OPERATOR(S) } & \multicolumn{2}{|c|}{ (Assumed= } & \multicolumn{2}{|l|}{3} & & \multicolumn{2}{|r|}{$x$} \\
\hline \multirow{3}{*}{\begin{tabular}{|c|} 
TRAVRSE \\
POINT \\
[inch]
\end{tabular}} & \multirow{3}{*}{$\begin{array}{l}\text { SAMPLE } \\
\text { TIME } \\
\text { [minute] }\end{array}$} & \multirow{3}{*}{$\begin{array}{c}\text { STATIC } \\
\text { PRESSURE } \\
{\left[" \mathrm{H}_{2} \mathrm{O}\right]} \\
\end{array}$} & \multirow{3}{*}{$\begin{array}{c}\text { STACK } \\
\text { TEMP } \\
\text { [ं. }\end{array}$} & \multirow{3}{*}{$\begin{array}{l}\text { PITOT } \\
\text { HEAD } \\
{\left[" \mathrm{H}_{2} \mathrm{O}\right]}\end{array}$} & \multirow{3}{*}{$\begin{array}{l}\text { ROTOMETER } \\
\text { SETTING }\end{array}$} & \multirow{3}{*}{$\begin{array}{c}\text { METER } \\
\text { READING } \\
{\left[\mathrm{ft}^{3}\right]}\end{array}$} & \multirow{2}{*}{\multicolumn{2}{|c|}{$\begin{array}{cc}\text { METER TEMP } \\
{[\mathrm{F}] \quad}\end{array}$}} & \multirow{3}{*}{$\begin{array}{c}\text { CONDENSER } \\
\text { TEMP } \\
{[\mathrm{F}]}\end{array}$} & \multirow{3}{*}{$\begin{array}{c}\text { PROBE } \\
\text { TEMP } \\
\text { ['F] }\end{array}$} & \multirow{3}{*}{$\begin{array}{c}\text { METER } \\
\text { VACUUM } \\
{[" \mathrm{Hg}]}\end{array}$} & \multirow{3}{*}{$\begin{array}{c}\mathrm{O}_{2} \\
\text { METER } \\
{[\%]}\end{array}$} & \multicolumn{2}{|c|}{ CONTROL ROOM } \\
\hline & & & & & & & & & & & & & $\mathrm{O}_{2}$ & DUCT TEMP \\
\hline & & & & & & & inlet & outlet & & & & & {$[\%]$} & [०F] \\
\hline & W & & & & & 43424 & & & & & & & & \\
\hline 8 & $0-10$ & -.503 & 187 & & 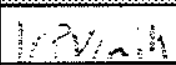 & $1.35,22$ & 55 & 54 & 143 & 529 & 9 & 81,6 & & \\
\hline $8^{\circ}$ & $10-20$ & & 186 & & ( & $2,36,21$ & 57 & 54 & 154 & 551 & 11 & 9.5 & & \\
\hline 8 & $20-30$ & -.57 & $\$ \delta \hat{6}$ & & & 437,29 & 158 & 55 & 157 & $5 \%$ & 1 & $\times 2.9 .6$ & & \\
\hline 8 & $30-40$ & & 186 & & & +138.26 & 58 & 56 & 151 & 561 & 1 & 9.6 & & . \\
\hline $8^{\circ}$ & $40-50$ & & 186 & & & 434.15 & 59 & 57 & 149 & $56<$ & 11 & 9,8 & & \\
\hline 8. & $50-60$ & $-i \delta \sigma_{i}$ & 187 & & & $40_{i} 19$ & 459 & 57 & 153 & 561 & 11 & 9,6 & & \\
\hline & & & & & & & & & & & & & & \\
\hline & & & & & & & & & & & . & & & \\
\hline & & & & & & & & & & & & & & \\
\hline & & & & & & & & & & & & & & \\
\hline AVERAGE & & $-0.5 \mathrm{~B}$ & 186.3 & & & 5.944 & 56. & 6 & 151.2 & & & 9.6 & & \\
\hline REMARKS & & Pre-Leak C & eck: & & & & & & Post-Leak & eck: & & & & \\
\hline
\end{tabular}

Condenser Temp $=140^{\circ} \mathrm{F}$

Sampling Rate $=3 \mathrm{lpm}=0.1 \mathrm{ft}^{3} / \mathrm{min}$

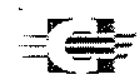

CONSOL ENERGY

GREENIDGE

STACK Test \#l

$3-2 E-i ; ?$

Start- s003Stop- 1103

NuTech \# M

T-UOF] 40

BAROMETRIC PRESSURE [" Hg] 30.06

$\% \mathrm{H}_{2} \mathrm{O}$ (Assumed) $1 \mathrm{O} / \mathrm{s}$

PROBE LENGTH [ft] 8

NOZZLE ID [inch] $\quad X X X X$

CALIBRATION FACTORS: delta H $\mathrm{XXXX}$

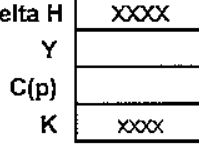

WATER BATH SETTING
PROBE HTR SETING

DUCT X-SECTION

POSITION OF PORT A

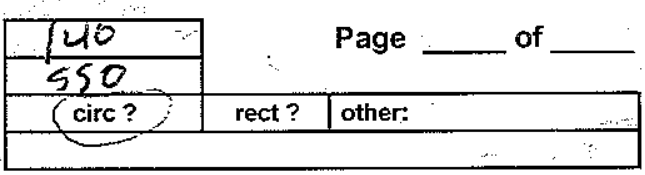

DRY MOLECULAR WEIGHT (Assumed)

WET MOLECULAR WEIGHT (Assumed)

$?$

\begin{tabular}{|l|l|l|l|l|l|l|}
\cline { 2 - 7 } \multicolumn{2}{c|}{} & ALIQUOT/ VOLUME & TITKATION (ml) & Ib/dscf & ppmv,act & ppmv,cor \\
\hline PLUG-SO & & & & & & \\
\hline PROBE-SO & & & & & & \\
\hline CONDENSER-SO & & & & & & \\
\hline BLANK & & & & & & \\
\hline MPINGER $\left(\mathrm{H}_{2} \mathrm{O}_{2}\right)-\mathrm{SO}_{2}$ & & & & & & \\
\hline
\end{tabular}

Gas Vol, dscf

$\mathrm{BaCl}_{2}$ NORMALITY 


\section{$\mathrm{SO}_{3}$ FIELD SAMPLING DATA SHEET}

PLANT

LOCATION

DUCT DIMENSIONS

DUCT AREA

DATE

TIME

SAMPLE BOX

METER BOX

PITOT TUBE DESC

OPERATOR(S)

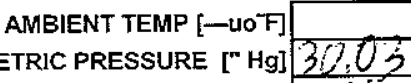

$\% \mathrm{H}_{2} \mathrm{O}$ (Assumed) 10 \%

PROBE LENGTH [ft] 8

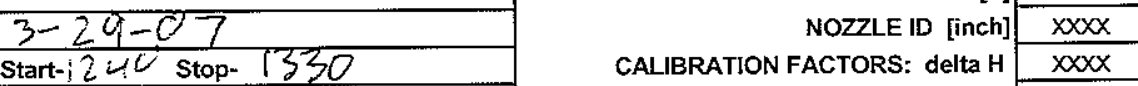

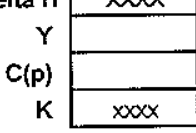

WATER BATH SETTING

PROBE HTR SETTING $S S 0$

DUCT X-SECTION

POSITION OF PORT A

DRY MOLECULAR WEIGHT (Assumed)

WET MOLECULAR WEIGHT (Assumed)

11.0

(Assumed $=$

\begin{tabular}{|c|c|c|c|c|c|c|c|c|}
\hline & & & \multirow{4}{*}{$\begin{array}{c}\text { CONDENSER } \\
\text { TEMP } \\
\text { [F] }\end{array}$} & \multirow{4}{*}{$\begin{array}{c}\text { PROBE } \\
\text { TEMP } \\
\text { [F] }\end{array}$} & \multirow{4}{*}{$\begin{array}{c}\text { METER } \\
\text { VACUUM } \\
\text { [" Hg] }\end{array}$} & \multirow{4}{*}{$\begin{array}{c}\mathrm{O}_{2} \\
\text { METER } \\
{[\%]}\end{array}$} & \multirow{2}{*}{\multicolumn{2}{|c|}{ CONTROL ROOM }} \\
\hline \multirow{3}{*}{$\begin{array}{c}\text { METER } \\
\text { READING } \\
{\left[\mathrm{ft}^{3}\right]}\end{array}$} & \multirow{2}{*}{\multicolumn{2}{|c|}{$\begin{array}{c}\text { METER TEMP } \\
\text { [ } \mathrm{F}] \\
\end{array}$}} & & & & & & \\
\hline & & & & & & & \multirow{2}{*}{$\begin{array}{c}\mathrm{O}_{2} \\
{[\%]}\end{array}$} & \multirow{2}{*}{$\begin{array}{c}\text { DUCT TEMP } \\
\left.{ }^{\circ} \mathrm{F}\right]\end{array}$} \\
\hline & inlet & outlet & & & & & & \\
\hline
\end{tabular}

\begin{tabular}{c|c|}
\hline ROTOMETER \\
SETTING
\end{tabular}

8

8

\begin{tabular}{l|l|l|l|l|l|l|}
\hline & $50-60$ & & & & \\
\hline & & & & & & \\
\hline & & & & & & \\
\hline & & & & & & \\
\hline & & & & & & \\
\hline AVERAGE & & & 185.6 & & & 4 \\
\hline
\end{tabular}

Pre-Leak Check:

\begin{tabular}{|c|c|c|c|c|c|c|c|c|c|}
\hline $\operatorname{lr}(\mathrm{v} / \mathrm{min}$ & 441.75 & 58 & 58 & 144 & 560 & 1 & 9.5 & & \\
\hline & 442.61 & 59 & 59 & 147 & 554 & 11 & 9.3 & & \\
\hline & 143.67 & 60 & 58 & $\mid 51$ & 562 & 11 & 9.4 & & \\
\hline & 411.50 & 60 & 98 & 153 & 554 & j1 & 9,4 & & \\
\hline & $4: 5.505$ & 6) & 59 & 15) & 565 & 11 & 9.6 & & \\
\hline & & & & & & & & & \\
\hline & & & & & & & & & \\
\hline & & & & & & & & & \\
\hline & & & & & & & & & \\
\hline & & & & & & & & & \\
\hline & 4.805 & 59.6 & 58.4 & 149.2 & 559.0 & & 9.44 & & \\
\hline
\end{tabular}

Condenser Temp $=140^{\circ} \mathrm{F}$

Sampling Rate $=3 \mathrm{lpm}=0.1 \mathrm{ft}^{3} / \mathrm{min}$

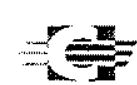

CONSOL ENERGY

\begin{tabular}{|l|l|l|l|l|l|l|}
\cline { 2 - 7 } \multicolumn{1}{c|}{} & ALIQUOT/ VOLUME & TITRATION (ml) & lb/dscf & ppmv,act & ppmv,cor \\
\hline PLUG-SO & & & & & & \\
\hline PROBE--SO & & & & & & \\
\hline CONDENSER-SO & & & & & & \\
\hline BLANK & & & & & & \\
\hline IMPINGER $\left(\mathrm{H}_{2} \mathrm{O}_{2}\right)-\mathrm{SO}_{2}$ & & & & & & \\
\hline
\end{tabular}

Gas Vol, dscf

$\mathrm{BaCl}_{2}$ NORMALITY

O:RdAppResiSTACKIdatasheetsISO3SHEET $03 / 20 / 07$ 
$\mathrm{SO}_{3}$ FIELD SAMPLING DATA SHEET

PLANT

LOCATION

DUCT DIMENSIONS

DUCT AREA

DATE

TIME

SAMPLE BOX

METER BOX

PITOT TUBE DESC

OPERATOR(S)

\begin{tabular}{|l|}
\hline GREENIDGE \\
\hline AIR HEATER-OUTLET \\
\hline \\
\hline $3 / 29 / 07$ \\
\hline Start- 1515 Stop- \\
\hline \\
\hline NuTech \#4 \\
\hline \\
\hline R. ODA \& D. OLSEN \\
\hline
\end{tabular}

AMBIENT TEMP [-UOF]

BAROMETRIC PRESSURE [" $\mathrm{Hg}] 29.97$

$\% \mathrm{H}_{2} \mathrm{O}$ (Assumed) 10

PROBE LENGTH [ft] 8

\begin{tabular}{r|l} 
NOZZLE ID [inch] & $X X X X$ \\
\cline { 2 - 2 } CALIBRATION FACTORS: delta $H$ & $X X X X$ \\
\cline { 2 - 2 } &
\end{tabular}

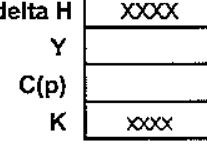

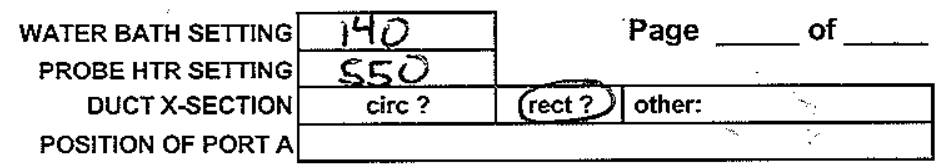

DRY MOLECULAR WEIGHT (Assumed)

1.8

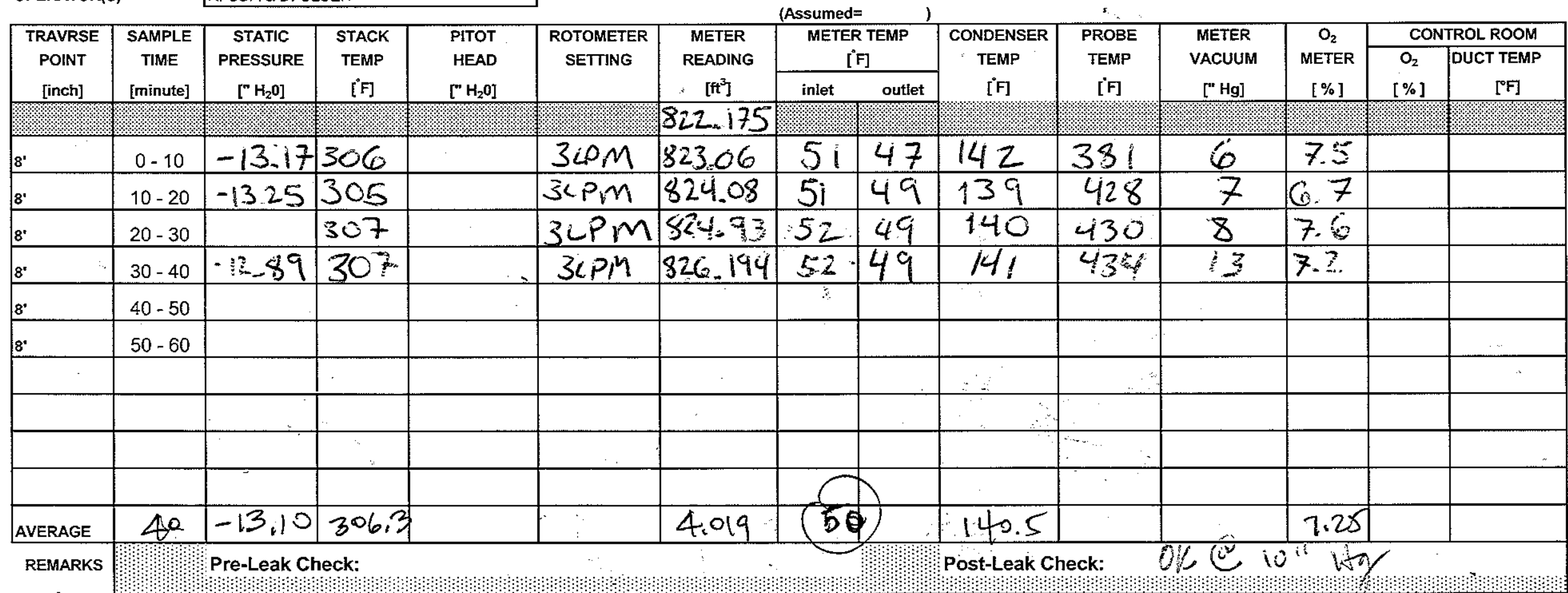

Condenser Temp $=140^{\circ} \mathrm{F}$

Sampling Rate $=3.1 \mathrm{pm}=0.1 \mathrm{ft}^{3} / \mathrm{min}$

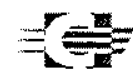

CONSOLENGRGY.

\begin{tabular}{|l|l|l|l|l|l|l|}
\cline { 2 - 7 } \multicolumn{1}{c|}{} & ALIQUOT / VOLUME & IITRATION (ml) & Ib/dscf & ppmv,act & ppmv,cor \\
\hline PLUG-SO & & & & & & \\
\hline PROBE-SO & & & & & & \\
\hline CONDENSER-SO & & & & & & \\
\hline BLANK & & & & & & \\
\hline IMPINGER $\left(\mathrm{H}_{2} \mathrm{O}_{2}\right)-\mathrm{SO}_{2}$ & & & & & & \\
\hline
\end{tabular}

Gas Vol, dscf

$\mathrm{BaCl}_{2}$ NORMALITY 


\section{$\mathrm{SO}_{3}$ FIELD SAMPLING DATA SHEET}

PLANT

LOCATION

DUCT DIMENSIONS

DUCT AREA

DATE

TIME

SAMPLE BOX

METER BOX

PITOT TUBE DESC

OPERATOR(S)

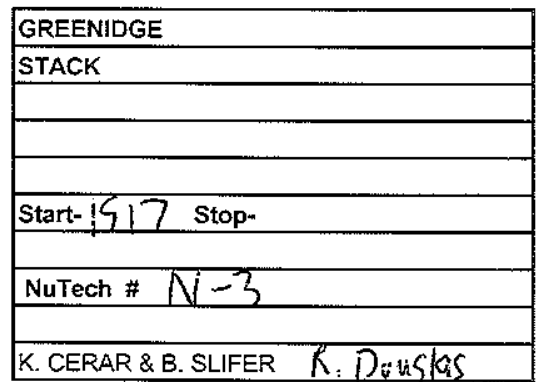

AMBIENT TEMP [- - uoF] BAROMETRIC PRESSURE [" Hg] 200

$\% \mathrm{H}_{2} \mathrm{O}$ (Assumed)

PROBE LENGTH [ft] $\frac{(1) ?}{8}$

NOZZLE ID [inch] $\quad X X X X$

CALIBRATION FACTORS: delta $\mathrm{H} X X X X$

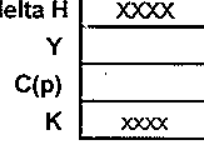

WATER BATH SETTING 140

PROBE HTR SETTING 5 SO

DUCT X-SECTION (circ 3

POSITION OF PORT

DRY MOLECULAR WEIGHT (Assumed)

10.5
WET MOLECULAR WEIGHT (Assumed)

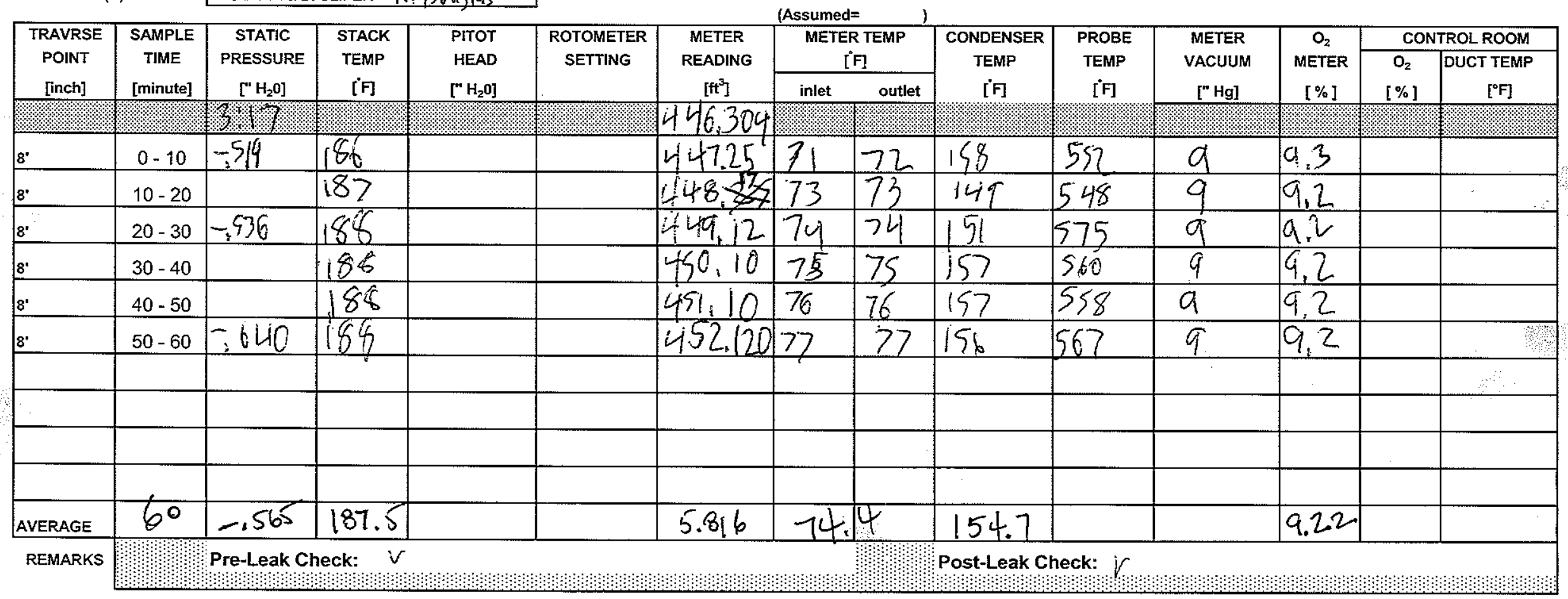

Condenser Temp $=140^{\circ} \mathrm{F}$

Sampling Rate $=3 \mathrm{lpm}=0.1 \mathrm{ft}^{3} / \mathrm{min}$

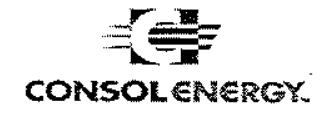

\begin{tabular}{|l|l|l|l|l|l|l|}
\cline { 2 - 7 } \multicolumn{1}{c|}{} & ALIQUOT/ VOLUME & TITRATION (m) & Ib/dscf & ppmv,act & ppmv,cor \\
\hline PLUG-SO & & & & & & \\
\hline PROBE-SO & & & & & & \\
\hline CONDENSER-SO & & & & & & \\
\hline BLANK & & & & & & \\
\hline IMPINGER $\left(\mathrm{H}_{2} \mathrm{O}_{2}\right)-\mathrm{SO}_{2}$ & & & & & & \\
\hline
\end{tabular}

Gas Vol, dscf

$\mathrm{BaCl}_{2}$ NORMALITY 
AES Greenldge Turbosorp $\mathrm{SO}_{3}$ Sampling Results

Guarantee Testing - May 2, 2007

\begin{tabular}{|c|c|c|c|c|c|c|}
\hline $\begin{array}{l}\text { DATE } \\
\text { START TIME } \\
\text { END TIME } \\
\text { RUN } \\
\end{array}$ & $\begin{array}{c}5 / 2 / 2007 \\
1050 \\
1202 \\
\text { AHO-1 }\end{array}$ & $\begin{array}{c}5 / 2 / 2007 \\
1044 \\
1215 \\
\text { STK-1 }\end{array}$ & $\begin{array}{c}5 / 2 / 2007 \\
1322 \\
1426 \\
\text { AHO-2 }\end{array}$ & $\begin{array}{c}5 / 2 / 2007 \\
1312 \\
1443 \\
\text { STK-2 }\end{array}$ & $\begin{array}{c}5 / 2 / 2007 \\
1527 \\
1635 \\
\text { AHO-3 }\end{array}$ & $\begin{array}{c}5 / 2 / 2007 \\
1517 \\
1648 \\
\text { STK-3 }\end{array}$ \\
\hline \multicolumn{7}{|l|}{ 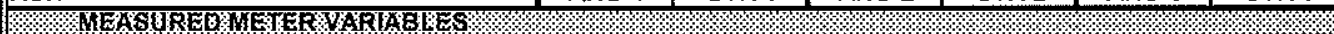 } \\
\hline $\begin{array}{l}\text { SAMPLE TIME [Minutes] } \\
\text { BAROMETRIC PRESSURE [" } \mathrm{Hal}\end{array}$ & $\begin{array}{r}60 \\
29.41\end{array}$ & $\begin{array}{r}90 \\
29.41\end{array}$ & $\begin{array}{r}60 \\
29.47\end{array}$ & $\begin{array}{r}90 \\
29.47\end{array}$ & $\begin{array}{r}60 \\
29.47\end{array}$ & $\begin{array}{r}90 \\
29.47\end{array}$ \\
\hline SAMPLE VOLUME $\left[\mathrm{ft}^{3}\right]$ & 5.53 & 9.70 & 5.73 & 9.04 & 5.89 & 9.39 \\
\hline METER TEMPERATURE $\left[{ }^{\circ} \mathrm{F}\right]$ & 62.2 & 63.5 & 67.2 & 73.2 & 66.5 & 78.7 \\
\hline |ORIFICE PRESSURE [" $\mathrm{H}_{2} \mathrm{O}$ ] (assumed) & 0.20 & 0.20 & 0.20 & 0.20 & 0.20 & 0.20 \\
\hline $\begin{array}{l}\text { Y FACTOR } \\
\text { DSCF SAMPLED }\end{array}$ & $\begin{array}{l}0.991 \\
5.447\end{array}$ & $\begin{array}{l}0.970 \\
9.330\end{array}$ & $\begin{array}{l}0.991 \\
5.597\end{array}$ & $\begin{array}{l}0.970 \\
8.551\end{array}$ & $\begin{array}{l}0.991 \\
5.766\end{array}$ & $\begin{array}{l}0.970 \\
8.789\end{array}$ \\
\hline APPROX CONDENSER TEMP $\left[{ }^{\circ} \mathrm{F}\right]$ & 143 & 140 & 144 & 141 & 146 & 140 \\
\hline $\begin{array}{l}\left.\text { WATER BATH TEMP [ }{ }^{\circ} \mathrm{F}\right] \\
\text { CCIMIN @ CONDENSER } \\
\text { DUCT STATIC PRESSURE, in } \mathrm{H}_{2} \mathrm{O}\end{array}$ & $\begin{array}{r}143 \\
2937\end{array}$ & $\begin{array}{r}140 \\
3335\end{array}$ & $\begin{array}{r}144 \\
3020\end{array}$ & $\begin{array}{r}141 \\
3061\end{array}$ & $\begin{array}{r}146 \\
3124\end{array}$ & $\begin{array}{r}140 \\
3144\end{array}$ \\
\hline $\begin{array}{l}\text { DUCT PRESSURE, " } \mathrm{Hg} \\
\text { DUCT MOISTURE, \% VOL }\end{array}$ & 29.41 & 29.41 & 29.47 & 29.47 & 29.47 & 29.47 \\
\hline DUCT OXYGEN [\%] (see note) & \multirow[t]{2}{*}{8.0} & 8.0 & 8.1 & 8.1 & 8.0 & 8.0 \\
\hline DUCT TEMP DURING TEST $\left[{ }^{\circ} \mathrm{F}\right]$ & & 172.1 & 299.5 & 172.0 & 306.0 & 172.0 \\
\hline \multicolumn{7}{|l|}{ \% } \\
\hline \multicolumn{7}{|l|}{$\mathrm{SO}_{3}$ in FILTER PLUG } \\
\hline Analytical Results: $\mathrm{mg} \mathrm{SO}_{4}{ }^{2-}$ & 3.02 & 0.41 & 2.27 & 0.03 & 3.39 & 0.11 \\
\hline PPMVD $\mathrm{SO}_{3}$ As Sampled & 4.9 & 0.4 & 3.6 & 0.0 & 5.2 & 0.1 \\
\hline $\begin{array}{l}\text { PPMVD SO } \mathrm{S}_{3} @ 3 \% \text { Oxygen } \\
\mathrm{SO}_{3} \text { In PROBE }\end{array}$ & 6.8 & 0.5 & 5.0 & 0.0 & 7.2 & 0.2 \\
\hline Analytical Results: $\mathrm{mg} \mathrm{SO}_{4}{ }^{2 \cdot}$ & 2.29 & 0.41 & 4.29 & 0.17 & 2.06 & 0.16 \\
\hline PPMVD $\mathrm{SO}_{3}$, As Sampled & 3.7 & 0.4 & 6.8 & 0.2 & 3.2 & 0.2 \\
\hline |PPMVD SO SO $_{3} @ 3 \%$ Oxygen & 5.2 & 0.5 & 9.5 & 0.2 & 4.4 & 0.2 \\
\hline \multicolumn{7}{|l|}{$\mathrm{SO}_{3}$ in CONDENSER } \\
\hline Analytical Results: $\mathrm{mg} \mathrm{SO}_{4}{ }^{2-}$ & 9.06 & 0.24 & 8.69 & 0.38 & 9.92 & 0.31 \\
\hline PPMVD $\mathrm{SO}_{3}$, As Sampled & 14.7 & 0.2 & 13.7 & 0.4 & 15.2 & 0.3 \\
\hline PPMVD SO ${ }_{31} @ 3 \%$ Oxygen & 20.4 & 0.3 & 19.2 & 0.5 & 21.1 & 0.4 \\
\hline GAS PHASE $\mathrm{SO}_{3}$ PPMVD as Sampled & 18.4 & 0.6 & 20.5 & 0.6 & 18.4 & 0.5 \\
\hline GAS PHASE $\mathrm{SO}_{3}$, PPMVD @ $3 \% \mathrm{O}_{2}$ & 25.5 & 0.8 & 28.7 & 0.8 & 25.4 & 0.7 \\
\hline TOTAL PHASE SO ${ }_{3}$, PPMVD as Sampled & 23.3 & 1.0 & 24.1 & 0.6 & 23.6 & 0.6 \\
\hline TOTAL PHASE $\mathrm{SO}_{3}$, PPMVD @ $3 \% \mathrm{O}_{2}$ & 32.3 & 1.4 & 33.7 & 0.8 & 32.6 & 0.8 \\
\hline$\% \mathrm{SO}_{3}$ in SOL.IDS [filter plug/total] & $21.0 \%$ & $38.6 \%$ & $14.9 \%$ & $5.2 \%$ & $22.1 \%$ & $19.0 \%$ \\
\hline
\end{tabular}

NOTE: The $\% \mathrm{O}_{2}$ at the stack was calculated from the $\% \mathrm{CO}_{2}$ measured by the plant's stack CEM and from the coal composition. $\mathrm{O}_{2}$ measurements at the air heater outlet were invalid; hence, the $\% \mathrm{O}_{2}$ at the air heater outlet was assumed to approximately equal the $\% \mathrm{O}_{2}$ at the stack. 
Ron One

$\mathrm{SO}_{3}$ FIELD SAMPLING DATA SHEET:

PLANT

LOCATION

DUCT DIMENSIONS

DUCT AREA

DATE

TIME

SAMPLE BOX

METER BOX

PITOT TUBE DESC

OPERATOR(S)
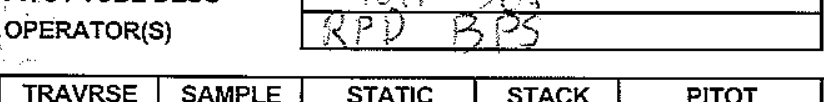

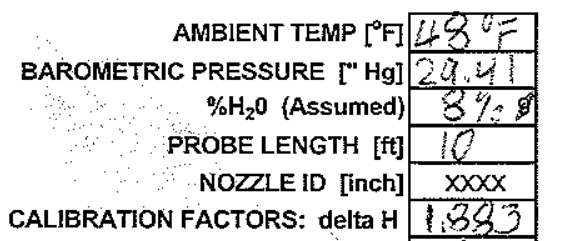

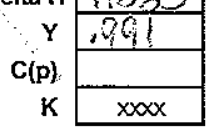

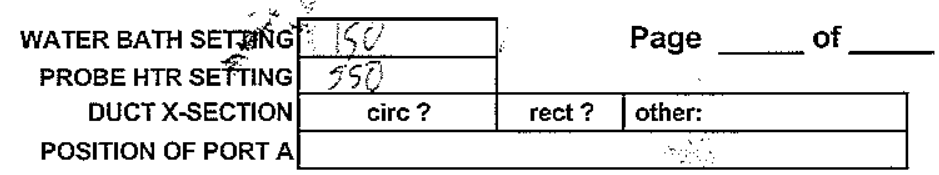

DRY MOLECULAR WEIGHT (Assumed)

WET MOLECULAR WEIGHT (Assumed)

\begin{tabular}{|c|c|c|c|c|c|c|c|c|c|c|c|c|c|c|c|}
\hline \multirow{4}{*}{$\begin{array}{l}\text { TRAVRSE } \\
24 \mathrm{POINT}^{2} \\
\text { [mch }^{2}\end{array}$} & \multirow{4}{*}{$\begin{array}{l}\text { SAMPLE } \\
\text { TIME } \\
\text { [minute] }\end{array}$} & \multirow{4}{*}{$\begin{array}{c}\text { STATIC } \\
\text { PRESSURE } \\
\left.\text { [" } \mathrm{H}_{2} \mathrm{O}\right]\end{array}$} & \multirow{4}{*}{$\begin{array}{l}\text { STACK } \\
\text { TEMP } \\
{\left[{ }^{\circ} \mathrm{F}\right]} \\
\end{array}$} & \multirow{4}{*}{$\begin{array}{l}\text { PITOT } \\
\text { HEAD } \\
{\left[" \mathrm{H}_{2} \mathrm{O}\right]} \\
\end{array}$} & \multirow{4}{*}{$\begin{array}{c}\text { ROTOMETER } \\
\text { SETTING }\end{array}$} & \multirow{4}{*}{$\begin{array}{c}\text { METER } \\
\text { READING } \\
{\left[\mathrm{ft}^{3}\right]} \\
\end{array}$} & \multicolumn{2}{|c|}{ (Assumed $=$ ) } & \multicolumn{7}{|c|}{ 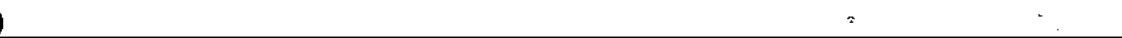 } \\
\hline & & & & & & & \multirow{2}{*}{\multicolumn{2}{|c|}{$\begin{array}{c}\text { METER TEMP } \\
{\left[{ }^{\circ} \mathrm{F}\right]} \\
\end{array}$}} & \multirow{3}{*}{$\begin{array}{c}\text { CONDENSER } \\
\text { TEMP } \\
{\left[{ }^{\circ} \mathrm{F}\right]}\end{array}$} & \multirow{3}{*}{$\begin{array}{c}\text { PROBE } \\
\text { TEMP } \\
{\left[{ }^{\circ} \mathrm{F}\right]}\end{array}$} & \multirow{3}{*}{$\begin{array}{c}\text { WATER BATH } \\
\text { TEMP } \\
\text { [₹] }\end{array}$} & \multirow{3}{*}{$\begin{array}{c}\text { METER } \\
\text { VACUUM } \\
{[" \mathrm{Hg}]}\end{array}$} & \multirow{3}{*}{$\begin{array}{c}\mathrm{O}_{2} \\
\text { METER } \\
{[\%]} \\
\end{array}$} & \multicolumn{2}{|c|}{ CONTROL ROOM } \\
\hline & & & & & & & & & & & & & & \multicolumn{2}{|c|}{\begin{tabular}{l|l}
$\mathrm{O}_{2}$ & DUCT TEMP \\
\end{tabular}} \\
\hline & & & & & & & inlet & outlet & & & & & & {$[\%]$} & [-UoF] \\
\hline $26^{\circ} / 0^{\prime}$ & & & & & & 86267 & & & & & & & & & \\
\hline$A B$ & $0-10$ & & & & & 608,26 & 60 & 63 & 1010 & 550 & NA & 10 & & & 5 \\
\hline$A-z$ & $10-20$ & & & & & 860,05 & 60 & 63 & 145 & 552 & & 13 & 12,7 & & \\
\hline$B-1$ & $20-30$ & & & & & 070.08 & 60 & 63 & 144 & fits & & 10 & 3.7 & & \\
\hline$B-2$ & $30-40$ & & & & & 67.10 & $6:$ & 63 & 11 & 35 & & 10 & 10.3 & & \\
\hline$K=1$ & $40-50$ & & & & & 872.15 & $6:$ & 65 & 114 & 655 & $\cdots$ & 10 & 196 & & \\
\hline-4 & $50-60$ & & & & & 273.15 & 62 & 65 & 43 & 943 & - & 11 & 10.5 & & 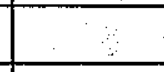 \\
\hline 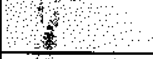 & & & & & & & & & & & & & & & \\
\hline s. & it pus & 15 & $\ln 7 n$ & hol & $62-1$ & & & & & & & & & & $\because$ \\
\hline (a) & & & & & & & & & & & & & & & \\
\hline 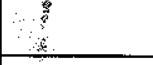 & & 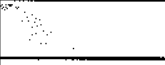 & & & & & & & & & & & & & \\
\hline AVERAGE & & & & & & 5.53 & 62 & 1.2 & 143.3 & & & $\cdots$ & 10.9 & & \\
\hline REMARKS & & Pre-Leak C & neck: $f$ & & & & & & Post-Leak C & heck: & & & 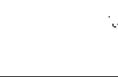 & & \\
\hline
\end{tabular}

Condenser Temp $=140^{\circ} \mathrm{F}$

Sampling Rate $=3 \mathrm{lpm}=0.1 \mathrm{ft}^{3} / \mathrm{min}$

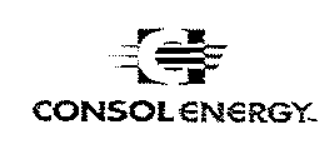

\begin{tabular}{|l|l|l|l|l|l|l|}
\cline { 2 - 7 } \multicolumn{2}{c|}{} & ALIQUOT I VOLUME & TITRATION (ml) & Ib/dscf & ppmv,act & ppmv,cor \\
\hline PLUG-SO & & & & & & \\
\hline PROBE-SO & & & & & & \\
\hline CONDENSER-SO & & & & & & \\
\hline BLANK & & & & & & \\
\hline IMPINGER $\left(\mathrm{H}_{2} \mathrm{O}_{2}\right)-\mathrm{SO}_{2}$ & $\vdots$ & & & & & \\
\hline
\end{tabular}


PLANT

LOCATION

DUCT DIMENSIONS

DUCT AREAA

DATE :

TIME

SAMPLE BOX

METER BOX

PITOT TUBE DESC

OPERATOR(S)

\begin{tabular}{|c|c|c|c|c|c|}
\hline \multicolumn{5}{|c|}{ OPERATOR(S) } & \multirow{2}{*}{$\begin{array}{c}\text { ROTOMETER } \\
\text { SETTING }\end{array}$} \\
\hline $\begin{array}{c}\text { TRAVRSE } \\
\text { POINT } \\
\text { [inch] }\end{array}$ & $\begin{array}{c}\text { SAMPLE } \\
\text { TIME } \\
\text { [minute] }\end{array}$ & $\begin{array}{c}\text { STATIC } \\
\text { PRESSURE } \\
{\left[" \mathrm{H}_{2} \mathrm{O}\right]} \\
\end{array}$ & $\begin{array}{c}\text { STACK } \\
\text { TEMP } \\
\left.{ }^{\circ} \mathrm{F}\right] \\
\end{array}$ & $\begin{array}{l}\text { PITOT } \\
\text { HEAD } \\
{\left[" \mathrm{H}_{2} \mathrm{O}\right]}\end{array}$ & \\
\hline $\cos A-1$ & $0-10$ & $N A$ & 171 & & $1 \mathrm{H}^{3} / \mathrm{min}$ \\
\hline $1-2$ & $10-20$ & & 172 & & it \\
\hline $2 \%$ & $20-30$ & & 172 & & 11 \\
\hline 3 & $30-40$ & & 172 & & if \\
\hline $3 / B-1$ & $40-50$ & & 172 & & il \\
\hline$B=1$ & $50-60$ & & 172 & & 11 \\
\hline 2 & $60-70$ & & 172 & & 11 \\
\hline $2 / B-3$ & $70-80$ & & 173 & & 11 \\
\hline$B-3$ & $80-90$ & $\downarrow$ & 173 & & 11 \\
\hline & 70-76 & $\downarrow$ & $\partial$ & & \\
\hline AVERAGE & & & 172.1 & & \\
\hline REMARKS & & $\mathbf{P}$ & . & & \\
\hline
\end{tabular}

$\mathrm{SO}_{3}$ FIELD SAMPLING DATA SHEET

GREENIDGE

STACK

$5 / 2 / 07$

CALIBRATION FACTORS: delta H 1 - 894

$$
\begin{array}{r|l|}
Y & 0.970 \\
K(p) & \\
& \\
&
\end{array}
$$

WATER BATH SETING 140 PROBE HTR SETTING 550 DUCTX-SECTION POSITION OF PORT A

DRY MOLECULAR WEIGHT (Assumed)

WET MOLECULAR WEIGHT (Assumed)

$$
15
$$

\begin{tabular}{|c|c|c|c|c|c|c|c|c|c|}
\hline \multirow{4}{*}{$\begin{array}{c}\text { METER } \\
\text { READING } \\
{\left[\mathrm{ft}^{3}\right]} \\
\end{array}$} & \multicolumn{2}{|c|}{ (Assumed $=\quad$ ) } & \multirow{3}{*}{$\begin{array}{c}\text { CONDENSER } \\
\text { TEMP }\end{array}$} & \multirow{3}{*}{$\begin{array}{c}\text { PROBE } \\
\text { TEMP }\end{array}$} & \multirow{4}{*}{$\begin{array}{c}\text { WATER BATH } \\
\text { TEMP } \\
\left.{ }^{\circ} \mathrm{F}\right]\end{array}$} & \multirow{4}{*}{$\begin{array}{c}\text { METER } \\
\text { VACUUM } \\
{[\mathrm{Hg}]} \\
\end{array}$} & \multirow{4}{*}{$\begin{array}{c}\mathrm{O}_{2} \\
\text { METER } \\
{[\%]}\end{array}$} & \multirow{2}{*}{\multicolumn{2}{|c|}{ Cottrinol-Baodil }} \\
\hline & \multirow{2}{*}{\multicolumn{2}{|c|}{$\begin{array}{c}\text { METER TEMP } \\
{\left[{ }^{\circ} \mathrm{F}\right]} \\
\end{array}$}} & & & & & & & \\
\hline & & & & & & & & $\mathrm{CO}_{2}$ & \&P \\
\hline & inlet & outlet & {$\left[{ }^{\circ} \mathrm{F}\right]$} & {$\left[{ }^{\circ} \mathrm{F}\right]$} & & & & [\%] & {$\left[-\mathrm{O}^{\circ} \mathrm{F}\right]$} \\
\hline 512.849 & & & & & & & & & \\
\hline 514.02 & 63 & 60 & 143 & 544 & NA & 5 & & & \\
\hline 518.88 & 63 & 61 & 140 & 508 & & 4 & 7.3 & & \\
\hline 515.96 & 64 & 61 & 138 & 497 & & 4 & 7.0 & & \\
\hline 517.05 & 64 & 62 & 140 & 495 & & 4 & 7.3 & & \\
\hline 518.12 & 66 & 63 & 142 & 499 & & 4 & 7.2 & & \\
\hline 519.24 & 65 & 63 & 139 & 503 & & 4 & 7.2 & & \\
\hline 520.32 & 65 & 63 & 139 & & & 4 & 7.2 & & \\
\hline 521.42 & 66 & 64 & 140 & 495 & & 4 & 7.2 & & \\
\hline 522.550 & 66 & 64 & 139 & 495 & & 4 & 7.3 & & \\
\hline 9.701 & 63.5 & & 1400 & & & & 7.2 & & \\
\hline
\end{tabular}

3 points per 2 ports. \& minetes per point / 10 pisucte readices

Condenser Temp $=140^{\circ} \mathrm{F}$

Sampling Rate $=3 \mathrm{lpm}=0.1 \mathrm{ft}^{3} / \mathrm{min}$

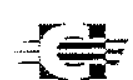

CONSOL ENERGX
Gas Vol, dscf

$\mathrm{BaCl}_{2}$ NORMALITY

\begin{tabular}{|l|l|l|l|l|l|l|}
\cline { 2 - 7 } \multicolumn{1}{c|}{} & ALIQUOT/ VOLUME & TITRATION (mi) & Ib/dscf & ppmv,act & ppmv,cor \\
\hline PLUG-SO & & & & & & \\
\hline PROBE-SO & & & & & & \\
\hline CONDENSER-SO & & & & & & \\
\hline BLANK & & & & & & \\
\hline IMPINGER $\left(\mathrm{H}_{2} \mathrm{O}_{2}\right)-\mathrm{SO}_{2}$ & & & & & & \\
\hline
\end{tabular}

port change at 45 minutes 
Run 2

$\mathrm{SO}_{3}$ FIELD SAMPLING DATA SHEET

PLANT

LOCATION

DUCT DIMENSIONS

DUCT AREA

DATE

TIME

SAMPLE BOX

METER BOX

PITOT TUBE DESC

OPERATOR(S)
AMBIENT TEMP [ $\left.{ }^{\circ} \mathrm{F}\right]$ \% $55^{\circ}$-WATER BATH SETTING

GREENIDGE

AIR HEATER OUTLET

$3-2-07$

Start 1322 stop- 126

NuTech \# $\mathrm{N}-\mathrm{S}$

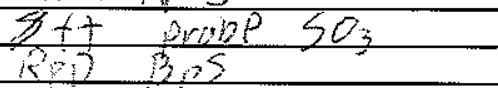

BAROMETRIC PRESSURE [" Hg] 20,47

$\% \mathrm{H}_{2} \mathrm{O}$ (Assumed) $8 \%$

PROBE LENGTH [ft] $\frac{\mathrm{ft}}{8}$

NOZZLE ID [inch] $X X X X$

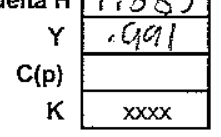

PROBE HTR SETTING

DUCT X-SECTION

POSITION OF PORT A

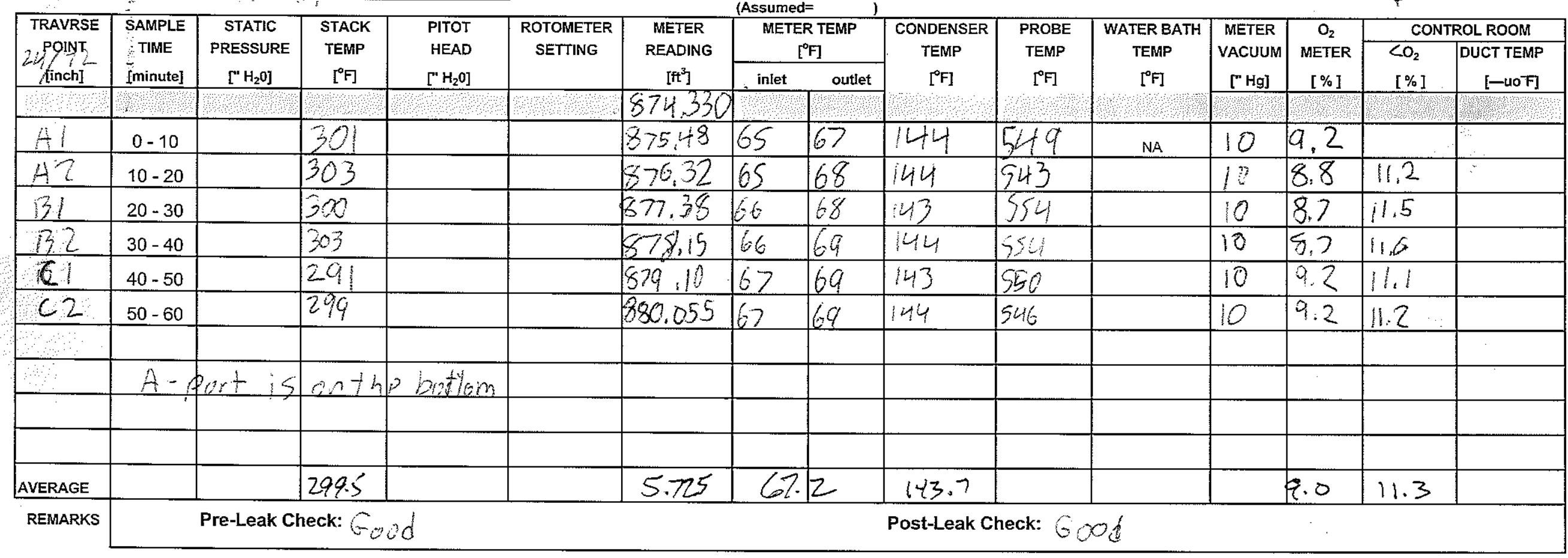

CALIBRATION FACTORS: delta $\mathrm{H} 1,883$

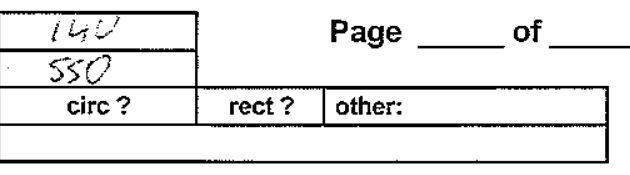

DRY MOLECULAR WEIGHT (Assumed)

WET MOLECULAR WEIGHT (Assumed)

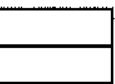

5 tack

$\mathrm{C}^{\mathrm{CO}_{2}}$

Condenser Temp $=140^{\circ} \mathrm{F}$

Sampling Rate $=3 \mathrm{lpm}=0.1 \mathrm{ft}^{3} / \mathrm{min}$

$r$

CONSOLENERGY.

\begin{tabular}{|l|l|l|l|l|l|l|}
\cline { 2 - 7 } \multicolumn{1}{c|}{} & ALIQUOT/ VOLUME & \multicolumn{2}{|l|}{ TITRATION (ml) } & Ib/dscf & ppmv,act & ppmV,cor \\
\hline PLUG-SO & & & & & & \\
\hline PROBE-SO & & & & & & \\
\hline CONDENSER-SO & & & & & & \\
\hline BLANK & & & & & & \\
\hline IMPINGER $\left(\mathrm{H}_{2} \mathrm{O}_{2}\right)-\mathrm{SO}_{2}$ & & & & & & \\
\hline
\end{tabular}

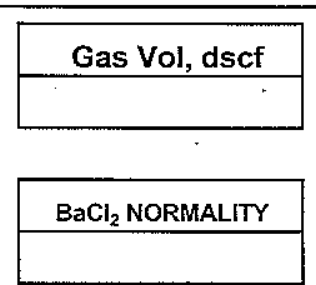

O:RdAppResISTACKIdatasheetsISO3SHEET 


\section{Run Two}

$\mathrm{SO}_{3}$ FIELD SAMPLING DATA SHEET

PLANT

LOCATION

DUCT DIMENSIONS

DUCT AREA

DATE

TIME

SAMPLE BOX

METER BOX

PITOT TUBE DESC

OPERATOR(S)

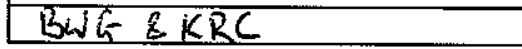

\section{$5 / 2 / 07$}

Start- 12 Stop- 1443
AMBIENT TEMP $\left[{ }^{\circ} \mathrm{F}\right] 60$ BAROMETRIC PRESSURE [" Hg] 29.47 $\% \mathrm{H}_{2} \mathrm{O}$ (Assumed) PROBE LENGTH [ft] NOZZLE ID [inch] $x X X X$ CALIBRATION FACTORS: delta H 1.844

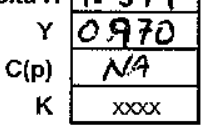

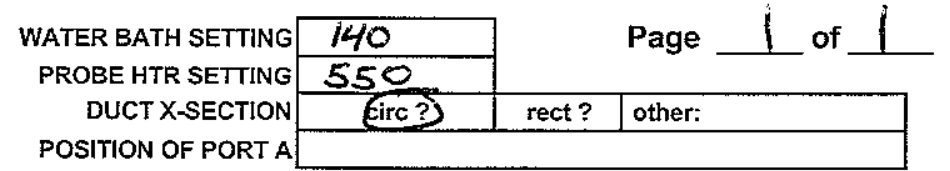

DRY MOLECULAR WEIGHT (Assumed) WET MOLECULAR WEIGHT (Assumed)

3

$3 /{ }^{2}$

$$
2
$$

AVERAGE

REMARKS

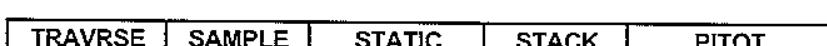

(Assumed $=$

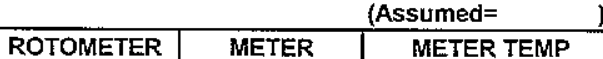

CONDENSER

\begin{tabular}{|c|c|c|c|c|}
\hline $\begin{array}{c}\text { POINT } \\
\text { [inch] }\end{array}$ & $\begin{array}{c}\text { TIME } \\
\text { [minute] }\end{array}$ & $\begin{array}{c}\text { PRESSURE } \\
{\left[" \mathrm{H}_{2} \mathrm{O}\right]}\end{array}$ & $\begin{array}{c}\text { TEMP } \\
{\left[{ }^{\circ} \mathrm{F}\right]}\end{array}$ & $\begin{array}{c}\text { HEAD } \\
{\left[{ }^{\prime \prime} \mathrm{H}_{2} \mathrm{O}\right]}\end{array}$ \\
\hline & & & &
\end{tabular}

\begin{tabular}{|c|c|c|c|c|}
\hline$B-1$ & $0-10$ & $N A$ & 172 & $.1 \mathrm{ft}^{3} / \min$ \\
\hline $1 / 2$ & $10-20$ & 1 & 171 & 11 \\
\hline 2 & $20-30$ & & 172 & 11 \\
\hline
\end{tabular}

2

$20-30$

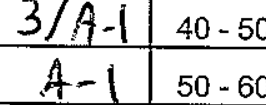

2 $60-70$

$70-80$

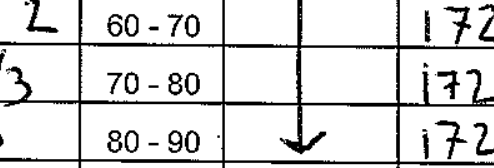

$-40$

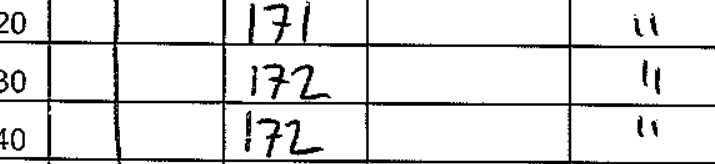

\begin{tabular}{l|l|l|l} 
& & 172 \\
\hline & & 172
\end{tabular}

Pre-Leak Check: Good

\begin{tabular}{|c|c|c|c|c|c|}
\hline & ALIQUOT / VOLUME & TITRATION (ml) & $\mathrm{lb} / \mathrm{dscf}$ & ppmv,act & ppmv,cor \\
\hline \multicolumn{6}{|l|}{ PLUG-SO } \\
\hline \multicolumn{6}{|l|}{ PROBE-SO } \\
\hline \multicolumn{6}{|l|}{ CONDENSER $-\mathrm{SO}_{3}$} \\
\hline \multicolumn{6}{|l|}{ BLANK } \\
\hline IMPINGER $\left(\mathrm{H}_{2} \mathrm{O}_{2}\right)-\mathrm{SO}_{2}$ & & & & & \\
\hline
\end{tabular}

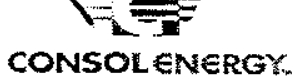

CONSOL ENERGY

\begin{tabular}{l|l|l}
173 & & ii \\
\hline 172 & & iा
\end{tabular}

\begin{tabular}{|c|c|} 
READING & \multicolumn{2}{|c|}{$\left[{ }^{\circ} \mathrm{F}\right]$} \\
\cline { 2 - 2 }$\left[\mathrm{ft}^{3}\right]$ & inlet outlet \\
\hline 526.300 &
\end{tabular}

$\begin{array}{llll}527.30 & 68 & 66\end{array}$

$\begin{array}{llll}528.34 & 69 & 67\end{array}$

529.33

530.367371

140

\begin{tabular}{ll|l|l}
531.52 & 76 & 73
\end{tabular}

141

\begin{tabular}{l|l|l|l|l|}
\hline 36 & 78 & 75 & 143 & 513 \\
\hline
\end{tabular}

\begin{tabular}{l|l|l|l|l|}
532.36 & 78 & 75 & 143 & 501 \\
533.34 & 78 & 75 & 145 & 515
\end{tabular}

498
492
10
532
513
01
15
23
525

140.8

NA

Condenser Temp $=140^{\circ} \mathrm{F}$

Sampling Rate $=3 \mathrm{lpm}=0.1 \mathrm{ft}^{3} / \mathrm{min}$

\begin{tabular}{|l|l|l|l|l|l|l|l|l|}
\hline 534.33 & 78 & 76 & 138 & 523 & & 4 & 7.2 & 13.0 \\
\hline 535.337 & 78 & 76 & 139 & 525 & & 4 & 7.2 & 13.0 \\
\hline & & & & & & & & \\
\hline 9.037 & 73.2 & 140.8 & & & & 7.1 & 13.1 \\
\hline
\end{tabular}

\begin{tabular}{|l|l|l|l|l|l|l|l|l|}
534.33 & 78 & 76 & 138 & 523 & & 4 & 7.2 & 13.0 \\
\hline 535.337 & 78 & 76 & 139 & 525 & & 4 & 7.2 & 13.0 \\
\hline 9.037 & 73.2 & 140.8 & & & & 7.1 & 13.1 \\
\hline
\end{tabular}

\begin{tabular}{l|c|c|c|c|c|c|} 
TEMP & TEMP & TEMP & VACUUM & METER & $\mathrm{CO}_{2}$ & DUCT TEMP \\
{$\left[{ }^{\circ} \mathrm{F}\right]$} & {$\left[{ }^{\circ} \mathrm{F}\right]$} & {$\left[{ }^{\circ} \mathrm{F}\right]$} & {$\left[\mathrm{N}^{\prime} \mathrm{Hg}\right]$} & {$[\%]$} & {$[\%]$} & {$\left[-\mathrm{Ho}^{2} \mathrm{~F}\right]$} \\
\hline
\end{tabular}

\section{METER}

DONFAOLAOM

4

\begin{tabular}{|c|c|c|}
\hline$[\%]$ & {$[\%]$} & {$[-407]$} \\
\hline & & \\
\hline & & \\
\hline
\end{tabular}




\section{Ron 3}

$\mathrm{SO}_{3}$ FIELD SAMPLING DATA SHEET

PLANT

LOCATION

DUCT DIMENSIONS

DUCT AREA

DATE

TIME

SAMPLE BOX

METER BOX

PITOT TUBE DESC

OPERATOR(S)

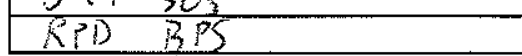

AMBIENT TEMP [0] $6 /$

BAROMETRIC PRESSURE [" Hg] 2447

$\% \mathrm{H}_{2} \mathrm{O}$ (Assumed) 8

PROBE LENGTH [ft] 8

NOZZLE ID [inch] $X X X X$

CALIBRATION FACTORS: delta $\mathrm{H}, 1,8 \mathrm{Z}^{3}$

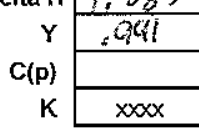

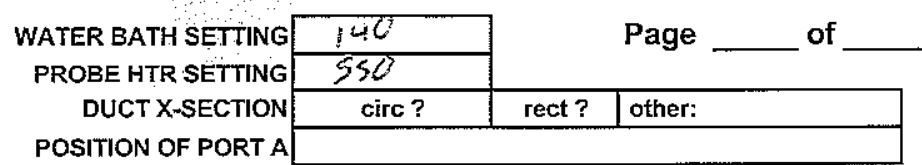

DRY MOLECULAR WEIGHT (Assume WET MOLECULAR WEIGHT (Assumed) $\square \mathrm{CO}_{2}$

\begin{tabular}{|c|c|c|c|c|c|c|c|c|c|c|c|c|c|c|c|}
\hline \multirow{4}{*}{\begin{tabular}{|c|} 
TRAVRSE \\
POINT \\
[inch]
\end{tabular}} & \multirow{4}{*}{$\begin{array}{l}\text { SAMPLE } \\
\text { TIME } \\
\text { [minute] }\end{array}$} & \multirow{4}{*}{$\begin{array}{c}\text { STATIC } \\
\text { PRESSURE } \\
{\left[" \mathrm{H}_{2} \mathrm{O}\right]} \\
\end{array}$} & \multirow{4}{*}{$\begin{array}{l} \\
\text { STACK } \\
\text { TEMP } \\
{\left[{ }^{\circ} \mathrm{F}\right]} \\
\end{array}$} & \multirow{4}{*}{$\begin{array}{r}\text { PITOT } \\
\text { HEAD } \\
{\left[" \mathrm{H}_{2} \mathrm{O}\right]} \\
\end{array}$} & \multirow{4}{*}{$\begin{array}{l}\text { ROTOMETER } \\
\text { SETTING }\end{array}$} & \multirow{3}{*}{\multicolumn{3}{|c|}{$\begin{array}{c}\text { (Assumed }= \\
\text { METER TEMP } \\
{\left[{ }^{\circ} \mathrm{F}\right]} \\
\end{array}$}} & \multirow{5}{*}{$\begin{array}{c}\text { CONDENSER } \\
\text { TEMP } \\
{\left[{ }^{\circ} \mathrm{F}\right]}\end{array}$} & \multirow{5}{*}{$\begin{array}{c}\text { PROBE } \\
\text { TEMP } \\
{\left[{ }^{\circ} \mathrm{F}\right]}\end{array}$} & \multirow{5}{*}{$\begin{array}{c}\text { WATER BATH } \\
\text { TEMP } \\
{\left[{ }^{\circ} \mathrm{F}\right]}\end{array}$} & \multirow{4}{*}{$\begin{array}{c}\text { METER } \\
\text { VACUUM } \\
{[" \mathrm{Hg}]} \\
\end{array}$} & \multirow{4}{*}{$\begin{array}{c}\mathrm{O}_{2} \\
\text { METER } \\
{[\%]} \\
\end{array}$} & \multirow{2}{*}{\multicolumn{2}{|c|}{ CONTROL ROOM }} \\
\hline & & & & & & & & & & & & & & & \\
\hline & & & & & & & & & & & & & & \multirow{2}{*}{$\begin{array}{c}\mathrm{O}_{2} \\
{[\%]}\end{array}$} & \multirow{2}{*}{$\begin{array}{r}\text { DUCT TEMP } \\
{[\because \text { Uo } \mathrm{F}]} \\
\end{array}$} \\
\hline & & & & & & \multirow{2}{*}{$\frac{\left[\mathrm{ft}^{3}\right]}{20 ! \mathrm{c5n}}$} & inlet & outlet & & & & & & & \\
\hline & & & & & & & & & & & & & & & \\
\hline & $0-10$ & & 301 & & & 682.71 & 65 & 66 & 154 & 354 & $\mathrm{NA}$ & 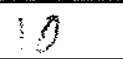 & 0.3 & 11.0 & \\
\hline & $10-20$ & & 303 & & & 583.72 & 66 & 67 & 150 & 552 & & 10 & 8.7 & 11.6 & \\
\hline & $20-30$ & & 301 & & & 834.53 & 66 & 67 & 142 & 362 & & 10 & 9.0 & 11,3 & \\
\hline & $30-40$ & & 303 & & & 685.44 & 66 & 67 & 143 & 574 & & 14 & 8.8 & 11,5 & \\
\hline & $40-50$ & & 292 & & & 886.47 & 66 & 68 & 144 & 652 & & 15 & 9.3 & 10.9 & \\
\hline & $50-60$ & & 300 & & & 557.410 & 66 & 68 & HU1 & 343 & & 15 & 9.0 & $11: 3$ & \\
\hline & & & & & & & & & & & & & & & $:$ \\
\hline & & & & & & & $\because$ & & & & & & & & \\
\hline & & & & & & & & & & & & & & & \\
\hline & & & & & & & & & & & & & & & \\
\hline AVERAGE & & & 306 & & & 5.890 & & 66.5 & 10.2 & & & & 9.0 & 11.3 & \\
\hline REMARKS & & e-Leak & ck: & & & & & & Pos & & & & & & \\
\hline
\end{tabular}

Condenser Temp $=140^{\circ} \mathrm{F}$

Sampling Rate $=3 \mathrm{lpm}=0.1 \mathrm{ft}^{3} / \mathrm{min}$

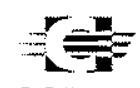

CONSOLENERGY

\begin{tabular}{|l|l|l|l|l|l|l|}
\cline { 2 - 7 } \multicolumn{1}{c|}{} & ALIQUOT/ VOLUME & TITRATION (ml) & lb/dscf & ppmv,act & ppmV,cor \\
\hline PLUG-SO & & & & & & \\
\hline PROBE-SO & & & & & & \\
\hline CONDENSER--SO & & & & & & \\
\hline BLANK & & & & & & \\
\hline IMPINGER $\left(\mathrm{H}_{2} \mathrm{O}_{2}\right)-\mathrm{SO}_{2}$ & & & & & & \\
\hline
\end{tabular}

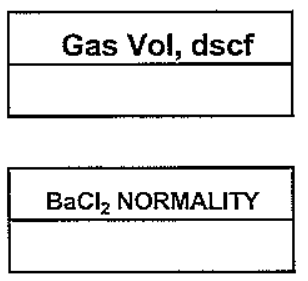

O.RdAPpResISTACKIdatasheetsISO3SHEET 
PLANT

LOCATION

DUCT DIMENSIONS

DUCT AREA

DATE

TIME

SAMPLE BOX

METER BOX

PITOT TUBE DESC

OPERATOR(S)

Run 3

$\mathrm{SO}_{3}$ FIELD SAMPLING DATA SHEET

\begin{tabular}{|l|}
\hline GREENIDGE \\
\hline STACK \\
\hline $512 / 07$ \\
\hline Start- 1517 Stop- 1645 \\
\hline NuTech 1 \\
\hline \\
\hline BWG 16 RRC \\
\hline
\end{tabular}

AMBIENT TEMP $\left[{ }^{\circ} \mathrm{F}\right) \quad \mathrm{C} 6$

BAROMETRIC PRESSURE [" Hg] 29.47 $\% \mathrm{H}_{2} \mathrm{O}$ (Assumed) PROBE LENGTH [ft] NOZZLE ID [inch] $\quad x \times x x$ CALIBRATION FACTORS: delta $H \frac{X X X}{1.394}$

\begin{tabular}{|c|c|}
\hline $\mathrm{Y}$ & 0.970 \\
\hline$C(p)$ & \\
\hline $\mathrm{K}$ & $x x$ \\
\hline
\end{tabular}

WATER BATH SETTING PROBE HTR SETTING DUCT X-SECTION POSITION OF PORT A

DRY MOLECULAR WEIGHT (Assumed) WET MOLECULAR WEIGHT (AsSUm

Page 1 of 1

$\frac{550}{50}$

$5 Q$
\begin{tabular}{|l|l|}
\hline rect? & other: \\
\hline
\end{tabular}

\section{\begin{tabular}{|l|l|l|l|l|}
\hline TRAVRSE & SAMPLE & STATIC & STACK & PITOT \\
\hline
\end{tabular}}
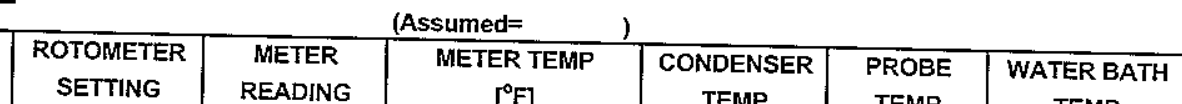

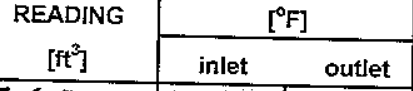

536.721
$.19+3 / \min 537.7279$

A

$$
2
$$

3

$3 / \$-1 \quad 40-50$

Tि- $250-60$

B-

$\frac{21}{3}$$$
\text { AVE }
$$

AVERAGE

REMARKS

\begin{tabular}{|l|l|l|}
\hline $60-70$ & & 172 \\
\hline $70-80$ & & 172 \\
\hline $80-90$ & & 172 \\
\hline
\end{tabular}

$70-80$

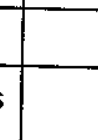

172

Pre-Leak Check: Good

\begin{tabular}{|l|l|l|l|}
\hline 538.73 & 79 & 78 & 146 \\
\hline 539 & 140 & 4 \\
\hline
\end{tabular}

\begin{tabular}{l|l|l|l|l|}
\hline 38.73 & 79 & 78 & 140 & 499 \\
\hline 539.75 & 78 & 78 & 139 & 492
\end{tabular}

\begin{tabular}{|l|l|l|l|l|}
539.75 & 78 & 78 & 139 & 492 \\
540.84 & 79 & 78 & 137 & 497
\end{tabular}

\begin{tabular}{ll|l|l|l}
541.99 & 78 & 78 & 143 & 498
\end{tabular}

$\begin{array}{lllllll}543.04 & 79 & 79 & 141 & 497\end{array}$

$\begin{array}{llllll}544.03 & 79 & 79 & 141 & 497\end{array}$

\begin{tabular}{ll|l|l|l}
544.03 & 79 & 79 & 141 & 497 \\
545.05 & 79 & 79 & 139 & 496
\end{tabular}

79

138

9.385

\begin{tabular}{ll}
78.7 & 140.4 \\
\hline
\end{tabular}

Post-Leak Check:

496

\begin{tabular}{l|l|l|l|l} 
& WATER BATH & METER & $\mathrm{O}_{2}$ & NFPOLROM
\end{tabular}

Condenser Temp $=140^{\circ} \mathrm{F}$

Sampling Rate $=3 \mathrm{lpm}=0.1 \mathrm{ft}^{3} / \mathrm{min}$

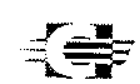

CONSOL ENERGY

\begin{tabular}{|l|l|l|l|l|l|l|}
\cline { 2 - 6 } \multicolumn{1}{c|}{} & ALIQUOT/ VOLUME & TITRATION (ml) & lb/dscf & ppmv,act & ppmv,cor \\
\hline PLUG-SO & & & & & & \\
\hline PROBE-SO & & & & & & \\
\hline CONDENSER-SO & & & & & & \\
\hline BLANK & & & & & & \\
\hline IMPINGER $\left(\mathrm{H}_{2} \mathrm{O}_{2}\right)-\mathrm{SO}_{2}$ & & & & & & \\
\hline
\end{tabular}

\begin{tabular}{|c|c|c|c|}
\hline $\begin{array}{l}\text { METER } \\
\text { vacuUM }\end{array}$ & $\mathrm{O}_{\mathrm{M}}^{\mathrm{O}_{2}}$ & & 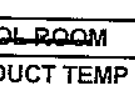 \\
\hline$\Gamma^{[\mathrm{Hg}]}$ & {$[\%]$} & {$[\%]$} & {$[-10 \%]$} \\
\hline 5 & 7.5 & 12.7 & \\
\hline 6 & 76 & 12.6 & \\
\hline 6 & 7.5 & 12.7 & \\
\hline 6 & 7.4 & 12.8 & \\
\hline 6 & 7.4 & $12-8$ & \\
\hline 6 & 7.4 & 12.8 & \\
\hline 6 & $7-4$ & 12.8 & \\
\hline 6 & $7-4$ & $12-8$ & \\
\hline 6 & 7.4 & 12.8 & \\
\hline & 7.4 & 128 & \\
\hline
\end{tabular}

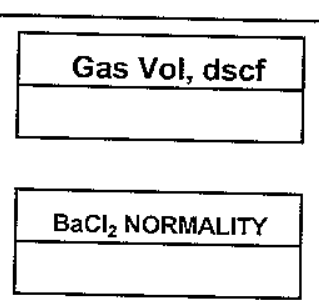

O:RdAppResISTACKIdatasheetsISO3SHEET 
AES GREENIDGE UNIT 4 GUARANTEE TESTING

$\mathrm{HCl} \& \mathrm{HF}$ Emission Summary

March 29, 2007

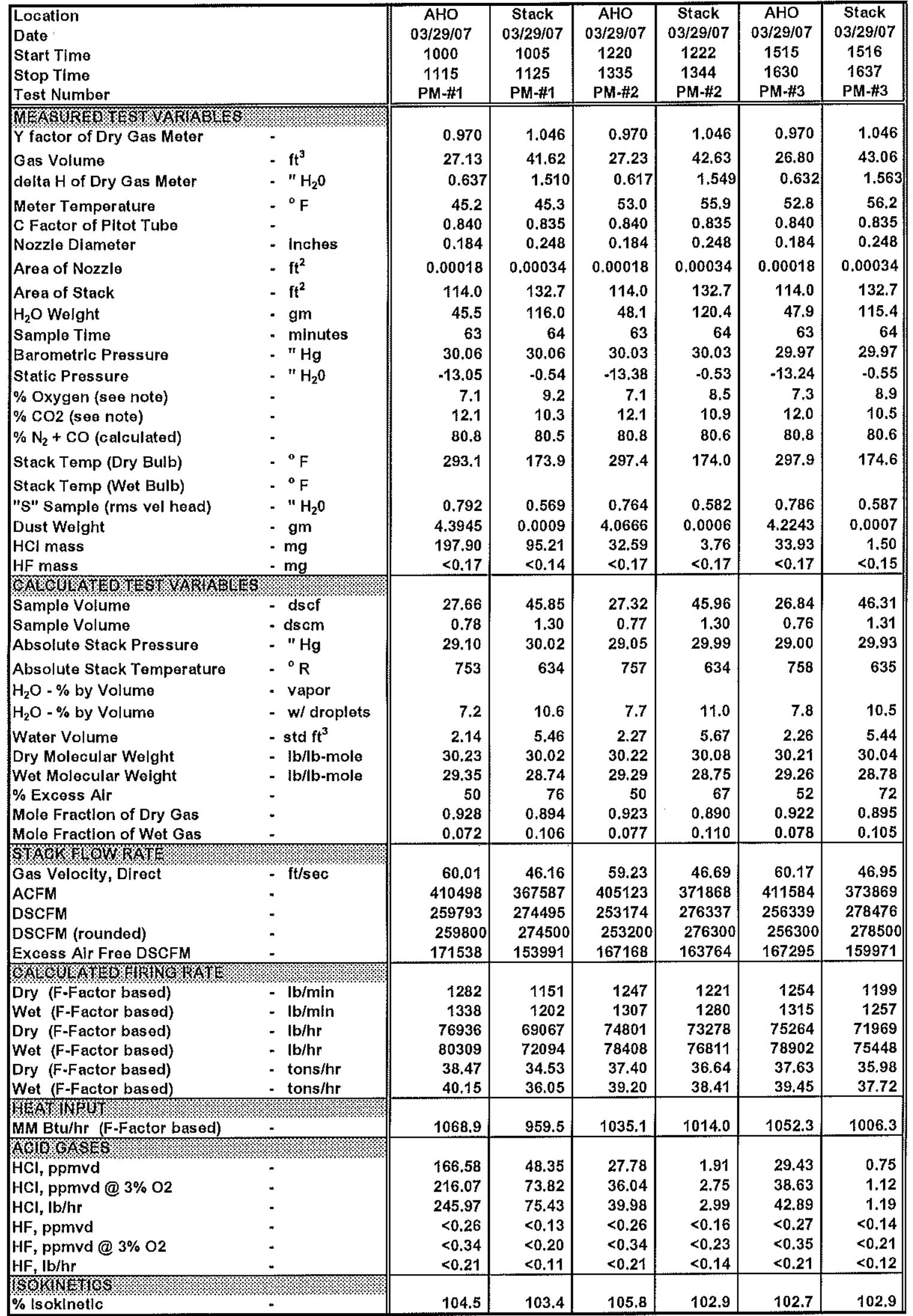

NOTE: The $\% \mathrm{O}_{2}$ at the alr heater oullet was measured by CONSOL using a Teledyne Max 5 porlable electrochemical $\mathrm{O}_{2}$ analyzer, and the $\% \mathrm{CO}_{2}$ at the air heater outlet was calculated from the measured $\mathrm{O}_{2}$ and coal compositlon. The $\% \mathrm{CO}_{2}$ al the stack was measured by the plant's stack CEM, and the $\% \mathrm{O}_{2}$ at the stack was calculated from the measured $\mathrm{CO}_{2}$ and coal composition. $\mathrm{HCl}$ concentrations measured at bolh the air heater outlet and stack during Tesi \#1 are invald because the sampling train was contaminated with $\mathrm{HCl}$. 


\section{ACID GAS / METHOD 5 PARTICULATE SAMPLING FIELD DATA SHEET}

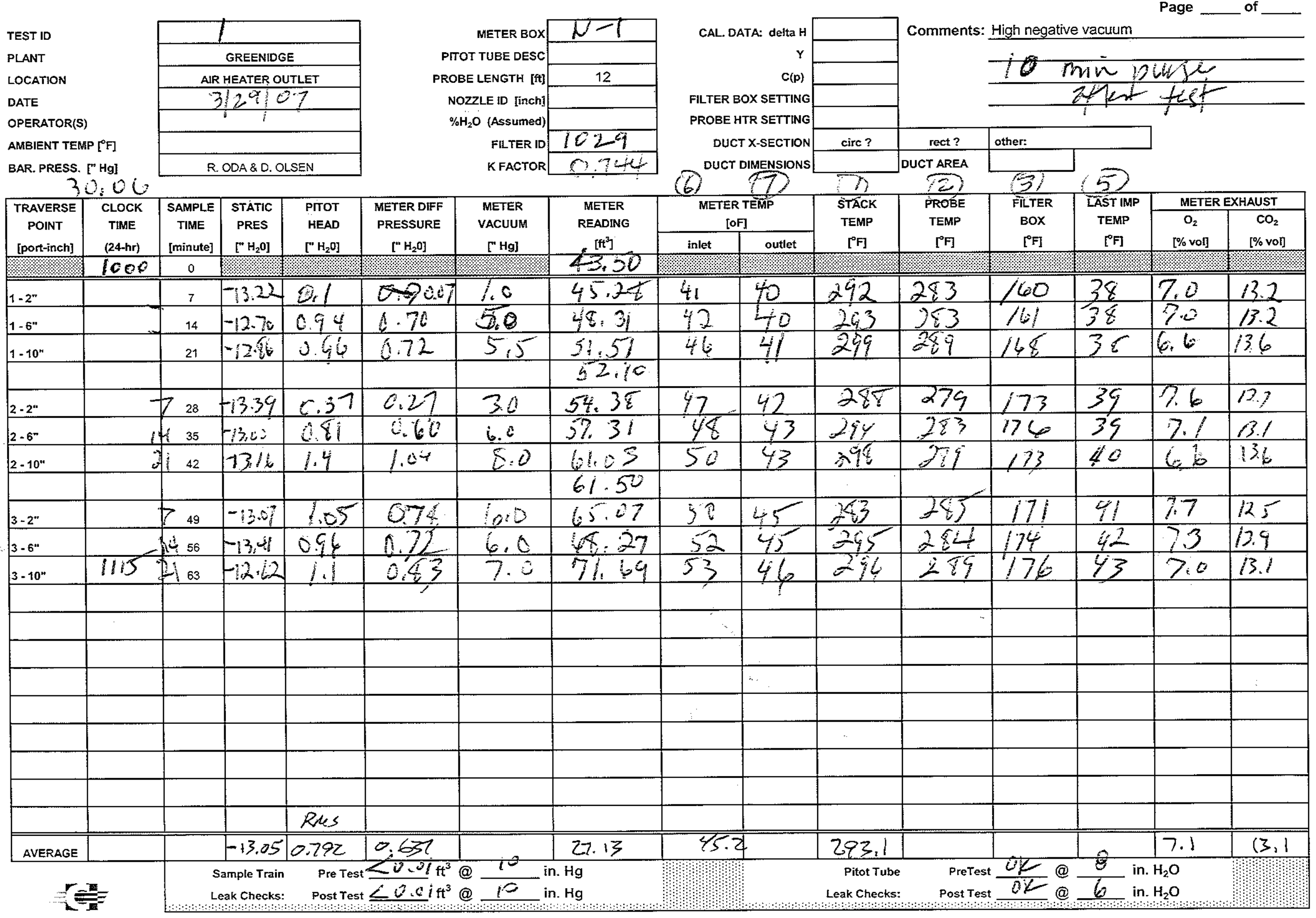


ACID GAS / METHOD 5 SAMPLING FIELD DATA SHEET

\begin{tabular}{|c|c|}
\hline TEST ID & $D N E$ \\
\hline PLANT & Greenidge \\
\hline LOCATION & STACK \\
\hline DATE & 0 \\
\hline OPERATOR(S) & K. CERAR \& B. SLIFER \\
\hline AMBIENT TEMP $\left[{ }^{\circ} \mathrm{F}\right]$ & 2310 \\
\hline
\end{tabular}
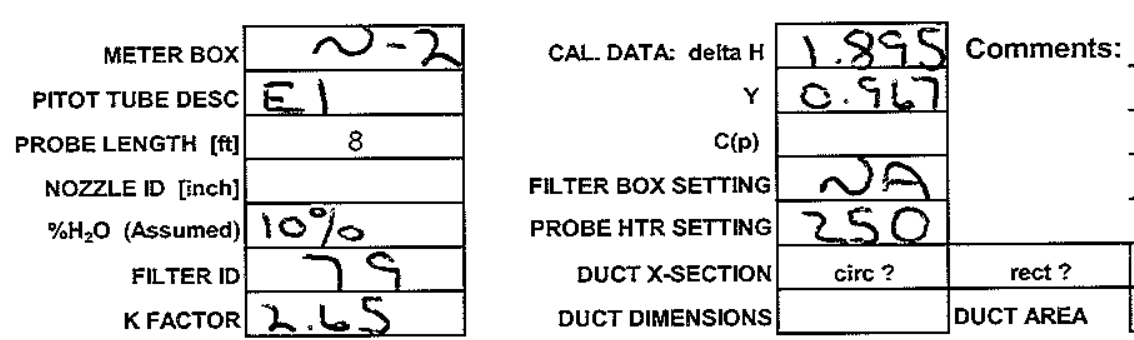

Page ___ of

BAR. PRESS. [" Hg]

\begin{tabular}{|c|c|c|c|c|c|c|c|c|c|c|c|c|c|c|c|}
\hline \multirow{2}{*}{\begin{tabular}{|c|c} 
TRAVERSE \\
POINT \\
[port-inch]
\end{tabular}} & \multirow{2}{*}{$\begin{array}{c}\text { CLOCK } \\
\text { TIME } \\
\text { (24-hr) }\end{array}$} & \multirow{2}{*}{\begin{tabular}{|c|} 
SAMPLE \\
TIME \\
[minute]
\end{tabular}} & \multirow{2}{*}{\begin{tabular}{|c|} 
STATIC \\
PRES \\
{$\left[" \mathrm{H}_{2} \mathrm{O}\right]$} \\
\end{tabular}} & \multirow{2}{*}{$\begin{array}{l}\text { PITOT } \\
\text { HEAD } \\
{\left[\mathrm{H}_{2}{ }^{0]}\right.} \\
\end{array}$} & \multirow{2}{*}{\begin{tabular}{|c|} 
METER DIFF \\
PRESSURE \\
{$\left[\mathrm{H}_{2} 0\right]$} \\
\end{tabular}} & \multirow{2}{*}{$\begin{array}{l}\text { METER } \\
\text { vacuUM } \\
{[" \mathrm{Hgl}} \\
\end{array}$} & \multirow{2}{*}{$\begin{array}{c}\text { METER } \\
\text { READING } \\
{\left[f^{\prime}{ }^{2}\right]} \\
\end{array}$} & \multirow{2}{*}{\multicolumn{2}{|c|}{$\begin{array}{l}\text { METER TEMP } \\
\text { [OF] }\end{array}$}} & \multirow{2}{*}{$\begin{array}{l}\text { STACK } \\
\text { TEMP } \\
\text { [0F] }\end{array}$} & \multirow{2}{*}{$\begin{array}{c}\text { PROBE } \\
\text { TEMP } \\
\text { PF }\end{array}$} & \multirow{2}{*}{ 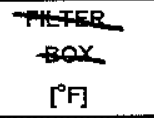 } & \multirow{2}{*}{$\begin{array}{c}\text { LAST IMP } \\
\text { TEMP } \\
\text { [F }] \\
\end{array}$} & \multicolumn{2}{|c|}{ METER EXHAUST } \\
\hline & & & & & & & & inlet & & & & & & $\begin{array}{c}\mathrm{O}_{2} \\
\% \mathrm{vol}\end{array}$ & $\begin{array}{c}\mathrm{CO}_{2} \\
{[\% \text { vol] }}\end{array}$ \\
\hline & 1005 & 0 & & & & & 910.60 & & & & & & & & \\
\hline $1-5^{n}$ & & 4 & & .44 & 1.16 & & 512 & & & 173 & $2-$ & & & & E \\
\hline $1-16.33^{\prime \prime}$ & & 8 & & .44 & 1.16 & 4 & 515.20 & & & 173 & $2+3$ & 188 & & & 10 \\
\hline $1-30.25^{n}$ & & 12 & 503 & .50 & 1.32 & & 917.57 & & 3 & $17 x$ & 251 & 155 & & S.S & 10. \\
\hline \multirow[t]{2}{*}{$1-50.38^{\prime \prime}$} & & 16 & & .50 & 1.32 & 4 & SIS.9O & & 38 & 174 & $2+2$ & 159 & & 9.5 & 10. \\
\hline & & & & & $\zeta \cdot c$ & $O K \leftrightarrow$ & 920.00 & & & & & & & & \\
\hline $2-5^{\prime \prime}$ & & 20 & & .54 & 1.43 & & $\$ 22.54$ & & & 173 & 249 & 230 & & & \\
\hline $2-16.38^{n}$ & & 24 & & 64 & 1.70 & & 525.30 & & 39 & 174 & $2+5$ & & 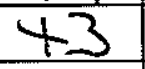 & 9.6 & 10 \\
\hline $2-30.25^{\prime \prime}$ & & 28 & 57) & .60 & 1.60 & & 927.98 & 5 & +10 & 174 & 236 & & 1 & 9.6 & 10. \\
\hline \multirow[t]{2}{*}{$2-50.38^{\prime \prime}$} & & 32 & & .54 & 1.43 & H & 930.52 & 52 & 4 & 174 & $2+1$ & & & 9.5 & 10. \\
\hline & & & & & L.C & $k \rightarrow$ & 930.60 & & & & & & & & \\
\hline $3-5^{n}$ & & 36 & & 64 & 1.70 & & 533.37 & 5 & 4 & 174 & $2+0$ & 243 & & $=$ & $E$ \\
\hline $3-16.38^{\prime \prime}$ & & 40 & & .70 & 1.85 & $S$ & 936.27 & & 43 & 174 & 248 & 244 & & S.t & 10.9 \\
\hline 3-30.25" & & 44 & 569 & 170 & 1.85 & 5 & 939.16 & 5 & +3 & 175 & 238 & $2+2$ & 5 & 9.6 & 10.7 \\
\hline \multirow[t]{2}{*}{$3-50.38 "$} & & 48 & & .60 & 1.60 & 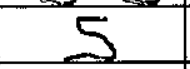 & 541.88 & 56 & 44 & 175 & $2+5$ & $2+3$ & & 9.7 & 10. \\
\hline & & & & & & $0 K \leftrightarrow$ & 542.00 & & & & & & & & \\
\hline $4-5^{\prime \prime}$ & & 52 & & .56 & 1.48 & 5 & 944.60 & $t$ & 44 & 174 & $2+0$ & $2+6$ & 62 & $=$ & \\
\hline 4 & & 56 & & .60 & 1.60 & & & I & 45 & 174 & 238 & 248 & 65 & 9.4 & 10.9 \\
\hline $4-30.25^{\prime \prime}$ & & 60 & .523 & 60 & 1.60 & $z$ & 949.57 & & 48 & 174 & 238 & 248 & 8 & 9.6 & 10 \\
\hline \multirow[t]{2}{*}{$4-50.38^{\prime \prime}$} & & 64 & & .54 & 1.43 & & 552.52 & 57 & 47 & 174 & $2+2$ & $24 x$ & 2) & 9.6 & 0 \\
\hline & 1125 & & & & & & & & & & & & & & \\
\hline AVERAC & & & $-0.54 \mathrm{z}$ & 0.569 & 1.51 & & 41.62 & 45.3 & & 173.9 & & & & 9.6 & 10.8 \\
\hline & & & ample Train & Pre Test & $\frac{O K}{O K}$ & 10 & & & & $\begin{array}{l}\text { Pitot Tube } \\
\text { Lear Check: }\end{array}$ & & K@ & 6 i & & \\
\hline
\end{tabular}


ACID GAS / METHOD 5 PARTICULATE SAMPLING FIELD DATA SHEET

\begin{tabular}{|c|c|c|c|c|c|c|c|c|c|c|c|c|c|c|c|}
\hline \multirow{8}{*}{\multicolumn{2}{|c|}{$\begin{array}{l}\text { TEST ID } \\
\text { PLANT } \\
\text { LOCATION } \\
\text { DATE } \\
\text { OPERATOR(S) } \\
\text { AMBIENT TEMP [P] } \\
\text { BAR. PRESS. [" Hg] }\end{array}$}} & & & & \multirow{3}{*}{\multicolumn{2}{|c|}{$\begin{array}{r}\text { METER BOX } \\
\text { PITOT TUBE DESC }\end{array}$}} & \multirow[b]{2}{*}{$\hat{N}-1$} & \multirow{2}{*}{\multicolumn{2}{|c|}{ CAL. DATA: delta $H$}} & & \multirow{2}{*}{\multicolumn{3}{|c|}{ Comments: High negative vacuum }} & \multicolumn{2}{|c|}{ Page ___ of } \\
\hline & & \multicolumn{3}{|c|}{2} & & & & & & & & & & & \\
\hline & & & GREENIDG & & & & & & $\mathrm{r}$ & & & & & & \\
\hline & & & HEATER O: & UTLET & & ELENGTH [ft] & 12 & & $\mathrm{C}(\mathrm{p})$ & & & $m$ & Pur & T & \\
\hline & & & $4 x^{2}$ & $\overline{C H}$ & & ZZLE ID [inch] & & FILTER & BOX SETTING & & & & $\sqrt{24}$ & $\tan$ & \\
\hline & & & & & & ${ }_{2} \mathrm{O}$ (Assumed) & & PROBE & ITR SETTING & & & & & & \\
\hline & & & & & & FILTER ID & 1030 & & TX-SECTION & circ? & rect? & other: & & & \\
\hline & & & ODA\&D.O & LSEN & & K FACTOR & 0.744 & DuC & DIMENSIONS & & DUCT AREA & & & & \\
\hline TRAVERSE & CLOCK & \begin{tabular}{|l|} 
SAMPLE \\
\end{tabular} & \begin{tabular}{l|} 
STATIC \\
\end{tabular} & Рாтт & METER DIFF & METER & METER & METE & TEMP & STACK & PROBE & FILTER & LAST IMP & METER & XXHAUST \\
\hline - POINT & TIME & TIME & PRES & HEAD & PRESSURE & VACUUM & READING & & 96 & TEMP & TEMP & Box & TEMP & $\mathrm{O}_{2}$ & $\mathrm{CO}_{2}$ \\
\hline [port-inch] & $(24-\mathrm{hr})$ & [minute] & {$\left[" \mathrm{H}_{2} \mathrm{O}\right]$} & {$\left[" \mathrm{H}_{2} \mathrm{O}\right]$} & {$\left[" \mathrm{H}_{2} \mathrm{O}\right]$} & {$\left[{ }^{*} \mathrm{Hg}\right]$} & {$\left[\mathrm{ft}^{3}\right]$} & inlet & outlet & {$[\circ \mathrm{F}]$} & {$[\circ \mathrm{F}]$} & & & {$[\%$ vol $]$} & {$[\%$ vol] } \\
\hline & $82: 20$ & 0 & l. & 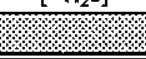 & / & la & 73.40 & 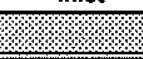 & 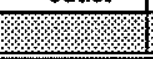 & 1. & & 9. & & & \\
\hline $1-2^{\prime \prime}$ & (x) & 7 & $-13: 2$ & 0.10 & 0.07 & 1.0 & 79.96 & 48 & 48 & 294 & 283 & 125 & 40 & 7.6 & 12.3 \\
\hline $\left.1-6^{\prime \prime} v\right)^{x}$ & & 14 & -3.65 & 3.74 & 0.55 & 2.5 & 42.79 & 49 & 48 & 300 & 253 & 137 & 40 & 8.0 & 13.2 \\
\hline $1-10^{\prime \prime}$ & & 21 & -13.43 & $1: 0$ & A. & 4.6 & $86,0 \%$ & 31 & 4 & 303 & 36 & 350 & 40 & 6.8 & 134 \\
\hline & & & & & $\therefore$ & & 96.70 & & & & & & & & \\
\hline $2-2^{n} \rightarrow$ & & 728 & -13.34 & Q 1 & $: 24$ & 1,5 & 88.79 & 53 & $4 c$ & 289 & 294 & 148 & 40 & 7.1 & 13.5 \\
\hline $2-6^{\prime \prime}+x^{2}$ & & 14 & $-1,9$ & 0.48 & $0.6 \mathrm{C}$ & 300 & 41.63 & 53 & 49 & 298 & 290 & 152 & 41 & 7.0 & 132 \\
\hline $2-10^{4}$ & & $1 \longdiv { 4 2 }$ & $-3,81$ & 1.4 & 081.47 & $5: 0$ & 95.49 & 55 & 30 & 301 & 296 & 153 & 42 & 6.6 & 13.7 \\
\hline & & & & & & & 95,97 & & 30 & & & & & & \\
\hline & $=$ & 749 & $=8297$ & 62 & 42 & 2,5 & 99.00 & 33 & 50 & $2 \varphi_{1}$ & 28 & 144 & 41 & 7.5 & 12.8 \\
\hline $3-6^{\prime \prime} x^{3}$ & & 56 & -13.65 & .96 & .72 & 40 & $102: 18$ & 56 & 51 & 300 & $2 \sqrt{9}$ & 149 & 43 & 7.5 & 12.8 \\
\hline $3-10^{n}$ & $1335 \%$ & 63 & 13.0. & $1: 1$ & 0.23 & 5,0 & 105.63 & 38 & 51 & 301 & 293 & 149 & 45 & 72 & $3 \pi$ \\
\hline & & & & & & & & & & & & & & & \\
\hline & & & & & & & & & & & & & & & \\
\hline & & & & & & & & & & & & & & & \\
\hline & & & & & & & & & & & & & & & \\
\hline & & & & & & & & & & & & & & & \\
\hline & & & & & & & & & & & & & & & \\
\hline & & & & & & & & & & & & & & & \\
\hline & & & & & & & & & & & & & & & \\
\hline & & & & 0.764 & & & & & & & & & & & \\
\hline AVERAGE & & & -13.38 & 20 & 0.617 & & 27.23 & 53.0 & 49.3 & 297.4 & 288.4 & & $4(.3$ & 7.14 & 13.1 \\
\hline & & & $\begin{array}{l}\text { ample Train } \\
\text { ak Checks: }\end{array}$ & $\begin{array}{r}\text { Pre Tes } \\
\text { Post Tes }\end{array}$ & $\begin{array}{l}\angle 0.01 \mathrm{ft}^{3} \\
\angle 0 . * 1 \mathrm{ft}^{3} \\
\end{array}$ & $\frac{1}{\frac{6}{4}}$ in & $\mathrm{Hg}$ & & & $\begin{array}{l}\text { Pitot Tube } \\
\text { Leak Checks: }\end{array}$ & $\begin{array}{r}\text { PreTe } \\
\text { Post Te }\end{array}$ & $\frac{O Y}{O Y} @$ & $\frac{9}{8}$ in & $\begin{array}{l}\mathrm{H}_{2} \mathrm{O} \\
\mathrm{H}_{2} \mathrm{O}\end{array}$ & \\
\hline
\end{tabular}




\section{ACID GAS / METHOD 5 SAMPLING FIELD DATA SHEET}

\begin{tabular}{|c|c|c|c|c|c|c|c|c|c|c|c|c|c|c|c|}
\hline \multirow{7}{*}{\multicolumn{2}{|c|}{$\begin{array}{l}\text { TEST ID } \\
\text { PLANT } \\
\text { LOCATION } \\
\text { DATE } \\
\text { OPERATOR(S) } \\
\text { AMBIENT TEMP PF] } \\
\text { BAR. PRESS. [" Hg] }\end{array}$}} & \multirow{2}{*}{\multicolumn{3}{|c|}{$T \omega 0$}} & \multirow{3}{*}{\multicolumn{2}{|c|}{$\begin{array}{r}\text { METER BOX } \\
\text { PTIOT TUBE DESC }\end{array}$}} & & \multirow{2}{*}{\multicolumn{2}{|c|}{ CAL. DATA: detta H }} & \multirow{6}{*}{$\begin{array}{l}1.895 \\
0.967 \\
.29 \\
2.50\end{array}$} & \multirow{6}{*}{ Comments: } & \\
\hline & & & & & & & $\sim-2$ & & & & & & & & \\
\hline & & \multirow{2}{*}{\multicolumn{3}{|c|}{$\begin{array}{c}\text { Greenidge } \\
\text { STACK }\end{array}$}} & & & EI & \multirow{4}{*}{\multicolumn{2}{|c|}{$\begin{array}{r}c(p) \\
\text { FLLTER BOX SETING } \\
\text { PROBE HTR SETTING }\end{array}$}} & & & & & & \\
\hline & & & & & \multirow{2}{*}{\multicolumn{2}{|c|}{$\begin{array}{l}\text { PROBE LENGTH [tt] } \\
\text { NOZZLE ID [inch] }]\end{array}$}} & \multirow{3}{*}{$10 \%$} & & & & & & & & \\
\hline & & \multirow{2}{*}{\multicolumn{3}{|c|}{\begin{tabular}{|c|}
$3-28 \cdot 07$ \\
K. CERAR \& B. SLIFER
\end{tabular}}} & & & & & & & & & & & \\
\hline & & & & & \multicolumn{2}{|c|}{$\begin{array}{l}\text { NOZZLE ID [inch] } \\
\% \mathrm{H}_{2} \mathrm{O} \text { (Assumed) }\end{array}$} & & & & & & \multirow{2}{*}{\multicolumn{2}{|c|}{ other: }} & & \\
\hline & & \multicolumn{3}{|c|}{$\frac{246}{30.03}$} & \multicolumn{2}{|r|}{$\begin{array}{l}\text { FILTER ID } \\
\text { K FACTOR }\end{array}$} & $\frac{80}{2.65}$ & \multicolumn{2}{|c|}{$\begin{array}{r}\text { DUCT X-SECTION } \\
\text { DUCT DIMENSIONS }\end{array}$} & circ? & $\begin{array}{l}\text { rect? } \\
\text { DUCT AREA }\end{array}$ & & & & \\
\hline \multirow{4}{*}{\begin{tabular}{|c|} 
TRAVERSE \\
POINT \\
[port-inch]
\end{tabular}} & CLOCK & SAMPLE & & & METER DIFF & METER & METER & METEF & & & PROBE & FFP & & METERE & EHAUST \\
\hline & TIME & TIME & PRES & HEAD & PRESSURE & vacuUm & READING & & & TEMP & TEMF & $\operatorname{sex}$ & & & $\mathrm{CO}_{2}$ \\
\hline & $(24-\mathrm{hr})$ & [minute] & {$\left[\mathrm{IH}_{2} \mathrm{O}\right]$} & {$\left[\mathrm{H}_{2} \mathrm{O}\right]$} & {$\left[\mathrm{r}^{\mathrm{N}} \mathrm{O}\right]$} & {$[" \mathrm{Hg}]$} & & inlet & outlet & {$[\mathrm{P}$} & {$[\mathrm{P}]$} & {$[\mathrm{F}]$} & & {$[\%$ vol] } & {$[\%$ vol] } \\
\hline & 1222 & 0 & & & & & 558.50 & & & & & & & & \\
\hline $1-5^{\prime \prime}$ & & 4 & & .60 & 1.60 & 7 & 961.21 & 48 & & 172 & 242 & 217 & & & $\longrightarrow$ \\
\hline $1-16.38^{\prime \prime}$ & & 8 & & .60 & 1.60 & 7 & 963.70 & 49 & 45 & 173 & 239 & 218 & & 9.6 & 10.7 \\
\hline $1-3025^{n}$ & & 12 & .504 & .60 & 1.60 & 7 & 966.57 & & 46 & 174 & $2+1$ & 219 & & 9.4 & 10.9 \\
\hline $1-50.38^{n}$ & & 16 & & .56 & 1.48 & 6.5 & 969.15 & 53 & 46 & 175 & $2+4$ & 218 & 45 & 9.4 & 10.9 \\
\hline & & & & & & $0 k \rightarrow$ & 569.25 & & & & & & & & \\
\hline $2-5^{\prime \prime}$ & & 20 & & .64 & 1.70 & 7.5 & 572.01 & & 47 & 172 & 248 & 215 & 44 & & $=$ \\
\hline $2-16.38^{\prime \prime}$ & & 24 & & .70 & 1.85 & 8 & 974.88 & 55 & 47 & 174 & 235 & 215 & +4 & q & 10.8 \\
\hline $2-3025^{n}$ & & 28 & 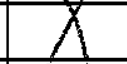 & 70 & 1.85 & 8 & 977.76 & 57 & 48 & 174 & $2+2$ & 216 & 45 & 9.3 & 11.0 \\
\hline $2.50 .38^{n}$ & & 32 & & .64 & 1.70 & 8 & 980.55 & 59 & 48 & 175 & 244 & 214 & & 9.3 & 11.0 \\
\hline & & & & & & $O K \rightarrow$ & 580.65 & & & & & & & & \\
\hline $3.5^{\prime \prime}$ & & 36 & & 6 & 1.48 & 6.5 & 983.29 & 55 & 48 & 171 & $2+1$ & 210 & +5 & - & \\
\hline 3. 16.38" & & 40 & .554 & .60 & 1.60 & 2 & 985.98 & 57 & 48 & 174 & $2+9$ & 185 & 44 & 9.5 & 10.8 \\
\hline $3-30.25^{n}$ & & 44 & & .60 & 1.60 & 7 & 588.68 & 59 & 49 & 175 & $2+2$ & 183 & 45 & 9.6 & 10.7 \\
\hline $3 .-50.38^{n}$ & & 48 & & .60 & 1.60 & 7 & 991.37 & 60 & 50 & 175 & $2+1$ & 182 & 45 & 9.4 & 10.5 \\
\hline & & & & & $L . C$ & $O K \rightarrow$ & SSI.KS & & & & & & & & \\
\hline $4-5^{n \prime}$ & & 52 & & 34 & 1.16 & $=$ & 593.78 & 59 & 50 & 175 & 242 & 165 & 43 & & $=$ \\
\hline $4-16.38^{n}$ & & 56 & & .50 & 1.32 & 6 & & 59 & 51 & 175 & 244 & 160 & 41 & 9.5 & 10.8 \\
\hline $4-30.25^{\prime \prime}$ & & 60 & .521 & 50 & 1.32 & 6 & 998.65 & 60 & 51 & 175 & 240 & 160 & +1 & 9.t & 10.9 \\
\hline $4-50.38^{\prime \prime}$ & & 64 & & .50 & 1.32 & 6 & 001.13 & 61 & 51 & 175 & 238 & 160 & +1 & 9.4 & 10.9 \\
\hline & 1344 & & & & & & & & & & & & 44.4 & 9.44 & 10.86 \\
\hline AVERAGE & & & -0.526 & 0,582 & 1.549 & & 42.63 & 55.9 & 48.1 & 174.0 & 242.3 & & $d$ & 1 & $\downarrow$ \\
\hline . & & & $\begin{array}{l}\text { ample Train } \\
\text { eak Crecks }\end{array}$ & $\begin{array}{l}\text { Pre T } \\
\text { Post T }\end{array}$ & OK $\mathrm{ft}^{3}$ & $@$ & 1. $\mathrm{Hg}$ & & & $\begin{array}{l}\text { Pitot Tube } \\
\text { Leak Checks: }\end{array}$ & $\begin{array}{l}\text { PreTest } \\
\text { Post Test }\end{array}$ & OK@ & & & \\
\hline
\end{tabular}


ACID GAS / METHOD 5 PARTICULATE SAMPLING FIELD DATA SHEET

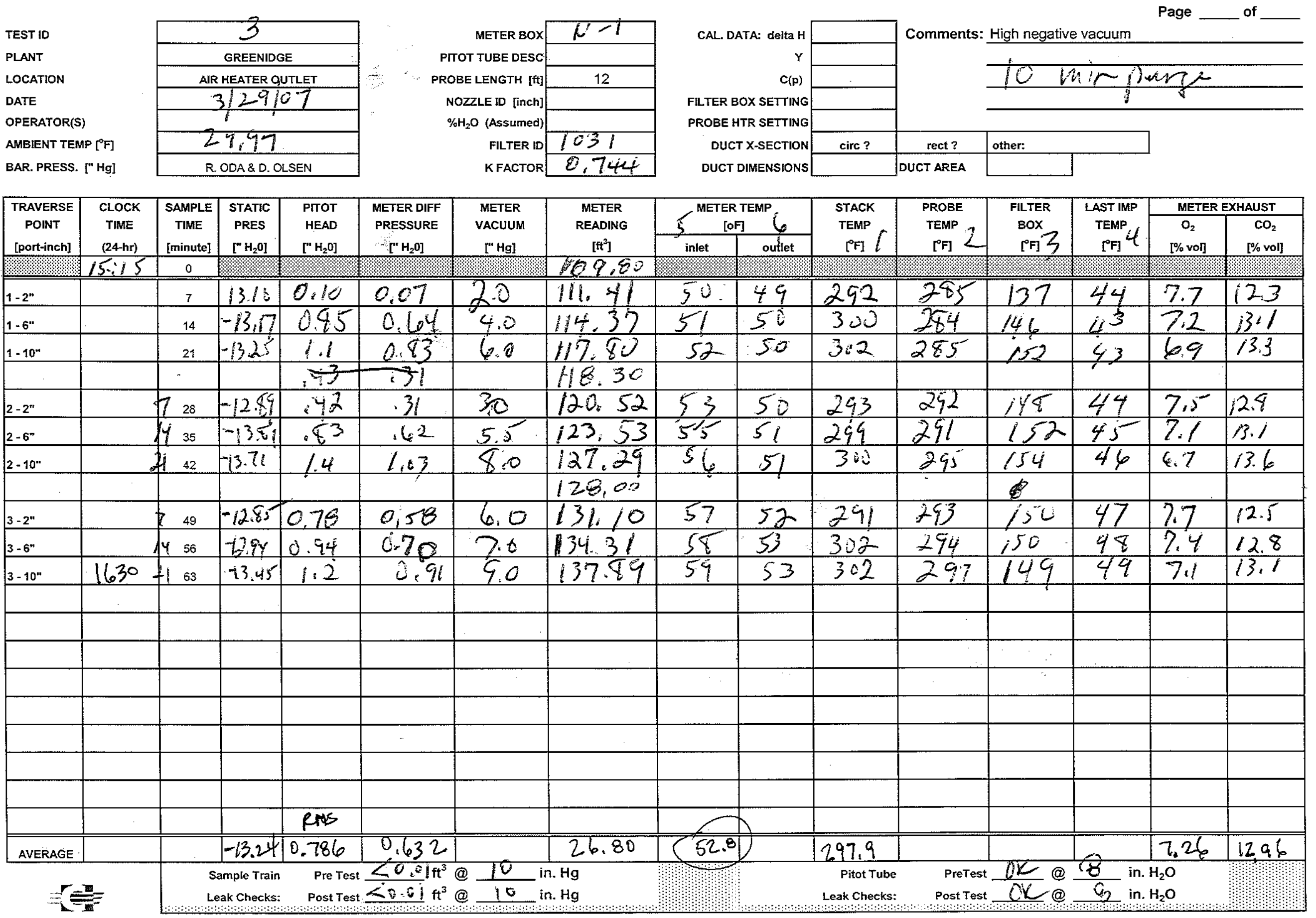


ACID GAS / METHOD 5 SAMPLING FIELD DATA SHEET

\begin{tabular}{|c|c|c|c|c|c|c|c|c|c|c|c|c|c|c|c|}
\hline \multirow{7}{*}{\multicolumn{2}{|c|}{$\begin{array}{l}\text { TEST ID } \\
\text { PLANT } \\
\text { LOCATION } \\
\text { DATE } \\
\text { OPERATOR(S) } \\
\text { AMBIENT TEMP [ }{ }^{\circ} \text { ] } \\
\text { BAR. PRESS. [" Hg] }\end{array}$}} & \multirow{2}{*}{\multicolumn{3}{|c|}{ WREE }} & \multicolumn{2}{|c|}{ METER BOX $\sim \cdots 2$} & & \multirow{2}{*}{\multicolumn{2}{|c|}{ CAL. DATA: delta $H$}} & \multirow{2}{*}{$\frac{1.895}{0.967}$} & \multirow[b]{2}{*}{ Comments: } & & \multicolumn{2}{|c|}{ Page ___ of } \\
\hline & & & & & & & $E$ & & & & & & & & \\
\hline & & & STACK & & & BEE LENGT & 8 & & $c(p)$ & & & & & & \\
\hline & & $3-$ & $29=$ & 0 & & OZZLE ID [inch] & & FILTER $B$ & $X$ SETTING & $\sim A$ & & & & & \\
\hline & & K. C & ERAR \& B. & SLFER & & $\mathrm{H}_{2} \mathrm{O}$ (Assumed) & $10 \%$ & PROBE H & R SETTING & 250 & & & & & \\
\hline & & & 50 & & & FILTER ID & 81 & DuC & X-SECTION & circ? & rect? & other: & & & \\
\hline & & 25 & 9.9 & & & K FACTOR & 2.65 & Duct & IMENSIONS & & DUCT AREA & & & & \\
\hline TRAVERSE & CLOCK & SAMPLE & STATIC & Pाтот & METER DIFF & METER & METER & METER & & & & TITER & LAST IMP & METER & XHAUST \\
\hline POINT & TIME & TIME & PRES & HEAD & PRESSURE & VACUUM & READING & & & TEMP & TEMP & $+0 x$ & TEMP & $\mathrm{O}_{2}$ & $\mathrm{~J}_{2}$ \\
\hline [port-inch] & & [minute] & {$\left[\mathrm{H}_{2} \mathrm{O}\right]$} & {$\left[{ }^{n} \mathrm{H}_{2} \mathrm{O}\right]$} & {$\left[" \mathrm{H}_{2} \mathrm{O}\right]$} & [" Hg] & {$\left[\mathrm{ft}^{2}\right]$} & inlet & outlet & {$\left[{ }^{\circ} \mathrm{F}\right]$} & {$\left[{ }^{\circ} \mathrm{F}\right]$} & {$\left[{ }^{\circ} \mathrm{F}\right]$} & {$\left[{ }^{\circ} \mathrm{F}\right]$} & {$[\%$ voll } & {$[\%$ vol] } \\
\hline & 1516 & 0 & & & & & $010 . \div 0$ & & & & & & & & \\
\hline $1-5^{\prime \prime}$ & & 4 & & 0.44 & 1.1 & 3 & 013.26 & & $\frac{15}{5}$ & 17 & 238 & 200 & & & \\
\hline $1-16.38^{\prime \prime}$ & & 8 & & 0.48 & 1.27 & 4 & 015.72 & & 49 & 174 & 251 & 206 & & & 11.0 \\
\hline $1.30 .25 "$ & & $12-$ & 519 & .50 & 1.32 & & 018.20 & & 50 & 174 & & 201 & & S. & 11.0 \\
\hline $1-50.38^{\prime \prime}$ & & 16 & & .56 & $1 .+8$ & & 020.82 & & 50 & 175 & & 201 & & & 11 \\
\hline & & & & & & $0 k<\rightarrow$ & 020.90 & & & & & & & & \\
\hline $2-5^{\prime \prime}$ & & 20 & & 56 & 1.48 & 48 & 023.54 & & 51 & 172 & 244 & 204 & & & $\longrightarrow$ \\
\hline $2-16.38^{\prime \prime}$ & & 24 & & .60 & 1.60 & & 026.27 & & & 17 & 237 & 205 & & 9.2 & 11.1 \\
\hline $2-30.25^{\prime \prime}$ & & 28 & 536 & .60 & 1.60 & & 025.00 & & & 176 & 236 & 201 & & 9.1 & 11.2 \\
\hline $2-50.38 "$ & & 32 & & .64 & 1.70 & & 031.80 & & $S$ & 175 & 251 & 204 & 46 & $\mathrm{G} .1$ & 11.2 \\
\hline & & & & & & $0 K \rightarrow$ & 032.00 & & & & & & & & \\
\hline $3-5^{\prime \prime}$ & & 36 & & .70 & 1.8 & & 034.90 & & & 1 & & 197 & & $=$ & - \\
\hline $3-16.38^{\prime \prime}$ & & 40 & & $: 7+$ & 1.95 & 5 & 037.90 & & & 175 & & 202 & & 9.2 & 11.1 \\
\hline $3-30.25 "$ & & 44 & 640 & .70 & 1.85 & $S$ & 040.80 & & & 175 & 239 & 201 & & $\mathrm{~S}$ & 11. \\
\hline $3-50.38^{\prime \prime}$ & & 48 & & .64 & 1.70 & 5 & 043.61 & & $S$ & 176 & 23 & 202 & 56 & & 11.2 \\
\hline & & & & & & $0)<\rightarrow$ & $0+3.70$ & & & & & & & & \\
\hline $4-5^{\prime \prime}$ & & 52 & & .56 & 1.48 & $S$ & 046.35 & & & 17 & & 197 & & 9.1 & 11.2 \\
\hline $4-16.38^{\prime \prime}$ & & 56 & & .60 & 1.60 & 5 & $045 .+6$ & & 55 & 175 & & 188 & & 9.2 & 11.1 \\
\hline $4-30.25^{\prime \prime}$ & & 60 & & .56 & 1.48 & 5 & 051.68 & 64 & 5.5 & 175 & 235 & 153 & $S S$ & S.O & 11.3 \\
\hline $4-50.38 "$ & 163 & 64 & 521 & .56 & 1.48 & 5 & 054.33 & & 55 & 175 & 252 & 190 & 57 & 9.0 & 11.3 \\
\hline & & & & exts & & & & & & & & & & & \\
\hline AVERAGE & & & 554 & 0.587 & 1.563 & & 43.06 & & & 174.6 & & & & 9.15 & $11+15$ \\
\hline & & & nple Train & $\begin{array}{r}\text { Pre Test } \\
\text { Post Test }\end{array}$ & OK $\mathrm{ft}^{3}$ & $\frac{10}{10}$ in & $\begin{array}{l}\mathrm{Hg} \\
\mathrm{Hg}\end{array}$ & & & $\begin{array}{r}\text { Pitot Tube } \\
\text { Leak Checks: }\end{array}$ & & $\frac{O K}{O K C}$ & 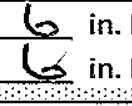 & & \\
\hline
\end{tabular}




\section{AES GREENIDGE UNIT 4 GUARANTEE TESTING}

\section{HF \& $\mathrm{HCl}$ Emission Summary}

May 4, 2007

\begin{tabular}{|c|c|c|c|c|c|}
\hline \multicolumn{2}{|l|}{\begin{tabular}{|l} 
Location \\
Date \\
Start Time \\
Stop Time \\
Test Number \\
\end{tabular}} & $\begin{array}{c}\text { AHO } \\
05 / 04 / 07 \\
831 \\
931 \\
\text { AGl\#1 } \\
\end{array}$ & $\begin{array}{c}\text { Stack } \\
05 / 04 / 07 \\
831 \\
940 \\
\text { AGO\#1 } \\
\end{array}$ & $\begin{array}{c}\text { AHO } \\
05 / 04 / 07 \\
1015 \\
1119 \\
\text { AGl\#2 } \\
\end{array}$ & $\begin{array}{c}\text { Stack } \\
05 / 04 / 07 \\
1015 \\
1130 \\
\text { AGO\#2 } \\
\end{array}$ \\
\hline \multicolumn{2}{|l|}{ 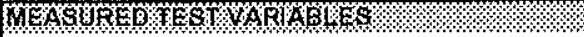 } & \multirow[b]{2}{*}{0.991} & \multirow[b]{2}{*}{0.970} & \multirow[b]{2}{*}{0.991} & \multirow[b]{2}{*}{0.970} \\
\hline Y factor of Dry Gas Meter & $\cdot$ & & & & \\
\hline Gas Volume & $-\mathrm{ft}^{3}$ & \multirow{2}{*}{$\begin{array}{c}36.61 \\
1.920\end{array}$} & \multirow{2}{*}{$\begin{array}{r}45.96 \\
1.770\end{array}$} & \multirow{2}{*}{$\begin{array}{r}42.76 \\
1.960\end{array}$} & \multirow{2}{*}{$\begin{array}{r}44.87 \\
1.673\end{array}$} \\
\hline delta $H$ of Dry Gas Meter & - " $\mathrm{H}_{2} \mathrm{O}$ & & & & \\
\hline $\begin{array}{l}\text { Meter Temperature } \\
\text { C Factor of Pitot Tube } \\
\text { Nozzle Diameter }\end{array}$ & $\begin{array}{l}={ }^{\circ} \mathrm{F} \\
=\text { inches }\end{array}$ & $\begin{array}{r}55.8 \\
0.840 \\
0.248\end{array}$ & $\begin{array}{r}55.4 \\
0.839 \\
0.250\end{array}$ & $\begin{array}{r}68.0 \\
0.840 \\
0.248\end{array}$ & $\begin{array}{r}63.7 \\
0.839 \\
0.250\end{array}$ \\
\hline Area of Nozzle & $-\mathrm{ft}^{2}$ & 0.00034 & 0.00034 & 0.00034 & 0.00034 \\
\hline Area of Stack & $-\mathrm{ft}^{2}$ & 114.0 & 132.7 & 114.0 & 132.7 \\
\hline $\mathrm{H}_{2} \mathrm{O}$ Welght & - gm & 69.0 & 149.3 & 68.9 & 142.7 \\
\hline Sample Time & - minutes & 55 & 64 & 60 & 64 \\
\hline Barometrlo Pressure & " " Hg & 29.74 & 29.74 & 29.74 & 29.74 \\
\hline Statlc Pressure & " " $\mathrm{H}_{2} \mathrm{O}$ & $\cdot 13,50$ & -0.46 & -13.30 & -0.40 \\
\hline$\%$ Oxygen (see note below) & $\cdot$ & 7.9 & 8.1 & 7.3 & 8.0 \\
\hline$\% \mathrm{co} 2$ (see note below) & $=$ & 11.4 & 11.2 & 11.9 & 11.2 \\
\hline$\% \mathrm{~N}_{2}+\mathrm{CO}$ (calculated) & $=$ & 80.7 & 80.7 & 80.8 & 80.7 \\
\hline Stack Temp (Dry Bulb) & $-{ }^{\circ} \mathrm{F}$ & 303.2 & 173.3 & 303.3 & 171.8 \\
\hline Stack Temp (Wet Bulb) & $-{ }^{\circ} \mathrm{F}$ & & & & \\
\hline "S" Sample (rms val head) & - " $\mathrm{H}_{2} \mathrm{O}$ & 0.709 & 0.544 & 0.737 & 0.521 \\
\hline Dust Welght & -gm & & & & \\
\hline $\mathrm{HCl}$ mass & $-\mathrm{mg}$ & 46.70 & 1.79 & 55.22 & $\begin{array}{r}1.36 \\
1.36\end{array}$ \\
\hline HF mass & $=m g$ & $<0.16$ & $<0.12$ & $<0.16$ & $<0.12$ \\
\hline 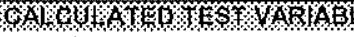 & & & & & \\
\hline $\begin{array}{l}\text { Sample Volume } \\
\text { Sample Volume } \\
\text { Absolute Stack Pressure }\end{array}$ & $\begin{array}{l}\text { dscf } \\
- \text { dsem } \\
-" \mathrm{Hg}\end{array}$ & $\begin{array}{r}37.08 \\
1.05 \\
28.75\end{array}$ & $\begin{array}{r}45.58 \\
1.29 \\
29.71\end{array}$ & $\begin{array}{r}42.30 \\
1.20 \\
28.76\end{array}$ & $\begin{array}{r}43.78 \\
1.24 \\
29.71\end{array}$ \\
\hline Absolute Stack Temperature & ${ }^{\circ}{ }^{\circ} R$ & 763 & 633 & 763 & 632 \\
\hline $\mathrm{H}_{2} \mathrm{O}=\%$ by Volume & - vapor & & & & \\
\hline $\mathrm{H}_{2} \mathrm{O}=\%$ by Volume & - w/ droplets & 8.1 & 13.4 & 7.1 & 13,3 \\
\hline Water Volume & $-s t d \mathrm{ft}^{3}$ & 3.25 & 7.03 & 3.25 & 6.72 \\
\hline Dry Molecular Weight & " $\mid \mathrm{b} / \mathrm{lb}-\mathrm{mole}$ & 30.14 & 30.12 & 30.19 & 30.12 \\
\hline Wet Molecular Welght & - lb/lb-mole & 29.16 & 28.50 & 29.33 & 28.50 \\
\hline$\%$ Excess Air & $\cdot$ & 59 & 61 & 52 & 61 \\
\hline Mole Fraction of Dry Gas & $"$ & 0.919 & 0.866 & 0.929 & 0.867 \\
\hline Mole Fraction of Wet Gas & $=$ & 0.081 & 0.134 & 0.071 & 0.133 \\
\hline $\mathrm{SHAOKOSOASAO}$ & & & & & \\
\hline Gas Velocity, Direct & ft/sec & 57.70 & 45.76 & 58.65 & 44.72 \\
\hline ACFM & - & 394643 & 364388 & 401146 & 356127 \\
\hline DSCFM & - & 241184 & 261313 & 247742 & 256203 \\
\hline DSCFM (rounded) & - & 241200 & 261300 & 247700 & 256200 \\
\hline Excess Air Free DSCFM & - & 150019 & 160183 & 161684 & 157557 \\
\hline 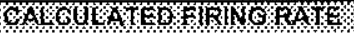 & & & & & \\
\hline Dry (F-Factor based) & - Ib/min & 1122 & 1198 & 1222 & 1191 \\
\hline Wet (F-Factor based) & - $\mathrm{lb} / \mathrm{m} \mid \mathrm{n}$ & 1193 & 1274 & 1300 & 1267 \\
\hline Dry (F-Factor based) & . Ib/hr & 67320 & 71881 & 73325 & 71453 \\
\hline Wet (F-Factor based) & * lb/hr & 71571 & 76421 & 77980 & 75990 \\
\hline Dry (F-Factor based) & - tons/hr & 33.66 & 35.94 & 36.66 & 35.73 \\
\hline Wet (F-Factor based) & - tons $/ \mathrm{hr}$ & 35.79 & 38.21 & 38.99 & 38,00 \\
\hline 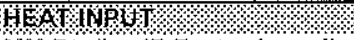 & & & & & \\
\hline MM Btu/hr (F-Factor based) & - & 924.4 & 987.1 & 1009.1 & 983.3 \\
\hline HOANAS & & & & & \\
\hline Gralns/DSCF & - & & & & \\
\hline $\mathrm{lb} / \mathrm{hr}$ & - & & & & \\
\hline Ib/MM Btu & $\therefore$ & & & & \\
\hline AOUOSSS & & & & & \\
\hline $\mathrm{HCl}$, ppmvd & 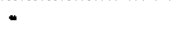 & 29.33 & 0.91 & 30.40 & 0.72 \\
\hline $\mathrm{HCl}$, ppmvd@3\% O2 & * & 40.38 & 1.28 & 39.89 & 1.01 \\
\hline $\mathrm{HCl}, \mathrm{lb} / \mathrm{hr}$ & - & 40.20 & 1.36 & 42.80 & 1.05 \\
\hline HF, ppmvd & - & $<0.18$ & $<0.11$ & $<0.16$ & $<0.12$ \\
\hline HF, ppmvd@3\% O2 & - & $<0.25$ & $<0,16$ & $<0.21$ & $<0.16$ \\
\hline $\mathrm{HF}_{\mathrm{I}} \mathrm{ib} / \mathrm{hr}$ & $=$ & $<0.14$ & $<0,09$ & $<0.12$ & $<0,09$ \\
\hline 1SOLASTS & & & & & \\
\hline$\%$ Isokinetic & - & 95.1 & 106.2 & 96.8 & 104.1 \\
\hline
\end{tabular}


EPA METHOD 5/26A PARTICULATE SAMPLING FIELD DATA SHEET

\begin{tabular}{|c|c|c|c|c|c|c|c|c|c|c|c|c|c|c|c|}
\hline \multirow{8}{*}{\multicolumn{2}{|c|}{$\begin{array}{l}\text { TEST ID } \\
\text { PLANT } \\
\text { LOCATION } \\
\text { DATE } \\
\text { OPERATOR(S) } \\
\text { AMBIENT TEMP PF] } \\
\text { BAR. PRESS. I" HgI }\end{array}$}} & \multirow{2}{*}{\multicolumn{3}{|c|}{ Run One }} & \multirow{3}{*}{\multicolumn{2}{|c|}{$\begin{array}{r}\text { METER BOX } \\
\text { PITOT TUBE DESC }\end{array}$}} & & \multirow{3}{*}{\multicolumn{2}{|c|}{\begin{tabular}{r|r} 
CAL. DATA: delta & \\
$Y$
\end{tabular}}} & \multirow{7}{*}{\begin{tabular}{|c|}
1.883 \\
0.991 \\
0.840 \\
$\mathrm{NA}$ \\
250 \\
circ? \\
\end{tabular}} & \multirow{6}{*}{ Comments: } & & & \\
\hline & & & & & & & $N-5$ & & & & & \multicolumn{2}{|c|}{ Page ___ of } & & \\
\hline & & \multirow{2}{*}{\multicolumn{3}{|c|}{\begin{tabular}{|c|} 
GREENIDGE \\
AIR HEATER OUTLET
\end{tabular}}} & & & $E-3$ & & & & & & & \\
\hline & & & & & \multirow{2}{*}{\multicolumn{2}{|c|}{$\begin{array}{l}\text { PROBE LENGTH [ft] } \\
\text { NOZZLE ID [inch] }\end{array}$}} & 12 & \multirow{3}{*}{\multicolumn{2}{|c|}{$\begin{array}{r}\text { C(p) } \\
\text { FILTER BOX SETING } \\
\text { PROBE HTR SETTING }\end{array}$}} & & & & & & \\
\hline & & \multirow{2}{*}{\multicolumn{3}{|c|}{$5 / 4 / 07$}} & & & $1 / 400.248$ & & & & & & & & \\
\hline & & & & & \multicolumn{2}{|c|}{$\% \mathrm{H}_{2} \mathrm{O}$ (Assumed) } & 40 & & & & & & & & \\
\hline & & \multicolumn{3}{|c|}{45} & \multirow{2}{*}{\multicolumn{2}{|c|}{$\begin{array}{r}\text { FLLTER ID } \\
\text { KFACTOR }\end{array}$}} & 1042 & \multirow{2}{*}{\multicolumn{2}{|c|}{$\begin{array}{r}\text { DUCT X-SECTION } \\
\text { DUCT DIMENSIONS }\end{array}$}} & & \multirow{2}{*}{\begin{tabular}{|l} 
rect? \\
DUCT AREA
\end{tabular}} & \multicolumn{2}{|l|}{ other: } & & \\
\hline & & \multicolumn{3}{|c|}{29.74} & & & 2.52 & & & & & \multicolumn{3}{|l|}{ LINE } & \\
\hline & & & & & & & & $\begin{array}{l}\text { METER } \\
\end{array}$ & & STACK & PROBE & PATter & LAST IMP & METER & XHAUST \\
\hline \begin{tabular}{|l|l|} 
POINT \\
\end{tabular} & TIME & TIME & \begin{tabular}{|l|} 
PRES \\
\end{tabular} & HEAD & PRESSURE & VACUUM & READING & & & TEMP & TEMP & sex- & TEMP & $\mathrm{O}_{2}$ & $\mathrm{CO}_{2}$ \\
\hline & (24-hr) & [minute] & {$\left[" \mathrm{H}_{2} \mathrm{0}\right]$} & {$\left[" \mathrm{H}_{2} \mathrm{O}\right]$} & {$\left[\mathrm{H}^{\mathrm{H}} \mathrm{H}_{2} \mathrm{O}\right]$} & {$[" \mathrm{Hg}]$} & {$\left[\mathrm{ft}^{3}\right]$} & inlet & outlet & {$[\mathrm{r} F]$} & {$\left[{ }^{\circ} \mathrm{F}\right]$} & {$\left[{ }^{\circ} \mathrm{F}\right]$} & {$[\mathrm{n}$} & {$[\% \mathrm{vol}]$} & {$[\%$ vol $]$} \\
\hline & 0831 & 0 & & & & & 909.400 & & & & & & & & \\
\hline$A-18^{n}$ & 0836 & 5 & -13.2 & 0.1 & 0.25 & 0 & 910.7 & 47 & 49 & 293 & 247 & 240 & 42 & & \\
\hline$A-54^{\prime \prime}$ & 0841 & 10 & & 0.57 & 1.4 & 4 & 913.6 & 47 & 49 & 307 & 246 & 246 & 40 & 8.1 & 12.1 \\
\hline$A-90 "$ & 0846 & 15 & -13.9 & 1.1 & 2.7 & 7 & 917.5 & 52 & 51 & 311 & 244 & 239 & 43 & 7.6 & 12.6 \\
\hline A-126" & 0851 & 20 & & 0.41 & 1.0 & 4 & 920.397 & 55 & $5 i$ & 313 & 247 & 239 & 47 & 7.5 & 12.7 \\
\hline & & & & & & & & & & & & & & & \\
\hline & 10854 & & & & & & & & & & 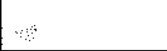 & & & & \\
\hline$B-18^{n}$ & 0859 & 25 & $-13,0$ & 0.30 & 0.75 & 2 & 922.7 & 56 & 54 & 298 & 244 & 240 & 47 & & \\
\hline B.54" & 0904 & 30 & & 0.70 & 1.8 & 5 & 926.3 & 58 & 55 & 302 & 247 & 240 & 47 & 8.1 & 12.1 \\
\hline B-90" & 0909 & 35 & -14.0 & 1.1 & 2.7 & 8 & 929.8 & 60 & 55 & 307 & 249 & 240 & 51 & 7.8 & 12.4 \\
\hline B-126" & 10914 & 40 & & 1.5 & 3.7 & 14 & 935.012 & 65 & 57 & 312 & 245 & 240 & 56 & 7.0 & 13.2 \\
\hline & & & & & & & & & & & & & & & \\
\hline & 0916 & & & 0.65 & \pm 6 & 7 & & & & & & & & & \\
\hline$c-18^{\prime \prime}$ & 0921 & 45 & $-13,4$ & 0.65 & 1.6 & 7 & 937.6 & 63 & 57 & 294 & 252 & 239 & 59 & & \\
\hline c-54" & 0926 & $\stackrel{50}{=}$ & & 1.1 & $2 . \overline{3}$ & 13 & 941.9 & 65 & 58 & 296 & 255 & 240 & 60 & 8.7 & 11.6 \\
\hline$c-90^{\circ}$ & 0931 & 55 & & 0.98 & 2.5 & 14 & 946.014 & 66 & 58 & 302 & 255 & 2.39 & 57 & 8.2 & $12: 1$ \\
\hline C-126" & & 60 & & & & & & & & & & & & & \\
\hline & & & & & & & & & & & & & & & \\
\hline & & & & & & 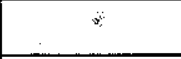 & & & & & & & & & \\
\hline & & & & & & & & & & & & & & & \\
\hline & & & & Rus & & & & & & & & & & & \\
\hline \begin{tabular}{|l|} 
AVERAGE \\
\end{tabular} & & 55 & -13.5 & 0.709 & 1.92 & & 36.614 & 55.8 & & 303.2 & & & & 7.9 & 12.4 \\
\hline$z=$ & & & $\begin{array}{l}\text { ample Train } \\
\text { ak Checks: }\end{array}$ & $\begin{array}{l}\text { Pre Tes } \\
\text { Post Tes }\end{array}$ & $\begin{array}{l}0.001 \mathrm{ft}^{2} \\
0.002 \mathrm{ft}^{2}\end{array}$ & $@ \frac{10}{16}$ & $\begin{array}{l}\text { in. } \mathrm{Hg} \\
\text { in. } \mathrm{Hg} \\
\end{array}$ & & & $\begin{array}{r}\text { Pitot Tube } \\
\text { Leak Checks: }\end{array}$ & Po: & $\frac{V}{V}$ & $\frac{7}{7}$ & $\mathrm{H}_{2} \mathrm{O}$ & \\
\hline
\end{tabular}


EPA METHOD 5/26A PARTICULATE SAMPLING FIELD DATA SHEET.

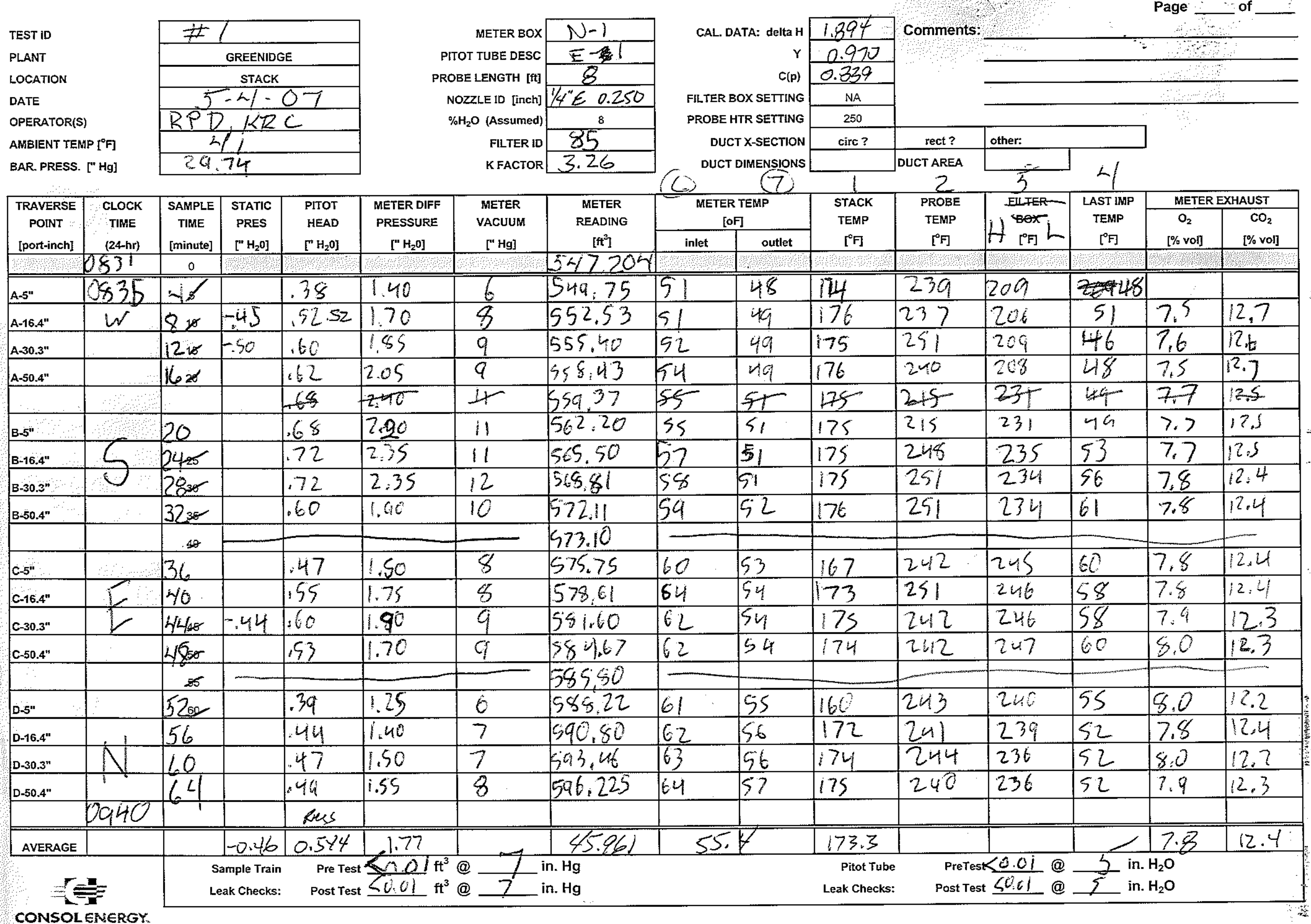




\section{EPA METHOD 5/26A PARTICULATE SAMPLING FIELD DATA SHEET}

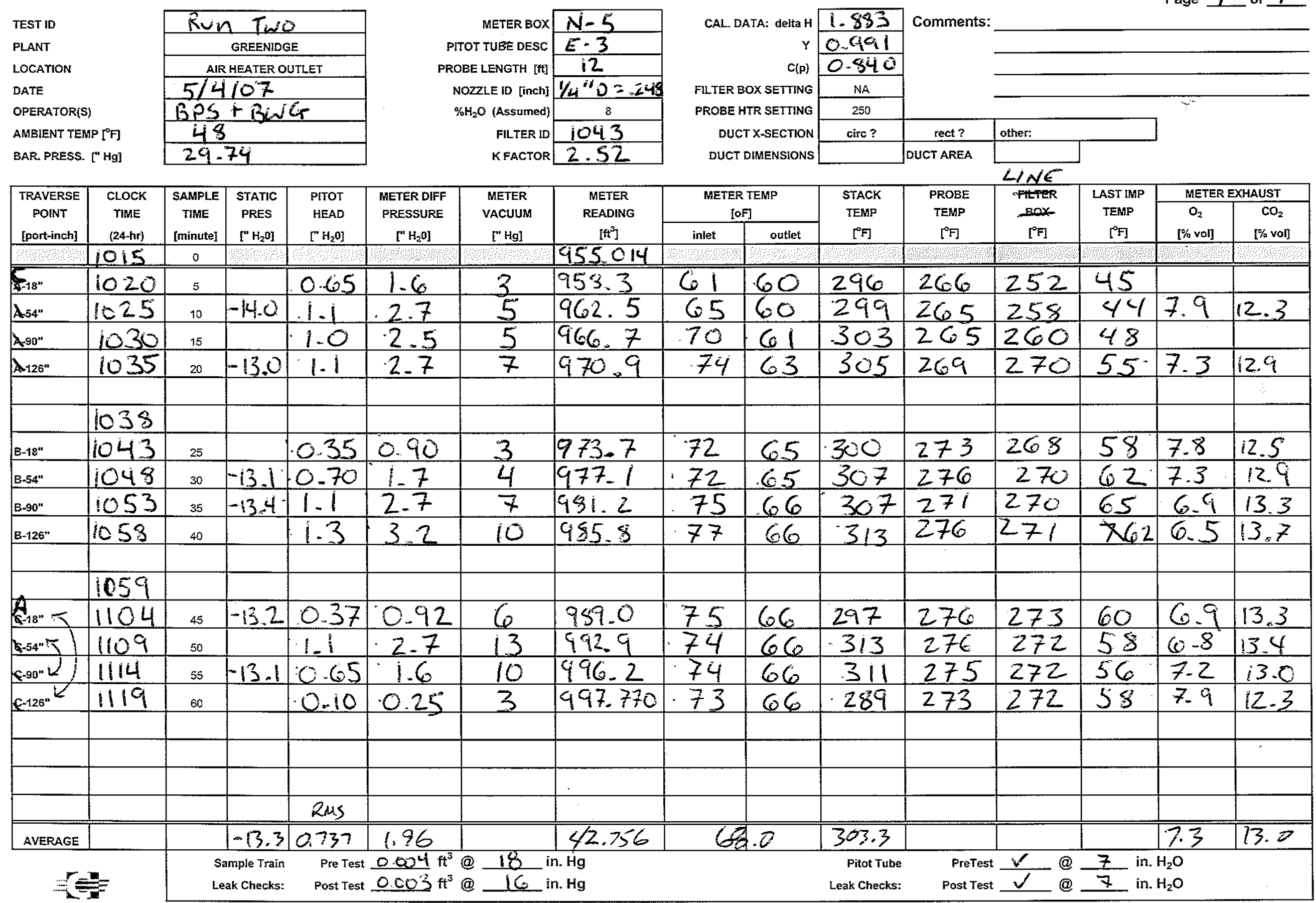


EPA METHOD 5/26A PARTICULATE SAMPLING FIELD DATA SHEET

\begin{tabular}{|c|c|c|c|c|c|c|c|c|c|c|c|c|c|c|c|}
\hline \multirow{7}{*}{\multicolumn{2}{|c|}{$\begin{array}{l}\text { TEST ID } \\
\text { PLANT } \\
\text { LOCATION } \\
\text { DATE } \\
\text { OPERATOR(S) } \\
\text { AMBIENT TEMP [م"F] } \\
\text { BAR. PRESS. [" Hg] }\end{array}$}} & \multirow{2}{*}{\multicolumn{3}{|c|}{52}} & \multirow{4}{*}{\multicolumn{2}{|c|}{$\begin{array}{r}\text { METER BOX } \\
\text { PITOT TUBE DESC } \\
\text { PROBE LENGTH SFrt }\end{array}$}} & & \multirow{3}{*}{\multicolumn{2}{|c|}{ CALL DATA: delta H }} & & \multirow[b]{2}{*}{ Comments } & & \\
\hline & & & & & & & $\mathrm{NI}$ & & & \begin{tabular}{|l|l|}
1.804 \\
\end{tabular} & & & & & \\
\hline & & \multicolumn{3}{|c|}{ GREENIDGE } & & & $E-1$ & & & \multirow{5}{*}{\begin{tabular}{|c|}
0.970 \\
0.3399 \\
$25 A$ \\
250 \\
circ? \\
\end{tabular}} & \multirow{5}{*}{\begin{tabular}{|c|} 
\\
rect? \\
DUCT AREA
\end{tabular}} & & \multirow{2}{*}{\multicolumn{2}{|c|}{$\mathrm{SP}$}} \\
\hline & & \multicolumn{3}{|c|}{ STACK } & & & & \multirow{4}{*}{\multicolumn{2}{|c|}{$\begin{array}{r}Y \\
C(p) \\
\text { FLLTER BOX SETTING } \\
\text { PROBE HTR SETTING } \\
\text { DUCT X-SECTION } \\
\text { DUCT DIMENSIONS }\end{array}$}} & & & & & & \\
\hline & & \multirow{2}{*}{\multicolumn{3}{|c|}{$5: 4.07$}} & \multirow{3}{*}{\multicolumn{2}{|c|}{$\begin{array}{r}\text { PROBE LENGTH [ft] } \\
\text { NOZZLE ID [inch) } \\
\% H_{2} \mathrm{O} \text { (Assumed) } \\
\text { FLLTER ID } \\
\text { K FACTOR }\end{array}$}} & $34 E=0.250$ & & & & & & & \multirow[b]{3}{*}{$\cdots$} & \\
\hline & & & & & & & 8 & & & & & \multirow{2}{*}{\multicolumn{2}{|c|}{ other: }} & & \\
\hline & & \multicolumn{3}{|c|}{ RB $\frac{k C}{59}$} & & & $\frac{8 / 4}{3,20}$ & & & & & & & & \\
\hline \multirow{3}{*}{\begin{tabular}{|c|} 
TRAVERSE \\
POINT \\
[port-inch] \\
\end{tabular}} & \multirow{3}{*}{$\begin{array}{c}\text { CLOCK } \\
\text { TIME } \\
(24 \mathrm{hr}) \\
O \mathrm{OLS}\end{array}$} & \multirow{2}{*}{\begin{tabular}{|c|} 
SAMPLE \\
TIME \\
[minute] \\
\end{tabular}} & \begin{tabular}{|l} 
STATIC \\
SopG
\end{tabular} & PITOT & \begin{tabular}{|l} 
METER DIFF \\
PRESSURE
\end{tabular} & METER & METER & METER & TEMP & STACK & PROBE & FXTER & LAST IMP & METER: & \\
\hline & & & 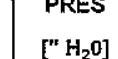 & 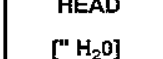 & \begin{tabular}{|l|l} 
PRESSURE \\
F" H,
\end{tabular} & VACUUM & 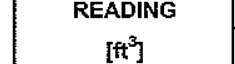 & inlet & outlet & $\begin{array}{l}\text { TEMP } \\
{ }^{\circ} \mathrm{F}\end{array}$ & $\begin{array}{l}{ }^{T} \text { TEMP } \\
{ }^{\circ} \mathrm{F}\end{array}$ & $+H_{r^{\circ}}^{B 0 x}$ & $\begin{array}{l}\text { TEMP } \\
\text { IPF }\end{array}$ & O\% & $\begin{array}{ll}\mathrm{CO}_{2} \\
\mathrm{roO}\end{array}$ \\
\hline & & 0 & & & & & 1596.904 & & & & & & & & \\
\hline$A-5^{n}$ & & 24 & 42 & 1.35 .42 & 1.35 & 8 & 1599.89 & 60 & 158 & $\mid 61$ & 274 & 1211 & 152 & 7.5 & $11^{2}, 7$ \\
\hline A-16.4" & $\Lambda$ & 8.19 & & 1.46 & 1.42 & 7 & 602,44 & 60 & 58 & 170 & 273 & 208 & 51 & 7.5 & 12.7 \\
\hline$A-30.3^{n}$ & 18 & 12 古 & -38 & ].46 & 1.42 & 7 & 605,10 & 62 & 58 & 173 & 270 & 206 & 52 & 7.4 & 12.8 \\
\hline 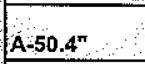 & & 1126 & & .48 & 1.52 & 7 & 607.72 & 63 & 59 & 174 & 270 & 214 & 52 & 7.5 & 12,7 \\
\hline$\therefore$ & & & & & & & 1605,25 & & & & & & & & \\
\hline B.5.5 & & 20 & & .42 & 1.35 & 6 & 610.35 & 64 & 59 & 159 & 277 & 232 & 54 & 8.2 & 11.9 \\
\hline B-16.4" & & $25^{\circ}$ & $-4 \mid$ & .55 & 1.75 & 6 & 613.14 & 64 & 59 & 172 & 270 & 230 & 56 & 7.5 & 12.7 \\
\hline B-30.3" & $\omega$ & $30 \div$ & & .55 & 1.75 & 7 & $616_{0}: 4$ & 65 & 60 & 174 & 272 & 229 & 57 & 1,6 & 12,6 \\
\hline B-50.4" & & $351^{2}$ & & .56 & 1.79 & 8 & 619.13 & 67 & 60 & 174 & 275 & 229 & 64 & 7.6 & 12.6 \\
\hline 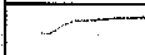 & - & 640 & & & & 2 & 9699.90 & $\cdots-5$ & + & 1 & $=$ & 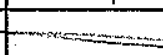 & 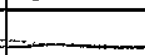 & 5 & 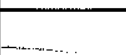 \\
\hline C.5" & & & & .59 & 1.39 & 8 & $622800^{\circ 2280}$ & 967 & 61 & 171 & 263 & 229 & 62 & 7.6 & 12.6 \\
\hline$c-16.4^{n}$ & & & & .68 & 2,20 & $q$ & 62599 & 68 & 61 & 174 & 270 & 231 & 65 & 7.5 & 12.7 \\
\hline$c-30.3^{n}$ & & 45 & & $: 67$ & 2.15 & 8 & 629.19 & 69 & 62 & 178 & 267 & 231 & 64 & $7: 6$ & 12,6 \\
\hline C.50.4" & & 50 & & .60 & 1.90 & 8 & 632.32 & 70 & 63 & 178 & 266 & 232 & 66 & 7,6 & 12.6 \\
\hline 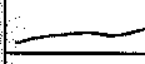 & & $F_{55}$ & & - & & & 633.68 & & & & & & & & $F$ \\
\hline D. $55^{n}$ & & 60 & & .50 & 1.60 & 6 & 636.29 & 70 & 63 & 170 & 273 & 243 & 63 & 7.5 & 12.7 \\
\hline D-16.4" & & & & .51 & 11.65 & 6 & 638.94 & 71 & 64 & 174 & 270 & 242 & 61 & 7,5 & 12.7 \\
\hline D-30.3. & 1 & & & .54 & 1.72 & 7 & 641.80 & 72 & 64 & 173 & 273 & 243 & 61 & 7.2 & 13.1 \\
\hline D. $50.4^{n}$ & 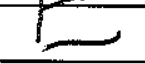 & & & .40 & 1,30 & 6 & 644.435 & 72 & 65 & 173 & 275 & 240 & 6 & 7.0 & $13: 1$ \\
\hline & 1130 & & & & Riu & & & & & & & & & & \\
\hline AVERAGE & & & & $(0.40)$ & 0.521 & 1.673 & 44,371 & 63.1 & & 171.3 & & & & 7.5 & 12.7 \\
\hline $2=$ & & & $\begin{array}{l}\text { sample Trai } \\
\text { eak Checks }\end{array}$ & $\begin{array}{r}\text { Pre Test } \\
\text { Post Test }\end{array}$ & $\mathrm{ft}$ & 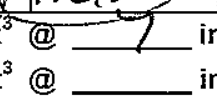 & & & & $\begin{array}{l}\text { Pitot Tub } \\
\text { Leak Checks }\end{array}$ & & 60.01 & & & \\
\hline
\end{tabular}


AES GREENIDGE UNIT 4 ONTARIO HYDRO SAMPLING TRAIN DATA

GUARANTEE TESTING - March 28, 2007 - NO CARBON INJECTION

\begin{tabular}{|c|c|c|c|c|c|c|c|}
\hline \begin{tabular}{|l} 
Location \\
Date \\
Start Time \\
Stop Time \\
Test Number \\
Sample Type \\
\end{tabular} & & $\begin{array}{c}\text { AHO } \\
3 / 28 / 07 \\
905 \\
1132 \\
1 \\
\text { OH-Hg } \\
\end{array}$ & $\begin{array}{c}\text { Stack } \\
3 / 28 / 07 \\
910 \\
1133 \\
1 \\
\mathrm{OH} \cdot \mathrm{Hg} \\
\end{array}$ & $\begin{array}{c}\text { AHO } \\
3 / 28 / 07 \\
1245 \\
1502 \\
2 \\
\mathrm{OH} \cdot \mathrm{Hg} \\
\end{array}$ & $\begin{array}{c}\text { Stack } \\
3 / 28 / 07 \\
1243 \\
1507 \\
2 \\
\mathrm{OH}-\mathrm{Hg} \\
\end{array}$ & $\begin{array}{c}\text { AHO } \\
3 / 28 / 07 \\
1558 \\
1817 \\
3 \\
\text { OH-Hg } \\
\end{array}$ & $\begin{array}{c}\text { Stack } \\
3 / 28 / 07 \\
1600 \\
1814 \\
3 \\
\mathrm{OH}-\mathrm{Hg} \\
\end{array}$ \\
\hline Y factor of dry gas meter & - & 0.970 & 1.046 & 0.970 & 1.046 & 0.970 & 1.046 \\
\hline $\begin{array}{l}\text { Gas Volume } \\
\text { Delta H of dry gas meter }\end{array}$ & $\begin{array}{l}-\mathrm{ft}^{3} \\
-\quad " \mathrm{H}_{2} \mathrm{O}\end{array}$ & $\begin{array}{r}50.95 \\
0.59\end{array}$ & $\begin{array}{r}82.93 \\
1.68\end{array}$ & $\begin{array}{r}52.21 \\
0.64\end{array}$ & $\begin{array}{r}82.90 \\
1.68\end{array}$ & $\begin{array}{r}51.67 \\
0.61\end{array}$ & $\begin{array}{r}80.15 \\
1.55\end{array}$ \\
\hline $\begin{array}{l}\text { Meter Temperature } \\
\text { C Factor of pitot tube } \\
\text { Nozzle Diameter }\end{array}$ & $\begin{array}{l}{ }^{\circ} \mathrm{F} \\
-\quad \text { inches }\end{array}$ & $\begin{array}{r}50.3 \\
0.840 \\
0.184\end{array}$ & $\begin{array}{r}46.6 \\
0.835 \\
0.248\end{array}$ & $\begin{array}{r}52.1 \\
0.840 \\
0.184\end{array}$ & \begin{tabular}{r||}
48.2 \\
0.835 \\
0.248
\end{tabular} & $\begin{array}{r}53.9 \\
0.840 \\
0.184\end{array}$ & $\begin{array}{r}51.6 \\
0.835 \\
0.248\end{array}$ \\
\hline A $n$ (area of nozzle) & $-\mathrm{ft}^{2}$ & 0.00018 & 0.00034 & 0.00018 & 0.00034 & 0.00018 & 0.00034 \\
\hline Area of Stack & $-\mathrm{ft}^{2}$ & 114.0 & 132.7 & 114.0 & 132.7 & 114.0 & 132.7 \\
\hline $\mathrm{H}_{2} \mathrm{O}$ Weight & $-g m$ & 65.7 & 230.5 & 69.0 & 197.5 & 76.7 & 218.5 \\
\hline Sample Time & - minutes & 126 & 120 & 126 & 120 & 126 & 120 \\
\hline Barometric Pressure & . " Hg & 29.83 & 29.83 & 29.85 & 29.85 & 29.85 & 29.85 \\
\hline Static Pressure & - " $\mathrm{H}_{2} \mathrm{O}$ & -12.94 & -0.56 & -13.09 & -0.60 & -12.93 & -0.58 \\
\hline$\%$ Oxygen (see note) & - & 6.9 & 8.0 & 7.0 & 8.2 & 7.0 & 8.3 \\
\hline$\% \mathrm{CO} 2$ (see note) & - & 12.2 & 11.2 & 12.2 & 11.1 & 12.2 & 11.1 \\
\hline$\% \mathrm{~N}_{2}+\mathrm{CO}$ (calculated) & $\cdot$ & 80.9 & 80.7 & 80.8 & 80.7 & 80.8 & 80.6 \\
\hline Stack Temp (Dry Bulb) & $-{ }^{\circ} \mathrm{F}$ & 293 & 175 & 300 & 176 & 298 & 174 \\
\hline $\begin{array}{l}\text { Stack Temp (Wet Bulb) } \\
\text { "S" sample (rms vel head) }\end{array}$ & $\begin{array}{l}-{ }^{\circ} \mathrm{F} \\
=" \mathrm{H}_{2} \mathrm{O}\end{array}$ & 0.719 & 0.568 & 0.792 & 0.578 & 0.751 & 0.565 \\
\hline Dust Wt. &. $\mathrm{gm}$ & 8.3021 & 0.0003 & 7.5722 & 0.0000 & 8.0255 & 0.0000 \\
\hline $\begin{array}{l}\text { Sample Volume } \\
\text { Sample Volume } \\
\text { ABS ST PRES }\end{array}$ & $\begin{array}{l}=\text { DSCF } \\
=\text { dscm } \\
. " \mathrm{Hg}\end{array}$ & \begin{tabular}{l|}
51.04 \\
1.445 \\
28.88
\end{tabular} & $\begin{array}{l}90.47 \\
2.562 \\
29.79\end{array}$ & $\begin{array}{l}52.15 \\
1.477 \\
28.89\end{array}$ & $\begin{array}{l}90.22 \\
2.555 \\
29.81\end{array}$ & $\begin{array}{l}51.43 \\
1.457 \\
28.90\end{array}$ & $\begin{array}{l}86.62 \\
2.453 \\
29.81\end{array}$ \\
\hline $\begin{array}{l}\text { ABS ST TEMP } \\
\mathrm{H}_{2} \mathrm{O}-\% \text { by } \mathrm{Vol}\end{array}$ & $\begin{array}{l}{ }^{\circ} \mathbf{R} \\
. \quad \text { vapor }\end{array}$ & $\begin{array}{r}753 \\
5.7\end{array}$ & $\begin{array}{r}635 \\
10.7\end{array}$ & $\begin{array}{r}760 \\
5.9\end{array}$ & $\begin{array}{r}636 \\
9.3\end{array}$ & $\begin{array}{r}758 \\
6.6\end{array}$ & $\begin{array}{r}634 \\
10.6\end{array}$ \\
\hline $\begin{array}{l}\text { Water Volume } \\
\text { Dry Molecular Weight } \\
\text { Wet Molecular Weight } \\
\% \text { EXCESS AIR } \\
\text { Dry Mole Frac. } \\
\text { Wet Mole Frac. }\end{array}$ & $\begin{array}{l}-\quad \text { std } \mathrm{ft}^{3} \\
=\mathrm{lb} / \mathrm{lb}-\mathrm{mole} \\
=\mathrm{lb} / \mathrm{lb}-\mathrm{mole} \\
- \\
= \\
=\end{array}$ & $\begin{array}{r}3.09 \\
30.23 \\
29.53 \\
47.7 \\
0.943 \\
0.057\end{array}$ & $\begin{array}{r}10.86 \\
30.12 \\
28.82 \\
60.4 \\
0.893 \\
0.107\end{array}$ & $\begin{array}{r}3.25 \\
30.23 \\
29.51 \\
48.8 \\
0.941 \\
0.059\end{array}$ & $\begin{array}{r}9.30 \\
30.10 \\
28.97 \\
62.9 \\
0.907 \\
0.093\end{array}$ & $\begin{array}{r}3.61 \\
30.23 \\
29.43 \\
49.2 \\
0.934 \\
0.066 \\
\end{array}$ & $\begin{array}{r}10.29 \\
30.11 \\
28.82 \\
63.5 \\
0.894 \\
0.106\end{array}$ \\
\hline Gas Velocity, Direct & $=\mathrm{ft} / \mathrm{sec}$ & 57.23 & 46.27 & 60.33 & $\begin{array}{r}46.58 \\
70071\end{array}$ & 58.73 & $\begin{array}{r}46.11 \\
367220\end{array}$ \\
\hline ACFM & - & $\begin{array}{l}391422 \\
249699\end{array}$ & $\begin{array}{l}368457 \\
272388\end{array}$ & $\begin{array}{l}412634 \\
260682\end{array}$ & $\begin{array}{l}370971 \\
278034\end{array}$ & $\begin{array}{l}401739 \\
252720\end{array}$ & $\begin{array}{l}367220 \\
272147\end{array}$ \\
\hline $\begin{array}{l}\text { DSCFM (rounded) } \\
\text { DSCMM }\end{array}$ & & $\begin{array}{c}249700 \\
7071\end{array}$ & $\begin{array}{c}272400 \\
7714\end{array}$ & $\begin{array}{c}260700 \\
7383\end{array}$ & $\begin{array}{r}278000 \\
7874\end{array}$ & $\begin{array}{r}252700 \\
7157\end{array}$ & $\begin{array}{r}272100 \\
7707\end{array}$ \\
\hline $\begin{array}{l}\text { Excess Air Free DSCFM } \\
\text { CALCULATED FIRING RATE }\end{array}$ & : & 167263 & 167788 & 173372 & 168642 & 167714 & 164457 \\
\hline Dry & - $\mathrm{lb} / \mathrm{min}$ & 1248 & 1252 & 1306 & 1271 & 1264 & 1239 \\
\hline Wet & - $\mathrm{lb} / \mathrm{min}$ & 1336 & 1340 & 1394 & 1356 & 1352 & 1326 \\
\hline Dry & - Ib/hr & 74858 & 75093 & 78382 & 76243 & 75838 & 74365 \\
\hline $\begin{array}{l}\text { Wet } \\
\text { CALCULATED FIRING RATE }\end{array}$ & : $\mathrm{lb} / \mathrm{hr}$ & 80165 & 80417 & 83634 & 81352 & 81145 & 79569 \\
\hline (a) & - tons/hr & 37.4 & 37.5 & 39.2 & 38.1 & 37.9 & 37.2 \\
\hline Wet & - tons/hr & 40.1 & 40.2 & 41.8 & 40.7 & 40.6 & 39.8 \\
\hline HEAT INPUT: & & & & & & & \\
\hline $\begin{array}{l}\text { MM Btu/hr } \\
\text { PARTICULATE LOADING: }\end{array}$ & - & 1031 & 1034 & 1082 & 1052 & 1055 & 1035 \\
\hline Grains/DSCF & * & 2.5100 & 0.0001 & 2.2402 & 0.0000 & 2.4078 & 0.0000 \\
\hline $\mathrm{lb} / \mathrm{hr}$ & $\cdot$ & 5374 & 0.12 & 5008 & 0.00 & 5217 & 0.00 \\
\hline Ib/MM Btu & $\cdot$ & 5.21 & 0.00 & 4.63 & 0.00 & 4.94 & 0.00 \\
\hline$\%$ ISOKINETIC & - & 100.3 & 109.6 & 98.1 & 107.1 & 99.8 & 105.1 \\
\hline
\end{tabular}

NOTE: The $\% \mathrm{O}_{2}$ at the air heater outlet was measured by $\mathrm{CONSOL}$ using a Teledyne Max 5 portable electrochemlcal $\mathrm{O}_{2}$ analyzer, and the $\% \mathrm{CO}_{2}$ at the air heater outlet was calculated from the measured $\mathrm{O}_{2}$ and coal composition. The $\% \mathrm{CO}_{2}$ at the stack was measured by the plant's stack $\mathrm{CEM}$, and the $\% \mathrm{O}_{2}$ at the stack was calculated from the measured $\mathrm{CO}_{2}$ and coal composition. 


\begin{tabular}{|c|c|c|c|c|c|c|}
\hline Impinger Components Wts \& Volumes & $\mathrm{AHO}-1$ & Stack - 1 & AHO - 2 & Stack - 2 & $\mathrm{AHO}-3$ & Stack - 3 \\
\hline $\begin{array}{l}\text { Filter Wt., g } \\
\text { ppm } \mathrm{Hg} \text { (thimbles) or ug/filter (filters) } \\
\text { total ug } \\
\text { ug/dscm }\end{array}$ & $\begin{array}{r}8.3021 \\
0.72 \\
6.01 \\
4.16 \\
\end{array}$ & $\begin{array}{r}0.0003 \\
<0.01 \\
<0.01 \\
<0.00\end{array}$ & $\begin{array}{r}7.5722 \\
0.56 \\
4.26 \\
2.89\end{array}$ & $\begin{array}{r}0.0000 \\
<0.01 \\
<0.01 \\
<0.00\end{array}$ & \begin{tabular}{r|}
8.0255 \\
0.69 \\
5.52 \\
3.79 \\
\end{tabular} & $\begin{array}{r}0.0000 \\
<0.01 \\
<0.01 \\
<0.00\end{array}$ \\
\hline $\begin{array}{l}\text { Probe Rinse volume, } \mathrm{ml} \\
\text { Analytical } \mathrm{Hg}, \mathrm{ng} / \mathrm{ml} \\
\mathrm{ug} / \mathrm{dscm}\end{array}$ & $\begin{array}{r}150 \\
1.15 \\
0.12 \\
\end{array}$ & $\begin{array}{r}80 \\
<1.40 \\
<0.04 \\
\end{array}$ & $\begin{array}{r}117 \\
1.59 \\
0.13 \\
\end{array}$ & $\begin{array}{r}136 \\
<1.40 \\
<0.07 \\
\end{array}$ & $\begin{array}{r}118 \\
<1.40 \\
<0.11 \\
\end{array}$ & $\begin{array}{r}110 \\
<1.40 \\
<0.06 \\
\end{array}$ \\
\hline $\begin{array}{l}\text { Line Rinse volume, } \mathrm{ml} \\
\text { Analytical } \mathrm{Hg}, \mathrm{ng} / \mathrm{ml} \\
\text { ug/dscm }\end{array}$ & $\begin{array}{r}160 \\
2.21 \\
0.24 \\
\end{array}$ & $\begin{array}{r}62 \\
<1.40 \\
<0.03 \\
\end{array}$ & $\begin{array}{r}117 \\
6.78 \\
0.54 \\
\end{array}$ & $\begin{array}{r}60 \\
<1.40 \\
<0.03 \\
\end{array}$ & $\begin{array}{r}73 \\
3.66 \\
0.18 \\
\end{array}$ & $\begin{array}{r}125 \\
<1.40 \\
<0.07 \\
\end{array}$ \\
\hline $\begin{array}{l}\mathrm{KCl} \text { volume, } \mathrm{ml} \\
\text { Analytical } \mathrm{Hg}, \mathrm{ng} / \mathrm{ml} \\
\text { ug/dscm }\end{array}$ & $\begin{array}{r}616 \\
5.97 \\
2.54 \\
\end{array}$ & $\begin{array}{r}676 \\
<0.28 \\
<0.07\end{array}$ & $\begin{array}{r}567 \\
10.65 \\
4.09 \\
\end{array}$ & $\begin{array}{r}522 \\
<0.28 \\
<0.06 \\
\end{array}$ & $\begin{array}{r}634 \\
7.85 \\
3.42 \\
\end{array}$ & $\begin{array}{r}523 \\
<0.28 \\
<0.06\end{array}$ \\
\hline $\begin{array}{l}\text { Nitric/Peroxide volume, } \mathrm{ml} \\
\text { Analytical } \mathrm{Hg}, \mathrm{ng} / \mathrm{ml} \\
\text { ug/dscm }\end{array}$ & $\begin{array}{r}125 \\
<1.40 \\
<0.12 \\
\end{array}$ & $\begin{array}{r}181 \\
<1.40 \\
<0.10\end{array}$ & $\begin{array}{r}178 \\
<1.40 \\
<0.17\end{array}$ & $\begin{array}{r}173 \\
<1.40 \\
<0.09\end{array}$ & $\begin{array}{r}181 \\
<1.40 \\
<0.17 \\
\end{array}$ & $\begin{array}{r}175 \\
<1.40 \\
<0.10 \\
\end{array}$ \\
\hline $\begin{array}{l}\text { KMnO4 volume, } \mathrm{ml} \\
\text { Analytical } \mathrm{Hg}, \mathrm{ng} / \mathrm{ml} \\
\text { ug/dscm }\end{array}$ & $\begin{array}{r}200 \\
1.99 \\
0.28 \\
\end{array}$ & $\begin{array}{r}244 \\
<0.28 \\
<0.03\end{array}$ & $\begin{array}{r}245 \\
<0.28 \\
<0.05\end{array}$ & $\begin{array}{r}248 \\
<0.28 \\
<0.03 \\
\end{array}$ & $\begin{array}{r}242 \\
1.16 \\
0.19 \\
\end{array}$ & $\begin{array}{r}244 \\
<0.28 \\
<0.03 \\
\end{array}$ \\
\hline $\begin{array}{l}\text { KMnO4-Acid Rinse volume, } \mathrm{ml} \\
\text { Analytical } \mathrm{Hg}, \mathrm{ng} / \mathrm{ml} \\
\text { ug/dscm }\end{array}$ & $\begin{array}{r}100 \\
<1.40 \\
<0.10 \\
\end{array}$ & $\begin{array}{r}100 \\
<1.40 \\
<0.05 \\
\end{array}$ & $\begin{array}{r}100 \\
<1.40 \\
<0.09 \\
\end{array}$ & $\begin{array}{r}100 \\
<1.40 \\
<0.05 \\
\end{array}$ & $\begin{array}{r}100 \\
<1.40 \\
<0.10 \\
\end{array}$ & $\begin{array}{r}100 \\
<1.40 \\
<0.06 \\
\end{array}$ \\
\hline $\begin{array}{l}\text { Particulate } \mathrm{Hg} \text { (ug/dscm) } \\
\text { Oxidized } \mathrm{Hg}(\mathrm{ug} / \mathrm{dscm} \text { ) } \\
\text { Elemental } \mathrm{Hg} \text { (ug/dscm) } \\
\text { Total } \mathrm{Hg} \text { (ug/dscm) }\end{array}$ & $\begin{array}{c}4.16 \\
2.91 \\
0.49 \\
7.56\end{array}$ & $\begin{array}{l}<0.00 \\
<0.15 \\
<0.18 \\
<0.33\end{array}$ & $\begin{array}{l}2.89 \\
4.75 \\
<0.31 \\
7.95\end{array}$ & $\begin{array}{l}<0.00 \\
<0.16 \\
<0.18 \\
<0.34\end{array}$ & $\begin{array}{c}3.79 \\
3.71 \\
0.46 \\
7.97\end{array}$ & $\begin{array}{l}<0.00 \\
<0.19 \\
<0.18 \\
<0.38\end{array}$ \\
\hline $\begin{array}{l}\text { Particulate Hg (ug/dscm @ } 3 \% \text { O2) } \\
\text { Oxidized Hg (ug/dscm @ 3\% O2) } \\
\text { Elemental Hg (ug/dscm @ 3\% O2) } \\
\text { Total Hg (ug/dscm @ 3\% 02) } \\
\end{array}$ & $\begin{array}{l}5.32 \\
3.72 \\
0.63 \\
9.67\end{array}$ & \begin{tabular}{l||}
$<0.00$ \\
$<0.21$ \\
$<0.25$ \\
$<0.46$
\end{tabular} & $\begin{array}{r}3.72 \\
6.12 \\
0.40 \\
10.23\end{array}$ & $\begin{array}{l}<0.00 \\
<0.23 \\
<0.25 \\
<0.49\end{array}$ & $\begin{array}{r}4.89 \\
4.79 \\
0.60 \\
10.28 \\
\end{array}$ & $\begin{array}{l}<0.00 \\
<0.27 \\
<0.26 \\
<0.54\end{array}$ \\
\hline Removal (\%) & \multicolumn{2}{|c|}{$>95.2$} & \multicolumn{2}{|c|}{$>95.3$} & \multicolumn{2}{|c|}{$>94.7$} \\
\hline
\end{tabular}


o

\begin{tabular}{|c|c|}
\hline \multirow{6}{*}{$\begin{array}{l}\text { TEST ID } \\
\text { PLANT } \\
\text { LOCATION } \\
\text { DATE } \\
\text { OPERATOR(S) } \\
\text { AMBIENT TEMP [F] }\end{array}$} & ONE \\
\hline & GREENIDGE \\
\hline & AIR HEATER OUTLET \\
\hline & 3132107 \\
\hline & R. ODA\& D. OLSEN \\
\hline & 29.83 \\
\hline
\end{tabular}

ONTARIO HYDRO Hg SAMPLING FIELD DATA SHEET $\frac{35}{1}: 2 \frac{7}{35^{-1}}$

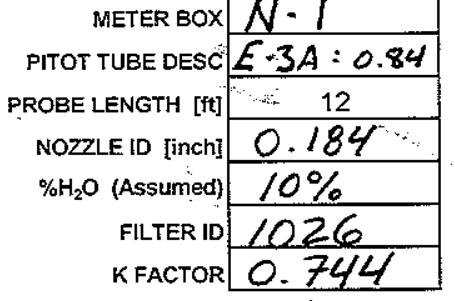

KFACTOR 0.744

\begin{tabular}{|c|c|c|c|c|c|c|c|c|c|c|c|c|c|c|c|}
\hline \begin{tabular}{|l|} 
TRAVERSE \\
\end{tabular} & CLOCK & \begin{tabular}{|l} 
SAMPLE \\
MuE
\end{tabular} & STATIC & PITOT & MEEER DIFF & METER & $\begin{array}{l}\text { METER } \\
\text { PEAN }\end{array}$ & 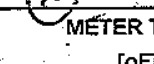 & & & PROBE & & $\begin{array}{l}\text { LAST IMP } \\
\text { TWMP } \\
\text { D. }\end{array}$ & MEIER & XHAUST \\
\hline \begin{tabular}{|c|} 
PoINT \\
[port-inch]
\end{tabular} & $\begin{array}{l}\text { TiME } \\
(24 \mathrm{hr})\end{array}$ & $\left.\mid \begin{array}{c}\text { TIISE } \\
\text { [minute] }\end{array}\right]$ & 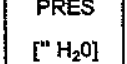 & 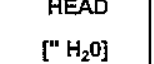 & $\begin{array}{c}\text { PRRSSSREE } \\
{\left[" \mathrm{H}_{2} 0\right]}\end{array}$ & $\begin{array}{c}\text { VACUUM } \\
\text { [" Hg] }\end{array}$ & 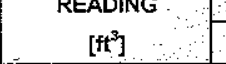 & inlet & outlet & $\begin{array}{l}\text { PEMP } \\
\text { "PA" }\end{array}$ & P & 邽 & $\mathrm{PF}_{\mathrm{F}}$ & {$[\%$ vol $]$.} & $\begin{array}{c}\mathrm{CO}_{2} \\
{[\%} \\
\mathrm{rol}\end{array}$ \\
\hline & 0.505 & 0 & & & & & $87+10$ & . & & & & & & & \\
\hline 1-2" & 0912 & 7 & $-12,6$ & 0.05 & 0.04 & 20 & 85.19 & 4.5 & 44 & 284 & 263 & 172 & 41 & & \\
\hline $1-2^{n}$ & 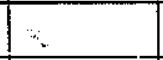 & 14. & -12.55 & औ心 & 5.44 & 2.0 & 876.33 & 46 & 4.5 & 289 & 275 & 172 & 40 & 6.7 & 13,5 \\
\hline$\overline{1-6^{\prime \prime}}$ & & 21 & -0.42 & 0.75 & 0,56 & 2.5 & 779 & 47 & 45 & 294 & 284 & 177 & 39 & 6.7 & 13.5 \\
\hline $1-6^{\prime \prime}$ & & 28 & $-12,95$ & 0.78 & 0.54 & 2.5 & 8.98 & 49 & 46 & 294 & 284 & 180 & 38 & 6.6 & 13.7 \\
\hline $1-10^{\prime \prime}$ & & 35 & $-3,01$ & 1.4 & 0.83 & 4.0 & 885.18 & 50 & 46 & 296 & 278 & 184 & 34 & 6.4 & 13.8 \\
\hline $1 \cdot 10^{\prime \prime}$ & & 42 & & 1.1 & 0.83 & 50 & 888.59 & 50 & 47 & 296 & 273 & 18 & 40 & 8.2 & 14.0 \\
\hline & 1000 & & & - & & & 889,20 & & & & & & & & \\
\hline $2 \cdot 2^{\prime \prime}$ & $\bar{z}$ & 749 & -12.68 & 0,30 & 0.22 & 20 & $89 \% .38$ & 51 & 48 & 285 & 250 & 180 & 40 & 9.1 & $B .1$ \\
\hline $2-2^{\prime \prime}$ & & 1456 & $=-13,0$ & $0.3 d$ & 0.22 & 2.0 & 893,26 & 51 & 48 & 291 & 260 & 182 & 40 & 2.1 & 13.1 \\
\hline $2-6^{n}$ & & $=4_{63}$ & $=12.26$ & 0.78 & 0.58 & 4.0 & 896.16 & 52 & 49 & 293 & 279 & 178 & 41 & 96.9 & 13.3 \\
\hline $2-6^{\prime \prime}$ & & $2 i_{70}$ & 43.0 & 0.78 & 0.58 & 45 & 899.03 & 54 & 49 & 293 & 284 & 178 & 40 & 6.8 & 13.4 \\
\hline $2-10^{\prime \prime}$ & & $35_{77}$ & $\Phi B .49$ & 1.3 & 0.94 & 6.0 & 90259 & 55 & 50 & 295 & 28 & 177 & 41 & 6.2 & 14.0 \\
\hline $2-10^{*}$ & & $42_{84}$ & $-72.4 \overline{7}$ & 1.3 & 0.96 & 7.0 & 906,26 & 56 & 50 & 295 & $2 \times 4$ & 180 & 41 & 6.2 & 14.0 \\
\hline & 1050 & & & & 0.61 & & 907.10 & & & & 87 & & & & \\
\hline $3-2^{n}$ & & 791 & $=13.48$ & 0.82 & 022 & 10.0 & 910.35 & 54 & 51 & 288 & $2 \frac{2 x^{2}}{20}$ & 175 & 42 & 2,6 & 12.6 \\
\hline $3-2^{\prime \prime}$ & & 498 & -13.27 & $0 \mathrm{~S}^{2}$ & 0.61 & 6.5 & 913.33 & 55 & 51 & 291 & 271 & 178 & 42 & 7.5 & 12.8 \\
\hline $3 \cdot 6^{n}$ & & 4105 & $4+136$ & 0.94 & 0.76 & 8.0 & 916.53 & 55 & 51 & $274 \leqslant$ & 7298 & 178 & 42 & 2.4 & $12, \bar{z}$ \\
\hline $3-6^{\prime \prime}$ & & $\underbrace{}_{112}$ & -13.01 & 0.94 & 0.70 & 8.8 & 919.72 & 56 & 31 & 298 & 281 & 17 & 42 & 7.4 & 12.8 \\
\hline $3-10^{\prime \prime}$ & & 85 & -12.75 & 1.1 & 0.83 & 9.5 & 923.11 & 56 & 51 & 298 & 285 & & 42 & 7.0 & 13.2 \\
\hline $3 \cdot 10^{\mathrm{n}}$ & 1132 & $2{ }_{126}$ & $-13,32$ & 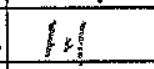 & 0.83 & 10.5 & 926.50 & 56 & 51 & 298 & 259 & 178 & 43 & 7.0 & 13.2 \\
\hline & & & & & & & & & & & & & & & \\
\hline A) & & & -1294 & 0.719 & 0.592 & & 50.95 & 50. & 3 & 293.2 & & & & 6.9 & 13.3 \\
\hline & & & $\begin{array}{l}\text { Sample Train } \\
\text { eak Checks }\end{array}$ & $\begin{array}{l}\text { Pre Test } \\
\text { Post Tes }\end{array}$ & $\angle 0.01 \mathrm{ft}^{2}$ & & Hg & & & $\mathrm{kCl}$ & & & & & \\
\hline
\end{tabular}

CONSOL ENERGK. 
ONTARIO HYDRO Hg SAMPLING FIELD DATA SHEET

\begin{tabular}{|c|c|c|c|c|c|c|c|c|c|c|c|c|c|c|c|}
\hline \multirow{7}{*}{\multicolumn{2}{|c|}{$\begin{array}{l}\text { TEST ID } \\
\text { PLANT } \\
\text { LOCATION } \\
\text { DATE } \\
\text { OPERATOR(S) } \\
\text { AMBIENT TEMP PF] } \\
\text { BAR. PRESS. [" Hg] }\end{array}$}} & \multirow{2}{*}{\multicolumn{3}{|c|}{$O n \varepsilon$}} & \multirow{3}{*}{\multicolumn{2}{|c|}{$\begin{array}{r}\text { METER BOX } \\
\text { PITOT TUBE DESC }\end{array}$}} & & \multirow{2}{*}{\multicolumn{2}{|c|}{ CAL. DATA: delta $H$}} & \multirow{2}{*}{\multicolumn{2}{|c|}{1.859 Comments: }} & & & \multirow{2}{*}{\multicolumn{2}{|c|}{- of }} \\
\hline & & & & & & & $N-2$ & & & & & & & & \\
\hline & & & GREENIDGE & & & & $E \cdot \mid A: 0.835$ & & & 1.046 & & & & & \\
\hline & & & $\frac{\text { STACK }}{29-1}$ & & & BE LENGTH [ $\mathrm{ft}]$ & $\begin{array}{r}10 \\
78 \\
\end{array}$ & & $c(p)$ & 0.835 & & & & & \\
\hline & & & $\frac{-28-C}{\text { ERAR \&B.S }}$ & 37 & & $\begin{array}{l}\text { OZZLE ID [inch] } \\
{ }_{0} \mathrm{H}_{2} \mathrm{O} \text { (Assumed) }\end{array}$ & $\frac{0.248}{10 \%}$ & KOBE H & PX SETTING & $\frac{N A}{250}$ & & & & & \\
\hline & & & 45 & & & & & DUCT & X-SECTION & circ? & rect? & other: & & & \\
\hline & & & .83 & & & & & & & & $\overbrace{\text { CTAREA }}$ & & & & \\
\hline \begin{tabular}{|l|} 
TRAVERSE \\
\end{tabular} & CLOCK & SAMPLE & STATIC & Pाтот & & & ME & & & & & & & ERE & $\overline{\mathrm{XHAL}}$ \\
\hline POINT & TIME & TIME & PRES & HEAD & & & READING & & & TEMP & TEMP & -80 & & & \\
\hline [port-inch] & $\frac{(24 n)}{0,05}$ & $\frac{\text { [minute] }}{0}$ & {$\left[\mathrm{H}_{2} \mathrm{O}\right]$} & {$\left[\begin{array}{rl} \\
{[N}\end{array}\right.$} & & $\mathrm{Hgl}$ & $\begin{array}{c}{\left[\mathrm{ft}^{2}\right]} \\
+0,101\end{array}$ & & outlet & & & {$[\pi]$} & & & {$[\%$ vol] } \\
\hline $1-5^{n}$ & 0910 & 7.5 & & 58 & 1.7 & 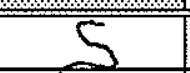 & 648.00 & 43 & 40 & 173 & 203 & 210 & & & $=$ \\
\hline 1-16.38" & & 15 & & .62 & 1.85 & $S$ & 653.31 & & 40 & 175 & 206 & 211 & 3 & 5. & 11.0 \\
\hline $1-30.25^{\prime \prime}$ & & 22.5 & .533 & .58 & 1.70 & $S$ & 658.67 & 48 & 42 & 175 & 201 & 211 & 4 & 9.2 & 11.0 \\
\hline $1.50 .38 "$ & & 30 & & .58 & 1.70 & 5 & 663.90 & 48 & +2 & 176 & 202 & 210 & & $=$ & $=$ \\
\hline & & & & & L. & $k \rightarrow$ & 664.10 & & & & & & & & \\
\hline $2.5^{\prime \prime}$ & & 37.5 & & .62 & 1.85 & & 669.52 & & 43 & 17 & 200 & 206 & & & \\
\hline $2-16.38^{n}$ & & 45 & & .68 & 2.00 & 5.5 & 675,17 & 0 & 44 & 175 & 208 & 207 & & & 10.8 \\
\hline $2-30.25^{\prime \prime}$ & & 52.5 & .582 & .70 & 2.05 & & 180.90 & & 45 & 175 & 204 & 207 & & & 10.9 \\
\hline $2-50.38^{\prime \prime}$ & & 60 & & .62 & 1.85 & 5.5 & 6.86 .38 & 53 & 46 & 176 & 208 & 202 & & 9,3 & 11.0 \\
\hline & & & & & L.C. & $0 \mathrm{~K} \rightarrow$ & 686.50 & & & & & & & & \\
\hline $3.5^{\prime \prime}$ & & 67.5 & & 54 & 1.58 & 4.5 & 691.56 & & 47 & 177 & 209 & 216 & 43 & & $=$ \\
\hline $3-16.38^{\prime \prime}$ & & 75 & & .58 & 1.70 & 5 & 696.78 & 53 & 47 & 174 & 202 & 218 & 43 & 9.4 & 10.5 \\
\hline 3-30.25" & & 82.5 & .588 & .58 & 1.70 & 5 & 101.98 & & 48 & 175 & 206 & 219 & 42 & 9.5 & 10.8 \\
\hline $3-50.38^{\prime \prime}$ & & 90 & & .54 & 1.58 & 5 & 707.05 & 55 & $\div 8$ & 178 & 200 & 220 & 43 & 9.4 & 10.9 \\
\hline $\bar{\sigma}$ & & & & & $a c$ & $O K \rightarrow$ & 707.20 & & & & & & & & \\
\hline$\overline{4-5^{\prime \prime}}$ & & 97.5 & & .46 & 1.35 & 4 & 711.87 & 54 & 48 & 174 & 194 & 246 & 43 & & - \\
\hline $4-16.38^{\prime \prime}$ & & 105 & & $: 46$ & 1.35 & 4 & 716.58 & 53 & 48 & 175 & 197 & 246 & 42 & 9.5 & 10.8 \\
\hline $4-30.25^{\prime \prime}$ & & 112.5 & .545 & $: 48$ & 1.40 & 4 & 721.36 & 54 & +9 & 176 & 203 & 247 & +3 & 5.3 & 11.0 \\
\hline $4-50.38^{\prime \prime}$ & & 120 & & .50 & 1.46 & 4.5 & 726.20 & 55 & 49 & 175 & 201 & 247 & 46 & 9.5 & 10.8 \\
\hline & 11133 & & & Rees & & & & & & & & & & & \\
\hline VERAGE & & & -0.564 & 0.563 & 1.676 & & 32.93 & 46.6 & & 174.9 & & & & 9.4 & 10.9 \\
\hline & & & mple Train & $\begin{array}{l}\text { Pre Te } \\
\text { Post Te }\end{array}$ & $\begin{array}{l}\frac{30.61}{50.01 \mathrm{ft}^{3}} \\
\end{array}$ & $@ \frac{20}{10}$ & & & & $\begin{array}{r}\text { Pitot } \\
\text { Leak } C h\end{array}$ & & & 6 & & \\
\hline
\end{tabular}


ONTARIO HYDRO Hg SAMPLING FIELD DQATA SHEET

\begin{tabular}{|c|c|c|c|c|c|c|c|c|c|c|c|c|c|c|c|}
\hline \multirow{8}{*}{\multicolumn{2}{|c|}{$\begin{array}{l}\text { TESTID } \\
\text { PLANT: } \\
\text { LOCATION } \\
\text { DATE } \\
\text { OPERATOR(S) } \\
\text { AMBIENT TEMP [F" } \\
\text { BAR. PRESS. [" Hg] }\end{array}$}} & \multirow{2}{*}{\multicolumn{3}{|c|}{2}} & \multirow{8}{*}{\multicolumn{3}{|c|}{ 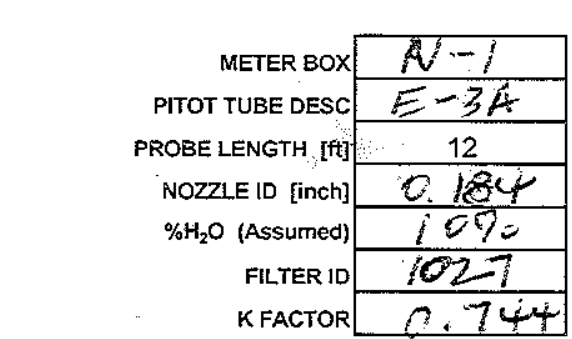 }} & \multirow{8}{*}{\multicolumn{2}{|c|}{$\begin{array}{r}\text { CAL DATA: detta H } \\
\text { C..C(P) } \\
\text { FLTER BOX SETTING } \\
\text { PROBE HTR SETTING } \\
\text { DUCT X-SECTION } \\
\text { DPUCT DIMENSIONS }\end{array}$}} & & \multirow{6}{*}{ Comments: } & \multirow{2}{*}{\multicolumn{4}{|c|}{ Page __ of }} \\
\hline & & & & & & & & & & $\theta$ & & & & & \\
\hline & & \multicolumn{3}{|c|}{ GREENIDGE. } & & & & & & 0.770 & & \\
\hline & & \multirow{2}{*}{\multicolumn{3}{|c|}{$\begin{array}{l}\text { AIR HEATER OUTLET } \\
3 / 281 \mathrm{C} 7\end{array}$}} & & & & & & & & \multicolumn{4}{|c|}{10 min piurge after tes } \\
\hline & & & & & & & & & & & & & & & \\
\hline & & & & & & & & & & \multirow{2}{*}{\multicolumn{2}{|c|}{ other: }} & \\
\hline & & & \multicolumn{2}{|c|}{ ODA\&D. OLSEN } & & & & & & \multirow{2}{*}{\begin{tabular}{|l|l|} 
circ? & rect? \\
3 & DUCT AREA \\
3
\end{tabular}} & \multirow{2}{*}{\begin{tabular}{|c|} 
rect? \\
Duct AREA \\
Df?
\end{tabular}} & & & & \\
\hline & & \multicolumn{3}{|c|}{29.85} & & & & & & & & \multicolumn{2}{|c|}{ sheding } & & \\
\hline TRAVERSE & & \multicolumn{2}{|c|}{$2,1.03$} & PITOT & \multirow{4}{*}{$\begin{array}{c}\text { METER DIFF } \\
\text { PRESSURE } \\
{\left[{ }^{n} \mathrm{H}_{2} 0\right]} \\
\end{array}$} & \multirow{3}{*}{$\begin{array}{c}\text { METER } \\
\text { VACUuM } \\
\text { I" HgI }\end{array}$} & \multirow{3}{*}{$\begin{array}{c}\text { METER } \\
\text { READING } \\
{\left[\left[t^{2}\right]\right.} \\
\end{array}$} & \multirow{2}{*}{\multicolumn{2}{|c|}{ (6) }} & $\frac{7}{s^{4} A c k}$ & PROBE & \multicolumn{2}{|c|}{ (3) } & METERE & XHAUST \\
\hline POINT & TIME & TIME & PRES & HEAD & & & & & & TEMP & TEMP & Box & TEMP & WLILKL & $\mathrm{CO}_{2}$ \\
\hline [port-inch] & & [minute] & {$\left[\mathrm{In}_{2} \mathrm{O}\right]$} & {$\left[\mathrm{H}_{2} \mathrm{O} \mathrm{O}\right]$} & & & & inlet & outlet & {$[\mathrm{F}]$} & {$[\mathrm{F}]$} & {$[\mathrm{F}]$} & {$[\mathrm{P}$} & {$[\%$ vol $]$} & {$[\%$ voli $]$} \\
\hline & 1240 & 0 & & & & & 430.20 & & & & & & & 2.2 & \\
\hline $1 \cdot 2^{n}$ & & 7 & $-j 2.58$ & 0.10 & 0.07 & 1.8 & $93 \% .51$ & $\frac{20}{60}$ & 48 & 295 & 246 & 132 & 942 & tक & 12.5 \\
\hline $1-2^{\prime \prime}$ & & 14 & $-13,1$. & $d, 10$ & 0.67 & 10 & 932.75 & 50 & 49 & 298 & 261 & 147 & 42 & 7.0 & 13.2 \\
\hline $1.6^{\prime \prime}$ & & 21 & -73.24 & $i, 1$ & $d .83$ & 40 & 635.91 & 51 & $49^{4}$ & 301 & 273 & 149 & 41 & 6.7 & 13.6 \\
\hline $1.6^{\prime \prime}$ & & 28 & $-13,04$ & 1.1 & $6: 83$ & 40 & 439.10 & 52 & 49 & 301 & $2 \varepsilon_{i}$ & 154 & $4 \pi$ & 6.6 & $\beta .6$ \\
\hline$\frac{1-10 "}{1-10}$ & & 35 & -73.39 & i. & 0.87 & 50 & 442.47 & 54 & 49 & 313 & 286 & 160 & 40 & 6.3 & $13 \times 8$ \\
\hline $1.10^{\mathrm{m}}$ & & 42 & -73.59 & .1 .1 & 93 & $5: 0$ & $9+5.90$ & 55 & $5 i$ & 303 & 292 & 160 & 41 & 6.3 & 13.8 \\
\hline & & & & & & & 946,20 & & & & & & & & \\
\hline $2 \cdot 2^{n "}$ & & 749 & -1223 & .38 & 28 & 3.0 & 940,49 & 33 & 51 & 291 & 277 & 155 & 42 & 7.6 & 12.7 \\
\hline $2 \cdot 2^{\prime \prime}$ & & 56 & -20.52 & 38 & $\not 2$ & 3.0 & 450.55 & 54 & 51 & 299 & 279 & 158 & 42 & 7.1 & $B: 1$ \\
\hline $2 \cdot 6^{\prime \prime}$ & & 63 & $-12,56$ & 0.53 & 0.63 & 4.5 & 453.39 & 54 & 51 & 299 & 280 & 159 & 42 & 6,9 & 13.3 \\
\hline $2 \cdot 6^{\prime \prime}$ & & $f_{70}$ & -121 & 0.85 & 0.3 & 4.5 & 956.39 & 55 & 51 & 299 & 288 & 161 & 42 & 6.9 & 133 \\
\hline $2-10^{\prime \prime}$ & & 557 & $-x 325$ & 14 & 1.04 & 7.0 & 960,20 & 56 & 7 & 30 & 284 & 163 & 43 & 6.6 & 13.5 \\
\hline $2-10^{n}$ & & 284 & $-13,24$ & 2,4 & 604 & 70 & 963,94 & 56 & 51 & 34 & 265 & 163 & 43 & 6.7 & 13.6 \\
\hline & 1420 & & & & & & 164.60 & & & & & & & & \\
\hline $3-2^{n}$ & & $7_{91}$ & 0.993 & 072 & 0.53 & 1,0 & 967.58 & 54 & 51 & 287 & 272 & 155 & 43 & 7.6 & 12.6 \\
\hline $3 \cdot 2^{\prime \prime}$ & & 498 & 12.73 & 0.72 & 0.53 & 6.0 & 970.32 & 55 & 51 & 298 & 277 & 198 & 43 & 7.5 & $\mid 2.7$ \\
\hline $3.6^{\prime \prime}$ & & 105 & $-B .72$ & 0,95 & 0.70 & 7.5 & 973.36 & 55 & 32 & 305 & 281 & 159 & +4 & 7.4 & 12.8 \\
\hline $3.6^{\prime \prime}$ & & 28 & -13.18 & 0.95 & 0.76 & 8,0 & 976.52 & .56 & 52 & 305 & 287 & 160 & 44 & 7.4 & 12,8 \\
\hline $3-10^{n}$ & & 5119 & $-(3), 2$ & 1.1 & 0.83 & 10.0 & 979.92 & 52 & 51 & 307 & 290 & 167 & 44 & 7.0 & 13.2 \\
\hline 3.-40" & $1502^{2}$ & 会 & 012.45 & 1.1 & 0.03 & 11.0 & 983.95 & 56 & 52 & 303 & 269 & 170 & 44 & 70 & 13.2 \\
\hline & & & & 0.792 & & & & & & & & & & & \\
\hline AVERAGE & & & $\mid-13.09$ & 0,854 & 0.638 & & 52.21 & 52.1 & & 698.6 & & & & 7.0 & 13.2 \\
\hline $2=$ & & & $\begin{array}{l}\text { Sample Train } \\
\text {-eak checks: }\end{array}$ & Post & $\frac{20,01}{40.02}$ & $@ \frac{10}{13}$ & & & & Leak Checks: & & $\frac{\partial E}{2}$ & $\frac{7}{6}$ & & \\
\hline
\end{tabular}




\section{ONTARIO HYDRO Hg SAMPLING FIELD DATA SHEET}

\begin{tabular}{|c|c|c|c|c|c|c|c|c|c|c|c|c|c|c|c|}
\hline \multirow{7}{*}{\multicolumn{2}{|c|}{$\begin{array}{l}\text { TEST ID } \\
\text { PLANT } \\
\text { LOCATION } \\
\text { DATE } \\
\text { OPERATOR(S) } \\
\text { AMBIENT TEMP PF] } \\
\text { BAR. PRESS. [" Hg] }\end{array}$}} & \multicolumn{3}{|c|}{ TwO } & \multirow{2}{*}{\multicolumn{2}{|c|}{$\begin{array}{r}\text { METER BOX } \\
\text { PITOT TUBE DESC }\end{array}$}} & $0-2$ & \multicolumn{2}{|c|}{ CAL. DATA: delta H } & .859 & \multirow[t]{2}{*}{ Comments } & \\
\hline & & \multicolumn{3}{|c|}{ GREENIDGE } & & & $E-1 A=0.8$ & & & 1.046 & & & & & \\
\hline & & \multirow{2}{*}{\multicolumn{3}{|c|}{$\begin{array}{c}\text { STACK } \\
3-28-07\end{array}$}} & \multirow{2}{*}{\multicolumn{2}{|c|}{$\begin{array}{l}\text { PTTOT TUBE DESC } \\
\text { PROBE LENGTH [ft] } \\
\text { NOZZLE ID [inch] }\end{array}$}} & 10 & \multirow{3}{*}{\multicolumn{2}{|c|}{$\begin{array}{r}\text { C(P) } \\
\text { FILTER BOX SETTNGG } \\
\text { PROBE HTR SETTING }\end{array}$}} & 0.835 & & & & & \\
\hline & & & & & & & $0.2+8$ & & & $\sim A$ & & & & & \\
\hline & & \multicolumn{3}{|c|}{ K. CERAR \& B. SSLIFER } & \multirow{3}{*}{\multicolumn{2}{|c|}{$\begin{array}{c}\text { NOZZLE ID linch] } \\
\% H_{2} \mathrm{O} \text { (Assumed) } \\
\text { FILTER D } \\
\text { K FACTOR }\end{array}$}} & & & & 250 & & \multirow{2}{*}{\multicolumn{2}{|c|}{ other: }} & & \\
\hline & & \multirow{2}{*}{\multicolumn{3}{|c|}{$\begin{array}{r}445 \\
2985\end{array}$}} & & & 77 & \multirow{2}{*}{\multicolumn{2}{|c|}{$(6)$}} & circ? & \multirow{2}{*}{ DUCTAREA } & \multirow{2}{*}{\multicolumn{4}{|c|}{ other: }} \\
\hline & & & & & & & 204 & & & & & & & & \\
\hline \multirow{4}{*}{\begin{tabular}{|c|} 
TRAVERSE \\
POIITT \\
[port-inch] \\
\end{tabular}} & \multirow{4}{*}{\begin{tabular}{|c|} 
CLOCK \\
TTME \\
$(24-\mathrm{hr})$ \\
$1243(3$ \\
\end{tabular}} & \multirow{4}{*}{\begin{tabular}{|c|} 
SAMPLE \\
TMEE \\
[minute] \\
0 \\
\end{tabular}} & \multirow{4}{*}{\begin{tabular}{|l|} 
STATIC \\
PRES \\
{$\left[" \mathrm{H}_{2} \mathrm{O}\right]$} \\
\end{tabular}} & \multirow{4}{*}{$\begin{array}{c}\text { PTIOT } \\
\text { HEAD } \\
{\left[{ }^{*} \mathrm{H}_{2} \mathrm{O}\right]} \\
\end{array}$} & \multirow{4}{*}{$\begin{array}{c}\text { METER DIFF } \\
\text { PRESSURE } \\
{ }^{\left[" \mathrm{H}_{2} 0\right]} \\
\end{array}$} & & METER & & & STACK & & $\operatorname{lom}_{x+T E R}$ & & METER E & XHAUST \\
\hline & & & & & & VACUUM & READING & & & TEMP & TE & an & & & $\mathrm{CO}_{2}$ \\
\hline & & & & & & {$[\mathrm{Hg}]$} & {$\left[\mathrm{ft}^{3}\right]$} & inlef & outlet & {$[\mathrm{F}$} & {$[\mathrm{F}]$} & [P] & & {$[\%$ vol $]$} & {$[\%$ vol $]$} \\
\hline & & & & & & & 133.30 & & & & & & & & \\
\hline$-5^{\prime \prime}$ & & 7.5 & & 45 & 1.32 & 4 & 737.71 & +S & +7 & 178 & 23 & 170 & & & $\mathrm{C}$ \\
\hline$-16.38^{n}$ & & 15 & & 48 & 1,40 & 4 & $7+2 .+1$ & 47 & 44 & 178 & 238 & 176 & & 9.0 & 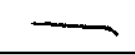 \\
\hline$-30.25^{\prime \prime}$ & & 22.5 & & .48 & 1.40 & 4 & 74.17 & 45 & 451 & 177 & 25 & 178 & & - & 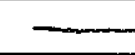 \\
\hline$-50.38^{*}$ & & $30-$ & 589 & .54 & 1.58 & 5 & 752.22 & 50 & 45 & 177 & $2+5$ & 168 & & 9.1 & 11.2 \\
\hline & & & & & $1, c$ & $0 k \rightarrow$ & 352.30 & & & & & & & & \\
\hline$-5^{n}$ & & 37.5 & & 54 & 1.58 & 5 & 757.36 & 49 & $\div 5$ & 176 & 240 & 194 & 39 & - & - \\
\hline $2-16.38^{\prime \prime}$ & & 45 & & 56 & 1.63 & $S$ & 762.76 & 50 & 45 & 175 & 243 & 201 & 40 & 9.0 & 11.3 \\
\hline$-30.25^{\prime \prime}$ & & 52.5 & 150 & .60 & 1.76 & 5 & 767.77 & 50 & 45 & 175 & 238 & 198 & 41 & 9.1 & 11.2 \\
\hline$-50.38^{\prime \prime}$ & & 60 & & .56 & $1.6-3$ & 5 & 772.91 & 51 & 46 & 175 & 243 & 197 & +2 & 9.0 & 11.3 \\
\hline & & & & & & $0 K \rightarrow$ & 73.80 & & & & & & & & \\
\hline$-5^{\prime \prime}$ & & 67.5 & & 1.4 & 1.88 & 5.5 & 779.31 & 49 & +5 & 176 & $2+3$ & 229 & 41 & - & $=$ \\
\hline $3-16.38^{n}$ & & 75 & & .72 & 2.10 & 6 & .85 .07 & 51 & 47 & 177 & 251 & 230 & 41 & 9.2 & 11.1 \\
\hline 3.-30.25" & & $82.5-$ & 635 & .72 & 2.10 & 6 & 790.87 & 5 & b) & 177 & $2+6$ & 232 & +2 & 9.2 & 11.1 \\
\hline $50.38^{n}$ & & 90 & & .64 & 1.88 & 6 & 796.40 & 53 & 47 & 176 & 247 & 231 & 43 & 9.2 & 11.1 \\
\hline & & & & & L.C.C & $0 K \rightarrow$ & 796.60 & & & & & & & & \\
\hline $4.5^{\prime \prime}$ & & 97.5 & & 56 & 1.63 & $S$ & 801.73 & 51 & 47 & 176 & 252 & 227 & 46 & $=$ & $=$ \\
\hline 4-16.38" & & 105 & & .60 & 1.76 & 5 & 807.04 & 52 & 47 & 176 & 238 & 227 & 49 & 9.2 & $1 ! 1$ \\
\hline $4-30.25^{n} K$ & $=2.75$ & $\overrightarrow{112.5}$ & & .62 & 1.70 & 5 & 812.27 & 53 & 48 & 175 & 246 & 228 & 51 & 9.2 & 11.1 \\
\hline $50.38^{n}$ & & $120-$ & 543 & .58 & 1.58 & 5 & 817.37 & 54 & +8 & 175 & $2+2$ & $2+5$ & 52 & 9.2 & 11.1 \\
\hline & 1507 & & & Rus & & & & & & & & & & & \\
\hline AVERAGE & & & -0.604 & 0.578 & 1.683 & & 82.90 & 48.2 & & 176.2 & & & & 9.1 & 11.2 \\
\hline & & & ample Tra & $\begin{array}{l}\text { Pre Te. } \\
\text { Post Te. }\end{array}$ & Q.ci $\mathrm{ft}^{3}$ & $\frac{8}{10}$ ir & & & & $\begin{array}{l}\text { Pitot Tube } \\
\text { Leaak Checks: }\end{array}$ & 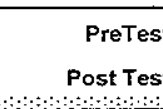 & $\frac{0.01}{0.0}$ & c & $0^{\circ}$ & \\
\hline
\end{tabular}


管

\begin{tabular}{|c|c|c|c|c|c|c|c|c|c|c|c|c|c|c|c|}
\hline \multirow{8}{*}{\multicolumn{2}{|c|}{$\begin{array}{l}\text { TEST ID } \\
\text { PLANT } \\
\text { LOCATION } \\
\text { DATE } \\
\text { OPERATOR(S) } \\
\text { AMBIENT TEMP ["F] } \\
\text { BAR. PRESS. [" Hg] }\end{array}$}} & \multirow{2}{*}{\multicolumn{3}{|c|}{3}} & \multirow{3}{*}{\multicolumn{2}{|c|}{ METER BOX }} & & \multirow{3}{*}{\multicolumn{2}{|c|}{ CAL DATA: delta $\mathrm{H}$}} & & & & & \multicolumn{2}{|c|}{ Page __ of } \\
\hline & & & & & & & $M$ & & & & & & & & \\
\hline & & \multicolumn{3}{|c|}{ GREENIDGE } & & & $\equiv-3 A$ & & & 0.970 & & \multicolumn{3}{|c|}{ io minpunger } & \\
\hline & & \multicolumn{3}{|c|}{ AIR HEATER OUTLET } & \multicolumn{2}{|c|}{$\begin{array}{r}\text { PITOT TUBE DESC } \\
\text { PROBE LENGTH [ft] }\end{array}$} & & \multirow{2}{*}{\multicolumn{2}{|c|}{$\begin{array}{r}c(p) \\
\text { FILTER BOX SETTING }\end{array}$}} & 0.840 & & \multirow{2}{*}{\multicolumn{4}{|c|}{$j$}} \\
\hline & & \multirow{2}{*}{\multicolumn{3}{|c|}{$\frac{3 / 28 / 17}{\text { R. ODÁ \& D. OLSEN }}$}} & \multicolumn{2}{|c|}{ NOZZLE ID [inch] } & 0.104 & & & & & & & & \\
\hline & & & & & \multicolumn{2}{|r|}{$\% \mathrm{H}_{2} \mathrm{O}$ (Assumed) } & & \multicolumn{2}{|c|}{ PROBE HTR SETANG } & & & & & & \\
\hline & & \multirow{2}{*}{\multicolumn{3}{|c|}{29.85}} & \multirow{2}{*}{\multicolumn{2}{|c|}{$\begin{array}{r}\text { FILTER ID } \\
\text { KFACTOR }\end{array}$}} & 1028 & \multirow{2}{*}{\multicolumn{2}{|c|}{$\begin{array}{l}\text { DUCT X-SECTION } \\
\text { DHCT DIMENSLONS }\end{array}$}} & circ? & rect? & \multicolumn{2}{|l|}{ other: } & & \\
\hline & & & & & & & 44 & & & & & & & & \\
\hline TRAVERSE & CLOCK & SAMPLE & STATIC & PITOT & \multirow{4}{*}{$\begin{array}{c}\text { METER DIFF } \\
\text { PRESSURE } \\
{\left[{ }^{\prime \prime} \mathrm{H}_{2} 0\right]} \\
\end{array}$} & \multirow{3}{*}{$\begin{array}{c}\text { METER } \\
\text { VACUUM } \\
{[" \mathrm{Hg}]} \\
\text { ' }\end{array}$} & \multirow{3}{*}{$\begin{array}{c}\text { METER } \\
\text { READNG } \\
\text { [ift'] }\end{array}$} & & & SIACKK & REOE & Fin: & 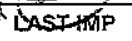 & METERE & XHAUST \\
\hline POINT & TIME & . TIME & PRES & HEAD & & & & & & TEMP & TEMP & & TEMP & $\mathrm{O}_{2}$ & $\mathrm{CO}_{2}$ \\
\hline [port-inch] & (24-hr) & [minute] & {$\left[" \mathrm{H}_{2} \mathrm{O}\right]$} & {$\left[" \mathrm{H}_{2} \mathrm{O}\right]$} & & & & inlet & outlet & {$\left[{ }^{\circ} \mathrm{F}\right]$} & {$[\circ]$} & {$[\mathrm{F}]$} & {$\left[{ }^{\circ} \mathrm{F}\right]$} & {$[\%$ vol $]$} & {$[\%$ vol $]$} \\
\hline$\sqrt[1]{1}$ : & 1553 & 0 & & & & רוm. & 984.10 & & & & & & & & \\
\hline $1-2^{\prime \prime}$ & & 7 & & 0.10 & $2: 27$ & 1.0 & 990.56 & 51 & 50 & $24 \%$ & 278 & 135 & 45 & 7.6 & 12.5 \\
\hline$-2^{m}$ & & 14 & -12.71 & $0.1 /$ & 0.67 & 1.0 & 951.67 & $5 i$ & 50 & 296 & 25 & 141 & 44 & 7.2 & 13.0 \\
\hline $1-6^{m}$ & & 21 & -13.38 & 0.85 & 0.44 & 3,5 & 94,61 & 52 & 57 & 299 & 25 & 148 & 44 & 6.8 & 13.4 \\
\hline $1.6^{m}$ & & 28 & $=12.65$ & 0.5 & 664 & 4,0 & 997.43 & 57 & 31 & $2 \% 9$ & 284 & 155 & 42 & 6.8 & 13.4 \\
\hline $1-10^{\pi}$ & & 35 & -12.60 & 1.1 & 6.83 & 5.0 & 1001.02 & 55 & 51 & 300 & 289 & 156 & 42 & 6.6 & 130 \\
\hline$-10^{n}$ & & 42 & -12.41 & 1.1 & 83 & $5 \cdot 0$ & $100 \% .47$ & 56 & 51 & 301 & 299 & 157 & 43 & 6.6 & 13.0 \\
\hline & 1643 & & & 725 & $2+6$ & & 5.00 & & & & & & & & \\
\hline $2-2^{n}$ & & 749 & -13.90 & 0.35 & $0: 26$ & 3.0 & 7.08 & 55 & 31 & $2 \sqrt{5}$ & 28 & 153 & 44 & 2,7 & 12.7 \\
\hline$-2 "$ & & 456 & $-12,55$ & 0.35 & A. 3 & 3.0 & 9.11 & 55 & 52 & 297 & 284 & 154 & 45 & 2,1 & 13,1 \\
\hline $2 \cdot 6^{\prime \prime}$ & & 63 & $-i 2.96$ & 0.78 & 0.5 & 5.0 & 11.94 & 75 & 52 & 297 & $28 \%$ & 155 & 4,5 & 6.9 & 13.3 \\
\hline $2-6^{\prime \prime}$ & & 70 & -13.70 & 2.78 & 0.58 & 5.0 & 14.90 & 57 & 52 & 297 & 254 & 156 & 44 & 6.9 & 13.3 \\
\hline $2 \cdot 10^{\prime \prime}$ & & $\sum \pi$ & -1327 & 1.3 & 0.94 & 2.0 & $=$ & 56 & 32 & 299 & $2 \longdiv { 8 7 }$ & 157 & 45 & 6.5 & 137 \\
\hline$-10^{\prime \prime}$ & is & 84 & 13.0 & 1.3 & 0.96 & 8.0 & 22.21 & 54 & 33 & 245 & 276 & 159 & 46 & 6.5 & 13.7 \\
\hline & 1135 & & & 375 & 0,56 & & $22,4=$ & & & & & & & & \\
\hline $3-2^{*}$ & & $\nabla_{91}$ & -13.4 & .0 .75 & $\cos 4$ & 4.5 & 25.39 & 58 & 53 & 290 & 281 & 149 & 47 & 7.5 & 12.7 \\
\hline $3 \cdot 2^{n}$ & & 98 & $\rightarrow 12.26$ & 0,75 & 0.56 & 5 & 26.19 & 51 & 53 & 295 & 285 & 153 & 47 & 7.5 & 12,7 \\
\hline $3-6^{n}$ & & 105 & $-13,0 ?$ & 0.97 & 0.72 & 6.8 & 31.39 & 58 & 53 & 300 & 251 & 153 & 46 & 7.2 & 130 \\
\hline $3-6^{\prime \prime}$ & & - 112 & 1346 & 097 & 0,72 & 65 & 34.59 & 50 & 54 & 301 & 295 & 154 & 47 & 7.3 & 12,9 \\
\hline $3.10^{\prime \prime}$ & 3 & $5 \quad 119$ & $12 i^{\circ}$ & 1.1 & 0.83 & 8.0 & $38.0 t$ & 58 & 54 & 302 & 293 & 157 & 47 & 71 & 13,1 \\
\hline $3-10^{\prime \prime}$ & & $Z_{126}$ & & 1,1 & 0.35 & 10.0 & 41.49 & 59 & 54 & 301 & 272 & 140 & $4 \div$ & $7.2^{7}$ & 13.2 \\
\hline & & & & (R+S) & & & & & & & & & & & \\
\hline AVERAGE & & 126 & -12.93 & 0.751 & 9.606 & & 51.67 & 53. & & 292.5 & & & & 7.03 & 13.09 \\
\hline & & & $\begin{array}{l}\text { ample Train } \\
\text { eak Checks: }\end{array}$ & Pre Test & 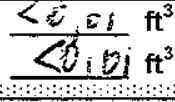 & $@ \frac{i}{0} i$ & $\begin{array}{l}\text { in. } \mathrm{Hg} \\
\text { in. } \mathrm{Hg}\end{array}$ & & & $\begin{array}{r}\text { Pitot Tube } \\
\text { Leak Checks: }\end{array}$ & Pre & $C^{\circ} F$ & $\frac{5}{9} i$ & $\begin{array}{l}\mathrm{H}_{2} \mathrm{O} \\
\mathrm{H}_{2} \mathrm{O}\end{array}$ & \\
\hline
\end{tabular}

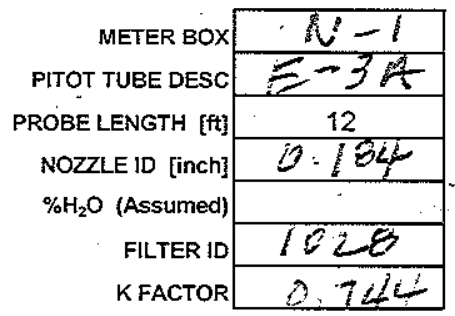

CONSOL ENETEY

\section{ONTARIO HYDRO Hg SAMPLING FIELD DATA SHEET}




\section{ONTARIO HYDRO Hg SAMPLING FIELD DATA SHEET}

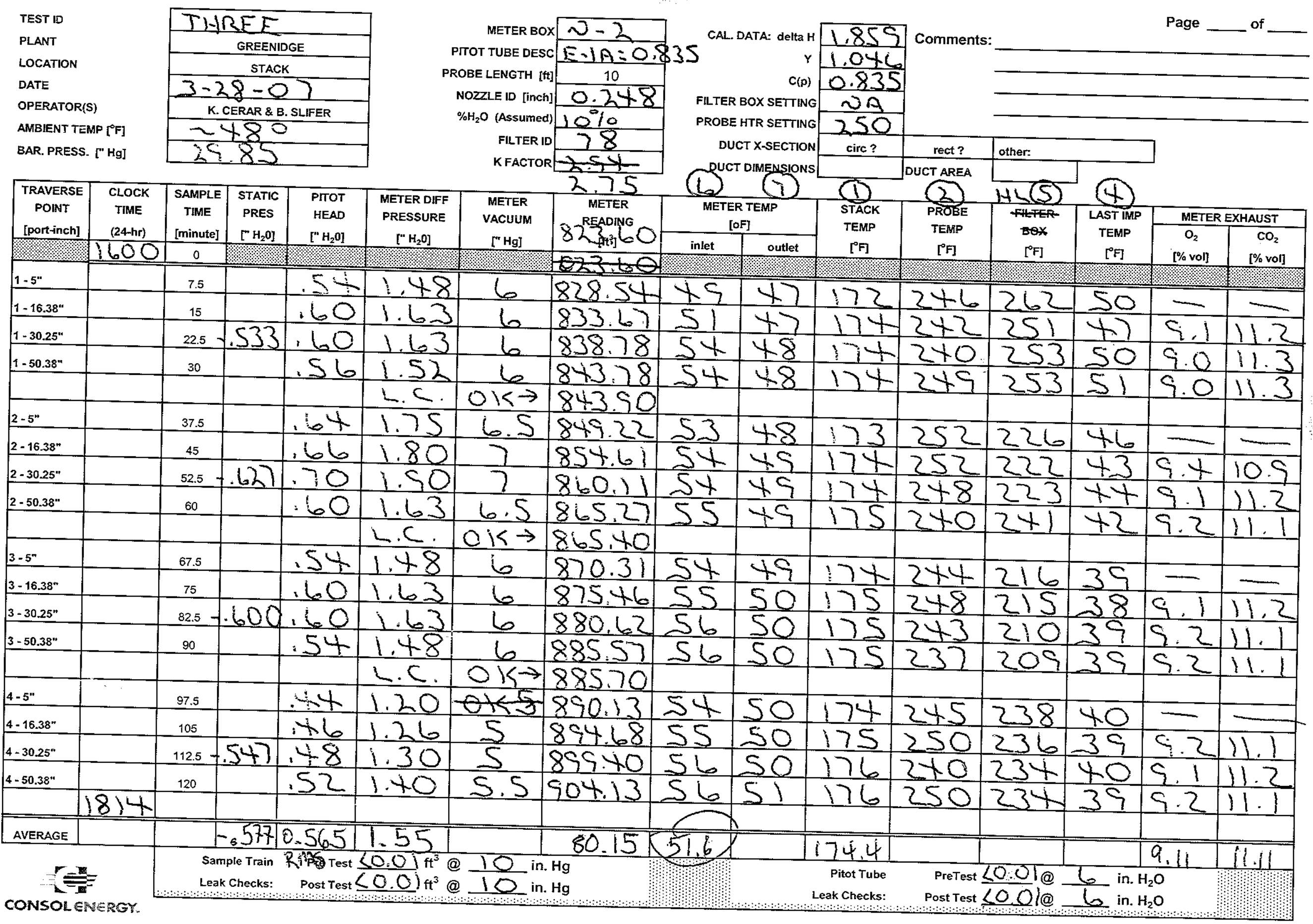


Distribution: JEL, BWG

Project No: $1621-85$

Sample Date: $3-28-07$

Location: AHO

Task:

Test: $1+(s-1$

Operator: Ro/Do

\begin{tabular}{|c|c|c|c|c|c|c|c|c|}
\hline Sample ID & Bottle \# & Description & $\begin{array}{c}\text { Initial Vol } \\
\mathrm{mL}\end{array}$ & $\begin{array}{c}\text { Rinse Vol } \\
\text { mL }\end{array}$ & $\begin{array}{c}\text { Gain } \\
\mathrm{mL}\end{array}$ & $\begin{array}{c}\text { Final Vol } \\
\mathrm{mL}\end{array}$ & $\mathrm{ppb} \mathrm{Hg}$ & $\begin{array}{c}\text { Total ug } \\
\text { of } \mathrm{Hg} \\
\end{array}$ \\
\hline 1 & $\mathbf{s}$ & Filter/Solids & 一 & - & - & - & & \\
\hline 2 & 1 & Probe \& Filter Rinse & 0 & 150 & 工 & 150 & & \\
\hline 3 & 2 & Sample Line Rinse & 0 & 160 & - & 168 & & \\
\hline 4 & 3 & $\mathrm{KCl}$ Impingers & 300 & 150 & $1+6160$ & 616 & & \\
\hline$S$ & 4 & $\mathrm{HNO}_{3} / \mathrm{H}_{2} \mathrm{O}_{2}$ Impinger & 100 & 75 & -50 & 125 & & \\
\hline 6 & 5 & $\mathrm{KMnO}_{4}$ Impingers & 200 & 50 & -50 & 200 & & \\
\hline 7 & 6 & $\mathrm{KMnO}_{4}$ Acid Rinse & 0 & 100 & 0 & 100 & & \\
\hline
\end{tabular}

Filter Gross wt:

Filter Net wt:

Filter Tare wt:

Probe/Line Rinse wt:

Filter Net wt:

Total Particulate wt:

Condensate Total: $\mathrm{ml}$

Location:

STK

Task:

\begin{tabular}{|c|c|c|c|c|c|c|c|c|}
\hline Sample ID & Bottle \# & Description & $\begin{array}{c}\text { Initial Vol } \\
\mathrm{mL}\end{array}$ & $\begin{array}{c}\text { Rinse Vol } \\
\mathrm{mL}\end{array}$ & $\begin{array}{c}\text { Gain } \\
\mathrm{mL}\end{array}$ & $\begin{array}{c}\text { Final Vol } \\
\mathrm{mL}\end{array}$ & $\mathrm{ppb} \mathrm{Hg}$ & $\begin{array}{c}\text { Total ug } \\
\text { of } \mathrm{Hg}\end{array}$ \\
\hline 8 & $\mathbf{S}$ & Filter/Solids & - & - & - & - & & \\
\hline $9 / 10$ & $1 / 1 A$ & Probe \& Filter Rinse/line & $0 / 0$ & 80162 & - & $8 0 \longdiv { 6 2 }$ & & \\
\hline 11 & 2 & KCl Impingers & 300 & 150 & 216 & 676 & & \\
\hline 12 & 3 & $\mathrm{HNO}_{3} / \mathrm{H}_{2} \mathrm{O}_{2}$ Impinger & 100 & 75 & 6 & 181 & & \\
\hline 13 & 4 & $\mathrm{KMnO}_{4}$ Impingers & 200 & 750 & -6 & $2 \times 4$ & & \\
\hline 14 & 5 & $\mathrm{KMnO}_{4}$ Acid Rinse & 0 & 100 & 0 & 100 & & \\
\hline
\end{tabular}

Filter Gross wt:

Filter Tare wt:

Filter Net wt:

Recovered By:

Recovered By:
Filter Net wt:

Probe/Line Rinse wt:

Total Particulate wt:
Date: $3128 / 2007$

Test: $H G-1$ Operator: BS/KC

\begin{tabular}{||c|c|c|c|}
\hline \hline Sample ID & Description & ppb Hg & $\begin{array}{c}\text { Total ug } \\
\text { of } \mathrm{Hg}\end{array}$ \\
\hline & 3 in. Filter Blank & & \\
\hline & $\mathrm{KCl}$ Blank & & \\
\hline & $\mathrm{HNO}_{3} / \mathrm{H} 2 \mathrm{O} 2$ Blank & & \\
\hline & $\mathrm{KMnO}_{4}$ Blank & & \\
\hline & $\mathrm{KMnO}_{4}$ Blank & & \\
\hline & $\mathrm{HNO}_{3} / \mathrm{HCl} \mathrm{Blank}$ & & \\
\hline
\end{tabular}

Date: $3 / 28 / 2007$

Date: 
Distribution: JEL, BWG

Project No.: $1621-85$

Sample Date: $3-28-2007$

Location: AHO

Task:

Test: H 1 Co-z Operator: Ro/00

\begin{tabular}{|c|c|c|c|c|c|c|c|c|}
\hline Sample ID & Bottle \# & Description & $\begin{array}{c}\text { Initial Vol } \\
\mathrm{mL}\end{array}$ & $\begin{array}{c}\text { Rinse Vol } \\
\mathrm{mL}\end{array}$ & $\begin{array}{c}\text { Gain } \\
\mathrm{mL}\end{array}$ & $\begin{array}{c}\begin{array}{c}\text { Final Vol } \\
\mathrm{mL}\end{array} \\
\end{array}$ & $\mathrm{ppb} \mathrm{Hg}$ & $\begin{array}{c}\text { Total ug } \\
\text { of } \mathrm{Hg}\end{array}$ \\
\hline 15 & $\mathrm{~s}$ & Filter/Solids & - & - & - & - & & \\
\hline 16 & 1 & Probe \& Filter Rinse & 0 & 136 & - & 136 & & \\
\hline 17 & 2 & Sample Line Rinse & 0 & 60 & - & 60 & & \\
\hline 18 & 3 & $\mathrm{KCl}$ Impingers & $3 \infty 0$ & 72 & 150 & 522 & & \\
\hline 19 & 4 & $\mathrm{HNO}_{3} / \mathrm{H}_{2} \mathrm{O}_{2}$ Impinger & 100 & -2 & 75 & 173 & & \\
\hline 20 & 5 & $\mathrm{KMnO}_{4}$ Impingers & 200 & $-2<$ & 50 & 248 & & \\
\hline 21 & 6 & $\mathrm{KMnO}_{4}$ Acid Rinse & 0 & 100 & 3 & 100 & & \\
\hline
\end{tabular}

Filter Gross wt:

Filter Tare wt:

Filter Net wt:

Recovered By: 0

Location:

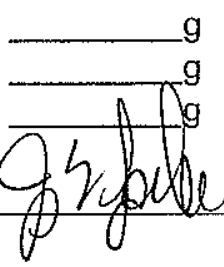

Probe/Line Rinse wt:

Total Particulate wt:

Condensate Total: $\mathrm{ml}$

Date: $3 / 28 / 2007$

Task:

1

Test: $1+q-2$ Operator: BSIXC

\begin{tabular}{|c|c|c|c|c|c|c|c|c|}
\hline Sample ID & Bottle \# & Description & $\begin{array}{c}\text { Initial Vol } \\
\mathrm{mL}\end{array}$ & $\begin{array}{c}\text { Rinse Vol } \\
\mathrm{mL}\end{array}$ & $\begin{array}{c}\text { Gain } \\
\mathrm{mL}\end{array}$ & $\begin{array}{c}\text { Final Vol } \\
\mathrm{mL}\end{array}$ & ppb Hg & $\begin{array}{c}\text { Total ug } \\
\text { of } \mathrm{Hg}\end{array}$ \\
\hline $2 z$ & $\mathrm{~s}$ & Filter/Solids & - & & & & & \\
\hline $23 / 24$ & $1 / 18$ & Probe \& Filter Rinse /lial & Rinse o 0 & $1 1 8 \longdiv { 7 3 }$ & - & $1.18 / 73$ & & \\
\hline 25 & 2 & $\mathrm{KCl}$ Impingers & 300 & 150 & 184 & 634 & & \\
\hline 26 & 3 & $\mathrm{HNO}_{3} / \mathrm{H}_{2} \mathrm{O}_{2}$ Impinger & 100 & 75 & 6 & 181 & & \\
\hline 27 & 4 & $\mathrm{KMnO}_{4}$ Impingers & 200 & 50 & -8 & 242 & & \\
\hline 28 & 5 & $\mathrm{KMnO}_{4}$ Acid Rinse & $D$ & 100 & 0 & 100 & & \\
\hline
\end{tabular}

Filter Gross wt:

Filter Tare wt:

Fecovered By:

Recovered By:
Filter Net wt:

Probe/Line Rinse wt:

Total Particulate wt:
Condensate Total: $\mathrm{ml}$

Date: $3|22| z 000$

Date:

\begin{tabular}{|c|c|c|c|}
\hline Sample ID & Description & ppb Hg & $\begin{array}{c}\text { Total ug } \\
\text { of } \mathrm{Hg}\end{array}$ \\
\hline & 3 in. Filter Blank & & \\
\hline & $\mathrm{KCl} \mathrm{Blank}$ & & \\
\hline & $\mathrm{HNO}_{3} / \mathrm{H} 2 \mathrm{O} 2 \mathrm{Blank}$ & & \\
\hline & $\mathrm{KMnO}_{4}$ Blank & & \\
\hline & $\mathrm{KMnO}_{4}$ Blank & & \\
\hline & $\mathrm{HNO}_{3} / \mathrm{HCl}$ Blank & & \\
\hline
\end{tabular}


Distribution: JEL, BWG

Project No.: 1621-85

Sample Date: $\frac{3-28-2687}{18}$
Location:
$\mathrm{AHO}$
Task:
Test: $\mathrm{H}(\mathrm{G}-3$
Operator: Ro / Do

\begin{tabular}{|c|c|c|c|c|c|c|c|c|}
\hline Sample ID & Bottle \# & Description & $\begin{array}{c}\text { Initial Vol } \\
\mathrm{mL}\end{array}$ & $\begin{array}{c}\text { Rinse Vol } \\
\mathrm{mL}\end{array}$ & $\begin{array}{c}\text { Gain } \\
\mathrm{mL}\end{array}$ & $\begin{array}{c}\text { Final Vol } \\
\mathrm{mL}\end{array}$ & ppb Hg & $\begin{array}{c}\text { Total ug } \\
\text { of } \mathrm{Hg}\end{array}$ \\
\hline 29 & $\mathbf{s}$ & Filter/Solids & - & - & - & $=$ & & \\
\hline 30 & 1 & Probe \& Filter Rinse & $\emptyset$ & 110 & - & $1 / 0$ & & \\
\hline 31 & 2 & Sample Line Rinse & 0 & 125 & - & 125 & & \\
\hline $3 z$ & 3 & $\mathrm{KCl}$ Impingers & 300 & 150 & 73 & 523 & & \\
\hline 33 & 4 & $\mathrm{HNO}_{3} / \mathrm{H}_{2} \mathrm{O}_{2}$ Impinger & 100 & 75 & 0 & 175 & & \\
\hline 34 & 5 & $\mathrm{KMnO}_{4}$ Impingers & 200 & 50 & -6 & 244 & & \\
\hline 35 & 6 & $\mathrm{KMnO}_{4}$ Acid Rinse & 0 & 100 & 0 & 100 & & \\
\hline
\end{tabular}

Filter Gross wt:

Filter Net wt:

Filter Tare wt:

Fecovered By:

Probe/Line Rinse wt:

Total Particulate wt:

Condensate Total: $\mathrm{ml}$

Location:

STK

Task:

1.

Test: $H G-\zeta$ Operator: BS (KC

\begin{tabular}{|c|c|c|c|c|c|c|c|c|}
\hline Sample ID & Bottle \# & Description & $\begin{array}{c}\text { Initial Vol } \\
\mathrm{mL}\end{array}$ & \begin{tabular}{|c|} 
Rinse Vol \\
$\mathrm{mL}$
\end{tabular} & $\begin{array}{c}\text { Gain } \\
\mathrm{mL}\end{array}$ & $\begin{array}{c}\text { Final Vol } \\
\mathrm{mL}\end{array}$ & ppb Hg & $\begin{array}{c}\text { Total ug } \\
\text { of } \mathrm{Hg} \\
\end{array}$ \\
\hline 36 & $\mathbf{s}$ & Filter/Solids & & & & & & \\
\hline $37 / 38$ & $1 / / A$ & Probe \& Filter Rinse/Line & Rivise o) 0 & $68 / 45$ & - & $68 / 45$ & & \\
\hline 399 & 2 & KCl Impingers & 300 & 150 & 208 & 658 & & \\
\hline 0 & 3 & $\mathrm{HNO}_{3} / \mathrm{H}_{2} \mathrm{O}_{2}$ impinger & 100 & 75 & 2 & 177 & & \\
\hline 4) & 4 & $\mathrm{KMnO}_{4}$ Impingers & 200 & 50 & -6 & 244 & & \\
\hline 42 & 5 & $\mathrm{KMnO}_{4}$ Acid Rinse & 0 & 100 & 0 & 100 & & \\
\hline
\end{tabular}

Filter Gross wt:

Filter Tare wt:

Filter Net wt: 9

g

$\mathrm{g}$
Filter Net wt:

Probe/Line Rinse wt:

Total Particulate wt:

Date: $3 / 29 / 2007$

Recovered By:

Recovered By:
Condensate Total: $\mathrm{ml}$

Date: 3/29) $20 x+4$

Date:

\begin{tabular}{|c|c|c|c|}
\hline Sample ID & Description & $\mathrm{ppb} \mathrm{Hg}$ & $\begin{array}{c}\text { Total ug } \\
\text { of } \mathrm{Hg}\end{array}$ \\
\hline 87 & 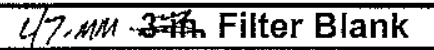 & & \\
\hline 44 & KCl Blank & & \\
\hline $4 / 3$ & $\mathrm{HNO}_{3} / \mathrm{H} 2 \mathrm{O} 2$ Blank & & \\
\hline $4 / 3$ & $\mathrm{KMnO}_{4}$ Blank & & \\
\hline 48 & Thinabl & & \\
\hline 46 & $\mathrm{HNO}_{3} / \mathrm{HCl}$ Blank & & \\
\hline
\end{tabular}


AES GREENIDGE UNIT 4 ONTARIO HYDRO SAMPLING TRAIN DATA

GUARANTEE TESTING - March 30, 2007 - ACTIVATED CARBON INJECTION

\begin{tabular}{|c|c|c|c|c|c|c|c|}
\hline \multicolumn{2}{|l|}{$\begin{array}{l}\text { Location } \\
\text { Date } \\
\text { Start Time } \\
\text { Stop Time } \\
\text { Test Number } \\
\text { Sample Type }\end{array}$} & $\begin{array}{c}\mathrm{AHO} \\
3 / 30 / 07 \\
855 \\
1110 \\
4 \\
\mathrm{OH} \cdot \mathrm{Hg} \\
\end{array}$ & $\begin{array}{c}\text { Stack } \\
3 / 30 / 07 \\
855 \\
1110 \\
4 \\
\mathrm{OH}-\mathrm{Hg} \\
\end{array}$ & $\begin{array}{c}\mathrm{AHO} \\
3 / 30 / 07 \\
1243 \\
1502 \\
5 \\
\mathrm{OH}-\mathrm{Hg} \\
\end{array}$ & $\begin{array}{c}\text { Stack } \\
3 / 30 / 07 \\
1243 \\
1454 \\
5 \\
\mathrm{OH}-\mathrm{Hg} \\
\end{array}$ & $\begin{array}{c}\text { AHO } \\
3 / 30 / 07 \\
1552 \\
1805 \\
6 \\
\text { OH-Hg } \\
\end{array}$ & $\begin{array}{c}\text { Stack } \\
3 / 30 / 07 \\
1552 \\
1803 \\
6 \\
\mathrm{OH} \cdot \mathrm{Hg} \\
\end{array}$ \\
\hline \multicolumn{2}{|l|}{ Y factor of dry gas meter } & 0.970 & 1.046 & 0.970 & 1.046 & 0.970 & 1.046 \\
\hline $\begin{array}{l}\text { Gas Volume } \\
\text { Delta } \mathrm{H} \text { of dry gas meter }\end{array}$ & $\begin{array}{l}\cdot \mathrm{ft}^{3} \\
" n \mathrm{H}_{2} \mathrm{O}\end{array}$ & $\begin{array}{r}50.26 \\
0.60\end{array}$ & $\begin{array}{r}78.34 \\
1.46\end{array}$ & $\begin{array}{r}52.46 \\
0.62\end{array}$ & $\begin{array}{r}81.05 \\
1.55\end{array}$ & $\begin{array}{r}51.54 \\
0.60\end{array}$ & $\begin{array}{r}80.07 \\
1.52\end{array}$ \\
\hline $\begin{array}{l}\text { Meter Temperature } \\
\text { C Factor of pitot tube } \\
\text { Nozzle Diameter }\end{array}$ & $\begin{array}{l}-{ }^{\circ} \mathrm{F} \\
-{ }^{-} \text {inches }\end{array}$ & $\begin{array}{r}46.5 \\
0.840 \\
0.184\end{array}$ & $\begin{array}{r}50.8 \\
0.835 \\
0.248\end{array}$ & $\begin{array}{r}58.5 \\
0.840 \\
0.184\end{array}$ & $\begin{array}{r}63.8 \\
0.835 \\
0.248\end{array}$ & $\begin{array}{r}60.4 \\
0.840 \\
0.184\end{array}$ & $\begin{array}{r}65.5 \\
0.835 \\
0.248\end{array}$ \\
\hline \multirow{4}{*}{$\begin{array}{l}\text { A n (area of nozzle) } \\
\text { Area of Stack } \\
\mathrm{H}_{2} \mathrm{O} \text { Weight } \\
\text { Sample Time } \\
\text { Barometric Pressure }\end{array}$} & $-\mathrm{ft}^{2}$ & 0.00018 & 0.00034 & 0.00018 & 0.00034 & 0.00018 & 0.00034 \\
\hline & $=\mathrm{ft}^{2}$ & 114.0 & 132.7 & 114.0 & 132.7 & 114.0 & 132.7 \\
\hline & . gm & 82.8 & 226.7 & 90.3 & 232.1 & 97.2 & 230.1 \\
\hline & - minutes & $\begin{array}{r}126 \\
29.80\end{array}$ & $\begin{array}{r}120 \\
29.80\end{array}$ & $\begin{array}{r}126 \\
29.77\end{array}$ & $\begin{array}{r}120 \\
29.77\end{array}$ & $\begin{array}{r}126 \\
29.71\end{array}$ & $\begin{array}{r}120 \\
29.71\end{array}$ \\
\hline \multirow{3}{*}{$\begin{array}{l}\text { Static Pressure } \\
\% \text { Oxygen (see note) } \\
\% \mathrm{CO} \text { (see note) } \\
\% \mathrm{~N}_{2}+\mathrm{CO} \text { (calculated) }\end{array}$} & - " $\mathrm{Hg}$ & -13.18 & -0.49 & -13.25 & -0.53 & .12 .84 & -0.55 \\
\hline & - & 7.1 & 8.6 & $\begin{array}{r}7.2 \\
7.2\end{array}$ & 8.7 & $\begin{array}{r}6.9 \\
12.3\end{array}$ & 8.3 \\
\hline & $\dot{-}$ & $\begin{array}{l}12.1 \\
80.8\end{array}$ & $\begin{array}{l}10.8 \\
80.6\end{array}$ & $\begin{array}{l}12.1 \\
80.7\end{array}$ & $\begin{array}{l}10.8 \\
80.6\end{array}$ & $\begin{array}{l}12.3 \\
80.8\end{array}$ & $\begin{array}{l}11.1 \\
80.6\end{array}$ \\
\hline \multirow{2}{*}{$\begin{array}{l}\text { Stack Temp (Dry Bulb) } \\
\text { Stack Temp (Wet Bulb) } \\
\text { "S" sample (rms vel head) }\end{array}$} & $={ }^{0} \mathrm{~F}$ & 295 & 174 & 296 & 175 & 298 & 174 \\
\hline & $\begin{array}{l}={ }^{0} \mathrm{~F} \\
={ }^{"} \mathrm{H}_{2} \mathrm{O}\end{array}$ & 0.742 & 0.562 & 0.774 & 0.597 & 0.750 & 0.581 \\
\hline \multirow{4}{*}{$\begin{array}{l}\text { Dust Wt. } \\
\text { Sample Volume } \\
\text { Sample Volume } \\
\text { ABS ST PRES }\end{array}$} & - gm & 8.1636 & 0.0010 & 8.1806 & 0.0000 & 7.3852 & 0.0024 \\
\hline & - DSCF & 50.67 & 84.63 & 51.62 & 85.32 & 50.42 & 83.84 \\
\hline & - dscm & 1.435 & 2.397 & 1.462 & 2.416 & 1.428 & 2.374 \\
\hline & - " $\mathrm{Hg}$ & 28.83 & 29.76 & 28.80 & 29.73 & 28.77 & 29.67 \\
\hline \multirow{2}{*}{$\begin{array}{l}\text { ABS ST TEMP } \\
\mathrm{H}_{2} \mathrm{O}-\% \text { by } \mathrm{Vol}\end{array}$} & - ${ }^{\circ} \mathrm{R}$ & 755 & 634 & 756 & 635 & 758 & 634 \\
\hline & - vapor & 7.2 & 11.2 & 7.6 & 11.4 & 8.3 & 11.4 \\
\hline \multirow{6}{*}{$\begin{array}{l}\text { Water Volume } \\
\text { Dry Molecular Welght } \\
\text { Wet Molecular Weight } \\
\text { \% EXCESS AIR } \\
\text { Dry Mole Frac. } \\
\text { Wet Mole Frac. }\end{array}$} & - std $\mathrm{ft}^{3}$ & 3.90 & 10.68 & 4.25 & 10.93 & 4.58 & 10.84 \\
\hline & - lb/lb-mole & 30.22 & 30.07 & 30.22 & 30.07 & 30.24 & 30.11 \\
\hline & - lb/b-mole & 29.35 & 28.72 & 29.29 & 28.70 & 29.22 & 28.72 \\
\hline & & 49.9 & 68.2 & 51.0 & 68.9 & 48.0 & 63.4 \\
\hline & - & 0.928 & 0.888 & 0.924 & 0.886 & 0.917 & 0.886 \\
\hline & - & 0.072 & 0.112 & 0.076 & 0.114 & 0.083 & 0.114 \\
\hline \multirow{2}{*}{$\begin{array}{l}\text { Gas Velocity, Direct } \\
\text { ACFM }\end{array}$} & $-\mathrm{ft} / \mathrm{sec}$ & 58.45 & 46.10 & 59.82 & 47.58 & 59.07 & 46.95 \\
\hline & - & 399774 & 367163 & 409187 & 378900 & 404014 & 373882 \\
\hline DSCFM & - & 249994 & 269932 & 254038 & 277555 & 247949 & 273251 \\
\hline \multicolumn{2}{|l|}{ DSCFM (rounded) } & 250000 & 269900 & 254000 & 277600 & 247900 & 273300 \\
\hline \multicolumn{2}{|l|}{ DSCMM } & 7080 & 7644 & 7194 & 7860 & 7022 & 7738 \\
\hline \multicolumn{2}{|c|}{$\begin{array}{l}\text { Excess Alr Free DSCFM } \\
\text { CALCULATED FIRING RATE: }\end{array}$} & 165068 & 158498 & 166523 & 162325 & 165853 & 165284 \\
\hline Dry & - $\mathrm{lb} / \mathrm{min}$ & 1242 & 1192 & 1250 & 1219 & 1243 & 1239 \\
\hline Wet & - $\mathrm{lb} / \mathrm{min}$ & 1330 & 1278 & 1316 & 1282 & 1307 & 1302 \\
\hline Dry & $=\mathrm{lb} / \mathrm{hr}$ & 74513 & 71547 & 75015 & 73124 & 74577 & 74321 \\
\hline Wet & - $\mathrm{lb} / \mathrm{hr}$ & 79830 & 76652 & 78938 & 76949 & 78395 & 78126 \\
\hline \multicolumn{8}{|c|}{ CALCULATED FIRING RATE: } \\
\hline Dry & - tons/hr & 37.3 & 35.8 & 37.5 & 36.6 & 37.3 & 37.2 \\
\hline Wet & - tons $/ \mathrm{hr}$ & 39.9 & 38.3 & 39.5 & 38.5 & 39.2 & 39.1 \\
\hline \multicolumn{8}{|l|}{ HEAT INPUT: } \\
\hline \multicolumn{2}{|l|}{$\begin{array}{l}\text { MM Btu/hr } \\
\text { PARTICULATE LOADING: }\end{array}$} & 1044 & 1003 & 1047 & 1021 & 1040 & 1037 \\
\hline Grains/DSCF & - & 2.4858 & 0.0002 & 2.4454 & 0.0000 & 2.2600 & 0.0004 \\
\hline $\mathrm{lb} / \mathrm{hr}$ & - & 5329 & 0.42 & 5326 & 0.00 & 4804 & 1.04 \\
\hline $\mathrm{Ib} / \mathrm{MM} \mathrm{Btu}$ & $=$ & 5.10 & 0.00 & 5.09 & 0.00 & 4.62 & 0.00 \\
\hline$\%$ ISOKINETIC & . & 89.4 & 103.5 & 99.7 & 101.5 & 99.7 & 101.3 \\
\hline
\end{tabular}

NOTE: The $\% \mathrm{O}_{2}$ at the air heater outlet was measured by CONSOL using a Teledyne Max 5 portable electrochemical $\mathrm{O}_{2}$ analyzer, and the $\% \mathrm{CO}_{2}$ at the air heater outlet was calculated from the measured $\mathrm{O}_{2}$ and coal composition. The $\% \mathrm{CO}_{2}$ at the stack was measured by the plant's stack $\mathrm{CEM}$ and the $\% \mathrm{O}_{2}$ at the stack was calculated from the measured $\mathrm{CO}_{2}$ and coal composition. 


\begin{tabular}{|c|c|c|c|c|c|c|}
\hline Impinger Components Wts \& Volumes & $\mathrm{AHO}-4$ & Stack - 4 & $\mathrm{AHO} \cdot 5$ & Stack - 5 & AHO - 6 & Stack - 6 \\
\hline $\begin{array}{l}\text { Filter Wt., g } \\
\text { ppm } \mathrm{Hg} \text { (thimbles) or ug/filter (filters) } \\
\text { total ug } \\
\text { ug/dscm }\end{array}$ & $\begin{array}{r}8.1636 \\
0.60 \\
4.88 \\
3.40 \\
\end{array}$ & $\begin{array}{r}0.0010 \\
<0.01 \\
<0.01 \\
<0.00 \\
\end{array}$ & $\begin{array}{r}8.1806 \\
0.66 \\
5.41 \\
3.70 \\
\end{array}$ & \begin{tabular}{r|}
0.0000 \\
$<0.01$ \\
$<0.01$ \\
$<0.00$ \\
\end{tabular} & \begin{tabular}{r|}
7.3852 \\
0.62 \\
4.60 \\
3.22 \\
\end{tabular} & \begin{tabular}{r|}
0.0024 \\
$<0.01$ \\
$<0.01$ \\
$<0.00$ \\
\end{tabular} \\
\hline $\begin{array}{l}\text { Probe Rinse volume, } \mathrm{ml} \\
\text { Analytical } \mathrm{Hg}, \mathrm{ng} / \mathrm{ml} \\
\text { ug/dscm }\end{array}$ & $\begin{array}{r}128 \\
<1.40 \\
<0.12 \\
\end{array}$ & $\begin{array}{r}140 \\
<1,40 \\
<0,08 \\
\end{array}$ & $\begin{array}{r}157 \\
<1.40 \\
<0.15 \\
\end{array}$ & $\begin{array}{r}138 \\
<1.40 \\
<0.08 \\
\end{array}$ & $\begin{array}{r}81 \\
<1.40 \\
<0.08 \\
\end{array}$ & $\begin{array}{r}117 \\
<1.40 \\
<0.07 \\
\end{array}$ \\
\hline $\begin{array}{l}\text { Line Rinse volume, } \mathrm{ml} \\
\text { Analytical } \mathrm{Hg}, \mathrm{ng} / \mathrm{ml} \\
\text { ug/dscm }\end{array}$ & $\begin{array}{r}150 \\
3.12 \\
0.33 \\
\end{array}$ & $\begin{array}{r}81 \\
<1.40 \\
<0.05 \\
\end{array}$ & $\begin{array}{r}128 \\
<1.40 \\
<0.12 \\
\end{array}$ & $\begin{array}{r}79 \\
<1.40 \\
<0.05 \\
\end{array}$ & $\begin{array}{r}140 \\
<1.40 \\
<0.14 \\
\end{array}$ & $\begin{array}{r}129 \\
<1.40 \\
<0.08 \\
\end{array}$ \\
\hline $\begin{array}{l}\mathrm{KCl} \text { volume, } \mathrm{ml} \\
\text { Analytlcal } \mathrm{Hg}, \mathrm{ng} / \mathrm{ml} \\
\mathrm{ug} / \mathrm{dscm}\end{array}$ & $\begin{array}{r}522 \\
8.86 \\
3.22 \\
\end{array}$ & $\begin{array}{r}649 \\
<0.28 \\
<0.08 \\
\end{array}$ & $\begin{array}{r}530 \\
8.45 \\
3.06 \\
\end{array}$ & $\begin{array}{r}664 \\
<0.28 \\
<0.08 \\
\end{array}$ & $\begin{array}{r}531 \\
8.04 \\
2.99 \\
\end{array}$ & $\begin{array}{r}658 \\
<0.28 \\
<0.08 \\
\end{array}$ \\
\hline $\begin{array}{l}\text { Nitric/Peroxide volume, } \mathrm{ml} \\
\text { Analytical } \mathrm{Hg}, \mathrm{ng} / \mathrm{ml} \\
\text { ug/dscm }\end{array}$ & $\begin{array}{r}176 \\
<1.40 \\
<0.17 \\
\end{array}$ & $\begin{array}{r}184 \\
<1.40 \\
<0.11 \\
\end{array}$ & $\begin{array}{r}175 \\
<1.40 \\
<0.17 \\
\end{array}$ & $\begin{array}{r}175 \\
<1.40 \\
<0.10 \\
\end{array}$ & $\begin{array}{r}175 \\
<1.40 \\
<0.17 \\
\end{array}$ & $\begin{array}{r}181 \\
<1.40 \\
<0.11\end{array}$ \\
\hline $\begin{array}{l}\mathrm{KMnO} 4 \text { volume, } \mathrm{ml} \\
\text { Analytical } \mathrm{Hg}, \mathrm{ng} / \mathrm{ml} \\
\text { ug/dscm }\end{array}$ & $\begin{array}{r}250 \\
<0.28 \\
<0.05 \\
\end{array}$ & $\begin{array}{r}254 \\
<0.28 \\
<0.03 \\
\end{array}$ & $\begin{array}{r}250 \\
0.40 \\
0.07 \\
\end{array}$ & $\begin{array}{r}250 \\
0.37 \\
0.04 \\
\end{array}$ & $\begin{array}{r}256 \\
1.55 \\
0.28 \\
\end{array}$ & $\begin{array}{r}250 \\
<0.28 \\
<0.03 \\
\end{array}$ \\
\hline $\begin{array}{l}\text { KMnO4-Acid Rinse volume, } \mathrm{ml} \\
\text { Analytical } \mathrm{Hg}, \mathrm{ng} / \mathrm{ml} \\
\text { ug/dscm }\end{array}$ & $\begin{array}{r}100 \\
<1.40 \\
<0.10 \\
\end{array}$ & $\begin{array}{r}100 \\
<1.40 \\
<0.06 \\
\end{array}$ & $\begin{array}{r}100 \\
<1.40 \\
<0.10 \\
\end{array}$ & $\begin{array}{r}100 \\
<1.40 \\
<0.06 \\
\end{array}$ & $\begin{array}{r}100 \\
<1.40 \\
<0.10 \\
\end{array}$ & $\begin{array}{r}100 \\
<1.40 \\
<0.06 \\
\end{array}$ \\
\hline $\begin{array}{l}\text { Particulate } \mathrm{Hg}(\mathrm{ug} / \mathrm{dscm}) \\
\text { Oxidized } \mathrm{Hg}(\mathrm{ug} / \mathrm{dscm}) \\
\text { Elemental } \mathrm{Hg}(\mathrm{ug} / \mathrm{dscm}) \\
\text { Total } \mathrm{Hg}(\mathrm{ug} / \mathrm{dscm})\end{array}$ & $\begin{array}{l}3.40 \\
3.67 \\
<0.32 \\
7.39\end{array}$ & $\begin{array}{l}<0.00 \\
<0.20 \\
<0.20 \\
<0.40\end{array}$ & $\begin{array}{c}3.70 \\
3.34 \\
<0.33 \\
7.37\end{array}$ & $\begin{array}{l}<0.00 \\
<0.20 \\
<0.20 \\
<0.40\end{array}$ & $\begin{array}{l}3.22 \\
3.21 \\
0.55 \\
6.98\end{array}$ & $\begin{array}{l}<0.00 \\
<0.22 \\
<0.20 \\
<0.42\end{array}$ \\
\hline $\begin{array}{l}\text { Partlculate Hg (ug/dscm @ 3\% O2) } \\
\text { Oxidized Hg (ug/dscm @ 3\% O2) } \\
\text { Elemental Hg (ug/dscm @ 3\% O2) } \\
\text { Total Hg (ug/dscm @ 3\% O2) } \\
\end{array}$ & $\begin{array}{r}4.41 \\
4.77 \\
<0.41 \\
9.59 \\
\end{array}$ & \begin{tabular}{l|}
$<0.00$ \\
$<0.30$ \\
$<0.29$ \\
$<0.59$ \\
\end{tabular} & $\begin{array}{r}4.83 \\
4.36 \\
<0.43 \\
9.63 \\
\end{array}$ & \begin{tabular}{l|}
$<0.00$ \\
$<0.30$ \\
$<0.29$ \\
$<0.59$ \\
\end{tabular} & $\begin{array}{l}4.13 \\
4.11 \\
0.70 \\
8.93\end{array}$ & $\begin{array}{l}<0.00 \\
<0.32 \\
<0.28 \\
<0.60 \\
\end{array}$ \\
\hline Removal (\%) & \multicolumn{2}{|c|}{$>93.9$} & \multicolumn{2}{|c|}{$>93.9$} & \multicolumn{2}{|c|}{$>93.3$} \\
\hline
\end{tabular}


ONTARIIO HYDRO Hg SAMPLING FIELD DATA SHEET

TEST ID

PLANT

LOCATION

DATE

OPERATOR(S)

AMBIENT TEMP [०F]

BAR. PRESS. [" Hg]

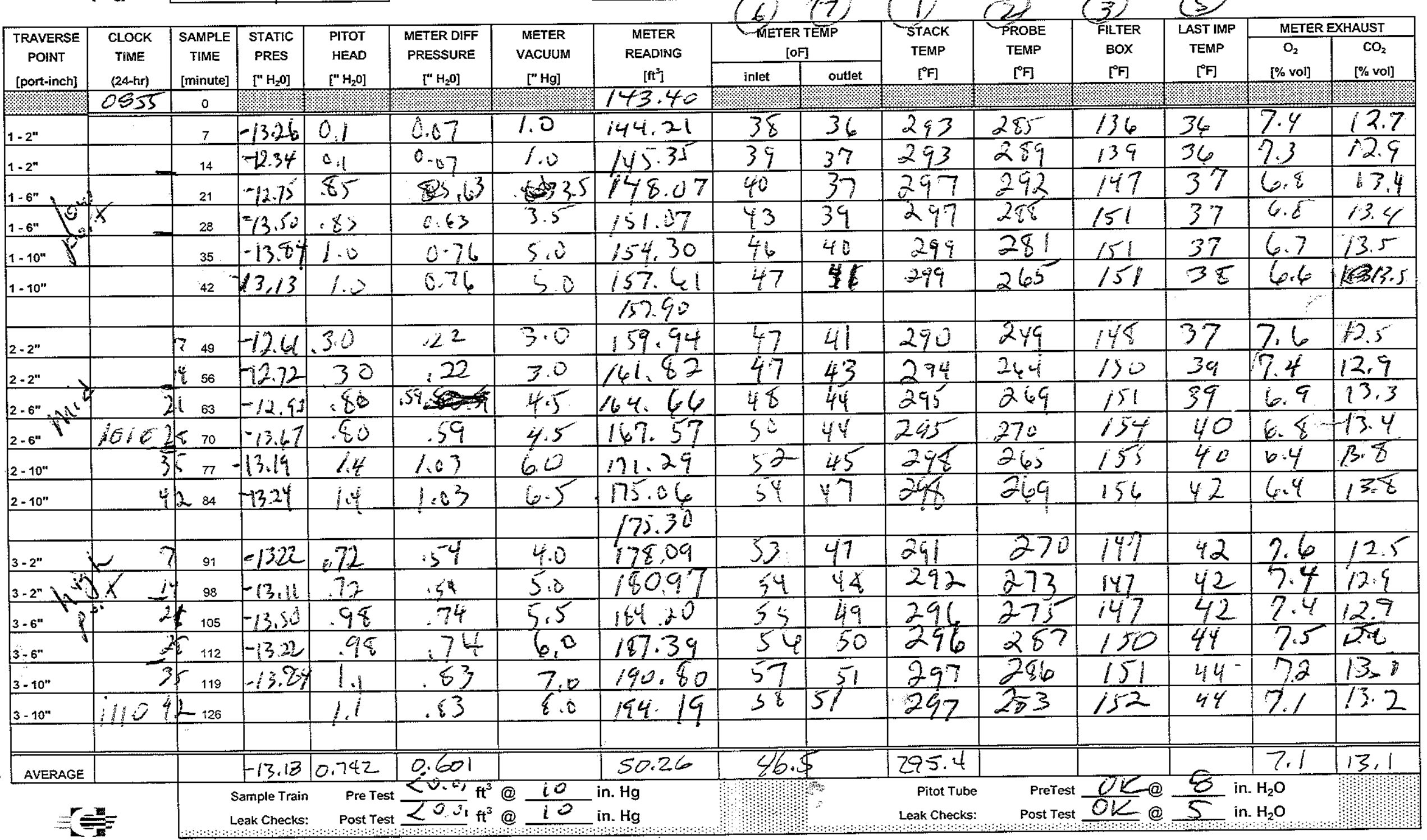

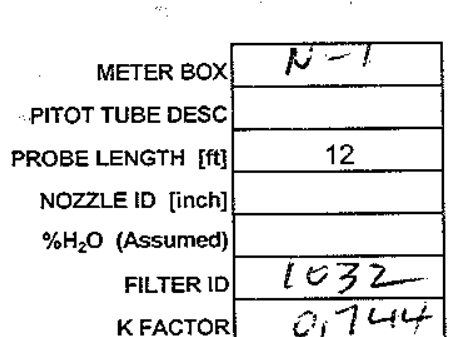

GREENIDGE

AIR HEATER OUTLET

$3 / 30 / 107$

R. ODA \& D.OLSEN

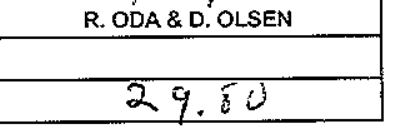

\begin{tabular}{r|r}
$Y$ & $P .970$ \\
& \\
$(\mathrm{p})$ &
\end{tabular}

FILTER BOX SETTING PROBE HTR SETTING

DUCT X-SECTION Bifore Testirc

$\longrightarrow$
10 min purgentertist

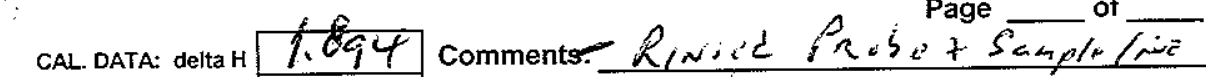


ONTARIO HYDRO Hg SAMPLING FIELD DATA SHEET

\begin{tabular}{|c|c|c|c|c|c|c|c|c|c|c|c|c|c|c|c|}
\hline \multirow{7}{*}{\multicolumn{2}{|c|}{$\begin{array}{l}\text { TEST ID } \\
\text { PLANT } \\
\text { LOCATION } \\
\text { DATE } \\
\text { OPERATOR(S) } \\
\text { AMBIENT TEMP PF] } \\
\text { BAR. PRESS. [" Hg] }\end{array}$}} & \multirow{2}{*}{\multicolumn{3}{|c|}{ FOUR }} & \multirow{2}{*}{\multicolumn{2}{|c|}{$\begin{array}{r}\text { METER BOX } \\
\text { PTIOT TUBE DESC }\end{array}$}} & & \multirow{2}{*}{\multicolumn{2}{|c|}{ CAL. DATA: delta $H$}} & \multirow{2}{*}{$\frac{0.895}{0.967}$} & \multirow{2}{*}{ Comments: } & & & \multicolumn{2}{|r|}{ - of } \\
\hline & & & & & & & Ei & & & & & & & & \\
\hline & & & STACK & & & BE LENGTH [fft] & 10 & & $c(p)$ & & & & & & \\
\hline & & $3-$ & 30 & -07 & & OZZLE ID [inch] & & ER! & :TING & vi & & & & & \\
\hline & & & ERAR \& $B$. & SLFER & & ${ }_{6} \mathrm{H}_{2} \mathrm{O}$ (Assumed) & $10 \%$ & ROBE & & 250 & & & & & \\
\hline & & $\approx$ & 30 & & & FILTER ID & 82 & & X-SECTION & circ? & & other: & & & \\
\hline & & & 15 & & & KFACT & 2.60 & & & (1) & $\overbrace{2 \text { CTAREA }}^{\text {Can }}$ & HLC & & & \\
\hline \begin{tabular}{|l} 
TRAVERSE \\
\end{tabular} & CLOCK & SAMPLE & \begin{tabular}{|l|} 
STATIC \\
\end{tabular} & РாОT & & & METER & TEF & & & & & & METER E & XHAUST \\
\hline \begin{tabular}{|l} 
POINT \\
\end{tabular} & TIME & TIME & \begin{tabular}{|l|l|} 
PRES \\
\end{tabular} & HEAD & ESSURE & VACUUM & READING & & & TEMP & TE & & & & \\
\hline [port-inch] & (24-hr) & & {$\left[\mathrm{H}_{2} \mathrm{O}\right]$} & {$\left[" \mathrm{H}_{2} \mathrm{O}\right]$} & {$\left[" \mathrm{H}_{2} \mathrm{O}\right]$} & {$[\mathrm{Hgl}$} & {$\left[t^{2}\right]$} & & & {$[\mathrm{F}]$} & {$\left[{ }^{\circ} \mathrm{F}\right.$} & {$[F]$} & & {$[\% \mathrm{vol}]$} & \\
\hline & 0855 & 0 & & & & & 060.20 & & & & & & & & \\
\hline $1-5^{n}$ & & 7.5 & & 0.54 & 1.4 & 4 & 065.10 & & & 172 & 250 & 221 & & 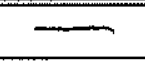 & $=$ \\
\hline $1-16.38^{\prime \prime}$ & & 15 & & .56 & 1.45 & 4 & 070.17 & 43 & 3 & 173 & 242 & 232 & & 8.7 & 1. \\
\hline $1-30.25^{\prime \prime}$ & & 22.5 & 496 & .56 & 1.45 & 4 & $07+.37$ & 4) & & 174 & 255 & 224 & & 8.8 & 11 \\
\hline 1-50.38" & & 30 & & .54 & 1.40 & 4 & 079.67 & SI & 3 & $17+$ & 239 & 221 & & 8.7 & 11. \\
\hline & & & & & & $0 k \rightarrow$ & 079.80 & & & & & & & & \\
\hline $2-5^{n}$ & & 37.5 & & .66 & 1. & 5 & 085.07 & 53 & 4 & 174 & 239 & 239 & & 工 & - \\
\hline$-16.38^{m}$ & & 45 & & .66 & 1.73 & 5 & 090.37 & 57 & 4 & 174 & $2 \times 5$ & $2+2$ & & 9.3 & 11.0 \\
\hline $2-30.25^{\prime \prime}$ & & 52.5 & 535 & .66 & 1.73 & 5 & 095.66 & 59 & 45 & 176 & $2+9$ & $24 x$ & 4 & 9.2 & 11.1 \\
\hline$\left.\right|_{2-50.38^{\prime \prime}}$ & & 60 & & .60 & $.5 S$ & 4.5 & 100.73 & 60 & 46 & 176 & 244 & 245 & +8 & 5.3 & 1.0 \\
\hline & & & & & L. & $O K \rightarrow$ & 100.80 & & & & & & & & \\
\hline $3-5^{n}$ & & 67.5 & & .60 & 1.55 & 4.5 & 105.80 & 59 & & 174 & $2+9$ & 248 & & $\overline{-}$ & $\sigma$ \\
\hline $3-16.38^{\prime \prime}$ & & 75 & & .60 & $1.5 S$ & 4.5 & 110.84 & 61 & 50 & 175 & 247 & $2+9$ & +6 & 8.9 & 11.4 \\
\hline $3.30 .25^{\prime \prime}$ & & 82.5 & .504 & .60 & 1.55 & $\div .5$ & 115.89 & 62 & 51 & 175 & $2+5$ & $2+5$ & & 9.0 & 11.3 \\
\hline $3.50 .38^{\prime \prime}$ & & 90 & & $: 56$ & $1 .+5$ & $\frac{4}{5}$ & 120.78 & 63 & 52 & 175 & 243 & 247 & 47 & 9.0 & 11.3 \\
\hline & & & & & $2 . c$ & $0 K \rightarrow$ & 120.90 & & & & & & & & \\
\hline $4-5^{n}$ & & 97.5 & & $\div 2$ & 1.08 & 3.5 & 125.17 & 60 & 53 & 174 & 238 & 254 & 46 & $=$ & - \\
\hline $4-16.38^{\prime \prime}$ & & 105 & & 146 & 1.19 & 4 & 129.62 & 61 & 53 & 174 & 244 & 254 & +6 & 9.0 & 11.3 \\
\hline $4-30.25^{\prime \prime}$ & & 112.5 & .438 & .50 & 1.30 & 4 & 134.24 & 62 & 53 & 175 & $2+5$ & 254 & +6 & 5.0 & 11.3 \\
\hline $4-50.38^{\prime \prime}$ & & 120 & & .50 & 1.30 & 4 & 138.86 & 62 & 53 & 175 & $2+8$ & 260 & 4) & 8.9 & 11.4 \\
\hline & 1111 & & & & & & & & & & & & & & \\
\hline AVERAGE & & & -0.493 & 0.562 & 1.46 & & 78.34 & 52.4 & 50.8 & 174.4 & & & & 9.0 & 11.3 \\
\hline & & & $\begin{array}{l}\text { mple Train } \\
\text { ak checks: }\end{array}$ & $\begin{array}{r}\text { Pre Test } \\
\text { Post Test }\end{array}$ & $\begin{array}{l}0 i<\mathrm{ft} \\
0 \mathrm{kt}\end{array}$ & $\frac{10}{10}$ & & & & & $\mathrm{Pr}$ & $\frac{O K}{O K}$ & 6 & 20 & \\
\hline
\end{tabular}
CONSOL ENERGY 


\section{ONTARIO HYDRO Hg SAMPLING FIELD DATA SHEET}

\begin{tabular}{|c|c|c|c|c|c|c|c|c|c|c|c|c|c|c|c|}
\hline \multirow{8}{*}{\multicolumn{2}{|c|}{$\begin{array}{l}\text { TEST ID } \\
\text { PLANT } \\
\text { LOCATION } \\
\text { DATE } \\
\text { OPERATOR(S) } \\
\text { AMBIENT TEMP ["F] } \\
\text { BAR. PRESS. [" Hg] }\end{array}$}} & \multirow{2}{*}{\multicolumn{3}{|c|}{5}} & \multirow{6}{*}{\multicolumn{2}{|c|}{$\begin{array}{r}\text { METER BOX } \\
\text { PITOT TUEE DESC } \\
\text { PROBE EENGTH [(t) } \\
\text { NOZZLE ID [inch) } \\
\text { \%HO (AAsumed) }\end{array}$}} & \multirow{2}{*}{$N-1$} & \multirow{3}{*}{\multicolumn{2}{|c|}{ CAL. DATA: delta $\mathrm{H}[$}} & \multicolumn{6}{|c|}{ Page ___ of } \\
\hline & & & & & & & & & & \multirow{2}{*}{$\begin{array}{l}1.894 \\
0.970\end{array}$} & \multirow{2}{*}{ Comments: } & \multirow{2}{*}{\multicolumn{4}{|c|}{ 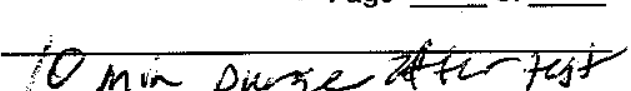 }} \\
\hline & & \multicolumn{3}{|c|}{ GREENIDGE } & & & & & & & & & & & \\
\hline & & \multicolumn{3}{|c|}{ AIR MEATER OUTLET } & & & 12 & & & & & & & & \\
\hline & & & & & & & & \multirow{2}{*}{\multicolumn{2}{|c|}{$\begin{array}{l}\text { FLLTER BOX SETIING } \\
\text { PROBE HTR SETTING }\end{array}$}} & & & & & & \\
\hline & & \multicolumn{3}{|c|}{ R. ODA\&D. OLLEN } & & & \multirow{3}{*}{$\frac{1033}{0.144}$} & & & & & & & & \\
\hline & & & \multirow{2}{*}{\multicolumn{2}{|c|}{$\begin{array}{r}\% \mathrm{H}_{2} \mathrm{O} \text { (Assumed) } \\
\text { FLTER ID } \\
\text { K FACTOR }\end{array}$}} & & \multirow{2}{*}{\multicolumn{2}{|c|}{$\begin{array}{l}\text { DUCT X-SECTION } \\
\text { RUCT DIMENSSONS }\end{array}$}} & circ? & rect? & \multicolumn{4}{|l|}{ other: } \\
\hline & & \multicolumn{3}{|c|}{29.77} & & & & & & & & & & & \\
\hline \begin{tabular}{|c|} 
TRAVERSE \\
POINT
\end{tabular} & CLOCK & SAMPLE & STATIC & PITOT & \multirow{3}{*}{$\begin{array}{l}\text { METER DIFF } \\
\text { PRESSURE } \\
{\left["{ }^{2} \mathrm{H}_{2} 0\right]}\end{array}$} & \multirow{3}{*}{\begin{tabular}{c|} 
METER \\
VACUUM \\
{$["$ Hg] }
\end{tabular}} & \multirow{3}{*}{\begin{tabular}{|c|} 
METER \\
READING \\
REAT \\
\end{tabular}} & \multirow{2}{*}{\multicolumn{2}{|c|}{$\begin{array}{l}\text { METER TI } \\
\text { [OF] } \\
\end{array}$}} & STACK & PROEE & FLFR & LAST IMP & \multicolumn{2}{|c|}{ METER EXHAUST } \\
\hline \begin{tabular}{|c|} 
POINT \\
poort-inchl
\end{tabular} & & TIME & PRES & HEAD & & & & & & TEMP & & Box & TEMP & & $\mathrm{CO}_{2}$ \\
\hline [ port-inch] & $\frac{(24-h r)}{12+3}$ & $\frac{\text { [minute] }}{0}$ & {$\left[\mathrm{r}^{\mathrm{H}} \mathrm{2}_{2}\right]$} & & & & & inlet & outlet & & {$\left[{ }^{\circ} \mathrm{F}\right]$} & {$[\mathrm{F}$} & {$[F]$} & {$[\%$ voll $]$} & $\%$ vol] \\
\hline $1-2^{n}$ & & 7 & -12.90 & 0.10 & 0.07 & 1.0 & 200.82 & 52 & 51 & 279 & 284 & 207 & 46 & 8.1 & 12.1 \\
\hline $1-2^{n}$ & & 14 & 13.7 & 0.10 & 7 & 1.0 & 701.98 & 53 & 52 & 289 & 206 & 204 & 44 & 8.1 & 12.1 \\
\hline $1-6^{\prime \prime}$ & & 21 & -1307 & 0.85 & 4 & 3.5 & $20 \% 91$ & 54 & 53 & 299 & 298 & 206 & 44 & 2.4 & $12 . \overline{8}$ \\
\hline$\overline{1-6^{n}}$ & & 28 & +12.69 & 8.48 & .066 & 3.5 & 28.99 & 54 & 53 & 299 & 297 & 208 & 42 & 7.2 & 2.9 \\
\hline 1-10" & & 35 & $-13.7 i$ & 1.1 & $0: 83$ & 4,5 & 211.47 & 59 & 54 & 302 & $3-\frac{1}{2} 4$ & 209 & 43 & 6.9 & 13.3 \\
\hline 1-10" & & 42 & -13.42 & 1,1 & 0.83 & 5,0 & 214.95 & 60 & 54 & 300 & 258 & 207 & $4 y$ & 6.6 & 13.5 \\
\hline & BL9 & & & & & & 215.20 & & & & & & & & \\
\hline$-2^{n}$ & & 749 & -12.29 & 43 & 30 & 3.0 & 217.47 & 60 & 55 & 289 & 290 & 217 & 46 & 2.6 & 17.6 \\
\hline$-2^{n}$ & & 姓56 & $-1, ;, \infty 5$ & 40 & 30 & 3.0 & $\infty$ & 60 & 56 & 294 & 294 & 210 & 46 & 7.2 & 2.9 \\
\hline $2-6^{\prime \prime}$ & & fi 63 & .1322 & 240 & .60 & 4.5 & 222.45 & $6 i$ & 57 & 267 & 258 & $2 i l$ & 44 & 710 & 3.1 \\
\hline $6^{\prime \prime}$ & 1357 & $x_{70}$ & & .80 & 60 & 5.0 & $225: 4$ & 62 & $5 ?$ & 297 & 295 & 211 & 46 & 6,9 & 13.3 \\
\hline $2-10^{\prime \prime}$ & & $b^{5} 77$ & $-73.6 i$ & 1.4 & 1.04 & 7.0 & 229.19 & 64 & 56 & 300 & 297 & 212 & 46 & 6.4 & $B .7$ \\
\hline $2-10^{n}$ & & $i_{84}$ & & 1.4 & 1.04 & 8.0 & 232.99 & 64 & 56 & $3 \cdot 2$ & 297 & 2,2 & 47 & 6.7 & 135 \\
\hline & 1420 & & 4 & & & & 233.40 & & & & & & & $\rightarrow$ & \\
\hline $3 \cdot 2^{\prime \prime}$ & & $7_{91}$ & -13.65 & .83 & .80 & 4.5 & 236.46 & 62 & 59 & 289 & 289 & 216 & 48 & 8 & 12.6 \\
\hline $3-2^{\prime \prime}$ & & fi 98 & & .80 & 0 & 5.0 & 239.38 & 64 & 60 & 296 & 257 & 214 & 47 & 2.4 & 12.6 \\
\hline $3.6^{n}$ & & fi 105 & -12.94 & .92 & 65,42 & $6.0,0$ & 242,54 & 65 & 60 & 299 & 295 & 211 & 47 & 73 & 12.9 \\
\hline $3.6^{\prime \prime}$ & & 2112 & & 192 & .68 & 6.0 & 245,70 & 45 & Co & 299 & 245 & 2,0 & 48 & 7.3 & 10.4 \\
\hline $3-10^{n}$ & & 35119 & 73.43 & $i .1$ & 0.83 & 5.0 & 249.14 & 65 & 60 & 300 & 294 & 206 & 48 & 2.1 & $B, 1$ \\
\hline$\overline{3 \cdot 10^{\prime \prime}}$ & & $42_{126}$ & & 1.1 & 3,83 & 9,5 & 252.62 & 45 & 60 & 300 & 254 & 2.3 & 48 & 9.0 & 132 \\
\hline & & & & & & & & & & & & & & & \\
\hline De & & & $\mid-13.25$ & 0.774 & 0.623 & & 52.46 & 58.4 & & 33296.2 & & & & 7.2 & 3.0 \\
\hline & & & $\begin{array}{l}\text { Sample Train } \\
\text { Leak Checks: }\end{array}$ & $\begin{array}{l}\text { Pre T } \\
\text { Post T }\end{array}$ & 0,0 & $\frac{10}{10}$ & $\begin{array}{l}\text { in. } \mathrm{Hg} \\
\text { in. } \mathrm{Hg}\end{array}$ & & & $\begin{array}{r}\text { Pitot } \\
\text { Leak Che }\end{array}$ & & $\frac{O K}{O K}$ & 6 & & \\
\hline
\end{tabular}


ONTARIO HYDRO Hg SAMPLING FIELD DATA SHEET

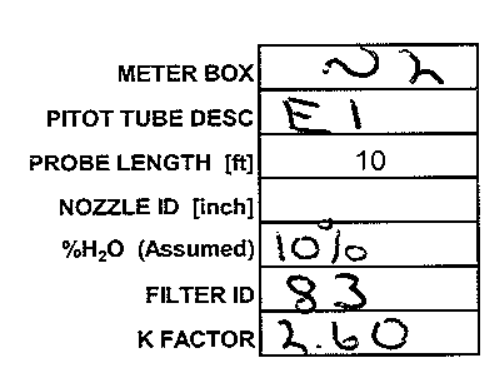

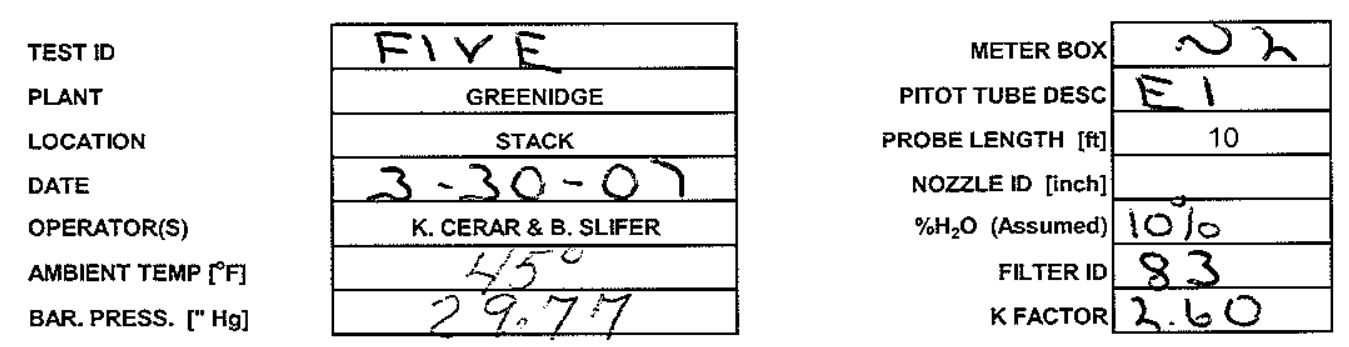

CAL. DATA: delta H 1.855 Comments:

Page

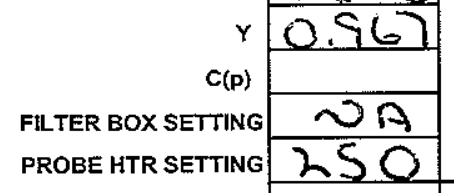

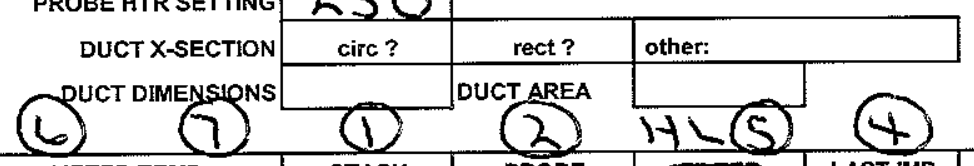

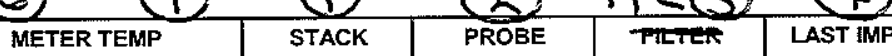
[oF]

[" $\left.\mathrm{H}_{2} \mathrm{O}\right]$

READING

[" Hg]

145.00

PF]

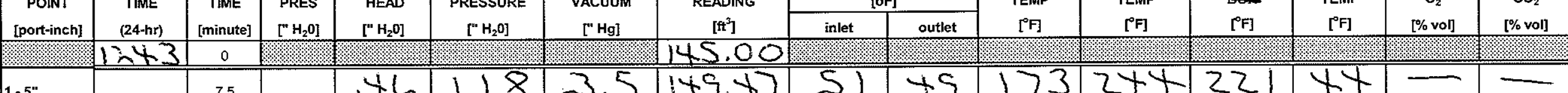
\begin{tabular}{ccc|c|c|c|c|c|c|c|}
.58 & 1.50 & + & 168.43 & 64 & 54 & $17+252$ & 232 & +5 & -
\end{tabular}

$$
\begin{array}{|l} 
\\
4-5 \\
4-1 \\
\hline
\end{array}
$$

\begin{tabular}{|c|c|}
\hline & \\
\hline 4-5" & \\
\hline $4-16.38 "$ & \\
\hline $4-30.25^{\prime \prime}$ & \\
\hline $4-50.38^{\prime \prime}$ & \\
\hline & 1454 \\
\hline
\end{tabular}$$
4
$$

\begin{tabular}{|l|l|l|l}
\hline \hline AVERAGE & \\
\hline
\end{tabular}

\subsection{5

81.05

Leak Checks:

ost Test $O K \mathrm{ft}^{3} @ 10$ in. $\mathrm{Hg}$

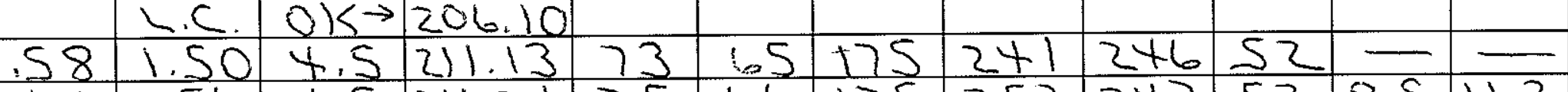

5) $49173324+122144=-$

$565017425222+4+8.811 .4$

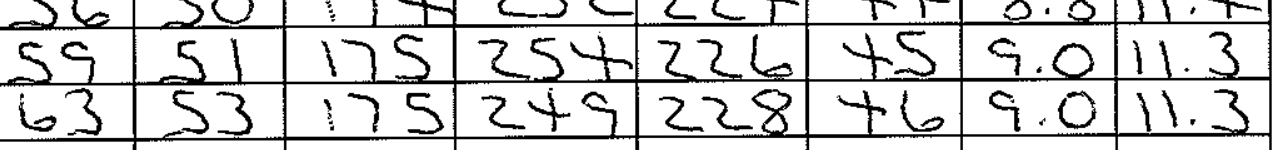

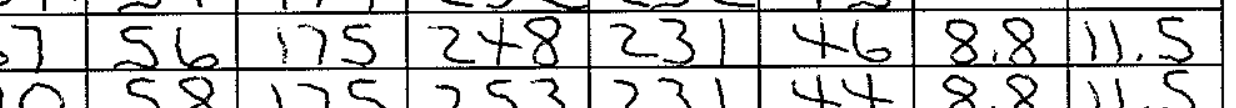
\begin{tabular}{|c|c|c|c|c|c|c|c|}
.60 & 1.56 & 4.5 & 178.57 & 70 & 58 & 175 \\
\hline 58 & 150 & 4.5 & 183.95 & 71 & 60 & +75
\end{tabular} \begin{tabular}{|l|l|l|l|l|}
253 & 231 & 44 & 8.8 & 11.5 \\
\hline 241 & 231 & 44 & 8.8 & 11.5 \\
\hline
\end{tabular} L.C. OK $\rightarrow 184.05$ \begin{tabular}{lll|l|l|l|l|l|l|l|l}
.70 & 1.80 & 5 & 189.52 & 70 & 61 & 175 & 251 & 244 & 45 & -
\end{tabular} \begin{tabular}{ll|l|l|l|l|l|l|l|l|}
5 & 195.13 & 73 & 62 & 175 & 255 & 251 & 47 & 8.5 & 11.4 \\
\hline
\end{tabular}

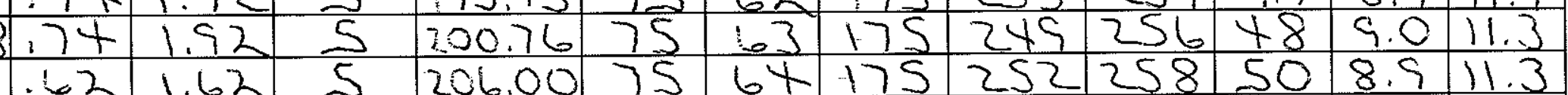
\begin{tabular}{llllllllllllll}
160 & 1.56 & 4.5 & 26.24 & 75 & 66 & 175 & 252 & 247 & 52 & 8.9 & 11.3 \\
\hline
\end{tabular} 
ONTARIO HYDRO Hg SAMPLING FIELD DATA SHEET

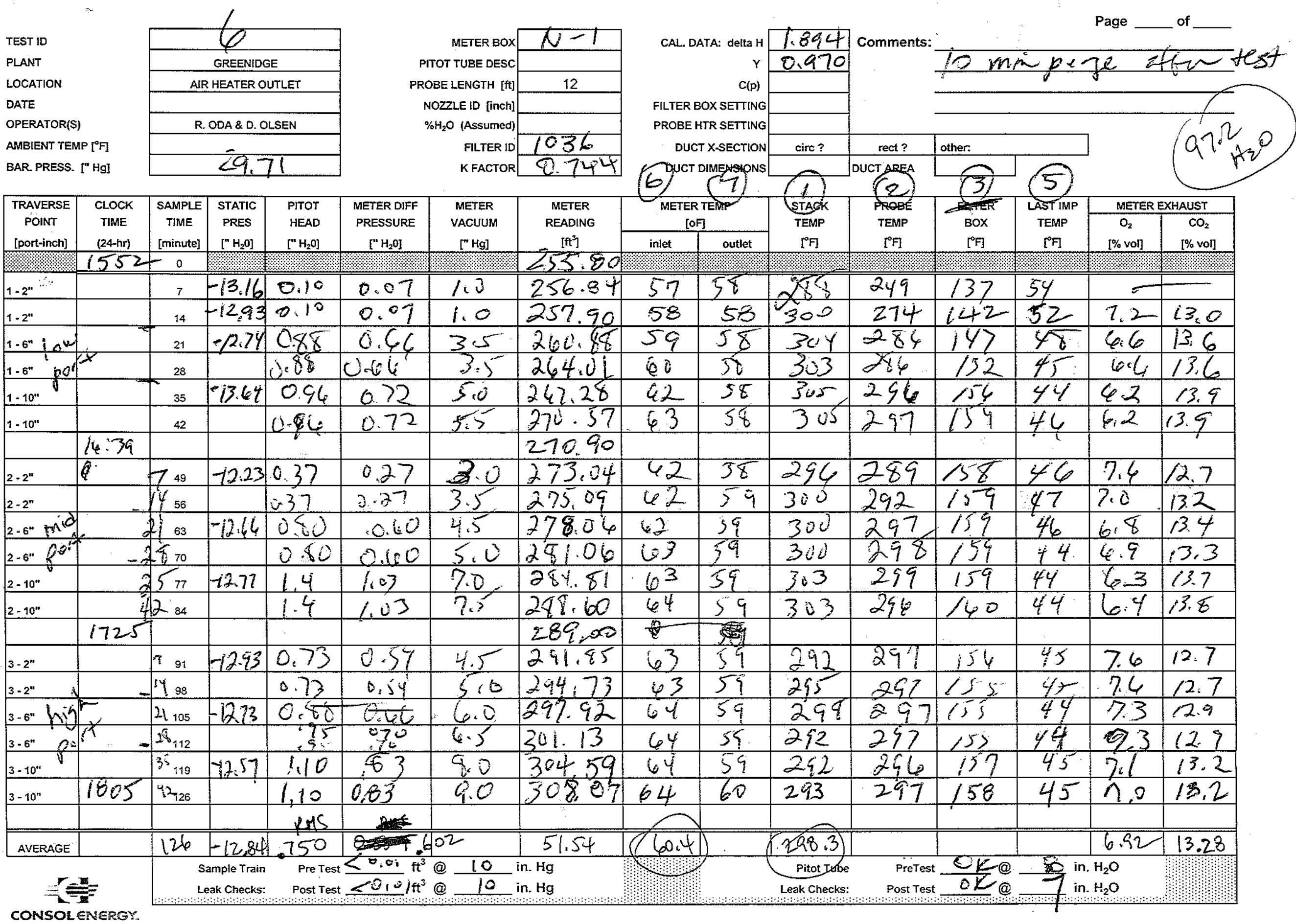




\section{ONTARIO HYDRO Hg SAMPLING FIELD DATA SHEET}

\begin{tabular}{|c|c|c|c|c|c|c|c|c|c|c|c|c|c|c|c|}
\hline \multirow{7}{*}{\multicolumn{2}{|c|}{$\begin{array}{l}\text { TEST ID } \\
\text { PLANT } \\
\text { LOCATION } \\
\text { DATE } \\
\text { OPERATOR(S) } \\
\text { AMBIENT TEMP [0 } \\
\text { BAR. PRESS. [" Hg] }\end{array}$}} & \multirow{2}{*}{\multicolumn{3}{|c|}{$51 x$}} & \multirow{2}{*}{\multicolumn{3}{|c|}{${ }_{\text {METER BOX }} \sim 2$}} & \multirow{2}{*}{\multicolumn{2}{|c|}{ CAL. DATA: detia H }} & \multirow{5}{*}{\begin{tabular}{|l|}
1.895 \\
0.567 \\
$2 A$ \\
250 \\
\end{tabular}} & \multirow{2}{*}{ Comments } & & \multirow{2}{*}{\multicolumn{2}{|c|}{ Page __ of }} \\
\hline & & & & & & & & & & & & & & & \\
\hline & & & GREENIDC & & & & & & & & & & & & \\
\hline & & $3=$ & STACK & 07 & & DBE LENGTH [ft] & 10 & $R_{1}$ & $c(p)$ & & & & & & \\
\hline & & & ERAR \& B. & SLIFER & & $\mathrm{KH}_{2} \mathrm{O}$ (Assumed) & $10 \%$ & ROBE & :TING & & & & & & \\
\hline & & & 60 & & & & 84 & & X-SECTION & \begin{tabular}{|c|} 
circ? \\
\end{tabular} & trect? & other: & & & \\
\hline & & & 5.7 & & & & 2.60 & & & & & & & & \\
\hline AVERSE & & & & & & & & & & STÁ & DPOBE & & & $R E$ & $x$ \\
\hline POINT & TIME & TIME & PRES & HEAD & ESSURE & VACUUN & $\begin{array}{l}\text { READING } \\
\text { RE }\end{array}$ & & & TEN & $\begin{array}{l}\text { PROBE } \\
\text { TEMP }\end{array}$ & & & & \\
\hline [port-inch] & (24-hr) & [minute] & {$\left[\mathrm{H}_{2} \mathrm{O}\right]$} & {$\left[" \mathrm{H}_{2} \mathrm{O}\right]$} & {$\left[\mathrm{H}_{2} \mathrm{O}\right]$} & [" Hg] & {$\left[\mathrm{rt}^{3}\right]$} & inlet & outlet & PF & {$[\mathrm{F}]$} & & & & \\
\hline & 1552 & 0 & & & & & 232.60 & & & & & & & & \\
\hline 1. $5^{\prime \prime}$ & & 7.5 & & .62 & 1.62 & 5 & 2.37 .76 & 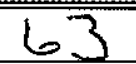 & & 172 & 245 & 208 & & - & \\
\hline - $16.38^{\prime \prime}$ & & 15 & & .62 & 1.62 & 5 & 242.90 & & 62 & & 250 & 212 & 4 & 9.5 & 10.8 \\
\hline $1-30.25^{\prime \prime}$ & & 22.5 & .571 & .60 & 1.58 & 5 & 248.01 & 68 & 62 & 17 & 245 & 212 & $S$ & 5.4 & 10.9 \\
\hline 1-50.38" & & 30 & & .58 & 1.50 & 5 & 252.98 & 70 & 62 & 175 & 238 & 215 & 54 & 9.3 & 11.0 \\
\hline & & & & & L.C. & $0 K \rightarrow$ & 253.10 & & & & & & & & \\
\hline $2-5^{\prime \prime}$ & & 37.5 & & .68 & 1.75 & 6 & 3.58 .50 & 67 & 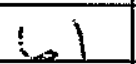 & 17 & $2+2$ & 231 & 5 & - & $=$ \\
\hline$-16.38^{\prime \prime}$ & & 45 & & 170 & 1.80 & 1 & 263.90 & 70 & 62 & $17 x$ & 253 & 233 & 5 & 8.9 & 11.4 \\
\hline$-30.25^{\prime \prime}$ & & $52.5-$ & .638 & 70 & 1.80 & & 269.37 & 71 & 62 & 175 & 253 & 233 & & 8.8 & 11.5 \\
\hline$-50.38^{"}$ & & 60 & & .60 & 1.58 & 5.5 & 274.49 & 71 & 62 & 175 & $2+9$ & 232 & & 8.7 & 11,5 \\
\hline & & & & & L.C. & $O K \rightarrow$ & 274.60 & & & & & & & & \\
\hline$-5^{n}$ & & 67.5 & & 60 & 1.58 & 5.5 & 279.73 & 68 & 62 & 175 & 236 & 232 & & - & \\
\hline 16.38" & & 75 & & .60 & 1.58 & 5.5 & 284.86 & 69 & 61 & 175 & 249 & 230 & & 9.0 & 11.3 \\
\hline$-30.25^{\prime \prime}$ & & 82.5 & -.508 & .60 & 1.58 & 5 & 290.00 & 69 & 61 & 175 & 251 & 232 & +8 & 8.9 & 11.4 \\
\hline 3-50.38" & & 90 & & .58 & 1.50 & 5.5 & 295.00 & 71 & 62 & 175 & 237 & 233 & +8 & 8.9 & 11.4 \\
\hline & & & & & L.C & $O K \leftrightarrow \rightarrow$ & 255.10 & & & & & & & & \\
\hline $4-5^{\prime \prime}$ & & 97.5 & & .44 & 1.14 & 4 & 299.47 & 69 & 62 & 124 & 244 & 234 & 46 & - & $=$ \\
\hline $4-16.38^{\prime \prime}$ & & 105 & & .46 & 1.18 & 4.5 & 303.94 & 70 & 62 & 174 & 249 & $2+3$ & 45 & 8.5 & 11.3 \\
\hline $4-30.25^{\prime \prime}$ & & 112.5 & -.470 & .46 & 1.18 & 4.5 & 308.40 & 71 & 62 & 174 & 252 & $2+2$ & +5 & 9.0 & 11.3 \\
\hline 4-50.38" & & 120 & & .50 & 1.28 & 5 & 313,00 & 71 & 63 & 174 & 242 & $2+1$ & 46 & 9.0 & 11.3 \\
\hline & 1803 & & & pass & & & & & & & & & & & \\
\hline AVERAGE & & & 0.547 & 0.581 & 1.517 & & 80.07 & 65. & & 174.4 & & & & 9.03 & 11.26 \\
\hline & & & $\begin{array}{l}\text { mple Train } \\
\text { ak Checks: }\end{array}$ & $\begin{array}{l}\text { Pre Test } \\
\text { Post Test }\end{array}$ & $\begin{array}{l}O K \mathrm{ft}^{3} \\
O K \mathrm{ft}^{3}\end{array}$ & $\begin{array}{l}10 \\
10\end{array}$ & & & & $\begin{array}{r}\text { Pitot Tube } \\
\text { Leak Checks: }\end{array}$ & & $\frac{0 k}{0 k}$ & & $\mathrm{H}_{2} \mathrm{O}$ & \\
\hline
\end{tabular}

CONSOL ENERGY 
Distribution: JEL, BWG

Project No.: 1621-85

Sample Date: $3 / 30 / 07$

\begin{tabular}{|c|c|c|c|c|c|c|c|c|}
\hline Location: & $\mathrm{AHO}$ & Task: & Tes & $H G-4$ & Operato & $0 / 00$ & & \\
\hline Sample ID & Bottle \# & Description & $\begin{array}{c}\text { Initial Vol } \\
\mathrm{mL}\end{array}$ & $\begin{array}{c}\text { Rinse Vol } \\
\mathrm{mL}\end{array}$ & $\begin{array}{l}\text { Gain } \\
\mathrm{mL}\end{array}$ & $\begin{array}{c}\text { Final Vol } \\
\mathrm{mL}\end{array}$ & ppb Hg & $\begin{array}{c}\text { Total ug } \\
\text { of } \mathrm{Hg}\end{array}$ \\
\hline 89 & $\mathbf{S}$ & Filter/Solids & - & $\cdots$ & - & & & \\
\hline 90 & 1 & Probe \& Filter Rinse & 0 & 128 & & & & \\
\hline 91 & 2 & Sample Line Rinse & 0 & 50 & & & & \\
\hline 92 & 3 & $\mathrm{KCl}$ Impingers & 300 & 150 & 6572 & 522 & & \\
\hline 93 & 4 & $\mathrm{HNO}_{3} / \mathrm{H}_{2} \mathrm{O}_{2}$ Impinger & 100 & \pm 5 & $\vdots$ & 176 & & \\
\hline 94 & 5 & $\mathrm{KMnO}_{4}$ Impingers & 200 & 50 & 0 & 250 & & \\
\hline 95 & 6 & $\mathrm{KMnO}_{4}$ Acid Rinse & 0 & 100 & 0 & 100 & & \\
\hline
\end{tabular}

Filter Gross wt:

Filter Tare wt:

Filter Net wt:
Filter Net wt:

Probe/Line Rinse wt:

Total Particulate wt:
Condensate Total: $\mathrm{ml}$

Recovered By: J. Locke

Date: $3 / 30 / 07$

Location: STK

Task:

Test: $4 / \mathrm{G} \cdot 5$ Operator: $B .5 / K C$

\begin{tabular}{|c|c|c|c|c|c|c|c|c|}
\hline Sample ID & Bottle \# & Description & $\begin{array}{c}\text { Initial Vol } \\
\mathrm{mL}\end{array}$ & $\begin{array}{c}\text { Rinse Vol } \\
\mathrm{mL}\end{array}$ & $\begin{array}{c}\text { Gain } \\
\mathrm{mL}\end{array}$ & $\begin{array}{c}\text { Final Vol } \\
\mathrm{mL}\end{array}$ & ppb Hg & $\begin{array}{c}\text { Total ug } \\
\text { of } \mathrm{Hg}\end{array}$ \\
\hline 96 & $S$ & Filter/Solids & & & & & & \\
\hline $97 / 98$ & 1 & Probe \& Filter Rinse / tollt & & 40,81 & & & & \\
\hline 99 & 2 & $\mathrm{KCl}$ Impingers & 300 & 150 & 99 & 649 & & \\
\hline 100 & 3 & $\mathrm{HNO}_{3} / \mathrm{H}_{2} \mathrm{O}_{2}$ Impinger & 100 & 75 & 9 & 184 & & \\
\hline 101 & 4 & $\mathrm{KMnO}_{4}$ Impingers & 200 & 50 & 4 & 2.54 & & \\
\hline 102 & 5 & $\mathrm{KMnO}_{4}$ Acid Rinse & 0 & 100 & 0 & 100 & & \\
\hline
\end{tabular}

Filter Gross wt:

Filter Tare wt:

Filter Net wt: g

g g
Filter Net wt:

Probe/Line Rinse wt:

Total Particulate wt:
Condensate Total: $\mathrm{ml}$

Recovered By: J. Locke

Date: $3 / 30 / 07$

Recovered By:

Date:

\begin{tabular}{|c|c|c|c|}
\hline Sample ID & Description & ppb Hg & $\begin{array}{c}\text { Total ug } \\
\text { of } \mathrm{Hg}\end{array}$ \\
\hline & 3 in. Filter Blank & & \\
\hline & $\mathrm{KCl} \mathrm{Blank}$ & & \\
\hline & $\mathrm{HNO}_{3} / \mathrm{H} 2 \mathrm{O} 2$ Blank & & \\
\hline & $\mathrm{KMnO}_{4}$ Blank & & \\
\hline $\mathrm{KMnO}_{4}$ Blank & & \\
\hline & $\mathrm{HNO}_{3} / \mathrm{HCl}$ Blank & & \\
\hline
\end{tabular}


Distribution: JEL, BWG

Project No.: 1621-85

Sample Date: $3 / 30 / 07$

Location: $\mathrm{AHO}$

Task:

Test: $K_{G} \cdot 5$ operator: RD/PO

\begin{tabular}{|c|c|c|c|c|c|c|c|c|}
\hline Sample ID & Bottle \# & Description & $\begin{array}{c}\text { Initial Vol } \\
\mathrm{mL}\end{array}$ & $\begin{array}{c}\text { Rinse Vol } \\
\mathrm{mL}\end{array}$ & $\begin{array}{c}\text { Gain } \\
\mathrm{mL}\end{array}$ & $\begin{array}{c}\text { Final Vol } \\
\mathrm{mL}\end{array}$ & ppb Hg & $\begin{array}{c}\text { Total ug } \\
\text { of } \mathrm{Hg}\end{array}$ \\
\hline 103 & $\mathbf{s}$ & Filter/Solids & - & - & - & & & \\
\hline 104 & 1 & Probe \& Filter Rinse & & 140 & 467 & 157 & & \\
\hline 105 & 2 & Sample Line Rinse & & $\sqrt{2}+1$ & & 128 & & \\
\hline 106 & 3 & $\mathrm{KCl}$ Impingers & 300 & 50 & 80 & 530 & & \\
\hline 107 & 4 & $\mathrm{HNO}_{3} / \mathrm{H}_{2} \mathrm{O}_{2}$ Impinger & 100 & 75 & 0 & 175 & & \\
\hline 108 & 5 & $\mathrm{KMnO}_{4}$ Impingers & 200 & 50 & 0 & 250 & & \\
\hline 105 & 6 & $\mathrm{KMnO}_{4}$ Acid Rinse & 0 & 100 & 0 & 100 & & \\
\hline
\end{tabular}

Filter Gross wt:

Filter Tare wt:

Filter Net wt:

Filter Net wt:

Probe/Line Rinse wt:

Total Particulate wt:

Condensate Total: $\mathrm{ml}$

Recovered By: I. Locke

Date: $3 / 30 / 07$

Location: STK

Task:

1

Test: $H G-5$ operator: $B S / K C$

\begin{tabular}{|c|c|c|c|c|c|c|c|c|}
\hline Sample ID & Bottle \# & Description & $\begin{array}{c}\text { Initial Vol } \\
\mathrm{mL}\end{array}$ & $\begin{array}{c}\text { Rinse Vol } \\
\mathrm{mL}\end{array}$ & $\begin{array}{c}\text { Gain } \\
\mathrm{mL}\end{array}$ & $\begin{array}{c}\text { Final Vol } \\
\mathrm{mL}\end{array}$ & ppb Hg & $\begin{array}{c}\text { Total ug } \\
\text { of } \mathrm{Hg}\end{array}$ \\
\hline 110 & $\mathbf{s}$ & Filter/Solids & & & & & & \\
\hline $111 / 112$ & 1 & Probe \& Filter Rinse $L$, , & & $138 / 79$ & & $138 / 79$ & & \\
\hline 113 & 2 & KCI Impingers & 300 & 150 & 214 & 664 & & \\
\hline 114 & 3 & $\mathrm{HNO}_{3} / \mathrm{H}_{2} \mathrm{O}_{2}$ Impinger & 100 & 75 & 0 & 175 & & \\
\hline 115 & 4 & $\mathrm{KMnO}_{4}$ Impingers & 200 & 50 & 0 & 250 & & \\
\hline 110 & 5 & $\mathrm{KMnO}_{4}$ Acid Rinse & 0 & 100 & 0 & 100 & & \\
\hline
\end{tabular}

Filter Gross wt:

Filter Tare wt:

Filter Net wt:

Filter Net wt:

Probe/Line Rinse wt:

Total Particulate wt:
Condensate Total: $\mathrm{ml}$

Date: $3 / 30 / 07$

Date:

\begin{tabular}{||c|c|c|c|}
\hline Sample ID & Description & ppb Hg & $\begin{array}{c}\text { Total ug } \\
\text { of } \mathrm{Hg}\end{array}$ \\
\hline & $3 \mathrm{in}$. Filter Blank & & \\
\hline & $\mathrm{KCl}$ Blank & & \\
\hline & $\mathrm{HNO}_{3} / \mathrm{H} 2 \mathrm{O} 2$ Blank & & \\
\hline $\mathrm{KMnO}_{4}$ Blank & & \\
\hline & $\mathrm{KMnO}_{4}$ Blank & & \\
\hline & $\mathrm{HNO}_{3} / \mathrm{HCl}$ Blank & & \\
\hline
\end{tabular}


Distribution: JEL, BWG

Project No.: 1621-85

Sample Date: $3 / 30 / 07$

Location: AHO

Task:

Test: $H G_{T} \cdot 6$ Operator: RO/DO

\begin{tabular}{|c|c|c|c|c|c|c|c|c|}
\hline Sample ID & Bottle \# & Description & $\begin{array}{c}\text { Initial Vol } \\
\mathrm{mL}\end{array}$ & $\begin{array}{c}\text { Rinse Vol } \\
\mathrm{mL}\end{array}$ & $\begin{array}{c}\text { Gain } \\
\mathrm{mL}\end{array}$ & $\begin{array}{c}\text { Final Vol } \\
\mathrm{mL}\end{array}$ & $\mathrm{ppb} \mathrm{Hg}$ & $\begin{array}{c}\text { Total ug } \\
\text { of } \mathrm{Hg}\end{array}$ \\
\hline 117 & $s$ & Filter/Solids & & & & & & \\
\hline 113 & 1 & Probe \& Filter Rinse & - & 81 & - & 81 & & \\
\hline 119 & 2 & Sample Line Rinse & - & 140 & - & 140 & & \\
\hline 120 & 3 & $\mathrm{KCl}$ Impingers & 300 & 150 & 81 & 531 & & \\
\hline 121 & 4 & $\mathrm{HNO}_{3} / \mathrm{H}_{2} \mathrm{O}_{2}$ Impinger & 100 & 75 & 0 & 175 & & \\
\hline 122 & 5 & $\mathrm{KMnO}_{4}$ impingers & 200 & 50 & 6 & 256 & & \\
\hline 123 & 6 & $\mathrm{KMnO}_{4}$ Acid Rinse & 0 & 100 & 0 & 100 & & \\
\hline
\end{tabular}

Filter Gross wt:

Filter Tare wt:

Filter Net wt:
Filter Net wt:

Probe/Line Rinse wt:

Total Particulate wt:

Recovered By: J. Locke

Location: STK

Task:

1

Test: $H G_{F}-6$ Operator:

Date: $3 / 30 / 07$

\begin{tabular}{|c|c|l|c|c|c|c|c|c|}
\hline Sample ID & Bottle \# & \multicolumn{1}{|c|}{ Description } & $\begin{array}{c}\text { Initial Vol } \\
\mathrm{mL}\end{array}$ & $\begin{array}{c}\text { Rinse Vol } \\
\mathrm{mL}\end{array}$ & $\begin{array}{c}\text { Gain } \\
\mathrm{mL}\end{array}$ & $\begin{array}{c}\text { Final Vol } \\
\mathrm{mL}\end{array}$ & $\begin{array}{c}\mathrm{ppb} \mathrm{Hg} \\
\text { of } \mathrm{Hg}\end{array}$ \\
\hline 124 & $\mathrm{~s}$ & Filter/Solids & & & & & & \\
\hline $125 / 126$ & 1 & Probe \& Filter Rinse & & $117 / 129$ & & $117 / 129$ & & \\
\hline 127 & 2 & $\mathrm{KCl}^{\prime}$ Impingers & 300 & 150 & $20 \%$ & 6.58 & & \\
\hline 128 & 3 & $\mathrm{HNO}_{3} / \mathrm{H}_{2} \mathrm{O}_{2}$ Impinger & 100 & 75 & 0 & 181 & & \\
\hline 129 & 4 & $\mathrm{KMnO}_{4}$ Impingers & 200 & 50 & 0 & 250 & & \\
\hline 130 & 5 & $\mathrm{KMnO}_{4}$ Acid Rinse & 0 & 100 & 0 & 100 & & \\
\hline
\end{tabular}

Filter Gross wt:

Filter Tare wt:

Filter Net wt: g g g
Filter Net wt:

Probe/Line Rinse wt:

Total Particulate wt:
Condensate Total: $\mathrm{ml}$
Recovered By: J. Locke

Recovered By: $\mathrm{ml}$
Condensate Total:

\begin{tabular}{|c|c|c|c|}
\hline Sample ID & Description & ppb Hg & $\begin{array}{c}\text { Total ug } \\
\text { of } \mathrm{Hg}\end{array}$ \\
\hline 132 & 3 in. Fliter Blank & & \\
\hline 133 & $\mathrm{KCl}^{3}$ Blank & & \\
\hline 31 & $\mathrm{HNO}_{3} / \mathrm{H} 2 \mathrm{O}$ Blank & & \\
\hline 134 & $\mathrm{KMnO}_{4}$ Blank & & \\
\hline 135 & $\mathrm{KMnO}_{4}$ Blank imp-ncie & & \\
\hline
\end{tabular}


APPENDIX D

Laboratory Analyses 


\begin{tabular}{|l|l|l|c|c|c|c|}
\hline ANALNUM & DESCR & SAMPLE & NH3 as N & NH3 as NH4 & & NH3 as NH4 \\
\hline & & & mg/L & mg/L & volume (L) & mg \\
\hline 20071759 & IMPINGER 1 NH3-1 & IMP 1 & 9.04 & 11.64 & 0.250 & 2.91 \\
\hline 20071760 & IMPINGER 2 NH3-1 & IMP 2 & $<0.10$ & $<0.13$ & 0.250 & $<0.03$ \\
\hline 20071761 & IMPINGER 3 NH3-1 & IMP 3 & $<0.10$ & $<0.13$ & 0.250 & $<0.03$ \\
\hline 20071762 & PROBE \& IMPINGER 1 NH3-2 & IMP 1 & 21.15 & 27.24 & 0.255 & 6.95 \\
\hline 20071763 & IMPINGER 2 NH3-2 & IMP 2 & $<0.10$ & $<0.13$ & 0.250 & $<0.03$ \\
\hline 20071764 & IMPINGER 3 NH3-2 & IMP 3 & $<0.10$ & $<0.13$ & 0.250 & $<0.03$ \\
\hline 20071765 & PROBE \& IMPINGER 1 NH3-3 & IMP 1 & 22.51 & 28.99 & 0.272 & 7.88 \\
\hline 20071766 & IMPINGER 2 NH3-3 & IMP 2 & $<0.10$ & $<0.13$ & 0.250 & $<0.03$ \\
\hline 20071767 & IMPINGER 3 NH3-3 & IMP 3 & $<0.10$ & $<0.13$ & 0.250 & $<0.03$ \\
\hline
\end{tabular}




\begin{tabular}{|c|c|c|c|c|c|c|}
\hline NUMBER & LOGATION & SAMPLE & TEST & $\mathrm{NH} 3$ as N & NH3 as N & $\mathrm{NH} 3$ as NH4 \\
\hline & -7 & & & ug & $\mathrm{mg}$ & $\mathrm{mg}$ \\
\hline 1 & $\mathrm{AHI}$ & PROBE/LINE RINSE & $\mathrm{NH} 3-1$ & 922 & 0.92 & 1.19 \\
\hline 2 & $\mathrm{AHI}$ & Impinger 1 & $\mathrm{NH3}-1$ & 619 & 0.62 & 0.80 \\
\hline 3 & $\mathrm{AHI}$ & Impinger 2 & $\mathrm{NH} 3-1$ & 53 & 0.05 & 0.07 \\
\hline 4 & $\mathrm{AHI}$ & Impinger 3 & $\mathrm{NH3}-1$ & $<25$ & $<0.03$ & $<0.03$ \\
\hline 5 & SCRO & PROBE/LINE RINSE & $\mathrm{NH3}-1$ & 3592 & 3.59 & 4.63 \\
\hline 6 & SCRO & Impinger 1 & $\mathrm{NH3}-1$ & 431 & 0.43 & 0.56 \\
\hline 7 & SCRO & Impinger 2 & $\mathrm{NH} 3-1$ & 26 & 0.03 & 0.03 \\
\hline 8 & SCRO & Impinger 3 & $\mathrm{NH3}-1$ & $<25$ & $<0.03$ & $<0.03$ \\
\hline 9 & $\mathrm{AHI}$ & PROBE/LINE RINSE & $\mathrm{NH} 3-2$ & 1050 & 1.05 & 1.35 \\
\hline 10 & $\mathrm{AHI}$ & Impinger 1 & $\mathrm{NH} 3-2$ & 920 & 0.92 & 1.18 \\
\hline 11 & $\mathrm{AHI}$ & Impinger 2 & $\mathrm{NH} 3-2$ & 77 & 0.08 & 0.10 \\
\hline 12 & $\mathrm{AHI}$ & Impinger 3 & $\mathrm{NH} 3-2$ & $<25$ & $<0.03$ & $<0.03$ \\
\hline 13 & SCRO & PROBE/LINE RINSE & $\mathrm{NH3-2}$ & 3063 & 3.06 & 3.94 \\
\hline 14 & SCRO & Impinger 1 & $\mathrm{NH} 3-2$ & 215 & 0.22 & 0.28 \\
\hline 15 & SCRO & Impinger 2 & $\mathrm{NH} 3-2$ & 41 & 0.04 & 0.05 \\
\hline 16 & SCRO & Impinger 3 & $\mathrm{NH} 3-2$ & $<25$ & $<0.03$ & $<0.03$ \\
\hline 17 & $\mathrm{AHI}$ & PROBE/LINE RINSE & $\mathrm{NH3}-3$ & 1099 & 1.10 & 1.42 \\
\hline 18 & $\mathrm{AHI}$ & Impinger 1 & $\mathrm{NH} 3-3$ & 578 & 0.58 & 0.74 \\
\hline 19 & AHI & Impinger 2 & $\mathrm{NH} 3-3$ & 80 & 0.08 & 0.10 \\
\hline 20 & $\mathrm{AHI}$ & Impinger 3 & $\mathrm{NH} 3-3$ & $<25$ & $<0.03$ & $<0.03$ \\
\hline 21 & SCRO & PROBE/LINE RINSE & $\mathrm{NH} 3-3$ & 4195 & 4.20 & 5.40 \\
\hline 22 & SCRO & Impinger 1 & $\mathrm{NH} 3-3$ & 767 & 0.77 & 0.99 \\
\hline 23 & SCRO & Impinger 2 & $\mathrm{NH} 3-3$ & 67 & 0.07 & 0.09 \\
\hline 24 & SCRO & Impinger 3 & $\mathrm{NH3}-3$ & $<25$ & $<0.03$ & $<0.03$ \\
\hline 25 & $\mathrm{AHI}$ & PROBE/LINE RINSE & $\mathrm{NH} 3-4$ & 1219 & 1.22 & 1.57 \\
\hline 26 & $\mathrm{AHI}$ & Impinger 1 & $\mathrm{NH3}-4$ & 517 & 0.52 & 0.67 \\
\hline 27 & $\mathrm{AHI}$ & Impinger 2 & $\mathrm{NH3-4}$ & 69 & 0.07 & 0.09 \\
\hline 28 & $\mathrm{AHI}$ & Impinger 3 & $\mathrm{NH} 3-4$ & $<25$ & $<0.03$ & $<0.03$ \\
\hline 29 & SCRO & PROBE/LINE RINSE & $\mathrm{NH3}-4$ & 3155 & 3.16 & 4.06 \\
\hline 30 & SCRO & Impinger 1 & $\mathrm{NH} 3-4$ & 321 & 0.32 & 0.41 \\
\hline 31 & SCRO & Impinger 2 & $\mathrm{NH} 3-4$ & 70 & 0.07 & 0.09 \\
\hline 32 & SCRO & Impinger 3 & $\mathrm{NH3}-4$ & $<25$ & $<0.03$ & $<0.03$ \\
\hline 33 & Blank & $0.1 \mathrm{NH} 2 \mathrm{SO} 4$ & & $<25$ & $<0.03$ & $<0.03$ \\
\hline 34 & Blank & $\mathrm{DI} \mathrm{H} 2 \mathrm{O}$ & & $<25$ & $<0.03$ & $<0.03$ \\
\hline
\end{tabular}


SO3 by Controlled Condensation - IC Analysis Results AES Greenidge Unit 4 - May 2, 2007

\begin{tabular}{|r|l|c|c|c|}
\hline Sample ID & Sample Description & $\mathrm{mg}^{\prime} \mathrm{L}_{\text {as } \mathrm{SO}_{4}{ }^{2-}}$ & $\mathrm{L}$ & $\mathrm{mg}$ as SO $_{4}{ }^{2-}$ \\
\hline 72427 & plug & 30.21 & 0.1 & 3.02 \\
\hline 72428 & probe & 22.91 & 0.1 & 2.29 \\
\hline 72429 & condenser & 90.56 & 0.1 & 9.06 \\
\hline 72432 & plug/probe & 8.14 & 0.1 & 0.81 \\
\hline 72433 & condenser & 2.41 & 0.1 & 0.24 \\
\hline 72436 & plug & 22.73 & 0.1 & 2.27 \\
\hline 72437 & probe & 42.94 & 0.1 & 4.29 \\
\hline 72438 & condenser & 86.93 & 0.1 & 8.69 \\
\hline 72441 & plug & 0.34 & 0.1 & 0.03 \\
\hline 72442 & probe & 1.65 & 0.1 & 0.17 \\
\hline 72443 & condenser & 3.77 & 0.1 & 0.38 \\
\hline 72446 & plug & 33.91 & 0.1 & 3.39 \\
\hline 72447 & probe & 20.59 & 0.1 & 2.06 \\
\hline 72448 & condenser & 99.18 & 0.1 & 9.92 \\
\hline 72451 & plug & 1.1 & 0.1 & 0.11 \\
\hline 72452 & probe & 1.56 & 0.1 & 0.16 \\
\hline 72453 & condenser & 3.1 & 0.1 & 0.31 \\
\hline 72456 & Condenser Blank & 0.34 & 0.1 & 0.03 \\
\hline 72457 & H2O2 blank & 0.17 & 0.1 & 0.02 \\
\hline 72458 & DI Blank & 0.11 & 0.1 & 0.01 \\
\hline
\end{tabular}


HCL

$s$

$S$

$v_{s}$

mw of $\mathrm{HCl} \mathrm{mw}$ of $\mathrm{Cl} \mathrm{HCl}$ mass $\mathrm{HCl}$ mass

Comments

$\mu \mathrm{g} / \mathrm{ml}$ of $\mathrm{Cl}-\mathrm{ml}$ of sample $\mu \mathrm{g} / \mu \mathrm{g}$-mole $\mu \mathrm{g} / \mu \mathrm{g}$-mole $\mu \mathrm{g}$

20071771 AHO ACID GAS AGl-1 PROBE \& LINE RINSE RUN 1

20071772 AHO ACID GAS AGI-1 IMPINGER SOLUTION

20071774 STACK ACID GAS AGO-1 IMPINGER SOLUTION

20071775 AHO ACID GAS AGI-2 PROBE RINSE

20071776 AHO ACID GASAGI-2 IMPINGER SOLUTION

20071777 STACK ACID GAS AGO-2 PROBE RINSE

20071778 STACK ACID GAS AGO-2 IMPINGER SOLUTION

20071779 AHO ACID GAS AGI-3 PROBE RINSE

20071780 |AHO ACID GAS AGI-3 IMPINGER SOLUTION

20071781 STACK ACID GAS AGO-3 PROBE RINSE

20071782 STACK ACID GAS AGO-3 IMPINGER SOLUTION

$200717830.1 \mathrm{~N}$ H2SO4 BLANK SOLUTION

\begin{tabular}{|c|c|c|c|c|c|c|l|}
\hline 49 & 137.40 & 270 & 36.46 & 35.45 & 38154.95 & 38.15 & Comtaminated with SO2 sample rinse, Used average of 2 replicates \\
\hline 50 & 310.64 & 500 & 36.46 & 35.45 & 159745.20 & 159.75 & Used average of 2 replicates \\
\hline 51 & 47.75 & 140 & 36.46 & 35.45 & 6875.46 & 6.88 & Used average of 2 replicates \\
\hline 52 & 168.41 & 510 & 36.46 & 35.45 & 88336.15 & 88.34 & Used average of 4 replicates \\
\hline 53 & 24.57 & 100 & 36.46 & 35.45 & 2527.00 & 2.53 & Used average of 2 replicates \\
\hline 54 & 59.66 & 490 & 36.46 & 35.45 & 30056.28 & 30.07 & Used average of 2 replicates \\
\hline 55 & 16.22 & 130 & 36.46 & 35.45 & 2168.68 & 2.17 & Used average of 3 replicates \\
\hline 56 & 2.71 & 570 & 36.46 & 35.45 & 1588.71 & 1.59 & Used average of 4 replicates \\
\hline 57 & 13.95 & 150 & 36.46 & 35.45 & 2152.12 & 2.15 & Used average of 4 replicates \\
\hline 58 & 61.79 & 500 & 36.46 & 35.45 & 31775.22 & 31.78 & Used average of 2 replicates \\
\hline 59 & 4.89 & 140 & 36.46 & 35.45 & 704.10 & 0.70 & Used average of 2 replicates \\
\hline 60 & 1.41 & 550 & 36.46 & 35.45 & 797.59 & 0.80 & Used average of 4 replicates \\
\hline 61 & $<0.20$ & 160 & 36.46 & 35.45 & $<32.9$ & $<0.03$ & Used average of 2 replicates \\
\hline
\end{tabular}




\begin{tabular}{|c|c|c|c|c|c|c|c|c|}
\hline & & & & & & & & \\
\hline 20071771|AHO ACID GAS AGI-1 PROBE \& LINE RINSE RUN 1 & $\frac{49}{50}$ & $<0.20$ & 270 & 20.01 & 18.99 & $<56.90$ & 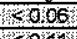 & comtaminated with $\mathrm{SO} 2$ sample rinse \\
\hline 20071772/AHO ACID GAS AGI-1 IMPINGER SOLUTION & 50 & $<0.20$ & 500 & 20.01 & 18.99 & $<105.37$ & 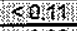 & \\
\hline 20071773 STACK ACID GAS AGO-1 PROBE RINSE & 51 & $<0.20$ & 140 & 20.01 & 18.99 & $<29.50$ & S:D:03: & \\
\hline 20071774 STACK ACID GAS AGO-1 IMPINGER SOLUTION & 52 & $\angle 0.20$ & 510 & 20.01 & 18.99 & $<107.48$ & 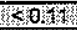 & \\
\hline 20071775 A.HO ACID GAS AGl-2 PROBE RINSE & 53 & 0.64 & 100 & 20.01 & 18.99 & 67.44 & 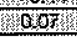 & Used average of 4 replicates \\
\hline 20071776 AHO ACID GAS AGI-2 IMPINGER SOLUTION & 54 & $<0.20$ & 490 & 20.01 & 18.99 & $<103.26$ & SOHO & \\
\hline 20071777 STACK ACID GAS AGO-2 PROBE RINSE & 55 & $<0.20$ & 130 & 20.01 & 18.99 & $<27.40$ & Ka.03: & \\
\hline 20071778 STACK ACID GAS AGO-2 IMPINGER SOLUTION & 56 & 0.23 & 570 & 20.01 & 18.99 & 138.14 & 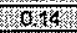 & Used average of 4 replicates \\
\hline 20071779 AHO ACID GAS AGI-3 PROBE RINSE & 57 & 0.35 & 150 & 20.01 & 18.99 & 55.32 & 40.05 & \\
\hline 20071780 AHO ACID GAS AGI-3 IMPINGER SOLUTION & 58 & $<0.20$ & 500 & 20.01 & 18.99 & $<105.37$ & 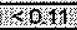 & \\
\hline 20071781 STACK ACID GAS AGO-3 PROBE RINSE & 59 & $<0.20$ & 140 & 20.01 & 18.99 & $<29.50$ & 50.03 & \\
\hline 20071782 STACK ACID GAS AGO-3 IMPINGER SOLUTION & 60 & $<0.20$ & 550 & 20.01 & 18.99 & $<115.91$ & 2042 & \\
\hline 2007178330.1 N H2SO4 BLANK SOLUTION & 61 & $<0.20$ & 160 & 20.01 & 18.99 & $<33.72$ & 2.0.03 & \\
\hline
\end{tabular}


$\mathrm{HCL}$

20072458 ACID GAS PROBE AHO TEST 1 20072459 ACID GAS IMPINGERS AHO TEST 1 20072460 ACID GAS PROBE STK TEST 1

20072461 ACID GAS IMPINGERS STK TEST 1 20072462 ACID GAS PROBE AHO TEST 2

20072463 ACID GAS IMPINGERS AHO TEST 2 20072464 ACID GAS PROBE STK TEST 2

20072465 ACID GAS IMPINGER STK TEST 2 20072466 ACID GAS 0.1N H2SO4 BLANK \begin{tabular}{|l|l|}
\hline 20072467 & ACID GAS DI H2O BLANK \\
\hline
\end{tabular}

\section{$1 \mathrm{~F}$}

20072458 ACID GAS PROBE AHO TEST 1 20072459 ACID GAS IMPINGERS AHO TEST 1 20072460 ACID GAS PROBE STK TEST 1 20072461 ACID GAS IMPINGERS STK TEST 1 20072462 ACID GAS PROBE AHO TEST 2 20072463 ACID GAS IMPINGERS AHO TEST 2 20072464 ACID GAS PROBE STK TEST 2 \begin{tabular}{l|l}
\hline 20072465 & ACID GAS IMPINGER STK TEST 2 \\
\hline
\end{tabular} 20072466 ACID GAS 0.1N H2SO4 BLANK \begin{tabular}{|l|l|}
\hline 20072467 & ACID GAS DI H2O BLANK \\
\hline
\end{tabular}
$S$ $V_{\mathrm{s}} \quad \mathrm{mw}$ of $\mathrm{HCl} \quad \mathrm{mw}$ of $\mathrm{Cl}^{-} \quad \mathrm{HCl}$ mass $\mathrm{HCl}$ mass $\mu \mathrm{g} / \mathrm{ml}$ of $\mathrm{Cl}-\mathrm{ml}$ of sample $\mu \mathrm{g} / \mu \mathrm{gg}$-mole $\mu \mathrm{g} / \mu \mathrm{g}$-mole

\begin{tabular}{|l|c|c|}
\hline 66 & 1.84 & 115 \\
\hline 67 & 84.48 & 535 \\
\hline 68 & 4.32 & 70 \\
\hline 69 & 2.64 & 545 \\
\hline 70 & 1.21 & 125 \\
\hline 71 & 113.9 & 470 \\
\hline 72 & 1.45 & 90 \\
\hline 73 & 2.45 & 490 \\
\hline 74 & $<0.20$ & 105 \\
\hline 75 & $<0.20$ & 280 \\
\hline
\end{tabular}

\begin{tabular}{|r|r}
\hline 36.46 \\
\hline 36.46 \\
\hline 36.46 \\
\hline 36.46 \\
\hline 36.46 \\
\hline 36.46 \\
\hline 36.46 \\
\hline 36.46 \\
\hline 36.46
\end{tabular}

\begin{tabular}{|c|c|c|}
\hline$\mu g-$ mole & \multicolumn{1}{c}{$\mu \mathrm{g}$} & $\mathrm{mg}$ \\
\hline 35.45 & 217.63 & 0.22 \\
\hline 35.45 & 46484.49 & 46.48 \\
\hline 35.45 & 311.02 & 0.31 \\
\hline 35.45 & 1479.79 & 1.48 \\
\hline 35.45 & 155.56 & 0.16 \\
\hline 35.45 & 55058.20 & 55.06 \\
\hline 35.45 & 134.22 & 0.13 \\
\hline 35.45 & 1234.70 & 1.23 \\
\hline 35.45 & $<21.60$ & $<0.02$ \\
\hline 35.45 & $<57.60$ & $<0.06$ \\
\hline
\end{tabular}

$S$

$\mathrm{V}_{\mathrm{s}}$

$\mathrm{mw}$ of $\mathrm{HF}$

mw of $\mathrm{F}^{-}$

HF mass HF mass

$\mu \mathrm{g} / \mathrm{ml}$ of $\mathrm{F}_{-}-\mathrm{ml}$ of sample $\mu \mathrm{g} / \mu \mathrm{g}$-mole $\mu \mathrm{g} / \mu \mathrm{g}$-mole

$\mu \mathrm{g}$ $\mathrm{mg}$

\begin{tabular}{|c|c|c|c|c|c|c|}
\hline & $\mu \mathrm{g} / \mathrm{ml}$ of $\mathrm{F}_{-}$ & ample & $\mu \mathrm{g} / \mu \mathrm{g}$-mole & $\mu \mathrm{g} / \mu \mathrm{g}-$ mole & $\mu \mathrm{g}$ & $\mathrm{mg}$ \\
\hline 66 & 0.40 & 115 & 20.01 & 18.99 & 48.47 & 0.05 \\
\hline 67 & $<0.20$ & 535 & 20.01 & 18.99 & $<112.7$ & C. 0.11 \\
\hline 68 & $<0.20$ & 70 & 20.01 & 18.99 & $<14.75$ & 601 \\
\hline 69 & $<0.20$ & 545 & 20.01 & 18.99 & $<114.9$ & 0.11 \\
\hline 70 & 0.43 & 125 & 20.01 & 18.99 & 56.63 & 0106 \\
\hline 71 & $<0.20$ & 470 & 20.01 & 18.99 & $<99.05$ & 80 \\
\hline 72 & $<0.20$ & 90 & 20.01 & 18.99 & $<19.00$ & 0.02 \\
\hline 73 & $<0.20$ & 490 & 20.01 & 18.99 & $<103.3$ & \% 10 \\
\hline 74 & $<0.20$ & 105 & 20.01 & 18.99 & $<22.13$ & (2002 \\
\hline 75 & $<0.20$ & 280 & 20.01 & 18.99 & $<59.01$ & \%006 \\
\hline
\end{tabular}




\section{F CONSOLENERGY}

\section{Greenidge \\ CONSOL Project: 1621-85 \\ Date: $\quad 4 / 13 / 2007$}

Results - Impingers, Rinses, Blanks

\begin{tabular}{|c|c|c|c|c|}
\hline Lab No. & 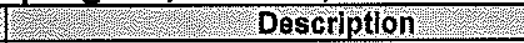 & Comments & Sample & $\mathrm{Hg}(\mu \mathrm{g} / \mathrm{L})$ \\
\hline 20071684 & PROBE \& FILTER RINSE & $\mathrm{AHO}-1-\mathrm{HG}-1-\mathrm{RO} / \mathrm{DO}$ & 2 & 1.15 \\
\hline 20071685 & SAMPLE LINE RINSE & $\mathrm{AHO}-1-\mathrm{HG}-1-\mathrm{RO} / \mathrm{DO}$ & 3 & 2.21 \\
\hline \multirow[t]{2}{*}{20071686} & KCL IMPINGER & AHO-1-HG-1-RO/DO & 4 & 5.59 \\
\hline & & & $4 a$ & 0.38 \\
\hline 20071687 & HNO3/H2O2 IMPINGER & AHO-1-HG-1-RO/DO & 5 & $<1.40$ \\
\hline 20071688 & KMNO4 IMPINGER & $\mathrm{AHO}-1-\mathrm{HG}-1-\mathrm{RO} / \mathrm{DO}$ & 6 & 1.99 \\
\hline 20071689 & KMNO4 ACID RINSE & AHO-1-HG-1-RO/DO & 7 & $<1.40$ \\
\hline \multirow[t]{2}{*}{20071690} & PROBE \& FILTER RINSE/LINE & STK-1-HG-1-BS/KC & 10 & $<1.40$ \\
\hline & & & 9 & $<1.40$ \\
\hline 20071691 & KCL IMPINGER & STK-1-HG-1-BS/KC & 11 & $<0.28$ \\
\hline 20071692 & $\mathrm{HNO} 3 / \mathrm{H} 2 \mathrm{O} 2$ IMPINGER & STK-1-HG-1-BS/KC & 12 & $<1.40$ \\
\hline 20071693 & KMNO4 IMPINGER & STK-1-HG-1-BS/KC & 13 & $<0.28$ \\
\hline 20071694 & KMNO4 ACID RINSE & STK-1-HG-1-BS/KC & 14 & $<1.40$ \\
\hline 20071695 & PROBE \& FILTER RINSE & AHO-1-HG-2-RO/DO & 16 & 1.59 \\
\hline 20071696 & SAMPLE LINE RINSE & AHO-1-HG-2-RO/DO & 17 & 6.78 \\
\hline 20071697 & KCL IMPINGER & $\mathrm{AHO}-1-\mathrm{HG}-2-\mathrm{RO} / \mathrm{DO}$ & 18 & 10.65 \\
\hline 20071698 & HNO3/H2O2 IMPINGER & $\mathrm{AHO}-1-\mathrm{HG}-2-\mathrm{RO} / \mathrm{DO}$ & 19 & $<1.40$ \\
\hline 20071699 & KMNO4 IMPINGER & AHO-1-HG-2-RO/DO & 20 & $<0.28$ \\
\hline 20071700 & KMNO4 ACID RINSE & AHO-1-HG-2-RO/DO & 21 & $<1.40$ \\
\hline \multirow[t]{2}{*}{20071701} & PROBE \& FILTER RINSE/LINE RINSE & STK-1-HG-2-BS/KC & 24 & $<1.40$ \\
\hline & & & 23 & $<1.40$ \\
\hline 20071702 & KCL IMPINGER & STK-1-HG-2-BS/KC & 25 & $<0.28$ \\
\hline 20071703 & HNO3/H2O2 IMPINGER & STK-1-HG-2-BS/KC & 26 & $<1.40$ \\
\hline 20071704 & KMNO4 IMPINGER & STK-1-HG-2-BS/KC & 27 & $<0.28$ \\
\hline 20071705 & KMNO4 ACID RINSE & STK-1-HG-2-BS/KC & 28 & $<1.40$ \\
\hline 20071706 & PROBE \& FILTER RINSE & AHO-1-HG-3-RO/DO & 30 & $<1.40$ \\
\hline 20071707 & SAMPLE LINE RINSE & AHO-1-HG-3-RO/DO & 31 & 3.66 \\
\hline 20071708 & KCL IMPINGER & $\mathrm{AHO}-1-\mathrm{HG}-3-\mathrm{RO} / \mathrm{DO}$ & 32 & 7.85 \\
\hline 20071709 & HNO3/H2O2 IMPINGER & AHO-1-HG-3-RO/DO & 33 & $<1.40$ \\
\hline 20071710 & KMNO4 IMPINGER & $\mathrm{AHO}-1-\mathrm{HG}-3-\mathrm{RO} / \mathrm{DO}$ & 34 & 1.16 \\
\hline 20071711 & KMNO4 ACID RINSE & AHO-1-HG-3-RO/DO & 35 & $<1.40$ \\
\hline \multirow[t]{2}{*}{20071712} & PROBE \& FILTER RINSE/LINE RINSE & STK-1-HG-3-BS/KC & 38 & $<1.40$ \\
\hline & & & 37 & $<1.40$ \\
\hline 20071713 & KCL IMPINGER & STK-1-HG-3-BS/KC & 39 & $<0.28$ \\
\hline 20071714 & HNO3/H2O2 IMPINGER & STK-1-HG-3-BS/KC & 40 & $<1.40$ \\
\hline 20071715 & KMNO4 IMPINGER & STK-1-HG-3-BS/KC & 41 & $<0.28$ \\
\hline 20071716 & KMNO4 ACID RINSE & STK-1-HG-3-BS/KC & 42 & $<1.40$ \\
\hline 20071717 & KMNO 4 BLANK & & 43 & $<0.28$ \\
\hline 20071718 & KCL BLANK & & 44 & $<0.28$ \\
\hline 20071719 & $\mathrm{HNO} 3 / \mathrm{H} 2 \mathrm{O} 2 \mathrm{BLANK}$ & & 45 & $<140$ \\
\hline 20071720 & HNO3/HCL BLANK & & 46 & $<1.40$ \\
\hline 20071721 & PROBE \& FILTER RINSE & $\mathrm{AHO}-1-\mathrm{HG}-4-\mathrm{RO} / \mathrm{DO}$ & 90 & $<1.40$ \\
\hline 20071722 & SAMPLE LINE RINSE & $\mathrm{AHO}-1-\mathrm{HG}-4-\mathrm{RO} / \mathrm{DO}$ & 91 & 3.12 \\
\hline 20071723 & KCL IMPINGER & AHO-1-HG-4-RO/DO & 92 & 8.86 \\
\hline 20071724 & HNO3/H2O2 IMPINGER & AHO-1-HG-4-RO/DO & 93 & $<1.40$ \\
\hline 20071725 & KMNO4 IMPINGER & AHO-1-HG-4-RO/DO & 94 & $<0.28$ \\
\hline 20071726 & KMNO4 ACID RINSE & $\mathrm{AHO}-1-\mathrm{HG}-4-\mathrm{RO} / \mathrm{DO}$ & 95 & $<1,40$ \\
\hline
\end{tabular}




\begin{tabular}{|c|c|c|c|c|}
\hline 20071727 & |PROBE \& FILTER RINSE/LINE & STK-1-HG-5-BS/KC & 98 & $<1.40$ \\
\hline & & & 97 & $<1.40$ \\
\hline 20071728 & KCL IMPINGER & STK-1-HG-5-BS/KC & 99 & $<0.28$ \\
\hline 20071729 & HNO3/H2O2 IMPINGER & STK-1-HG-5-BS/KC & 100 & $<1.40$ \\
\hline 20071730 & KMNO4 IMPINGER & STK-1-HG-5-BS/KC & 101 & $<0.28$ \\
\hline 20071731 & KMNO4 ACID RINSE & STK-1-HG-5-BS/KC & 102 & $<1.40$ \\
\hline 20071732 & PROBE \& FILTER RINSE & AHO-1-HG-5-RO/DO & 104 & $<1.40$ \\
\hline 20071733 & SAMPLE LINE RINSE & AHO-1-HG-5-RO/DO & 105 & $<1.40$ \\
\hline 20071734 & KCL IMPINGER & AHO-1-HG-5-RO/DO & 106 & 8.45 \\
\hline 20071735 & HNO3/H2O2 IMPINGER & AHO-1-HG-5-RO/DO & 107 & $<1.40$ \\
\hline 20071736 & KMNO4 IMPINGER & AHO-1-HG-5-RO/DO & 108 & 0.40 \\
\hline 20071737 & KMNO4 ACID RINSE & AHO-1-HG-5-RO/DO & 109 & $<1.40$ \\
\hline \multirow[t]{2}{*}{20071738} & PROBE \& FILTER RINSE/LINE & STK-1-HG-5-BS/KC & 112 & $<1.40$ \\
\hline & & & 111 & $<1.40$ \\
\hline 20071739 & KCL IMPINGER & STK-1-HG-5-BS/KC & 113 & $<0.28$ \\
\hline 20071740 & HNO3/H2O2 IMPINGER & STK-1-HG-5-BS/KC & 114 & $<1.40$ \\
\hline 20071741 & KMNO4 IMPINGER & STK-1-HG-5-BS/KC & 115 & 0.37 \\
\hline 20071742 & KMNO4 ACID RINSE & STK-1-HG-5-BS/KC & 116 & $<1.40$ \\
\hline 20071743 & PROBE \& FILTER RINSE & AHO-1-HG-6-RO/DO & 118 & $<1.40$ \\
\hline 20071744 & SAMPLE LINE RINSE & AHO-1-HG-6-RO/DO & 119 & $<1.40$ \\
\hline 20071745 & KCL IMPINGER & AHO-1-HG-6-RO/DO & 120 & 8.04 \\
\hline 20071746 & HNO3/H2O2 IMPINGER & AHO-1-HG-6-RO/DO & 121 & $<1.40$ \\
\hline 20071747 & KMNO4 IMPINGER & AHO-1-HG-6-RO/DO & 122 & 1.55 \\
\hline 20071748 & KMNO4 ACID RINSE & AHO-1-HG-6-RO/DO & 123 & $<1.40$ \\
\hline \multirow[t]{2}{*}{20071749} & PROBE \& FILTER RINSE & STK-1-HG-6 & 126 & $<1.40$ \\
\hline & & & 125 & $<1,40$ \\
\hline 20071750 & KCL IMPINGER & STK-1-HG-6 & 127 & $<0.28$ \\
\hline 20071751 & HNO3/H2O2 IMPINGER & STK-1-HG-6 & 128 & $<1.40$ \\
\hline 20071752 & KMNO4 IMPINGER & STK-1-HG-6 & 129 & $<0.28$ \\
\hline 20071753 & KMNO4 ACID RINSE & STK-1-HG-6 & 130 & $<1.40$ \\
\hline 20071754 & KMNO4 BLANK & & 131 & $<0.28$ \\
\hline 20071755 & KCL BLANK & & 132 & $<0.28$ \\
\hline 20071756 & HNO3/H2O2 BLANK & & 133 & $<1.40$ \\
\hline 20071757 & KMNO4 BLANKIMPINGER & & 134 & $<0.28$ \\
\hline 20071758 & HNO3/HCLBLANK & & 135 & $<1.40$ \\
\hline
\end{tabular}

Results - Filters/Blank Thimble

\begin{tabular}{|c|c|c|c|}
\hline ANALNUM & DESCR & SAMPLE & $\mathrm{Hg}$, ug/filite \\
\hline 20071671 & FILTER/SOLIDS & 8 & $<0.007$ \\
\hline 20071673 & FILTER/SOLIDS & 22 & $<0.007$ \\
\hline 20071675 & FILTER/SOLIDS & 36 & $<0.007$ \\
\hline 20071677 & FILTER/SOLIDS & 96 & $<0.007$ \\
\hline 20071679 & FILTER/SOLIDS & 110 & $<0.007$ \\
\hline 20071681 & FILTER/SOLIDS & 124 & $<0.007$ \\
\hline 20071682 & 47 MM FILTER BLANK & 47 & $<0.007$ \\
\hline 20071683 & THIMBLE BLANK & 48 & $<0.007$ \\
\hline
\end{tabular}

Results - Loose Particulate in the Thimble

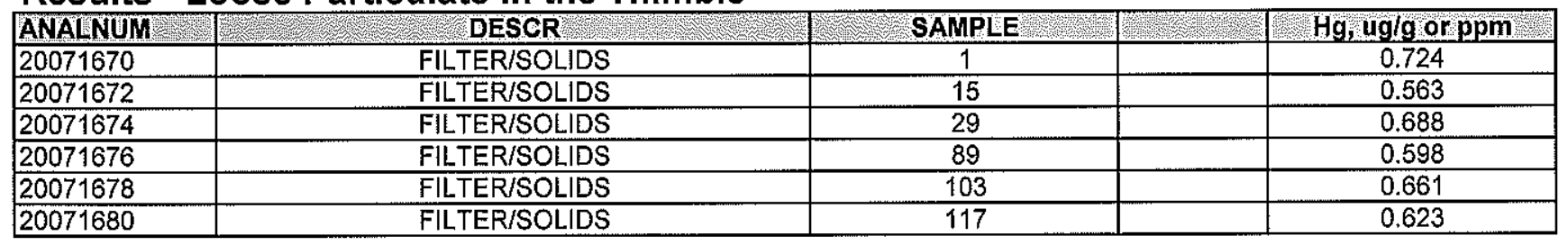




\section{YONSOL ENERGY}

\section{QAQC}

Mercury Duplicate Analyses RPD (Limit of 10\%).... Impingers, Rinses, Blanks

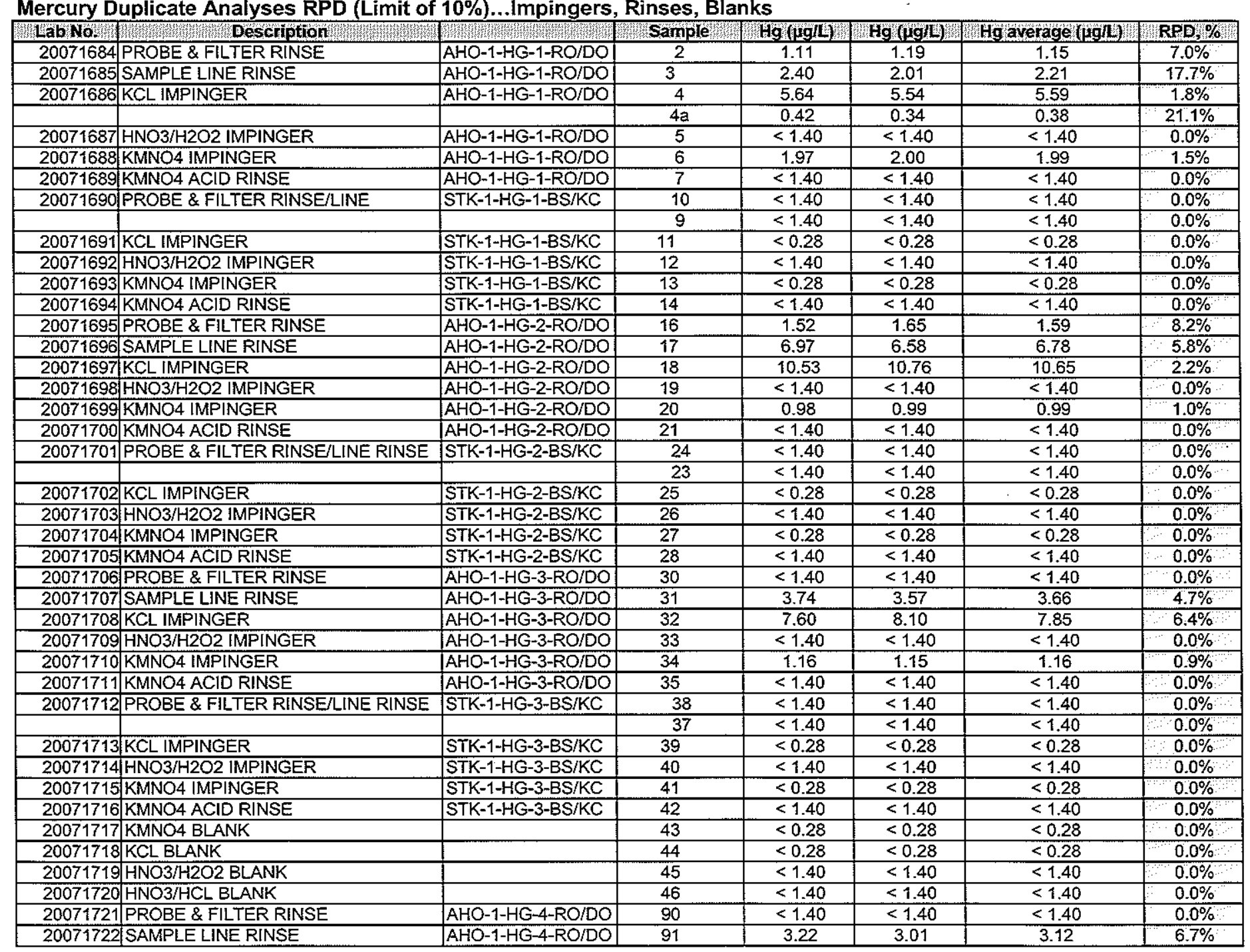




\begin{tabular}{|c|c|c|c|c|c|c|c|}
\hline 20071723 & KCL IMPINGER & $\mathrm{AHO}-1-\mathrm{HG}-4-\mathrm{RO} / \mathrm{DO}$ & 92 & 8.72 & 8.99 & 8.86 & $3.0 \%$ \\
\hline 20071724 & $\mathrm{HNO} / \mathrm{H} 2 \mathrm{O} 2$ IMPINGER & $\mathrm{AHO}-1-\mathrm{HG}-4-\mathrm{RO} / \mathrm{DO}$ & 93 & $<1.40$ & $<1.40$ & $<1.40$ & $0.0 \%$ \\
\hline 20071725 & KMNO4 IMPINGER & AHO-1-HG-4-RO/DO & 94 & $<0.28$ & $<0.28$ & $<0.28$ & $0.0 \%$ \\
\hline 20071726 & KMNO4 ACID RINSE & AHO-1-HG-4-RO/DO & 95 & $<1.40$ & $<1.40$ & $<1.40$ & $0.0 \%$ \\
\hline \multirow[t]{2}{*}{20071727} & PROBE \& FILTER RINSE/LINE & STK-1-HG-5-BS/KC & 98 & $<1.40$ & $<1.40$ & $<1.40$ & $0.0 \%$ \\
\hline & & & 97 & $<1.40$ & $<1.40$ & $<1.40$ & $0.0 \%$ \\
\hline 20071728 & KCL IMPINGER & STK-1-HG-5-BS/KC & 99 & $<0.28$ & $<0.28$ & $<0.28$ & $0.0 \%$ \\
\hline 20071729 & HNO3/H2O2 IMPINGER & STK-1-HG-5-BS/KC & 100 & $<1.40$ & $<1.40$ & $<1.40$ & $0.0 \%$ \\
\hline 20071730 & KMNO4 IMPINGER & STK-1-HG-5-BS/KC & 101 & $<0.28$ & $<0.28$ & $<0.28$ & $0.0 \%$ \\
\hline 20071731 & KMNO4 ACID RINSE & STK-1-HG-5-BS/KC & 102 & $<1.40$ & $<1.40$ & $<1.40$ & $0.0 \%$ \\
\hline 20071732 & PROBE \& FILTER RINSE & AHO-1-HG-5-RO/DO & 104 & $<1.40$ & $<1.40$ & $<1.40$ & $0.0 \%$ \\
\hline 20071733 & SAMPLE LINE RINSE & $\mathrm{AHO}-1-\mathrm{HG}-5-\mathrm{RO} / \mathrm{DO}$ & 105 & $<1.40$ & $<1.40$ & $<1.40$ & $0.0 \%$ \\
\hline 20071734 & KCL IMPINGER & AHO-1-HG-5-RO/DO & 106 & 8.32 & 8.57 & 8.45 & $3.0 \%$ \\
\hline 20071735 & HNO3/H2O2 IMPINGER & AHO-1-HG-5-RO/DO & 107 & $<1.40$ & $<1.40$ & $<1.40$ & $0.0 \%$ \\
\hline 20071736 & KMNO4 IMPINGER & AHO-1-HG-5-RO/DO & 108 & 0.37 & 0.42 & 0.40 & $12.7 \%$ \\
\hline 20071737 & KMNO4 ACID RINSE & AHO-1-HG-5-RO/DO & 109 & $<1.40$ & $<1.40$ & $<1.40$ & $0.0 \%$ \\
\hline \multirow[t]{2}{*}{20071738} & PROBE \& FILTER RINSE/LINE & STK-1-HG-5-BS/KC & 112 & $<1.40$ & $<1.40$ & $<1.40$ & $0.0 \%$ \\
\hline & & & 111 & $<1.40$ & $<1.40$ & $<1.40$ & $0.0 \%$ \\
\hline 20071739 & KCL IMPINGER & ISTK-1-HG-5-BS/KC & 113 & $<0.28$ & $<0.28$ & $<0.28$ & $0.0 \%$ \\
\hline 20071740 & HNO3/H2O2 IMPINGER & ISTK-1-HG-5-BS/KC & 114 & $<1.40$ & $<1.40$ & $<1.40$ & $0.0 \%$ \\
\hline 20071741 & KMNO4 IMPINGER & |STK-1-HG-5-BS/KC & 115 & 0.42 & 0.31 & 0.37 & $30.1 \%$ \\
\hline 20071742 & KMNO4 ACID RINSE & ISTK-1-HG-5-BS/KC & 116 & $<1.40$ & $<1.40$ & $<1.40$ & $0.0 \%$ \\
\hline 20071743 & PROBE \& FILTER RINSE & $\mathrm{AHO}-1-\mathrm{HG}-6-\mathrm{RO} / \mathrm{DO}$ & 118 & $<1.40$ & $<1.40$ & $<1.40$ & $0.0 \%$ \\
\hline 20071744 & SAMPLE LINE RINSE & $\mathrm{AHO}-1-\mathrm{HG}-6-\mathrm{RO} / \mathrm{DO}$ & 119 & $<1.40$ & $<1.40$ & $<1,40$ & $0.0 \%$ \\
\hline 20071745 & KCL IMPINGER & $\mathrm{AHO}-1-\mathrm{HG}-6-\mathrm{RO} / \mathrm{DO}$ & 120 & 7.96 & 8.12 & 8.04 & $2.0 \%$ \\
\hline 20071746 & $\mathrm{HNO} 3 / \mathrm{H} 2 \mathrm{O} 2$ IMPINGER & $\mathrm{AHO}-1-\mathrm{HG}-6-\mathrm{RO} / \mathrm{DO}$ & 121 & $<1.40$ & $<1.40$ & $<1.40$ & $0.0 \%$ \\
\hline 20071747 & KMNO4 IMPINGER & AHO-1-HG-6-RO/DO & 122 & 1.54 & 1.55 & 1.55 & $0.6 \%$ \\
\hline 20071748 & KMNO4 ACID RINSE & AHO-1-HG-6-RO/DO & 123 & $<1.40$ & $<1.40$ & $<1.40$ & $0.0 \%$ \\
\hline \multirow[t]{2}{*}{20071749} & PROBE \& FILTER RINSE & STK-1-HG-6 & 126 & $<1.40$ & $<1.40$ & $<1.40$ & $0.0 \%$ \\
\hline & & & 125 & $<1.40$ & $<1.40$ & $<1.40$ & $0.0 \%$ \\
\hline 20071750 & KCL IMPINGER & STK-1-HG-6 & 127 & $<0.28$ & $<0.28$ & $<0.28$ & $0.0 \%$ \\
\hline 20071751 & IHNO3/H2O2 IMPINGER & STK-1-HG-6 & 128 & $<1.40$ & $<1.40$ & $<1.40$ & $0.0 \%$ \\
\hline 20071752 & KMNO4 IMPINGER & STK-1-HG-6 & 129 & $<0.28$ & $<0.28$ & $<0.28$ & $0.0 \%$ \\
\hline 20071753 & KMNO4 ACID RINSE & STK-1-HG-6 & 130 & $<1.40$ & $<1.40$ & $<1.40$ & $0.0 \%$ \\
\hline 20071754 & KMNO4 BLANK & & 131 & $<0.28$ & $<0.28$ & $<0.28$ & $0.0 \%$ \\
\hline 20071755 & KCL BLANK & & 132 & $<0.28$ & $<0.28$ & $<0.28$ & $0.0 \%$ \\
\hline 20071756 & $\mathrm{HNO} 3 / \mathrm{H} 2 \mathrm{O} 2 \mathrm{BLANK}$ & & 133 & $<1.40$ & $<1.40$ & $<1.40$ & $0.0 \%$ \\
\hline 20071757 & KMNO4 BLANK IMPINGER & & 134 & $<0.28$ & $<0.28$ & $<0.28$ & $0.0 \%$ \\
\hline 20071758 & HNO3/HCL BLANK & & 135 & $<1.40$ & $<1.40$ & $<1.40$ & $0.0 \%$ \\
\hline
\end{tabular}


Mercury Duplicate Analyses RPD (Limit of 10\%)..Filters/Blank Thimble

\begin{tabular}{|c|c|c|c|c|c|c|c|}
\hline WabNo. & U Description & 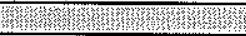 & Sample & Wg, ugifilter: & Hg ughtilter & Hg average, ug/filted & RPQ $\%$ \\
\hline 20071671 & FILTER/SOLIDS & & 8 & $<0.007$ & $<0.007$ & $<0.007$ & $0.0 \%$ \\
\hline 20071673 & FILTER/SOLIDS & & 22 & $<0.007$ & $<0.007$ & $<0.007$ & $0.0 \%$ \\
\hline 20071675 & FILTER/SOLIDS & & 36 & $<0.007$ & $<0.007$ & $<0.007$ & $0.0 \%$ \\
\hline 20071677 & FILTER/SOLIDS & & 96 & $<0.007$ & $<0.007$ & $<0.007$ & $0.0 \%$ \\
\hline 20071679 & FILTER/SOLIDS & & 110 & $<0.007$ & $<0.007$ & $<0.007$ & $0.0 \%$ \\
\hline 20071681 & FILTER/SOLIDS & & 124 & $<0.007$ & $<0.007$ & $<0.007$ & $0.0 \%$ \\
\hline 20071682 & 47 MM FILTER BLANK & & 47 & $<0.007$ & $<0.007$ & $<0.007$ & $0.0 \%$ \\
\hline 20071683 & THIMBLE BLANK & & 48 & $<0.007$ & $<0.007$ & $<0.007$ & $0.0 \%$ \\
\hline
\end{tabular}

\section{Mercury Duplicate Analyses RPD (Limit of 10\%)..Loose Particulate in Thimbles}

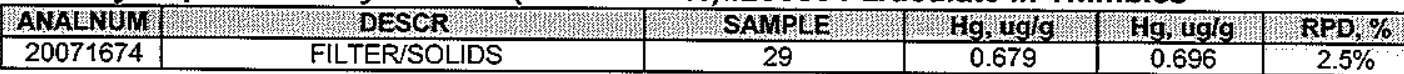

Mercury Triplicate Analyses RSD (Limit of 10\%)...Impingers, Rinses, Blanks

\begin{tabular}{|c|c|c|c|c|c|c|c|}
\hline Lab No. & 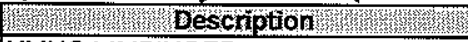 & 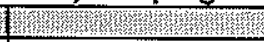 & Sample & 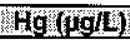 & Hg (ugn) & Hg $(\mu g)$ & RST: $\%$ \\
\hline 20071752 & KMNO4 IMPINGER & STK-1-HG-6 & 129 & $<0.28$ & $<0.28$ & $<0.28$ & $0.0 \%$ \\
\hline 20071697 & KCL IMPINGER & AHO-1-HG-2-RO/DO & 18 & 10.53 & 10.76 & 10.71 & $1.1 \%$ \\
\hline 20071705 & KMNO4 ACID RINSE & STK-1-HG-2-BS/KC & 28 & $<1.40$ & $<1.40$ & $<1.40$ & $0.0 \%$ \\
\hline 20071748 & KMNO4 ACID RINSE & AHO-1-HG-6-RO/DO & 123 & $<1.40$ & $<1.40$ & $<1.40$ & $0.0 \%$ \\
\hline 20071690 & PROBE \& FILTER RINSE/LINE & STK-1-HG-1-BS/KC & 10 & $<1.40$ & $<1.40$ & $<1.40$ & $0.0 \%$ \\
\hline & & & 37 & $<1.40$ & $<1.40$ & $<1.40$ & $0.0 \%$ \\
\hline 20071727 & PROBE \& FILTER RINSE/LINE & STK-1-HG-5-BS/KC & 98 & $<1.40$ & $<1.40$ & $<1.40$ & $0.0 \%$ \\
\hline 20071704 & KMNO4 IMPINGER & STK-1-HG-2-BS/KC & 27 & $<0.28$ & $<0.28$ & $<0.28$ & $0.0 \%$ \\
\hline 20071730 & KMNO4 IMPINGER & STK-1-HG-5-BS/KC & 101 & $<0.28$ & $<0.28$ & $<0.28$ & $0.0 \%$ \\
\hline 20071723 & KCL IMPINGER & AHO-1-HG-4-RO/DO & 92 & 8.72 & 8.99 & 9.02 & $1.9 \%$ \\
\hline 20071703 & HNO3/H2O2 IMPINGER & STK-1-HG-2-BS/KC & 26 & $<1.40$ & $<1.40$ & $<1.40$ & $0.0 \%$ \\
\hline 20071751 & HNO3/H2O2 IMPINGER & STK-1-HG-6 & 128 & $<1.40$ & $<1.40$ & $<1.40$ & $0.0 \%$ \\
\hline
\end{tabular}


Matrix (Standard Addition) Spikes (recovery of $90-110 \%$ )

The following samples were spiked with a standard solution of $2 p p$.

\begin{tabular}{|c|c|c|c|c|}
\hline LabNo & Description & 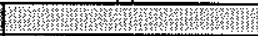 & Sample & \%Recovery \\
\hline 20071747 & KMNO4 IMPINGER & AHO-1-HG-6-RO/DO & 122 & 105.5 \\
\hline 20071700 & KMNO4 ACID RINSE & AHO-1-HG-2-RO/DO & 21 & 96.5 \\
\hline 20071753 & KMNO4 ACID RINSE & STK-1-HG-6 & 130 & 95.5 \\
\hline & & & 9 & 100 \\
\hline 20071712 & PROBE \& FILTER RINSE/LINE RINSE & STK-1-HG-3-BS/KC & 38 & 90.5 \\
\hline 20071702 & KCL IMPINGER & STK-1-HG-2-BS/KC & 25 & 96 \\
\hline & & & 97 & 93.5 \\
\hline 20071749 & PROBE \& FILTER RINSE & STK-1-HG-6 & 126 & 98 \\
\hline 20071713 & KCL IMPINGER & STK-1-HG-3-BS/KC & 39 & 95.5 \\
\hline 20071698 & $\mathrm{HNO} 3 / \mathrm{H} 2 \mathrm{O} 2$ IMPINGER & $\mathrm{AHO}-1-\mathrm{HG}-2-\mathrm{RO} / \mathrm{DO}$ & 19 & 101 \\
\hline 20071746 & HNO3/H2O2 IMPINGER & AHO-1-HG-6-RO/DO & 121 & 106 \\
\hline 20071699 & KMNO4 IMPINGER & AHO-1-HG-2-RO/DO & 20 & 103.5 \\
\hline 20071736 & KMNO4 IMPINGER & AHO-1-HG-5-RO/DO & 108 & 101 \\
\hline 20071673 & FILTER/SOLIDS & & 22 & 103.5 \\
\hline
\end{tabular}

NIST SRM 1633B Fly Ash...Digested/Analyzed with Filters and Analyzed with Loose Particulate ( $90-110 \%$ of Certified Value)

NIST SRM has a cerrtified value of $141 \mathrm{ng} / \mathrm{g}$.

Lab No.

\begin{tabular}{|l|c|c|c|c|}
\hline LRM & NA & $1633 \mathrm{~B}$ & & $97.9 \%$ \\
\hline SRM & NA & $1633 \mathrm{~B}$ & & $94.4 \%$ \\
analyzed with digestates of filters/blank thimble
\end{tabular}

Digestion Duplicates and Digestion Spikes...Impingers

Digestion 1 and 2 results represent an average of duplicate analyses.

\begin{tabular}{|c|c|c|c|c|c|}
\hline W & Sample & Comments & Digestion 1 & Digestion 2 & RPDE\% \\
\hline 20071691 KCL IMPINGER & STK-1-HG-1-BS/KC & 11 & $<0.28$ & $<0.28$ & 0.0 \\
\hline 20071715 KMNO4 IMPINGER & STK-1-HG-3-BS/KC & 41 & $<0.28$ & $<0.28$ & 0.0 \\
\hline 20071740| $\mathrm{HNO} / \mathrm{H} 2 \mathrm{O} 2$ IMPINGER & STK-1-HG-5-BS/KC & 114 & $<1.40$ & $<1.40$ & 0.0 \\
\hline
\end{tabular}

The following samples were spiked with a standard solution of 2 ppb before digestion.

\begin{tabular}{|c|c|c|c|c|}
\hline LabNo: & Description & 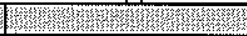 & Sample & W Recoven \\
\hline 2007169 & KCL IMPINGER & STK-1-HG-1-BS/KC & 11 & 104.5 \\
\hline 2007171 & KMNO4 IMPINGER & STK-1-HG-3-BS/KC & 41 & 102.5 \\
\hline 2007174 & HNO3/H2O2 IMPINGER & STK-1-HG-5-BS/KC & 114 & 106.5 \\
\hline
\end{tabular}




\section{F CONSOLENERGY}
Research and Development
www.consolenergy.com
4000 Brownsville $\mathrm{Rd}$.
South Park, PA 15129

Sample Description.: COAL MARCH 28 09:00-10:00

Sample No.: TEST 1

Date Received: 04/03/2007

Analytical No.:

20071795

Project No.: $1621 \quad-085 \quad-000$

Date Completed: 04/19/2007

Submitted By: D. CONNELL

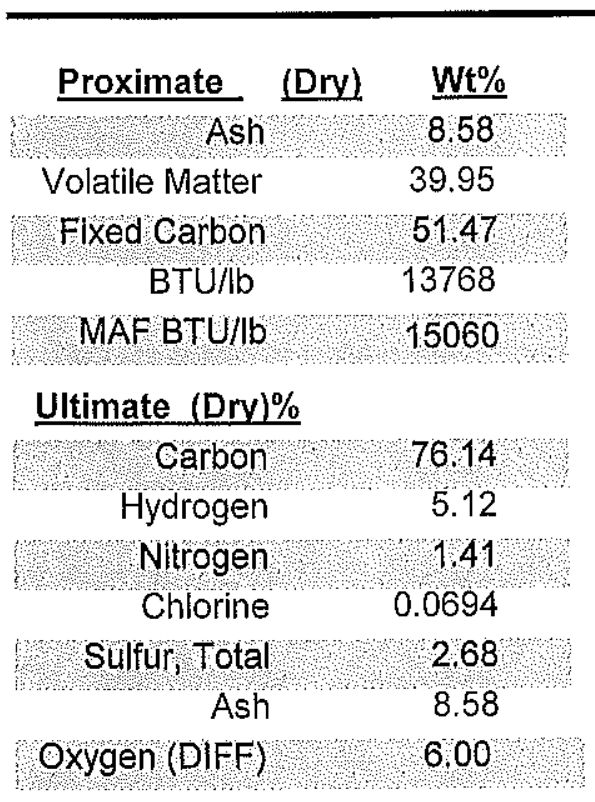

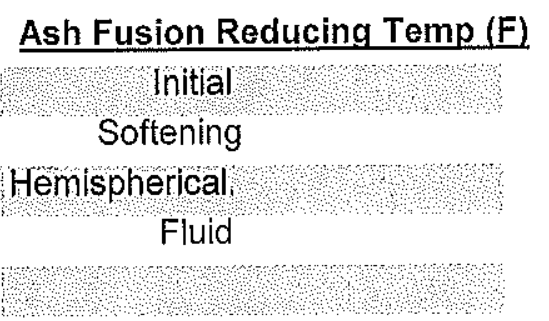

\section{Ash Fusion Oxidizing}

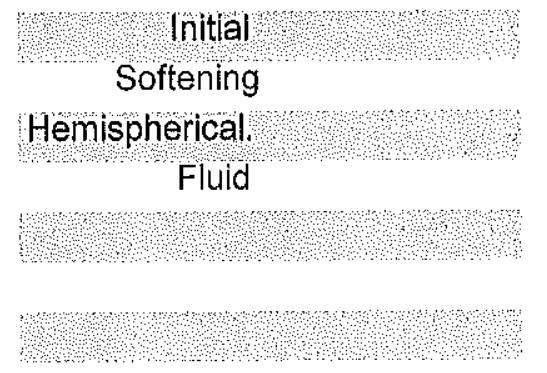

Major Ash Elem. (Ignited)

\begin{tabular}{|c|c|}
\hline $\mathrm{SiO} 2$ & 44.98 \\
\hline $\mathrm{Al} 2 \mathrm{O} 3$ & 20.75 \\
\hline $\mathrm{TiO}_{2}$ & 0.92 \\
\hline $\mathrm{Fe} 2 \mathrm{O} 3$ & 20.51 \\
\hline $\mathrm{CaO}$ & 4.98 \\
\hline $\mathrm{MgO}$ & 0.85 \\
\hline $\mathrm{Na} 2 \mathrm{O}$ & 0.80 \\
\hline $\mathrm{K} 2 \mathrm{O}$ & 1.46 \\
\hline$P 205$ & 0.36 \\
\hline $\mathrm{SO} 3$ & 4.81 \\
\hline Undëtermined & 0.42 \\
\hline Total Moisture & 6.62 \\
\hline \multicolumn{2}{|c|}{ Sulfur Forms (Dry) } \\
\hline \multicolumn{2}{|l|}{ Pyritic Sulfur } \\
\hline Organic & \\
\hline Sulfur, Total & 2.68 \\
\hline
\end{tabular}

As Determined Moisture $1.305 \%$

These values have been reviewed and are approved for transmission
HGI/FSI

$\mathrm{HGI}$

FSI

\section{Analysis Value}

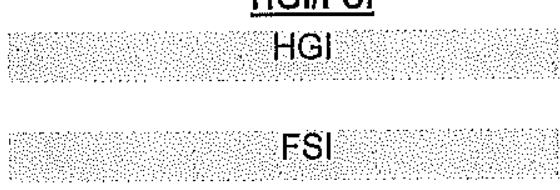

Trace Elements (ppm (Drv)

\begin{tabular}{|c|c|}
\hline $\mathrm{Hg}$ & 0.101 \\
\hline$F$ & 66.77 \\
\hline As & 6.17 \\
\hline $\mathrm{Ba}$ & 66.7 \\
\hline $\mathrm{Be}$ & .801 \\
\hline $\mathrm{Cd}$ & .06 \\
\hline co & 2.93 \\
\hline $\mathrm{Cr}$ & 12.90 \\
\hline $\mathrm{Cu}$ & 7.06 \\
\hline $\mathrm{Li}$ & 9.01 \\
\hline $\mathrm{Mn}$ & 18.00 \\
\hline Mo & .87 \\
\hline $\mathrm{Ni}$ & 8.60 \\
\hline $\mathrm{Pb}$ & 3.60 \\
\hline $\mathrm{Sb}$ & .48 \\
\hline Se & 1.26 \\
\hline $\mathrm{Sn}$ & .51 \\
\hline Th & 1.64 \\
\hline $\mathrm{TI}$ & .28 \\
\hline U & .57 \\
\hline $\mathrm{V}$ & 21.10 \\
\hline $\mathrm{Zn}$ & 10.00 \\
\hline
\end{tabular}

Seive Analysis SIZE 


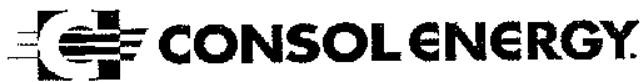

Research and Development

www.consolenergy.com

4000 Brownsville Rd.

South Park, PA 15129

Sample Description.: COAL MARCH 28 13:00-14:00

Sample No.: TEST 2

Date Received: 04/03/2007

Date Completed: 04/19/2007

Analytical No.:

Project No.: $1621-085-000$

Submitted By: D. CONNELL

\begin{tabular}{|c|c|}
\hline Proximate (Dry) & $\underline{W t} \%$ \\
\hline Ash & 8.36 \\
\hline Volatile Matter & 39.95 \\
\hline Fixed Carbon & 51.69 \\
\hline $\mathrm{BTU} / \mathrm{Ib}$ & 13799 \\
\hline MAF BTU/Ib & 15058 \\
\hline \multicolumn{2}{|l|}{ Ultimate (Dry)\% } \\
\hline Carbon & 75.66 \\
\hline Hydrogen & 5.04 \\
\hline Nitrogen & 1.45 \\
\hline Chlorine & 0.0753 \\
\hline Sulfur, Total & 2.69 \\
\hline Ash & 8.36 \\
\hline Oxygen (DIFF) & 6.72 \\
\hline
\end{tabular}

Major Ash Elem. (Ignited)

\begin{tabular}{|c|c|}
\hline $\mathrm{SiO} 2$ & 42.69 \\
\hline $\mathrm{Al} 2 \mathrm{O} 3$ & 20.16 \\
\hline $\mathrm{T}_{\mathrm{O}} \mathrm{O}$ & 0.88 \\
\hline $\mathrm{Fe} 2 \mathrm{O} 3$ & 22.46 \\
\hline GaO & 5,29 \\
\hline $\mathrm{MgO}$ & 0.85 \\
\hline $\mathrm{Na} 2 \mathrm{O}$ & 0.76 \\
\hline $\mathrm{K} 2 \mathrm{O}$ & 1.39 \\
\hline $\mathrm{P} 205$ & 0.38 \\
\hline $\mathrm{SO} 3$ & 4.97 \\
\hline Undetermined & 0.17 \\
\hline Total Moisture & 6.28 \\
\hline \multicolumn{2}{|c|}{ Sulfur Forms (Dry) } \\
\hline \multicolumn{2}{|l|}{ Pyritic Sulfur } \\
\hline \multicolumn{2}{|l|}{ Sulfate } \\
\hline Organic & \\
\hline Sulfur, Total & 2.69 \\
\hline
\end{tabular}

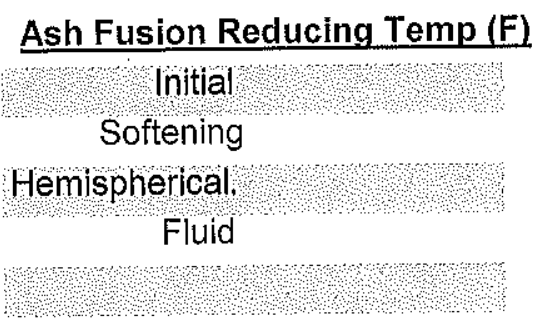

\section{Ash Fusion Oxidizing}

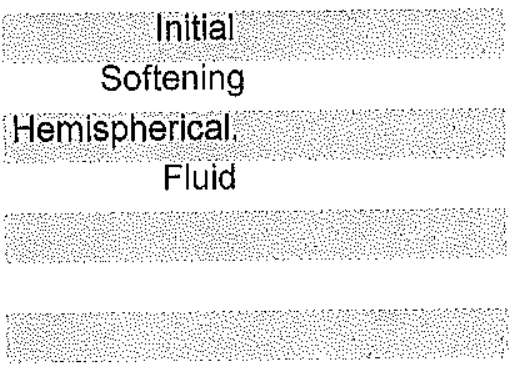

Misc.

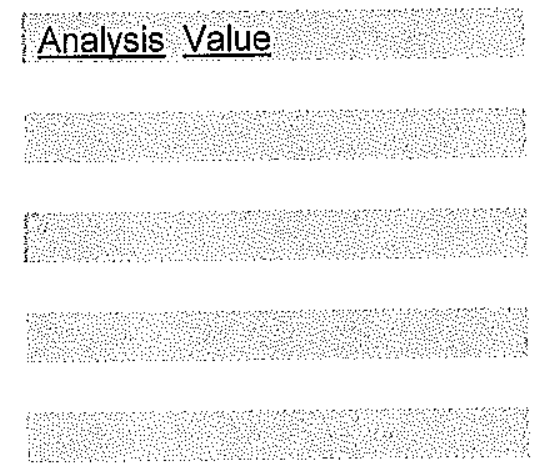

Trace Elements (ppm (Dry)

\begin{tabular}{|c|c|}
\hline $\mathrm{Hg}$ & 0.113 \\
\hline$F$ & 67.07 \\
\hline As & 7.48 \\
\hline $\mathrm{Ba}$ & 64.70 \\
\hline $\mathrm{Be}$ & .77 \\
\hline $\mathrm{Cd}$ & .06 \\
\hline Co & 2.97 \\
\hline $\mathrm{Cr}$ & 13.20 \\
\hline $\mathrm{Cu}$ & 5.91 \\
\hline $\mathrm{Li}^{\circ}$ & 8.37 \\
\hline $\mathrm{Mn}$ & 17.60 \\
\hline Mo & .96 \\
\hline $\mathrm{Ni}$ & 9.99 \\
\hline $\mathrm{Pb}$ & 3.60 \\
\hline $\mathrm{Sb}$ & .35 \\
\hline $\mathrm{Se}$ & 1.15 \\
\hline Sn & .49 \\
\hline Th & 1.54 \\
\hline $\mathrm{TI}$ & .28 \\
\hline U & .53 \\
\hline V & 44.90 \\
\hline $\mathrm{Zn}$ & 9.50 \\
\hline
\end{tabular}

Seive Analysis SIZE
HGI/FSI

$\mathrm{HGl}$

FSI

As Determined Moisture $1.29 \%$
These values have been reviewed and are approved for transmission. An? 


\section{WONSOLENERGY.}

Research and Development
www.consolenergy.com
4000 Brownsville Rd.
South Park, PA 15129

Sample Description.: COAL MARCH 28 16:15

Sample No.: TEST 3

Date Received: 04/03/2007

Analytical No.:

20071797

Project No.: $1621-085-000$

Date Completed: 04/19/2007

Submitted By: D. CONNELL

\begin{tabular}{|c|c|}
\hline Proximate (Dry) & $\underline{W t} \%$ \\
\hline Ash & 799 \\
\hline Volatile Matter & 39.92 \\
\hline Fixed Carbon & 52.09 \\
\hline $\mathrm{BTU} / \mathrm{lb}$ & 13916 \\
\hline MAF BTU/D & 15124 \\
\hline \multicolumn{2}{|l|}{ Ultimate (Dry)\% } \\
\hline Carbon & 75.86 \\
\hline Hydrogen & 5.01 \\
\hline Nitrogen & 1.44 \\
\hline Chlorine & 0.0745 \\
\hline Sulfur, Total & 2.57 \\
\hline Ash & 7.99 \\
\hline Oxygen (DIFF) & 706 \\
\hline
\end{tabular}
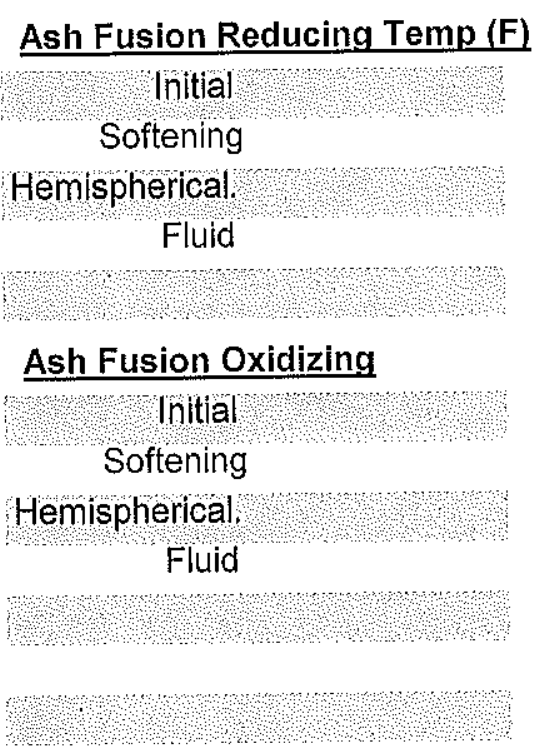

Major Ash Elem. (Ignited)

\begin{tabular}{|c|c|}
\hline $\mathrm{SiO} 2$ & 44.62 \\
\hline $\mathrm{Al} 2 \mathrm{O} 3$ & 22.46 \\
\hline $\mathrm{TIO} 2$ & 0.99 \\
\hline $\mathrm{Fe} 2 \mathrm{O} 3$ & 20.52 \\
\hline $\mathrm{CaO}$ & 4.59 \\
\hline $\mathrm{MgO}$ & 0.92 \\
\hline $\mathrm{Na} 20$ & 0.81 \\
\hline $\mathrm{K} 2 \mathrm{O}$ & 1.57 \\
\hline $\mathrm{P} 205$ & 0.48 \\
\hline $\mathrm{SO} 3$ & 3.78 \\
\hline Undetermined & -0.74 \\
\hline Total Moisture & 6.54 \\
\hline \multicolumn{2}{|c|}{ Sulfur Forms (Dry) } \\
\hline \multicolumn{2}{|l|}{ Pyritic Sulfur } \\
\hline Sulfate & \\
\hline Y Organic & \\
\hline Sulfur, Total & 2.57 \\
\hline
\end{tabular}

Misc.

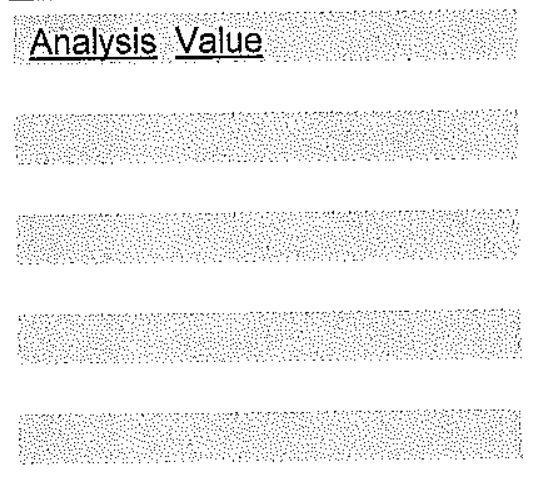

HGI/FSI

$\mathrm{HGI}$

FSI
Trace Elements (ppm (Dry)

\begin{tabular}{|c|c|}
\hline $\mathrm{Hg}$ & 0.097 \\
\hline $\mathrm{F}$ & 70.61 \\
\hline As & 6.22 \\
\hline $\mathrm{Ba}$ & 70.00 \\
\hline $\mathrm{Be}$ & 81 \\
\hline $\mathrm{Cd}$ & .06 \\
\hline Co & 287 \\
\hline $\mathrm{Cr}$ & 13.20 \\
\hline $\mathrm{Cu}$ & 5.73 \\
\hline $\mathrm{Li}$ & 8.67 \\
\hline $\mathrm{Mn}$ & 16,40 \\
\hline Mo & .96 \\
\hline $\mathrm{Ni}$ & 8.42 \\
\hline $\mathrm{Pb}$ & 3.70 \\
\hline $\mathrm{Sb}$ & .38 \\
\hline Se & 1,25 \\
\hline Sn & .53 \\
\hline Th & 1.59 \\
\hline $\mathrm{TI}$ & .28 \\
\hline $\mathrm{U}$ & 60 \\
\hline $\mathrm{V}$ & 21.30 \\
\hline $\mathrm{Zn}$ & 8.86 \\
\hline
\end{tabular}

\section{Seive Analysis} SIZE
WT \%

As Determined Moisture $1.43 \%$
These values have been reviewed and are approved for transmission? 


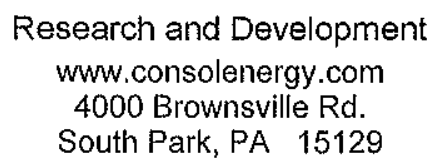

Sample Description.: COAL MARCH 28 18:25

Sample No.: TEST 3

Date Received: 04/03/2007

Date Completed: 04/19/2007

Analytical No.:

Project No.: $1621 \quad-085 \quad-000$

Submitted By: D. CONNELL

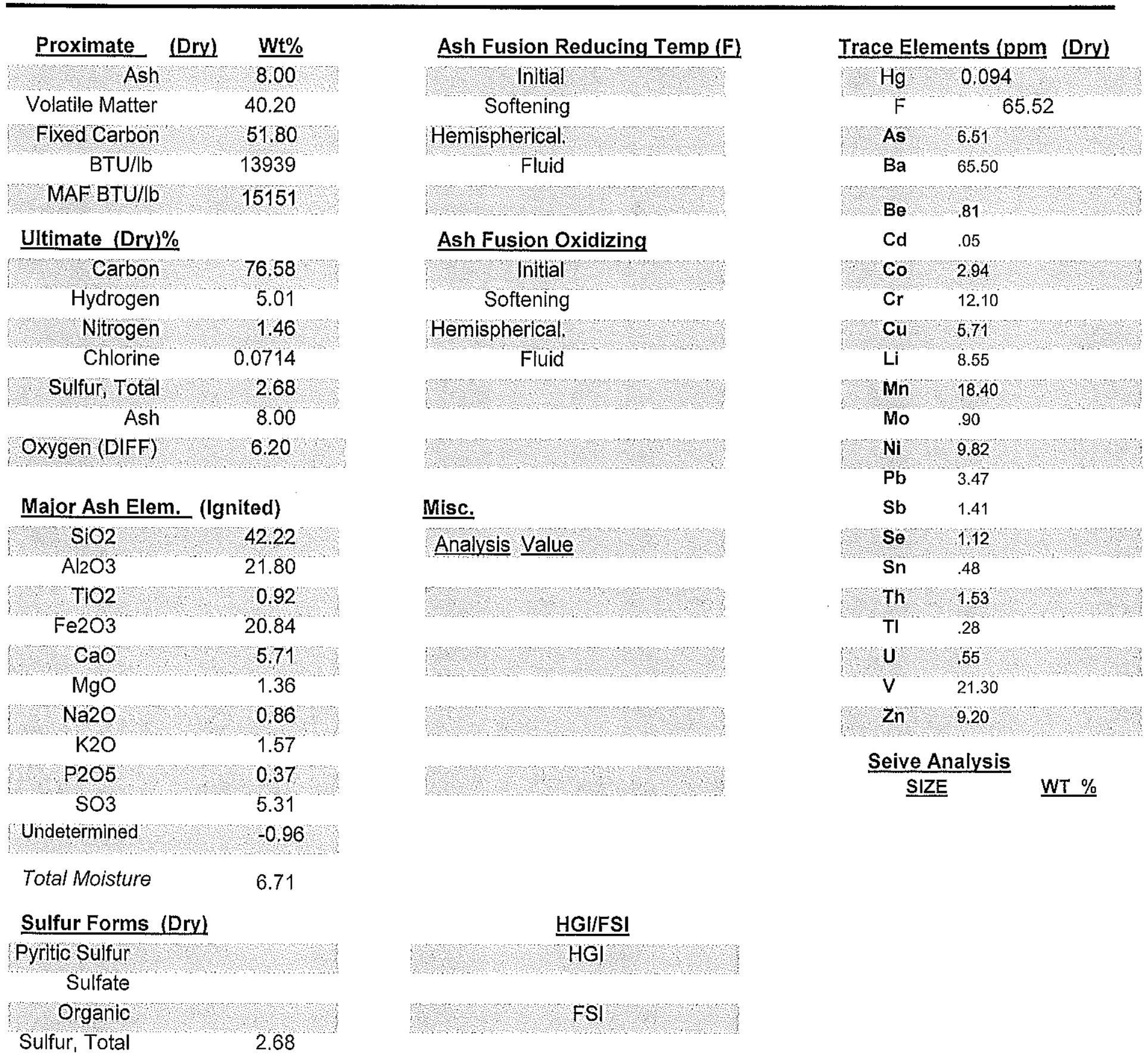

As Determined Moisture $1.41 \quad \%$

These values have been reviewed and are approved for transmission. $M 1 \mathcal{X}$ 
Research and Development

www.consolenergy.com

4000 Brownsville Rd.

South Park, PA 15129

Sample Description.: COAL MARCH 29 9:30

Sample No:: TEST 1

Date Received: 04/03/2007

Date Completed: 04/19/2007

Analytical No.: $\quad 20071814$

Project No.: $1621-085 \quad-000$

Submitted By: D. CONNELL

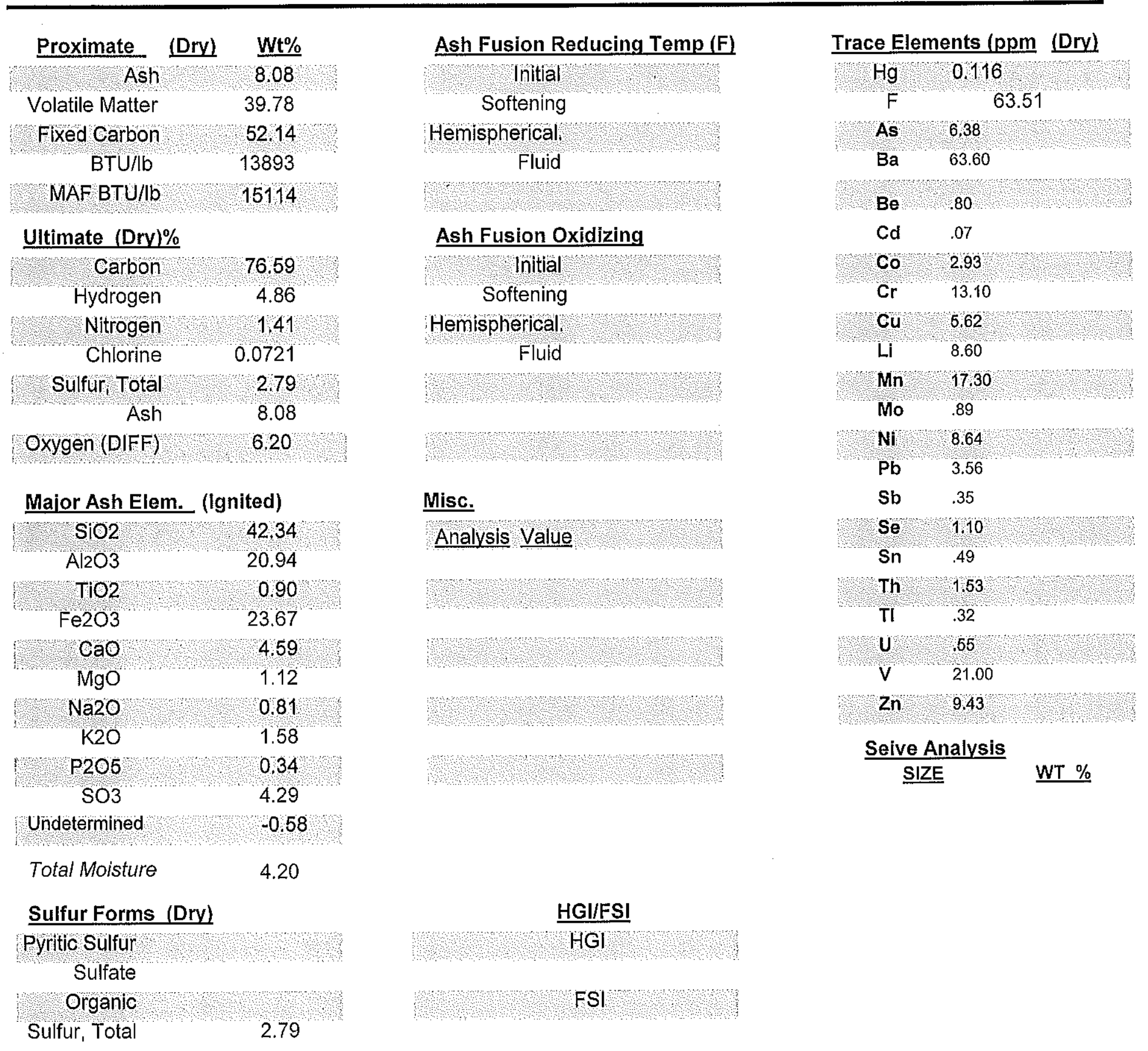

As Determined Moisture $1.75 \quad \%$

These values have been reviewed and are approved for transmission. ThA 


\title{
Y CONSOLENERGY.
}

\author{
Research and Development \\ www.consolenergy.com \\ 4000 Brownsville Rd. \\ South Park, PA 15129
}

Sample Description.: COAL MARCH 29 13:30

Sample No.: TEST 2

Analytical No.:

20071815

Date Received: 04/03/2007

Project No: $1621-085-000$

Date Completed: 04/19/2007

Submitted By: D. CONNELL

\begin{tabular}{|c|c|}
\hline Proximate (Dry) & $\mathbf{W t} \%$ \\
\hline Ash & 8.54 \\
\hline Volatile Matter & 40.36 \\
\hline Flxed Carbon & 51,10 \\
\hline BTU/lb & 13838 \\
\hline MAF BTU/lb & 15130 \\
\hline \multicolumn{2}{|l|}{ Ultimate (Dry)\% } \\
\hline Carbon & 76.62 \\
\hline Hydrogen & 4.93 \\
\hline Nitrogen & 1.50 \\
\hline Chlorine & 0.0727 \\
\hline Sülfur, Total & 2.50 \\
\hline Ash & 8.54 \\
\hline Oxygen (DIFF) & 5.84 \\
\hline
\end{tabular}

Ash Fusion Reducing Temp (F)

Initial

Softening

Hemispherical.

Fluid

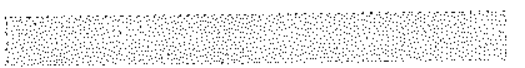

Ash Fusion Oxidizing

Initial

Softening

Hemispherical.

Fluid

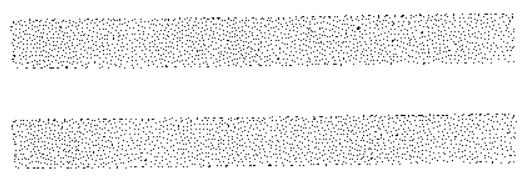

Misc.

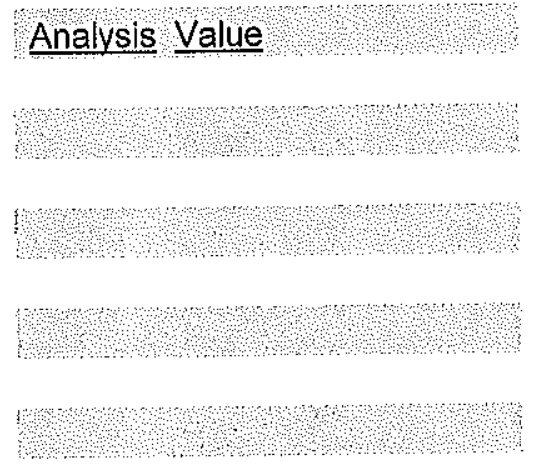

Trace Elements (ppm (Dry)

\begin{tabular}{|c|c|}
\hline $\mathrm{Hg}$ & 0.097 \\
\hline$F$ & 63.83 \\
\hline As & 5.78 \\
\hline $\mathrm{Ba}$ & 65.00 \\
\hline $\mathrm{Be}$ & 77 \\
\hline$C d$ & .06 \\
\hline Co & 2.80 \\
\hline $\mathrm{Cr}$ & 13.00 \\
\hline $\mathrm{Cu}$ & 5.69 \\
\hline $\mathrm{Li}$ & 8,50 \\
\hline $\mathrm{Mn}$ & 17.90 \\
\hline Mo & .91 \\
\hline $\mathrm{Ni}$ & 8.57 \\
\hline $\mathrm{Pb}$ & 3.49 \\
\hline $\mathrm{Sb}$ & .33 \\
\hline $\mathrm{Se}$ & 1.04 \\
\hline Sn & .48 \\
\hline Th & 1.53 \\
\hline $\mathrm{Tl}$ & .29 \\
\hline 0 & .52 \\
\hline V & 19.40 \\
\hline $\mathrm{Zn}$ & 9,47 \\
\hline
\end{tabular}

Seive Analysis SIZE
HGIFSI

$\mathrm{HGl}$

FSI

As Determined Moisture $1.61 \quad \%$

These values have been reviewed and are approved for transmission. 11 ? 
Research and Development

www.consolenergy.com

4000 Brownsville Rd.

South Park, PA 15129

Sample Description.: COAL MARCH 29 16:30

Sample No.: TEST 3

Analytical No.:

20071816

Date Received: 04/03/2007

Project No.: $1621 \quad-085 \quad-000$

Date Completed: 04/19/2007

Submitted By: D. CONNELL

\begin{tabular}{|c|c|}
\hline Proximate (Dry) & $\underline{w t} \%$ \\
\hline Ash & 7.99 \\
\hline Volatile Matter & 39.66 \\
\hline Fixed Carbon & 52.35 \\
\hline $\mathrm{BTU} / \mathrm{lb}$ & 13982 \\
\hline MAF BTU//b & 15196 \\
\hline \multicolumn{2}{|l|}{ Ultimate (Dry)\% } \\
\hline Carbon & 76.23 \\
\hline Hydrogen & 4.97 \\
\hline Nitrogen & 1.46 \\
\hline Chlorine & 0.0759 \\
\hline Sulfur, Total & 2.66 \\
\hline Ash & 7.99 \\
\hline Oxygen (DIFF) & 6.61 \\
\hline
\end{tabular}

Ash Fusion Reducing Temp (F)

Initial

Softening

Hemispherical.

Fluid

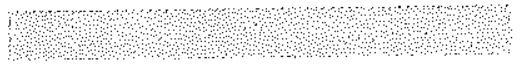

\section{Ash Fusion Oxidizing}

Softening

Hemispherical.

Fluid

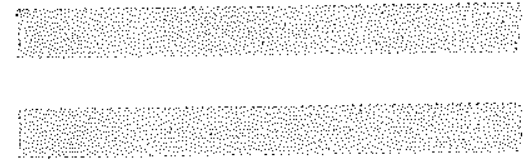

Misc.

\begin{tabular}{|c|c|}
\hline \multicolumn{2}{|c|}{ Major Ash Elem. (Ignited) } \\
\hline $\mathrm{SiO} 2$ & 43.52 \\
\hline $\mathrm{Al} 2 \mathrm{O} 3$ & 21.48 \\
\hline $\mathrm{THO} 2$ & 0.91 \\
\hline $\mathrm{Fe} 2 \mathrm{O} 3$ & 23.20 \\
\hline $\mathrm{CaO}$ & 4.28 \\
\hline $\mathrm{MgO}$ & 0.86 \\
\hline $\mathrm{Na} 2 \mathrm{O}$ & 0.83 \\
\hline $\mathrm{K} 2 \mathrm{O}$ & 1.61 \\
\hline$P 205$ & 0.40 \\
\hline $\mathrm{SO} 3$ & 3.83 \\
\hline Undetermined & -0.92 \\
\hline Total Moisture & 4.61 \\
\hline
\end{tabular}

Sulfur Forms (Dry)

Pyritic Sulfur

Sulfate

Organic

Sulfur, Total

2.66
HGI/FSI

$\mathrm{HGI}$

FSI

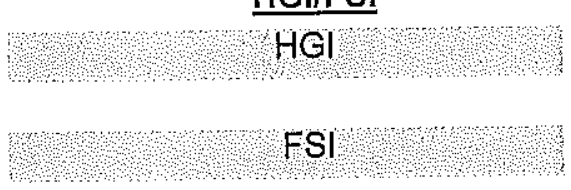

Trace Elements (ppm (Dry)

\begin{tabular}{|c|c|}
\hline $\mathrm{Hg}$ & $\begin{array}{r}0.108 \\
66.23\end{array}$ \\
\hline As & 7.23 \\
\hline $\mathrm{Ba}$ & 65.50 \\
\hline $\mathrm{Be}$ & 81 \\
\hline $\mathrm{Cd}$ & .05 \\
\hline Co & 2.98 \\
\hline $\mathrm{Cr}$ & 13.50 \\
\hline cu & 6.76 \\
\hline $\mathrm{Ll}$ & 8.86 \\
\hline $\mathrm{Mn}$ & 1770 \\
\hline Mo & .89 \\
\hline $\mathrm{NI}$ & 10.50 \\
\hline $\mathrm{Pb}$ & 3.55 \\
\hline $\mathrm{Sb}$ & .70 \\
\hline $\mathrm{Se}$ & 1,12 \\
\hline Sn & .50 \\
\hline Th & 1.56 \\
\hline $\mathrm{TI}$ & .33 \\
\hline $\mathrm{O}$ & 54 \\
\hline $\mathrm{V}$ & 21.50 \\
\hline $\mathrm{Zn}$ & 9.55 \\
\hline
\end{tabular}

Seive Analysis SIZE

As Determined Moisture $1.7 \quad \%$

These values have been reviewed and are approved for transmission: 
Research and Development

www.consolenergy.com

4000 Brownsville Rd.

South Park, PA 15129

Sample Description: COAL MARCH 30 9:05-13:35

Sample No: TEST 1

Analytical No.:

20071829

Date Received: 04/03/2007

Project No.: $1621 \quad-085-000$

Date Completed: 04/19/2007

Submitted By: D. CONNELL

\begin{tabular}{|c|c|}
\hline Proximate (Dry) & $\mathrm{Wt} \%$ \\
\hline Ash & 792 \\
\hline Volatile Matter & 40.30 \\
\hline Fixed Carbon & 5178 \\
\hline $\mathrm{BTU} / \mathrm{lb}$ & 14015 \\
\hline MAF BTU/L & 15220 \\
\hline \multicolumn{2}{|l|}{ Ultimate (Dry) $\%$} \\
\hline Carbon & 76.02 \\
\hline Hydrogen & 4.98 \\
\hline Nitrogen & 1.48 \\
\hline Chlorine & 0.0755 \\
\hline Sulfur; Total & 2.61 \\
\hline Ash & 7.92 \\
\hline Oxygen (DIFF) & 6.91 \\
\hline
\end{tabular}

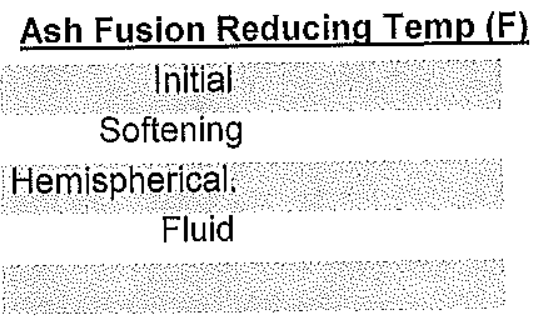

\section{Ash Fusion Oxidizing}

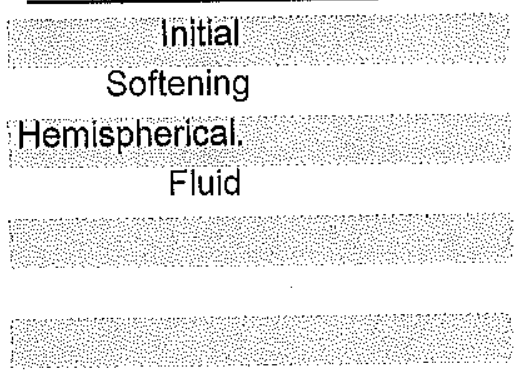

Misc.

Major Ash Elem. (lgnited)

\begin{tabular}{|c|c|}
\hline $\mathrm{SiO} 2$ & 4181 \\
\hline $\mathrm{Al} 2 \mathrm{O} 3$ & 20.75 \\
\hline $\mathrm{TiO}_{2}$ & 0.87 \\
\hline $\mathrm{Fe} 2 \mathrm{O} 3$ & 21.95 \\
\hline $\mathrm{CaO}$ & 6.42 \\
\hline $\mathrm{MgO}$ & 0.95 \\
\hline $\mathrm{Na} 2 \mathrm{O}$ & 0.87 \\
\hline $\mathrm{K} 2 \mathrm{O}$ & 1.53 \\
\hline $\mathrm{P} 205$ & 0.37 \\
\hline $\mathrm{SO} 3$ & 5.14 \\
\hline Undetermined & -0.66 \\
\hline Total Moisture & 6.66 \\
\hline \multicolumn{2}{|c|}{ Sulfur Forms (Dry) } \\
\hline \multicolumn{2}{|l|}{ Pyritic Sulfur } \\
\hline \multicolumn{2}{|l|}{ Sulfate } \\
\hline \multicolumn{2}{|l|}{ OY Organic } \\
\hline Sulfur, Total & 2.61 \\
\hline
\end{tabular}

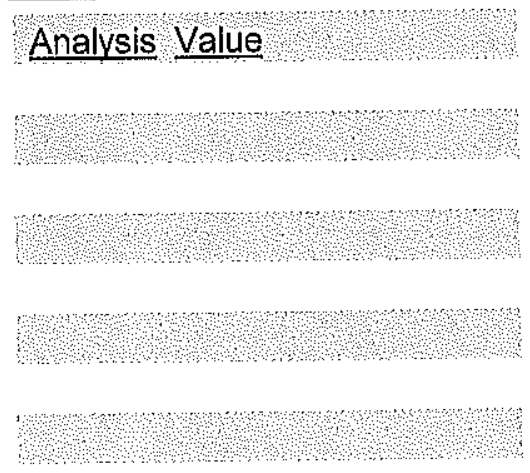

$\underline{\text { HGI/FSI }}$

$\mathrm{HGl}$

FSI
Trace Elements (ppm (Drv)

\begin{tabular}{cc}
$\mathrm{Hg}$ & 0.097 \\
$\mathrm{~F}$ & \multicolumn{1}{c}{59.27} \\
$\mathrm{As}$ & 4.97 \\
$\mathrm{Ba}$ & 58.10 \\
$\mathrm{Be}$ & .69 \\
$\mathrm{Cd}$ & .04 \\
$\mathrm{Co}$ & 2.43 \\
$\mathrm{Cr}$ & 10.60 \\
$\mathrm{Cu}$ & 5.41 \\
$\mathrm{Li}$ & 7.76 \\
$\mathrm{Mn}$ & 16.00 \\
$\mathrm{Mo}$ & .73 \\
$\mathrm{Nl}$ & 6.89 \\
$\mathrm{~Pb}$ & 2.91 \\
$\mathrm{Sb}$ & .27 \\
$\mathrm{Se}$ & 1.01 \\
$\mathrm{Sn}$ & .42 \\
$\mathrm{Th}$ \\
$\mathrm{Tl}$
\end{tabular}

Seive Analysis SIZE

As Determined Moisture $1.3 \quad \%$

These values have been reviewed and are approved for transmission $/ 4$ T 
Research and Development www.consolenergy.com 4000 Brownsville Rd. South Park, PA 15129

Sample Description.: COAL MARCH 30 12:45-14:00

Sample No.: TEST 2

Analytical No.:

20071830

Date Received: 04/03/2007

Project No.: $1621-085-000$

Date Completed: 04/19/2007

Submitted By: D. CONNELL

\begin{tabular}{|c|c|}
\hline Proximate (Dry) & $\mathrm{Wt} \%$ \\
\hline Ash & 8.18 \\
\hline Volatile Matter & 40.32 \\
\hline Flxed Carbon & 51.50 \\
\hline BTU/lb & 13956 \\
\hline MAF BTU/Ib & 15199 \\
\hline \multicolumn{2}{|l|}{ Ultimate (Dry)\% } \\
\hline Carbon & 76.41 \\
\hline Hydrogen & 4.83 \\
\hline Nitrogen & 1.40 \\
\hline Chlorine & 0.0748 \\
\hline Sulfur, Total & 2.65 \\
\hline Ash & 8.18 \\
\hline Oxygen (DIFF) & 6.46 \\
\hline
\end{tabular}

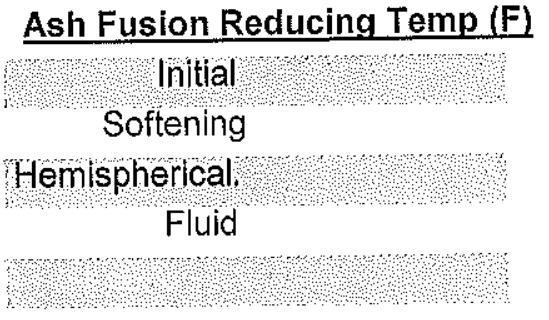

\section{Ash Fusion Oxidizing}

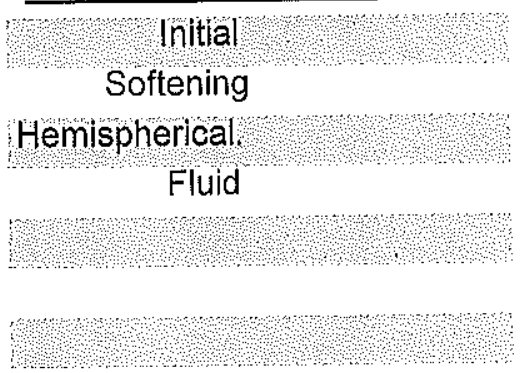

Misc.

Analysis Value

\begin{tabular}{|c|c|}
\hline $\mathrm{SiO} 2$ & 42.67 \\
\hline $\mathrm{Al} 2 \mathrm{O} 3$ & 20.97 \\
\hline $\mathrm{TIO}_{2}$ & 0.90 \\
\hline $\mathrm{Fe} 2 \mathrm{O} 3$ & 21.39 \\
\hline $\mathrm{CaO}$ & 5.59 \\
\hline $\mathrm{MgO}$ & 0.98 \\
\hline $\mathrm{Na} 2 \mathrm{O}$ & 0.84 \\
\hline $\mathrm{K} 2 \mathrm{O}$ & 1.46 \\
\hline$P 205$ & 0,38 \\
\hline $\mathrm{SO} 3$ & 4.92 \\
\hline Undetermined & -0.10 \\
\hline Total Moisture & 4.97 \\
\hline
\end{tabular}

Sulfur Forms (Dry)

Pyritic Sulfur

Sulfate

Organic

Sulfur, Total

2.65
HGI/FSI

HGI

FSI
Trace Elements (ppm (Dry)

\begin{tabular}{|c|c|}
\hline $\mathrm{Hg}$ & 0.090 \\
\hline$F$ & 69.47 \\
\hline As & 3.76 \\
\hline $\mathrm{Ba}$ & 40.10 \\
\hline $\mathrm{Be}$ & 51 \\
\hline $\mathrm{Cd}$ & .03 \\
\hline Co & 1.65 \\
\hline $\mathrm{Cr}$ & 7.31 \\
\hline $\mathrm{Cu}$ & 3.39 \\
\hline $\mathrm{Li}$ & 5.56 \\
\hline $\mathrm{Mn}$ & 12.10 \\
\hline Mo & .50 \\
\hline $\mathrm{Ni}$ & 4.90 \\
\hline $\mathrm{Pb}$ & 2.15 \\
\hline Sb & .20 \\
\hline $\mathrm{Se}$ & 1.02 \\
\hline Sn & .29 \\
\hline Th & .922 \\
\hline $\mathrm{Tl}$ & .17 \\
\hline U. & .31 \\
\hline $\mathrm{V}$ & 11.90 \\
\hline $\mathrm{Zn}$ & 5.23 \\
\hline
\end{tabular}

Seive Analysis SIZE

As Determined Moisture $1.69 \%$

These values have been reviewed and are approved for transmission. WT 
Research and Development www.consolenergy.com 4000 Brownsville Rd. South Park, PA 15129

Sample Description.: COAL MARCH 30 15:45-16:45

Sample No.: TEST 3

Analytical No.:

20071831

Date Received: 04/03/2007

Project No.: $1621-085 \quad-000$

Date Completed: 04/19/2007

Submitted By: D. CONNELL

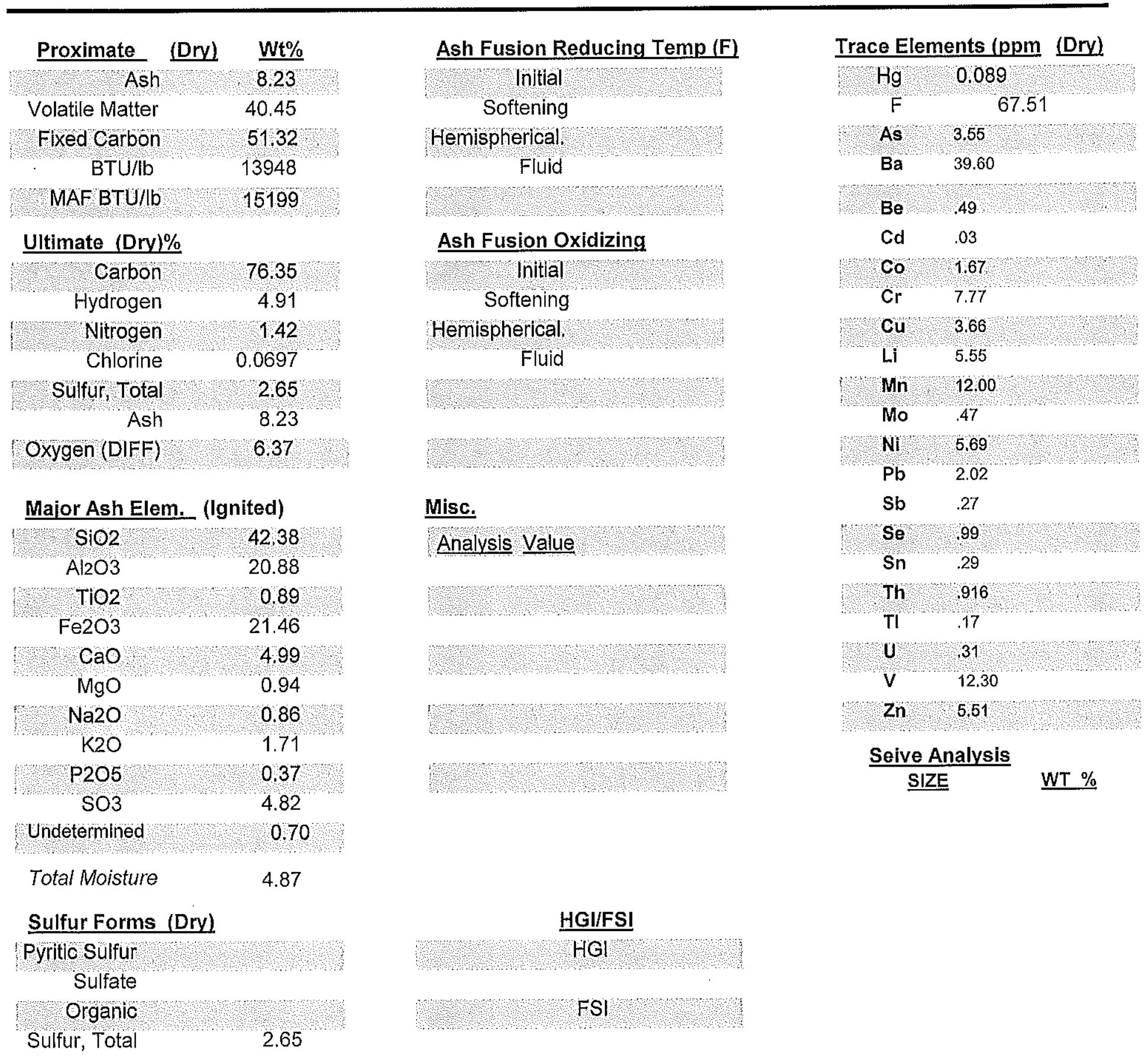

As Determined Moisture $1.495 \quad \%$

These values have been reviewed and are approved for transmission/ $/$ m 


\title{
Y CONSOLENERGY
}

\author{
Research and Development \\ www.consolenergy.com \\ 4000 Brownsville Rd. \\ South Park, PA 15129
}

Sample Description.: GREENIDGE COAL 5/1/07

Sample No.: NH3-1

Analytical No.:

20072513

Date Received: $05 / 11 / 2007$

Date Completed: 05/31/2007

Project No.: $1621-085-000$

Submitted By: J. LOCKE/D. CONNELL

\begin{tabular}{|c|c|c|}
\hline Proximate (Dry) & $\mathbf{W t} \%$ & Ash Fusion Reducing Temp (F \\
\hline Ash & 8.64 & Initial \\
\hline Volatile Matter & 40.81 & Softening \\
\hline Fixed Carbon & 50.55 & Hemispherical: \\
\hline $\mathrm{BTU} / \mathrm{lb}$ & 13796 & Fluid \\
\hline MAF BTU/lb & 15101 & \\
\hline Ultimate (Dry) \% & & Ash Fusion Oxidizing \\
\hline Carbon & 75.37 & Initial \\
\hline Hydrogen & 5.26 & Softening \\
\hline Nitrogen & 138 & Hemispherical \\
\hline Chlorine & 0.0795 & Fluid \\
\hline Sulfur, Total & 3.05 & entu \\
\hline Ash & 8.64 & \\
\hline Oxygen (DIFF) & 6.22 & \\
\hline
\end{tabular}

Major Ash Elem. (Ignited)

\begin{tabular}{|c|c|}
\hline $\mathrm{SiO} 2$ & 42.89 \\
\hline $\mathrm{Al}_{2} \mathrm{O} 3$ & 21.86 \\
\hline $\mathrm{T} 102$ & 0.90 \\
\hline $\mathrm{Fe} 2 \mathrm{O} 3$ & 21.64 \\
\hline $\mathrm{CaO}$ & 4.98 \\
\hline $\mathrm{MgO}$ & 0.81 \\
\hline $\mathrm{Na} 2 \mathrm{O}$ & 0.79 \\
\hline $\mathrm{K} 2 \mathrm{O}$ & 1.46 \\
\hline $\mathrm{P} 205$ & 0.26 \\
\hline $\mathrm{SO} 3$ & 4.88 \\
\hline Undetermined & 0.47 \\
\hline Total Moisture & 6.04 \\
\hline
\end{tabular}

Misc.

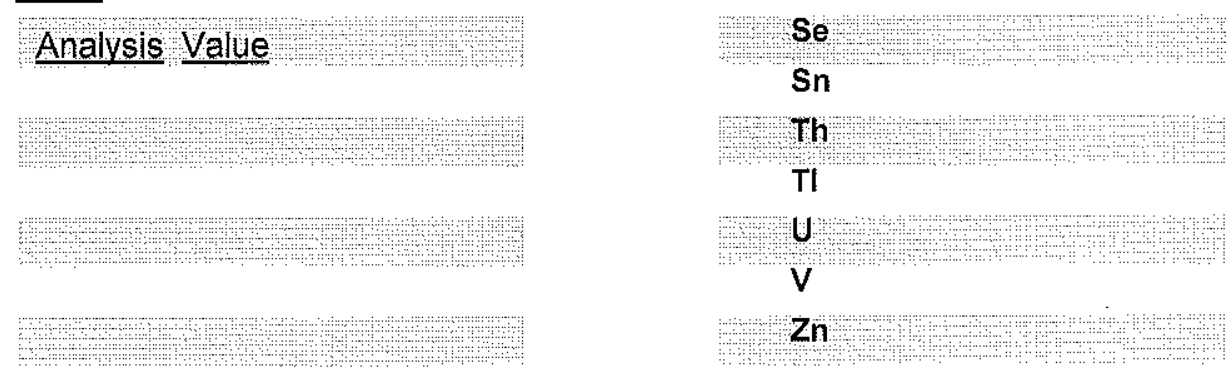

Trace Elements (ppm (Dry)

$\mathrm{Hg}$

$\mathrm{F}$

As

$\mathrm{Ba}$

Be

Cd

co

$\mathrm{Cr}$

ca

$\mathrm{Li}$

$\mathrm{Mn}$

Mo

Ni

$\mathrm{Pb}$

$\mathrm{Sb}$

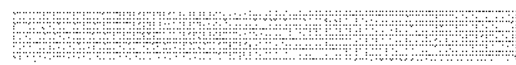

Seive Analysis SIZE WT \%

\section{Sulfur Forms (Dry)}

Pyritic Sulfur

Sulfate

Organic

Sulfur, Total

3.05

HGI/FSI

HGl

$\mathrm{FSI}$

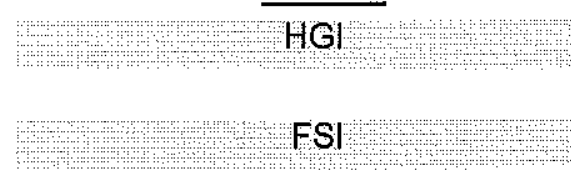

As Determined Moisture $1.255 \%$

These values have been reviewed and are approved for transmission.

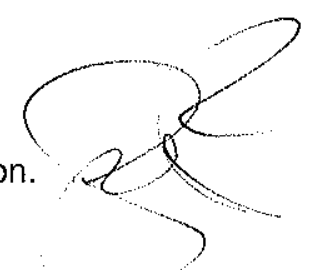




\title{
需ECONSOLENERGY
}

\author{
Research and Development \\ www.consolenergy.com \\ 4000 Brownsville Rd. \\ South Park, PA 15129
}

Sample Description.: GREENIDGE COAL 5/1/07

Sample No.: $\mathrm{NH} 3-2$

Date Received: 05/11/2007

Date Completed: 05/31/2007

Analytical No.:

Project No: $1621-085-000$

Submitted By: J. LOCKE/D. CONNELL

\begin{tabular}{|c|c|c|c|}
\hline Proximate (Drv) & $\underline{W t} \%$ & Ash Fusion Reducing Temp (F) & Trace Elements (ppm (Dry) \\
\hline Ash & 8.95 & Initial & Hg \\
\hline Volatile Matter & 41.02 & Softening & $\mathrm{F}$ \\
\hline Fixed Carbon & 50.03 & Hemispherical: & As: \\
\hline $\mathrm{BTU} / \mathrm{lb}$ & 13765 & Fluid & $\mathrm{Ba}$ \\
\hline$\triangle \mathrm{MAF} \mathrm{BT} / / \mathrm{lb}$ & 15118 & & Be \\
\hline Ultimate (Dry)\% & & Ash Fusion Oxidizing & Cd \\
\hline Carbon & 75.59 & $=3.4$ Initial & Co \\
\hline Hydrogen & 5.01 & Softening & $\mathrm{Cr}$ \\
\hline Nitrogen & 138 & Hemispherical. & $\mathrm{cu}$ \\
\hline Chlorine & 0.0681 & Fluid & $\mathrm{Li}$ \\
\hline Sulfur, Total & 3.14 & 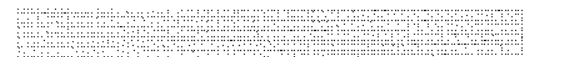 & $\mathrm{Mn}$ \\
\hline Ash & 8.95 & & Mo \\
\hline Oxygen (DIFF) & 586 & +4 & $\mathrm{NI}$ \\
\hline Major Ash Elem. (Ig & ited) & Misc. & $\mathbf{S b}$ \\
\hline $\mathrm{SiO} 2$ & 43.12 & Analysis value & Se \\
\hline $\mathrm{Al} 2 \mathrm{O} 3$ & 21.81 & & Sn \\
\hline T102 & 0.90 & & Th \\
\hline $\mathrm{Fe} 2 \mathrm{O} 3$ & 21.37 & & $\mathrm{TI}$ \\
\hline $\mathrm{CaO}$ & 4.87 & 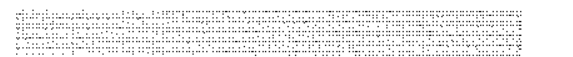 & U. \\
\hline $\mathrm{MgO}$ & 0.79 & & V \\
\hline $\mathrm{Na} 2 \mathrm{O}$ & 0.80 & 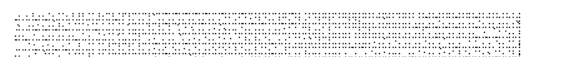 & (4) \\
\hline $\mathrm{K} 2 \mathrm{O}$ & 1.41 & & Seive Analysis \\
\hline $\mathrm{P} 205$ & 0.27 & 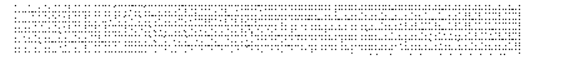 & SIZE \\
\hline $\begin{array}{c}\mathrm{SO} 3 \\
\text { Undetermined }\end{array}$ & $\begin{array}{l}4.93 \\
-0.27\end{array}$ & & \\
\hline Total Moisture & 6.14 & & \\
\hline Sulfur Forms (Dry) & & HGI/FSI & \\
\hline $\begin{array}{c}\text { Pyritic Sulfur } \\
\text { Sulfate }\end{array}$ & 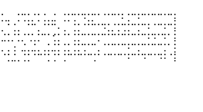 & -HGl & \\
\hline $\begin{array}{l}\text { Organic } \\
\text { Sulfur, Total }\end{array}$ & 3.14 & FSI & \\
\hline
\end{tabular}

As Determined Moisture $1.32 \quad \%$

These values have been reviewed and are approved for transmission.

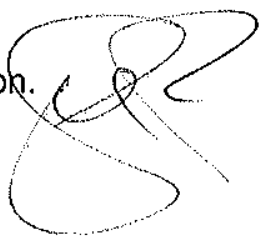




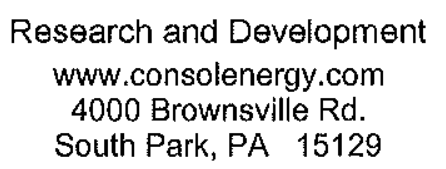

Sample Description.: GREENIDGE COAL 5/1/07

Sample No:: NH3-3

Analytical No.:

20072515

Date Received: 05/11/2007

Date Completed: 05/31/2007

Project No.: $1621-085-000$

Submitted By: J. LOCKE/D. CONNELL

\begin{tabular}{|c|c|}
\hline Proximate (Dry) & $\mathbf{W t} \%$ \\
\hline Ash & 8.53 \\
\hline Volatile Matter & 41.11 \\
\hline Fixed Carbon & 50.36 \\
\hline $\mathrm{BTU} / \mathrm{lb}$ & 13818 \\
\hline MAF BTU/lb & 15107 \\
\hline \multicolumn{2}{|l|}{ Ultimate (Dry)\% } \\
\hline Carbon & 75.69 \\
\hline Hydrogen & 5.00 \\
\hline Nitrogen & 1.38 \\
\hline Chlorine & 0.0755 \\
\hline Sulfur, Total & 3,11 \\
\hline Ash & 8.53 \\
\hline Oxygen (DIFF) & 6.21 \\
\hline
\end{tabular}

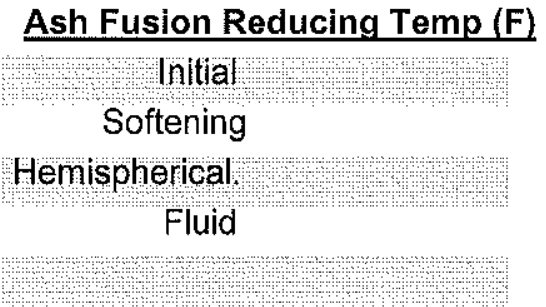

\section{Ash Fusion Oxidizing}

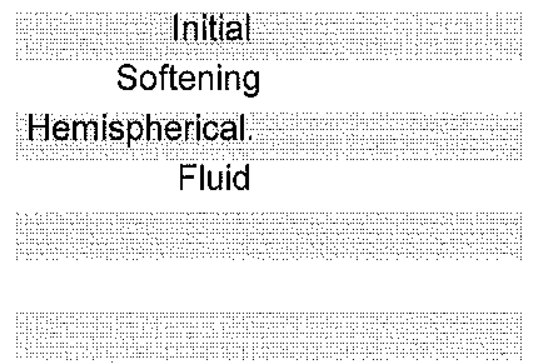

Maior Ash Elem. (Ignited)

$\begin{array}{cc}\mathrm{S} 1 \mathrm{O} 2 & 42.72 \\ \mathrm{Al} 2 \mathrm{O} 3 & 21.69 \\ \mathrm{~T} 1 \mathrm{O} 2 & 0.89 \\ \mathrm{Fe} 2 \mathrm{O} 3 & 20.91 \\ \mathrm{CaO} & 4.58 \\ \mathrm{MgO} & 0.79 \\ \mathrm{Na} 2 \mathrm{O} & 0.79 \\ \mathrm{~K} 2 \mathrm{O} & 1.38 \\ \mathrm{P} 2 \mathrm{O} 5 & 0.24 \\ \mathrm{SO} 3 & 3.74 \\ \text { determined } & \\ & \end{array}$

Total Moisture

\subsection{7}

Sulfur Forms (Dry)

Pyritic Sulfur

Sulfate

Organic

Sulfur, Total

3.11
Misc.
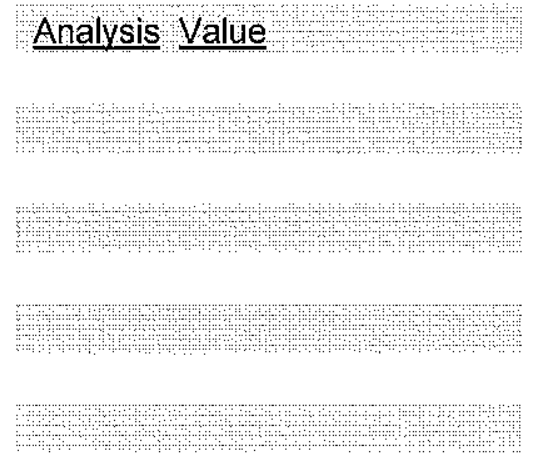

Trace Elements (ppm (Drv)

$\mathrm{Hg}$

F

As

$\mathrm{Ba}$

$\mathrm{Be}$

$\mathrm{Cd}$

co

$\mathrm{Cr}$

Cu

$\mathrm{Li}$

$\mathrm{Mn}$

Mo

$\mathrm{Ni}$

$\mathrm{Pb}$

$\mathrm{Sb}$

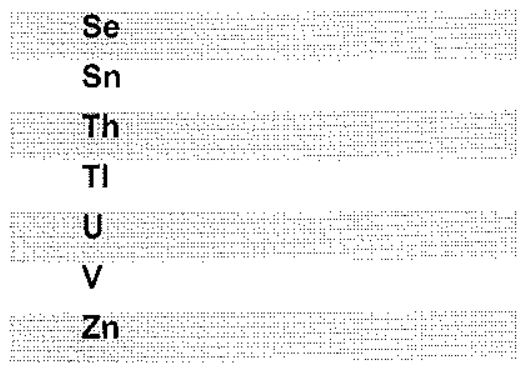

Seive Analysis SIZE

As Determined Moisture $1.255 \quad \%$ These values have been reviewed and are approved for transmission. 


\title{
Y CONSOL ENERGY
}

\author{
Research and Development \\ www.consolenergy.com \\ 4000 Brownsville Rd. \\ South Park, PA 15129
}

Sample Description.: GREENIDGE COAL 5/1/07

Sample No.: $\mathrm{NH} 3-4$

Analytical No.:

20072516

Date Received: $05 / 11 / 2007$

Date Completed: 05/31/2007

Project No.: $1621 \quad-085 \quad-000$

Submitted By: J. LOCKE/D. CONNELL

\begin{tabular}{|c|c|c|}
\hline Proximate & (Dry) & $\mathbf{W t} \%$ \\
\hline Ash & & 871 \\
\hline Volatile Matter & & 41.07 \\
\hline Fixed Carbon & & 5022 \\
\hline $\mathrm{BTU} / \mathrm{lb}$ & & 13784 \\
\hline MAF BTU/L & & 15099 \\
\hline \multicolumn{3}{|c|}{ Ultimate (Dry)\% } \\
\hline Carbon & & 75.61 \\
\hline Hydrogen & & 5.26 \\
\hline Nitrogen & & 1.37 \\
\hline Chlorine & & 0.0882 \\
\hline Sulfur, Total & & 3.08 \\
\hline Ash & & 8.71 \\
\hline Oxygen (DIFF) & & 588 \\
\hline
\end{tabular}

Ash Fusion Reducing Temp (F)

Initial

Softening

Hemispherical:

Fluid

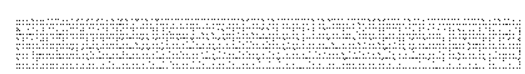

Ash Fusion Oxidizing

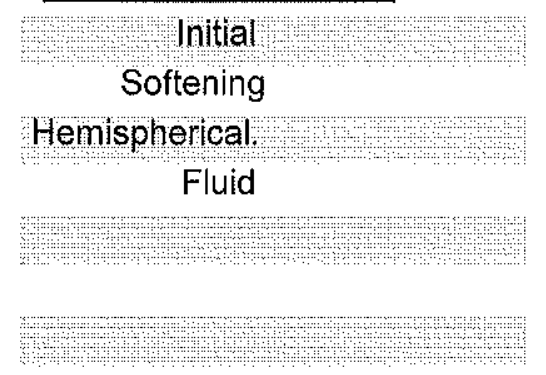

Misc.

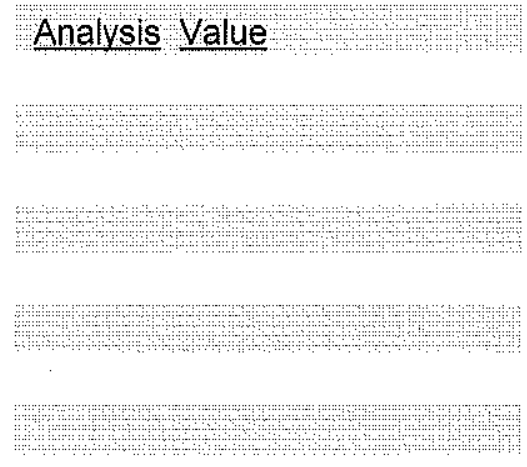

Trace Elements (ppm (Drv)

$\mathrm{Hg}$

$\mathrm{F}$

As

$\mathrm{Ba}$

Be

$\mathrm{Cd}$

co

$\mathrm{Cr}$

$\mathrm{Cu}$

$\mathrm{Li}$

$\mathrm{Mn}$

Mo

Ni

$\mathrm{Pb}$

$\mathrm{Sb}$

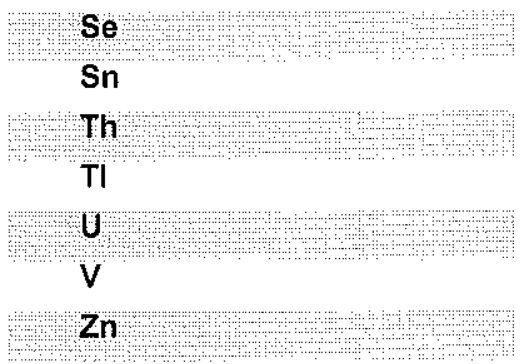

Seive Analysis SIZE

\section{Total Moisture \\ 5.85}

Sulfur Forms (Dry)

Pyritic Sulfur

Sulfate

Organic

Sulfur, Total

3.08
HGI/FSI

$\mathrm{HGl}$

FSI

As Determined Moisture $1.195 \quad \%$

These values have been reviewed and are approved for transmission. 


\section{F CONSOLENERGY}

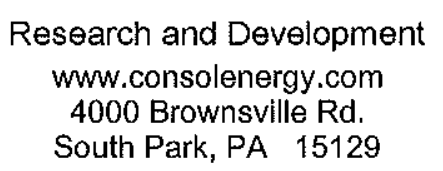

Sample Description.: GREENIDGE COAL 5/2/07

Sample No.: SO3-1

Analytical No.:

20072517

Date Received: 05/11/2007

Date Completed: 05/31/2007

Project No.: $1621-085-000$

Submitted By: J. LOCKE/D. CONNELL

\begin{tabular}{|c|c|c|c|}
\hline Proximate (Dry) & $\underline{W t} \%$ & Ash Fusion Reducing Temp (F) & Trace Elements (ppm (Dry) \\
\hline Ash & 8.53 & Initial & $\mathrm{Hg}$ \\
\hline Volatile Matter & 41.30 & Softening & $\mathrm{F}$ \\
\hline Fixed Carbon & 50.17 & Hemispherical. & As \\
\hline $\mathrm{BTU} / \mathrm{lb}$ & 13819 & Fluid & $\mathrm{Ba}$ \\
\hline MAF BTU/L & 15108 & 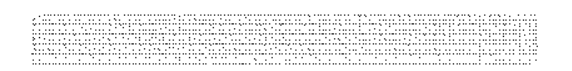 & $\mathrm{Be}$ \\
\hline Ultimate (Drv)\% & & Ash Fusion Oxidizing & $\mathrm{Cd}$ \\
\hline Carbon & 75.19 & Initial & Co \\
\hline Hydrogen & 5.12 & Softening & $\mathrm{Cr}$ \\
\hline Nitrogen & 1.39 & Hemispherical. & $\mathrm{Cu}$ \\
\hline Chlorine & 0.0636 & Fluid & $\mathrm{Li}$ \\
\hline Sulfur, Total & 3.20 & 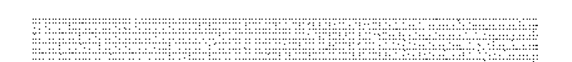 & $\mathrm{Mn}$ \\
\hline Ash & 8.53 & & Mo \\
\hline Oxygen (DIFF) & 6.51 . & tut & $\mathrm{Ni}$ \\
\hline \multirow{2}{*}{\multicolumn{2}{|c|}{ Major Ash Elem. (Ignited) }} & & $\mathrm{Pb}$ \\
\hline & & Misc. & Sb \\
\hline $\mathrm{SiO} 2$ & 43.40 & Analysis Value & Se \\
\hline $\mathrm{Al}_{2} \mathrm{O} 3$ & 22.08 & & Sn \\
\hline $\mathrm{TiO} 2$ & 0.91 & ma & Th \\
\hline $\mathrm{Fe} 2 \mathrm{O} 3$ & 20.48 & & TI \\
\hline $\mathrm{CaO}$ & 4.36 & $=$ & 0 \\
\hline $\mathrm{MgO}$ & 0.78 & & $\mathrm{v}$ \\
\hline $\mathrm{Na} 2 \mathrm{O}$ & 0.76 & 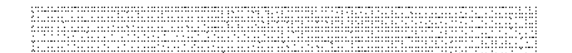 & $\mathrm{Zn}$ \\
\hline $\mathrm{K} 2 \mathrm{O}$ & 1.40 & & Seive Analvsis \\
\hline $\mathrm{P} 2 \mathrm{O} 5$ & 0.25 & 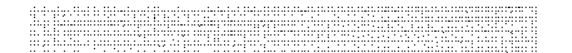 & SIZE \\
\hline $\mathrm{SO} 3$ & 4.16 & & \\
\hline Undetermined & 1.42 & & \\
\hline Total Moisture & 5.51 & & \\
\hline \multicolumn{2}{|l|}{ Sulfur Forms (Dry) } & \multicolumn{2}{|l|}{ HGI/FSI } \\
\hline Pyritic Sulfur & + & $\mathrm{HGl}$ & \\
\hline \multicolumn{4}{|l|}{ Sulfate } \\
\hline Organic & 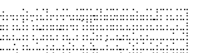 & FSI & \\
\hline Sulfur, Total & 3.20 & & \\
\hline
\end{tabular}

As Determined Moisture $1.185 \quad \%$ These values have been reviewed and are approved for transmission.

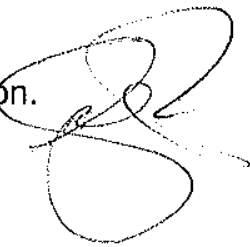




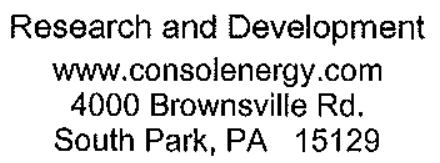

Sample Description.: GREENIDGE COAL 5/2/07

Sample No.: SO3-2

Date Received: $05 / 11 / 2007$

Date Completed: 05/31/2007

Analytical No.:

Project No.: $1621 \quad-085 \quad-000$

Submitted By: J. LOCKE/D. CONNELL

\begin{tabular}{|c|c|c|}
\hline Proximate & (Dry) & $\underline{W t} \%$ \\
\hline Ash & & 875 \\
\hline Volatile Matter & & 41.37 \\
\hline Fixed Carbon & & 49.88 \\
\hline BTU//b & & 13741 \\
\hline MAF BTU/lb & & 15059 \\
\hline
\end{tabular}

Ultimate (Drv)\%

$\begin{array}{cr}\text { Carbon } & 75.14 \\ \text { Hydrogen } & 4.84 \\ \text { Nitrogen } & 1.35 \\ \text { Chlorine } & 0.0878\end{array}$

Sulfur Total $\bullet \longrightarrow 3.38$

Ash $\quad 8.75$

Oxygen (DIFF)

Major Ash Elem. (Ignited)

$\mathrm{SiO} 2,42.03$

$\mathrm{Al} 2 \mathrm{O} 3 \quad 21.33$

$\mathrm{TiO2} . \quad 0.86$

$\mathrm{Fe} 2 \mathrm{O} 3 \quad 22.42$

$\mathrm{CaO}, \longleftarrow \square \square .42$

$\mathrm{MgO} \quad 0.76$

$\mathrm{Na} 2 \mathrm{O} ?$

$\mathrm{K} 2 \mathrm{O} \quad 1.38$

$\mathrm{P} 2 \mathrm{O} 5 ? 0.23$

$\mathrm{SO} 3 \quad 4.56$

Undetermined,$\lrcorner, 1 / 13$

Total Moisture

5.66

Sulfur Forms (Dry)

Pyritic Sulfur

Sulfate

Organic

Sulfur, Total

3.38
Ash Fusion Reducing Temp (F)

Initial

Softening

Hemispherical:

Fluid

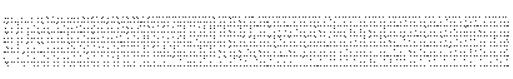

Ash Fusion Oxidizing

Initial

Softening

Hemispherical:

Fluid

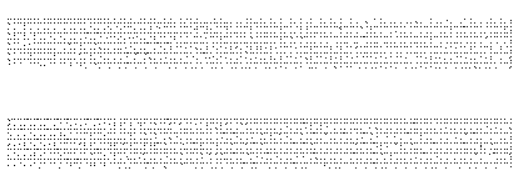

Misc.
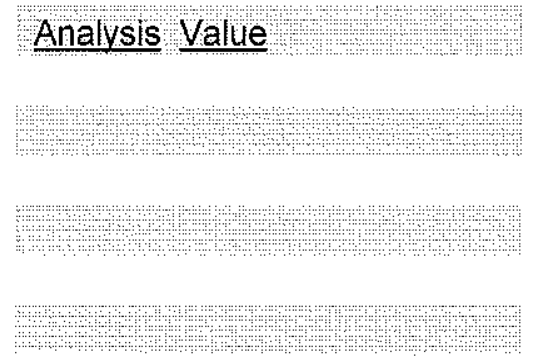

?t:
Trace Elements (ppm (Dry)

Hg

$\mathrm{F}$

As

$\mathrm{Ba}$

$\mathrm{Be}$

$\mathrm{Cd}$

co

$\mathrm{Cr}$

cu

Li

$\mathrm{Mn}$

Mo

$\mathrm{Ni}$

$\mathrm{Pb}$

$\mathrm{Sb}$

Se

Sn

Th

TI

U

v

$\mathrm{Zn}$

Seive Analysis

SIZE

WT \%
HGI/FSI

$\mathrm{HGl}$

FSI

As Determined Moisture $1.155 \quad \%$

These values have been reviewed and are approved for transmission. 


\section{흘 CONSOL ENERGY.}

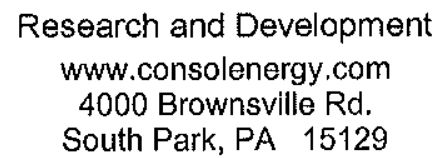

Sample Description.: GREENIDGE COAL 5/2/07

Sample No.: $\mathrm{SO} 3-3$

Analytical No.:

20072519

Date Received: 05/11/2007

Project No.: $1621 \quad-085 \quad-000$

Date Completed: 05/31/2007

Submitted By: J. LOCKE/D. CONNELL

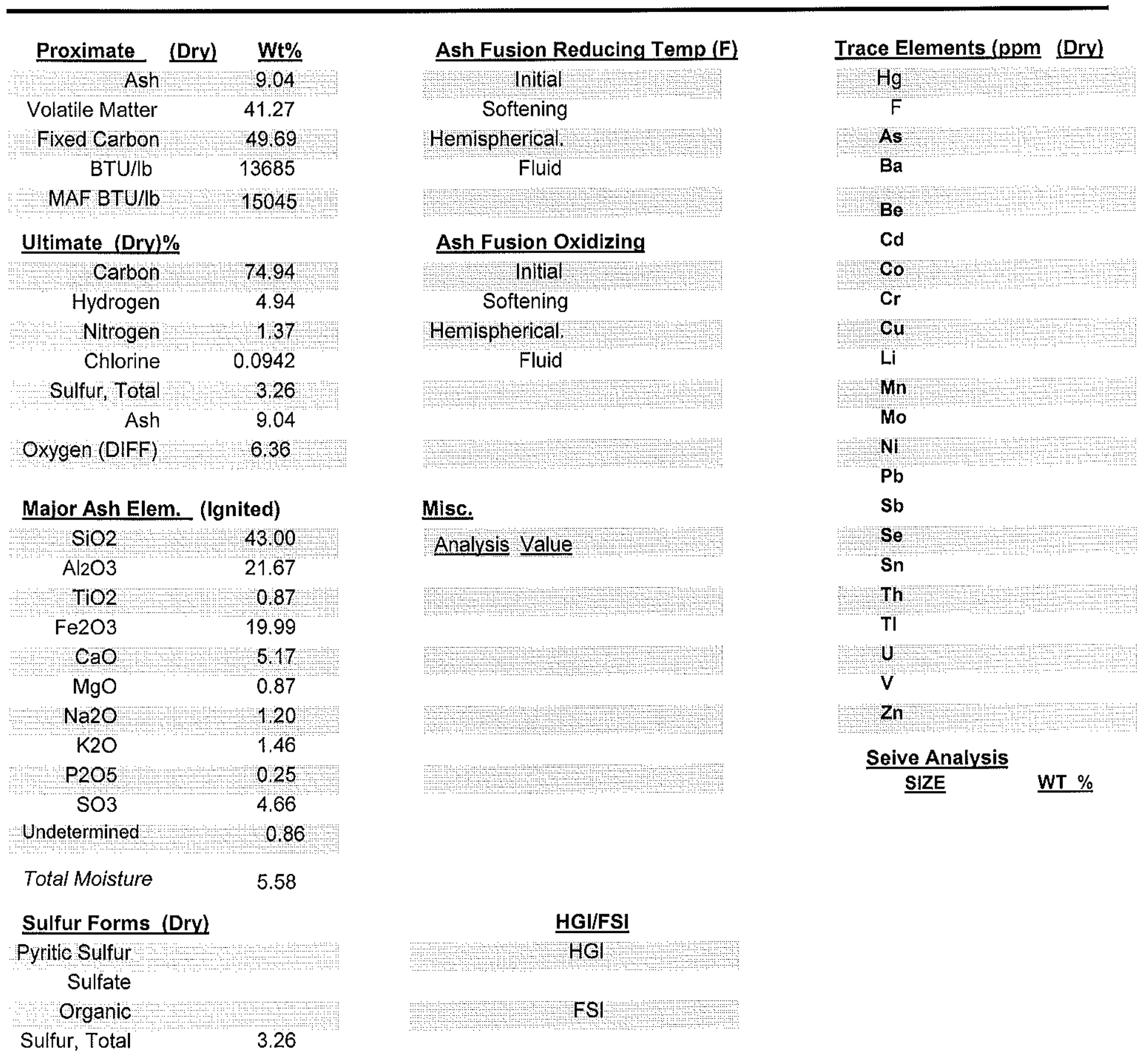

As Determined Moisture $1.3 \quad \%$ These values have been reviewed and are approved for transmission. 


\title{
SONSOLENERGY
}

\author{
Research and Development \\ www.consolenergy.com \\ 4000 Brownsville Rd. \\ South Park, PA 15129
}

Sample Description.: GREENIDGE COAL 5/4/07

Sample No:: $A G-1$

Analytical No.: $\quad 20072520$

Date Received: 05/11/2007

Date Completed: 05/31/2007

Project No.: $1621 \quad-085 \quad-000$

Submitted By: J. LOCKE/D. CONNELL

\begin{tabular}{|c|c|c|}
\hline Proximate & (Drv) & $W_{t} \%$ \\
\hline Ash & & 860 \\
\hline Volatile Matter & & 41.39 \\
\hline Fixed Carbon & & 50.01 \\
\hline $\mathrm{BTU} / \mathrm{lb}$ & & 13732 \\
\hline MAF BTU/b & & 15024 \\
\hline
\end{tabular}

\section{Ultimate (Dry)\%}

\begin{tabular}{cr} 
Carbon & 76.24 \\
Hydrogen & 4.83 \\
Nitrogen & 1.35 \\
Chlorine & 0.0662 \\
ulfur, Total & 3.32 \\
Ash & 8.60 \\
gen (DIFF) & 5.59 \\
\hline
\end{tabular}

Major Ash Elem. (Ignited)

\begin{tabular}{|c|c|}
\hline $\mathrm{S} 1 \mathrm{O} 2$ & 43,14 \\
\hline $\mathrm{Al}_{2} \mathrm{O} 3$ & 22.03 \\
\hline $\mathrm{TlO}_{2}$ & 0.90 \\
\hline $\mathrm{Fe} 2 \mathrm{O} 3$ & 21.13 \\
\hline $\mathrm{CaO}$ & 4.77 \\
\hline $\mathrm{MgO}$ & 0.83 \\
\hline $\mathrm{Na} 2 \mathrm{O}$ & 0.75 \\
\hline $\mathrm{K} 2 \mathrm{O}$ & 1.40 \\
\hline P205 & 0.27 \\
\hline $\mathrm{SO} 3$ & 3.88 \\
\hline determined & 0.90 \\
\hline
\end{tabular}

Total Moisture

5.94

Sulfur Forms (Dry)

Pyritic Sulfur

Sulfate

Organic

Sulfur, Total

3.32
HGI/FSI

$\mathrm{HGI}$

$\mathrm{FSI}$

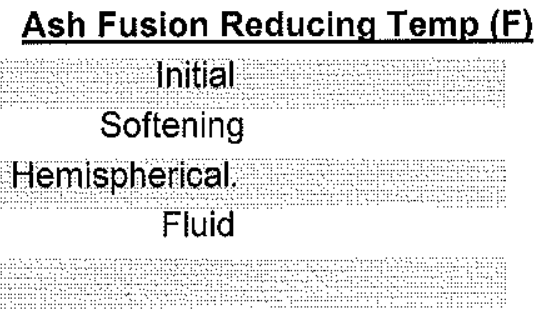

Ash Fusion Oxidizing

Trace Elements (ppm (Drv)

$\mathrm{Hg}$

F

As

$\mathrm{Ba}$

$\mathrm{Be}$

$\mathrm{Cd}$

co

$\mathrm{Cr}$

Softening

Hemispherical:

Fluid

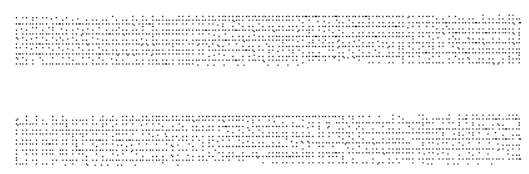

Misc.

\section{Analysis Value}
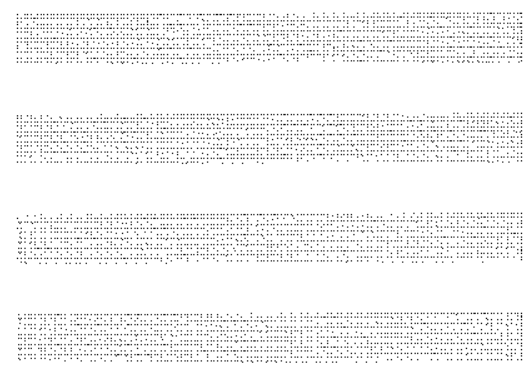

$\mathrm{Cu}$

$\mathrm{Li}$

$\mathrm{Mn}$

Mo

$\mathrm{Ni}$

$\mathrm{Pb}$

$\mathrm{Sb}$

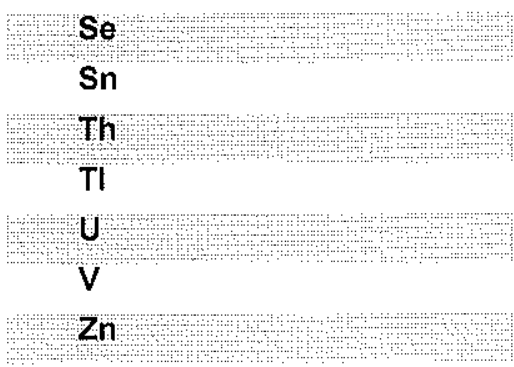

Seive Analysis SIZE

As Determined Moisture $1.295 \quad \%$

These values have been reviewed and are approved for transmission. 


\title{
Y CONSOL ENERGY.
}

\author{
Research and Development \\ wuw.consolenergy.com \\ 4000 Brownsville Rd. \\ South Park, PA 15129
}

Sample Description.: GREENIDGE COAL 5/4/07

Sample No:: AG-2

Date Received: $05 / 11 / 2007$

Analytical No.:

20072521

Project No.: $1621 \quad-085 \quad-000$

Date Completed: 05/31/2007

Submitted By: J. LOCKE/D. CONNELL

\begin{tabular}{|c|c|c|}
\hline Proximate & (Dry) & $\underline{W t} \%$ \\
\hline Ash & & 8.85 \\
\hline Volatile Matter & & 41.31 \\
\hline Fixed Carbon & & 49.84 \\
\hline BTU/lb & & 13762 \\
\hline MAF BTU/lb & & 15098 \\
\hline \multicolumn{3}{|c|}{ Ultimate (Dry)\% } \\
\hline Carbon & & 75.13 \\
\hline Hydrogen & & 5.01 \\
\hline Nitrogen & & 1.39 \\
\hline Chlorine & & 0.0957 \\
\hline Sulfur, Total & & 3.25 \\
\hline Ash & & 8.85 \\
\hline Oxygen (DIFF) & & 6.27 \\
\hline
\end{tabular}

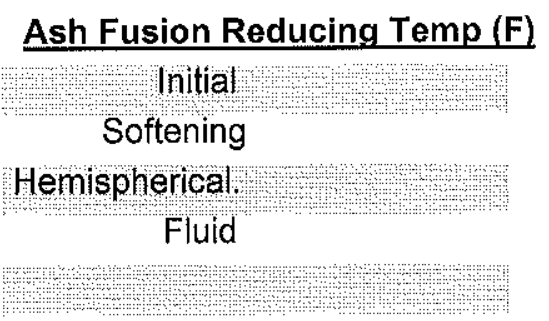

\section{Ash Fusion Oxidizing}

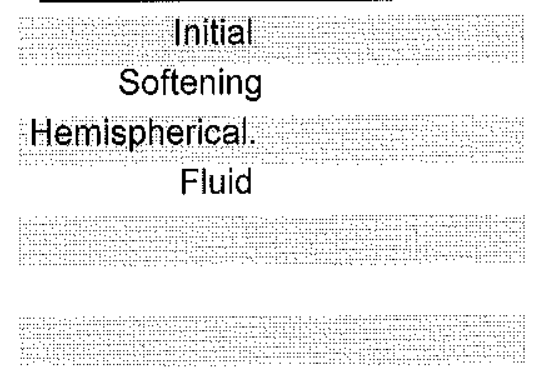

Major Ash Elem. (Ignited)

\begin{tabular}{cc}
$\mathrm{SiO} 2$ & 43.06 \\
$\mathrm{Al} 2 \mathrm{O} 3$ & 21.81 \\
$\mathrm{TlO} 2$ & 0.87 \\
$\mathrm{Fe} 2 \mathrm{O} 3$ & 20.24 \\
$\mathrm{CaO}$ & 4.99 \\
$\mathrm{MgO}$ & 0.81 \\
$\mathrm{Na} 2 \mathrm{O}$ & 0.99 \\
$\mathrm{~K} 2 \mathrm{O}$ & 1.43 \\
$\mathrm{P} 2 \mathrm{O} 5$ & 0.24 \\
$\mathrm{SO} 3$ & 4.66 \\
determined & 0.90 \\
\hline
\end{tabular}

Total Moisture

5.97

Sulfur Forms (Dry)

Pyritic Sulfur

Sulfate

Organic

Sulfur, Total

3.25
Misc.

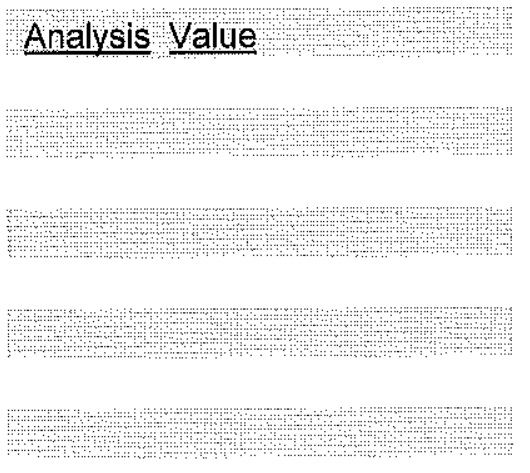

\section{HGI/FSI}

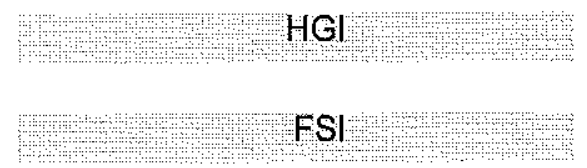

Trace Elements (ppm (Dry)

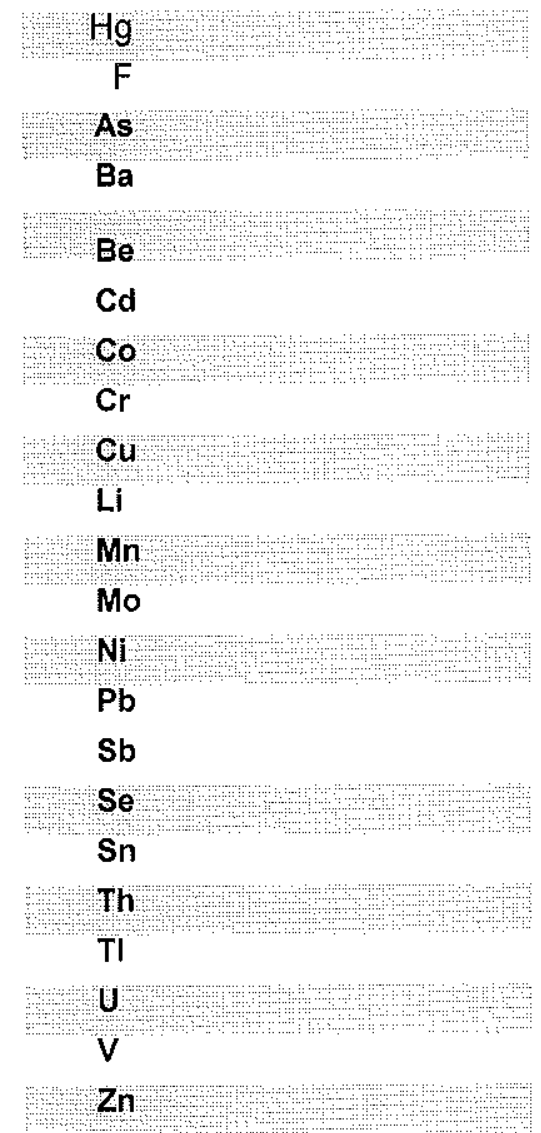

Seive Analysis SIZE As Determined Moisture $1.315 \%$
These values have been reviewed and are approved for transmission 


\title{
흘 CONSOL ENERGY.
}

\author{
Research and Development \\ www.consolenergy.com \\ 4000 Brownsville Rd. \\ South Park, PA 15129
}

Sample Description.: AHO ASH 5/1/07

Sample No: $\mathrm{NH} 3-1$

Date Received: 05/11/2007

Analytical No.:

20072522

Project No.: $1621 \quad-085 \quad-000$

Date Completed: 05/31/2007

Submitted By: J. LOCKE/D. CONNELL

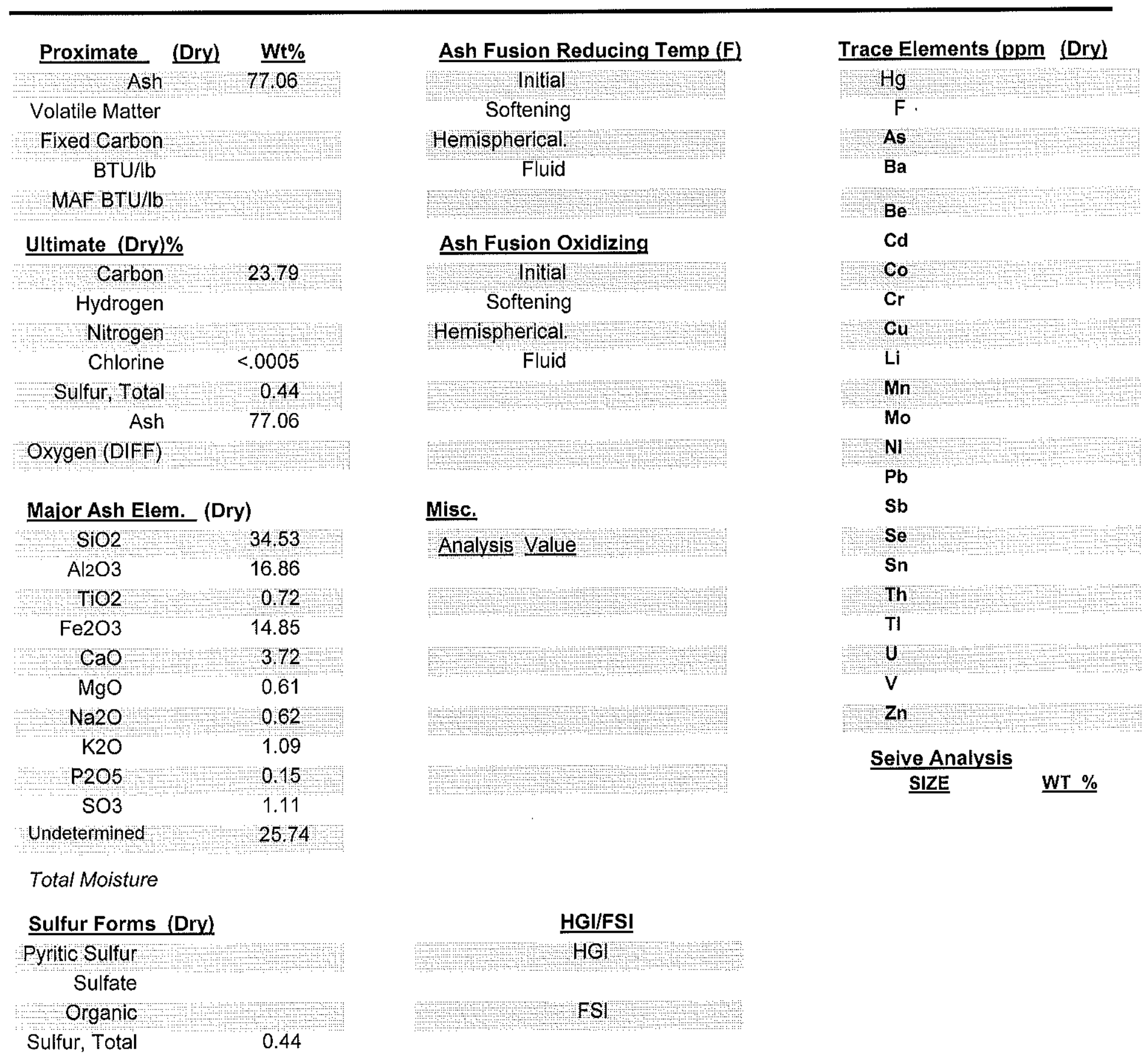

As Determined Moisture $\quad 0.2 \quad \%$ These values have been reviewed and are approved for transmissign.

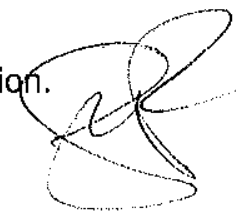




\title{
FONSOLENERGY
}

\author{
Research and Development \\ www,consolenergy.com \\ 4000 Brownsville Rd. \\ South Park, PA 15129
}

Sample Description: AHO ASH 5/1/07

Sample No.: $\mathrm{NH} 3-2$

Date Received: 05/11/2007

Date Completed: $05 / 31 / 2007$

Analytical No.: $\quad 20072523$

Project No.: $1621 \quad-085 \quad-000$

Submitted By: J. LOCKE/D. CONNELL

\begin{tabular}{|c|c|c|}
\hline Proximate (Dry) & $\underline{W t} \%$ & Ash Fusion Reducing Temp (F \\
\hline Ash & 83.09 & Initial \\
\hline Volatile Matter & & Softening \\
\hline Fixed Carbon & & Hemispherical. \\
\hline $\mathrm{BTU} / \mathrm{lb}$ & & Fluid \\
\hline MAF BTU/lb & & \\
\hline Ultimate (Dry) \% & & Ash Fusion Oxidizing \\
\hline Carbon & 1626 & Initial \\
\hline Hydrogen & & Softening \\
\hline Nitrogen & & Hemispherical: \\
\hline Chlorine & 0.0138 & Fluid \\
\hline Sulfur, Total & 0.54 & +4 \\
\hline Ash & 83.09 & \\
\hline Oxygen (DIFF) & & \\
\hline
\end{tabular}

Major Ash Elem. (Dry)

$$
\mathrm{SiO} 2
$$

39.20

$\mathrm{Al} 2 \mathrm{O} 3$

19.74

$\mathrm{TiO} 2$

0.89

$\mathrm{Fe} 2 \mathrm{O} 3$

14.74

$\mathrm{CaO}$

3.90

$\mathrm{MgO}$

0.73

$\mathrm{Na2O}$

0.78

$\mathrm{K} 2 \mathrm{O}$

1.35

$\mathrm{P} 205$

$\mathrm{SO} 3$

0.22

1.34

1711
Misc.

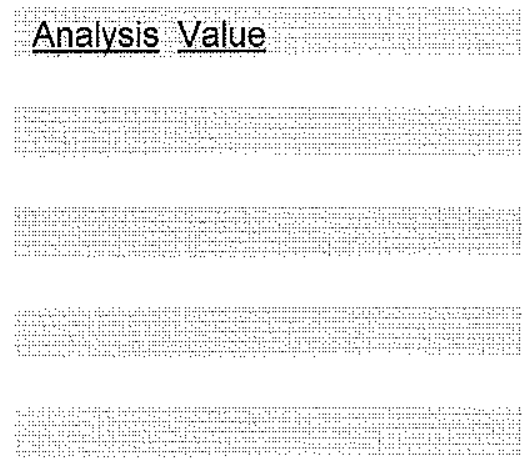

Trace Elements (ppm (Drv)

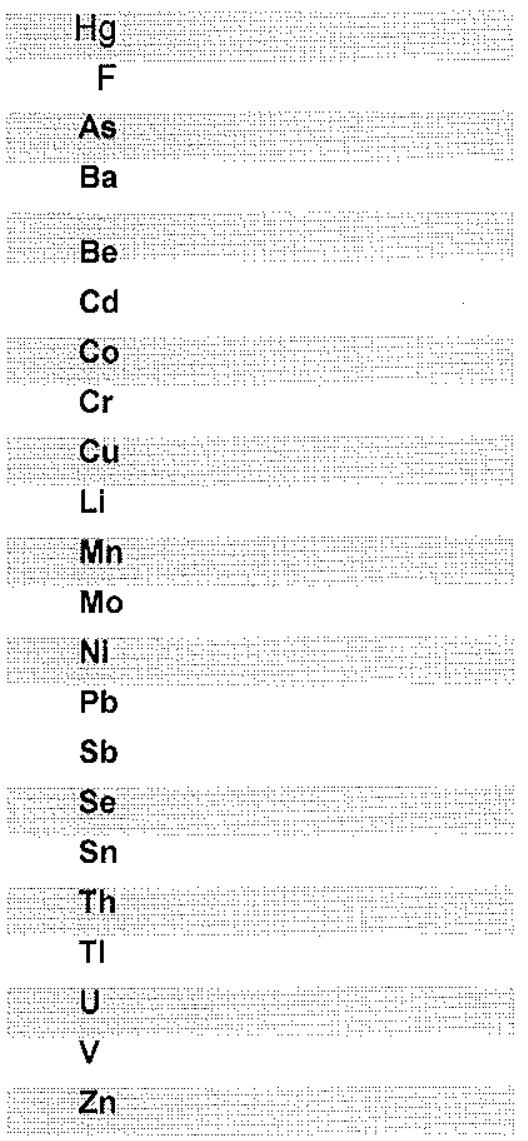

Seive Analysis SIZE

\section{Total Moisture}

\section{Sulfur Forms (Dry)}

Pyritic Sulfur

Sulfate

Organic

Sulfur, Total

\section{HGI/FSI}

$\mathrm{HGl}$

FSI

As Determined Moisture $1.07 \quad \%$ These values have been reviewed and are approved for transmission. 


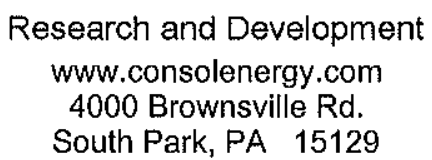

Sample Description.: AHO ASH 5/1/07

Sample No: $\mathrm{NH} 3-3$

Analytical No.:

20072524

Date Received: $05 / 11 / 2007$

Project No.: $1621-085-000$

Date Completed: 05/31/2007

Submitted By: J. LOCKE/D. CONNELL

\begin{tabular}{|c|c|}
\hline Proximate (Dry) & $\mathbf{W t} \%$ \\
\hline Ash & 84.20 \\
\hline \multicolumn{2}{|l|}{ Volatile Matter } \\
\hline \multicolumn{2}{|l|}{ Fixed Carbon } \\
\hline \multicolumn{2}{|l|}{$\mathrm{BTU} / \mathrm{b}$} \\
\hline \multicolumn{2}{|l|}{ MAF BTU/lb } \\
\hline \multicolumn{2}{|l|}{ Ultimate (Dry)\% } \\
\hline Carbon & 15.09 \\
\hline \multicolumn{2}{|l|}{ Hydrogen } \\
\hline Nitrogen & \\
\hline Chlorine & 0.0065 \\
\hline Sulfur, Total & 0.53 \\
\hline Ash & 84.20 \\
\hline Oxygen (DIFF) & \\
\hline
\end{tabular}

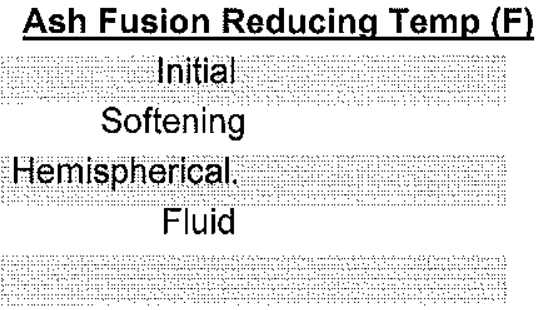

\section{Ash Fusion Oxidizing}

Initial

Softening

Hemispherical.

Fluid
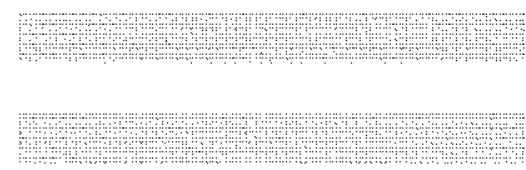

Major Ash Elem. (Dry)

$\mathrm{SiO} 2$

39.63

$\mathrm{Al} 2 \mathrm{O} 3$

20.07

$\mathrm{TiO} 2$

0.90

$\mathrm{Fe} 2 \mathrm{O} 3$

14.87

$\mathrm{CaO}$

4.07

$\mathrm{MgO}$

0.75

$\mathrm{Na} 2 \mathrm{O}$

0.79

$\mathrm{K} 2 \mathrm{O}$

1.38

$\mathrm{P} 205$

$\mathrm{SO} 3$

0.21

1.30

16.03
Misc.

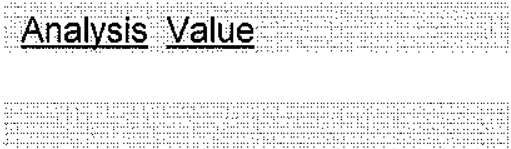

Analysis Value
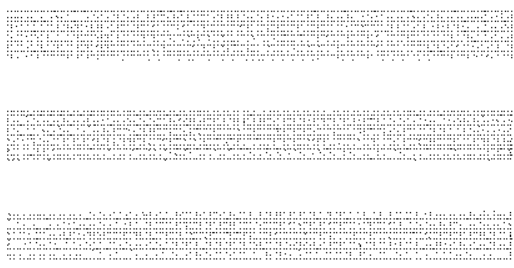

Trace Elements (ppm (Dry)

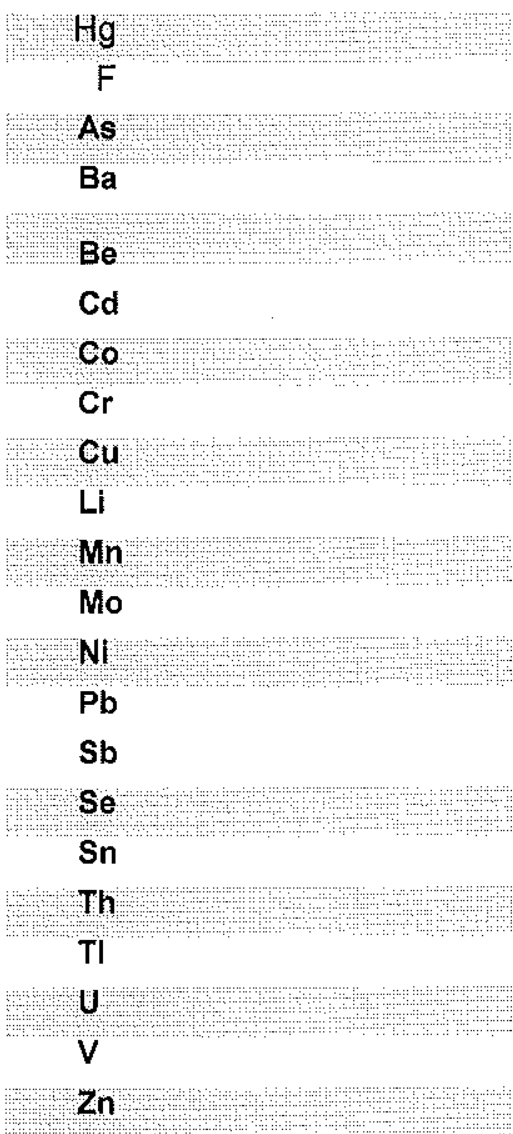

Seive Analysis

SIZE

\section{HGI/FSI}

HGI

$\mathrm{FSI}$

Pyritic Sulfur

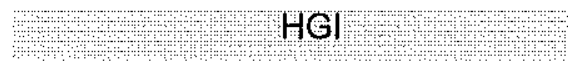

Sulfate

Organic

Sulfur, Total

0.53

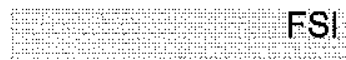

As Determined Moisture $1.08 \quad \%$ These values have been reviewed and are approved for transmission.

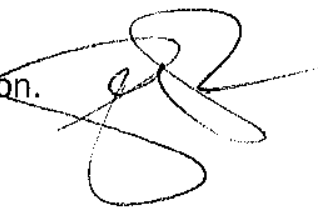




\section{Research and Development www.consolenergy.com 4000 Brownsville Rd. \\ South Park, PA 15129}

Sample Description.: AHO ASH 5/1/07

Sample No.: $\mathrm{NH} 3-4$

Analytical No.: $\quad 20072525$

Date Received: 05/11/2007

Date Completed: 05/31/2007

Project No.: $1621-085-000$

Submitted By: J. LOCKE/D. CONNELL

\begin{tabular}{|c|c|c|}
\hline Proximate & (Drv) & $\mathbf{W t} \%$ \\
\hline Ash & & 8032 \\
\hline \multicolumn{3}{|l|}{ Volatile Matter } \\
\hline \multicolumn{3}{|l|}{ Fixed Carbon } \\
\hline \multicolumn{3}{|l|}{$\mathrm{BTU} / \mathrm{lb}$} \\
\hline \multicolumn{3}{|l|}{ MAF BTU/lb } \\
\hline \multicolumn{3}{|c|}{ Ultimate (Dry)\% } \\
\hline Carbon & & 20.83 \\
\hline \multicolumn{3}{|l|}{ Hydrogen } \\
\hline \multicolumn{3}{|l|}{ Nitrogen } \\
\hline Chlorine & & 0.0041 \\
\hline Sulfur, Total & & 0.52 \\
\hline Ash & & 80.32 \\
\hline Oxygen (DIFF) & & \\
\hline
\end{tabular}

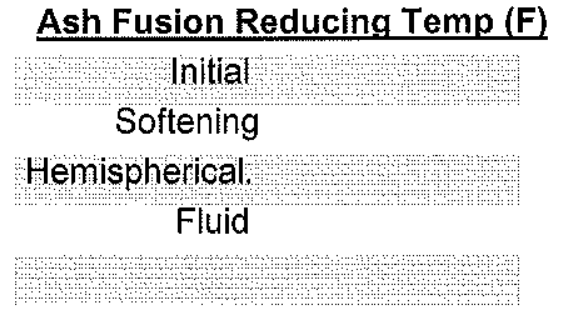

\section{Ash Fusion Oxidizing}

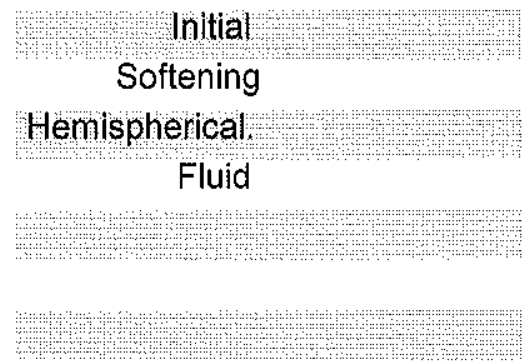

Major Ash Elem. (Dry)

$\mathrm{SiO} 2.38 .90$

$\mathrm{Al}_{2} \mathrm{O} 3 \quad 19.38$

$\mathrm{THO} 2,0.87$

$\mathrm{Fe} 2 \mathrm{O} 3 \quad 15.29$

$\mathrm{CaO} \cup \quad 4.01$

$\mathrm{MgO} \quad 0.69$

$\mathrm{Na} 2 \mathrm{O} \cup u \quad 0.71$

$\mathrm{K} 2 \mathrm{O} \quad 1.26$

$\mathrm{P} 2 \mathrm{O} 5 ? \quad 0.18$

$\mathrm{SO} 3 \quad 1.29$

Undetermined $\square \simeq \quad 17.42$

Total Moisture

Sulfur Forms (Dry)

Pyritic Sulfur

Sulfate

Organic

Sulfur, Total

0.52
Misc.
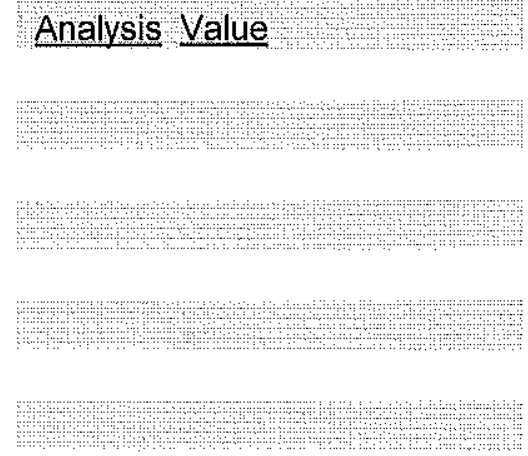

HGI/FSI

HGI

FSI
Trace Elements (ppm (Dry)

$\mathrm{Hg}$

$\mathrm{F}$

As

$\mathrm{Ba}$

$\mathrm{Be}$

$\mathrm{Cd}$

co

$\mathrm{Cr}$

cu

$\mathrm{Li}$

$\mathrm{Mn}$

Mo

$\mathrm{Ni}$

$\mathrm{Pb}$

$\mathrm{Sb}$

Se

$\mathrm{Sn}$

Th

$\mathrm{TI}$

0

V

$\mathrm{Zn}$

Seive Analysis

SIZE

WT \%

As Determined Moisture $0.38 \quad \%$

These values have been reviewed and are approved for transmissiøn.

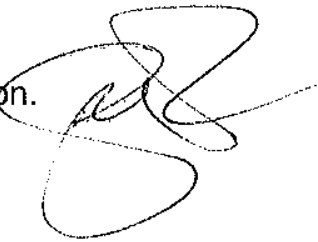




\title{
F CONSOLENERGY.
}

\author{
Research and Development \\ www.consolenergy.com \\ 4000 Brownsville Rd. \\ South Park, PA 15129
}

Sample Description.: AHO ASH 5/2/07

Sample No.: SO3-1

Analytical No.: $\quad 20072526$

Date Received: $05 / 11 / 2007$

Project No.: $1621 \quad-085-000$

Date Completed: 05/31/2007

Submitted By: J. LOCKE/D. CONNELL

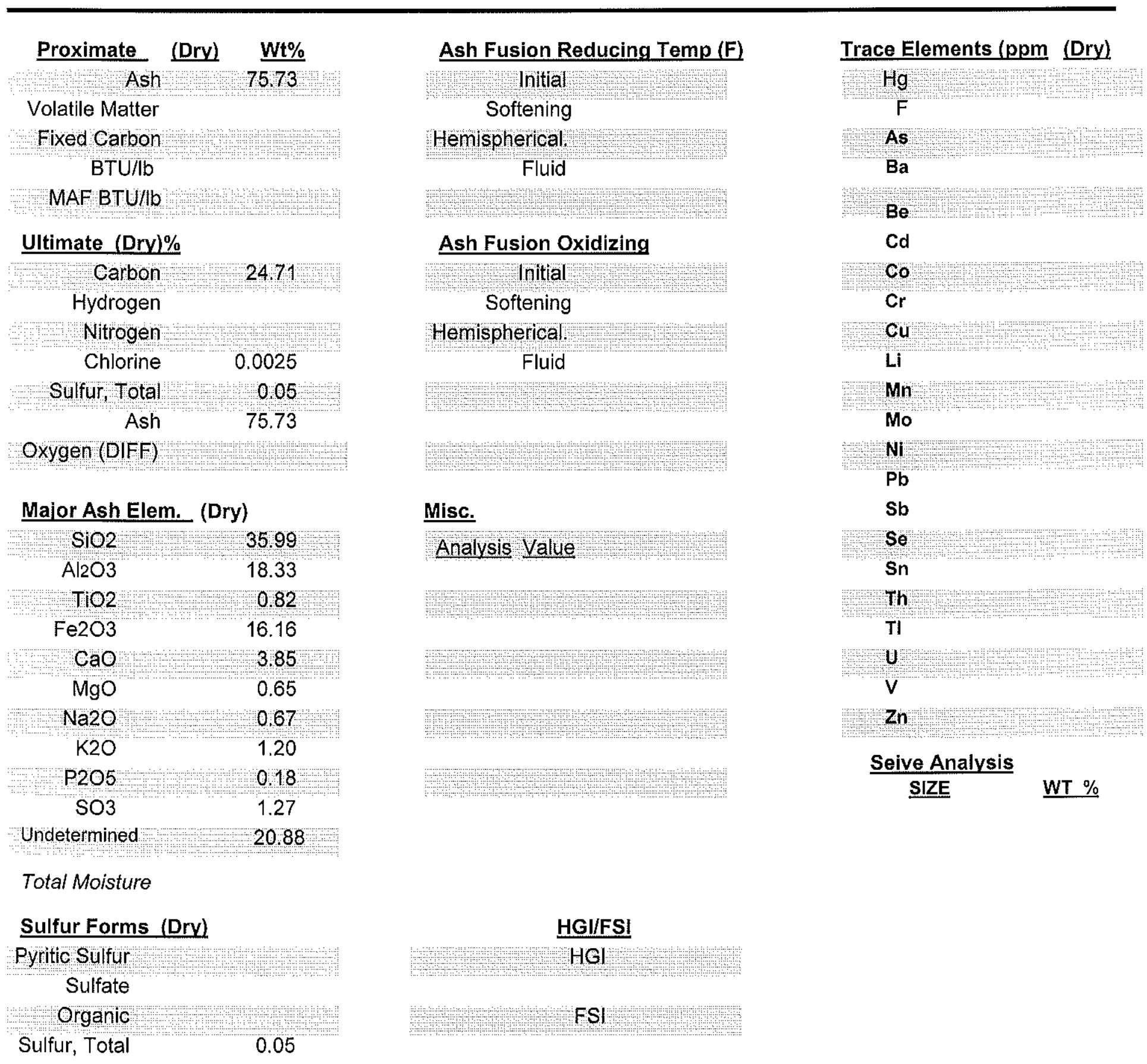

As Determined Moisture $0.165 \quad \%$

These values have been reviewed and are approved for transmission.

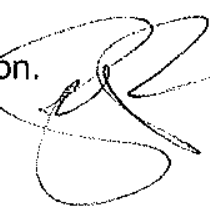




\section{言: CONSOL ENERGY}

Research and Development www.consolenergy.com 4000 Brownsville Rd. South Park, PA 15129

Sample Description.: AHO ASH 5/2/07

Sample No.: SO3-2

Analytical No.:

20072527

Date Received: 05/11/2007

Project No.: $1621 \quad-085 \quad-000$

Date Completed: 05/31/2007

Submitted By: J. LOCKE/D. CONNELL

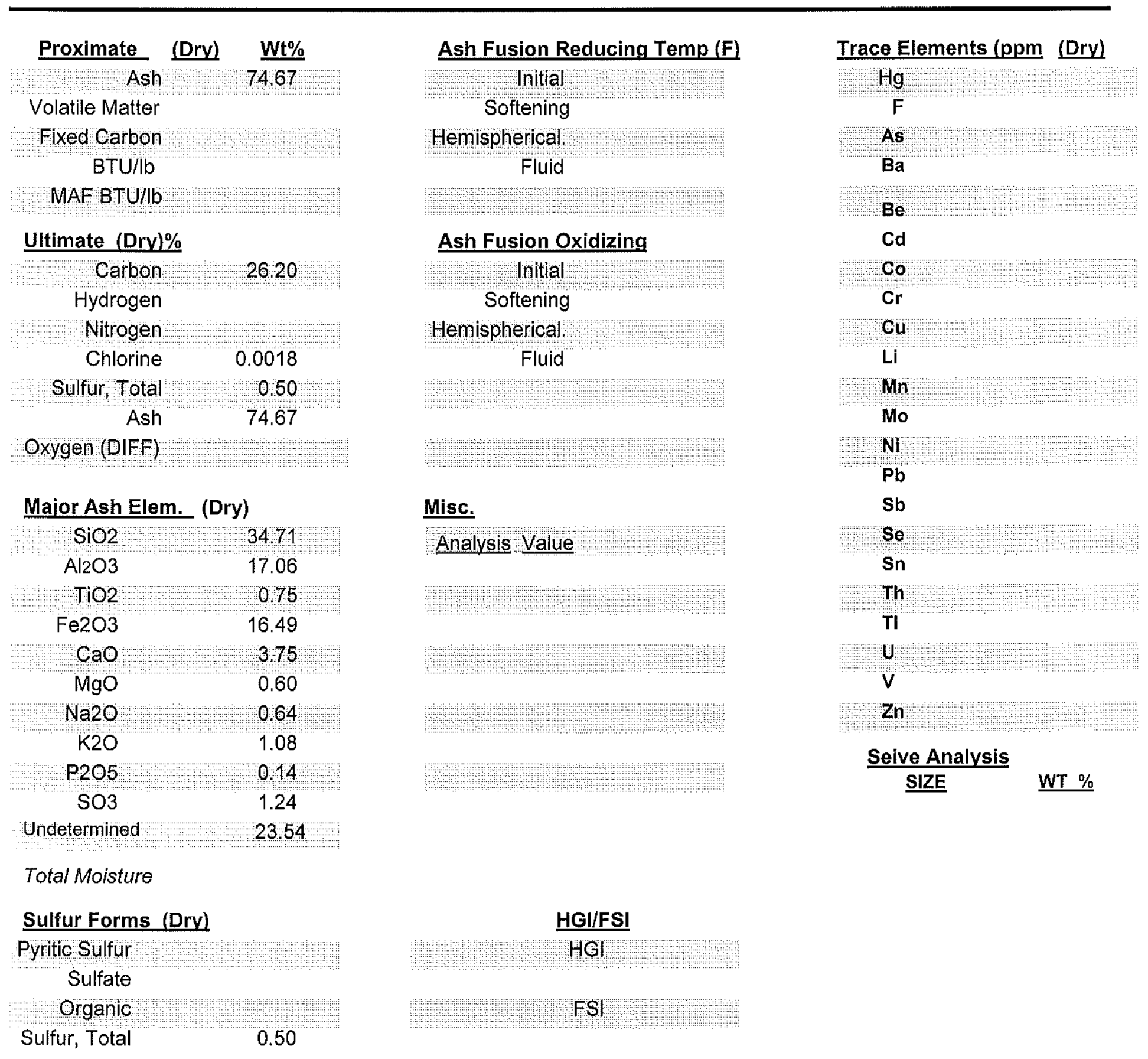

As Determined Moisture $0.11 \quad \%$ These values have been reviewed and are approved for transmission. C.

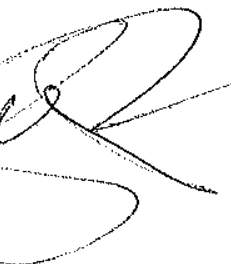


Research and Development

www.consolenergy.com

4000 Brownsville Rd.

South Park, PA 15129

Sample Description: AHO ASH 5/2/07

Sample No.: SO3-3

Date Received: $05 / 11 / 2007$

Date Completed: 06/01/2007

Analytical No.:

20072528

Project No.: $1621 \quad-085 \quad-000$

Submitted By: J. LOCKE/D. CONNELL

\begin{tabular}{|c|c|c|c|}
\hline Proximate (Dry) & $\underline{W t} \%$ & Ash Fusion Reducing Temp (F) & Trace Elements (ppm (Drv) \\
\hline Ash & 79.08 & Initial & $\mathrm{Hg}$ \\
\hline Volatile Matter & & Softening & $F$ \\
\hline Fixed Carbon & & Hemispherical & As \\
\hline BTU/lb & & Fluid & $\mathrm{Ba}$ \\
\hline MAF BTU/L & & १क & $\mathrm{Be}$ \\
\hline Ultimate (Dry) $\%$ & & Ash Fusion Oxidizing & Cd \\
\hline Carbon & 2110 & Initial & Co \\
\hline Hydrogen & & Softening & $\mathrm{Cr}$ \\
\hline Nitrogen & & Hemispherical. & Cu \\
\hline Chlorine & 0.0137 & Fluid & $\mathrm{Li}$ \\
\hline Sulfur, Total & 0.52 & & $\mathrm{Mn}$ \\
\hline Ash & 79.08 & & Mo \\
\hline Oxygen (DIFF) & & & $\mathrm{Ni}$ \\
\hline & & & $\mathrm{Pb}$ \\
\hline Major Ash Elem. (Dr & & Misc. & $\mathrm{Sb}$ \\
\hline $\mathrm{SiO} 2$ & 3721 & Analysis Value & Se \\
\hline $\mathrm{Al} 2 \mathrm{O} 3$ & 17.91 & & Sn \\
\hline $\mathrm{T} / \mathrm{O} 2$ & 0.80 & & Th \\
\hline $\mathrm{Fe} 2 \mathrm{O} 3$ & 14.82 & & $\mathrm{TI}$ \\
\hline $\mathrm{CaO}$ & 4.12 & & $\mathrm{O}$ \\
\hline $\mathrm{MgO}$ & 0.71 & & V \\
\hline $\mathrm{Na} 2 \mathrm{O}$ & 0.72 & ? & Zn \\
\hline $\mathrm{K} 2 \mathrm{O}$ & 1.31 & & Seive Analysis \\
\hline$P 205$ & 0.19 & & SIZE \\
\hline $\mathrm{SO} 3$ & 1.30 & & \\
\hline Undetermined & 20.91 & & \\
\hline \multicolumn{4}{|l|}{ Total Moisture } \\
\hline \multicolumn{3}{|l|}{ Sulfur Forms (Dry) } & \\
\hline $\begin{array}{l}\text { Pyritic Sulfur } \\
\text { Sulfate }\end{array}$ & & \multirow[t]{3}{*}{$\mathrm{HGI}$} & \\
\hline Y Organic & & & \\
\hline Sulfur, Total & 0.52 & & \\
\hline
\end{tabular}

As Determined Moisture $0.79 \quad \%$

These values have been reviewed and are approved for transmission. grr 

Research and Development
uww.consolenergy.com
4000 Brownsville Rd.
South Park, PA 15129

Sample Description.: AHO ASH 5/4/07

Sample No:: $A G-1$

Analytical No:: $\quad 20072529$

Date Received: 05/11/2007

Project No.: $1621-085-000$

Date Completed: 06/01/2007

Submitted By: J. LOCKE/D. CONNELL

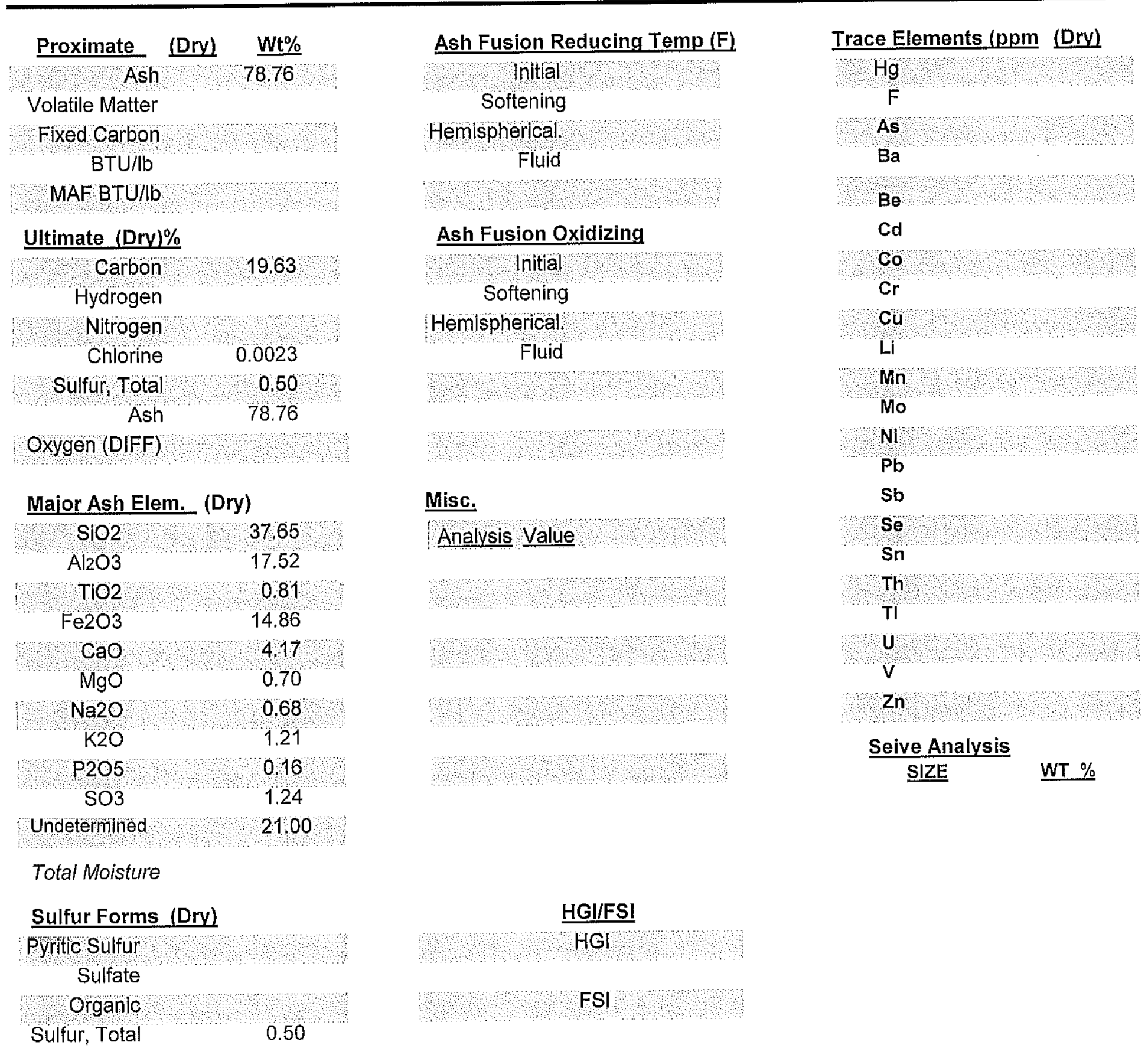

As Determined Moisture $0.19 \quad \%$

These values have been reviewed and are approved for transmission. 


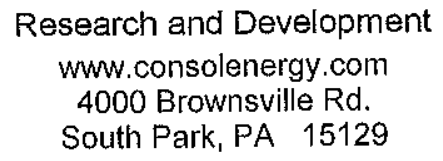

Sample Description.: AHO ASH 5/4/07

Sample No.: $A G-2$

Analytical No.: $\quad 20072530$

Date Received: 05/11/2007

Project No.: $1621 \quad-085 \quad-000$

Date Completed: 06/01/2007

Submitted By: J. LOCKE/D. CONNELL

\begin{tabular}{|c|c|}
\hline Proximate (Dry) & $\underline{W t} \%$ \\
\hline Ash & 80.01 \\
\hline \multicolumn{2}{|l|}{ Volatile Matter } \\
\hline \\
\hline \multicolumn{2}{|l|}{ BTU/b } \\
\hline \multicolumn{2}{|l|}{ MAF BTU/b } \\
\hline \multicolumn{2}{|l|}{ Ultimate (Dry) $\%$} \\
\hline Carbon & 1771 \\
\hline \multicolumn{2}{|l|}{ Hydrogen } \\
\hline \multicolumn{2}{|l|}{ Nitrogen } \\
\hline Chlorine & 0.0060 \\
\hline Sulfur, Total & 0.65 \\
\hline Ash & 80.01 \\
\hline \multicolumn{2}{|l|}{ Oxygen (DIFF) } \\
\hline \multicolumn{2}{|c|}{ Major Ash Elem. (Dry) } \\
\hline SiO2 & 38.72 \\
\hline $\mathrm{Al} 2 \mathrm{O}_{3}$ & 18.73 \\
\hline $\mathrm{TIO} 2$ & 0.89 \\
\hline $\mathrm{Fe} 2 \mathrm{O} 3$ & 12.99 \\
\hline $\mathrm{CaO}$ & 4.23 \\
\hline $\mathrm{MgO}$ & 0.76 \\
\hline $\mathrm{Na} 20$ & 075 \\
\hline $\mathrm{K} 2 \mathrm{O}$ & 1.32 \\
\hline $\mathrm{P} 205$ & 0.18 \\
\hline $\mathrm{SO} 3$ & 1.60 \\
\hline Undetermined & 19.83 \\
\hline
\end{tabular}

Ash Fusion Reducing Temp (F)

Initial

Softening

Hemispherical:

Fluid

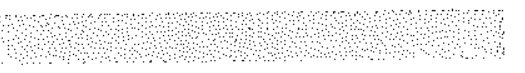

Ash Fusion Oxidizing

Initial

Softening

Hemispherical:

Fluid

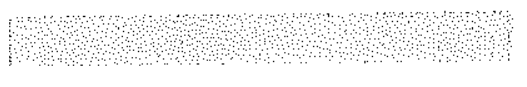

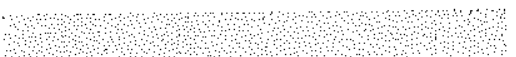

Misc.

Analysis Value
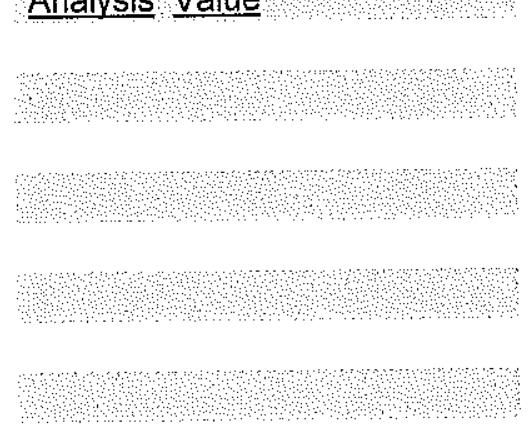

Trace Elements (ppm (Dry)

$\mathrm{Hg}$

$\mathrm{F}$

As

$\mathrm{Ba}$

$\mathrm{Be}$

$\mathrm{Cd}$

co

$\mathrm{Cr}$

$\mathrm{Cu}$

$\mathrm{Li}$

$\mathrm{Mn}$

Mo

Ni

$\mathrm{Pb}$

$\mathrm{Sb}$

Se

Sn

Th

TI

$\mathrm{U}$

V

Zn

Seive Analysis SIZE
HGI/FSI

$\mathrm{HGI}$

FSI
Organic

Sulfur, Total

As Determined Moisture $0.905 \quad \%$

These values have been reviewed and are approved for transmission. 7 a 
Research and Development

www.consolenergy.com

4000 Brownsville Rd.

South Park, PA 15129

Sample Description.: TURBOSORB MARCH 28 11:30

Sample No.: TEST 1

Date Received: 04/03/2007

Analytical No.:

20071805

Project No.: $1621-085-000$

Date Completed: 04/19/2007

Submitted By: D. CONNELL

Proximate $\frac{\text { (Drv) }}{\text { Wt } \%}$
81.48
Volatile Matter
Fixed Carbon
BTU/lb
MAFBTU/lb

Ultimate (Dry)\%

Carbon

19.10

Hydrogen

Nitrogen

Chlorine

0.0270

Sulfur, Total

Ash

81.48

Oxygen (DIFF)

Major Ash Elem. (Dry)

$\mathrm{SiO} 2$

25.86

$\mathrm{Al} 2 \mathrm{O} 3$

11.66

$\mathrm{TiO} 2$

0.46

$\mathrm{Fe} 2 \mathrm{O} 3$

37.54

$\mathrm{CaO}$

4.91

$\mathrm{MgO}$

0.46

$\mathrm{Na} 2 \mathrm{O}$

0.38

$\mathrm{K} 2 \mathrm{O}$

0.69

$\mathrm{P} 205$

0.28

$\mathrm{SO} 3$

1.50

16.26
Ash Fusion Reducing Temp (F)

Initial

Softening

Hemispherical.

Fluid

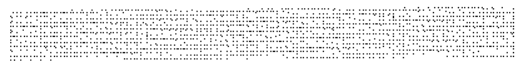

Ash Fusion Oxidizing

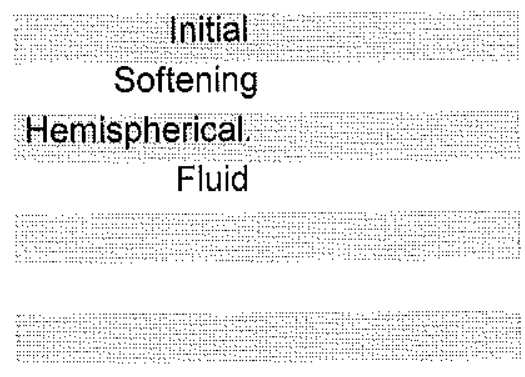

Misc.

\section{Analysis Value}
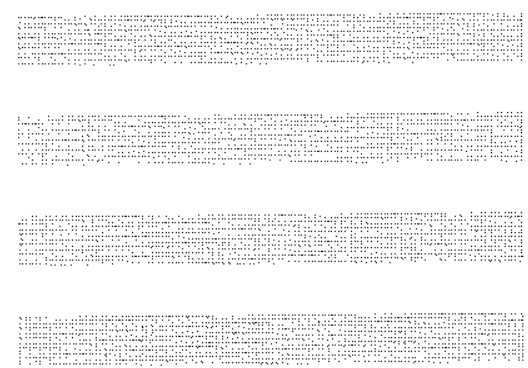

Trace Elements (ppm (Dry)

$\mathrm{Hg} .0 .134$

$\begin{array}{ll}F & 58.16\end{array}$

As

$\mathrm{Ba}$

$\mathrm{Be}$

$\mathrm{Cd}$

co

$\mathrm{Cr}$

$\mathrm{Cu}$

$\mathrm{Li}$

$\mathrm{Mn}$

Mo

Ni

$\mathrm{Pb}$

$\mathrm{Sb}$

Se

Sn

Th

$\mathrm{TI}$

u

V

Zn

Seive Analysis

SIZE

\section{HGI/FSI}

HGI

FSI

Organic

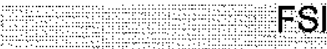

Sulfur, Total

As Determined Moisture $0.1 \quad \%$

These values have been reviewed and are approved for transmission. 7 f 
Research and Development

www.consolenergy.com

4000 Brownsville Rd.

South Park, PA 15129

Sample Description.: TURBOSORB MARCH 28 16:00

Sample No.: TEST 2

Date Received: 04/03/2007

Date Completed: 04/19/2007

Analytical No.: $\quad 20071806$

Project No.: $1621 \quad-085 \quad-000$

Submitted By: D. CONNELL

\begin{tabular}{|c|c|}
\hline Proximate (Dry) & $\underline{\mathrm{Wt}} \%$ \\
\hline Ash & 82.45 \\
\hline \multicolumn{2}{|l|}{ Volatile Matter } \\
\hline \multicolumn{2}{|l|}{ Fixed Carbon } \\
\hline \multicolumn{2}{|l|}{ BTU $/ \mathrm{lb}$} \\
\hline \multicolumn{2}{|l|}{ MAF BTU/lb } \\
\hline \multicolumn{2}{|l|}{ Ultimate (Dry)\% } \\
\hline C Carbon & 1799 \\
\hline \multicolumn{2}{|l|}{ Hydrogen } \\
\hline \multicolumn{2}{|l|}{ Nitrogen } \\
\hline Chlorine & 0.0180 \\
\hline \multicolumn{2}{|l|}{ Sulfur, Total } \\
\hline Ash & 82.45 \\
\hline \multicolumn{2}{|l|}{ Oxygen (DIFF) } \\
\hline \multicolumn{2}{|c|}{ Major Ash Elem. (Dry) } \\
\hline $\mathrm{SIO} 2$ & 25.48 \\
\hline $\mathrm{Al} 2 \mathrm{O} 3$ & 10.36 \\
\hline $\mathrm{TiO} 2$ & 0.46 \\
\hline $\mathrm{Fe} 2 \mathrm{O} 3$ & 40.28 \\
\hline $\mathrm{CaO}$ & 7.06 \\
\hline $\mathrm{MgO}$ & 0.54 \\
\hline $\mathrm{Na} 2 \mathrm{O}$ & 0.31 \\
\hline $\mathrm{K} 2 \mathrm{O}$ & 0.54 \\
\hline $\mathrm{P} 205$ & 0.35 \\
\hline $\mathrm{SO} 3$ & 2.10 \\
\hline Undetermined & 12.52 \\
\hline
\end{tabular}

Ash Fusion Reducing Temp (F)

Initial

Softening

Hemispherical.

Fluid

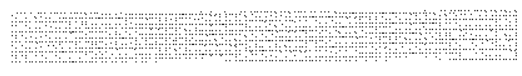

Ash Fusion Oxidizing

Initial

Softening

Hemispherical

Fluid

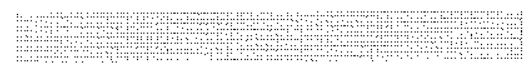

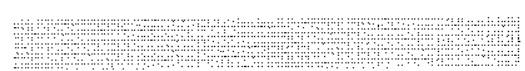

Misc.
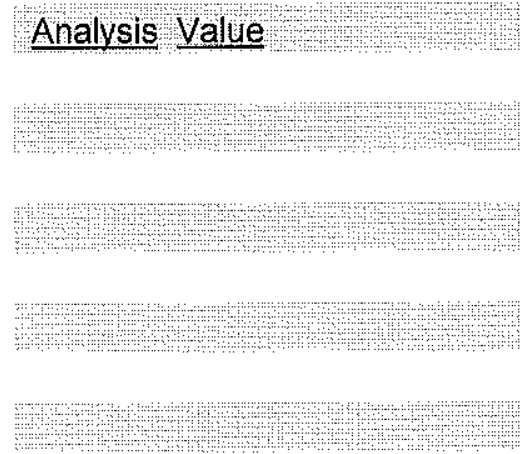

Trace Elements (ppm (Drv)

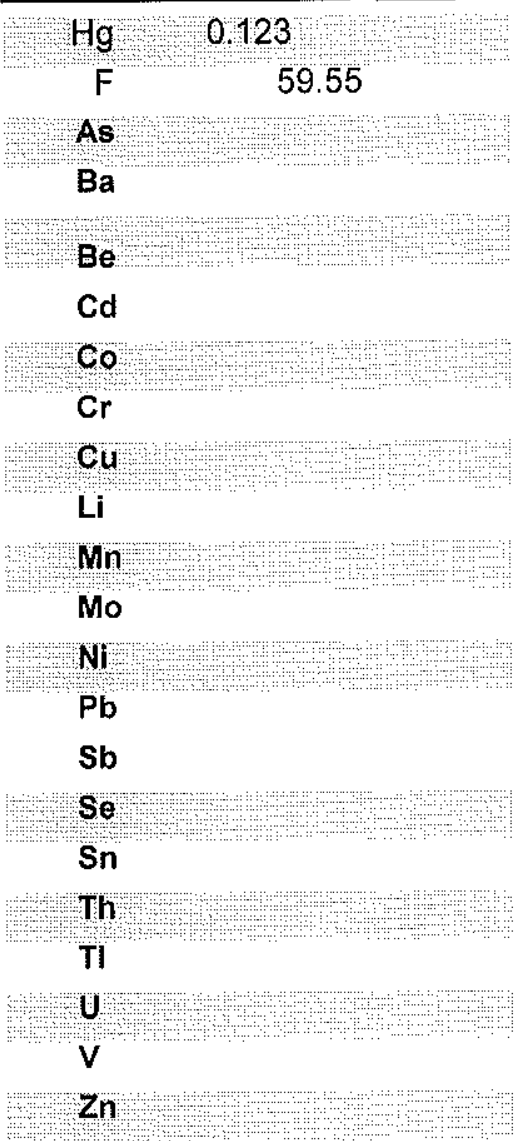

Seive Analysis SIZE
HGI/FSI

$\mathrm{HGI}$

FSI

Sulfur Forms (Drv)

Pyritic Sulfur

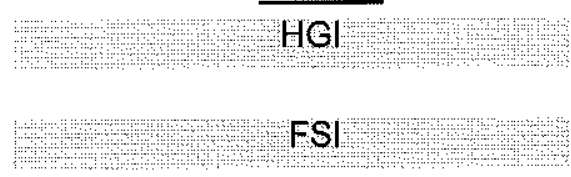

Sulfur, Total

Sulfate

Organic

As Determined Moisture $0.09 \quad \%$

These values have been reviewed and are approved for transmission. $7 m$ 


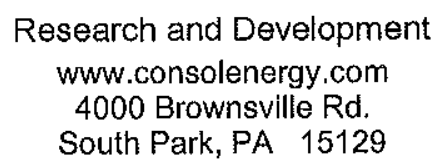

Sample Description.: TURBOSORB MARCH 28 20:00

Sample No.: TEST 3

Analytical No.:

20071807

Date Received: 04/03/2007

Project No.: $1621-085-000$

Date Completed: $04 / 19 / 2007$

Submitted By: D. CONNELL

Proximate (Dry) $\frac{\text { Wt\% }}{\text { Ash }} \quad 79.20$
Volatile Matter
Fixed Carbon
BTU $/ \mathrm{lb}$
MAF BTU/lb

Ultimate (Dry)\%

Carbon
Hydrogen
Nitrogen
Chlorine
C

Sulfur, Total Ash $\quad 79.20$

Oxygen (DIFF)

Major Ash Elem. (Dry)

\begin{tabular}{cc}
$\mathrm{SiO} 2$ & 26.31 \\
$\mathrm{Al} 2 \mathrm{O} 3$ & 11.96 \\
$\mathrm{~T} 1 \mathrm{O} 2$ & 0.47 \\
$\mathrm{Fe} 2 \mathrm{O} 3$ & 32.91 \\
$\mathrm{CaO}$ & 5.10 \\
$\mathrm{MgO}$ & 0.49 \\
$\mathrm{Na} 2 \mathrm{O}$ & 0.38 \\
$\mathrm{~K} 2 \mathrm{O}$ & 0.70 \\
$\mathrm{P} 2 \mathrm{O} 5$ & 0.32 \\
$\mathrm{SO} 3$ & 1.52 \\
determined & \\
\hline
\end{tabular}

Total Moisture

\section{Sulfur Forms (Dry)}

Pyritic Sulfur

$$
\text { Sulfate }
$$

Organic

Sulfur, Total

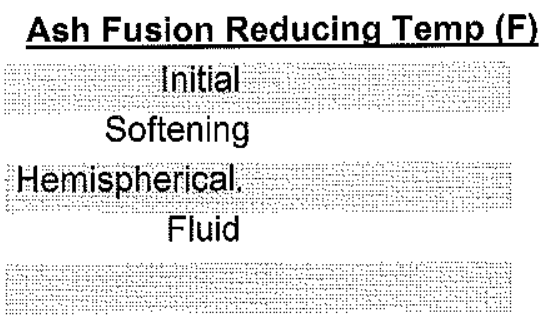

\section{Ash Fusion Oxidizing}

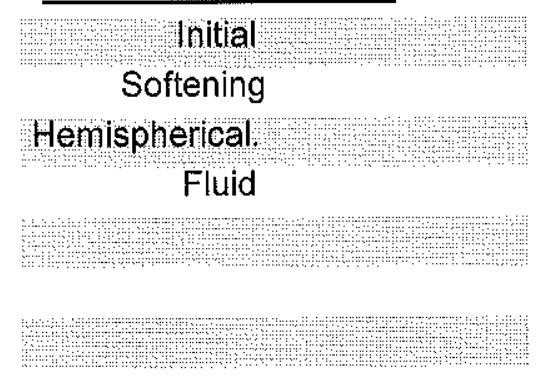

Misc.

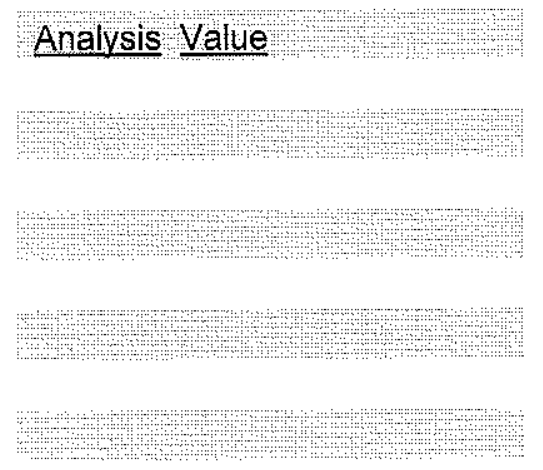

Trace Elements (ppm (Dry)

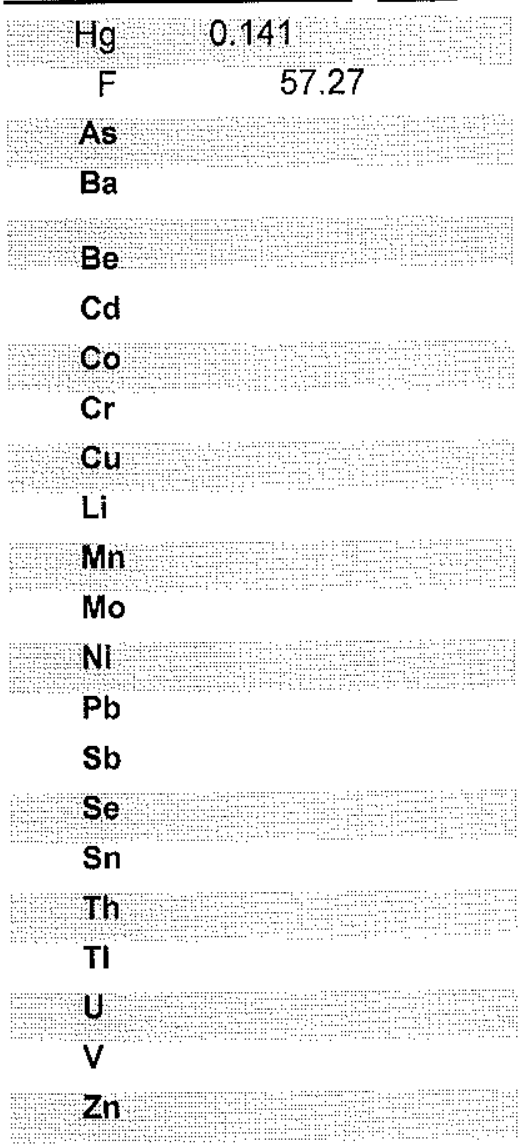

Seive Analysis SIZE
HGI/FSI

$\mathrm{HGl}$

FSI

As Determined Moisture $0.12 \quad \%$

These values have been reviewed and are approved for transmission. $\mathrm{A} \gamma$ 
Research and Development

www.consolenergy.com

4000 Brownsville Rd.

South Park, PA 15129

Sample Description.: TURBOSORB MARCH 30 10:00

Sample No.: TEST 1

Date Received: 04/03/2007

Date Completed: $04 / 19 / 2007$

Analytical No::

20071835

Project No.: $1621-085-000$

Submitted By: D. CONNELL

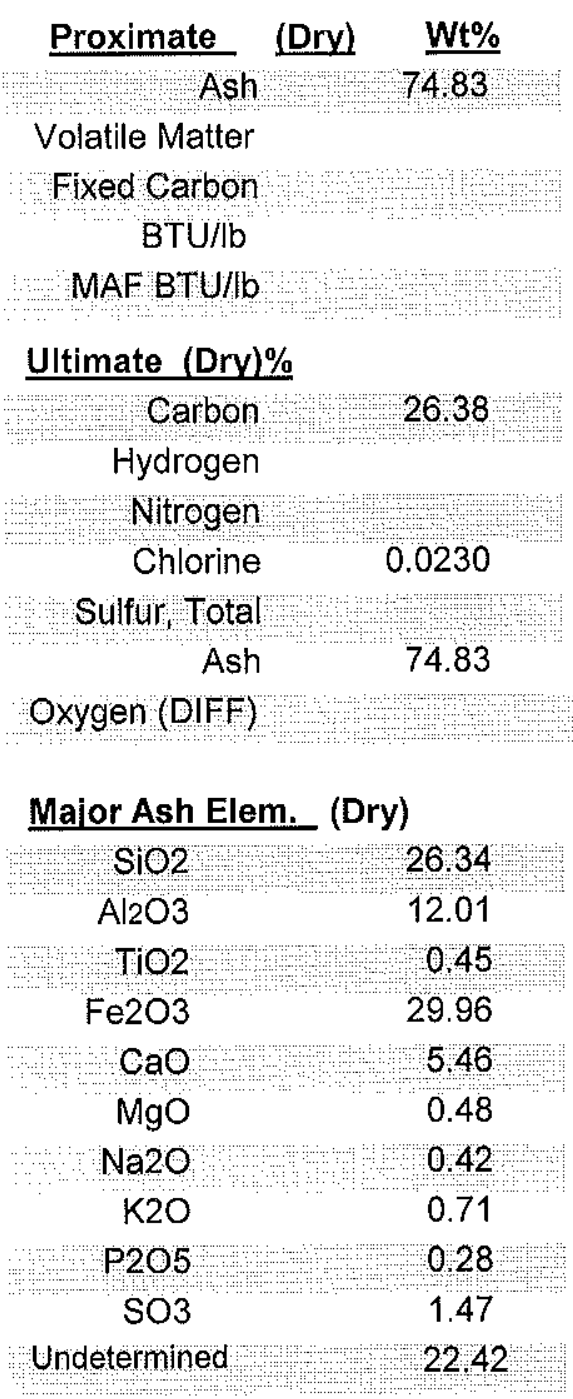

Ash Fusion Reducing Temp (F)

Initial.
Softening

Hemispherical.

Fluid

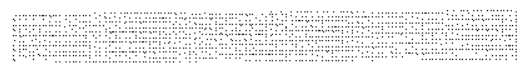

\section{Ash Fusion Oxidizing}

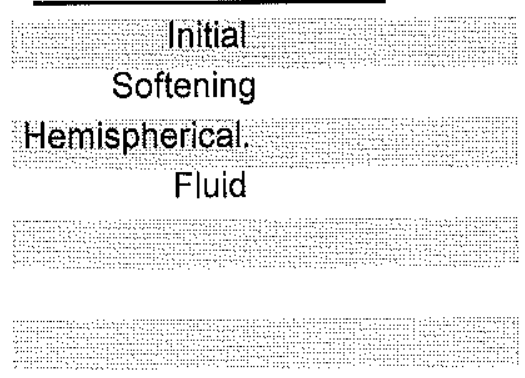

Misc.
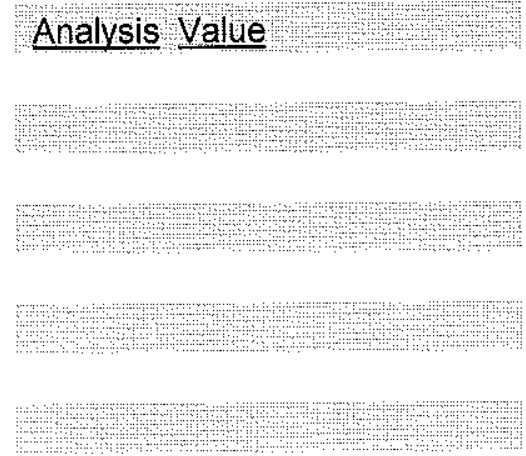

Trace Elements (ppm (Drv)

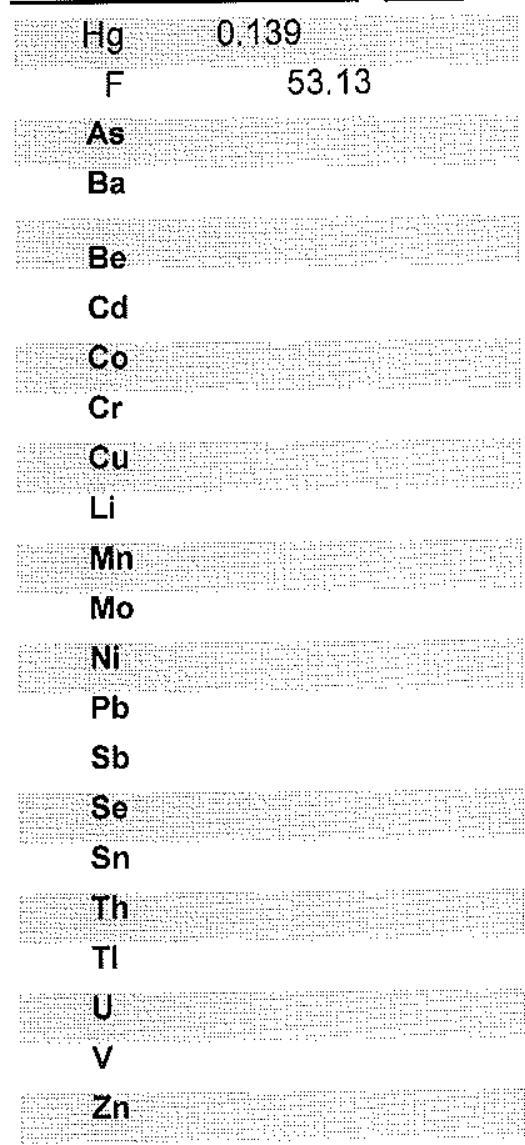

Seive Analysis SIZE
HGI/FSI

$\mathrm{HGl}$

FSI

Organic

Sulfur, Total

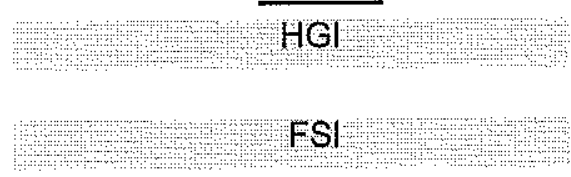

As Determined Moisture $0.06 \quad \%$

These values have been reviewed and are approved for transmission? 
Research and Development www.consolenergy.com 4000 Brownsville Rd.

South Park, PA 15129

Sample Description.: TURBOSORB MARCH 30 13:45

Sample No.: TEST 2

Analytical No:

20071836

Date Received: $04 / 03 / 2007$

Project No.: $1621 \quad-085-000$

Date Completed: 04/19/2007

Submitted By: D. CONNELL

\begin{tabular}{|c|c|c|}
\hline Proximate & (Dry) & $\mathbf{W t} \%$ \\
\hline Ash & & 82.78 \\
\hline \multicolumn{3}{|l|}{ Volatile Matter } \\
\hline \multicolumn{3}{|l|}{ Fixed Carbon } \\
\hline \multicolumn{3}{|l|}{$\mathrm{BTU} / \mathrm{lb}$} \\
\hline \multicolumn{3}{|l|}{$\mathrm{MAF} \mathrm{BTU} / \mathrm{lb}$} \\
\hline \multicolumn{3}{|c|}{ Ultimate (Drv)\% } \\
\hline Carbon & & 16.36 \\
\hline \multicolumn{3}{|l|}{ Hydrogen } \\
\hline \multicolumn{3}{|l|}{ Nitrogen } \\
\hline Chlorine & & 0.0160 \\
\hline \multicolumn{3}{|l|}{ Sulfur, Total } \\
\hline Ash & & 82.78 \\
\hline Oxygen (DIFF) & & \\
\hline
\end{tabular}

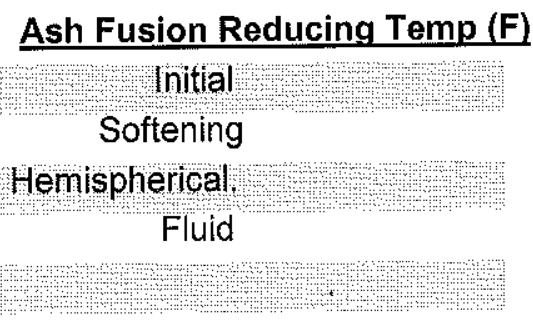

Ash Fusion Oxidizing

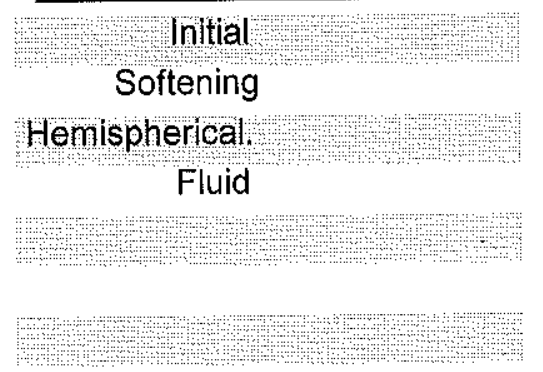

Misc.

Major Ash Elem. (Dry)

$\begin{array}{cc}\mathrm{SiO} 2 & 26.73 \\ \mathrm{Al} 2 \mathrm{O} 3 & 12.18 \\ \mathrm{TlO} 2 & 0.48 \\ \mathrm{Fe} 2 \mathrm{O} 3 & 34.00 \\ \mathrm{CaO} & 8.09 \\ \mathrm{MgO} & 0.54 \\ \mathrm{Na} 2 \mathrm{O} & 0.42 \\ \mathrm{~K} 2 \mathrm{O} & 0.71 \\ \mathrm{P} 2 \mathrm{O} 5 & 0.26 \\ \mathrm{SO} 3 & 2.38 \\ \text { determined } & \\ \end{array}$

Total Moisture

\section{Sulfur Forms (Dry)}

Pyritic Sulfur

$$
\text { Sulfate }
$$

Organic

Sulfur, Total
HGI/FSI

HGI

FSI
Trace Elements (ppm (Drv)

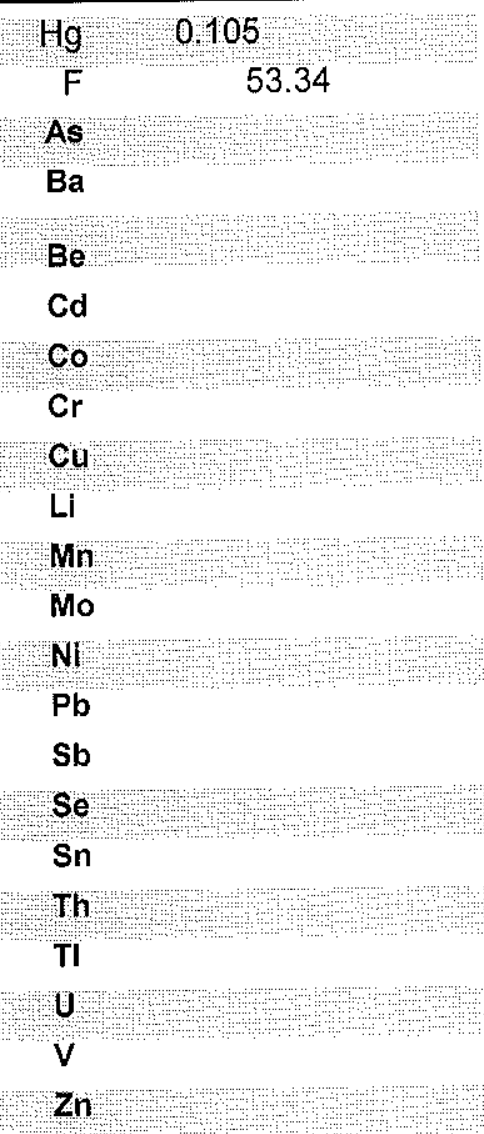

Seive Analysis SIZE

As Determined Moisture $0.08 \quad \%$

These values have been reviewed and are approved for transmission. 


\title{
TONSOLENERGY
}

\author{
Research and Development \\ www.consolenergy.com \\ 4000 Brownsville Rd. \\ South Park, PA 15129
}

Sample Description.: TURBOSORB MARCH 30 16:45

Sample No.: TEST 3

Analytical No.:

Date Received: 04/03/2007

Project No.: $1621 \quad-085-000$

Date Completed: 04/19/2007

Submitted By: D. CONNELL

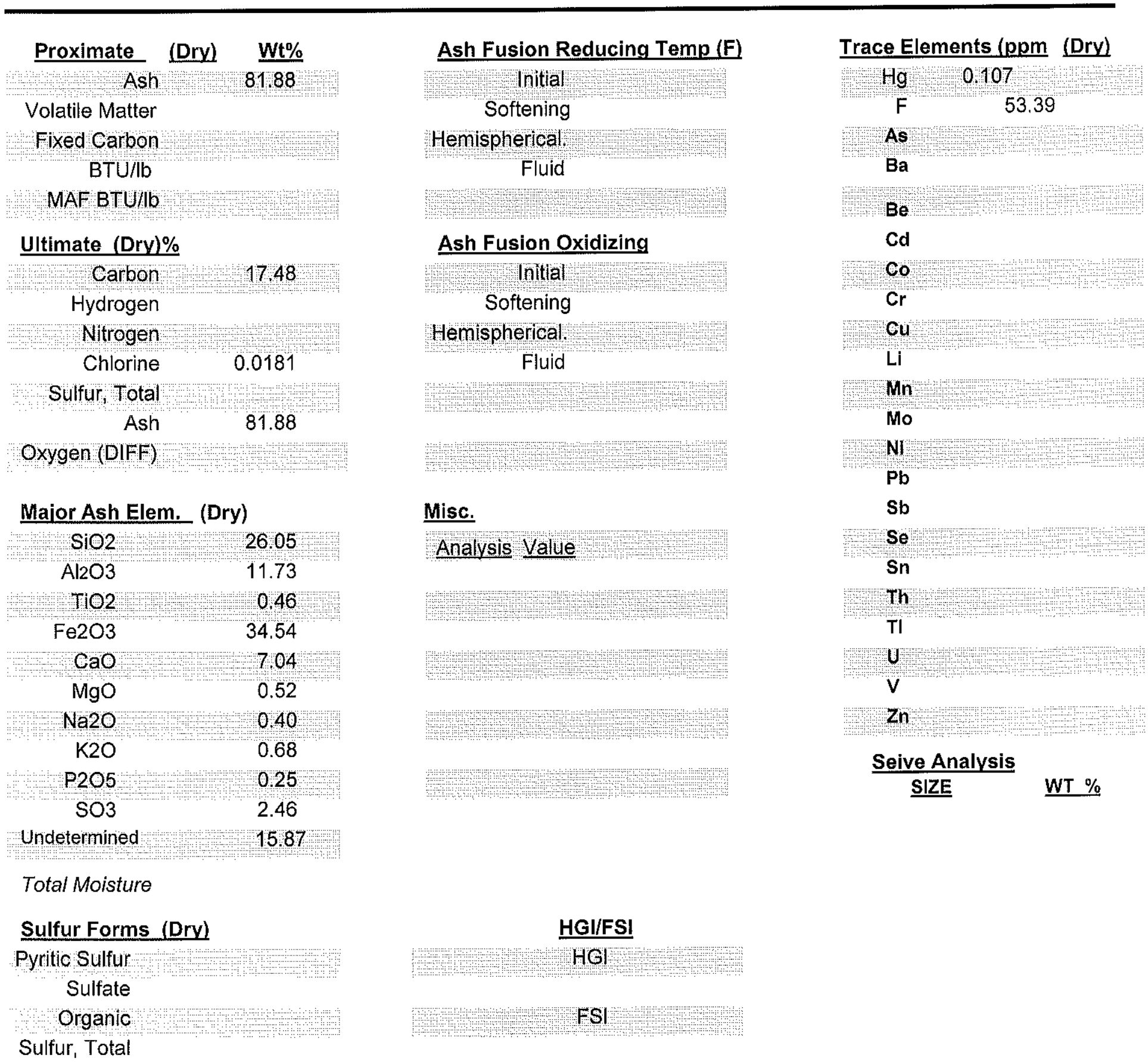

As Determined Moisture $0.35 \quad \%$
These values have been reviewed and are approved for transmission. 


\section{击E CONSOLENERGY.}

Research and Development

www.consolenergy.com

4000 Brownsville Rd.

South Park, PA 15129

Sample Description.: PRODUCT/FLY ASH MARCH 28 10:00

Sample No.: TEST 1

Date Received: 04/03/2007

Analytical No.:

20071799

Project No.: $1621-085-000$

Date Completed: 04/19/2007

Submitted By: D. CONNELL

\begin{tabular}{|c|c|c|c|}
\hline Proximate (Dry) & $\underline{\mathbf{w}} \%$ & Ash Fusion Reducing Temp (F) & Trace Elements (ppm (Dry) \\
\hline Ash & 84.57 & Initial & $1 \mathrm{Hg}=0.346$ \\
\hline Volatile Matter & & Softening & $\begin{array}{ll}F & 80.74\end{array}$ \\
\hline Fixed Carbon & & Hemispherical. & As \\
\hline $\mathrm{BTU} / \mathrm{lb}$ & & Fluid & $\mathrm{Ba}$ \\
\hline MAF BTU/b & +4 & 3 & $\mathrm{Be}$ \\
\hline Ultimate (Drv)\% & & Ash Fusion Oxidizing & Cd \\
\hline Carbon & 8.05 & $\ln \mid \operatorname{lial}$ & Co \\
\hline Hydrogen & & Softening & $\mathrm{Cr}$ \\
\hline Nitrogen & & Hemispherical & $\mathrm{Cu}$ \\
\hline Chlorine & 0.2762 & Fluid & $\mathrm{Li}$ \\
\hline Sulfur, Total & 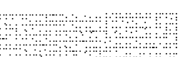 & & Mn \\
\hline Ash & 84.57 & & Mo \\
\hline Oxygen (DIFF) & $\mathrm{Sutu}_{4}$ & 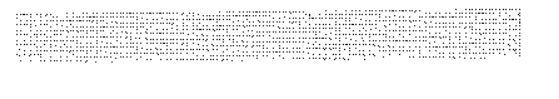 & $\begin{array}{r}\mathrm{Ni} \\
\mathrm{Pb}\end{array}$ \\
\hline Major Ash Elem. ( & & Misc. & $\mathrm{Sb}$ \\
\hline $\mathrm{SiO} 2$ & 12.28 & Analysis Value & Se \\
\hline $\mathrm{Al} 2 \mathrm{O} 3$ & 6.08 & & Sn \\
\hline $\mathrm{THO} 2$ & 0.26 & is & Th \\
\hline $\mathrm{Fe} 2 \mathrm{O} 3$ & 4.55 & & $\mathrm{TI}$ \\
\hline $\mathrm{CaO}$ & 37.53 & ; & U \\
\hline $\mathrm{MgO}$ & 0.64 & & V \\
\hline $\mathrm{Na} 2 \mathrm{O}$ & 0.32 & + & $\mathrm{Zn}$ \\
\hline $\mathrm{K} 2 \mathrm{O}$ & 0.45 & & Seive Analysis \\
\hline $\mathrm{P} 205$ & 0.14 & & SIZE \\
\hline $\mathrm{SO} 3$ & 22.79 & & \\
\hline Undetermined & 14.96 & & \\
\hline \multicolumn{4}{|l|}{ Total Moisture } \\
\hline \multicolumn{2}{|l|}{ Sulfur Forms (Drv) } & HGI/FSI & \\
\hline \multicolumn{2}{|l|}{ Pyritic Sulfur } & $\mathrm{HGl}$ & \\
\hline $\begin{array}{l}\text { Organic } \\
\text { Sulfur, Total }\end{array}$ & 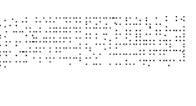 & $\mathrm{FSl}$, & \\
\hline
\end{tabular}

As Determined Moisture $0.79 \quad \%$

These values have been reviewed and are approved for transmission 7 m 


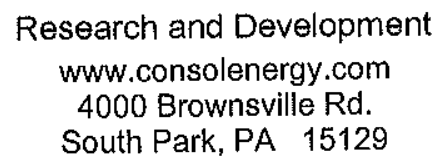

Sample Description.: PRODUCT/FLY ASH MARCH 28 11:00

Sample No:: TEST 1

Date Received: 04/03/2007

Analytical No: $\quad 20071800$

Project No.: $1621-085-000$

Date Completed: $04 / 19 / 2007$

Submitted By: D. CONNELL

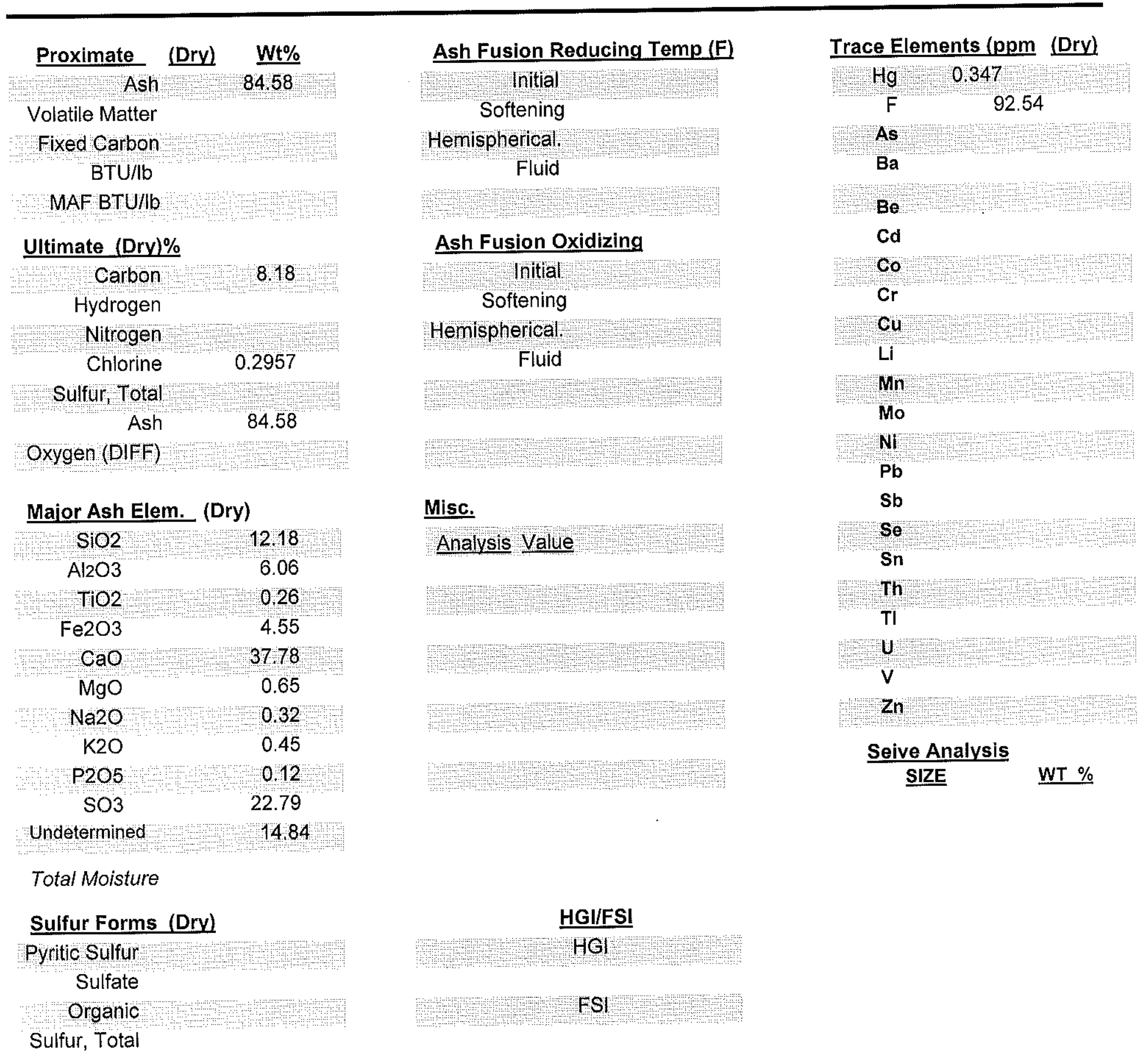

As Determined Moisture $0.91 \quad \%$

These values have been reviewed and are approved for transmission. J $m$ P 


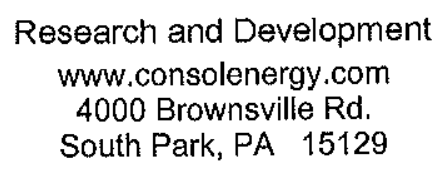

Sample Description.: PRODUCT/FLY ASH MARCH 28 14:00

Sample No.: TEST 2

Date Received: 04/03/2007

Analytical No.: $\quad 20071801$

Project No.: $1621-085-000$

Date Completed: 04/19/2007

Submitted By: D. CONNELL

\begin{tabular}{|c|c|}
\hline Proximate (Dry) & $\underline{\text { Wt } \%}$ \\
\hline Ash & 84,37 \\
\hline \multicolumn{2}{|l|}{ Volatile Matter } \\
\hline \multicolumn{2}{|l|}{ Fixed Carbon } \\
\hline \multicolumn{2}{|l|}{$\mathrm{BTU} / \mathrm{b}$} \\
\hline \multicolumn{2}{|l|}{ MAF BTU/L } \\
\hline \multicolumn{2}{|l|}{ Ultimate (Drv)\% } \\
\hline Carbon & 8.13 \\
\hline \multicolumn{2}{|l|}{ Hydrogen } \\
\hline \multicolumn{2}{|l|}{ Nitrogen } \\
\hline Chlorine & 0.2909 \\
\hline \multicolumn{2}{|l|}{ Sulfur, Total } \\
\hline Ash & 84.37 \\
\hline \multicolumn{2}{|l|}{ Oxygen (DIFF) } \\
\hline \multicolumn{2}{|c|}{ Major Ash Elem. (Dry) } \\
\hline $\mathrm{SiO} 2$ & 11.85 \\
\hline $\mathrm{Al} 2 \mathrm{O} 3$ & 5.93 \\
\hline $\mathrm{TiO} 2$ & 0.25 \\
\hline $\mathrm{Fe} 2 \mathrm{O} 3$ & 4.40 \\
\hline $\mathrm{CaO}$ & 38.14 \\
\hline $\mathrm{MgO}$ & 0.64 \\
\hline $\mathrm{Na} 2 \mathrm{O}$ & 0.30 \\
\hline $\mathrm{K} 2 \mathrm{O}$ & 0.43 \\
\hline $\mathrm{P} 205$ & 0.12 \\
\hline $\mathrm{SO} 3$ & 23.11 \\
\hline Undetermined & 14.83 \\
\hline
\end{tabular}

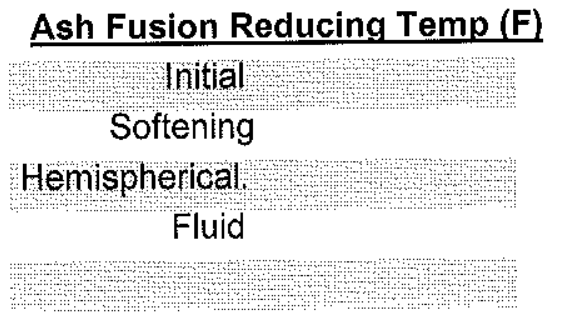

Ash Fusion Oxidizing

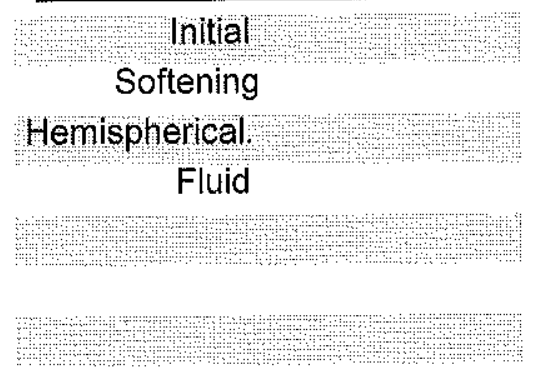

Misc.

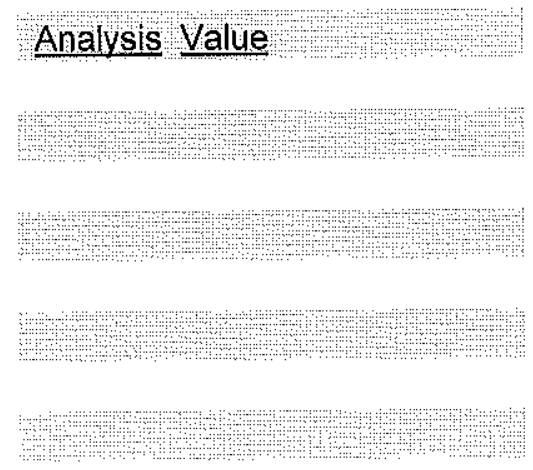

Trace Elements (ppm (Dry)

$\mathrm{Hg} .0 .360$

$F \quad 83.95$

As

$\mathrm{Ba}$

$\mathrm{Be}$

Cd

Co

$\mathrm{Cr}$

Cu

Li

\section{$\mathrm{Mn}$}

Mo

Ni

$\mathrm{Pb}$

$\mathrm{Sb}$

Se

Sn

Th

TI

u

V

Zn

Seive Analysis SIZE

Total Moisture

Sulfur Forms (Dry)

Pyritic Sulfur

HGI/FSI

Sulfate

Organic

Sulfur, Total
$\mathrm{HGl}$

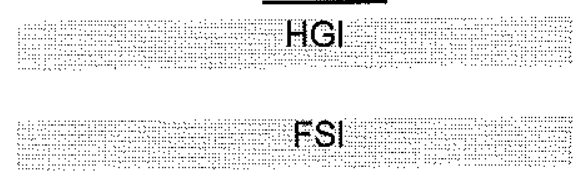

As Determined Moisture $0.65 \quad \%$

These values have been reviewed and are approved for transmission. Yhif 
Research and Development

www.consolenergy.com

4000 Brownsville Rd.

South Park, PA 15129

Sample Description.: PRODUCT/FLY ASH MARCH 28 15:50

Sample No.: TEST 2

Date Received: 04/03/2007

Analytical No.:

20071802

Project No.: $1621-085 \quad-000$

Date Completed: 04/19/2007

Submitted By: D. CONNELL

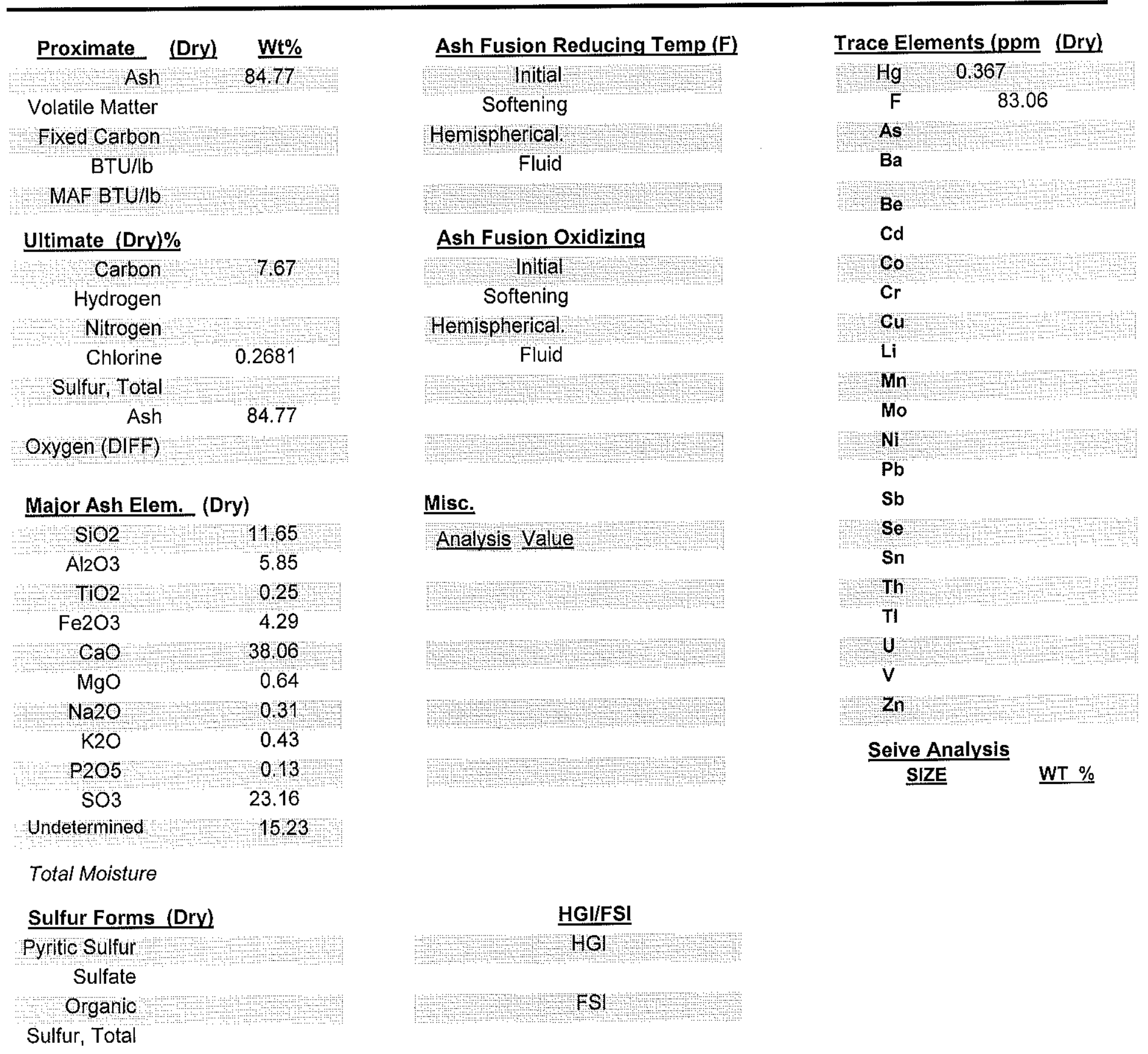

As Determined Moisture $0.8 \quad \%$

These values have been reviewed and are approved for transmission. 

Research and Development
www.consolenergy.com
4000 Brownsville Rd.
South Park, PA 15129

Sample Description.: PRODUCT/FLY ASH MARCH 28 17:15

Sample No.: TEST 3

Date Received: 04/03/2007

Date Completed: 04/19/2007

Analytical No.: $\quad 20071803$

Project No.: $1621-085-000$

Submitted By: D. CONNELL

\begin{tabular}{|c|c|c|c|}
\hline Proximate (Dry) & $\underline{\mathbf{W t} \%}$ & Ash Fusion Reducing Temp (F) & Trace Elements (ppm (Drv) \\
\hline S A Ash & 8478 & Initial & 0.363 \\
\hline Volatile Matter & & Softening & 87.36 \\
\hline Fixed Carbon & 3 & Hemispherical, & As \\
\hline $\mathrm{BTU} / \mathrm{lb}$ & & Fluid & $\mathrm{Ba}$ \\
\hline MAF BTU/G & ?a & 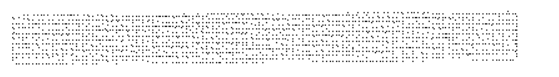 & $\mathrm{Be}$ \\
\hline Ultimate (Dry)\% & & Ash Fusion Oxidizing & $\mathrm{Cd}$ \\
\hline Carbon & 761 & Initial & co \\
\hline Hydrogen & & Softening & $\mathrm{Cr}$ \\
\hline Nitrogen & & Hemispherical. & Cu \\
\hline Chlorine & 0.2781 & Fluid & $\mathrm{Li}$ \\
\hline Sulfur, Total & 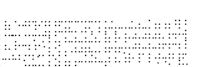 & 3 & $\mathrm{Mn}$ \\
\hline Ash & 84.78 & & Mo \\
\hline Oxygen (DIFF) & +4 & 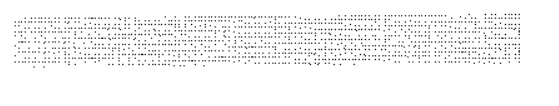 & $\begin{array}{l}\mathrm{NI} \\
\mathrm{Pb}\end{array}$ \\
\hline Major Ash Elem. (L & & Misc. & Sb \\
\hline $\mathrm{SiO} 2$ & 1182 & Analysis Value & Se \\
\hline $\mathrm{Al} 2 \mathrm{O} 3$ & 5.94 & & Sn \\
\hline $\mathrm{T} 102$ & 0.26 & (a) & Th \\
\hline $\mathrm{Fe} 2 \mathrm{O} 3$ & 4.35 & & $\mathrm{TI}$ \\
\hline $\mathrm{CaO}$ & 3777 & & $\mathrm{U}$ \\
\hline $\mathrm{MgO}$ & 0.63 & & v \\
\hline $\mathrm{Na} 20$ & 0.30 & & $\mathrm{Zn}$ \\
\hline $\mathrm{K} 2 \mathrm{O}$ & 0.43 & & Seive Analysis \\
\hline $\mathrm{P} 205$ & 0.11 u & 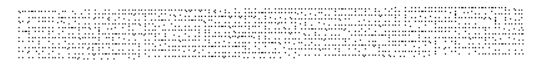 & SIZE \\
\hline $\mathrm{SO} 3$ & 23.16 & & \\
\hline Undetermined & 15.23 & & \\
\hline \multicolumn{4}{|l|}{ Total Moisture } \\
\hline Sulfur Forms (Dry) & & HGI/FSI & \\
\hline $\begin{array}{l}\text { Pyritic Sulfur } \\
\text { Sulfate }\end{array}$ & $+?$ & 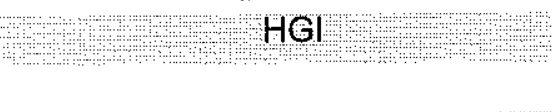 & \\
\hline $\begin{array}{c}\text { Organic } \\
\text { Sulfur, Total }\end{array}$ & + & $F S \mid$ & \\
\hline
\end{tabular}

As Determined Moisture $0.75 \quad \%$

These values have been reviewed and are approved for transmission. In 


\section{을 CONSOLENERGY.}

Research and Development

www.consolenergy.com

4000 Brownsville Rd.

South Park, PA 15129

\section{Sample Description: PRODUCT/FLY ASH MARCH 28 19:20}

Sample No.: TEST 3

Date Received: 04/03/2007

Analytical No.:

Project No.: $1621-085 \quad-000$

Date Completed: $04 / 19 / 2007$

Submitted By: D. CONNELL

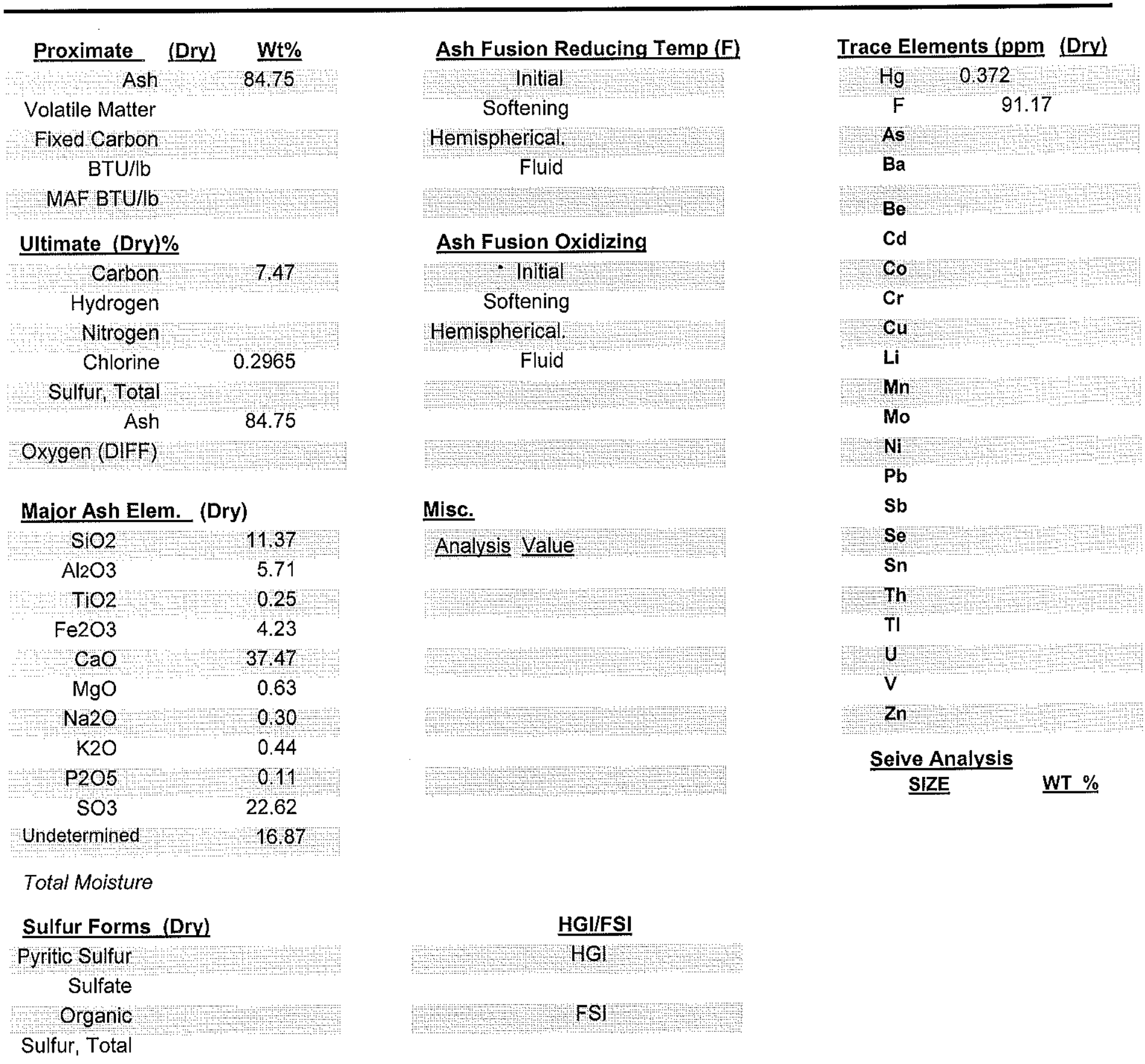

As Determined Moisture $0.84 \quad \%$

These values have been reviewed and are approved for transmission. Th 


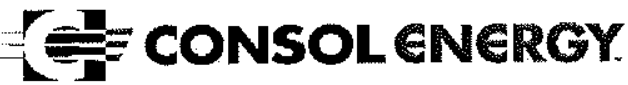

Research and Development

www.consolenergy.com

4000 Brownsville Rd.

South Park, PA 15129

Sample Description.: PRODUCT/FLY ASH MARCH 29 9:30

Sample No.: TEST 1

Date Received: 04/03/2007

Date Completed: 04/19/2007

Analytical No.: $\quad 20071817$

Project No.: $1621-085 \quad-000$

Submitted By: D. CONNELL

\begin{tabular}{|c|c|}
\hline Proximate (Dry) & $\underline{W t} \%$ \\
\hline Ash & 83.72 \\
\hline \multicolumn{2}{|l|}{ Volatile Matter } \\
\hline \multicolumn{2}{|l|}{ Flxed Carbon } \\
\hline \multicolumn{2}{|l|}{$\mathrm{BTU} / \mathrm{lb}$} \\
\hline \multicolumn{2}{|l|}{ MAF BTU//b } \\
\hline \multicolumn{2}{|l|}{ Ultimate (Drv)\% } \\
\hline Carbon & 8.23 \\
\hline \multicolumn{2}{|l|}{ Hydrogen } \\
\hline \multicolumn{2}{|l|}{ Nitrogen } \\
\hline \multicolumn{2}{|l|}{ Chlorine } \\
\hline Sulfur, Total & \\
\hline Ash & 83.72 \\
\hline Oxygen (DIFF) & \\
\hline
\end{tabular}

Major Ash Elem. (Dry)

\begin{tabular}{cc}
$\mathrm{SiO} 2$ & 12.41 \\
$\mathrm{Al} 2 \mathrm{O} 3$ & 6.17 \\
$\mathrm{THO} 2$ & 0.26 \\
$\mathrm{Fe} 2 \mathrm{O} 3$ & 4.62 \\
$\mathrm{CaO}$ & 38.84 \\
$\mathrm{MgO}$ & 0.66 \\
$\mathrm{Na} 2 \mathrm{O}$ & 0.32 \\
$\mathrm{~K} 2 \mathrm{O}$ & 0.46 \\
$\mathrm{P} 2 \mathrm{O} 5$ & 0.11 \\
$\mathrm{SO} 3$ & 21.14 \\
determined & 15.01 \\
\hline
\end{tabular}

Total Moisture

Sulfur Forms (Dry)

Pyritic Sulfur

Sulfate

Organic

Sulfur, Total
Ash Fusion Reducing Temp (F)

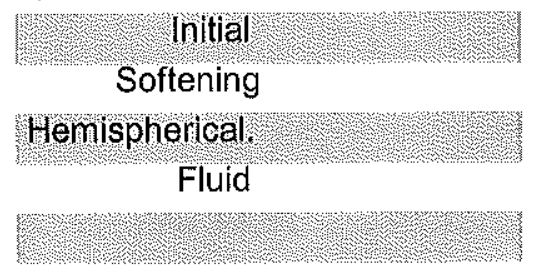

Ash Fusion Oxidizing
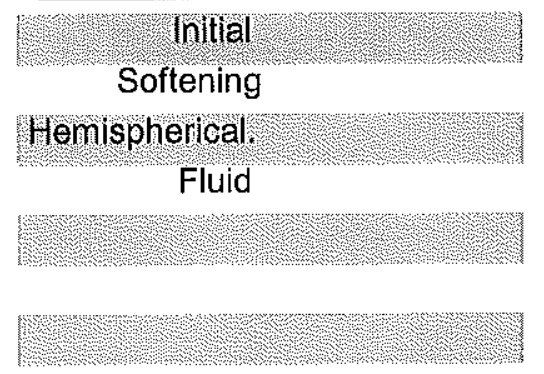

Misc.
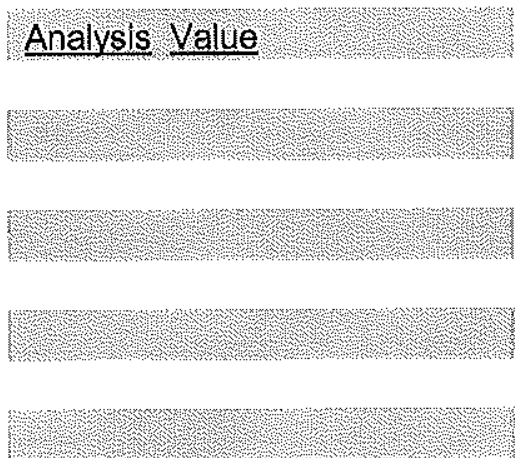

Trace Elements (ppm (Drv)

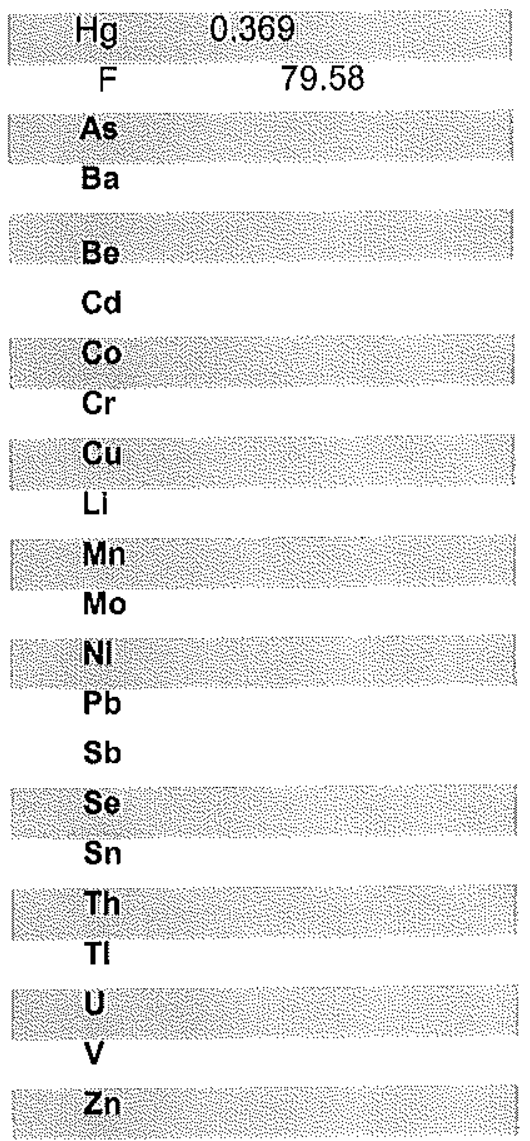

Seive Analysis

SIZE

WT \%
HGI/FSI

461

FSI

As Determined Moisture $0.98 \quad \%$

These values have been reviewed and are approved for transmission 1 i 
Research and Development

www.consolenergy.com

4000 Brownsville Rd.

South Park, PA 15129

Sample Description.: PRODUCT/FLY ASH MARCH 29 13:30

Sample No:: TEST 2

Date Received: $04 / 03 / 2007$

Analytical No.:

Date Completed: $04 / 19 / 2007$

Submitted By: D. CONNELL

\begin{tabular}{|c|c|}
\hline Proximate (Drv) & $\underline{\mathbf{W t}} \%$ \\
\hline Ash & 84.23 \\
\hline \multicolumn{2}{|l|}{ Volatile Matter } \\
\hline \multicolumn{2}{|l|}{ Fixed Carbon } \\
\hline \multicolumn{2}{|l|}{ BTU/lb } \\
\hline \multicolumn{2}{|l|}{ MAF BTU/LO } \\
\hline \multicolumn{2}{|l|}{ Ultimate (Drv)\% } \\
\hline Carbon & 8.07 \\
\hline \multicolumn{2}{|l|}{ Hydrogen } \\
\hline \multicolumn{2}{|l|}{ Nitrogen } \\
\hline \multicolumn{2}{|l|}{ Chlorine } \\
\hline \multicolumn{2}{|l|}{ Sulfur, Total } \\
\hline Ash & 84.23 \\
\hline \multicolumn{2}{|l|}{ Oxygen (DIFF) } \\
\hline \multicolumn{2}{|c|}{ Major Ash Elem. (Dry) } \\
\hline $\mathrm{SIO} 2$ & 12.06 \\
\hline $\mathrm{Al} 2 \mathrm{O} 3$ & 6.05 \\
\hline TTO2 & 0.26 \\
\hline $\mathrm{Fe} 2 \mathrm{O} 3$ & 4.49 \\
\hline $\mathrm{CaO}$ & 38.45 \\
\hline $\mathrm{MgO}$ & 0.65 \\
\hline $\mathrm{Na} 2 \mathrm{O}$ & 0.31 \\
\hline $\mathrm{K} 2 \mathrm{O}$ & 0.45 \\
\hline P2O5 & 0.13 \\
\hline $\mathrm{SO} 3$ & 21.90 \\
\hline Undetermined & 15.25 \\
\hline
\end{tabular}

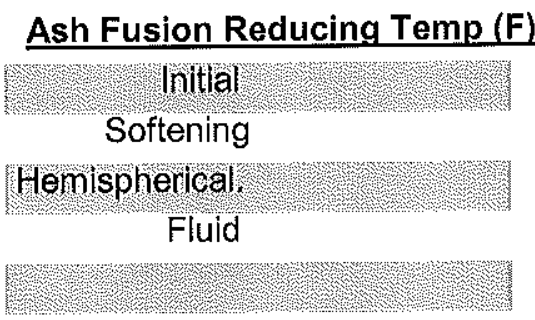

Ash Fusion Oxidizing

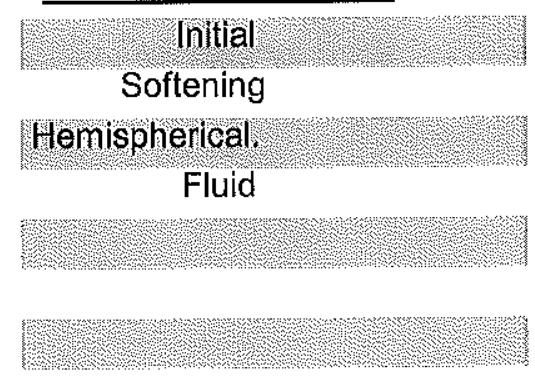

Misc.

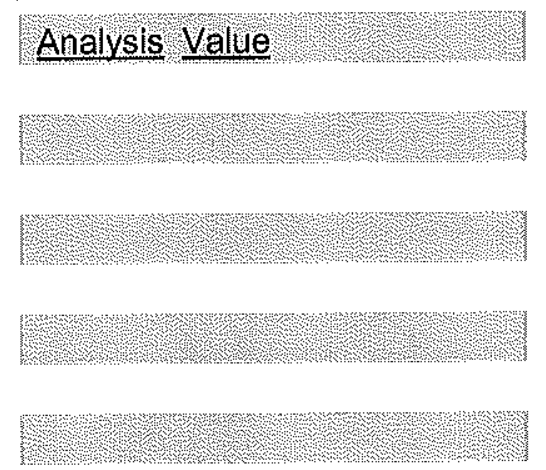

Trace Elements (ppm (Dry)

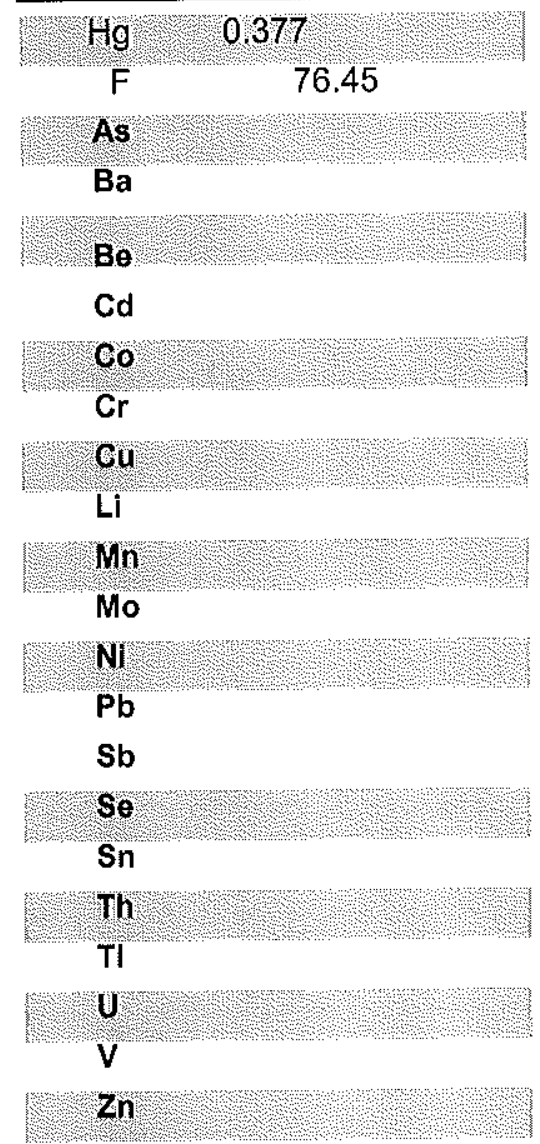

Seive Analysis SIZE

WT $\%$

\section{HGI/FSI}

101

FSI

Organic

Sulfur, Total

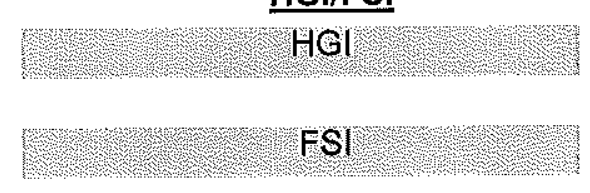

As Determined Moisture $0.98 \quad \%$

These values have been reviewed and are approved for transmission. MP 


\section{F $=$ CONSOL ENERGY}
Research and Development www.consolenergy.com 4000 Brownsville Rd.
South Park, PA 15129

Sample Description.: PRODUCT/FLY ASH MARCH 29 16:30

Sample No.: TEST 3

Date Received: 04/03/2007

Date Completed: 04/19/2007
Analytical No.: $\quad 20071819$

Project No.: $1621 \quad-085-000$

Submitted By: D. CONNELL

\begin{tabular}{|c|c|}
\hline Proximate (Dry) & $\underline{\mathbf{W t} \%}$ \\
\hline Ash & 83.30 \\
\hline \multicolumn{2}{|l|}{ Volatile Matter } \\
\hline \multicolumn{2}{|l|}{ Fixed Carbon } \\
\hline \multicolumn{2}{|l|}{$\mathrm{BTU} / \mathrm{lb}$} \\
\hline \multicolumn{2}{|l|}{ MAF BTU/Ib } \\
\hline \multicolumn{2}{|l|}{ Ultimate (Dry)\% } \\
\hline Carbon & 7.96 \\
\hline \multicolumn{2}{|l|}{ Hydrogen } \\
\hline \multicolumn{2}{|l|}{ Nitrogen } \\
\hline \multicolumn{2}{|l|}{ Chlorine } \\
\hline \multicolumn{2}{|l|}{ Sülfur, Total } \\
\hline Ash & 83.30 \\
\hline \multicolumn{2}{|l|}{ Oxygen (DIFF) } \\
\hline \multicolumn{2}{|c|}{ Major Ash Elem. (Dry) } \\
\hline $\mathrm{SiO} 2$ & 12.03 \\
\hline $\mathrm{Al} 2 \mathrm{O} 3$ & 6.06 \\
\hline $\mathrm{TiO} 2$ & 0.26 \\
\hline $\mathrm{Fe} 2 \mathrm{O} 3$ & 4.33 \\
\hline $\mathrm{CaO}$ & 38.18 \\
\hline $\mathrm{MgO}$ & 0.63 \\
\hline $\mathrm{Na} 2 \mathrm{O}$ & 0.31 \\
\hline $\mathrm{K} 2 \mathrm{O}$ & 0.44 \\
\hline $\mathrm{P} 205$ & 0.14 \\
\hline $\mathrm{SO} 3$ & 21.91 \\
\hline Undetermined & 15.71 \\
\hline
\end{tabular}

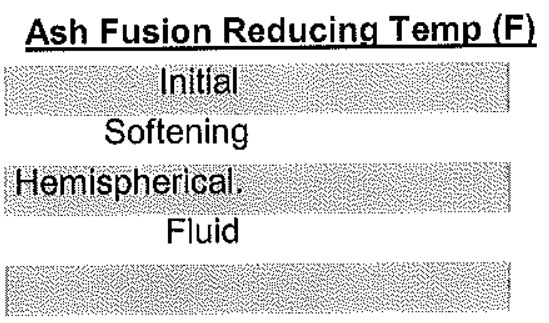

\section{Ash Fusion Oxidizing}

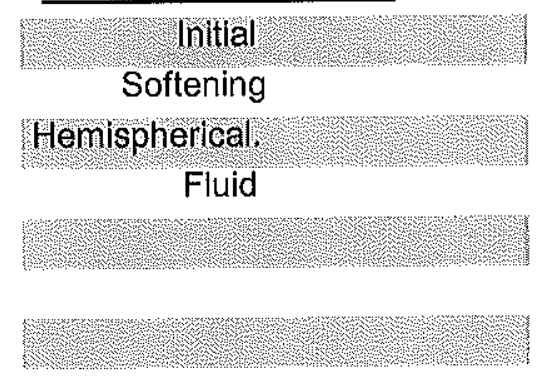

Misc.

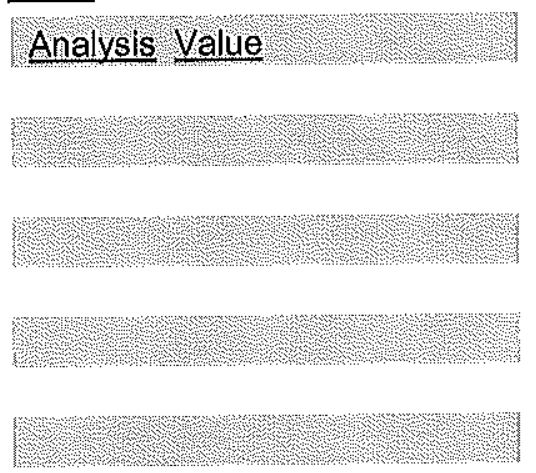

Trace Elements (ppm (Dry)

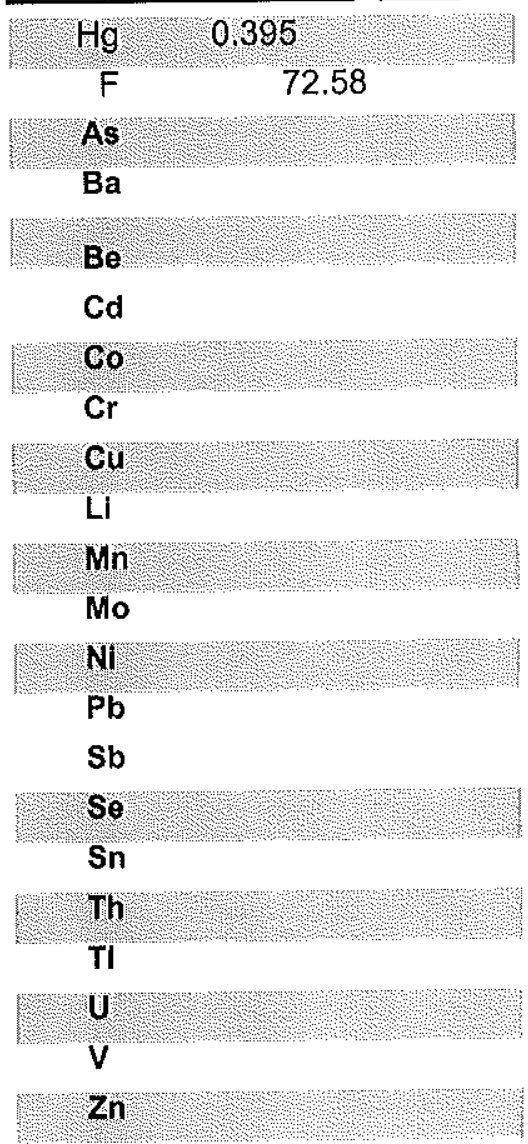

Seive Analysis SIZE

WT $\%$
HGI/FSI

$\mathrm{HGl}$

FSI

\section{Sulfate \\ Organic}

Sulfur, Total

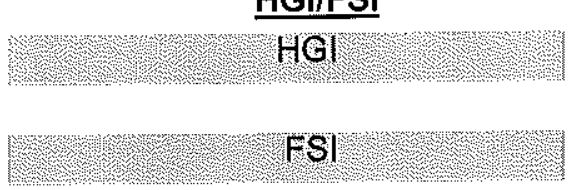

As Determined Moisture $0.66 \quad \%$
These values have been reviewed and are approved for transmission. yin 


\section{을 CONSOL ENERGY.}

Research and Development

www.consolenergy.com

4000 Brownsville Rd.

South Park, PA 15129

Sample Description.: PRODUCT/FLY ASH MARCH 30 11:15

Sample No.: TEST 1

Date Received: 04/03/2007

Analytical No.:

20071832

Project No.: $1621 \quad-085 \quad-000$

Date Completed: 04/19/2007

Submitted By: D. CONNELL

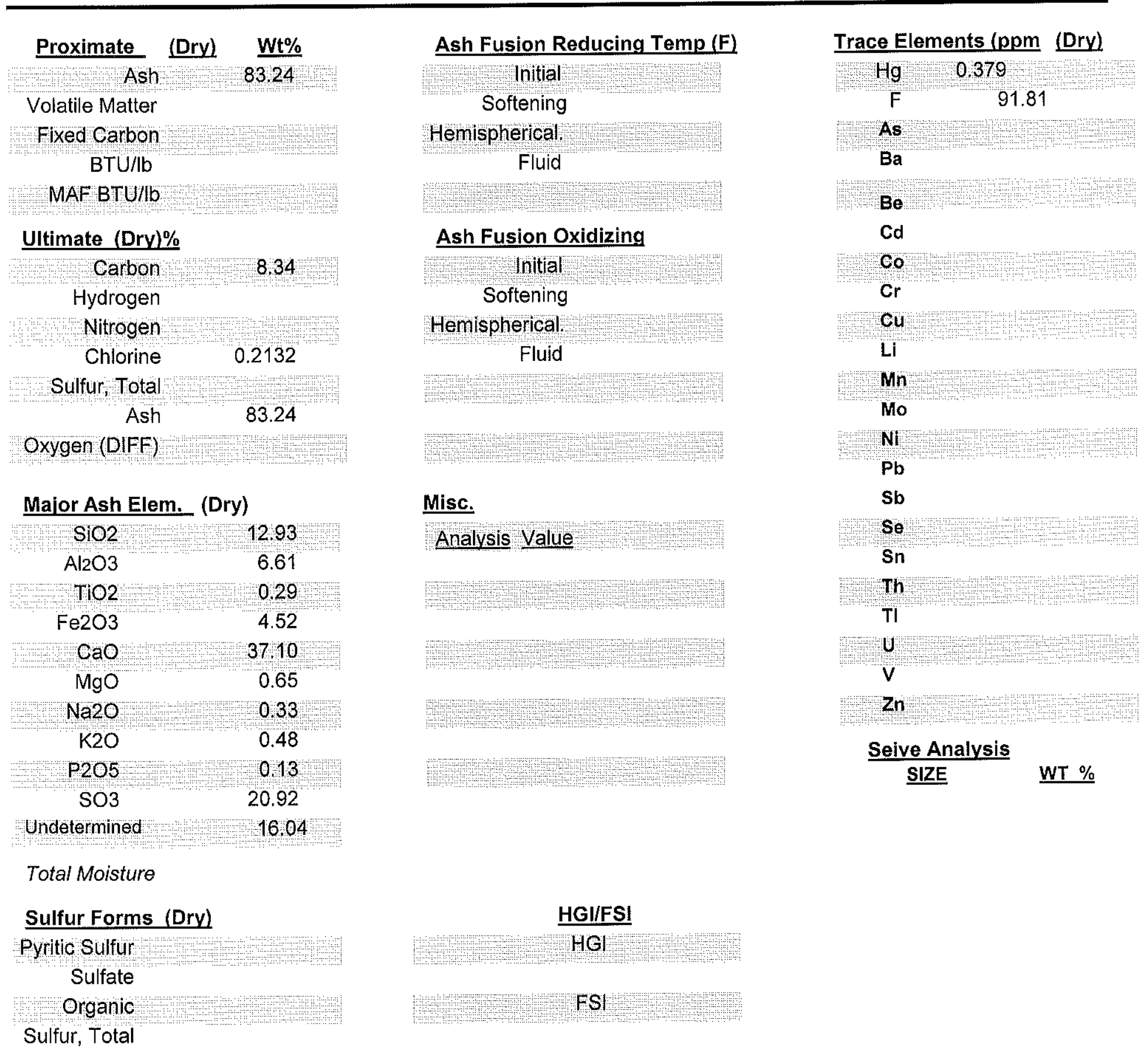

As Determined Moisture $0.56 \quad \%$

These values have been reviewed and are approved for transmission. inf 


\section{Y $=$ CONSOL ENERGY.}

Research and Development

www.consolenergy.com

4000 Brownsville Rd.

South Park, PA 15129

Sample Description.: PRODUCT/FLY ASH MARCH 30 13:45

Sample No:: TEST 2

Date Received: 04/03/2007

Date Completed: 04/19/2007

Analytical No.:

20071833

Project No.: $1621 \quad-085 \quad-000$

Submitted By: D. CONNELL

\begin{tabular}{|c|c|c|c|}
\hline Proximate (Dry) & $\underline{\mathbf{W t} \%}$ & Ash Fusion Reducing Temp (F) & Trace Elements (ppm (Dry) \\
\hline+4 Ash & 83.64 & Inital & 0.349 \\
\hline Volatile Matter & & Softening & 82.12 \\
\hline Fixed Carbon & & Hemispherical: & As \\
\hline $\mathrm{BTU} / \mathrm{b}$ & & Fluid & $\mathrm{Ba}$ \\
\hline MAFBTU//6 & & 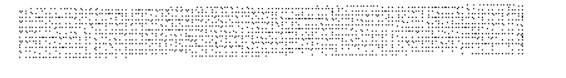 & $\mathrm{Be}$ \\
\hline Ultimate (Dry)\% & & Ash Fusion Oxidizing & $\mathrm{Cd}$ \\
\hline Carbon & 8.16 & Initial & Co \\
\hline Hydrogen & & Softening & $\mathrm{Cr}$ \\
\hline Nitrogen & 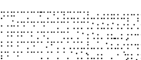 & Hemispherical. & $\mathrm{cu}$ \\
\hline Chlorine & 0.2697 & Fluid & $\mathrm{Li}$ \\
\hline Sulfur, Total & tan & & $\mathrm{Mn}$ \\
\hline Ash & 83.64 & & Mo \\
\hline Oxygen (DIFF) & 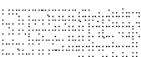 & & $\mathrm{Ni}$ \\
\hline & & & $\mathrm{Pb}$ \\
\hline Major Ash Elem. (L & & Misc. & $\mathrm{Sb}$ \\
\hline $\mathrm{SiO} 2$ & 12.59 & Analysis Value & Se \\
\hline $\mathrm{Al} 2 \mathrm{O} 3$ & 6.28 & & Sn \\
\hline $\mathrm{TiO} 2$ & 0.27 & H & Th \\
\hline $\mathrm{Fe} 2 \mathrm{O} 3$ & 4.73 & & $\mathrm{TI}$ \\
\hline $\mathrm{CaO}$ & 38.21 & & U: \\
\hline $\mathrm{MgO}$ & 0.66 & & V \\
\hline $\mathrm{Na} 2 \mathrm{O}$ & 0.33 & en & $\mathrm{zn}$ \\
\hline $\mathrm{K} 2 \mathrm{O}$ & 0.48 & & Seive Analysis \\
\hline$=\mathrm{P} 205$ & 0.13 & & SIZE \\
\hline $\mathrm{SO} 3$ & 21.57 & & \\
\hline Undetermined & 1475 & & \\
\hline \multicolumn{4}{|l|}{ Total Moisture } \\
\hline \multicolumn{2}{|l|}{ Sulfur Forms (Dry) } & \multicolumn{2}{|l|}{ HGI/FSI } \\
\hline \multicolumn{2}{|l|}{ Pyritic Sulfur } & \multicolumn{2}{|c|}{ Sulfate } \\
\hline $\begin{array}{l}\text { Organic } \\
\text { Sulfur Total }\end{array}$ & $+u$ & FSI & \\
\hline
\end{tabular}

As Determined Moisture $0.63 \quad \%$

These values have been reviewed and are approved for transmission. JM 


\section{夏= CONSOL ENERGY.}
Research and Development www.consolenergy.com 4000 Brownsville Rd.
South Park, PA 15129

Sample Description.: PRODUCT/FLY ASH MARCH 30 16:45

Sample No.: TEST 3

Analytical No.:

20071834

Date Received: $04 / 03 / 2007$

Date Completed: $04 / 19 / 2007$

Project No.: $1621-085-000$

Submitted By: D. CONNELL

\section{Proximate (Dry) Wt\% Ash $\because 83.69$ \\ Volatile Matter \\ Fixed Carbon \\ BTU/lb \\ MAF BTU/Ib}

Ultimate (Dry)\%

Carbon

8.12

Hydrogen

Nitrogen

Chlorine

0.2804

Sulfur, Total $\begin{array}{ll}\text { Ash } & 83.69\end{array}$

Oxygen (DIFF)

Major Ash Elem. (Dry)

$\mathrm{SiO} 2$

12.12

$\mathrm{Al}_{2} \mathrm{O} 3$

6.06

$\mathrm{TIO} 2$

0.26

$\mathrm{Fe} 2 \mathrm{O} 3$

4.57

$\mathrm{CaO}$

38.77

$\mathrm{MgO}$

0.66

$\mathrm{Na2O}$

0.33

$\mathrm{K} 2 \mathrm{O}$

0.47

$\mathrm{P} 2 \mathrm{O} 5$

0.12

$\mathrm{SO} 3$

21.84

Undetermined 14.80
Ash Fusion Reducing Temp (F) Initial Softening

Hemispherical.

Fluid

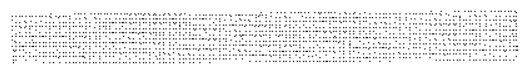

Ash Fusion Oxidizing

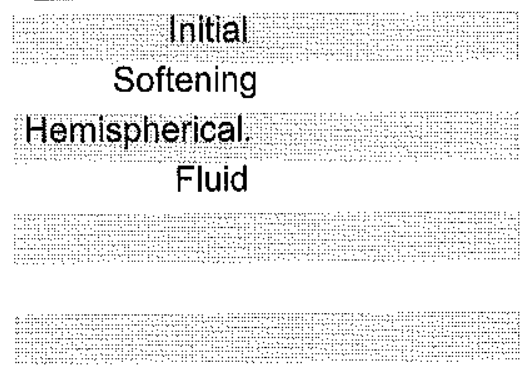

Misc.

\section{Analysis Value}
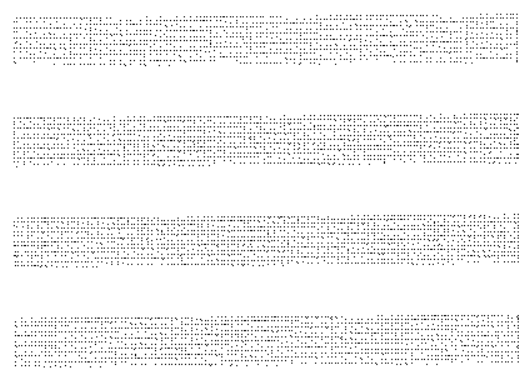

Trace Elements (ppm (Dry)

$\mathrm{Hg} \quad 0.352$

F $\quad 80.31$

As

$\mathrm{Ba}$

$\mathrm{Be}$

$\mathrm{Cd}$

co

$\mathrm{Cr}$

$\mathrm{Cu}$

$\mathrm{Li}$

$\mathrm{Mn}$

Mo

$\mathrm{NI}$

$\mathrm{Pb}$

$\mathrm{Sb}$

Se

Sn

Th

$\mathrm{TI}$

u

V

$\mathrm{Zn}$

Seive Analysis SIZE

Total Moisture

Sulfur Forms (Dry)

Pyritic Sulfur

Sulfate

Organic

Sulfur, Total
HGI/FSI

$1 \mathrm{GI}$

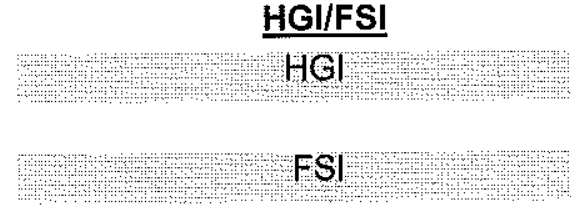

As Determined Moisture $0.51 \quad \%$ These values have been reviewed and are approved for transmission. 


\section{Y $=$ CONSOL ENERGY}
Research and Development
www.consolenergy.com
4000 Brownsville Rd.
South Park, PA 15129

Sample Description.: PEBBLE LIME MARCH 28 10:00

Sample No.: TEST 1

Date Received: 04/03/2007

Date Completed: 04/19/2007

Analytical No.:

Project No.: $1621 \quad-085-000$

Submitted By: D. CONNELL

\begin{tabular}{|c|c|c|c|}
\hline Proximate (Dry) & Wt\% & Ash Fusion Reducing Temp (F) & Trace Elements (ppm (Drv) \\
\hline Ash & 97.74 & Initial & $\mathrm{Hg}<<0.005$ \\
\hline Volatile Matter & & Softening & $F$ \\
\hline Fixed Carbon & & Hemispherical: & As \\
\hline $\mathrm{BTU} / \mathrm{lb}$ & & Fluid & $\mathrm{Ba}$ \\
\hline MAF BTU/L & 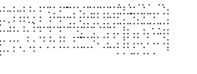 & 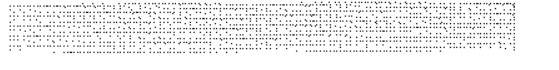 & $\mathrm{Be}$ \\
\hline Ultimate (Dry)\% & & Ash Fusion Oxidizing & $\mathrm{Cd}$ \\
\hline Carbon & 0.37 & Initial & Co \\
\hline Hydrogen & & Softening & $\mathrm{Cr}$ \\
\hline Nitrogen & & Hemispherical. & $\mathrm{Cu}$ \\
\hline Chlorine & & Fluid & $\mathrm{Li}$ \\
\hline Sulfur, Total & + & & Mn \\
\hline Ash & 97.74 & & Mo \\
\hline Oxygen (DIFF) & 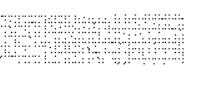 & 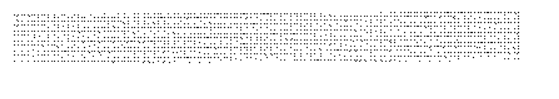 & $\begin{array}{l}\mathrm{NI} \\
\mathrm{Pb}\end{array}$ \\
\hline Major Ash Elem. (As & Det) & Misc. & Sb \\
\hline $\mathrm{SiO} 2$ & 1.06 & Analysis Value & Se \\
\hline $\mathrm{Al}_{2} \mathrm{O} 3$ & 0.48 & & Sn \\
\hline $\mathrm{TiO} 2$ & 0.02 & & Th \\
\hline $\mathrm{Fe} 2 \mathrm{O} 3$ & 0.20 & & $\mathbf{T I}$ \\
\hline $\mathrm{CaO}$ & 95.22 & $\sqrt{3}$ & U \\
\hline $\mathrm{MgO}$ & 1.11 & & v \\
\hline $\mathrm{Na} 2 \mathrm{O}$ & 0.08 & + & $\mathrm{Zn}$ \\
\hline $\mathrm{K} 2 \mathrm{O}$ & 0.07 & & Seive Analysis \\
\hline $\mathrm{P} 205$ & 0.01 & 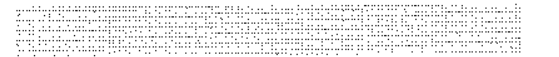 & SIZE \\
\hline SO3 & 0.16 & & \\
\hline Undetermined & 159 & & \\
\hline \multicolumn{4}{|l|}{ Total Moisture } \\
\hline Sulfur Forms (Drv) & & HGI/FSI & \\
\hline $\begin{array}{l}\text { Pyritic Sulfur } \\
\text { Sulfate }\end{array}$ & tus & $\mathrm{HGl}$ & \\
\hline $\begin{array}{c}\text { Organic } \\
\text { Sulfur, Total }\end{array}$ & 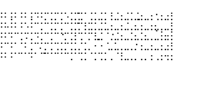 & 4 & \\
\hline
\end{tabular}

As Determined Moisture 0

$\%$

These values have been reviewed and are approved for transmission. 
Research and Development

www.consolenergy.com

4000 Brownsville Rd.

South Park, PA 15129

Sample Description.: PEBBLE LIME MARCH 28 14:30

Sample No.: TEST 2

Date Received: 04/03/2007

Analytical No.:

20071812

Project No.: $1621 \quad-085 \quad-000$

Date Completed: 04/19/2007

Submitted By: D. CONNELL

\begin{tabular}{|c|c|c|c|}
\hline Proximate (Dry) & $\underline{W t \%}$ & Ash Fusion Reducing Temp (F) & Trace Elements (ppm (Dry) \\
\hline $\mathrm{Ash}$ & 95.88 & Initial & $<0.005$ \\
\hline Volatile Matter & & Softening & $\mathrm{F}$ \\
\hline Fixed Carbon & 4 & Hemispherical. & As \\
\hline $\mathrm{BTU} / \mathrm{lb}$ & & Fluid & $\mathrm{Ba}$ \\
\hline $\mathrm{MAF} \mathrm{BTU} / \mathrm{L}$ & & ?tu & $\mathrm{Be}$ \\
\hline Ultimate (Dry)\% & & Ash Fusion Oxidizing & Cd \\
\hline Carbon & 1.36 & Initial & Co \\
\hline Hydrogen & & Softening & $\mathrm{Cr}$ \\
\hline Nitrogen & & Hemispherical. & $\mathrm{Cu}$ \\
\hline Chlorine & & Fluid & Li \\
\hline Sulfur, Total & & & $\mathrm{Mn}$ \\
\hline Ash & 95.88 & & Mo \\
\hline Oxygen (DIFF) & & & $\frac{\mathrm{Nl}}{\mathrm{Pb}}$ \\
\hline Major Ash Elem. (A & Det) & $\underline{\text { Misc. }}$ & Sb \\
\hline $\mathrm{SiO} 2$ & 1.07 & Analysis Value & Se \\
\hline $\mathrm{Al} 2 \mathrm{O} 3$ & 0.49 & & Sn \\
\hline $\mathrm{T} 102$ & $0.02 \rightarrow$ & & Th \\
\hline $\mathrm{Fe} 2 \mathrm{O} 3$ & 0.25 & & $\mathrm{TI}$ \\
\hline $\mathrm{CaO}$ & 91.37 & is & U \\
\hline $\mathrm{MgO}$ & 2.88 & & v \\
\hline $\mathrm{Na} 2 \mathrm{O}$ & 0.06 & 3 & $2 n$ \\
\hline $\mathrm{K} 2 \mathrm{O}$ & 0.06 & & Seive Analysis \\
\hline $\mathrm{P} 205$ & 0.01 & & SIZE \\
\hline SO3 & 0.18 & & \\
\hline Undetermined & 3.61 & & \\
\hline \multicolumn{4}{|l|}{ Total Moisture } \\
\hline Sulfur Forms (Dry) & & HGI/FSI & \\
\hline $\begin{array}{l}\text { Pyritic Sulfur } \\
\text { Sulfate }\end{array}$ & ?tus & $\mathrm{HGl}$ & \\
\hline $\begin{array}{l}\text { Organic } \\
\text { Sulfur, Total }\end{array}$ & 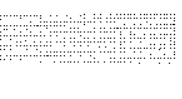 & FSI & \\
\hline
\end{tabular}

As Determined Moisture $0 \quad \%$

These values have been reviewed and are approved for transmission. M7 


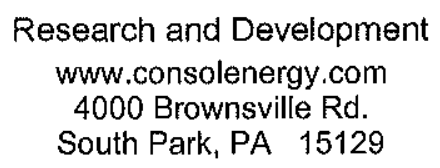

Sample Description: PEBBLE LIME MARCH 28 17:15

Sample No.: TEST 3

Date Received: 04/03/2007

Date Completed: $04 / 19 / 2007$

Analytical No.: $\quad 20071813$

Project No.: $1621-085-000$

Submitted By: D. CONNELL

\begin{tabular}{|c|c|c|c|}
\hline Proximate (Dry) & $\mathbf{W t} \%$ & Ash Fusion Reducing Temp (F) & Trace Elements (ppm (Drv) \\
\hline Ash & 98.69 & Initial & $\mathrm{Hg}=<0.005$ \\
\hline Volatile Matter & & Softening & $\mathrm{F}$ \\
\hline Fixed Carbon & & Hemispherical & As \\
\hline BTU/lb & & Fluid & $\mathrm{Ba}$ \\
\hline MAF BTU/Ib & +4 & Butustututututat & $\mathrm{Be}$ \\
\hline Ultimate (Dry)\% & & Ash Fusion Oxidizing & Cd \\
\hline Carbon & 014 & Initial & Co \\
\hline Hydrogen & & Softening & $\mathrm{Cr}$ \\
\hline Nitrogen & $t=$ & Hemispherical. & $\mathrm{Cu}$ \\
\hline Chlorine & & Fluid & $\mathrm{Li}$ \\
\hline Sulfur, Total & i & 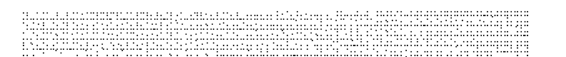 & $\mathrm{Mn}$ \\
\hline Ash & 98.69 & & Mo \\
\hline \multirow[t]{2}{*}{ Oxygen (DIFF) } & 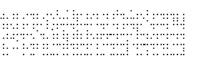 & ? & $\mathrm{NI}$ \\
\hline & & & $\mathrm{Pb}$ \\
\hline \multicolumn{2}{|c|}{ Major Ash Elem. (AsDet) } & Misc. & $\mathbf{S b}$ \\
\hline $\mathrm{SiO} 2$ & 0.98 & Analysis Value & Se \\
\hline $\mathrm{Al}_{2} \mathrm{O} 3$ & 0.48 & & Sn \\
\hline $\mathrm{T} 1 \mathrm{O} 2$ & 0.02 & eas & Th \\
\hline $\mathrm{Fe} 2 \mathrm{O} 3$ & 0.21 & & $\mathrm{TI}$ \\
\hline $\mathrm{CaO}$ & 96.28 & an & $\mathrm{U}$ \\
\hline $\mathrm{MgO}$ & 1.04 & & V \\
\hline $\mathrm{Na} 2 \mathrm{O}$ & 0.08 & 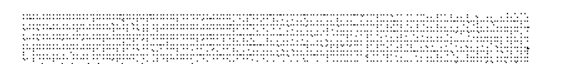 & $\mathbf{z n}_{\mathbf{n}}$ \\
\hline $\mathrm{K} 2 \mathrm{O}$ & 0.06 & & Seive Analysis \\
\hline $\mathrm{P} 205$ & 0.01 & जutu & SIZE \\
\hline $\mathrm{SO} 3$ & 0.17 & & \\
\hline Undetermined & 0.67 & & \\
\hline \multicolumn{4}{|l|}{ Total Moisture } \\
\hline \multicolumn{2}{|l|}{ Sulfur Forms (Dry) } & HGI/FSI & \\
\hline Pyritic Sulfur & Sulfate & 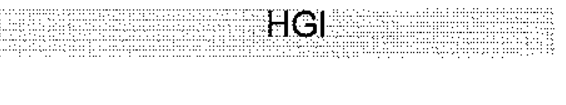 & \\
\hline $\begin{array}{l}\text { Organic } \\
\text { Sulfur, Total }\end{array}$ & 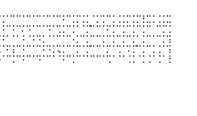 & $\mathrm{FSI}$ & \\
\hline
\end{tabular}

As Determined Moisture 0

$\%$

These values have been reviewed and are approved for transmission $7 \mathrm{~m}$. 


\title{
Y $=$ CONSOL ENERGY
}

\author{
Research and Development \\ www.consolenergy.com \\ 4000 Brownsville Rd. \\ South Park, PA 15129
}

Sample Description.: PEBBLE LIME MARCH 29 9:30

Sample No.: TEST 1

Date Received: 04/03/2007

Analytical No:: $\quad 20071826$

Project No.: $1621 \quad-085 \quad-000$

Date Completed: 04/19/2007

Submitted By: D. CONNELL

\begin{tabular}{|c|c|}
\hline Proximate (Dry) & $\underline{W t} \%$ \\
\hline Ash & 9787 \\
\hline \multicolumn{2}{|l|}{ Volatile Matter } \\
\hline \multicolumn{2}{|l|}{ Fixed Carbon } \\
\hline \multicolumn{2}{|l|}{$\mathrm{BTU} / \mathrm{lb}$} \\
\hline \multicolumn{2}{|l|}{ MAF BTU//b } \\
\hline \multicolumn{2}{|l|}{ Ultimate (Dry)\% } \\
\hline Carbon & 0.31 \\
\hline \multicolumn{2}{|l|}{ Hydrogen } \\
\hline \multicolumn{2}{|l|}{ Nitrogen } \\
\hline \multicolumn{2}{|l|}{ Chlorine } \\
\hline \multicolumn{2}{|l|}{ Sulfur, Total } \\
\hline Ash & 97.87 \\
\hline \multicolumn{2}{|l|}{ Oxygen (BIFF) } \\
\hline \multicolumn{2}{|c|}{ Major Ash Elem. (AsDet) } \\
\hline $\mathrm{SIO} 2$ & 1,03 \\
\hline $\mathrm{Al} 2 \mathrm{O} 3$ & 0.54 \\
\hline TiO2 & $<0.03$ \\
\hline $\mathrm{Fe} 2 \mathrm{O} 3$ & 0.23 \\
\hline CaO & 95.21 . \\
\hline $\mathrm{MgO}$ & 1.04 \\
\hline $\mathrm{Na2O}$ & 0.05 \\
\hline $\mathrm{K} 2 \mathrm{O}$ & $<0.03$ \\
\hline $\mathrm{P} 205$ & $<0.03$ \\
\hline $\mathrm{SO} 3$ & 0.14 \\
\hline Undetermined & 1.76 \\
\hline
\end{tabular}

Ash Fusion Reducing Temp (F)

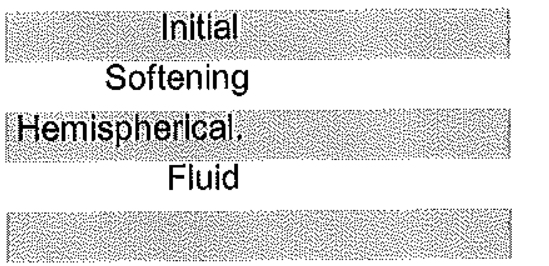

Ash Fusion Oxidizing

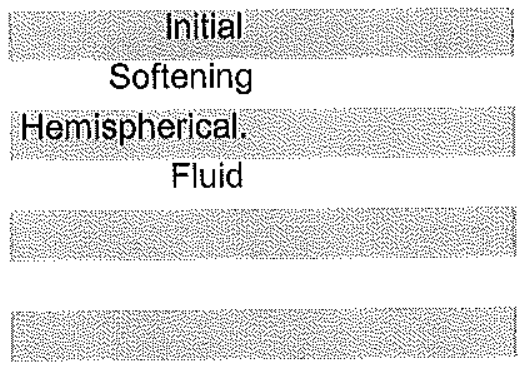

Misc.

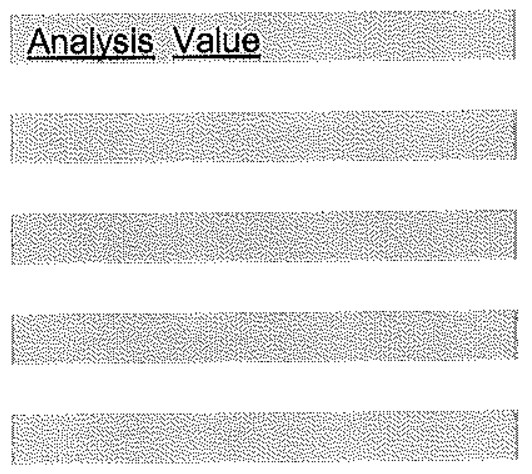

Trace Elements (ppm (Dry)

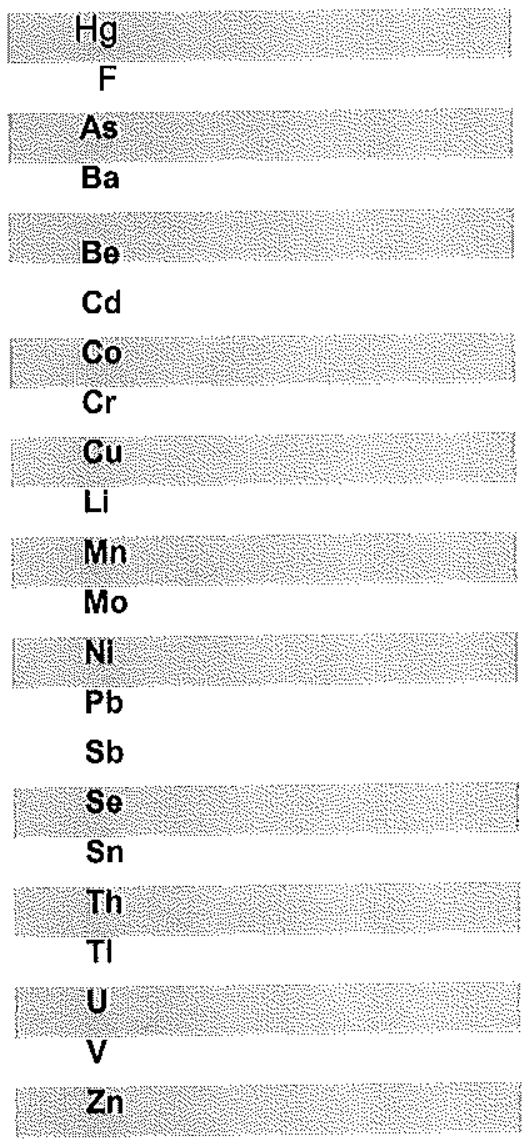

Seive Analysis SIZE

WT $\%$

\section{HGI/FSI}

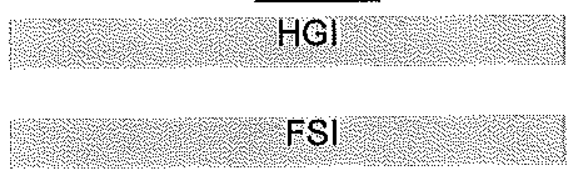

Sulfur Forms (Dry)

Pyritic Sulfur Sulfate

Organic

Sulfur, Total

As Determined Moisture 0

$\%$

These values have been reviewed and are approved for transmission. $3 m$ X 


\section{严= CONSOL ENERGY}

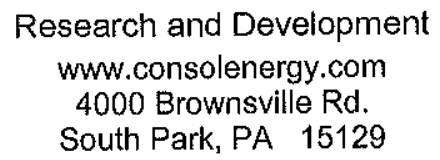

\section{Sample Description.: PEBBLE LIME MARCH 29 13:30}

Sample No:: TEST 2

Date Received: 04/03/2007

Analytical No.:

20071827

Project No.: $1621-085 \quad-000$

Date Completed: 04/19/2007

Submitted By: D. CONNELL

\begin{tabular}{|c|c|}
\hline Proximate (Dry) & $\underline{W} \%$ \\
\hline Ash & 97.51 \\
\hline \multicolumn{2}{|l|}{ Volatile Matter } \\
\hline \multicolumn{2}{|l|}{ Fixed Carbon } \\
\hline \multicolumn{2}{|l|}{ BTU/lb } \\
\hline \multicolumn{2}{|l|}{ MAF BTU/L } \\
\hline \multicolumn{2}{|l|}{ Ultimate (Drv)\% } \\
\hline Carbon & 0.30 \\
\hline \multicolumn{2}{|l|}{ Hydrogen } \\
\hline \multicolumn{2}{|l|}{ Nitrogen } \\
\hline \multicolumn{2}{|l|}{ Chlorine } \\
\hline \multicolumn{2}{|l|}{ Sulfur, Total } \\
\hline Ash & 97.51 \\
\hline \multicolumn{2}{|l|}{ Oxygen (DIFF) } \\
\hline \multicolumn{2}{|c|}{ Major Ash Elem. (AsDet) } \\
\hline SIO2 & 1.61 \\
\hline $\mathrm{Al} 2 \mathrm{O} 3$ & 0.49 \\
\hline TIO2 & 002 \\
\hline $\mathrm{Fe} 2 \mathrm{O} 3$ & 0.21 \\
\hline $\mathrm{CaO}$ & 96.64 \\
\hline $\mathrm{MgO}$ & 1.05 \\
\hline Na20. & 0.09 \\
\hline $\mathrm{K} 2 \mathrm{O}$ & 0.07 \\
\hline $\mathrm{P} 205$ & $<0.01$ \\
\hline $\mathrm{SO} 3$ & 0.15 \\
\hline Undetermined & -0.33 \\
\hline
\end{tabular}

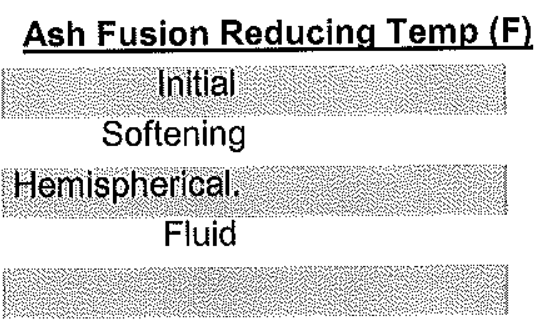

\section{Ash Fusion Oxidizing}
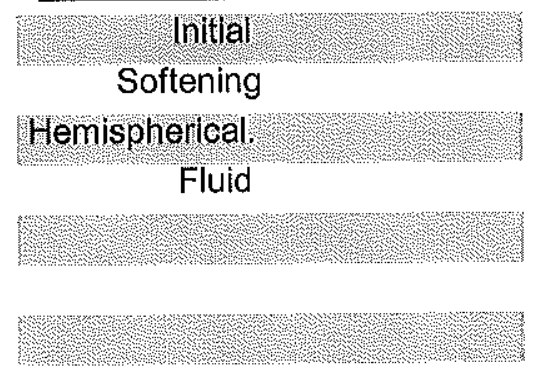

Misc.

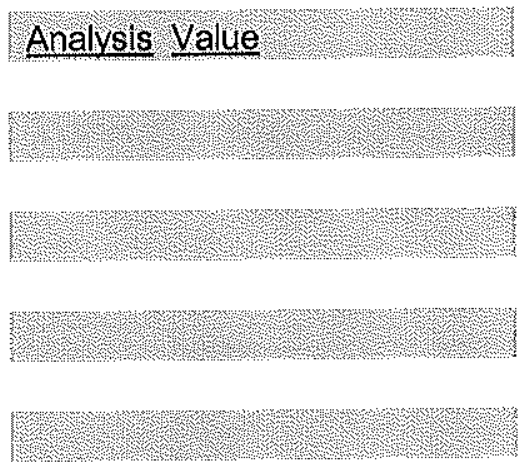

Trace Elements (ppm (Drv)

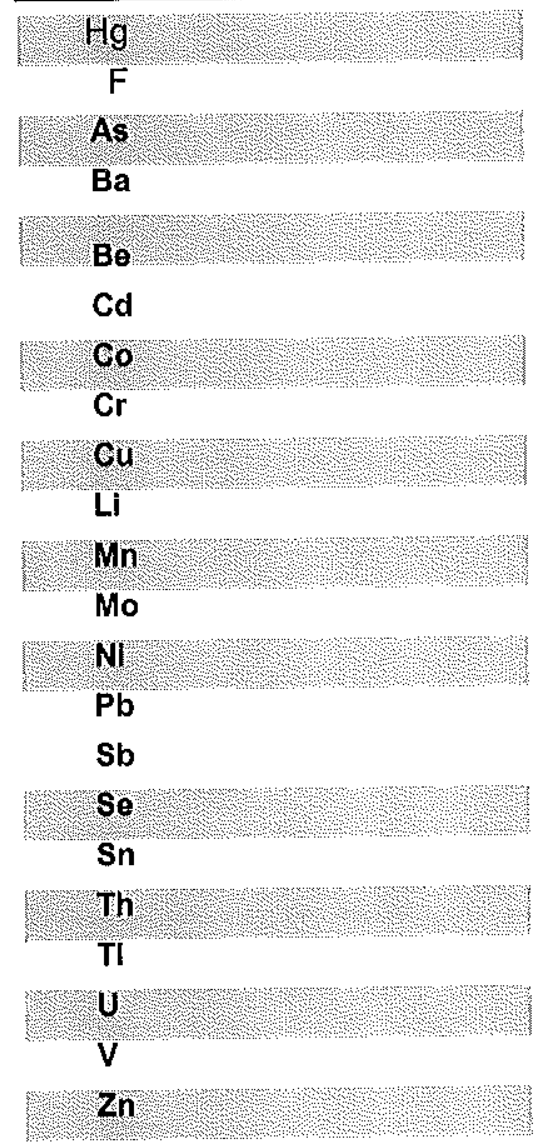

Seive Analysis SIZE

WT \%

\section{Sulfate \\ Organic}

Sulfur, Total
HGI/FSI

$\mathrm{HGl}$

FSI

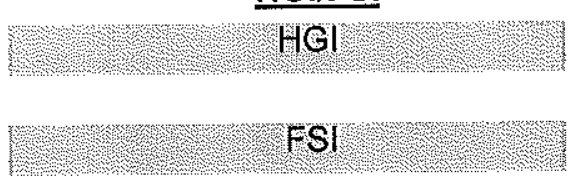

As Determined Moisture 0

$\%$

These values have been reviewed and are approved for transmission. Jim 7 


\section{Y宩= CONSOL ENERGY.}

Research and Development

www.consolenergy.com

4000 Brownsville Rd.

South Park, PA 15129

\section{Sample Description.: PEBBLE LIME MARCH 29 16:30}

Sample No.: TEST 3

Date Received: 04/03/2007

Date Completed: $04 / 19 / 2007$

Analytical No.: $\quad 20071828$

Project No.: $1621-085 \quad-000$

Submitted By: D. CONNELL

\begin{tabular}{|c|c|}
\hline Proximate (Drv) & $\underline{\mathrm{Wt}} \%$ \\
\hline Ash & 96.01 \\
\hline \multicolumn{2}{|l|}{ Volatile Matter } \\
\hline \multicolumn{2}{|l|}{ Flxed Carbon } \\
\hline \multicolumn{2}{|l|}{ BTU/lb } \\
\hline \multicolumn{2}{|l|}{ MAFBTU/L } \\
\hline \multicolumn{2}{|l|}{ Ultimate (Dry)\% } \\
\hline Carbon & 0.38 \\
\hline \multicolumn{2}{|l|}{ Hydrogen } \\
\hline \multicolumn{2}{|l|}{ Nitrogen } \\
\hline \multicolumn{2}{|l|}{ Chlorine } \\
\hline \multicolumn{2}{|l|}{ Sulfür, Total } \\
\hline Ash & 96.01 \\
\hline \multicolumn{2}{|l|}{ Oxygen (DIFF) } \\
\hline \multicolumn{2}{|c|}{ Major Ash Elem. (AsDet) } \\
\hline $\mathrm{SiO} 2$ & 1.33 \\
\hline $\mathrm{Al} 2 \mathrm{O} 3$ & 0.43 \\
\hline 7102 & 0.02 \\
\hline $\mathrm{Fe} 2 \mathrm{O} 3$ & 0.19 \\
\hline $\mathrm{CaO}$ & 95.51 \\
\hline $\mathrm{MgO}$ & 0.98 \\
\hline $\mathrm{Na} 2 \mathrm{O}$ & 0.08 \\
\hline $\mathrm{K} 2 \mathrm{O}$ & 0.06 \\
\hline $\mathrm{P} 205$ & $<0.01$ \\
\hline $\mathrm{SO} 3$ & 0.17 \\
\hline Undetermined & 1.23 \\
\hline
\end{tabular}

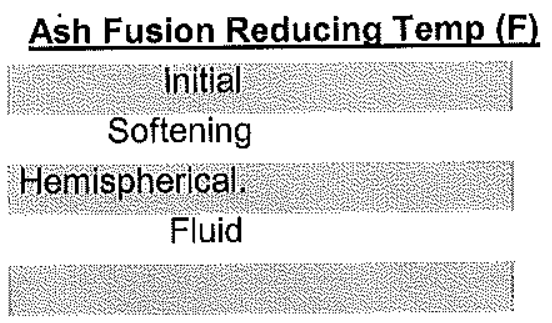

Ash Fusion Oxidizing

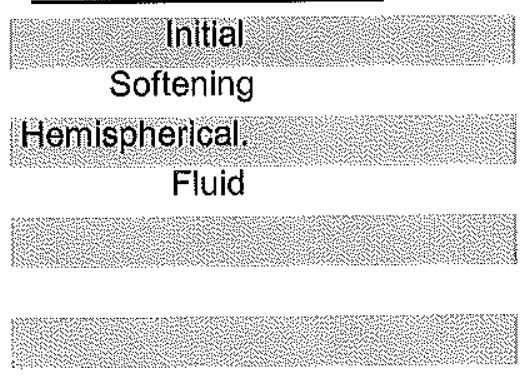

Misc.

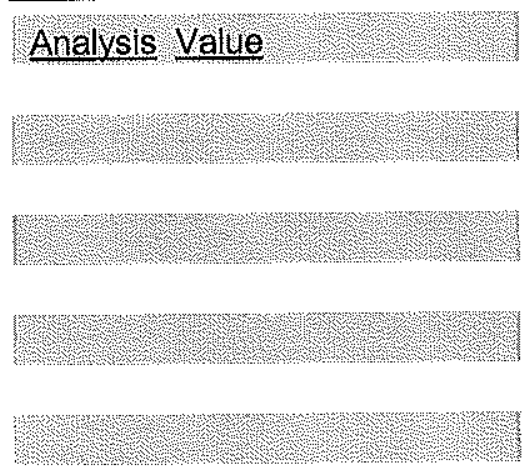

Trace Elements (ppm (Dry)

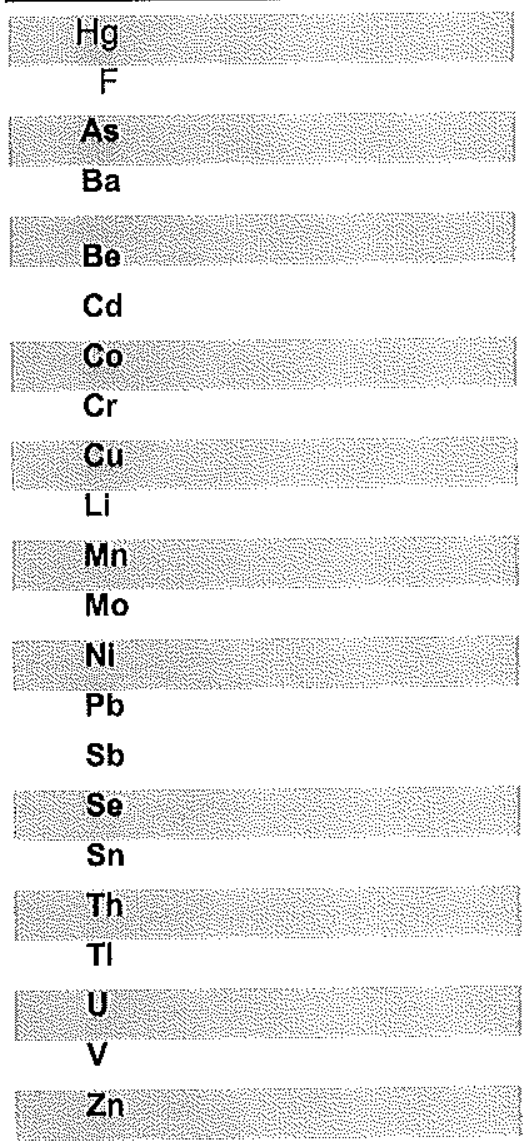

Seive Analysis SIZE

WT \%

\section{HGI/FSI}

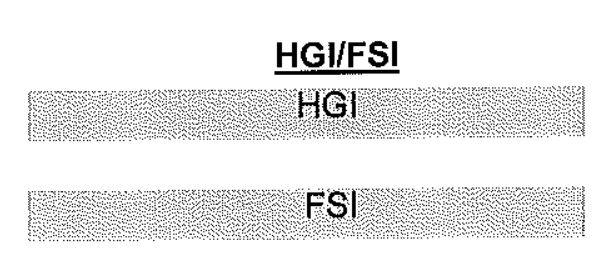

Sulfur Forms (Dry)

Pyitic Sulfur

Sulfate

Organic

Sulfur, Total

As Determined Moisture 0

$\%$

These values have been reviewed and are approved for transmission? 


\title{
을 CONSOL ENERGY.
}

\author{
Research and Development \\ wuw.consolenergy.com \\ 4000 Brownsville Rd. \\ South Park, PA 15129
}

\section{Sample Description.: PEBBLE LIME MARCH 30 10:00}

Sample No:: TEST 1

Date Received: $04 / 03 / 2007$

Analytical No.:

Project No.: $1621-085-000$

Date Completed: 04/19/2007

Submitted By: D. CONNELL

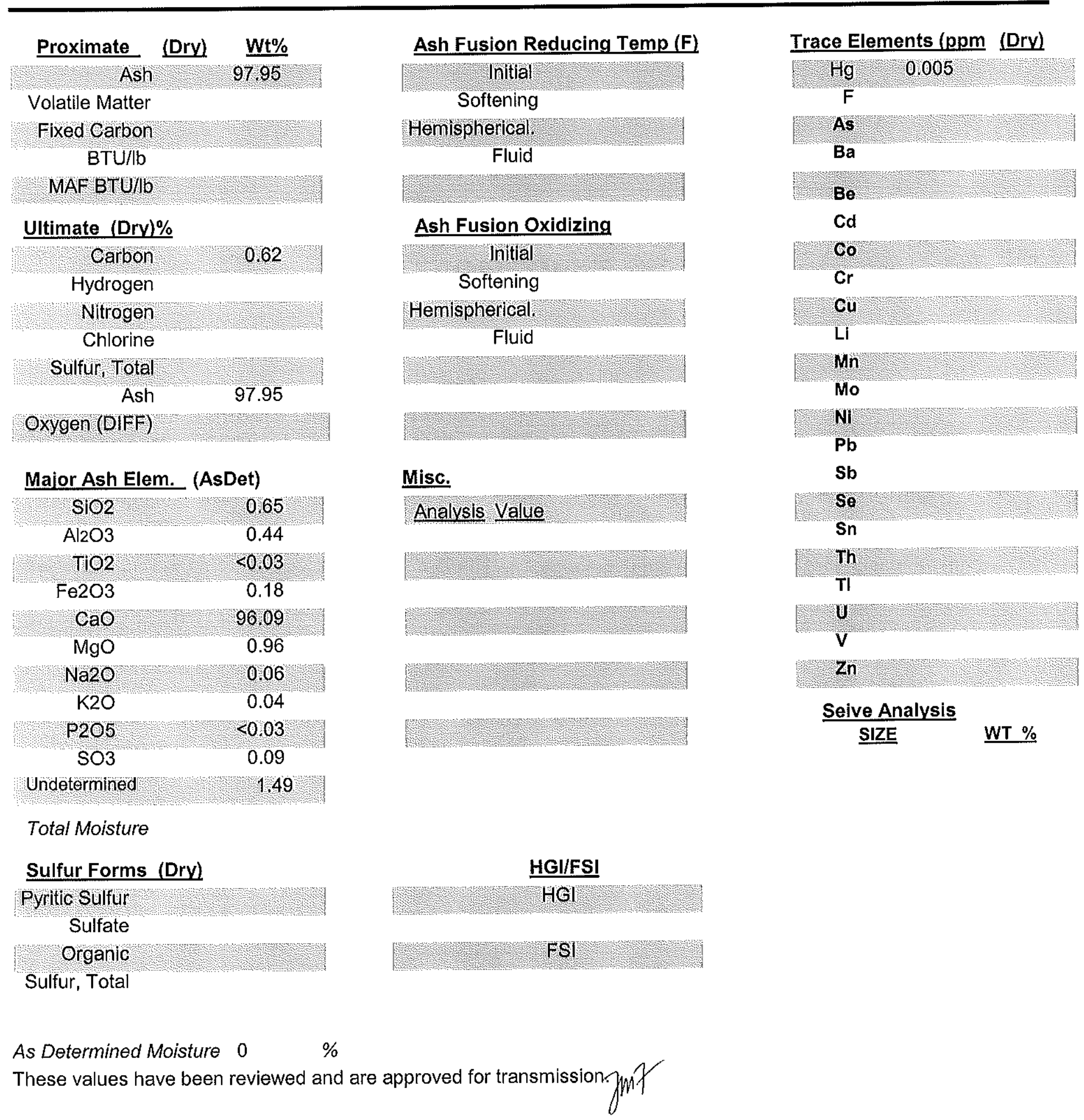




\section{严 CONSOL ENERGY}

Research and Development

www.consolenergy.com

4000 Brownsville Rd.

South Park, PA 15129

Sample Description.: PEBBLE LIME MARCH 30 13:45

Sample No.: TEST 2

Date Received: 04/03/2007

Analytical No.:

20071842

Project No.: $1621-085-000$

Date Completed: 04/19/2007

Submitted By: D. CONNELL

\begin{tabular}{|c|c|}
\hline Proximate (Dry) & Wt \% \\
\hline Ash & 98.11 \\
\hline \multicolumn{2}{|l|}{ Volatile Matter } \\
\hline \multicolumn{2}{|l|}{ Fixed Carbon } \\
\hline \multicolumn{2}{|l|}{$\mathrm{BTU} / \mathrm{lb}$} \\
\hline \multicolumn{2}{|l|}{ MAF BTU/Ib } \\
\hline \multicolumn{2}{|l|}{ Ultimate (Dry)\% } \\
\hline Carbon & 0.48 \\
\hline \multicolumn{2}{|l|}{ Hydrogen } \\
\hline \multicolumn{2}{|l|}{ Nitrogen } \\
\hline \multicolumn{2}{|l|}{ Chlorine } \\
\hline \multicolumn{2}{|l|}{ Sulfur, Total } \\
\hline Ash & 98.11 \\
\hline Oxygen (DIFF) & \\
\hline
\end{tabular}

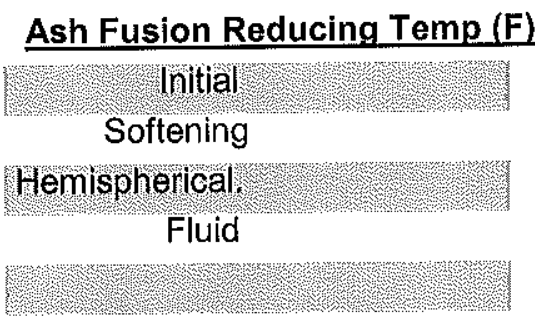

\section{Ash Fusion Oxidizing}
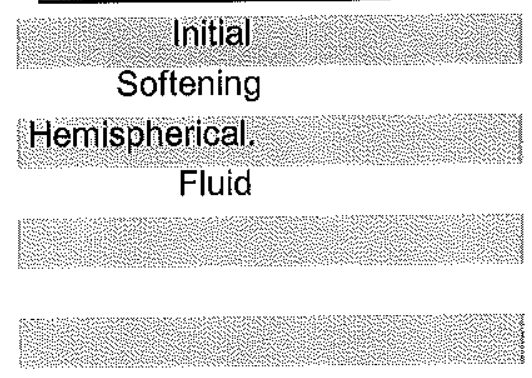

Misc.

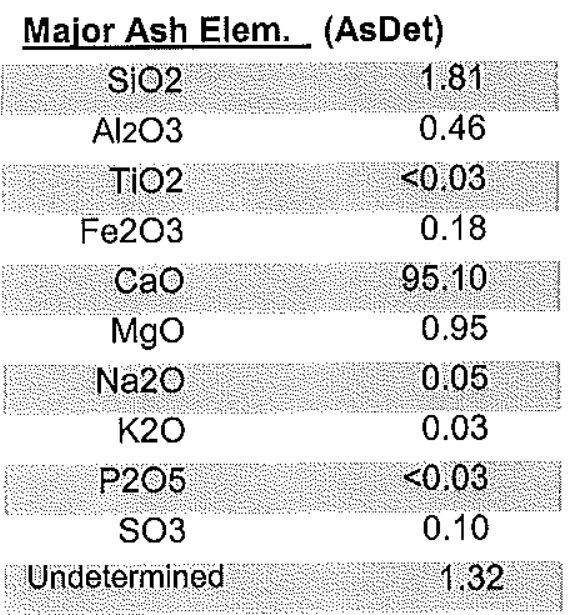

Total Moisture

\section{Sulfur Forms (Dry)}

Pyritic Sulfur

Sulfate

Organic

Sulfur, Total

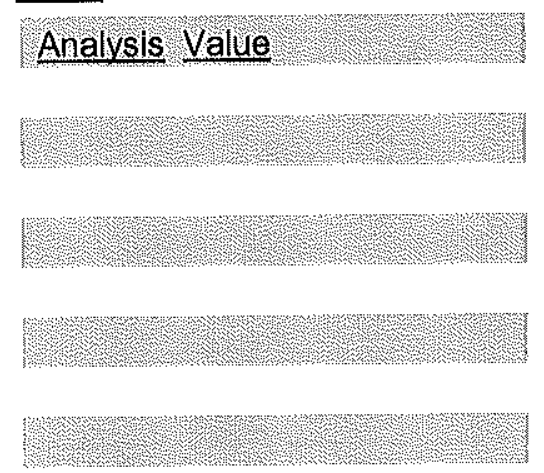

HGI/FSI

HGI

FSI
Trace Elements (ppm (Dry)

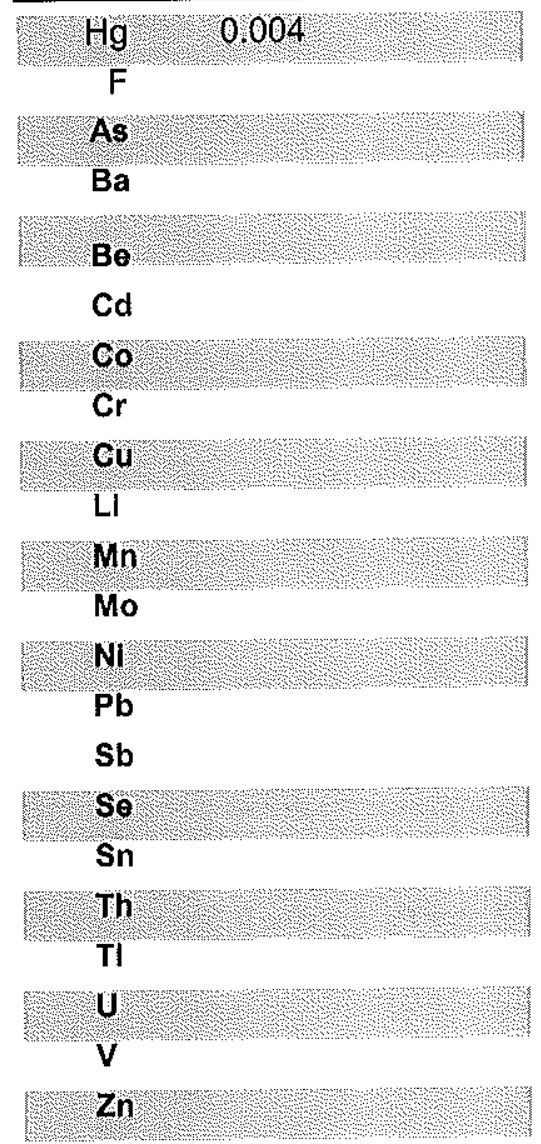

Seive Analysis SIZE

WT \%

As Determined Moisture 0

$\%$

These values have been reviewed and are approved for transmission. W 


\section{Y贯: CONSOL ENERGY}
Research and Development
www.consolenergy.com 4000 Brownsville Rd.
South Park, PA 15129

\section{Sample Description.: PEBBLE LIME MARCH 30 16:45}

Sample No:: TEST 3

Date Received: 04/03/2007

Date Completed: 04/19/2007

Analytical No.: $\quad 20071843$

Project No.: $1621-085-000$

Submitted By: D. CONNELL

\begin{tabular}{|c|c|}
\hline Proximate (Dry) & $\mathbf{W t} \%$ \\
\hline Ash & 99.08 \\
\hline \multicolumn{2}{|l|}{ Volatile Matter } \\
\hline \multicolumn{2}{|l|}{ Fixed Garbon } \\
\hline \multicolumn{2}{|l|}{ BTU/lb } \\
\hline \multicolumn{2}{|l|}{ MAF BTU/lb } \\
\hline \multicolumn{2}{|l|}{ Ultimate (Drv)\% } \\
\hline Carbon & 0.31 \\
\hline \multicolumn{2}{|l|}{ Hydrogen } \\
\hline \multicolumn{2}{|l|}{ Nitrogen } \\
\hline \multicolumn{2}{|l|}{ Chlorine } \\
\hline \multicolumn{2}{|l|}{ Sulfur, Total } \\
\hline Ash & 99.08 \\
\hline Oxygen (DIFF) & \\
\hline
\end{tabular}

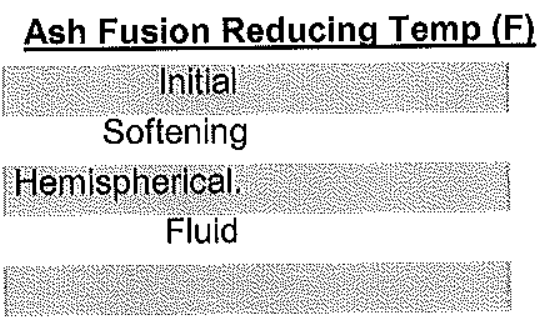

\section{Ash Fusion Oxidizing}
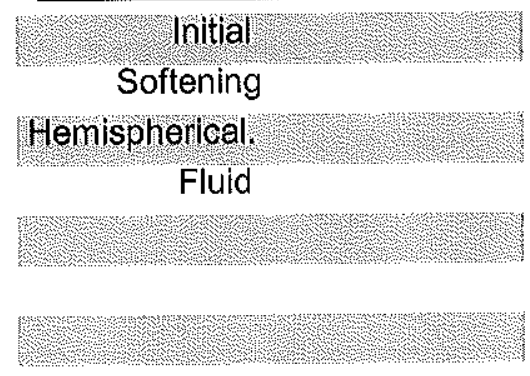

Misc.

\begin{tabular}{cc} 
Major Ash Elem. & (AsDet) \\
\hline $\mathrm{SiO} 2$ & 2.34 \\
$\mathrm{Al} 2 \mathrm{O} 3$ & 0.46 \\
\hline $\mathrm{TlO} 2$ & $<0.03$ \\
$\mathrm{Fe} 2 \mathrm{O} 3$ & 0.18 \\
$\mathrm{CaO}$ & 95.17 \\
$\mathrm{MgO}$ & 0.99 \\
$\mathrm{Na2O}$ & 0.06 \\
$\mathrm{~K} 2 \mathrm{O}$ & $<0.03$ \\
$\mathrm{P} 2 \mathrm{O} 5$ & $<0.03$ \\
$\mathrm{SO} 3$ & 0.12 \\
\hline Undetermined & 0.68 \\
\hline
\end{tabular}

Total Moisture

\section{Sulfur Forms (Dry)}

Pyritic Sulfur Sulfate

Organic Sulfur, Total

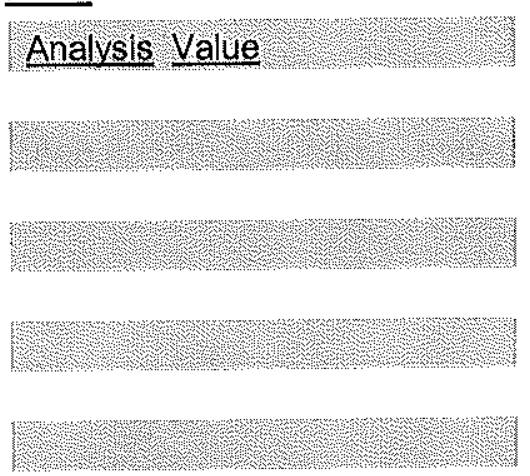

\section{HGI/FSI}

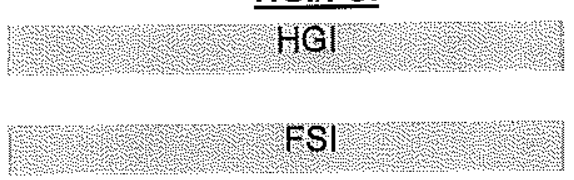

Trace Elements (ppm (Drv)

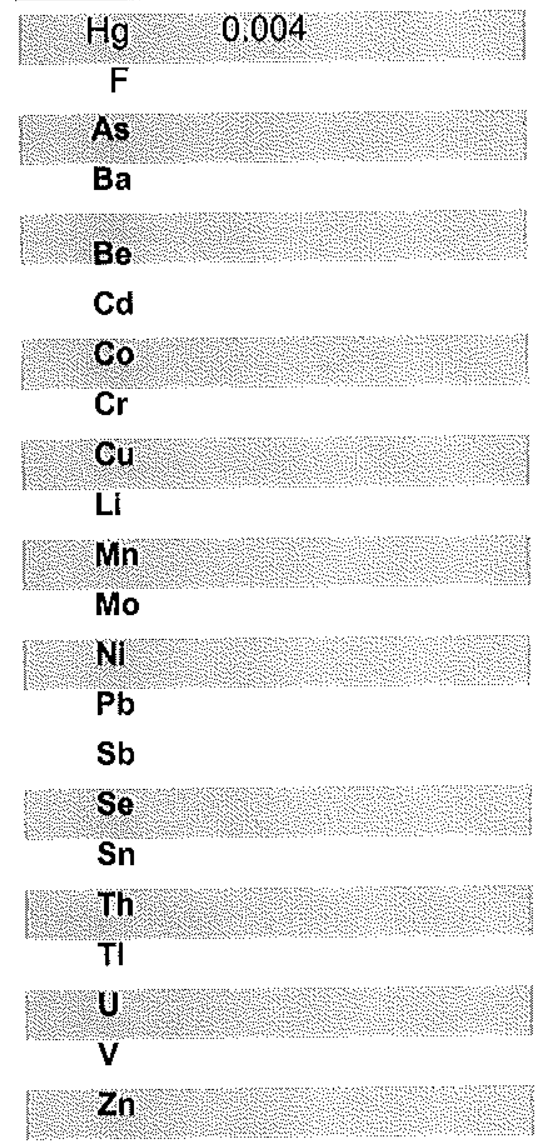

Seive Analysis SIZE

\section{WT \%}

As Determined Moisture 0

$\%$

These values have been reviewed and are approved for transmission. Jin 7 


\section{Y CONSOLENERGY}
Research and Development
www.consolenergy.com
4000 Brownsville Rd.
South Park, PA 15129

Sample Description.: HYDRATED LIME MARCH 28 11:00

Sample No.: TEST 1

Date Received: 04/03/2007

Date Completed: 04/19/2007

Analytical No.:

Project No.: $1621-085-000$

Submitted By: D. CONNELL

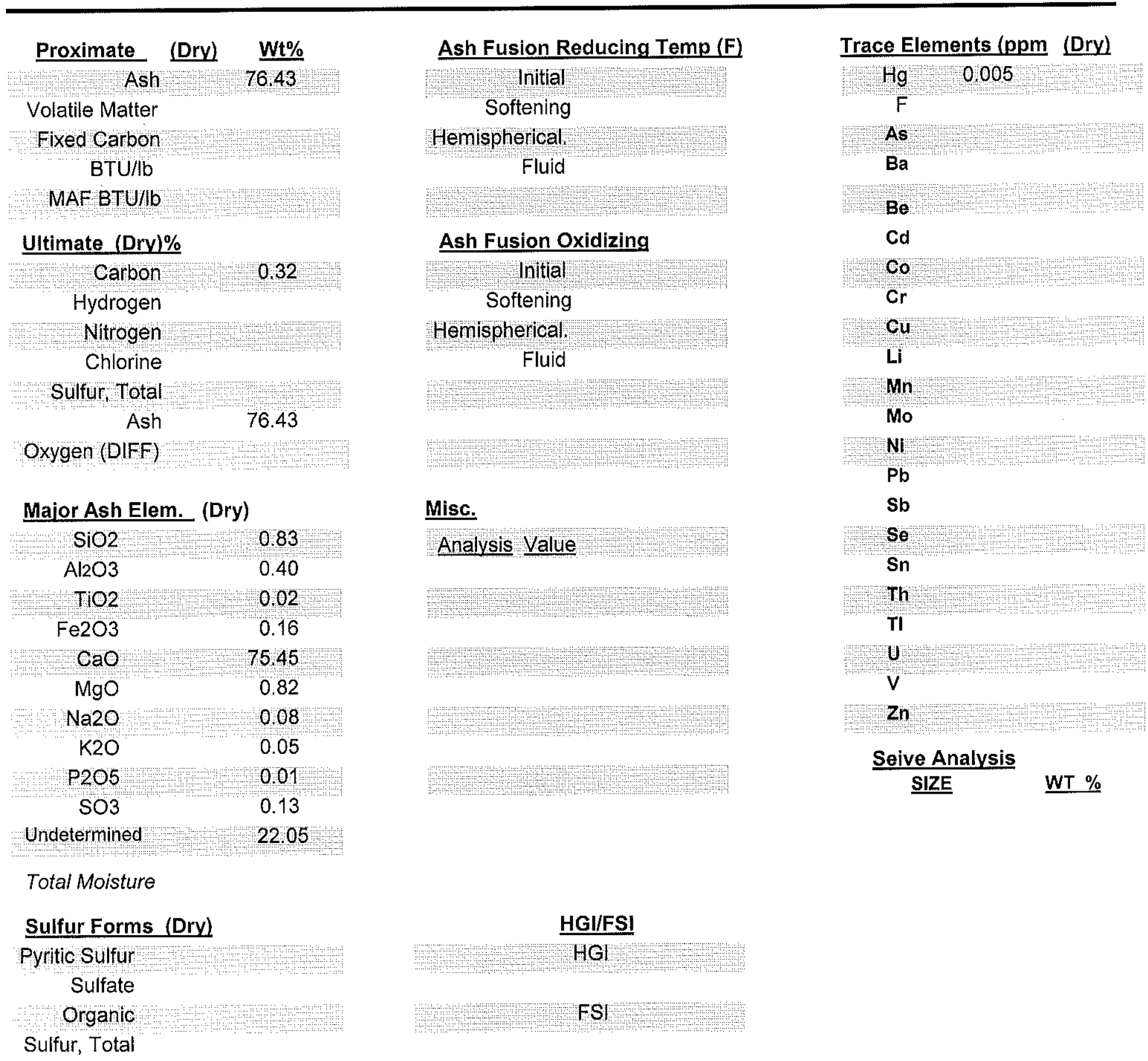

As Determined Moisture $\quad 0.27 \quad \%$

These values have been reviewed and are approved for transmission. 197 


\section{Y $=$ CONSOL ENERGY.}
Research and Development
www.consolenergy.com
4000 Brownsville Rd.
South Park, PA 15129

Sample Description.: HYDRATED LIME MARCH 28 14:30

Sample No.: TEST 2

Date Received: 04/03/2007

Analytical No.:

20071809

Project No.: $1621 \quad-085-000$

Date Completed: 04/19/2007

Submitted By: D. CONNELL

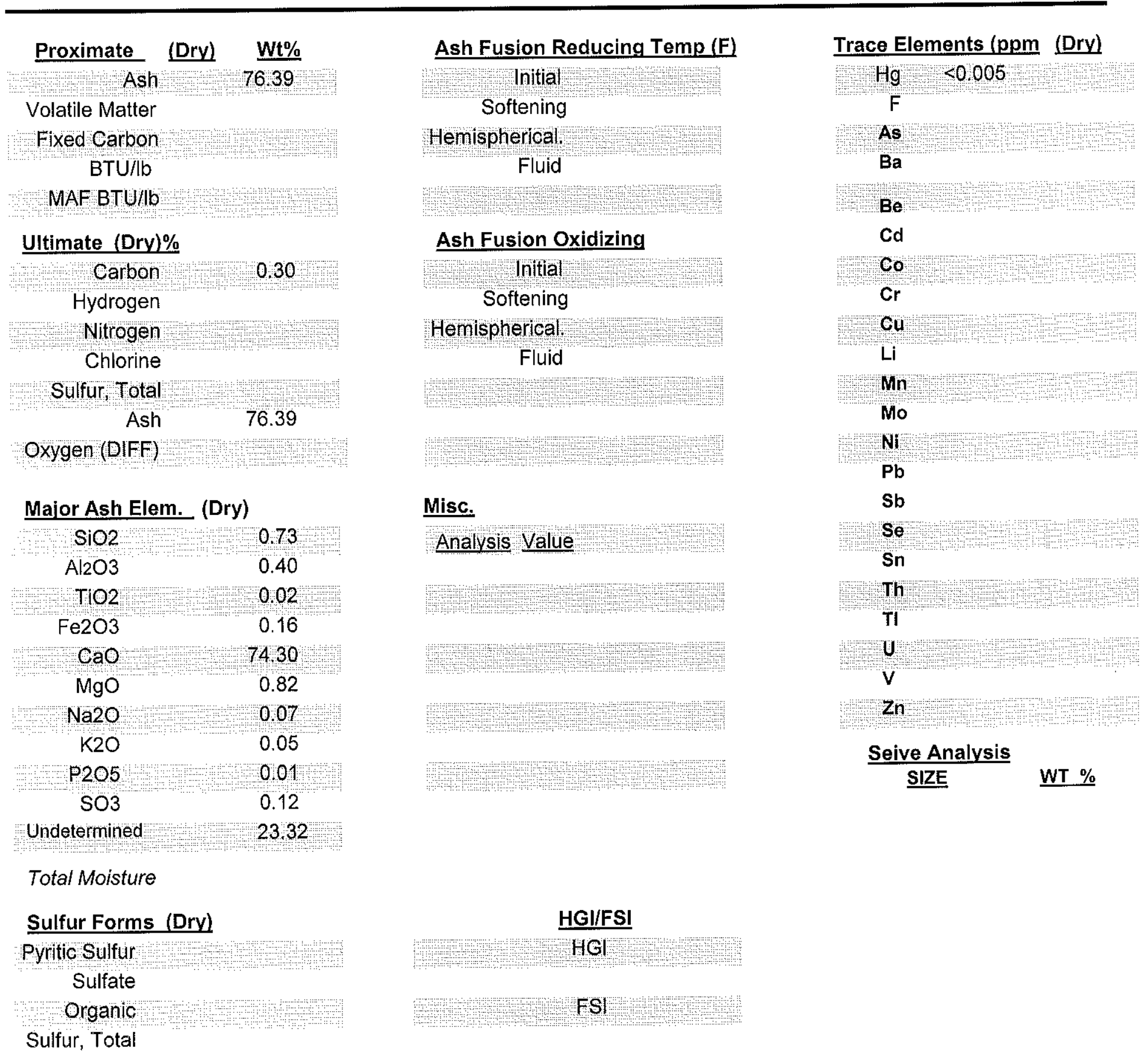

As Determined Moisture $0.29 \quad \%$

These values have been reviewed and are approved for transmission. Jhi 7 . 


\section{严 CONSOL ENERGY}

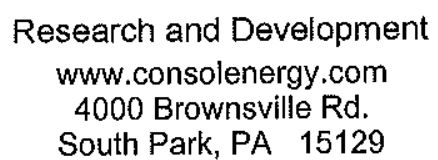

Sample Description.: HYDRATED LIME MARCH 28 17:00

Sample No.: TEST 3

Date Received: 04/03/2007

Analytical No.:

20071810

Project No.: $1621 \quad-085-000$

Date Completed: 04/19/2007

Submitted By: D. CONNELL

\begin{tabular}{|c|c|c|c|}
\hline Proximate (Dry) & $\mathbf{W t} \%$ & Ash Fusion Reducing Temp (F) & Trace Elements (ppm (Drv) \\
\hline Ash & 76.39 & susunitial & $\mathrm{Hg}<<0.005$ \\
\hline Volatile Matter & & Softening & $\mathrm{F}$ \\
\hline Fixed Carbon & & Hemispherical. & As \\
\hline $\mathrm{BTU} / \mathrm{lb}$ & & Fluid & $\mathrm{Ba}$ \\
\hline $\mathrm{MAFBTU} / \mathrm{B}$ & & 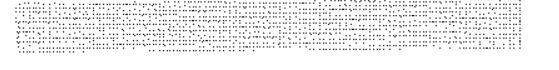 & $\mathrm{Be}$ \\
\hline Ultimate $($ Dry $) \%$ & & Ash Fusion Oxidizing & Cd \\
\hline Carbon & 0.32 & U Initial & Co \\
\hline Hydrogen & & Softening & $\mathrm{Cr}$ \\
\hline Nitrogen & 3 & Hemispherical. & $\mathrm{Cu}$ \\
\hline Chlorine & & Fluid & $\mathrm{Li}$ \\
\hline Sulfur, Total & +4 & & Mn \\
\hline Ash & 76.39 & & Mo \\
\hline Oxygen (DIFF) & 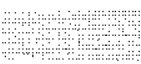 & ta & $\mathrm{Nl}$ \\
\hline & & & $\mathrm{Pb}$ \\
\hline Major Ash Elem. (D & & Misc. & $\mathrm{Sb}$ \\
\hline $\mathrm{SiO} 2$ & 0.71 & Analysis Value & Se \\
\hline $\mathrm{Al} 2 \mathrm{O} 3$ & 0.41 & & Sn \\
\hline $\mathrm{TTO} 2$ & 0.02 & & Th \\
\hline $\mathrm{Fe} 2 \mathrm{O} 3$ & 0.16 & & $\mathrm{TI}$ \\
\hline $\mathrm{CaO}$ & 74,19 & Ia & U \\
\hline $\mathrm{MgO}$ & 0.81 & & v \\
\hline $\mathrm{Na} 2 \mathrm{O}$ & 0.07 & 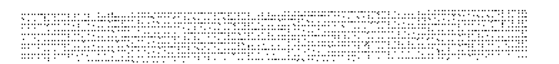 & $\mathrm{Zn}$ \\
\hline $\mathrm{K} 2 \mathrm{O}$ & 0.05 & & Seive Analysis \\
\hline $\mathrm{P} 205$ & 0.01 & $(4+4+4=$ & SIZE \\
\hline $\mathrm{SO} 3$ & 0.17 & & \\
\hline Undetermined & 23.40 & & \\
\hline \multicolumn{4}{|l|}{ Total Moisture } \\
\hline \multicolumn{2}{|l|}{ Sulfur Forms (Drv) } & \multicolumn{2}{|l|}{ HGI/FSI } \\
\hline \multicolumn{2}{|l|}{ Pyritic Sulfur } & \multicolumn{2}{|c|}{ Sulfate } \\
\hline Organic & us & FSI & \\
\hline
\end{tabular}

As Determined Moisture $0.35 \quad \%$

These values have been reviewed and are approved for transmission. $\gamma_{M}$ 


\section{击= CONSOLENERGY.}

Research and Development www.consolenergy.com 4000 Brownsville Rd. South Park, PA 15129

Sample Description.: HYDRATED LIME MARCH 29 9:30

Sample No.: TEST 1

Analytical No::

20071823

Date Received: 04/03/2007

Project No.: $1621-085-000$

Date Completed: 04/19/2007

Submitted By: D. CONNELL

\begin{tabular}{|c|c|}
\hline Proximate (Dry) & $\underline{\mathbf{W t} \%}$ \\
\hline जutush & 75.73 \\
\hline \multicolumn{2}{|l|}{ Volatile Matter } \\
\hline \multicolumn{2}{|l|}{ Fixed Carbon } \\
\hline \multicolumn{2}{|l|}{$\mathrm{BTU} / \mathrm{lb}$} \\
\hline \multicolumn{2}{|l|}{$\mathrm{MAFBTU} / \mathrm{lb}$} \\
\hline \multicolumn{2}{|l|}{ Ultimate (Drv)\% } \\
\hline Carbon & 0.44 \\
\hline \multicolumn{2}{|l|}{ Hydrogen } \\
\hline \multicolumn{2}{|l|}{ Nitrogen } \\
\hline \multicolumn{2}{|l|}{ Chlorine } \\
\hline \multicolumn{2}{|l|}{ Sulfur, Total } \\
\hline Ash & 75.73 \\
\hline \multicolumn{2}{|l|}{ Oxygen (DIFF) } \\
\hline \multicolumn{2}{|c|}{ Major Ash Elem. (Dry) } \\
\hline $\mathrm{SiO} 2$ & 1.31 \\
\hline $\mathrm{Al}_{2} \mathrm{O} 3$ & 0.38 \\
\hline $\mathrm{TlO} 2$ & 0.02 \\
\hline $\mathrm{Fe} 2 \mathrm{O} 3$ & 0.14 \\
\hline $\mathrm{CaO}$ & 70.01 \\
\hline $\mathrm{MgO}$ & 0.80 \\
\hline $\mathrm{Na} 20$ & 0.01 \\
\hline $\mathrm{K} 2 \mathrm{O}$ & 0.04 \\
\hline $\mathrm{P} 205$ & 0.01 \\
\hline $\mathrm{SO} 3$ & 0.20 \\
\hline Undetermined & 27.08 \\
\hline
\end{tabular}

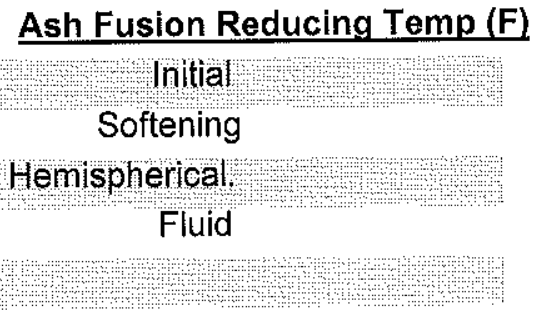

\section{Ash Fusion Oxidizing}

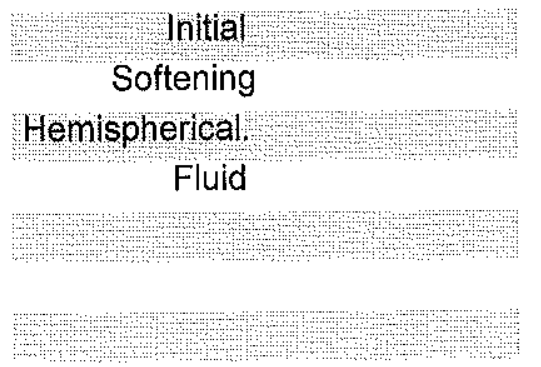

Misc.
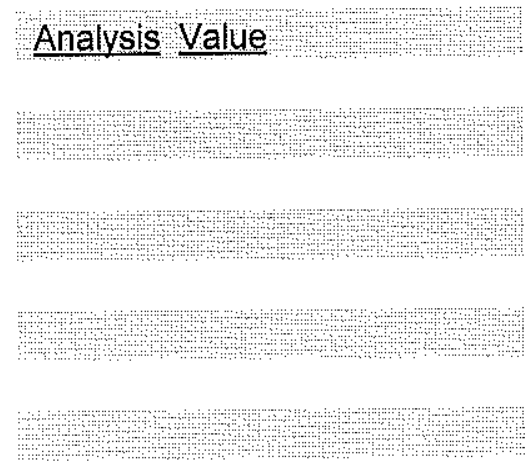

Trace Elements (ppm (Dry)

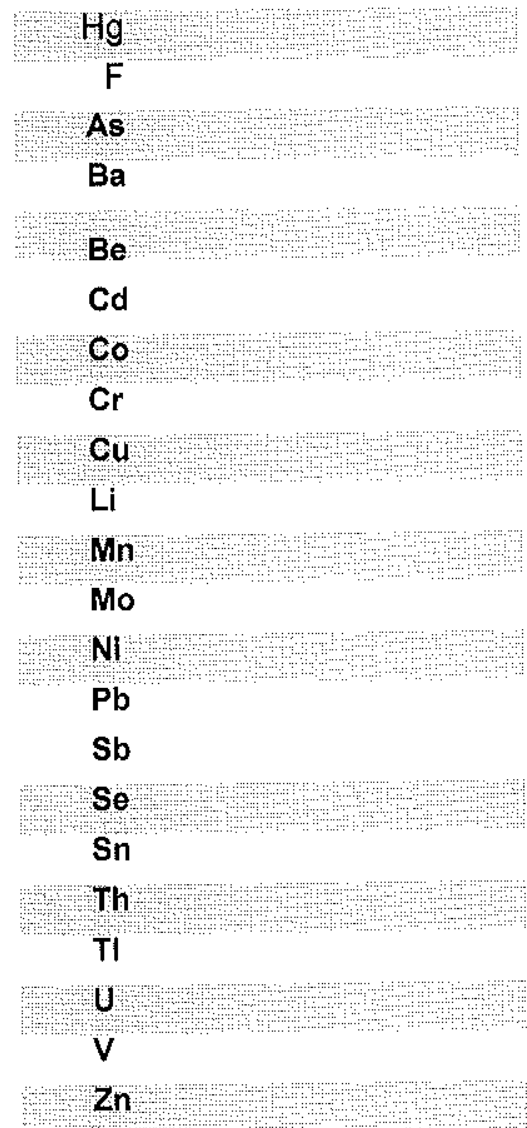

Seive Analysis SIZE
HGI/FSI

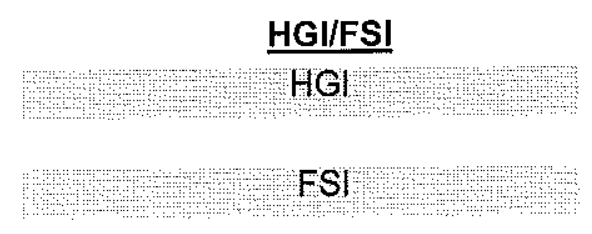

FSI

\section{Sulfur Forms (Dry)}

Pyritic Sulfur

Sulfate

Organic

Sulfur, Total

As Determined Moisture $0.09 \quad \%$ These values have been reviewed and are approved for transmission. 7 X 


\title{
YeC CONSOL ENERGY
}

\author{
Research and Development \\ uww.consolenergy.com \\ 4000 Brownsville Rd. \\ South Park, PA 15129
}

Sample Description.: HYDRATED LIME MARCH 29 13:30

Sample No.: TEST 2

Date Received: 04/03/2007

Analytical No.: $\quad 20071824$

Project No.: $1621-085-000$

Date Completed: $04 / 19 / 2007$

Submitted By: D. CONNELL

\begin{tabular}{|c|c|}
\hline Proximate (Dry) & $\underline{\mathrm{Wt}} \%$ \\
\hline Ash & 75.52 \\
\hline \multicolumn{2}{|l|}{ Volatile Matter } \\
\hline \multicolumn{2}{|l|}{ Fixed Carbon } \\
\hline \multicolumn{2}{|l|}{ BTU/lb } \\
\hline \multicolumn{2}{|l|}{ MAF BTU/L } \\
\hline \multicolumn{2}{|l|}{ Ultimate (Dry)\% } \\
\hline Carbon & 0.58 \\
\hline \multicolumn{2}{|l|}{ Hydrogen } \\
\hline \multicolumn{2}{|l|}{ Nitrogen } \\
\hline \multicolumn{2}{|l|}{ Chlorine } \\
\hline \multicolumn{2}{|l|}{ Sulfur, Total } \\
\hline Ash & 75.52 \\
\hline \multicolumn{2}{|l|}{ Oxygen (DIFF) } \\
\hline \multicolumn{2}{|c|}{ Major Ash Elem. (AsDet) } \\
\hline SiO2 & 1.31 \\
\hline $\mathrm{Al} 2 \mathrm{O} 3$ & 0.38 \\
\hline TIO2 & 0,02 \\
\hline $\mathrm{Fe} 2 \mathrm{O} 3$ & 0.18 \\
\hline $\mathrm{CaO}$ & 6905 \\
\hline $\mathrm{MgO}$ & 0.80 \\
\hline $\mathrm{Na2O}$ & 0.02 \\
\hline $\mathrm{K} 2 \mathrm{O}$ & 0.04 \\
\hline P2O5 & 0.02 \\
\hline SO3 & 0.21 \\
\hline Undetermined & 2797 \\
\hline
\end{tabular}

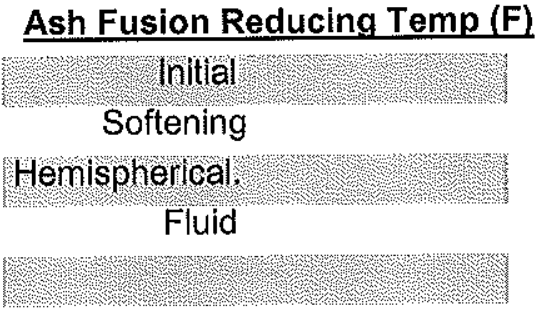

Ash Fusion Oxidizing

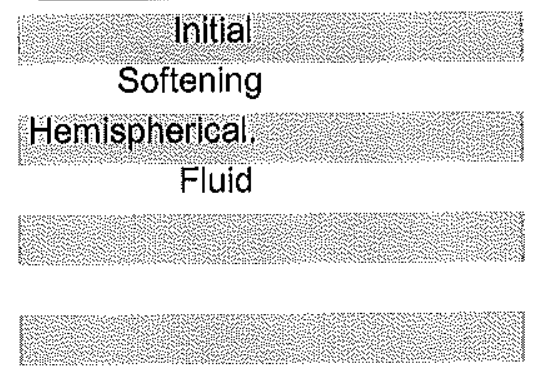

Misc.

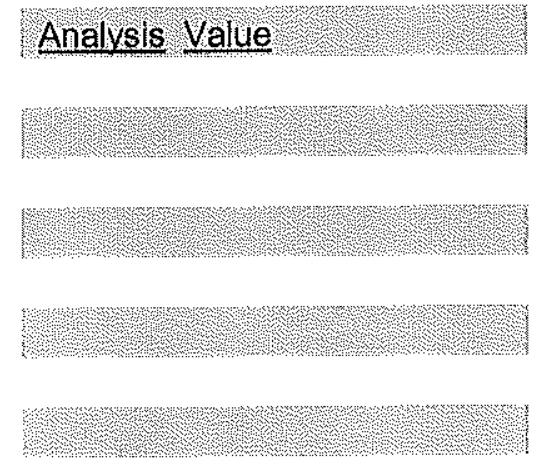

Trace Elements (ppm (Dry)

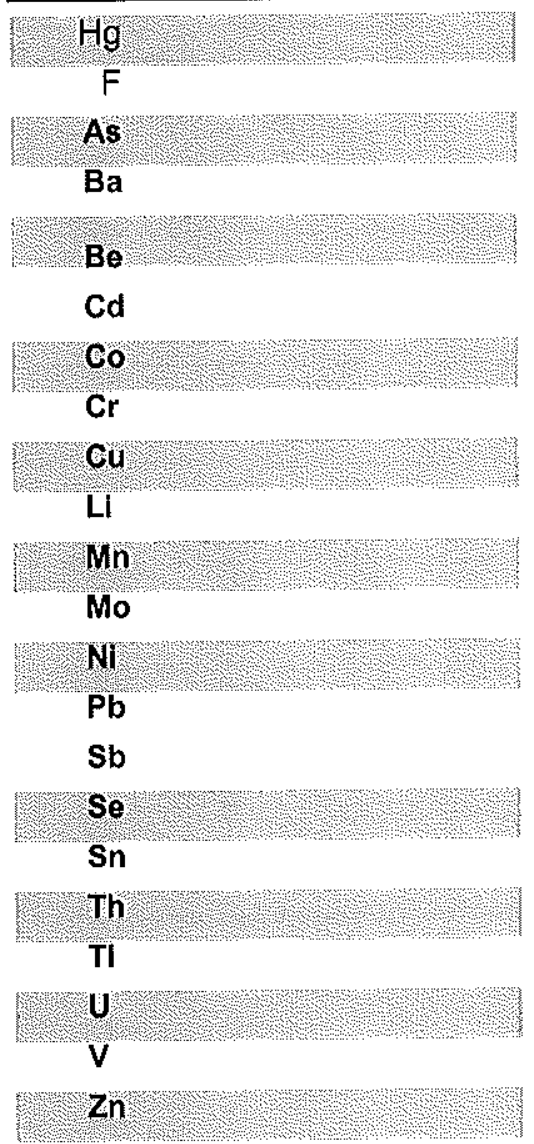

Seive Analysis SIZE
HGI/FSI

HGI

FSI

\section{Sulfate}

Organic

Sulfur, Total
As Determined Moisture 0

$\%$

These values have been reviewed and are approved for transmission. $\mathrm{y}$. 


\section{을 CONSOL ENERGY.}

Research and Development

www.consolenergy.com

4000 Brownsville Rd.

South Park, PA 15129

Sample Description.: HYDRATED LIME MARCH 29 16:30

Sample No.: TEST 3

Date Received: 04/03/2007

Analytical No.:

20071825

Project No.: $1621 \quad-085-000$

Date Completed: 04/19/2007

Submitted By: D. CONNELL

\begin{tabular}{|c|c|}
\hline Proximate (Dry) & $\mathbf{W t} \%$ \\
\hline Ash & 75.92 \\
\hline \multicolumn{2}{|l|}{ Volatile Matter } \\
\hline \multicolumn{2}{|l|}{ Fixed Carbon } \\
\hline \multicolumn{2}{|l|}{ BTU/b } \\
\hline \multicolumn{2}{|l|}{$\mathrm{MAF} B \mathrm{BT} / \mathrm{lb}$} \\
\hline \multicolumn{2}{|l|}{ Ultimate (Drv)\% } \\
\hline Carbon & 0.42 \\
\hline \multicolumn{2}{|l|}{ Hydrogen } \\
\hline \multicolumn{2}{|l|}{ Nitrogen } \\
\hline \multicolumn{2}{|l|}{ Chlorine } \\
\hline \multicolumn{2}{|l|}{ Sulfur, Total } \\
\hline Ash & 75.92 \\
\hline Oxygen (D|FF) & \\
\hline
\end{tabular}

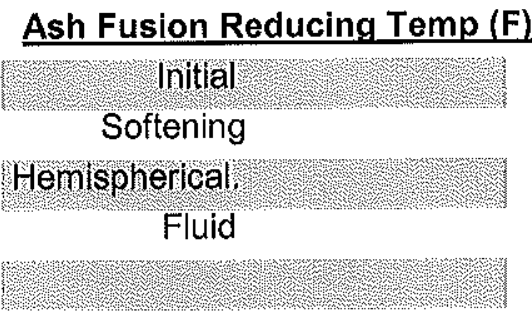

\section{Ash Fusion Oxidizing}

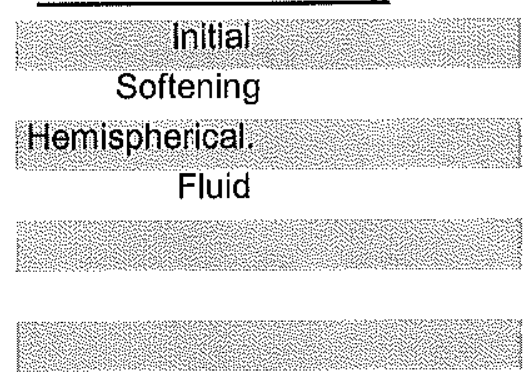

Misc.

\begin{tabular}{cc} 
Major Ash Elem. & (AsDet) \\
\hline $\mathrm{SiO} 2$ & 1.31 \\
$\mathrm{Al} 2 \mathrm{O} 3$ & 0.37 \\
$\mathrm{~T} 1 \mathrm{O} 2$ & 0.02 \\
$\mathrm{Fe} 2 \mathrm{O} 3$ & 0.14 \\
$\mathrm{CaO}$ & 70.83 \\
$\mathrm{MgO}$ & 0.81 \\
$\mathrm{Na2O}$ & 0.02 \\
$\mathrm{~K} 2 \mathrm{O}$ & 0.04 \\
$\mathrm{P} 2 \mathrm{O} 5$ & 0.01 \\
$\mathrm{SO} 3$ & 0.18 \\
Undetermined & 26.27 \\
\hline
\end{tabular}

Total Moisture

\section{Sulfur Forms (Dry)}

Pyritic Sulfur

Sulfate

Organic

Sulfur, Total
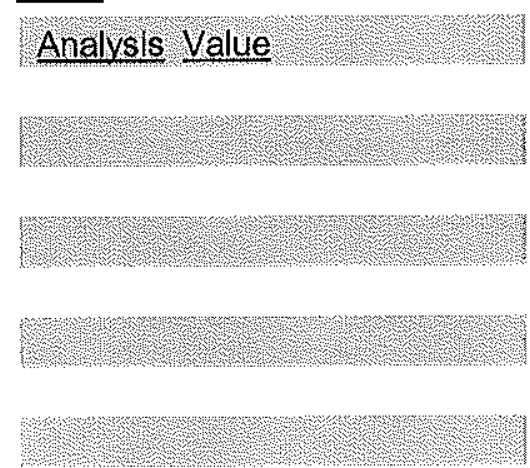

HGI/FSI

HGI

FSI
Trace Elements (ppm (Dry)

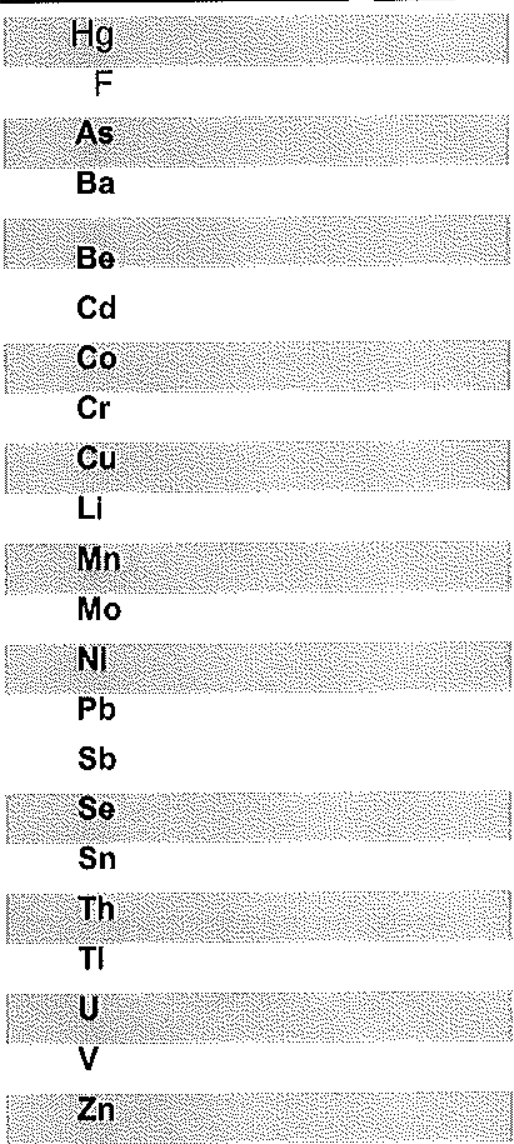

Seive Analysis SIZE

As Determined Moisture $0 \quad \%$

These values have been reviewed and are approved for transmission. $\mathrm{m} M$ ? 
Research and Development

www.consolenergy.com

4000 Brownsville Rd.

South Park, PA 15129

Sample Description.: HYDRATED LIME MARCH 30 10:00

Sample No:: TEST 1

Date Received: 04/03/2007

Analytical No.:

Project No.: $1621 \quad-085 \quad-000$

Date Completed: 04/19/2007

Submitted By: D. CONNELL

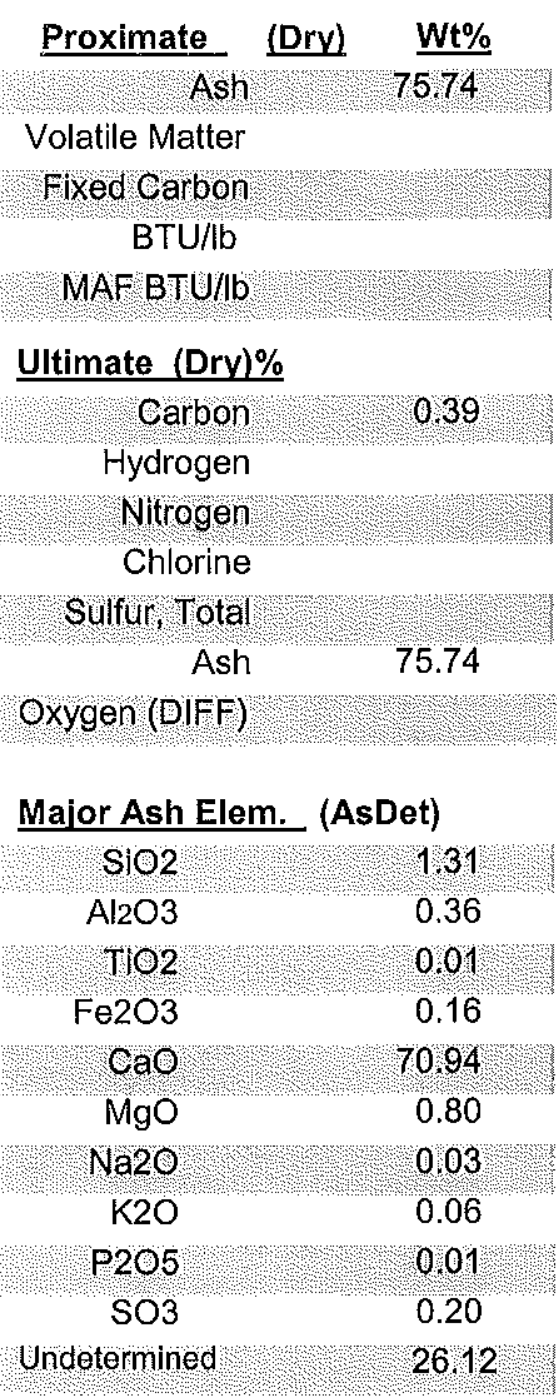

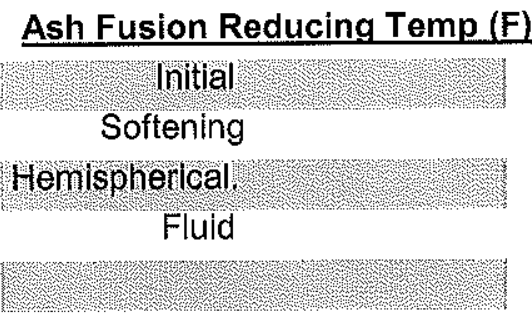

Ash Fusion Oxidizing

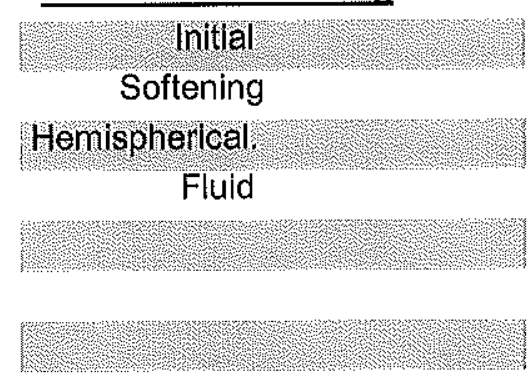

Misc.

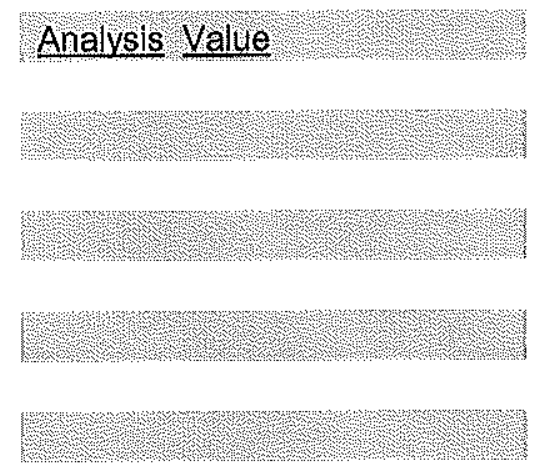

Trace Elements (ppm (Drv)

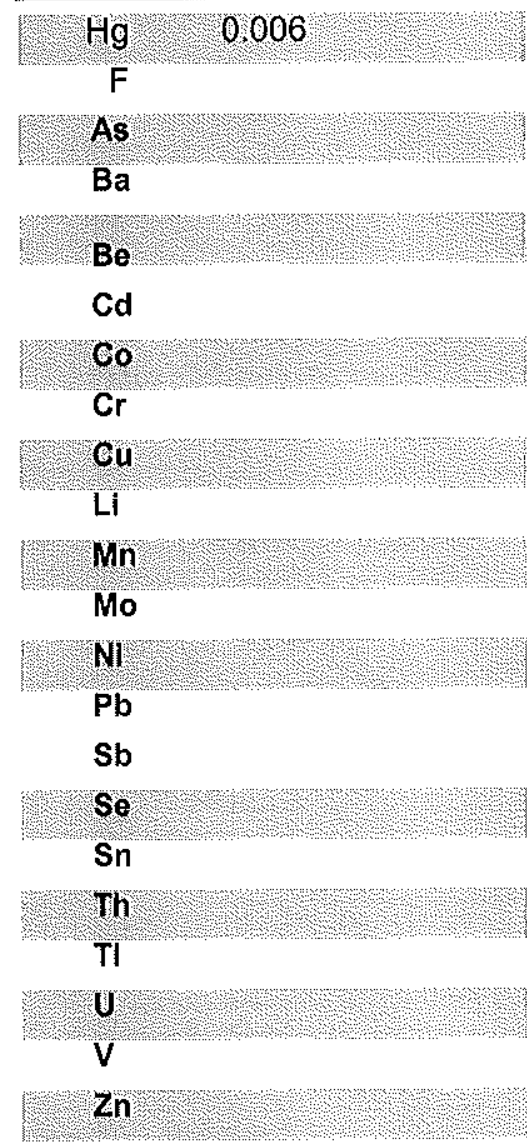

\section{Seive Analysis} SIZE

Total Moisture

\section{Sulfur Forms (Dry)}

Pyritic Suilfur

Sulfate

Organic

Sulfur, Total

HGI/FSI

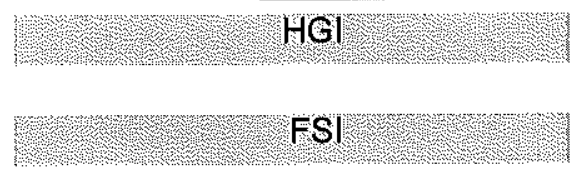

As Determined Moisture 0

$\%$

These values have been reviewed and are approved for transmission $\mathrm{XuY}$ 


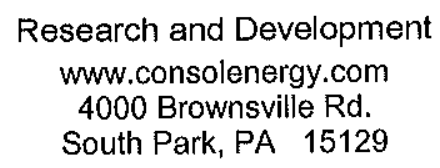

Sample Description.: HYDRATED LIME MARCH 30 13:45

Sample No.: TEST 2

Analytical No.:

Date Received: 04/03/2007

Project No.: $1621-085-000$

Date Completed: $04 / 19 / 2007$

Submitted By: D. CONNELL

\begin{tabular}{|c|c|}
\hline Proximate (Dry) & $\mathbf{W t} \%$ \\
\hline Ash & 76.00 \\
\hline \multicolumn{2}{|l|}{ Volatile Matter } \\
\hline \multicolumn{2}{|l|}{ Fixed Carbon } \\
\hline \multicolumn{2}{|l|}{ BTU/lb } \\
\hline \multicolumn{2}{|l|}{ MAF BTU/L } \\
\hline \multicolumn{2}{|l|}{ Ultimate (Drv)\% } \\
\hline Carbon & 0.45 \\
\hline \multicolumn{2}{|l|}{ Hydrogen } \\
\hline \multicolumn{2}{|l|}{ Nitrogen } \\
\hline \multicolumn{2}{|l|}{ Chlorine } \\
\hline \multicolumn{2}{|l|}{ Sulfur, Total } \\
\hline Ash & 76.00 \\
\hline \multicolumn{2}{|l|}{ Oxygen (DIFF) } \\
\hline \multicolumn{2}{|c|}{ Major Ash Elem. (AsDet) } \\
\hline $\mathrm{SIO} 2$ & 134 \\
\hline $\mathrm{Al} 2 \mathrm{O} 3$ & 0.36 \\
\hline T1O2 & 0.01 \\
\hline $\mathrm{Fe} 2 \mathrm{O} 3$ & 0.14 \\
\hline $\mathrm{CaO}$ & 71.24 \\
\hline $\mathrm{MgO}$ & 0.80 \\
\hline $\mathrm{Na2O}$ & 0.02 \\
\hline $\mathrm{K} 2 \mathrm{O}$ & 0.05 \\
\hline $\mathrm{P} 2 \mathrm{O} 5$ & $<0,01$ \\
\hline $\mathrm{SO} 3$ & 0.22 \\
\hline Undetermined & 25.82 \\
\hline
\end{tabular}

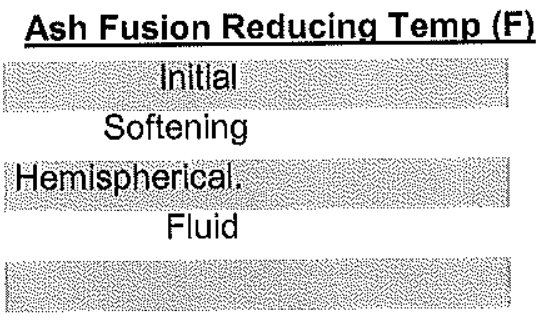

\section{Ash Fusion Oxidizing}

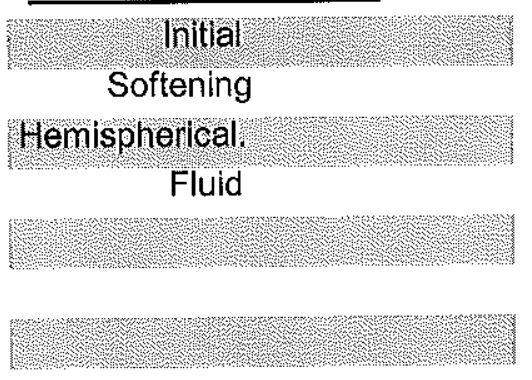

Misc.
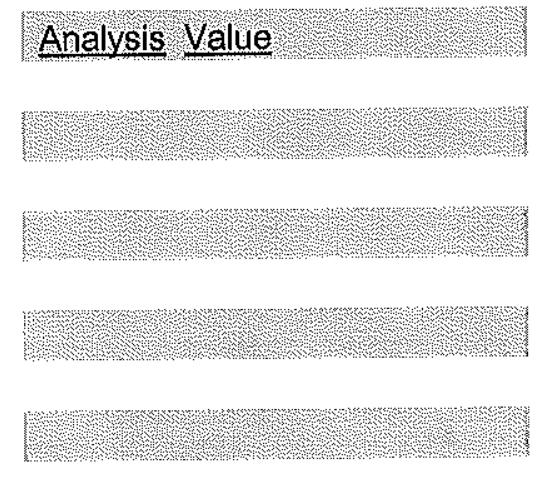

\section{Total Moisture}

Sulfur Forms (Dry)

Pyritic Sulfur Sulfate Organic Sulfur, Total

HGI/FSI

HGl

FSI
Trace Elements (ppm (Drv)

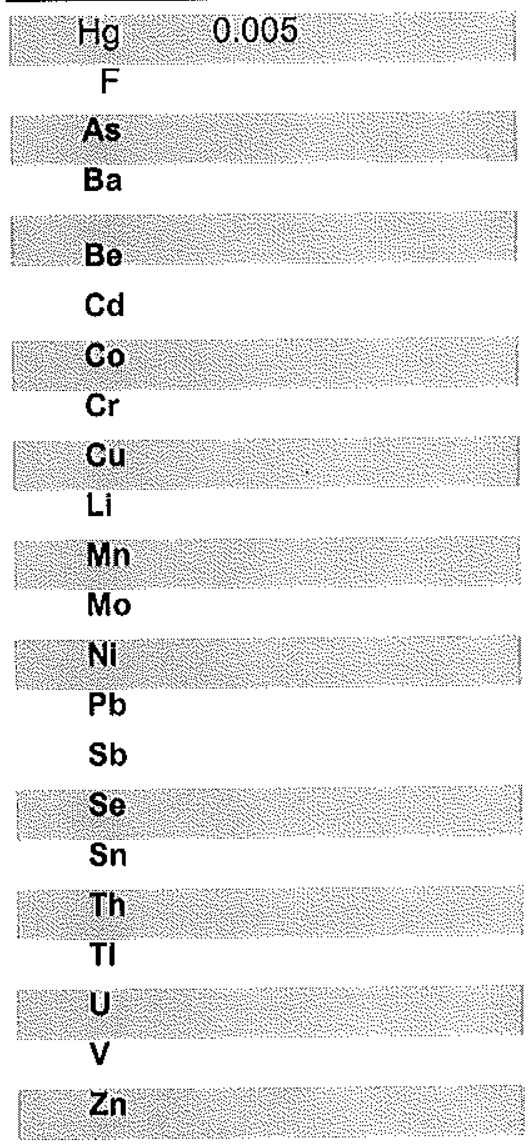

Seive Analysis SIZE

As Determined Moisture $0 \quad \%$

These values have been reviewed and are approved for transmission. Mht 
Research and Development

www.consolenergy.com

4000 Brownsville Rd.

South Park, PA 15129

Sample Description.: HYDRATED LIME MARCH 30 16:45

Sample No.: TEST 3

Date Received: 04/03/2007

Date Completed: 04/19/2007

Analytical No:: $\quad 20071840$

Project No:: $1621 \quad-085 \quad-000$

Submitted By: D. CONNELL

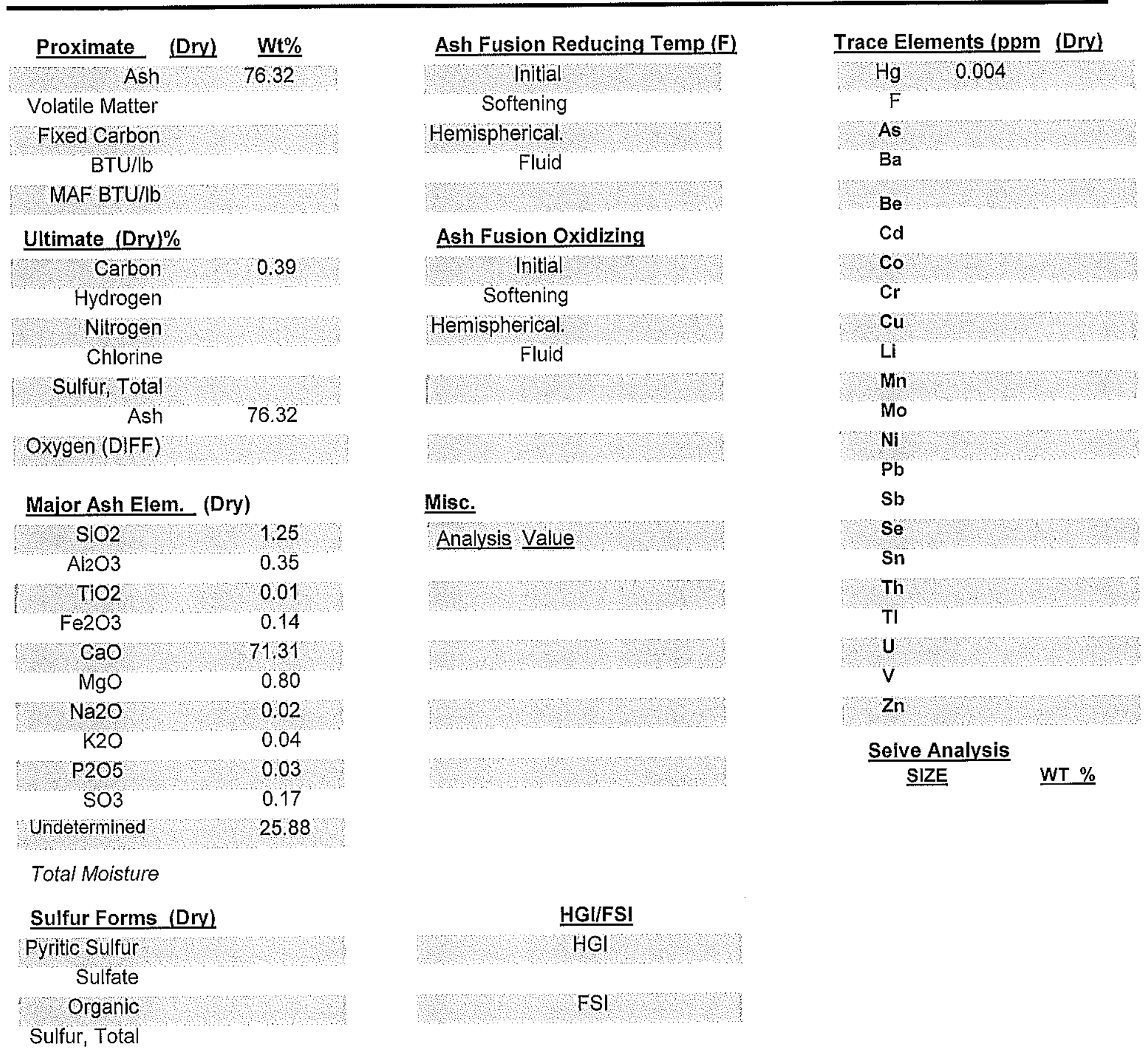

As Determined Moisture $\quad 0.06 \quad \%$

These values have been reviewed and are approved for transmission: J $X$ X 
Research and Development

www.consolenergy.com

4000 Brownsville Rd.

South Park, PA 15129

Sample Description.: ACTIVATED CARBON 3/29/07

Sample No.: $15: 30$

Analytical No:

20071845

Date Received: 04/04/2007

Project No.: $1621 \quad-085-000$

Date Completed: 04/20/2007

Submitted By: D. CONNELL

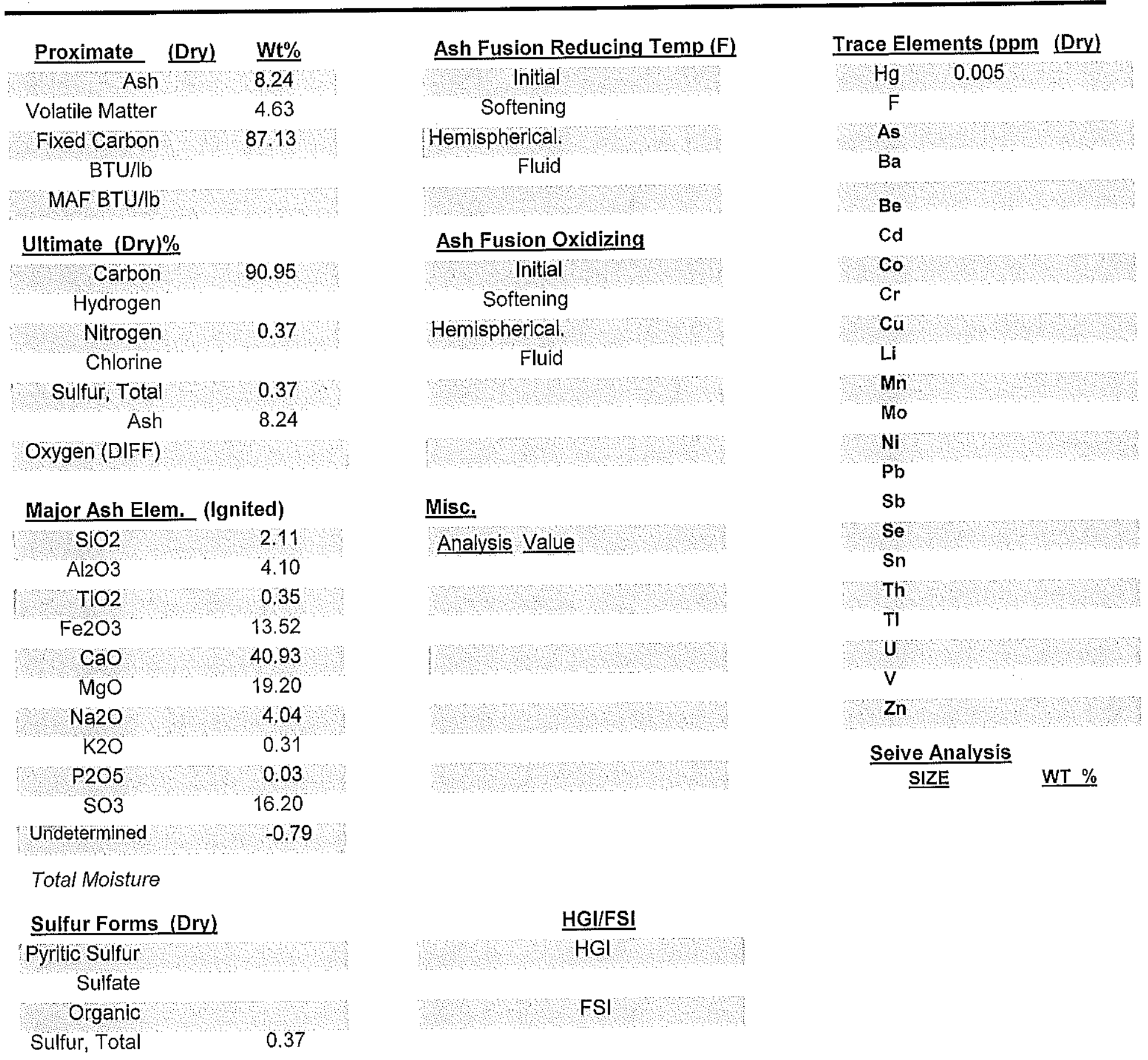

As Determined Moisture $1.21 \%$
These values have been reviewed and are approved for transmission. 


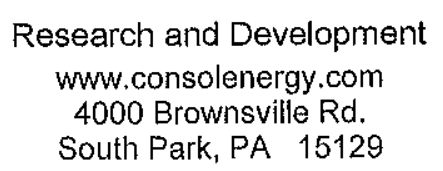

Sample Description.: ACTIVATED CARBON 3/30/07

Sample No.: $12: 00$

Analytical No.:

20071844

Date Received: 04/04/2007

Date Completed: 04/20/2007

Project No.: $1621-085 \quad-000$

Submitted By: D. CONNELL

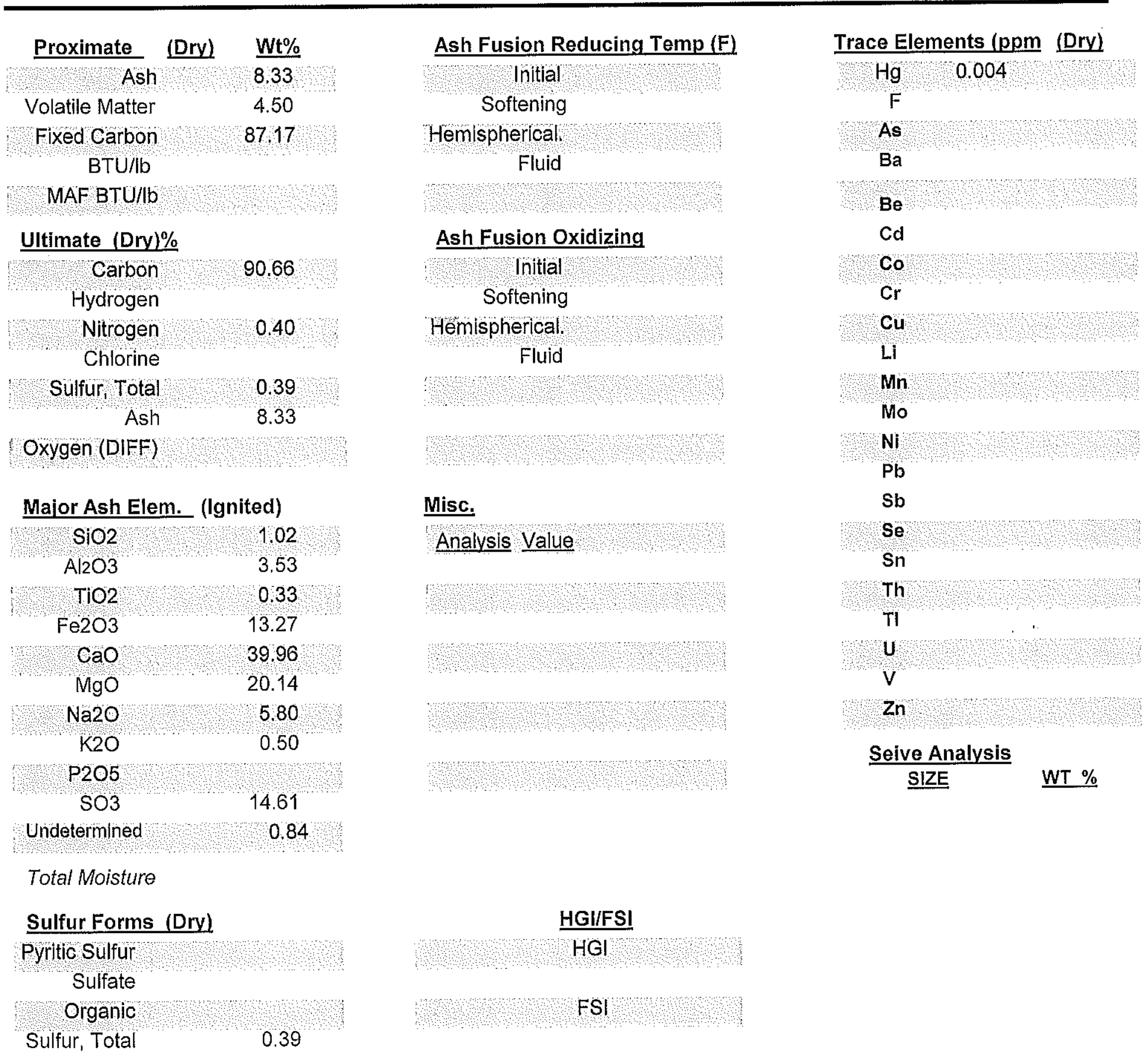

As Determined Moisture $1.17 \quad \%$

These values have been reviewed and are approved for transmission. $\mathrm{AP}_{\text {P }}$ 
Research and Development www.consolenergy.com 4000 Brownsville $\mathrm{Rd}$.

South Park, PA 15129
AES GREENIDGE UREA COMP 3/28/2007

Sample No.: $3 / 28 / 2007$

Date Received: 04/18/2007

Date Completed: 07/19/2007
Analytical No: 20071971

Project No.: $1621 \quad-085 \quad-000$

Submitter: D. CONNELL

\begin{tabular}{|c|c|c|c|c|c|c|c|}
\hline \multirow[b]{2}{*}{ Parameter } & \multicolumn{4}{|c|}{$\begin{array}{c}\text { Water Result } \\
\text { (mg/L unless noted otherwise) }\end{array}$} & & & \\
\hline & Value & Value & Units & Avg Value & \multicolumn{3}{|c|}{ Quality Control Calculation } \\
\hline $\mathrm{pH}$ & 9.56 & & & & Ion Sum & & 0.00 \\
\hline Acidity, $\mathrm{CaCO}_{3}$ & & & & & Cation Sum & & 0.00 \\
\hline Alkalinity, $\mathrm{CaCO}_{3}$ & & & & & & & \\
\hline Hydroxide, $\mathrm{CaCO}_{3}$ & & & & & Anion Sum & & 0.00 \\
\hline Carbonate, $\mathrm{CaCO}_{3}$ & & & & & Ion Balance & & 0.00 \\
\hline Bicarbonate, $\mathrm{CaCO} 3$ & & & & & & & \\
\hline Total Suspended Sollds & $<6$ & & & & $\%$ Ion Imbe & alance & 0.00 \\
\hline Total Dissolved Solids & & & & & & & \\
\hline Specific Conductivity & & & & & & & \\
\hline Hardness & & & & & & & \\
\hline Turbidity & & & & & & & \\
\hline Osmotic Pressure & & & & & & & \\
\hline Dissolved Oxygen & & & & & Analysis & & \\
\hline Ammonia, $\mathrm{N}$ & 595 & & & & Orthophosp & 45.20 & \\
\hline Total Elements & & & & & & & \\
\hline Aluminum & & & & & & & \\
\hline Calcium & & & & & & & \\
\hline Iron & & & & & & & \\
\hline Magnesium & & & & & & & \\
\hline Manganese & & & & & & & \\
\hline Potassium & & & & & & & \\
\hline Phosphorous & & & & & & & \\
\hline Silicon & & & & & & & \\
\hline Sodium & & & & & & & \\
\hline Chromium & & & & & & & \\
\hline Anions: & & & & & & & \\
\hline Sulfate & & & & & & & \\
\hline Chloride & & & & & & & \\
\hline Nitrate, N & & & & & & & \\
\hline Nitrite, $\mathrm{N}$ & & & & & & & \\
\hline Bromide & & & & & & & \\
\hline Fluoride & & & & & & & \\
\hline
\end{tabular}

COD

These values have been reviewed and are approved for transmissiong $\mathcal{Y}$ 
Research and Development www.consolenergy.com 4000 Brownsville Rd.

South Park, PA 15129

AES GREENIDGE UREA COMP 3/30/2007

Sample No: $3 / 30 / 2007$

Analytical No.: 20071972

Date Received: 04/18/2007

Date Completed: 07/19/2007

Project No.: $1621 \quad-085 \quad-000$

Submitter: D. CONNELL

Water Result

(mg/L unless noted otherwise)

Parameter

\section{Value}

Value

9.40

Acidity, $\mathrm{CaCO} 3$

Alkalinity, $\mathrm{CaCO}_{3}$

Hydroxide, $\mathrm{CaCO}_{3}$

Carbonate, $\mathrm{CaCO}_{3}$

Bicarbonate, $\mathrm{CaCO}_{3}$

Total Suspended Solids

Total Dissolved Solids

Specific Conductivity

Hardness

Turbidity

Osmotic Pressure

Dissolved Oxygen

Ammonia, $\mathrm{N}$

Total Elements

Aluminum

Calcium

Iron

Magnesium

Manganese

Potassium

Phosphorous

Silicon

Sodium

Chromium

Anions:

Sulfate

Chloride

Nitrate, $\mathrm{N}$

Nitrite, N

Bromide

Fluoride

$<6$

\section{Units Avg Value}

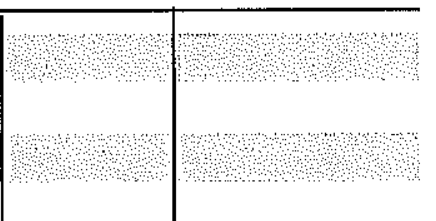

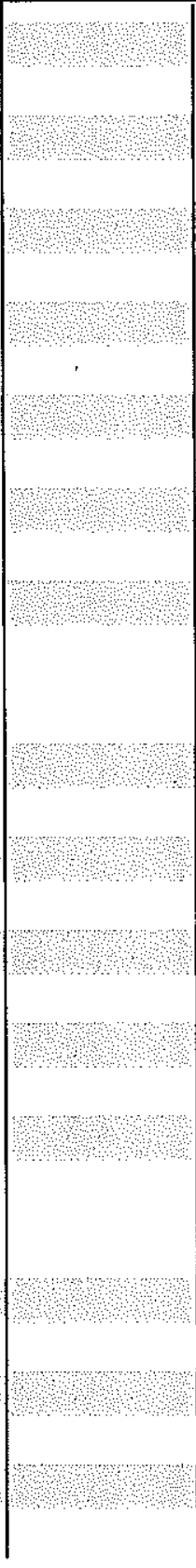

Quality Control Calculations

Ion Sum

0.00

Cation Sum

0.00

Anion Sum

0.00

Ion Balance

0.00

$\%$ Ion Imbalance

0.00
Analysis

Density

Orthophosp $\quad 79.20$

COD

These values have been reviewed and are approved for transmission $y$. 
APPENDIX E

Unit 4 Stack CEM Data 
Daily Stack Calibration Report

Generated: 10/16/2007

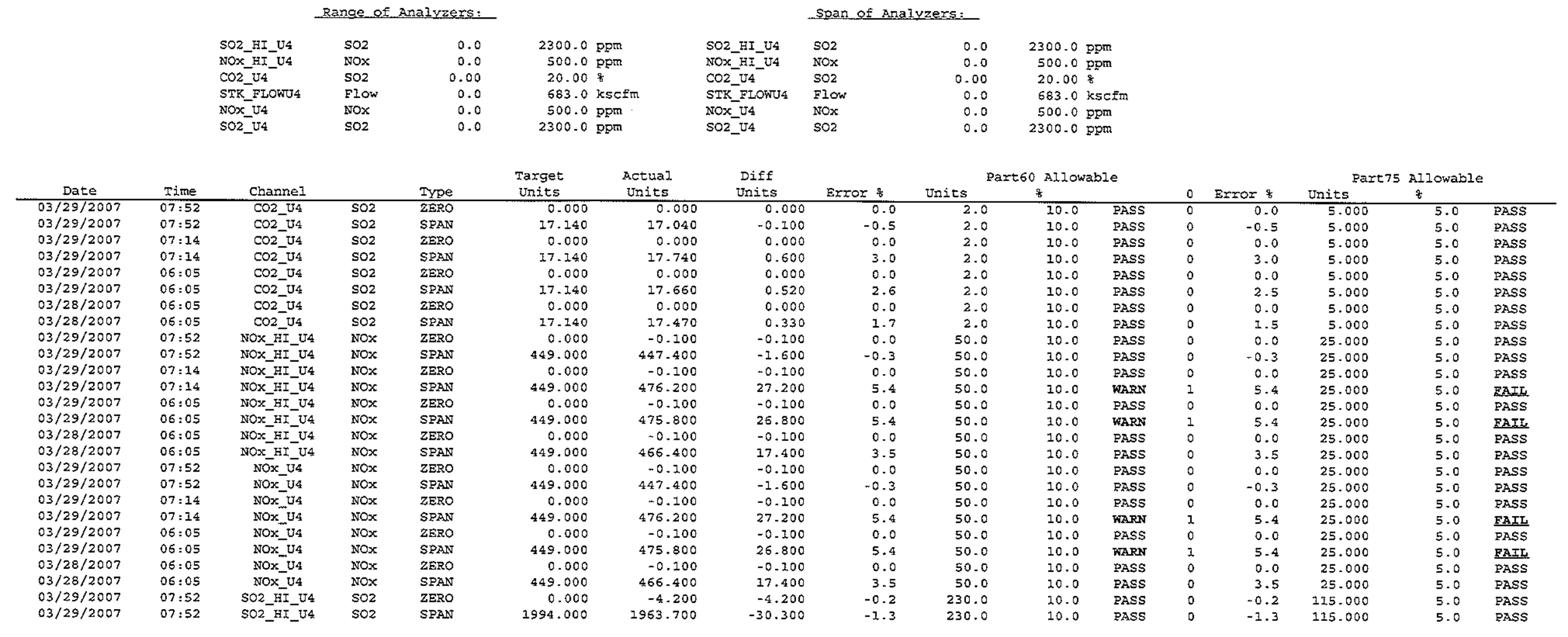


Period Start: $3 / 28 / 2007$ Period End: $3 / 30 / 2007$ Source: STACK 4
Included Calibrations: Daily (40CFR60)/(40CFR75)

\begin{tabular}{|c|c|c|c|c|c|c|c|c|c|c|c|c|c|c|c|c|}
\hline & & & & & Target & Actual & Diff & \multicolumn{6}{|c|}{ Part60 Allowable } & \multirow{2}{*}{\multicolumn{3}{|c|}{ Part75 Allowable }} \\
\hline$\frac{\text { Date }}{03 / 29 / 2007}$ & $\frac{\text { Time }}{07: 14}$ & $\begin{array}{l}\text { Charmel } \\
\text { S02 }\end{array}$ & & $\frac{\text { Type }}{2 \text { ppo }}$ & Units & Units & Units & Error \& & Units & 8 & & WD & Error $q$ & & & \\
\hline & $07: 14$ & & 502 & ZERO & 0.000 & -3.800 & -3.800 & -0.2 & 230.0 & 10.0 & PASS & 0 & -0.2 & 115.000 & 5.0 & PASS \\
\hline $03 / 29 / 2007$ & $07: 14$ & $\mathrm{SO}_{2} \mathrm{HI}+\mathrm{U} 4$ & 502 & SPAN & 1994.000 & 2066.400 & 72.400 & 3.1 & 230.0 & 10.0 & PASS & 0 & 3.1 & 115.000 & 5.0 & RASS \\
\hline $03 / 29 / 2007$ & $06=05$ & SO2_HI_U4 & $\mathrm{SO} 2$ & ZERO & 0.000 & -6.500 & -6.500 & -0.3 & 230.0 & 10.0 & PASS & 0 & -0.3 & 115.000 & 5.0 & RASS \\
\hline $03 / 29 / 2007$ & $06: 05$ & SO2_HII_U4 & $\mathrm{SO} 2$ & SPAN & 1994.000 & 2110.100 & 116.100 & 5.0 & 230.0 & 10.0 & PASS & 0 & 5.0 & 115.000 & 5.0 & PASS \\
\hline $03 / 28 / 2007$ & $07: 52$ & $\mathrm{SO} 2 \mathrm{HI}$-U4 & $\mathrm{SO} 2$ & ZERO & 0.000 & -4.700 & -4.700 & -0.2 & 230.0 & 10.0 & PASS & 0 & -0.2 & 115.000 & 5.0 & PASS \\
\hline $03 / 28 / 2007$ & $07: 52$ & $\mathrm{SO} 2$ HI_U4 & $\mathrm{SO2}$ & SPAN & 1994.000 & 2014.400 & 20.400 & 0.9 & 230.0 & 10.0 & PASS & 0 & 0.9 & 115.000 & 5.0 & PASS \\
\hline $03 / 28 / 2007$ & $06: 22$ & SO2_HI_U4 & 502 & ZERO & 0.000 & -1.900 & +1.900 & -0.1 & 230.0 & 10.0 & PASS & 0 & -0.1 & 115.000 & 5.0 & PASS \\
\hline $03 / 28 / 2007$ & $06: 22$ & $\mathrm{SO} 2 \mathrm{HI}$ _U4 & $\mathrm{SO} 2$ & SPAN & 1994.000 & 2046.400 & 52.400 & 2.3 & 230.0 & 10.0 & PASS & 0 & 2.3 & 2.15 .000 & 5.0 & PASS \\
\hline $03 / 28 / 2007$ & $06: 05$ & $\mathrm{SO} 2 \mathrm{HI} \mathrm{H} 4$ & $\mathrm{SO} 2$ & ZERO & 0.000 & 0.900 & 0.900 & 0.0 & 230.0 & 10.0 & PASS & 0 & 0.0 & 115.000 & 5.0 & PASS \\
\hline $03 / 28 / 2007$ & $06=05$ & $\mathrm{SO} 2_{-} \mathrm{HI}{ }_{-} \mathrm{U} 4$ & $\mathrm{SO} 2$ & SPAN & 1994.000 & 2344.500 & 350.500 & 15.2 & 230.0 & 10.0 & $R D G$ & OOC & 15.2 & 115.000 & 5.0 & $R D G$ \\
\hline $03 / 29 / 2007$ & $07: 52$ & $\mathrm{SO} 2$ U4 4 & $\mathrm{SO}_{2}$ & ZERO & 0.000 & -4.200 & -4.200 & -0.2 & 230.0 & 20.0 & $\overline{\text { PASS }}$ & 0 & -0.2 & 115.000 & 5.0 & PASS \\
\hline $03 / 29 / 2007$ & $07: 52$ & $\mathrm{SO}_{2}=\mathrm{U} 4$ & 502 & SPAN & 1994.000 & 1963.700 & -30.300 & -1.3 & 230.0 & 10.0 & PASS & 0 & -1.3 & 115.000 & 5.0 & PASS \\
\hline $03 / 29 / 2007$ & $07: 14$ & $\mathrm{SO}_{2}$ U4 4 & $\mathrm{SO} 2$ & ZERO & 0.000 & -3.800 & -3.800 & -0.2 & 230.0 & 20.0 & PASS & 0 & -0.2 & 115.000 & 5.0 & PASS \\
\hline $03 / 29 / 2007$ & $07: 14$ & $\mathrm{SO} 2$ _U4 & $\mathrm{SO}_{2}$ & SPAN & 1994.000 & 2066.400 & 72.400 & 3.1 & 230.0 & 20.0 & PASS & 0 & 3.1 & 115.000 & 5.0 & PASS \\
\hline $03 / 29 / 2007$ & $06=05$ & $\mathrm{SO}_{2} \mathrm{U} 4$ & $\mathrm{SO} 2$ & ZERO & 0.000 & -6.500 & -6.500 & -0.3 & 230.0 & 20.0 & PASS & 0 & -0.3 & 115.000 & 5.0 & PASS \\
\hline $03 / 29 / 2007$ & $06=05$ & $\mathrm{SO}_{2} \mathrm{UU}_{4}$ & $\mathrm{SO2}$ & SPAN & 1994.000 & 2110.100 & 116.100 & 5.0 & 230.0 & 10.0 & PASS & 0 & 5.0 & 115.000 & 5.0 & PASS \\
\hline $03 / 28 / 2007$ & $07: 52$ & $\mathrm{SO} 2$ U 4 & $\mathrm{SO2}$ & ZERO & 0.000 & -4.700 & -4.700 & -0.2 & 230.0 & 10.0 & PASS & 0 & -0.2 & 115.000 & 5.0 & RASS \\
\hline $03 / 28 / 2007$ & $07: 52$ & $\mathrm{SO}_{2} \mathrm{U}_{4}$ & $\mathrm{SO} 2$ & SPAN & 1994.000 & 2014.400 & 20.400 & 0.9 & 230.0 & 20.0 & PASS & 0 & 0.9 & 115.000 & 5.0 & PASS \\
\hline $03 / 28 / 2007$ & $06: 22$ & $\mathrm{SO} 2$-U4 & $\mathrm{SO} 2$ & ZERO & 0.000 & -1.900 & -1.900 & -0.1 & 230.0 & 10.0 & PASS & 0 & -0.1 & 115.000 & 5.0 & PASS \\
\hline $03 / 28 / 2007$ & $06: 22$ & SO2_U4 & $\mathrm{SO} 2$ & SPAN & 1994.000 & 2046.400 & 52.400 & 2.3 & 230.0 & 10.0 & PASS & 0 & 2.3 & 115.000 & 5.0 & PASS \\
\hline $03 / 28 / 2007$ & $06=05$ & $\mathrm{SO} 2$-U4 & $\mathrm{SO} 2$ & ZERO & 0.000 & 0.900 & 0.900 & 0.0 & 230.0 & 10.0 & PASS & 0 & 0.0 & 115.000 & 5.0 & PASS \\
\hline $03 / 28 / 2007$ & $06=05$ & $\mathrm{SO}_{2} \mathrm{U}_{4}$ & SO2 & SPAN & 1994.000 & 2344.500 & 350.500 & 15.2 & 230.0 & 10.0 & $R R G$ & OOc & 15.2 & 115.000 & 5.0 & $\underline{R D C}$ \\
\hline $03 / 29 / 2007$ & $06=07$ & STK_FLOWU4 & Flow & ZERO & 0.000 & -0.900 & -0.900 & -0.2 & 82.0 & 12.0 & PASS & 0 & -0.1 & 40.980 & 6.0 & RASS \\
\hline $03 / 29 / 2007$ & $06=07$ & STK_FLOWU4 & Elow & SPAN & 410.000 & 410.700 & 0.700 & 0.1 & 82.0 & 12.0 & PASS & 0 & 0.2 & 40.980 & 6.0 & PASS \\
\hline $03 / 28 / 2007$ & $06: 07$ & STK_FLOWU4 & Flow & ZERO & 0.000 & 0.000 & 0.000 & 0.0 & 82.0 & 12.0 & PASS & 0 & 0.0 & 40.980 & 6.0 & pASS \\
\hline $03 / 28 / 2007$ & $06: 07$ & STK_ELOWU4 & Flow & SRAN & 410.000 & 410.900 & 0.900 & 0.1 & 82.0 & 12.0 & RASS & 0 & 0.2 & 40.980 & 6.0 & PASS \\
\hline
\end{tabular}

FAII = Difference Erxox $>$ Regulations Allow

Whra Error < Daily Allowed but > 5 Consecutive Days Allowed

TARG = Invalia larget (not within regulatory specs)

$R D G=$ Reading exceeds "Range of Analyzer"

WD = Number of Consecutive Days in Warning - ('?' Nor Available) - ('00c' No Passed Cal. since a Failed Daily or 5 Days in warning)

Note: 4 OCFR75 pass/fail determination is performed after rounding the value of Error:, ox Drift, to one decimal place 
Part 60 Calibration (Absolute Average DIFF and Calibration Exror)

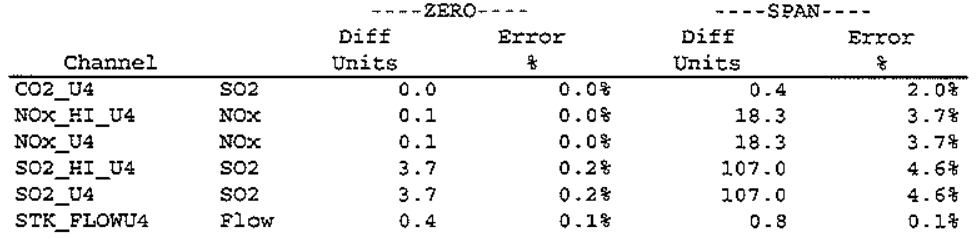

Part 75 Calibration (Absolute Avexage DIFF and Calibration Error)

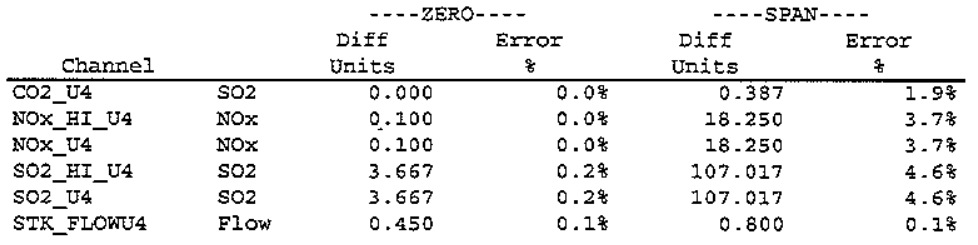

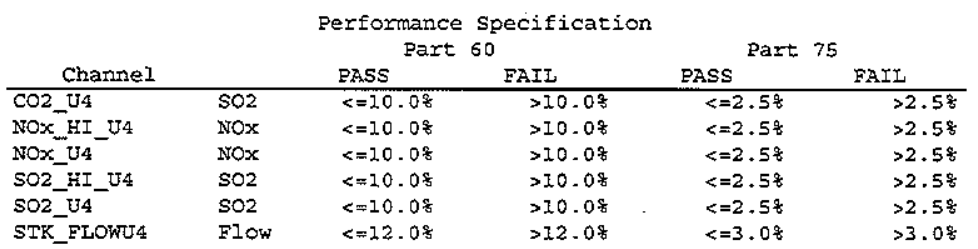

Perf: [Part75 Daily S02] zero $=5.0$ \&Range, Span $=5.0$ \&Range, [Part60 Daily SO2] zero $=10.0$ \&Range, Span

AitPerf: (Pa)t75 Daily SO2] Zero $=5$ \&SO2 (Range $<=50$ \&) $/ 10$ \&SO2 (50 \& $<$ Range $<=200$ \&), Span $=5$ \&SO2 (Range $<=50$

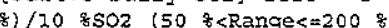

Perf: [Part75 Daily NOx] Zero $=5.0$ \&Range, Span $=5.0$ sRange, $[$ Part60 Daily NOx] Zero $=10.0$ sRange, span I0.0 sRange

AItPerf: $[$ Part 75 Daily NOx] Zero $=5 \mathrm{ppm}($ Range $<=50 \mathrm{ppm}) / 10 \mathrm{ppm}(50$ ppm $<$ Range $<=200 \mathrm{ppm})$, Span $=5$ ppm (Range $<=50 \mathrm{ppm}) / 10 \mathrm{ppm}(50$ ppm<Range $<=200 \mathrm{ppm})$

Perf: [Raxt75 Daily NOx] Zero $=5.0$ \&Range, Span $=5.0$ \&Range, [Part60 Daily NOx] Zero $=10.0$ sRange, Span 10 .

AltPerf: [Rart75 Daily NOx] Zero $=5 \mathrm{ppm}$ (Range $<=50 \mathrm{ppm}) / 10 \mathrm{ppm}(50$ ppm<Range $<=200 \mathrm{ppm})$, Span $=5 \mathrm{ppm}$ Range $<=50$ ppra $) / 10$ ppm $(50$ ppm<Range $<=200 \mathrm{ppm}$ )

Pexf: [Part75 Daily S02] Zero $=5.0$ forange, Span $=5.0$ \&Range, $[$ Fart60 Daily S02] Zero $=10.0$ sRange, Span

AItPerf: $[$ Part75 Daily SO2] zexo $=5$ ppm (Range $<=50$ ppm) $/ 10$ ppm $(50$ ppm $<$ Range $<=200$ ppm), Span $=5$ ppm (Range $<=50 \mathrm{ppm}$ ) $/ 10 \mathrm{ppm}(50 \mathrm{ppm}<$ Range $<=200 \mathrm{ppm}$ )

Perf: [Part75 Daily SO2] Zero $=5.0$ \&Range, Span $=5.0$ \&Range, [Part60 Daily S02] Zero $=10.0$ \&Range, Span

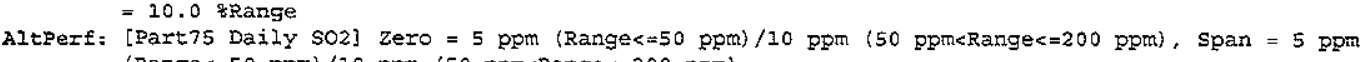

Drange, [part60 paily flow\} zero = 12.0 onange Pan $=12.0$ \& Range 
Company: AEs Greenidge Plant: 590 Plant Road
City/St: Dresden, NY 14441 Source: STACKA
Period start : 3/28/2007 00:00 Period End: 3/30/2007 00,00 validation Type: 1/1 min Averaging period: 1 min Type: Block Avg

\begin{tabular}{|c|c|c|c|c|}
\hline \multicolumn{2}{|c|}{ period Start, } & $\begin{array}{l}\text { Average } \\
\text { NoxLBMM US } \\
\text { lb } / \operatorname{mm} \bar{B}\end{array}$ & $\begin{array}{l}\text { Average } \\
\text { SO2LBMM U4 } \\
\text { 1b/mmB }\end{array}$ & $\begin{array}{c}\text { Average } \\
\text { U4_NFR_NW } \\
\text { WW - } \\
\end{array}$ \\
\hline $03 / 28 / 2007$ & $00: 00$ & $\mathrm{~N} / \mathrm{A}$ & $\mathrm{N} / \mathrm{A}$ & 60.32 \\
\hline $03 / 28 / 2007$ & 00,01 & $\mathrm{~N} / \mathrm{A}$ & N/A & 60.84 \\
\hline $03 / 28 / 2007$ & 00,02 & $N / A$ & N/A & 61.57 \\
\hline $03 / 28 / 2007$ & 00,03 & 0.459 & 0.221 & 62.74 \\
\hline $03 / 28 / 2007$ & $00: 04$ & 0.452 & 0.337 & 63.45 \\
\hline $03 / 28 / 2007$ & $00: 05$ & 0.439 & 0.307 & 64,41 \\
\hline $03 / 28 / 2007$ & $00: 06$ & 0.428 & 0.265 & 65.29 \\
\hline $03 / 28 / 2007$ & $00: 07$ & 0.420 & 0.212 & 65.86 \\
\hline $03 / 28 / 2007$ & $00: 08$ & 0.439 & 0.129 & 66.82 \\
\hline $03 / 28 / 2007$ & $00: 09$ & 0.461 & 0.226 & 68.06 \\
\hline $03 / 28 / 2007$ & $00: 10$ & 0.466 & 0.368 & 68.73 \\
\hline $03 / 28 / 2007$ & $00: 11$ & 0.498 & 0.446 & 69.86 \\
\hline $03 / 28 / 2007$ & $00: 12$ & 0.548 & 0.539 & 71.16 \\
\hline $03 / 28 / 2007$ & $00: 13$ & 0.540 & 0.606 & 72.49 \\
\hline $03 / 28 / 2007$ & $00: 14$ & 0.467 & 0.674 & 74.15 \\
\hline $03 / 28 / 2007$ & 00115 & 0.491 & 0.623 & 75.12 \\
\hline $03 / 28 / 2007$ & $00 \backslash 16$ & 0.525 & 0.478 & 76.01 \\
\hline $03 / 28 / 2007$ & $00: 17$ & 0.482 & 0.434 & 76.71 \\
\hline $03 / 28 / 2007$ & $00: 18$ & 0.418 & 0.378 & 77.33 \\
\hline $03 / 28 / 2007$ & $00: 19$ & 0.400 & 0.264 & 78.03 \\
\hline $03 / 28 / 2007$ & $00: 20$ & 0.429 & 0.378 & 78.95 \\
\hline $03 / 28 / 2007$ & $00: 21$ & 0.438 & 0.365 & 79.66 \\
\hline $03 / 28 / 2007$ & 00.22 & 0.423 & 0.388 & 80.38 \\
\hline $03 / 28 / 2007$ & $00: 23$ & 0.394 & 0.378 & 81.17 \\
\hline $03 / 28 / 2007$ & 00,24 & 0.384 & 0.422 & 81.80 \\
\hline $03 / 28 / 2007$ & 00,25 & 0.368 & 0.401 & 82.48 \\
\hline $03 / 28 / 2007$ & 00,26 & 0.306 & 0.332 & 82.99 \\
\hline $03 / 28 / 2007$ & 00,27 & 0.267 & 0.354 & 84.22 \\
\hline $03 / 28 / 2007$ & $00: 28$ & 0.246 & 0.263 & 84.67 \\
\hline $03 / 28 / 2007$ & $00 \div 29$ & 0.227 & 0.370 & 85.83 \\
\hline $03 / 28 / 2007$ & $00: 30$ & 0.226 & 0.376 & 86.27 \\
\hline $03 / 28 / 2007$ & $00: 31$ & 0.225 & 0.434 & 85.83 \\
\hline $03 / 2 B / 2007$ & $00: 32$ & 0.213 & 0.317 & 85.07 \\
\hline $03 / 28 / 2007$ & $00: 33$ & 0.210 & 0.260 & 84.19 \\
\hline $03 / 28 / 2007$ & $00: 34$ & 0.207 & 0.229 & 83.31 \\
\hline $03 / 28 / 2007$ & $00: 35$ & 0.191 & 0.213 & 82.20 \\
\hline $03 / 28 / 2007$ & $00: 36$ & 0.186 & 0.146 & 81.39 \\
\hline $03 / 28 / 2007$ & $00: 37$ & 0.183 & 0.102 & 80.48 \\
\hline $03 / 28 / 2007$ & $00: 38$ & 0.183 & 0.118 & 79.56 \\
\hline $03 / 28 / 2007$ & $00: 39$ & 0.183 & 0.100 & 78.70 \\
\hline $03 / 28 / 2007$ & $00: 40$ & 0.186 & 0.071 & 77.80 \\
\hline $03 / 28 / 2007$ & $00: 41$ & 0.197 & 0.104 & 76.66 \\
\hline $03 / 28 / 2007$ & $00: 42$ & 0.205 & 0.058 & 75.70 \\
\hline $03 / 28 / 2007$ & $00: 43$ & 0.160 & 0.059 & 74.64 \\
\hline $03 / 28 / 2007$ & $00: 44$ & 0.213 & 0.060 & 73.82 \\
\hline $03 / 28 / 2007$ & $00: 45$ & 0.279 & 0.054 & 72.47 \\
\hline $03 / 28 / 2007$ & $00: 46$ & 0.312 & 0.107 & 71.45 \\
\hline $03 / 28 / 2007$ & $00: 47$ & 0.303 & 0.079 & 70.65 \\
\hline $03 / 28 / 2007$ & $00: 48$ & 0.315 & 0.039 & 69.51 \\
\hline $03 / 28 / 2007$ & $00: 49$ & 0.302 & 0.037 & 68.75 \\
\hline $03 / 28 / 2007$ & $00: 50$ & 0.279 & 0.042 & 67.79 \\
\hline $03 / 28 / 2007$ & $00: 51$ & 0.271 & 0.044 & 67.02 \\
\hline $03 / 28 / 2007$ & 00,52 & 0.235 & 0.045 & 66.08 \\
\hline $03 / 28 / 2007$ & 00,53 & 0.212 & 0.033 & 65.58 \\
\hline $03 / 28 / 2007$ & 00,54 & 0.281 & 0.066 & 64.39 \\
\hline $03 / 28 / 2007$ & 00155 & 0.333 & 0.091 & 63.44 \\
\hline $03 / 28 / 2007$ & 00,56 & 0.341 & 0.062 & 62.71 \\
\hline $03 / 28 / 2007$ & $00: 57$ & 0.368 & 0.040 & 61.53 \\
\hline $03 / 28 / 2007$ & 00,58 & $\mathrm{~N} / \mathrm{A}$ & $\mathrm{N} / \mathrm{A}$ & 60.87 \\
\hline $03 / 28 / 2007$ & 00,59 & N/A & $\mathrm{N} / \mathrm{A}$ & 60.14 \\
\hline $03 / 28 / 2007$ & 01,00 & $\mathrm{~N} / \mathrm{A}$ & N/A & 59.01 \\
\hline $03 / 28 / 2007$ & $01: 01$ & $\mathrm{~N} / \mathrm{A}$ & N/A & 58.37 \\
\hline $03 / 28 / 2007$ & 01,02 & $N / A$ & N/A & 57.49 \\
\hline $03 / 28 / 2007$ & 01,03 & 0.456 & 0.056 & 56.58 \\
\hline $03 / 28 / 2007$ & $01: 04$ & 0.462 & 0.046 & 55.47 \\
\hline $03 / 28 / 2007$ & $01: 05$ & 0.478 & 0.061 & 54.58 \\
\hline $03 / 28 / 2007$ & $01: 06$ & 0.511 & 0.025 & 53.52 \\
\hline $03 / 28 / 2007$ & $01: 07$ & 0.521 & 0.046 & 52.69 \\
\hline $03 / 28 / 2007$ & $01: 0 B$ & 0.536 & 0.135 & 52.43 \\
\hline $03 / 28 / 2007$ & $01: 09$ & 0.539 & 0.118 & 52.14 \\
\hline $03 / 28 / 2007$ & $01: 10$ & 0.525 & 0,052 & 50.43 \\
\hline $03 / 28 / 2007$ & $01: 11$ & 0.525 & 0.026 & 49.66 \\
\hline $03 / 28 / 2007$ & $01: 12$ & 0.545 & 0,046 & 49.44 \\
\hline $03 / 28 / 2007$ & $01: 13$ & 0.555 & 0.094 & 49.56 \\
\hline $03 / 28 / 2007$ & $01: 14$ & 0.562 & 0.059 & 49,45 \\
\hline $03 / 28 / 2007$ & $01: 15$ & 0.566 & 0.072 & 49.83 \\
\hline $03 / 28 / 2007$ & $01: 16$ & 0.575 & 0.160 & 50.79 \\
\hline $03 / 28 / 2007$ & $01: 17$ & 0.565 & 0.130 & 51.21 \\
\hline $03 / 28 / 2007$ & $01: 18$ & 0.552 & 0.109 & 50.67 \\
\hline $03 / 28 / 2007$ & $01: 19$ & 0.553 & 0.103 & 50,16 \\
\hline $03 / 28 / 2007$ & $01: 20$ & 0.555 & 0.133 & 50,05 \\
\hline $03 / 28 / 2007$ & 01.21 & 0.554 & 0.092 & 49.75 \\
\hline $03 / 28 / 2007$ & $01: 22$ & 0.558 & 0.052 & 50.47 \\
\hline $03 / 2 B / 2007$ & $01: 23$ & 0.560 & 0.115 & 50.55 \\
\hline $03 / 28 / 2007$ & $01: 24$ & 0.555 & 0.216 & 50.40 \\
\hline $03 / 28 / 2007$ & $01: 25$ & 0.551 & 0.171 & 50.53 \\
\hline $03 / 28 / 2007$ & $01: 26$ & 0.548 & 0,116 & 50.71 \\
\hline $03 / 28 / 2007$ & $01: 27$ & 0.548 & 0.157 & 50.68 \\
\hline $03 / 28 / 2007$ & $01: 28$ & 0.551 & 0.071 & 50.25 \\
\hline $03 / 28 / 2007$ & $01: 29$ & 0.558 & 0.107 & 50.44 \\
\hline
\end{tabular}




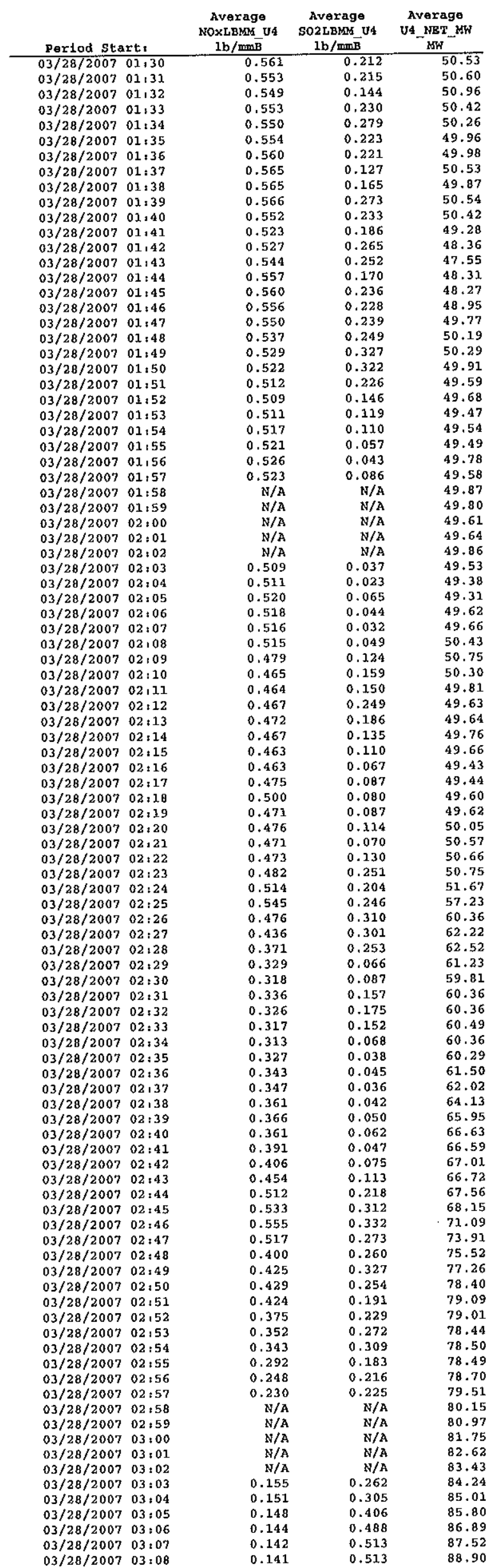




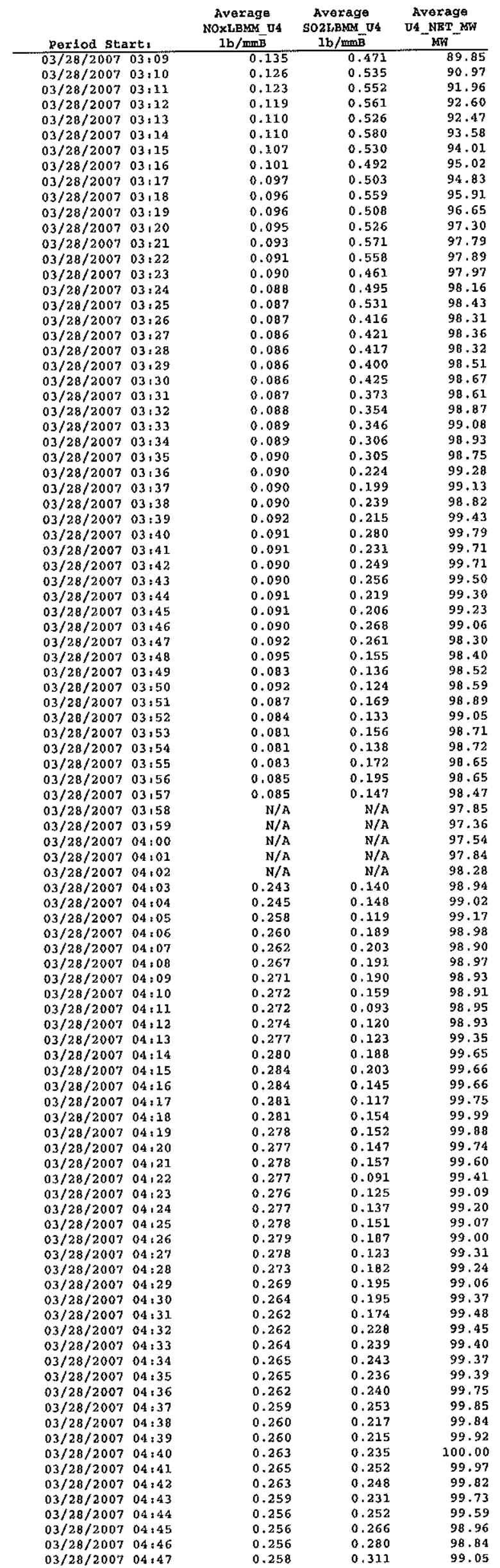




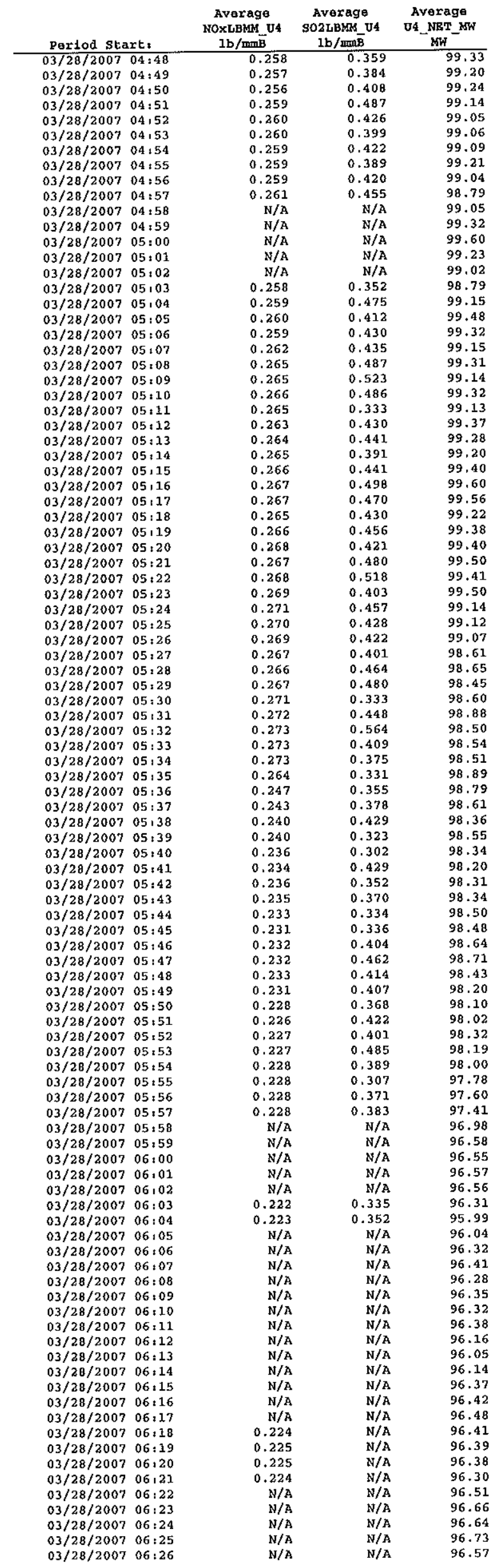




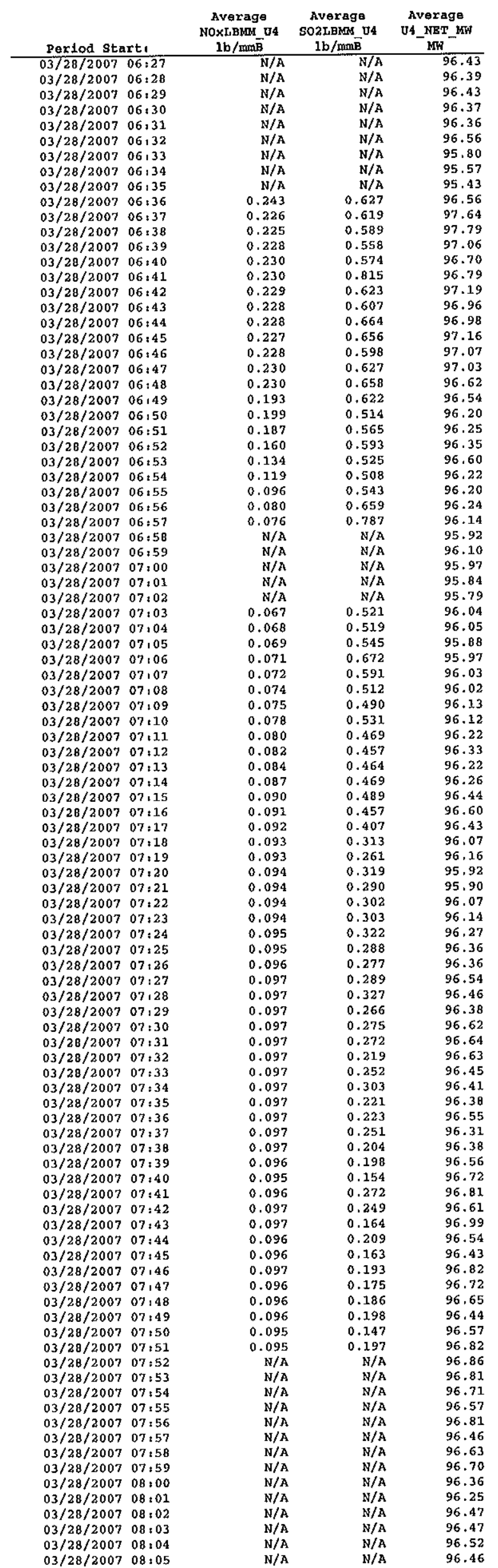




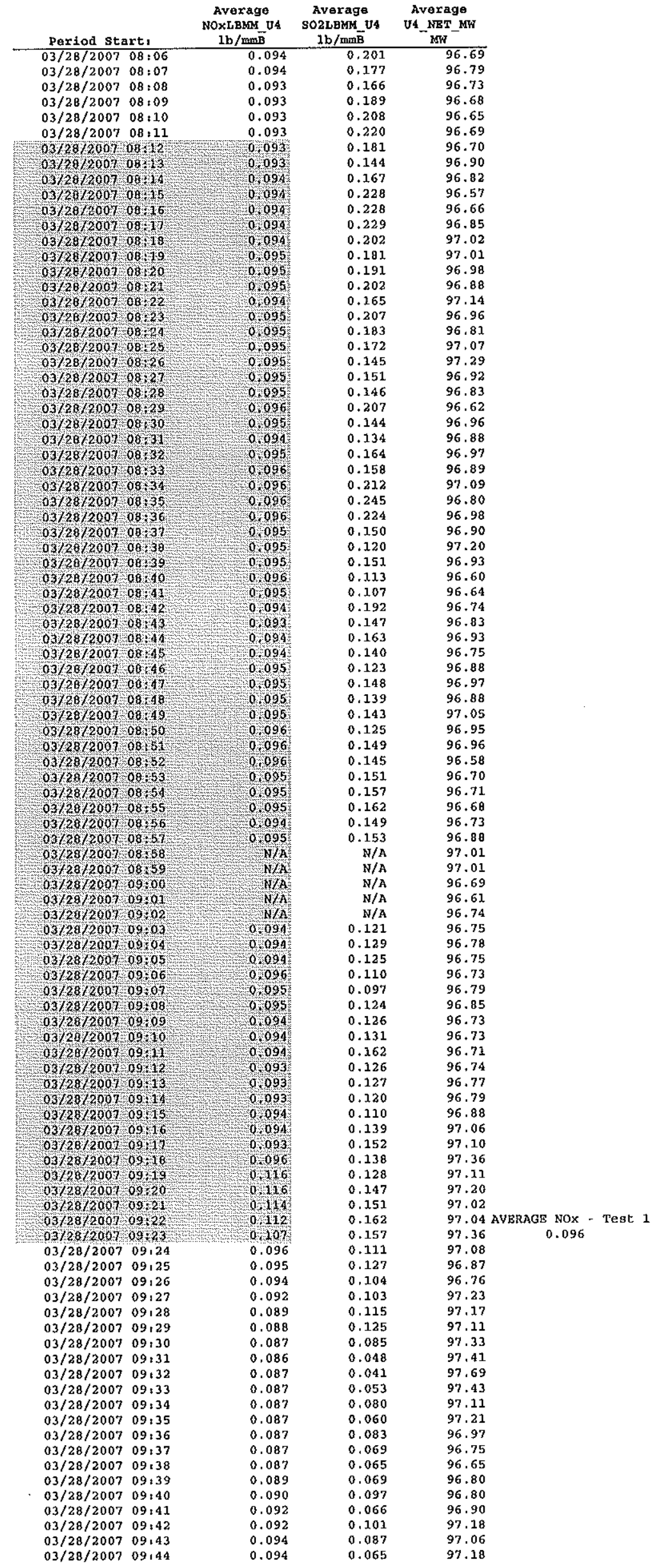




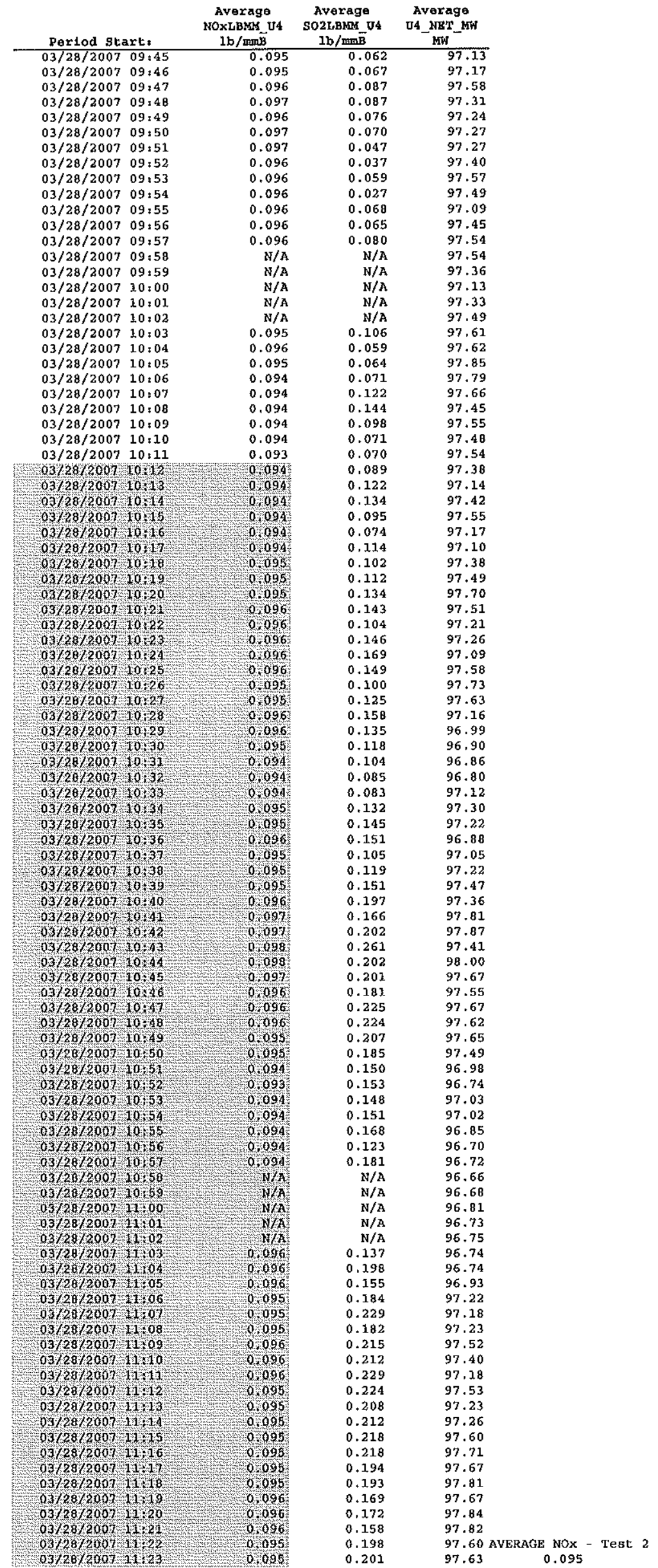




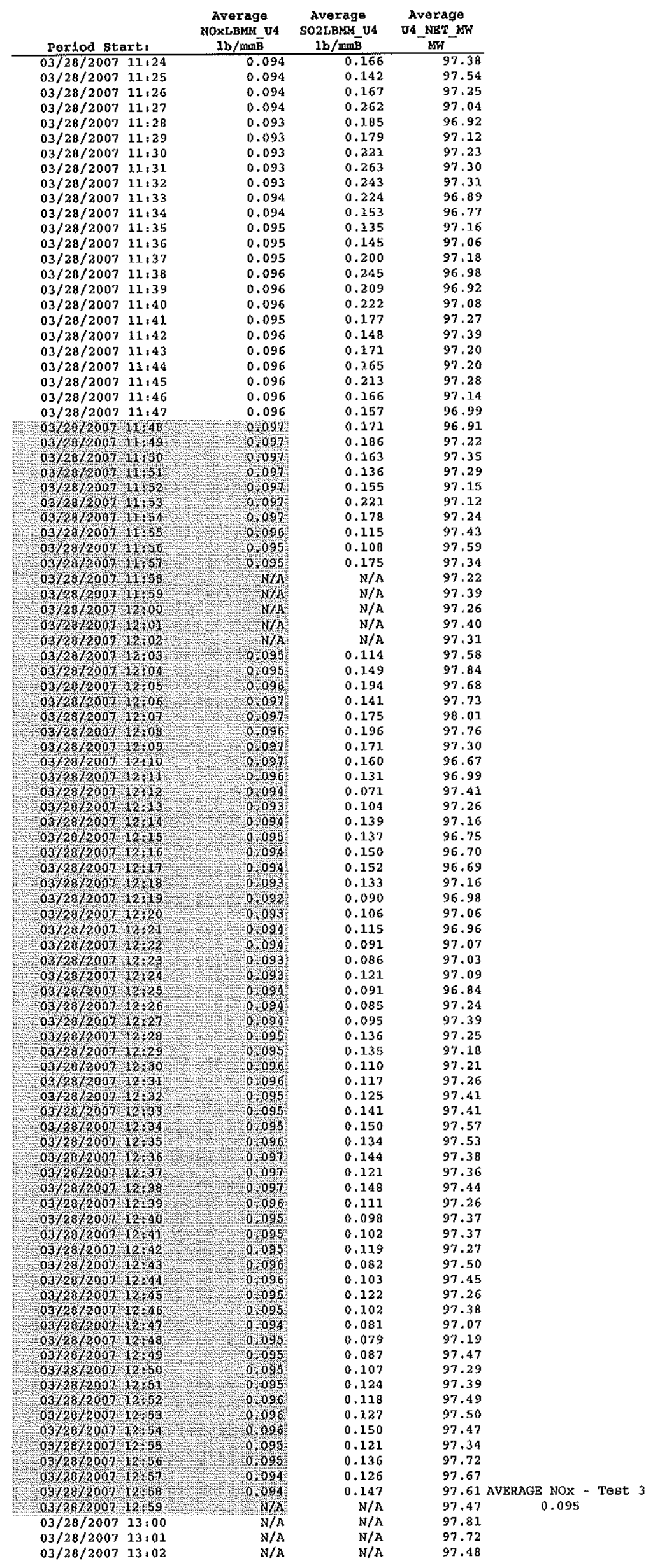




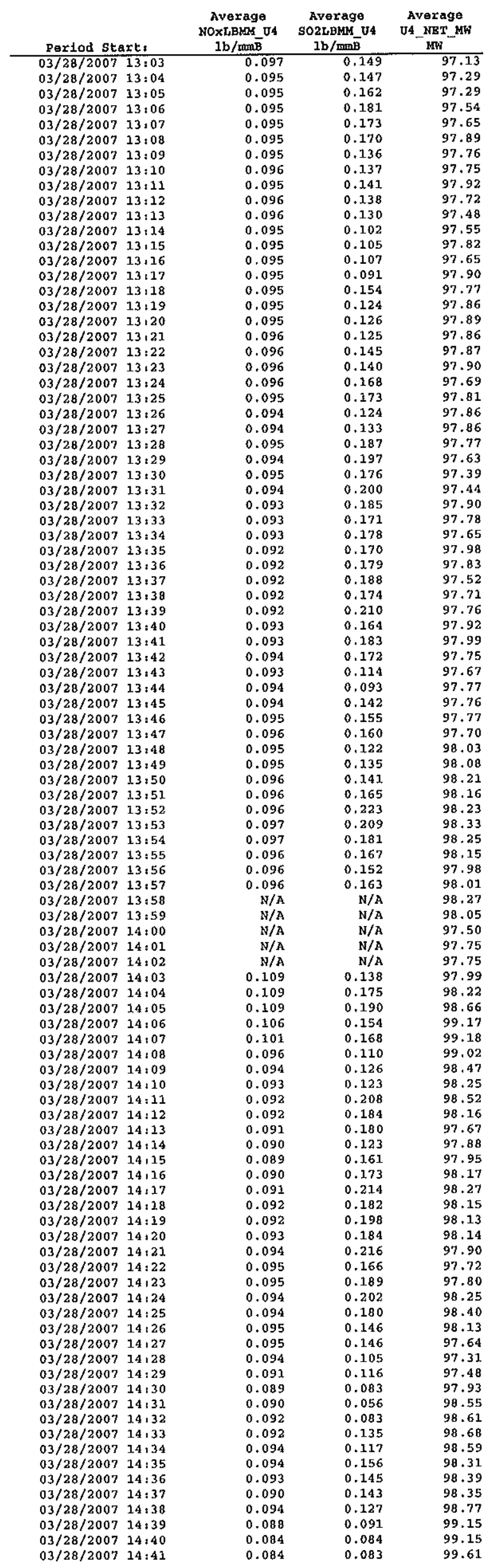




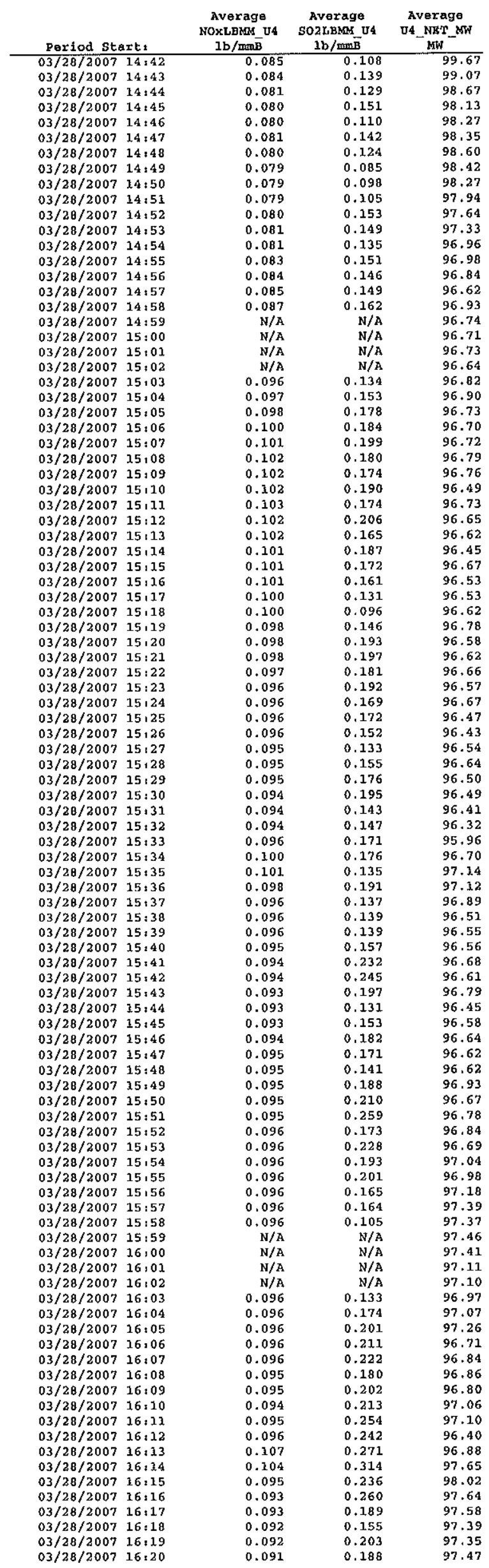




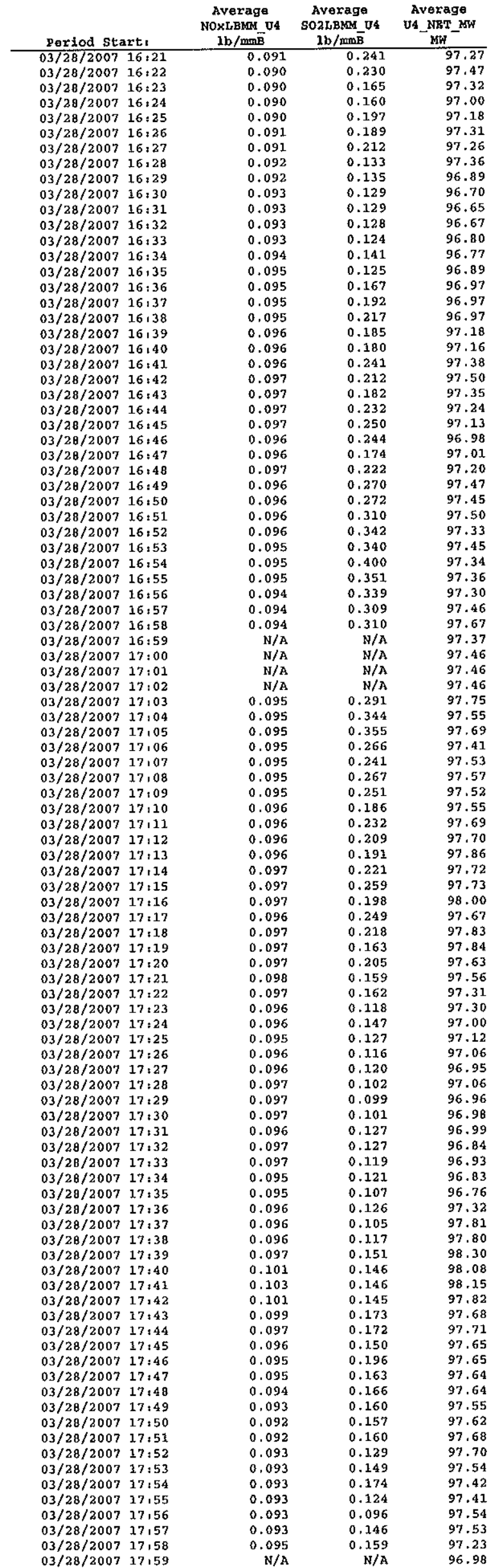




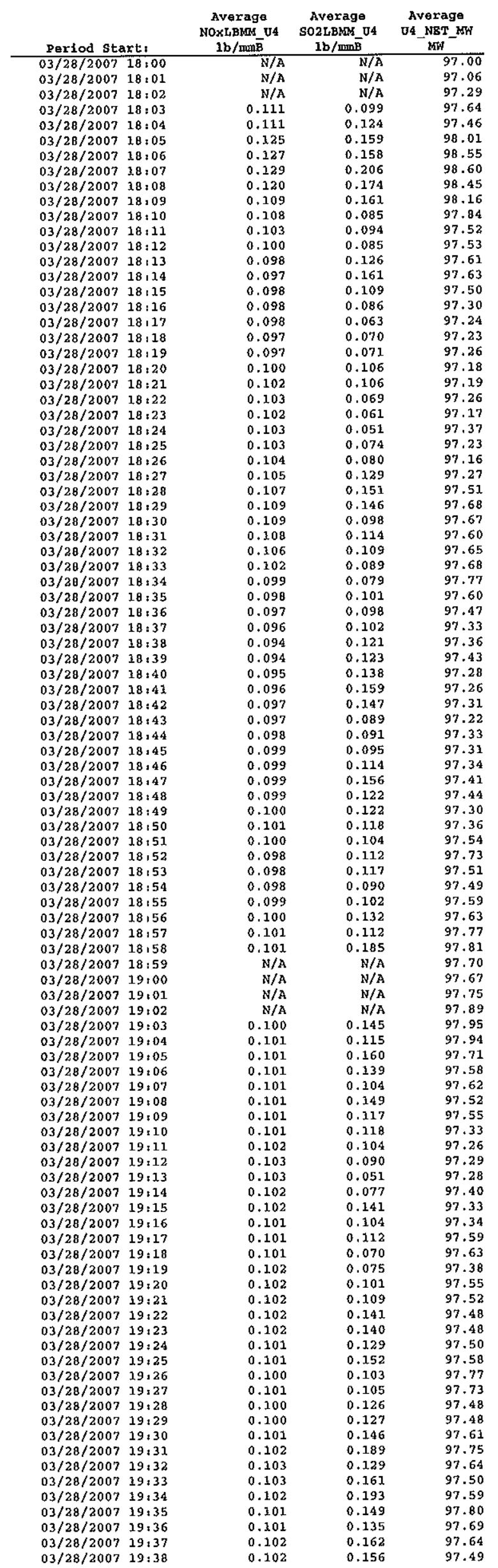




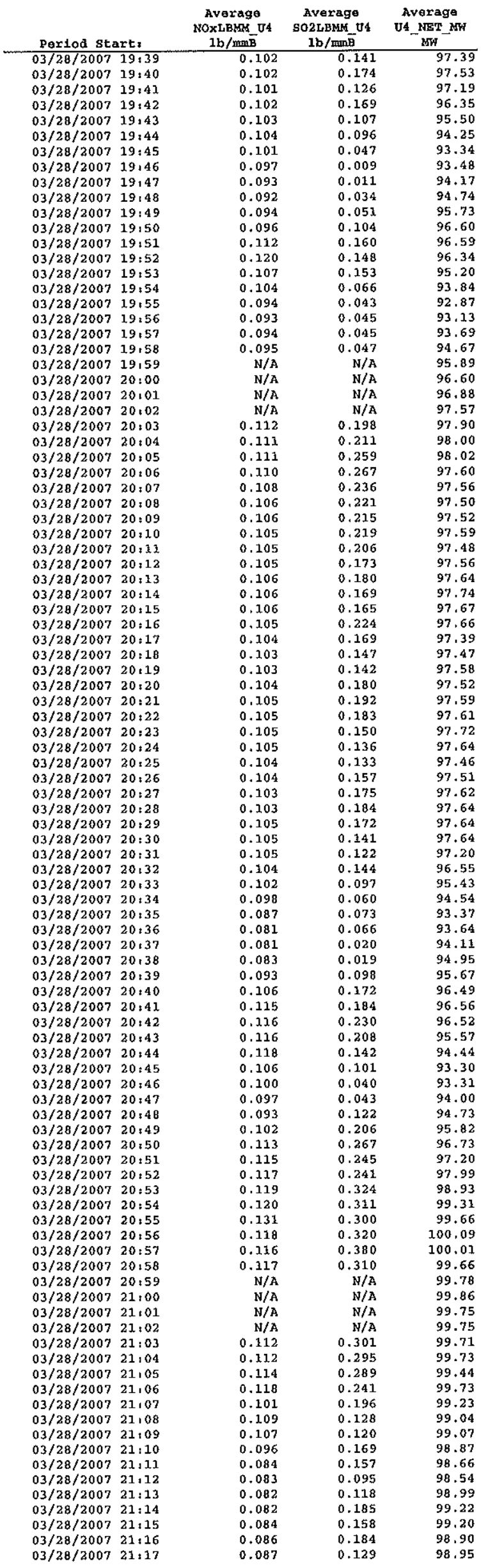




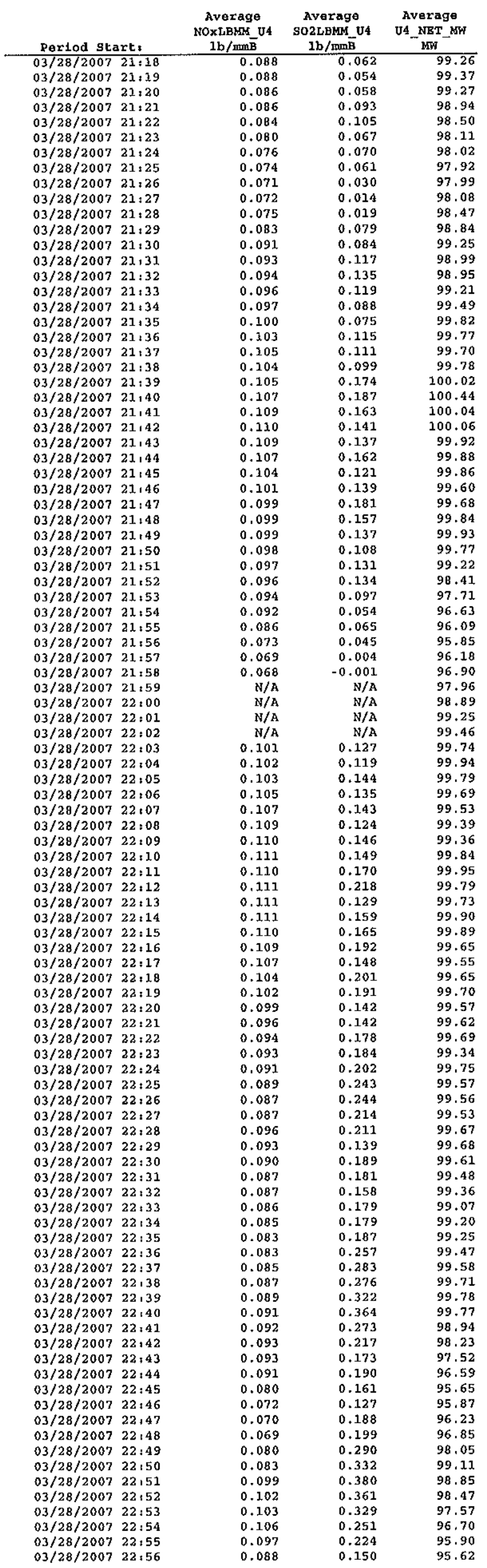




\begin{tabular}{|c|c|c|c|c|}
\hline Pertod sta & $a x t:$ & $\begin{array}{c}\text { Average } \\
\text { NOxLBMM U4 } \\
1 \mathrm{~b} / \mathrm{mmB} \bar{B}\end{array}$ & $\begin{array}{l}\text { Average } \\
\text { SO2LBMR UA } \\
1 \mathrm{~b} / \operatorname{minB} \bar{B}\end{array}$ & $\begin{array}{l}\text { Average } \\
\text { U4_NET_LW } \\
\text { NHA }^{\text {NST }}\end{array}$ \\
\hline $03 / 28 / 2007$ & $22: 57$ & 0.083 & 0.128 & 96.09 \\
\hline $03 / 28 / 2007$ & $22: 58$ & 0.081 & 0.095 & 96.79 \\
\hline $03 / 28 / 2007$ & $22: 59$ & $\mathrm{~N} / \mathrm{A}$ & $N / A$ & 97.97 \\
\hline $03 / 28 / 2007$ & $23: 00$ & N/A & $\mathrm{N} / \mathrm{A}$ & 99.01 \\
\hline $03 / 28 / 2007$ & $23: 03$ & $\mathrm{~N} / \mathrm{A}$ & $\mathrm{N} / \mathrm{A}$ & 99.40 \\
\hline $03 / 28 / 2007$ & $23: 02$ & N/A & N/A & 99.82 \\
\hline $03 / 28 / 2007$ & $23: 03$ & 0.112 & 0.356 & 100.14 \\
\hline $03 / 28 / 2007$ & $23: 04$ & 0.113 & 0.333 & 100.26 \\
\hline $03 / 28 / 2007$ & $23: 05$ & 0.116 & 0.312 & 100.06 \\
\hline $03 / 28 / 2007$ & $23: 06$ & 0.117 & 0.296 & 99.91 \\
\hline $03 / 28 / 2007$ & $23: 07$ & 0.117 & 0.347 & 99.90 \\
\hline $03 / 28 / 2007$ & $23: 08$ & 0.117 & 0.308 & 99.99 \\
\hline $03 / 28 / 2007$ & $23: 09$ & 0.117 & 0.330 & 100.06 \\
\hline $03 / 28 / 2007$ & $23: 10$ & 0.116 & 0.367 & 99.82 \\
\hline $03 / 28 / 2007$ & $23: 11$ & 0.113 & 0.307 & 99.70 \\
\hline $03 / 28 / 2007$ & $23: 12$ & 0,109 & 0.304 & 99.92 \\
\hline $03 / 28 / 2007$ & $23: 13$ & 0.106 & 0.253 & 99.68 \\
\hline $03 / 28 / 2007$ & $23: 14$ & 0.105 & 0.286 & 99.74 \\
\hline $03 / 28 / 2007$ & $23: 15$ & 0.104 & 0.302 & 99.73 \\
\hline $03 / 28 / 2007$ & $23: 16$ & 0.101 & 0.305 & 99.70 \\
\hline $03 / 28 / 2007$ & $23: 17$ & 0.098 & 0.255 & 99.67 \\
\hline $03 / 28 / 2007$ & $23: 18$ & 0.096 & 0.322 & 99.56 \\
\hline $03 / 28 / 2007$ & $23: 19$ & 0.095 & 0.186 & 99.68 \\
\hline $03 / 28 / 2007$ & $23: 20$ & 0,093 & 0.275 & 99.56 \\
\hline $03 / 28 / 2007$ & $23: 21$ & 0.092 & 0.224 & 99.68 \\
\hline $03 / 28 / 2007$ & $23: 22$ & 0.090 & 0.233 & 99.62 \\
\hline $03 / 28 / 2007$ & $23: 23$ & 0.089 & 0.277 & 99.38 \\
\hline $03 / 28 / 2007$ & 23,24 & 0.087 & 0.277 & 99.38 \\
\hline $03 / 28 / 2007$ & 23,25 & 0.086 & 0.242 & 99.49 \\
\hline $03 / 28 / 2007$ & $23: 26$ & 0.083 & 0.227 & 99.65 \\
\hline $03 / 28 / 2007$ & $23: 27$ & 0.082 & 0.293 & 99.46 \\
\hline $03 / 28 / 2007$ & $23: 28$ & 0.083 & 0.235 & 99.43 \\
\hline $03 / 28 / 2007$ & 23,29 & 0.083 & 0.241 & 99,28 \\
\hline $03 / 28 / 2007$ & 23,30 & 0.082 & 0.249 & 99.14 \\
\hline $03 / 28 / 2007$ & $23: 31$ & 0.081 & 0.215 & 99.05 \\
\hline $03 / 28 / 2007$ & 23,32 & 0.081 & 0.192 & 98.87 \\
\hline $03 / 28 / 2007$ & $23: 33$ & 0.080 & 0.197 & 98.79 \\
\hline $03 / 28 / 2007$ & 23,34 & 0.080 & 0.230 & 98.70 \\
\hline $03 / 28 / 2007$ & $23 ; 35$ & 0.080 & 0.254 & 98.87 \\
\hline $03 / 28 / 2007$ & $23: 36$ & 0.080 & 0.231 & 99.04 \\
\hline $03 / 28 / 2007$ & $23: 37$ & 0.081 & 0.181 & 99.08 \\
\hline $03 / 28 / 2007$ & $23: 38$ & 0.083 & 0.243 & 99.19 \\
\hline $03 / 28 / 2007$ & $23: 39$ & 0.085 & 0.257 & 99.27 \\
\hline $03 / 28 / 2007$ & $23: 40$ & 0.088 & 0.233 & 99.34 \\
\hline $03 / 28 / 2007$ & $23: 41$ & 0.090 & 0.249 & 99.43 \\
\hline $03 / 28 / 2007$ & $23: 42$ & 0.092 & 0.258 & 99.48 \\
\hline $03 / 28 / 2007$ & $23: 43$ & 0.093 & 0.215 & 99.46 \\
\hline $03 / 28 / 2007$ & $23: 44$ & 0.094 & 0.264 & 99.53 \\
\hline $03 / 28 / 2007$ & $23: 45$ & 0.096 & 0.263 & 99.45 \\
\hline $03 / 28 / 2007$ & $23: 46$ & 0.096 & 0.227 & 99.63 \\
\hline $03 / 28 / 2007$ & $23: 47$ & 0.096 & 0.243 & 99.68 \\
\hline $03 / 28 / 2007$ & $23: 48$ & 0.097 & 0.269 & 99.66 \\
\hline $03 / 28 / 2007$ & $23: 49$ & 0.098 & 0.312 & 99.56 \\
\hline $03 / 28 / 2007$ & 23150 & 0.097 & 0.249 & 99.37 \\
\hline $03 / 28 / 2007$ & 23,51 & 0.097 & 0.197 & 99.56 \\
\hline $03 / 28 / 2007$ & $23: 52$ & 0.097 & 0.253 & 99.46 \\
\hline $03 / 28 / 2007$ & 23,53 & 0.097 & 0.253 & 99.66 \\
\hline $03 / 28 / 2007$ & $23: 54$ & 0.098 & 0.205 & 99.57 \\
\hline $03 / 28 / 2007$ & $23: 55$ & 0.098 & 0.227 & 99.59 \\
\hline $03 / 28 / 2007$ & 23,56 & 0.097 & 0.279 & 99.64 \\
\hline $03 / 28 / 2007$ & 23,57 & 0.096 & 0.197 & 99.71 \\
\hline $03 / 28 / 2007$ & $23: 58$ & 0.097 & 0.196 & 99.82 \\
\hline $03 / 28 / 2007$ & $23: 59$ & $\mathrm{~N} / \mathrm{A}$ & N/A & 99.90 \\
\hline Daily & Average & 0.155 & 0.199 & 93.06 \\
\hline & Maximum* & 0.575 & 0.815 & 100.44 \\
\hline & & $03 / 28 / 2007$ & $03 / 28 / 2007$ & $03 / 28 / 2007$ \\
\hline & & $1: 16$ & $6: 41$ & $21: 40$ \\
\hline & kinfmum* & 0.067 & -0.001 & 47.55 \\
\hline & & $03 / 28 / 2007$ & $03 / 28 / 2007$ & $03 / 28 / 2007$ \\
\hline & & $7: 03$ & $21: 58$ & $1: 43$ \\
\hline
\end{tabular}

* Does not include Invalid Averaging Periods ("N/A") 
Average values Report

Generated: $9 / 27 / 2007 \quad 11: 12$

Company: AES Greenidge

City/St: Dresden, NY 14441

Source! STACKA
Period Staxt: 3/28/2007 00:00 Period End: 3/30/2007 00:00 validation Type: $1 / 1 \mathrm{~min}$ Averaging Period: $1 \mathrm{~min}$ Type: Block Avg

\begin{tabular}{|c|c|c|c|c|}
\hline \multicolumn{2}{|c|}{ period start: } & $\begin{array}{c}\text { Average } \\
\text { NOxLBNG U4 } \\
\text { lb/mmB }\end{array}$ & $\begin{array}{l}\text { Average } \\
\text { SO2LBMA_U4 } \\
\text { lb/man }\end{array}$ & $\begin{array}{c}\text { Average } \\
\text { U4_NET_MW } \\
\text { NW - }\end{array}$ \\
\hline $03 / 29 / 2007$ & $00: 00$ & $\mathrm{~N} / \mathrm{A}$ & $\mathrm{N} / \overline{\mathrm{A}}$ & 99.93 \\
\hline $03 / 29 / 2007$ & $00: 01$ & $\mathrm{~N} / \mathrm{A}$ & $\mathrm{N} / \mathrm{A}$ & 99.82 \\
\hline $03 / 29 / 2007$ & $00: 02$ & N/A & $\mathrm{N} / \mathrm{A}$ & 99.79 \\
\hline $03 / 29 / 2007$ & $00: 03$ & 0.097 & 0.266 & 99.57 \\
\hline $03 / 29 / 2007$ & 00,04 & 0.097 & 0.256 & 99.58 \\
\hline $03 / 29 / 2007$ & 00,05 & 0.096 & 0.258 & 99.49 \\
\hline $03 / 29 / 2007$ & 00,06 & 0.096 & 0.252 & 99.39 \\
\hline $03 / 29 / 2007$ & $00: 07$ & 0.096 & 0.177 & 99.53 \\
\hline $03 / 29 / 2007$ & $00: 08$ & 0.095 & 0,192 & 99.47 \\
\hline $03 / 29 / 2007$ & $00: 09$ & 0.094 & 0.200 & 99.45 \\
\hline $03 / 29 / 2007$ & $00: 30$ & 0.094 & 0.243 & 99.51 \\
\hline $03 / 29 / 2007$ & $00: 11$. & 0.094 & 0.197 & 99.57 \\
\hline $03 / 29 / 2007$ & $00: 12$ & 0.093 & 0.190 & 99.74 \\
\hline $03 / 29 / 2007$ & $00: 13$ & 0.093 & 0.225 & 99.88 \\
\hline $03 / 29 / 2007$ & $00: 14$ & 0.093 & 0.298 & 99.78 \\
\hline $03 / 29 / 2007$ & $00: 15$ & 0.094 & 0.252 & 99.57 \\
\hline $03 / 29 / 2007$ & $00: 16$ & 0.095 & 0.243 & 99.80 \\
\hline $03 / 29 / 2007$ & $00: 17$ & 0.096 & 0.228 & 99.74 \\
\hline $03 / 29 / 2007$ & $00: 18$ & 0.096 & 0.268 & 99.80 \\
\hline $03 / 29 / 2007$ & $00: 39$ & 0.097 & 0.228 & 99,77 \\
\hline $03 / 29 / 2007$ & $00: 20$ & 0.097 & 0.227 & 99.71 \\
\hline $03 / 29 / 2007$ & $00: 21$ & 0.097 & 0.296 & 99.82 \\
\hline $03 / 29 / 2007$ & $00: 22$ & 0.097 & 0.226 & 99.84 \\
\hline $03 / 29 / 2007$ & $00: 23$ & 0.098 & 0.215 & 99,82 \\
\hline $03 / 29 / 2007$ & $00: 24$ & 0.098 & 0.200 & 99.73 \\
\hline $03 / 29 / 2007$ & $00: 25$ & 0.098 & 0.206 & 99.65 \\
\hline $03 / 29 / 2007$ & $00: 26$ & 0.097 & 0.213 & 99.67 \\
\hline $03 / 29 / 2007$ & $00: 27$ & 0.097 & 0.252 & 99.72 \\
\hline $03 / 29 / 2007$ & $00: 28$ & 0.097 & 0.239 & 99.92 \\
\hline $03 / 29 / 2007$ & $00: 29$ & 0.097 & 0.270 & 99.78 \\
\hline $03 / 29 / 2007$ & $00: 30$ & 0.098 & 0.231 & 99.69 \\
\hline $03 / 29 / 2007$ & 00,31 & 0.098 & 0.215 & 99.85 \\
\hline $03 / 29 / 2007$ & $00: 32$ & 0.098 & 0.235 & 99.94 \\
\hline $03 / 29 / 2007$ & $00: 33$ & 0.099 & 0.249 & 99.71 \\
\hline $03 / 29 / 2007$ & $00: 34$ & 0.100 & 0.224 & 99.65 \\
\hline $03 / 29 / 2007$ & $00: 35$ & 0.099 & 0.255 & 99.44 \\
\hline $03 / 29 / 2007$ & 00,36 & 0.097 & 0.221 & 99.43 \\
\hline $03 / 29 / 2007$ & $00: 37$ & 0.095 & 0.222 & 99.58 \\
\hline $03 / 29 / 2007$ & $00: 38$ & 0.095 & 0.217 & 99.77 \\
\hline $03 / 29 / 2007$ & $00: 39$ & 0.095 & 0.242 & 99.33 \\
\hline $03 / 29 / 2007$ & $00: 40$ & 0.095 & 0,199 & 99.39 \\
\hline $03 / 29 / 2007$ & $00: 41$ & 0.094 & 0,202 & 99.39 \\
\hline $03 / 29 / 2007$ & $00: 42$ & 0.093 & 0.203 & 99.47 \\
\hline $03 / 29 / 2007$ & 00,43 & 0.093 & $0.23 B$ & 99.55 \\
\hline $03 / 29 / 2007$ & 00,44 & 0.093 & 0.238 & 99.60 \\
\hline $03 / 29 / 2007$ & 00,45 & 0.095 & 0.201 & 99.57 \\
\hline $03 / 29 / 2007$ & 00146 & 0.094 & 0.184 & 99.42 \\
\hline $03 / 29 / 2007$ & 00,47 & 0.094 & 0.149 & 99.58 \\
\hline $03 / 29 / 2007$ & 00,48 & 0.095 & 0.219 & 99.60 \\
\hline $03 / 29 / 2007$ & 00,49 & 0.095 & 0.158 & 99.79 \\
\hline $03 / 29 / 2007$ & $00: 50$ & 0.095 & 0.155 & 99.72 \\
\hline $03 / 29 / 2007$ & 00,51 & 0.097 & 0.150 & 99.96 \\
\hline $03 / 29 / 2007$ & $00: 52$ & 0.097 & 0.202 & 99.83 \\
\hline $03 / 29 / 2007$ & 00,53 & 0.097 & 0.204 & 99.84 \\
\hline $03 / 29 / 2007$ & $00: 54$ & 0.098 & 0.149 & 99.93 \\
\hline $03 / 29 / 2007$ & 00,55 & 0.097 & 0.145 & 99.87 \\
\hline $03 / 29 / 2007$ & $00+56$ & 0.096 & 0.158 & 99.80 \\
\hline $03 / 29 / 2007$ & 00,57 & 0.095 & 0.198 & 99.84 \\
\hline $03 / 29 / 2007$ & $00: 58$ & 0.095 & 0.223 & 99.76 \\
\hline $03 / 29 / 2007$ & $00: 59$ & $\mathrm{~N} / \mathrm{A}$ & N/A & 99.60 \\
\hline $03 / 29 / 2007$ & $01: 00$ & $N / A$ & N/A & 99.92 \\
\hline $03 / 29 / 2007$ & $02: 01$ & $\mathrm{~N} / \mathrm{A}$ & $\mathrm{N} / \mathrm{A}$ & 100.04 \\
\hline $03 / 29 / 2007$ & 01.02 & $\mathrm{~N} / \mathrm{A}$ & N/A & 99.48 \\
\hline $03 / 29 / 2007$ & $01: 03$ & 0.088 & 0.149 & 98.88 \\
\hline $03 / 29 / 2007$ & $01: 04$ & 0.086 & 0.152 & 99.26 \\
\hline $03 / 29 / 2007$ & $01: 05$ & 0.084 & 0.138 & 99.23 \\
\hline $03 / 29 / 2007$ & $01: 06$ & 0.083 & 0.122 & 99.22 \\
\hline $03 / 29 / 2007$ & $01: 07$ & 0.083 & 0.221 & 98.89 \\
\hline $03 / 29 / 2007$ & $01: 08$ & 0.083 & 0.113 & 98,87 \\
\hline $03 / 29 / 2007$ & $01: 09$ & 0.080 & 0.078 & 98.84 \\
\hline $03 / 29 / 2007$ & $01: 10$ & 0.078 & 0.046 & 98.83 \\
\hline $03 / 29 / 2007$ & $01: 11$ & 0.079 & 0.077 & 99.23 \\
\hline $03 / 29 / 2007$ & $01: 12$ & 0.081 & 0.070 & 99.38 \\
\hline $03 / 29 / 2007$ & $01: 13$ & 0.082 & 0.052 & 99.34 \\
\hline $03 / 29 / 2007$ & $01: 14$ & 0.084 & 0.049 & 99.14 \\
\hline $03 / 29 / 2007$ & $01: 15$ & 0.086 & 0.066 & 99.01 \\
\hline $03 / 29 / 2007$ & $01: 16$ & 0.087 & 0.077 & 99.61 \\
\hline $03 / 29 / 2007$ & 01,17 & 0.090 & 0.077 & 99.87 \\
\hline $03 / 29 / 2007$ & 01,18 & 0.092 & 0.066 & 99.76 \\
\hline $03 / 29 / 2007$ & 01.19 & 0.094 & 0.116 & 99.93 \\
\hline $03 / 29 / 2007$ & 01,20 & 0.097 & 0.084 & 100.00 \\
\hline $03 / 29 / 2007$ & $01: 21$ & 0.098 & 0.106 & 99.98 \\
\hline $03 / 29 / 2007$ & $01: 22$ & 0.095 & 0.075 & 99.69 \\
\hline $03 / 29 / 2007$ & $01: 23$ & 0.094 & 0.052 & 99.68 \\
\hline $03 / 29 / 2007$ & $01: 24$ & 0.092 & 0.070 & 99.42 \\
\hline $03 / 29 / 2007$ & 01.25 & 0.091 & 0.074 & 99.10 \\
\hline $03 / 29 / 2007$ & $01: 26$ & 0.089 & 0.085 & 98.92 \\
\hline $03 / 29 / 2007$ & $01: 27$ & 0.087 & 0.104 & 98.88 \\
\hline $03 / 29 / 2007$ & $01: 28$ & 0.087 & 0.098 & 99.31 \\
\hline $03 / 29 / 2007$ & $01: 29$ & 0.088 & 0.073 & 99.48 \\
\hline
\end{tabular}




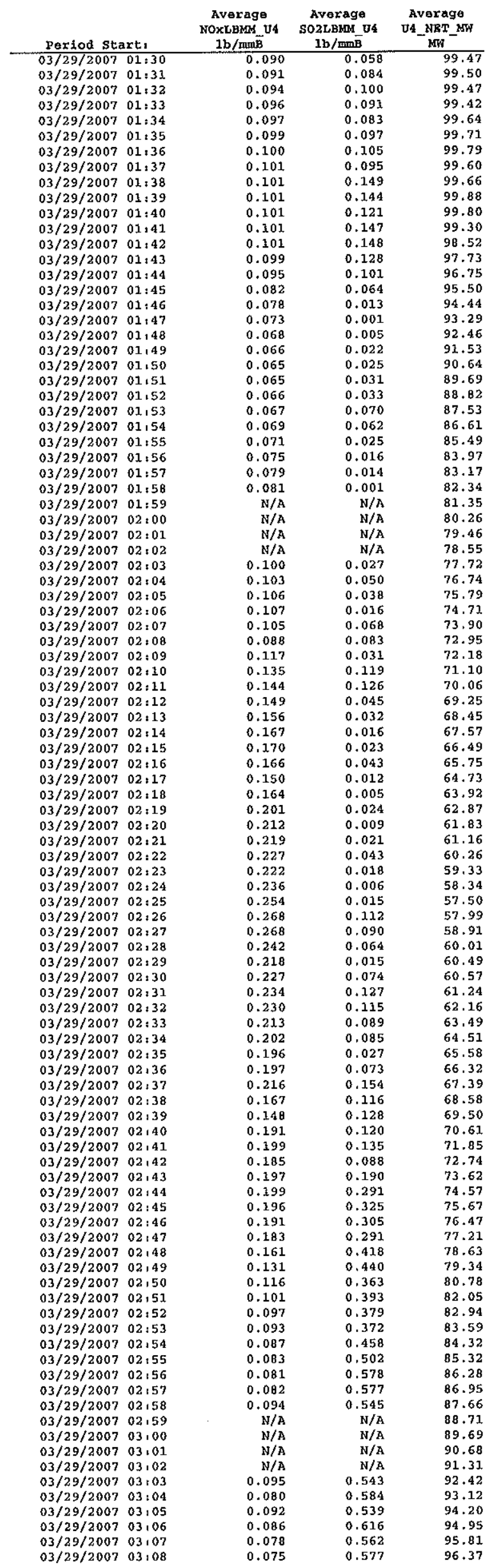




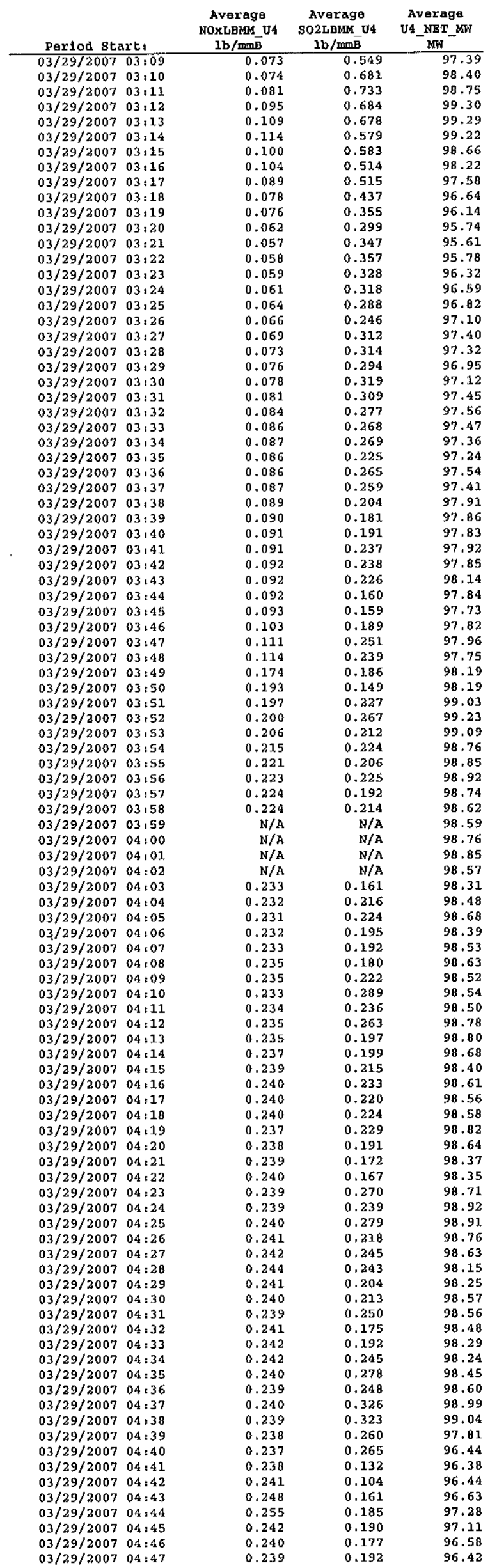




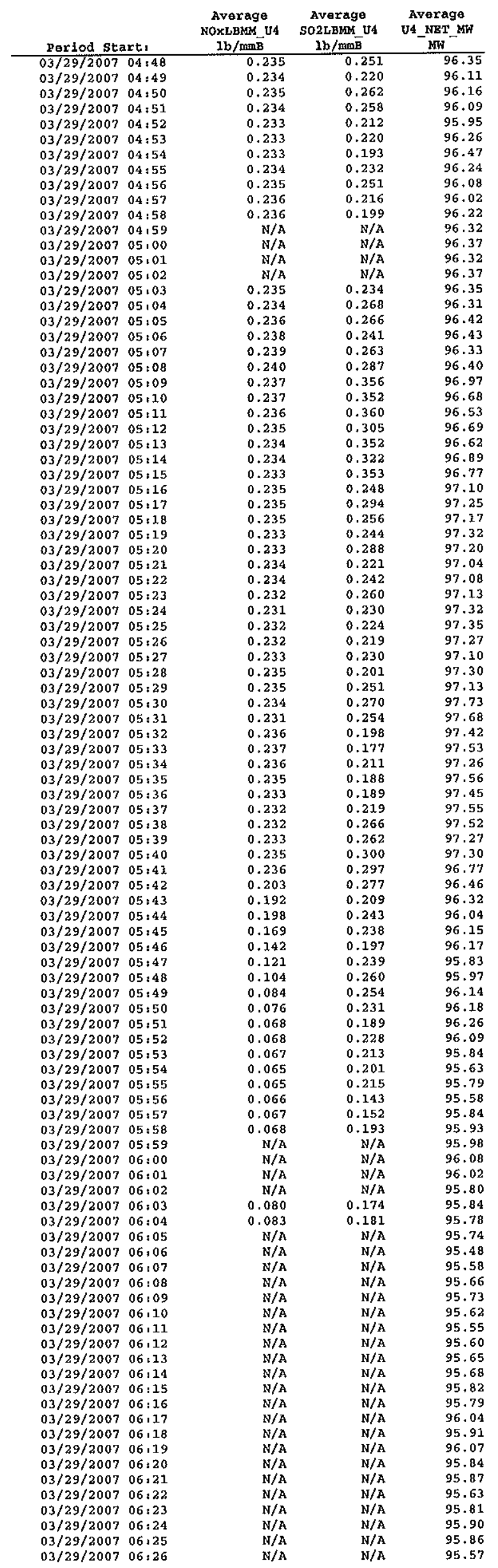




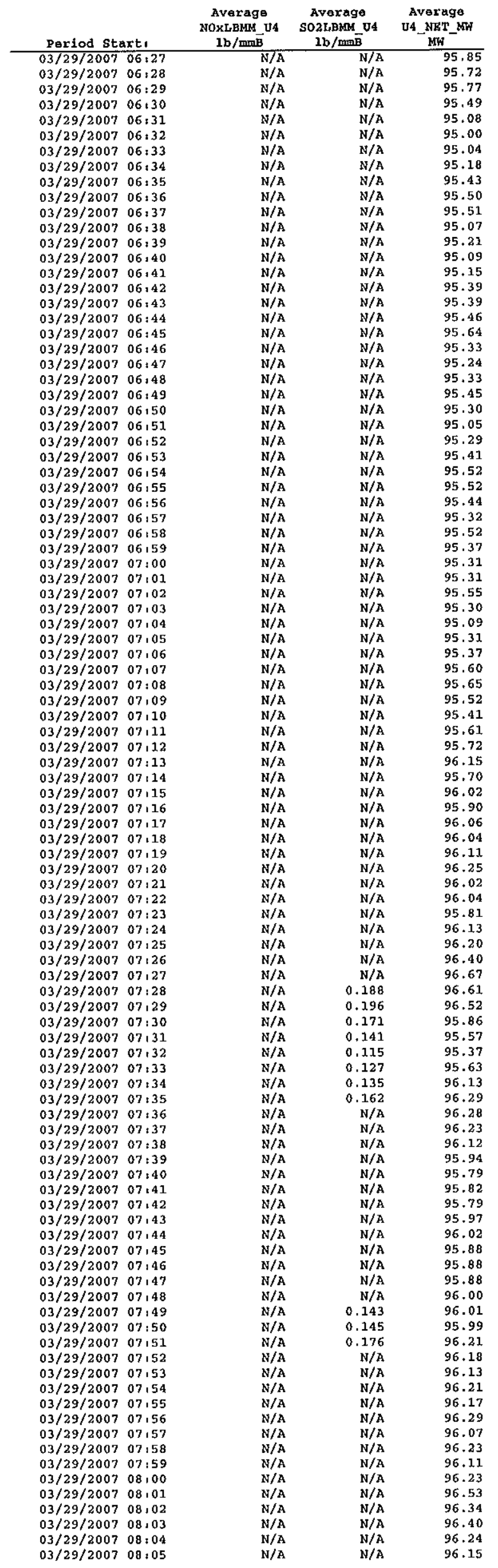




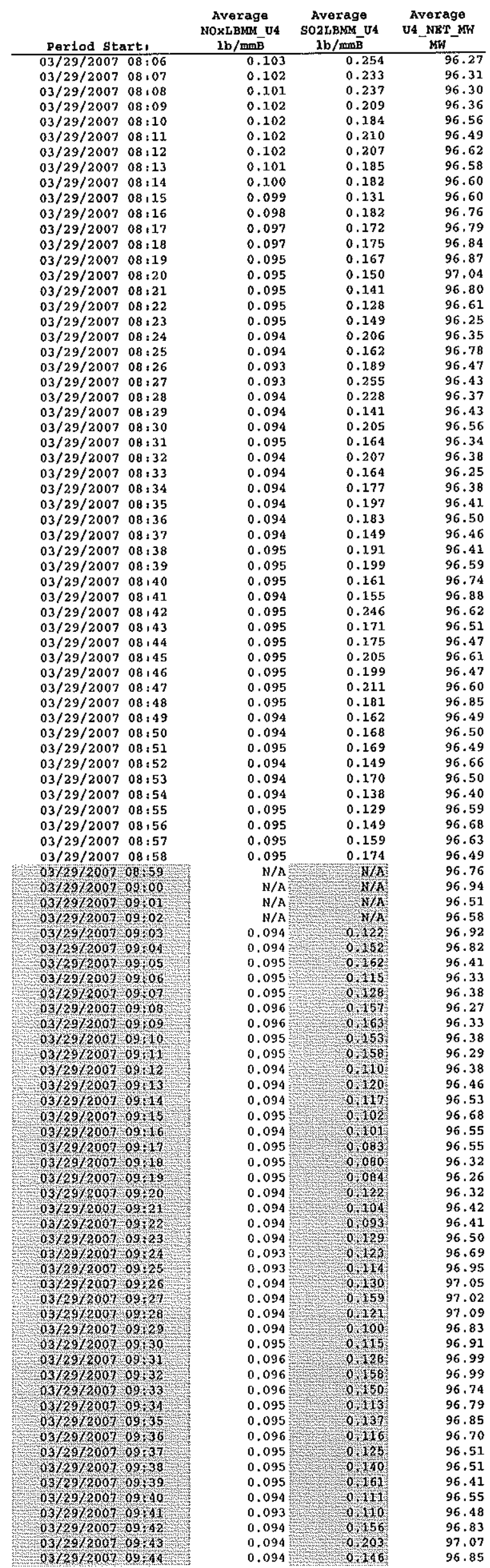




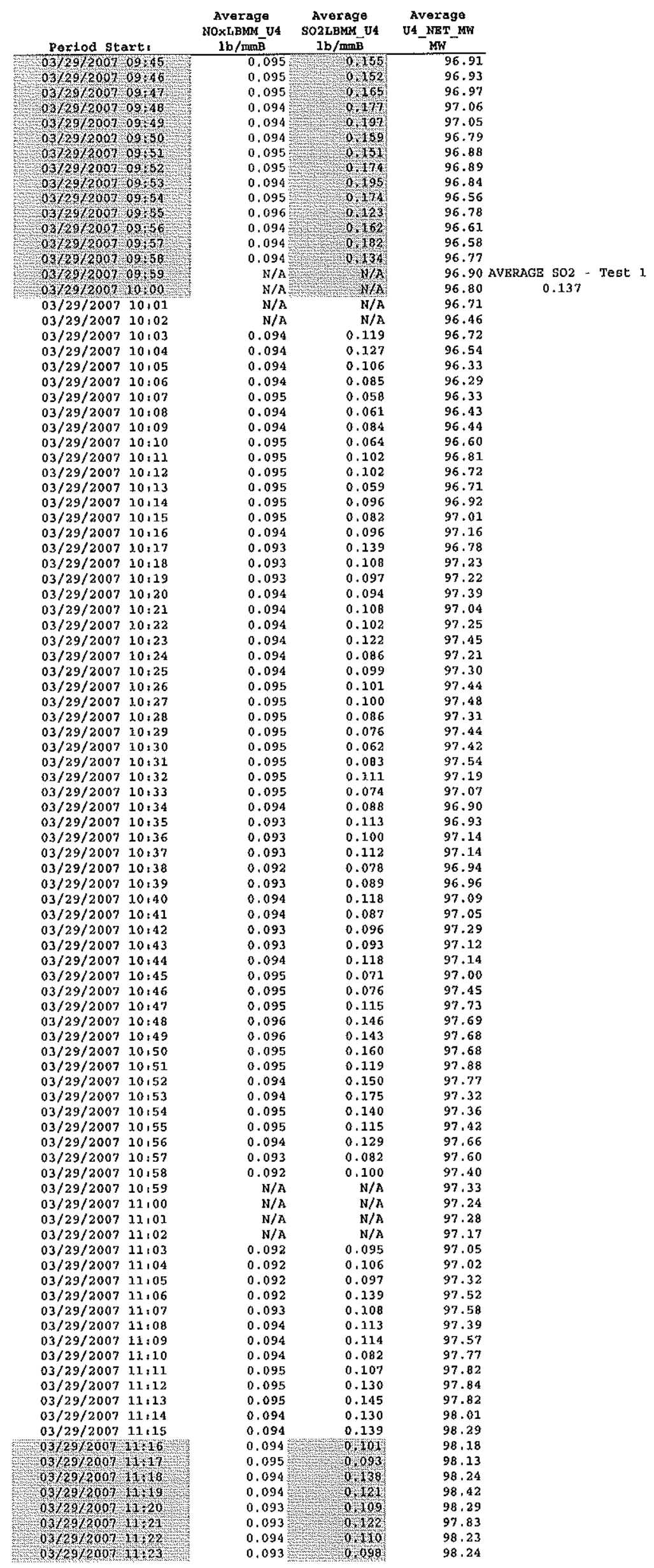




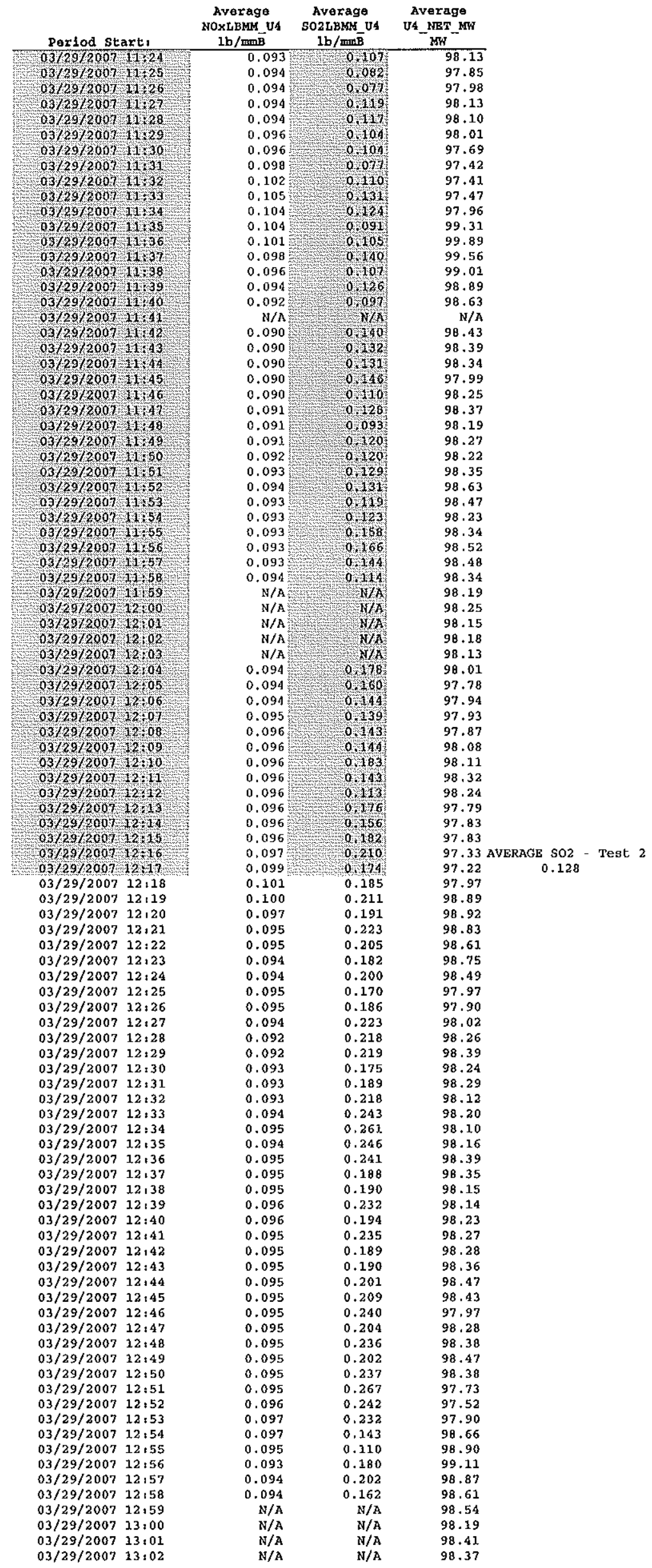




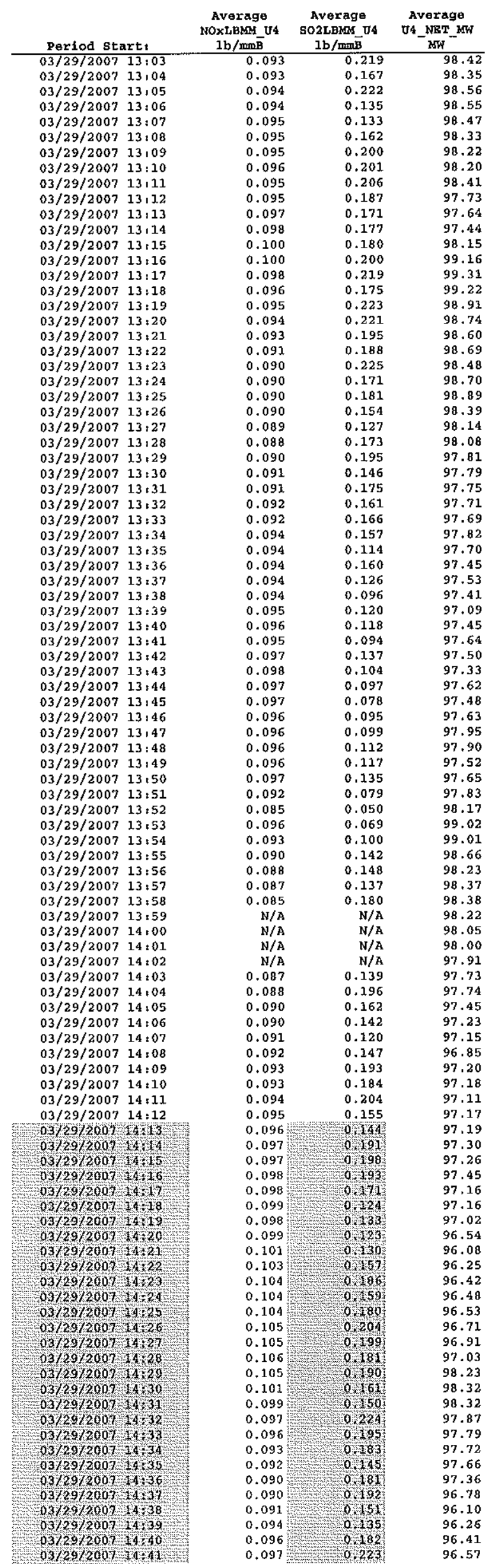




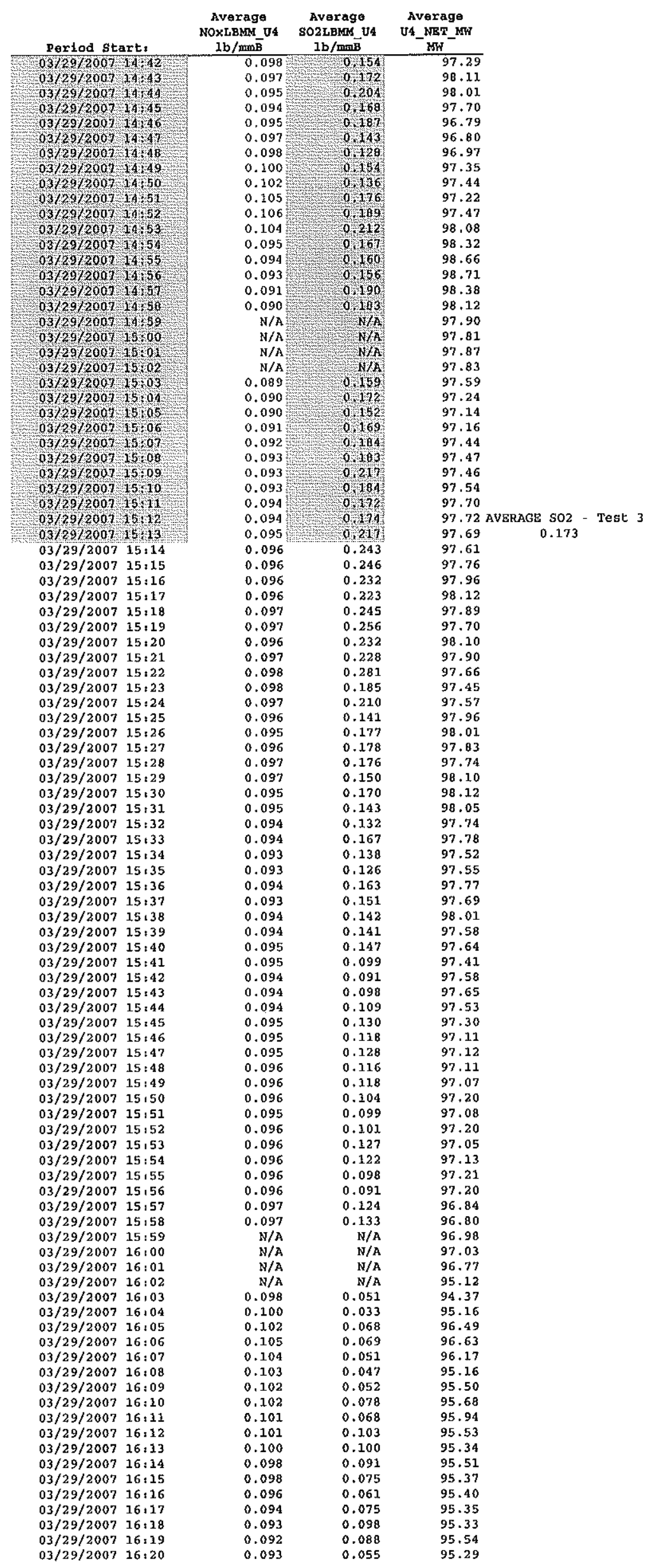




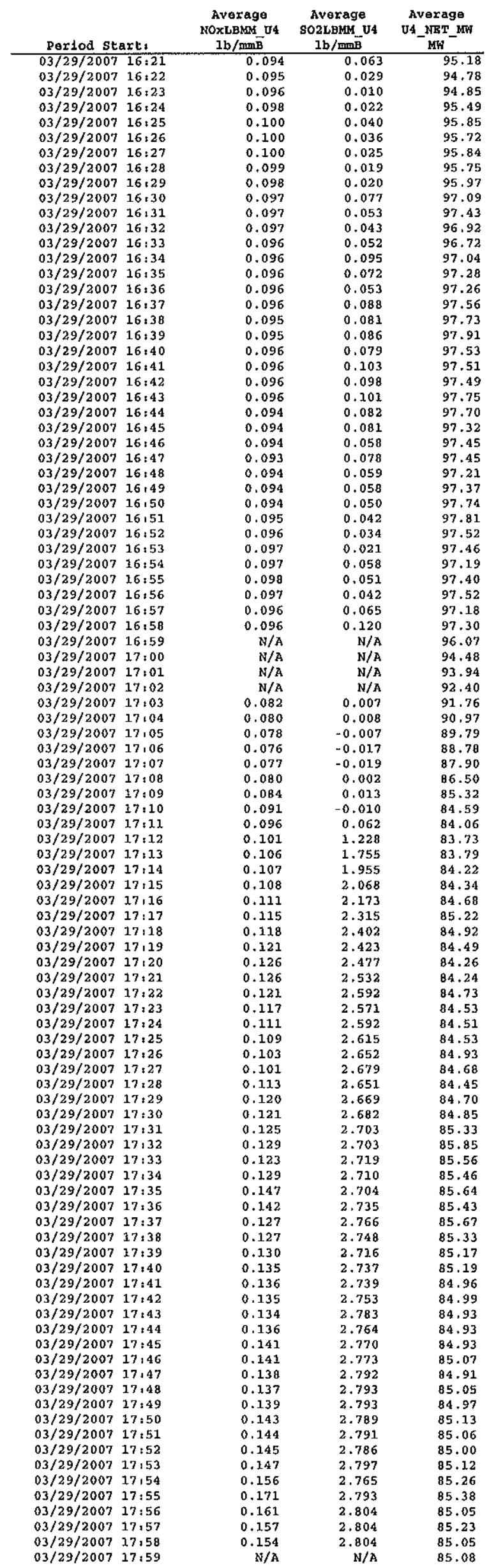




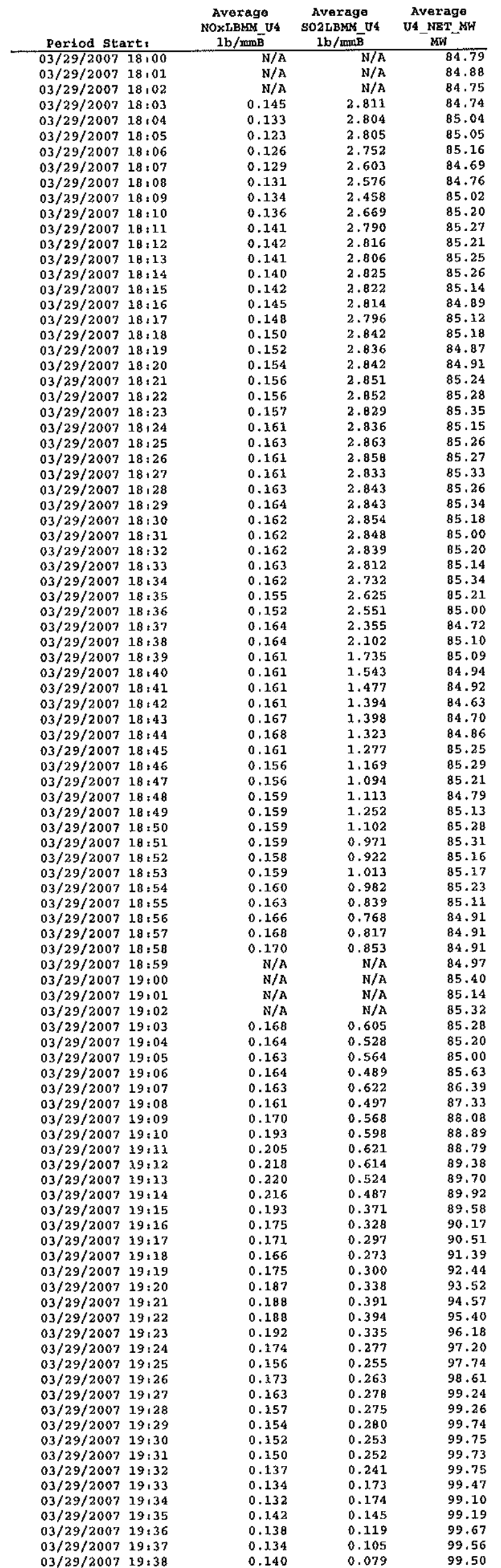




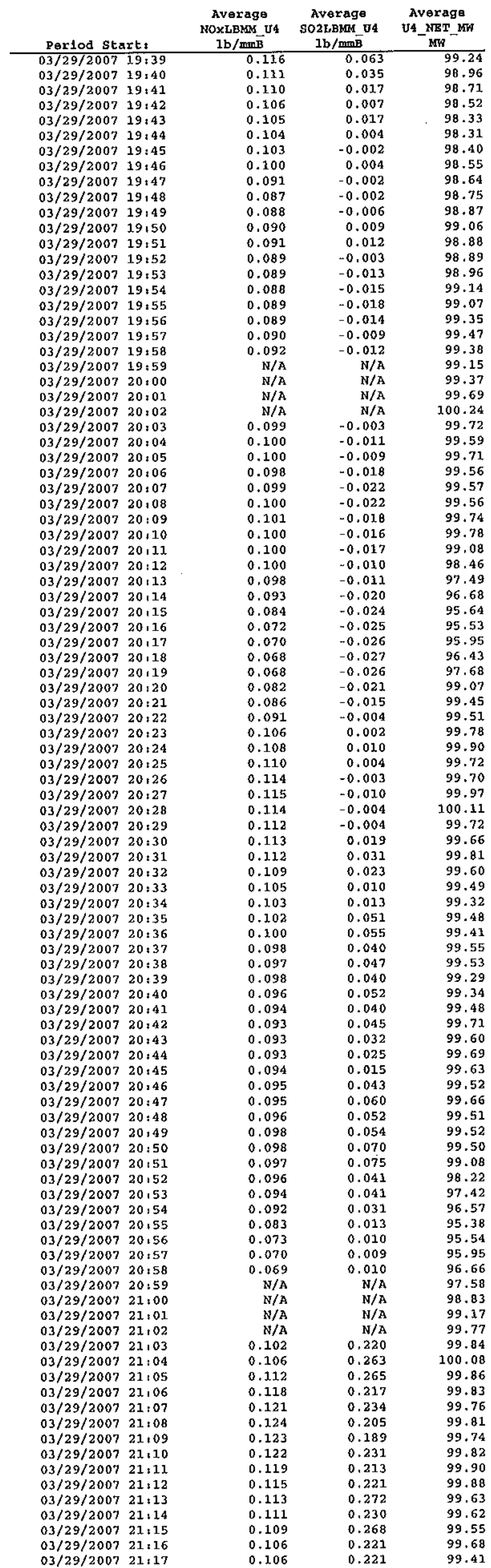




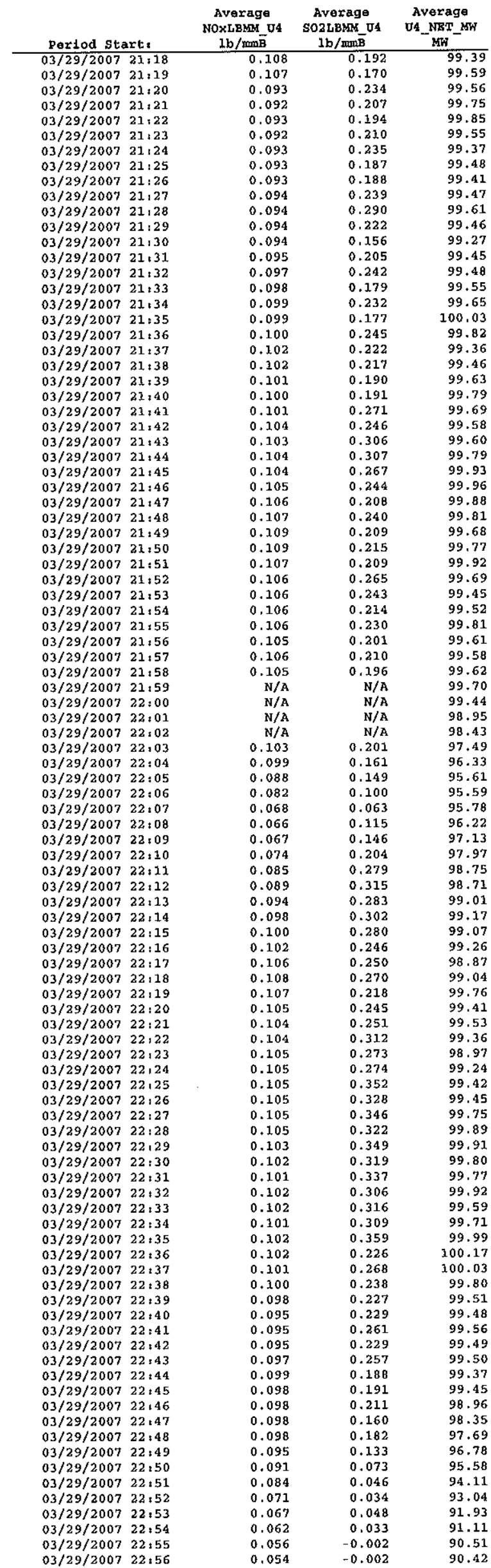




\begin{tabular}{|c|c|c|c|c|}
\hline Pariod St & $2 \times t_{2}$ & $\begin{array}{l}\text { Average } \\
\text { NOxLBMR_U4 } \\
1 \mathrm{~b} / \operatorname{manB}\end{array}$ & $\begin{array}{l}\text { Average } \\
\text { SO2LBMA UA } \\
\text { lb } / \mathrm{mmB}\end{array}$ & $\begin{array}{c}\text { Average } \\
\text { U4_NET_NW } \\
\text { NWW }^{\text {Nat }}\end{array}$ \\
\hline $03 / 29 / 2007$ & $22: 57$ & 0.054 & 0.007 & 90.97 \\
\hline $03 / 29 / 2007$ & $22: 58$ & 0.055 & 0.012 & 92.04 \\
\hline $03 / 29 / 2007$ & $22: 59$ & $\mathrm{~N} / \mathrm{A}$ & $\mathrm{N} / \mathrm{A}$ & 93.07 \\
\hline $03 / 29 / 2007$ & $23: 00$ & N/A & $\mathrm{N} / \mathrm{A}$ & 93.93 \\
\hline $03 / 29 / 2007$ & $23: 01$ & $\mathrm{~N} / \mathrm{A}$ & $\mathrm{N} / \mathrm{A}$ & 94.78 \\
\hline $03 / 29 / 2007$ & $23: 02$ & $\mathrm{~N} / \mathrm{A}$ & $\mathrm{N} / \mathrm{A}$ & 95.54 \\
\hline $03 / 29 / 2007$ & 23,03 & 0.103 & 0.171 & 96.67 \\
\hline $03 / 29 / 2007$ & 23,04 & 0.118 & 0.288 & 97,93 \\
\hline $03 / 29 / 2007$ & $23: 05$ & 0.121 & 0,287 & 98.74 \\
\hline $03 / 29 / 2007$ & 23,06 & 0.139 & 0.368 & 99.25 \\
\hline $03 / 29 / 2007$ & 23,07 & 0.143 & 0.366 & 99,73 \\
\hline $03 / 29 / 2007$ & 23,08 & 0.145 & 0.434 & 100.48 \\
\hline $03 / 29 / 2007$ & $23: 09$ & 0.150 & 0.396 & 100.91 \\
\hline $03 / 29 / 2007$ & $23: 10$ & 0.143 & 0.408 & 100.81 \\
\hline $03 / 29 / 2007$ & $23: 11$ & 0.130 & 0.327 & 100.45 \\
\hline $03 / 29 / 2007$ & $23: 12$ & 0.117 & 0.283 & 100.38 \\
\hline $03 / 29 / 2007$ & $23: 13$ & 0.107 & 0.292 & 100.17 \\
\hline $03 / 29 / 2007$ & $23: 14$ & 0.105 & 0.310 & 100.42 \\
\hline $03 / 29 / 2007$ & $23: 15$ & 0.100 & 0.343 & 100.09 \\
\hline $03 / 29 / 2007$ & 23,16 & 0.096 & 0.302 & 99.59 \\
\hline $03 / 29 / 2007$ & $23: 17$ & 0.093 & 0.280 & 99.00 \\
\hline $03 / 29 / 2007$ & $23: 18$ & 0.087 & 0.202 & 98.08 \\
\hline $03 / 29 / 2007$ & 23,29 & 0.082 & 0.162 & 97.04 \\
\hline $03 / 29 / 2007$ & $23: 20$ & 0.069 & 0.106 & 95.58 \\
\hline $03 / 29 / 2007$ & 23,21 & 0.062 & 0.052 & 94.30 \\
\hline $03 / 29 / 2007$ & 23,22 & 0.059 & 0.041 & 93.13 \\
\hline $03 / 29 / 2007$ & $23: 23$ & 0.056 & 0.052 & 92.16 \\
\hline $03 / 29 / 2007$ & $23: 24$ & 0.055 & 0.049 & 91.42 \\
\hline $03 / 29 / 2007$ & $23: 25$ & 0.056 & 0.046 & 90.61 \\
\hline $03 / 29 / 2007$ & $23: 26$ & 0.057 & 0.019 & 89.51 \\
\hline $03 / 29 / 2007$ & $23: 27$ & 0.058 & 0.048 & 88.59 \\
\hline $03 / 29 / 2007$ & $23: 28$ & 0.060 & 0.068 & 87.31 \\
\hline $03 / 29 / 2007$ & 23,29 & 0.062 & 0.034 & 86.57 \\
\hline $03 / 29 / 2007$ & 23,30 & 0.064 & 0.005 & 85.37 \\
\hline $03 / 29 / 2007$ & 23,31 & 0.068 & 0.012 & 84.30 \\
\hline $03 / 29 / 2007$ & 23,32 & 0.072 & 0.036 & 83.13 \\
\hline $03 / 29 / 2007$ & 23,33 & 0.075 & 0.006 & 82.33 \\
\hline $03 / 29 / 2007$ & $23: 34$ & 0.078 & 0.033 & 81.29 \\
\hline $03 / 29 / 2007$ & 23,35 & 0.083 & 0.076 & 80.52 \\
\hline $03 / 29 / 2007$ & 23,36 & 0.086 & 0.052 & 79.63 \\
\hline $03 / 29 / 2007$ & $23: 37$ & 0.088 & 0.025 & 78.69 \\
\hline $03 / 29 / 2007$ & 23,38 & 0.089 & 0.004 & 77.63 \\
\hline $03 / 29 / 2007$ & 23,39 & 0.091 & -0.013 & 76.61 \\
\hline $03 / 29 / 2007$ & $23: 40$ & 0.092 & 0.064 & 75.49 \\
\hline $03 / 29 / 2007$ & $23: 41$ & 0.077 & 0.036 & 74.87 \\
\hline $03 / 29 / 2007$ & 23,42 & 0.106 & -0.002 & 74.08 \\
\hline $03 / 29 / 2007$ & 23,43 & 0.125 & -0.006 & 73.74 \\
\hline $03 / 29 / 2007$ & 23,44 & 0.136 & 0.015 & 74.22 \\
\hline $03 / 29 / 2007$ & 23,45 & 0.145 & 0.047 & 74.08 \\
\hline $03 / 29 / 2007$ & $23: 46$ & 0.146 & 0.060 & 74.92 \\
\hline $03 / 29 / 2007$ & $23: 47$ & 0.148 & 0.049 & 75.57 \\
\hline $03 / 29 / 2007$ & $23: 48$ & 0.154 & 0.056 & 76.62 \\
\hline $03 / 29 / 2007$ & $23: 49$ & 0.154 & 0.154 & 77.62 \\
\hline $03 / 29 / 2007$ & 23,50 & 0.153 & 0.196 & 78.76 \\
\hline $03 / 29 / 2007$ & $23: 51$ & 0.152 & 0.182 & 80.03 \\
\hline $03 / 29 / 2007$ & $23: 52$ & 0.153 & 0.152 & 81,42 \\
\hline $03 / 29 / 2007$ & $23: 53$ & 0.150 & 0.134 & 82,17 \\
\hline $03 / 29 / 2007$ & $23: 54$ & 0.128 & 0.276 & 83.31 \\
\hline $03 / 29 / 2007$ & $23: 55$ & 0.117 & 0.303 & 84.37 \\
\hline $03 / 29 / 2007$ & $23: 56$ & 0.103 & 0.252 & 85.24 \\
\hline $03 / 29 / 2007$ & $23: 57$ & 0.099 & 0.335 & 86.55 \\
\hline $03 / 29 / 2007$ & $23: 58$ & 0.098 & 0.472 & 87.07 \\
\hline $03 / 29 / 2007$ & $23: 59$ & $\mathrm{~N} / \mathrm{A}$ & $\mathrm{N} / \mathrm{A}$ & 87.72 \\
\hline $03 / 30 / 2007$ & $00: 00$ & N/A & $N / A$ & 88.62 \\
\hline \multirow[t]{7}{*}{ Dally } & Average* & 0.116 & 0.351 & 94.87 \\
\hline & Maximun* & 0.268 & 2.863 & 100.91 \\
\hline & & $03 / 29 / 2007$ & $03 / 29 / 2007$ & $03 / 29 / 2007$ \\
\hline & & $2: 27$ & $18: 25$ & $23: 09$ \\
\hline & Minimum* & 0.054 & -0.027 & 57.50 \\
\hline & & $03 / 29 / 2007$ & $03 / 29 / 2007$ & $03 / 29 / 2007$ \\
\hline & & $22: 57$ & $20: 18$ & 2,25 \\
\hline \multirow[t]{7}{*}{ Final } & Average * & 0.136 & 0.274 & 93.96 \\
\hline & Maximum* & 0.575 & 2.863 & 100.91 \\
\hline & & $03 / 28 / 2007$ & $03 / 29 / 2007$ & $03 / 29 / 2007$ \\
\hline & & $1: 16$ & $18: 25$ & 23,09 \\
\hline & Min1mim* & 0.054 & -0.027 & 47.55 \\
\hline & & $9 / 2007$ & $03 / 29 / 2007$ & $03 / 28 / 2007$ \\
\hline & & 22,57 & $20: 18$ & 1.143 \\
\hline
\end{tabular}

* Does not include Invalid Averaging Periods ("N/A") 